
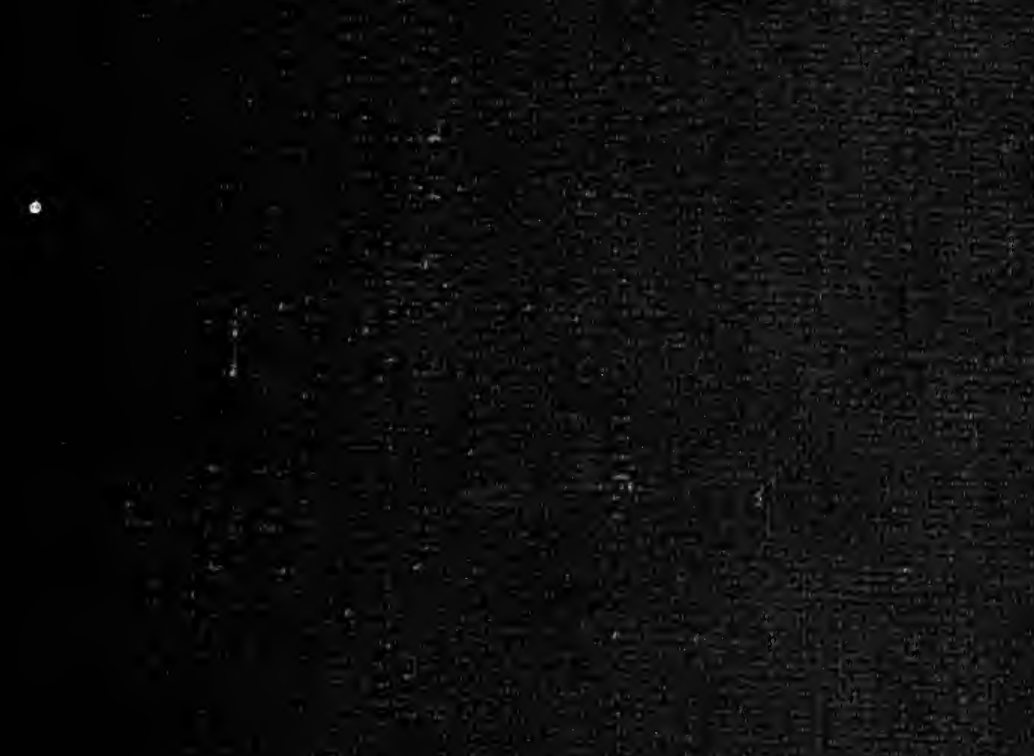


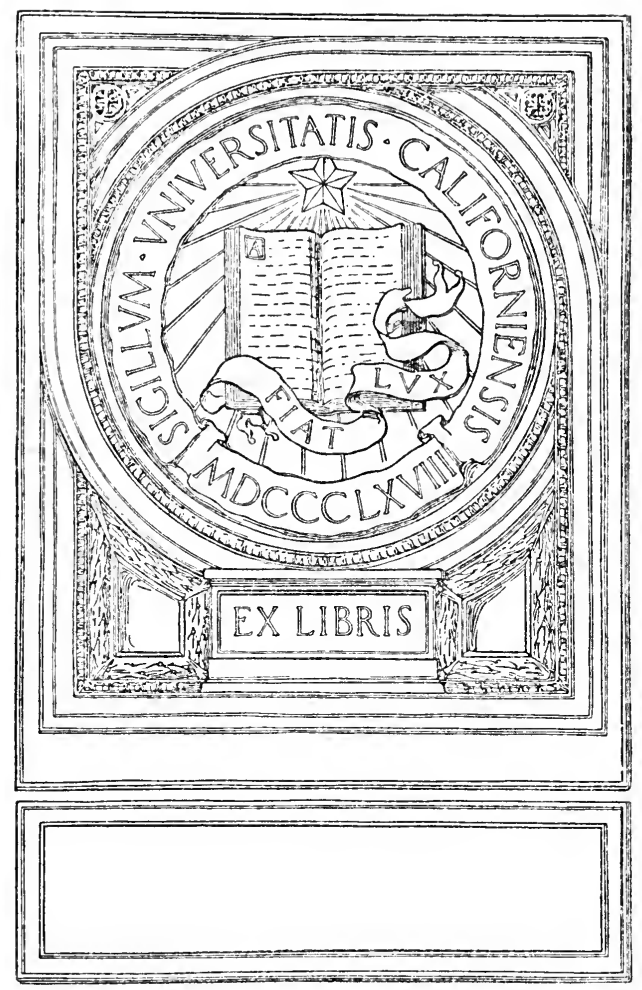


W.

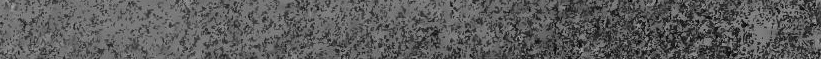

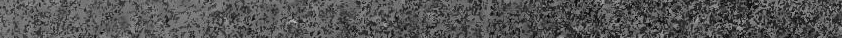

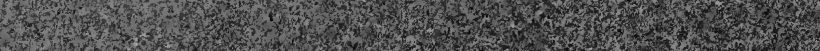

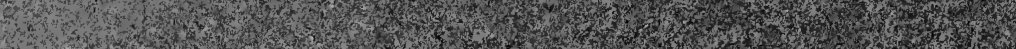
2.1.7.

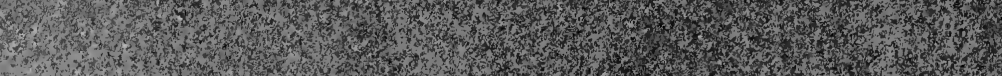

1. W

1.4
7

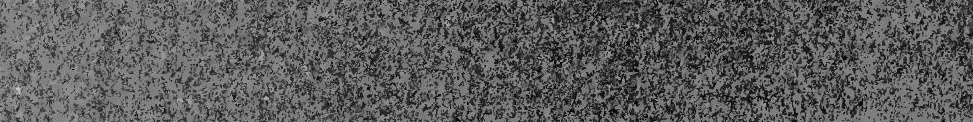

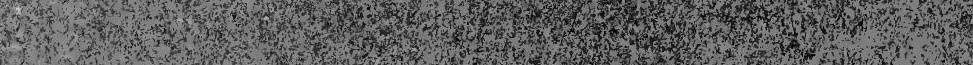

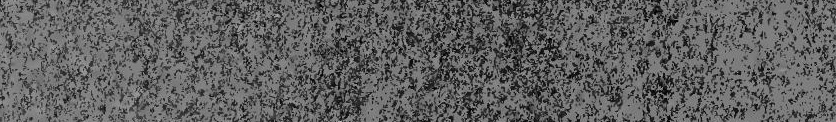

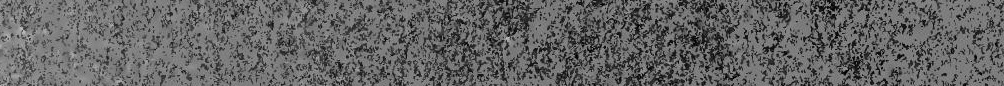

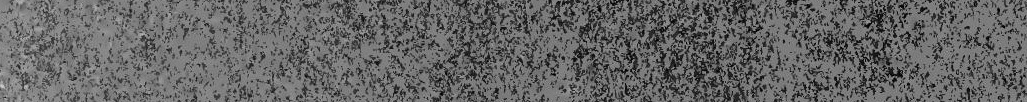

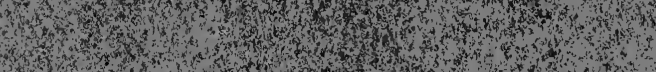

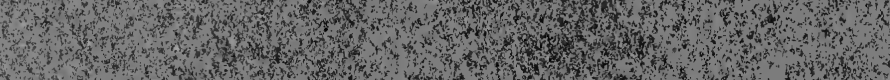

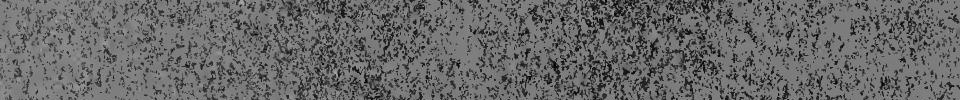

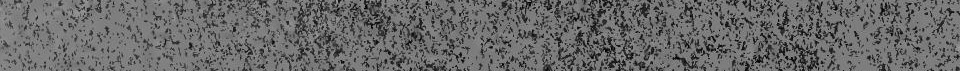

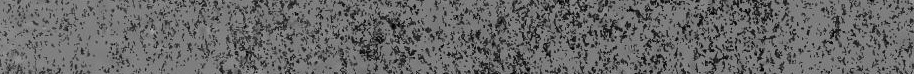

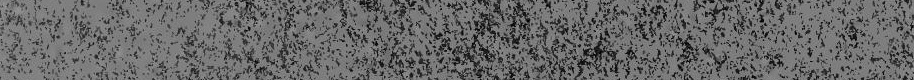

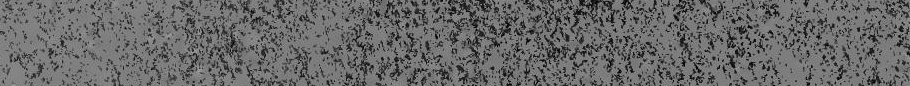

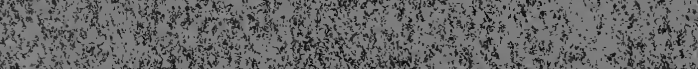

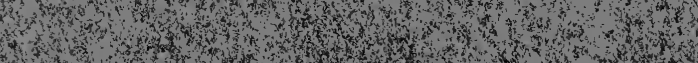

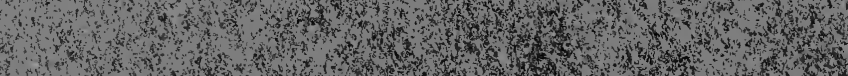

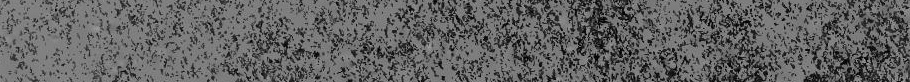

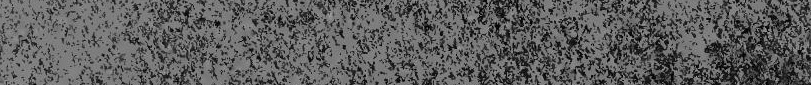

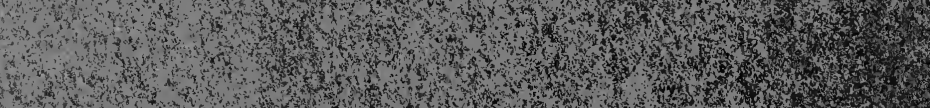

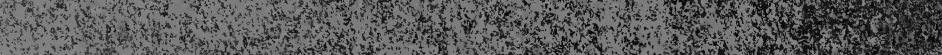

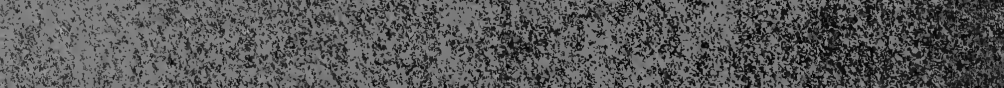

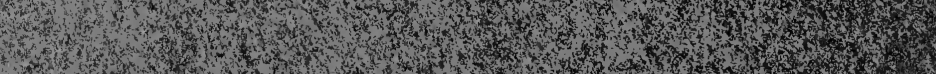
3.t.

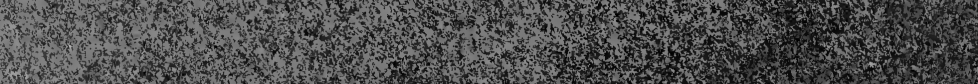

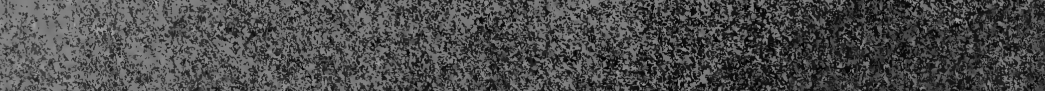

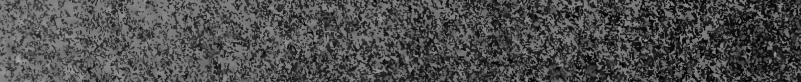

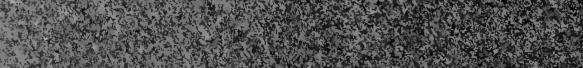

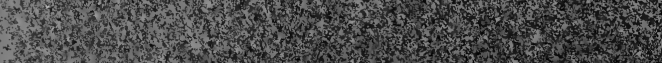
1 





\section{- HYDRAULICS AND}

\section{ITS APPLICATIONS.}

BY

A. H. Gibson, M. Sc. Assoc. Mem. Inst. C.E. SENIOR DEMONSTRATOR AND ASSISTANT LECTURER IN ENGINEERING IN THE UNIVERSITY OF MANCHESTER
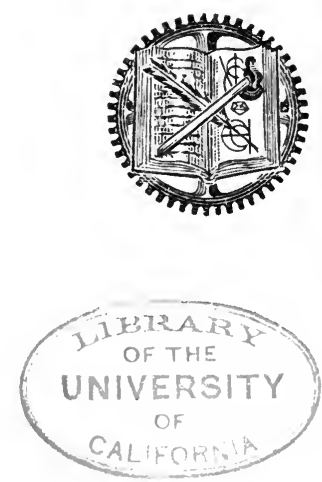

NEW YORK

D. VAN NOSTRAND COMPANY

23 MURRAY AND 27 WARREN STREETS 1908 


$$
\underset{G C 6}{T C 6}
$$

GENERA:

174569 


\section{PREFACE.}

Were water a perfectly non-viscous, inelastic fluid, whose particles, when in motion, always followed sensibly parallel paths, Hydraulics would be one of the most exact of the sciences.

But water satisfies none of these conditions, and the result is that in the majority of cases brought before the engineer, motions and forces of such complexity are introduced as baffle all attempts at a rigorous solution.

This being so, the best that can be done is to discuss each phenomenon on the assumption that the fluid in motion' is perfect, and to modify the results so obtained until they fit the results of experiment, by the introduction of some empirical constant which shall involve the effect of every disregarded factor.

It is worth while here impressing on the student of the science that, apart from these experimentally-deduced constants, his theoretical results are, at the best, only approximations to the truth, and may, if care be not taken in their interpretation, be actually misleading.

On the other hand, it may be well to answer the criticism of those who would cavil at such theoretical treatment, by pointing out that the results so obtained provide the only rational framework on which to erect the more complete structure of Hydraulies.

In the following pages an attempt has been made to consider the science, and its application to the design of Hydraulic 
Machinery, in a manner suitable for a student who has some initial knowledge of mechanics.

While written primarily with the needs of a student in view, it is, however, hoped that the book may prove of value to such as are actively engaged in the practice and profession of Hydraulic Engineering. Although it has not been attempted to elude the largely imaginary difficulties of a mathematical treatment involving some knowledge of the Differential and Integral Calculus, the knowledge of this subject which is necessary for a thorough grasp of the greater part of the book is very slight.

Where, as in Arts. 23, 24, 35 and 62, a somewhat more extended mathematical knowledge is required, the work is such as may safely be left by all but the more advanced student of the subject.

In the section devoted to Hydraulic Machinery, it has not been attempted to deal in any way exhaustively with the subject, and only such machines have been illustrated or described as are typical of their class, represent good modern design, and illustrate some definite principle of construction. For many of these illustrations the Author is indebted to the manufacturers, and while reference has been made to these in the text, he would take this opportunity of thanking them collectively for the help which they have so courteously tendered.

As was essential, reference has been freely made to the minutes of the proceedings of the various English and American societies, and to the English and German technical press, as well as to standard works on the subject. Of these the Author is particularly indebted to the Councils of the Institution of Civil Engineers, the Institution of Mechanical Engineers, the American Society of. Civil Engineers, the Zeitschrift des Vereins deutscher Ingenieure, The Engineer, and Engineering.

The greatest debt of all is, however, owing to the teachings and published papers of his old professor and some time chief, Osborne Reynolds. Old students of the Professor will readily 
recognise to what extent any slight merit which the book may possess is due, directly or indirectly, to the influence of one to whom the science of Hydraulics owes so much.

In conclusion, the Author would tender his thanks to Mr. S. Chapman, to Mr. E. Magson, and to his colleagues, Mr. C. H. Lander and Mr. F. Pickford, of the Manchester University, each of whom has revised a portion of the proois, and to whose lindly criticism and suggestion the book owes much.

\section{A. H. GIBSON.}

Manchester,

Felmary, 190 s. 



\title{
CONTENTS.
}

\author{
SECTION I. \\ Physical Properties of Water-Hydrostatics.
}

\section{CHAP'TER I.}

ART.

1. Historical Resumé

2. Physical Properties of Water.

3. Cohesion; Adhesion; Capillarity ; Surface Tension

4. Viscosity.

\section{CHAPTER II.}

5. Hydrostatics

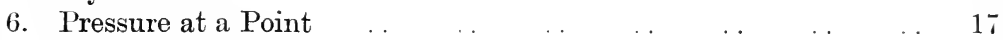

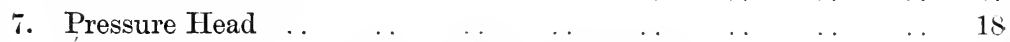

8. Transmissibility of Pressure-Hydraulic Press $\quad \ldots \quad$. . $\quad$..

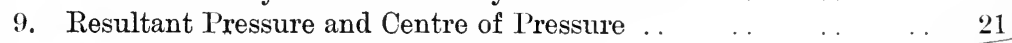

10. Masonry Dams ..

11. Equilibrium of Floating Bodies

12. Oscillations of Ships

13. Strength of Pipes and Cylinders

\section{SECTION II.}

\section{Hydraulics.}

\section{CHAPTER III}

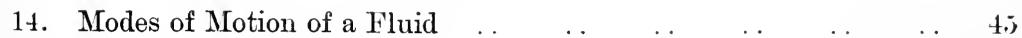

15. Vortices ..

16. Conditions Regulating the Two Modes of Motion _. $\quad \ldots \quad$. 49

17. Critical Velocity. $\quad \begin{array}{lllllllll} & \ldots & \ldots & \ldots & \ldots & \ldots & \ldots & \ldots & 51\end{array}$

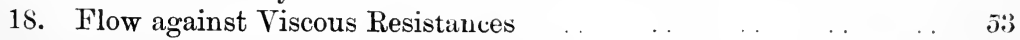

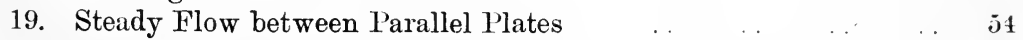

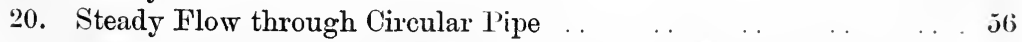

21. Steady Flow through Circular Pipe, assuming slip at Boundaries 58 
CHAPTER IV.

AKT.

22. Motion of Fluids

23. Equations of Motion for a Viscous Fluid

24. Application to Stream Line Motion ..

25. Effect of Heat Motion . .

26. Bernouilli's Theorem

27. Elementary Proof of Bernoulli's Theorem .

28. Application to Unsteady Motion

29. Experimental Verification of Bernoulli's Theorem . .

30. Venturi Meter .

31. Sudden Enlargement of Section

32. Sudden Contraction of Area

33. Initiation and Stoppage of Motion

34. Flow in Converging Passages-Radial Flow

35. Change of Pressure across a Stream Tube ..

36. Vortex Motion-Forced, Free and Compound Vortices

\section{CHAPTER V.}

37. Flow from a Small Orifice

38. Determination of Coefficients of Velocity, Discharge, and Contraction

39. Equation of Momentum

40. Borda's Mouthpiece ..

41.. Orifice in Flat Plate ..

42. Bell-mouthed Orifice .

43. Velocity of Approach

44. Time of Discharge from Small Orifices

45. Form of Effluent Streams

46. Large Orifices

47. Rectangular Orifice

48. Circular Orifice ..

49. Submerged Orifice

50. Law of Comparison for Orifices

51. Notches and Weirs

52. Theoretical Formula for Flow over a Notch

53. Submerged Weirs

54. Shape of Weir Sill

55. Rise in Surface Level produced by a Dam

56. Use of Weir as a Measuring Appliance

57 . Time of Discharge through Orifices and Notches

\section{CHAPTER VI.}

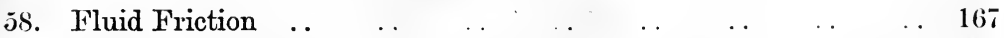

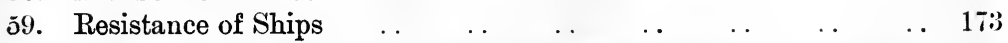

60. Propulsion of Ships - Power Necessary - Screw PropellersPaddle Wheels 
CHAP'TER VII.

ART.

61. Pipe Flow

PAGE

62. Critical Velocity.. $\quad \ldots \quad$.

63. Formulae for Pipe Flow-Rational and Empirical. .

64. Flow in Rivetted Pipes. .

65. Values of $f$ in Formula, $h=\frac{f l i^{2}}{2 g m}$.

66. Accuracy of Determination of $v$

67. Mean Velocity and Distribution of Velocity in Pipe

68. Measurement of Discharge .. $\quad \ldots \quad \ldots \quad$..

69. Relation between Diameter and Discharge ..

70. Water Hammer in Pipes of Uniform Bore

\section{CHAPTER VIII.}

71. Pipe Iine Losses-Hydraulic Gradient

72. Losses at Valves-Bends-Elbows-Tees, etc.

73. Flow in Long Pipes

74. Time of Discharge through Long Pipe Line..

75. Equivalent Diameter of Uniform Main

76. Branch Mains ..

77. Multiple Supply. .

78. Flow along a Bye-Pass. .

79. Pipes Coupled in Parallel

so. Flow through Nozzles-Conditions for Maximum Delivery of Energy

\section{CHAPTER IX.}

s3. Flow in an Open Channel

St. Most suitable Form of Channel

85. General Equation of Flow in an Open Channel _. $\ldots \ldots$. .

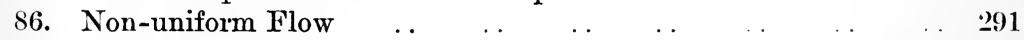

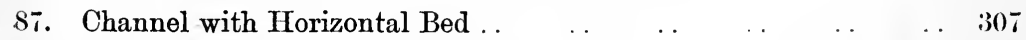

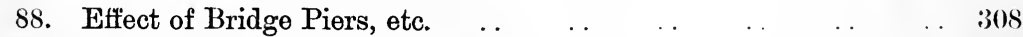

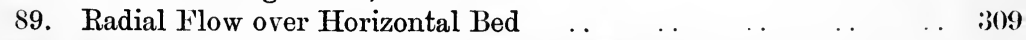

90. Change of Level produced by Passage of Boat through Canal . 310

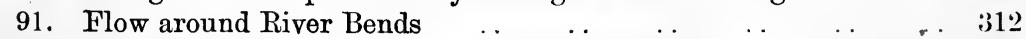

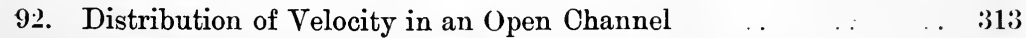

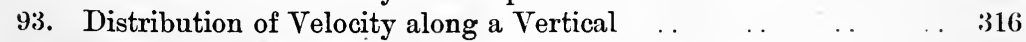

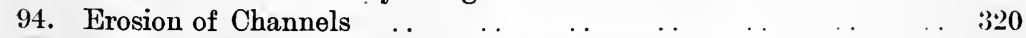

95. Gauging of Flow in Rivers or Open Channels $\quad \ldots \quad \ldots \ldots \ldots$. . 32.2 


\section{CHAPTER X.}

ART.

96. Impact of Jets on Fixed Vanes

97. Actual Force of Impact

98. Distribution of Pressure across a Jet

99. Plate near to Orifice of Nozzle-Ball Nozzle

100. Impact on Moving Vanes

101. Graphical Construction for Pressure on Vane and for Centre of Pressure

10.2. Compounding of Jets .

103. Jet Propulsion ..

104. Hydraulic Mining

105. Resistance to Motion of Submerged Planes

106. Rudder Action . .

10\%. Resistance to Motion of Submerged Bodies

\section{SECTION III.}

\section{Hydraulic Machinfiy.}

\section{CHAPTER XI.}

10S. Iydraulic Prime Movers

109. Utilization of Water Powers .

110. Overshot Water Wheel

111. Breast Wheel . .

112. Sagebien Wheel

113. Undershot Wheel

114. Poncelet Wheel

115. Pelton Wheel - Form - Construction - Form of Buckets-

Number of Buckets-Speed Regulation. .

\section{CHAPTER NII.}

116. Turbines-Classification of ..

117. Impulse 'Turbines

118. Girard and Haenel " Limit" Turbine

119. Pressure Turbines-Barker's Mill ..

120. Borda Turbine ..

121. Fourneyron Turbine ..

12.2. Jonval Turbine

123. Suction Tube . .

124. Francis Turbine

125. Thomson Vortex Turbine

12(i. Compound Turbine

127. American Mixed Flow Turbine

12S. Governing of Turbine Plants-Stand Pipes-Relief Valves-

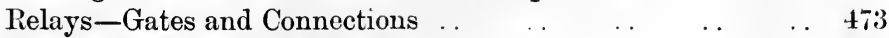

129. I)esign of Head and 'Tail-Races 


\section{CHAPTER XIII.}

130. General Considerations of Turbine Design .

131. $\left\{\begin{array}{l}\text { General Case of Inward Radial Flow Pressure Turbine } \\ \text { Inward Radial Flow Turbine with Radial Vanes-Limitations of } \\ \text { Theory }\end{array}\right\}$

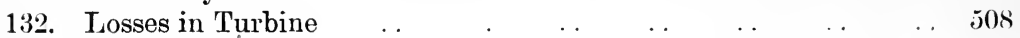

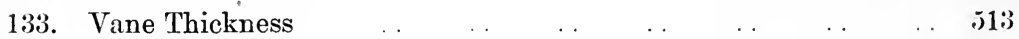

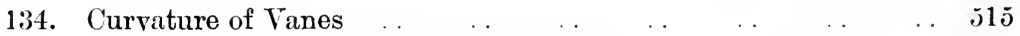

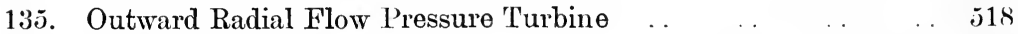

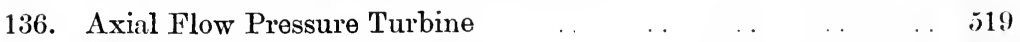

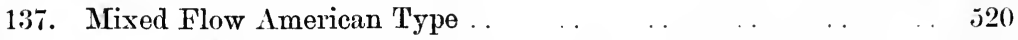

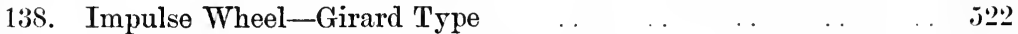

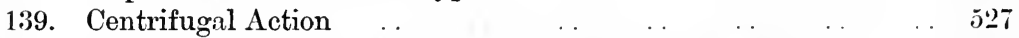

140. General Comparison of Impulse and Pressure Turbines .. . . 5 5

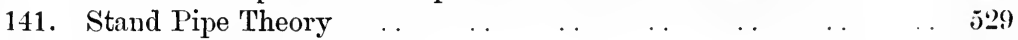

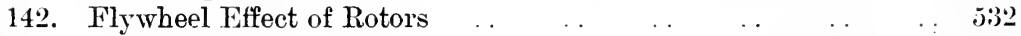

CHAPTER XIV.

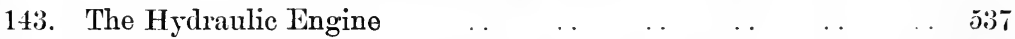

144. Theory of the Hydraulic Engine-Yort Areas _. $\quad \ldots \quad \ldots \quad \ldots \quad j 41$

CHAPTER XV.

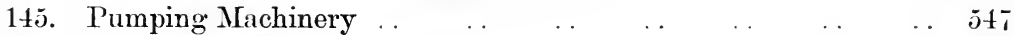

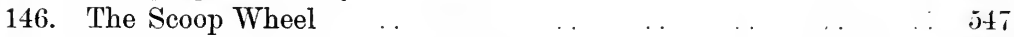

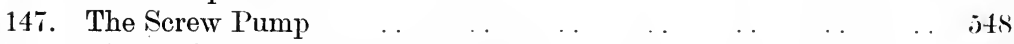

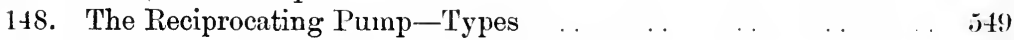

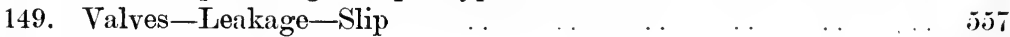

150. Displacement Curves ..

151. Tariation of Pressure in Cylinder-Coefficient of Discharge-

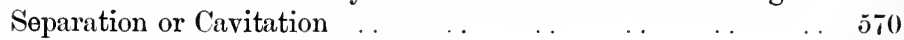

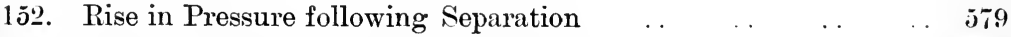

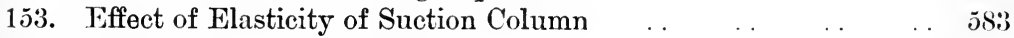

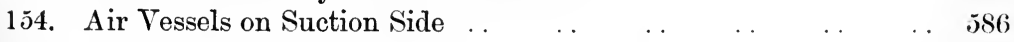

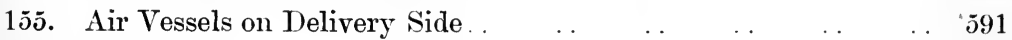

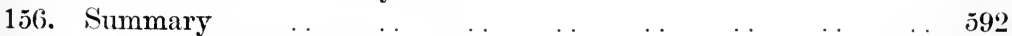

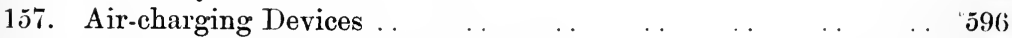

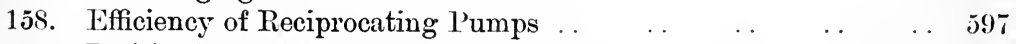

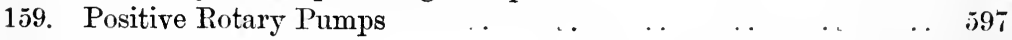

CHAPTER XVI.

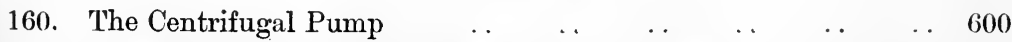

161. Losses in the Centrifugal Pump-Volute Chamber-Vortex or

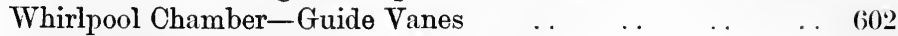

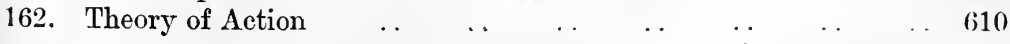


$\triangle \mathrm{RT}$.

163. Pump with Inefficient Collecting Chamber

164. Pump with Whirlpool Chamber .. .

165. Pump with Guide Tanes

166. Most suitable Peripheral Speed in Practice

167. Compound Multiple Chamber High-Lift Pumps .

625

168. Speed at which Pumping commences

626

169. Size of Pump for given Discharge-Similar Pumps

627

170. Suction and Delivery Pipes . .

171. Examples of Design

$6: 30$

172. Types of Pump. .

$6: 34$

173. Balancing of End Thrust on Impeller Shaft

\section{CHAPTER XVII.}

174. Water Hoisting from Mines ..

175. The Hydraulic Ram-Types-Theory-the Pearsall Ram-Ram for Air Compression

176. The Jet Pump ..

177. The Injector Hydrant. .

178. The Air Lift Pump

179. The Hydraulic Air Compressor

\section{CHAPTER XVIII.}

- 180. Hydraulic Power Transmission

181. Accumulators

$\begin{array}{llllll}\ldots & \ldots & \ldots & \ldots & \ldots & 677\end{array}$

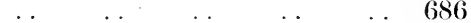

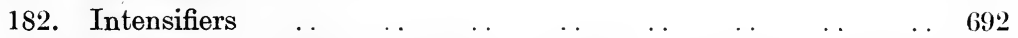

183. Friction of Leather Collars for Rams and Pistons-Hemp

Packings
184. Water Meters

\section{CHAPTER XIX.}

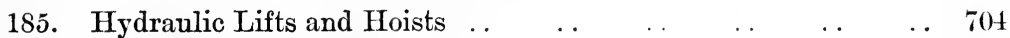

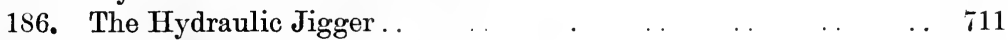

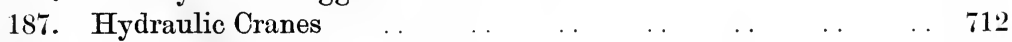

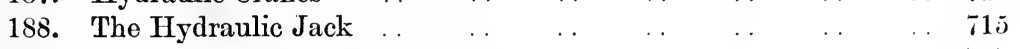

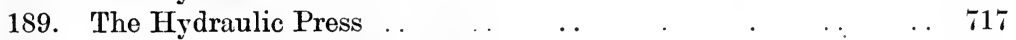

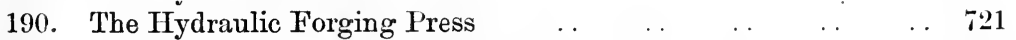

191. Hydraulic Rivetters $\ldots \begin{array}{lllllllll} & \ldots & \ldots & \ldots & \ldots & \ldots & \ldots & 723\end{array}$

192. Hydraulic Brakes and Buffer stops. . $\quad \ldots \quad \ldots \quad \ldots \quad \ldots \quad \ldots 727$

193. Hydraulic Dynamometers . .

$\begin{array}{lllllllllll}\operatorname{APPENDIX} & \ldots & \ldots & \ldots & \ldots & \ldots & \ldots & \ldots & \ldots & \ldots & 745\end{array}$

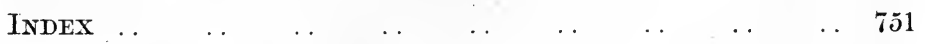




\section{LIST OF SYMBOLS AS GENERALLY ADOPTED THROUGHOUT THE BOOK.}

Where, for any reason, this notation has been lepurted from, special notice is given.

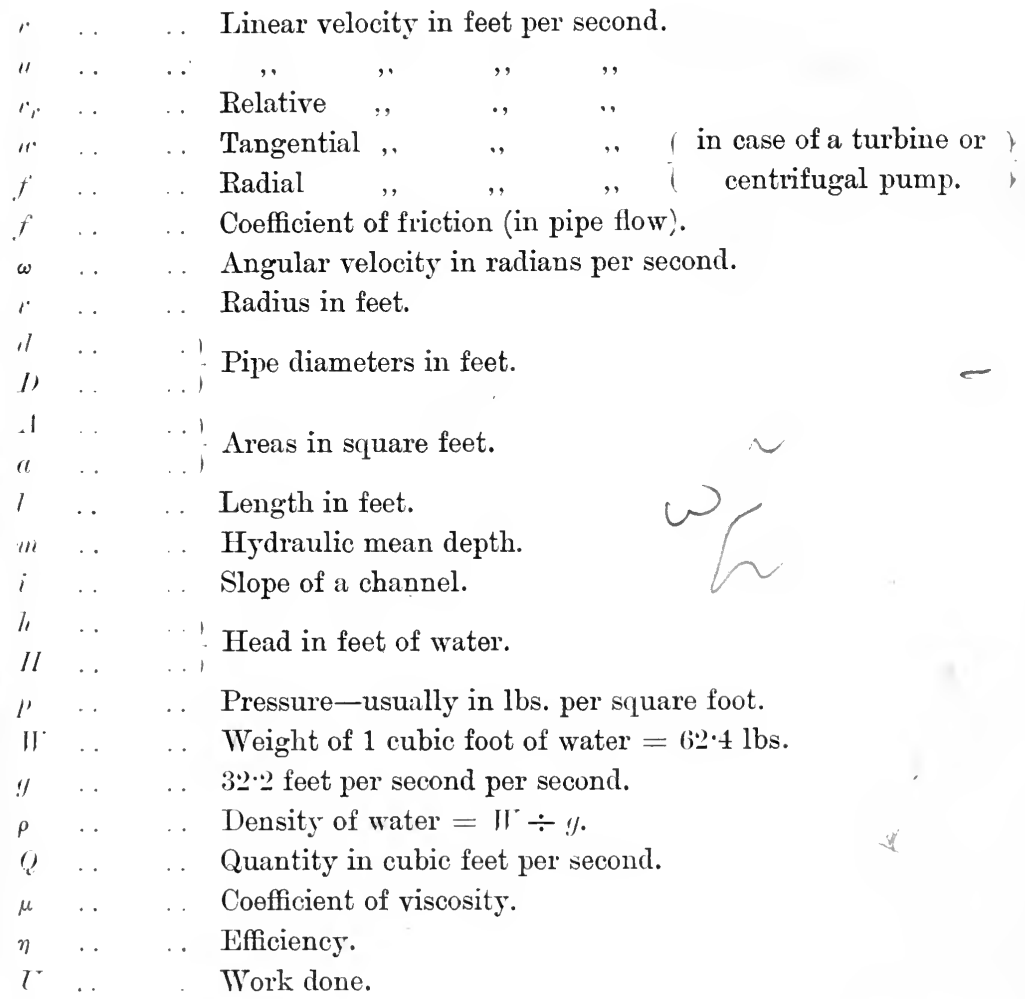




\section{CORRIGENDA.}

Page 14. Footnote. Substitute Proc. Roy. Soc., Vol. 80, 1908, p. 11 .

. 4.). Line $3, p u t$ "three points not in the same straight line " $f\left({ }^{\prime}, "\right.$ two point.."

.. 60. Insert "incompressible" before "fluid," on line 18.

. $\quad 90$. Line $3, p u t-\left(\frac{s}{g} \alpha+A\right) f o r-\left(\frac{s}{g}+A\right)$

.. 195.. Art. 63, line 3. After "Reynolds" insert " and may be obtained."

, 400. On last line put plus sign for minus before $k(r-u) \cos \alpha$.

.. 401. On top line insert the factor $\frac{1}{y}$ before $(v-u)(1-k \cos \alpha)$. 


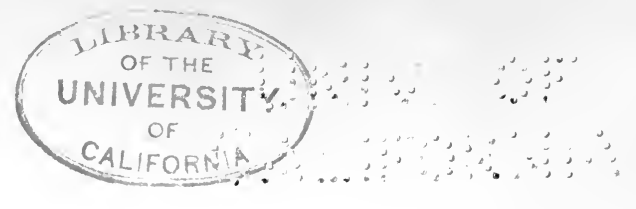

\section{HYDRAULICS AND ITS APPLICATIONS.}

\section{SECTION I.}

\section{CHAPTER I.}

Introductory-Historical Résumé-Physical Properties of Water-Density-Com. pressibility-Cohesion-Adhesion-Capillarity—Surface Tension-Viscosity.

Introductory-Physical Properties of Water.

\section{Art. 1.-Hydronechanics.}

The science which deals with liquids at rest or in motion may be divided into two branches: Hydrostatics, which deals with the equilibrium of liquids at rest; and Hydrodynamics, which deals with the problems connected with their motion. The term Iydraulics is usually broadly applied to that portion of the latter branch which deals with the motion of water in so far as this is of importance in the problems brought directly under the notice of the engineer.

A knowledge of the fundamentals of Hydrostatics is however so essential to a thorough grasp of the principles of Hydrodynamics, and is of such direct importance to the hydraulic engineer, that a treatise on Hydraulics would not be complete without some preliminary treatment of this branch of the subject.

The origin of the science is of great antiquity, and no attempt will be made to give a detailed historical résumé of its growth. Some few of the principles of Hydrostatics were enuneiated by 
Archimedes (в. 250 ) and it is a remarkable fact that for 1,800 years from this date-until the time of Stevinus, Galileo, and Torricelli-practically no further progress was made.

The construction of the elaborate series of aqueducts and of service pipes for supplying Rome with water indeed shows that the Romans possessed some knowledge of the properties of water when at rest and when in motion in pipes and open channels, but we have no record that this knowledge was based on any quantitative laws.

A treatise by Stevinus, written about 1585 , would appear to follow historically that of Archimedes. In this the method of obtaining the pressure of a liquid on the sides and base of a containing vessel was first demonstrated.

Galileo, in a treatise published in 1612, discussed the Hydrostatic Paradox and also the flotation of bodies in water.

Shortly afterwards Torricelli made an important investigation into the behaviour of a jet when issuing vertically from an orifice, while, since the middle of the seventeenth century, numerous investigators have been at work deducing by experimental observation and theoretical reasoning the laws governing the various manners of motion of liquids, and applying these laws to the development of the science of Hydraulics. Of these experimentalists perhaps Mariotte, Bernoulli, and D'Alembert, with Poiseuille, Darcy, and Bazin in France; Rankine, Froude, Osborne Reynolds and James Thomson in England; Eytelwein, Weisbach, and Hagen in Germany; Venturi in Italy, with Francis and Hamilton Smith in America, are most worthy of note.

In spite however of all the work which has been so ably accomplished by these and other observers, Hydraulics cannot yet be classed as an exact science. The laws governing many of its phenomena are still imperfectly understood, and the difficulties - chiefly analytical-- to be overcome before all the disturbing factors can be taken fully into account, are very great. In such cases, experience, based on the results of experiment, forms the only safe guide. In other cases, however, the deductions of theory are found to be perfectly in accord with observed phenomena, and an attempt will be made in the course of this 
work to indicate to what extent our knowledge of the forces controlling any phenomenon is sufficiently accurate and comprehensive to enable theory to be an exact guide, and where, on the other hand, theory, based on insufficient data, is only useful as indicating in what direction a true solution is to be found.

\section{Art. 2.-Physical Properties of Water.}

In its pure state water is an almost colourless, transparent, odourless liquid and one of the best solvents to be found in Nature. Its maximum density occurs at $4^{\circ} \mathrm{C}$., or $39 \cdot 1^{\circ} \mathrm{F}$., and under atmospheric pressure it freezes at $32^{\circ} \mathrm{F}$. and boils at $212^{\circ} \mathrm{F}$. The freezing point is lowered, and the boiling point raised by an increase in pressure, the opposite being true of a reduction of pressure. The specific gravity at maximum density is unity, and the specific heat varies slightly with temperature, increasing from 1.000 at $32^{\circ} \mathrm{F}$. to $1^{\circ} 013$ at $212^{\circ} \mathrm{F}$.

The latent heat of fusion of ice at $32^{\circ} \mathrm{F}$. is about 142 B.T.U., while the latent heat of evaporation at $212^{\circ} \mathrm{F}$. is $966^{\circ} 6$ B.T.U.

Weight of Water.-Authorities differ as to the precise value of the weight at maximum density, the lowest value given being about $62 \cdot 379 \mathrm{lbs}$. per cubic foot and the highest $62.425 \mathrm{lbs}$. The latter value - that of Rankine-is commonly adopted as being most nearly correct. The following table, calculated from

\begin{tabular}{|c|c|c|c|c|c|c|c|}
\hline $\begin{array}{l}\text { Temp. } \\
\text { Fahr. }\end{array}$ & $\begin{array}{l}\text { Weight per } \\
\text { cubic foot. }\end{array}$ & $\begin{array}{l}\text { Temp. } \\
\text { Fahr. }\end{array}$ & $\begin{array}{l}\text { Weight per } \\
\text { cubic foot. }\end{array}$ & $\begin{array}{l}\text { Temp. } \\
\text { Fahr. }\end{array}$ & $\begin{array}{l}\text { Weight per } \\
\text { cubic foot. }\end{array}$ & $\begin{array}{l}\text { Temp. } \\
\text { Fahr. }\end{array}$ & $\begin{array}{l}\text { Weight per } \\
\text { cubic foot. }\end{array}$ \\
\hline $32^{\circ}$ & $62 \cdot 42$ & $80^{\circ}$ & $62 \cdot 23$ & $130^{\circ}$ & $61 \cdot 56$ & $180^{\circ}$ & $60 \cdot \tilde{5}$ \\
\hline $40^{\circ}$ & $62 \cdot 42$ & $90^{\circ}$ & $62 \cdot 13$ & $140^{\circ}$ & $61 \cdot 37$ & $190^{\circ}$ & $60 \cdot 32$ \\
\hline $50^{\circ}$ & $62 \cdot 41$ & $100^{\circ}$ & $62 \cdot 02$ & $150^{\circ}$ & $61 \cdot 18$ & $200^{\circ}$ & 60.07 \\
\hline $60^{\circ}$ & $62 \cdot 37$ & $110^{\circ}$ & $61 \cdot 89$ & $160^{\circ}$ & 60.98 & $210^{\circ}$ & $59 \cdot 82$ \\
\hline $70^{\circ}$ & $62 \cdot 31$ & $120^{\circ}$ & $61 \cdot 7 t$ & $170^{\circ}$ & $60 \cdot 77$ & & \\
\hline
\end{tabular}

Rankine's formula, gives the weight per cubic foot at different temperatures. At $212^{\circ} \mathrm{F}$. values by different experimenters vary from $59.56 \mathrm{lbs}$. to $59.84 \mathrm{lbs}$. Above this temperature the 
exact values are not so well known and are unimportant to the engineer. For the purpose of all calculations relating to Hydraulics it is sufficiently accurate to take the weight per cubic foot at 62.4 lbs., more especially as the water with which the engineer has to deal is never perfectly pure, but contains more or less of the soluble salts. Unless otherwise stated, the above value will be adopted throughout this treatise.

The density of sea water varies slightly with the locality, but is about 1.026 times that of fresh water. Its weight, at the temperatures commonly met with in practice, may be taken as $64 \cdot 0$ lbs. per cubic foot.

Compressibility.-Water is very slightly compressible, the compressibility varying with the temperature and with the amount of air in solution. When pure, the decrease in volume $\delta \mathrm{V}$, due to an increment in pressure $\delta P$, of one atmosphere, increases from 000040 to $\cdot 000051$ as the temperature decreases from $212^{\circ} \mathrm{F}$. to $35^{\circ} \mathrm{F}$. (Grassi).

This gives values of the bulk modulus $K$, which equals $-\frac{\delta P}{\frac{\delta V}{V}}$,

varying from 368,000 to $288,000 \mathrm{lbs}$. per square inch under this temperature variation, the modulus increasing with an increase in temperature.

The value of $K$ at $50^{\circ} \mathrm{F}$. may be taken as approximately 300,000 lbs. per square inch.

The compressibility is so slight that in all practical calculations concerning water at rest or in a state of steady motion it may be assumed to be an incompressible fluid. In certain important phenomena, however, notably those involving a sudden initiation or stoppage of motion, this compressibility becomes an important, and often the predominating. factor, and in the treatment of such cases the above mean value of $K$ will be adopted.

At ordinary temperatures and pressures, water is capable of dissolving comparatively large volumes of air. As the temperature is raised this is set free, and at atmospheric pressure a temperature of $180^{\circ} \mathrm{F}$. is sufficiently high to liberate almost all the dissolved gases. Any reduction in pressure also tends to the same end, and the 
hissing which is so often noticeable in jet pumps and injectors, or where water is escaping at high velocities past the restricted area of a valve seat, is due to the reduction of pressure and subsequent liberation of bubbles of air, which occurs under these circumstances.

In common with all other liquids water also possesses the properties of Cohesion, Adhesion, and Viscosity.

\section{Art. 3.-Cohesion}

is that property of a liquid, or solid, which enables neighbouring molecules to resist any stress of the nature of a tension. Adhesion is that property which enables it to adhere to a solid body with which it may be in contact. Thus a drop of water exhibits cohesion in its hanging together, and adhesion in virtue of its clinging to a solid body, the force of gravity being overcome both by cohesion and by adhesion. Both phenomena are due to molecular attraction, cohesion between neighbouring molecules of the liquid, and adhesion between those of the solid and liquid. A consideration of the molecular theory of matter indicates that if $a$ is the distance between any two molecules, their mutual attractive force is approximately equal to $\frac{k}{a^{6}}$; and it follows that a rise in temperature, by increasing the molecular distance, diminishes both cohesion and adhesion.

Their relative values vary for different combinations of solid and liquid, the adhesive force being greater than that of cohesion when the liquid wets the solid and vice versî. Also the adhesive force, unlike that of cohesion, is found to vary largely with the time of contact, increasing as this is increased.

That water, under suitable conditions, may exist in a state of tensile stress may be shown in many ways, and experiments ${ }^{1}$ show that this may attain considerable proportions. For example Achard and Gay-Lussac each obtained values of about 1,024 lbs. per square foot, while Defour ${ }^{2}$ succeeded in raising water in the

1 For an account of some experiments on cohesion see a paper by Osborne Reynolds in "Proceedings of the Manchester Literary and Philosophical Society," $1800-1$.

Maxwell's " Heat," p. 259. 
spherical state and exposed to atmospheric pressure, to a temperature of $356^{\circ} \mathrm{F}$. without boiling. Since this corresponds to a vapour pressure of $132 \mathrm{lbs}$. per square inch the cohesion must have attained this value for rupture not to have taken place. This high value is probably due to the absence of dissolved air at this temperature.

With water in bulk, the great difficulty in demonstrating cohesion is to get it into such a state that it may be exposed to a direct tensile stress over any appreciable area.

The method of rupture, as it commonly occurs, may be likened to that of a sheet of paper by a tear extending from one edge. Could the water be brought into such a state that its rupture was similar to that of the same sheet of paper when pulled directly into two parts, the effect of cohesion would be more strikingly apparent. Such an action however, involving the simultaneous rupture over any appreciable area, is extremely difficult to procure except where the water exists in the state of a film, and on this account the effect of cohesion on the behaviour of water in bulk is negligible. Its importance to the engineer is then confined to its effects as shown in the phenomena of capillarity and surface tension.

That the idea of cohesion is not incompatible with the exceeding mobility of the particles of water may be indirectly demonstrated by the behaviour of two iron surface plates, when brought into contact. Here, if the plates are clean, the resistance to sliding of one over the other is very small, while the force necessary to overcome cohesion and to separate the plates by normal motion is surprisingly great.

The phenomenon known as Capillarity, which appears in the tendency of a liquid to rise or fall from its normal level in a tube of fine bore, according as the liquid does or does not wet the tube, would appear to be due to cohesion and adhesion; while it is probable that cohesion between the surface molecules of a liquid gives rise to Surface Tension, the property of a liquid which gives it the appearance of having an elastic skin at its surface of separation from a gas, or from any other liquid. Experiment shows that the tension in any such surface film is everywhere the same, and that the tension across any imaginary 
line in the surface is normal to that line. Also the tension is independent of the curvature of the surface; decreases with an increase in temperature; is constant for a given temperature, for the surface of separation of any particular liquid and gas, or of any two given liquids; and for any given liquid and solid in the presence of air, the angle of inclination, $a$, of the surfaces is constant.

Thus, for water and glass at a temp. of $68^{\circ} \mathrm{F} . \quad . a=25^{\circ} 32^{\prime}$ for mercury and glass . . . . . $a=128^{\circ} 52^{\prime}$ while the surface tension, expressed in pounds per foot run of the line of contact, and at the above temperature, has the following values (from Quincke's experiments) :-

For water and air . . $T=\cdot 005548$ lbs. per foot

For mercury and air . . $T=\cdot 03698 \quad$,,

No satisfactory explanation of the precise nature of surface tension has been formulated. Beyond doubt however it is a molecular phenomenon and is inseparable from that of cohesion, the intensity of the one probably determining that of the other.

Many theories have been educed to explain the phenomena of capillarity. Of these, the two which have been most generally accepted are due respectively to Poisson and Young. Briefly outlined, the former assumes that when a liquid wets a plate the attraction between the layer of molecules in intimate contact with the plate and the plate itself is greater than the intermolecular attraction of the liquid. The molecular motion of the molecules forming the surface film is thus reduced, while, in consequence of their greater freedom, that of the molecules immediately distant from this film is increased. The density of this secondary layer of fluid is thus reduced below the normal, and the resultant upward pressure of the surrounding fluid causes it to rise up the plate until a state of statical equilibrium is attained between the cohesive forces and the action of gravity on the supported fluid.

Where a liquid does not wet, the plate the density of this secondary film is increased by the molecular action between the plate and fluid, with a consequent fall in the surface level in the immediate neighbourhood of the plate. That this variation of 
density actually occurs has been indirectly proved in other ways, while results obtained by the application of this theory are amply confirmed by experiment.

The second theory attributes the phenomena to the action of a series of surface tensions, which are assumed to exist at every surface of contact of any liquid or gas with any solid, and also at the surface of contact of any liquid with any other liquid, or with a gas. Thus at the common line of intersection of a solid, liquid, and gas, i.e., at the line passing through $P$ (Fig. 1) and perpendicular to the plane of the paper, three surface tensions, of intensity $T_{a s}, T_{a l}$. and $T_{l s}$ per unit length of this line, are in existence. For equilibrium then, their directions and magnitudes will be related according to the ordinary laws of statical

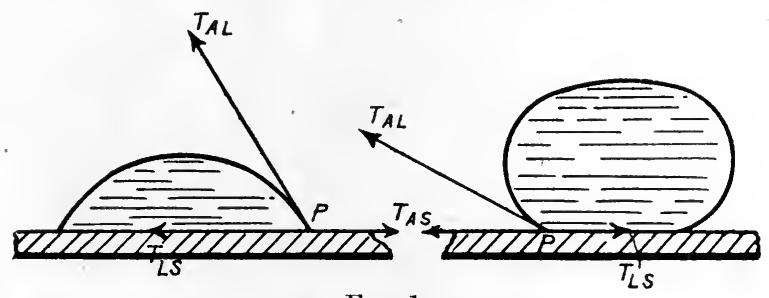

Fig. 1.

equilibrium. Thus for contact with a plane surface we have$T_{a s}^{\prime}=T_{l s}+T_{a l} \cos a$,

and the angle $a$ will be acute or obtuse according as $T_{a s}-T_{l s}$ is positive or negative.

If $T_{a s}=T_{l s}+T_{a l}, a=0$ and for this, and all greater relative values of $T_{a s}$, the fluid will immediately spread to cover the surface, the effect being as though the liquid were pulled outwards in every direction by the tension $T_{c s}$, the resultant of the tensions $T_{l s}$ and $T_{c l}$ being insufficient to resist this motion. On the other hand if $T_{a s}-T_{l s}$ is negative, $\cos \alpha$ is negative and the angle $a$ is obtuse. If $T_{l s}-T_{a s}=T_{a l}^{\prime}, a=180^{\circ}$, and the liquid, if in sufficiently small masses, assumes the spherical state.

On these assumptions, justified in so far as the results obtained by their application go, the various phenomena of capillary action easily lend themselves to mathematical treatment.

Rise of Liquid in a Capillary Tube.-Let $h$ be the height of the 
liquid in the tube, of diameter $d$ feet, and let $w$ be the weight of unit volume of the liquid. Here $h$ is measured to a horizontal plane tangential to the curved surface at the top of the column (Fig. 2). Let $V=$ the (small) volume of liquid above this plane.

The height of the column when in a state of equilibrium may be deduced from the principle of virtual work, ${ }^{1}$ i.e., by equating the work done against gravity in any small vertical displacement of the column, to that done by the surface tensions.

If $\delta x$ is this displacement we have, for equilibrium

$$
\begin{gathered}
\left(T_{l s}-T_{a s}\right) \pi d \delta x+w \delta x\left\{\frac{\pi d^{2} h}{4}+V\right\}=0 \\
\therefore h=\frac{4}{w d}\left(T_{a s}-T_{l s}\right)-\frac{4 V}{\pi d^{2}}
\end{gathered}
$$

But $T_{a s}-T_{s}=T_{a l} \cos a$

$$
\therefore h=\frac{4 T_{a l} \cos a}{w d}-\frac{4 V}{\pi d^{2}}
$$

In general $V^{r}$ is very small, so that without sensible error (1) may be written

$$
h=\frac{4 T \cos a}{w d}
$$

Similarly it may be shown that the vertical rise or fall between two parallel plates at a distance $d$ apart $=\frac{2 T \cos a}{u \cdot d}$

It is with the former case of capillary action that we are chiefly concerned in hydraulics, as affect-

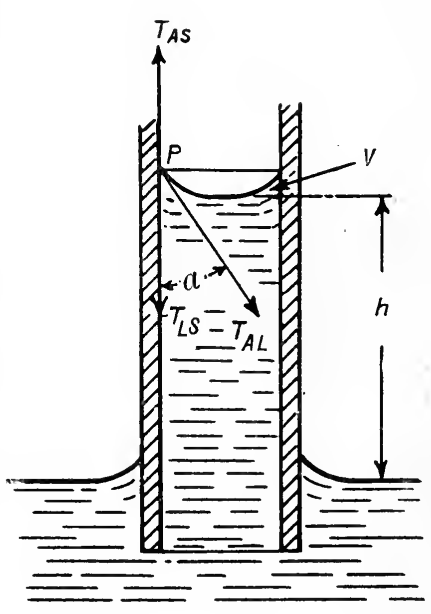

FIG. 2. ing the accuracy of measurements of pressure in a liquid, when

1 More simply, it may be considered that the whole weight of the supported column is carried by the surface films, and that this weight is equal to the vertical component of the surface tension. This leads, as before, to the equation

$$
\begin{aligned}
\pi d \cdot T \cos \alpha & =w \frac{\pi d^{2}}{4} h, \\
\therefore \quad h & =\frac{4 T \cos \alpha}{w d}
\end{aligned}
$$

With liquid in its spherical state we have $2 \pi r T=\pi r^{2} p$, or $p=\frac{2 T}{r}$, giving $p$, the excess of internal over external pressure. Thus with small values of $r$ a comparatively small value of $T$ may be accompanied by a large value of $p$. 
these depend upon the height of a supported column of the liquid. Thus with a piezometer, in which pressure is measured by means of a water column, the artificial elevation of the pressure column by capillary action at about $68^{\circ} \mathrm{F}$. is given by

$$
h=\frac{4 \times \cdot 005548 \cos 25^{\circ} 32^{\prime}}{62 \cdot 4 d} \text { feet. }
$$

If $d$ be measured in inches, this artificial elevation in inches is given by

$$
h=\frac{04624}{d} \text { inches. }
$$

In the case of a mercury column, taking the specific gravity of mercury to be $13 \cdot 596$, we have $\cos a=-\cdot 6276$

$$
\begin{aligned}
\therefore \quad h & =-\frac{4 \times 03698 \times 6276 \times 144}{62.4 \times 13.596 d} \text { inches. } \\
& =-\frac{.01576}{d} \text { inches. }
\end{aligned}
$$

where $d$ is measured in inches.

The negative sign here indicates that the surface of the mercury column is depressed below the level corresponding to the statical

\begin{tabular}{|c|c|c|c|c|c|c|c|c|c|c|c|c|}
\hline & & $\cdot 02$ & $\cdot 04$ & $\cdot 06$ & $\cdot 08$ & $\cdot 10$ & $\cdot 125$ & $\cdot 15$ & $\cdot 20$ & $\cdot 2 \tilde{\jmath}$ & $\cdot 30$ & .50 \\
\hline \multirow{2}{*}{$\begin{array}{l}h \text { in } \\
\text { inches }\end{array}$} & $\begin{array}{l}\text { Water in } \\
\text { glass tube }\end{array}$ & $2 \cdot 312$ & $1 \cdot 156$ & $: 771$ & .578 & $\cdot 462$ & $\cdot 370$ & $\cdot 308$ & $\cdot 231$ & $\cdot 185$ & $\cdot 154$ & \\
\hline & $\begin{array}{l}\text { Mercury in } \\
\text { glass tube }\end{array}$ & 788 & $\cdot 394$ & $\cdot 263$ & $\cdot 197$ & $\cdot 158$ & $\cdot 126$ & $\cdot 105$ & $\cdot 079$ & $\cdot 063$ & $\cdot 053$ & \\
\hline
\end{tabular}
pressure alone. - The following table gives values of $h$ at $68^{\circ} \mathrm{F}$. corresponding to various tube diameters.

Diameter of 'Tube in Inches.

Evidently then, tubes of small bore - below about $\cdot 3$ ins. internal diameter-are not advisable for use in such pressuremeasuring apparatus, especially where small pressures are to be measured, except where the difference of height in two similar tubes is used to indicate the pressure. Here the effects of capillarity, being the same for each tube, neutralise each other. Again, since the specific gravity of mercury is $13 \cdot 596$, the proportional error introduced by neglecting capillarity in the case 
of a mercury column will be $\frac{13.596 \times \cdot 01576}{\cdot 04624}=4 \cdot 63$ times that introduced with a water column, and will be in the opposite direction. ${ }^{1}$

The effect of surface tension in liquid in motion may be strikingly shown by allowing two jets of water, moving steadily in the same straight line and in opposite directions, to meet. No splash occurs, but a perfectly clear and steady circular film of water is produced in a plane perpendicular to that of the jets, while under favourable circumstances the diameter of this may be extended to some feet before it breaks up into a series of detached drops.

ArT. 4.

Every known fluid offers a resistance, analogous to friction, to the relative sliding motion of any two adjacent layers, and the physical property of the fluid to which this is due is termed Viscosity. This property - only noticeable when the fluid is in motion-is the cause of all so-called fluid friction and gives the fluid the appearance of being able to withstand a shear stress between adjacent layers. The analogy between the viscosity of a fluid and the transverse elasticity of a solid may be shown by introducing a band of aniline dye solution across a beaker containing water at rest, and by then rotating the beaker very slowly. The outer layers of water move with the glass and transmit their motion gradually to the inner layers, the band forming a spiral. If now the motion be reversed, the colour band returns to its old position and again forms a straight line.

The magnitude of this shear or distortive stress over any plane is proportional to the rate of distortion, and hence to the rate of change of velocity with space perpendicular to the plane. Thus if coordinate axes $O X, O Y, O Z$ (Fig. 3) be taken at some point $O$, of a stream, and it $f_{s}$ be the distortive stress accompanying relative motion of adjacent layers in a direction parallel to the axis of $x$,

1 Lack of space prevents any further investigation into the properties of surface films. These are, however, except in so far as they affect the stability of jets, only of slight importance in hydraulics. For further information the reader is referred to any textbook of physics or hydrostatics, or to the article on Capillary Action in the " Encyclopædia Britannica." 
the velocity in this direction at any point $\mathrm{P}$, distant $y$ from that axis being denoted by $v$, and if the velocity in the direction of $O Z$ is zero, we have, at the point $P$

$$
f_{s} \propto \frac{d v}{d y}
$$

If a curve, having ordinates representing values of $v$, be plotted

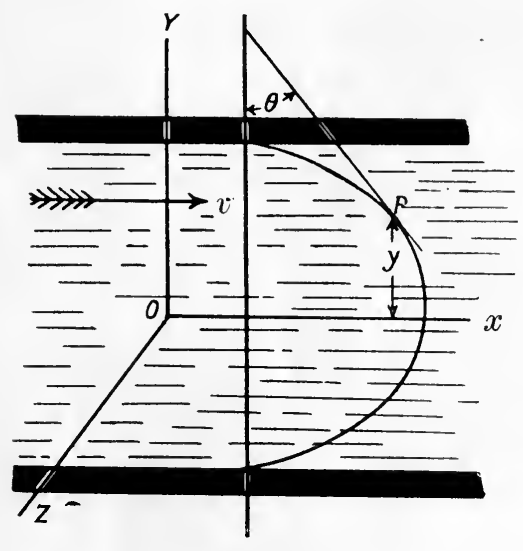

FIG. 3. on a base parallel to $O Y$, and if $\theta$ be the angle which the tangent to the curve at $P$ makes with this base line, the values which $f_{s}$ adopts as $y$ varies will be represented to scale by the corresponding values of $\tan \theta$

Putting $f_{s}=\mu \frac{d v}{d y}$, the coefficient $\mu$ is termed the Coefficient of Viscosity. ${ }^{1}$

The value of this coefficient varies with different fluids, and varies with temperature

for any particular fluid.

For water, experiments by Poiseuille indicate that it follows the law

$$
\mu=\frac{\cdot 00003716}{\cdot 4712+\cdot 01435 T+\cdot 0000682 T^{\prime 2}}
$$

where $' T$ ' is in degrees $\mathrm{F}$., and the corresponding value of the distortive stress $f_{s}$ is given in pounds per square foot. Using the Fahrenheit scale and taking the poundal and the foot aso units of force and space, this formula becomes

$$
\mu=\frac{\cdot 001197}{\cdot 4712+\cdot 01435 T^{\prime}+\cdot 0000682 T^{2}}
$$

With the pound, foot, and degree Centigrade as units we get

1 Expressed dimensionally we have $\mu=f_{s} \div \frac{d v}{d y}=$ stress $\div \frac{\text { vel. }}{\text { space }}=\frac{\text { stress }}{\text { time. }}$

$$
\begin{aligned}
& \text { But stress }=\frac{\text { force }}{\text { area }}=\frac{\text { mass } \times \text { acceleration }}{\text { area }}=\frac{M I}{L^{2}} \times \frac{V}{T^{\prime}}=\frac{M}{L T^{2}} . \\
& \therefore \mu \text { is of dimension } \frac{M I}{L T^{2}} \div \frac{1}{T}=\frac{M I}{L T} .
\end{aligned}
$$




$$
\mu=\frac{\cdot 00003716}{1+\cdot 03368 T^{\prime}+\cdot 000221 T^{2}}
$$

while using the poundal, foot, and degree Centigrade this becomes

$$
\mu=\frac{\cdot 001197}{1+\cdot 03368 T+\cdot 000221 T^{2}}
$$

In C. G. S. units, the unit of force being taken as the weight of 1 gramme, and the temperature in degrees Centigrade we have

$$
\mu=\frac{\cdot 0000181}{1+03368 T^{\prime}+\cdot 000221 T^{\prime 2}} .
$$

The following table indicates the values of $\mu$ for water corresponding to different temperatures, $f_{s}$ being expressed in lbs. per square foot.

\begin{tabular}{c|c|c}
\hline \multicolumn{2}{c|}{ Temperature. } & $\mu$. \\
\hline Fahrenheit. & Centigrade. & \\
\hline $32^{\circ}$ & $0^{\circ}$ & $\cdot 00003716$ \\
$50^{\circ}$ & $10^{\circ}$ & $\cdot 00002735$ \\
$68^{\circ}$ & $20^{\circ}$ & $\cdot 00002109$ \\
$86^{\circ}$ & $30^{\circ}$ & $\cdot 00001685$ \\
$104^{\circ}$ & $40^{\circ}$ & $\cdot 00001376$ \\
$122^{\circ}$ & $50^{\circ}$ & $\cdot 00001150$ \\
$140^{\circ}$ & $60^{\circ}$ & $\cdot 00000971$ \\
\hline
\end{tabular}

Experiments by Hauser indicate that above $32^{\circ} \mathrm{F}$. the viscosity of water, in addition to varying with temperature, also increases very slightly with an increase in pressure, and this would appear to be borne out by the critical velocity experiments of Messrs. Barnes \& Coker at the McGill University. It may be noted that whereas with liquids the viscosity decreases with an increase in temperature, with gases the reverse is the case. Researches by Dr. Grindley and the author show that in the case of air the viscosity is independent of the pressure and, if temperatures are measured in degrees Centigrade, increases with the temperature according to the law 
$\mu=\left\{355 \cdot 3+1 \cdot 168 T^{\prime}-{ }^{\cdot} 00248 T^{2}\right\} \times 10^{-9} \mathrm{ft}$. lb. sec. units, or approximately

$$
\mu=\{358+\cdot 932 T\} \times 10^{-9} \mathrm{ft} . \mathrm{lb} \text {. sec. units }
$$

between the limits of temperature $0^{\circ} \mathrm{C}$. and $100^{\circ} \mathrm{C} .^{1}$

This is as might be inferred from the kinetic theory of fluids, for layers of fluid moving with different velocities are continually interchanging molecules by diffusion. Thus the more rapidly moving layers are continually losing momentum by interchange with the slower layers and a continually applied force is necessary to maintain this state of motion. Since the diffusivity increases with an increase of temperature both in liquids and gases, it would be expected that in both cases the viscosity would increase with temperature.

It is however extremely probable that cohesion plays a great part in producing viscosity in liquids, and while in gases the molecules exert forces on each other by collision only, so that here diffusivity is the important factor in producing viscosity, in liquids the greater cohesion at lower temperatures more than counterbalances the diminished diffusivity and hence increases the viscosity.

Since the interchange of molecules will be proportional to the area over which such interchange takes place the resistance to distortion will be proportional to this area. Evidently then if eddies are formed in the course of a stream of fluid, since the area over which interchange of momentum may take place is greatly increased and in an indefinite manner, the viscous resistance to motion will also be increased.

Viscosity, being a physical property of a fluid, is independent of the velocity of translation of its particles. The resistance to motion can however only be directly inferred from the viscosity when the motion of the fluid is everywhere steady and in parallel lines.

When eddies are set up, although the viscosity is unaltered yet the motion is so irregular, and in such different directions, that it is impossible to determine the total work done against viscosity, and hence impossible to directly calculate the resistance to motion solely from a knowledge of the viscosity.

1 "Phil. Trans. Royal Society." 
The energy absorbed in overcoming viscous resistance in any fluid motion finally appears in the form of heat and is usually dissipated by conduction and radiation.

\section{Examples.}

(1) If it be assumed that the top of a circular capillary column assumes an hemispherical form, show that the height of the column is given by

$$
h=\frac{4 T \cos a}{w d}-\frac{d}{6} .
$$

(2) Determine the true readings, and the percentage errors introduced by capillarity in the measurement of a pressure, when this is measured

(a) by a mercury column whose apparent reading is $\quad 50 \mathrm{ins}$.

(c) ", water

(d) ,

Ans. (a) True reading =

$$
\begin{aligned}
& \text { (b) }, \quad, \quad= \\
& \text { (c) },, \quad, \quad= \\
& \text { (d) } ", \quad=9 \mathrm{ft} .11 .815, \\
& .563 \text { ins. Error }=11.2 \%
\end{aligned}
$$

(3) Show, from a consideration of surface tension, that the form of the capillary surface in the neighbourhood of a vertical plate is given by the equation

$$
y^{2}=\frac{2 T \cos \theta}{w}+\text { constant }
$$

Where $y$ is the vertical distance of some point $P$ on this surface from the horizontal tangent plane to the same surface, and $\theta$ is the angle of inclination to the horizontal of the tangent to the surface at $P$.

(4) Show that the coefficient of viscosity $\mu$ is of the dimensions $\left[\frac{M}{L T}\right]$ where $M=$ mass, $L=$ length, $T=$ time. 


\section{CHAPTER II.}

Hydrostatics - Principles - Pressure Intensity-Transmissibility of PressureAtmospheric Pressure-Resultant Pressure-Centre of Pressure-Stability of Masonry Dams-Equilibrium of Floating Bodies-Metacentric HeightOscillations of Ships-Strength of Pipes and Cylinders.

\section{Art. 5.-Hydrostatics.}

Commonsy, any substance which, at ordinary temperatures, possesses in a marked degree the property of accommodating itself to the shape of any vessel into which it may be placed, is termed a fuid.

Fluids may be divided into two classes; gases and liquids; according as they are easily, or with difficulty, compressible.

Definition of a Perfect Fluid.-By a perfect fluid we mean a substance such that the pressure exerted by it on any surface with which it may be in contact is everywheren ormal to that surface.

The laws governing the action and reaction, and generally the statical equilibrium, of such a fluid may be easily deduced from theoretical considerations. Before, however, extending these laws to the case of such a fluid as water it becomes necessary to determine to what extent this differs, in its essentials, from our conceptions of a perfect fluid.

From the above definition it follows that with a perfect fluid any action of the nature of friction between solid and fluid is impossible, since this would necessitate the action of some force tangential to the surface. It follows that there can be no frictional resistance to the motion of a solid body through the fluid, or to the steady motion of the fluid through any pipe or channel having solid boundaries, and that in consequence any portion of the fluid may be separated from any other portion by a force however small, if applied for a sufficient length of time. Further, the perfect fluid is incapable of existing in a state of tension. 
Such a conception is useful, although no such substance as a perfect fluid is known in Nature. All known fluids, in virtue of their properties of cohesion and viscosity, offer some resistance of the nature of friction to the motion of any solid surface with which they may be in contact.

Evidently then the laws governing the behaviour of a perfect fluid when in motion, are not applicable, without some modification, to water; nor are the laws governing its statical equilibrium applicable to that of water in cases where, as when under the influence of capillary action, the effect of cohesion is great. When dealing however with water in bulk, impregnated as it usually is with air in solution, the effect of cohesion may be neglected, and when at rest water may be considered as satisfying the essential condition of a perfect fluid, viz. that it exerts a normal pressure on all surfaces with which it may be in contact. All the laws governing the statical equilibrium of a perfect fluid, depending as they do solely on this property, can then be applied to that of water, and are included among the principles of Hydrostatics.

\section{Art. 6.-Pressure at a Point.}

The average pressure intensity over any area $A$ equals the total pressure $P$ on the area divided by the area, or equals $\frac{P}{A}$. If the pressure varies from point to point of the area, and if this be divided into a large number $n$ of small areas $\delta A$, the pressure on any one of these containing a given point being $\delta P$, then the limiting value to which the ratio $\frac{\delta P}{\delta A}$ tends, as $n$ is made infinitely large, is taken as the pressure intensity--or more shortly the pressure-at the point under consideration.

The units in which pressure intensity is measured depend on those of force and space.

In English practice the unit is usually the pound per square inch, or per square foot. In the metric system of units the usual unit is the kilogramme per square centimeter.

These units are connected by the relationship that 1 kilogramme per square $\mathrm{cmm}$. $=14 \cdot 223 \mathrm{lbs}$. per square inch.

H.A. 
Art. 7.-In a Liquid at Rest, the Pressure Intexsity is eVErywhere the save at the Save Depth, aNd is the same in all Directions.

The truth of the first of these propositions may be seen by

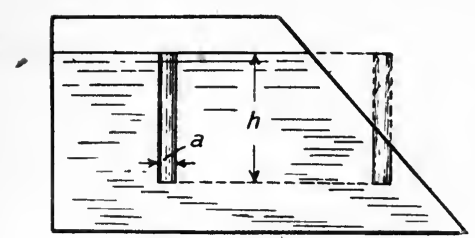

FIG. 4. considering the equilibrium of a small vertical column of the liquid of cross sectional area $a$ and having its base at the depth $h$ below the surface (Fig. 4). Wherever this column is taken in the liquid its weight will be the same and must be balanced by the vertical upward pressure on the base, which will therefore be the same. Since the sectional area of the column is unaltered, it follows that the pressure intensity on the base must everywhere be the same.

If $W$ is the weight of unit volume, or the intrinsic weight of the liquid, the weight of the column $=W a h$.

If $p$ is the pressure intensity at a depth $h$, the upward pressure on the base $=p a$.

$$
\begin{aligned}
\therefore & p a & =W a h . \\
\therefore & p & =W h .
\end{aligned}
$$

The pressure of still water against the sides or bottom of any

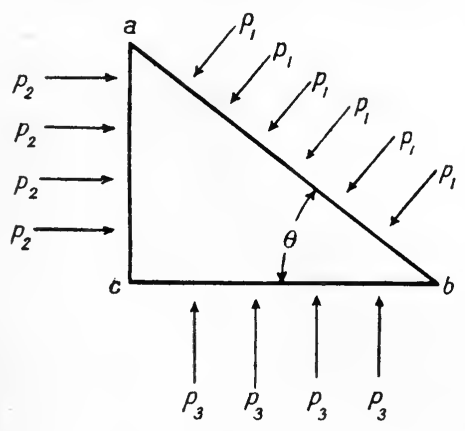

FIG. 5. vessel is then simply due to the " head," or height of the level of the free surface of the water above the point considered. Each square foot of the surface at a depth $h$ may be considered as supporting a column of the water of one square foot cross sectional area, and of height $h$, and therefore of weight $62 \cdot 4$ $h$ lbs.

$\therefore \quad$ Pressure per square foot, per foot of head $=62.4 \mathrm{lbs}$.

$$
\text { , , , inch, , , }=.433 \mathrm{lbs} \text {. }
$$

$\therefore$ Head equivalent to a pressure of $1 \mathrm{lb}$. per square inch $=$ $2 \cdot 308$ feet. 
The second of the above propositions may be deduced by considering the equilibrium of a triangular prism of the liquid of unit length having its edges horizontal, and its ends perpendicular to the sides. If $a b c$ (Fig. 5) be a cross section of the prism, $a c b$ being a right angle and $b c$ being horizontal, and $p_{1}, p_{2}, p_{3}$, be the mean intensities of pressure on the sides $a b, a c, c b$, we have for equilibrium, resolving parallel to the sides $b c$ and $c a$

$$
\begin{aligned}
& p_{2} a c=p_{1} a b \sin \theta \\
& p_{3} c b=p_{1} a b \cos \theta+W \frac{a c c b}{2}
\end{aligned}
$$

Putting $a c=a b \sin \theta ; b c=a b \cos \theta$ we get

$$
\begin{aligned}
& p_{2}=p_{1} \\
& p_{3}=p_{1}+\frac{W}{2} a b^{2} \sin \theta \cos \theta .
\end{aligned}
$$

If now the sides of the prism be indefinitely diminished $p_{1}, p_{2}$, and $p_{3}$ become, in the limit, the pressure intensities at the same point but in different directions. Also $a b^{2}$ being of the second order of small quantities will vanish, so that in the limit

$$
p_{1}=p_{2}=p_{3} \text {. }
$$

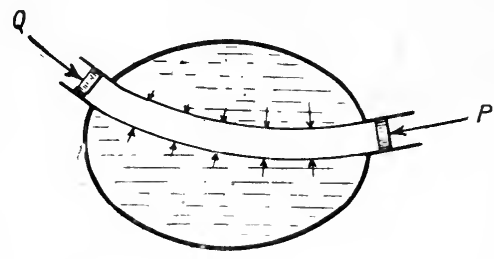

Fig. 6.

It follows from these theorems that the free surface of still water is a surface of equal pressure and that all equipotential surfaces in the fluid are parallel to this. This surface is not plane, but is everywhere normal to the direction of the force of gravitation, and therefore to the direction of a plumb line.

\section{Art. 8.-Transmissibility of Pressure in a Fluid.}

If a-pressure be applied to the surface of a fluid this pressure is transmitted equally to all parts of the fluid. This may be seen by considering a closed vessel filled with water, and fitted with a piston of area $A$, to which a force $P$ is applied, producing a pressure intensity at this point of $\frac{P}{A}$. Considering any other area $A$ of the surface, the two may be supposed connected by a cylinder of the fluid, having imaginary boundaries (Fig. 6). 
Suppose piston $P$ to be displaced through a small distance $x$. Then in virtue of the incompressibility of the fluid, $Q$ will be displaced through the same distance. Also, since the reaction of the cylinder walls is everywhere perpendicular to the direction of motion of the contained fluid, no work is done against this reaction and in consequence the work done by $P=$ work done on

$Q$. Since the areas

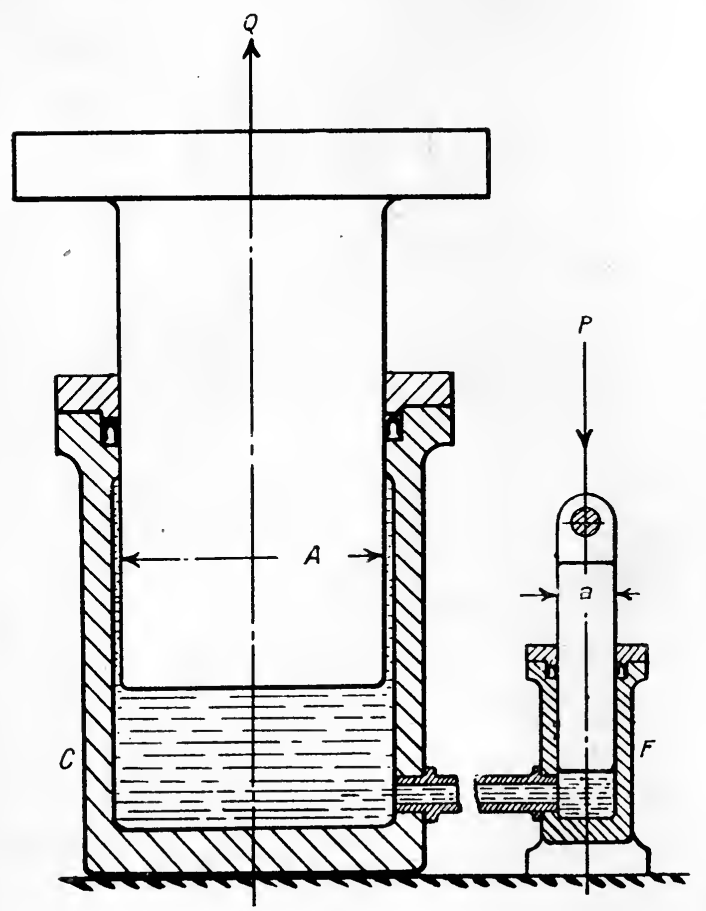

Fig. 7.-Bramah's Press.

and displacements of $P$ and $Q$ are the same, the pressure intensities over their surfaces, introduced by the action of the external forces, must also be equal. The total pressure intensity at either $P$ or $Q$ will then be obtained by adding to the pressure produced by the force $P$, that due to the weight of the liquid. Altering the plane of the pistons at $P$ and $Q$ will evidently not affect the pressure intensity on either surface.

This property is taken advantage of in many hydraulic machines, notably in Bramah's Hydraulic Press, and in machines of a like type.

In the Hydraulic Press, illustrated diagramatically in Fig. 7, water is forced by means of a small pump $F$, whose plunger has an area $a$, into the cylinder $C$ of the press whose area is $A$. Neglecting friction, a force $P$, applied to the plunger of the force pump, will then produce a pressure intensity of $\frac{P}{a}$ in the pump 
and press eylinder, and hence a force of $Q=P \frac{A}{a}$ on the press plunger. A particular case of the transmissibility of pressure is found in the transmission of the pressure of the atmosphere on the surface of water to every part of its depth. Thus the pressure intensity at any depth, $h$, is strictly that due to the weight of the superposed water, together with the atmospheric pressure.

Atmospheric Pressure.-The pressure of the atmosphere varies from day to day at the same place, and from place to place at the same time. Its mean value at sea level is about equivalent to that at the base of a mercury column 30 inches in height, or $14.7 \mathrm{lbs}$. per square inch. This is equivalent to the pressure at the base of a water column of approximately 34 feet in height, and this is usually taken as the height of the water barometer. Thus the true pressure intensity at a depth $h$ below the free surface of water is given by $62 \cdot 4(h+34)$ lbs. per square foot.

In most hydrostatic problems however it is the pressure in excess of that due to the atmosphere which is required, so that the pressure at a submerged point is commonly taken as being that due to the head of water alone.

\section{Art. 9.-Resultant Pressure and Centre of Pressure of a subuerged Area.}

If $p$ denote the mean pressure intensity over a small element of area $\delta A$, the total pressure on this element will be given by $p \delta A$ and the total pressure on the whole submerged area will be the sum of all these small normal pressures, and will be represented by $\Sigma p \delta A$.

The resultant of all these elementary forces is termed the Resultant Pressure on the area, while the point in which the line of action of this resultant meets the area is termed the Centre of Pressure.

If the centre of gravity-or centroid-of an element $\delta A$, be at a depth $x$ below the free surface of the liquid, the total pressure on the whole submerged area will thus be given by

$$
\Sigma W x \delta A \text {. }
$$


If $\bar{x}$ be the depth of the centroid of this area it can be easily shown that $\Sigma x \delta A=\bar{x} \Sigma \delta A=\bar{x} A$.

$$
\therefore \quad \Sigma W x \delta A=W A \bar{x}
$$

i.e., the total pressure on a single face of any submerged area is equal to the area multiplied by the depth of its centroid below the free surface and by the intrinsic weight of the fluid.

Example 1.-Calculate the total pressure on the internal curved surface of an

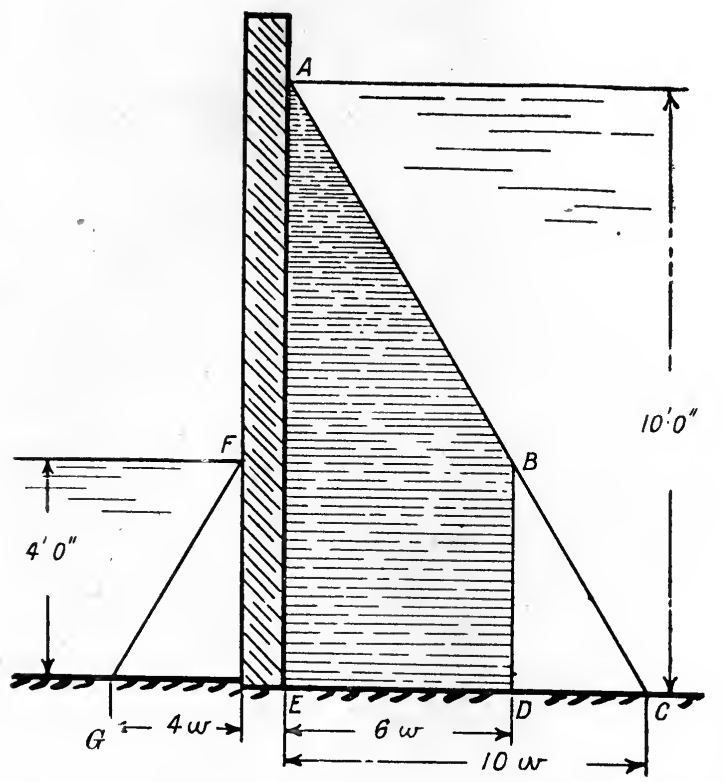

FIG. 8.

hemispherical bowl of radius $r$, placed with its diametrical plane horizontal, and just filled with water.

Here $\vec{x}=\frac{r}{2} ; A=2 \pi r^{2}$.

$$
\therefore \quad \text { Total pressure }=62.4 \times 2 \pi r^{2} \times \frac{r}{2}=62.4 \pi r^{3} \mathrm{lbs} .
$$

In this case the resultant pressure will, for equilibrium, be vertical and equal to the weight of the water;

$$
\therefore \quad \text { Resultant pressure }=62.4 \times \frac{2}{3} \pi r^{3} \mathrm{lbs} .
$$

In the case of a plane surface, such as a dock gate, the total pressure on a face will be the same as the resultant pressure, both being normal to the face. 
Example 2.-In the dock gate shown in Fig. 8, the width of gate being 20 feet, the depth of the centroid of the submerged portion on the right-hand side is 5 feet, and on the left-hand side is 2 feet. The areas of these submerged surfaces are 200 feet and 80 feet respectively, so that the total pressures, and also the resultant pressures on the two faces are $5 \times 200 \times 62.5=62,500 \mathrm{lbs}$. and $2 \times 80 \times 62.5=$ $10,000 \mathrm{lbs}$. The resultant of the two pressures will then be a single force of $62,500-10,000=52,500 \mathrm{lbs}$. acting from right to left. The magnitude of the resultant pressure intensity, and of its distribution over the gate are indicated in Fig. 8. Here the pressure intensity at any depth is indicated by the horizontal distance between the surface of the gate and the straight lines $A C$ and $F G$. The resultant pressure at any depth is then to the left, and is represented by the horizontal width of the shaded area. Evidently at all points below the lower surface level, the resultant pressure intensity will be constant, since the pressure intensity increases at equal rates on both sides of the gate. The resultant force to the left per foot run of the gate is represented by the shaded area.

The determination of the position of the Centre of Pressure of an area is the same as that of the line of action of the resultant of a series of statical forces each normal to the surface under consideration. Referring this to rectangular co-ordinates () $X$ and $O \quad Y$ (Fig. 9) of which $O X$ is vertical and $O Y^{\prime}$ in the surface, we have, taking moments about these axes and representing the

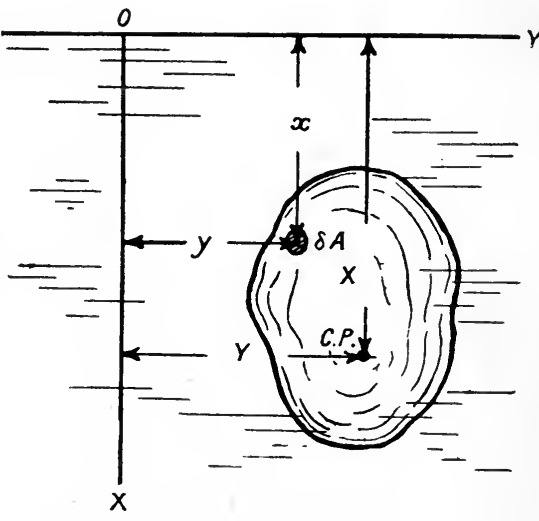

FIG. 9. co-ordinates of the centre of pressure by $X Y$

$$
\left.\begin{array}{l}
\Sigma(p \delta A \cos \theta) X=\Sigma(p \delta A \cos \theta x) \\
\Sigma(p \delta A \cos \theta) Y=\Sigma(p \delta A \cos \theta y)
\end{array}\right\}
$$

writing $p=W x$ these become

$$
\left.\begin{array}{l}
\Sigma(I \delta A \cos \theta x) X=\Sigma\left(W \delta A \cos \theta x^{2}\right) \\
\Sigma(W \delta A \cos \theta x) Y=\Sigma(W \delta A \cos \theta x y)
\end{array}\right\}
$$

Here $\theta$ is the angle between the tangent plane to the surface at the point $(X Y)$ and the plane $X O Y$. It follows that $\Sigma(\delta A \cos \theta)$ is the projection of the. surface on this plane. Call this $A^{\prime}$, and let $\bar{x}^{\prime}$ be the co-ordinate of the centroid of this projection on the reference plane. 
Then (3) becomes

Similarly

$$
\begin{aligned}
w A^{\prime} \bar{x}^{\prime} X & =w \Sigma\left(\delta A \cos \theta x^{2}\right) \\
\therefore \quad X & =\frac{\Sigma\left(\delta A \cos \theta x^{2}\right)}{A^{\prime} \bar{x}^{\prime}} \\
Y & =\frac{\Sigma(\delta A \cos \theta x y)}{A^{\prime} \bar{x}^{\prime}}
\end{aligned}
$$

It follows that the centre of pressure of any curved surface has the same co-ordinates as that of its projection on the plane of reference.

Example.-The centre of pressure of the curved surface of a hemisphere having its diametrical plane vertical, and immersed with its upper edge in the surface of the water, is at the same depth as that of the vertical diametrical plane.

Since $\Sigma\left(\delta A \cos \theta x^{2}\right)$ is the moment of inertia of the projection of the surface, about the axis $O Y$, expression (5) may be written

$$
X=\frac{A^{\prime} k^{\prime 2}}{A^{\prime} \bar{x}^{\prime}}=\frac{k^{\prime 2}}{\bar{x}^{\prime}}
$$

where $k^{\prime}$ is the radius of gyration about the axis $O Y$ of the projection of the surface on the plane $X O Y$.

Where the surface is plane, the axes $O X, O Y$ may be taken in the plane itself, when $\theta=0^{\circ}$, and the above expressions reduce to

$$
\begin{aligned}
& X=\frac{A k^{2}}{A \bar{x}}=\frac{k^{2}}{x} \\
& Y=\frac{\sum(\delta a x y)}{A x}
\end{aligned}
$$

where $A$ is the area and $k$ its radius of gyration about the axis $O Y$ in the surface, $x$ now being the distance of the centroid of the area from the surface, measured in the plane of the area.

In the case of the hemisphere just considered-radius $r$ -

$$
\begin{aligned}
\bar{x}^{\prime} & =r \quad A^{\prime}=\pi r^{2} \\
A^{\prime} k^{2} & =\frac{\pi r^{4}}{4}+\pi r^{2} \cdot r^{2}=\pi r^{2}\left\{\frac{5}{4} r^{2}\right\} \\
\therefore \quad X & =\frac{5}{4} r .
\end{aligned}
$$

In the case of a rectangle, having its side $b$ in the surface, and its side $d$ inclined at an angle $\theta$ to the vertical, 


$$
\begin{aligned}
A k^{2} & =\frac{1}{12} b d^{3}+\frac{b d^{3}}{4}=b d\left(\frac{d^{2}}{3}\right) \\
\bar{x} & =\frac{d}{2} \\
\therefore \quad X & =\frac{2}{3} d,
\end{aligned}
$$

i.e., whatever the inclination, the C. P. is on the median line at a point distant $\frac{2}{3}$ the length of the rectangle from the surface.

Where the upper edge of the rectangle is horizontal and at a depth $h$ below the surface (Fig. $10 a$ )

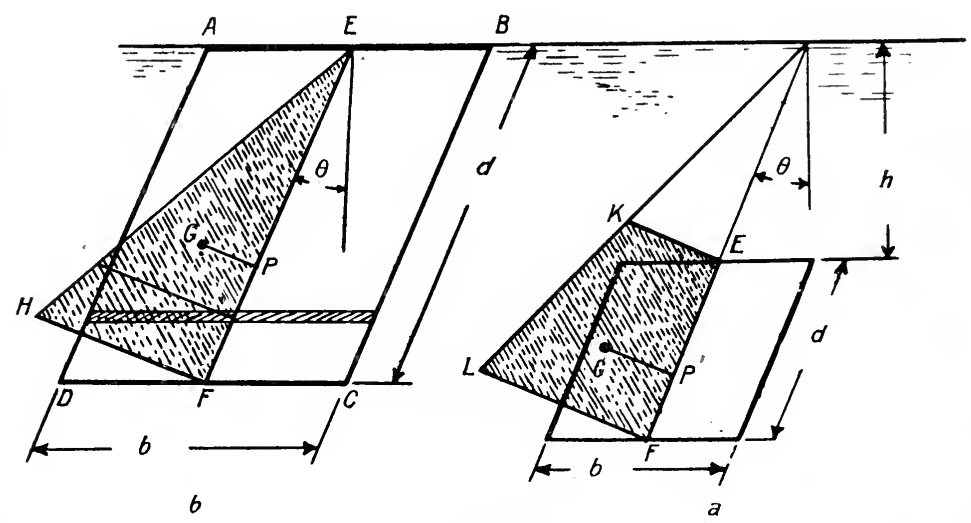

FIG. 10.

$$
\begin{aligned}
& k^{2}=\frac{d^{2}}{12}+\left(h \sec \theta+\frac{d}{2}\right)^{2} \\
& \bar{x}=h \sec \theta+\frac{d}{2} \\
& \therefore \quad X=\frac{\frac{d^{2}}{12}+\left(h \sec \theta+\frac{d}{2}\right)^{2}}{h \sec \theta+\frac{d}{2}}
\end{aligned}
$$

Where the plane of the rectangle is vertical $\theta=0^{\circ}$ and the above becomes

$$
X=\frac{\frac{d^{2}}{12}+\left(h+\frac{d}{2}\right)^{2}}{h+\frac{d}{2}}
$$


- From the form of this result it is clear that as $h$ increases, $X$ becomes more nearly equal to $\left(h+\frac{d}{2}\right)$ i.e, the C. P. approaches more nearly to the centroid of the area.

The position of the Centre of Pressure may, in some instances, be deduced by elementary methods.

E.g., Parallelogram with base in surface (Fig. 10 b). Divide the surface into a series of elementary horizontal strips of equal width. The pressure on each is proportional to its distance from

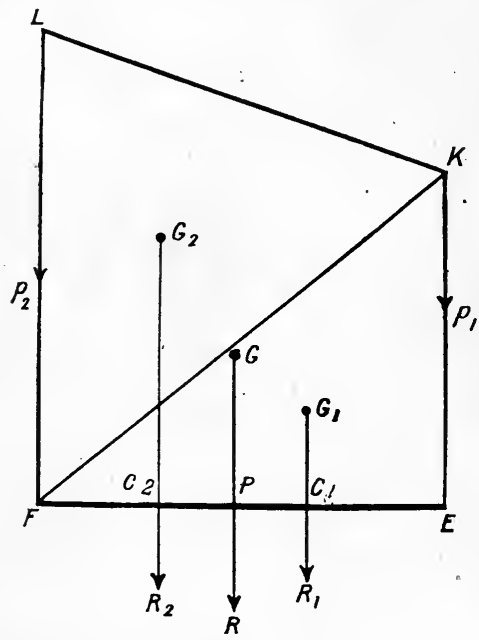

FIG. 11. the surface, and will be represented by the ordinate of the triangle $E F H$ erected on the strip and perpendicular to the area as shown. This triangle may be taken to represent the load diagram. The single resultant of this load will pass through the C. G. of the load area; will be perpendicular to the surface; and will therefore cut the median line $E F$ at a distance from $E$ equal to $\frac{2}{3} E F$, i.e., at $P$.

If the upper edge of the parallelogram be at a depth $h$

below the surface, the load diagram will now be a quadrilateral $E K L F$, such that $L K$ when produced meets $F E$ produced in the surface.

If $E K=p_{1}$ and $F L=p_{2}$, considering the pressure per unit width, we may divide the load area into two, $E K F$ and $F K L$ (Fig. 11).

The resultant of first is $R_{1}=\frac{p_{1}}{2} \cdot E F$.

$$
\text { , }, \quad \text {, second is } R_{2}=\frac{n_{2}}{2} \cdot E F^{\prime} \text {. }
$$

This varying pressure may then be replaced by two single forces $R_{1}$ and $R_{2}$ acting through the C. $\mathrm{G}^{\mathrm{s}}$. $G_{1}$ and $G_{2}$ of the 
two load areas, i.e., at points distance $\frac{1}{3} E F^{\prime}$ from $E$ and $F^{l}$ respectively.

The resultant of these will pass through $P$, where

$$
\frac{C_{1} P}{C_{2} P}=\frac{R_{2}}{R_{1}}=\frac{p_{2}}{p_{1}}
$$

i.e., the centre of pressure divides the middle third of the median line in the inverse ratio of the pressures at the two ends.

The magnitude of the resultant $R=\frac{p_{1}+p_{2}}{2} . E F$.

Also

$$
\begin{gathered}
\frac{C_{1} P+C_{2} P}{C_{2} P}=\frac{C_{1} C_{2}}{C_{2} P}=\frac{\frac{1}{3} E F}{C_{2} P}=\frac{p_{2}+p_{1}}{p_{1}} . \\
\therefore \quad C_{2} P=\frac{\frac{p_{1}}{3} \cdot E F}{p_{1}+p_{2}} \\
\therefore \quad F P=\frac{1}{3} E F\left\{1+\frac{p_{1}}{p_{1}+p_{2}}\right\}=\frac{E F^{2}}{3} \frac{\left(p_{2}+2 p_{1}\right)}{2 R}
\end{gathered}
$$$$
=\frac{E F^{2}}{6 R}\left(p_{2}+2 p_{1}\right)
$$

$$
\therefore p_{2}+2 p_{1}=\frac{6 R}{E H^{\prime 2}} \cdot F P \text {. }
$$

Also

$$
\begin{gathered}
p_{2}+p_{1}=\frac{2 R}{E F}=\frac{2 R \times E F}{E F^{2}} . \\
\therefore \quad p_{1}=\frac{2 R}{E F^{2}}\{3 E P-E F\} \\
p_{2}=\frac{2 R}{E F^{2}}\left\{2 E F-3 F^{\prime} P\right\}
\end{gathered}
$$

If $F P=\frac{E F}{3}$ then $p_{1}=0$.

If $F P=\frac{2 E F}{3}$ then $p_{2}=0$.

I.e., when the resultant acts through either extremity of the middle third of $E F$, the pressure at one end is zero.

For both $p_{1}$ and $p_{2}$ to be positive the C. P. must lie within the middle third. Wherever the pressure intensity varies uniformly acrosis a surface as in this case, this deduction holds true. One particular case, of great importance to the hydraulic engineer, occurs in a masonry dam or retaining wall exposed to 
water pressure on one side. Here the pressure across any horizontal joint varies uniformly, and in order that the jointing material should not be exposed to a tensile stress the C. P. of each joint should lie within its middle third.

Although failure to comply with this condition does not necessarily imply instability, yet this point should be kept in view when designing the dam. This subject is of such importance from the engineering point of view that it is now proposed to discuss briefly the theory of the stability of such structures.

\section{Art. 10.--Stability of Masonry Dams.}

The forces acting on any section of a masonry dam exposed to water pressure on one face are the weight of the section acting vertically downwards, and the pressure of the water everywhere normal to its face. The dam may yield

(1) by overturning about any horizontal section,

(2) by sliding of one horizontal section over that below it,

(3) by yielding of the footings or crushing at any joint, due to excessive compressive stress,

(4) by shear in a vertical plane parallel to the water face of the dam.

Consider a section of the dam, of unit width (Fig. 12). The resultant $R$ of the forces $P$ and $W$ acting on the section above any joint $F E$, and also its line of action $O R$, may be determined by graphical composition of forces as indicated in the figure. Here the force $W$, equal to the weight of the masonry above the joint $E F$, acts vertically through $G$, the C. G. of the mass. The water pressure $P$ is normal to the surface and acts through the centre of pressure of the area from $Q$ to $E$, i.e., at a point $\frac{2}{3} Q E$ from $Q$. The resultant $R$ therefore passes through $O$, the point of intersection of the lines of action of $P$ and $W$. Since $O R$ meets $F E$ in $C$, this is the centre of pressure of the joint, and for stability should lie within its middle third. This ensures that no portion of the joint is under tension. The only condition necessary in order that actual overturning should not take place, is that the centre of pressure should lie somewhere within the joint, so that a joint in which this lies within the middle third cannot yield by overturning. 
For the dam shown in the sketch, $H C P$ shows the line of action of the resultant forces at each joint, taking the weight per cubic foot of masonry to be $175 \mathrm{lbs}$., and since the lines $A L K, B D N$, mark the boundaries of the middle thirds, evidently the first requisite for stability is fulfilled.

A graphical method of determining the complete line of pressures is indicated in Fig. 13. The section is first divided into a series of horizontal strips and the pressures $P_{1} P_{2}$, etc., on each, and the weights $W_{1}, W_{2}$, of each are calculated. I'he directions of $P_{1}, P_{2}$, etc., being known, the magnitudes and directions of the resultant pressures $R_{1}, K_{2}$, etc., on the joints (1), (2), etc., may be graphically determined as indicated in Fig. $13 a$.

Thus $R_{2}$ is the resultant of $W_{2}$, $R_{1}$, and $P_{2}$, i.e., of all the external forces acting about the joint (2).

The direction of the resultant $S_{1}$, $S_{2}$, etc., of each pair of forces

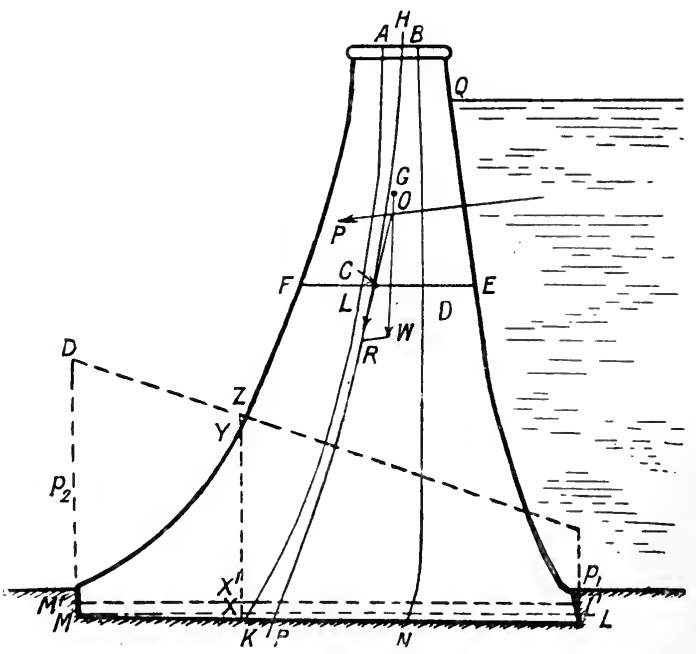

FIG. 12. $W$ and $P$, may next be graphically obtained as shown in Fig. $13 b$. Next, from the intersections of the lines representing the directions of each $P$ and $W$, in the sectional elevation of the dam, draw lines parallel to $S_{2}, S_{3}, S_{4}$, etc., as shown. From the intersection of $P_{1}$ and $W_{1}$ draw a line parallel to $R_{1}$. From the intersection of this with $S_{2}$, draw a parallel to $R_{2}$. From the intersection of this with $S_{3}$, draw a parallel to $R_{3}$ and so on. Then the parallel to $R_{3}$ represents the line of action of the resultant force on the joint (3), both in direction and position. Similarly for the parallels to $R_{1}$ and $R_{2}$, etc.

These lines, if produced to eut their respective joints, will do 
so in the centre of pressure of the joint, these points $C_{1}, C_{3}$, etc., being indicated in Fig. 13 by circles. The smooth curve drawn through these points then gives the line of pressure for the section.

The resultant pressure on any horizontal joint may be resolved into a normal and a tangential component $N$ and $T$, and if $\mu$ be the value of the coefficient of friction at the joint, then in

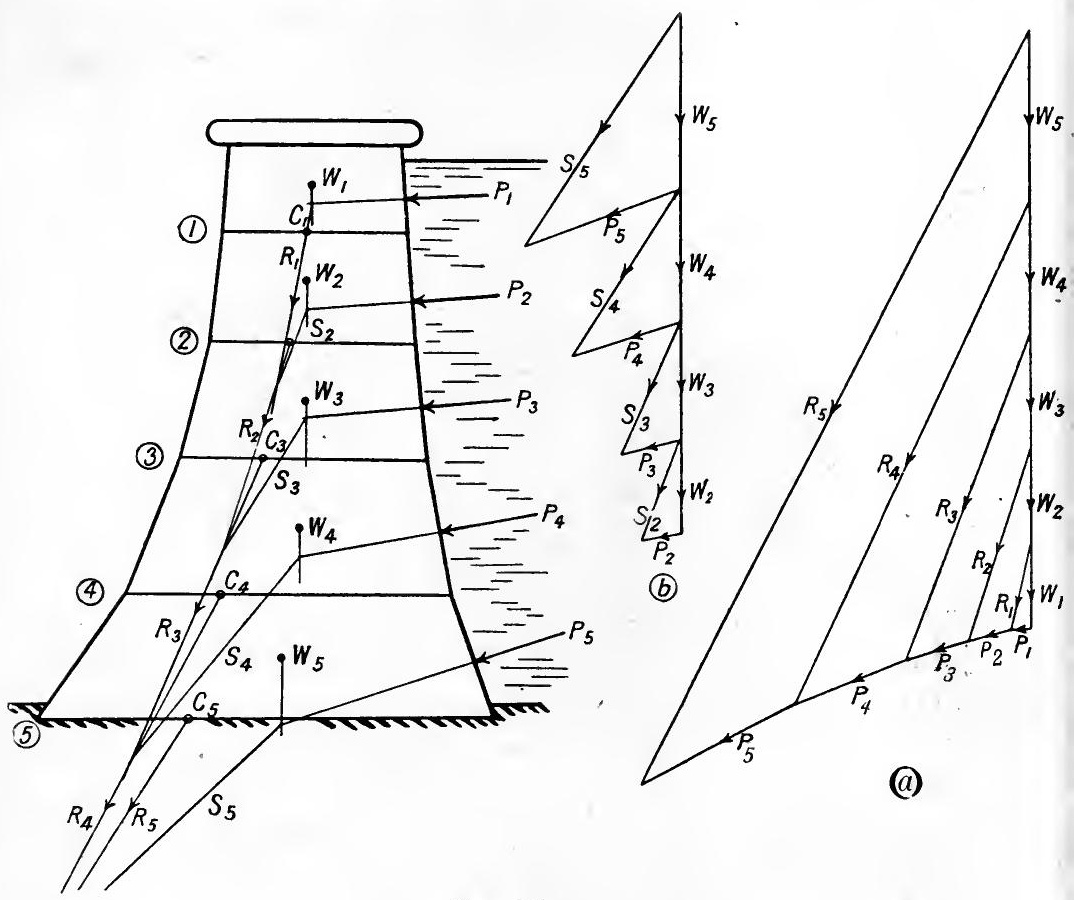

FIG. 13.

order that rupture may not take place by bodily sliding of the upper over the lower portion of the dam, $\mu N$ must be greater than $T$. Special care should be taken to prevent the percolation of water through the foundations, as this may produce a large upward statical pressure over the footings, and will thus tend to relieve the footing of a large proportion of the weight $W$. The effective value of $N$ is thus reduced, and rupture by bodily sliding of the dam may occur.

The normal component $N$, is equivalent to a loading which 
varies uniformly across the joint, and has its maximum value $p_{2}$ at the free end $F$, of the section (Fig. 12), where

$$
p_{2}=\frac{2 N}{E F^{2}}\{2 E F-3 F C\}
$$

and this pressure must not exceed the safe compressive stress which the material can stand. Yielding by crushing will be most liable to take place at the toe $M$ of the dam, and here, in general, the stress should not exceed 6 tons per square foot, this depending on the nature of the soil and of the foundation, and on the depth of $M$ below the surface.

To investigate the conditions for rupture by shear in a vertical plane parallel to the water face, consider any horizontal section $L M$ (Fig. 12), having a centre of pressure $P$. Then if $p_{1}$ and $p_{2}$ be the pressure intensities at $L$ and $M$, we have

$$
\begin{aligned}
& p_{1}=\frac{2 R}{L M^{2}}\{3 M P-L M\} \\
& p_{2}=\frac{2 R}{L M^{2}}\{2 L M-3 M P\}
\end{aligned}
$$

and since the pressure varies uniformly from $L$ to $M$, the area I) $M X \dot{Z}$ represents the total upward pressure over the section $M X$, to the left of any vertical plane $X Y$. The downward force over the same section $=$ weight of masonry $Y M X$ and therefore we are left with a resultant upward fore equal to the difference of these two. This force can only be equilibrated by a shear over the plane $X Y$. The magnitude of this shear stress varies with the height of the point considered, and may be determined if a second horizontal section $L^{\prime} M^{\prime}$ be taken to $L M$, and if the total shear force over the section $X^{\prime} Y$ be found. The difference gives the shear over the small area from $X$ to $X^{\prime}$ of the vertical plane, from which the mean shear stress over this small area may be determined. A similar procedure enables the shear stress at any height in the plane $X Y$ to be found, and for stability the maximum intensity of this shear should not be greater than the jointing material is easily capable of withstanding. ${ }^{1}$

It may be shown that this shear is also accompanied by an

1 For a further investigation into this method of rupture see a paper by L. W. Atcherley on "Some disregarded points in the stability of Masonry dams" (Dulan \& Co., London), and also ar article by Prof. Unwin in Engineering, April 21, 1905. 
equal shear in the horizontal plane passing through the same point.

\section{Art. 11.-Equilibrium OF $^{\circ}$ Floating Bodies.}

When a body is freely floating in a liquid, the conditions of equilibrium may be inferred by imagining the body removed and the space occupied by it to be filled with the liquid. The reactions of the surrounding liquid will be unaltered by the change, the whole being still in equilibrium, and it is clear that the resultant pressure of the surrounding liquid is vertically upwards, and is equal to the weight of the displaced liquid, and also that

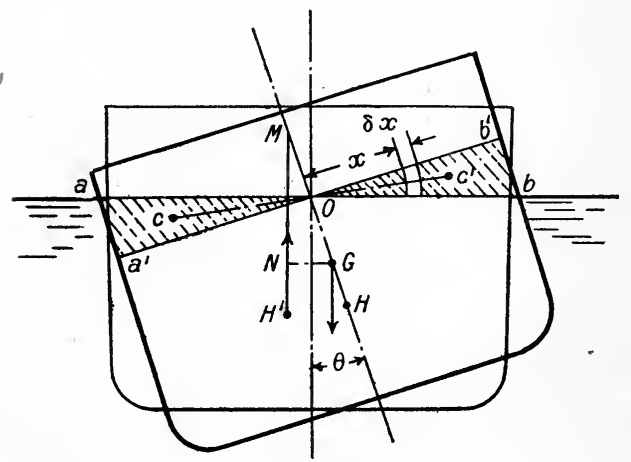

FIG. 14.

the line of action of this resultant pressure passes through the Centre of Gravity of the displaced liquid. It follows that for equilibrium the weight of the floating body is equal to the weight of the liquid which it displaces, and that the Centres of Gravity of the body and of the

displaced liquid are in the same vertical line.

The Centre of Gravity of the displaced liquid is called the Centre of Buoyancy.

Stability of Equilibrium.-If a floating body be slightly displaced from its equilibrium position so that the line joining. the C. G. and the Centre of Buoyancy is no longer vertical, the forces now acting may tend to restore the body to its original position ; to move it still further from that position ; or to maintain it in equilibrium. In the first case the equilibrium is said to be stable; in the second, unstable; and in the third, neutral.

Let $G$ (Fig. 14) be the C. G. of the floating body; $H$ the Centre of Buoyancy when in the equilibrium position; and $H^{\prime}$ the C. B. in the displaced position shown. Through $H^{\prime}$ draw a vertical $H^{\prime} M$, to meet $H G$ in $M$. 
The weight of the body now acts vertically downwards through $G$, and the equal force of buoyancy vertically upwards through $H^{\prime}$, each of these forces being equal to $W$, the weight of the body, and together forming a couple whose arm is $G N$, the perpendicular from $G^{\prime}$ on to $H^{\prime} M$.

Obviously if $M$ is above $G$ this couple tends to restore the body to its equilibrium position, so that the equilibrium is stable, unstable, or neutral, according as $M$ is above, below, or coincides with $G$.

If the angle of displacement $=\theta$, the magnitude of the righting moment $=W \cdot G N=$ $W . G M \sin \theta$.

As $\theta$ is increased the position of the intersection $M$ of the verticals through $H$ and $H^{\prime}$ will in general move and will approach or recede from $G$. The point $M$, for an infinitely small angle of displacement, is called the Metacentre of the body, and the distance $G M$ is called the Metacentric Height.

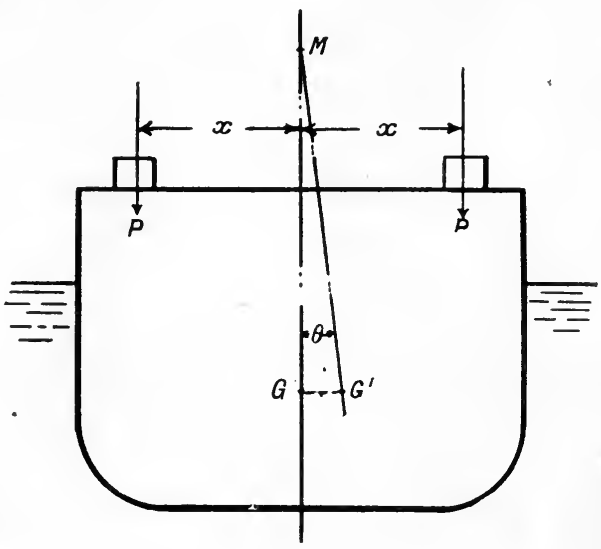

FIG. 15.

Evidently in ship design it is of the highest importance that the metacentre should be above $G$, under all conditions of loading and under any circumstances of rolling.

The height $G M$ may be determined experimentally by placing two equal weights $P$ at equal distances $x$ from the centre line of the vessel, when floating on an even keel and in its equilibrium position.

Let $W$ be the weight of the vessel, including the weights $P$.

Then if both weights be moved through a distance $\delta x$ to the right, the C. G. of the vessel will move through a distance $G G^{\prime}$ (Fig. 15) where

$$
G^{\prime} G=\frac{2 P}{W} \cdot \delta x
$$

H.A. 
Also $G G^{\prime}$ will be parallel to the direction in which $P$ is moved, i.e., will be perpendicular to $G M$, since if any portion of a body be moved in a given direction, the C. G. of the whole moves in the same direction. The truth of these theorems becomes evident if moments be taken, firstly about a vertical line in the plane containing $M G G^{\prime}$, and secondly in the same plane, about a line parallel to the positions $P P$ of the two weights.

If $\theta$ be the angle of heel produced by this shift of the weights $G G^{\prime}=G M \tan \theta$.

$$
\therefore \quad G M=G G^{\prime} \cot \theta=\frac{2 P}{W} \cdot \delta x \cot \theta \text {. }
$$

$\theta$ may be measured by noting the change in inclination of a long pendulum as the weights are moved. The experiment should

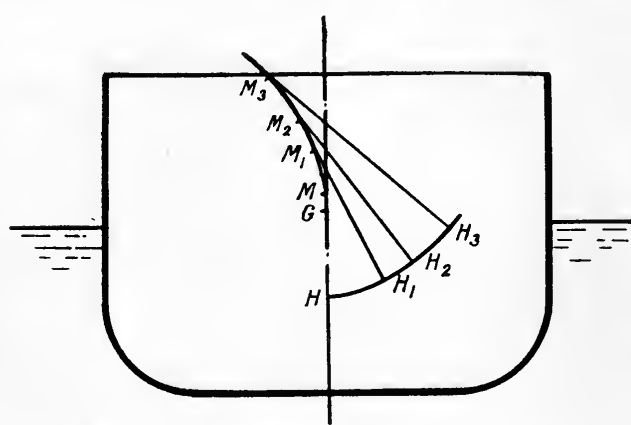

FIG. 16. be repeated for different values of $\delta x$, measured both to the right and to the left and a curve may then be drawn on an angle base, showing values of $G M$. By exterpolation the value of $G M$ in the limit when $\theta=o$ can then be determined.

Since the righting couple $=W \cdot G M \sin \theta$, this equals $2 P \delta x \cos \theta$, so that the same experiment enables us to draw the Stability Curve, showing the value of this righting couple for different angles of heel.

If the body be moved about, its weight, and therefore the volume of water displaced remaining constant, the locus of its Centre of Buoyancy is termed the Surface of Buoyancy. Since, for equilibrium, the vertical through the C.B. must pass through the C. G., and since for small displacements, the line joining two successive positions of the $\mathrm{C}$. B. is parallel to the surface, it follows that the tangent plane to the surface of buoyancy at the C. B. is parallel to the water surface, and therefore that the vertical through the C. G. of the body is normal to the surface of 
buoyancy. In other words, any curve of buoyancy $H_{1} H_{2} H_{3}$ is an involute of the corresponding curve of metacentres $M_{1} M_{2} M_{3}$ (Fig. 16).

It follows that the possible positions of equilibrium of a floating body can be determined by drawing normals from its C. G. to the surface of buoyancy, some such normal being vertical in any position of equilibrium.

In general, in the case of a ship, owing to the fact that the under water contours are not symmetrical about an amidships section, as they are about a longitudinal section, the vertical through the centre of buoyancy in the displaced position will not intersect the line $H G$, since the C. B. is now displaced in a different plane to that of the rotation of the boat. By projecting the verticals through the successive centres of buoyancy on to two vertical planes, one running fore and aft, and the other perpendicular to this, we get one series of intersections on each plane, and thus get two

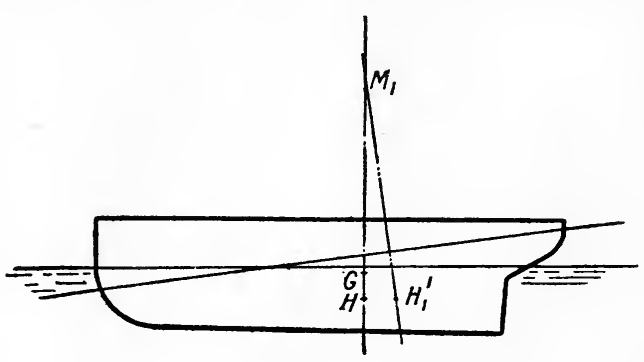

Frg. 17. metacentric heights, the first for pitching displacements (Fig. 17), and the other, previously obtained, for rolling displacements.

The latter is in general, for the ordinary type of ship, by far the more important, although the stability in a longitudinal direction may be seriously affected by the flooding of one or more watertight compartments. In the case of vessels of the submarine type, the longitudinal stability becomes of the greatest importance, lack of such stability causing a tendency to dive suddenly.

The metacentric height for fore and aft displacements may be experimentally determined just as for roiling displacements.

The position of the metacentre may be determined theoretically as follows, if the positions $G$ and $H$ of the C. G. of the vessel and of the centre of buoyancy are known. 
Let $A=$ area of section of vessel made by the plane of water line, or plane of flotation.

, $K=$ radius of gyration of this area about a longitudinal axis through its C. G.

, $V=$ volume of water displaced by vessel.

" $G, H, H^{\prime}, M$, have the meanings previously attached to them.

, O be the elevation of the point of intersection of the planes of flotation in the equilibrium and displaced positions (Fig. 14).

Then if a small angular displacement $\theta$ be given to the vessel,

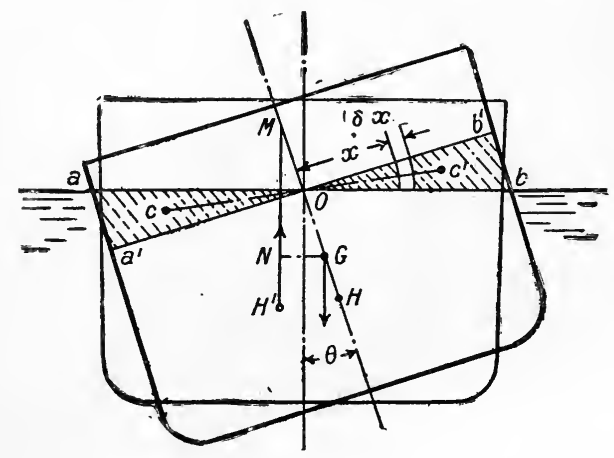

FIg. 14. the volume displaced, being proportional to the weight of the vessel, does not change.

$\therefore$ Volume of wedge a $\circ a^{\prime}=$ volume of $b o b^{\prime}$.

Again if $y$ be the depth, perpendicular to the paper, of any element $\delta x$ of the wedge, at a distance $x$ from $o$, the volume of this element $=y x \theta \delta x$.

$\therefore$ Volume of wedge $a$ o $a^{\prime}=\theta \int_{o}^{a} x y d x$

$\therefore \quad$ Since volume displaced is unaltered we have

$$
\begin{aligned}
& \int_{0}^{a} x y d x=\theta \int_{0}^{b} x y d x \\
\therefore & \int_{-b}^{a} x y d x=0
\end{aligned}
$$

while for this to be true the axis through $O$ must pass through the C.G. of the water line section. Also, due to the immersion of the wedge $a \quad o \quad a^{\prime}$ and the emersion of $b o b^{\prime}$, we get an upward force of buoyancy transferred from C. G. of wedge $b o b^{\prime}$ to that of 
a o $a^{1}$, or since if $w=w t$ of 1 cubic foot of water, the moment of buoyancy due to the immersion of any element of the wedge as shown is $w, x^{2}, \theta, y \delta x$.

$\left.\therefore \quad \begin{array}{l}\text { Moment of buoyancy of } \\ \text { wedges } a \text { o } a^{\prime} \text { and } b \text { o } b^{\prime}\end{array}\right\}=w \theta \int_{-b}^{a} y x^{2} d x$ $=w \theta A K^{2}$

But by the transference of the wedge of water, the centre of buoyancy is moved parallel to $c c^{\prime}$; $c$ and $c^{\prime}$, being the C. $\mathrm{G}^{\mathrm{s}}$. of the wedges; through a distance $H H^{\prime}$, where $H H^{\prime} \cdot V w=w$ $\theta A K^{2}$ (as before).

$$
\therefore \quad H H^{\prime}=\frac{A K^{2}}{V} \theta \text {. }
$$

Also, if $\theta$ is small $\frac{H H^{\prime}}{H M}=\theta$ very nearly.

$$
\therefore \quad H M=\frac{A K^{2}}{V} \text {. }
$$

Knowing $H M$, and determining $G M$ experimentally, $H G$ can be determined, from which, if the position of $H$ is known, that of $G$ may then be found. $H$ may in general be determined with comparative ease, since the contours of a vessel at various levels, and hence the volumes displaced between these levels, are usually accurately known. The problem then simply resolves itself into finding the C. G. of these volumes by taking moments about the water line.

Art. 12.-Tine of Oscillation of a Rolling Ship in Still WATER.

When a floating body is freely oscillating, the resistance of the water being neglected, its motion is similar to that of a pendulum except that the body does not now oscillate about a fixed axis.

Just however as in the case of a pendulum, the righting coup!e is proportional to the sine of the angle of displacement, so that the time of a complete double oscillation is given-as in the case of a pendulum-by

$$
T=2 \pi \sqrt{\frac{\text { Moment of inertia of body about axis of oscillation }}{g \times \text { righting moment for unit angle of displacement. }}}
$$

As the displacement increases, the curve, to which the plane 
of flotation is everywhere tangent, is known as the Curve of Flotation, and evidently in rolling the motion of the body is exactly the same as if an imaginary curve of flotation fixed in the vessel were to roll on a fixed horizontal surface. The position of the instantaneous axis of oscillation may then be determined by noting that since the weight of the vessel and the buoyancy, both vertical, are the only forces acting on the body, the C. G. of the vessel must move vertically, if at all, so that the instantaneous axis is in the horizontal line through $G$ (Fig. 18). Again since the curve of flotation rolls on a horizontal surface, the instantaneous centre must also be in the vertical through the centre of flotation $F$, i.e., the axis is at $O$, the point of inter-

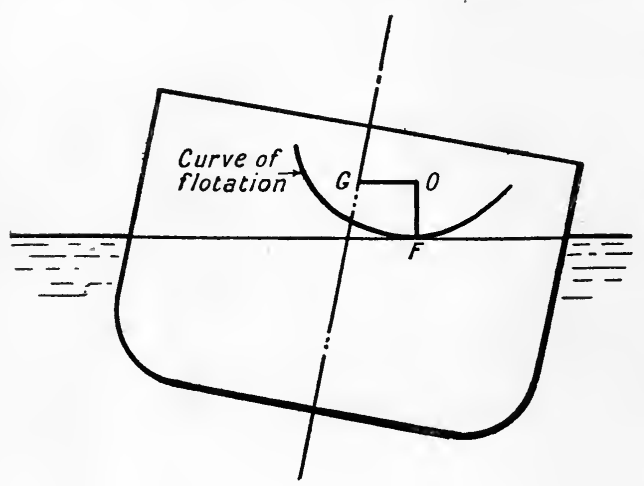

FIG. 18. section of $G \quad O$ and $F^{\prime} O$. The oscillation may then be looked upon as compounded of a rotation about a longitudinal axis through $G$, together with a vertical oscillation of the C. G., the period of rolling being given above.

If $k=$ radius of gyration of the body about an axis through its C. G. and if $m=$ metacentric height for rolling displacements, the equation of motion may be written

$$
\frac{d^{2} \theta}{d t^{2}} \cdot W k^{2}+W m \sin \theta=0
$$

or for small displacements

$$
\frac{d_{2} \theta}{d t^{2}} \cdot \frac{W k^{2}}{g}+W m \theta=0
$$

from which we get $T=2 \pi \sqrt{\frac{k^{2}}{g m}}$, the relation given above.

Although a certain unknown mass of water will move along with the vessel, increasing the inertia of the moving mass without increasing the restoring couple and thus tending to 
increase the time of oscillation, yet in practice very close agreement is found between the calculated and experimental periods.

Ex. In the Devastation, the calculated time was 7 secs.

$$
\begin{aligned}
& \text {, experimental ", } 6.75 \text { secs. } \\
& \text { " Monitor(U.S.N.) calculated " } 2.5 \text { secs. } \\
& \text { " experimental ", } 2.7 \text { secs. }
\end{aligned}
$$

Froude, experimenting on ships fitted with and without bilge keels, ${ }^{1}$ found that the effect of the keel is to extinguish the oscillations more rapidly, but that the effect on the period of rolling is very slight, the average difference produced by the addition of these keels being about 6 per cent. Also the extinctive effect is greater when the ship is moving than when stationary.

The effect of an increase in the metacentric height is to stiffen the vessel and to diminish the period of the oscillation, while any increase in its radius of gyration tends to increase the period. Too great stiffness is inadvisable because of the tendency of the forces brought into play to strain the vessel, and it is often advisable in the case of a cargo vessel to arrange the cargo so that its heavier portions are as near to the skin of the vessel as possible. This increases the radius of gyration, and thus the period of rolling, without seriously affecting the stability.

The metacentric height in the case of merchant ships varies of course with the loading, but when fully loaded is usually between $1 \frac{1}{2}$ and 4 feet. For small vessels such as tugs and torpedo boats it varies from about 1 to 2 feet.

\section{Art. 13.-Strength of Pipes and Cylinders.}

This will be briefly discussed as being of great importance to the hydraulic engineer.

In a thin pipe the stress over the whole thickness of metal may be taken as being sensibly uniform, and by considering a section made by a diametrical plane we see that the force per unit length of pipe tending to rupture it across this plane $=2 r p$, where

1 For an investigation into the action of bilge keels see a paper by Dr. G. H. Bryan, "'Trans. Inst. Naval Architects," 1900. 
$r=$ pipe radius in inches.

$p=$ internal pressure in lbs. per square inch.

If $\quad t=$ thickness of pipe in inches and $f=$ stress per square inch in metal, we have the force resisting rupture along this plane $=2 f t$.

\section{$\therefore$ for equilibrium $f=\frac{p r}{t}$ or $t=\frac{p r}{f^{\prime}}$.}

For steel or wrought-iron pipes this rule is sufficiently accurate, and here the working value of $f$ varies from 7,500 to $8,500 \mathrm{lbs}$. per sq. inch for wrought iron, and 10,000 to 12,000 lbs. per sq. inch for steel, increasing with the size of pipe. In a riveted pipe the efficiency of the riveted joint must be taken into account in determining this thickness. This may be taken at about

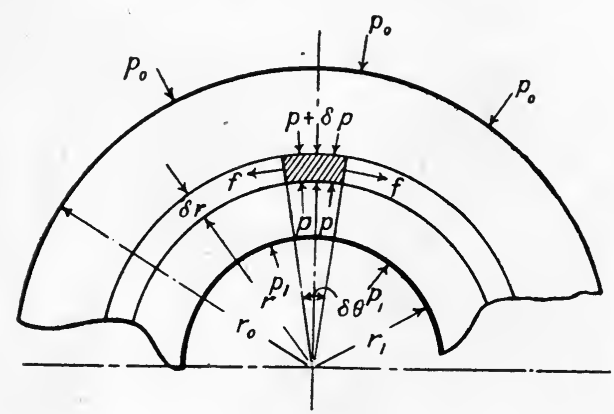

Fig. 19.

55 per cent. for single and 70 per cent. for double riveting. A minimum thickness of about $\frac{3}{16}$ inch should be adopted to allow for any reduction by corrosion.

Where the pipes are to be exposed to considerable pressure, and especially when made of cast iron, the distribution of pressure over the walls is not so simple, those fibres of the metal nearer the centre being more heavily stressed than those further removed. In this case consider the equilibrium of a portion of an elementary ring of metal concentric with the pipe, having inner and outer radii $r$ and $r+\delta r$, and subtending an angle $\delta \theta$ at the centre (Fig. 19). Let the radial pressure on the faces of this element be $p$ and $p+\delta p$, and the circumferential stress in the metal be $f$.

Then, for equilibrium of this element we have

$$
\begin{gathered}
(p+\delta p)(r+\delta r) \delta \theta-p r \delta \theta+f \delta r \delta \theta=0 \\
\therefore \quad r \delta p+p \delta r+f \delta r=0 \\
r \frac{\delta p}{\delta r}=-f-p .
\end{gathered}
$$

or 
Again, if we assume that the plane ends of the pipe remain plane during extension, we get the further condition that

$$
p-f=\text { constant }=2 \mathrm{~A} .
$$

Combining this with (2) we have, in the limit :-

$$
\begin{gathered}
r \frac{d p}{d r}+2 p=2 A \\
r^{2} \frac{d p}{d r}+2 p r=2 A r \\
\therefore \frac{d\left(p r^{2}\right)}{d r}=2 A r .
\end{gathered}
$$

Integrating this expression we get

$$
\begin{aligned}
& p r^{2}=A r^{2}+B \\
& \therefore \quad p=A+\frac{B}{r^{2}}
\end{aligned}
$$

and, from (3)

$$
f=-A+\frac{B}{r^{2}} \text {. }
$$

Assuming, as is generally permissible in practice, that when $r=r_{0}$, i.e., at the outer circumference, $p=o$, and putting $p=p_{1}$ when $r=r_{1}$ i.e., at the inner circumference we get on substituting

$$
\left\{\begin{array}{l}
A=p_{1}\left\{\frac{r_{1}^{2}}{r_{1}^{2}-r_{0}^{2}}\right\} \\
B=p_{1}\left\{\frac{r_{0}^{2} r_{1}^{2}}{r_{0}^{2}-r_{1}^{2}}\right\}
\end{array}\right.
$$

Substituting these values in (6) we have

$$
f=p_{1} \frac{r_{1}^{2}}{r^{2}}\left\{\frac{r^{2}+r_{0}^{2}}{r_{0}^{2}-r_{1}^{2}}\right\}
$$

Evidently this will have its maximum value when $r=r_{1}$, i.e., at the inner circumference, and at this point.

$$
f_{\text {(max. })}=p_{1} \frac{r_{1}^{2}+r_{0}^{2}}{r_{0}^{2}-r_{1}^{2}}
$$

This may be written

$$
r_{1}=r_{0} \sqrt{\frac{f+p_{1}}{f-p_{1}}}
$$

The value adopted for the working stress $f$ varies with the pipe diameter, and with cast-iron pipes increases gradually from about 2,000 lbs. per sq. inch in a 2-inch pipe to 3,000 lbs. per sq. inch 
in an 8-inch pipe, and 3,500 lbs. per sq. inch in a pipe or cylinder of 24 inches diameter.

The following table indicates the thickness of cast-iron pipes and cylinders for heavy pressures, as calculated on the above assumption.

\begin{tabular}{|c|c|c|c|}
\hline \multirow{2}{*}{$\begin{array}{l}\text { Pipe diameter } \\
\text { in inches. }\end{array}$} & \multicolumn{3}{|c|}{ Working pressure, lbs. per square inch. } \\
\hline & 750 & 1000 & 1120 \\
\hline 2 & $\frac{1}{2}$ & $\frac{3}{4}$ & $\frac{7}{8}$ \\
\hline 3 & $\frac{11}{16}$ & $\frac{15}{15}$ & $11^{8}$ \\
\hline 4 & $\frac{10}{16}$ & $1_{8}^{\frac{1}{1}}$ & $1 \frac{8}{8}$ \\
\hline 5 & $\frac{29}{32}$ & $1 \frac{5}{16}$ & $1 \frac{9}{16}$ \\
\hline 6 & $1 \frac{1}{32}$ & $1 \frac{7}{16}$ & $1 \frac{23}{32}$ \\
\hline 8 & $1 \frac{5}{32}$ & $1 \frac{10}{1} \frac{1}{6}$ & $1 \frac{29}{32}$ \\
\hline 10 & $1 \frac{13}{32}$ & $2 \cdot 0$ & $2 \frac{5}{16}$ \\
\hline 12 & $1 \frac{23}{32}$ & $2 \frac{5}{16}$ & $2 \frac{2}{3} \frac{1}{2}$ \\
\hline 16 & $2 \frac{5}{32}$ & $2 \frac{1}{1} \frac{5}{6}$ & $3 \frac{1}{3} \frac{3}{2}$ \\
\hline 20 & $2 \frac{5}{8}$ & $3 \frac{9}{16}$ & $4 \frac{1}{8}$ \\
\hline 24 & $3 \frac{1}{32}$ & $4 \frac{1}{8}$ & $4 \frac{3}{4}$ \\
\hline
\end{tabular}

\section{Examples.}

1. In an hydraulic press the ram is $\mathbf{1 0}$ ins. and the pump plunger 1 in. diam., the leverage for working the pump 16 to 1 . What is the velocity ratio of the plunger and ram? Actually a force of $30 \mathrm{lbs}$. exerts a pressure of $44,000 \mathrm{lbs}$. on the press table What is the efficiency?

$$
\text { Ans. : } 1,600 ; 91 \cdot 7 \text { per cent. }
$$

2. A dock gate is $12 \mathrm{ft}$. broad and $14 \mathrm{ft}$. deep, and the water rises $12 \mathrm{ft}$. on one side and $3 \mathrm{ft}$. on the other side above its lower edge. Find the resultant pressure and centre of pressure for each side of the gate and find the magnitude and position of the resultant of these.

Ans.: 53,914 lbs.; 3,370 lbs.; centres of pressure $4 \mathrm{ft} . ; 1 \mathrm{ft}$. from lower edge.

Single resultant $50,544 \mathrm{lbs}$. at $4 \cdot 20 \mathrm{ft}$. from lower edge.

3. A rectangular sluice gate, $5 \mathrm{ft}$. broad and $6 \mathrm{ft}$. deep, having its upper edge at a depth of 4 feet, is inclined at $45^{\circ}$ to the 
vertical and admits water to an empty penstock. The sluice is lifted by a force applied parallel to its plane. Determine the necessary magnitude of this force if the coefficient of friction between gate and guides is $\cdot 12$.

\section{Ans. : 1,375 lbs.}

4. A circular conduit, $6 \mathrm{ft}$. diam., which just runs full, is fitted with a sluice, and it is required to balance this about a horizontal axis. Show that this axis should be placed $2 \mathrm{ft} .3 \mathrm{ins}$. above the bottom of the conduit.

5. A vertical wall, $10 \mathrm{ft}$. high and $3 \mathrm{ft}$. thick, is exposed to water pressure on one side. What is the maximum depth of water in order that the lowest joint should not be anywhere under tension? Weight of masonry per cubic ft. $=170 \mathrm{lbs}$.

Ans. : $6 \cdot 26 \mathrm{ft}$.

6. The Furens Dam has a height of $164 \mathrm{ft}$. The breadths corresponding to various heights are :-

\begin{tabular}{|c|c|c|c|c|c|}
\hline Height (ft.) . & 0 & 50 & 100 & 150 & 164 \\
\hline Breadth (ft.) . & 161 & 88 & 40 & 20 & 18 \\
\hline $\begin{array}{c}\text { Batter of front face } \\
\text { (ft.) }\end{array}$ & 0 & 20 & 35 & 38 & 38 \\
\hline
\end{tabular}

Sketch the line of pressure for the dam, both full and empty.

7. 'The new Croton Dam has a total depth of $224 \mathrm{ft}$., the lower $72 \mathrm{ft}$. of which is imbedded back and front in earth. The dimensions are approximately as follows :-

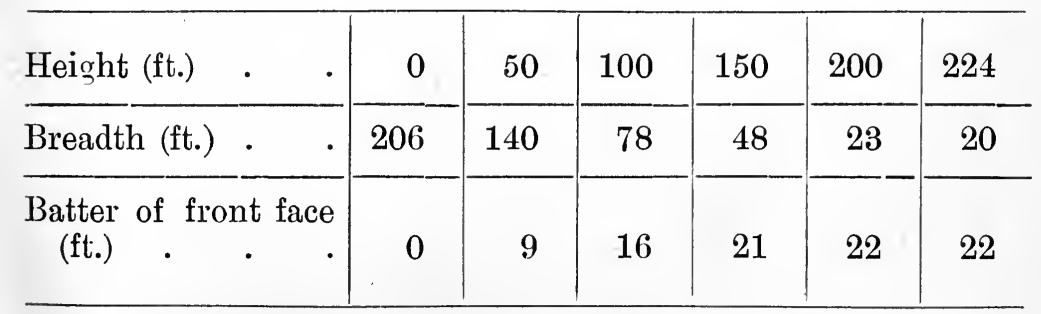

Sketch the line of pressure for the dam when full. 
8. A battleship weighs 13,000 tons. On filling the ship's boats on one side with water-this weighing 60 tons and its mean distance from the centre of the boat being $30 \mathrm{ft}$. - the angle of displacement of a plumb line is $2^{\circ} 16^{\prime}\left(\tan \theta={ }^{0396}\right)$. Determine the metacentric height for rolling displacements. Ans.: $3 \mathrm{ft} .6$ ins.

9. Show that a solid cylinder of length $l$, radius $r$, and specific gravity $s$, floating with its axis vertical, is in stable equilibrium if

$$
r>l \sqrt{2 s(1-s)} .
$$

10. A single-riveted steel pipe, 30 ins. internal diameter, is exposed to a head of 450 feet of water. Taking $f=12,000 \mathrm{lbs}$. per square inch, and the efficiency of the joints $=60$ per cent., what should be its thickness?

$$
\text { Ans. : } t=\cdot 407 \text { in. }=\frac{13}{32} \text { in. }
$$

11. A cast-iron pressure pipe, 4 ins. diameter, is exposed to a pressure of $1,100 \mathrm{lbs}$. per square inch. Taking $f=2,800 \mathrm{lbs}$. per square inch what is the requisite thickness?

$$
\text { Ans. : } t=1.032 \text { in. }=1 \frac{1}{32} \text { in. }
$$

12. The mean pressure over the horizontal section of a dam is 5 tons per square foot. The centre of pressure over this section is at a point distant $\frac{3}{8}$ of its width from the down-stream edge. Determine the maximum and minimum pressures on the section.

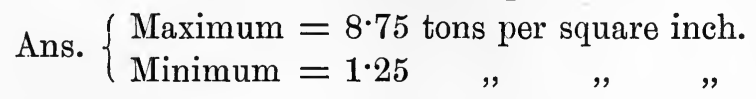

13. It is proposed to subject the lower $9 \mathrm{ft}$. of a wall $17 \mathrm{ft}$. high and $5 \mathrm{ft}$. thick, weighing $150 \mathrm{lbs}$. per cubic foot, to water pressure. Determine the centre of pressure on the lowest horizontal section.

Ans. : At a distance from the centre of the section $=7 \cdot 14$ ins. 


\section{SECTION II.}

\section{CHAPTER III.}

Motion of Viscous Fluids-Stream Line and Eddy Motion-Vortices-Conditions regulating the two modes of Motion-Reynolds's Researches-Critical Velocity -Flow against Viscous Resistance-Between Parallel Plates-Through Circular Tube.

\section{Art. 14.-Motion of Viscous Fluids.}

When a rigid body moves, the motion at any particular point, whether inside or on the surface of the body, can be accurately. determined once the motions of any two points in the body are fixed. In this case then, when the boundary conditions are known, the determination of the internal motion is simple. But with a fluid such as water the motion of the mass of fluid as a whole does not necessarily give the internal motion. While giving the mean motion, it does not fix the absolute motion of each particle of the fluid relatively to any fixed point. Even when a mass of water is apparently at rest its internal molecules are usually in a state of relative motion. This may be shown by the careful introduction of a drop of dye-coloured water to the interior of such a mass. The boundaries of this drop are at first sharply defined, and the first indication of motion is that shoots are gradually sent off from its surface, these ultimately terminating in small vortices. The boundaries in the meantime gradually lose their sharpness of definition owing to diffusion, and finally the colouring matter will be found diffused uniformly over the whole mass of water.

By the earlier experimentalists the motion of water in mass was imperfectly understood. On the assumption of a motion simple and analogous to that of a rigid body, it was impossible to reconcile the results experimentally obtained by many observers, while the very transparency, or uniform opacity 
of most fluids made it impossible to see the internal motion. Probably the first indication of there being more than one kind of fluid motion was obtained from the appearance of the free surface of flowing water. Where not otherwise disturbed this may have two appearances, corresponding to different modes of motion. In the one, objects are reflected from the surface without distortion, while with the other an irregular motion of the surface may be noted, and reflection is accompanied by distortion. Where motion occurs in a passage having solid lateral boundaries however, even this indication is absent, and the introduction of floating particles of solid matter does not help to any extent in showing the nature of the action which is taking place.

Matters remained in this state for many years, and it remained for Professor Reynolds, by his method of colour bands, ${ }^{1}$ to prove conclusively that the motion in a mass of water may be of two kinds; to make clear the simplicity of the one, and the complexity of the other; and to demonstrate the reasons for, and the laws governing each kind of motion.

The conclusions to be drawn from Professor Reynolds's experiments are as follows:-firstly, we may have a continuous steady motion of the particles, in which the motion at a fixed point always remains constant; and secondly, we may have unsteady or $e d d y$ motion, when the motion at any fixed point varies according to no definite law. This is due to the formation of eddies or vortices in the fluid.

Introducing the idea of stream lines, i.e., of imaginary lines in the fluid, such that at any point the direction of motion is tangential to the line, it follows that in steady motion these stream lines become fixed, and this type of motion is therefore linown as stream line motion. Certain properties of these stream lines are of interest. They must always have a continuous curvature, except where the motion is zero, since to cause an infinite change of curvature an infinite force acting perpendicularly to the direction of curvature would be necessary. It follows that in steady motion a fluid will always move in a curve round any

1 For a full account of this method of investigating the two manners of motion of water see a paper by Osborne Reynolds, " Phil. Trans. Royal Society," 1883. 
sharp corner, and that the stream lines will be tangential to any such boundaries, as indicated in Fig. $20 a$ and $b$, in which the general form of the stream lines for steady flow out of two forms of orifice are shown. With a very viscous fluid an approximation to this infinite force may be introduced by the effect of cohesion, and the radius of curvature may then become very small. This was clearly shown in a series of experiments by Professor Hele Shaw, ${ }^{1}$ who, by producing flow between two parallel glass plates at an extremely small distance apart, produced a state of affairs in which viscosity was the predominating factor. In ordinary cases however, this is not so, and the effect of the inertia of the fluid is more marked than that of cohesion. On this account it

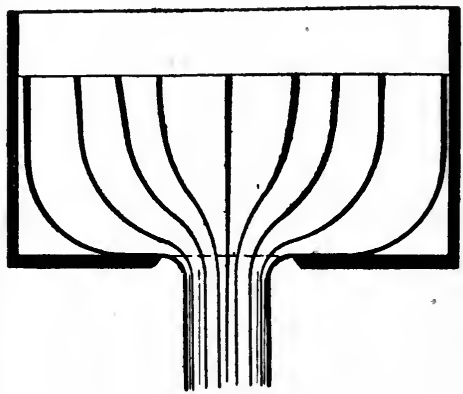

(a)

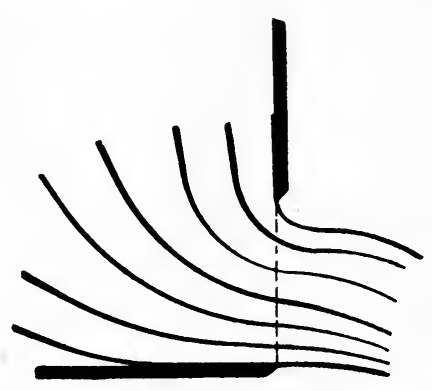

(b)

FIG. 20.

is always necessary for easy flow to design any pipe or passage for conveying fluids with curves having as gradual a curvature as possible.

If the stream line be imagined to form the axis of a tube of finite sectional area having imaginary boundaries, and such that its area, at different points in its length, is inversely proportional to the velocity at those points, this is known as a stream tube.

If the motion at a fixed point varies, and if it is still possible to find a definite motion for these points such that the motion of the fluid relative to the points may remain constant, the latter may be reduced to steady motion by considering the motion relative to these moving points. The fluid may then be said to have a continuous, steady, but unequal motion.

' "Trans. Inst. Naval Architects," 1897-8, 1900. 
In almost all the cases of fluid motion which are of practical importance in hydraulics, the motion is found to be unsteady.

\section{Art. 15.-Vortices.}

A mass of fluid, rotating about some axis in the fluid itself and forming a closed circuit, is termed a vortex or eddy.

All unsteady fluid motion is accompanied by the production of vortices, for as is usual in nature the fluid adopts the method of motion which necessitates the least loss of energy, and which is found in vortex motion.

An instance of this may be found in the case of the ordinary smoke ring, which, by the expenditure of a comparatively small amount of energy, may be projected for great distances through the surrounding air. A still more striking instance may be found in the behaviour of a similar vortex ring, a vortex having an endless circular axis, in water. If this vortex ring be coloured by the admission of aniline dye to the generating box, and if the ring be then projected through a tank of clear water, it is seen to travel with a motion of uniform rotation about its circular axis through the surrounding liquid, its outer layers moving, relatively to the axis, in the opposite direction to that of its own motion of translation. Relatively to the surrounding water however the motion at the outer layers is very small, so that the vortex moves through the surrounding fluid with a resistance almost akin to rolling friction. 'This accounts for the small resistance experienced. The surrounding water is displaced in a direction at right angles to that of the ring's translation, and thus with a ring moving horizontally waves are produced on the surface of the water.

That the relative motion between the outside layers of the vortex and the surrounding water is very small is shown by the slowness with which the coloured water of the ring diffuses.

An attempt to propel a solid sphere of approximately the same size and mass as the ring through the fluid by means of a sudden blow, will show very forcibly the relative loss of energy as compared with that of vortex motion.

The whole subject of the translatory motion of vortices is 
fraught with difficulty, and no attempt will be made here to discuss this motion analytically. 'The following may be taken as a partial explanation of the reason of this translation. Consider a vortex ring at the instant of formation. The velocity at the interior of the aperture is greater than that outside; the pressure inside is therefore less than that outside; and in. consequence the ring begins to contract. The effect of this contraction of the aperture is to set up motion in the surrounding fluid, which, combined with the cyclic motion inseparable from vortex production, increases the velocities in front of, and decreases those behind the ring.

This sets up a difference of pressure at similar points before and behind the ring, which urges the latter forward with an increasing velocity. A limit to this velocity is reached when the velocity within the aperture approximates to that without. ${ }^{1}$

The mass of fluid forming a vortex has the property that its momentum is unaffected by its angular motion, just as the momentum of a fly-wheel in any direction is unaltered by the fact of the wheel itself rotating about its own axis, the momentum of the mass in any direction being equal to the mass multiplied by the resolved part of the velocity of its mass centre in that direction.

\section{Art. 16.-Conditions regulating the two manners} of Motion.

Several conditions combine to determine whether in any particular instance the motion of water shall be steady or unsteady. Osborne Reynolds, "Phil. Trans. Royal Society," 1883, came to the conclusion that the conditions tending to stability and steadiness of motion are :-

(1) An increase in viscosity.

(2) Converging solid boundaries.

(3) Free (exposed to air) surfaces.

(4) Curvature of the path, with the greatest velocity at the outside of the curve.

1 For an extension of this idea and for an investigation into the motion of vortices, consult a paper by Mr. W. M. Hicks, "Phil. Trans. Royal Society," 1884 , p. 161.

H.A. 
Experiments carried out at McGill University show that an increase in pressure also tends to stability of motion.

The conditions tending to instability and unsteadiness of motion are :-

(1) A decrease in viscosity.

(2) Solid (rigid tangentially) boundaries in general and particularly diverging solid boundaries.

(3) A stream of fluid flowing through fluid at rest.

(4) Curvature with the greatest velocity at the inside of the curve.

(5) Greater density of the fluid.

Thus an increase in the temperature of water, by diminishing its viscosity, increases its liability to unsteady motion.

The effect of solid boundaries in producing instability would

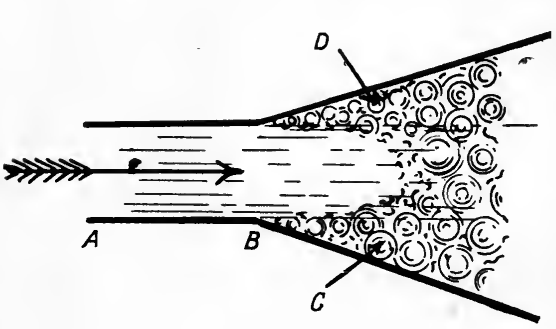

FIG. 21. appear to be due rather to their tangential than their lateral stiffness. One very remarkable instance of this effect of boundaries possessing tangential stiffness, however small this may be, occurs when a film of oil is allowed to form on the surface of water. Here the oil film exerts a small but appreciable tangential constraint with the result that motion which was originally stable becomes unstable.

This results in the formation of eddies below the surfaces of the oil and water, and the energy which was originally imparted by the action of the wind to form and maintain stable wave motion is now applied to the institution of this eddy motion, with the well-known result as to the stilling of the waves.

This effect may be studied on a large sheet of water by introducing colour bands between the surfaces of the oil and water.

Where one stream of liquid is in contact with a second stream moving with a different velocity, the common surface of separation is found to be in a most unstable condition. This was shown by allowing the two liquids, Carbon Bisulphide and water, to form 
a horizontal surface of separation in a long horizontal tube. The tube was then slightly tilted so as to produce a relative axial motion of the fluids, when it was found that for extremely small values of the relative velocity the motion was unstable.

In this manner may be explained why diverging boundaries are a cause of instability. In such a case as that shown in Fig. 21, the motion from $\mathrm{A}$ to $\mathrm{B}$ in the parallel portion of the channel may be perfectly steady, depending on the dimensions of the channel and the velocity of flow. On leaving B, however, at any but the smallest velocities the water appears to be projected, in the form of a core of the same dimensions as the channel, through the mass of dead water at $\mathrm{C}$ and $\mathrm{D}$. Here all the conditions necessary for instability are present.

The determination of the constants involved in the case of the stability of motion in flow through a parallel tuke has been accurately determined, but not in the case of a converging or a diverging tube. At sufficiently low velocities we know that the motion is steady in any case, but in converging or diverging tubes the angle of inclina-

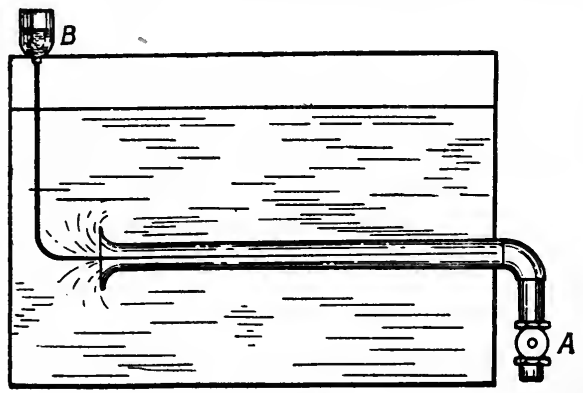

FIG. 22. tion of the sides has a great deal to do with determining the velocity at which stable becomes unstable motion.

The exact effect of altering this inclination is still unknown, except that increasing the angle of divergence rapidly diminishes the stability, while in converging tubes stability of motion rapidly increases with the angle of convergence. At all ordinary velocities, in fact, the motion in tubes or nozzle shaving more than a few degrees of convergence may be considered as being steady.

\section{Art. 17.-Critical Velocity.}

The experiments by which Professor Reynolds demonstrated the nature of the two modes of motion of water were carried 
out on glass tubes of various diameter up to 2 in., and abont $4 \mathrm{ft} .6$ in. long. These were fitted with bell-mouth entrances, and were immersed horizontally in a tank of clear water having glass sides (Fig. 22). In carrying out the experiment the water in the tank is allowed to come absolutely to rest, and the valve $\mathrm{A}$ is then slightly opened, allowing water to flow slowly through the tube. A little water, coloured with aniline dye, is introduced into the mouthpiece through a fine tube supplied from the vessel $B$.

At first this coloured water is drawn out into a single stream tube, extending through the whole length of the tube, as shown in Fig. 22, the whole appearing to be motionless unless a slight motion of oscillation is given to the water in the supply tank,
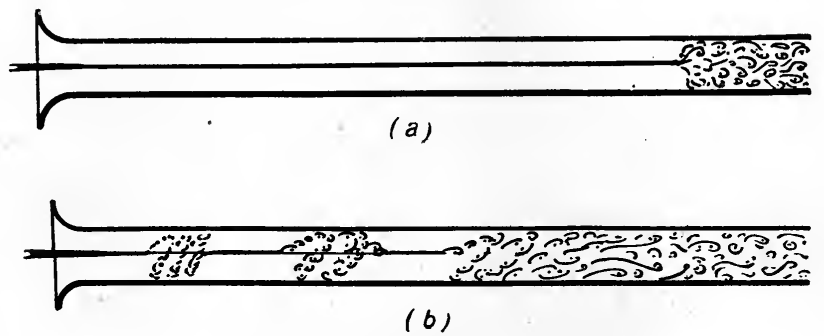

Fig. 23.

when the stream line sways gently from side to side, but without in the least losing its definition. As the valve $A$ is further opened the velocity through the tube increases, and the stream tube is drawn out more and more, still retaining its definition, until at a certain velocity eddies begin to be formed intermittently near the end of the tube more remote from the mouthpiece (Fig. $23 a$ ).

The formation of these eddies is very clearly denoted and is accompanied by the almost instantaneous diffusion of the colour band. As the velocity is still further increased the point of initiation of eddy motion advances towards the mouthpiece, this point being fixed for any one velocity. Finally the motion throughout becomes unsteady (Fig. $23 \mathrm{~b}$ ).

Any initial disturbance of the water tends to reduce the velocity at which the motion changes from steady to unsteady, and which is termed the Critical Velocity. Further reference will be made to the results of these experiments (Art. 62), but in the 
meantime it may be stated that they point to the fact that when water is in motion in any pipe or channel, its motion is either steady or sinuous according as the velocity is below or above a certain critical value, this value depending largely on the dimensions of the channel; also that the law of resistance to the motion of such a body of water changes suddenly when the critical velocity is attained, the resistance being directly proportional to the velocity for steady motion, and approximately proportional to the square of the velocity for sinuous motion.

\section{Art. 18.-Flow against Viscous Resistance.}

The resistance to steady motion of a fluid through a pipe or channel is simply due to the viscosity of the fluid, and not to any actual slip at the boundary of solid and fluid if the adhesive force at the boundaries is sufficiently great to overcome the shear in the fluid at these points. That this is true, at all events in the case of water at pressures slightly above atmospheric, may be shown by immersing a glass tube horizontally in water and introducing a drop of aniline dye to the bottom of the tube. If now water be allowed to flow through the tube, the coloured matter in contact with the side will not be removed so long as the motion remains steady. Directly the motion becomes unsteady, however, rapid diffusion takes place, and the colouring matter at once disappears. It follows from this that with steady motion the resistance is independent of the nature of the solid surface. With unsteady motion, on the other hand, there would appear to be an actual interchange, by the breaking down of adhesion, of molecules in intimate contact with the boundaries (Art. 67), and since any such interchange will be greater as the roughness of the surface increases, and will vary with the material of the pipe, it might be inferred that resistance to unsteady motion will depend on the state and material of the surface, and will increase as its roughness increases. ${ }^{1}$

1 Experiments by Mr. J. Morrow, "Proc. Royal Soc.," vol. 76, 1905, p. 205, seem to show that at velocities slightly in excess of the critical slip does not take place at the boundary, but that near this point the velocity falls very rapidly to zero. Other experiments, however (see Art. 67), indicate that at higher velocities slip actually does take place, and on any other assumption it appears difficult to explain why the material of a pipe, apart from its roughness, affects the resistance to flow. 
Art. 19.-Steady Flow between Horizontal Parallel Plates.

Take $O X, O Y, O Z$, co-ordinate axes in the fluid (Fig. 24). Let the direction of motion be parallel to the axis of $x$, and the plates perpendicular to that of $y$. Suppose the boundaries in the direction of $O Z$ to be infinitely distant, so as not to affect the motion, and neglect the effect of gravity. Let $2 h=$ distance between plates, and let $O X$ bisect this distance.

Let $u, v$, and $w$ denote velocities of flow in directions $O X, O Y, O Z$. Then $v=o, \therefore$ if $p$ is the intensity of normal pressure on any plane perpendicular to $O X$ we have $\frac{d p}{d y}=0$. Also the variations of velocity in the direction $O Z$ may be neglected, since the boundaries in this direction, and therefore the points at which

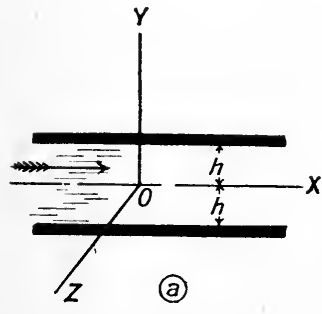

FIG. 24.

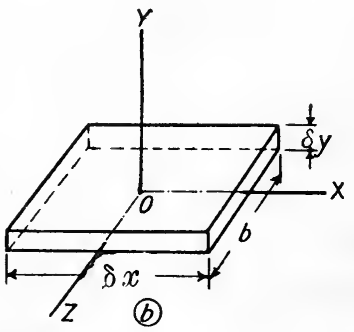

(b) the pressure may be zero, are at an infinite distance.

It follows that $\frac{d p}{d z}=0$, so that on any plane perpendicular to $O X$ the intensity of normal pressure is uniform.

The tractive-or shear-force on any plane perpendicular to $O Y$ and of area $A$ is $\mu \frac{d u}{d y} \cdot A$, where $\mu=$ coefficient of viscosity (Art. 4).

The difference of tractive force on the two faces of a stratum of thickness $\delta y$, and of area $A$ (Fig. $24 b$ ), will thus be given by

$$
\frac{d}{d y}\left\{\mu \frac{d u}{d y} \cdot A\right\} \delta y=\mu \frac{d^{2} u}{d y^{2}} A \delta y,
$$

i.e., by the rate of change of this force with respect to $y$, multiplied by the change in $y$.

But if $b$ is the width of the stratum, and $\delta x$ its length, the area of each end $=b \delta y$, while its area $A=b \delta x$.

Also, since for equilibrium the difference of traction on the two faces of the stratum is equal to the difference of normal pressures on the two ends, we get 


$$
\begin{gathered}
b . \delta y \cdot \frac{d p}{d x} \cdot \delta x=\mu \frac{d^{2} u}{d y^{2}} \cdot A \delta y=\mu \frac{d^{2} u}{d y^{2}} \cdot \delta \delta x \delta y \\
\therefore \frac{d p}{d x}=\mu \frac{d^{2} u}{d y^{2}} .
\end{gathered}
$$

Integrating this expression, we get

$$
u=C+B y+\frac{1}{2 \mu} \cdot y^{2} \cdot \frac{d p}{d x}: \begin{array}{r}
\text { where } C \text { and } B \\
\text { are constants. }
\end{array}
$$

Since the motion is symmetrical about the axis of $x$, i.e., is the same for equal positive and negative values of $y$, the term involving the first power of $y$ must vanish, since this would change signs with $y$, so that $B=o$. Again, for uniformity of flow $\frac{d p}{d x}$ is constant, while, assuming no slip at the boundaries, we have $u=o$ for $y= \pm h$.

Determining the constant $C$, so that these boundary conditions are satisfied, we get

$$
u=-\frac{1}{2 \mu}\left\{l^{2}-y^{2}\right\} \frac{d p}{d x}
$$

i.e., the pressure diminishes as $x$ increases. Evidently, too, from the form of the equation the curve of velocities is a parabola.

The flux over unit width of the plates is given by

$$
\int_{-h}^{h} u d y=-\frac{2}{3} \frac{h^{3}}{\mu} \cdot \frac{d p}{d x} .
$$

If the measurements are taken in pounds, feet, and seconds, the volume per second in cubic feet, $Q$, is given by

$$
Q=\frac{2}{3} \frac{h^{3}}{\mu} \cdot \frac{d p}{d x} .
$$

- If $p_{1}-p_{2}=$ fall of pressure between two points at a distance $l$ apart, this becomes

$$
Q=\frac{2}{3} \frac{h^{3}}{\mu} \cdot \frac{p_{1}-p_{2}}{l}
$$

The maximum velocity occurs at the axis, where $y=0$, and equals $\frac{1}{2 \mu} \cdot l^{2} \cdot \frac{d p}{d x}$.

The mean velocity $=\frac{\text { flux over a section }}{\text { area of section }}=\frac{Q}{2 h}=\frac{1}{3 \mu} \dot{h}^{2} \frac{d p}{d x}$ 


$$
\therefore \frac{\text { Maximum velocity }}{\text { Mean velocity }}=\frac{3}{2}
$$

The shear stress on the boundaries is given by

$$
\mu \frac{d u}{d y}=y \frac{d p}{d x}=h \cdot \frac{p_{1}-p_{2}}{l} .
$$

Art. 20.--Stéady. Flow through a Circular Pipe.

Suppose the pipe to be horizontal, and, neglecting the effect of gravity, assume the flow to be produced by a uniform difference of pressure head along its length. Let the axis of $x$ be the axis of the tube, and let $a$ be its radius. Using the same notation as in the preceding example, and assuming the velocity everywhere parallel to $O X$, we have $v=o, w=o$

$$
\therefore \quad \frac{d p}{d y}=0 ; \frac{d p}{d z}=0 \text {, as in the previous case. }
$$

The velocity $u$ will be a function of the radius $r$.

The tangential stress, or tractive force per unit area, on a plane perpendicular to a radius $=\mu \frac{d u}{d v}$.

Hence for a cylindrical shell concentric with the pipe, of length $\delta x$ and having inner and outer radii $r$ and $r+\delta r$, the difference of the tangential force on the inner and outer surfaces will be $-\frac{d}{d r}\left\{2 \pi r . \delta x \cdot \mu \frac{d u}{d r}\right) \delta r$, and this must be balanced by the difference of normal pressure on the two ends of the shell.

Since $\frac{d p}{d r}=o$, the pressure intensity $p$ at any point of the section is uniform.

$$
\begin{gathered}
\therefore \quad 2 \pi r . \delta r \cdot \frac{d p}{d x} \cdot \delta x=-\frac{d}{d r}\left\{2 \pi r . \delta x \cdot \mu \frac{d u}{d r}\right\} \delta r \\
\therefore \frac{d}{d r}\left\{r \frac{d u}{d r}\right\}=-\frac{d p}{d x} \cdot \frac{r}{\mu} .
\end{gathered}
$$

Integrating, we get

$$
u=-\left\{\frac{1}{4 \mu} \frac{d \dot{p}}{d x}\right\} r^{2}+A \log r+B
$$

Since the velocity at the axis, where $r=o$, cannot be infinite and since $\log o=$ inf., we get $A=\dot{o}$. 
Determining $B$, so that $u=o$ when $r= \pm a$, i.e., for no slip at the boundaries, we get

$$
u=-\frac{1}{4 \mu} \cdot \frac{d p}{d x} \cdot\left\{a^{2}-r^{2}\right\}
$$

So that the flux through the pipe, which

$$
=\int_{0}^{a} u \cdot 2 \pi r d r,=\frac{\pi a^{4}}{8 \mu} \cdot \frac{d p}{d x} \text { cubic feet per second. }
$$

Writing $\frac{d p}{d x}$ as $\frac{p_{1}-p_{2}}{l}$, where $p_{1}$ and $p_{2}$ are the pressure intensities at a distance $l$ apart, along the axis of the pipe, we have

$$
Q=\frac{\pi a^{t}}{8 \mu} \cdot \frac{p_{1}-p_{2}}{l} \text { cubic feet per second. }
$$

The maximum velocity is obtained by putting $r=o$ in equation (2) and $=\frac{p_{1}-p_{2}}{4 \mu l} \cdot a^{2}$ feet per: second.

The mean velocity $=\frac{Q}{\text { area of section }}=\frac{p_{1}-p_{2}}{8 \mu l} \cdot a^{2}$ feet per second.

$\therefore \quad$ Maximum velocity $=2$ (mean velocity)

From equation (2) we see that the curve of velocities across a diameter is a parabola, and that the surface of velocities for the pipe will be a paraboloid of revolution (Fig. 25). ${ }^{1}$

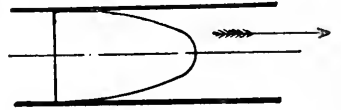

FIG. 25.

If $v=$ mean velocity, we have $p_{1}-p_{2}=\frac{32 \mu l v}{d^{2}}$, Poiseuille's form of the equation.

Here the shear stress at the boundary is given by

$$
\mu \frac{d u}{d r}=\frac{r}{2} \cdot \frac{d p}{d x}=\frac{a}{2} \cdot \frac{p_{1}-p_{2}}{l} .
$$

On equating expressions (2) and (3) it is readily shown that the filament of mean velocity is found at a radius $707 a$.

1 For a curve showing the variations in velocity for stream line flow through a two-inch pipe, see a paper by Morrow, "Proc. Roy. Soc.," vol. 76, 1905. 
Art. 21.-Steady Flow through a Circular Tube, assuming Slip at the Boundaries.

If we assume slip at the boundaries of a circular tube, some assumption must be made as to the magnitude of the slip before any determination' of the pressure fall along the tube can be made. Assuming, as seems rational, that the velocity at the boundaries is proportional to the tangential stress $u\left(\frac{d u}{d r}\right)_{r=a \text {, }}$ we have if $u^{\prime}=$ velocity at boundaries, then $u^{\prime} \propto \mu\left(\frac{d u}{d r}\right)_{r=a}$ or $\quad u^{\prime}=-k\left(\frac{d u}{d r}\right)_{r=a}=\frac{k a}{2 \mu} \cdot \frac{d p}{d x} \quad$ from (4), Art. 20.

Using this to determine $B$ in equation (1), Art. 20, and proceeding as before, we finally get

$$
Q=\frac{\pi a^{4}}{8 \mu}\left\{1+\frac{4 k}{a} ! \frac{p_{1}-p_{2}}{l}\right. \text { cub. ft. per sec. }
$$




\section{CHAPTER IV.}

The Motion of a Fluid-Equations of Motion for a viscous fluid-Application to Stream Line Motion-Bernoulli's Equation-Elementary Proof-Experimental Verification-Applications-Venturi Meter-Loss due to sudden change in Section of a Stream-Special Cases-Initiation and Stoppage of MotionFlow in converging Channel-Change of Pressure across Stream LinesVortex Motion-Forced Vortex-Free Vortex-Compound Vortex.

\section{Art. 22.-Motion of a Fluid.}

THe motion of any particle of fluid acted upon by external forces and by its own weight may be considered from two points of view. In the first, by equating the work done on the mass to the increase of energy in the potential, pressure, and kinetic form, together with the loss by dissipation, as by internal friction, which converts mechanical energy into heat, we get an expression for the velocity in terms of the applied forces. With steady motion of a non-viscous fluid this method is always applicable.

Where a mass of fluid has unsteady-sinuous-motion, however, the impossibility of determining the kinetic energy possessed by these eddies in virtue of their whirling motion renders the application of the energy equation impossible, and it becomes necessary to consider the force from the point of view of the production of momentum, since the momentum of the fluid forming a vortex is unaltered by its motion of rotation.

With unsteady motion, moreover, of a viscous fluid, the magnitude and direction of the forces-including those due to viscosity -acting at any particular point become indeterminate, so that the molecular motion at the point is then indeterminate, and the general equations of motion become in general impossible of application. Even in the case of the steady motion of a viscous fluid, these, when stated in terms of the viscosity, become so unwieldy that except in one or two particular cases, e.g., those of steady flow between parallel plates or through a circular pipe, 
they are unfitted for application to the solution of any practical problem, although where so applicable the solution becomes perfectly accurate.

A simplification of these equations may be obtained by neglecting the effect of viscosity-i.e., assuming the liquid to be a perfect fluid-and it is in this form that they are usually stated. Evidently the solution of any such equation can only be made to apply to the results of any given problem by the introduction of some constant obtained by experiment, which itself has the effect of modifying the solution so as to take into account the effect of viscosity, and it is to this extent that hydraulics is to be considered an experimental science. If it were possible in every case to apply the equations of motion in full, the science would become exact.

The equations in full are deduced in the following pages, and the terms involving viscosity are afterwards eliminated, so as to give the form as usually stated for a non-viscous fluid.

Afterwards, in considering the motion of fluids, it will be assumed that with uniform steady motion the distribution of pressure is unaffected, so that the pressure at any point is the equivalent of the hydrostatic pressure due to its depth. This appears from the general equations of motion. Also the further assumption is made, that if the moving particles have the acceleration which they would have if acted upon by their external forces alone-i.e., if independent of the surrounding particlesthe pressure throughout is uniform. Thus, in a jet falling freely through the atmosphere under the action of gravity, the pressure throughout is sensibly uniform and equal to that of the atmosphere.

'The principles on which the following demonstrations are based may be briefly indicated, and are as follow :-

The Principle of Linear Momentum.-The rate of change of the component of the linear momentum of any system in any direction is equal to the parallel component of the applied forces.

The Principle of Angular Momentum.-The rate of change of the component of angular momentum of a system about any 
axis is equal to the moment of the applied forces about that axis.

The Principle of the Conservation of Energy.-The sum of the kinetic and potential energies of any system is constant, except for the effect of such dissipative forces as friction which convert mechanical energy into heat.

\section{Art. 23.-Equations of Motion for a Viscous Fluid.}

Taking a fixed point $O$ in the fluid as the origin of co-ordinates, let $O X, O Y, O Z$ be three co-ordinate axes, and let $u, v, w$ be the components of the velocity of a particle parallel to these three axes. $u, v$, and $w$ will be supposed finite and continuous, and, since they vary with the position of the particle and the time, are functions of $x, y, z$ and $t$.

The velocity of a particle may be considered from two points of view. Considering any fixed point, the velocity of successive particles as they pass that point may vary, and since $x, y, z$ are now constant, the rates of variation parallel to the three axes are represented by the partial differentials $\frac{\partial u}{\partial t}$, etc. For steady motion these are separately zero.

If, however, we consider the variation of velocity of any one particle as it moves from point to point of its path, since this now also includes the variation of $x, y, z$ with $t$, its components will now be represented by $\frac{d u}{d t}$, etc., and are the components of the true acceleration of the particle. These are not necessarily zero for steady motion.

If then a particle moves from a point $(x, y, z)$ to a second point $(x+\delta x ; y+\delta y ; z+\delta z)$ in time $\delta t$, its change of velocity $\delta u$ is given by

$$
\delta u=\frac{\partial u}{\partial x} \delta x+\frac{\partial u}{\partial y} \delta y+\frac{\partial u}{\partial z} \cdot \delta z+\frac{\partial u}{\partial t} \cdot \delta t
$$

and in the limit

$$
\frac{d u}{d t}=\frac{\partial u}{\partial x} \cdot \frac{d x}{d t}+\frac{\partial u}{\partial y} \cdot \frac{d y}{d t}+\frac{\partial u}{\partial z} \cdot \frac{d z}{d t}+\frac{\partial u}{\partial t} .
$$


But from the definitions of $u, v, w$, we have

$$
\begin{aligned}
& u=\frac{d x}{d t}: v=\frac{d y}{d t}: w=\frac{d z}{d t} \\
& \therefore \quad \frac{d u}{d t}=u \frac{\partial u}{\partial x}+v \frac{\partial u}{\partial y}+v \frac{\partial u}{\partial z}+\frac{\partial u}{\partial t} \\
\text { similarly } & \frac{d v}{d t}=u \frac{\partial v}{\partial x}+v \frac{\partial v}{\partial y}+v \frac{\partial v}{\partial z}+\frac{\partial v}{\partial t} \\
\text { and } & \frac{d w}{d t}=u \frac{\partial w}{\partial x}+v \frac{\partial w}{\partial y}+w \frac{\partial w}{\partial z}+\frac{\partial w}{\partial t}
\end{aligned}
$$

and

Next consider an elementary parallelopiped in the fluid

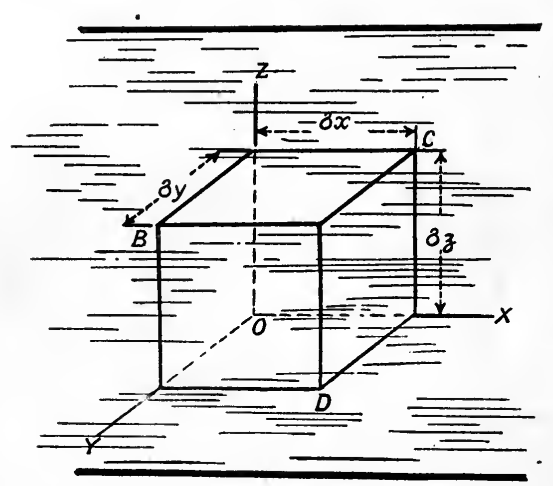

Fig. 26: bounded by edges $\delta x, \delta y$, $\delta z$ (Fig. 26). For continuity of motion the difference between the amounts of fluid which flow in and out of these surfaces during time $\delta t$ must be equal to the increase in the mass which the surface encloses. Expressing this analytically, we get the equation of continuity.

Now the mass of fluid entering across the face $O B$ in time $\delta t=\rho u \delta y . \delta z . \delta t$.

And the mass of fluid leaving across the face $C D$ in time $\delta t$ $=\rho u \delta y . \delta z . \delta t+\frac{\partial}{\partial x}(\rho u) \delta x . \delta y . \delta z . \delta t$.

$\therefore \quad$ Gain across these faces $=-\frac{\partial}{\partial x}(\rho u) \delta x . \delta y . \delta z . \delta t$.

$\therefore$ Total gain

$=-\left\{\frac{\partial}{\partial x}(\rho u)+\frac{\partial}{\partial y}(\rho v)+\frac{\partial}{\partial z}(\rho u)\right\} \delta x . \delta y \cdot \delta z . \delta t$.

But the mass contained at time $t=\rho \delta x . \delta y . \delta z$. and $\quad, \quad, \quad, t+\delta t=\left(\rho+\frac{\partial \rho}{\partial t} \cdot \delta t\right)$ $\delta x . \delta y . \delta z$.

$\therefore \quad$ Gain in time $\delta t=\frac{\partial \rho}{\partial t} \cdot \delta t . \delta x . \delta y . \delta z$. 
Equating these expressions for the gain, we have

$$
\frac{\partial \rho}{\partial t}+\frac{\partial(\rho u)}{\partial x}+\frac{\partial(\rho v)}{\partial y}+\frac{\partial(\rho u)}{\partial z}=0
$$

from which, if $\rho$ is constant, i.e., if the fluid is incompressible, we have

$$
\frac{\partial u}{\partial x}+\frac{\partial x}{\partial y}+\frac{\partial w}{\partial z}=0
$$

as the equation of' continuity for an incompressible fluid.

In the case of a gas $\rho$ is not constant, but we may have $\frac{\partial \rho}{\partial t}=$ constant, in which case we have regular motion in the gas.

Now if the stresses in a viscous fluid (which follows the same stress strain law as an elastic solid) be represented by the notation $p_{x x}, p_{x y}, p_{y z}$, etc., where each of these symbols denotes a stress on the plane perpendicular to the axis of co-ordinates represented by the first suffix, in the direction of the second suffix, so that, for example, the stress $p_{x y}$ is a stress on the plane perpendicular to $O \mathrm{X}$, in the direction $O \mathrm{Y}$, and is therefore a tangential stress on this plane, the relations holding between the various stresses for equilibrium are given by the following equations. ${ }^{1}$

$$
\begin{aligned}
& \left.p_{x x}=-p-\frac{2}{3} \mu\left(\frac{\hat{c} u}{\hat{c} x}+\frac{\partial r}{\partial y}+\frac{\partial u}{\partial z}\right)+2 \mu \frac{\partial u}{\partial x}\right) \\
& p_{y y}=-p-\frac{2}{3} \mu\left(\frac{\hat{c} u}{\hat{c} x}+\frac{\partial v}{\partial y}+\frac{\partial u}{\partial z}\right)+2 \mu \frac{\partial v}{\partial y} \\
& \left.p_{z z}=-p-\frac{2}{3} \mu\left(\frac{\hat{c} u}{\hat{c} x}+\frac{\partial v}{\partial y}+\frac{\partial w}{\partial z}\right)+2 \mu \frac{\partial w}{\partial z}\right) \\
& \left.p_{x y}=p_{y x}=\mu\left(\frac{\partial r}{\partial x}+\frac{\partial u}{\partial y}\right)\right) \\
& \left.p_{y z}=p_{z y}=\mu\left(\frac{\partial w}{\partial y}+\frac{\partial v}{\partial z}\right)\right\} \\
& \left.p_{z x}=p_{x z}=\mu\left(\frac{\partial u}{\partial z}+\frac{\partial w}{\partial x}\right)\right)
\end{aligned}
$$

where $p=\frac{p_{x x}+p_{y y}+p_{z z}}{3}$, and $\mu=$ Coefficient of Viscosity.

1 Stokes on "Theories of the internal friction of fluids in motion and of the equilibrium and motion of elastic solids" ; also Lamb"s "Motion of Fluids," p. 219. 
Again, considering the elementary volume $\delta x . \delta y . \delta z$, we see that the total force acting on this volume due to any variation of stress on opposite faces of the volume in the direction $O X$ is $\left\{\frac{\partial p_{x x}}{\partial x}+\frac{\partial p_{y x}}{\partial y}+\frac{\partial p_{z x}}{\partial z}\right\} \delta x . \delta y . \delta z^{1}$

So that if an external force, having a component $X$ per unit mass, in this direction, also act on the element, we have $\begin{gathered}\text { Total force on element } \\ \quad \text { in direction } O X\end{gathered}=\left[\left(\frac{\partial p_{x x}}{\partial x}+\frac{\partial}{\partial y}+\frac{\partial p_{z x}}{\partial z}\right)+\rho X\right]$ $\delta x . \delta y . \delta z$. $\left.\begin{array}{c}\text { This equals mass } \times \text { acceleration } \\ \text { of element in direction } O X\end{array}\right\}=\rho . \delta x . \delta y . \delta z \times \frac{d u}{d t}$.

$$
\therefore \quad \rho X+\left(\frac{\partial p_{x x}}{\partial x}+\frac{\partial p_{y x}}{\partial y}+\frac{\partial p_{z x}}{\partial z}\right)=\rho \frac{d u}{d t} .
$$

Similarly; considering the accelerations in directions $\{Y$ and $O Z$, we get

$$
\begin{aligned}
& \rho Y+\left(\frac{\partial p_{x y}}{\partial x}+\frac{\partial p_{y y}}{\partial y}+\frac{\partial p_{z y}}{\partial z}\right)=\rho \frac{d v}{d t} \\
& \rho Z+\left(\frac{\partial p_{x z}}{\partial x}+\frac{\partial p_{y z}}{\partial y}+\frac{\partial p_{z z}}{\partial z}\right)=\rho \frac{d w}{d t}
\end{aligned}
$$

1 This may be proved as follows. The difference of normal stress on the faces $O B$ and $C D$ (Fig. $26 \mathrm{~A})=\frac{\partial p_{x x}}{\partial x} \delta x$.

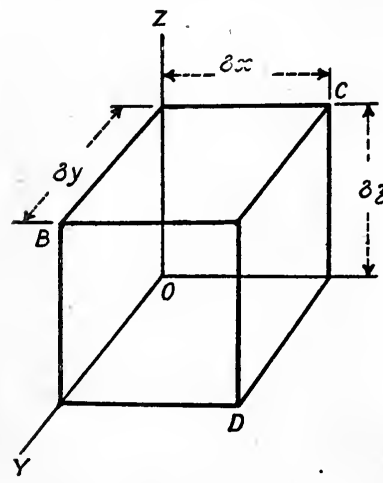

Fig. 26 A.

$\therefore$ Difference of normal force on these faces $=\frac{\partial p_{x x}}{\partial x} \delta x \cdot \delta y \cdot \delta:$.

Also the difference of tangential stress in the direction $O \mathrm{X}$ on the faces $O C$ and $B D=$ $\frac{\partial p_{y x}}{\partial y} \delta y$, while the resultant force due to these tangential stresses in this direction $=\frac{\partial p_{y x}}{\partial y}$ $. \delta x . \delta y . \delta z$.

Similarly the tangential stresses on the faces $O D$ and $B C$ in the direction $O X$ give rise to a resultant force $\frac{\partial p_{z x}}{\partial z} \delta x . \delta y . \delta:$.

These include all the forces due to variation of stress across the element, which have a component in the direction $O X$. Summing these, we get the resultant force in the direction of increasing $x$. Similarly for the forces in the directions $O X$ and $O Y$. 


\section{UNIVERSITY \\ EQUATIONS OF MOTION.}

Differentiating the equations (7) and (8), so as to obtain the values of $\frac{\partial p_{x x}}{\partial x}$, etc., in the case of a viscous fluid, and inserting the values thus obtained in equations (9), the first of these becomes

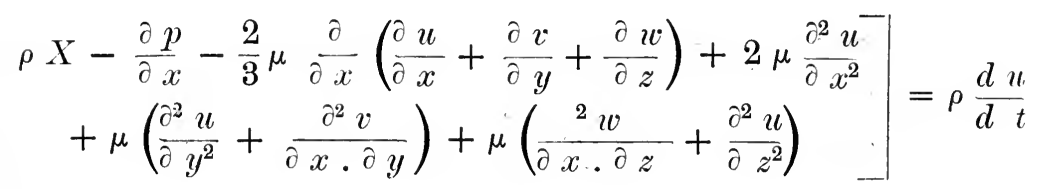

From the equation of continuity we have

$$
\frac{\partial u}{\partial x}+\frac{\partial v}{\partial y}+\frac{\partial w}{\partial z}=O
$$

Also $\frac{\partial^{2} u}{\partial x^{2}}+\frac{\partial^{2} v}{\partial x \partial y}+\frac{\partial^{2} u}{\partial x \partial z}=\frac{\partial}{\partial x}\left(\frac{\partial u}{\partial x}+\frac{\partial v}{\partial y}+\frac{\partial u}{\partial z}\right)=0$

So that the equations become

$$
\left.\begin{array}{l}
\rho \frac{d u}{d t}=\rho X-\frac{\partial p}{\partial x}+\mu\left(\nabla^{2} u\right) \\
\rho \frac{d v}{d t}=\rho Y-\frac{\partial p}{\partial y}+\mu\left(\nabla^{2} v\right) \\
\rho \frac{d w}{d t}=\rho Z-\frac{\partial p}{\partial z}+\mu\left(\nabla^{2} w\right)
\end{array}\right)
$$

where $\nabla^{2}$ denotes the operator $\left(\frac{\hat{\sigma}^{2}}{\partial x^{2}}+\frac{\hat{\partial}^{2}}{\partial y^{2}}+\frac{\partial^{2}}{\partial z^{2}}\right)$.

The terms involving $\mu$ in these equations are complex, and for purposes of practical application to hydraulic problems are usually neglected. If this is done, the equations of motion for a non-viscous, incompressible fluid become

$$
\left.\begin{array}{l}
\rho \frac{d u}{d t}=\rho X-\frac{\partial p}{\partial x} \\
\rho \frac{d v}{d t}=\rho Y-\frac{\partial p}{\partial y} \\
\rho \frac{d w}{d t}=\rho Z-\frac{\partial p}{\partial z}
\end{array}\right\}
$$

or, writing $\frac{d u}{d t}$ in terms of $\frac{\partial u}{\partial x}$, etc., from equation (2), and 
dividing throughout by $\rho$,

$$
\left.\begin{array}{l}
X-\frac{1}{\rho} \frac{\partial p}{\partial x}=u \frac{\partial u}{\partial x}+v \frac{\partial u}{\partial y}+w \frac{\partial u}{\partial z}+\frac{\partial u}{\partial t} \\
Y-\frac{1}{\rho} \frac{\partial p}{\partial y}=u \frac{\partial v}{\partial x}+v \frac{\partial v}{\partial y}+w \frac{\partial v}{\partial z}+\frac{\partial v}{\partial t} \\
Z-\frac{1}{\rho} \frac{\partial p}{\partial z}=u \frac{\partial v}{\partial x}+v \frac{\partial w}{\partial y}+w \frac{\partial w}{\partial z}+\frac{\partial w}{\partial t}
\end{array}\right\}
$$

the Eulerian equations of motion.

Here the axes of co-ordinates have been taken to coincide with

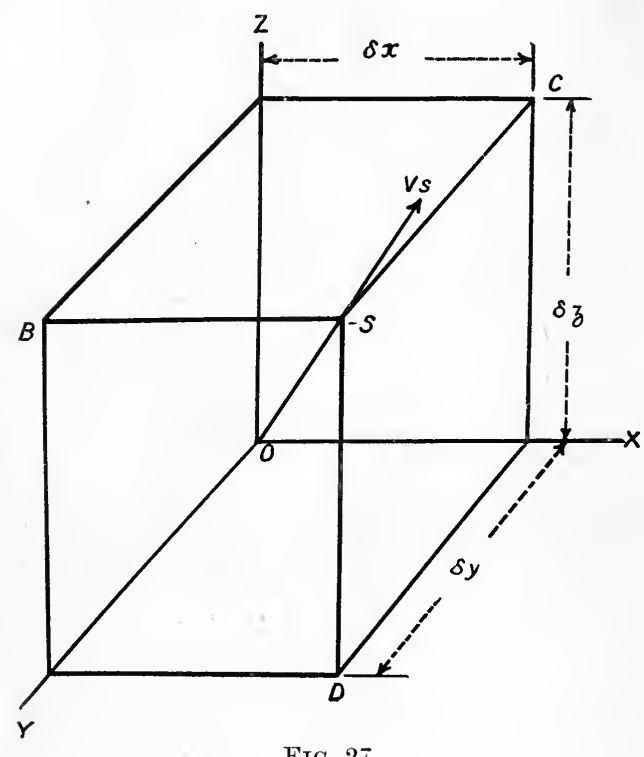

FIG. 27. the principal axes of stress. If, however, these are transferred, exactly similar equations are obtained.

In the case where gravity is the only external force acting on the fluid, $X=0$, $Y=o$, and $Z=-$ $g, g$ acting in the opposite direction to that of $z$ increasing.

So far, the equations are applicable to motion of any kind, whether steady or unsteady, but in their present form are not obviously useful for the solution of any practical problem. So long as the motion is sinuous and irregular nothing further can be done with the equations.

\section{Art. 24.-Application to Stream Line Motion.}

If, however, the fluid be moving with definite stream line motion, these equations can be considerably simplified. Suppose a particle moving with stream line motion from $O$, in the direction $O S$ (Fig. 27), with velocity $V_{s}$, the space $O S$ being oss. The 


\section{EQUATIONS OF MOTION.}

direction cosines of this motion in the directions $O X, O Y, O Z$, are $\frac{d x}{d s} \cdot \frac{d y}{d s} \cdot \frac{d z}{d s}$.

Also

$$
\left\{\begin{array}{l}
\frac{d x}{d s}=\frac{u}{V_{s}}=l \\
\frac{d y}{d s}=\frac{v}{V_{s}}=m \\
\frac{d z}{d s}=\frac{w}{V_{s}}=n
\end{array}\right.
$$

Again, geometrically we get

And

$$
\begin{aligned}
& V_{s}^{2}=u^{2}+v^{2}+w^{2} \\
& V_{s}=l u+m v+n w .
\end{aligned}
$$

For stream line motion, and with only gravity acting, the general equations (12) may then be written

$$
\left.\begin{array}{r}
-\frac{1}{\rho} \frac{\partial p}{\partial x}=\frac{\partial u}{\partial t}+V_{s}\left(l \frac{\partial u}{\partial x}+m \frac{\partial u}{\partial y}+n \frac{\partial u}{\partial z}\right) \\
-\frac{1}{\rho} \frac{\partial p}{\partial y}=\frac{\partial v}{\partial t}+V_{s}\left(l \frac{\partial v}{\partial x}+m \frac{\partial v}{\partial y}+n \frac{\partial v}{\partial z}\right) \\
-g-\frac{1}{\rho} \frac{\partial p}{\partial z}=\frac{\partial w}{\partial t}+V_{s}\left(l \frac{\partial w}{\partial x}+m \frac{\partial w}{\partial y}+n \frac{\partial w}{\partial z}\right)
\end{array}\right\}
$$

Now $\left(l \frac{\partial u}{\partial x}+m \frac{\partial u}{\partial y}+n \frac{\partial u}{\partial z}\right)=\frac{d x}{d s} \cdot \frac{\partial u}{\partial x}+\frac{d y}{d s} \cdot \frac{\partial u}{\partial y}+\frac{d z}{d s} \cdot \frac{\partial u}{\partial z}$

$$
=\frac{d u}{d s}
$$

$$
\left.\begin{array}{r}
\therefore \quad-\frac{1}{\rho} \frac{\partial p}{\partial x}=\frac{\partial u}{\partial t}+V_{s} \frac{d u}{d s} \\
-\frac{1}{\rho} \frac{\partial p}{\partial y}=\frac{\partial v}{\partial t}+V_{s}^{r} \frac{d v}{d s} \\
-g-\frac{1}{\rho} \frac{\partial p}{\partial z}=\frac{\partial w}{\partial t}+V_{s} \frac{d w}{d s}
\end{array}\right\}
$$

Multiplying these equations respectively by $l, m$, and $n$, and adding, we get

$-n g-\frac{1}{\rho}\left(l \frac{\partial p}{\partial x}+m \frac{\partial p}{\partial y}+n \frac{\partial p}{\partial z}\right)=l \frac{\partial u}{\partial t}+m \frac{\partial v}{\partial t}+n \frac{\partial w}{\partial t}$

$$
+u \frac{d u}{d s}+v \frac{d v}{d s}+w \frac{d w}{d s} .
$$


From (14) we get $\frac{\partial V_{s}}{\partial t}=l \frac{\partial u}{\partial t}+m \frac{\partial v}{\partial t}+n \frac{\partial w}{\partial t}$.

From (13) we get $\frac{d V_{8}^{2}}{d s}=2\left(u \frac{d u}{d s}+v \frac{d v}{d s}+w \frac{d w}{d s}\right)$,

also

$$
l \frac{\partial p}{\partial x}+m \frac{\partial p}{\partial y}+n \frac{\partial p}{\partial z}=\frac{d p}{d s} .
$$

$\therefore$ Substituting, equation (17) becomes

$$
-n g-\frac{1}{\rho} \frac{d p}{d s}=\frac{\partial V_{s}}{\partial t}+\frac{1}{2} \frac{d}{d s}\left(V_{s}^{2}\right)
$$

or, since $n=\frac{d z}{d s}$

$$
-g \frac{d z}{d s}-\frac{1}{\rho} \frac{d p}{d s}=\frac{\partial V_{s}}{\partial t}+\frac{1}{2} \frac{d}{d s}\left(V_{s}^{2}\right)
$$

or,

$$
\frac{d}{d s}\left(\frac{p}{\rho}+\frac{V_{s}^{2}}{2}+g z\right)=-\frac{\partial V_{s}}{\partial t}
$$

If $W=$ weight of unit volume of the fluid, we have $W=\rho g$, so that the equation may be written

$$
\frac{d}{d s}\left(\frac{p}{W}+\frac{V_{8}^{2}}{2 g}+z\right)=-\frac{1}{g} \frac{\partial V_{8}}{\partial t} .
$$

Integrating this, we get $\frac{p}{W}+\frac{V_{s}^{2}}{2 g}+z=-\frac{1}{g} \int \frac{\partial V_{8}}{\partial t} \cdot d s$

In steady stream line motion, the velocity at any fixed point remains constant, so that $\frac{\partial V_{s}}{\partial t}=o$, and the equation then becomes

$$
\frac{p}{W}+\frac{V_{s}^{2}}{2 g}+z=\text { constant. }
$$

This equation holds throughout any particular stream line, but the constant varies from one stream line to another.

\section{Art. 25.}

Taking the general equations of motion (10), then by an algebraic transformation we obtain an equation giving the rate of increase of kinetic energy, which becomes equal to the sum of two terms. The first is the rate of increase of kinetic energy due to work done by the stresses, and the second has been called the rate of increase of kinetic energy in the form of heat. It follows that the $u, v, w$, which have been defined as the component 
velocities at the point $O$, must strictly also take into account and include these heat motions. From this point of view we may say that if the heat motion dissipated by radiation and conduction is equal to the relative molecular motion, the motion as a whole is steady. If, however, the relative molecular motion is greater than that dissipated in this way, we, have eddies formed.

\section{ART. 26.}

The theorem expressed in the equation $\frac{p}{W}+\frac{V^{2}}{2 g}+z=$ constant, is commonly known, from its discoverer, as Bernoulli's theorem.

It expresses the fact that the total energy of the fluid per lb. in any stream tube is constant. The three terms denote (1) the pressure energy per lb. $\frac{p}{W}$; (2) the kinetic energy per lb. $\frac{V^{2}}{2 g}$; (3) the potential energy per $1 \mathrm{~b}$. $z$, where $z$ is the height above some datum to be fixed for any particular problem. The significance of the second and third of these terms is obvious, but some difficulty is often experienced in grasping the precise significance of the first, or pressure energy term. If $p$ be the pressure intensity in pounds per square foot, and $W$ the weight per cubic foot, the expression $\frac{p}{W}$ gives the height in feet of a column of water which would produce the statical pressure " $p . "$

Now, if water is compressed in a cylinder fitted with a movable piston its pressure is enormously increased by an extremely small movement of the piston. Exactly the same thing would occur if some elastic solid, such as indiarubber, were compressed in the cylinder, and just as with rubber, so with the water, the work done on the substance during compression would be returned during a slow retrograde motion of the piston.

Since, due to an increase in pressure of $p$ lbs. per square foot, the decrease in volume of water per cubic foot or

$$
\frac{\delta V}{V}=\frac{p}{31 \times 144 \times 10^{4}}=\frac{p}{\bar{K}}
$$

$\therefore \quad$ Work expended per cubic foot in compressing water to this 
pressure $=\frac{p}{2} \cdot \frac{p}{K}=\frac{p^{2}}{2 K}$, and this is the amount of work stored in the water, in virtue of its pressure alone, and which would be given out during expansion. If, in the cylinder in question, the piston were fixed, and the water allowed to escape through a small nozzle, the kinetic energy of the issuing jet would equal the above expression, while the pressure would, with the removal of the first few drops of water, fall to that of the atmosphere.

The amount of energy thus stored in the water per lb. in virtue of its pressure alone is $\frac{p^{2}}{2 K W}$ foot lbs., and if water were a perfectly incompressible fluid, so that $K=\infty$, would be zero. But this is not what we mean in hydraulics by the pressure energy of water.

Suppose, however, the piston pressed home with a continuous pressure of $p$ lbs. per square foot, while the water escapes from the cylinder. The work done on the water per cubic foot is now $p$ foot lbs., and per lb. is $\frac{p}{W}$.

The pressure of the water is exactly the same as before, but now, so long as the piston is moving, the water is capable of doing work, in virtue of this pressure, at the rate of $\frac{p}{W}$ foot lbs. per $1 \mathrm{~b}$., and this is what we mean by the pressure energy.

The idea of pressure energy only becomes applicable when we have a continuous supply of water under pressure, as is the case, for example, in the supply pipes of an hydraulic power company. Here a continuous supply of pressure water is pumped into the mains, with a velocity which is in general so low that the kinetic energy is negligible. The potential energy is also in general negligible, so that it is in virtue of the pressure energy alone that the water is capable of doing work. If, however, the pumps are stopped and the accumulators disconnected, the withdrawal of a very few cubic feet of water from the mains will reduce the pressure to that corresponding to the statical head at any point, and the capacity for doing work to almost zero.

Or consider an element of a stream tube of weight $W$, at a depth $h$ below the free surface, and at a height $z$ above some 
datum. Its potential energy is $W z$ foot lbs., while, in virtue of its position, its pressure energy is $W h$, its pressure " $p$ " being $W h$.

Removed from its connection with the surrounding mass of fluid, which guarantees the permanence of the pressure conditions for a finite period, the potential energy is unaltered, while its available pressure energy $\frac{p}{W}$ is now practically zero-absolutely zero in the case of a perfectly incompressible fluid.

Art. 27.-Flementary Proof of Bernoulli's Theorem.

Beginning with the assumption that the fluid is non-viscous, and exerts only normal forces on any bounding surface, the proof of this theorem is easily deduced. Consider the element-length $\delta s$-of a stream tube in such a fluid, the only forces tending to produce motion of this element being its own weight and the normal pressures of the surrounding fluid (Fig. 28).

Let its cross sectional area at the top be " $a+\delta a$, , and at the bottom " $a$," the pressure intensities at the top and bottom being $p-\delta p$ and $p$ respectively. Let the average normal pressure on the sides of the element be $q$. Actually $q$ will lie between $p$ and $p-\delta p$.

This normal pressure will have an unbalanced component $q . \delta a$ along

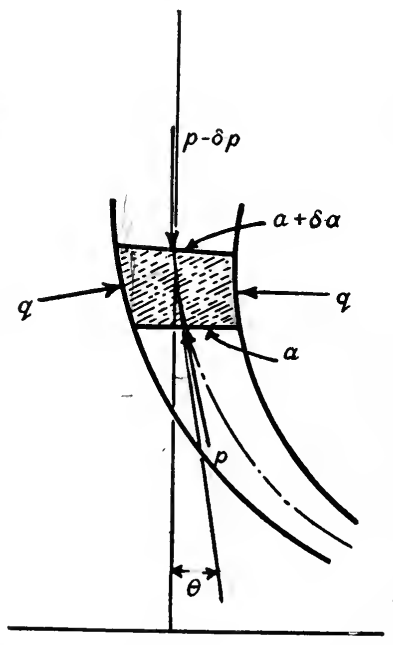

F'IG. 28. the axis, and, since the fluid is non-viscous, the only effect of this pressure in producing motion in the direction of the axis will be due to this component.

Let the direction of the axis make an angle $\theta$ with the vertical.

Then the magnitude of the resultant $\left.\begin{array}{l}\text { of forces on top and bottom faces of } \\ \text { element, in the direction of its motion }\end{array}\right\}=\left\{\begin{array}{c}(p-\delta p)(a+\delta a)- \\ p a=p \delta a-a \delta p .\end{array}\right.$ 
Resultant of normal pressures on sides of element, in same direction $\}=-q \delta a$.

The only other force acting? on the element is its weight, and the resolved part of this in the same direction

$$
=\left\{W\left(a+\frac{\delta a}{2}\right) \delta s \cos \theta\right\}
$$

$\therefore \begin{aligned} & \text { Resultant force in } 1 \\ & \text { direction of motion }=p \delta a-a \delta p-q \delta a+W\left(a+\frac{\delta a}{2}\right) \delta s \cdot \cos \theta\end{aligned}$

$$
=\text { mass } \times \text { acceleration }
$$

$$
=\frac{W\left(a+\frac{\delta a}{2}\right) d s}{g} \times \frac{d v}{d t}, \begin{gathered}
\text { where } v=\text { velocity } \\
\text { of element. }
\end{gathered}
$$

Putting $q=p-k \delta p$, and neglecting small quantities of the second order, we get

$$
\frac{W a \delta s}{g} \cdot \frac{\delta v}{\delta t}=W a \delta s \cos \theta-a \delta p .
$$

But $v=\frac{\delta s}{\delta t}$, and $\delta s \cos \theta=-\delta z, z$ being measured vertically upwards, and $\delta z$ being the difference of level of the two ends of the element.

$\therefore$ Equation (1) becomes $\frac{W}{g} \cdot v \delta v=-W \delta z-\delta p$,

or

$$
\frac{W}{g} v \delta v+W \delta z+\delta p=0 \text {. }
$$

When $\delta s, \delta p$, are indefinitely small we get, in the limit, on integrating

or

$$
\begin{aligned}
& \frac{W}{g} \cdot \frac{v^{2}}{2}+W z+p=\text { constant } \\
& \frac{v^{2}}{2 g}+\frac{p}{W}+z=\text { constant. }
\end{aligned}
$$

ART. 28.

Since $v$ is the velocity along a stream line, any attempt to apply the equation to the motion of a mass of fluid by taking $v$ as the mean velocity of the mass will obviously lead to error unless the square of this mean velocity, multiplied by the mass itself, is equal to the sum of the squares of the various stream tube velocities, each multiplied by the mass contained in its own 
stream tube; and this is only true when all the stream lines are parallel and have the same velocity, and when, in consequence, no internal work is being performed against viscosity.

While this state of affairs is never accurately realised in the case of the motion of water in pipes or open channels, yet the equation may be made to apply to such eases by the introduction of a term involving losses of energy due to viscous resistance.

E.g., if the suffixes (1) and (2) refer to two successive positions of a particle of viscous fluid, we may say that

$$
\frac{p_{1}}{W}+\frac{v_{1}{ }^{2}}{2 g}+Z_{1}=\frac{p_{2}}{W}+\frac{v_{2}^{2}}{2 g}+Z_{2}+{ }_{1} H_{2},
$$

where ${ }_{1} H_{2}$ represents this loss of energy between the positions (1) and (2).

Where we have unsteady motion set up between (1). and (2), this equation may still be usefully applied, ${ }_{1} H_{2}$ now including the loss of energy in eddy formation. Applying this to the case of flow through a pipe or channel of varying area, it is usual to assume that the equation still holds when $v_{1}$ and $v_{2}$ are the mean velocities of flow in the direction of the axis at the two sections (1) and (2).

Where the flow changes from steady to unsteady between (1) and (2) the term ${ }_{1} H_{2}$ will then include the kinetic energy due to motion in directions perpendicular to the axis of the pipe.

Art. 29.-Experingental Verification of Bernoulli's Theorem.

The apparatus shown (Fig. 29) consists of a horizontal passage having parallel vertical sides of plate-glass, the first half forming a converging, and the second half a diverging channel. The passage connects two reservoirs, $A$ and $B$, and pressure tubes are erected at frequent intervals.

Water is fed into the tank $A$, which is of large area compared with the passage, and is led away by the overflow $C$. By suitable regulation of the flow into $A$, and of the height of the overflow $C$, the velocity of flow through the channel and the pressure at any point may be regulated as required. Aniline dye may be 
introduced into the incoming stream of water by means of the capillary tube $T$.

At very low speeds the motion throughout the passage is steady, and the curve joining the tops of the pressure columns is as shown in $(a)$. At any point to the right of the throat the pressure head will be less than that at the corresponding point to the left, because of viscous resistance, so that, since the velocity head is the same at the two points, the total head is less on the right. The curve is discontinuous-has a cusp-at the throat.

As the velocity is increased, the first indication of unsteady

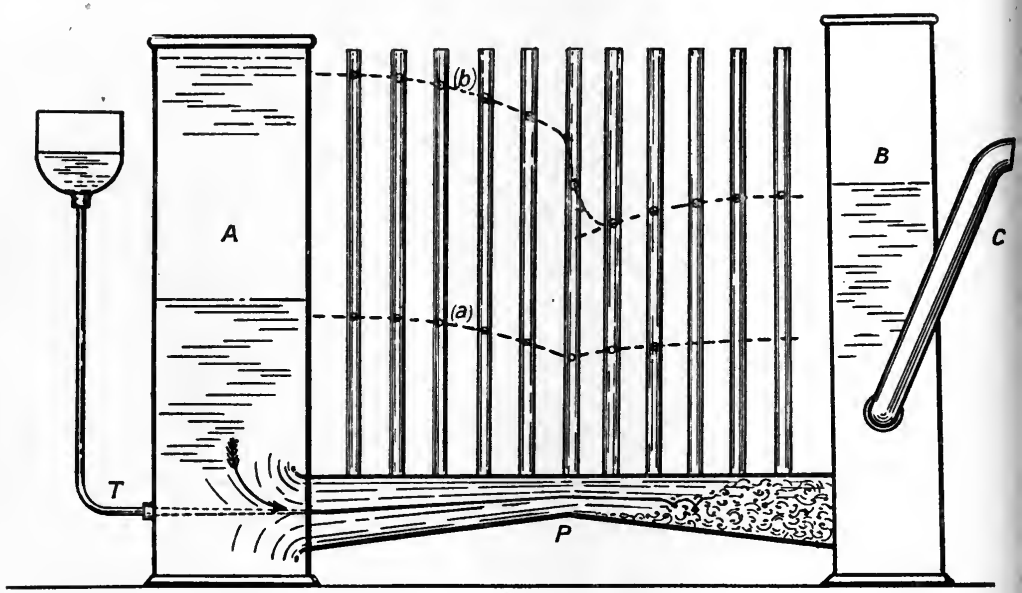

FIG. 29.

motion is provided by an occasional eddying of such colour bands as get below the horizontal through the lowest point of the throat.

The velocity at which this occurs depends on the angle of divergence of the sides of the passage. As the velocity is increased this unsteady motion extends to the whole of the water in the diverging portion of the passage, the motion in the converging part being uniformly steady. The pressure curve now appears as shown in (b), and is discontinuous at the throat.

On plotting the energy curve, $\frac{p}{W^{\gamma}}+\frac{r^{2}}{2 !}+z=y$, the curve 
shown in Fig. 30 is obtained, and shows very clearly the magnitude of the various losses.

Drawing smooth curves between the plotted points from $A$ to $P$ and from $P$ to $B$, it will be noted that the observed point $E$ at $P$ lies on neither of these curves. In the figure, $H_{1}$ represents loss of head, due to viscous resistance between $A$ and $P ; H_{2}$, the loss due to eddy formation at the throat; and $H_{3}$, the loss due to viscous resistance and to eddy formation between $P$ and $B$. Evidently from the position of $\dot{E}$ and the form of the curve $P B$, almost the whole energy of eddy formation is absorbed at and within a very small distance of

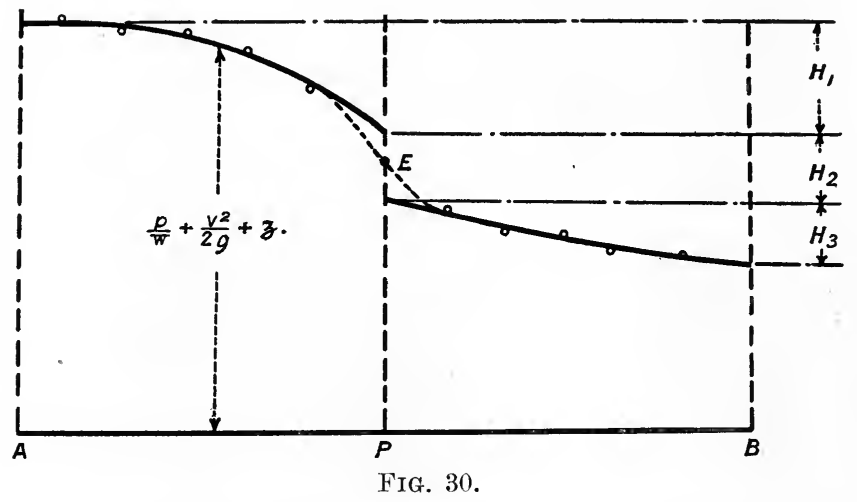

the throat. With speeds below the critical velocity $H_{2}{ }^{\prime}=O$ and $H_{1}=H_{3}$ (approx.).

With speeds exceeding the critical, $H_{3}$ becomes less or greater than $H_{1}$, depending on the velocity, as, with fairly low velocities, the eddies formed at the throat die out to some extent before reaching $B$, their kinetic energy being transformed into available pressure energy and thus reducing $H_{3}$. At suitable velocities indeed $H_{3}$ may be negative, indicating that the energy rendered available by the dying out of eddy motion is more than equal to that absorbed in overcoming viscous resistance after the throat is passed.

With high velocities however the process of eddy formation goes on after the throat is passed, part of the available pressure energy being expended to this end, and $H_{3}$ is in consequence 
increased. At a suitable velocity, a balance is obtained between the energy absorbed from $P$ to $B$ in eddy formation and that made re-available by the dying out of eddies, and in this case $H_{3}$ will equal $H_{1}$.

In every case however the head loss $\left(\mathrm{H}_{2}+\mathrm{H}_{3}\right)$ is greater than $H_{1}$, and experiments show very clearly that while it is possible to change pressur head to velocity head without appreciable loss of energy, it is impossible to change velocity head to pressure head by the reverse operation without loss, except at velocities too low to be considered in practice.

This is the important factor in the difference between the efficiencies of centrifugal pumps and turbines.

The following table indicates results of experiments carried out by the author on an apparatus similar to that shown in Fig. 29, and having angles of convergence and divergence equal to $3^{\circ} 55^{\prime}$. ' The length from entrance to throat and from throat to exit is $36 \cdot 4$ times the depth at the throat.

\begin{tabular}{c|c|c|c|c|c}
\hline $\begin{array}{c}\text { Velocity at Throat. } \\
\text { Feet per secorid. }\end{array}$ & $H_{1} \mathrm{ft.}$ & $H_{2} \mathrm{ft.}$ & $H_{3} \mathrm{ft.}$ & $H_{2}+H_{3}$ & $\begin{array}{c}\text { Total Energy at Throat } \\
\text { expressed in foot lbs. } \\
\text { per lb. }\end{array}$ \\
\hline $4 \cdot 38$ & $\cdot 070$ & $\cdot 190$ & $-\cdot 035$ & $\cdot 155$ & $\cdot 298$ \\
\hline $5 \cdot 56$ & $\cdot 115$ & $\cdot 143$ & $+\cdot 042$ & $\cdot 185$ & $\cdot 481$ \\
\hline $6 \cdot 40$ & $\cdot 125$ & $\cdot 125$ & $+\cdot 140$ & $\cdot 265$ & $\cdot 637$ \\
\hline $7 \cdot 30$ & $\cdot 146$ & $\cdot 142$ & $+\cdot 162$ & $\cdot 304$ & $\cdot 828$ \\
\hline $8 \cdot 30$ & $\cdot 150$ & $\cdot 190$ & $+\cdot 160$ & $\cdot 350$ & $1 \cdot 071$ \\
\hline $8 \cdot 52$ & $\cdot 170$ & $\cdot 240$ & $+\cdot 160$ & $\cdot 400$ & $1 \cdot 126$ \\
\hline
\end{tabular}

More experiments on this point are needed, but so far as they go these appear to indicate that the loss of energy in a diverging channel is practically constant for all angles of divergence from $2^{\circ}$ up to a certain limiting value, which for a circular section is apparently between $5^{\circ}$ and $6^{\circ}$. Above this limiting value it increases rapidly. Experiments by Dr. Stanton on a circular pipe having walls diverging at an angle of $5^{\circ}$ showed a loss, due 
to eddy formation and to friction, of $2 \frac{v^{2}}{2 g}$, where $v$ is the velocity at the throat. With a rectangular pipe, having two, sides parallel and the remaining sides diverging at an angle not exceeding 5 ., this loss was approximately $28 \frac{v^{2}}{2 g}$, this being about three times as great as the loss in ac corresponding converging tube. ${ }^{1}$

\section{Art. 30.-Applications of Bernoului 's Theorem. Venturi Meter.}

The Venturi water meter, invented by Herschell in 1881, and called by him after Venturi because of the experimental work of

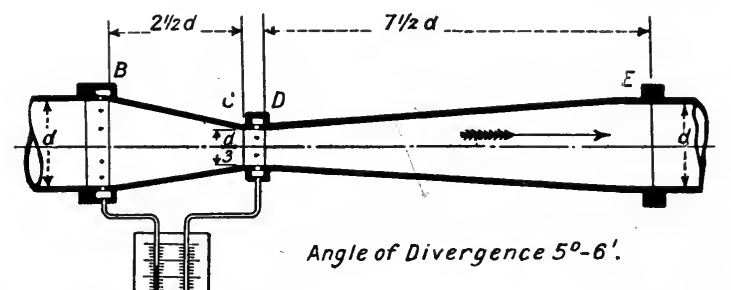

FIG. 31.-Venturi Meter.

the latter on the physical properties of diverging tubes, depends in its principles on the truth of Bernoulli's theorem.

It is at once the simplest, and for large quantities of water the most satisfactory, meter yet designed, and simply consists (Fig. 31) of a pipe passing the whole quantity of water to be measured, and fitted with a portion $B C$, uniformly converging to a short parallel throat $C D$. At $D$ the pipe again diverges to its full diameter at $E$. The usual proportions of the meter are indicated in the figure in terms of the pipe diameter, experiments showing that an angle of divergence of $5^{\circ} 6^{\prime}$ gives the best results in the reconversion of kinetic to pressure energy from $D$ to $E$. If the pipe ie horizontal, and if $A$ and $a$ be the areas of main pipe and of

1 Engineering, vol. 7t, p. 66t, November 21st, 1902. 
throat, then since-neglecting viscosity - we have no loss of head in the converging portion of the meter, we get

$$
\begin{aligned}
\frac{p_{A}}{W}+\frac{V_{A}{ }^{2}}{2 g} & =\frac{p_{a}}{W}+\frac{V_{a}^{2}}{2 g} \\
\therefore \quad \frac{p-p_{a}}{W} & =\frac{V_{a}^{2}-V_{A}^{2}}{2 !}
\end{aligned}
$$

But for continuity of flow $V_{a}=V_{A} \cdot \frac{A}{u}$

$$
\begin{aligned}
\therefore \quad \frac{p_{A}-p_{a}}{W} & =\frac{V_{A}^{2}}{2 g}\left\{\left(\frac{A}{a}\right)^{2}-1\right\} \\
\therefore V_{A} & =\sqrt{\frac{2 g\left(p_{A}-p_{a}\right)}{W\left\{\left(\frac{A}{a}\right)^{2}-1\right\}}}
\end{aligned}
$$

Owing to viscosity, the true velocity accompanying a given fall of pressure $\left(p_{A}-p_{a}\right)$ is less than this, the true velocity being given by

$$
V_{A}=c \sqrt{\frac{2 g\left(p_{A}-p_{a}\right)}{W\left\{\left(\frac{A}{a}\right)^{2}-1\right\}}}
$$

Here $c$ is a coefficient of velocity which depends slightly on the diameter and material of the pipes and on the velocity of flow, increasing with the diameter and velocity of flow and diminishing as the surface roughness increases. ${ }^{1}$

1 More correctly, inserting a term $\frac{4 f l \bar{\imath}^{2}}{2 g d}$ for the effect of viscosity, so that $\frac{p_{A}}{W}+\frac{V_{A}{ }^{2}}{2 g}=\frac{p_{a}}{W}+\frac{V_{a}{ }^{2}}{2 g}+\frac{4 f l r^{2}}{2 g d}$, equation (2), above, becomes

$$
V_{A}=\sqrt{\frac{2 g\left(p_{A}-p_{a}\right)}{W\left\{\left(\frac{A}{u}\right)^{2}-1+\frac{k^{\prime \prime}+f l}{d}\right\}}}
$$

where, if $\bar{r}^{2}=$ mean square of velocities between points (1) and (2), $k^{\prime \prime}=\left(\frac{v}{V_{A}}\right)^{2}$.

In the ordinary meter $\frac{V_{a}}{V_{A}}=\frac{A}{\iota}=9$.

$$
\therefore \frac{V_{a}{ }^{2}}{V_{A}{ }^{2}}=81 \quad \therefore \quad \bar{\imath}^{2}=V_{A^{2}}\left(1+\frac{2}{3} \cdot 81\right) \equiv \tilde{5} 5 V_{A^{2}} \quad \therefore \quad k^{\prime \prime}=5 \tilde{5} .
$$

Also this velocity occurs at a point where the diameter $=\sqrt[4]{\frac{d}{55}}=\frac{d}{2 \cdot 73}$. Again, $l=2 \cdot 5 d$, so that we have the coefficient of velocity given by

$$
c=\sqrt{\frac{80}{80+550 f}}=\sqrt{\frac{1}{1+6 \cdot 9 f}}
$$


Experiments by Herschell ${ }^{1}$ show that $c$ varies from $\cdot 94$ to unity, the great majority of tests giving values between $\cdot 96$ and .99. In a $48^{\prime \prime}$ meter $c$ had the value 995 .

For any meter $\left(\frac{A}{a}\right)$ is fixed, the ratio commonly being $9: 1$, so that

$$
\sqrt{\frac{2 g}{\left(\frac{A}{a}\right)^{2}-1}} \text { is constant }=k \text {. }
$$

Taking $\frac{A}{a}=9$, then $k:=\sqrt{\frac{2 g}{80}}=\cdot 8972$.

$\left.\begin{array}{l}\text { Then the volume in cubic feet } \\ \text { per second through meter }\end{array}\right\}=V_{A} A=c k A \sqrt{\frac{p_{A}-p_{a}}{W}}$

$$
=c k^{1} \sqrt{\frac{p_{A}-p_{a}}{W}}
$$

Here $\frac{p_{A}-p_{a}}{W}$ or $h_{A}-h_{a}$ is the difference in pressure at the points (1) and (2) expressed as a head in feet of water, and is directly measured in the meter. The constants $c$ and $k^{1}$ being determined for the instrument, the volume passing per second may be directly inferred.

This meter has the advantage of registering at almost any velocity, the permissible range of velocities depending on the permissible loss of head in passing the meter. Generally, a maximum velocity of up to sixteen times the minimum is permissible. It will register with velocities so low as $\cdot 2$ foot per second, and is exceedingly accurate when fitted to large mains, but is not suitable without careful calibration for use in small pipes below

where $f$ has the value given by the curves of Art. 65 for a pipe of diameter $\frac{d}{2 \cdot 73}$ and for a velocity of flow $=\sqrt{\overline{5} 0} V_{A}=7 \cdot 4 V_{A}$.

Ex. $12^{\prime \prime}$ meter. $V_{A}=2 \mathrm{f} . \mathrm{s}$. Here, taking the surface as that of a clean iron pipe, we have $f=\cdot 0055$.

$$
\therefore \quad c=\sqrt{\frac{1}{1 \cdot 0380}}=\cdot 982 .
$$

${ }^{1}$ For results of tests on meters fitted to pipes of 12 inches and 108 inches diameter, see "Trans. American Soc. Civil Engineers," November, 1887. For tests on a small meter for a $1 \frac{5}{8}$ pipe, see "Proc. Canadian Soc. Civil Engineers," 1902. 
about 4 " diameter, because of the greater proportional effect of viscosity in such pipes. It is moreover not well adapted for use in pipes where the water is subjected to periodic pulsations, as, for example, in the discharge pipe from a reciprocating pump, since this is likely to set up oscillations in the recording mechanism.

For measuring the difference of head $h_{A}-h_{a}$, a differential gauge, consisting of a U-tube containing mercury, may be used (Fig. 31), ${ }^{1}$ though where small differences of pressure are to be measured a preferable device is that shown in Fig. 32, where the difference of pressure head is directly measured in feet of water.

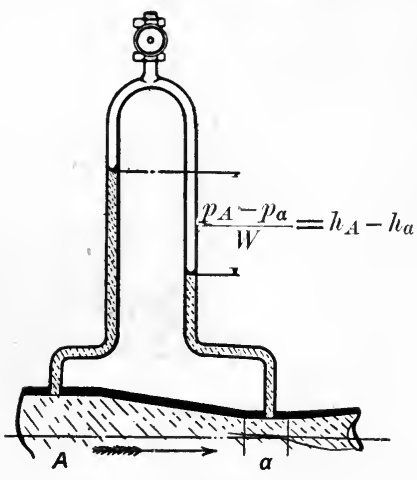

FIG. 32. Here compressed air must be supplied to the higher portion of the inverted U-tube. A continuous automatic record of the quantity of water passing is easily obtained, either by arranging for the continuous photographing of the difference in height of the pressure columns on a strip of sensitised paper mounted on a drum driven at a known rate by clockwork, or from the record of a pencil receiving vertical motion from a float asindicated in Fig. 33, which shows a form of automatic recording mechanism very commonly adopted. These records, by suitable adjustment of the pressure scale, give a curve showing volumes per second on a time base, so that the area under the curve gives the total volume passing the meter.

If the meter tube be not horizontal, and if $z$ be the difference in level at the entrance and throat, so that we have

$$
\frac{p_{A}}{W}+\frac{V_{A}^{2}}{2 g}=\frac{p_{a}}{W}+\frac{V_{a}^{2}}{2 g}+z
$$

it is easily shown that equation (4) becomes:-

$$
\text { Volume per second }=c k^{1} \sqrt{\left(h_{A}-h_{a}\right)-z} \text {. }
$$

1 Where a mercury gauge is used having the connecting pipes full of water, it is easily shown that the effective gauge reading is less than the apparent in the ratio $\frac{13 \cdot 6-1}{13 \cdot 6}=\cdot 926$. 
If the connecting tubes be shut off from the main, and connection be made so that the water may attain a common level in the two tubes, and if now the pencil of the recorder be put to zero, the effect is to automatically add $z$ to the observed head, and on cutting off the connection between the tubes and coupling up to the mains, true readings will be given on the ordinary record sheet.

It should be noted that the converging portion of the main is the only part really essential to the meter action. The diverging cone simply ensures that the reconversion of kinetic into potential energy shall take place without undue loss. Where the meter is required to measure the flow in either direction, the angles of convergence and divergence are each made equal to $5^{\circ} 6^{\prime}$.

Since this increases the length of meter tube it necessitates a

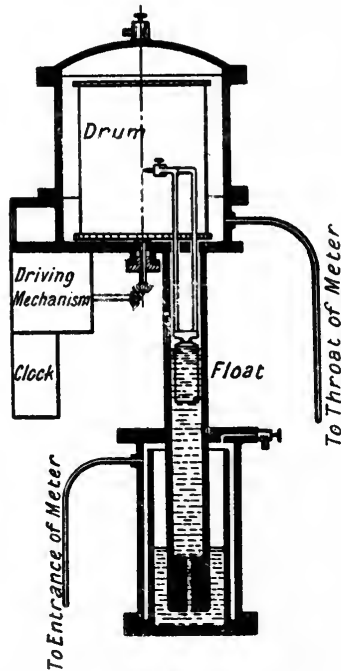

Fic. 33-Recording Mechanisin for Venturi Meter.

reduced value of $c$, the difference being about $1 \frac{1}{2}$ per cent. See the footnote to page 79 .

Art. 31.-Loss due to the Sudden Enlargenent of Cross Section of a Streau.

Whenever the cross section of a pipe or channel increases abruptly, the water, on passing the enlargement, is thrown into a state of unsteady motion, with a consequent loss of available energy. If the mean velocities before and after passing the enlargement are known, the equations of momentum may be applied to determine the magnitude of this loss.

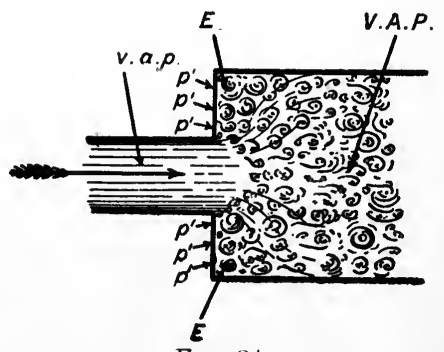

FIG. 34. 
For, consider the case of flow through a horizontal pipe (Fig. 34), suffering a sudden change of area from $a$ to $A$.

Let $p$ and $v$ be the pressure and mean velocity immediately before the change of section.

Let $P$ and $V$ be the pressure and mean velocity in the large pipe when the motion has steadied after impact.

Let $p^{\prime}$ be the mean pressure on the end of the pipe at $E E$.

Neglecting frictional effects, which will be small, the forces tending to produce a change of momentum in the direction of flow between the sections at which $p$ and $P$ are measured are :-

(1) the pressure over the area at $p=p a .^{1}$

(2) " , " , , , $E E=p^{\prime}(A-a)$.

(3) " " , , , at $P=P A$.

$\therefore \quad$ The force producing change of momentum in the direction of motion $=\left\{p a+p^{\prime}(A-a)-P A\right\}$ lbs.

This must equal the change of momentum per second in the direction of this force

$$
=\frac{W A V^{2}}{g}-\frac{W a v^{2}}{g}=\frac{W}{g}\left\{A V^{2}-a v^{2}\right\} .
$$

The assumption is commonly made that the pressure $p^{\prime}$ over the face $E E$ is uniform, and is sensibly equal to $p$. Although the actual distribution and magnitude of this pressure probably vary with the ratio of the areas and relative position of the two branches, and with the velocity, we have not sufficient experimental data to frame any definite law connecting the two. The above assumption, however, appears to be fairly well justified by the results of such experiments as are to hand, and making use of this we have, on combining equations (1) and (2)

$$
(p-P) A=\frac{W}{g}\left\{A V^{2}-a v^{2}\right\}
$$

Also for continuity of flow $A V=a v \quad \therefore v=\frac{A V}{a}$

$$
\therefore \quad(p-P) A=\frac{W}{g}\left\{1-\frac{A}{a}\right\} A V^{2} .
$$

1 The pressure $p$ being measured at the mean depth of the section. 
If now $H^{\prime}=$ loss of head due to shock at the enlargement, we have

$$
\begin{aligned}
& \frac{p}{W}+\frac{v^{2}}{2 g}=\frac{P}{W}+\frac{V^{2}}{2 g}+H^{\prime} \\
\therefore \quad & \frac{p-P}{W}+\frac{V^{2} A^{2}}{2 a^{2} g}-\frac{V^{2}}{2 g}=H^{\prime}
\end{aligned}
$$

Substituting for $\frac{p-P}{W}$ from (4) we get

$$
\begin{aligned}
H^{\prime} & =\frac{V^{2}}{2 g}\left(\frac{A}{a}-1\right)^{2} \\
& =\frac{v^{2}}{2 g}\left(1-\frac{a}{A}\right)^{2} \\
& =\frac{(v-V)^{2}}{2 g}
\end{aligned}
$$

Writing $m$ for $\frac{A}{a}$, relations (7) and (8) become:-

$$
\begin{aligned}
H^{\prime} & =\frac{V^{2}}{2 g}(m-1)^{2} \\
& =\frac{v^{2}}{2 g}\left(1-\frac{1}{m}\right)^{2}
\end{aligned}
$$

The following are results of experiments carried out by the author on a pipe having a sudden enlargement of diameter from $\cdot 65$ inches to $2 \cdot 15$ inches, the ratio $\frac{A}{a}$ being accurately 10.96. The positions at which gauges were inserted are shown

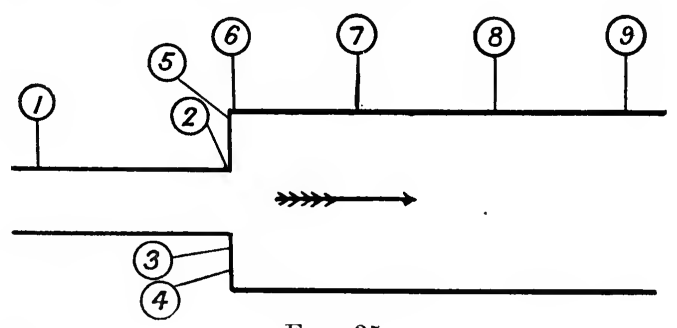

FIG. 35.

in Fig. 35. In the experiments the gauge pressures $3,4,5$ and 6 at different radii over the end of the tube were found to differ by not more than $\cdot 004$ feet of water, and the mean of these has been adopted as the value of $p^{\prime}$. The pressure at 2 is adopted as being $p$, and the pressure at (9) as $P$. 
HYDRAULICS AND ITS APPLICATIONS.

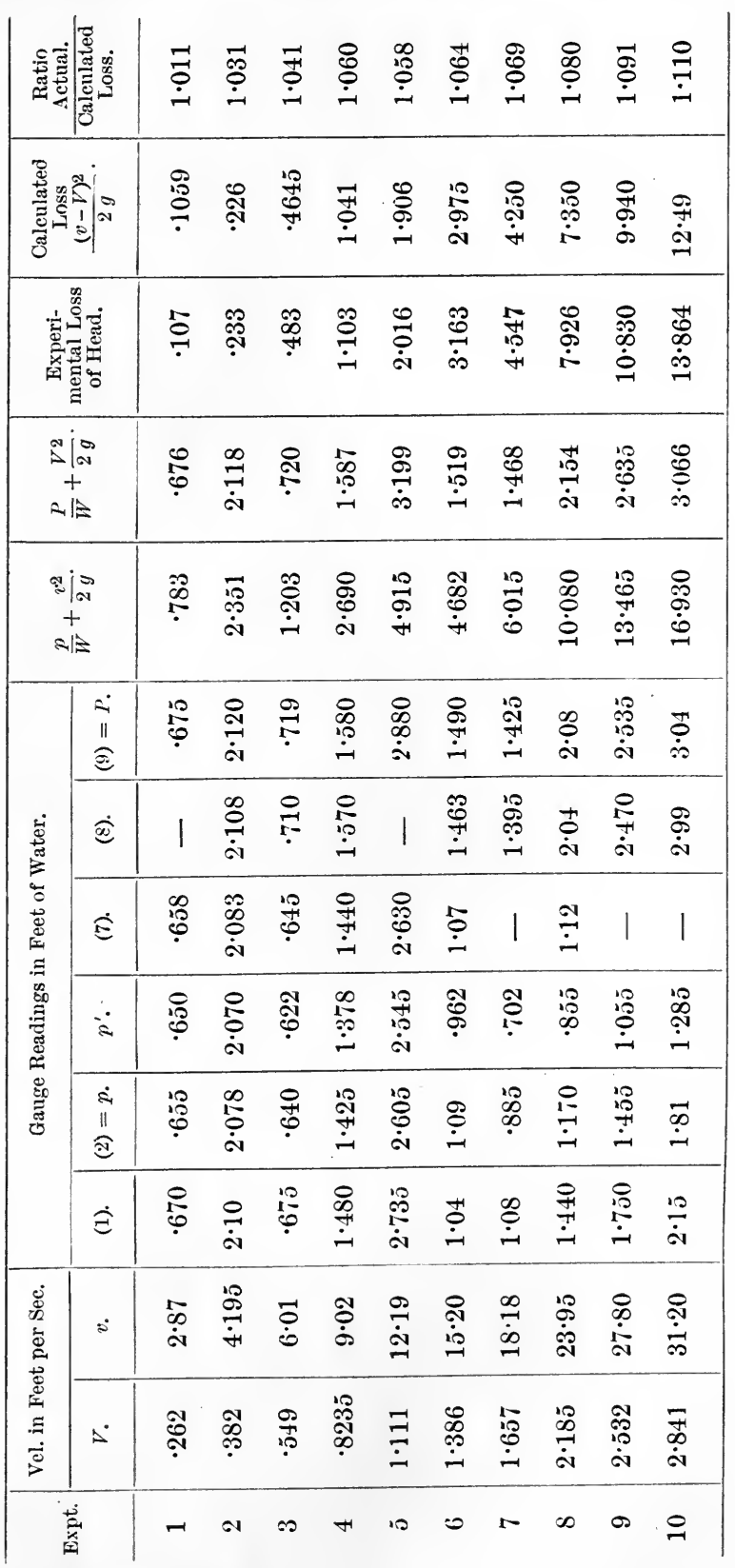


An examination of these results shows that in every case $p^{\prime}$ was less than $p$, the difference between $p$ and $p^{\prime}$ increasing with the velocity. Neglecting the assumption that $p^{\prime}=p$, equation (4) of this article becomes

$$
\begin{gathered}
(p-P)\left\{\frac{K A-a}{K-1}\right\}=\frac{W}{g} A V^{2}\left(1-\frac{A}{a}\right) \\
K=\frac{P-p^{\prime}}{p-p^{\prime}}
\end{gathered}
$$

where

Substituting this value in equation (6) we have finally

$$
H^{\prime}=\frac{V^{2}}{2 g}\left\{\left(\frac{A}{a}\right)^{2}-1+2 \frac{\left(1-\frac{A}{a}\right)(K-1)}{K-\frac{a}{A}}\right\}
$$

In these experiments $\frac{A}{a}=10 \cdot 96$, so that this becomes

$$
H^{\prime}=\frac{V^{2}}{2 g}\left\{119-\frac{19 \cdot 92(K-1)}{K-\cdot 0912}\right\}
$$

\begin{tabular}{|c|c|c|c|c|c|c|c|c|c|c|}
\hline $\begin{array}{l}\text { apr of } \\
\text { seriment }\end{array}$ & 1 & 2 & 3 & 4 & 5 & 6 & 7 & 8 & 9 & 10 \\
\hline $\begin{array}{l}\text { head as } \\
\text { wlated. }\end{array}$ & $\cdot 1089$ & $\cdot 238$ & $\cdot 480$ & $1 \cdot 090$ & $1 \cdot 965$ & $3 \cdot 059$ & $4 \cdot 44$ & $7 \cdot 70$ & $10 \cdot 40$ & $13 \cdot 20$ \\
\hline $\begin{array}{l}\text { css of } \\
\text { :al over } \\
\text { apretical } \\
\text { s. . }\end{array}$ & $-1 \cdot 1 \%$ & $-2.1 \%$ & $+\cdot 62 \%$ & $+1 \cdot 2 \%$ & $+25 \%$ & $+3 \cdot 3 \%$ & $+2 \cdot 2 \%$ & $+2.85 \%$ & $+4 \cdot 1 \%$ & $+4 \cdot 8 \%$ \\
\hline
\end{tabular}

The values of $H^{\prime}$ calculated from this formula, and also the excess of the actual loss over that thus calculated, are tabulated below :-

As the measured loss of head includes frictional losses between gauges (2) and (9), which are 10 inches apart, the coincidence between actual and calculated losses is very close indeed.

It is worthy of note that if the pressure at gauge (7), which is 4 inches from the enlargement, be taken as $P$, the loss of head is approximately 20 per cent. greater than the theoretical. 
Throughout the remainder of the book, the loss due to an enlargement of section will be taken as being given with sufficient accuracy by the simple formula $H^{\prime}=\frac{(v-V)^{2}}{2 g}$ feet.

This is usually taken as applying wherever two streams of fluid moving in the same direction combine to form a single stream of velocity $V$. If $v_{1}$ and $v_{2}$ are the initial velocities of the streams, their loss of energy per lb. at impact is taken as $\frac{\left(v_{1}-V\right)^{2}}{2 g}$ in the one and $\frac{\left(v_{2}-V\right)^{2}}{2 g}$ in the other case. Where the streams are inclined to each other and to the direction of the final motion, the above equation may be applied separately to their component velocities in, and perpendicular to, the direction

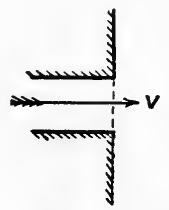

(a)

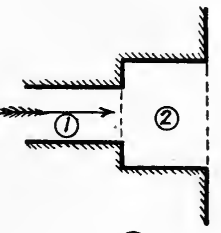

(b)

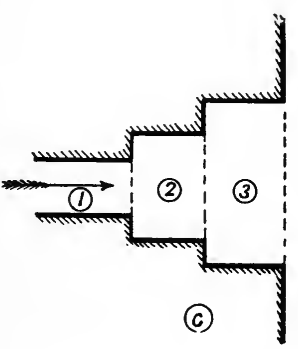

(c)

FIG. 36.

of the combined stream. The sum of the losses of head thus obtained will then give the total loss due to the impact.

The loss of head at a sudden enlargement is usefully applied to reduce leakage in the system of grooves for the water packing of small pistons and plungers.

Special Cases.-(a) A very large: Ex-pipe discharging directly into a reservoir (Fig. 36, a).

Here $V$ may be taken as zero.

Then the loss $H^{\prime}=\frac{(v-o)^{2}}{2 g}=\frac{v^{2}}{2 g}$ i.e., the whole of the kinetic energy is lost.

(b) Pipe discharging into a reservoir in two stages (Fig. 36, b). 
Here the loss at first enlargement ${ }_{1} H_{2}=\frac{\left(v_{1}-v_{2}\right)^{2}}{2 g}$

$$
" \quad, \quad \text { second } \quad " \quad{ }_{2} H_{3}=\frac{\left(v_{2}-v_{3}\right)^{2}}{2 g}
$$

and since $v_{3}=o$, the total loss ${ }_{1} H_{3}=\frac{\left(v_{1}-v_{2}\right)^{2}+v_{2}^{2}}{2 g}$

Putting $\frac{a_{2}}{a_{1}}=m$ this becomes $\frac{v_{1}^{2}}{2 g}\left\{1-\frac{2}{m}+\frac{2}{m^{2}}\right\}$

Differentiating with respect to $m$ and expressing the fact that $\frac{\left.d{ }_{1} H_{3}\right)}{d m}=0$ for ${ }_{1} H_{3}$ to be a minimum, we get, for the loss to be a minimum, $\frac{2}{m^{2}}-\frac{4}{m^{3}}=0 \quad \therefore m=2$.

$\therefore$ loss is a minimum when $m=2$ and then equals $\frac{1}{2} \cdot \frac{v_{1}{ }^{2}}{2 g}$

(c) If the pipe discharges in three stages (Fig. 36, c), the ratios $\frac{a_{2}}{a_{1}}, \frac{a_{3}}{a_{2}}$ being equal and each equal to $m$, the total loss, ${ }_{1} H_{4}$ may be shown to equal $\frac{v_{1}^{2}}{2 g}\left\{1-\frac{2}{m}+\frac{2}{m^{2}}-\frac{2}{m^{3}}+\frac{2}{m^{4}}\right\}$ which is a minimum when $m=1 \cdot 65$, and then equals $368 \frac{v_{1}^{2}}{2 g}$.

This is only true when each change of area is so far from the preceding one as to allow of the motion steadying between changes.

To reduce the loss as much as possible, the number of steps should be as large and the increment of area at each step as small as possible, the limit being reached when a continuously diverging outlet pipe is used.

With an outlet of this type having a final area of 3 times the initial, the loss due to shock at exit may be reduced to about $\cdot 3 \frac{v^{2}}{2 g}$ (Reynolds), this with an angle of divergence not exceeding $6^{\circ}$.

The rate of this divergence is all-important. If the angle is greater than the vertical angle of the conical stream naturally formed during sudden enlargement, the pressure over the 
diverging boundaries will be the same as that over the blank end of the enlarged pipe, and the resistance will be unaltered.

If, however, the boundaries intersect this cone, the pressure over these faces is increased, and the resistance is in consequence diminished. The less the angle of divergence, the greater this pressure and the less the resistance.

As will be readily understood, the effect depends largely on the initial velocity, being the less as this increases.

It appears probable that, as this natural angle of stream divergence increases as the velocity decreases, the most efficient adjutage would be trumpet-shaped, the rate of divergence of its boundaries increasing towards the outlet.

\section{Art. 32.-Loss due to Sudden Contraction of Areas of a Stream.}

When water flowing along a channel or through a pipe meets with a sudden contraction of the area of the pipe, as shown in

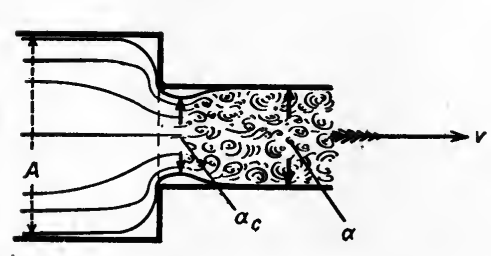

FI(x. 37.

Fig. 37, we also get a loss of energy which is due, not directly to the contraction of the stream, but to the subsequent re-enlargement which always takes place.

The stream, after passing the contraction, suffers a further diminution in area, and for a short distance fails to fill the pipe, afterwards re-expanding as shown. ${ }^{1}$ Up to the contracted section the loss is solely that due to simple viscous resistance.

If $A$ represents the larger stream area

$\begin{array}{llll}\| a & , & \text { smaller } & ,\end{array}$

$V$, $v$ and $v_{c}$ representing the velocities at these sections, the loss of energy due to this enlargement will be approximately

$$
\frac{\left(v_{c}-v\right)^{2}}{2 g} \text { ft. lbs. per lb. }
$$

1 This action may occur even though the pipe is full of water, the space shown empty in Fig. 37 being now occupied by dead water which takes no part in the flow through the pipe. 


$$
\text { If } m=\frac{A}{a} \text { we have } v=m V \text {. }
$$

Also

$$
\frac{v_{c}}{V}=\frac{A}{a_{c}}=K_{c} \text { (say) }
$$

$$
\begin{aligned}
\therefore \quad \text { loss }=\frac{\left(K_{c} V-m V\right)^{2}}{2 g} & =\left(K_{c}-m\right)^{2} \frac{V^{2}}{2 g} . \\
& =F \cdot \frac{V^{2}}{2 g} \text { ft. lbs. per lb. }
\end{aligned}
$$

Here $F$ is called the coefficient of hydraulic resistance, and can only be determined experimentally.

Writing $C_{c}$ for $\frac{a^{c}}{a}$, the above may be expressed in the form

$$
\frac{\left(v_{c}-v\right)^{2}}{2 g}=\left(\frac{1}{C_{c}}-1\right)^{2} \cdot \frac{v^{2}}{2 g}=F^{1} \frac{v^{2}}{2 g} .
$$

If $C_{c}$ has the value 586 , the loss $=\cdot 50 \frac{r^{2}}{2 g}$. This is approximately the value of $C_{c}$, where the area $A$ is so large as not to effect the stream line production, and gives the loss usually found where a pipe is led out of a reservoir by means of a sharp-edged junction.

A series of experiments carried out by the author on the apparatus shown in Fig. 35, having a value of $m=10 \cdot 96$, and having pipe diameters of $2 \cdot 15$ inches and 65 inch, gave a mean value of 600 for $C_{c}$, over a range of values of $v$ from 1.35 to $8 \cdot 69$ feet per second. The value diminished from $\cdot 610$ with the lowest to 587 with the highest of these velocities.

\section{ARt. 33.-Initiation and Stoppage of Motion.}

The general equations of motion of Art. (24) lead to the final result

$$
\frac{d}{d s}\left\{\frac{p}{W}+\frac{v^{2}}{2 g}+z\right\}=-\frac{1}{g} \frac{d v}{d t}
$$

as applying to the accelerated motion of a frictionless and incompressible fluid, and if the acceleration $\frac{d v}{d t}$ be represented by $a$, this expression may be integrated where $a$ is uniform or is known in terms of $s$ or of $v$. 
Example 1.-Suppose the acceleration to be uniform and independent of $s$, on integrating we now obtain the expression

$$
\frac{p}{W}+\frac{v^{2}}{2 g}+z=-\frac{s}{g} \text { 計 } A
$$

where $A$ is a constant of integration.

Example 2. - Imagine the motion to be simple harmonic. This would be the case if the fluid were contained in a pipe and followed the motion of a piston driven from a uniformly rotating crank of radius $r$, by means of a long connecting rod. If the angular velocity of the crank is $w$ radians per second $\left(w=\frac{2 \pi n}{60}\right)$ where $n=$ revolutions per minute, and if $\theta$ represent the crank angle described from the dead centre in time $t(\theta=w t)$, we have : $v=w r \sin \theta ; a=w^{2} r \cos \theta ; s=r(1-\cos \theta)$, and equation (1) becomes on integrating

$$
\begin{aligned}
\frac{p}{W}+\frac{v^{2}}{2 g}+z & =-\int \frac{v^{2} r \cos \theta}{g} \cdot d s \\
& =-\frac{w^{2} r^{2}}{g} \int \cos \theta \sin \theta d \theta \\
& =\frac{w^{2} r^{2}}{4 g} \cos 2 \theta+A .
\end{aligned}
$$

As a further example of the handling of this equation, determine the pressure produced by the uniform closing of a valve fitted to the outlet from a 6 -inch pipe. This pipe is 1,066 feet long, and is discharging with a velocity of 7.5 feet per second. The valve is at a depth of $\mathbf{1 0 0}$ feet below the surface level in the supply reservoir, and the time of closing the valve is 03 seconds.

Here $s$ is to be measured along the pipe from the entrance. Let suffix 0 refer to entrance. Let $p, v, z$, refer to valve. Then $s_{0}=0$. From equation (2) we have

$$
\begin{gathered}
\frac{p_{0}}{W}+\frac{v_{0}^{2}}{2 g}+z_{0}=A . \quad \text { Since } v_{0}=v \\
\therefore \frac{p-p_{0}}{W}+z-z_{0}=-\frac{s}{g} a .
\end{gathered}
$$

Taking the datum level at the valve we have $z=0$; also $a=\frac{d v}{d t}=-\frac{7 \cdot 5}{.03}=-250$ f.s.s., the negative sign being taken since the acceleration is negative. 
$\therefore \quad$ (4) becomes

$$
\frac{p-p_{0}}{W}-z_{0}=\frac{1066}{32 \cdot 2} \times 250
$$

or

$$
\frac{p}{W}=\frac{p_{0}}{W}+z_{0}+8,276 \text { feet of water, }
$$

i.e., the pressure at the valve is equal to the statical head $\left(\frac{p_{0}}{W}+z_{0}\right)$ together with an additional head of 8,276 feet, or $3,585 \mathrm{lbs}$. per square inch, the latter being due to the closing of the valve.

This problem may be considered from another point of view, and the pressure determined from a consideration of the force necessary to produce a retardation of $7 \cdot 5$ feet per second in -03 seconds, in a column of water 1,066 feet in length. Expressed mathematically we have:-Force = mass of column $\times$ retardation.

$$
\therefore \quad F=\frac{\pi \imath^{2} l W}{g} \times a \text { pounds, }
$$

where $r=$ radius ; $l=$ length of pipe in feet.

This force can only be produced by a pressure at the valve in excess of that due to the statical head and of magnitude $p$ lbs. per square foot, where $\pi r^{2} p=F$.

$$
\begin{aligned}
\therefore \quad p & =\frac{W l}{g} \text { a lbs. per sq. foot. } \\
& =\frac{62 \cdot 4 \times 1066 \times 250}{32 \cdot 2} \\
& =516,300 \mathrm{lbs} . \text { per sq. foot. } \\
& =3,585 \mathrm{lbs} . \text { per sq. inch. }
\end{aligned}
$$

Expressed in another way, the retarding force at the valve must be sufficient to completely destroy the momentum of the water column in 03 seconds. Expressing this mathematically, we again get the preceding equation.

The method of treatment so far outlined, while giving results which are rigorously true for a perfect incompressible fluid, fails to account for many of the phenomena actually observed during the stoppage of motion in a long column of water, since these are largely due to the elasticity of the water column. For example, an examination of equations (4) or (5) indicates that 
an instantaneous stoppage of motion, involving an infinite retardation, will necessitate an infinite retarding force, and hence an infinite pressure at the closed end of the pipe, a conclusion which is not at all borne out by the result of experiment.

Actually, when the column is brought instantaneously to rest, compression takes place; a wave of compression is reflected from the closed end of the pipe; and the initial kinetic energy of the water is transformed partly into kinetic energy of wave propagation and partly into resilient energy or energy of strain.

When the retardation is gradual, part of the kinetic energy is absorbed in doing work against the retarding force, and part in compressing the column, the latter factor becoming increasingly important as the rate of retardation is increased.

The phenomena accompanying such stoppage of motion, involving as they do wave

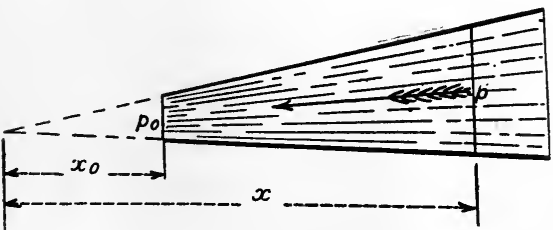

FIG. 38. motions of some complexity, do not in their entirety readily lend themselves to mathematical treatment. In ordinary cases however, theory, though imperfect, gives a reasonable approximation to actual results, and the more important cases which occur in practice will be briefly considered from this point of view in Art. 70 .

Art. 34.-Flow in Converging Channels. Radial Flow.

Where flow takes place in a converging channel, the motion is steady and, neglecting viscosity, the energy throughout any stream tube is constant, so that we may write

$$
\frac{p}{W}+\frac{v^{2}}{2 g}+z=\text { constant. }
$$

But for continuity of flow, if $A=$ area of channel at some point where the velocity is $v$, we have

$$
A v=\text { constant }=A_{0} v_{0}
$$

where $A_{0}$ and $v_{0}$ are the area and velocity at some point distant $x_{0}$ from the point of convergence of the boundaries (Fig. 38). 
Putting $A=k x$ we have $v=\frac{x_{0} v_{0}}{x}$, and if $z$ is constant, i.e., if the stream lines are horizontal, we get

$$
\begin{aligned}
& \frac{p}{W}+\frac{x_{0}{ }^{2} v_{0}{ }^{2}}{2 g x^{2}}=c \\
\therefore \quad & \frac{p}{W}=c-\frac{x_{0}^{2} v_{0}{ }^{2}}{2 g x^{2}} .
\end{aligned}
$$

But from (2) $\frac{p_{0}}{W}=c-\frac{v_{0}^{2}}{2 g}$

$$
\therefore \quad c=\frac{p_{0}}{W}+\frac{v_{0}^{2}}{2 g}
$$

$$
\therefore \quad \frac{p-p_{0}}{W}=\frac{v_{0}^{2}}{2 g}\left\{1-\frac{x_{0}^{2}}{x^{2}}\right\} \text { ft. of water }
$$

This applies to flow through a converging channel having parallel upper and lower boundaries and to the case of (inward) radial flow towards a centre. The result gives the fall in pressure between two points radially distant $x$ and $x_{0}$ from the origin, and may be applied to flow in an inward flow radial turbine. Since with outward flow between diverging boundaries the motion is unsteady, Bernoulli's equation ceases to hold, so that the formula is inapplicable to the case of a radial (outward flow) turbine. ${ }^{1}$

In the case of flow through a circular converging pipe or nozzle, $A=k x^{2}$.

$$
\therefore \quad v=\frac{x_{0}^{2} v_{0}}{x^{2}} \text {, and assuming } z \text { constant, }
$$

we have

$$
\begin{gathered}
\frac{p}{W}+\frac{x_{0}{ }^{4} v_{0}{ }^{2}}{2 g x^{4}}=c . \\
c=\frac{p_{0}}{W}+\frac{v_{0}{ }^{2}}{2 g} \\
\therefore \quad \frac{p-p_{0}}{W}=\frac{v_{0}{ }^{2}}{2 g}\left\{1-\frac{x_{0}{ }^{4}}{x^{4}}\right\}
\end{gathered}
$$

1 Steady motion is, however, possible with radial outward flow without solid boundaries. This is shown when two equal and steady vertical jets impinge directly on each other. Here a circular disc of water with radial outward flow and having perfectly steady stream-line motion, as may be shown by the introduction of colour bands, is produced. 
Art. 35.-Change of Pressure across Streau Lines.

If water be moving with stream line motion, these stream lines being curved, we shall experience a change in pressure as we move radially inwards or outwards from one stream tube to another. This change in pressure may be determined as

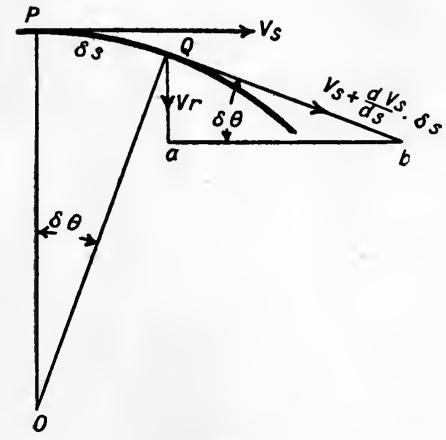

Fig. 39.

follows from the general equations of motion :-

Let $P Q$ be a portion of an elementary stream tube.

Suppose $V_{8}$ to be the stream line velocity at any point $P$ (Fig. 39).

Suppose $V_{r}$ to be the velocity in the direction of the radius at $P$.

Let $P Q=\delta s$.

, $\widehat{P O Q}=\delta \theta$.

,$r=$ radius of curvature of stream line at $P$.

Then the stream line velocity at $Q=\left(V_{8}+\frac{d V_{8}}{d s} \cdot \delta s\right)$.

The component of this in the direction $P O=-\frac{d V_{r}}{d s} \cdot \delta s$.

$\therefore$ From the figure we have

$$
-\frac{\frac{d V_{r}}{d s} \cdot \delta s}{V+\frac{d V_{8}}{d s} \cdot \delta s}=\frac{a Q}{b Q}=\sin \delta \theta .
$$

In the limit when $\delta s$ is infinitely small, $\sin \delta \theta=\delta \theta$ and $V_{s}+\frac{d V_{s}}{d s} \cdot \delta s=V_{s} . \quad$ Also $\delta s=v \delta \theta$.

$\therefore \quad$ in the limit $-\frac{d V_{r}}{d s}=\frac{V_{s}}{\frac{d s}{d \theta}}=\frac{V_{s}}{r}(1)$.

Taking equations (16), Art. 24, of the general equations of motion and multiplying these by $l^{1}, m^{1}$, and $n^{1}$, the direction cosines of the radius of curvature, we get in place of equation (17) 


$$
\begin{gathered}
-n^{1} g-\frac{1}{\rho} \frac{d p}{d r}=l^{1} \frac{\partial u}{\partial t}+m^{1} \frac{\partial v}{\partial t}+n^{1} \frac{\partial w}{\partial t} \\
+V_{s}\left(l^{1} \frac{d u}{d s}+m^{1} \frac{d v}{d s}+n^{1} \frac{d w}{d s}\right) .
\end{gathered}
$$

Whence from (13) and (14) we get

$$
-n^{1} g-\frac{1}{\rho} \frac{d p}{d r}=\frac{\partial V_{r}}{\partial t}+V_{s} \frac{d V_{r}}{d s} .
$$

Substituting $-\frac{V_{s}}{r}$ for $\frac{d V}{d s}$ from (1) above, we have

$$
\begin{aligned}
-n^{1} g-\frac{1}{\rho} \frac{d p}{d r} & =\frac{\partial V_{r}}{\partial t} \cdot \frac{V_{8}^{2}}{r} \\
\therefore \quad \frac{d}{d r}\left\{g z+\frac{p}{\rho}\right\} & =-\frac{\partial V_{r}}{\partial t}+\frac{V_{8}^{2}}{r} .
\end{aligned}
$$

For steady motion $\frac{\partial V_{r}}{\partial t}=0$

$$
\therefore \frac{d}{d r}\left\{z+\frac{p}{W}\right\}=+\frac{V_{s}^{2}}{g r} .
$$

Taking points in the same horizontal plane we have

$$
\frac{d p}{d r}=+\frac{W v^{2}}{g r}
$$

This may be more easily seen by considering the equilibrium of an elementary column of fluid of sectional area $\delta$ a (Fig. 40), having its axis radial, and resting with its two ends in two stream tubes, whose pressures are $p$ and $\left(p+\frac{d p}{d r} \cdot \delta r\right)$ respectively. Th $\mathrm{e}$

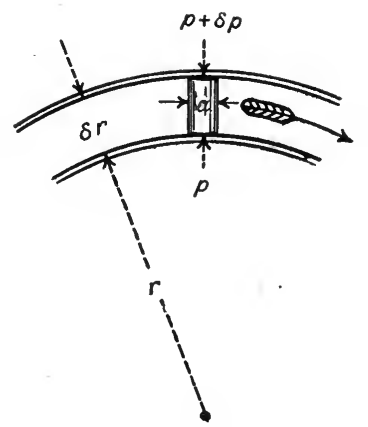

Fig. 40. centrifugal force on the column is balanced by the difference of pressure on the two ends, and we have, for equilibrium,

$$
\begin{gathered}
W . \delta a . \delta r \cdot \frac{v^{2}}{g r}=\frac{d p}{d r} \cdot \delta r \cdot \delta a \\
\therefore \frac{W v^{2}}{g r}=\frac{d p}{d r}
\end{gathered}
$$


Art. 36.-Vortex Motion. Pressure in a Rotating Liquid.

Where a mass of liquid moves as a whole with vortex motion, this may occur in either of two ways.

The first is seen when a vessel containing water is rapidly rotated, or when the contained water is stirred so as to make it rotate as a solid body, the velocity increasing with the radius. Here the motion is unsteady throughout, and the vortex is termed a Forced Vortex.

The second is seen when water flows through a hole in the bottom of a vessel. Here a vortex is usually formed naturally; some initial disturbance of the water determining the direction

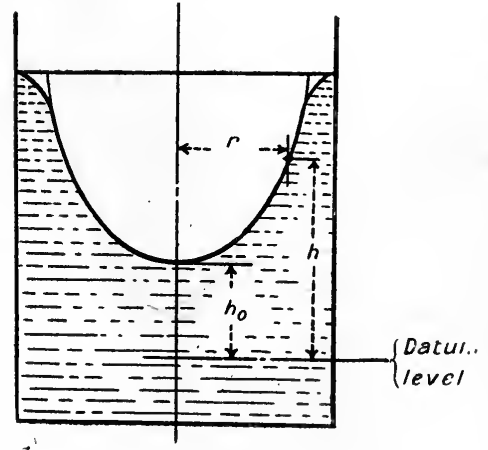

Fig. 41.-Forced Vortex. of rotation, although in the northern hemisphere the earth's rotation would itself tend to cause a rotation in an anti-clockwise direction as viewed from above. This is termed a Free Spiral Vortex. The water moves spirally towards the centre with stream line motion, so that, neglecting viscosity, its energy per unit mass is everywhere the same. If, while the mass is rotating, the hole be stopped, the motion becomes one of simple rotation in horizontal planes, and forms a Free Cylindrical Vortex.

Forced Vortex Motion.-Since the angular velocity $w$ is constant, we have at any radius $r, v=w r$.

The increase in pressure radially is given by

$$
\frac{d p}{d r}=\frac{W}{a} \cdot \frac{w^{2} r^{:}}{r}=\frac{W}{g} w^{2} r
$$

Integrating between the limits $r_{1}$ and $r_{2}$ we have

$$
\frac{p_{1}-p_{2}}{W}=\frac{w^{2}}{2 g}\left(r_{1}^{2}-r_{2}^{2}\right)
$$

If $p=p_{0}$ where $r=o$, we get

$$
\frac{p-p_{0}}{W}=\frac{w^{2}}{2 g} \cdot r^{2}
$$


or, putting $\frac{p}{W}==h$ (Fig. 41),

$$
h-h_{0}=\frac{w^{2}}{2 g} \cdot r^{2} \quad \therefore \quad h=h_{0}+\frac{w^{2} r^{2}}{2 g}
$$

which is the equation to a parabola.

Since the pressure at any point is that equivalent to the column of water supported at the point, it follows that all surfaces of equal pressure, including the free surface of the vortex, form paraboloids of revolution having the axis of rotation as their common axis. Near the sides of the vessel the liquid lags owing to viscosity, and here the surface level will fall below that of the paraboloid.

Free Cylindrical Vortex Motion.-Here, since we have stream line motion, the equation $\frac{p}{W}+\frac{v^{2}}{2 g}+z=$ constant; holds.

$\therefore$ in any horizontal plane $\frac{p}{W}+\frac{v^{2}}{2 g}=$ constant.

Differentiating we have

$$
\frac{1}{W} \cdot \frac{d p}{d r}+\frac{v}{g} \cdot \frac{d v}{d r}=0
$$

Introducing the condition for rise in pressure across a stream tube, viz., $\frac{d p}{d r}=\frac{W v^{2}}{g r}$, this becomes

$$
\begin{gathered}
\frac{v^{2}}{g r}+\frac{v}{g} \frac{d v}{d r}=0 \\
\therefore \frac{d v}{d r}+\frac{v}{r}=0, \text { or }-\frac{d r}{r}=\frac{d v}{v}
\end{gathered}
$$

Integrating we get $\log _{e} r+\log _{e} v=$ constant $=B$.

$$
\begin{gathered}
\therefore v r=\text { constant }=e^{B}=B_{1} . \\
\therefore \quad v=\frac{B_{1}}{r}
\end{gathered}
$$

i.e., in a free vortex the velocity varies inversely as the distance from the axis of rotation.

It follows that the increase of pressure with radius is identical with that in inward radial flow.

H.A. 
Thus if $p_{1}, v_{1}, r_{1}$, are the attributes of a point in the same horizontal plane as $p, v, r$, we have

$$
\frac{p-p_{1}}{W}=\frac{v_{1}^{2}}{2 g}\left\{1-\frac{r_{1}^{2}}{r_{2}}\right\}
$$

Putting $\frac{p}{W}$ constant in Bernoulli's equation we get the equation to the curve of equal pressure, and substituting for $v$ in terms of $r$ from (2) we have, since $\frac{v^{2}}{2 g}+z=$ constant $=C$

$$
\begin{aligned}
& \frac{B_{1}^{2}}{2 g r^{2}}+z=C \\
& \because z=C-\frac{B_{1}}{2 g r^{2}}
\end{aligned}
$$

the equation to a hyperbolic curve of the nature $y x^{2}=A$, and

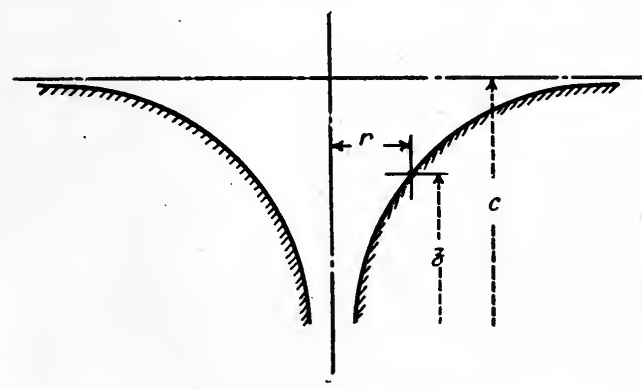

Fig. 42.-Firee Vortex. which is asymptotic to the axis of rotation and to the horizontal through $z=C$ (Fig. 42).

A Free Spiral Vortex may be considered as a case of cylindrical vortex and radial motion combined in the required proportions, since in each case the velocity is inversely proportional to the radius. The angle between the stream line and the corresponding radius vector at any point will then be constant, and the streams line will form a series of equiangular or logarithmic spirals. The difference of pressure between any two points may then be found by adding the pressure differences due to the two methods of flow taken separately.

Strictly this should only be applied to cases of inward flow, since outward flow causes instability.

Since the velocity varies inversely as the radius, and since this velocity cannot be infinite at the axis where $r=o$, we must have an air column at the centre of a free vortex. When this air column cannot be maintained, we get a combination of a forced 
vortex-at and near the axis-and a free vortex at points further removed (Fig. 43).

If $a$ be the radius at which the two surface curves intersect, the depth of the central depression below the general level of the surface may be shown to be given by $\frac{w^{2} a^{2}}{g}$ feet.

This is termed a Compound Vortex, and the state of affairs there existing is of importance in its application to the theory of the flow of water in a centrifugal pump fitted with a Vortex Chamber. Here the water in the impeller forms a forced vortex with outward radial flow, while when free of the vanes an approximation to a free spiral vortex is formed, and the pressure increases as the velocity diminishes outwards. In this free vortex we have theoretically no loss of energy, and we thus get a change from kinetic to pressure energy which is of great importance as affecting the efficiency of the pump.

Certain properties of the free vortex are of interest. If a film of oil be formed on the surface, owing to its density

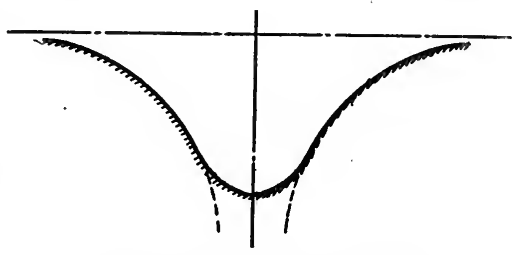

FIG. 43.-Compound Vortex. being less than that of water this at once approaches the axis of rotation and disappears down the funnel of the vortex. The same thing happens to any small floating body. If of moderate dimensions, however, the portion nearer the axis of rotation comes into a region of higher velocity than those portions further removed, and a series of frictional forces are thus called into play, acting on the body, which are greater as the points at which they act are nearer the axis of rotation. The effect of this is to produce a rotation of the body about some point near its outer edge, and away from the centre of the vortex. The body is thus alternately attracted to and repelled from this centre, the action being repeated as long as the vortex is in existence. A large floating body destroys the funnel in its upper part, and, if air is prevented from entering through the exit, in the lower part also. Since in the floating body $v \propto r$, while in the 
liquid $v \propto \frac{1}{r}$, this, by friction, tends to destroy the vortex motion of the upper layers of liquid, and, by viscosity, also of the lower layers.

The formation of a vortex in a discharging vessel increases the time of discharge by diminishing the effective area of the orifice, and by reducing the inclination of the issuing particles to the plane of the orifice.

\section{Examples.}

(1) Taking equations (10), Art. 23, for the motion of a viscous fluid, show that if the velocity be uniform in the direction of $x$, and be zero in the direction of $y$ and $z$, these reduce to

$$
\frac{d p}{d x}=\mu \cdot \frac{d^{2} u}{d y^{2}} ; \quad \frac{d p}{d y}=0 ; \quad \frac{d p}{d z}=0,
$$

the equations deduced by another method in Art. 19 in considering the steady flow between two parallel plates.

(2) In a 12 inch Venturi meter the throat diameter is 4 inches. Taking $C$ to be constant $=\cdot 982$, determine the difference of head at the entrance and throat when discharging

$\left.\begin{array}{ll}\text { (a) } & 100 \\ \text { (b) } & 500 \\ \text { (c) } & 1000\end{array}\right\}$ gallons per minute.

N.B. -1 gallon $=10 \mathrm{lbs}$.

Answer. (a) Vel. $=\cdot 340 f . s-\cdot 0920$ feet.

(b) , $=1 \cdot 701 \mathrm{f} . \mathrm{s}-2 \cdot 302$ feet.

(c),,$=3 \cdot 402 f . s-9 \cdot 208$ feet.

(3) Allowing for the variation of $f$ with velocity as indicated by the curve Fig. 84, for a cast iron pipe, determine the actual loss of head corresponding to the rates of flow given in the preceding question.

Answer. (a) 0923 feet of water.

(b) $2 \cdot 302$, , ,

(c) $9 \cdot 200$, , ,

(4) A uniform pipe, 200 feet long, is fitted with a plunger which, originally moving at 6 feet per second, is brought to rest 
uniformly in 1.5 second. Assuming the water to be incompressible, determine the pressure on the piston caused by the retardation.

Answer. 10.76 lbs. per square inch.

(5) If, in the above example, the plunger is driven from a crank 1 foot long, and making 100 revolutions per minute, and with S. H. motion, determine the pressure produced by retardation at the end of the strolse.

\section{Answer. 295 lbs. per square inch.}

(6) Inward radial flow takes places between two parallel circular plates, 3 feet diameter and 6 inches apart. Discharge takes place through an orifice 18 inches diameter in the centre of the lower plate. Neglecting viscous resistances, determine the pressure at the entrance when 1000 cubic feet per minute pass the plates, assuming the pressure at the edge of the discharge orifice to be atmospheric.

Answer. $\quad \cdot 585$ feet of water $=\cdot 254$ lbs. per square inch.

(7) If in the previous example the plates are fitted with vanes and rotate at 400 revolutions per minute, determine the difference of pressure at inlet and outlet due solely to the production of a forced vortex.

Answer. 46.04 feet of water $=20$ lbs. per square inch.

(8) Taking the above (6) and (7), as an example of an inward radial flow turbine, determine the total pressure drop between inlet and outlet.

\section{Answer. $46 \cdot 62$ feet of water.}

(9) A centrifugal pump has an impeller 12 inches internal, 24 inches external diameter and runs at 800 revolutions, discharging into a vortex chamber where a free vortex is formed. If the outer diameter of the vortex chamber is 3 feet, determine the rise in pressure due to the vortex motion
(a) in the wheel ;
(b) in the vortex chamber.

Neglect all losses and assume a perfectly efficient rortex chamber.

Answer. (a) $81 \cdot 9$ feet of water.

(b) $60 \cdot 6$, , ,

(10) A flat cylindrical disc, 18 inches diameter, keyed on to the lower end of a 3 inch vertical shaft serves as a hydraulic footstep 
bearing. Its lower face is plane and bears against radiating ribs cast in the pressure cyclinder, while its upper face carries a series of radial ribs which bear against the plane upper lid of the pressure cylinder. Thus water above the disc rotates with the disc, while the water below is at rest. The upper and lower sides are in free communication around the periphery of the disc. Determine the resultant upward pressure on the shaft at a speed of 400 revolutions per minute.

Answer. $844 \mathrm{lbs}$.

(11) A pipe 4 inches diameter is suddenly enlarged to 6 inches diameter. Determine the consequent loss of energy per lb. of water with a velocity of flow in the small pipe of 6 feet per second.

Answer. $\quad \cdot 173$ foot lbs. per $1 \mathrm{~b}$. 


\section{CHAPTER V.}

Flow from a Small Orifice-Co-efficients of Contraction, Velocity and DischargeBorda s Mouthpiece - Sharp edged Orifice - Converging Mouthpiece - Bellmouthed orifice-Diverging Outlet-Velocity of Approach-Time of emptying Reservoir-Submerged Orifice-Form of Jets - Large Orifices - Partially submerged Orifice-Notches and Weirs-Theoretical Formulae-Francis-Law of Comparison-Submerged Weirs-Weirs with wide Sill-Rise in surface level produced by Weir-Measurement of flow by Weir-'Times of Discharge.

\section{Art. 37.-Flow from a Suall Orifice. ${ }^{1}$}

IF an opening be made in the side or base of a tank containing water, the introduction of a few drops of aniline dye shows that steady stream line motion is set up in the mass of fluid, these stream lines converging towards the orifice from every side. At the boundary of the issuing jet the stream lines are, as already explained (Art. 14), tangential to the edges of the orifice, and with a sharp edged orifice the general stream line motion is as shown in Fig. 20. It follows that, after passing the plane of the orifice, the section of the jet gradually diminishes, and its boundaries do not become parallel until some finite distance from the orifice. The section at which the jet becomes parallel is termed the vena contracta, or contracted vein, and with a small circular orifice is at a distance from the orifice equal to about $\cdot 498$ times the diameter.

Now suppose a particle of weight $w \mathrm{lbs}$. to travel along a stream line from the surface to the orifice and on to the vena contracta (Fig. 44). Since the motion is steady, the energy throughout is constant (neglecting viscosity); and if we suppose the surface area to be large, so that the surface velocity may be neglected, when in the surface its kinetic energy is zero. If the

1 By a small orifice we mean one whose dimensions are small in comparison with the head of water above its centre, so that at any point in its area the head may be taken as equal to that at its centre without sensible error. The subjectmatter of Arts $37-46$ refers to such orifices. 
orifice is at a mean depth $h$ below the free surface, and if its level be taken as datum, the potential energy of the particle is wh foot lbs., while its pressure energy is zero, its pressure being that of the atmosphere which is taken as datum pressure.

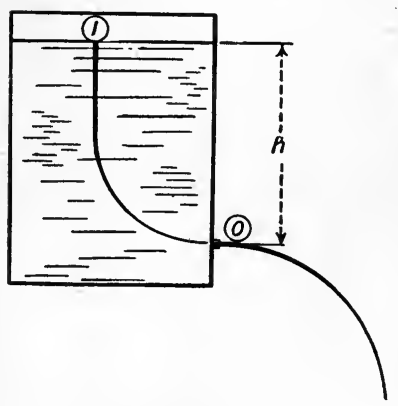

FIG. 44.

Immediately after passing the orifice, its potential energy is zero, so that if we can determine its pressure energy, its kinetic energy and therefore its velocity may be inferred. Now on passing the orifice the jet is exposed to atmospheric pressure, and if the pressure throughout is the same its pressure energy is zero. If the jet is parallel this condition is satisfied, but while its boundary is curved the centrifugal action of the outer layers necessitates an increase in pressure along the radius of curvature towards the centre of the jet. It follows that in the plane of the orifice, and up to the vena contracta, the pressure in the interior of the jet is greater than that of the atmosphere.

At the rena contracta, however, the pressure across the jet is

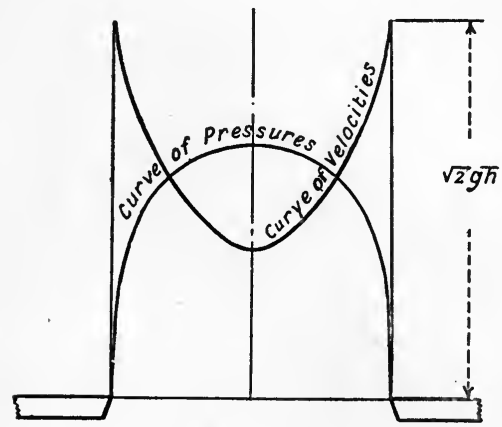

FIG. 45. uniform and atmospheric, the pressure energy at the orifice having bəen converted into kinetic energy, with a consequent increase in velocity from orifice to vena contracta.

The distribution of pressure and of velocity across a horizontal diameter in the plane of the orifice is substantially as shown in Fig. 45 .

The pressure energy at the vena contracta then is zero, the potential energy is zero, while the velocity energy is

$$
\frac{v^{2}}{2 g} \text { foot lbs. per } \mathrm{lb} \text {. }
$$


Denoting the surface by the suffix ${ }_{(1)}$, and the orifice-or strictly the vena contracta-by the suffix $\left({ }_{0}\right)$ we have

$$
\begin{aligned}
& w\left[\frac{p_{0}}{W}+\frac{v_{0}^{2}}{2 g}+z_{0}\right]=w\left[\frac{p_{1}}{W}+\frac{v_{1}^{2}}{2 g}+z_{1}\right] \\
& \text { where } p_{1}=p_{0}=0 ; z_{0}=0 ; v_{1}=0 \\
& \therefore \quad \frac{v_{0}^{2}}{2 g}=z_{1} \\
& \therefore \quad v_{0}=\sqrt{2 g z_{1}}=\sqrt{2 g h} \text { ft. per sec. }
\end{aligned}
$$

i.e., theoretically the velocity of efflux at the vena contracta is the same as if the particles had fallen freely through a height $h$ feet under the action of gravity.

The truth of the above theorem was demonstrated by Torricelli, ${ }^{1}$ who showed that a vertical jet of fluid would rise very approximately to the free level in the vessel from which it was supplied.

Due, however, to the viscosity of the liquid, and to the resistance of the air, the velocity is always slightly less than this theoretical value, and is given by $C_{v} \sqrt{2 g h}$, where $C_{v}$ is called the Coefficient of Velocity. The value of $C$ can only be determined

\begin{tabular}{|c|c|c|c|c|c|}
\hline$h$ feet & $\cdot 066$ & $1 \cdot 64$ & $11 \cdot 5$ & $56 \cdot 0$ & 338 \\
\hline & $\cdot 959$ & $\cdot 967$ & $\cdot 975$ & 994 & ·994 \\
\hline
\end{tabular}
experimentally, but with a sharp lipped orifice increases with $h$ from about 96 to $\cdot 994$. Weisbach gives the following values for an orifice of $\cdot 033$ feet diameter :-

At the vena contracta the stream lines for the first time become parallel and perpendicular to the cross section of the stream, so that if $a_{c}$ be the sectional area of the vena contracta in square feet, the volume discharged per second $=C_{v} \sqrt{2 g h} \times a_{c}$ cubic ft. If $a$ is the area of the orifice, the ratio $\frac{a_{c}}{a}$ is termed the Coefficient of Contraction, and is denoted by $C_{c}$. The discharge is thus given by $C_{c} C_{v} \sqrt{2 g h} \times a=C \sqrt{2 g h} \times a$ cub. ft. sec.

1 De motu gravium naturaliter accelerato (1643). 
Here $C$ is termed the coefficient of discharge.

These coefficients vary with the head $h$; with the area of the orifice; and with the shape of the orifice and its position.

The following table, giving values of $C$, abridged from Hamilton Smith's "Hydraulics," indicates the nature of these variations. In each case the orifice had full contraction, had free discharge into air, and the inner face of the plate in which the orifice was formed had sharp corners, so that the escaping jet only touched these inner edges.

\begin{tabular}{c|c|c|c|c|c}
\hline \multirow{2}{*}{$\begin{array}{c}\text { Head from } \\
\text { centre of orifice } \\
\text { in feet. }\end{array}$} & \multicolumn{5}{|c}{ Square Orifices ; lengths of side of square in feet. } \\
\cline { 2 - 6 } & $\cdot 02$ & $\cdot 04$ & $\cdot 07$ & $\cdot 12$ & $\cdot 20$ \\
\hline$\cdot 4$ & - & $\cdot 643$ & $\cdot 628$ & $\cdot 616$ & - \\
$\cdot 6$ & $\cdot 660$ & $\cdot 636$ & $\cdot 623$ & $\cdot 61:$ & $\cdot 605$ \\
$1 \cdot 0$ & $\cdot 648$ & $\cdot 628$ & $\cdot 618$ & $\cdot 610$ & $\cdot 605$ \\
$6 \cdot 0$ & $\cdot 623$ & $\cdot 612$ & $\cdot 607$ & $\cdot 605$ & $\cdot 604$ \\
$20 \cdot 0$ & $\cdot 606$ & $\cdot 604$ & $\cdot 602$ & $\cdot 602$ & $\cdot 602$ \\
$100 \cdot 0$ & $\cdot 599$ & $\cdot 598$ & $\cdot 598$ & $\cdot 598$ & $\cdot 598$ \\
\hline
\end{tabular}

\begin{tabular}{c|c|c|c|c|c}
\hline \multirow{2}{*}{$\begin{array}{c}\text { Head above } \\
\text { centre of orifice } \\
\text { in feet. }\end{array}$} & \multicolumn{5}{|c}{ Circular Orifices; diameters in feet. } \\
\cline { 2 - 6 } & $\cdot 02$ & $\cdot 04$ & $\cdot 07$ & $\cdot 12$ & $\cdot 20$ \\
\hline$\cdot 4$ & - & - & $\cdot 628$ & $\cdot 612$ & - \\
$\cdot 6$ & $\cdot 655$ & $\cdot 630$ & $\cdot 618$ & $\cdot 609$ & $\cdot 601$ \\
$1 \cdot 0$ & $\cdot 644$ & $\cdot 623$ & $\cdot 612$ & $\cdot 605$ & $\cdot 600$ \\
$6 \cdot 0$ & $\cdot 618$ & $\cdot 607$ & $\cdot 602$ & $\cdot 599$ & $\cdot 598$ \\
$20 \cdot 0$ & $\cdot 601$ & $\cdot 599$ & $\cdot 597$ & $\cdot 596$ & $\cdot 596$ \\
$100 \cdot 0$ & $\cdot 593$ & $\cdot 592$ & $\cdot 592$ & $\cdot 592$ & $\cdot 592$ \\
\hline
\end{tabular}

With a.rectangular orifice, the coefficient is rather greater than with a square orifice of the same area, and generally increases with the ratio of the perimeter to the area of an orifice.

Any interference with the free production of stream lines, such as occurs, for example, where the depth of water above the orifice is too small, or where this is near the sides or 
bottom of a tank as indicated in Fig. $20 \mathrm{~b}$, curtails the full flow to the orifice, and tends to prevent the full contraction of section at the vena contracta. In any such case the value of $C_{c}$ and of $C$ will be greater than indicated above, the true values being entirely dependent on the circumstances governing the flow to the orifice.

Where an orifice is made in the thick sides of a vessel, or is fitted with an external pipe so that the jet, after springing clear of its inner edges, again touches the sides of the orifice before escaping, or where fitted with re-entrant mouthpiece, the physical conditions governing the flow are entirely changed, and the coefficients assume new values. Thus the coefficient of contraction may vary from $\cdot 5$ to $1 \cdot 0$ under different conditions of working, with the same size of orifice and the same head. There is, however, nothing mysterious about this change-it is governed entirely by the conditions of flow, and, as will be seen, may be predetermined with some exactitude when those conditions are known.

The following may be taken as mean values of the coefficients for small circular orifices where the head of water is between three times and twenty times the diameter, and where the stream line formation is unaffected by the sides or bottom of the containing vessel.

\begin{tabular}{|c|c|c|c|c|}
\hline \multirow{2}{*}{ Coefficient. } & \multicolumn{2}{|c|}{$\begin{array}{c}\text { Sharp-edged Orifice in plane side } \\
\text { of Tank. }\end{array}$} & \multicolumn{2}{|c|}{ Borda's (re-entrant) Mouthpiece } \\
\hline & Running freely. & $\begin{array}{l}\text { With external pipe } \\
\text { of same diameter. }\end{array}$ & Running freely. & Running full. \\
\hline$C_{v}$ & $\cdot 97$ & $\cdot 82$ & $\cdot 97$ & $\cdot 773$ \\
\hline$C_{c}$ & $\cdot 62$ & $1 \cdot 00$ & $\cdot 50$ & $1 \cdot 00$ \\
\hline$C$ & $\cdot 61$ & $\cdot 82$ & $\cdot 48$ & $\cdot 773$ \\
\hline
\end{tabular}

Submerged Orifices.-If an orifice discharges below the surface of the water in a second vessel instead of into the air, this has the effect of increasing the pressure against which discharge takes place, and thus of reducing the effective head, and also of slightly reducing the value of the coefficient of discharge. 
In the sketch (Fig. 46), if the suffix (1) refer to the free surface in the upper vessel and suffix $\left(_{0}\right)$ to the vena contracta, we have, neglecting viscosity

$$
\frac{p_{1}}{W}+\frac{v_{1}^{2}}{2 !}+z_{1}=\frac{p_{0}}{W}+\frac{v_{0}^{2}}{2 g}+z_{0} .
$$

Taking the datum level at the centre of the orifice, and assuming the area at the surface in the upper vessel to be large, we have

$$
\begin{gathered}
p_{1}=0 ; v_{1}=0 ; z_{0}=0 ; z_{1}=H_{1} ; \frac{p_{0}}{W}=H_{2} \\
\therefore \quad H_{1}-H_{2}=\frac{v_{0}^{2}}{2 g} \text { feet } \\
\therefore \quad v_{0}=\sqrt{2 g\left(H_{1}-H_{2}\right)}=\sqrt{2 g h},
\end{gathered}
$$

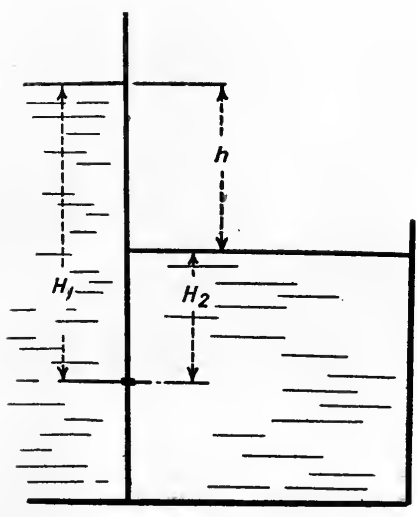

FIG. 46.

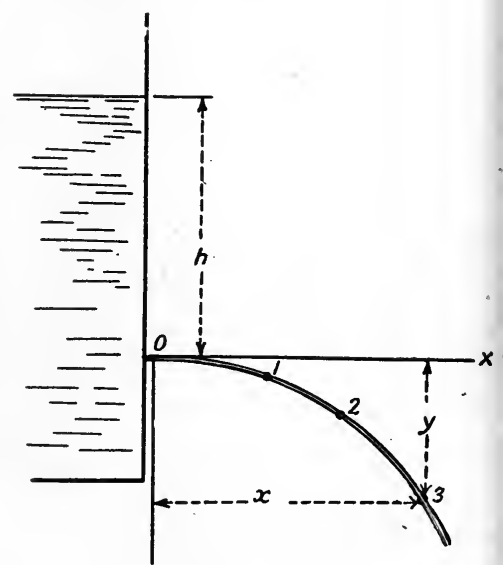

Fig. 47 .

where $h=$ difference in level of the free surfaces on the two sides of the orifice.

Art. 38.-Experinental Determination of the Coefficients.

(1) Coefficient of Velocity, for an orifice in a vertical plate. Keeping $h$ constant, mark on a flat board parallel to the plane of the jet, points $1,2,3,4$, etc., by means of a set square, these points marking as nearly as possible the centre of the jet. Draw a smooth curve through the points, and draw the horizontal $O X$ through $O$ (Fig. 47). Taking any point on the curve we get, if $v$ 
is the velocity of efflux, measuring horizontal distances from the vena contracta:-

$$
\begin{gathered}
x=v t ; y=\frac{1}{2} g t^{2} \\
\therefore \quad v=\frac{x}{t}=\frac{x}{\sqrt{\frac{g}{2 y}}}=\sqrt{\frac{g x^{2}}{2 y}}
\end{gathered}
$$

$$
\text { But } \frac{v}{\sqrt{2 g h}}=C_{v} . \quad \therefore C_{v}=\sqrt{\frac{x^{2}}{4 y h}} .
$$

If the plane of the orifice make an angle $a$ with the horizontal, then

$$
x=v \cos a \cdot t ; y=\frac{1}{2} g t^{2}
$$

horizontal and vertical axes of co-ordinates being taken through the highest point of the curve

$$
\begin{aligned}
& \therefore \quad v=\frac{x}{t \cdot \cos ^{2} a}=\frac{x}{\sqrt{\frac{2 y}{g} \cdot \cos ^{2} a}} \\
& \therefore \quad v=\sqrt{\frac{g x^{2}}{2 y \cos ^{2} a}} .
\end{aligned}
$$

Generally speaking, if $a$ be the angle made by any stream tube of area $\delta a$ and of velocity $v$, at the orifice, the total quantity discharged per second $=\Sigma v \cos a \delta a$.

The mean velocity of efflux $\bar{v}=\frac{\Sigma v \cos a \delta a}{a}$ where $a$ is the area of the orifice, and we then have the coefficient of discharge

$$
C=\frac{\Sigma(v \cos a \delta a)}{a \sqrt{2 g h}} .
$$

A second method of determining the coefficient of velocity is to allow a jet of water to escape from an orifice in the vertical side of a tank supported on knife edges (Fig. 48), the level of the water in the tank being maintained constant by the influx of a vertical stream. The position of a pointer fixed to the tank is noticed before the orifice is opened, and when the jet is allowed to escape this pointer is brought back to the same mark by means of the weight $W$, placed at a leverage $x$.

The weight of water $w$ leaving the vessel per second is 
determined by weighing. Then if $v$ be its mean velocity in a horizontal direction, the momentum per second leaving the tank in this direction $=\frac{w v}{g}$. To produce this flux of momentum

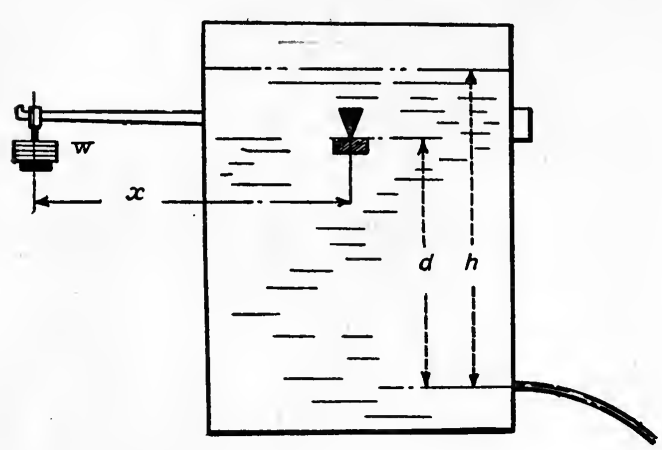

FIG. 48.

a constant horizontal force of magnitude $\frac{w v}{g}$ lbs. must be impressed on the jet, a force which can only be due to an otherwise unbalanced pressure on the side of the tank opposite to the orifice.

We have then,

taking moments about the knife edge :-

$$
\begin{gathered}
W x=\frac{w v}{g} \cdot d \text { where } d=\text { depth of orifice below knife edge } \\
\therefore \quad v=\frac{W}{w} \cdot g \cdot \frac{x}{d} \text { feet per second } \\
\therefore \quad C_{v}=\frac{W g x}{w d \sqrt{2 g h}} .
\end{gathered}
$$

(2) The coefficient of contraction, $C_{c}$, may be measured by

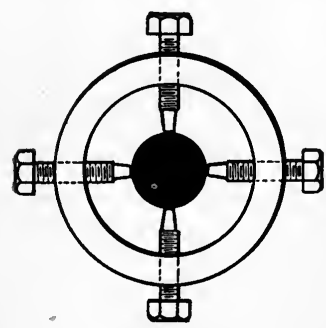

Fig. 49. using a ring (Fig. 49), surrounding the jet at the vena contracta, and fitted with micrometer measuring screws, which may be adjusted until just touching its surface. Owing, however, to the difficulty in keeping the jet absolutely steady, this method is not so suitable as that of deducing the value of this coefficient from those of the coefficients of velocity and discharge.

(3) The coefficient of discharge, $C$, may be determined directly by measuring the quantity $Q$ in cubic feet discharged per second from the orifice $a$, under a head $h$.

Then

$$
C=\frac{Q}{a \sqrt{2 g h}}
$$


Art. 39.

Theoretically we may always calculate the flow from an orifice in the side or bottom of a vessel, by equating the flux of momentum across the plane of the orifice per second to the unbalanced statical pressure on the opposite vertical side in the one case, and to the weight of the unbalanced column of water in the other, i.e., by expressing the fact that the total unbalanced force in the direction of flow is taken up in producing this flow. With an ordinary opening in the side or bottom of a vessel, however, the calculation of this unbalanced force is impossible, since, due to the fact that

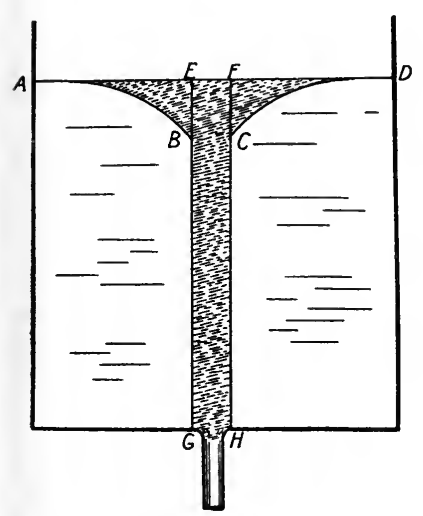

FIG. 20.

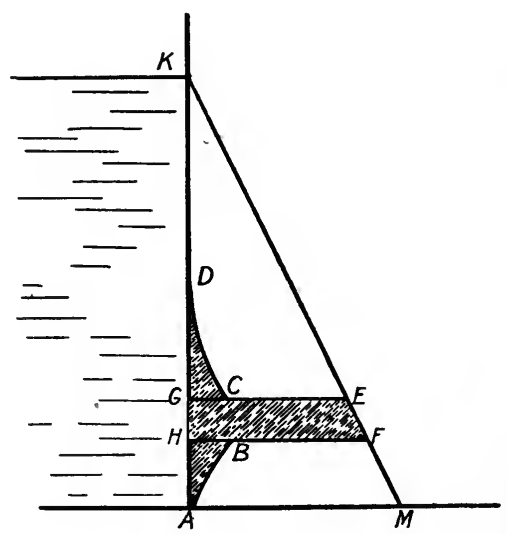

FIG. 51.

there is stream line motion along the face containing the opening, giving the water kinetic head, the pressure head at any point in this face is less than that simply due to the statical head at that point by an amount which is indeterminate. ${ }^{1}$ The resultant unbalanced pressure is therefore in this case indeterminate. In the case of an opening in the flat, horizontal bottom of a vessel, the pressure over this face is not uniform, since the kinetic head is greatest near the orifice, the distribution of pressure being as represented in Fig. 50, where the ordinates from the base to the curve $A B C D$ measure the pressure on the base.

When the orifice $G H$ is closed, the pressure on $G H$ is that represented by the shaded area $E F H G$, but when open,

1 Since part of the head produces acceleration towards the point. 
the unbalanced pressure becomes that represented by the whole shaded area $A B G H C D$.

Similarly in the case of an opening in the vertical side $A K$ of a vessel (Fig. 51). Here the area $G E F H$ represents the pressure on $G H$ when the orifice is closed, while the horizontal distances between the line $K M$ and the curves $A B$ and $C D$ measure the pressures on the side of the vessel with an issuing jet. The shaded area then repre-

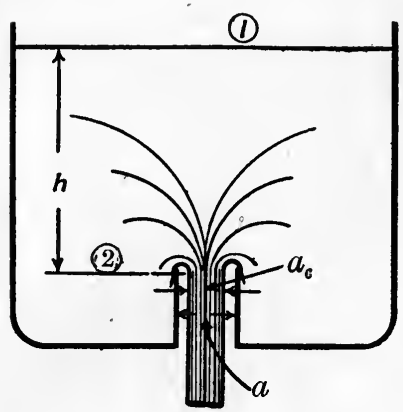

Frg. 52. sents the unbalanced pressure, which is to be equated to the outflow of momentum per second from the orifice.

By returning the mouthpiece for some distance into the vessel, as in the Borda mouthpiece (Fig. 52), this flow over the face containing the orifice is prevented, and the pressure on this face now approximates to that due to the statical head alone. The equations of momentum can therefore be easily applied.

\section{Art. 40.-Borda's Mouthpiece.}

Let $a$ be the area of the orifice and $a_{c}$ the area of the stream as soon as it has assumed a parallel cylindrical form, i.e., at the vena contracta. Let $h$ be the depth of this point below the surface. If the suffixes $\left(_{1}\right)$ refer to the surface, and $\left(_{2}\right)$ to the vena contracta we have, neglecting viscosity

$$
\frac{p_{1}}{\bar{W}}+\frac{v_{1}^{2}}{2 g}+z_{1}=\frac{p_{2}}{W}+\frac{v_{2}^{2}}{2 g}+z_{2} \text {. }
$$

Also $p_{2}=p_{1}=0 ; z_{1}-z_{2}=h ; v_{1}=0$ if surface area is large,

$$
\therefore v_{2}^{2}=2 g h \text {. }
$$

Mass carried away per second $=\frac{W}{g} \cdot a_{c} v_{2}$.

$\therefore \quad$ Momentum carried away per second $=\frac{W}{g} \cdot a_{c} v_{2}^{2}$. 
This momentum is produced by a force equal to the weight of a column of water of area $a$ and of height $h$ (approximately).

$\therefore$ Force producing motion $=W a h$ lbs.

$$
\begin{gathered}
\therefore \quad W a h=\frac{W}{g} \cdot a_{c} v_{2}{ }^{2} \\
=\frac{W}{g} \cdot a_{c} \cdot 2 g h \\
\therefore \quad a=2 a_{c}{ }^{1}
\end{gathered}
$$

i.e., the co-efficient of contraction in the case of a Borda's mouthpiece, where the effluent stream does not touch the sides of the mouthpiece, is one half.

Borda's mouthpiece running full.-With a vertical Borda, where the water in the vessel is initially quite steady, the issuing jet after becoming parallel does not again come into contact with the sides of the mouthpiece. If, however, the water in the vessel be agitated, the jet, after contracting to form a vena contracta again expands to fill the mouthpiece (Fig. 58). This is termed running full as opposed to running freely as in the previous

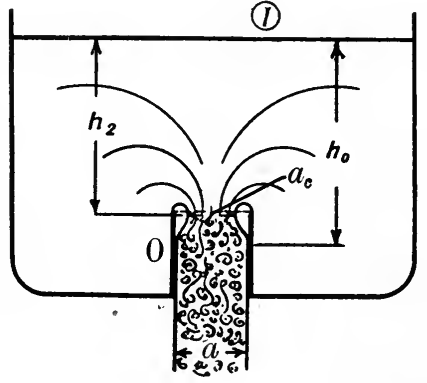

FIG. 53. case. The issuing motion is now turbulent, not steady as is the case with a free Borda. It is worthy of note that under no circumstances with a free Borda, or indeed with any jet having non-sinuous motion, is any splash produced on meeting a solid surface. With sinuous motion splashing always occurs.

We now get a loss of head due to the expansion of the stream from $a_{c}$ to $a$ after contracting, so that

$$
\frac{p_{1}}{W}+\frac{r_{1}^{2}}{2 !}+z_{1}=\frac{p_{0}}{W}+\frac{r_{0}^{2}}{2 g}+z_{0}+\text { loss due to expansion. }
$$

1 Actually $r_{2}^{2}=C_{v}^{2} \times 2 g h$, so that $\frac{a}{a_{c}}<2$. Approximately $a=2 C_{v}^{2} a_{c}$. The co-efficient of contraction for the Borda running full is modified in a similar manner.

H.A. 


$$
\begin{aligned}
& \text { I'he loss due to expansion (Art. 31) }=\frac{v_{0}^{2}}{2 g}(m-1)^{2}, \\
& \text { where } m=\frac{a}{a_{c}} \\
& \therefore \quad \frac{p_{1}}{W}+\frac{v_{1}^{2}}{2 g}+z_{1}=\frac{p_{0}}{W}+\frac{v_{0}^{2}}{2 g}\left[(m-1)^{2}+1\right]+z_{0} \\
& \text { But } p_{1}=0 \text { and } v_{1}=0 . \text { Also } z_{1}-z_{0}=h_{0} . \\
& \therefore \quad \frac{p_{0}}{W}+\frac{v_{0}^{2}}{2 g}\left[(m-1)^{2}+1\right]=h_{0} .
\end{aligned}
$$

Now equating the momentum passing the section at $O$ per second, to the force producing it, we get

$$
\begin{gathered}
W a h_{0}-p_{0} a=\frac{W}{g} a r_{0}^{2} \\
\therefore \quad h_{0}-\frac{p_{0}}{W}=\frac{r_{0}^{2}}{g}
\end{gathered}
$$

But $h_{0}-\frac{\mu_{0}}{W}=\frac{v_{0}^{2}}{2 g}\left[(m-1)^{2}+1\right]$ from (1)

$\therefore \quad$ Equating these values we get $\frac{(m-1)^{2}+1}{2}=1$

$$
\therefore \quad(m-1)^{2}=1 \quad \text { or } m=2,
$$

i.e., the coefficient of contraction at the neck in a Borda's mouthpiece running full, is one-half.

If the jet discharges into the atmosphere so that $p_{0}=0$ we have

$$
v_{0}^{2}=g h_{0}, \text { so that } v_{0}=\frac{\sqrt{2 g h}}{\sqrt{2}} \text { (approx.), }
$$

i.e., the velocity of discharge is $\frac{1}{\sqrt{2}}$ times the velocity when flowing free, and since the area of full jet $=2$ (area of free jet).

$\begin{aligned} & \therefore \text { Quantity flowing per) } \\ & \text { cond from full Borda }\end{aligned}=\sqrt{2}$ (quantity from free Borda).

Denoting the contracted section by the suffix $\left(_{2}\right)$ we have

$$
\frac{p_{2}}{W}+\frac{r_{2}^{2}}{2 g}+z_{2}=\frac{p_{0}}{W}+\frac{r_{0}^{2}}{2 g}+z_{0}+\text { loss due to expansion, }
$$


But loss due to expansion $=\frac{r_{0}^{2}}{2 g}(2-1)^{2}=\frac{v_{0}{ }^{2}}{2 g}$

$$
\begin{gathered}
\text { Also } v_{2}=2 v_{0}, \\
\therefore \quad \frac{p_{0}-p_{2}}{W}=\frac{4 v_{0}^{2}-2 v_{0}^{2}}{2 g}+\left(z_{2}-z_{0}\right) \\
\therefore \quad \frac{p_{0}-p_{2}}{W}=\frac{r_{0}^{2}}{g}+\left(z_{2}-z_{0}\right) . \\
\text { Again } v_{0}^{2}=g h_{0}, \\
\therefore \quad \frac{p_{0}-p_{2}}{W}=h_{0}+z_{2}-z_{0} .
\end{gathered}
$$

In a horizontal mouthpiece $z_{2}=z_{0}$,

$$
\therefore \frac{p_{0}-p_{2}}{W}=h_{0} \text { feet, }
$$

i.e., the gain of pressure from $(2)$ to $(0)$ is equal to a head of $h_{0}$ feet.

If the jet issues into the atmosphere $p_{0}=0$.

$$
\therefore \quad \frac{p_{2}}{W}=-h_{0} \text { feet. }
$$

Or the pressure at $\left({ }_{2}\right)$ is less than that of the atmosphere by an amount equivalent to a head $h_{0}$ of water.

This reduction of pressure at section (2) explains why the velocity at this point, and hence the discharge, is greater with a full Borda than with one running free. The conditions from the surface to the section at $(2)$ are exactly the same whether the mouthpiece runs full or free, but in one case the jet is discharging at this point against atmospheric pressure, while in the other case this pressure is in part removed.

The effect is then substantially the same as if the free Borda were subjected to an additional head $h_{0}$.

The theoretical limit of $h_{0}$ is 34 feet, but practically, the liberation of dissolved air as the pressure falls, prevents the formation of anything approaching an absolute vacuum.

\section{Art. 41.-Sharp-edged Orifice in a flat Plate, with External 'I'ube.}

With an external tube of the same diameter as the orifice, the effluent stream, after forming a vena contracta, always re-expands to fill the tube (Fig. 54). 
In this case, as already explained, the coefficient of contraction cannot be theoretically deduced by an application of the equations of momentum.

Its value is experimentally found to be about 620 , i.e., $\frac{a}{a}$ $=\cdot 620$.

The final area of the jet $=a$, and the coefficient of velocity at the exit is experimentally found to be $\cdot 825$.

It follows that the coefficient of discharge $=\cdot 825$.

If suffixes $\left(_{0}\right)$ and $\left(_{2}\right)$ refer to outlet and to vena contracta, since

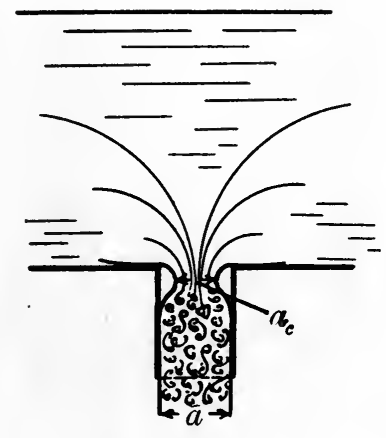

(a)

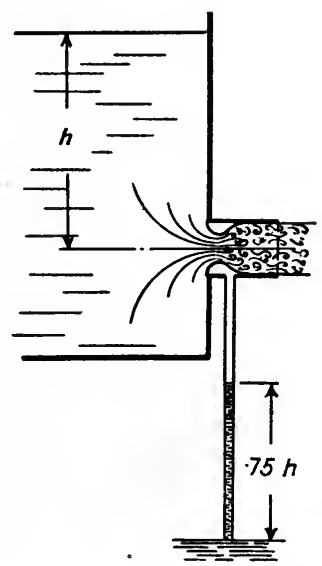

(b)

FIG. 5t.

we have no loss of head from the surface to the section at ${ }_{2}$ we get

$$
\iota=\frac{p_{2}}{W}+\frac{v_{2}^{2}}{2 g}
$$

Again, since the only loss of energy between section $\left(_{2}\right)$ and the outlet (neglecting friction) is that due to the enlargement of section from $a_{c}$ to $a$, i.e.,

$$
\frac{v_{0}^{2}}{2 g}(m-1)^{2}, \quad \text { where } m=\frac{a}{a_{c}},
$$

we have $h=\frac{p_{2}}{W}+\frac{v_{2}^{2}}{2 g}=\frac{v_{0}^{2}}{2 g}\left\{1+(m-1)^{2}\right\}$ 
Writing $v_{0}=C_{v} \sqrt{2 g h}$ we get $\frac{1}{C_{v}^{2}}=m^{2}-2 m+2$.

Giving $C_{v}$ its observed value, $\cdot 825$, this makes $m=1 \cdot 685 .=\frac{1}{\cdot 594}$ while giving $\frac{1}{m}$ the value $\cdot 62$, as observed for a similar orifice without the external tube, it makes $C_{v}=\cdot 852$. Actually we have $C_{v}=\cdot 825$, and the difference between this value and $\cdot 852$ is probably due to the loss due to expansion being somewhat greater than indicated above. Assuming this loss to be given by 1.25 $\frac{v_{0}^{2}}{2 g}\{m-1\}^{2}, c f$. Art. 31, and introducing this into equation (2), equation (3) becomes

$$
\frac{8}{C_{v}^{2}}=m^{2}-2 m+1 \cdot 8,
$$

while giving $C_{v}$ the value $\cdot 825$ this makes $m=1 \cdot 61 ; \frac{1}{m}=\cdot 620$.

If, in equation (1), we substitute $m C_{v} \sqrt{2 g h}=m v_{0}$ for $v_{2}$ this becomes $\frac{p_{2}}{W}=h\left(1-m^{2} C_{v}^{2}\right)$,

and giving $C_{v}$ and $m$ the above values we have $\frac{p_{2}}{W}=-\cdot 763 \mathrm{~h}$.

This agrees very closely with the observed value of $\frac{p_{2}}{W}$ which is approximately $-\cdot 75 \mathrm{~h}$.

If then an orifice be made in the mouthpiece, at the vena contracta (Fig. 54 b), a tube coupled to this and having its lower end open and in water will support a column of height approximately $\cdot 75 h$. Theoretically, this will hold until $\cdot 75 h=$ height of the water barometer $=34$ feet, i.e., until $h=\frac{34}{.75}=45^{\circ} 4$ feet. The effect then of adding the external mouthpiece is to reduce the pressure on the discharge side of the orifice, and so to increase the effective head, and therefore the velocity of discharge. Thus with a stream of the assumed proportions we have $v_{2}=\frac{825}{620}$ $\sqrt{2 g h}=1.33 \sqrt{2 g h,}$ a velocity greatly in excess of that 
obtainable with the same orifice when discharging directly into the atmosphere, while the effective head is increased in the ratio $\left\{\frac{1 \cdot 33}{1}\right\}^{2}=1 \cdot 77$.

For values of $h$ greater than about 40 feet, the negative pressure $\frac{p_{2}}{W}$, being constant, will have a less proportional effect as the head increases, and for very high heads the effect of the external tube will be small. Also, as $h$ increases above about 40 feet, the coefficient of discharge will diminish. In the limit, with a very high head, the issuing velocity would become so great that the jet would not come into contact with the sides of the tube, and the coefficient of discharge would become $C_{v} \times \cdot 64=\cdot 620$ (approx.).

If a diverging tube be placed at the discharge end of the mouthpiece, or indeed at the outlet from any pipe fed by the mouthpiece, the pressure at the vena contracta will, so long as this is greater than the absolute zero, be reduced, and the discharge thereby increased if this outlet runs full.

This follows since the pressure difference at the vena contracta and at the outlet increases with the ratio of the velocities and hence of the areas. If however the final area of the tube be so large that the pressure at the vena contracta is reduced to absolute zero ( -34 feet of water), continuity of motion becomes impossible and the diverging tube ceases to run full. Even with a moderate ratio of areas the angle of divergence of the sides $\theta$ (Fig. $55 \mathrm{~b}$ ), should not exceed $16^{\circ}$ (Venturi), while the maximum effect is obtained with an angle of about $6^{\circ}$, the diameter increasing from 1 to $1 \cdot 8$ in a length $=9$ diameters.

The same increased discharge may be obtained, within limits, by the substitution of a discharge pipe of greater area than the discharge orifice.

Roman law recognised the latter fact, and prohibited the attachment of pipes of greater diameter than the aperture provided at the reservoir, for a distance of 50 feet. The possibility of evasion of the law by the application of a conical frustrum at the open end of the pipe was, however, not apparently perceived. 
Sharp-edged Orifice with Converging Mouthpiece.-If a conical converging pipe be fitted external to the orifice, with its larger diameter equal to that of the orifice, the coefficients of velocity at exit and of discharge depend on the angle of convergence.

The following values are taken from experiments by Castel, and indicate that a maximum discharge is to be obtained when the angle of convergence is about $13^{\circ} 20^{\prime}$.

Diameter of mouthpiece $=\cdot 61$ inches.

\begin{tabular}{|c|c|c|c|c|c|c|c|c|c|c|c|c|c|}
\hline $0^{\circ} \cdot 0^{\prime}$ & $1^{\circ} \cdot 36^{\prime}$ & $3^{\circ} \cdot 10^{\prime}$ & $5^{\circ} 26^{\prime}$ & $7^{\circ} \cdot 52^{\prime}$ & $10^{\circ} \cdot 20^{\prime}$ & $12^{\circ} \cdot 4^{\prime}$ & $13^{\circ} \cdot 24^{\prime}$ & $14^{\circ} \cdot 2 \delta^{\prime}$ & $16^{\circ} \cdot 36^{\prime}$ & $21^{\circ} \cdot 0^{\prime}$ & $29^{\circ} \cdot 5 \mathrm{~S}^{\prime}$ & $40^{\circ} \cdot 20^{\prime}$ & $49^{\circ} \cdot 0^{\prime}$ \\
\hline 830 & $\cdot 866$ & $\cdot 594$ & $\cdot 924$ & 931 & $\cdot 950$ & .955 & $\cdot 962$ & $• 966$ & $\cdot 971$ & $\cdot 9 \pi 1$ & 975 & $\cdot 980$ & .984 \\
\hline$\cdot 829$ & $\cdot 866$ & $\cdot 895$ & $\cdot 920$ & $\cdot 929$ & $\cdot 938$ & $\cdot 942$ & $\cdot 946$ & $\cdot 941$ & $\cdot 938$ & $\cdot 918$ & $\cdot 896$ & $\cdot 869$ & -847 \\
\hline
\end{tabular}

Diameter $=.787$ inches.

\begin{tabular}{c|c|c|c|c|c|c|c|c|c}
\hline $\begin{array}{c}\text { Augle of } \\
\text { Convergence. }\end{array}$ & $2^{\circ} \cdot 50^{\prime}$ & $5^{\circ} \cdot 26^{\prime}$ & $6^{\circ} \cdot 54^{\prime}$ & $10^{\circ} \cdot 30^{\prime}$ & $12^{\circ} \cdot 10^{\prime}$ & $13^{\circ} \cdot 40^{\prime}$ & $15^{\circ} \cdot 2^{\prime}$ & $18^{\circ} \cdot 10^{\prime}$ & $33^{\circ} \cdot 52^{\prime}$ \\
\hline$C_{v}$ & $\cdot 906$ & $\cdot 930$ & $\cdot 938$ & $\cdot 953$ & $\cdot 957$ & $\cdot 964$ & $\cdot 967$ & $\cdot 970$ & $\cdot 979$ \\
\hline$C$ & $\cdot 910$ & $\cdot 928$ & $\cdot 938$ & $\cdot 945$ & $\cdot 949$ & $\cdot 956$ & $\cdot 949$ & $\cdot 939$ & $\cdot 920$ \\
\hline
\end{tabular}

Art. 42.-Bell-Mouth Orifice.

With a bell-mouth orifice (Fig. 55 a) having a curvature approximately the same as that of the natural stream lines, the pressure over the walls of the mouthpiece will be zero; the coefficient of contraction, unity; and the only loss of head,

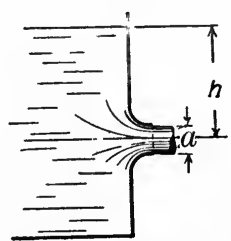

(2)

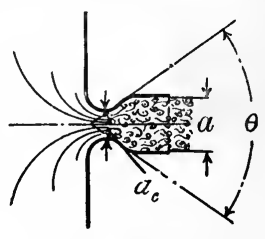

(b)

Fig. รัต. that due to viscosity. Here $C_{c}=1 ; C_{v}=\cdot 975$ (experimental);

$$
\therefore \quad C=\cdot 975
$$

$$
\text { Since } v=\cdot 975 \sqrt{2 g h} ; \frac{r^{2}}{2 g}=\cdot 95 h
$$

$\therefore \quad$ loss of energy due to this type of mouthpiece $=\cdot 05 h$ $=\cdot 05 \frac{v^{2}}{2 g} \mathrm{ft}$. lbs. per lb. (approx.). 
Experiments by Weisbach indicate that the general proportions of such a mouthpiece should be as shown in Fig. 56, the sides of the mouthpiece making an angle of $67^{\circ}$ with the plane of the orifice at a point distant about $\frac{d}{10}$ from that plane.

Bell-mouth Orifice with Diverging Outlet.-If a diverging pipe

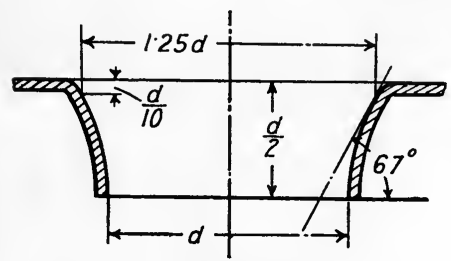

FIG. 56. be fitted to the bell-mouth as shown in Fig. $55 \mathrm{~b}$, so long as this pipe runs full, the pressure at the throat is reduced just as at the vena contracta in the previous forms of mouthpiece. The effect of the adjutage is therefore to increase the velocity of flow through the throat, and hence the discharge. The loss of energy due to the divergence may be calculated as in the previous case.

\section{Art. 43.--Velocity of Approach.}

Where the surface area of a vessel is not so large as to allow of the velocity of approach of the water to an orifice being neglected, if the suffixes $\left(_{1}\right)$ and $\left(_{2}\right)$ refer to the surface and to the vena contracta respectively, we have

$$
\frac{p_{1}}{W}+\frac{r_{1}^{2}}{2 g}+z_{1}=\frac{p_{2}}{W}+\frac{r_{2}^{2}}{2 g}+z_{2} .
$$

Putting $p_{1}=0$ and $p_{2}=0$, we have

$$
\begin{aligned}
& \frac{v_{2}^{2}}{2 g}=\frac{v_{1}^{2}}{2 g}+z_{1}-z_{2} \\
& \quad=\frac{v_{1}^{2}}{2 g}+h \quad(h=\text { head over orifice }) \\
& \therefore \quad v_{2}^{2}=2 g h+r_{1}^{2} .
\end{aligned}
$$

Here $v_{1}$ is the velocity of approach.

In words, the kinetic energy at efflux is equivalent to the potential energy at the surface, together with the kinetic energy due to the velocity of approach. 
The effective head which would give the same velocity of efflux is now

$$
H=\left[h+\frac{v_{1}^{2}}{2 g}\right]=h+h^{\prime} .
$$

So that the effect of the velocity of approach in increasing the outflow is theoretically the same as that of an additional head $h^{\prime}$ where $h^{\prime}=\frac{v_{1}^{2}}{2 g}$.

If $\frac{a_{c}}{A}$ be the ratio of the vena contracta and surface areas, we have

$$
\begin{gathered}
v_{1}=v_{2} \frac{a_{c}}{A}, \\
\therefore v_{2}^{2}=2 g h+v_{2}^{2} \frac{a_{c}{ }^{2}}{A^{2}}=\frac{2 g h}{1-\frac{a_{c}{ }^{2}}{A^{2}}}
\end{gathered}
$$

i.e., the effective head is increased in the ratio $\frac{1}{1-\frac{a_{c}^{2}}{A^{2}}}$.

The discharge is therefore increased in the ratio $\frac{A}{\sqrt{A^{2}-a^{c^{2}}}}$

$$
=\frac{1}{1-\frac{a^{c 2}}{2 A^{2}}} \text { (approximately). }
$$

Art. 44.

Time of emptying a Vessel through a small, freely discharging Orifice.-(1) Suppose the surface area $A$ of the vessel to be uniform and large compared with the area $a$ of the orifice.

Let $v=$ velocity of efflux at any instant, and $h$ the height of free surface above the rena contracta at the same instant.

Now assuming that $v=c_{v} \sqrt{2 g h}$, we have, since

$$
\begin{gathered}
\frac{\text { velocity of fall of surface }}{\text { velocity of efflux }}=\frac{a^{c}}{A}, \text { that } \\
\text { velocity of surface }=-\frac{d h}{d t}=C_{v} \frac{a_{c}}{A} \sqrt{2 g h} \\
\therefore \quad C_{v} \frac{a_{c}}{A} \sqrt{2 g} \cdot d t=-\frac{d h}{\sqrt{ } \bar{h}}
\end{gathered}
$$


Integrating between any two limits of height $h_{1}$ and $h_{2}$ we get the time $t_{2}-t_{1}=t$, necessary to lower the surface through the distance $h_{1}-h_{2}$.

$$
\begin{gathered}
\therefore \quad C_{v} \frac{a_{c}}{A} \sqrt{2 g} \cdot t=2\left\{h_{1}^{\frac{1}{2}}-h_{2}^{\frac{1}{2}}\right\} \\
\therefore \quad t=\frac{2 A}{C_{v} a_{c} \sqrt{2 g}}\left\{h_{1}^{\frac{1}{2}}-h_{2}^{\frac{1}{2}}\right\}
\end{gathered}
$$

If $C$ be the coefficient of discharge for the orifice the time will then be given by

$$
t=\frac{2 A}{C a \sqrt{2 g}}\left\{h_{1}^{\frac{1}{2}}-h_{2}^{\frac{1}{2}}\right\}
$$

Example.

With Borda's mouthpiece running freely

$$
t=\frac{2 A}{.48 a \sqrt{2 g}}\left\{h_{1}^{\frac{1}{2}}-h_{2}^{\frac{1}{2}}\right\} .
$$

Time of emptying a Reservoir of varying Cross-section, by small freely discharging Orifice.- Here $A$ is no longer constant but will be a function of $h$, so that equation (2)

$$
-\frac{d h}{d t}=\frac{c a}{A} \sqrt{2 g h}
$$

may be integrated if $A$ is an integrable function of $h$.

\section{Example I.}

Reservoir with uniformly varying cross-sectional area. Let $k D=$ area of reservoir at the orifice.

Then $A=k(D+h)$,

$$
\begin{aligned}
& \text { and }-k \int_{h_{2}}^{h_{1}}\left(\frac{D+h}{\sqrt{h}}\right) d h=C \int_{t_{2}}^{t_{1}}(a \sqrt{2 g)} d t \\
& \therefore \quad t_{2}-t_{1}=t=\frac{k}{C}\left[\frac{2 D\left(h_{1}^{\frac{1}{2}}-h_{2}^{\frac{1}{2}}\right)+\frac{2}{3}\left(h_{1}^{\frac{3}{2}}-h_{2}^{\frac{3}{2}}\right)}{a \sqrt{2 g}}\right]
\end{aligned}
$$

Where $C=$ coefficient of discharge. 


\section{ExampLe II.}

Hemispherical boul-radins r-emptied through hole in the bottom.

$$
\begin{gathered}
\text { Here } A=\pi\left\{r^{2}-(r-h)^{2}\right\}=\pi\left\{l^{2}-2 r h\right\} \\
\therefore \quad \int_{h_{2}}^{h_{1}} \frac{A}{\sqrt{h}} d h=\pi \int_{h_{2}}^{h_{1}}\left(h^{\frac{3}{2}}-2 r h^{\frac{1}{2}}\right) d h \\
\left.=\pi \frac{2}{5}\left(h_{1}^{\frac{5}{2}}-h_{2}^{\frac{5}{2}}\right)-\frac{4}{3} r\left(h_{1}^{\frac{3}{2}}-h_{2}^{\frac{3}{2}}\right)\right\} \\
\therefore \quad t_{2}-t_{1}=t=\frac{\pi}{C}\left[\frac{\frac{2}{5}\left(h_{1}^{\frac{5}{2}}-h_{2}^{\frac{5}{2}}\right)-\frac{4}{3} r\left(h_{1}^{\frac{3}{2}}-l_{2} \frac{3}{2}\right)}{a \sqrt{2} g}\right]
\end{gathered}
$$

Where the lower limit of height is less than about four times the diameter of the orifice, a vortex, with an air core, is usually formed, with the effect that the area of discharge is reduced.

Time of discharge from a Submerged Orifice.-If two vessels whose surface areas are $A_{1}$ and $A_{2}$ (Fig. 57) are connected by an orifice of area $a$, and if at any instant $H_{1}$ and $H_{2}$ are the heights

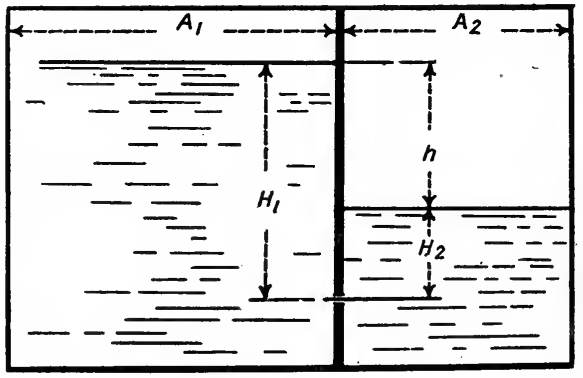

FIG. 57. of their free surfaces above the level of the orifice, we have, neglecting viscosity, and assuming

$$
v=\sqrt{2 g\left(H_{1}-1 I_{2}\right)}=\sqrt{2 g h}
$$

Velocity of surface $A_{1}=v \cdot \frac{a}{A_{1}}$; velocity of $A_{2}=v \frac{a}{A_{2}}$

$$
\therefore \quad-\frac{d h}{d t}=C \sqrt{2 g h} \cdot a \cdot\left\{\frac{1}{A_{1}}+\frac{1}{A_{2}}\right\}
$$

$$
\therefore \quad t_{2}-t_{1}=t=\frac{2}{C\left\{\frac{1}{A_{1}}+\frac{1}{A_{2}}\right\} a \cdot \sqrt{2 g}}\left\{h_{1}^{\frac{1}{2}}-h_{2}^{\frac{1}{2}}\right\}
$$

where $C=$ coefficient of discharge. 
If $t=$ time for surfaces to come to a common level we have

$$
\begin{gathered}
\left\{\begin{array}{l}
h_{1}=H_{1}-H_{2}, \\
h_{2}=0,
\end{array}\right. \\
\therefore \quad t=\frac{2}{C\left\{\frac{1}{A_{1}}+\frac{1}{A_{2}}\right\} a \sqrt{2 g}} \sqrt{H_{1}-H_{2}} .
\end{gathered}
$$

Where the upper surface remains at the same level we have the state of affairs which holds during the filling of a canal lock through a submerged orifice in the lock gate. The upper surface is in effect now of infinite area, so that putting $A_{1}=\infty$ in equation (1), we get the time of filling the lock.

$$
\therefore \quad t=\frac{2 A_{2}}{C a \sqrt{2 g}} \sqrt{H_{1}-H_{2}} \text {. }
$$

With submerged orifices the value of $C$ is about $\cdot 61$, diminishing slightly with an increase in the effective head $\mathrm{H}_{1}-\mathrm{H}_{2}$.

\section{ART. 45.-Fori of JET.}

Where a jet issues from an orifice in the horizontal base of a vessel, its sectional area gradually diminishes as the velocity increases, until it finally breaks up into a series of detached drops. With a circular orifice this is the most noticeable feature. With any other form of orifice however, the jet, after escaping, suffers a continuous change in the form of its cross section. This is due to the effect of the tension of its surface film, which tends in the first place to bring the jet into the circular form. The inertia of the particles of water causes this effect to be slightly overdone, so that a continual alteration and realteration of section takes place, the jet finally-if sufficiently large in diameter and of sufficient height-settling down into a circular section. The action is periodic and approximately isochronous, and consequently with a steady jet the section at any fixed point is constant.

Where a jet escapes from an orifice in the vertical side of a vessel a second disturbing factor now affects its form, for since the velocities of efflux at points on a vertical diameter of the orifice increase with the depth, the trajectories of any two 
particles situated in the same vertical plane will intersect if produced.

This has the effect of narrowing the jet in the direction of its depth, and, by the consequent impact of particles, causing it to become wider in a horizontal direction. Surface tension prevents dispersion of the stream, and brings the diverging particles back towards the axis of the jet and in a horizontal plane. The consequent impact causes the jet to become narrower in a horizontal direction and increases its depth, and a continual succession of
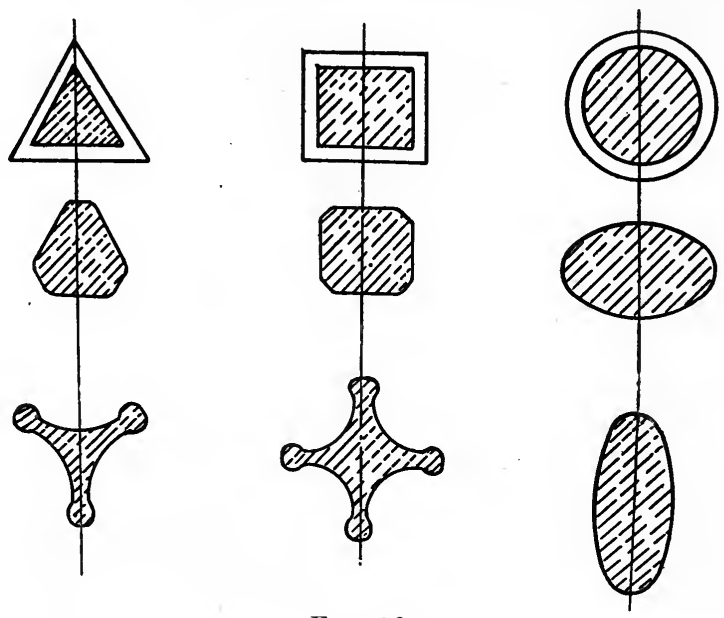

Fig. 58.

such reactions produces a continual state of change of section along its length. The action is periodic and approximately isochronous, and consequently, with a steady jet, the points at which the cross section is of the same general shape as the orifice are relatively stationary. With a circular orifice, the section varies from an ellipse with major axis horizontal near the vena contracta to the same with major axis vertical. With a polygonal orifice of $n$ sides the section ultimately becomes a star of $n$ points, these points having thickened extremities. The angular points either coincide in direction with those of the orifice or intersect a corresponding face, always being symmetrical with respect to the orifice. Figure 58 illustrates the general form taken by the jet from a triangular, square, or circular orifice, the upper section 
in each case being at, and the second slightly past the vena contracta, and the lower giving the star form assumed by the jet. ${ }^{1}$

Figure 59 shows the form taken by a stream issuing from a
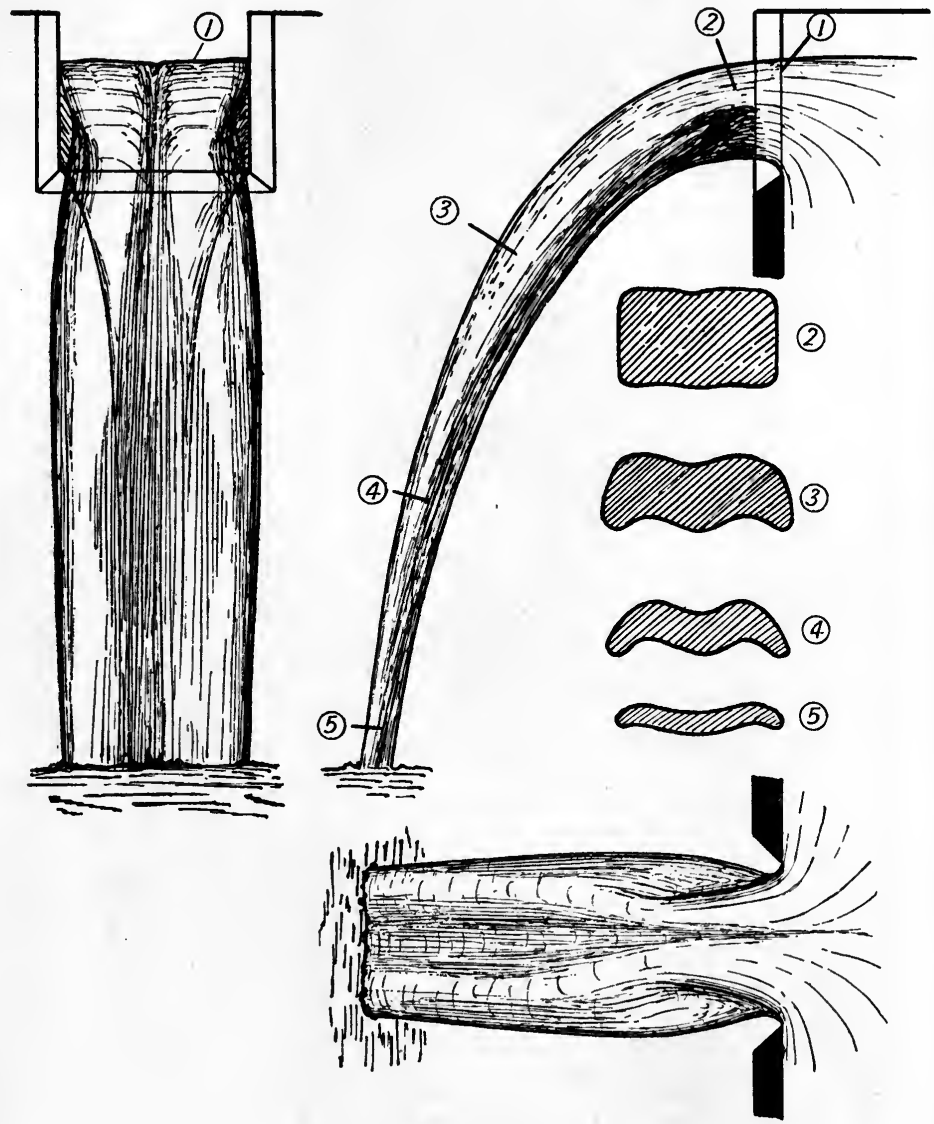

FIG. 59.

sharp-edged rectangular notch. The effect of the impact of converging particles is here strongly marked. The cross sections at various points on the jet are indicated in the figure.

1 For further information, a paper by Lord Rayleigh (" Proceedings Royal Society," vol. 71) should be consulted. 


\section{Art. 46.-Large Orifices.}

Where an orifice is large, except where formed in the horizontal base of a vessel, the assumption that the mean velocity over its whole area is sensibly equal to that at its centre of area is no longer true, and it becomes necessary to take account of the variation of velocity at different depths in its plane. As in the case of a small orifice, a vena contracta is formed of approximately the same sectional shape as the orifice, and depending for its magnitude on the shape, dimensions, and head above the latter, and on the circumstances governing the formation of stream lines in the approaching vein.

In the usual theoretical discussion of the flow from such an orifice, the two fundamental assumptions on which the theory is based are themselves false and quite misleading, and while the results obtained are not without value as forming the basis of useful empirical formulae, the treatment cannot be looked upon as scientific.

Briefly outlined, the method consists in assuming that at all points at the same depths in the plane of an orifice the velocity of efflux is the same, being that corresponding to the head of water above the point, and that the direction of flow at each point is perpendicular to the plane of the orifice.

Calculating the discharge over a small element $\delta a$ of the area, i.e., $\sqrt{2 g h} . \delta a$, and summing suchdischarges over the elements which go to make up the whole area, gives what is termed the theoretical discharge $\int \sqrt{2 g h} . \delta a$, and this, when multiplied by an empirical constant termed the coefficient of discharge, gives the true discharge.

Figure 45, which indicates roughly how the velocity at the orifice varies at different points in the cross section, sufficiently shows the error involved in the first, while a consideration of the stream line formation shows the error of the second assumption. Figure 60 indicates how the velocity perpendicular to the orifice varies and how it differs from the theoretical velocity $\sqrt{2 g h}$, as given by the horizontal ordinates of the parabola $m n q$. Here the actual velocity is represented in elevation and plan 
by the corresponding ordinates of the curves $a, c, b$, and $a^{\prime}, c^{\prime}, a^{\prime \prime}$.

The solid represented in plan and elevation by the shaded areas will then represent to scale the volume discharged per second, while the solid bounded by the plane of the orifice; the

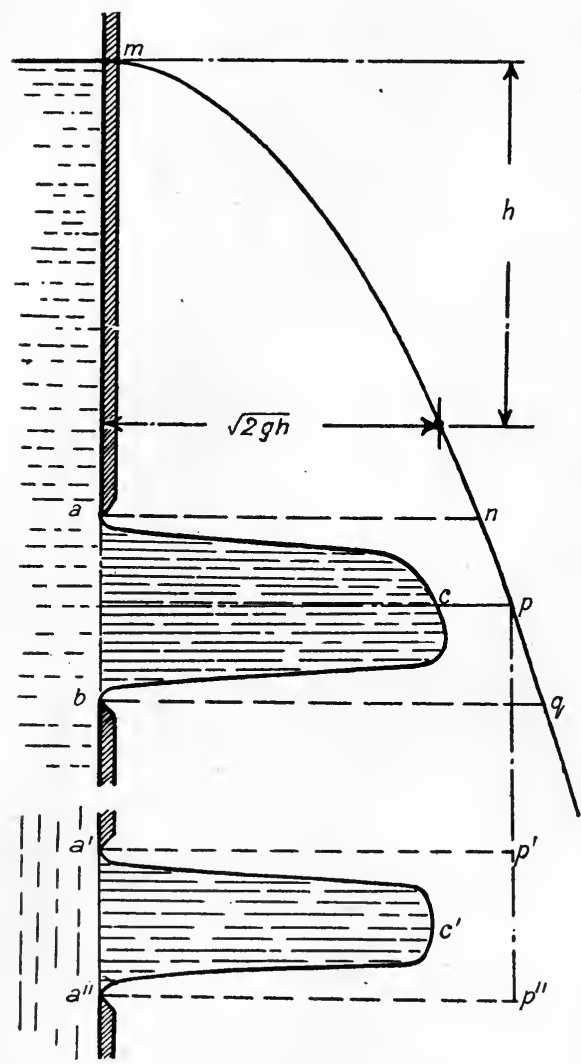

FIG. 60. perpendiculars an, $b q$, $a^{\prime} p^{\prime}, a^{\prime \prime} p^{\prime \prime}$; and the curved surface of the parabola, represented by $n p q$, will represent to the same scale the theoretical discharge. The ratio of these volumes thus gives the coefficient of discharge.

In spite of the recognised fallacies embodied in the method of treatment outlined above, the difficulties encountered in a rigorous treatment are so many and the results obtained so cumbrous, that we are still compelled to fall back on the more simple, though inaccurate, formulae, together with these experimentally deduced coefficients.

The assumptions, however, become more rational

if the state of affairs at the vena contracta be considered instead of at the orifice. Here we may assume with some reason that the flo: is every where perpendicular to the cross section of the stream, and that the pressure throughout is sensibly uniform and equal to that of the atmosphere, so that, except for the retarding effect of the atmosphere at the boundaries, the velocity at any point 
in this section, at a depth $h$, below the free surface in the vessel, is sensibly equal to $\sqrt{2 ! h}$. The more important cases of flow will now be considered on this assumption.

Art. 47.-Large Vertical Rectangular Orifice, neglecting Veiocity of Approach.

Let $H^{\prime}=$ height of free surface above top of vena contracta. ,,$b=$ breadth of orifice.

, , $=$ depth ,

,$b^{\prime}=$ breadth of venc contracta assumed to be

$, d^{\prime}=\operatorname{depth} \quad, \quad, \quad$ a rectangle.

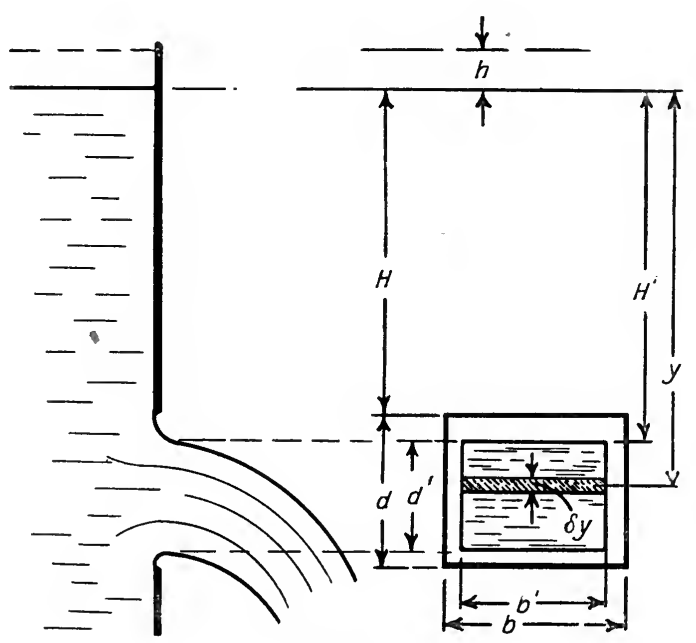

FiG. 61.

Consider the flow over any element of the rena contracta at a depth $y$ below the free surface, and having an area $b^{\prime} \delta y$ (Fig. 61).

The velocity at this depth $=C_{v} \sqrt{2 g y}$ feet per second.

$\therefore$ Flow over this area per second $=C_{v} b^{\prime} \delta y \sqrt{2 g y}, c_{f}$.

Integrating this expression, and giving $y$ the limits $H^{\prime}+d^{\prime}$ and $H^{\prime}$ we get the flow over the whole area.

H.A. 


$$
\begin{gathered}
\therefore Q=C_{v} b^{\prime} \sqrt{2 g} \int_{H^{\prime}}^{H^{\prime}+d^{\prime}} \sqrt{y} d y=\frac{2}{3} C_{v} b^{\prime} \sqrt{2 g}\left[y^{\frac{3}{2}}\right]_{H^{\prime}}^{H^{\prime}+d^{\prime}} \\
\left.=\frac{2}{3} C_{v} b^{\prime} \sqrt{2 !}{ }^{\prime}\left(H^{\prime}+d^{\prime}\right)^{\frac{3}{2}}-H^{\prime \frac{3}{2}}\right\}
\end{gathered}
$$

The difficulty of obtaining accurate measurements of $b^{\prime}, H^{\prime}$ and $d^{\prime}$ now arises, since these vary with, but do not bear a fixed ratio to $b, H$, and $d$, while experimental evidence as to the precise nature of the variation is wanting. The best way out of the difficulty appears to be to write

$$
C=C_{a} \frac{b^{\prime}\left\{\left(H^{\prime}+d^{\prime}\right)^{\frac{3}{2}}-H^{\prime \frac{3}{2}}\right\}}{\left.b \int_{1}^{\prime}(H+d)^{\frac{3}{2}}-H^{\frac{3}{2}}\right\}}
$$

where $H=$ depth of top of orifice below the free surface. This then gives us

$$
\left.Q=\frac{2}{3} C b \sqrt{2 !} \cdot(H+l)^{\frac{3}{2}}-H^{\frac{3}{2}}\right)
$$

The reason for this substitution is that the value of $C$ in this formula has been determined with some accuracy for various types of orifice and for different heads.

Formula (2) is that obtained by the inaccurate process of reasoning outlined at the beginning of this article, for, assuming that the flow through any element $b \delta y$ of the orifice is the same as that through the corresponding element of the rena contracta of area $b^{\prime} \frac{d^{\prime}}{d} \cdot \delta y$, and that this flow is therefore given by $C b \delta y \sqrt{2 g y}$, where $C$ has the same value for each element, and therefore for the whole area, we have

$$
\begin{gathered}
Q=C b \sqrt{2 g} \int_{H}^{H+d} \sqrt{y \cdot d} y \\
=\frac{2}{3} C b \sqrt{2 !}\left[(H+d)^{\frac{3}{2}}-H^{\frac{3}{2}}\right]
\end{gathered}
$$


Comparing this with the simple formula

$$
Q=C b d \sqrt{2 g}\left(H+\frac{d}{2}\right),
$$

obtained by considering the head over the section as sensibly equal to that at its centroid, we see that the two values of $Q$ are in the ratio $\frac{2}{3} \frac{(H+d)^{\frac{3}{2}}-H^{\frac{3}{2}}}{d \sqrt{H+\frac{d}{2}}}$. For values of $\frac{H}{d}$ greater $\operatorname{than} \frac{1}{2}$ the difference amounts to less than $1 \cdot 0$ per cent., so that for all larger values of $H$, the simpler formula is to be preferred.

The velocity of approach may be taken into account by increasing the head by an amount $h$ as explained in Art. 43, thus making the effective head $=H+h$.

$$
\text { Then } Q=\frac{2}{3} C b \sqrt{2 !}\left[(H+h+d)^{\frac{3}{2}}-(H+h)^{\frac{3}{2}}\right]
$$

As is clear from the expression assumed above for the value of $C$, this is not a constant, but varies both with the depth of the water and with the dimensions of the orifice, and can only be determined by experiment.

With sharp-edged orifices, and a free discharge into air, the values of $C$ for a square opening are very approximately as given below (Hamilton Smith).

\begin{tabular}{c|c|c|c|c}
\hline & \multicolumn{4}{|c}{ Length of side of square in feet. } \\
\cline { 2 - 4 } $\begin{array}{c}\text { Head above centre } \\
\text { of orifice in feet. }\end{array}$ & $\cdot 40$ & $\cdot 60$ & $\cdot 80$ & $1 \cdot 0$ \\
\hline$\cdot 6$ & $\cdot 601$ & $\cdot 598$ & - & - \\
$1 \cdot 0$ & $\cdot 603$ & $\cdot 601$ & $\cdot 600$ & $\cdot 599$ \\
$3 \cdot 0$ & $\cdot 605$ & $\cdot 604$ & $\cdot 603$ & $\cdot 603$ \\
$6 \cdot 0$ & $\cdot 604$ & $\cdot 603$ & $\cdot 602$ & $\cdot 602$ \\
$10 \cdot 0$ & $\cdot 603$ & $\cdot 602$ & $\cdot 602$ & $\cdot 601$ \\
$20 \cdot 0$ & $\cdot 601$ & $\cdot 601$ & $\cdot 601$ & $\cdot 600$ \\
$100 \cdot 0$ & $\cdot 598$ & $\cdot 598$ & $\cdot 598$ & $\cdot 598$ \\
\hline
\end{tabular}

For vertical rectangular orifices the value of $C$ varies with the ratio of the width $b$ to the depth $d$. 
The following table gives the value of the coefficient for orifices 1 foot wide.

\begin{tabular}{|c|c|c|c|c|c|c|c|}
\hline \multirow{2}{*}{$\begin{array}{l}\text { Head in feet from } \\
\text { centre of orifice. }\end{array}$} & \multicolumn{7}{|c|}{ Values of $d$ in feet. } \\
\hline & $\frac{1}{8}$ & $\frac{1}{4}$ & $\frac{1}{2}$ & $\frac{3}{4}$ & $1 \cdot 0$ & $1 \cdot 5$ & $2 \cdot 0$ \\
\hline $1 \cdot 0$ & ·632 & $\cdot 632$ & 618 & $\cdot 612$ & $\cdot 606$ & $\cdot 626$ & - \\
\hline $3 \cdot 0$ & $\cdot 627$ & $\cdot 627$ & $\cdot 615$ & $\cdot 610$ & .605 & $\cdot 614$ & $\cdot 619$ \\
\hline 6.0 & $\cdot 615$ & $\cdot 615$ & .609 & .604 & .602 & $\cdot 606$ & $\cdot 610$ \\
\hline $10 \cdot 0$ & $\cdot 606$ & $\cdot 603$ & 601 & $\cdot 601$ & $\cdot 601$ & $\cdot 601$ & $\cdot 602$ \\
\hline 20.0 & $\cdot 602$ & $\cdot 601$ & $\cdot 601$ & $\cdot 601$ & $\cdot 601$ & $\cdot 601$ & $\cdot 602$ \\
\hline
\end{tabular}

Art. 48.-Flow through a Circular Orifice. Let $H=$ depth of centre of orifice (Fig. 62).

Let $R=$ radius of orifice.

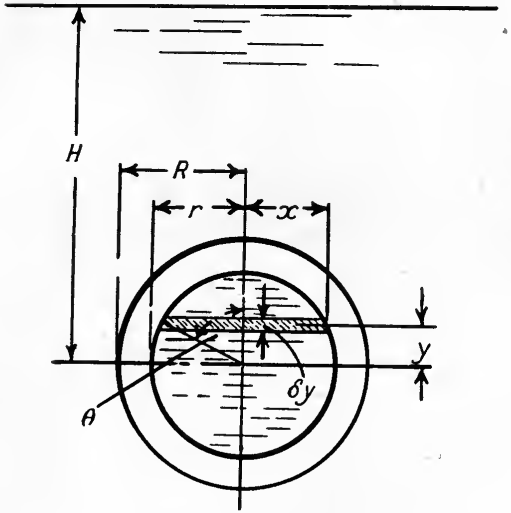

FIG. 62.

,$r=$, , rena contracta.

'Then the area of an elementary strip taken in the rena contracta at a distance $y^{\circ}$ from the centre, as shown, is $2 x \delta y$.

'The head over the element $=H-y$,

$\therefore$ Flow over this element $=$ $\sqrt{2 !(H-y)} \times 2 x \delta y$

$\therefore \delta Q=2 \sqrt{2 g}$

$\sqrt{\left(r^{2}-y^{2}\right)(H-y)} \delta y$.

For convenience in integrating, expand $\sqrt{H-y}$ by the Binomial Theorem, i.e., put

$$
\begin{gathered}
\left.\sqrt{H-y}=\sqrt{H} \sqrt{1-\frac{y}{H}}=\sqrt{H} 1-\frac{y}{2 H}-\frac{y^{2}}{8 H^{2}}+\text { etc. }\right) \\
\left(\text { since }(1-x)^{n}=1-n x+\frac{n(n-1)}{2} x^{2}-\text { etc. }\right)
\end{gathered}
$$

Now put $y=r \cos \theta$. 
Then $\quad \frac{d y}{d \theta}=-r \sin \theta . \quad \therefore \delta y=-r \sin \theta \delta \theta$,

and $\quad \sqrt{r^{2}-y^{2}}=\sqrt{r^{2}\left(1-\cos ^{2} \theta\right)}=r \sin \theta$.

So that $\delta Q=2 \sqrt{2 g} \bar{H}\left\{-r^{2} \sin ^{2} \theta\left(1-\frac{r \cos \theta}{2 H}-\right.\right.$

$$
\left.\frac{r^{2} \cos ^{2} \theta}{8 H^{2}}+- \text { etc. }\right) ; \delta \theta \text {. }
$$

Integrating this expression and giving $\theta$ the limits $\pi$ and $o$, since these are the values of $\theta$ corresponding to the values $\mp r$ of $y$, we get, on introducing $C_{v}$, the total flow $Q$ over the whole section.

$$
\begin{gathered}
\therefore Q=2 C_{v} \sqrt{2 g H} \int_{o}^{\pi} r^{2} \sin ^{2} \theta\left(1-\frac{r \cos \theta}{2 H}-\frac{r^{2} \cos ^{2} \theta}{8 H^{2}}+- \text { etc. }\right) d \theta \\
\left.=C_{v} \pi r^{2} \sqrt{2 ! H} \int_{i} 1-\frac{1}{32} \frac{r^{2}}{H^{2}}+\text { - etc. }\right\}
\end{gathered}
$$

the succeeding terms being negligible.

If $C$ be the coefficient of discharge for the orifice, then since $C_{c}=\frac{\pi r^{2}}{\pi R^{2}}$ we may write

$$
\left.Q=C \pi R^{2} \sqrt{2 g H} ; 1-\frac{1}{32} \frac{r^{2}}{H^{2}}\right\}
$$

As in the case of a rectangular orifice, the coefficient in general decreases as the orifice increases and also as the head increases.

The following table gives the approximate values of $C$ for circular orifices (Hamilton Smith) :-

\begin{tabular}{c|c|c|c|c} 
& \multicolumn{4}{|c}{ Diameter of orilice in feet. } \\
\cline { 2 - 3 } $\begin{array}{c}\text { Ifead above centre } \\
\text { of orifice (feet). }\end{array}$ & $\cdot 40$ & $\cdot 60$ & $\cdot 80$ & $1 \cdot 0$ \\
\hline $1 \cdot 0$ & $\cdot 596$ & $\cdot 595$ & $\cdot 593$ & $\cdot 591$ \\
$2 \cdot 0$ & $\cdot 598$ & $\cdot 597$ & $\cdot 596$ & $\cdot 595$ \\
$4 \cdot 0$ & $\cdot 599$ & $\cdot 597$ & $\cdot 597$ & $\cdot 596$ \\
$6 \cdot 0$ & $\cdot 598$ & $\cdot 597$ & $\cdot 596$ & $\cdot 596$ \\
$10 \cdot 0$ & $\cdot 597$ & $\cdot 596$ & $\cdot 596$ & $\cdot 595$ \\
$20 \cdot 0$ & $\cdot 596$ & $\cdot 596$ & $\cdot 595$ & $\cdot 594$ \\
$100 \cdot 0$ & $\cdot 592$ & $\cdot 592$ & $\cdot 592$ & $\cdot 592$ \\
\hline
\end{tabular}




\section{Art. 49.-Submerged Orifices.}

If the water level on the discharge side is above highest point of the opening we get a submerged orifice.

Let $H_{1}=$ depth of water above upper edge of orifice on the incoming side, in feet.

Let $H_{2}=$ similar depth on discharge side.

Then the effective depth at all points in the orifice is the same, viz., $H_{1}-H_{2}$ (Art. 37).

$\therefore$ Theoretical velocity of efflux $=\sqrt{2 g\left(H_{1}-H_{2}\right)} \mathrm{ft}$. sec.

$\therefore$ Quantity per second through orifice $=C A \sqrt{2}$ !! $\sqrt{H_{1}-H_{2}}$, where $A$ is the area of the orifice.

Taking into account the velocity of approach, $v_{1}$, the effective head will now be $H_{1}+h-H_{2}$ feet, where $h=\frac{v_{1}^{2}}{2 g}$, and the discharge will be given by

$$
Q=C A \sqrt{2 g} \sqrt{H_{1}+h-H_{2}} \text { cub. ft. sec. }
$$

The value of $C$ for a submerged orifice may be taken as being about 1 per cent. less than its value for the same orifice under the same effective head, and when discharging freely into air.

Hanbury Brown ("Irrigation ") $)^{1}$ gives the following as being approximate values of $C$ for sluices and regulator openings in masonry piers.

Ordinary lock sluices _. . . . $C=\cdot 62$

Small regulator openings with shallow) water.

Regulator openings up to 6 feet wide) with recesses in the piers. .

Ditto between 6 feet and 13 feet wide , , $\quad 72$

Ditto above 13 feet wide . . . , 92

Regulator openings up to 6 feet wide! with straight and continuous piers

Ditto between 6 and 13 feet wide . , , 92

Ditto above 13 feet wide . . . . . , 992

1 Constable \& Co., Iondon, 1907. 
Partially Submerged Orifice (Fig. 63).-Here the portion of the orifice $l \mathrm{~m}$, below the normal water level on the discharge side, may be considered as a submerged orifice, while the portion $k l$ above this level may be looked upon as discharging freely into air, the quantity passing each portion being calculated separately on these assumptions.

Thus in a rectangular orifice of breadth $b$ and depth $d$, having a head of water on the supply side $=H$ above the top of the orifice, with free discharge over a depth $d_{1}$,

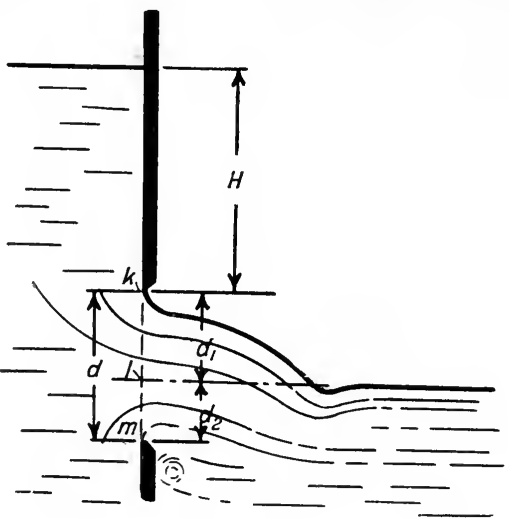

FIG. 63. and submerged over the remainder $d_{2}$ of its depth we have Discharge over depth $l_{1}=Q_{1}=\frac{2}{3} C_{1} b \sqrt{2 g}\left\{\left(H+d_{1}\right)^{\frac{3}{2}}-H^{\frac{3}{2}}\right\}$

$$
\begin{gathered}
", \quad, \quad d_{2}=Q_{2}=C_{2} b d_{2} \sqrt{2 g}\left(H+l_{1}\right)^{\frac{1}{2}} . \\
\therefore \quad \text { Total discharge } Q=Q_{1}+Q_{2}=b \sqrt{2 g} \\
{\left[\frac{2}{3} C_{1}\left\{\left(H+d_{1}\right)^{\frac{3}{2}}-H^{\frac{3}{2}}\right)+d_{2} C_{2}\left(H+l_{1}\right)^{\frac{1}{2}}\right]}
\end{gathered}
$$

If $C_{1}=C_{2}=C$ we get

$$
\left.Q=C b \sqrt{ } 2 g\left[\left(H+d_{1}\right)^{\frac{1}{2}}: \frac{2}{3}\left(H+d_{1}\right)+d_{2}\right\}-\frac{2}{3} H^{\frac{3}{2}}\right]
$$

If $h=$ the head equivalent to the velocity of approach, the formula becomes

$$
Q=C b \sqrt{2 y}\left[\begin{array}{c}
\left.\left(H+d_{1}+h\right)^{\frac{1}{2}}: \frac{2}{3}\left(H+d_{1}+h\right)+d_{2}\right) \\
-\frac{2}{3}(H+h)^{\frac{3}{2}} .
\end{array}\right]
$$

The difficulty in exactly estimating $d_{1}$ and $d_{2}$, and the undulatory motion of the surface which is usually found in a partially submerged orifice, renders this a most unsatisfactory method of estimating the flow of a stream.

The coefficients so far given only apply where the jet is wholly 
submerged or where discharge takes place freely into the air. If the issuing stream, instead of springing clear of the orifice, adheres to its face, the formation of a true vena contracta is prevented and we have an abnormal increase in $C$. Thus if the jet is discharging freely into air, and if the level on the discharge side is allowed to rise, at some point-before the level reaches that of the notch sill the discharge ceases to be free and a sudden increase in the value of $C$ results. As $d_{2}$ increases, $C$ resumes its normal value.

\section{Art. 50.-Law of Conparison for Orifices.}

Similar orifices are such as may be represented by the same drawing to different scales. If similarly situated, the free water surface will be represented by the same line, whatever the scale of the drawing.

If the scale ratio for any two orifices, i.e., the ratio of any two corresponding linear dimensions, is $S$, the ratio of the areas of corresponding elements of the orifices will be $S^{2}$, while if similarly situated with respect to the water surface, their depths are proportional to $S$.

That the value of $C$ may be expected to be very approximately the same for two such similar orifices was shown by Professor James Thomson in a discussion of which the following is a brief résumé.

Consider two similar and similarly situated particles of fluid (1) and (2). The masses of these and their weights are in the ratio of their volumes, so that we have $u_{1}=S^{3} u_{2}$.

Now if these particles are to trace out paths which shall be similar, all corresponding forces acting upon them must have the same ratio, and it follows that this ratio must be the ratio of their weights since this is fixed. Apart from the forces due to the statical pressure corresponding to the depth of the particle, which are proportional to its depth multiplied by its area, and therefore follow the required law, the only other forces are those due to centrifugal action and to the effect of viscosity. Neglecting the latter, ${ }^{1}$ and terming $F$ the centrifugal force, we have

since the force introduced by viscosity $=\mu \frac{d r}{d s}$ per unit area, we have, if $P$ is

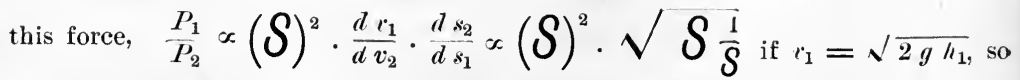
that $\frac{P_{1}}{P_{2}} \propto(S)^{\frac{3}{2}}$. Viscosity therefore tends to prevent exact similarity of flow. 


$$
\frac{F_{1}}{F_{2}}=\frac{w_{1} v_{1}^{2}}{r_{1}} \cdot \frac{r_{2}}{u_{2} v_{2}{ }^{2}}=S^{3} \cdot \frac{r_{2}^{-}}{r_{1}}\left(\frac{v_{1}}{r_{2}}\right)^{2}
$$

But if the paths of the particles are similar, $r_{1}=S r_{2}$,

$$
\therefore \frac{r_{1}}{r_{2}}=S^{2}\left(\frac{r_{1}}{r_{2}}\right)^{2}
$$

It follows as a necessary condition that $\left(\frac{v_{1}}{r_{2}}\right)^{2}=S$, and this condition will evidently be fulfilled if $\left(\frac{v_{1}}{v_{2}}\right)^{2}=\frac{h_{1}}{h_{2}} i . e$., if $v^{2}=2 g h$, and if all corresponding particles are similarly situated with respect to the free surface. In such a case, the paths of all corresponding particles being similar, the contractions of the two jets will also be similar and the values of $C$ will become identical. A simple ratio thus connects the relative discharge of the two orifices.

Example.

In two triangular notches having the same value of $\theta$, we have two similar and similarly situated orifices, so that $\frac{Q_{1}}{Q_{2}}=\left(\frac{H_{1}}{H_{2}}\right)^{\frac{5}{2}}$.

\section{Art. 51.-Flow over Notches and Weirs.}

Where the free surface on the supply side is below the level of the upper edge of an orifice, this is termed a notch, and, if of large dimensions, a weir:

The theoretical treatment for flow over such notches follows exactly similar lines to that for flow through large orifices, and is subject to the same erroneous assumptions. The errors involved in assuming that the velocity of efflux perpendicular to

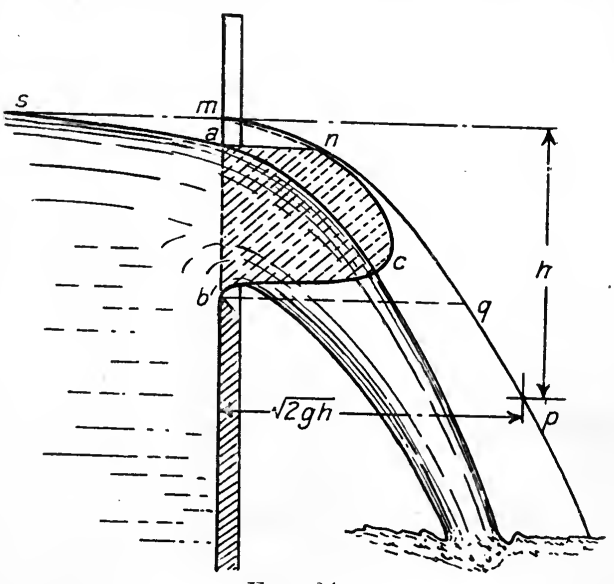

FIG. 64. the plane of the notch, at the notch, at any depth $h$, is 
proportional to $\sqrt{2 g h}$, is indicated in Fig. 64 , where the horizontal ordinates of the parabola $m n q$ show the theoretical velocity at any depth below the free surface of the still water at $S$, while the ordinates of the curve $a n c b$ denote the actual velocities in this direction in the plane of the notch. The coefficient of discharge will then be equal to the ratio of the volumes represented in elevation by the shaded area $a n c b$ and the area $m \quad n \quad q b$.

The difficulty of determining the point in the stream at which the pressure over a cross-section is uniform, renders a rigorous treatment impossible, and the next best method is to consider the state of affairs in the plane of the notch.

An examination of the contour of the escaping stream shows that in the plane of the notch its upper surface is lower than the free surface at a point a short distance up stream where the motion is steady.

This fall from $s$ to $a$ (Fig. 64) is essential if the surface filaments are to have the required velocity of efflux at the notch, and since their motion is approximately perpendicular to the plane of the orifice and is unaffected save by the resistance of the air, their velocity may be taken as sensibly equal to $\sqrt{2 g(a m)}=\sqrt{2 g H^{\prime}}$ (Fig. 65).

In general $H^{\prime}$ is approximately equal to $\cdot 20 \dot{H}$, where $H=$ height of water measured to still water level, above the crest of the notch or weir, so that $\left(H-H^{\prime}\right)=$ depth of water at crest in feet.

Ar'T. 52.-Theoretical Foruulae for Flow over Weirs.

(1) Rectangular Weirs (Fig. 65).

Let $b=$ length of weir in feet.

Consider the flow across any element of area $b \delta x$, in the plane of the weir, at a depth $x$ below the still water surface of the supply.

The volume passing this element per second $=\delta Q=C^{\prime} b \delta x$ $\sqrt{2 g x}$ cub. ft. per sec.

Integrating this expression and giving $x$ the upper and lower 
limits $H$ and $H^{\prime}$, and assuming that $C^{\prime}$ has the same value for each element, we have

$$
Q=\frac{2}{3} C^{\prime} b \sqrt{2 g}\left\{H^{\frac{3}{2}}-H^{\frac{13}{2}}\right\} \text { cub. ft. per sec. }
$$

The value of this coefficient $C^{\prime}$ has not been experimentally determined, so that the formula in its present form cannot be applied to determine the discharge in any practical case. If, however, we write

$$
C^{\prime}\left\{H^{\frac{3}{2}}-H^{\prime \frac{3}{2}}\right\}=C H_{2}^{\frac{3}{2}}
$$

the formula becomes

$$
Q=\frac{2}{3} C b \sqrt{2 g} H^{\frac{3}{2}} \text { cub. ft. per sec. }
$$

and the value of this coefficient is known with fair accuracy for different values of $H$.

Formula (2) may be deduced with less reason by assuming the

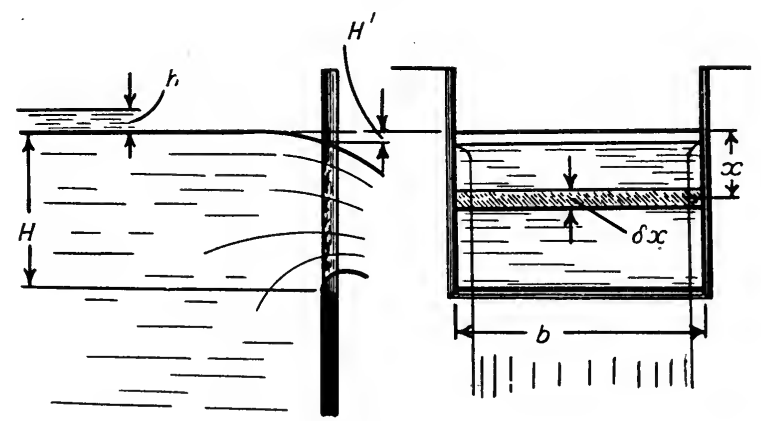

FIG. 65.

depth of water in the plane of the notch to be $H$, i.e., neglecting the fall from still water level, and assuming that the velocity of the surface filaments is zero, and therefore by integrating the expression $\delta Q=C \quad b \delta x \sqrt{2 g x}$ between the limits $H$ and 0 . It might also be deduced from equation (2) of p. 130 by writing $\ddot{H}=0 ; d=H$.

The value of $C$, in addition to varying with the breadth and depth of the weir, varies largely with its position with respect to the sides and bottom of the approach channel. Where the sides of this channel are so far removed as not to affect the contraction 
of section at either side of the weir, this is said to have two end contractions, while where the channel is of the same breadth as the weir, the latter has no end contractions, and is termed a Suppressed Weir. The bottom contraction is also affected by the nearness of the sill of the weir to the bottom of the channel of approach and by the inclination of the weir face.

Where the "nappe," or falling sheet of water, comes into contact with the top or downstream face of the weir, as shown in Figs. 69 and 73, a further and in general irregular variation takes place in $C$. In what immediately follows, only those weirs will be considered in which the nappe springs clear of the crest and discharges freely into the air, the crest being vertical, narrow in comparison with the head, and having a sharp upstream edge.

Adopting the base formula $Q=K b H^{\frac{3}{2}}$ cub. ft. per sec., where $K=\frac{2}{3} \sqrt{2 g} C$, many experiments have been carried out to determine the value of $K$. Of these, perhaps those of Francis, Fteley and Stearns, Smith, and Bazin are the most reliable.

Francis, ${ }^{1}$ in a preliminary series of experiments carried out in 1848 and 1851, assumed the formula $Q=K l H^{x}$, and found $x$ to vary slightly with the head, having a mean value $1 \cdot 47$.

In his later experiments, however, carried out in 1852, he adopted, for convenience, the constant value $1 \cdot 5$, and determined the corresponding values of $K$.

From these experiments, carried out on weirs from 8 to $10 \mathrm{ft}$. long, and with heads ranging from $\cdot 62$ to $1 \cdot 56 \mathrm{ft}$., he deduced the empirical formula

$$
Q=3 \cdot 33\left(l-\frac{n}{10} I I\right) H^{\frac{3}{2}} \text { cub. ft. per sec. }
$$

for the flow over a sharl-edged rectangular notch, where $i=2,1$, or 0 , according as the stream has 2,1 , or 0 end contractions, the bottom contraction being free in every case. 
Thus for two end contractions $Q=3 \cdot 33(b-\cdot 2 H) H^{\frac{3}{2}}$ c.f.s.

$$
\begin{aligned}
& \text {, one , contraction } Q=3 \cdot 33(b-\cdot 1 H) H^{\frac{3}{2}} \text { c.f.s. } \\
& \text {, no }, \quad, \quad Q=3 \cdot 33 \text { b } H^{\frac{3}{2}} \text { c.f s. }
\end{aligned}
$$

The experiments lead to the conclusion that at all depths the contraction of the stream in a vertical direction is approximately constant, having a mean value of 623 . The side contraction is also constant so long as $b$ is greater than $3 H$.

That this formula, though empirical, has a rational basis may be seen if we consider the state of affairs in a long and shallow notch having two end contractions (Fig. 66).

If the notch is sufficiently broad, a certain section in the middle will have stream lines which, as seen from above, are parallel, while the two end sections have stream lines which suffer an amount of curvature depending on their distance from the ends. The distance over which this curvature extends is found to be approximately $m H$, where $m$ $=1.5$. This end contraction

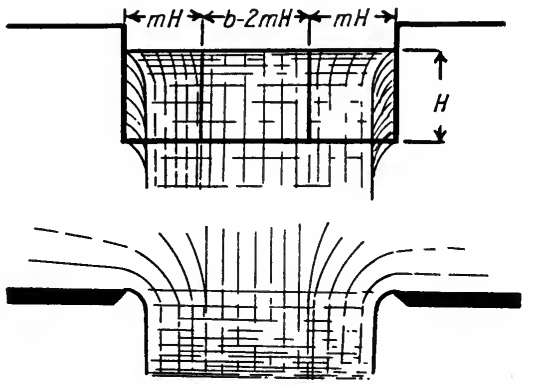

FIG. 66. evidently diminishes the discharge by an amount which is constant for a given depth, and it is reasonable to assume that it therefore diminishes the effective breadth of the notch by an amount which is also constant for a given depth, and which depends only on the depth. The law of its variation with the depth has not been accurately determined, but it was assumed, as appears to be approximately true, that the diminution in effective length is directly proportionai to the depth and that the effective length is therefore equal to $(b-n k H)$, where $n$ is the number of contractions and $l_{i}$ is a constant to be determined experimentally. Francis then assumed (though not on theoretical grounds) that the discharge from notches of equal effective length varies according to a constant power of the depth, so that the formula became ${ }^{1}$

$$
Q=K\{b-n k H\} H^{x} \text {. }
$$

1 A formula of this form was suggested by Boyden in 1846 . 
Though neither of the assumptions made is quite correct, yet if $x$ be given the value $1 \cdot 5, K$ the value $3 \cdot 33$, and $k$ the value $\frac{1}{10}$, they lead to results closely in accord with experiment.

Recent experiments at Cornell University ${ }^{1}$ on a suppressed weir $6.56 \mathrm{ft}$. wide and with heads ranging from $2 \cdot 0$ to $4.85 \mathrm{ft}$. give mean results $98.4 \mathrm{per}$ cent. of those calculated by Francis's formula, and indicate its reliability for heads up to $5 \mathrm{ft}$. In general it is suitable for use for all heads above 6 ins. where the bottom contraction and end contractions, if any, are free, and where the length of crest is greater than three times the head.

For perfect contractions Francis specifies a distance to side of approach channel $=2 H$, and a depth below the weir crest $=3 H$, and states that a reduction of the bottom clearance to $2 H$ and of the side clearance to $H$ increases the flow by about one per cent.

Hamilton Smith specifies a minimum side clearance $=2 \mathrm{H}$.

Effect of the Velocity of Approach.-As in the case of a submerged orifice, the fact that the stream has linetic energy in virtue of its velocity of approach to the weir, may be taken into account, and correction made for this by adding to the measured head $H$ a supplementary head $h$, where $h=\frac{v^{2}}{2}, j$. Here $v$ is the velocity of approach, and is necessarily the computed mean velocity over the whole section of the approach channel. But the distribution of velocity over this section is not at all uniform, the filaments nearer the surface and nearer the centre having a velocity greater than the mean, depending on the depth, width, and surface condition of the channel. The particles which suffer the least change in their direction of motion on passing the weir are thus those which have a velocity of approach greater than the mean, and since the velocity of approach in these particles has a greater proportional effect in increasing the flow past the weir than in those which approach its plane in a more oblique direction, the effective velocity of approach will be greater than that corresponding to the calculated head $h$.

1 Trans. Am. Soc. C. E., Vol.44, p. 397. The maximum variation from Francis's formula, viz., four per cent., occurred with $2 \cdot 64 \mathrm{ft}$. head. 
Most experimenters, therefore, put the effective head as equal to $I I+a h$, where $a$ has some value greater than unity, and write ()$=K b(H+a h)^{\frac{3}{2}}=K l D^{\frac{3}{2}}$.

Francis, however, allows for the velocity of approach by putting $H+h$ and $h$ as his limits of integration in formula (1) of this article, and thus gets

For a suppressed weir

$$
\begin{aligned}
& Q=3.33 b\left\{(H+h)^{\frac{3}{2}}-h^{\frac{3}{2}}\right\} \\
& Q=3.33(b-\cdot 1 H)\left\{(H+h)^{\frac{3}{2}}-h^{\frac{3}{2}}\right\}
\end{aligned}
$$

With one end contraction

With two end contractions ()$=3 \cdot 33(b-\cdot 2 H)\left\{(H+h)^{\frac{3}{2}}-l^{\overline{2}}\right\}$.

In using Francis's formula, care should be taken to apply the correction in this manner.

The value of $h=\frac{v^{2}}{2 g}$, is determined by approximation. Thus $Q$ is first determined from the measured $H$, and this value of $Q$ is used to determine $v$ from the-known area of the channel.

The second approximation to the true value of $Q$, obtained by inserting the value of $h$ thus found, in the expressions given above, is always sufficiently near for all practical purposes.

Since in this formula we have $D^{\frac{3}{2}}=\left\{(H+h)^{\frac{3}{2}}-h^{\frac{3}{2}}\right\}$

$$
\therefore \quad D=\left\{(H+h)^{\frac{3}{2}}-h^{\frac{3}{2}}\right\}^{\frac{2}{3}} \text {. }
$$

Expanding this as a series and omitting all terms containing a small quantity of the second or higher order, we get

$$
D=H+h-\frac{2}{3} \sqrt{\frac{h^{3}}{H}}=H+a h, \text { where } a=1-\frac{2}{3} \sqrt{\frac{h}{H}} .
$$

Fteley and Stearns, ${ }^{1}$ from experiments on suppressed weirs. 5 feet and 19 feet long, and with heads ranging from 56 feet to 95 feet, and also from a discussion of Francis's results, obtained the formula for a suppressed weir

$$
Q=3.31 b H^{\frac{3}{2}}+\cdot 007 b \text { cub. ft. per sec. }
$$

For a weir with end contractions, $b$ is to be replaced by 1 'Trans. Am. Soc. C. E., 1883, pp. 1-118. 
$(b-\cdot 1 n H)$. The velocity of approach is to be allowed for by putting the effeetive head $=H+a h$, where

$a=1.5$ for a suppressed weir.

$a=2.05$ for a weir having two end contractions.

These are mean values. Actually the experiments showed that a varies with $H$, as shown in the following table:-

\begin{tabular}{|c|c|c|c|c|c|c|}
\hline \multirow{2}{*}{$\begin{array}{c}\text { Deptli of } \\
\text { channel of } \\
\text { approach } \\
\text { below crest. }\end{array}$} & \multicolumn{6}{|c|}{ Suppressed weir-channel $5 \mathrm{ft}$. wide, depth on crest in feet. } \\
\hline & $\because 2$ & $\cdot 4$ & $\cdot 6$ & $1 \cdot 0$ & $1 \cdot 5$ & $2 \cdot 0$ \\
\hline$\cdot 5$ & $1 \cdot 70$ & $1 \cdot 5.3$ & $1 \cdot 52$ & $1 \cdot 48$ & - & - \\
\hline $1 \cdot 0$ & $1 \cdot 87$ & $1 \cdot 79$ & $1 \cdot 71$ & $1 \cdot 61$ & $1 \cdot 52$ & - \\
\hline $1 \cdot 7$ & $1 \cdot 66$ & $1 \cdot 63$ & $1 \cdot 60$ & $1 \cdot 54$ & $1 \cdot 46$ & $1 \cdot 38$ \\
\hline $2 \cdot 6$ & $1 \cdot 51$ & $1 \cdot 49$ & $1 \cdot 47$ & $1 \cdot 43$ & $1 \cdot 38$ & $1 \cdot 33$ \\
\hline
\end{tabular}

Hamilton Smith, ${ }^{1}$ from experiments by Fteley and Stearns, Francis, and self, with weir lengths from $\cdot 66$ feet up to 19 feet, heads up to 2 feet, altered Francis's formula so as to take a correction $H+a h=D$, for the velocity of approach, giving $a$ the values 1.4 for a contracted, and 1.33 for a suppressed weir.

Then $Q=3.29 ; b+\frac{D}{7} ; D^{\frac{3}{2}}$ for a suppressed weir.

$$
\begin{aligned}
& Q=3.29 b D^{\frac{3}{2}} \text { for one end contraction. } \\
& Q=3.29
\end{aligned}
$$

Smith also suggests determining $v$, and therefore $h$, by measuring the surface velocity in the approach channel, the ratio of surface velocity to mean velocity being usually approximately equal to $a$.

$B a z i n,{ }^{2}$ from his own experiments on suppressed weirs from $1 \cdot 65$ feet to 6.56 feet long, and with heads ranging from $\cdot 17$ feet to 1.97 feet, and also from an examination of the results of Fteley and Stearns, deduced the formula

$$
Q=\left\{\cdot 405+\frac{\cdot 00984}{H}\right\} b \sqrt{2 g} H^{\frac{3}{2}} \text {. c.f.s. }
$$

for a weir with no velocity of approach. For a weir with velocity

1 Hamilton Smith, "Hydraulics," pp. 99 and 137.

2 "Annales des Ponts et Chaussées," 1898, 2 me trimestre. 
of approach, the effective head $=H+1 \cdot 69 h$, and on substituting for $h$ in terms of the depth of approach channel Bazin finally gets

$$
Q=\left\{1+\cdot 55\left(\frac{H}{P+H}\right)^{2}\right\}\left\{\cdot 405+\frac{\cdot 00984}{H}\right\} b \sqrt{2 g} H^{\frac{3}{2}}
$$

cub. ft. per sec.

Approximately, for heads from 4 inches to 1 foot we have

$$
Q=\left\{\cdot 425+\cdot 21\left(\frac{H}{P+H}\right)^{2}\right\} b \sqrt{2 g} H^{\frac{3}{2}} \text { cub. ft. per sec. }
$$

correct within about $2 \frac{1}{2}$ per cent. In these formulae $P=$ height of the weir crest above the bed of the approach channel. All dimensions are in feet. Expressing the formula in the form $Q=K b H^{\frac{3}{2}}$, the following values of $K$ are deduced from Bazin's experimental results :-

\begin{tabular}{c|c|c|c|c|c}
\hline \multirow{2}{*}{$\begin{array}{c}\text { Head in feet. } \\
H .\end{array}$} & \multicolumn{5}{|c}{ Height of notch sill above channel bed, in feet. } \\
\cline { 2 - 6 } & $\cdot 66 \mathrm{ft}$. & $1 \cdot 00 \mathrm{ft}$. & $1 \cdot 50 \mathrm{ft}$. & $2 \cdot 00 \mathrm{ft}$. & $5 \cdot 00 \mathrm{ft}$. \\
\hline$\cdot 164$ & $3 \cdot 667$ & $3 \cdot 636$ & $3 \cdot 610$ & $3 \cdot 604$ & $3 \cdot 593$ \\
$\cdot 230$ & $3 \cdot 652$ & 3.593 & $3 \cdot 561$ & $3 \cdot 550$ & 3.529 \\
$\cdot 656$ & $3 \cdot 850$ & $3 \cdot 667$ & $3 \cdot 555$ & $3 \cdot 501$ & $3 \cdot 406$ \\
1.050 & - & $3 \cdot 823$ & $3 \cdot 661$ & 3.555 & $3 \cdot 394$ \\
1.575 & - & - & $3 \cdot 796$ & $3 \cdot 655$ & $3 \cdot 394$ \\
1.969 & - & - & 3.877 & $3 \cdot 733$ & 3.422 \\
\hline
\end{tabular}

The following table shows the ratio of the velocity-of-approach correction applied by various experimenters for a suppressed weir.

\begin{tabular}{c|c|c|c}
\hline Bazin. & $\begin{array}{c}\text { Fteley and } \\
\text { Stearns. }\end{array}$ & Smith. & Francis. \\
\hline 1.0 & $\cdot 887$ & $\cdot 789$ & $\cdot 454$ \\
\hline
\end{tabular}

This large difference is, however, more apparent than real, as the larger velocity corrections are compensated by smaller weir coefficients. Actually the final agreement is, in general, fairly 
close, as is shown by the following table, which shows the comparative values of $Q$ per foot of crest length for a suppressed weir.

\begin{tabular}{|c|c|c|c|c|c|c|c|}
\hline Length. & $\begin{array}{c}\text { Height } \\
\text { of crest. }\end{array}$ & Head. & $\begin{array}{l}\text { Velocity of } \\
\text { approach. }\end{array}$ & Francis. & $\begin{array}{l}\text { Fteley and } \\
\text { Stearns. }\end{array}$ & Bazin. & $\begin{array}{l}\text { Smith- } \\
\text { Francis. }\end{array}$ \\
\hline $10 \mathrm{ft}$. & $2 \mathrm{ft}$. & $1 \cdot 0 \mathrm{ft}$. & $1 \cdot 16$ f.s. & $1 \cdot 00$ & $1 \cdot 015$ & $\cdot 985$ & $1 \cdot 018$ \\
\hline 10, & 4 , & $1 \cdot 0$, & $\cdot 68$ & $1 \cdot 00$ & $1 \cdot 001$ & $1 \cdot 010$ & $1 \cdot 002$ \\
\hline 10, & 4, & $4 \cdot 0$, & $2 \cdot 15$, & $1 \cdot 00$ & $1 \cdot 050$ & $1 \cdot 050$ & $1 \cdot 115$ \\
\hline
\end{tabular}

It may be shown that neglect to allow for the velocity of approach will lead to an error which may amount to 6 per cent. where $A$ (the area of approach channel) $=2 b \mathrm{H}$, but is reduced to $\cdot 7$ per cent. if $A=6 \mathrm{bH}$, and to $\cdot 25$ per cent. if $A=10 \mathrm{bH}$. Thus with a suppressed weir it is in general important that this should be taken into account, while with two end contractions it is usually unnecessary.

Braschmann's formula,

$$
Q=\left\{\cdot 3838+\cdot 0386 \frac{b}{B}+\frac{\cdot 00174}{H}\right\} b \sqrt{2 g} H^{\frac{3}{2}}, \text { c.f.s. }
$$

the dimensions being in feet, is much used in Germany. Here $B$ is the width of the approach channel. The formula only applies to rectangular weirs with two end contractions and free bottom contraction, and agrees well with that of Francis, where $B>4 b$.

The following values of $C$ were determined by Professor Dwelshauvers-Devy, of Liège, from experiments on a small rectangular notch 2.593 inches wide, and with heads varying from $\cdot 4$ to $4 \cdot 3$ inches, ${ }^{1}$ with no velocity correction.

\begin{tabular}{c|c|c|c|c|c|c|c|c|c|c}
\hline $\begin{array}{c}\text { Head in } \\
\text { inches. }\end{array}$ & $\cdot 4$ & 5 & 6 & $\cdot 8$ & $1 \cdot 0$ & $1 \cdot 5$ & $2 \cdot 0$ & $2 \cdot 5$ & $3 \cdot 0$ & $4 \cdot 0$ \\
\hline$C$ & $\cdot 629$ & $\cdot 622$ & $\cdot 618$ & $\cdot 6125$ & $\frac{\cdot 609}{\cdot 6042}$ & $\frac{\cdot 6029}{\cdot 6023}$ & $\frac{\cdot 6021}{\cdot 6021}$ \\
\hline
\end{tabular}

1 "Proceedings Inst. Civil Engineers," vol. 94, p. 333. 
Effect of a Change in Head on the Discharge.

Since

$$
Q=K H^{\frac{3}{2}}, \therefore \frac{d Q}{d H}=1.5 K H^{\frac{1}{2}},
$$

$\therefore \quad \delta Q=1.5 K H^{\frac{3}{2}} \delta H$, and $\frac{\delta Q}{Q}=1.5 \frac{\delta H}{H}$, i.e., the proportional change in $Q$ is 1.5 times that in $H$.

Effect of Inclination of Weir Face.

As might be expected from its effect on the crest contraction, an up-stream inclination of the weir face (Fig. 67 a) reduces,

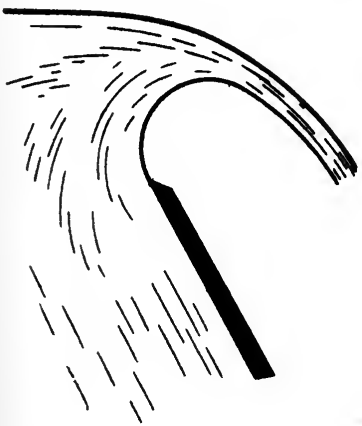

(a)

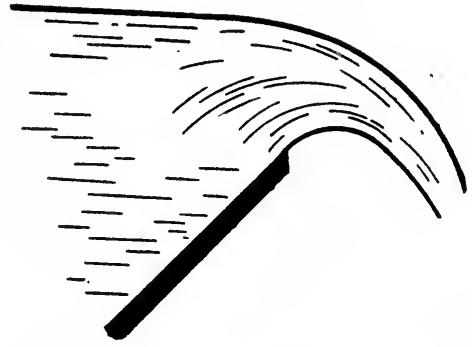

(b)

FIG. 67.

while a down-stream inclination (Fig. 67 b) increases, the discharge.

Bazin obtained the following comparative results with inclined weirs.

\begin{tabular}{|c|c|c|c|c|c|c|c|c|c|}
\hline \multirow{2}{*}{$\begin{array}{l}\begin{array}{c}\text { Direction of } \\
\text { inclination. }\end{array} \\
\begin{array}{l}\text { Slope: horizon- } \\
\text { tal distance } \\
\text { given first }\end{array}\end{array}$} & \multicolumn{3}{|c|}{ Up-stream. } & \multirow{2}{*}{$\begin{array}{c}\begin{array}{c}\text { Weir, } \\
\text { Vertical. }\end{array} \\
0 \\
0\end{array}$} & \multicolumn{5}{|c|}{ Down-stream. } \\
\hline & 1 to 1 & 2 to 3 & 1 to 3 & & 1 to 3 & 2 to 3 & 1 to 1 & 2 to 1 & 4 to 1 \\
\hline $\begin{array}{l}\text { Relative dis- } \\
\text { charge. }\end{array}$ & $\cdot 93$ & $\cdot 94$ & $\cdot 96$ & $1 \cdot 00$ & $1 \cdot 04$ & $1 \cdot 07$ & $1 \cdot 10$ & $1 \cdot 12$ & $1 \cdot 09$ \\
\hline
\end{tabular}

The maximum discharge was obtained with a down-stream inclination of 7 to 4 . 
Effect of a Sloping Apron on the Down-stream Side of a SharpCrested Weir.

If such a weir, having a vertical up-stream face and a sharpcrest, is fitted with a sloping apron on the down-stream side, the nappe is usually affected to an extent depending on the slope of the apron. Bazin, experimenting on such weirs having heights of 1.64 and 2.64 feet and with heads ranging from 3 to 1.5 feet, deduced the approximate formula $K=\frac{3 \cdot 85}{S^{\cdot 12}}$ for values of the slope $S$ between 1 and 12 .

\section{Effect of Rounding the Up-stream Edge.}

A slight rounding of the up-stream edge, by diminishing the contraction of section as indicated in Fig. 68 a, always causes an

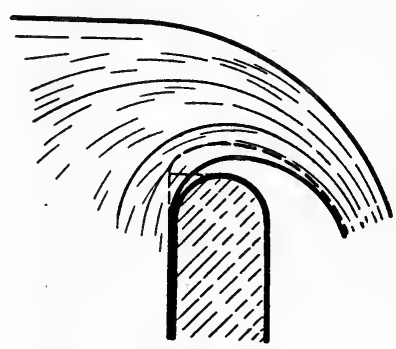

(a)

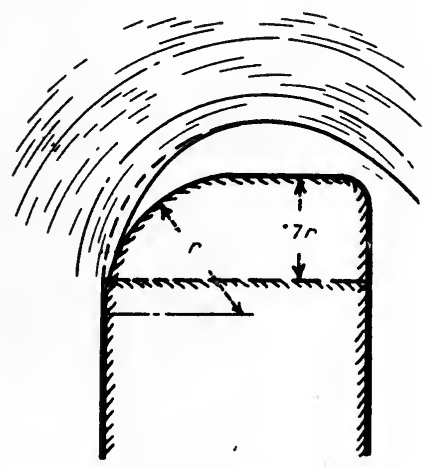

(b)

FiG. 68.

increased discharge. Messrs. Fteley and Stearns, from experiments with crest radii up to 1 inch, found that, so long as the nappe was fully aerated, the effect of the rounding was to increase the effective head by $h^{\prime}$, where $h^{\prime}=\cdot 7 r$. (Fig. 68 b.)

\section{Effect of Clinging Nappe.}

The experiments of Bazin showed very clearly the importance of taking into consideration the particular form assumed by the nappe. In general the nappe may either 
(1) Discharge freely into the air.

(2) Adhere to the top of the crest. (Fig. 73 a.)

(3) Adhere to the down-stream face of crest. (Fig. 69 a.)

(4) Adhere to both top and down-stream face. (Fig. 73 b.)

(5) Adhere to top and to sides of discharge channel, but remain detached from face. In this case a partial vacuum is produced underneath the nappe. (Fig. 69 b.)

Bazin gives the following as the relative

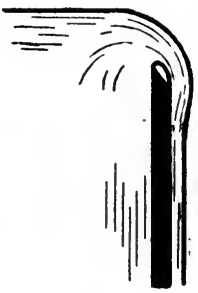

()

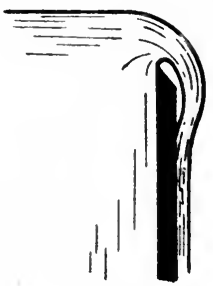

(b)

FIG. 69. discharges from a thin-edged weir $2 \cdot 46$ feet high and with a head of $\cdot 656$ feet under different nappe conditions.

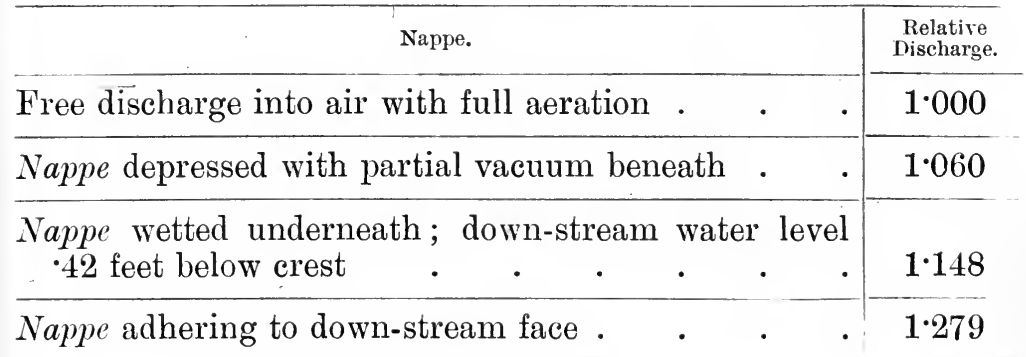

For steep down-stream slopes where the nappe tends to break free from the apron, the discharge is increased by the partial vacuum formed beneath the nappe. As this slope becomes flatter the conditions approach those for a hori-

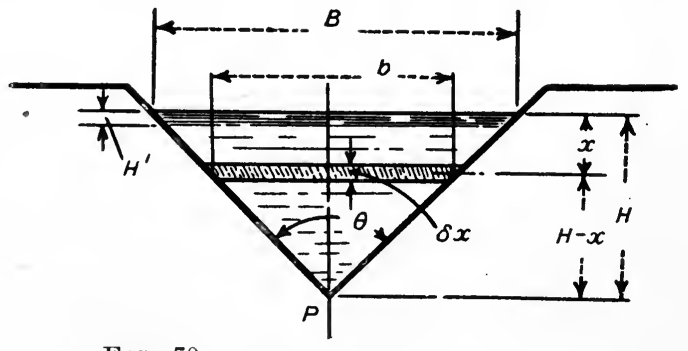

Fig. 70. zontal crest, while for slopes of $3: 1$ the discharge is approximately the same as for a thin-crested freely discharging weir. 
(2) Triangular Notch.-Let $H$ be the head of water above the vertex $P$ of the notch (Fig. 70), $H$ being measured to the level of the still water surface behind the notch. Let $B=$ breadth of notch at height $H$.

Then assuming the velocity of efflux at any depth $x$ to be given by $\sqrt{2 g x}$, a formula for the discharge can be deduced as for the rectangular notch.

Thus, the area of an element of the area at a depth $x$ and of width $\delta x$ is $b \delta x=B \cdot \frac{H-x}{H} \cdot \delta x$.

$\therefore$ Flow over this element

$$
\begin{aligned}
& =\hat{o} Q=B \sqrt{2 g} x \cdot \frac{H-x}{H} \cdot \delta x \text { c.f.s. } \\
\therefore \quad Q & =C^{\prime} B \sqrt{2 g} \int_{H^{\prime}}^{H}\left(1-\frac{x}{H}\right) x^{\frac{1}{2}} d x \\
& =C^{\prime} B \sqrt{2 g}\left[\frac{2}{3}\left\{H^{\frac{3}{2}}-H^{\prime \frac{3}{2}}\right\}-\frac{2}{5}\left\{H^{\frac{3}{2}}-\frac{H^{\prime \frac{5}{2}}}{H}\right\}\right] \\
& =C^{\prime} B \sqrt{2 g}\left[\frac{4}{15} H^{\frac{3}{2}}-H^{\prime \frac{3}{2}}\left\{\frac{2}{3}-\frac{2}{5} \frac{H^{\prime}}{H}\right\}\right]
\end{aligned}
$$

To get this into a form available for practical use we must write

$$
\begin{aligned}
& \left.C^{\prime}\left[\frac{4}{15} H^{\frac{3}{2}}-H^{\prime \frac{3}{2}}: \frac{2}{3}-\frac{2}{5} \frac{H^{\prime}}{H}\right\}\right]=\frac{4}{15} C H^{\frac{3}{2}} \\
& \text { So that } C=C^{\prime}\left\{1-\left(\frac{H^{\prime}}{H}\right)^{\frac{3}{2}}\left(2.5-1.5 \frac{H^{\prime}}{H}\right)\right\}
\end{aligned}
$$

Equation (2) then becomes

$$
Q=\frac{4}{15} C B \sqrt{2 g} H^{\frac{3}{2}}
$$

the formula obtained by integrating the expression (1) between the limits $H$ and 0 .

Putting $\frac{B}{2}=H \tan \frac{\theta}{2}$, where $\theta$ is the angle included between 
the sides of the notch, this becomes

$$
\begin{aligned}
Q & =\frac{8}{15} C \sqrt{2 g} \tan \frac{\theta}{2} H^{\frac{5}{2}} \\
& =4.28 C \tan \frac{\theta}{2} H^{\frac{5}{2}}
\end{aligned}
$$

The co-efficient $C$ here includes both variables $C^{\prime}$ and $H^{\prime}$. Since the ratio $\frac{B}{H}$ is constant for any one notch, it is to be expected that the value of $C$ with different heads will be more nearly constant than in the case of a rectangular notch. Experiments by Professor James Thomson ${ }^{1}$ indicate that this is so, the value increasing very slightly as the head diminishes. With a rightangled notch the variation was less than 1 per cent. under a range of heads from 2 inches to 7 inches, while with a notch having sides inclined at 2 horizontal to 1 vertical ( $\tan \frac{\theta}{2}=2$ ) the value of $C$ increased by about 2 per cent. as the head was reduced.

As the result of these experiments Professor Thomson estimated the mean value of $C$ for a right-angled notch as 593 , thus giving a discharge

$$
Q=2.536 H^{\frac{5}{2}} \text { cubic feet per second. }
$$

With a notch having side inclinations of 2 to $1\left(\tan \frac{\theta}{2}=2\right)$, the mean value of $C$ was found to be $\cdot 618$, making

$$
Q=5 \cdot 29 H^{\frac{5}{2}} \text { cubic feet per second, }
$$

while as the angle is still further increased $C$ appears to approach a limiting value $\cdot 620$.

With these wide-angled notches the value of $C$, however, fluctuates between much wider limits than in the case of the right-angled notch, and this renders the former type not so suitable for measuring purposes.

While suffering from the disadvantage that only small quantities of water are passed with a comparatively high head, the triangular has the advantage over the rectangular notch where the flow is very variable, that with either large or small heads it is equally

$$
1 \text { "British Association Report," 1861, p. } 351 .
$$


easy to take accurate measurements of the head, while with the rectangular notch with very low heads this is practically impossible. Also, the effect of the bottom of the approach channel in altering the value of $C$ is found to be negligible in the triangular notch.

On allowing for the velocity of approach, equation (4) becomes

$$
Q=\frac{8}{15} C \tan \frac{\theta}{2} \sqrt{2 g}\left\{(H+h)^{\frac{5}{2}}-h^{\frac{5}{2}}\right\} \text { cub. ft. per sec. }
$$

Here $h$ may be taken as $1 \cdot 4 \frac{v^{2}}{2 g}$, where $v=$ velocity of approach.

In using the notch, care should be taken that the sides are equally inclined to the vertical.

Variation of Discharge with Head.

Since

$$
\begin{aligned}
Q & =K H^{\frac{5}{2}} \\
\therefore \quad \frac{d Q}{d H} & =2.5 K H^{\frac{3}{2}} \\
\therefore \quad \frac{\delta Q}{Q} & =2.5 \frac{\delta H}{H}
\end{aligned}
$$

It follows that a small change in, or error in estimating $H$, produces $2 \cdot 5$ times the percentage change or error in $Q$.

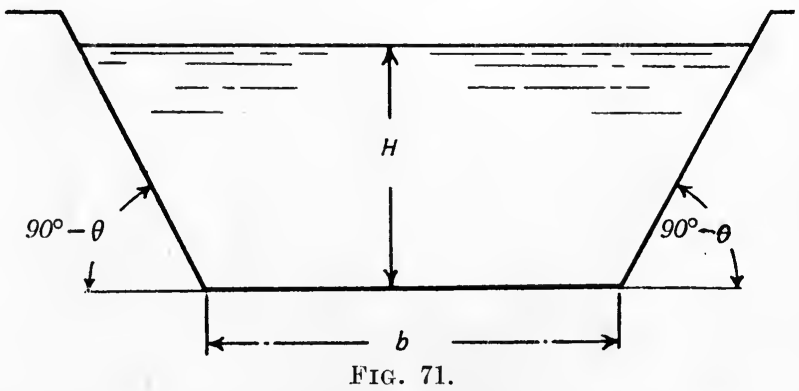

(3) Trapezoidal Notch (Fig. 71).-Let $b$ be the bottom breadth of the notch. Let $\theta$ be the inclination to the vertical, of the sides.

The notch then becomes equivalent to a rectangular notch of breadth $b$, together with a triangular notch having an angle 
$2 \theta$ between its inclined sides, and the discharge $Q$ due to a head $H$ is obtained by adding the values of $Q$, as calculated for two such notches.

$$
\therefore Q=\sqrt{2 g}\left\{\frac{2}{3} b C_{1} H^{\frac{3}{2}}+\frac{8}{15} C_{2} \tan \theta H^{\frac{5}{2}}\right\}
$$

Assuming $C_{1}=C_{2}=C$, this becomes

$$
Q=C \sqrt{2 g} H^{\frac{3}{2}}\left\{\frac{2}{3} b+\frac{8}{15} H \tan \theta\right\} .
$$

Francis's formula for a rectangular notch shows that the two end contractions reduce the effective breadth by $\cdot 2 H$, and the discharge by an amount

$$
\cdot 2 H \times \frac{2}{3} C \cdot \sqrt{2 g} \cdot H^{\frac{3}{2}}=\frac{2}{15} C \sqrt{2 g} \cdot H^{\frac{5}{2}} .
$$

If then the ends of the weir, instead of being vertical, are inclined outwards so that the added area counterbalances the increased contraction of section of the stream, the coefficient $K$ in the formula $Q=K b H^{\frac{3}{2}}$ should be independent of the head.

For this to be so we have

$$
\frac{2}{15} C \sqrt{2 g} \cdot H^{\frac{5}{2}}=\frac{8}{15} C \sqrt{2 g} \tan \theta H^{\frac{5}{2}},
$$

so that $\tan \theta=\frac{1}{4}$, or the sides are to be inclined outwards with a slope of 1 in 4 . This is termed a Cippoletti ${ }^{1}$ weir.

From his own experiments and those of Francis on heads from 3 to 24 inches, Cippoletti made $Q=3.367 b H^{\frac{3}{2}}$ cub. ft. per sec., the Francis velocity correction being used.

Experiments by Messrs. Flinn and Dyer ${ }^{2}$ on weirs having sill lengths of from 3 feet to 9 feet, and heads from 3 feet to 1.25 feet, gave, as the mean of 32 experiments, $K=3 \cdot 283$. In this formula, however, the velocity correction of Hamilton Smith (effective head $=H+1.4 h$ ) was used. Had the Francis correction been applied, this coefficient would have been in close accord with that of Cippoletti.

1 First described by C. Cippoletti. See "Giornale del Genies Civiles," 1886.

2 Trans. Am. Soc. C.E., vol. 32, 1894, pp. 9-33. 
Art. 53.-Submerged Weirs.

Where the water on the down-stream side of a weir rises above the level of the sill we get a submerged weir.

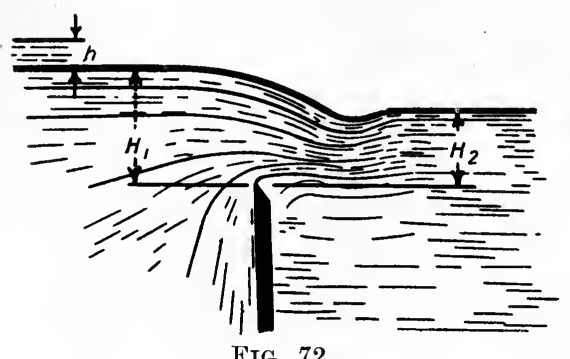

FIG. 72.

If $H_{1}$ and $H_{2}$ (Fig. 72) be the heights of the free surfaces above the sill of a rectangular weir, the flow over the upper part of the section, of a depth $H_{1}-H_{2}$, may be considered as a free discharge into air, and that over the lower part, of depth $H_{2}$,

as a discharge through a submerged orifice.

Thus for the upper portion $Q_{1}=\frac{2}{3} C_{1} b \sqrt{2 g}\left(H_{1}-H_{2}\right)^{\frac{3}{2}}$

$$
\text { " }, \quad \text { lower } \quad, \quad Q_{2}=C_{2} b \sqrt{2 g} H_{2}\left(H_{1}-H_{2}\right)^{\frac{1}{2}}
$$

If we assume $C_{1}=C_{2}=C$ we have for the total flow

$$
\begin{aligned}
Q & =C b \sqrt{2 g\left(H_{1}-H_{2}\right)}\left\{\frac{2}{3}\left(H_{1}-H_{2}\right)-H_{2}\right\} \\
& =C b \sqrt{2 g\left(H_{1}-H_{2}\right)}\left\{\frac{2}{3} H_{1}+\frac{1}{3} H_{2}\right\} .
\end{aligned}
$$

Taking into account the velocity of approach, we have

$$
\begin{aligned}
& Q_{1}=\frac{2}{3} C_{1} b \sqrt{2 g}\left\{\left(H_{1}-H_{2}+h\right)^{\frac{3}{2}}-h^{\frac{3}{2}}\right\} \\
& Q_{2}=C_{2} b \sqrt{2 g} H_{2}\left(H_{1}-H_{2}+h\right)^{\frac{1}{2}} \\
& \therefore \quad Q=C b \sqrt{2 g\left(H_{1}-H_{2}+h\right)}\left\{\frac{2}{3}\left(H_{1}-H_{2}+h\right)+H_{2}\right. \\
&-\frac{2}{3} h^{\frac{3}{2}} \vdots \\
&\left(H_{1}-H_{2}+h\right)^{\frac{1}{2}}
\end{aligned}
$$

As a very close approximation we have

$$
Q=C b \sqrt{2 g\left(H_{1}-H_{2}+h\right)}\left\{\frac{2}{3}\left(H_{1}+h\right)+\frac{1}{3} H_{2}\right\} .
$$


The value of $C$ varies with the ratio $\frac{H_{2}}{H_{1}+h}$, and can only be deduced by experiment.

The following mean values are obtained from the results of experiments by Francis, and by Messrs. Fteley and Stearns.

Francis.-Depth of water at sill $1-2 \cdot 3$ feet.

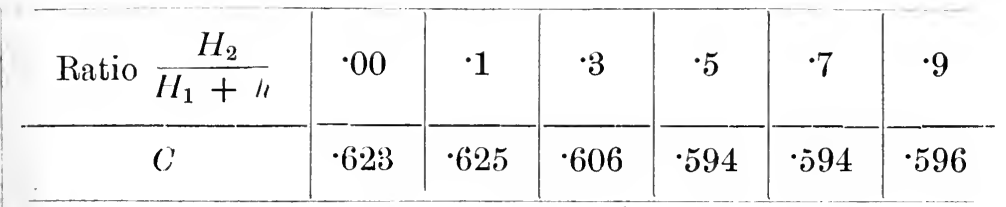

Messis. Fteley and Stearns.

\begin{tabular}{c|c|c|c|c|c}
\hline$H_{2}$ & $\cdot 1$ & $\cdot 3$ & 5 & $\cdot 7$ & $\cdot 9$ \\
\hline$H_{1}+h$ & & & & & \\
\hline$C$ & $\cdot 630$ & $\cdot 605$ & $\cdot 590$ & $\cdot 585$ & .595
\end{tabular}

The difficulty of obtaining accurate values of $H_{1}$ and $H_{2}$, combined with the fact that $\mathrm{H}_{2}$ is in a continual state of change owing to the undulatory motion of the surface, renders the submerged weir very unsatisfactory as a means of measuring the flow of water.

\section{Art. 54.-Broad-crested Weirs. (Fig. 73.)}

In general the nappe will clear the crest of a sharp-edged weir if this is of less width than $5 H$. For widths between $\cdot 5 H$ and $66 \mathrm{H}$ the condition is unstable, and any variation in the flow will cause the nappe to cling or to break free, with a corresponding variation in the discharge, while for widths greater than this the nappe clings to the crest in every case.

$B a z i n$, experimenting on weirs having crests ranging from $\cdot 164$ to 6.56 feet in width, found that where the nappe adheres to the top but not to the down-stream face of such a flat-crested weir, the discharge may be expressed by

$$
Q=K^{\prime} b^{\frac{3}{2}}, \text { where } K^{\prime}=K\left\{\cdot 70+\cdot 185 \frac{H}{w}\right\},
$$


$w$ being the width or thickness of the crest. Here $K$ is the coefficient for the corresponding thin-crested weir. This formula appears to give accurate results for values of $w$ up to 3 feet, but for wider crests, and for heads above 1.5 feet, gives rather low results.

Fteley and Stearns, using crest widths of from 2 to 10 inches, and heads from $\cdot 116$ to 894 feet, deduced the formula $Q=K^{\prime} b$ $(H+k)^{\frac{3}{2}}$, where $k=\cdot 2016 \sqrt{(\cdot 807 b-H)^{2}+\cdot 2146 b^{2}}-\cdot 1876 b$, and $K$ is the coefficient for a thin-crested weir.

A very extensive series of experiments were carried out in 1899 for the U.S. Board of Engineers on Deep Waterways, and again

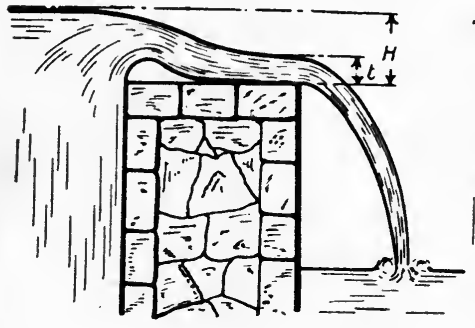

(a)

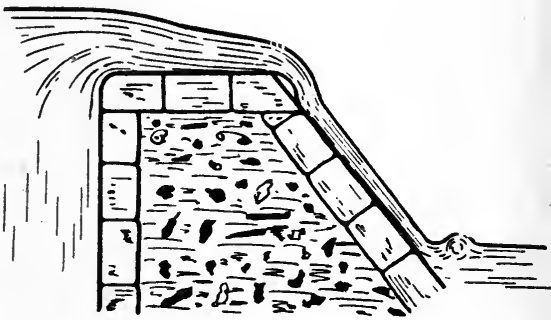

(b)

FIG. 73.

in 1903 for the U.S. Geological Survey, at Cornell University, on weirs with broad crests and weirs with ogee sections. ${ }^{1}$

The chief deductions to be drawn from these experiments are as follow :-

(1) For depths less than from 3 to $\cdot 5$ feet the nappe is very unstable owing to varying aeration or adhesion to the downstream weir face.

(2) For heads from 5 up to 1 or 2 feet for very broad weirs, or to the point of detachment in narrow weirs, the coefficient is somewhat variable. For broad weirs the variation of $K^{\prime}$ between the depths indicated is however narrow, $K^{\prime}$ being between $2 \cdot 73$ and $2 \cdot 62$.

1 For a full discussion of these results, as well as of those of Bazin, see "Water Supply and Irrigation," Paper No. 200, U.S. Geological Survey ; "Weir Experiments, Coefficients and Formulas," by R. E. Horton. 
(3) Should the nappe become detached, the coefficient becomes practically identical with that for a thin-crested weir.

(4) On broad weirs, for depths exceeding 1 to 2 feet up to the limit of the experiments ( 5 feet), the coefficient is sensibly uniform for all depths. If anything, $K^{\prime}$ increases with $H$.

With weirs from 5 to 16 feet wide, however, $K^{\prime}$ lies between 2.62 and $2 \cdot 64$, and is practically independent of either $H$ or $w$. Probably $2 \cdot 64$ is correct for all weirs with horizontal crests more than 3 feet wide, under heads exceeding 2 feet, and for weirs of 5 feet or more crest-width with considerably lower heads, with but small error.

A more rational formula for flow over a weir of this type may be deduced if we assume the crest to be so wide that the filaments form a parallel stream of thickness $t$ (Fig. 73 a) before leaving the crest, and that in this stream the pressure at any point is that statically corresponding to its depth. We then have the velocity at the surface and at every point in this stream = $\sqrt{2 g(H-t)}$, while $Q=b t \sqrt{2 g(H-t)}$.

Although $t$ cannot be determined from theoretical considerations, we may determine its theoretical value for maximum flow by equating $\frac{d Q}{d t}$ to zero. This gives $t=\frac{2}{3} H$, and substituting this value, we get $Q=\cdot 385 b \sqrt{2 g} H^{\frac{3}{2}}$ as the maximum possible discharge. Writing this in the usual form $Q=\frac{2}{3} C b \sqrt{2 g} H^{\frac{3}{2}}$, we get $C^{\prime}=\cdot 578$, and $K^{\prime}=3 \cdot 087$.

The true discharge is less than this because of frictional losses and because $t$ is usually less than $66 \mathrm{H}$. The following results were obtained in the United States Deep Waterways experiments at Cornell in 1899.

Weir crest horizontal -6.56 feet wide -4.56 feet high.

(1) Sharp up-stream edge.

\begin{tabular}{c|c|c|c|c|c|c}
\hline$H \mathrm{ft}$. & $\cdot 90$ & $1 \cdot 15$ & $1 \cdot 80$ & $2 \cdot 60$ & $3 \cdot 55$ & $5 \cdot 15$ \\
\hline$t \div H$ & $\cdot 58$ & $\cdot 59$ & $\cdot 63$ & $\cdot 67$ & $\cdot 71$ & $\cdot 61$ \\
\hline
\end{tabular}


(2) Crest edge rounded to 4 inches radius.

\begin{tabular}{r|r|r|r|r|r|r}
$H$ ft. & $1 \cdot 00$ & $1 \cdot 32$ & $1 \cdot 98$ & $2 \cdot 85$ & $3 \cdot 90$ & $4 \cdot 65$ \\
\hline$t \div H$ & $\cdot 50$ & $\cdot 50$ & $\cdot 50$ & $\cdot 60$ & $\cdot 64$ & $\cdot 61$ \\
\hline
\end{tabular}

A sloping crest increases the discharge, as does any rounding of the up-stream corner. The effect of this rounding is not so pronounced as with a thin-crested weir, diminishing as the crest width increases and also as the head increases. Fteley and Stearns, experimenting on a crest 4 inches wide, with radii of one-fourth, one-half, and one inch respectively, found the effective head to be increased in the ratio $\left\{1+\frac{\cdot 41 R}{H}\right\}$, this correction being applicable for heads of not less than 17 and 26 feet on weirs with radii of one-fourth and one-half inch respectively.

Bazin's experiments on crests having widths of 2.62 and 6.56 feet, heads from 25 to 1.50 feet, with an up-stream crest radius of 4 inches, showed a mean increased discharge of 13.5 per cent. with the narrower and 10 per cent. with the broader crest, while the United States Deep Waterways experiments on a weir 22 feet wide, with $6: 1$ slope on each face, showed an average increase of 2 per cent. with an up-stream edge rounded to a radius of 4 inches. The condition of the crest as regards roughness is found not to influence the discharge by more than about 2 per cent.

\section{Dams with Ogee Cross Section.}

From experiments on weirs of ogee cross section for the United States Geological Survey (ante loq.), the formula

$$
K^{\prime}=\{3 \cdot 62-\cdot 16(s-1)\} H^{\frac{1}{20}}
$$

was found to give the coefficient with reasonable accuracy. Here $s$ is the slope of the up-stream face.

Example: $s=2: 1 ; H=4 \cdot 0 ; \therefore K^{\prime}=3 \cdot 46 \times 4^{\frac{1}{20}} ; \log .4^{\frac{1}{20}}=\cdot 0301 ; \therefore K^{\prime}=$ $3 \cdot 46 \times 1 \cdot 0716=3 \cdot 70$. Experiments gave $K^{\prime}=3 \cdot 74$.

This formula holds for weirs with an up-stream slope more than from 3 to 4.5 feet broad, and with a down-stream radius 
(above from 2 to 3 feet) great enough to retain the nappe in contact, and yet not so large as to simulate a broad flat crest.

The effect of a raising of the down-stream level to that of the weir sill is much less in the case of a wide-crested weir than with one having a narrow crest. In the former case the effect is almost inappreciable until the down-stream level rises to that of the sill itself.

\section{Art. 55.-Rise in Surface Level Produced by a Weir.}

A dam or weir is usually placed across a stream with the idea of raising the surface level and increasing the depth of water up stream. The distance to which this effect may be felt is sometimes considerable (see Art. 86), and can only be determined when the rise in level in the neighbourhood of the dam is known. This may be obtained if the discharge of the stream before the introduction of the dam is known (since this discharge will be unaffected by the presence of the dam) by equating this, in cubic feet per second, to the flow over a dam of the required length $b$ and under a head $H .^{1}$ This determines $H$ and therefore the total rise in level when the height of dam is given. Thus if $h=$ mean depth of water before the introduction of the dam, and if $h_{d}=$ height of dam,

we have

$$
\begin{aligned}
Q & =\frac{2}{3} C b \sqrt{2 g} H^{\frac{3}{2}} \\
\therefore \quad H & =\left\{\frac{1 \cdot 5 Q}{C b \sqrt{2 g}}\right\}^{\frac{2}{3}} \text { feet, }
\end{aligned}
$$

1 Where the discharge is not known, the mean velocity of flow may be approximately estimated from equation (5) of Art. 85, viz., $v=\sqrt{\frac{2 g i h}{f}}$ or $r=C^{1} \sqrt{\hbar i}$, where $f$ and $C^{1}$ are coefficients depending on the condition of the bed of the stream (see Art. 83).

Then $Q=A r=b h r=b C^{1} i^{\frac{1}{2}} h^{\frac{3}{2}}$, and the rise in surface level becomes

$$
\begin{aligned}
& h_{d}-h+\left\{1.5 \frac{C^{1}}{C} \sqrt{\frac{i}{2 g}}\right\}^{\frac{2}{3}} h \text { feet } \\
= & h_{d}-h\left\{1-\left(1.5 \frac{C^{1}}{O} \sqrt{\frac{i}{2 g}}\right)^{\frac{2}{3}}\right\} \text { feet. }
\end{aligned}
$$

This velocity may also be detcrmined by current meter or float observations. See Art. 95. 
$\begin{aligned}\left.\therefore \quad \begin{array}{l}\text { Rise in surface level } \\ \text { produced by the dam }\end{array}\right\} & =h_{d}+H-h \\ & =h_{d}-h+\left\{\frac{1.5 Q}{C b \sqrt{2} g}\right\}^{\frac{2}{3}} \text { feet. (1) }\end{aligned}$

The same reasoning applies to the case of a submerged weir thrown across a stream, or to the rise in level produced by the erection of bridge piers in the stream (Art. 88).

Art. 56.-Use of the Weir as a Water Measuring Appliance.

The standard sharp-edged weir having a free discharge, or, for small quantities, the right-angled triangular notch, are the only types for which the coefficients have been determined with sufficient accuracy to admit of their use for accurate measurement of flow without previous calibration.

For accurate measurement we must have :-

1. Sharp-edged weir sill, fixed so as to be incapable of vibration, having its face vertical and perpendicular to the direction of the stream, and, if rectangular, having its sill horizontal.

2. Clear discharge into air, no adherence of vein to weir face.

3. Weir long in proportion to its depth, i.e., $b>3 H$.

4. $H$ small in comparison with the depth of the approach channel, and sectional area of vein $(b H)$ not greater than $\frac{1}{6}$ that of this channel.

5. Suitable channel of approach. This should be as long and of as uniform section as possible so as to allow of the motion becoming steady before reaching the weir. The length should, if possible, exceed $30 \mathrm{H}$, this ratio being increased where the length of weir is largely in excess of $3 H$. In the Cavour Canal an approach channel 66 feet in length is found to give good results with a head of 19 inches, giving a length $=42 \mathrm{H}$. Where such a length is impossible, one or more perforated diaphragms should be placed across the stream so as to steady the motion as far as possible.

6. Accurate determination of the head $H$. To measure $H$, water should if possible be run off by an auxiliary channel until exactly level with the notch sill. This level may be determined with 
great accuracy by observing the reflection at the surface in the immediate neighbourhood of the sill, since the absence of any curvature of the surface at this point, indicated as it is by nondistortion of reflected objects, shows that the correct level has been obtained. This level may then be read off, either on a graduated staff fixed vertically in the bed of the stream some 6 or 7 feet above the weir, or on a hook gauge (Fig. 128), the point of which is adjusted until exactly in the surface.

By levelling a straight-edge resting with one end on the sill and the other on the point of the hook gauge, the latter may be adjusted to the level of the sill with an accuracy which is probably greater than with the preceding method.

When the weir is discharging steadily, the head can be determined, either by direct reading of the graduated staff, or by taking the reading on the hook gauge when its point is again adjusted so as to be in the surface. Care should be taken when using the graduated staff that allowance is made for the increase in height over the up-stream face of the staff, and the decrease over the down-stream face, due to the piling up of the water which occurs at a solid obstacle. This effect will be more pronounced in a rapidly moving stream, and here the hook gauge is the only suitable appliance. With this, when fitted with adjusting screw and vernier, results accurate to $\frac{1}{50}$ of an inch may be easily obtained, and with practice the possible error may be reduced to about $\frac{1}{2} 0 \overline{0}$ of an inch. Note should always be made of the effect of capillary action in raising the apparent height of the surface at the hook.

Where possible, it is preferable to take gauge readings in a pit from 18 inches to 2 feet square, in communication with the main stream through a pipe a few inches in diameter, which opens out flush with the bed of the approach channel, and is perpendicular to the direction of flow. 'This was the method adopted by Bazin, the pit being situated about 16.5 feet above the weir.

Where it is impossible to stop flow past the weir, and where the depth is large, a vertical staff fixed at the weir face may be graduated to give heights above the sill in the neighbourhood of the surface. These heights may then be transferred by means of straight-edge and level to the measuring staff, or may be used

H.A. 
to give the datum level to which to adjust the zero reading of the hook gauge.

In selecting a formula for use in any particular case, it should be remembered that that of Francis gives accurate results for weirs with perfect bottom contractions and with heads above 6 inches. The formulae of Smith, Fteley and Stearns, and Bazin are better for very small heads, or where the bottom contraction is imperfect, this element tenaing to decrease the discharge, being included in the larger velocity-of-approach correction. Under such circumstances Bazin's formula is probably most reliable.

Although in expert hands the method of measurement by weirs will give results which may be relied upon to be correct within about 2.0 per cent., this degree of exactitude is not to be expected with any but the most careful measurements and consideration of the special conditions of each case.

\section{Art. 57.-Tine of Enptying a Vessel through a Large}

\section{Orifice.}

If the orifice be situated in the horizontal base of the vessel, the formulae relating to the time of discharge are the same as those for a small orifice (p. 121), except that now the effect of the velocity of approch is to be taken into account. Thus if $a_{c}=$ area of vena contracta and $A$ that of the vessel we have the velocity of efflux corresponding to a head $H$, given by

$$
\begin{aligned}
& v=C_{n} \sqrt{\frac{2 g H}{1-\frac{a_{c}{ }^{2}}{A^{2}}}} \\
& \therefore \quad-\frac{d H}{d t}=C_{v} \frac{a_{c}}{A} \sqrt{\frac{2 y H}{1-\frac{a_{c}^{2}}{A^{2}}}} \\
& \therefore \quad \frac{C_{v} a_{c} \sqrt{2} ! !}{A \sqrt{1-\frac{a_{c}}{A^{2}}}} \cdot d t=-\frac{d H}{\sqrt{ } H}
\end{aligned}
$$

And on integrating we have, if $t=t_{2}-t_{1}$, the time necessary to lower the surface through the distance $H_{1}-H_{2}$, 


$$
\begin{aligned}
t & =\frac{2 \sqrt{A^{2}-a_{c}^{2}}}{C_{v} a_{c} \sqrt{2 g}}\left\{H_{1}^{\frac{1}{2}}-H_{2}^{\frac{1}{2}}\right\} \\
& =\frac{2 \sqrt{A^{2}-a_{c}^{2}}}{a C \sqrt{2 !}}\left\{H_{1}^{\frac{1}{2}}-H_{2}^{\frac{1}{2}}\right\}
\end{aligned}
$$

where $C=$ coefficient of contraction for the orifice.

With an orifice in the vertical side of a vessel the effect of the variation of velocity at different depths in the orifice must be considered.

'Thus with a large rectangular orifice of depth $d$, the rate of discharge at the instant when the head of water above the upper edge is $H$ feet, is given by

$$
\begin{aligned}
() & =\frac{2}{3} C b \sqrt{2 g}\left[(H+d)^{\frac{3}{2}}-H^{\frac{3}{2}}\right] \text { cubic feet per second } \\
& =5 \cdot 76 C b\left[(H+d)^{\frac{3}{2}}-H^{\frac{3}{2}}\right] \text { cubic feet per second, }
\end{aligned}
$$

and the velocity of fall of the surface $\left(-\frac{d H}{d t}\right)$ is therefore equal to

$$
\frac{Q}{A}=\frac{5 \cdot 76 C b}{A}\left[(H+d)^{\frac{3}{2}}-H^{\frac{3}{2}}\right] \text { feet per second. }
$$

Thus equation (1) above becomes

$$
-\frac{d H}{d t}=\frac{5 \cdot 76 C b}{A}\left[(H+d)^{\frac{3}{2}}-H^{\frac{3}{2}}\right]
$$

and on integrating this between the required limits, the time occupied in lowering the surface through any required distance may be found.

Time of lowering the Level in a Reservoir through a Rectangular Notch.-With the usual notation the volume discharged per second with a head $H$ behind the notch is given by

$$
Q=K b H^{\frac{3}{2}} \text { cubic feet. }
$$

$\therefore$ At this instant we have the velocity of the free surface in the reservoir given by

$$
-\frac{d H}{d t} \doteq \frac{K b H^{\frac{3}{2}}}{A}
$$


Integrating this, the time $\left(t_{2}-t_{1}\right)$ seconds, to lower the level through a distance $H_{1}-H_{2}$ feet, is given by

$$
t_{2}-t_{1}=t=\frac{2 A}{\mathrm{~K} b}\left\{\frac{1}{H_{2}^{\frac{1}{2}}}-\frac{1}{H_{1}^{\frac{1}{2}}}\right\} \text { seconds. }
$$

\section{Examples.}

(1) A lock, 1,000 square feet area, is filled through a submerged orifice 3 feet long by 2 feet deep. The depth of water above the centre of this is initially 19 feet on the outside and 7 feet on the inside. Assuming a cotfficient of discharge of $\cdot 61$, determine the time occupied in filling the lock if the outside level remains constant.

\section{Answer. 236 seconds.}

(2) Assuming the level on the lower side of the above lock to remain constant, determine the time of emptying the lock, the sluice being the same size as on the entry side.

\section{Answer. 236 seconds.}

(3) Using Francis' formula, determine the discharge over a rectangular notch 36 inches long, and with heads of 3,6 , and 12 inches.

$\left.\begin{array}{l}\text { (a) With no side contractions } \\ \text { (b) , one , , , , , two , } \\ \text { (c) , tw }\end{array}\right\}$

Answer. \begin{tabular}{c|c|c|c}
\hline & \multicolumn{3}{|c}{ Head. } \\
\cline { 2 - 4 } & 3 inches. & 6 inches. & 12 inches. \\
\hline$a$ & $1 \cdot 25$ c.f.s. & 3.53 c.f.s. & $10 \cdot 0$ c.f.s. \\
\hline$b$ & $1 \cdot 24$ c.f.s. & $3 \cdot 47$ c.f.s. & $9 \cdot 66$ c.f.s. \\
\hline$c$ & 1.23 c.f.s. & $3 \cdot 41$ c.f.s. & $9 \cdot 33$ c.f.s. \\
\hline
\end{tabular}

(4) A submerged weir, 10 feet long, has a depth of water on the up-stream side of 17 inches, on the down-stream side of 9 inches. The velocity of approach $=1.96$ feet per second. 
Assuming the head equivalent to this velocity of approach to be given by $h=1.4 \frac{v^{2}}{2 g}$, determine the discharge in cubic feet per minute. 'Assume $c={ }^{5} 592$.

Answer. 3,090 cubic feet per minute.

(5) Show that in a triangular right-angled notch, discharging from a tank of sectional area $A$ square feet, the time of lowering the surface level from $H_{1}$ feet to $H_{2}$ feet above the vertex of the notch is given by

$$
t=\frac{1 \cdot 25 \mathrm{~A}}{C \sqrt{2 g}}\left\{\frac{1}{H_{2}^{\frac{3}{2}}}-\frac{1}{H_{1}^{\frac{3}{2}}}\right\} \text { seconds, }
$$

and taking $C={ }^{5} 593$, determine the time of lowering the surface level of a tank of 500 square feet sectional area from a depth of 1.5 feet to 1.0 feet above the vertex of the notch.

Answer. $t=60$ seconds.

(6) Find the time required to empty a swimming bath through a flat grating in the bottom of the deep end.

$\begin{aligned} \text { Depth of water at deep end } & =6 \text { feet. } \\ \text {,, shallow end } & =3 \text { feet. } \\ \text { Length of bath } & =80 \text { feet. } \\ \text { Breadth } & =30 \text { feet. }\end{aligned}$

Area of grating $=2$ square feet. Coefficient of discharge $\cdot 65$. Answer. 596 seconds.

(7) Two cylindrical tanks $A$ (5 feet diameter) and $B$ (10 feet diameter) are connected by a short pipe 4 inches diameter with bell-mouth inlet. At the beginning the level in $A$ is 10 feet and in $B$ is 1 foot above the centre line of the pipe. In what time will the surface levels be the same?

Answer. 138 seconds.

(8) A circular orifice 1 square inch in area is made in the vertical side of a large tank. If the jet fall vertically through $1 \frac{2}{3}$ feet while moving horizontally through 5 feet, at the same time discharging 16 gallons per minute, determine the horizontal force on the tank.

Answer. $\quad 1 \cdot 286 \mathrm{lbs}$. 
(9) If the tank of the preceding example is suspended from knife edges 5 feet alove the level of the orifice, and if, when the head of water in the tank is 4 feet, the discharge is $251.5 \mathrm{lbs}$. per minute, while a weight of 10.24 lbs., with a horizontal leverage of 1 foot is required to keep the tank vertical, determine the coefficients of velocity, contraction, and discharge.

Answer. $C_{v}=\cdot 98 ; C_{c}=\cdot 64 ; C_{d}=\cdot 627$.

(10) A boat, having jet propulsion, moves at 10 miles per hour. The water leaves the nozzles with a relative velocity of 40 feet per second. If $1,500 \mathrm{lbs}$. per second are passed through the pumps, determine the propelling force on the vessel.

Answer. 1,180 lbs. 


\section{CHAPTEP VI.}

Fluid Friction-Froude's Experiments-Resistance of Ships-Propulsion of ShipsThe Paddle Wheel-The Screw Propeller-Power necessary for Propulsion.

\section{Art. 58.-Fluid Friction.}

Whenever a liquid flows over a solid surface, or when a submerged plane moves in the direction of its length through a liquid, a resistance to motion is experienced. This is commonly termed fluid friction, and should not be confused with the wavemaking resistance which is experienced owing to the formation of surface waves, when a partially submerged body is in motion.

Though initially due to viscosity, the laws governing fluid friction are usually very different from those of simple viscous resistance, because of the fact that except at extremely low speeds the motion of the fluid becomes unsteady, eddies are formed, and the energy absorbed in fluid friction now also includes and chiefly consists of the energy of formation of these eddies. This energy is then finally absorbed in overcoming the viscous resistance of the fluid at points remote from the surface at which the eddies are generated.

As previously indicated, the laws of fluid friction for steady and unsteady motion are widely different.

With Steady Motion-Stream-line Motion :-

(1) The frictional resistance is directly proportional to the relocity.

(2) Is independent of the pressure in the fluid (probably not quite accurately so for high pressures).

(3) Is directly proportional to the area of the wetted surface if this is large, i.e., if the resistance is not sensibly affected by the edges of the surface.

(4) Is independent of the nature of the wetted surface. 
(5) Is directly proportional to the viscosity of the fluid, and so varies greatly with temperature.

These laws may be most easily verified by experiments on the flow of water through capillary tubes.

With Unsteady or Eddy Motion :-

(1) The frictional resistance varies with a higher power of the velocity than the first, and is usually approximately proportional to $V^{2}$.

(2) Is independent (within wide limits) of the pressure in the fluid.

(3) Where a submerged plane moves through still water, the resistance is not proportional to the area of the surface, but, per unit area of the surface, decreases as the length of the latter increases, and approaches a lower limiting value. In the case of flow through a pipe, the length is usually such as to allow of this limiting constant value being attained, so that here the frictional resistance does become practically proportional to the area of the wetted surface.

(4) Varies with the nature of the wetted surface.

(5) Varies only slightly with temperature, but is directly proportional to the density of the fluid.

By far the most important series of experiments to determine the resistance to the motion of submerged planes, are those carried out in 1872 by Mr. Froude, at Torquay. Here a series of flat boards, having differently prepared surfaces, were held vertically and suspended from a carriage which was driven at an uniform speed, and were thus towed endwise through the still water in a large basin. The carriage was fitted with a dynamometer and automatically recorded the velocity and resistance of the board. These boards were $\frac{3}{16}$ inch wide, 19 inches deep, and varied in length from 1 to 50 , feet. The top edge was submerged to a depth of $1 \frac{1}{2}$ inches, and the boards were fitted with a cutwater, the resistance to this being determined separately. In these experiments Mr. Froude determined that-

(1) The resistance varies greatly with the condition of the surface, the resistance for boards 50 feet long at a velocity of 10 feet per secord being with a coating of 
Varnish or smooth paint of such composition)

as is found on the bottom of iron ships

Tinfoil

Fine sand .

Calico

Sand of medium coarseness

250 lbs. per sq. ft.

(2) The resistance is proportional to $v^{n}$, where

$n\left\{\begin{array}{l}\text { (a) depends on the surface, } \\ \text { (b) decreases, up to a certain limit, with an increase in } \\ \quad \text { length, } \\ (c) \text { is independent of the velocity. }\end{array}\right.$

(3) The total resistance increases with the length, though the resistance per square foot decreases as the length increases.

Writing $:$ Resistance $=f S v^{n}$, where $S=$ area of surface, it was found that

(a) depends on the surface.

(b) decreases with an increase in length, becoming approximately constant when the length is large.

(c) is independent of the pressure.

(d) is proportional to the density of the fluid and diminishes very slightly as the temperature increases.

The following is a short resume of Mr. Froude's results, ${ }^{1}$ these particular experiments being carried out at a velocity of 10 feet per second.

The fact of the resistance per square foot over the aft part of the surface being less than at a point nearer the prow, may be explained as follows. ${ }^{1}$ The first portion of the surface, in passing through the water experiences resistance and communicates motion, in its own direction, to the water. 'The succeeding portion of the surface is then in contact with a body of water having a smaller relative velocity, and hence producing a smaller resistance per unit area, while it would appear that the velocity of the accompanying current increases until at some point in the surface a balance is obtained between the amount of energy given to the accompanying stream per second, and the energy dissipated by eddy formation in the surrounding

1 From the British Association Report, 1874." 


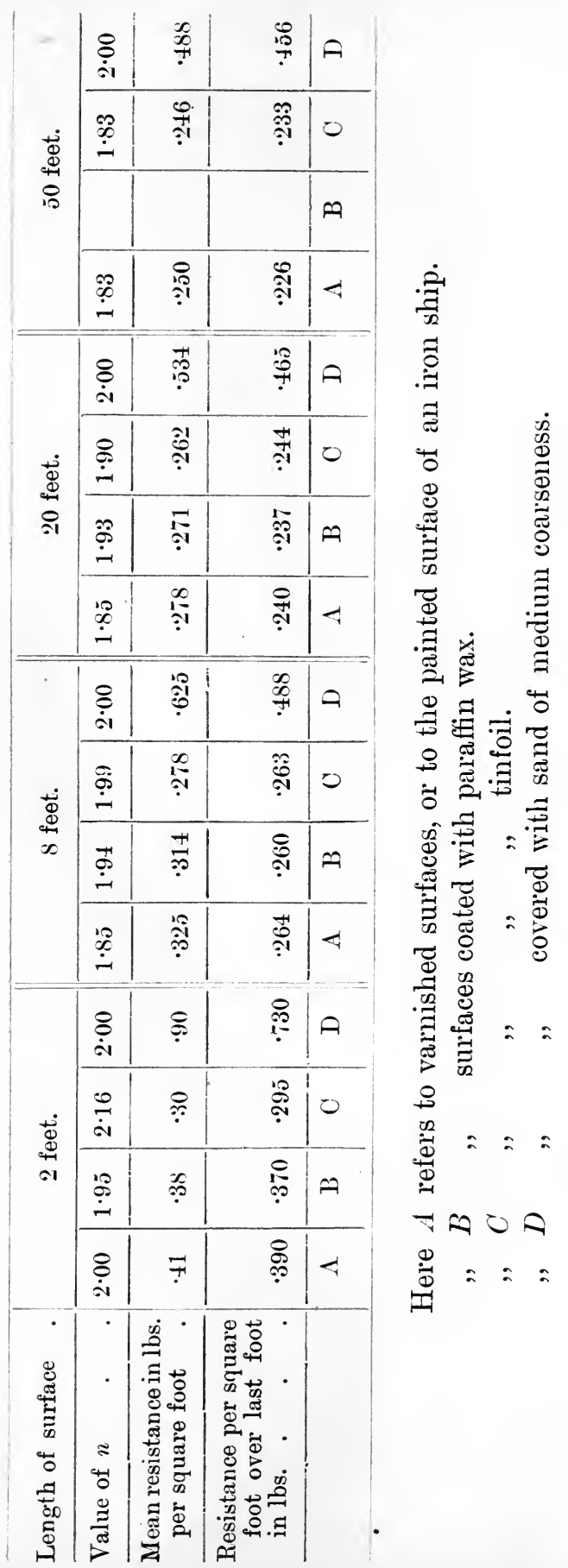


fluid and in producing motion of a greater volume of this water against viscous resistances. After this point is reached,

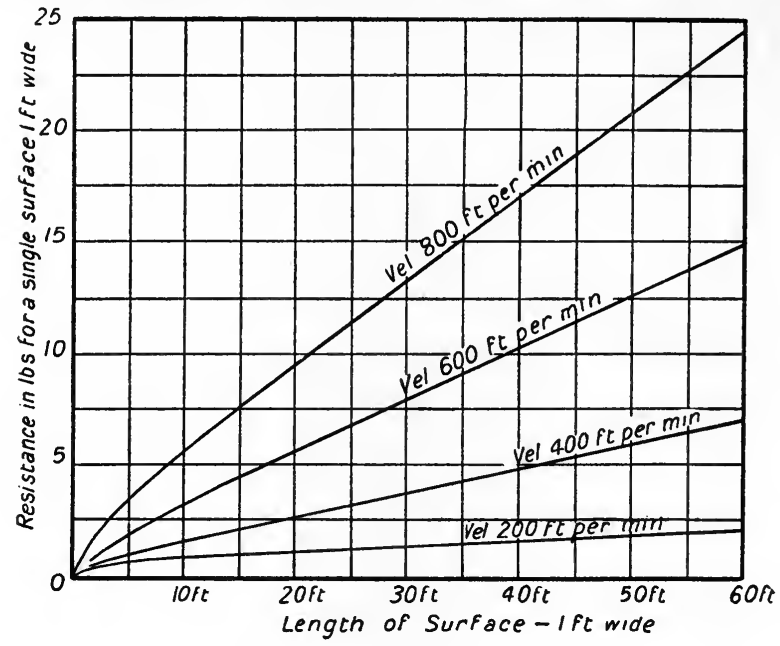

Fig. $7 t$.

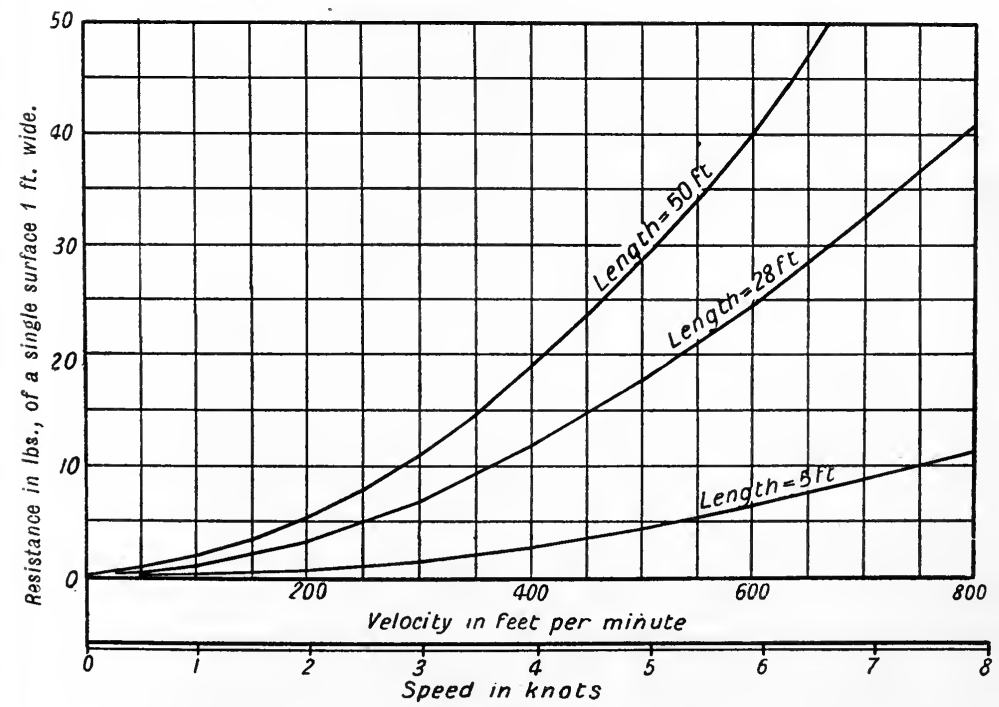

FIG. 75 .

the velocity of the accompanying current and the resistance per square foot of surface remain approximately constant. It 
will be noted that the mean resistance per square foot of area diminishes very slightly for lengths above 50 feet.

Curves (Figs. 74 and 75) have been prepared from the results of these experiments, and show respectively the variation of resistance with length at constant speed, and with speed for a given length, for a varnished or painted iron surface. In Fig. 76 values of $f$, for a painted iron surface, in the formula, Resistance $=f S v^{n}$, have been plotted from the experimental results for lengths up to 50 feet, and the curve thus obtained has been produced to give approximate values for lengths up to 330 feet.

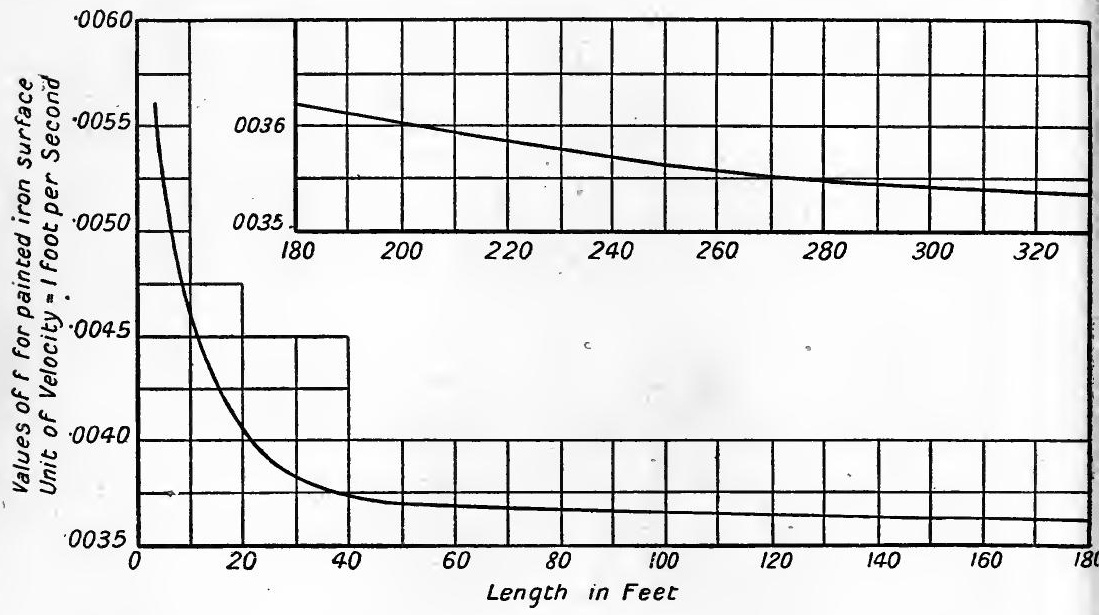

HII. 76.

The results may thus be extended for application to the determination of the frictional resistance of vessels of great length. For ships, the value of $n$ may be taken as $1 \cdot 83$, and the following table indicates results obtained by exterpolation from the above curve.

\begin{tabular}{|c|c|c|c|c|c|c|}
\hline \multicolumn{2}{|c|}{ Length in feet } & . & $\tilde{50}$ & 100 & 200 & 300 \\
\hline \multirow{2}{*}{$\begin{array}{c}\text { Values } \\
\text { of } f\end{array}$} & \multicolumn{2}{|c|}{$\left(\begin{array}{l}\text { With the unit of velocity }= \\
1 \text { foot per sec. }\end{array}\right.$} & $\cdot 00371$ & $\cdot 00361$ & $\cdot 00356$ & $\cdot 00353$ \\
\hline & \multicolumn{2}{|c|}{$\mid \begin{array}{c}\text { With the unit of velocity }= \\
1 \text { knot }=1 \cdot 69 \text { foot per sec. }\end{array}$} & $\cdot 00968$ & $\cdot 00941$ & $\cdot 00929$ & $\cdot 00922$ \\
\hline
\end{tabular}


The following values of $f$ are given by J. Hamilton. ${ }^{1}$

\begin{tabular}{c|c|c|c}
\hline & & \multicolumn{2}{|c}{ Copper Sheathing. } \\
\cline { 2 - 3 } Length in feet. & $\begin{array}{c}\text { Iron bottom } \\
\text { (painted). }\end{array}$ & Smooth. & Rough. \\
\cline { 2 - 3 } 200 & $\cdot 00944$ & $\cdot 00943$ & $\cdot 01170$ \\
300 & $\cdot 00923$ & $\cdot 00930$ & $\cdot 01152$ \\
400 & $\cdot 00910$ & $\cdot 00926$ & $\cdot 01140$ \\
500 & $\cdot 00904$ & $\cdot 00926$ & $\cdot 01136$ \\
\hline
\end{tabular}

Art. 59.-Resistance of Ships.

The resistance to the motion of a ship is due mainly to surface friction, but also to the formation of surface waves and of eddies (chiefly at the stern). Mr. Froude found that although the velocities of gliding vary largely at different points of the hull of a ship, no sensible error is involved if the frictional resistance be calculated on the assumption that the wetted surface is equivalent to that of a plane of equal area and length in the direction of motion, and moving at the same speed.

The variation in speed of the particles at different points of the surface may thus be neglected, and the frictional resistance for the ship calculated from an extension of the foregoing results. With a frictionless fluid, the particles displaced laterally by the prow would move without any frictional losses and without any tendency to eddy formation over surrounding particles, and would immediately return to exert a pressure on the stern equivalent to that on the bows. Thus the only resistance to uniform motion through such a fluid would be that due to wave formation, and, with a deeply immersed body, would be zero. A geometrical construction for the stream lines in a perifect fluid has been laid down by Professor Rankine, while Professor HeleShaw has shown these and has verified the accuracy of this construction in the case of a viscous fluid flowing past an obstacle, the motion taking place between parallel glass plates at a very small distance apart. ${ }^{2}$ Here the motion is governed almost

1 "Proc. Inst. Naval Architects," March, 1898.

2 British Association Report, 1898 ; also "Transactions Inst. Naval Architects," 1898 , vol. 85 . 
entirely by viscosity, all eddy motion is prevented, and, as proved by Professor Sir G. G. Stokes, ${ }^{1}$ the effect as regards stream-line formation is the same as in the case of a perfect fluid.

Wave production is a very complicated phenomenon, and depends in magnitude and in form largely on the form and speed of the boat. 'In general it may be taken that the motion is accompanied by the formation of bow and stern waves, and by a fall of the water surface amidships (Fig. 77). The fact that outward stream-line flow must commence in front of the bows, and that the head necessary to produce this can only be obtained by a relative elevation of the surface level, accounts for the bow wave, while the reduction in

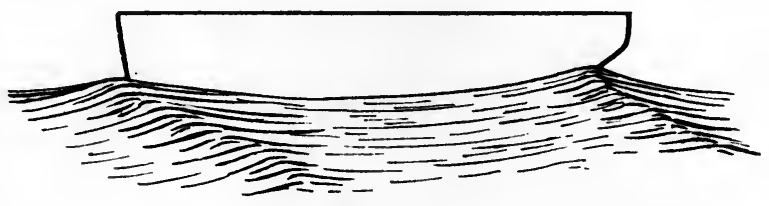

FIG. 77.

velocity of the accompanying stream at the stern and the consequent increase in pressure accounts for the stern wave. ${ }^{2}$ Also the increased velocity amidships is accompanied by a diminution in pressure, and accounts for the lowering of the surface level at this point. With a wholly submerged body the displacements produced on the surrounding mass of water are identical in form at all velocities, and are similar for similar bodies. Also, as pointed out by $\mathrm{Mr}$. Froude, and subsequently confirmed by experiment, this holds for partially submerged bodies in spite of the effect of gravity on the vertical displacements, if the similar bodies move with velccities proportional to $\sqrt{ } \bar{D}$, where $D$ is the ratio of their linear dimensions. Assuming then, as appears to be approximately true, that the height of the waves is proportional to $v^{2}$, and that their breadth is proportional to their height, we have their mass proportional to $v^{4}$, and

1 British Association Report, 1898 ; also "Transactions Inst. Naval Architects," 1898 , vol. 85 .

2 For an article on the changes of level around a vessel, see an article in the Eingineer, vol. 63, p. 252. 
the energy of formation to $v^{6}$. This energy is largely dependent on the form and relative length of entrance and run of a ship, and every vessel would appear to have some limiting speed beyond which any increase is accompanied by an altogether disproportionate increase in wave-making resistance. $\mathrm{Mr}$. Scott Russell states that this limit is somewhat less than that corresponding to the length of the wave which the ship tends to form, which length depends on the length of entrance and run, and gives the following formula ${ }^{1}$ for the maximum velocity obtainable without abnormal resistance :-

$$
V=1.03 \sqrt{L_{1}+L_{2}} \text {. }
$$

Where $V=$ velocity in knots

$L_{1}$ and $L_{2}$ are lengths of entrance and run in feet.

Also $L_{1}$ should not be less than $562 V^{2}$

and $L_{2} \quad, \quad, \quad, \quad 375 V^{2}$.

Thus for a speed of 10 knots, $L_{1}+L_{2}>93 \cdot 7$ feet.

$$
\begin{array}{llllll}
\prime & \quad & , & 20 & , & L_{1}+L_{2}>375 \quad, \\
, & \quad & 30 & , & L_{1}+L_{2}>843 \quad,
\end{array}
$$

While agreeing fairly well with observed results, more recent investigations ${ }^{2}$ point to the incompleteness of this rule, and indicate that the length of the middle body of a ship also affects the wave resistance.

Eddy formation, apart from that due to skin friction, is largely confined to the stern, and is due to flow in what is in effect a channel with diverging boundaries. The reduction thus produced in the forward pressure on the stern is analogous to that in a diverging, as opposed to a converging pipe, and is thus an indirect cause of resistance.

Mr. Froude, in his experiments, found it necessary not only to use a cutwater, but also to taper off the stern of his planks in order to get a resistance-length curve, which should pass through the origin of co-ordinates, the effect being as indicated in Fig. 78.

As a factor in reducing resistance in fact, a finely tapered stern or run is of much greater value than a fine stem. With a model

1 "Transactions Inst. Naval Architects," vols. 1 and 2.

2 "Transactions Inst. Naval Architects," 1881. 
as shown in Fig. 79, the directions of motion for least and greatest resistances are as indicated.

Taking $S_{s}$ to be the projected area of the stern, the reduction

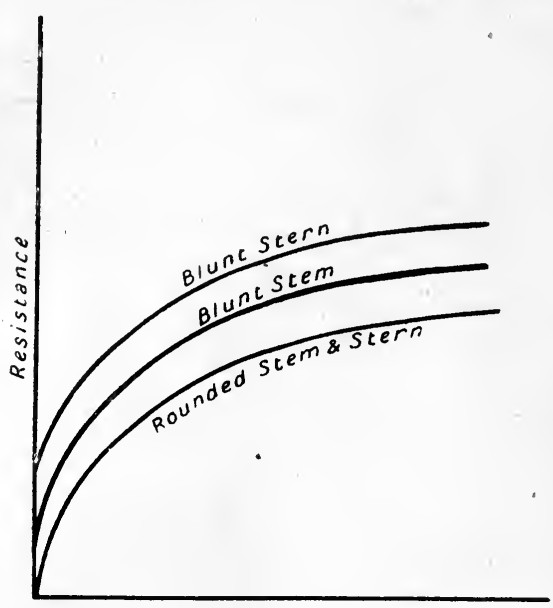

Lenglh

.Fig. 78. in pressure due to eddy production is approximately proportional to $v^{2}$, and the resistance thus produced proportional to $S_{s} v^{2}$.

In general, however, skin friction accounts for the greater part of the total resistance, varying from 80-90 per cent. of the total for speeds of 6 to 8 knots, to 50 or 60 per cent. at very high speed-this with clean bottoms. Eddy production, in a well - formed ship, accounts for 8-10 per cent.

of the total, and wave-making resistance for the remainder.

The total resistance, at speeds up to about $10 \mathrm{knots}$, is approximately proportional to $v^{2}$, while Sir William White ${ }^{1}$ states that as the speed increases further, the power of the velocity to which

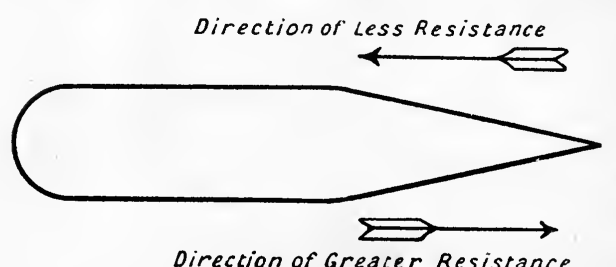

FIG. 79. the resistance is proportional increases to a maximum value of about 3 , owing to the increasing magnitude of the wavemaking resistance, and then falls again to a value slightly below 2 .

Thus in the case of an 80-foot boat exampled by Sir William White The resistance up to 10 knots was approximately $\propto v^{2}$

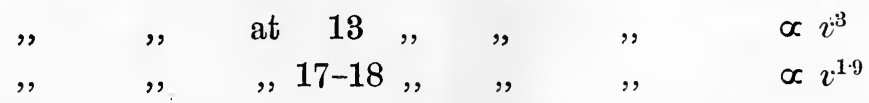

1 "Manual of Naval Architecture," by. W. H. White, p. 4ó6. London, John Murray. 
while with the Iris-300 feet long,

the resistance up to 13 knots was approximately $\propto v^{2}$.

,,$\quad$ at $18,, \quad, \quad \propto v^{28}$.

The total resistance of a ship may be deduced from that of a scale model, and the skin friction alone from Froude's results, so that it becomes possible to deduce the eddy and wavemaking resistances experimentally.

Before proceeding to describe Mr. Froude's methods of doing this, we may note that, if the relative scales of a ship and its model are as $D: 1$, the relative wetted areas are as $D^{2}: 1$, and if the suffix $_{1}$ refers to the ship and ${ }_{2}$ to the model, we have the frictional resistances given by

$$
\left.\begin{array}{l}
R_{1}=f_{1} S_{1} V_{1}^{2} \\
R_{2}=f_{2} S_{2} V_{2}^{2}
\end{array}\right\} \text { assuming resistance } \propto r^{2} .
$$

and, neglecting for the time the difference between $f_{1}$ and $f_{2}$, we have

$$
\frac{R_{1}}{\overline{R_{2}}}=\frac{S_{1}}{S_{2}}\left(\frac{V_{1}}{V_{2}}\right)^{2}=D^{2}\left(\frac{V_{1}}{V_{2}}\right)^{2}
$$

Again, the ratio of the wave-making resistances is given by

$$
\left(\frac{V_{1}}{V_{2}}\right)^{6}
$$

while the ratio of eddy resistances

$$
=\frac{S_{1}}{S_{2}}\left(\frac{V_{1}}{V_{2}}\right)^{2}=D^{2}\left(\frac{V_{1}}{V_{2}}\right)^{2} \text {. }
$$

Putting $\left(\frac{V_{1}}{V_{2}}\right)^{2}=D$, each of these resistances, and therefore the total becomes proportional to $D^{3}$, so that, to quote Mr. Froude, "If a ship be $D$ times the dimensions of the model, and if at speeds $V_{1}, V_{2}, V_{3}$, the measured resistances of the model are $R_{1}, R_{2}, R_{3}$, then for speeds $V_{1} \sqrt{\bar{D}}, V_{2} \sqrt{D}, V_{3} \sqrt{D}$, the resistances of the ship will be $D^{3} R_{1}, I^{3} R_{2}, D^{3} R_{3}$. To the speeds of the model and ship thus related it is convenient to apply the term 'corresponding speeds.'

In determining the resistance of any proposed ship, a scale model is made usually of paraffin wax, and is towed through still water, the resistance corresponding to any speed being noted. Thus in Fig. 80, $A A$ represents a resistance curve, the vertical 
ordinates from the line $O \mathrm{X}$ representing to scale the total resistance of the model.

The area and length of the wetted surface being known, and the coefficient of friction determined, the frictional resistance can now be calculated for any speed, and the curve $B B$ of frictional resistances drawn.

If now the horizontal scale be increased in the ratio $\sqrt{D}: 1$, and the vertical scale in the ratio $D^{3}: 1$, the curve $A A$ serves

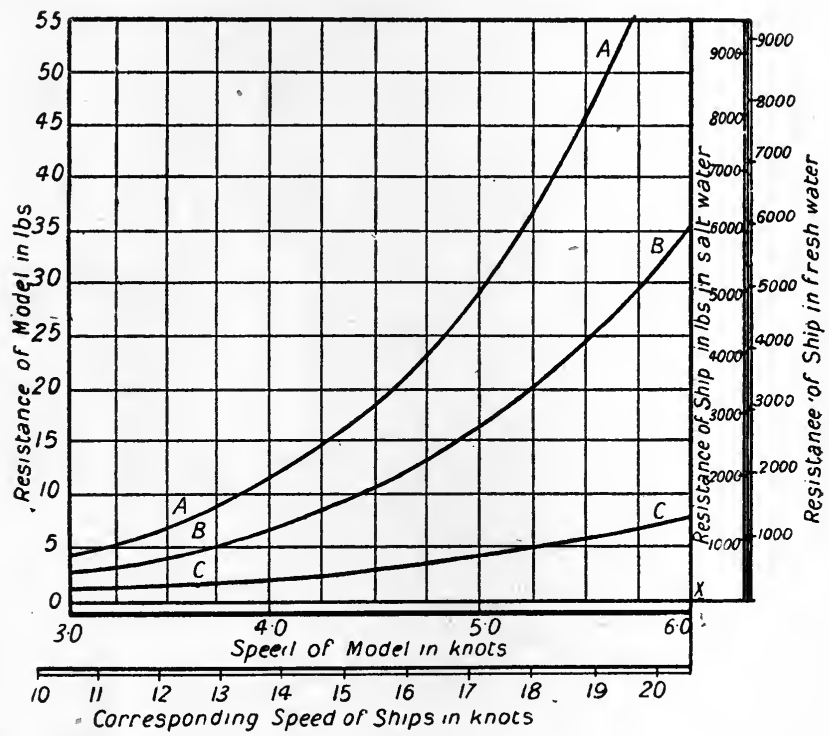

Fig. 80.

as the total resistance curve for the ship. Thus if the model is $\frac{1}{12}$ the size of the ship, $D=12$; the corresponding speeds of ship and model are in the ratio $\sqrt{12}: 1=3 \cdot 461: 1$, and at this corresponding speed the resistances are in the ratio $12^{3}: 1$ $=1728: 1$.

If fresh water is used in the experimental tank the vertical scale is to be increased again in the ratio of the densities of salt and fresh water, while a further final correction is necessary because of the different values of $f$ for the ship and model. This is got over by calculating the actual frictional resistance of the ship for her true length at various speeds, and by setting the 
values thus obtained as ordinates downwards from the curve $B B$, to form the curve $C C$. The true resistance of the ship at any speed is then given by the corresponding vertical intercept between $A A$ and $C C$.

\section{Art. 60.-The Propulsion of Ships.}

Of the many systems of mechanical propulsion which have from time to time been devised, only three have attained any measure of success. These respectively use the paddle wheel, the screw propeller, and the hydraulic jet as their propelling agent.

The principle underlying all these systems is the same, the propeller being devised so as to create a sternwards current of water, and the corresponding reaction on the propeller providing the propelling force on the ship. Thus, whatever system be adopted, if a mass of water of weight $C$ lbs. per second have its sternward velocity increased by $v$ feet per second by the action of the propeller, the change of momentum per second in this direction $=\frac{C v}{g}$ foot-pound units, and the thrust on the propeller is of the same magnitude, $\frac{C r}{g}$ lbs., and in the opposite direction.

Since the thrust varies as the product of $C$ and $x$, any one of these factors may be changed without affecting the thrust if the other factor suffer a corresponding change, so that a given thrust may be obtained by the comparatively slow movement of a large mass of water, or by the quick movement of a smaller mass. Other things being equal then, an increase in the area of the moving stream and a diminution in its velocity will tend to efficiency by reducing shock and kinetic losses.

While this conclusion is verified in practice, in the case of the paddle wheel and of the hydraulic jet (see Art. 103) practical considerations soon limit the maximum possible area (sooner in the case of the jet than with the paddle wheel). In the case of the screw propeller, moreover, frictional losses rapidly increase with the area of the screw, while an attempt to increase the area by increasing the radius, increases the centrifugal action of the water, 
and by reducing the pressure near the centre of the propeller, tends to reduce the effective thrust.

Paddle Wheels.-These give excellent results in smooth water, and where the draught and therefore the depth of immersion of the paddles is not likely to vary largely. They are well adapted for river navigation, particularly for shallow draught vessels, but are quite unfitted for use in any ocean-going boat which may be exposed to rough weather, and whose draught may differ by some feet on the outward-bound and homeward-bound journeys.

If $R=$ mean float radius,

, $N=$ number of revolutions per second, then $2 \pi R N=V_{P}=$ velocity of paddles relative to the ship in feet per second.

If $V_{s}$ be the absolute velocity of the ship, $\left(V_{P}-V_{s}\right)$ is termed the slip, or more correctly the apparent slip, and $100 \frac{V_{P}-V_{s}}{V_{P}}$ is termed the percentage slip. This in general varies from 20 to 30 per cent. The chief losses are due to shock at entrance and exit from the water and are reduced as far as possible by the use of feathering floats arranged so as to enter the water without shock. Where, as is usual, the paddle wheels are placed amidships, the stream-line motion over the run of the ship is greatly affected, at least over those layers between the surface and the bottom of the wheel, and since the relative velocity of water over this portion of the ship is largely increased, the effect is to augment the resistance as compared with the towing resistance with paddles removed.

The Screw Propeller is by far the most important of the three types, and has replaced the paddle wheel for all ocean going vessels. Its advantages consist in its possibilities of adoption to high speed prime movers; its depth of immersion, which makes its efficiency largely independent of a varying draught, and its reduced liability to accident, in virtue of its sheltered position.

If now $P=$ mean pitch of propeller blades, i.e., the distance the boat would travel per revolution if the screw were supposed to rotate in a fixed solid nut, and if $V_{P}$ is the corresponding axial velocity so that $V_{P}=N P$, then $V_{P}-V_{s}$ is usually termed the slip, 
or more correctly the apparent slip of the screw, and, if this worked in still water, would give the sternward velocity in the propeller race relative to the surrounding water. The real slip is the change actually produced by the screw on the velocity of the water in the propeller race, and since the propeller works in an accompanying current of water which has an initial absolute velocity $V_{w}$ in the direction of the ship, this is given by $\left(V_{P}-V_{s}+V_{v}\right)$.

Evidently the apparent is always less than the true slip, and may, if $V_{v}$ is sufficiently great, become negative. In general, however, it is positive and, expressed as a percentage of $V_{P}$, has a value of about 20 per cent.

The real slip is always positive if the screw is to have any propelling effect, as will be evident if it be remembered that it measures the change in the sternward momentum of the water.

Owing to the centrifugal action of the water, the pressure over the front face of the propeller is negative, so that part of the total change of velocity occurs before the water actually reaches the propeller. There is thus a tendency to draw water from the stern of the vessel and to reduce the forward pressure over this portion of its surface, with the result that the resistance, as compared with the towing resistance, is considerably augmented.

The maximum efficiency of the propeller would appear to be about 75 per cent., the magnitude of the various losses then probably approximating to the following values :-

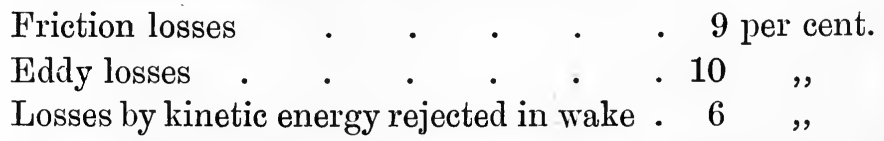

Assuming an efficiency for the engine and shafting of 80 per cent. this gives a combined efficiency of 60 per cent.

In spite of much experimental work and theoretical investigation, very little is actually known as to the best design of screw for any given conditions. Mr. Froude, after an extended series of experiments, concluded that the mean effective angle of the screw blade should be $45^{\circ}$, this making the pitch equal to about twice the extreme diameter, and that the true slip should be about $12 \frac{1}{2}$ per cent. The blade area, projected on a plane perpendicular 
to the axis of the screw should then be equal to $\frac{8 \cdot 9 R}{V_{8}^{2}}$ square feet, where $R=$ resistance of ship in pounds at the maximum velocity $V_{g^{*}}{ }^{1}$

The question of Jet Propulsion is treated in some detail in Art. 103.

Power necessary for Propulsion.

If $R=$ resistance of ship in lbs., and $V_{8}$ its velocity in feet per second, we have

Work done in propelling vessel $=R r_{s}$ foot-pounds per second.

$\therefore$ Horse-power to overcome resistance $=\frac{R V_{8}}{550}$.

The I.H.P. of the ship's engines will be considerably more than this, because of mechanical friction losses in the engine and propeller shaft and, to a still greater extent, because of the inefficiency of the propeller itself. In general it may be taken that from 45 per cent. to 60 per cent. of the energy developed in the engine cylinders is utilised in doing useful work.

Since $R$ is approximately proportional to $V_{s}^{2}$, the horse-power will vary as $V_{s}^{3}$, except at very high speeds, where the wavemaking resistance becomes abnormal.

\section{Examples.}

In the Greyhound, length 160 feet; breadth $33 \frac{1}{6}$ feet ; draught $13 \frac{3}{4}$ feet; displacement, 1,160 tons; the wetted area is 7,540 square feet. The following corresponding values of resistance and speed were experimentally obtained :-

\begin{tabular}{l|c|c|c|c|c}
\hline Speed (knots) . & 4 & 6 & 8 & 10 & 12 \\
\hline Resistance (tons) & $\cdot 6$ & $1 \cdot 4$ & $2 \cdot 5$ & $4 \cdot 7$ & $9 \cdot 0$
\end{tabular}

Taking $f=\cdot 00935$ (the unit of velocity being 1 knot), and assuming eddy making resistance to form 8 per cent. of the whole,

1 For a further investigation into the action of the propeller the reader is referred to a paper on the action of propellers by Professor Rankine, "Transactions Inst. Naval Architects," vol. 6 ; also papers by Mr. Froude in vols. 6, 8 and 19 of the same "Transactions," and to White's "Naval Architecture," p. 543. 
determine the magnitude of the frictional, eddy, and wavemaking resistances, and express these as a percentage of the whole.

Answer.

\begin{tabular}{|c|c|c|c|c|c|c|}
\hline & \multicolumn{2}{|c|}{4 knots. } & \multicolumn{2}{|c|}{8 knots. } & \multicolumn{2}{|c|}{12 knots. } \\
\hline & Lbs. & $\%$ & Lbs. & $\%$ & Lhs. & $\%$ \\
\hline $\begin{array}{l}\text { Frictional resistances } \\
\text { Eddy resistances }\end{array}$ & $\begin{array}{l}891 \\
107\end{array}$ & $\begin{array}{r}66 \cdot 3 \\
8 \cdot 0\end{array}$ & $\begin{array}{r}3169 \\
415\end{array}$ & $\begin{array}{r}56 \cdot 6 \\
8 \cdot 0\end{array}$ & $\begin{array}{l}665 \tilde{5} \\
1613\end{array}$ & $\begin{array}{r}33 \cdot 0 \\
8 \cdot 0\end{array}$ \\
\hline Wave-making resistances & 346 & $25 \cdot 7$ & 2016 & $35 \cdot 4$ & 11892 & $59 \cdot 0$ \\
\hline
\end{tabular}

(2) In the previous example, determine the energy actually absorbed in propulsion, and assuming that 45 per cent. of the I.H.P. of the engine is utilised, determine the I.H.P. at speeds of 4,8 , and 12 knots.

Answer.

\begin{tabular}{lc|c|c|c} 
& 4 knois. & 8 knots. & 12 knot. \\
\hline Useful horse power & $\cdot$ & 16.5 & 138 & 744 \\
\hline I.H.P. & $\cdot$ & 36.7 & 307 & 1651
\end{tabular}

(3) In the Merkiara, length 360 feet; breadth $37 \cdot 2$ feet; draught 16.25 ; displacement 3,980 tons, the wetted area is 18,660 feet. The following are experimental results -

\begin{tabular}{l|c|c|c|c|c|c}
\hline Speed (knots) & 4 & 6 & 8 & 10 & 12 & 13 \\
\hline Resistance (tons) & $1 \cdot 0$ & $2 \cdot 3$ & $3 \cdot 9$ & $-\frac{6 \cdot 0}{9 \cdot 0}$ & $11 \cdot 5$
\end{tabular}

Taking $f=\cdot 00917$, determine the percentage resistance due to frictional and to eddy and wave-making resistances, at 4, 8, and 12 knots. Also, assuming 42 per cent. of energy developed in engines to be utilised in propulsion, determine the I.H.P. at these speeds. 
Answer.

\begin{tabular}{l|c|c|c}
\hline & 4 knots. & 8 knots. & 12 knots. \\
\hline Frictional resistance & $96 \cdot 6 \%$ & $87 \cdot 8 \%$ & $75 \cdot 9 \%$ \\
\hline Eddy and wave-making $\cdot$ & $3 \cdot 4 \%$ & $12 \cdot 2 \%$ & $24 \cdot 1 \%$ \\
\hline I.H.P. of engines . & $65 \cdot 6$ & 511 & 1770 \\
\hline
\end{tabular}

(4) In the Greyhound the length of entrance and of run are each 75 feet; in the Merkara they are each 144 feet. Determine the "limiting" speed in each case.

$$
\text { Answer. }\left\{\begin{array}{l}
\text { Greyhound, } 1 \cdot 03 \sqrt{150}=12 \cdot 63 \text { knots. } \\
\text { Merkara, 1.03 } \sqrt{288}=17 \cdot 5 \text { knots. }
\end{array}\right.
$$

Note how these values bear out the abnormal increase in wave-making resistance at the limiting speed as determined in the preceding examples. In the Merkara the wave-making resistance at 19 knots was found to be over 60 per cent. of the total.

(5) Experiments on a 20 -feet model are carried out to determine the probable horse-power required in a ship 600 feet long when doing 24 knots. Determine the corresponding speed of the model, and, assuming the value of $f$ for the ship to be $\cdot 00910$ and that for the model to be $\cdot 00935$, determine the probable horsepower of the engines if the resistance of the model at the corresponding speed is $7 \cdot 54 \mathrm{lbs}$. Assume 50 per cent. of the I.H.P. to do useful work at the propeller, and assume wetted area of ship $=48,000 \mathrm{sq} . \mathrm{ft}$.

$$
\text { Answer. }\left\{\begin{array}{l}
\text { Corresponding speed }=4 \cdot 38 \text { knots. } \\
\text { I.H.P. }=21,920 .
\end{array}\right.
$$




\section{CHAPTER VII.}

Pipe flow - Experimental Formulae-Darcy-Hagen - D'Aubuisson - Prony Eytelwein - Weisbach - Kutter-Reynolds-Critical velocity experimentsCritical velocity formula-Rational formula for pipe flow-Unwin-LawtonThrupp-Mean velocity-Distribution of velocity-Measurement of discharge - Relation of pipe diameter to volume discharged-Sudden stoppage of motion in an uniform pipe-Water hammer-Stoppage by gradual closing of a valve.

\section{Art. 61.-Pipe Flow.}

ONE very important effect of fluid friction is experienced in the resistance to the flow of water through a pipe. This resistance can only be overcome by a gradual fall of pressure in the liquid, in the direction of motion, and, reasoning from analogy to the resistance experienced by a plane surface moving through water, it might be inferred that, with sinuous motion, the total resistance $R$ would equal $f S v^{n}$,

$f$ depends chiefly on the surface of the pipe and to a smaller extent on viscosity.

where $\{S=$ area of wetted surface.

$n$ depends on the pipe surface, and is approximately equal to 2.

Putting $A=$ sectional area of pipe in square feet.

, $\quad P=$ length of perimeter of pipe.

,$\quad p_{1}-p_{2}=$ fall in pressure in lbs. per square foot over a length $l$ feet of pipe.

This becomes

$$
\begin{aligned}
\left(p_{1}-p_{2}\right) A & =f P l v^{n} \\
\therefore \quad p_{1}-p_{2} & =f \frac{P}{A} \cdot l v^{n} .
\end{aligned}
$$

Here $\frac{A}{P}=\frac{\text { area }}{\text { perimeter }}$ is termed the " hydraulic mean depth,"

1 If the mass of water in the pipe be imagined as distributed over a horizontal surface of the same area as the walls of the pipe, its depth will then be the same as the hydraulic mean depth for the pipe. 
and is commonly denoted by $m$, so that (1) may be written

$$
p_{1}-p_{2}=\frac{f l v^{i}}{m} \text {. }
$$

In the case of a circular pipe $m=\frac{\pi r^{2}}{2 \pi r^{2}}=\frac{r}{2}=\frac{d}{4}$, so that, if in addition we express $p_{1}-p_{2}$ as a difference $h$ of head in feet of water, equation (2) becomes

$$
h=\frac{f l v^{n}}{62 \cdot 4 m}=\frac{f^{\prime} l v^{n}}{l} .
$$

The analogy between the two cases is, however, not exact, in that, while with a solid moving through a large body of water any disturbance set up at the surface may be propagated over any unknown distance, becoming less marked as the distance from the solid increases, and finally dying out altogether, any such disturbance in a pipe has a strictly limited range of extension, but is in general communicated to the whole mass of water in motion.

A consideration of the relative direction of rotation of eddies formed at opposite ends of a diameter indicates that these, on meeting in the interior of the pipe, will tend to destroy each other's angular motion and thus to augment the effective resistance, so that it might be inferred that the diameter of the pipe will have a further effect on the resistance, this diminishing to a greater extent than indicated in expression (3), with an increase in the diameter, and possibly being inversely proportional to $d^{c}$, where $x$ has some value greater than unity.

Where the motion through the pipe is everywhere steady, it is entirely governed by the law of simple viscous resistance, and the resistance is accurately given by the formula of Art. 20. Thus Poiseuille (1845), experimenting on tubes of very fine bore (between -02 and $\cdot 10$ millimetres), found the resistance to motion to be directly proportional to the velocity and to the pipe length, and inversely to the square of the diameter, and deduced the law (see p. 57):-

$$
\text { loss of pressure }=\frac{32 \mu l \cdot v}{d^{2}} .
$$

Many experimental researches have been carried out from 
time to time to determine the law of resistance with sinuous or unsteady motion, and it is now proposed to consider the results of these somewhat in detail, and to see in how far the above reasoning may be justified.

Probably the most complete series of experiments ever carried out on this subject is that of Darcy, who, in 1857, experimenting on cast-iron pipes having diameter's ranging from 5 inches to 20 inches, and lengths of 110 yards, concluded that the resistance is proportional to the length and to the square of the velocity, and is inversely proportional to the diameter. Thus expressed, the law becomes

$$
\iota=\frac{v^{2} l}{c^{2} l}
$$

$c$ being a constant for any one type of surface.

It was found however that this formula was not strictly applicable to all diameters of pipe, since, as $d$ increases, the resistance diminishes according to a slightly higher power of $d$ than the first. Darcy found that, within the range of his experiments, keeping $c$ a constant, this exponential value of $d$ imight be replaced by $\left\{\frac{d}{1+\frac{1}{12 d}}\right\}$, and thus obtained the law

$$
\iota=\frac{r^{2} l}{c^{2} d}\left\{1+\frac{1}{12 d}\right\}
$$

( where $d=$ diameter of pipe in feet.

,$\quad v=$ velocity in feet per second.

,$\quad h=$ head loss expressed in feet of water.

Assuming, with Darcy, that the loss is proportional to the kinetic energy of the stream, and replacing $d$ by its value $4 m$, the above becomes

$$
h=f\left(1+\frac{1}{12 d}\right) 2 \frac{l r^{2}}{2 g m}
$$

(where $f=\frac{g}{2 c^{2}}$ ), in which form the equation is often stated.

Darcy's experiments, carried out with C. I. pipes, gave the following values of the coefficient $f$.

f For clean and bare metal surfaces . $f=\cdot 005$.

For old and incrusted ,, , , . $f=\cdot 010$. 
Hagen (1854) deduced from experiments by Bossut, Couplet, and Dubuat, the formula-

$$
h=\frac{f l v^{175}}{d^{125}}
$$

but did not discover any law of variation of these indices or of the coefficient $f$, with the surface or diameter of the pipe. Most of the other formulae deduced by the earlier experimentalists are merely modifications of the formula-

$$
h=\frac{f l r^{2}}{2 g m}
$$

or, to put it in the form adopted by De Chezy-

$$
v=c \sqrt{\frac{m h}{l}}
$$

The more important of these are stated below, and for ease of comparison have been reduced, where possible, to one of these forms. Where the original formula did not contain the factor $2 g$, in introducing this the value 64.4 has been adopted, and other numerical factors altered correspondingly. Dimensions throughout are in feet.

Thus, D'Aubuisson, Prony, and Eytelwein, assumed that the resistance to motion is composed of two parts, one due to simple frictional resistance at the boundaries and proportional to the velocity, and the second due to eddy production and proportional to $v^{2}$. The constants $a$ and $b$ in the formula-

$$
h=\frac{l}{2 g m}\left\{b v+a v^{2}\right\}
$$

\begin{tabular}{|c|c|c|}
\hline & a. & . \\
\hline D'Aubuisson & $\cdot 00670$ & $\cdot 00121$ \\
\hline Prony. & $\cdot 00684$ & .00112 \\
\hline Eytelwein . & $\cdot 00503$ & $\cdot 00131$ \\
\hline
\end{tabular}

where $f=a+\frac{b}{v}$, were then determined on this assumption.

These values were as follows:- 
Weisbach, on the other hand, assuming the first part of the resistance proportional to $v^{\frac{3}{2}}$, obtained the formula-

$$
h=\frac{f l v^{2}}{2 g m}, \text { where } f=\cdot 00360+\frac{\cdot 00429}{\sqrt{v}} .
$$

Adopting the same formula, Bazin put $f=00294\left(1+\frac{\cdot 3736}{d}\right)$, while Kutter and Ganguillet put

$$
f=64 \cdot 4\left\{\frac{1+\left(41 \cdot 6+\frac{.00281 l}{h}\right) \frac{N}{\sqrt{ }}}{\frac{1 \cdot 811}{N}+41 \cdot 6+\frac{.00281 l}{h}}\right\}
$$

where $N$ is a coefficient depending on the roughness of the pipe surface and varying from 009 to 017 , its value for clean cast-iron or asphalted pipes being 013 .

These formulae, excepting that of Kutter, neglecting as they do any variation in resistance produced by a variation in the physical condition of the pipe surface, are obviously only of value where the experimental conditions can be reproduced. Moreover the fundamental assumption in the formulae of D'Aubisson, Prony, and Eytelwein, as to the resistance depending on two powers of the velocity, has been clearly demonstrated to be unsound by Reynolds. This since in every case where the logarithms of the resistances and velocities have been plotted, these are found to lie on accurately straight lines (p. 191).

Kutter's formula, introducing as it does a roughness coefficient $N$, has a much wider range of application, and gives much more consistent results than those previously mentioned, from Hagen to Weisbach. This formula, however, assumes the resistance to be always proportional to $v^{2}$, whereas experiment indicates that for smooth surfaces the power of the velocity is always less than the second, and we are led to the conclusion that none of these formulae, while giving good results within their own particular range of application, can be looked upon as representing the general state of affairs in pipe flow. 
ART. 62.

Before proceeding further, the work done by Professor Osborne Reynolds, in reconciling the widely divergent results as to the law of resistance obtained by Poiseuille and by Darcy; determining the constants involved in the critical velocity; and in deducing a rational formula for pipe flow, whether with steady or unsteady motion, must be considered.

This work is all the more noteworthy in that the formulae obtained, although initially deduced entirely from theoretical considerations, coincide so closely with the results of observa-

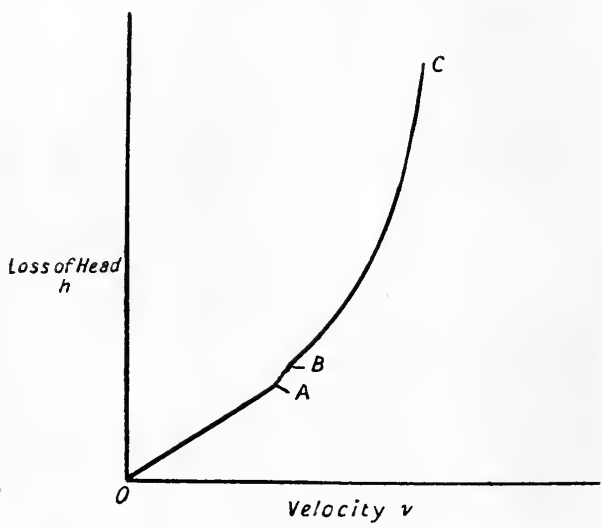

FIG. 81. tion, while from a student's point of view it is worth considering somewhat in detail as an example of the valuable results possible by purely inductive reasoning, when guided by a sound insight into the fundamental laws governing the phenomenon.

Having determined the nature of the two manners of motion possible in a parallel pipe, and the existence of a "critical velocity," below which the motion is steady, and above which it is unsteady, Reynolds determined the law of resistance in the two cases and the points at which the change takes place, by measuring the loss of head accompanying different velocities of "flow. On plotting a curve showing velocities and losses of head (Fig. 81) it is found that up to a certain velocity, for any given tube, these points lie on a straight line passing through the origin of co-ordinates. Above this velocity, the points lie more or less on a smooth curve, indicating that the loss of head is possibly proportional to $v^{n}$.

To test this, and if so to determine the value of $n$, the 
logarithms of the loss of head and of the velocity were plotted (Fig. 82), since if

$$
\begin{gathered}
h=k v^{n} \\
\log h=\log k+n \log v
\end{gathered}
$$

which is the equation to a straight line, inclined at an angle $\theta$ to the axis of $\log r$ (where $\tan \theta=n$ ), and cutting off an intercept $=\log k$ on the axis of $\log h$.

It is then found that with velocities increasing between each pair of experiments, the plotted points lie on a straight line up to a certain point $A$, the value of $\theta$ for this line being $45^{\circ}$. $\mathrm{Up}_{p}$ to this point $n$ is unity and $h$ is proportional to $v$. At $A$, which marks the higher. critical velocity, or the point at which motion, initially steady, becomes sinuous, the law suddenly changes and $h$ increases very rapidly. The relation between $h$ and $v$, however, follows no definite law, until the point $B$ is

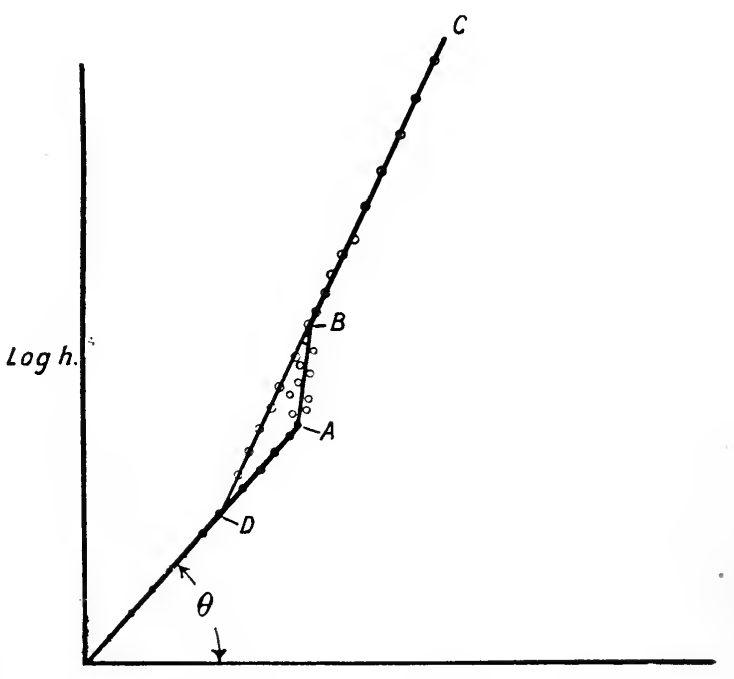

$\log v$.

FIG. 82. reached, where the velocity is about 1.3 times that at $A$. Above this point a perfectly definite law holds, the plotted points from $B$ to $C$ and onwards lying on a straight line.

The angle of inclination $\theta$ of this line varies with the material and surface of the pipe, but is constant for any one pipe.

With a lead pipe, $\tan \theta=1 \cdot 722$, so that here the law of resistance above the critical point is $h \propto v^{1722}$.

When the velocities are decreased between each pair of experiments, it is found that the plotted points follow the straight 
line $C B D$ to its intersection $D$ with $D O$,indicating that eddies once initiated do not die out until the velocity is reduced to that corresponding to $D$. The velocity at $D$, which is less than that at $A$, is termed the lower or true critical velocity.

For velocities between $D$ and $A$ the motion, if steady, is unstable, and any initial disturbance will produce eddy motion which will not die out.

A consideration of the part of the $\log h, \log v$ curve between $A$ and $B$ shows that here the value of $n$ is greater than before $A$ or after $B$, and that with motion of this intermittently unsteady or unstable type the increased resistance accompanying an increase in velocity is greater than even when the motion is altogether sinuous. This would appear to be due to the fact that within this range of velocities eddies are being initiated in the tube, and the loss of head in the tube is due, not only to the actual resistance to motion, but also to the absorption of energy in eddy formation. After $B$ is passed the eddies fill the whole length of tube, and the loss of head is then simply due to resistance to motion through the tube, i.e., to the maintenance, as opposed to the initiation, of eddy motion. ${ }^{1}$

The reason for the sudden change from steady to sinuous motion is not clear. It has been suggested that the change takes place when the shear stress, accompanying the varying rate of flow across the pipe, becomes greater than that which the liquid is capable of withstanding. That this theory is untenable is clear if we remember that in viscous flow through a pipe the maximum shear occurs at the boundaries, and has the value $\frac{r}{2} \frac{d p}{d x}$ (p. 57). For the theory to be true, this shear stress

1 Messrs. H. T. Barnes and E. G. Coker, "Proceedings Royal Society," vol. 74, determined the critical velocity by allowing water to flow through the given tube, which was jacketed with water at a higher temperature. A delicate thermometer indicated the temperature of the discharge water at exit. So long as the motion is steady, the transmission of heat through the water takes place entirely by conduction and is extremely slow, so that the thermometer gives a steady reading practically identical with that in the supply tank. Immediately the critical velocity is attained, however, the temperature of discharge increases rapidly, and the change from steady to unsteady motion is manifested by a sudden jump of the mercury thread of the thermometer. 
must have the same value in any pipe for the initiation of eddy motion, so that $r \frac{d p}{d x}$ must be constant.

But $r \frac{d p}{d x}=-\frac{8 \mu Q}{\pi r^{3}}=-\frac{8 \mu v}{r}$ (p. 57), and therefore the critical velocity will vary directly as the radius of the pipe. This result is directly opposed to the results of observations, experiments indicating that $v$ is inversely proportional to $r$.

The law governing the position of the critical point was inferred by Reynolds from a consideration of the general equations of motion for a viscous fluid.

Here the first equation of $(9)$, p. 64 , is

$$
\begin{gathered}
\rho X+\left(\frac{\partial p_{x x}}{\partial x}+\frac{\partial p_{y x}}{\partial y}+\frac{\partial p_{z x}}{\partial z}\right)=\rho \\
\left\{u \frac{\partial u}{\partial x}+v \frac{\partial u}{\partial y}+w \frac{\partial u}{\partial z}+\frac{\partial u}{\partial t}\right\} .
\end{gathered}
$$

Also for continuity we have

$$
\begin{gathered}
\frac{\partial u}{\partial x}+\frac{\partial v}{\partial y}+\frac{\partial w}{\partial z}=0 \\
\therefore \quad u \frac{\partial u}{\partial x}+u \frac{\partial v}{\partial y}+u \frac{\partial w}{\partial z}=0 .
\end{gathered}
$$

Adding this to the right-hand side of the above equation, this becomes

$$
\begin{gathered}
\rho\left\{2 u \frac{\partial u}{\partial x}+\left(v \frac{\partial u}{\partial y}+u \frac{\partial v}{\partial y}\right)+\left(w \frac{\partial u}{\partial z}+u \frac{\partial w}{\partial z}\right)+\frac{\partial u}{\partial t}\right\} \\
=\rho\left\{\frac{\partial(u u)}{\partial x}+\frac{\partial(u v)}{\partial y}+\frac{\partial(u w)}{\partial z}+\frac{\partial u}{\partial t}\right\}
\end{gathered}
$$

So that the equation may be written

$$
\begin{aligned}
\rho X+ & \left\{\frac{\partial}{\partial x}\left(p_{x x}-\rho u u\right)+\frac{\partial}{\partial y}\left(p_{y x}-\rho u v\right)\right. \\
+ & \left.+\frac{\partial}{\partial z}\left(p_{z x}-\rho u w\right)\right\}=\rho \frac{\partial u}{\partial t},
\end{aligned}
$$

the left-hand side of this equation expressing the force producing the acceleration $\frac{\partial u}{\partial t}$, in the direction $O X$. Taking this to be the 
direction of mean flow in a tube, and neglecting the external force $\rho X$, and ( $p_{x x}-\rho u u$ ), which simply marks the variation in the direction of flow, we are left with two terms which represent the variations in directions at right-angles to this. Since these involve the shear on parallel layers of the fluid, it is probable that the conditions involving steady or sinuous motion depend in some definite manner on these terms, and since, in a parallel tube, terms in $y$ and $z$ are similar and symmetrical if the effect of gravity be neglected, we may consider any one of these, and note how any variation in this may affect the conditions of flow.

Considering the term $\frac{\partial}{d y}\left(p_{y x}-\rho v u\right)$, substituting from equation (8), p. 63 , this may be written

$$
\frac{\partial}{\partial y}\left\{\mu\left(\frac{\partial v}{\partial x}+\frac{\partial u}{\partial y}\right)-\rho v u\right\} \text {. }
$$

The first of these terms involves the coefficient of viscosity $\mu$, and is of the nature $\mu \times \frac{\text { velocity }}{\text { space }}=\frac{\mu V}{L}$, while the second is of the nature $\rho V^{2}$. It was inferred then, that since the relative value of these terms probably determines the critical velocity, the latter will depend on the relation $\frac{\mu}{\rho V L}$.

Putting $L=\frac{d}{k}$ where $d$ is the diameter of pipe, experiments were devoted to determining whether the critical velocity $v_{c}$ was given by $\frac{k \mu}{\rho d}$. These experiments conclusively justified the inference and led to the formula

$$
v_{c}=\frac{1}{b} \cdot \frac{P}{d}
$$

where $b$ is a numerical constant.

$$
\text { , } P \propto \frac{\text { viscosity }}{\text { density }} \text { and therefore depends on the tempera- }
$$
ture of the water.

If $t=$ temperature in degrees centigrade, then for water the value of $P$ is given by

$$
P=\frac{1}{1+03368 t+000221 t^{2}}
$$


Thus when $t=32^{\circ} \mathrm{F} .=0^{\circ} \mathrm{C} . \quad . P=1$.

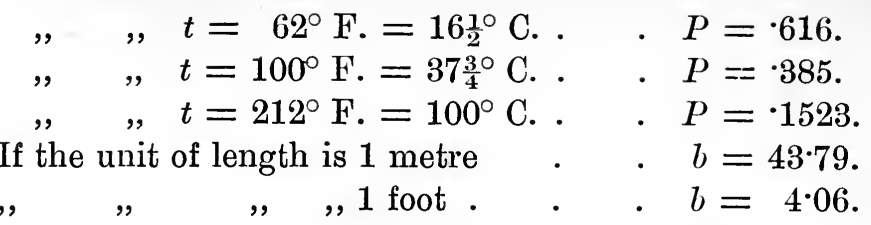

This gives the higher $\mathrm{C}$. V. at which steady becomes eddy motion.

Exactly the same formula, but with a different numerical coefficient, was found to hold for the lower (true) C. V. at which eddy becomes steady motion. ${ }^{1}$

Here if the unit of length is 1 metre . . $b=278$.

E.g., with water at $10^{\circ}$ C. $\Longrightarrow 50^{\circ}$ F.-

Motion is steady if $x d<\cdot 02$

,$\quad$ unstable if $v d \stackrel{>}{<\cdot 02}, 127$

" unsteady if $r d>\cdot 127$,

the unit of length being 1 foot.

\section{ARt. 63.}

A rational law of resistance to pipe flow, applicable to either steady or unsteady motion, was deduced by Professor Osborne Reynolds from general principles, the only assumptions made being that the fall of pressure per unit length depends on the radius of the pipe, the viscosity and density of the fluid, and on the velocity of flow, or, if $K$ is some constant,

$$
\delta p=K r^{x} \mu^{y} \rho^{z} v^{n} l \text {. }
$$

Expressed dimensionally this becomes

$$
\begin{aligned}
& \frac{\text { Force }}{\text { Area }}=K[L]^{x} \cdot\left[\frac{M}{L T}\right]^{y} \cdot\left[\frac{M}{L^{3}}\right]^{z}\left[\frac{L}{T}\right]^{n} \cdot L \\
\therefore & {\left[\frac{M}{L T^{2}}\right]=K[L]^{(x-y-3 z+n+1)}[M]^{(y+z)}[T\rceil^{-(y+n),} }
\end{aligned}
$$

1 Phil. Trans. Roy. Soc., 1883. Also "Scientific Papers," Osborne Reynolds, vol. 2, pp. 51-103. 
and equating indices of like quantities we get

$$
x-y-3 z=-2-n ; y+z=1 ; y=2-n,
$$

from which we have

$$
\left\{\begin{array}{l}
x=n-3 \\
y=2-n \\
z=n-1
\end{array}\right.
$$

The formula then becomes

$$
\delta p=g h \rho=K r^{n-3} \mu^{2-n} \rho^{n-1} v^{n} . l .
$$

If $i$ represents the loss of head in units of length of a water column per unit length of pipe, so that $i=\frac{h}{l}, i$ is called the hydraulic gradient of the pipe, and gives the slope at which this would need to be laid in order to give the required flow without any external head. Evidently $i$ is independent of the units used, and the formula may now be written

$$
i=\frac{B^{n} \cdot P^{2-n} v^{n}}{A \cdot d^{3-n}}
$$

where $P$ has the value given above, and $A$ and $B$ are constants.

1 A similar rational law may be deduced for the flow of compressible fluids by writing $\frac{p_{m}}{c \tau}$ for $\rho$ in equation (2). Here $\tau$ is the absolute temperature of the gas, while $c$ is the constant obtained from the relationship $p V=c \tau$, its value for air being 53.2 when the units are feet and pounds, and degrees on the absolute Fahrenheit scale. We then get

$$
\delta p=\frac{k \mu^{2-n} \stackrel{n}{r_{m}^{2 z}} l}{d^{3-n}}\left(\frac{p_{m}}{c \tau}\right)^{n-1} .
$$

An examination, by the author, of the results of experiments on the flow of air through pipes, by several experimenters, and with diameters ranging from -125 inches to 98 feet-velocities from 10 to 40 feet per second, confirms the assumptions made in deducing this formula. From these experiments the following values of $n$. have been deduced :-

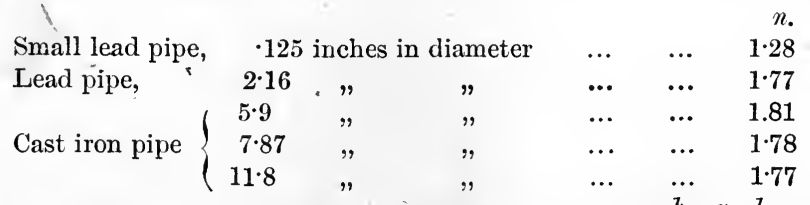

Below the C. V. $n=1$, and the formula becomes $\delta p=\frac{\Re_{\mu} r_{m} l}{d^{2}}$, indicating that the pressure drop is now independent of the absolute pressure in the pipea result verified by the experiments. 
The values of the constants $A$ and $B$ have been determined and if the units are feet and degrees centigrade $\left\{\begin{array}{l}A=1.93 \times 10^{6} \\ B=36.9\end{array}\right.$ $" \quad, \quad \quad$ metres and degrees centigrade $\left\{\begin{array}{l}A=67 \cdot 7 \times 10^{6} \\ B=396\end{array}\right.$ The formula is found to hold, with fair accuracy, for all velocities above or below the critical points by a suitable substitution for $n$.

Reynolds gives the following mean values of $n$ for velocities above the critical, those for cast iron being deduced from Darcy's results.

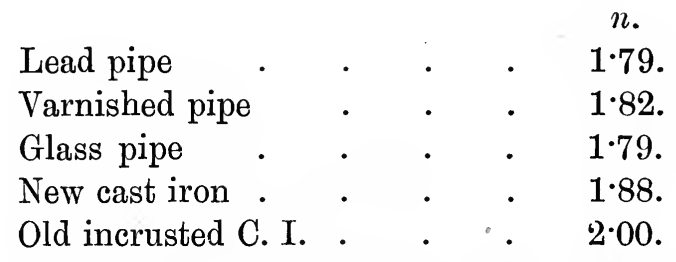

Below the C. V., $n=1$ for all surfaces and the formula becomes

$$
h=\frac{B}{A} \cdot \frac{P l v}{d^{2}} .
$$

Poiseuille's formula $h=\frac{p_{1}-p_{2}}{g \rho}=\frac{32 \mu l v}{g \rho d^{2}}$, the head being expressed in centimetres of water and the units in the C. G. S. system, becomes, on transforming to the metre as the unit of length, $h=\frac{278}{47 \cdot 7 \times 10^{6}} \cdot \frac{v P}{d^{2}}=5.83 \times 10^{-6} \frac{v I}{d^{2}}$. This is practically identical with the Reynolds formula on substitution for the constants $A$ and $B$.

From the form of equation (3), it appears that if $n$ is less than 2, the resistance depends on the temperature. If $n=2$, the term containing $P$ and involving the temperature becomes unity, and therefore for old and incrusted pipes the resistance is independent of the temperature, and the formula becomes

$$
\begin{aligned}
h & =\frac{B^{2} v^{2} l}{A d} \\
& =\cdot 000709 \frac{l v^{2}}{d} \text { feet of water. }
\end{aligned}
$$


Comparing this with the simplified form of Darcy's formula

$$
h=\frac{f l v^{2}}{2 g m} \text { or } \frac{4 f l v^{2}}{2 g d}
$$

we have coincidence if $\cdot 000709=\frac{4 f}{64 \cdot 4}$,

$$
\text { i.e., if } f=\cdot 0114 \text {, }
$$

which is the value given by Darcy's formula for rough pipes $7 \frac{1}{8}$ inches diameter.

It will be noted that in the general formula, the sum of the indices of $d$ and $v$ is always 3 .

Where the motion is sinuous, but the loss of head proportional to a lower power of $v$ than the second, as with clean or asphalted pipes, the formula indicates that variations in temperature affect the flow. This variation is, however, so small that it may safely be neglected. It is worth noting in passing, that while with sinuous motion the difference between the discharge at $5^{\circ}$ and $45^{\circ}$ centigrade is negligible, below the C. V. the discharge at $5^{\circ}$ centigrade is only one-half that at $45^{\circ}$ centigrade under the same head.

On applying the formula in its original form to the results of other experiments, it is found to be somewhat lacking in adaptability, while experience tends to show that in the particular case where $n=2$ the resistance does not, as indicated, vary inversely as the first, but as a slightly higher power of the diameter.

Professor Unwin, neglecting the small effect of temperature change at velocities above the critical, wrote this formula in the form

$$
h=\frac{f l v^{n}}{d^{x}}
$$

and deduced values for $f, n$, and $x$, from the results of experiments made by many different observers, and on pipes ranging in diameter from 2 inches to 48 inches.

Adopting the form $h=\frac{f l r^{\cdot n}}{2 g d^{x}}$ his deductions were as follows. ${ }^{1}$ $f$ varies from $\cdot 0215$ to $\cdot 0440$, increasing with the roughness of the surface. 
$n$ varies from $1 \cdot 75$ to $2 \cdot 00$, increasing with the roughness of the surface.

$x$ varies with the diameter and the surface from $1 \cdot 127$ to $1 \cdot 39$. It is always greater than unity and increases as the diameter increases. In general the relation $n+x=3$ is not quite true, no definite law appearing to connect $u$ and $x$.

The following are Unwin's mean values for $f, n$, and $x$.

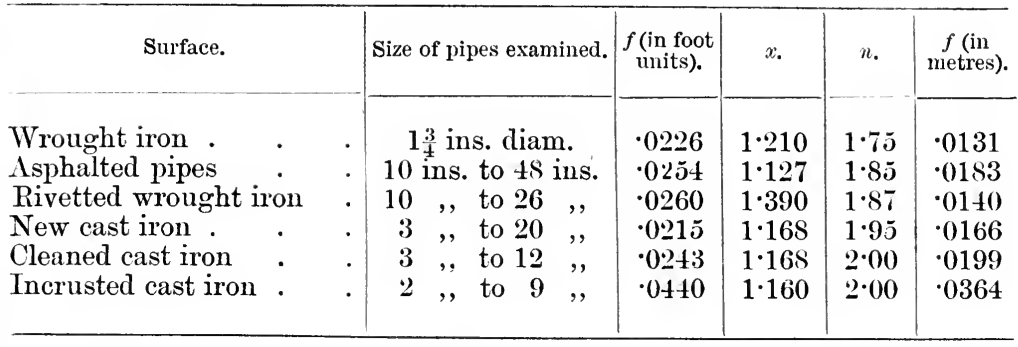

G. M. Lauton, ${ }^{1}$ comparing the latter formula with many recorded observations of more recent date, states that by writing the formula in the form

$$
h=k \cdot \frac{\cdot 0254 l v^{1 \cdot 87}}{2 g l^{1 \cdot 27}}
$$

and by giving $k$ the following values, the necessity for altering the index of $x$ with increasing diameter is obviated, while the formula gives good results for asphalted pipes of all diameters from 3 inches to 48 inches diameter.

\begin{tabular}{|c|c|c|c|c|c|}
\hline Diameter of pipe. & l. & Diameter of pipe. & l. & Diameter of pipe. & $k$. \\
\hline $\begin{array}{r}3 \text { ins. } \\
6 \quad,, \\
9 \quad,, \\
12,, \\
15 \quad,\end{array}$ & $\begin{array}{r}2 \cdot 031 \\
1 \cdot 486 \\
1 \cdot 220 \\
1.062 \\
\cdot 964\end{array}$ & $\begin{array}{l}18 \text { ins. } \\
21 \quad, \\
24 \quad, \\
27 \quad, \\
30 \quad,\end{array}$ & $\begin{array}{l}.904 \\
.880 \\
.858 \\
.839 \\
.823\end{array}$ & $\begin{array}{l}33 \text { ins. } \\
36 \quad, \\
39 \quad, \\
42, \\
45, \\
48,\end{array}$ & $\begin{array}{l}.806 \\
.797 \\
\cdot 78 \\
.765 \\
.754 \\
.744\end{array}$ \\
\hline
\end{tabular}

A further modification of the Reynolds formula, proposed by Thrupp, ${ }^{2}$ is given by

$$
h=\frac{C^{m} r^{n} l}{m} \text { where } m=\frac{l}{4} \text {. }
$$

1 "Proceedings Inst. Civil Engineers," vol. 153, p. 297.

2. Society of Engineers, 1887. 
The following are values of $c, x$, and $n$ for pipes of various materials,

\begin{tabular}{|c|c|c|c|c|c|}
\hline \multicolumn{3}{|c|}{ Surface. } & $n$. & c. & $x$. \\
\hline \multirow{2}{*}{\multicolumn{3}{|c|}{$\begin{array}{l}\text { Lead } \\
\text { Smooth wrought iron }\end{array}$}} & $1 \cdot 75$ & $\cdot 00522$ & $1 \cdot 085$ \\
\hline & & & $1 \cdot 80$ & $• 00479$ & $1 \cdot 170$ \\
\hline \multirow{2}{*}{\multicolumn{2}{|c|}{$\begin{array}{l}\text { Rivetted ," } \\
\text { New cast iron }\end{array}$}} & , & $1 \cdot 825$ & $\cdot 00567$ & $1 \cdot 235$ \\
\hline & & & $1 \cdot 850$ & $\cdot 00535$ & $1 \cdot 240$ \\
\hline Old & , & & $2 \cdot 00$ & $\cdot 00675$ & $1 \cdot 260$ \\
\hline
\end{tabular}

and for pipes of between 2 and 15 inches diameter the formula gives good results. For wrought iron pipes of less than 2 inches diameter a correction should be applied by writing

$$
x+a \sqrt{\frac{b}{m}-1} \text { for } x, \text { where } a=\cdot 0324 ; b=\cdot 07 .
$$

ART. 64.

Where wrought iron or steel pipes are used, consisting of single plates bent into the form of a circle and rivetted together, the value of $f$ is increased by the presence of the rivet heads and by the pipe joints, except in the case where butt joints are used, having a lap plate on the outside and having rivets with counter-sunk heads, on the inside of the pipe.

Experiments carried out by Herschell, on rivetted pipes consisting of alternate large and small rings, with projecting rivet heads, and which were coated with asphaltum, gave values of $f$ in the formula $h=\frac{f l v^{2}}{2 g m}$, for a $3 \frac{1}{2}$ feet diameter pipe, ranging from $\cdot 00675$ with a velocity of 1 foot per second, to $\cdot 0055$ with a velocity of 6 feet per second. With a similar 4 -feet pipe, $f$ varied from $\cdot 00675$ to $\cdot 0056$ with the same range of velocities.

After four years' use, the latter pipe gave values of $f$ ranging from 0085 to 0065 with the same range of velocities.

Experiments $^{1}$ on a bare rivetted pipe with butt joints and 6 feet in diameter, when new gave $f=\cdot 0052$ for all velocities

1 "Transactions American Society Civil Engineers," 1898 and 1900. 
from 1 to 4 feet per second. After two years' use, the values of $f$ were found to range from $\cdot 0139$ at $\cdot 5$ feet per second to $\cdot 00975$ at 1 foot per second, and 00618 at 4 feet per second.

J. Duane, ${ }^{1}$ as a result of experiments on a new 48-inch rivetted main of length 5,992 feet, obtained a value of $f=$ .00358 in the same formula with a velocity of $2 \cdot 28$ feet per second. After seven years, considerable tuberculation was found to have taken place on the interior surface of the pipe, and a second series of experiments gave $f=\cdot 00698$.

As a result of experience it may be laid down as established that a metal pipe prepared before laying by a coating of hot asphaltum on its inner surface will not deteriorate so as to cause any appreciable increase in the value of $f$. On the other hand a bare metal pipe will, by internal corrosion, cause the value of $f$ to largely increase-in a small pipe to double-in very few years, and allowance should always be made for this in computing the diameter necessary to give a required discharge.

The results of several experiments on wooden pipes ranging from 1 to 6 feet in diameter, are recorded in the "Transactions American Society of Civil Engineers," for 1902, and show values of $f$ ranging from 0065 at velocities of 1 foot per second to 00.40 at velocities of 5 feet per second. These experiments also demonstrate the fact that the value of $f$ in a wooden pipe is less for an old than for a new pipe.

Experiments by Messrs. Marx, Wing, and Hoskins ${ }^{2}$ on a 6 -feet wooden pipe gave a value of $f=\cdot 00468$ at a velocity of 2 feet per second, this value being approximately constant.

A series of experiments by Freeman ${ }^{3}$ on the flow through fire hose, give the following mean values of $f$, at velocities ranging from 10 to 30 feet per second.

$\begin{array}{lll}\text { Unlined linen hose . } & \cdot & f=\cdot 0084 . \\ \text { Smooth rubber lined hose } & \cdot & f=\cdot 0044 .\end{array}$

It is probable that at such velocities as are common in practice, riz., about 100 feet per second, these values would become about $\cdot 0080$ for the unlined, and $\cdot 0040$ for the rubber-lined hose.

1 "Transactions American Society Civil Engineers," 1892.

2 "Transactions American Society Civil Engineers," 1900, p. 108.

3 "Transactions American Society Civil Engineers," 1889, p. 303. 
Art. 65.

While the formulae of Kutter, Unwin, or Thrupp are more complicated than the old formula of the Chezy type, the increased accuracy rendered possible by their use more than counterbalances

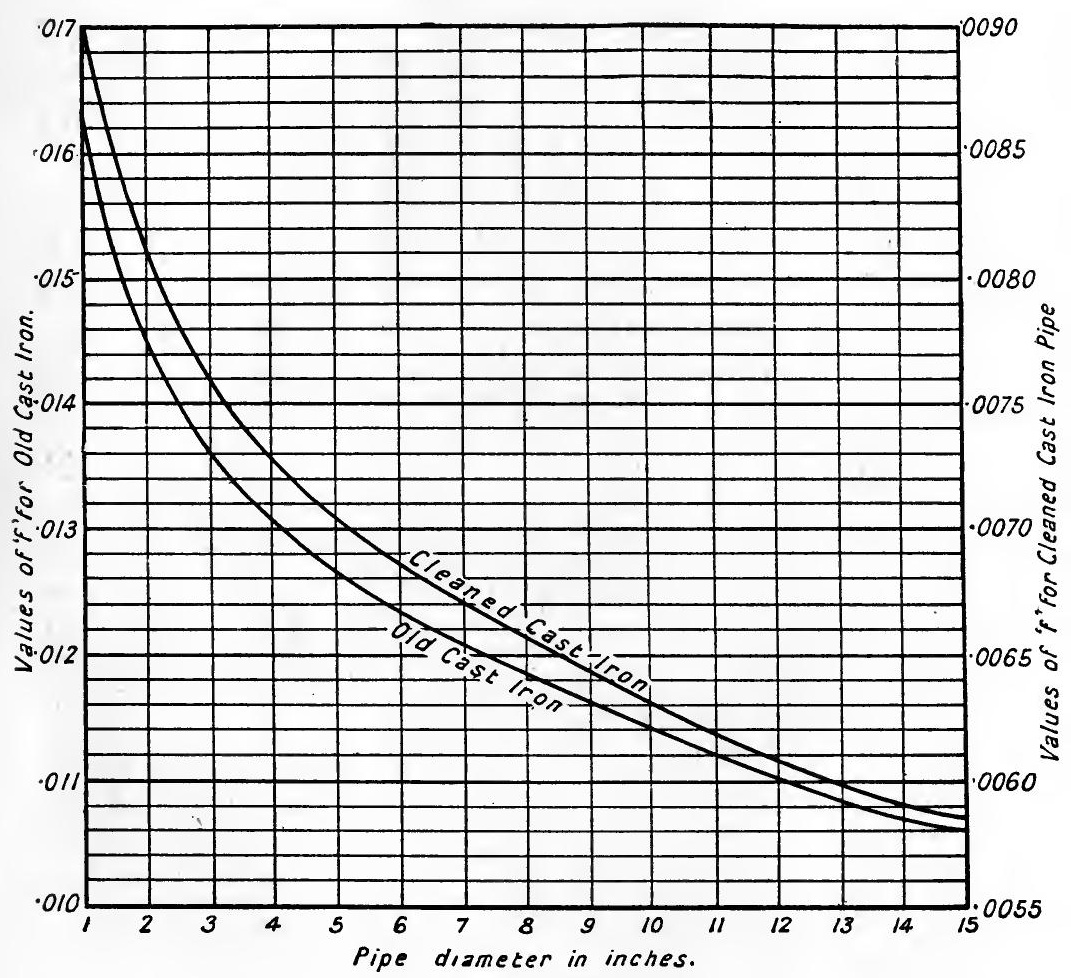

Fig. 83.

Curves showing values of $f$ in formula $\ell=\frac{f l x^{2}}{2 g m}$, for cleaned and old Cast Iron Pipes.

any inconvenience in handling. With the exception of the Kutter formula, which is exceedingly cumbrous to handle without the aid of specially prepared tables, this inconvenience is indeed more apparent than real since the formulae are in a form suitable for logarithmic calculation. 
For asphalted pipes with butt joints $\int f$ should be increased and projecting rivet heads $\quad\{$ by 25 per cent.

For asphalted pipes with in-and-out $f f$ should be increased rivetting and projecting rivet heads $\mid$ by 75 per cent.

In all cases of asphalted pipes having projecting rivets, a further allowance of $\mathbf{2 5}$ per cent. should be made where vegetable

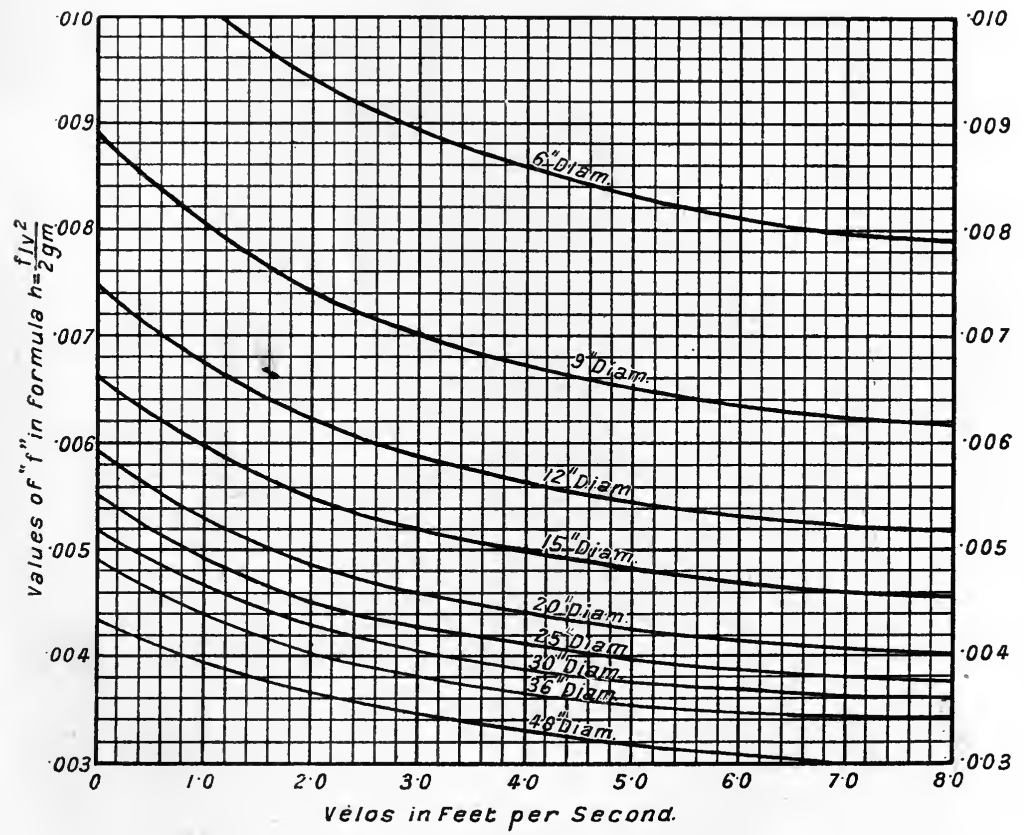

Fig. 85.

Curve showing values of $f$ for a clean Asphalted Pipe.

growth is likely to take place, while with smooth asphalted pipes the allowance should be 50 per cent.

Where, in the exponential formula, the index of $v$ is 2 , as for cleaned and old cast iron, a single curve gives the values of $f$ for all velocities.

This latter assertion is, however, not strictly correct, and where the pipe is very badly tuberculated, the value of $f$ would appear to increase as the velocity diminishes. Experiments by the author on such a pipe, 4 inches diameter and 600 feet long, 
gave values of $f$ which increase from $\cdot 0156$ at 2 feet per second, to $\cdot 0184$ at $\cdot 4$ feet per second.

ART. 66.

Since the velocity in a pipe is inversely proportional to $\sqrt{f}$, an error in the assumed value of $f$ will only lead to approximately half the percentage error in $r$, and hence in the calculated discharge. Still, since the physical condition of a pipe surface, and any deviation from straightness of the pipe vitally affect the resistance, and since the determination of the relative physical condition of two surfaces, even though of the same material, is a matter of the greatest difficulty, no pipe flow formula is to be relied upon as giving the discharge corresponding to a given loss of head, with any great degree of accuracy in a proposed pipe line. The difficulty in exactly reproducing the conditions of the experiments from which the formulae have been deduced prevents this, and the engineer who prognosticates to within 10 per cent. the discharge from a pipe under given pressure conditions has every reason to be satisfied with his choice of constants.

Art. 67.-Mean Velocity in a Pipe.

With unsteady motion in a pipe, the velocity in the direction of flow, throughout a cross section, is more nearly uniform than when the motion is steady, though the effect of the boundaries is still to cause the velocity to diminish from the centre.

Many experiments have been carried out to determine the variation of velocity along a diameter, notably by Darcy, Freeman, ${ }^{1}$ Cole, ${ }^{2}$ G. S. Williams ${ }^{3}$ and others, the pipes used having diameters ranging from $1 \frac{1}{8}$ inches to 48 inches. These tend to show that the velocity at the boundary is about one-half that at the centre for velocities ranging up to 6 feet per second, while for much higher velocities the ratio increases to about $\cdot 75$.

1 "Transactions American Society Civil Engineers," 1889, vol. 21, p. 412.

2 "Transactions American Society Civil Engineers," 1902, vol. 47, p. 63-276.

3 "Transactions American Society Civil Engineers," 1901, May, p. 314. 
Darcy obtained the relationship

$$
v=v_{\text {max }}-29 \sqrt{m i}\left(\frac{x}{a}\right)^{\frac{3}{2}}
$$

giving the velocity $v$ at any radius $x$ of a pipe of radius $a$.

Since this is a minimum

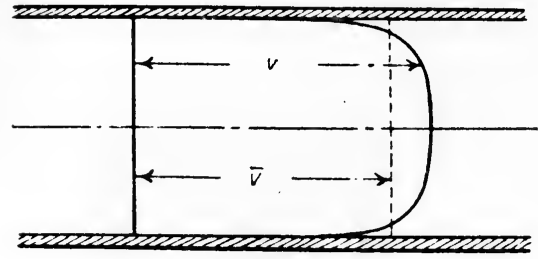

FIG. 86. when $x=a$ we have $v_{\text {max }}-v_{\text {min }}=29 \sqrt{m i}$.

The volume of the solid of revolution (Fig. 86) whose height represents the velocity at any radius is given by

$$
\text { Vol. }=\int_{o}^{a} 2 \pi x d x \cdot v
$$

$$
\begin{aligned}
& \therefore \quad \text { Mean height }=\frac{2 \pi \int_{o}^{a} v x d x}{\pi a^{2}} \\
& =\frac{2 \int_{0}^{a}\left(A x-B \frac{x^{\frac{5}{2}}}{a^{\frac{3}{2}}}\right) d x}{a^{2}} \\
& =\frac{1}{a^{2}}\left\{A a^{2}-\frac{4}{7} B a^{2}\right\} \\
& \text { where } A=v_{\max } \\
& B=29 \sqrt{m i} \\
& \therefore \quad \bar{c}=\text { mean velocity }=r_{m a x}-\frac{4}{7} \times 29 \sqrt{ } \overline{m i} . \\
& \text { Writing } \bar{r}=v_{\text {max }}-29 \sqrt{m i}\left(\frac{x}{a}\right)^{\frac{3}{2}} \text { from (1) we have } \\
& \frac{4}{7}=\left(\frac{x}{a}\right)^{\frac{3}{2}} \\
& \therefore \quad x=\cdot 689 a \text {, }
\end{aligned}
$$

giving the radius at which the velocity is equal to the mean throughout the cross-section. 
Assuming $\frac{h}{l}=i=\frac{f \bar{r}^{2}}{2 g m}$ so that $\bar{v}=\sqrt{\frac{2 g m i}{f}}$, and assuming $f=\cdot 01$ as being approximately true for a rough pipe, we get

$$
\begin{aligned}
\bar{r} & =80 \sqrt{m i} \\
& =r_{\text {max }}-16.57 \sqrt{m i} \\
\therefore \quad r_{\max } & =96.57 \sqrt{m i} \\
\therefore \quad \frac{\bar{r}}{r_{\max }} & =\frac{80}{96.57}=\cdot 829 .
\end{aligned}
$$

Taling $f=\cdot 006$ as the approximate value for a smooth pipe, the ratio $\frac{\bar{r}}{r_{\text {max }}}=\cdot 861$, while we also get

\begin{tabular}{c|c|c}
\hline & $f=\cdot 01$. & $f=\cdot 006$. \\
\hline$\frac{v_{\min }}{v^{\max }}$ & $\cdot 700$ & $\cdot 758$ \\
\hline$\frac{v_{\text {mix }}}{\bar{r}}$ & $\cdot 845$ & $\cdot 880$ \\
\hline
\end{tabular}

It is indeed to be expected that, as here indicated, the ratio $\frac{\bar{t}}{r_{\text {max }}}$, will vary with the surface conditions of the pipe. Experimentally this ratio is found to range from $\bullet 83$ to $\bullet 86$, and as in a large pipe the surface effect is of less importance than in one smaller, it may be taken that for pipes above 24 inches diameter the ratio is sensibly constant and equal to $\cdot 84$.

More recent experiments indicate that the curve of velocities in a diametrical plane is more nearly an ellipse than a parabola. Taling this former curve we find that the mean velocity occurs at a radius $x$ where $x=\cdot 75$ a, while if the surface velocity is onehalf that at the centre, i.e., $\frac{v_{\text {min }}}{v_{\text {mux }}}=\cdot 5$, the mean velocity is $\cdot 83$ times the maximum ${ }^{1}$. Figs. 86A $(a$ and $b$ ) show velocity curves as obtained by G. S. Williams and others on a 30 -inch pipe respectively on the straight and at a bend.

1 " Hydraulics" (Merriman), p. 201. 
E. S. Prentice, ${ }^{1}$ in a series of experiments on a fire hydrant, found that, taking the velocity at a radius $5 a$ to be equal to the mean, the discharge agreed within 5 per cent. with the results as calculated from the formula $v^{2}=\sqrt{2 g h}, h$ being the statical head equivalent to the pressure behind the orifice.

The radius of mean velocity is of importance since in gauging
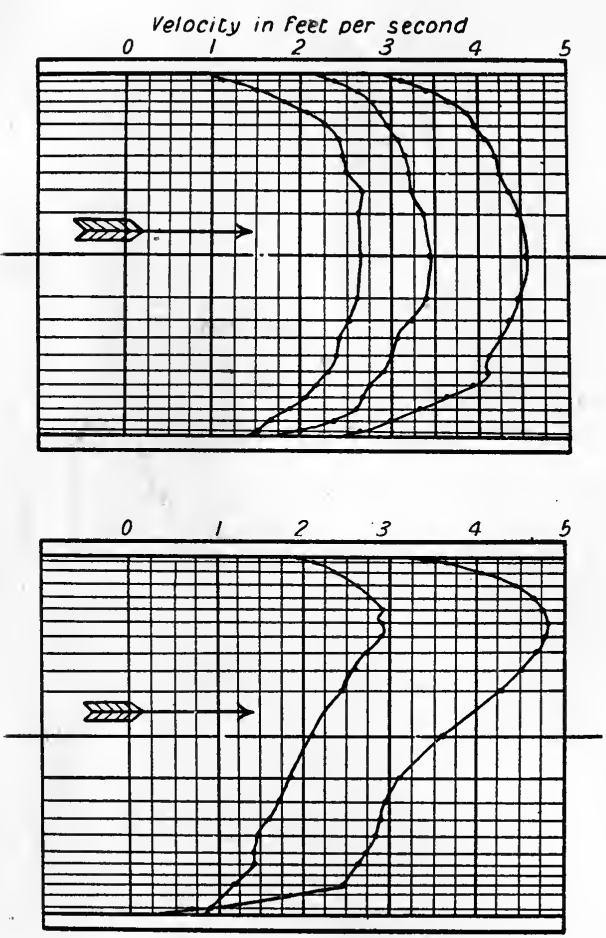

FIG. 86A. the discharge of a pipe by means of a Pitot tube (Art. 68), it is at this radius that the orifice of the tube should be placed. In general it may be taken that this radius varies from $69 a$ in small, to $.75 a$ in large pipes.

Experiments by $R$. Threlfall, ${ }^{2}$ on the flow of air through gas pipes from 6 to 36 inches diameter, indicate that here the radius of the circle of mean velocity is about 0.775 of the pipe radius, though in one case of a gas main, $15 \frac{3}{4}$ inches diameter, the radius of mean velocity was about 9 of the pipe radius.

The ratio of mean to maximum velocity in these experiments was very constant over a wide range of velocities-22 f.s. to 41 f.s. -and had the mean value 0.873 . 


\section{Art. 68.-Measurenent of Pipe Discharge.}

The volume actually discharged by a pipe may be determined approximately in several ways.

(1) The most accurate method is that of collecting and weighing the quantity discharged in a noted time, but this is impossible with any but the smallest of pipes.

(2) The mean velocity may be computed from a knowledge of the hydraulic gradient and of the diameter and internal condition of the pipe by an application of one or other of the formulae of Arts. 63-65. Where these data can be accurately obtained an error not exceeding 10 per cent. may be expected, with pipes ranging from 3 inches to 6.0 feet in diameter.

The hydraulic gradient may be obtained by observing the difference in the free level of the columns in two piezometers, or pressure tubes, placed at a known distance apart. Where the pressure in the pipe is less than atmospheric the type of piezometer shown in Fig. 87 (the vacuum piezometer) may be used. The point of attachment of these pressure tubes to the main is immaterial, the free surface

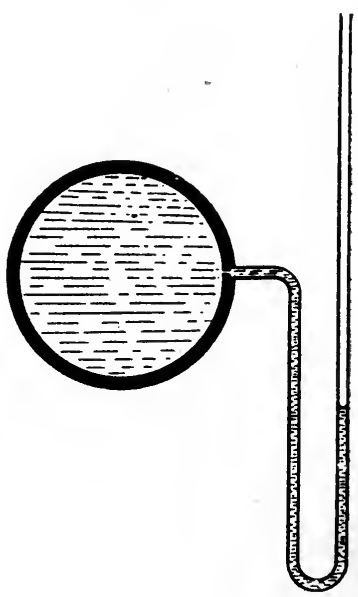

FIG. 87. rising to the same height whether the point of attachment is at the highest or lowest point of the diameter of the main.

In a series of experiments carried out to confirm this, Messrs. Marx, Wing, and Hoskins ${ }^{1}$ showed that whether with a pressure or vacuum piezometer, the pressure at any level in the main may be inferred from statical considerations, if the free level in the open branch of the piezometer is known.

It is of importance that the pressure opening should be perpendicular to the wall of the pipe, and that any internal burr caused by the drilling of this hole should be removed. Since the difference of head at any two points is unaffected by the pressure

$$
1 \text { “" Transactions American Society Civil Engineers," } 1898 .
$$

H.A. 
above the water column so long as this is constant, the upper ends of the piezometer tubes may be connected if convenient, and this pressure may then be maintained above or below that of the atmosphere. With such a device, whatever the pressure in the main, a comparatively short scale may be used to measure the difference of pressure at two points.

(3) The mean velocity may be obtained by'means of a Pitot tube, this being arranged either to obtain the velocity at the centre, when the mean velocity may be taken as 84 times this, or at a point 72 times the radius from the centre, when the observed velocity will approximately equal the mean for the whole tube.

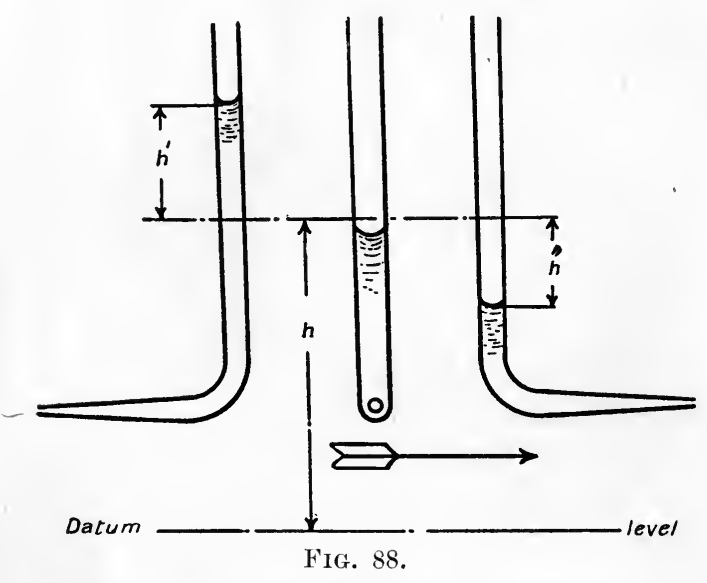

'The Pitot tube consists of a tube of tine bore bent at right angles (Fig. 88), having both ends open, and so arranged that while one leg remains vertical, the other may be rotated so as to point either up, down, or across stream, thus placing the plane of its orifice either at right angles to or parallel to the direction of flow.

When pointed up-stream, the statical pressure immediately inside the entrance to the tube must balance the statical head of water outside together with the pressure equivalent to the velocity head, so that if $h^{\prime}$ feet be the height of water in the tube above the free surface level, when turned up-stream, we have $\iota^{\prime}=k \frac{v^{2}}{2 g}$, where $k$ is a constant very approximately equal to unity.

Similarly, if turned down-stream, and if $l^{\prime \prime}$ is now the depression in the tube, we should liave 


$$
\begin{aligned}
& l^{\prime \prime}=l^{\prime}-\frac{t^{2}}{2 g} \\
\therefore \quad & h^{\prime}+h^{\prime \prime}=\frac{r^{2}}{g} \text { (very approximately) } \\
& r=\sqrt{g\left(h^{\prime}+h^{\prime \prime}\right) .}
\end{aligned}
$$

or

By using two tubes facing in opposite directions and having their upper ends connected, the head $\left(h^{\prime}+h^{\prime \prime}\right)$ may be directly obtained, while by altering the pressure above the water columns, their upper levels may be brought to any convenient part of the scale.

Theoretically, with the orifice pointing across the stream, the level inside the tube should indicate $h$, the statical head alone. Owing, however, to eddy formation and to a consequent reduction of pressure at the orifice, the level inside the tube will be slightly less than $h$.

When turned down-stream, eddy formation also affects the reading $h^{\prime \prime}$, giving this a lower value than the theoretical, while even when pointing up-stream the reading $h^{\prime}$ is only accurate when the tube is of very fine bore, and is given a conical form. so as to divert the oncoming stream with a minimum of disturbance. For this reason, the tube should be calibrated by observations in water moving with known velocities. Otherwise, even with a well constructed apparatus, an error of about 2 per cent. is probable, the calculated being greater than the actual velocity.

For measuring the velocity in streams from a fire nozzle, the point of a stylographic pen has been found to give good results as the entrance branch of a Pitot tube.

(4) The velocity may be obtained by Venturi meter (Art. 30), and for fairly large pipes an error not exceeding 1.5 per cent. is to be expected. With small pipes, one or other of the types of meter described in Art. 184 may be used.

(5) A chemical method of determining the discharge is described by F. Van Iterson. ${ }^{1}$ A given weight per minute of sodium thiosulphate being introduced into the pipe, a sample of the discharge is taken at some distance nearer the exit, and the quantity of 
chemical present is measured. The chemical being diluted in proportion to the volume of discharge, a measure of the latter is obtained. The sample is titrated with standard iodine, starch being used as an indicator. Several cocks arranged at different points on the circumference of the pipe should be used for withdrawing the samples, so as to obtain as far as possible a fair sample of the mixture. This method has been used with success for determining the discharge of sewage pumps at La Haye.

Where a colourless discharge is expected, coloured liquid may be introduced into the pipe in known quantities, and the colour of the discharge compared with that of standard admixtures. The method is, however, only to be looked upon as giving approximate results.

Art. 69.--Relation of Dianeter óf Pipe to Quantity Discharged.

With rough pipes, since $Q=\frac{\pi d^{2}}{4} \cdot r$, and since the loss of head $H$ in any length $l$ is given by

$$
\begin{gathered}
H=\frac{r^{2}}{2 g}\left\{\frac{f l}{m}\right\} \\
\therefore \quad r=\sqrt{\frac{2 g H m}{f l}} \\
\therefore \quad Q=\frac{\pi d^{2}}{4} \sqrt{\frac{2 g H d}{4 f l}}=\frac{\pi}{4} \sqrt{\frac{2 g H}{4 f l}} \cdot d^{\frac{5}{2}} \\
\therefore \quad Q=C \sqrt{\frac{H}{l}} \cdot d^{\frac{5}{2}} .
\end{gathered}
$$

If the unit of length is one foot, and the unit of time one second, $C=31 \cdot 6, f$ being taken as 01 .

For smooth pipes, the loss of head is proportional to a lower power of the velocity than the second, and to a power of the diameter slightly different from the first, i.e.,

$$
\begin{aligned}
H & =k \frac{v^{n} l}{d^{x}} \\
\therefore \quad r & =\left(\frac{H d^{x}}{k l}\right)^{\frac{1}{n}} \\
\therefore \quad Q & =C^{\prime}\left(\frac{H}{l}\right)^{\frac{1}{n}} \cdot d^{2+\frac{x}{n}}
\end{aligned}
$$


Professor Unwin gives coefficients for formulae deduced by Hagen, which for clean cast iron pipes make

$$
Q=C^{\prime}\left(\frac{H}{l}\right)^{\frac{1}{2}} d^{2 \cdot 58}
$$

$C^{\prime}$ having the value 41.9.

In experiments carried out by M. Vallot, $Q$ was found to be proportional to $d^{2 \cdot 66}$.

Art. 70.-Stoppage of Motion in a Pipe of Uniforil Bore.

(1) Sudden Stoppage. ${ }^{1}$

Let $a=$ area of pipe in square feet; $l=$ length in feet.

$" v=$ initial velocity of water in feet per second.

" $K=$ modulus of cubical elasticity of water.

$=300,000 \times 144 \mathrm{lbs}$. per square foot (approx.).

$, p,=$ pressure in lbs. per square inch, caused by stoppage.

Suppose instantaneous stoppage of flow in a rigid pipe.

The sequence of events is then as follows: At the instant of closure the pressure rises by an amount $p$; the velocity becomes zero; and a wave of pressure $p$ above the normal, and of zero velocity, is transmitted along the pipe to the open end, reaching this after a time, $t=l \div V_{p}$ seconds, where $V_{p}$ is the velocity . of propagation of pressure waves in water. This being the same as the velocity of sound in water is approximately 4,700 feet per second. At the open end the pressure is maintained constant; the compressed column instantly begins to re-expand, and the wave is reflected as a wave of normal pressure and of velocity $(-\imath)$. On reaching the closed end it is again reflected as a wave of negative pressure ( $p$ below the normal) and of zero velocity, to be reflected at the open end with normal pressure and velocity $(v)$. On reaching the closed end again, the pressure rises once more to $p$ above normal and the whole cycle of propagation is repeated, the wave formation being isochronous, and the time elapsing between any two consecutive states of maximum pressure at a point being given by $t=\frac{4 l}{4,700}$ seconds.

1 The reader who wishes for a rigorous mathematical treatment of this branch of the subject will find guidance from Love's "Treatise on the Theory of Elasticity," vol. ii., p. 126, or from Todhunter and Pearson's "History of the Theory of Elasticity," vol. ii., p. 294. 
At the stoppage the initial kinetic energy of the column is transformed into energy of strain and into kinetic energy of vibratory motion, and since, at the instant when a crest of the pressure wave reaches any point in the pipe the vibratory motion is zero, the whole energy at the point is in the form of strain energy.

But if a volume $a \delta l$ of water is compressed to a pressure $P$ lbs. per square foot, it may be easily shown that its strain energy $=\frac{a \delta l P^{2}}{2 K} \mathrm{ft.-lbs.,} \mathrm{so} \mathrm{that,} \mathrm{equating} \mathrm{the} \mathrm{original} \mathrm{kinetic} \mathrm{energy} \mathrm{of}$

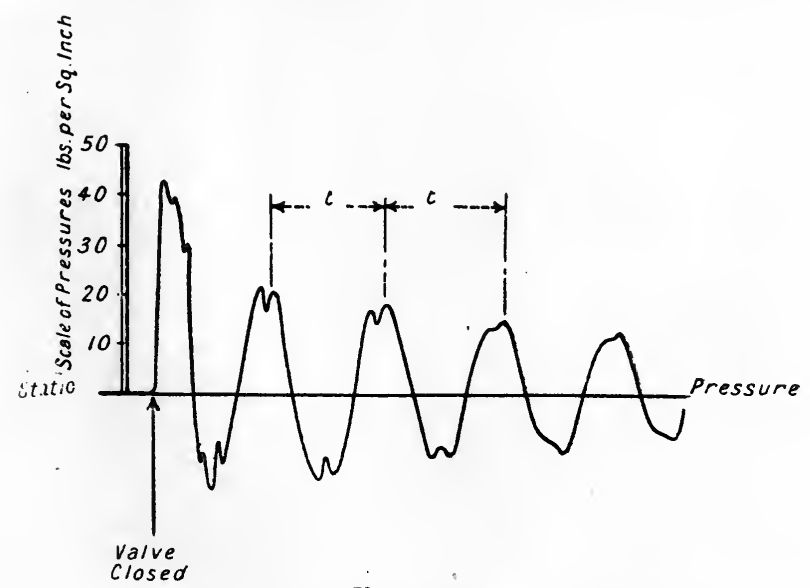

FIG. 89.

any such volume to its strain energy, when the wave of pressure is initiated we have

$$
\begin{aligned}
\frac{W a \delta l}{2 g} v^{2} & =\frac{a \delta l p^{2}}{2 K} \\
\therefore \quad P & =r \sqrt{\frac{K W}{g}} \text { lbs. per sq. ft. } \\
& =r \sqrt{\frac{300,000 \times 144 \times 62 \cdot 4}{32 \cdot 2}} \text { lbs. per sq. ft. } \\
& =9,160 r \text { lbs. per sq. ft. (approximately), } \\
\therefore \quad p & =637 r \text { lbs. per sq. in. }
\end{aligned}
$$

It should be noted that substantially the same maximum pressure is obtained at all points in the pipe length, and that this pressure is independent of the length of pipe. 
Actually, because of the elasticity of the pipe walls and joints, part of the kinetic energy of the moving column is expended in stretching these, with a resultant reduction in the maximum pressure attained, this reduction depending entirely on the form, material, and construction of the pipe line. ${ }^{1}$ Obviously an air vessel or any such device near the closed end will considerably reduce the pressure, and its action may be considered as being due either to a reduction in the effective modulus of elasticity of the pipe as a whole, or to an increase in the time occupied in bringing the moving column to rest. Owing to this, and to the viscosity of the water, the motion gradually dies out.

Since the effect of a disturbance at the end of the pipe is not again apparent at the same end until an interval of time $\frac{2 l}{4,700}$ seconds has elapsed, it would appear that if a valve, though not closing instantaneously, completes its closure in less time than thï,

1 A rough approximation to the effect of this may be obtained as follows :-

Let $\boldsymbol{R}=$ pipe radius in inches.

,$T=$, thickness in inches.

$" E=$ modulus of elasticity of material of pipe walls in lbs. per square inch.

Then the longitudinal stress in pipes $=\frac{r p}{2 T}$ lb:. per square inch,

and the circumferential $, \quad, \quad=\frac{r p}{T} \quad, \quad, \quad$,

$\therefore$ Work done in stretching the pipe

$$
\begin{aligned}
& =\frac{\text { volume of metal }+(\text { stress })^{2}}{2 E} \\
& =\frac{2 \pi r T l}{2 E^{2}}\left\{\frac{r^{2} p^{2}}{T^{2}}+\frac{r^{2} p^{2}}{4 T^{2}}\right\} \\
& =\frac{\pi}{4} \frac{\pi r^{3} l p^{2}}{E T}
\end{aligned}
$$

Work done in compressing water $=\frac{\pi r^{2} l p^{2}}{2 K}$,

$\therefore \quad \frac{\text { Work done in compressing water }}{\text { Total work absorbed }}=\frac{1}{1+\frac{5}{2} \frac{K r}{E T}}$,

For a steel pipe, taking $E=3 \times 10^{7} \mathrm{lbs}$. per square inch the ratio becomes

$$
\frac{1}{1+\frac{r}{40} T} \text {. }
$$

Taking this into account, the calculated pressure will be reduced in the ratio

$$
\sqrt{\frac{1}{1+\frac{r}{40 T}}}
$$


the pressure attained will be substantially the same as with instantaneous closure. The stoppage is then said to be "sudden."

Figure 89 represents a typical diagram obtained by the author from a pipe 3.6 inches diameter and about 610 feet long. The outlet from this pipe is regulated by a $2 \frac{1}{2}$-inch valve. In each experiment this was opened through a quarter of a turn, and on the flow becoming steady was closed as quickly as possible by hand. Although impossible to accurately measure the time of closing, this was probably about $\cdot 10$ second. The following table indicates results obtained in these experiments :-

\begin{tabular}{l|c|c|c|c|c|c|c}
\hline $\begin{array}{l}\text { Velocity of flow (feet } \\
\text { per second) }\end{array}$ & $\cdot 094$ & $\cdot 189$ & $\cdot 283$ & $\cdot 378$ & 566 & $\cdot 75.5$ & $\cdot 944$ \\
\hline $\begin{array}{l}\text { Maximum pressure } \\
\text { obtained (lbs. per } \\
\text { square inch) }\end{array}$ & $6 \cdot 0$ & $11 \cdot 9$ & $18 \cdot 0$ & $23 \cdot 2$ & $31 \cdot 0$ & $38 \cdot 5$ & $43 \cdot 5$ \\
\hline $\begin{array}{l}\text { Pressure as calculated } \\
p=63.7 \mathrm{r} .\end{array}$ & $6 \cdot 1$ & $12 \cdot 02$ & $18 \cdot 03$ & $24 \cdot 04$ & $36 \cdot 06$ & $48 \cdot 1$ & $60 \cdot 1$ \\
\hline
\end{tabular}

The period of a complete vibration of the indicator lever was .52 second, corresponding to a pipe length of about 613 feet. The pipe joints were of the ordinary spigot and faucet type with lead caulking, the effect of the yielding of these joints at high pressure being very marked.

The following values are taken from the results of a series of experiments carried out by M. Joukowsky. ${ }^{1}$ The pipes experimented upon were horizontal, of 4 and 6 inches diameter, and were 1,050 and 1,066 feet long respectively. In each experiment the outlet valve was closed in 03 second.

4-inch Pipe.

\begin{tabular}{|c|c|c|c|c|c|}
\hline Velocity in feet per second . . . & 5 & $2 \cdot 0$ & $3 \cdot 0$ & $4 \cdot 0$ & $9 \cdot 0$ \\
\hline Observed pressure in lbs. per square inch & 31 & 119 & 172 & 228 & 511 \\
\hline Calculated pressure taking $p=63.7 \mathrm{r}$ & $31 \cdot 8$ & 127 & 191 & 254 & 573 \\
\hline $\begin{array}{l}\text { Calculated pressure as modified for pipe } \\
\text { elasticity, taking } E^{\prime}=3+10^{7} \mathrm{lbs} \text {. per } \\
\text { square inch and } \frac{r}{T}=8 \quad . \quad . \quad .\end{array}$ & $29 \cdot 1$ & 116 & 175 & 232 & 52.5 \\
\hline
\end{tabular}

1 "Stoss in Wasserleitungsröhren," St. Petersburg, 1900. 
6-inch Pipe.

\begin{tabular}{lll|c|c|c|c}
\hline Velocity in feet per second .. &. &. & $\cdot 6$ & $2 \cdot 0$ & $3 \cdot 0$ & $7 \cdot 5$ \\
\hline Observed pressure in lbs. per square inch & $\cdot$ & $\cdot$ & 43 & 113 & 173 & 426 \\
\hline Calculated pressure taking $p=63 \cdot 7 r$ &. & $\cdot$ & 38 & 127 & 191 & 478 \\
\hline Calculated pressure modified for pipe elasticity & $\cdot$ & 35 & 116 & 175 & 436
\end{tabular}

The pressure in the last experiment, calculated on the assumption that the water is incompressible, is 3,585 lbs. per square inch (p. 91), a result which sufficiently indicates the nature of the errors involved in extreme cases by neglecting the effect of elasticity.

These sudden increases of pressure, commonly known as water hammer, may evidently become most serious with large values of $v$, and in such cases the ill effects due to the too sudden closing of a valve should be guarded against by a relief valve or other similar device placed as near as convenient to the main valve. In the case of a pipe line for a power plant, where the head is not excessive it is usual to provide a stand pipe, or simple vertical pipe capable of taking the whole discharge of the main, and open at the top to the atmosphere. This should be coupled to the supply main just before its entrance to the powerhouse, and the height should be so arranged that when standing under the statical pressure of the supply reservoir, the water level is within a short distance of the top. Under these conditions, the maximum pressure which may occur in the pipe line is that due to the statical pressure in the stand pipe, together with that necessary to produce motion up this pipe.

It is usual in practice to make some allowance for possible water hammer by designing the pipes to withstand a pressure $100 \mathrm{lbs}$. per square inch in excess of that due to the statical head, thus, in effect, allowing for an instantaneous stoppage at a velocity of $1 \cdot 6$ feet per second.

(2) Stoppage by the gradual closing of a valve.

Here the problem is complicated by the necessity for determining the retardation of the moving column produced at any instant by the closing of the valve. 
Calling $r$ the velocity of outlet from valve.

, $\quad$ a the area of vena contracta of discharge stream.

,, A the area of main.

,$\quad h$ the head at pipe entrance in feet.

On expressing the fact that the energy at entrance is absorbed in producing flow along the pipe and in giving kinetic energy at discharge, we have

$$
h=\frac{f l r^{2}}{2 g m}+\frac{v_{v}{ }^{2}}{2 g} .
$$

Also for continuity of flow we have $r A=v_{v} a$

$$
\therefore \quad h=\frac{r^{2}}{2 g}\left\{\frac{f l}{m}+\frac{A^{2}}{a^{2}}\right\}
$$

from which the pipe velocity corresponding to any given valve opening may be obtained.

Differentiating this expression with respect to $t$, we have

$$
\frac{d v}{d t}\left\{\frac{f l}{m}+\left(\frac{A}{a}\right)^{2}\right\}=v \frac{A^{2}}{a^{3}} \frac{d a}{d t} \text {. }
$$

Putting

$$
\begin{aligned}
& r=\sqrt{\frac{2 g h}{\frac{f^{\prime} l}{m}+\left(\frac{A}{a}\right)^{2}}}, \text { this becomes } \\
& \frac{d r}{d t}=\frac{A^{2} \sqrt{2 g h}}{a^{3}\left\{\frac{f l}{m}+\left(\frac{A}{a}\right)^{2}\right\}^{\frac{3}{2}}} \cdot \frac{d a}{d t},
\end{aligned}
$$

from which the retardation of the moving column may be calculated for any valve opening if the rate of closing of the valve be known. If the valve be closed uniformly in time $T$ seconds, and if $a_{1}$ be the area of discharge stream on passing the valve seat when the latter is wide open, we have $\frac{d a}{d t}=\frac{a_{1}}{T}$, while at any time $t$ before the instant at which the valve comes to its seat we have $a=\frac{a_{1} t}{T}$. Thus at this instant we have

$$
v=\sqrt{\frac{2 g h}{\frac{f l}{m}+\left(\frac{A}{a_{1}} \cdot \frac{T}{t}\right)^{2}}} \text { ft. per sec. }
$$


$\frac{d v}{d t}=\frac{A^{2} \sqrt{2 g h}}{\left\{\frac{a_{1} t}{T}\right)^{3}\left(\frac{f l}{m}+\left(\frac{A}{a_{1}} \cdot \frac{T}{t}\right)^{2}\right)^{\frac{3}{2}}} \cdot \frac{a_{1}}{T}$ ft. per sec. per sec. (5)

$=\frac{\sqrt{2 g h}}{\left\{\frac{a_{1}}{A} \cdot \frac{t}{T}\right)^{3}\left(\frac{t^{\prime} l}{m}+\left(\frac{A}{a_{1}} \cdot \frac{T}{t}\right)^{2}\right)^{\frac{3}{2}}} \cdot \frac{a_{1}}{A^{\prime} T^{\circ}}$

A curve may be drawn showing the retardation of the moving column at any instant after beginning to close the valve, and, if the effect of the elasticity of the column be neglected, the pressure necessary at the valve to produce this retardation may be calculated (p. 91). From equation (3), above, it appears that for an uniform rate of valve closing, the acceleration $\frac{d v}{d t}$ increases as the valve opening diminishes and will have a maximum value at the instant of closing, when $a=o$, given by

$$
\begin{aligned}
\frac{d r}{d t} & =\frac{\sqrt{2 g h}}{A} \cdot \frac{d a}{d t} \mathrm{ft} . \text { per sec. per sec. } \\
& =\frac{\sqrt{2 g h}}{A} \cdot \frac{a_{1}}{T} \mathrm{ft} \text {. per sec. per sec. }
\end{aligned}
$$

If $a_{v}$ be the actual valve opening at the instant of beginning to close the valve, then writing $a_{1}=c a_{v}$, this becomes

$$
\frac{d r}{d t}=\frac{c \sqrt{2 ! h}}{T} \cdot \frac{a_{v}}{A} \mathrm{ft} \text {. per sec. per sec. }
$$

and the maximum pressure would be given by

$$
\begin{aligned}
p & =\frac{W l}{144 \times 32 \cdot 2} \cdot \frac{d r}{l l t} \\
& =\frac{624 l}{1+4 \times 32 \cdot 2} \cdot \frac{c \sqrt{2 g h}}{T} \cdot \frac{a_{v}}{A} \text { lbs. per sq. in. }
\end{aligned}
$$

\section{ExaMPLE.}

Pipe 1,000 ft. long fitted with full way valve. Coefficient of contraction at time of closing $\cdot 8$. Head at entrance 10 feet above that at exit. Pipe diameter 6 in. Determine excess pressure produced when valve is uniformly closed in four seconds. $f=006$. 
Here when valve is wide open we have

$$
\begin{aligned}
h & =\frac{f l v^{2}}{2 g m}+\frac{r_{v}^{2}}{2 g}, \text { and } v_{v}=v \\
\therefore \quad 10 & =\frac{r^{2}}{2 g}\left\{\frac{006 \times 1,000 \times 4}{\left.\frac{1}{2}+1\right\}}\right. \\
& =\frac{49 v^{2}}{64 \cdot 4} \\
\therefore \quad v & =3.62 \mathrm{ft} . \text { per sec. }
\end{aligned}
$$

Maximum retardation of column $=\frac{\sqrt{2 g h}}{a} \cdot \frac{d\left(a^{v}\right)}{d t}$

$$
\begin{aligned}
& =\frac{\sqrt{2 g h}}{a} \cdot c \cdot \frac{d a}{d t} \\
& =\frac{\sqrt{644} \times 8 \times a}{a \times 4}=5.08 \mathrm{ft} \text {. per sec. per sec. }
\end{aligned}
$$

If $p=$ pressure at valve in lios. per square inch necessary to produce this retardation we have

$$
\begin{aligned}
144 p a & =\frac{62 \cdot 4 \times a \times l}{32 \cdot 2} \times 5 \cdot 08 \\
\therefore p & =\frac{62 \cdot 4 \times 1,000 \times 5 \cdot 08}{32 \cdot 2 \times 144}=68 \cdot 4 \text { lbs. per sq. in. }
\end{aligned}
$$

Actually, the effect of the elasticity of the column modifies the above result considerably. To take this into account, it must be recognised that if the time taken in closing a valve be less than $2 l \div V_{p}$ seconds, the excess pressure produced by water hammer is given by $p=63.7 \bar{v}$, where $\bar{r}$ is the mean velocity of flow in the pipe when the valve begins to close.

Since, if the flow is being retarded, the change of velocity at the open end of the pipe will lag behind that at the valve by a time interval of $l \div V_{p}$ seconds, the velocity at the open end will be greater than that at the valve by an amount $\frac{l}{4,700} \cdot \frac{d r}{d t}$ $\left[\right.$ since $\left.\delta v=\frac{d v}{d t} \cdot \delta t\right]$, and the mean velocity in the pipe will be equal to that at the valve, plus $\frac{l}{9,400} \cdot \frac{d v}{d t}$.

$$
\therefore \quad \bar{v}=v+\frac{l}{9,400} \cdot \frac{d r}{d t} \text {. f.s. }
$$

As the valve is closed then, the retardation of the moving column, and the consequent pressure, increase as previously indicated, until an instant $2 l \div V_{p}$ seconds before the valve comes to its seat. At this point the valve, in effect, closes suddenly and 
we get an additional rise in pressure of the nature of hammer, the excess over that produced by the retardation being given by

$$
\left.63 \cdot 7 r+\frac{l}{9,400} \cdot \frac{d v}{d t}\right) \text { lbs. per sq. inch, }
$$

where $r$ and $\frac{d r}{d t}$ are the velocity and acceleration of the moving column at the valve.

Putting $t=\frac{2 l}{4,700}$ in equations (4) and (6), we get $r$ and $\frac{d r}{d}$ at this instant.

Thus

$$
v=\sqrt{\frac{2 g h}{\frac{f l}{m}+\left\{\frac{A \times 4,700 T}{2 a_{1} l}\right\}^{2}}} \text { ft. per sec. }
$$

while the acceleration

$$
\frac{d v}{d t}=\frac{\sqrt{2 g h}}{\left\lfloor\frac{a_{1}}{A} \cdot \frac{2 l}{4,700 T} !^{3}\left(\frac{f l}{m}+\left(\frac{A}{a_{1}} \cdot \frac{4,700 T}{2 l}\right)^{2}\right)^{\frac{3}{2}}\right.} \cdot \frac{a_{1}}{T A} \text { f.s.s. (10) }
$$

Denoting these by $r^{\prime}$ and $\frac{d v^{\prime}}{d t}$ we have the maximum pressure produced by closing the valve given by

$$
p=\left[63.7\left\{v^{\prime}+\frac{l}{9,400 d t} d v^{\prime}\right\}+\frac{62 \cdot 4 l}{144 \times 32 \cdot 2} \cdot \frac{d v^{\prime}}{d t}\right] \text { sq. in. }
$$

\section{Example.}

Pipe 6 inches diameter, 1,000 feet long, full way valve, time of closing 4 seconds. Coefficient of contraction at time of closing $=\cdot 8, f=\cdot 006$. Head at entrance 10 feet above that at exit.

$$
\text { Here } \quad v^{\prime}=\frac{25 \cdot 38}{\sqrt{48+\left\{\frac{4700 \times 4}{2 \times 800}\right\}^{2}}}=1.86 \text { f.s. }
$$

and

$$
\begin{gathered}
\frac{d r^{1}}{d t}=\frac{25.38 \times 11 \cdot 77^{3}}{(186 \cdot 1)^{\frac{3}{2}}} \times \frac{8}{4}=3.245 \text { f.s.s. } \\
\therefore \quad p=63.7\{1.860+\cdot 345\}+\frac{62 \cdot 4 \times 1,000}{144 \times 32 \cdot 2} \times 3.245 \\
=140.5+43.7=184 \cdot 2 \text { lbs. per sq. in. }
\end{gathered}
$$


as compared with the value $68 \cdot 4 \mathrm{lbs}$. determined by neglecting the effect of elasticity and using formula (8) of this article.

The fact that the pipe line is elastic, and that the theory does not take account of the slight difference between the energy leaving, and that entering the pipe during the secondary period of closure, will, especially at high pressures, tend to give a calculated pressure in excess of that actually obtained. Experiments by the author, however, indicate that for moderate pressures, the calculated and experimental values agree closely, certainly very much more closely that do results obtained by the use of the usual change-of-momentum formula.

The following (p. 223) are some results of these experiments on a pipe line $3 \cdot 6$ inches diameter and 610 feet long, fitted with a $2 \frac{1}{2}$ inch outlet valve. The pipe joints were of the ordinary socket type with lead caulking, and the fact that at pressures much in excess of $30 \mathrm{lbs}$. per square inch, the observed are considerably below the calculated values, is attributed mainly to the yielding of these joints under such pressures. In each case the valve was closed uniformly in the time stated.

The error involved in using formula (8) of this article

$$
p=\frac{62 \cdot 4 l}{144 \times 32 \cdot 2} \cdot \frac{c \sqrt{2 g h}}{T} \cdot \frac{a_{v}}{A} \text { lbs. per sq. inch }
$$

will be evident from the following table, which gives the observed results from experiments (1) and (3) above, together with values of $p$ calculated from this formula.

\begin{tabular}{|c|c|c|c|c|}
\hline \multirow{3}{*}{$\begin{array}{l}\text { Times in } \\
\text { seconds. }\end{array}$} & \multicolumn{2}{|c|}{ Experiment 1.} & \multicolumn{2}{|c|}{ Experiment 3.} \\
\hline & \multicolumn{2}{|c|}{ Pressures. } & \multicolumn{2}{|c|}{ Pressures. } \\
\hline & Observed. & Calculated. & Observed. & Calculated. \\
\hline 10 & 15 & $4 \cdot 3$ & 6 & $1 \cdot 5$ \\
\hline 8 & 18 & $5 \cdot 4$ & 7 & $1 \cdot 8$ \\
\hline 6 & 22 & $7 \cdot 2$ & 10 & $2 \cdot 5$ \\
\hline 4 & 29 & $10 \cdot 8$ & 15 & $3 \cdot 7$ \\
\hline 2 & - & - & 24 & $7 \cdot 4$ \\
\hline
\end{tabular}

A typical pressure diagram taken during the closing of the valve is shown in Fig. $89 \mathrm{~A}$. Here $A A^{\prime}$ marks the pressure 


\begin{tabular}{|c|c|c|c|c|c|}
\hline \multirow{3}{*}{ } & \multirow{3}{*}{\multicolumn{2}{|c|}{ 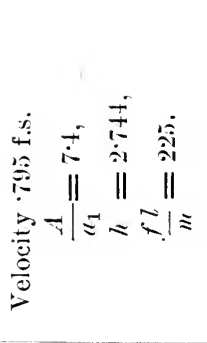 }} & \multirow{2}{*}{ 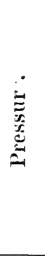 } & & 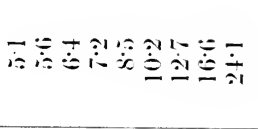 \\
\hline & & & & 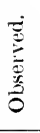 & 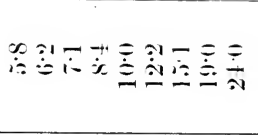 \\
\hline & & & \multicolumn{2}{|c|}{ 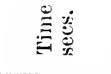 } & $00, \infty 11=0.0 .01$ \\
\hline \multirow{3}{*}{ 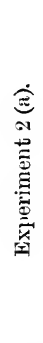 } & \multirow{3}{*}{\multicolumn{2}{|c|}{ 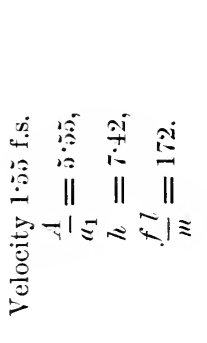 }} & \multirow{2}{*}{ 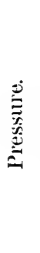 } & 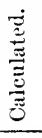 & 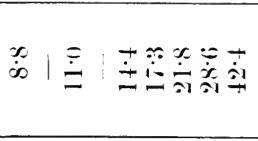 \\
\hline & & & & 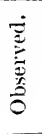 & 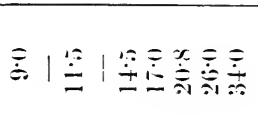 \\
\hline & & & \multicolumn{2}{|c|}{ 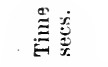 } & $\equiv=x+=10+\infty x_{1}$ \\
\hline \multirow{3}{*}{ 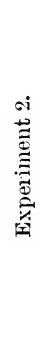 } & \multirow{3}{*}{\multicolumn{2}{|c|}{ 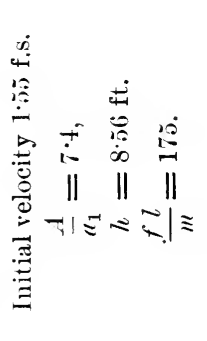 }} & \multirow{2}{*}{ 竞 } & 离 & 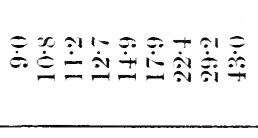 \\
\hline & & & & 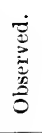 & 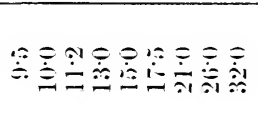 \\
\hline & & & \multicolumn{2}{|c|}{ 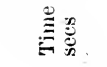 } & $\equiv=x+=x-x>10$ \\
\hline \multirow{3}{*}{ 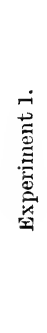 } & \multirow{3}{*}{\multicolumn{2}{|c|}{ 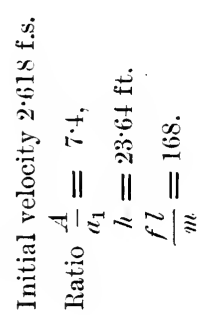 }} & \multirow{2}{*}{ 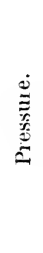 } & 离 & 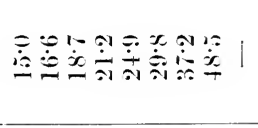 \\
\hline & & & & 突 & 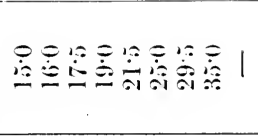 \\
\hline & & & \multicolumn{2}{|c|}{ 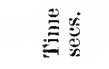 } & $0=x+=x+\infty$ \\
\hline
\end{tabular}


in the main before opening the valve, $B B^{\prime}$ the pressure at the valve with steady flow, so that the distance from $A A^{\prime}$ to $B B^{\prime}$ represents $\frac{f l r^{2}}{2 g m}$. $C$ marks the instant of closing of the valve and $C C^{\prime}$ indicates the excess pressure $p^{\prime}$ due to closure.

Both theory and practice emphasise the importance of a

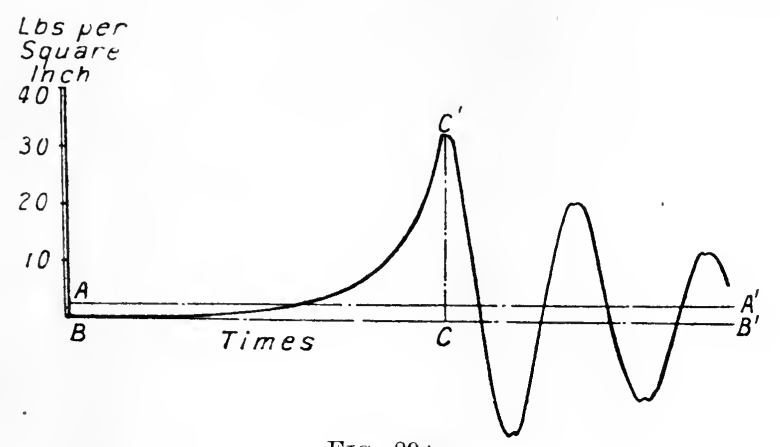

FIG. 89 A.

relatively slow motion of the valve as it approaches its seat, and particularly at the instant of closing.

Examples.

(1) Experiments by Dr. Lampe on the 16-inch asphalted pipes of the Dantzic main gave results which for velocities between 1.6 and $3 \cdot 0$ feet per second agree with the value as deduced from the formula $\frac{h}{l}=000371 \frac{r^{185}}{d^{1 \cdot 15}}$. Determine the corresponding values of $f$ in the formulae $h=\frac{f l v^{2}}{2 g m}$ when $v=1 \cdot 6$ and 3.0 feet per second.

$$
\text { Answer. }\left\{\begin{array}{l}
00531 \\
00485
\end{array}\right.
$$

(2) Darcy's experiments on a cast-iron pipe $\cdot 617$ feet diameter give the result $\frac{h}{l}=000380 \frac{v^{1 \cdot 95}}{d^{1 \cdot 05}}$ with fair accuracy. Determine the velocity of flow and the discharge per minute from a similar pipe. 1,000 feet long under a head of 50 feet.

Answer. $\left\{\begin{array}{l}\text { Velocity }=2.893 \mathrm{ft} . \text { per sec. } \\ \text { Volume }=51 \cdot 9 \mathrm{cub} . \mathrm{ft} \text {. per min. }\end{array}\right.$ 
(3) The following is a convenient mnemonic for flow in clean cast-iron pipes of diameters between 2 and 6 inches with a velocity of 3 feet per second $h=\frac{l}{25 d}$ where $h$ is in feet, $d$ in inches. Taking Unwin's exponential formula as being correct, determine the percentage error for pipe diameters $3,6,9$, and 12 inches.

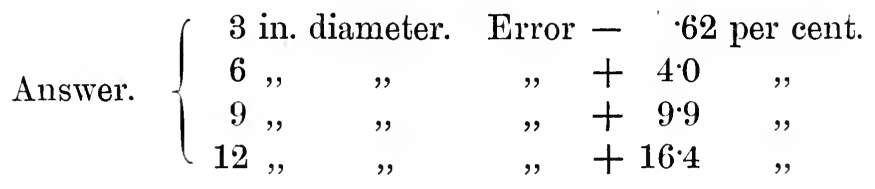

(4) W. Cox (Engineering (1892), p. 613) gives the following formula for flow in clean C. I. pipes :- $h=\frac{C l}{d \text { (in.) }}$, where $C$ has the following values :-

\begin{tabular}{c|c|c|c|c|c|c|c|c|c|c}
$\begin{array}{c}\text { Velocity (ft. } \\
\text { per sec.) }\end{array}$ & 1 & 2 & 3 & 4 & 5 & 6 & 7 & 8 & 9 & 10 \\
\hline$C$ & $\cdot 00583$ & -020 & $\cdot 0408$ & $\cdot 0683$ & $\cdot 1025$ & $\cdot 143$ & $\cdot 191$ & $\cdot 245$ & $\cdot 306$ & $\cdot 373$ \\
\hline
\end{tabular}

Compare these with the values obtained by using the curves (Fig. 84), and determine the per cent. difference for a 3 inch and 6 inch pipe at velocities of $2,4,6$, and 10 feet per second.

Answer.

\begin{tabular}{|c|c|c|c|c|c|}
\hline & & \multicolumn{4}{|c|}{ Velocity f.s. } \\
\hline \multirow{3}{*}{$\begin{array}{l}\text { The per cent. error } \\
\text { of the approximate } \\
\text { formula is }\end{array}$} & & 2 & 4 & 6 & 10 \\
\hline & 3 in. pipe & $+10 \%$ & $-2 \cdot 38 \%$ & $-7 \cdot 13 \%$ & $-11 \cdot 15 \%$ \\
\hline & 6 in. pipe & $+14.7 \%$ & $+2 \cdot 13 \%$ & $-2 \cdot 0 \%$ & $-7 \cdot 0 \%$ \\
\hline
\end{tabular}

(5) Using the curves of Figs. 84, 85, 86, determine the loss of head per 100 feet of pipe in each of the following cases :-

(a) 36 inch asphalted pipe, butt joints and countersunk rivets, $v=3$ f.s.

H.A. 
(b) 36 inches asphalted pipe, in and out rivetting, projecting rivets, new pipe, $v=3$ f.s.

(c) 36 inches asphalted years, $v=3$ f.s.

(d) 4 inch clean C. I. pipe, $v=4$ f.s.

(e) 4 inch old $\quad, \quad r=4$ f.s.

$$
\begin{gathered}
\text { Answer. } a=\cdot 071 \text { feet } ; b=\cdot 124 \text { feet } c=\cdot 142 \text { feet } ; \\
d=2 \cdot 39 \text { feet; } e=5 \cdot 21 \text { feet. }
\end{gathered}
$$

(6) The jet of a Pelton wheel has an effective diameter of 2 inches. The supply pipe is 6,000 feet long and is an asphalted pipe, 15 inches diameter, and the supply head is 800 feet. Determine the probable discharge in cubic feet per second, and the horse-power of the jet. Assume $c_{v}=\cdot 972: f=\cdot 0050$.

Answer. Velocity of jet $=218$ f.s.; discharge $=4 \cdot 75$ cubic feet per second; 396 horse-power.

(7) With a given slope, a clean cast iron pipe 9 inches diameter, is found to give a discharge of 25,000 gallons per hour. Determine the necessary diameter of pipe, having twice the slope, to give a discharge of 50,000 gallons per hour. (Take Unwin's value for the index of $d$ and of $i$, Art. 69.)

Answer. 10.3 inches.

(8) The outlet valve from a main 5,000 feet long is closed in 1 second. Determine the rise in pressure if the initial velocity of flow is 4 feet per second.

Answer. $254.8 \mathrm{lbs}$. square inch.

(9) If the pipe of the preceding question is fitted with an outlet valve of the same effective area as the pipe, and if this valve is uniformly closed in 5 seconds, determine the rise in pressure due to this closing. Take the coefficient of contraction for the valve as having a value of 8 just before closing. Head at pipe entrance $=3.25$ feet, $f=\cdot 006$, diameter $=12$ inches.

\section{Answer.}

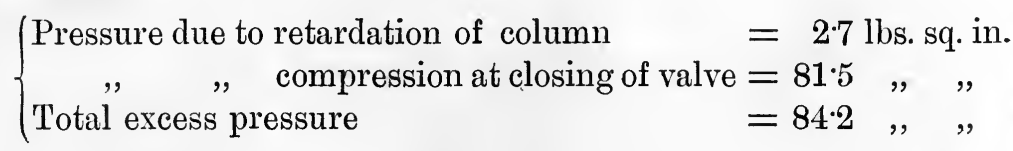


(10) If in the preceding example the head at the pipe entrance $=30.1$ feet, so as to give the water an initial velocity of flow of 4 feet per second as in example (8), determine the rise in pressure due to the valve being uniformly closed in 5 seconds.

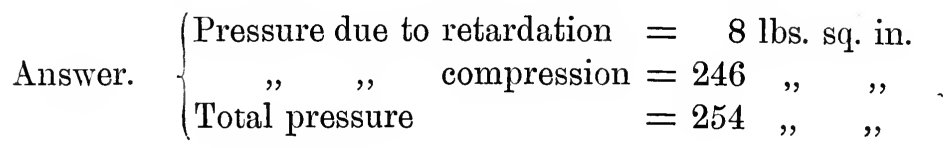




\section{CHAPTER VIII.}

Losses in a pipe line-Hydraulic gradient-Losses at Valves-Bends-ElbowsExit-Flow in long pipes-Time of discharge through pipe line-Equivalent diameter of uniform main-Branch mains-Multiple supply-Bye-pass-Flow through nozzles-Form of nozzle-Maximum discharge of kinetic energy through a nozzle-Syphons-Inverted syphon.

\section{Art. 71.-Pipe Line Losses.}

Is constructing a pipe line to connect two reservoirs, or to distribute the discharge from any reservoir, the problem which usually presents itself to the engineer is that of determining the minimum size of pipe which, under given conditions of head loss, shall be capable of discharging a given quantity of water per minute. In the construction of a supply pipe line for a power station, the problem is much the same, and in every case it is first of all necessary to determine the conditions involving loss of head, and the magnitude of these losses.

Commencing at the supply end of the pipe, we have

(1) Loss due to production of kinetic energy, and in some cases also to eddy formation at the entrance to the pipe.

(2) Frictional losses in the pipe itself.

(3) Losses at valves, sluices, etc.

(4) Losses at all elbows, bends, or deviations from the straight.

(5) Losses at pipe junctions.

(6) Losses at sudden enlargements, or contractions in the area of the pipe.

(7) Loss at exit, due to the rejection of kinetic energy.

In every case these losses are approximately proportional to the square of the velocity, so that if $v$ be the velocity we may write total loss of head $={ }_{1} H_{7}=F v^{2}$.

Where a pipe line connects two reservoirs, the difference of 
head between their free surfaces must then equal $F v^{2}$, for the pipe to run full with velocity $r$.

If in Fig. 90 a horizontal $A B$ be drawn through the upper free surface, and if a series of ordinates be drawn vertically downwards from $A B$ representing on the vertical scale of the drawing the total loss of pressure energy per lb. from the pipe entrance to the particular point considered, the ends of all such ordinates being connected give a curve called the hydraulic gradient for the pipe line. If now any datum line $C D$ be taken, such that the height $C A$ represents the potential energy of the water at $A$, the distance at any point from $C D$ to the hydraulic gradient

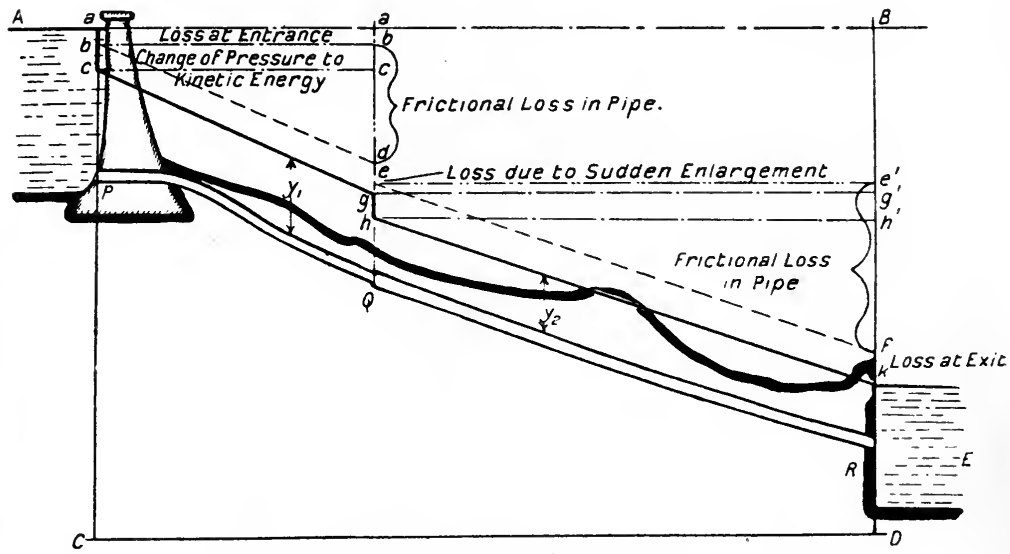

FIG. 90.-Hydraulic Gradient.

will evidently represent the head available for producing flow at that point, while the pressure energy at all points on the hydraulic gradient will be zero. It follows that if a series of open stand pipes are erected at various points on the pipe line the free surfaces in these pipes will lie on the gradient line, while the pressure in the pipe at any point will be measured by the vertical distance $y_{1}, y_{2}$, etc., of that point below the gradient.

The slope of the hydraulic gradient is termed the "virtual slope " of the pipe.

Figure 90 represents the hydraulic gradient for the pipe line 
shown in elevation, which consists of a parallel pipe $P Q$, having a sudden enlargement of section at $Q$, remaining parallel from $Q$ to $R$, and discharging at $R$ into the service reservoir $E$.

Here $a b$ represents frictional and eddy loss at entrance $: b d$ represents frictional loss from $P$ to $Q=\frac{f l_{1} v_{1}^{2}}{2 g m_{1}}$ ft.-lbs. per lb. : de represents loss due to sudden enlargement of section $=\frac{\left(v_{1}-v_{2}\right)^{2}}{2 g}$ ft.-lbs. per lb. : $e^{\prime} f$ represents loss due to friction between $Q$ and $R=\frac{f l_{2} r_{2}^{2}}{2 g m_{2}}$ ft.-lbs. per lb. $: f k$ represents loss due to rejection of kinetic energy at exit.

The vertical distance from the datum to the broken line $a b d$ e $f k$, now represents the sum of the (kinetic + pressure + potential) energies at any point, and if $c g$ be drawn parallel to $b d$ at a vertical distance $b c$ below $b d$, equal to $\frac{r_{1}^{2}}{2 g}$, the distance from the datum to the line $c g$ now gives the sum of the (pressure + potential) energies at any point from $P^{\prime}$ to $Q$. If similarly a line $h k$ be drawn parallel to $e f$, at a vertical distance below this equal to $\frac{r_{2}^{2}}{2 g}$, the whole line $a b c g h k$ now represents the hydraulic gradient for the pipe.

The pressure in the pipe line will be everywhere greater than that of the atmosphere, so long as the pipes nowhere rise above the hydraulic gradient. If part of the pipe line be laid above the gradient line, the pressure in this portion of the pipe will be less than atmospheric, and any leakage at a joint allows air to be drawn in, with a possible stoppage of flow. If the pipe rises above the gradient line by a distance equivalent to the barometric height, 34 feet, the flow will of necessity stop completely. Owing to the discharge of occluded air from water at low pressures, the maximum height practically attainable is however much less than this. The syphon (Art. 81) is an instance of the pipe line being above the hydraulic gradient.

If then the suffixes $A$ and $E$ refer to the surfaces in the two reservoirs and if $Z_{A}$ and $Z_{E}$ are the heights of these free surfaces 
above some datum, we have, if the pipe discharges below the surface in the lower reservoir

$$
\begin{aligned}
Z_{A}-Z_{E}={ }_{A} H_{E} & =\text { losses at entrance }+ \text { losses in pipe } \\
& + \text { losses at exit. }
\end{aligned}
$$

Art. 72.-Detalled Losses in Pipe Line.

\section{(1) Losses at Entrance.-}

These depend on the form of entrance adopted. Thus with a bell mouthpiece the loss of head is about $05 \frac{r^{2}}{2 g}$ feet of water (Art. 42), while with a pipe projecting into the reservoir and

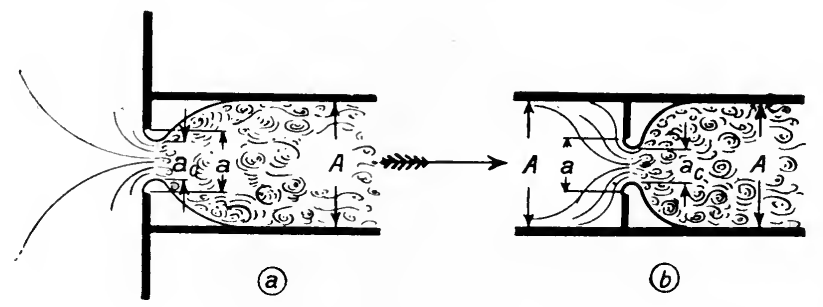

Fig. 91.

forming a re-entrant mouthpiece, the loss, when running full, is $\frac{r^{2}}{2 g}$ feet of water (Art. 40).

Where the pipe opens flush with the side or bottom of the reservoir the loss of head becomes about $375 \frac{v^{2}}{2 g}$ feet (Art. 41).

From what has already been said, it will be seen that this loss of energy occurs simply in getting the water into the pipe, due to the formation of a vena contracta and the subsequent re-enlargement of the stream to fill the pipe. This reduces the energy available for producing motion along the pipe and gives a reduced flow with the same total head.

(2) Frictional Losses in the Pipe.-

These have been considered in Arts. 63 to 65 .

(3) Losses at Valres, etc.-

The loss of energy caused by the presence of a valve or sluice 
in a pipe line may be looked upon as being due to the sinuous motion set up by the expansion of the stream to fill the pipe, after its contraction in passing the valve, and, by an application of the formulae deduced on p. 83, this loss can, except for irregularities in design which themselves cause sinuous motion, be approximately determined.

Much work in this direction has been done by Weisbach, to whom the following experimental results are due.

With a diaphragm having an aperture $a$ leading from a reservoir of large area into a pipe of area $A$ (Fig. $91 \mathrm{a}$ ), calling the loss $\left.\frac{A}{\left(C_{c} a\right.}-1\right)^{2} \frac{v^{2}}{2 g}=F \frac{r^{2}}{2 g}$, the following values were obtained. Here $C_{c} a=$ area of rena contracta.

\begin{tabular}{c|c|c|c|c|c|c|c|c|c|c}
\hline$\frac{a}{A}$ & $\cdot 1$ & $\cdot 2$ & $\cdot 3$ & $\cdot 4$ & $\cdot 5$ & $\cdot 6$ & $\cdot 7$ & $\cdot 8$ & $\cdot 9$ & $1 \cdot 0$ \\
\hline$C_{c}$ & $\cdot 616$ & $\cdot 614$ & $\cdot 612$ & $\cdot 610$ & $\cdot 607$ & $\cdot 605$ & $\cdot 603$ & $\cdot 601$ & $\cdot 598$ & $\cdot 596$ \\
\hline$F$ & $\frac{\cdot 61 \cdot 7}{231 \cdot 7}$ & $\frac{50 \cdot 99}{19 \cdot 78}$ & $\frac{9 \cdot 612}{5 \cdot 256}$ & $\frac{3 \cdot 07}{3 \cdot 0}$ & $\frac{1 \cdot 876}{1 \cdot 169}$ & $\frac{\cdot 734}{\cdot 480}$ \\
\hline
\end{tabular}

With a diaphragm inserted in a pipe of area $A$ (Fig. $91 b$ ) the following values were obtained.

$$
\text { Loss }=\left(\frac{A}{C_{c} a}-1\right)^{2} \frac{v^{2}}{2 g}=F \frac{r^{2}}{2 g} .
$$

\begin{tabular}{|c|c|c|c|c|c|c|c|c|c|c|}
\hline$\frac{a}{A}$ & $\cdot 1$ & $\cdot 2$ & $\cdot 3$ & $\cdot 4$ & כ) & $\cdot 6$ & $\cdot \tau$ & $\cdot 8$ & $\cdot 9$ & $1 \cdot 0$ \\
\hline$C_{c}$ & $\cdot 624$ & $\cdot 632$ & $\cdot 643$ & 659 & $\cdot 681$ & $\cdot 712$ & -75.5 & $\cdot 813$ & $\cdot 892$ & $1 \cdot 00$ \\
\hline$F$ & ():5:5 & $47 \cdot 77$ & $30 \cdot s 3$ & $7 \cdot 80$ & $3 \cdot 753$ & $1 \cdot 796$ & $\cdot 797$ & $\cdot 29$ & $\cdot 06$ & $\cdot 00$ \\
\hline
\end{tabular}

A formula connecting the area of the rena contracta with that of the aperture in the diaphragm, in terms of the relative area of pipe and of aperture, is given by Rankine, and is

$$
C_{c}=\frac{a_{c}}{a}=\frac{618}{\sqrt{1-618 \frac{a^{2}}{d^{2}}}} .
$$


Thus when $a=A, C_{c}=1$.

" $\quad a$ is very small, $C_{c}$ has the limiting value $\cdot 618$.

The effect of the portion of the pipe before the orifice is to produce a closer approximation to parallelism of the stream lines, and in consequence a vena contracta of larger area is obtained, with a reduced after-enlargement, and hence less loss than where this constraint is absent.

Loss caused by Sluice in Rectangular Pipe (Fig. 92 a).-

Depth of pipe $D$. Depth of sluice opening $d$.

\begin{tabular}{c|c|c|c|c|c|c|c|c|c|c}
\hline$\frac{d}{D}$ & $\cdot 1$ & $\cdot 2$ & $\cdot 3$ & $\cdot 4$ & $\cdot 5$ & $\cdot 6$ & $\cdot 7$ & $\cdot 8$ & $\cdot 9$ & $1 \cdot 0$ \\
\hline$F$ & 193 & $44 \cdot 5$ & $17 \cdot 8$ & $8 \cdot 12$ & $4 \cdot 02$ & $2 \cdot 08$ & $\cdot 95$ & $\cdot 39$ & $\cdot 09$ & $\cdot 00$ \\
\hline
\end{tabular}

Here contraction is prevented in three directions, and for small

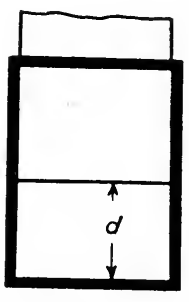

(a)

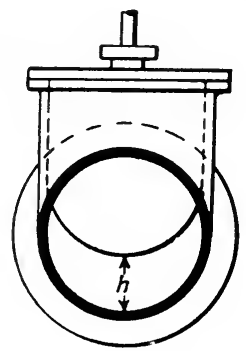

(b)

FIG. 92.

openings the result of this is marked by the reduction in the value of $F$.

Gate Valve in Circular Pipe (Fig. $92 b$ ).

Let $h=$ ratio of height of opening to diameter of pipe.

\begin{tabular}{|c|c|c|c|c|c|c|c|c|}
\hline$h$ & $\frac{1}{8}$ & $\frac{1}{4}$ & $\frac{3}{8}$ & $\frac{1}{2}$ & $\frac{5}{8}$ & $\frac{3}{4}$ & $\frac{7}{8}$ & $1 \cdot 0$ \\
\hline$F^{\prime}$ & $97 \cdot 8$ & $17 \cdot 00$ & $5 \div 2$ & $2 \cdot 06$ & $\cdot 81$ & $\cdot 26$ & .07 & .00 \\
\hline
\end{tabular}


Cock in Cylindrical Pipe (Fig. 93 a).

Here $a=$ area of section through valve : $A=$ area of pipe : $\theta=$ angle through which valve is turned.

\begin{tabular}{c|c|c|c|c|c|c|c|c|c}
\hline$\theta$ & $5^{\circ}$ & $10^{\circ}$ & $15^{\circ}$ & $20^{\circ}$ & $25^{\circ}$ & $30^{\circ}$ & $355^{\circ}$ & $40^{\circ}$ & $45^{\circ}$ \\
\hline$\frac{a}{A}$ & $\cdot 93$ & $\cdot 85$ & $\cdot 77$ & $\cdot 69$ & $\cdot 61$ & $\cdot 535$ & $\cdot 46$ & $\cdot 385$ & $\cdot 315$ \\
\hline$F$ & $\cdot 05$ & $\cdot 29$ & $\cdot 75$ & $1 \cdot 56$ & $3 \cdot 1$ & $5 \cdot 47$ & $9 \cdot 68$ & $17 \cdot 3$ & $31 \cdot 2$ \\
\hline
\end{tabular}

\begin{tabular}{c|c|c|c|c|c}
\hline$\theta$ & $50^{\circ}$ & $55^{\circ}$ & $60^{\circ}$ & $65^{\circ}$ & $82^{\circ}$ \\
\hline$\frac{a}{A}$ & $\cdot 25$ & $\cdot 19$ & $\cdot 14$ & $\cdot 09$ & $\begin{array}{c}\text { Valve } \\
\text { closed. }\end{array}$ \\
\hline$F$ & $52 \cdot 6$ & 106 & 206 & 486 & - \\
\hline
\end{tabular}

Throttle Valve in Cylindrical Pipe (Fig. 93 b).

\begin{tabular}{c|c|c|c|c|c|c|c|c|c}
\hline$\theta$ & $\circ$ & $10^{\circ}$ & $20^{\circ}$ & $30^{\circ}$ & $40^{\circ}$ & $50^{\circ}$ & $60^{\circ}$ & $70^{\circ}$ & $90^{\circ}$ \\
\hline$F^{\prime}$ & $\cdot 24$ & $\frac{52}{1 \cdot 54}$ & $\frac{3 \cdot 91}{10 \cdot 8}$ & $32 \cdot 6$ & 118 & 751 & - \\
\hline
\end{tabular}

These experiments were carried out on pipes and valves of slightly under 2 inches diameter.

Experiments carried out on a gate valve for a 24 -inch pipe, ${ }^{1}$

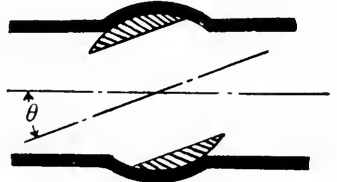

(a)

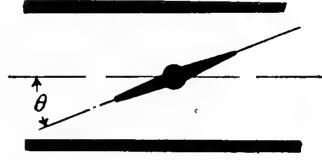

(b)

FiG. 93.

gave values of $F$ about 50 per cent. greater than those of Weisbach, down to a quarter of full gate.

This loss due to alteration in area and shape of section is serious in all hydraulic machinery when working at high

J Kuichling, "Transactions American Society Civil Engineers," 1892, vol. 26. 
velocities, and especially in machines of the piston type. Here, for efficiency of working, it is above all things important that the velocities should be kept as low as possible.

(4) Losses at Bends and Elbous.

Whenever the direction of motion of a stream is abruptly changed, as at a sharp bend or elbow, a loss of head is experienced which appears to be due to the formation of a vena-contracta on passing the elbow and to the subsequent re-enlargement and shock which then takes place (Fig. 94). With an easy bend recent experiments ${ }^{1}$ by Dr. Brightmore show that the state of affairs is very different, the water flowing round the bend with a

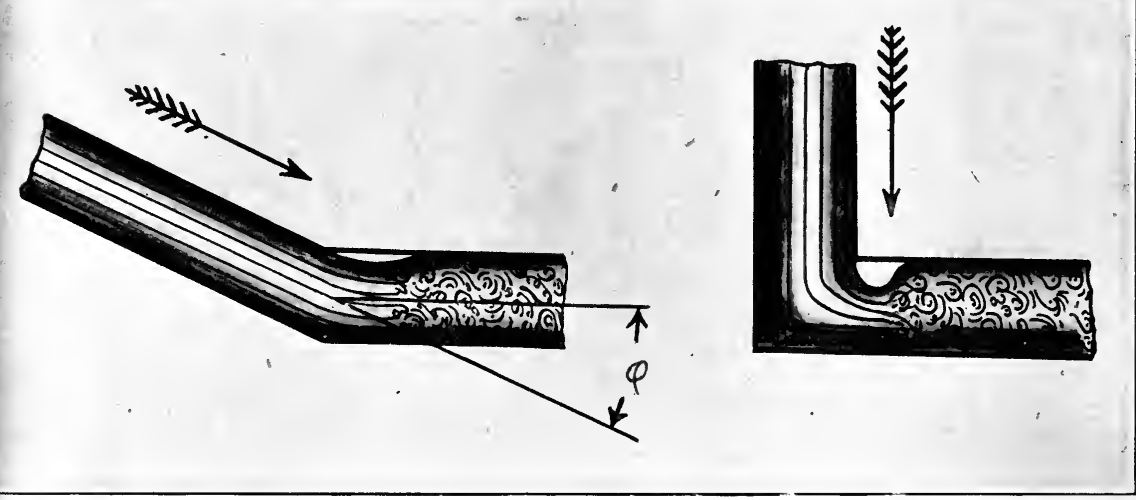

Fig. 94.

motion approximating to that in a free vortex, its velocity being greatest at the inside and least at the outside of the bend. In these experiments it was shown that, in such a case the loss due to the bend does not occur so much in the bend itself as in the portion of straight pipe immediately following, where the equalization of velocities in the stream of water gives rise to eddy production. Using cast iron pipes of $3^{\prime \prime}$ and $4^{\prime \prime}$ diameter, the loss in the bend itself was found to be almost identical with (sometimes less than) the loss in a corresponding length of straight pipe." From this point 1 "Proceedings Institute Civil Engineers," vol. 169, 1906-07, p. 315. 
of view it is probable that the loss occurring with a bend of uniform curvature will be largely independent of the angle through which the bend is taken, and that a bend of say $45^{\circ}$ will cause little less resistance than one of $90^{\circ}$. Also, as is confirmed by experiment, the resistance caused by a reflex bend will be much greater than that caused by two similar bends having continuous curvature in one direction.

The first experiments of any note on the resistance of bends are due to Weisbach, who from experiments on pipes of $1 \frac{1}{4}$ inches diameter, deduced the formula.

$$
h_{B}=\text { loss of head due to bend }=F \frac{v^{2}}{2 g} \times \frac{\theta^{\circ}}{180^{\circ}} \text { feet, }
$$

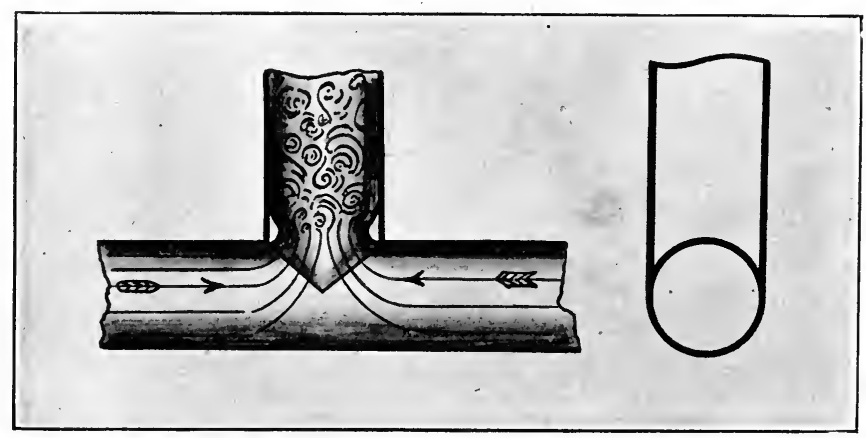

FIG. 95.

where $\theta$ is the angle through which the bend is carried.

For circular pipes, $F^{\prime}$ has, the value $\left[131+1.847\left(\frac{r}{R}\right)^{\frac{7}{2}}\right]$ where $r$ is the radius of the pipe, and $R$ the radius of the bend.

For pipes of rectangular section, $F=\cdot 124+3 \cdot 104\left(\frac{S}{2 R}\right)^{\frac{1}{2}}$ where $S$ is the length of side of a section parallel to the radius of curvature of the bend.

These experiments were, however, not numerous and were only carried out on bends of small radius, so that not much reliance is to be placed in the results. 
For sharp bends or elbows (Fig. 94), Weisbach deduced the formula

$$
\begin{gathered}
\text { Loss }=F \frac{v^{2}}{2 g} \text { feet } \\
\text { where } F^{\prime}=.946 \sin ^{2} \frac{\theta}{2}+2.05 \sin ^{4} \frac{\theta}{2},
\end{gathered}
$$

$\theta$ being the angle of deviation of the elbow.

With a tee branch pipe (Fig. 95) the loss is much the same as in a right-angled elbow.

Experiments by Brightmore ${ }^{1}$ on right-angled elbows on 3-inch and 4-inch pipes, gave a value of $F=1 \cdot 17$.

Experiments by Alexander ${ }^{2}$, by Williams ${ }^{3}$, and by Brightmore, the former using varnished wooden pipes of $1 \frac{1}{4}$ inches diameter, Williams using asphalted pipes of 12, 16 and 30 inches diameter, and Brightmore using cast iron pipes of 4 inches diameter, indicate that the additional loss due to the curvature of a pipe does not, as might be expected, diminish uniformly as the radius of curvature increases, but, after attaining a minimum value for a value of $R=5 r$ (Williams and Alexander), $R=7.5 r$ (Brightmore), increases slightly to a point where $R=$ about $13 r$, and afterwards diminishes to zero with a curve of infinite radius. The experiments further show that the power of $v$, to which the loss is proportional in a gradual bend, is the same for the bend as for the straight pipe. Alexander, as the result of his own and of Williams' experiments, concludes that the additional loss due to a bend of radius $R=5 r$, is equivalent to that offered by a length of straight pipe equal to $3 \cdot 38 l$, where $l$ is the length of the curved portion of the pipe. Thus if the angle of the bend is $90^{\circ}$ this makes the equivalent length equal to $13.3 d$, where $d=$ pipe diameter in feet. This agrees with the results of a number of experiments made at the Yorkshire Colleget, from which it was concluded that the resistance of an easy right-angled bend is equivalent to that in

1 "Proceedings Institute Civil Engineers," vol. 169, 1906-07, p. 315.

2 " Proceedings Institute Civil Engineers," vol. 159, p. 341.

3 "Transactions American Society Civil Engineers," 1901, p. 314.

4 Engineering, September 25 1896, p. 390. 
a straight pipe 10 to 15 diameters in length, while for a sharp right-angled bend or elbow the equivalent length is from 30 to 36 diameters.

Brightmore's results point to the fact that for all curves of the best radius, for all diameters of cast iron pipes and for all velocities, the additional loss of head due to the curvature in a right-angled bend is equal to $3 \frac{v^{2}}{2 g}$ feet.

(5) Losses at Sudden Changes in Section of the Pipe.

These have been already considered in Art. 31 .

(6) Losses at the Exit from the Pipe.

Where a pipe discharges below the surface, it is possible by using a diverging outlet, to convert a certain proportion, up to about $\frac{2}{3}$, of the kinetic energy of the escaping water into pressure energy. With this exception the whole of the kinetic energy is dissipated in eddy production in the surrounding mass of water.

With a given difference of surface level in the two reservoirs, the depth of immersion of the pipe entrance or of its exit does not in the least affect the flow, since, although the difference of level between entrance and exit is increased by lowering the exit or by reducing the depth of immersion of the inlet, yet the pressure at which the water is discharged is increased, and that at which it enters the pipe line is reduced, by a corresponding amount, so that the effective head producing flow is unchanged.

Where discharge takes place above the free surface, the pressure at the exit is now atmospheric, and the energy at this point exists solely in the potential and kinetic form. If $h_{e}$ represents the height of the outlet above the free surface $E$, the equation of energy now becomes

$$
Z_{A}-Z_{E}={ }_{A} H_{E} \doteq F \frac{v^{2}}{2 g}+h_{e}
$$

or $Z_{A}-\left[Z_{E}+h_{e}\right]=$ loss at entrance + losses in pipe $+\frac{\eta^{2}}{2 g}$, the whole of the kinetic energy being rejected in this case.

Where a pipe is composed of a series of lengths having different 
diameters, the total loss of head in the pipe will be given by ะ $\left(F \frac{r^{2}}{2 g}\right)$, where $F \frac{r^{2}}{2 g}$ represents the loss in any length of pipe having the velocity of flow $r$. Since the velocities in the different sections of the pipe are inversely as the areas of these sections, the whole series of losses can then be expressed in terms of a single velocity, and this having been determined the discharge may be calculated.

As an example of the application of this reasoning to the solution of a specific case consider the following.

\section{Example.}

A 6 -inch main takes water directly out of the side of a reservoir at a depth of 10 feet. At the end of the first mile, in the course of which there are six right-angled bends having a radius of curvature of 6 inches, it is suddenly contracted to 3 inches diameter, and remains of this diameter for 2 miles, until its exit directly into the side of a second reservoir at a point 2 feet below the surface. The difference of surface level in the two reservoirs is 220 feet. Determine the flow through the pipe per minute.

Taking the losses in the order in which they occur we have

(1) Loss at entrance $=\cdot 5 \frac{v_{1}^{2}}{2 g}$ (in eddy formation), where $v_{1}=$ velocity in 6 -inch pipe.

(2) Frictional losses in one mile of 6 -inch pipe. Taking

$$
\begin{aligned}
f=010 \text { this becomes } \frac{f l v_{1}^{2}}{2 g m} & =\frac{010 \times 5280 \times r_{1}^{2}}{2 g \times\left(\frac{1}{2}\right)} \mathrm{ft} . \\
& =422 \cdot 4 \frac{r_{1}^{2}}{2 g} \mathrm{ft} .
\end{aligned}
$$

(3) Loss at six bends in 6-inch pipe.

$$
\begin{aligned}
& =6\left[\cdot 065+923\left[\frac{3}{6}\right]^{\frac{7}{2}}\right] \frac{v_{1}^{2}}{2 g} \mathrm{ft.} \\
& =88 \frac{v_{1}^{2}}{2 g} \mathrm{ft.}
\end{aligned}
$$

(4) Loss at sudden change of section. 
Assuming a vena contracta to be formed of sectional area equal to 66 that of the 3 -inch pipe, we have, if $v_{2}$ be the velocity in this pipe,

$$
\text { Loss }=\frac{v_{2}^{2}}{2 g}\left\{\frac{1}{66}-1\right\}^{2}=\frac{.266 v_{2}^{2}}{2 g} \mathrm{ft} .
$$

Since $\frac{v_{2}}{v_{1}}=\frac{6^{2}}{3^{2}}=4$, we have loss at change of section $=4 \cdot 25$ $\frac{v_{1}^{2}}{2 g}$ feet.

(5) Friction loss in 3-inch pipe.

This equals $\frac{f l r_{2}^{2}}{2 g m}=\frac{16 f l v_{1}^{2}}{2 g m}$

$$
\begin{aligned}
& =\frac{16 \times 01 \times 2 \times 5,280}{\left(\frac{1}{4}\right)} \cdot \frac{r_{1}^{2}}{2 g} \text { feet } \\
& =27033 \cdot 6 \frac{r_{1}^{2}}{2 g} \text { feet. }
\end{aligned}
$$

(6) Loss at exit.

Since the whole of the kinetic energy is thrown away, the loss will be $\frac{v_{2}^{2}}{2 g}=16 \frac{v_{1}^{2}}{2 g}$ feet.

$$
\begin{aligned}
\left.\therefore \begin{array}{c}
\text { The total loss } \\
\text { of head }
\end{array}\right\} & =\frac{v_{1}^{2}}{2 g}\{\cdot 5+422 \cdot 4+\cdot 9+4 \cdot 2+27033 \cdot 6+16\} \\
& =27477 \cdot 6 \frac{\dot{v}_{1}{ }^{2}}{2} \text { feet. }
\end{aligned}
$$

Since the pressures at the entrance $B$ and exit $D$ are those corresponding to their depths below the free surfaces, viz., 10 feet and 2 feet, we have, neglecting the velocity before and after leaving the pipe-

Head before entering pipe $=Z_{B}+10=Z_{A}$.

Head after leaving pipe $=Z_{D}+2=Z_{E}$.

$\therefore \quad$ Loss of head in pipe $=Z_{A}-Z_{E}=220$ feet.

$$
\begin{aligned}
\therefore \quad 27,477 \frac{v_{1}^{2}}{2 g} & =220 \\
\therefore \quad v_{1} & =\sqrt{\frac{64 \cdot 4 \times 220}{27,477}}=\cdot 717 \text { feet per second, } \\
\therefore \quad v_{2} & =2.868 \text { feet per second. }
\end{aligned}
$$


$\therefore$ Quantity flowing per second $=\frac{717 \times \pi}{16}$ cubic feet,

$=\cdot 141$ cubic feet per second.

In gallons per minute this gives $\{\cdot 141 \times 6 \cdot 24 \times 60\}$.

$=52 \cdot 8$ gallons per minúte.

Art. 73.-Flow in Long Pipes.

A consideration of the preceding example will show that in a long pipe the losses at bends, at entrance and exit, and at ehanges of section, are usually so small in comparison with the friction losses as to be negligible, so that for a long pipe connecting two reservoirs, the whole resistance may be taken to be given by

$\Sigma \frac{f l v^{2}}{2 ! m}=\frac{f}{2 g}\left\{\frac{l_{1} v_{1}^{2}}{m_{1}}+\frac{l_{2} r_{2}^{2}}{m_{2}}+\frac{l_{3} r_{3}^{2}}{m_{3}}+---\right\}$ feet head $v_{1}, v_{2}$, etc., being the velocities, and $m_{1}, m_{2}$, etc., the hydraulic mean depths, in the lengths $l_{1}, l_{2}$, etc., of the pipe.

In short pipes the losses due to velocity changes become of greater importance as the length of pipe diminishes, and for pipes of lengths less than 50 diameters will, in general, be allimportant.

It may be required to find the diameter of pipe necessary to give a certain discharge between two reservoirs, the difference of level in the two reservoirs being fixed.

Here, expressing the fact that the total difference of head is equal to the sum of the pipe losses, we have, if

$l=$ length $; d=$ diameter of pipe $; v=$ velocity of flow;

$H=$ difference of head in reservoirs; $Q=$ quantity required per second in cubic feet.

$$
H=\frac{K^{\prime} v^{2}}{2 g} \text { (at entrance) }+\frac{4 f l v^{2}}{2 g l} \text { (in friction) }+\frac{K^{\prime \prime} v^{2}}{2 g} \text { (at }
$$
exit).

If we have any bends or obstructions in the pipe, the losses caused by these may all be expressed as $K^{\prime \prime \prime} \frac{r^{2}}{2 g}$, so that if 
$K=\left(K^{\prime}+K^{\prime \prime}+K^{\prime \prime \prime}\right)$ we have

$$
H=\frac{v^{2}}{2 !}\left\{K+\frac{4 f l}{d}\right\} \text { feet. }
$$

Also $Q=v \times$ area of pipe, $\therefore v=4 Q \div \pi d^{2}$

$$
\therefore \quad H=\frac{16 Q^{2}}{2 g \pi^{2} d^{4}}\left\{K+\frac{4 f l}{d}\right\} \text { feet, }
$$

an equation from which the value of $d$ may be obtained by graphical solution, by trial, or by successive approximations.

\section{Art. 74.-Time of Discharge Through an Uniforis Pipe Line.}

If two reservoirs of area $A_{1}$ and $A_{2}$ are connected by a single pipe of diameter $d$ and length $l$, and if $v$ be the velocity in the pipe when $h$ is the difference in surface level in the two reservoirs, we have

$$
h=\frac{v^{2}}{2 g}\left\{K+\frac{4 f l}{D}\right\} \text { feet }
$$

where $K=$ coefficient of loss at entrance and exit.

If the pipe is long, this may be written

$$
h=\frac{r^{2}}{2 g}\left\{\frac{4 f l}{d}\right\} \text { without sensible erior. }
$$

In this case $r=\sqrt{\frac{2 g h d}{4 f l}}$ feet per second.

Also $-\frac{d h}{d t}=$ relative velocity of surfaces $A_{1}$ and $A_{2}$

$$
\begin{aligned}
& =v \cdot \frac{\pi d^{2}}{4}\left\{\frac{1}{A_{1}}+\frac{1}{A_{2}}\right\} \\
\therefore \quad-\frac{d h}{d t} & =\sqrt{\frac{2 g d}{4 f l} \cdot \frac{\pi d^{2}}{4}} \cdot\left\{\frac{1}{A_{1}}+\frac{1}{A_{2}}\right\} \sqrt{h_{0}} .
\end{aligned}
$$

The time $\left(t_{2}-t_{1}=t\right)$ necessary to reduce the difference in level from $H_{1}$ to $H_{2}$ is then got by integrating this expression between the given limits

$$
\begin{aligned}
\therefore \quad t & =\frac{8 \sqrt{4 f} l}{\pi d^{2}\left\{\frac{1}{A_{1}}+\frac{1}{A_{2}}\right\} \sqrt{2 g d}}\left\{H_{1}^{\frac{1}{2}}-H_{2}^{\frac{1}{2}}\right\} \text { secs. } \\
& =\frac{2 \sqrt{f l}}{\pi\left\{\frac{1}{A_{1}}+\frac{1}{A_{2}}\right\} d^{\frac{5}{2}}}\left\{H_{1}^{\frac{1}{2}}-H_{2}^{\frac{1}{2}}\right\} \text { secs. }
\end{aligned}
$$


Thus the time of filling a reservoir $A_{2}$ from a second reservoir $A_{1}$, whose level remains constant, i.e., $A_{1}=\infty$ is given by

$$
t=\frac{2 \sqrt{f} l \cdot A_{2}}{\pi d^{\frac{5}{2}}}\left\{H_{1}^{\frac{1}{2}}-H_{2}^{\frac{1}{2}}\right\} \text { secs. }
$$

Art. 75.-Equivalent Diameter of an Unifori Main.

The diameter of an uniform pipe which will give the same discharge as that of a pipe of variable diameter when laid between the same points and under the same conditions as to head, may be determined by equating the resistances of the two pipes.

If $l_{1}, l_{2}, l_{3}$, etc., be the length of portions of the pipe having diameters $d_{1}, d_{2}, d_{3}$, etc., and velocities of flow $v_{1}, v_{2}, v_{3}$, and if $L$, $D$, and $V$ refer to the uniform pipe, we have for the same flow

$$
\begin{gathered}
\frac{f^{\prime} L V^{2}}{2 ! m}=\frac{f}{2 !}\left\{\frac{l_{1} v_{1}^{2}}{m_{1}}+\frac{l_{2} v_{2}^{2}}{m_{2}}+\frac{l_{3} r_{3}^{2}}{m_{3}}+\text { etc. }\right\} \\
\frac{L V^{2}}{D}=\left\{\frac{l_{1} v_{1}^{2}}{d_{1}}+\frac{l_{2} v_{2}^{2}}{d_{2}}+\frac{l_{3} r_{3}^{2}}{d_{3}}+\text { etc. }\right\}
\end{gathered}
$$

The discharge $Q$ in cubic feet per second is given by

$$
\begin{gathered}
\frac{\pi D^{2}}{4} \cdot V=\frac{\pi d_{1}^{2}}{4} \cdot v_{1}=\frac{\pi d_{2}^{2}}{4} \cdot v_{2}, \text { etc., } \\
\therefore \quad V=\frac{4 Q}{\pi D^{2}}: r_{1}=\frac{4 Q}{\pi d_{1}^{2}}: r_{2}=\frac{4 Q}{\pi d_{2}^{2}}, \text { etc. }
\end{gathered}
$$

So that equation (2) becomes

$$
\begin{aligned}
\frac{L}{D^{5}} & =\left\{\frac{l_{1}}{d_{1}{ }^{5}}+\frac{l_{2}}{d_{2}{ }^{5}}+\frac{l_{3}}{d_{3^{5}}}+\text { etc. }\right\} \\
\therefore \quad D & =\left\{\frac{L}{\Sigma\left(\frac{l}{d^{5}}\right)}\right\}^{\frac{1}{5}} .
\end{aligned}
$$

This neglects losses due to the changes of section between the lengths $l_{1}, l_{2}$, etc., and will only be true when the pipe is so long, and the number of changes of section so small or so gradual as to render this loss sensibly negligible. 
With a pipe which has a large number of sudden enlargements in the direction of flow, we get

$$
\begin{gathered}
\frac{l^{2}}{2 !}+\frac{f L V^{2}}{2 g m}=\frac{f}{2 g} ; \frac{l_{1} r_{1}^{2}}{m_{1}}+\frac{l_{2} r_{2}^{2}}{m_{2}}+\frac{l_{3} r_{3}^{2}}{m_{3}}+\text { etc. } \\
\quad+\frac{1}{2 g}\left\{\left(r_{1}-r_{2}\right)^{2}+\left(r_{2}-r_{3}\right)^{2}+\text { etc. }\right\}+\frac{r_{1}^{2}}{2 g}
\end{gathered}
$$

The last two terms may be written as

$$
\begin{aligned}
& \frac{1}{2 g}\left\{2\left(v_{1}^{2}+v_{2}^{2}+-\right)-2\left(v_{1} v_{2}+v_{2} v_{3}+r_{3} r_{4}+\text { etc. }\right)\right\} \\
& \therefore \frac{I^{2}}{2 g}\left(1+\frac{f L}{m} ;=\frac{1}{2 g}\left[\Sigma \cdot \frac{f l \imath^{2}}{m}+2 \Sigma \imath^{2}-2\left(v_{1} v_{2}+r_{2} r_{3}+\text { etc. }\right)\right]\right. \\
& \therefore \frac{1+\frac{4 f L}{D}}{l^{4}}=\left[\Sigma \frac{4 f^{\prime} l}{l^{5}}+2 \Sigma \frac{1}{d^{*}}-2\left(\frac{1}{d_{1}^{2} d_{2}{ }^{2}}+\frac{1}{l_{2}^{2} d_{3}^{2}}+\text { etc. }\right)\right]
\end{aligned}
$$

from which, when $d_{1}, d_{2}$, etc., are known, the value of $D$ may be determined.

\section{Art. 76.-Braxch Maixs.}

Where a large main is divided into a number of branches, $d_{1}, d_{2}, d_{3}$, etc., of different lengths and discharging into reservoirs

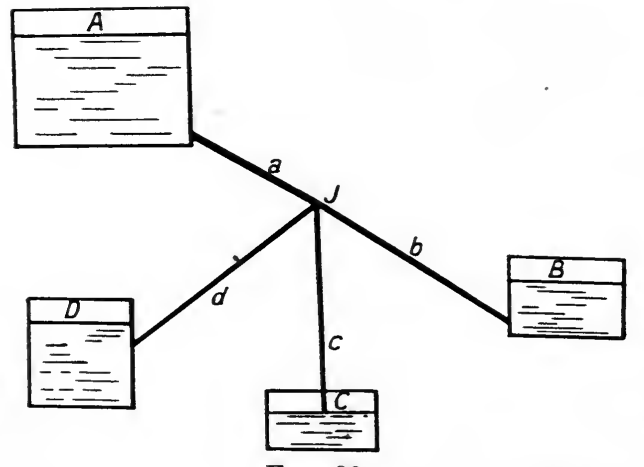

FIG. 96. against different heads, the flow along each of these branches may be determined as follows. Let Fig. 96 represent the arrangement diagrammatically, the reservoir $A$ supplying the pipe $a$, which in turn supplies the reservoirs $B$, $C$, and $D$ through pipes $b, c$, and $d$.

Let $Z_{A}, Z_{B}, Z_{C}, Z_{D}$ represent the heights above some common datum, of the free surfaces in the respective reservoirs, and let $Z_{J}$ represent the height of the junction $J$. Let $r_{a}, r_{b}$, $r_{c}$, etc., represent the velocities in the various pipes, and let $A_{\imath}, A_{b}, A_{c}$, etc., represent their areas. 
Then

$Z_{A}=Z_{J}+\frac{p_{J}}{W}+\frac{v_{u}^{2}}{2 g}+{ }_{A} H_{J}\left\{\begin{array}{l}\text { where }{ }_{A} H_{J} \text { represents the } \\ \text { loss of head from } A \text { to } J .\end{array}\right.$

Similarly

$$
\begin{aligned}
& Z_{J}+\frac{p_{J}}{W}+\frac{r_{b}^{2}}{2 g}=Z_{b}+{ }_{J} H_{B} \\
& Z_{J}+\frac{p_{J}}{W}+\frac{r_{c}^{2}}{2 g}=Z_{c}+{ }_{J} H_{C} \\
& Z_{J}+\frac{p_{J}}{W}+\frac{v^{2}}{2 g}=Z_{D}+{ }_{J} H_{D}
\end{aligned}
$$

Also if all the pipes run full we have for continuity of flow

$$
A_{a} v_{u}=A_{b} v_{b}+A_{c} v_{c}+A_{l} v_{d} \text {. }
$$

Since, in these five equations, the only unknowns are the four relocities and the pressure at $J$ (the losses ${ }_{A} H_{J},{ }_{J} H_{B}$, etc., being determinate in terms of the velocities), the equations are perfectly determinate and a solution will give the velocities in the various pipes in terms of known quantities. The quantities discharged through each pipe may then be determined.

\section{ExaMPLe.}

A reserroir $A$ supplies three supplementary reservoirs $B, C, D$ through a single 24 -inch pipe divided at $J$ into two 12-inch and one 18-inch pipe leading respectively to reservoirs $B, C$, and $D$. If the lengths of these pipes are $l_{a}=500$ feet, $l_{b}=1,000$ feet, $l_{c}=1,500$ feet, $l_{d}=3,000$ feet ; and if $Z_{A}=50$ feet, $Z_{B}=$ 30 feet, $Z_{c}=10$ feet, $Z_{D}=20$ feet, $Z_{J}=40$ feet, determine the velocities of flow in each pipe. Take $f=\cdot 005$, and neglect all except friction losses.

Here ${ }_{1} H_{J}$ in equation (1) above becomes

$$
\frac{005 \times 500 \times 4}{2} \frac{r_{a}{ }^{2}}{2 g}=\frac{5 r_{a}^{2}}{2 g} .
$$

Determining all such values, and substituting, equations (1) to (5) above become

$$
\begin{aligned}
& \frac{p_{J}}{W}+6 \frac{v_{a}{ }^{2}}{2 g}=10 \\
& \frac{p_{J}}{W}-19 \frac{v_{\iota}{ }^{2}}{2 g}=-10
\end{aligned}
$$




$$
\begin{array}{r}
\frac{p_{J}}{W}-29 \frac{v_{c}{ }^{2}}{2 g}=-30 \\
\frac{p_{J}}{W}-39 \frac{v_{d}^{2}}{2 g}=-20 \\
4 v_{a}=v_{b}+v_{c}+2 \cdot 25 v_{d} .
\end{array}
$$

Subtracting (2) from (1), (3) from (4), and (3) from (2) we eliminate $\frac{p_{J}}{W}$, and get

$$
\begin{array}{r}
6 r_{a}^{2}+19 v_{b}^{2}=40 g \\
29 r_{c}^{2}-39 r_{d}^{2}=20 g \\
29 v_{c}^{2}-19 v_{b}^{2}=40 g
\end{array}
$$

Determining $v_{b}, v_{c}$ and $v_{a}$ in terms of $v_{a}$ from equations (6), (7), and (8), and substituting these values in (5), this becomes, on writing $g=32 \cdot 2$, and simplifying

$$
\begin{gathered}
4 v_{a}=\sqrt{67 \cdot 75-\cdot 316 v_{a}^{2}}+\sqrt{88 \cdot 8-\cdot 207} v_{a}^{2}+ \\
2 \cdot 25 \sqrt{49 \cdot 5-\cdot 154 v_{a}^{2}} .
\end{gathered}
$$

Writing this as $4 v_{a}-\phi\left(v_{a}\right)=F\left(v_{a}\right)$ (where $\phi\left(v_{a}\right)$ is the right-hand side of this equation) and giving $v_{a}$ the value

$6 \cdot 0$ this makes $F\left(v_{a}\right)=-7 \cdot 32$.

Putting $v_{a}=6.5 \quad, \quad, \quad F\left(v_{a}\right)=-5 \cdot 13$.

$$
\text { , } v_{a}=7 \cdot 5 \quad, \quad, \quad F\left(v_{a}\right)=+\cdot 26 .
$$

Plotting these values of $v_{a}$ and $F\left(v_{a}\right)$ we find that $v_{a}=7 \cdot 48$ makes $F\left(v_{a}\right)=0$, and therefore satisfies the above equation (9).

Substituting this value of $v_{a}$ in equations $(6),(7)$ and (8) we get $v_{b}=7 \cdot 08, v_{c}=8 \cdot 79, v_{d}=6.39$.

Also from equation (1) we have

$$
\frac{p_{J}}{W}=10-6 \frac{v_{a}^{2}}{2 g}=4 \cdot 78 \text { feet of water. }
$$

\section{Art. 77.-Multiple Supply.}

Where more than one reservoir or source of supply feeds into one dommon pipe, the surfaces of the water in the supply reservoirs being at different levels, it becomes necessary to determine what share of the total flow each of these sources of supply contributes. E.g., in the case illustrated in Fig. 97 the two reservoirs $A$ and $B$, with surface levels $Z_{A}$ and $Z_{B}$ above datum, feed 
through pipes of areas $a$ and $b$, into a common pipe of area $c$, at $J$, the joint flow passing into a rəservoir at $C$, with its surface at a height $Z_{c}$ above datum level.

Here we have

$$
\begin{aligned}
& Z_{A}=Z_{J}+\frac{p_{J}}{W}+\frac{v_{a}{ }^{2}}{2 g}+{ }_{A} H_{J}\left\{\begin{array}{c}
{ }_{A} H_{J} \text { representing the } \\
\text { loss from } A \text { to } J .
\end{array}\right. \\
& Z_{B}=Z_{J}+\frac{p_{J}}{W}+\frac{v_{b}^{2}}{2 g}+{ }_{B} H_{J} \\
& Z_{C}+{ }_{J} H_{C}=Z_{J}+\frac{p_{J}}{W}+\frac{v_{c}{ }^{2}}{2 g}
\end{aligned}
$$

Also if all the pipes run full we have for continuity of flow

$$
a v_{a}+b v_{b}=c v_{c} \text {. }
$$

Then since ${ }_{A} H_{J},{ }_{B} H_{J},{ }_{J} H_{C}$ are respectively proportional to $r_{a}^{2}, r_{b}^{2}, r_{c}^{2}$, and may be determined in terms of these velocities when the construction and sizes of pipes are known, the only unknowns are the three velocities $v_{a}, v_{b}, v_{c}$, and the pressure $p_{J}$. These equations may then be solved and the unknown factors determined. A similar method of solution will apply to any case of multiple supply, with

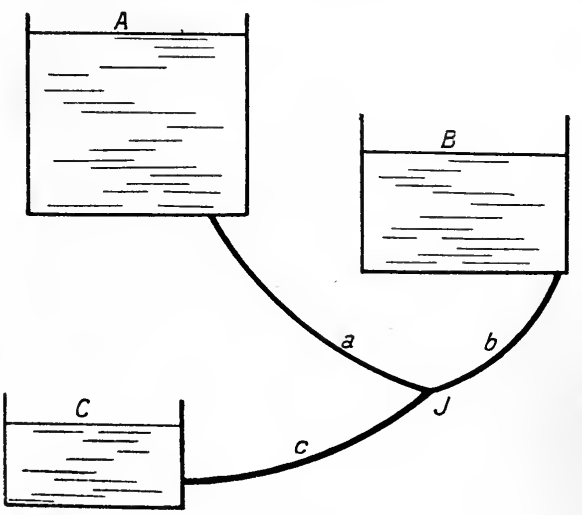

FIG. 97. any number $n$ of sources of supply. Here we shall have $n+2$ equations formed, from which the $(n+1)$ velocities and the pressure at the junction may be determined.

\section{Art. 78.-Flow along a Bye-Pass.}

If $p_{1}$ and $p_{2}$ lbs. per square foot are the pressures in the main pipe at the entrance to and exit from the bye-pass pipe or diversion (Fig. 98), we have, putting $V$ and $v$ for

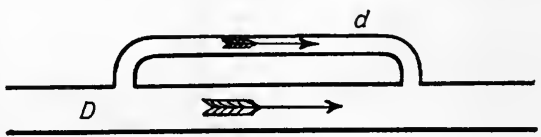

FIG. 98. 
the velocities in the large and small pipes respectively,

$$
\frac{p_{1}-p_{2}}{W}=\frac{4 f L V^{2}}{2 ! D}=\frac{4 f l v^{2}}{2 g l}+K \frac{v^{2}}{2 !}
$$

where $K \frac{v^{2}}{2 g}$ represents the loss in the bye-pass at entrance and exit and at any valves.

From this we have, assuming $f$ to have the same value in the two pipes,

$$
\begin{gathered}
\frac{L T^{2}}{L}=\frac{l v^{2}}{d}+K^{\prime} v^{2}=\left(\frac{l}{d}+K^{\prime}\right) v^{2} \\
\therefore \quad \frac{T^{2}}{v_{2}}=\frac{D}{L}\left\{\frac{l}{d}+K^{\prime}\right) .
\end{gathered}
$$

If $Q$ and $q$ are the quantities flowing along the two pipes

$$
\begin{aligned}
Q & =\frac{V \pi D^{2}}{4} \quad q=\frac{v \pi d^{2}}{4} \\
\therefore \quad \frac{Q}{q} & =\frac{V^{2} D^{2}}{v d^{2}}=\sqrt{\frac{D}{L}\left(\frac{l}{d}+K^{\prime}\right)} \cdot \frac{J^{2}}{d^{2}} \\
& =\sqrt{\left(\frac{D}{d}\right)^{5} \cdot \frac{l+K^{\prime} d}{L}} .
\end{aligned}
$$

The proportion of the whole flow which passes along the diversion, or $\frac{q}{Q+q}$ is given by

$$
q=\frac{1}{1+\sqrt{\left(\frac{D}{d}\right)^{5} \cdot \frac{l+K^{\prime} d}{L}}}(Q+q) .
$$

Art 79.-Flow through Pipes coupled up in Parallel (FIG. 99).

Here pipes $d_{1}, d_{2}$, etc., of length $l_{1}, l_{2}$, etc., couple up two vessels whose pressures at the points of exit and inlet are $p_{1}$ and $p_{2}$ lbs. per square foot. We now have

$$
\frac{p_{1}-p_{2}}{W}=4 f \frac{l_{1} r_{1}^{2}}{2 g d_{1}}=4 f \frac{l_{2} r_{2}^{2}}{2 g d_{2}}=4 f \frac{l_{3} r_{3}^{2}}{2 ! d_{3}}=\text { etc. }
$$

neglecting all losses except those due to friction. 
If $Q=$ total flow through pipes in cubic feet per second, we have

$$
Q=\frac{\pi}{4}\left\{v_{1} d_{1}^{2}+v^{2} d_{2}^{2}+v_{3} d_{3}^{2}+\text { etc. }\right\}
$$

$\therefore \quad$ Writing $v d^{2}=\sqrt{\frac{l v^{2}}{d}} \cdot \frac{d^{\frac{5}{2}}}{l^{\frac{1}{2}}}$ we get

$$
Q=\frac{\pi}{4} \sqrt{\frac{l_{1} v_{1}^{2}}{d_{1}}}\left\{\frac{d_{1}^{\frac{5}{2}}}{l_{1}^{\frac{1}{2}}}+\frac{d_{2}^{\frac{5}{2}}}{l_{2}^{\frac{1}{2}}}+\frac{d_{3}^{\frac{5}{2}}}{l_{3}^{\frac{1}{2}}}+\text { etc. }\right\} .
$$

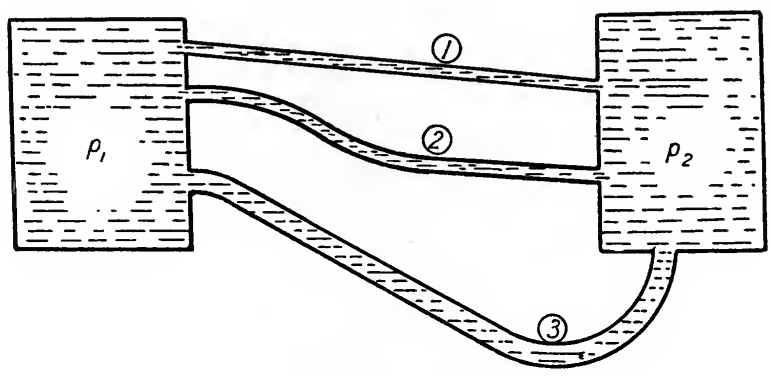

FIG. 99.

And since $\frac{p_{1}-p_{2}}{W}=\frac{4 f}{2 g} \cdot \frac{l_{1} r_{1}^{2}}{d_{1}}$, this becomes

$$
Q=\frac{\pi}{4} \sqrt{\frac{p_{1}-p_{2}}{W} \cdot 2 g}\left\{\Sigma \frac{d^{\frac{\pi}{2}}}{l^{\frac{1}{2}}}\right\} .
$$

ExaMple.

Determine the diameter of a pipe of length $l$, to give the same discharge as two parallel pipes of the same length and, of diameter $d$.

Discharge from two small pipes $=\frac{\pi}{4} \sqrt{\frac{p_{1}-p_{2}}{W} \cdot \frac{g}{2 f}}\left\{\frac{2 d^{\frac{5}{2}}}{l^{\frac{1}{2}}}\right\}$

$$
" \quad, \quad \text { large pipe }=\frac{\pi}{4} \sqrt{\frac{p_{1}-p_{2}}{W} \cdot \frac{g}{2 f}}\left\{\frac{D_{2}^{\frac{5}{2}}}{l^{\frac{1}{2}}}\right\}
$$

$\therefore \quad$ For same discharge $D^{\frac{5}{2}}=2 d^{\frac{5}{2}}$

$$
\therefore \quad D=2^{\frac{2}{5}} d=1.32 d \text {. }
$$


Thus, assuming the resistance to vary as $v^{2}$, one pipe $15 \cdot 84$ inches diameter would give the same discharge as two 12-inch pipes.

Assuming more correctly the results of the experiments quoted in Art. 69, and taking

$$
Q=C\left(\frac{H}{l}\right)^{\frac{1}{2}} d^{258}=C l^{\frac{1}{2}} \sqrt{\frac{p_{1}-p_{2}}{W}} d^{258},
$$

we should have in the above example, $D^{2 \cdot 58}=2 d^{2 \cdot 58}$

$$
\therefore \quad D=2^{\frac{1}{2 \cdot 58}} \cdot d=1 \cdot 308 d,
$$

or one pipe $15 \cdot 70$ inches would now give the same discharge as the two 12-inch pipes.

This duplication in parallel of supply pipes is very common in large water supply systems, and is also of use where, in the case of a fire main, the pressure at the nozzle is insufficient to give the required velocity of flow. By coupling a second line of hose between the supply main and the hydrant box, the fall in pressure at the nozzle is much reduced and consequently a higher jet is obtained.

\section{Art. 80.-Flow through Nozzles.}

Where a supply of water moving with a high velocity is required, as for fire extinguishing, or for the jets of an impulse turbine or Pelton wheel, a converging nozzle is fitted to the delivery end of the supply pipe.

The issuing jet now possesses high velocity and its store of energy is almost entirely in the kinetic form.

Theoretically, if pointed vertically upwards, and if no energy losses were experienced, the height of the jet would be the same as that of the free surface of the supply reservoir, or that corresponding to the pressure head inside the nozzle. The various frictional resistances, however, reduce the nozzle pressure and the issuing velocity, after which the resistance of the air and that due to the impact of faliing particles of water tend to retard the upward motion of the rising particles, so that the height to which 
the jet rises is considerably less than that given by $h=\frac{v^{2}}{2 g}, v$ being the velocity of efflux.

The following results are deduced from experiments carried out by J. T. Fanning ${ }^{1}$ with ordinary converging fire nozzles and with a stream slightly inclined to the vertical, so that the effect of the falling particles would not be so great as with a vertical stream.

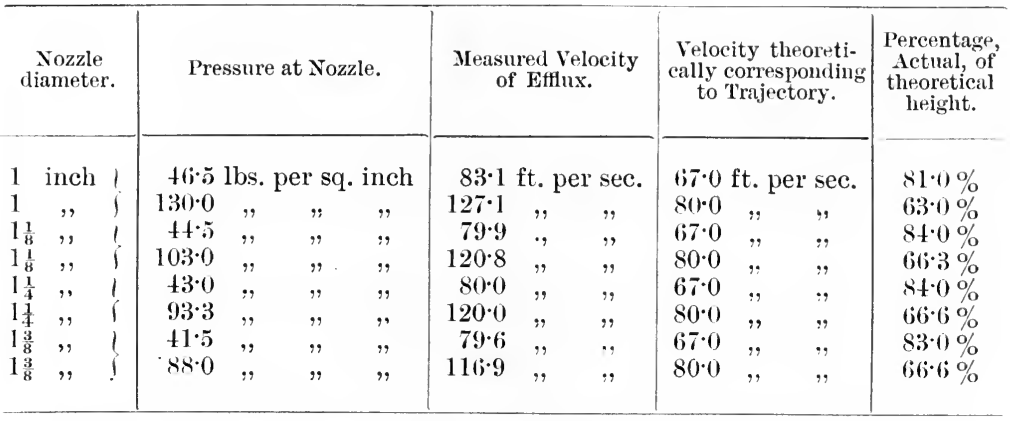

Forms of Nozzle.-These are usually of circular section and may either converge uniformly to a short parallel neck at the

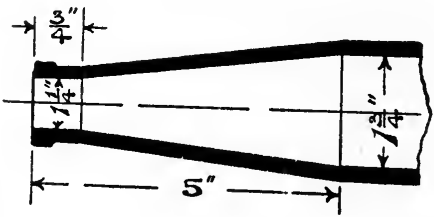

(a)

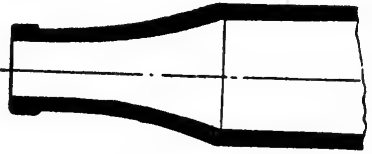

(b)

FIG. 100.

orifice (Fig. $100 a$ ), or have a convergence which becomes more gradual as the outlet is approached (Fig. 100b).

In either case, the coefficient of contraction is unity, and the coefficient of discharge about $\cdot 98$.

The following values of the latter coefficient are deduced from experiments by Freeman ${ }^{2}$ on smooth conical nozzles.

\begin{tabular}{lc|c|c|c|c|c}
\hline Diameter of nozzle & $\cdot$ & $\frac{3}{4} \mathrm{in}$. & $\frac{7}{8} \mathrm{in}$. & $1 \mathrm{in}$. & $1 \frac{1}{8} \mathrm{in.}$ & $1_{4}^{1} \mathrm{in.}$ \\
\hline Value of $C$. & $\cdot$ & $\cdot 983$ & $\cdot 982$ & $\cdot 972$ & $\cdot 976$ & $\cdot 971$ \\
\hline
\end{tabular}

1 Engineering News, July 14, 1892.

2 Appendix, Table D. 
A form known as the ring nozzle is used to a less extent, and consists of a converging nozzle having a circular orifice, which is fitted with a short annular ring of square section (Fig. 101a). This, with a ring $\frac{1}{8}$-inch square, gives a stream having a vena contracta of area about $\cdot 764$ times that of the orifice. The value of $C_{v}$ is about $\cdot 97$, the coefficient of discharge being about $\cdot 74$. Since the efficiency of a nozzle depends entirely on the value of its coefficient of velocity $C_{v}$ the ring nozzle would appear to have no advantage over the ordinary smooth nozzle, and in practice, with the same pressure head and size of nozzle, throws a jet to a slightly less height. It has been proposed to use a small reentrant mouthpiece, so as to form a Borda orifice in connection

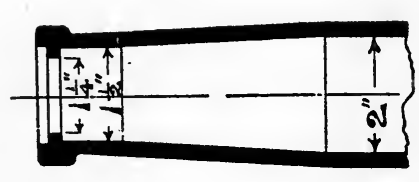

(a)

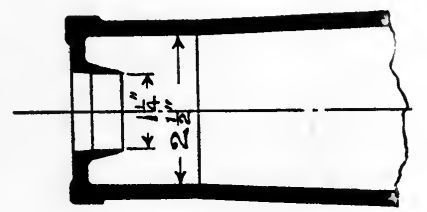

(b)

- FIg. 101.

with this nozzle (Fig. $101 \mathrm{~b}$ ), and to use this as a standard nozzle to experimentally determine the horse-power of a pumping engine, by measuring the pressure inside a nozzle or series of nozzles supplied by the engine. The coefficient of discharge of such a nozzle as shown in Fig. $101 \mathrm{l}$ is given by Freeman as ${ }^{\circ 582}$. Since, however, the ordinary type of nozzle shown in Fig. $100 a$ and $b$ may be relied upon to give discharges which are identical within the limits of practical measurement under given pressure conditions, certainly within 1 per cent., these nozzles are very satisfactory for such a purpose, this accuracy being quite as high as that attending the use of a standard weir for measuring purposes.

\section{Telocity of Flow through a Nozzle.}

Let $A, L$, and $v_{A}$ refer to the supply pipe, and $a$ and $v_{a}$ to the nozzle. Then $\frac{v_{A}}{v_{a}}=\frac{a}{A}$. 
If $\mathrm{H}=$ supply head in feet we have

$$
H=K \frac{v_{A}{ }^{2}}{2 g}+\frac{f l v_{A}{ }^{2}}{2 g m}+\frac{v_{a}^{2}}{2 g}+k \frac{v_{a}{ }^{2}}{2 g}
$$

where $K$ and $k$ represent the coefficients of loss at the entrance to the pipe and at the nozzle.

Putting $r_{A}=v_{a} \cdot \frac{a}{A}$ we get

$$
\begin{aligned}
& H=\left\{K \frac{a}{A}+\frac{f l a^{2}}{m A^{2}}+1+k\right\} \frac{v_{a}{ }^{2}}{2 g} \\
& \therefore \quad v_{a}=\sqrt{\frac{2 g H}{1+k+K \frac{a}{A}+\frac{f l a^{2}}{m A^{2}}}}
\end{aligned}
$$

Since $k=\cdot 05$ (approximately), while $K \frac{a}{A}$ is in general small, neglecting these terms we get

$$
v_{a}=\sqrt{\frac{2 g H}{1+\frac{f l a^{2}}{m A^{2}}}}=\sqrt{\frac{2 g H}{1+\frac{4 f l d^{4}}{D^{5}}}}
$$

If $p=$ pressure in pounds per square inch at the entrance to the nozzle we have, if $\frac{k v_{a}^{2}}{2 g}=$ loss in nozzle

$$
\begin{gathered}
\frac{p \times 144}{W}+\frac{v_{A}{ }^{2}}{2 g}=\frac{v_{a}{ }^{2}}{2 g}(1+k) \\
\therefore \quad 2 \cdot 3 p=\frac{v_{a}{ }^{2}}{2 g}\left\{1+k-\frac{a^{2}}{A^{2}}\right\} \\
\therefore \quad v_{a}=\sqrt{\frac{4 \cdot 6 p g}{1+k-\frac{a^{2}}{A^{2}}}}
\end{gathered}
$$

This formula may be used to determine $k$, and hence the coefficient of velocity $\sqrt{(1-k)}$, by measurement of $p$ and of the quantity discharged.

With a ring nozzle the preceding formula becomes

$$
v_{a}=\sqrt{\frac{4 \cdot 6 p g}{1+k-\frac{a_{c}^{2}}{A^{2}}}}
$$


where $a_{c}$ is now the area of the rena contracta of the issuing stream.

In any case, when $k$ and the coefficient of contraction for any given nozzle are known, the value of $v_{a}$ can be directly determined in terms of $p$. Then since the energy discharged at the nozzle per second

$$
=\frac{W a_{c} v_{a}^{3}}{2 g} \mathrm{ft} . \mathrm{lbs} .
$$

we have the horse-power delivered at the nozzle given by

$\mathrm{H} . \mathrm{P} .=\frac{W a_{c} v_{a}{ }^{3}}{2 g \times 550}=\frac{62 \cdot 4 a_{c} v_{a}{ }^{3}}{1,100 g}=\cdot 00176 a_{c} v_{a}{ }^{3}$

$$
=3 \cdot 14 a_{c}\left\{\frac{p}{1+k-\frac{a_{c}^{2}}{A^{2}}}\right\}^{\frac{3}{2}} \text {. }
$$

Exaniple.

With a uniformly converging nozzle, $1 \frac{1}{2}$ inch diameter, taling $l=03$, and assuming a supply pipe of 3 inches diameter, we have $a_{c}=a=$ area of $1 \frac{1}{2}$ inch pipe $=\cdot 01227$ square feet, while $\frac{a_{c}^{2}}{A^{2}}=\frac{1}{4}$. Thus if the pressure at the nozzle is $80 \mathrm{lbs}$. per square inch, we have

$$
\begin{aligned}
\text { H.P. at nozzle } & =3.139 \times 01227\left\{\frac{80}{1 \cdot 03-\cdot 25}\right\}^{\frac{3}{2}} \\
& =0385\{102.7\}^{\frac{3}{2}} \\
& =40 \mathrm{H} . \mathrm{P} .
\end{aligned}
$$

If water is supplied at the pipe entrance under a constant pressure $P$ lbs. per square foot, neglecting the difference in head between pipe entrance and nozzle, we have

$$
\begin{aligned}
& \frac{P}{W}=H, \\
& \therefore \quad v_{a}=\sqrt{\frac{2 g H}{\left(1+\frac{4 f l}{D^{5}}\right)}} \mathrm{ft} \text {. per sec. }
\end{aligned}
$$


Without the nozzle we should have $a=A, r_{a}=r_{A}$,

so that

$$
v_{A}=\sqrt{\frac{2 g H}{1+\frac{4 f l}{D}}} \text { ft. per sec. }
$$

so that the velocity with, is greater thon without the nozzle.

The discharge per second without the nozzle is then given by

$$
r_{A} \times A=\sqrt{\frac{2 g H}{1+\frac{4 f l}{D}}} \times A \text { cub. ft. }
$$

and the kinetic energy by $\frac{r_{A}{ }^{3}}{2 g} \cdot A \times 62 \cdot 4 \mathrm{ft}$. lbs.

$$
=\left\{\frac{2 ! H}{1+\frac{4+f l}{I} l}\right\}^{\frac{3}{2}} \cdot \frac{62 \cdot 4}{2 !} A \text { ft. lbs. }
$$

With the nozzle, the quantity per second is

$$
v_{a} \times a=\sqrt{\frac{2 ! H}{1+\frac{4 \cdot t^{5} l d^{4}}{D^{5}}}} \times a
$$

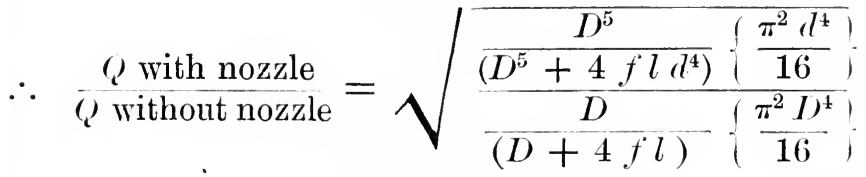

$$
\begin{aligned}
& =\sqrt{\frac{d^{4}(D+4 f l)}{\left(D^{5}+4 f l d^{4}\right)}}=\sqrt{\frac{I) d^{4}+4 f l d^{4}}{D^{5}+4 f l d^{4}}}
\end{aligned}
$$

or the quantity passing the pipe is always less with, than without the nozzle.

Since the kinetic energy varies as the product of two factors (the quantity and the (velocity) ${ }^{2}$ ), one of which increases, while the other diminishes as the nozzle area is increased, it would appear probable that a maximum amount of kinetic energy will be delivered at the nozzle with some definite ratio of pipe and nozzle areas. 
To obtain this ratio we have K.E. with nozzle $=\frac{v_{a}^{3}}{2 g} \times 62.4 a$ ft. lbs. per sec.

$$
\begin{aligned}
& =\left\{\frac{2 g H}{1+\frac{4 f l d^{4}}{D^{5}}}\right\}^{\frac{3}{2}} \times \frac{62 \cdot 4 \pi d^{2}}{2 g \times 4} \\
& =395 d^{2}\left\{\frac{H}{1+\frac{4 f l d^{4}}{D^{5}}}\right\}^{\frac{3}{2}} \text { ft. lbs. per sec. } \\
& =395 \frac{a}{A} D^{2}\left\{\frac{H}{1+\frac{4 f l}{D} \cdot \frac{a^{2}}{A^{2}}}\right\}^{\frac{3}{2}} \text { ft. lbs. per sec. }
\end{aligned}
$$

Differentiating this with respect to $\frac{a}{A}$, and equating the result to zero we have the condition that the K. E. should be a maximum. This gives $\mathrm{us}^{1}$

or

$$
\begin{aligned}
\left\{\frac{d}{D}\right)^{4} & =\frac{D}{8 f l} \\
\frac{a}{A} & =\sqrt{\frac{D}{8 f l}}
\end{aligned}
$$

the dimensions being taken in feet.

1 Put $\frac{d^{2}}{D^{2}}=\frac{a}{A}$. Then K. E. $=C\left\{\frac{H}{1+\frac{+f l}{D} \cdot \frac{a^{2}}{A^{2}}}\right\}^{\frac{3}{2}} \cdot \frac{a}{A} D^{2}$

$$
\begin{gathered}
\therefore \frac{d(K E)}{d\left(\frac{a}{A}\right)}=C D^{2} H^{\frac{3}{2}}\left[-\frac{3}{2}\left\{1+\frac{4 f l}{D} \cdot \frac{a^{2}}{A^{2}}\right\}^{-\frac{5}{2}} \times \frac{8 f l}{D} \cdot \frac{a^{2}}{A^{2}}+\right. \\
\left.\left\{1+\frac{4 f l}{D} \cdot \frac{a^{2}}{A^{2}}\right\}^{-\frac{3}{2}}\right] .
\end{gathered}
$$

Putting this equal to zero we have

$$
\begin{gathered}
\frac{12 f l}{D} \cdot \frac{a^{2}}{A^{2}}=1+\frac{4 f l}{D} \frac{a^{2}}{A^{2}} \\
\therefore \frac{a^{2}}{A^{2}}=\frac{D}{8 f l} .
\end{gathered}
$$

With a circular nozzle this makes $d=\sqrt[4]{\frac{D^{5}}{8 f} i}$ 
If for example the pipe supplying the nozzle of a Pelton wheel be 1 foot in diameter and 500 feet long, the maximum K. E. is delivered when

$$
\frac{a}{A}=\sqrt{\frac{1}{8 \times 500 f}}
$$

and if $f=\cdot 01$ this becomes

$$
\frac{a}{A}=\sqrt{\frac{1}{40}}=\cdot 158 \text {. }
$$

By substituting this value of $\frac{a}{A}$ or of $\frac{d^{2}}{D^{2}}$ in equation (7) above, the value of this $K$. E. is given.

Thus (K. E.) $\max$,

$$
\begin{aligned}
& =395 \times 158\left\{\frac{H}{1+\frac{4 \times 01 \times 500 \times(\cdot 158)^{2}}{1}}\right\}^{\frac{3}{2}} \text { ft. lbs. } \text { per sec. } \\
& =62 \cdot 4\left(\frac{H}{1 \cdot 50}\right)^{\frac{3}{2}} \\
& =34 H^{\frac{3}{2}} \text { ft. lbs. per second. }
\end{aligned}
$$

If the Pelton wheel have an efficiency of 80 per cent. the work done by the wheel per second will be given by

$$
\begin{aligned}
& \cdot 8 \times 34 H^{\frac{3}{2}} \text { ft. lbs. } \\
= & \frac{27 \cdot 2}{550} H^{\frac{3}{2}} \text { horse-power } \\
= & \cdot 0495 H^{\frac{3}{2}} \text { horse-power. }
\end{aligned}
$$

Thus with a head of 100 feet the wheel would develop $49 \cdot 5$ horse-power.

As the nozzle area is increased, starting from zero, the power developed thus increases to a maximum of $49.5 \mathrm{H} . \mathrm{P}$. with a nozzle opening $a=\cdot 158 \mathrm{~A}$, and then again diminishes.

If in the above problem $f$ be taken $=\cdot 005$, we get the maximum K. E. when $\frac{a}{A}=\cdot 224$. The value of this $\mathrm{K}$. E. $=48 \cdot 1 H^{\frac{3}{2}}$ foot lbs. per second, so that with the same head and wheel efficiency the maximum power developed would be $70.0 \mathrm{H}$.P.

In a fire nozzle, the problem is to obtain a stream moving with H.A. 
as high a velocity as possible, and this is evidently to be obtained by making the expression $\left[1+k+K \frac{a}{A}+\frac{f a^{2}}{m A^{2}}\right]$, the denominator of the fraction expressing the velocity, as small as possible. This is done by increasing, as far as practicable, the ratio $\frac{A}{a}$,

In the case of the supply to a Pelton wheel, a stream carrying the maximum possible amount of kinetic energy is required, and as just demonstrated, this can only be obtained by having a correct ratio of pipe and nozzle area. With a smaller nozzle area the weight issuing per second is unduly restricted, while with a greater area the loss due to increased friction in the pipe, due to the greater velocity of flow, causes the velocity of efflux to be reduced to such an extent as to more than counterbalance the increased weight passing the nozzle.

This fact may be of importance in the regulation of a Pelton wheel, which regulates its supply of water by automatic opening or throttling at the nozzle (Art. 115).

So long as this nozzle area has less than the critical value for the particular pipe, any further opening will admit more water and a greater supply of linetic energy to the wheel. Above this point any further opening of the valve admits more water, but gives a smaller supply of kinetic energy to the wheel, which will consequently slow down. On the other hand, with a nozzle area greater than the critical value, throttling leads to an increased supply of energy being given to the wheel and to an increased speed.

For successful governing the maximum nozzle area should not exceed that corresponding to the critical value, so that everywhere within the range of opening $\frac{d(K E)}{d(a)}$ may be positive, and the supply of energy may increase with the nozzle opening. The curves (Fig. 102) derived from equation (6) of this article, show the change of K. E. (expressed as horse-power) delivered from pipes of diameters $1 \cdot 0$ and $2 \cdot 0$ feet, 1,000 feet long, under a head of 200 feet, with different values of the ratio $\frac{a}{A}$. The value of $f$ 
has been taken at 005 . In each case the maximum nozzle opening for successful governing is that corresponding to the point $E .^{1}$ These curves are worth study as denoting the great increase in available energy with the larger pipes.

While the above investigation is interesting, it is not usual, in a well-designed pipe line, to come across a case in which the nozzle area is greater than the maximum for successful governing

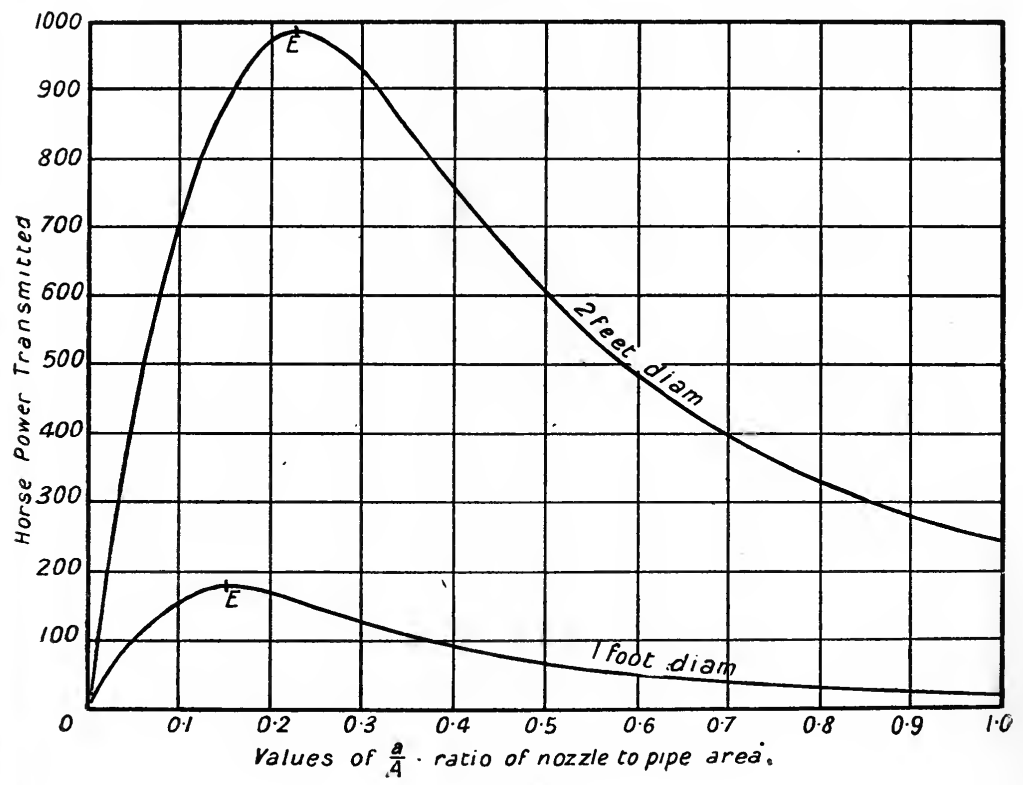

Fig. 102.

since this would entail a velocity of flow in the main much greater in general than that ( 3 to 6 feet per second) adopted in practice.

\section{ArTr 81.-Syphons.}

Where a pipe line is to be laid to connect two reservoirs at different levels, over ground which is higher than either water level, the cost of excavation is often so great as to preclude the use of a pipe line which shall fall below the hydraulic gradient, and in such cases a syphon (Fig. 103) is commonly used.

.${ }^{1}$ For a further consideration of this subject see an article by Professor John Goodman in Engineering, November 4, 1904. 
In its simplest form this consists of an inverted U-tube (Fig. 104), both legs being full of water, and the flow is generally calculated by equating the total head producing flow, i.e., the head due to the unbalanced column of water $Z_{A}-Z_{C}$, or the difference of head in the two reservoirs, to the sum of the frictional and other losses in the pipe and of the velocity head produced.

Thus $Z_{A}-Z_{C}=$ loss at entrance and exit $+\frac{f l r^{2}}{2 g m}, l$ being the total length of the syphon $A^{\prime} B C^{\prime}$.

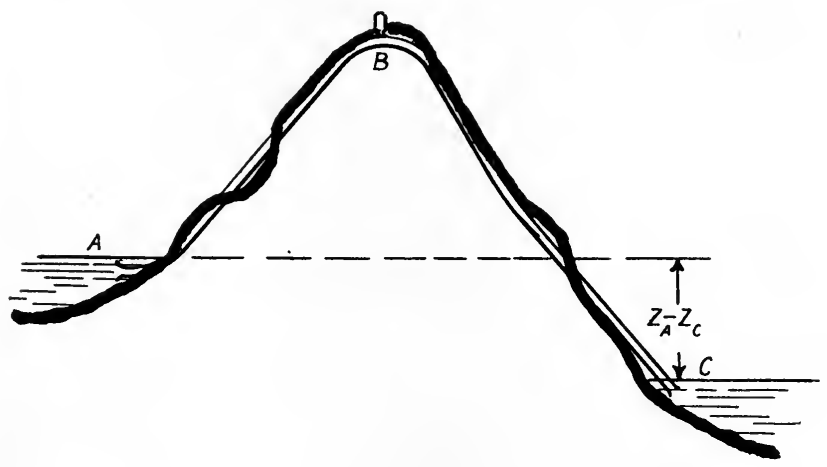

FIG. 103.

F: This may be seen by considering the flow along each leg of the syphon separately.

Along $A^{\prime} B$, we have

$$
\begin{gathered}
\frac{p_{A^{\prime}}}{W}+Z_{A^{\prime}}=\frac{p_{B}}{W}+Z_{B}+\frac{r^{2}}{2 !}+\frac{f l_{A^{\prime} B} r^{2}}{2 ! m}+\text { loss at entrance, } \\
\therefore \quad \frac{p_{A^{\prime}}}{W}+Z_{A^{\prime}}-Z_{B}-\frac{p_{B}}{W^{\prime}}=\frac{r^{2}}{2 !}\left\{1+\frac{f^{l} A^{\prime} B}{m}+K\right\}
\end{gathered}
$$

Along $B C^{\prime}$ we have

$$
\begin{gathered}
Z_{B}+\frac{v^{2}}{2 g}+\frac{p_{B}}{W^{\prime}}=Z_{C^{\prime}}+\frac{r^{2}}{2 !}+\frac{p_{C^{\prime}}}{W}+\frac{f l_{B C^{\prime} r^{2}}}{2 g m} \\
\therefore Z_{B}-Z_{C^{\prime}}+\frac{p_{B}}{W}=\frac{p_{C^{\prime}}}{W^{\prime}}+\frac{f l_{B C^{\prime} v^{2}}}{2 g} .
\end{gathered}
$$

$\therefore$ From (1) and (2) we get on adding

$$
\left.Z_{A^{\prime}}-h_{C^{\prime}}=\frac{p_{c^{\prime}}-p_{A^{\prime}}}{W}+\frac{\iota^{2}}{2 !} ! 1+\frac{f l}{m}+K\right)
$$


But

$$
\begin{gathered}
\left\{\begin{array}{l}
Z_{A^{\prime}}+\frac{p_{A^{\prime}}}{W}=Z_{A} \\
Z_{C^{\prime}}+\frac{p_{C^{\prime}}}{W}=Z_{C}
\end{array}\right. \\
\therefore Z_{A}-Z_{C}=\frac{r^{2}}{2 g}\left\{1+\frac{f l}{m}+K\right\}
\end{gathered}
$$

$=$ loss at exit + friction loss + loss at entrance.

The assumption that both legs of the syphon run full is one which is not always justified in practice. Evidently the height $B A$ must be less than the barometric height by an amount $-\frac{v^{2}}{2 g}\left\{1+\frac{f l_{A^{\prime} B}}{m}+K\right\}$ or we should have an absolute vacuum formed at points below $B$, and the flow would cease. Theoretically, the limit of possible flow up the inlet leg is reached when the pressure at the summit $B$ is absolute zero ( -34 feet of water), ${ }^{1}$ and is then given by the equation

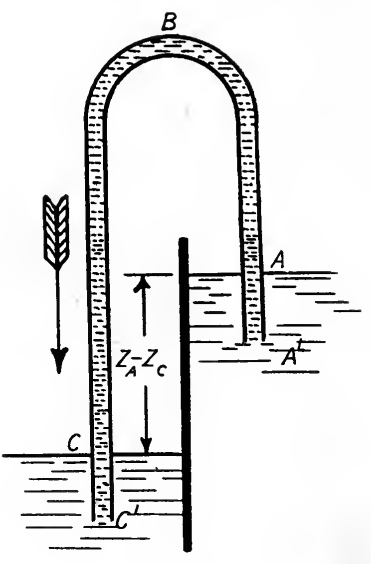

FIG. 104.

$$
34+\frac{p_{A^{\prime}}}{W}-\left(Z_{B}-Z_{A^{\prime}}\right)=\frac{v_{1}{ }^{2}}{2 g}\left\{1+\frac{f l_{1}}{m}+K\right\}
$$

or writing $K=\cdot 5$ and $Z_{A}$ for $Z_{A^{\prime}}+\frac{p_{A}{ }^{\prime}}{W}$ we get

$$
34-\left(Z_{B}-Z_{A}\right)=\frac{r_{1}^{2}}{2 !}\left\{1 \cdot 5+\frac{f l_{1}}{m}\right\}
$$

the suffix (1) referring to the inlet leg.

If the actual flow is less than this, $p_{B}$ is greater than zero, and we get an additional head aiding the flow down the outlet leg.

The velocity of flow down the outlet leg with maximum flow up the inlet is given by the equation

$$
Z_{B}-Z_{C}-34=\frac{f l_{2} v_{2}^{2}}{2 ! m}
$$

the suffix (2) referring to this leg.

1 Actually less than this, because of liberation of air at low pressures. 
If the syphon is to run full, we must have $v_{2}$ not greater than $v_{1}$.

$$
\begin{gathered}
\text { i.e., } \frac{2 g m}{f l_{2}}\left\{Z_{B}-Z_{C}-34\right\} ;>\frac{2 g m}{1.5 m+f l_{1}}\left\{34-\left(Z_{B}-Z_{A}\right)\right\} \\
\text { or } \quad \frac{\left(Z_{B}-Z_{C}\right)-34}{34-\left(Z_{B}-Z_{A}\right)} \ngtr \frac{f l_{2}}{1.5 m+f l_{1}}
\end{gathered}
$$

If the syphon be long, so that $1.5 \mathrm{~m}$ may be neglected $i_{1}$ comparison with $f l_{1}$ we have

or

$$
\begin{aligned}
& \frac{\left(Z_{B}-Z_{C}\right)-34}{34-\left(Z_{B}-Z_{A}\right)} \ngtr \frac{l_{2}}{l_{1}} \\
& \frac{Z_{A}-Z_{C}}{34-\left(Z_{B}-Z_{A}\right)} \ngtr \frac{l_{1}+l_{2}}{l_{1}} \\
\therefore & \frac{l_{1}}{l} \ngtr \frac{34-\left(Z_{B}-Z_{A}\right)}{\left(Z_{A}-Z_{C}\right)}
\end{aligned}
$$

ExAMIPLE.

$$
\begin{aligned}
& \text { If }\left\{\begin{aligned}
Z_{A}-Z_{C} & =50 \mathrm{ft} . \\
l & =1,000 \mathrm{ft} . \\
Z_{B}-Z_{A} & =10 \mathrm{ft} .
\end{aligned}\right. \\
& l_{1} \ngtr \frac{1,000 \times 24}{50}=480 \mathrm{ft} .
\end{aligned}
$$

or the outlet leg will not run full if the inlet leg is more than 480 feet in length.

With this length of inlet leg we then get the maximum flow. A shorter inlet and a consequently longer outlet will reduce the possible velocity along the outlet and therefore throughout the syphon. With a longer inlet and shorter outlet the flow up the inlet will not be able to keep pace with that down the outlet, and this will then run only partly full. Also the velocity up the inlet will not now be so great as with a shorter inlet, so that the discharge will be less.

Evidently, then, the position of the apex of the syphon has a great influence on the discharge.

In practice it is necessary to place an air chamber at the highest point of the syphon, into which air gradually accumulates during its working. This air is then removed at frequent 
intervals, either by some form of air pump, or by means of a steam ejector.

Where the syphon discharges into the atmosphere, any failure of the outlet leg to run full, by admitting air to the apex, at once breaks the vacuum and stops the flow.

Figure 105 shows the hydraulic gradient for a syphon, the straight line $A C$ being the gradient line. In drawing this the only losses taken into account have been those due to friction.

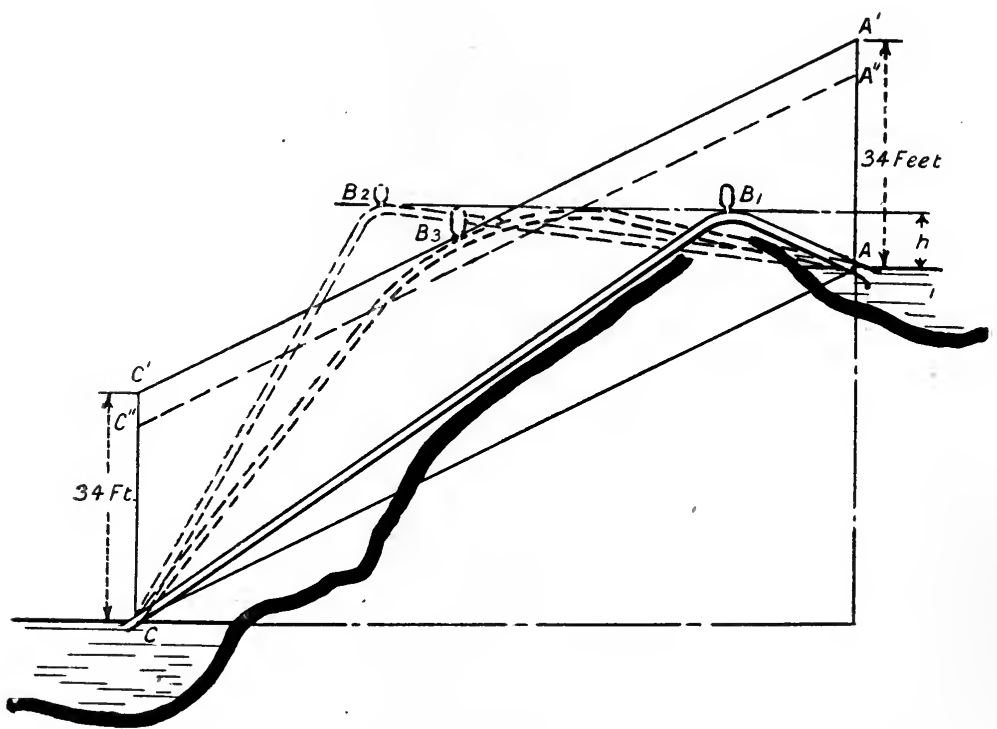

Fig. 105.

If a second line $A^{\prime} C^{\prime}$ be drawn parallel to and at a vertical distance from $A C$ equal to the barometric height, the distance of the syphon below $A^{\prime} C^{\prime}$ will give the absolute pressure at any point. In the sketch, syphons $A B_{1} C, A B_{3} C$, and $A B_{2} C$ are shown connecting $A$ and $C$, all rising to the same height $h$, above the surface at $A$. Here, although $B_{2}$ is not nearly 34 feet above $A$, an absolute vacuum would be attained before reaching $B_{2}$ and the syphon will consequently not work. A comparison of (1) and (3) shows that there is a greater pressure in the air vessel at $B_{1}$ than at $B_{3}$, and the syphon (1) will thus run longer without removal 
of air from this chamber than will (3). Leakage at joints is not likely to have so serious an effect as with (3). With the same pipe diameter, however, (3) will probably give a greater discharge than (1) depending on the ratios of $A B: B C$.

Any accumulation of air at the highest part of the pipe has the effect of increasing the pressure, and hence of lowering the line $A^{\prime} B^{\prime}$. Directly this line is lowered sufficiently to cut the pipe line the syphon ceases to flow. The air vessel should always be placed at the point of least pressure, i.e., at the point nearest to the hydraulic gradient line $A^{\prime} C^{\prime}$. If $A^{\prime} A^{\prime \prime}=C^{\prime} C^{\prime \prime}=\frac{v^{2}}{2 g}$, the line $A^{\prime \prime} C^{\prime \prime}$ will give the hydraulic gradient, taking into account change of potential into linetic energy at the entrance to the pipe. The effect of this is to reduce the value of the ratio $A B: B C$, for both branches of the syphon to run full with maximum discharge, while the true pressure in the pipe is now measured by the vertical distance between $A^{\prime \prime} C^{\prime \prime}$ and the pipe line.

\section{Art. 82.-Inverted Syphon.}

Where the pipes connecting two reservoirs cross a deep valley it is usual to use an inverted syphon (Fig. 106). The main pipe

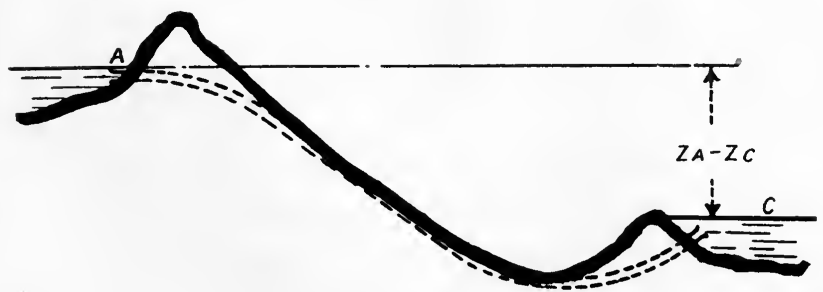

FIG. 106.

line is led into a storage tank at $A$, and out of a second tank at $C$, the connection between $A$ and $C$ being made by means of the inverted syphon. This has the advantage of reducing the maximum pressure which may be brought to bear on the pipe line by the sudden closing of a valve, since the pressure at $A$ cannot now exceed that of the atmosphere. 
Here, as in the case of the ordinary syphon, the velocity of flow is given by

or if $Z_{A}-Z_{C}=H$

$$
Z_{A}-Z_{0}=\frac{r^{2}}{2 g}\left\{1+\frac{f l}{m}+K\right\}
$$

$$
r=\sqrt{\frac{2 g H}{\left(1+\frac{f l}{m}+K\right)}} .
$$

With an inverted syphon, an automatic valve should always be placed at the entrance, so that if, due to the bursting of a pipe, the velocity of flow attains more than its normal value, water may be automatically cut off from the pipe line.

\section{Examples.}

(1) Two reservoirs are connected by a pipe 500 yards long. For the first 250 yards its diameter is 4 inches, increasing for the second 250 yards to 8 inches. A re-entrant mouthpiece is fitted at the entrance, while discharge takes place directly into the side of the lower reservoir at a depth of 6 feet below the surface. If the difference in the surface level in the reservoirs is 10 feet, determine the discharge in gallons per minute. $f=\cdot 005$.

Answer. $119 \cdot 6$ gallons per minute.

(2) A gate valve in a 12-inch cylindrical C. I. pipe is lifted through $\frac{3}{8}$ of its full opening. Determine the equivalent length of a straight pipe offering the same resistance. $f={ }^{\prime} 005$.

\section{Answer. $l=276$ feet.}

(3) A 6-inch pipe contains 6 right-angled bends, 12 inches in radius, 4 right-angled bends of 6 inches radius, and 2 sharp rightangled bends. Determine the equivalent length of straight pipe offering the same resistance to flow as this combination of bends. $f^{\prime}=\cdot 007$.

$$
\text { Answer. } l=53 \cdot 3 \text { feet. }
$$

(4) Two reservoirs of 80,000 and 10,000 square feet area are connected by a 6 -inch pipe, 500 feet long. Initially, the difference 
of surface level is $\mathbf{1 6}$ feet. Determine how soon the level in the two reservoirs will be the same. Take $f=\cdot 0075$.

\section{Answer. About 69 hours.}

(5) Two reservoirs, surfaces 15 and 25 feet above a common datum feed through a 12-inch and an 18-inch pipe respectively, into a common 24 -inch pipe at a height 5 feet above datum level, while this in turn feeds a reservoir whose surface level is taken as datum. If the lengths of the 12-inch, 18-inch, and 24-inch pipes are respectively 1,000 feet, 1,500 feet, and 2,000 feet, determine the velocity of flow in and the discharge through each pipe, neglecting all losses of head except those due to friction. Also determine the pressure at the junction of the three pipes. Take $t=\cdot 005$.

Answer. $\left\{\begin{array}{c}12 \text { in. pipe. } \quad r=4 \cdot 62 \text { f.s. } \quad Q=3 \cdot 62 \text { c.f.s. } \\ 18,, \quad, \quad r=7 \cdot 22 \text { f.s. } \quad Q=12 \cdot 76 \text { c.f.s. } \\ 24,, \quad, \quad r=5 \cdot 21 \text { f.s. } \quad Q=16 \cdot 38 \text { c.f.s. } \\ \text { Pressure at junction }=3 \cdot 03 \text { ft. of water. }\end{array}\right.$

(6) Assuming ( ) $\propto \sqrt{\frac{H}{l}} d^{2 \cdot 58}$ determine the discharge from a 12-inch pipe, if a similar 8-inch pipe of half the length gives a discharge of 10,000 gallons per hour under the same head.

Answer. 20,153 gallons per hour.

(7) A pumping engine supplies water at a pressure of $75 \mathrm{lbs}$. per square inch through a short pipe 20 inches diameter to a hydrant box, from which it is discharged by four parallel nozzles 2 inches in diameter. Taking the coefficient of loss in the nozzles to be $\cdot 03$, determine the water horse-power of the engine.

Answer.' 181 horse-power.

(8) The difference of surface level in two reservoirs which are connected by a syphon is 25 feet. The length of the syphon is 2,000 feet and its diameter is 12 inches. Assuming $f=005$, and that the syphon runs full, determine the discharge. Also if the vertex of the pipe line is 16 feet above the surface level in the upper reservoir, determine the maximum length of inlet leg for the pipe to run full.

Answer. $Q=4 \cdot 9$ c.f.s.

$=1,840$ gallons per minute.

Maximum length of inlet $=1,440$ feet 


\section{CHAPTER IX.}

Hlow in an open channel--Formulae-Darcy and Bazin-Prony-EytelweinKutter-Manning-Fidler-Thrupp-Form of channel-Channel of constant mean velocity-General equation of flow-Non-uniform flow-Standing waves-Backwater function-Effect of dams-Bridge piers-Passage of boat through a narrow canal-Flow round river bends-Distribution of velocity in an open channel-Gauging of flow in streams and channels-Current metersFloats.

\section{Art. 83.-Flow in an Open Channel.}

By the term "open channel" we include all rivers, artificial canals, aqueducts, and conduits, and, in addition, sewers and pipes of whatever section which run partially full, and which consequently do not present a solid boundary on every side of the contained liquid. The force producing flow cannot now be provided by any external head, but is solely due to the slope or gradient of the channel.

If a circular pipe be laid almost horizontally and if the surface level of water flowing through the pipe be allowed to rise, the change from the state in which the flow is governed by the laws appertaining to an open channel, to that in which the ordinary laws of pipe flow hold, is not abrupt, and it is to be inferred that a general formula is deducible which by satisfactory adjustment of constants shall fit either type of flow.

Much experimental work has been carried out in this branch of the subject, and to what extent experimental results justify this inference will be considered in the following pages.

The comparative simplicity of the conditions holding in the case of a circular pipe, the complications which must of necessity be introduced where water flows through a channel of uneven section, and the ease with which accurate observations are made in the one case and the difficulty with which even such a fundamental observation as the difference in level at points widely distant is accurately determined in the other, render it impossible 
that the laws governing the flow in open channels should be so definite and of such universal application as those already considered.

Assuming the resistance, $R$, to flow, to be proportional to the wetted perimeter $P$ of the channel, this may be expressed as

$$
R=f^{\prime} S v^{n}
$$

where $f^{\prime}$ is a coefficient depending on the condition of the surface and probably, from analogy to pipe flow, also on the velocity $v$, while $n$ is a number probably varying from 1.79 to $2 \cdot 00$, depending on the surface and on the velocity $v$, and $S=P \times l$, where $l=$ length of channel.

If $A=$ sectional area of channel beneath water line, and if we assume the resistance to be equally divided over the area we have, if $p=$ resistance per unit area of the stream

$$
p=f^{\prime} \cdot \frac{P}{A} \cdot l v^{n}
$$

Here $\frac{P}{A}$ or $m$ is the hydraulic mean depth of the section.

If the channel be of uniform slope $\frac{h}{l}$, where $\frac{h}{l}=i=\sin \theta$ ( $\theta$ being the angle of inclination), then the weight of water. in this length $l$, per unit area of the channel, being $W l$ lbs., the resolved part of this weight in the direction of motion $=W l \frac{h}{l}=W h \mathrm{lbs}$.

$\therefore$ If the velocity is constant so that this force is entirely expended in overcoming friction and not in producing acceleration, we have

$$
\begin{aligned}
W h & =W f^{\prime} \frac{P}{A} l r^{\cdot} \\
h & =\frac{f^{\prime} l v^{n}}{m} \\
\text { or } h & =\frac{f l v^{n}}{2 g m}
\end{aligned}
$$

In an open channel $n$ may be taken as being approximately equal to 2 , so that the formula becomes

$$
h=\frac{f l v^{2}}{2 g m}
$$


This may be written in the form adopted by Chezy, riz.,

where

$$
r=\sqrt{\frac{2 g}{f} \cdot \frac{h}{l} m}=C \sqrt{m i}
$$

Many experiments have been devoted to determining the values of $C$ or of $f$ for channels having different physical characteristics, and the results of the more important of these are as follow, the numerical values of the coefficients obtained by the various observers being collected and tabulated on pp. 271-273.

Darcy and Bazin (1855-9), as the results of experiments carried out on the Bourgoyne Canal, gave $C$ the value $\frac{1}{\sqrt{a+\frac{b}{m}}}$ where $a$ and $b$ (p. 271) vary only with the material and condition of the bed and sides of the channel.

'T'hese channels were of many different forms and dimensions; were lined with different materials, and had slopes varying from $\cdot 001$ to $\cdot 01$.

Promy, from experiments by Chezy and Dubuat on earthen channels and on wooden channels of small section put

$\mathrm{Ol}$

$$
\begin{gathered}
a r+b r^{2}=m i \\
C=\sqrt{\frac{1}{b+\frac{a}{r}}} .
\end{gathered}
$$

The corresponding value of $f=2 g\left(b+\frac{a}{v}\right)=A+\frac{B}{r}$

$$
\text { where } \begin{cases}\frac{1}{a}=22,472 . & A=\cdot 00607 . \\ \frac{1}{b}=10,607 . & B=\cdot 00286 .\end{cases}
$$

Eytelwein, from experiments on the Rhine channel, gave the same type of formula, his coefficients being

$$
\begin{array}{ll}
\frac{1}{a}=41,211 . & A=\cdot 00719 . \\
\frac{1}{b}=8,975 . & B=\cdot 00156 .
\end{array}
$$


In both these cases the unit of length is 1 foot. With moderate velocities $a v$ is small compared with $b r^{2}$ and may be neglected, when the formulae reduce to

$$
\begin{aligned}
r & =\frac{1}{\sqrt{b}} \sqrt{m i}=103 \sqrt{m i} . \quad f=\cdot 00607 . \\
\text { Eyteluein } r & =---\quad=95 \sqrt{m i} . \quad f=\cdot 00714 .
\end{aligned}
$$

These coefficients, being independent of the condition of the surface are obviously only applicable to channels having the same physical characteristics as those experimented upon.

Bazin (1897), as the result of a very large number of experiments on canals and conduits of all sections and dimensions deduced for $C$ the value

$$
\frac{157 \cdot 6}{1+\frac{N}{\sqrt{m}}} \text { in foot units. }
$$

$N$ varying with the character of the surface.

Values of $N$ for different types of surface are given on p. 271 .

This gives a value of $f=000259\left\{1+\frac{2 N}{\sqrt{m}}+\frac{N^{2}}{m}\right\}$.

This form of equation is in common use in France, and has given good results with velocities not exceeding 4 feet per second.

Ganguillet and Kutter deduced the coefficient

$$
C^{\prime}=\frac{41 \cdot 6+\frac{\cdot 00281}{i}+\frac{1 \cdot 8112}{N}}{1+\left(41 \cdot 6+\frac{00281}{i}\right) \frac{N}{\sqrt{m}}} \text { in foot units, }
$$

the equation being identical with that used for pipe flow. $N$ depends on the character of the surface and has values given on p. 271. This formula is of general application in Great Britain, Germany, and the United States, and probably gives, more reliable results than any other formula in general use.

By altering the value of $i$ in this formula from 001 to $\cdot 01$, the value of $C$ is altered by less than 1 per cent. For streams of 
fairly rapid slope the value of $i$ may then be taken as sensibly equal to 001 , in which case the value of $C$ simplifies to

$$
C=\frac{44 \cdot 4+\frac{1 \cdot 811}{N}}{1+44 \cdot 4 \frac{N}{\sqrt{ } m}}
$$

In very large rivers the flow is sensibly independent of the character of the bed, and for such a case Manning ${ }^{1}$ gives $C$ the value

$$
\begin{aligned}
& C=62\left\{1+\frac{\sqrt{m}}{7}-\frac{.05}{\sqrt{m}}\right) \text { in foot units. } \\
& C=34\left\{1+\frac{\sqrt{m}}{4}-\frac{.03}{\sqrt{m}}\right) \text { in metre units. }
\end{aligned}
$$

From the nature of the case it would appear hopeless to obtain any strictly mathematical solution for flow in open channels and rivers of irregular section, and even to observe and record correctly the physical data required is almost impossible. An examination of the results given by an application of the various formulae shows that for abnormal sections or velocities a difference of 50 per cent. is not uncommon. The formulae of Kutter and Bazin may be relied upon to give fairly consistent results in normal cases, that of Bazin being more suitable for small channels under 20 feet wide, and that of Kutter for moderately large

\begin{tabular}{|c|c|c|c|c|}
\hline \multirow{2}{*}{ Character of Surface. } & \multicolumn{2}{|c|}{ Darcy. } & Bazil. & Kutter. \\
\hline & $a$. & b. & $N$ & $N$ \\
\hline Simooth cement or planed timber . & $\cdot 000046$ & $\cdot 0000045$ & $\cdot 109$ & $\cdot 009$ to $\cdot 010$ \\
\hline $\begin{array}{l}\text { Unplaned timber, flumes, slightly } \\
\text { tuberculated iron, ashlar and } \\
\text { well-laid brickwork . }\end{array}$ & $\cdot 000058$ & $\cdot 0000133$ & ' 290 & $\cdot 012$ to $\cdot 013$ \\
\hline $\begin{array}{l}\text { Rubble masonry and brickwork in } \\
\text { an inferior condition. Fine } \\
\text { gravel well rammed. }\end{array}$ & $\cdot 000073$ & $\cdot 000060$ & 833 & $\cdot 017$ \\
\hline
\end{tabular}
channels.

Coefficients for Flow in Open Channels.

1 Inst. Civil Engineers of Ireland, December 4, 1889 
Coefficients for Flow in Open Channels-(continued).

\begin{tabular}{|c|c|c|c|c|}
\hline Character of Surface. & \multicolumn{2}{|c|}{ Darcy. } & Buzin. & Kutter \\
\hline $\begin{array}{l}\text { Rubble in inferior condition. Canals } \\
\text { with earth beds in very good } \\
\text { condition . . . . . }\end{array}$ & & & & $\cdot() 20$ \\
\hline $\begin{array}{c}\text { Canals with earth beds in good } \\
\text { condition } .\end{array}$ & & & $1 \cdot 540$ & (1)22.5 \\
\hline Ditto in moderate condition . & $\cdot 00008 j$ & $\cdot 00035$ & & $\cdot 025$ \\
\hline $\begin{array}{l}\text { Canals and rivers in rather bad } \\
\text { order } \cdot \text {. } \\
.\end{array}$ & & & $2 \cdot 355$ & $\cdot 030$ \\
\hline Ditto in very bad order . & $\cdot 00012$ & $\cdot 00070$ & $3 \cdot 170$ & 0.5 \\
\hline
\end{tabular}

Here a channel is said to be in very good order when it is free from boulders, hollows in its bed and banks, sharp bends, snags and weeds. When badly choked with weeds the value of $N$ in Kutter's formula may become much greater than 035 . The following values of $N$ in the latter formula are taken from Jackson's tables and are probably slightly more accurate than those given above.

Planed timber accurately jointed-glazed or enamelled surfaces . . . . . . . . . . 009

Smooth cement or plaster . . . . . . . 010

Unplaned timber, well jointed-new brickwork well laid . . . . . . . . . . 012

Unglazed stonework-iron-brick and ashlar _ . . 013

Wooden troughs with battens inside, $\frac{1}{2}$ inch apart . 015

Rubble set in cement . . . . . . . . 017

If any of these are in bad order the next higher value for $N$ is to be taken.

For convenience in applying the results of these formulae the values of $f$ in the formula $h=\frac{f l v^{2}}{2 g m}$, and of $C$ in the formula $v=C \sqrt{m i}$ have been calculated and are tabulated opposite. 
FLOW IN OPEN CHANNELS.

\begin{tabular}{|c|c|c|c|c|c|c|c|c|c|}
\hline \multirow{3}{*}{ 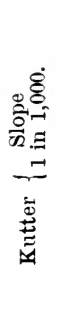 } & $\begin{array}{l}N \\
\cong\end{array}$ & 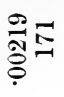 & 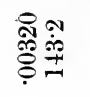 & 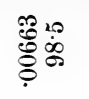 & 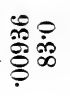 & 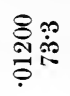 & 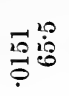 & స్ํำ & 㑒总 \\
\hline & $\begin{array}{l}\overrightarrow{\|} \\
\tilde{\Xi}\end{array}$ & 苑哭 & 兽芯 & 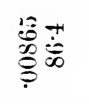 & 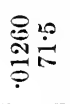 & 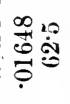 & 亩范 & 我早 & 窝曷 \\
\hline & $\begin{array}{l}0 \\
\approx\end{array}$ & 芬节 & 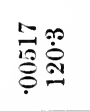 & 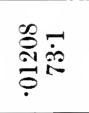 & 통요 & 总苛 & 羿 & 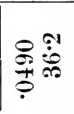 & 옹 \\
\hline \multirow{3}{*}{ 胥 } & $\underset{\Xi}{\|}$ & 串 & 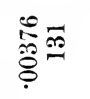 & 惫灾 & & 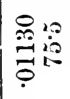 & & 感家 & 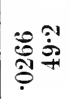 \\
\hline & $\begin{array}{l}\overrightarrow{\|} \\
\approx\end{array}$ & 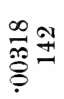 & 㸓 & 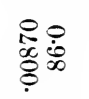 & & 兽 & & 色 & 量 \\
\hline & $\begin{array}{l}\dddot{10} \\
\approx\end{array}$ & 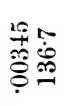 & $\begin{array}{l}20 \\
\frac{10}{8} \\
\frac{1}{1}\end{array}$ & 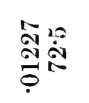 & & 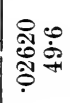 & & 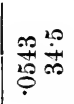 & 念京 \\
\hline \multirow{3}{*}{ 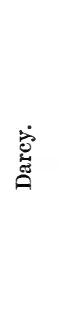 } & $\stackrel{N}{\|}$ & 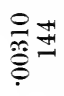 & 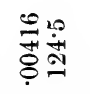 & 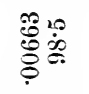 & & & 过 & & 菅审 \\
\hline & $\stackrel{\widetilde{\|}}{\approx}$ & 突 & 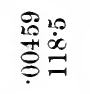 & $\begin{array}{l}0 \\
20 \\
00 \\
0 \\
0\end{array}$ & & & 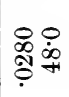 & & م. \\
\hline & $\stackrel{\dddot{?}}{\|}$ & 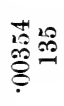 & $\begin{array}{l}\text { 車。 } \\
8 \\
8\end{array}$ & 索㝒 & & & مَ & & 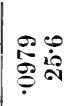 \\
\hline \multirow[b]{2}{*}{ 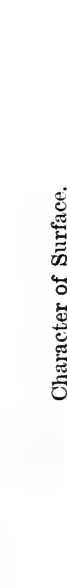 } & & $\left\|_{x}\right\|$ & $\underset{x}{\| !}$ & $\underbrace{\|}_{0}$ & \|\|$_{x} \|$ & $\left\|_{x}\right\|$ & $\underset{x \rightarrow 0}{\|} \|$ & \|\|$_{x} \|$ & $\left\|_{x \rightarrow 0}\right\|$ \\
\hline & & 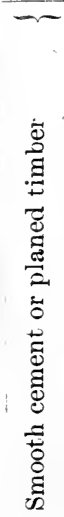 & 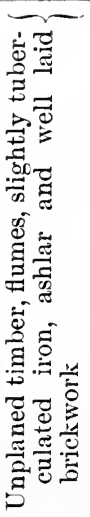 & 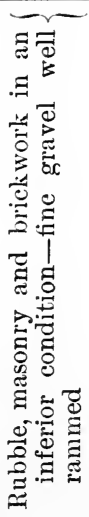 & 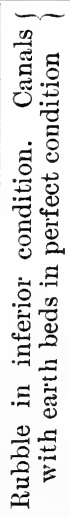 & 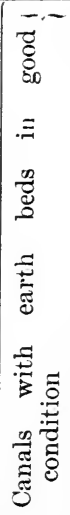 & 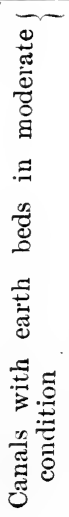 & 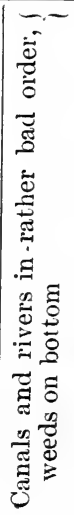 & 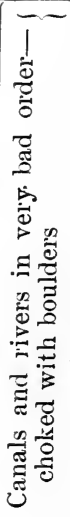 \\
\hline
\end{tabular}

H.A. 
Manning's formula gives :-

\begin{tabular}{c|c|c|c|c}
\hline$m$ & 1 & 2 & 4 & 16 \\
\hline$f$ & $\cdot 0140$ & $\cdot 0123$ & $\cdot 0105$ & $\cdot 00677$ \\
\hline$c$ & $67 \cdot 9$ & $72 \cdot 4$ & $78 \cdot 4$ & $97 \cdot 5$
\end{tabular}

'Taking $m=16$, Kutter's formula makes $f=\cdot 0077, C=914$, and for large and deep rivers having a depth of 15 feet and over, $f$ may be taken as approximately equal to 0072 , irrespective of the condition of the channel. 'This makes $C=94{ }^{\circ} 6$.

The change in the values of $C$ in Kutter's formula with changes in the values of $m$ and of the slope are indicated in the following tables :-1

Talues of $C$ (slope 1 in 5,000).

hydraulic Mean Depth $m$.

\begin{tabular}{c|c|c|c|c|c|c|c|c|c|c|c} 
& $\frac{1}{4}$ & $\frac{1}{2}$ & $\frac{3}{4}$ & 1 & $1 \cdot 5$ & 2 & $t$ & 6 & 8 & 10 & 20 \\
\hline$N=\cdot 020$ & 45 & 57 & 64 & 69 & 77 & 82 & 94 & 101 & 105 & 108 & 117 \\
\hline$N=\cdot 025$ & 34 & 43 & 49 & 54 & 60 & 64 & 76 & 82 & 86 & 89 & 95 \\
\hline$N=\cdot 030$ & 27 & 35 & 40 & 43 & 49 & 58 & 63 & 69 & 73 & 76 & 54
\end{tabular}

$$
N^{r}=\cdot 025 .
$$

HyDiÁUlic MEAN DEPTH $m$.

\begin{tabular}{ll|c|c|c|c|c|c|c|c|c|c}
\hline & 50 & 75 & $1 \cdot 0$ & $1 \cdot 5$ & $2 \cdot 0$ & $4 \cdot 0$ & $6 \cdot 0$ & $8 \cdot 0$ & $10 \cdot 0$ & $20 \cdot 0$ \\
\hline Slope $\frac{1}{10,000} \cdot$ & 41 & 45 & 52 & 59 & 64 & 76 & 83 & 88 & 92 & 102 \\
\hline Slope $\frac{1}{5,000} \cdot$ & $\cdot$ & 43 & 49 & 54 & 60 & 64 & 76 & 82 & 86 & 89 & 98 \\
\hline Slope $\frac{1}{2,500} \cdot$ & 45 & 50 & 5.5 & 61 & 6.5 & 75 & 81 & 85 & 88 & 95 \\
\hline Slope $\frac{1}{1,000} \cdot$ & + & 45 & 51 & 55 & 61 & 65 & 75 & 80 & 84 & 86 & 93 \\
\hline
\end{tabular}

1 A complete set of values of $C$ are given both in Jackson's " Canal and Culvert Tables" and in Higham's "Hydraulic Tables." 
With this value of $N(\cdot 025)$ the value of $C$ is independent of the slope when $m=3.22$ feet. The curves in Fig. 107 may be used to obtain values of $C$ for intermediate values of $m$ by interpolation

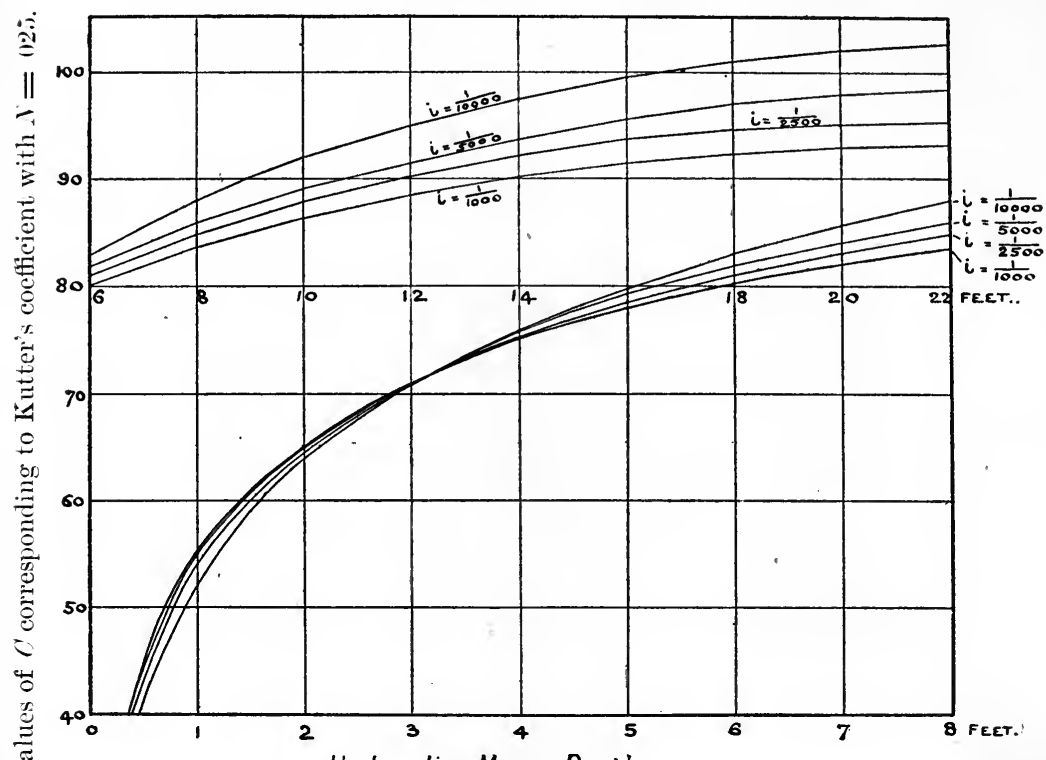

Hydraulic Mean Depth

Curve showing variation in $C$ in the formula $x=C \sqrt{m i}$, with a variation in $m$ and in $i$.

Fig. 107 .

and indicate how $C$ varies with the slope and with the hydraulic mean depth.

\section{Rational Formula.}

From analogy with pipe flow it would appear probable that a formula of the type

$$
h=\frac{f^{\prime} l v^{n}}{\left(\frac{A}{P}\right)^{x}}=\frac{f l v^{n}}{m^{x}}
$$

where, as in the case of a pipe, $n$ is in general less than 2 , would most nearly represent the law of channel flow.

Claxton Fidle ${ }^{1}$ has determined the values of $f, n$, and $x$ from many experimental results of Darcy, Bazin, Smith, Stearns, and

1 Fidler, "Calculations in Hydraulic Engineering," Part II. (Longmans \& Co.), 1902. 
other observers, and the following table is abridged from values given by him :-

\begin{tabular}{|c|c|c|c|c|c|}
\hline Form of Section. & Naterial of Surface & & $n$ & $x$ & $f$ \\
\hline Circular & Smooth neat cement & & $1 \cdot 75$ & $1 \cdot 167$ & $\cdot 0000676$ \\
\hline Rectangular & $", \quad$, & . & & & \\
\hline Circular & Cement and sand & · & $"$ & $"$ & $\cdot 000078 \pi$ \\
\hline Rectangulär & $\begin{array}{l}\text { Smooth brick } \\
\text { Smooth ashlar . }\end{array}$ & & & & $\cdot 0000904$ \\
\hline Circular . & Bare metal pipes with rive & joints & $1 \cdot 77$ & $\ddot{1} \cdot 18$ & .0000871 \\
\hline & Rough brickwork & . & $1 \cdot 80$ & $1 \cdot 20$ & $\cdot 000097 i$ \\
\hline Rectangular & Unplaned timber & t & $"$ &, & $\cdot 0000944$ \\
\hline & Rough brickwork or ashla & & & & $\cdot 0001122$ \\
\hline Circular & Lined with fine gravel & & $1 \cdot 90$ & $1 \cdot 33$ & $\cdot 0001202$ \\
\hline Rectangular & $" \quad, \quad$ fine gravel & . & $1 \cdot 96$ & $1 \cdot 40$ & $\cdot 0001521$ \\
\hline$"$ & $", \quad$ coarse $"$ & . & $2 \cdot 10$ & 1.50 & .0001862 \\
\hline$"$ & Rubble masonry & - & $"$ & $"$ & $\cdot 0002240$ \\
\hline
\end{tabular}

While further investigation may slightly alter these values, it is extremely probable that the final solution of the problem of flow in regular channels will be found in this type of formula.

The pipe flow formula of Thrupp

$$
h=\frac{C^{n} v^{n} l}{m^{x}}
$$

where $m$ is the hydraulic mean depth is also applicable to channel flow, the following being the values of the quantities $C^{n}, n$, and $x$. When $m$ is small, $x+a \sqrt{\frac{b}{m}-1}$ should be substituted for $x$.

\begin{tabular}{|c|c|c|c|c|c|c|}
\hline \multicolumn{2}{|l|}{ Surface. } & $n$. & $c^{n}$ & $x:$ & a. & b. \\
\hline \multicolumn{2}{|c|}{ Neat cement $\left\{\begin{array}{l}\text { semi-circular section. } \\
\text { rectangular section } .\end{array}\right.$} & $\begin{array}{l}1 \cdot 74 \\
1 \cdot 95\end{array}$ & $\begin{array}{l}\cdot 0000680 \\
\cdot 0000494\end{array}$ & $\begin{array}{l}1 \cdot 165 \\
1 \cdot 190\end{array}$ & & \\
\hline Brickwork, well laid . &.$\quad \cdot \quad$. & $2 \cdot 00$ & $\cdot 0000600$ & $1 \cdot 220$ & & \\
\hline $\begin{array}{l}\text { Unplaned plank } \\
\text { Brickwork, rough }\end{array}$ & - & $\begin{array}{l}\mathbf{2} \cdot 00 \\
2 \cdot 00\end{array}$ & $\begin{array}{l}\cdot 0000714 \\
\cdot 0000780\end{array}$ & $\begin{array}{l}1 \cdot 230 \\
1 \cdot 250\end{array}$ & $\begin{array}{l}\cdot 0670 \\
.0245\end{array}$ & .50 \\
\hline $\begin{array}{l}\text { Brickwork, rough } \\
\text { Lined with fine gravel }\end{array}$ & $\dot{.}$ & $\begin{array}{l}2 \cdot 00 \\
2 \cdot 00\end{array}$ & .0001399 & 1.320 & .0788 & .60 \\
\hline Chiselled masonry . & . & $2 \cdot 00$ & $\cdot 000124 t$ & $1 \cdot 320$ & $\cdot 1565$ & $1 \cdot 00$ \\
\hline Lined with coarse gravel & - & 200 & $\cdot 0002005$ & $1 \cdot 410$ & $\cdot 1518$ & $1 \cdot 00$ \\
\hline Earth in fair condition & . & $2 \cdot 00$ & $\cdot 0002360$ & $1 \cdot 440$ & & \\
\hline Rough earth & - & $2 \cdot 00$ & $\cdot 0002600$ & $1 \cdot 560$ & & \\
\hline
\end{tabular}

The results obtained by this formula compare very well with those of Fidler, the latter probably on the whole giving the better results. 


\section{Art. 84.-Form of Channel.}

Since for a channel of given sectional area $A$, the hydraulic mean depth $A \div P$ varies with the form of its section, while the resistance to flow increases as $A \div P$ diminishes, it becomes important to determine what form of channel shall give the maximum value of $A \div P$ for a given value of $A$, since this will be the channel of maximum discharge for a given slope. Further, if this sectional area is a minimum, the cost of excavation is a minimum, and since in general the perimeter increases with the sectional area, the cost of pitching the faces of the channel is also a minimum. Theoretically, the best form of channel is one in which the bed is a circular arc, since this gives a minimum ratio of wetted perimeter to sectional area.

An investigation into the properties of different sections will be simplified if the coefficient $C$ in the formula $v \doteq C \sqrt{\bar{A}} \cdot i$, be assumed constant for a given surface. On this assumption we have :-

$$
Q=A v=C \sqrt{\frac{A^{3}}{P} \cdot i} \text { cub. ft. per sec. }
$$

For,$v$ to be a maximum, $\frac{A}{P}$ must be a maximum, so that $d\left(\frac{A}{P}\right)=0 . \quad \therefore \quad P d A-A d P=0$.

Again, for $Q$ to be a maximum, $\frac{A^{3}}{\bar{P}}$ is to be a maximum, so. that $d\left(\frac{A^{3}}{P}\right)=0$.

$$
\therefore \quad 3 P A^{2} d A-A^{3} d P=0 \text {. }
$$

Where $A$ is fixed, $d A=0$ and both conditions are satisfied if $d P=0$.

\section{Example.}

(1) Rectungular Channel.-Breadth $2 b$ - depth $d$. Here $A=2 b d: P=2 b+2 d=\frac{A}{d}+2 d$

$$
\therefore \frac{d P}{d(d)}=-\frac{A}{d^{2}}+2 \text {. }
$$


Putting $A=2 b d$ and equating $\frac{d P}{d(d)}$ to zero, we have $b=d$. Putting the full breadth $=B$, we have $d=\frac{B}{2}$, i.e., for maximum flow the depth must equal one-half the breadth.

We then have

$$
\begin{aligned}
Q & =C \sqrt{\frac{A^{3}}{P} \cdot i} \\
& =C \sqrt{\frac{8 b^{3} d^{3}}{2 b+2 d} \cdot i} \\
& =C \sqrt{2 i} \cdot b^{\frac{5}{2}} \\
& =\frac{C}{4} \sqrt{i B^{\frac{5}{2}}}
\end{aligned}
$$

(2) Trapezoidal Channel.-Fig. 108.

Let $b=$ half bottom

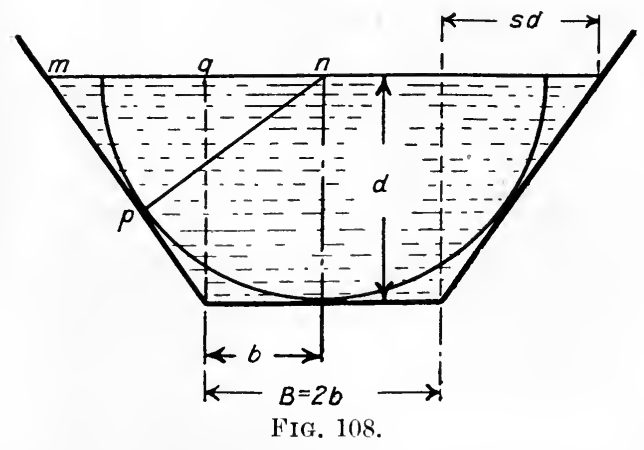
breadth ; $d=$ depth; $s=$ cotangent of angle of slope of sides.

Then $A=2 b d+$ $s d^{2}$. $P=2\left(b+d \sqrt{\left.1+s^{2}\right)}\right.$.

For $Q$ to be a maximum with a given area of channel or for the cost of construction to

be a minimum, it is necessary that $\frac{d P}{d(d)}=0$.

$$
\begin{aligned}
& \text { But } \quad P=2\left\{\frac{A}{2 d}-\frac{s d}{2}+d \sqrt{1+s^{2}}\right\} \\
& \left.\therefore \quad \frac{d P}{d(d)}=2 !-\frac{A}{2 d^{2}}-\frac{s}{2}+\sqrt{1+s^{2}}\right\} \\
& \therefore \text { For maximum value } \sqrt{1+s^{2}}=\frac{2 b d+s d^{2}}{2 d^{2}}+\frac{s}{2} \\
& =\frac{b}{d}+s \\
& \therefore \quad\left(1+s^{2}\right) d^{2}=(b+s d)^{2}
\end{aligned}
$$

But from the figure it will be seen that if a circle having its 
centre in the surface of the water can be drawn to touch the sides and bottom, we have

$$
m n=b+s d ; m p=m q=s d ; n p=d,
$$

and since $(m n)^{2}=(m p)^{2}+(n p)^{2}$, the above equation is satisfied. These proportions then give the best results.

T'he value of $s$ depends largely on the material in which the channel is excavated. The following may be taken as the minimum permissible values.

Earthen canal with faced sides $s=10^{\circ}$.

$$
\begin{aligned}
& , \quad, \quad \text { in light soil }, \quad s=1 \cdot 5 . \\
& , \quad s=2 \cdot 0 .
\end{aligned}
$$

The latter value is usually adopted for all unfaced earthen sides.

Substituting this value of $s$ in equation (2) we have

$$
\begin{aligned}
5 d^{2} & =(b+2 d)^{2} \\
\therefore \quad d & =4 \cdot 24 b
\end{aligned}
$$

$\therefore$ For maximum discharge $d=2 \cdot 12 B$, where $B$ is the bottom breadth.

We then have

$$
\begin{aligned}
Q & =C \sqrt{\frac{A^{3}}{P} \cdot i} \\
& =C \sqrt{\frac{\left(2 b d+s d^{2}\right)^{3}}{2\left(b+d \sqrt{\left.1+s^{2}\right)}\right.} \cdot i}
\end{aligned}
$$

Substituting for $d$ from (2) we have

$$
Q=C b^{\frac{5}{2}} \frac{2 \sqrt{1+s^{2}}-s}{\sqrt{2}\left(\sqrt{1+s^{2}}-s\right)^{\frac{5}{2}}} \cdot \sqrt{i}
$$

and giving $s$ the value 2

$$
Q=204 C b^{\frac{5}{2}} \text { cub. ft. per sec. }
$$

if $C$ and $b$ are taken in foot units

or

$$
Q=36 \cdot 1 C B^{\frac{5}{2}} \text {. }
$$

Whatever the slope of the sides, the trapezium of best shape will be that in which the sides are made tangent to the circle of radius $=d$ having its centre in the surface, and as may be readily shown, all such channels have the same hydraulic mean depth $=\frac{d}{2}$. It follows then that the velocity of flow when the channel 
is full will be independent of the slope of the sides, and will depend solely on the gradient of the bed. The discharge of any two trapezoidal channels of the best form and of the same gradient and depths, will, when running full, be proportional to their respective mean widths.

The following table indicates how the top and bottom widths for a section of this type, vary with the slope of the sides :-

\begin{tabular}{l|c|c|c}
\hline & \multicolumn{2}{|c}{ Width. } \\
& $\begin{array}{c}\text { Angle of inclination of } \\
\text { sides. }\end{array}$ & Top. & Bottom. \\
\hline 0 to 1 & $90^{\circ}$ & $2 \cdot 000 d$ & $2 \cdot 000 d$ \\
.25 to 1 & $75^{\circ} \cdot 58^{\prime}$ & $2 \cdot 062 d$ & $1 \cdot 562 d$ \\
$\cdot 5$ to 1 & $63^{\circ} \cdot 26^{\prime}$ & $2 \cdot 236 d$ & $1 \cdot 236 d$ \\
$\cdot 75$ to 1 & $53^{\circ} \cdot 8^{\prime}$ & $2 \cdot 500 d$ & $1 \cdot 000 d$ \\
$1 \cdot 0$ to 1 & $45^{\circ} \cdot 0^{\prime}$ & $2 \cdot 828 d$ & $\cdot 828 d$ \\
$1 \cdot 5$ to 1 & $33^{\circ} \cdot 41^{\prime}$ & $3 \cdot 606 d$ & $\cdot 606 d$ \\
$2 \cdot 0$ to 1 & $26^{\circ} \cdot 34^{\prime}$ & $4 \cdot 472 d$ & $\cdot 472 d$ \\
$2 \cdot 5$ to 1 & $21^{\circ} \cdot 48^{\prime}$ & $5 \cdot 385 d$ & $\cdot 385 d$ \\
3.0 to 1 & $18^{\circ} \cdot 26^{\prime}$ & $6 \cdot 325 d$ & $\cdot 325 d$ \\
\hline
\end{tabular}

Circular Section.-Fig. 109.

Let $d=$ diameter of circle.

,$\theta=$ angle at centre subtended by wetted perimeter.

Then $\quad A=\frac{d^{2}}{8}(\theta-\sin \theta) ; P=\frac{d}{2} \theta$

$$
\therefore \quad \frac{A}{P}=\frac{d}{4}\left(1-\frac{\sin \theta}{\theta}\right) . \quad \text { Suppose } d \text { fixed. }
$$

For maximum velocity $\frac{d\left(\frac{A}{P}\right)}{d \theta}=0$

$$
\begin{aligned}
& \therefore \quad \theta=\tan \theta \\
& \therefore \quad \theta=257 \frac{1}{2}^{\circ} .
\end{aligned}
$$

For maximum discharge $\frac{d\left(\frac{A^{3}}{P}\right)}{d \theta}=0$

$$
\begin{array}{ll}
\therefore & 3 P \frac{d A}{d \theta}-A^{d} \frac{P}{d \theta}=0 \\
\therefore \quad & 3 \theta(1-\cos \theta)=\theta-\sin \theta \\
\therefore \quad & 2 \theta-3 \theta \cos \theta+\sin \theta=0 .
\end{array}
$$


The value of $\theta$ which satisfies this equation is $308^{\circ}$, so that a circular conduit will give its maximum discharge when the depth of water is about 95 of the diameter, the discharge then being about 5 per cent. greater than when completely full.

'The discharge corresponding to any depth of water is given by

$$
\begin{aligned}
Q & =C \sqrt{\frac{A^{3}}{P} \cdot i} \\
& =C \sqrt{\frac{d^{5}}{256} \frac{(\theta-\sin \theta)^{3}}{\theta} \cdot i} \\
& =\frac{C}{16} d^{\frac{5}{2}} i^{\frac{1}{2}} \sqrt{\frac{(\theta-\sin \theta)^{3}}{\theta}}
\end{aligned}
$$

when $\theta=180^{\circ}=: \because$.

$$
Q=\frac{C}{5 \cdot 1} d^{\frac{5}{2}} \sqrt{i} . \text { cub. ft. per sec. }
$$

The semi-circular section when running full has a hydraulic mean depth of $\frac{d}{4}$, and since this is greater than that of any other form of channel of the same area, this section is well fitted for an open channel.

Where a polygonal channel is used, the hydraulic mean depth is greatest when the sides and bottom of the channel are designed so as to be

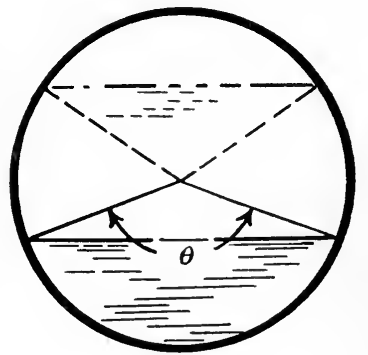

FIG. 109. tangent to a circle having its centre in the water line. The trapezoidal section and rectangular section of greatest flow, are particular cases of this. Where vertical sides are to be used the most suitable form of bottom consists of a circular arc, concave upwards.

Channel of Constant Mean Velocity.-Where the depth of water in a channel may vary within wide limits, it is in general desirable to design this so that the velocity of flow may be as nearly as possible independent of the depth. Otherwise, in an open canal, the velocity may become so great as to damage the sides and bottom by scouring (Art. 94), while in a sewer, with low 
heads, the velocity may become insufficient to produce the necessary flushing. On the assumption that $r=C \sqrt{m i}$, where $C=$ constant, the only essential condition to be satisfied for $v$ to be independent of the depth is that the hydraulic mean depth shall also be independent of the depth of water.

Thus the required channel must have sides formed by a continuous curve such that the area bounded by the sides, and any two horizontals varies as the length of the arcs intercepted between these horizontals. No curve can be found to satisfy these conditions, though close approximations may be obtained.

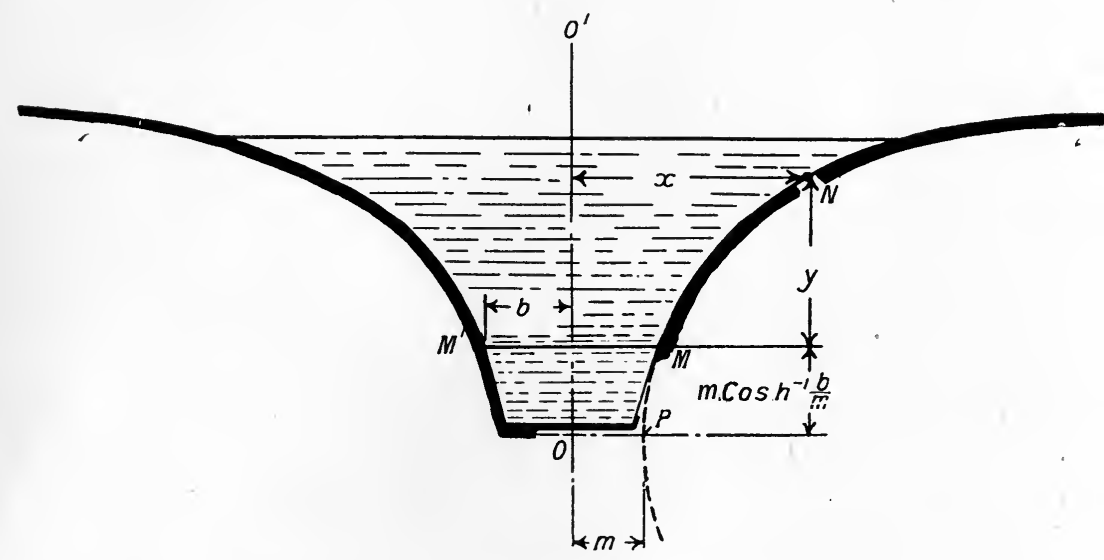

F19. 110 .

Obviously, a rectangular section of great depth compared with its width would approximately satisfy the conditions, and especially if its bottom were constructed so as to offer less resistance per unit area than its sides.

A construction which gives accurate results between certain limits may, however, be obtained as follows.

In Fig. 110 let $x$ be the half breadth of the section at a height $y$ above $M^{\prime} M$ where the half breadth is $b$, and let $s$ be the length of the arc $M N$.

'The position of the axis $M^{\prime} M$ and the breadth $b$ are usually fixed from a consideration of the minimum discharge to be expected through the channel, a trapezoidal channel having an upper breadth $M^{\prime} M(=2 b)$ being designed to take this 
minimum discharge when running full. Let $m$ be the hydraulic mean depth of this small channel, let $p$ be its half perimeter, and $a$ its half area. It is required to continue the sides of this channel so as to give a section for which the hydraulic mean depth $A \div P$ shall be equal to $m$ for all depths of water.

Considering the section of the complete channel to one side of the axis $O O^{\prime}$, we have

$$
\begin{gathered}
\frac{A}{P}=\frac{a+j x d y}{p+s}=\text { constant }=m \\
\therefore \quad a+\int x d y=m(p+s) .
\end{gathered}
$$

Differentiating, this gives $x=m \frac{d s}{d y}$.

But

$$
\begin{aligned}
\frac{d \cdot s}{d y} & =\sqrt{1+\left(\frac{d x}{d y}\right)^{2}} \\
\therefore \quad x^{2} & =m^{2}\left\{1+\left(\frac{d x}{d y}\right)^{2}\right\} \\
\therefore \quad \frac{d y}{m} & =\frac{d x}{\sqrt{x^{2}-m^{2}}} .
\end{aligned}
$$

Integrating this we have

$$
\begin{aligned}
y & =m \cosh ^{-1} \frac{x}{m}+D \\
& =m \log _{e}\left\{x+\sqrt{x^{2}-m^{2}}\right\}+D^{\prime \prime} .
\end{aligned}
$$

But $x=b$ when $y=0$

$$
\begin{aligned}
\therefore \quad D & =-m \cosh ^{-1} \frac{b}{m} \\
D^{\prime} & =-m \log _{e}\left\{b+\sqrt{b^{2}-m^{2}}\right\} \\
\therefore \quad y & =m\left(\cosh ^{-1} \frac{x}{m}-\cosh ^{-1} \frac{b}{m}\right) \\
& =m \log _{e}\left\{\frac{x+\sqrt{x^{2}-m^{2}}}{b+\sqrt{b^{2}-m^{2}}}\right\} .
\end{aligned}
$$

From equation (2) the curve of the side may be plotted by calculating values of $y$, corresponding to a series of values of $x$.

Since $v=C \sqrt{m i}$ this velocity with any given gradient may be adjusted to any given value by designing the small channel so as to give the required value of $m$. The only restriction is 
that $m$ cannot exceed $\frac{b}{2}$, this being its value when the lower channel is semicircular, or rectangular with a breadth equal to twice its depth.

\section{Exaniple.}

To design a channel to give an uniform velocity of flow of 4 feet per second, the half breadth $b$ being 2.5 feet, and $C$ having a value 90 .

Here, assuming a rectangular section for the lower channel, of depth 2.5 feet, we have $m=1.25$ feet.

$$
\begin{aligned}
& \therefore \quad 4=90 \sqrt{1.25 i} \\
& \therefore \quad i=\cdot 00158 .
\end{aligned}
$$

Again when $x=5$ we have

$$
\begin{aligned}
y & =1 \cdot 25 \log _{e}\left\{\frac{5+\sqrt{25-1 \cdot 5625}}{2 \cdot 5+\sqrt{6 \cdot 25-1 \cdot 5625}}\right\} \\
& =1 \cdot 25 \log _{e}\left\{\frac{5+\sqrt{23 \cdot 4375}}{2 \cdot 5+\sqrt{4 \cdot 6875}}\right\} \\
& =1 \cdot 25 \log _{e} 2 \cdot 108 \\
& =1 \cdot 25+2 \cdot 302 \log _{10}(2 \cdot 108) \\
& =.933 \text { feet. }
\end{aligned}
$$

Obtaining a series of such values of $y$, corresponding to definite values of $x$, the section may be constructed. For this particular example the following table shows how the half breadth of the section increases with the depth :-

\begin{tabular}{c|c|c|c|c|c|c|c}
\hline$x$ & $2 \cdot 5$ & $5 \cdot 0$ & $10 \cdot 0$ & $15 \cdot 0$ & $20 \cdot 0$ & $30 \cdot 0$ & $50 \cdot 0$ \\
\hline$y$ & 0 & $\cdot 933$ & $1 \cdot 817$ & $2 \cdot 320$ & $2 \cdot 520$ & $2 \cdot 832$ & $3 \cdot 300$ \\
\hline
\end{tabular}

The curve is a portion of a catenary, and, writing its equation in the form $\frac{y-D}{m}=\cosh ^{-1} \frac{x}{m}$,

or

$$
m \cosh \left(\frac{y-D}{m}\right)=x,
$$

it will be seen that this catenary has its axis parallel to and at 
a distance $(-D)=m \cosh ^{-1} \frac{b}{m}$ below the axis $M^{\prime} M$, while its vertex $P$ is at a horizontal distance $m$ from the centre line $O O^{\prime}$ (Fig. 110).

In a closed channel, or sewer, it is impossible to make the mean depth, and therefore the velocity, constant for all depths of water. To approximate to this as far as possible the egg shaped sewer (Hig. 111) is often used. In section this consists of two circular ares centred at $A$ and $B$, and connected by a second pair of circular ares centred at $C$ and $C^{\prime}$. The proportions often adopted in practice are indicated in Fig. 111.

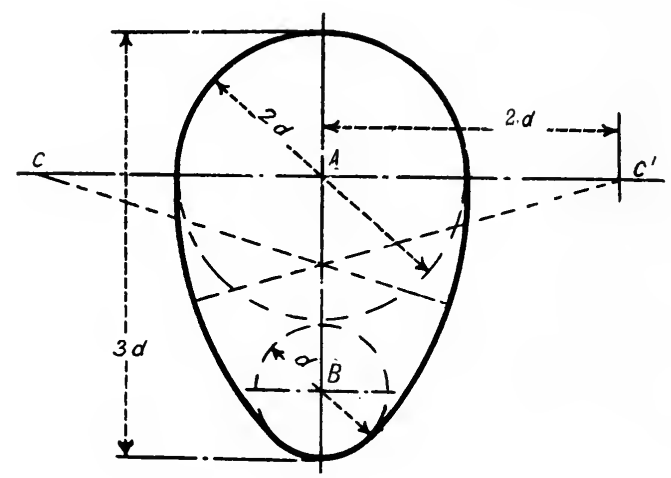

FIG. 111.

Figure $112 a$ and $b$ shows sections which are sometimes adopted for large sewers to the same end, the hydraulic mean depth being fairly high even with a small discharge.

Effect of varying $m, l$, or $i$.

Assuming

we have

or

$$
\begin{gathered}
v=C \sqrt{m i} \\
\frac{d v}{d m}=\frac{C}{2} \sqrt{\frac{i}{m}} \\
d v=\frac{C}{2} \sqrt{\frac{i}{m}} \cdot d m=\frac{v}{2 m} \cdot d m \\
\therefore \frac{d v}{v}=\frac{d m}{2 m} .
\end{gathered}
$$


Thus a small increase in $m$ produces one-half its percentage increase in $v$.

Similarly it may be shown that $\frac{d v}{v}=\frac{d i}{2 i}$, so that in calculating $v$, and therefore the discharge, from this formula, any error in the value assumed for the slope will lead to one-half the proportional error in the estimated discharge.

Again, since

$$
\begin{gathered}
Q=C \sqrt{\frac{A^{3}}{P} \cdot i} \\
\frac{d Q}{d A}=\frac{3}{2} C \sqrt{\frac{i}{P !}} \cdot A^{\frac{1}{2}} \\
\therefore \quad \frac{d Q}{Q}=\frac{3}{2} \frac{d A}{A},
\end{gathered}
$$

so that, neglecting the small increase in the wetted perimeter

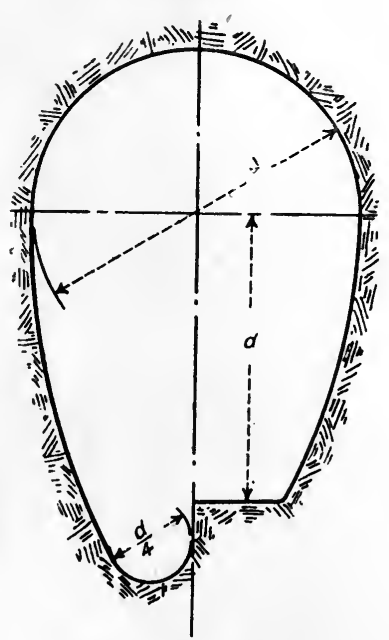

(a)

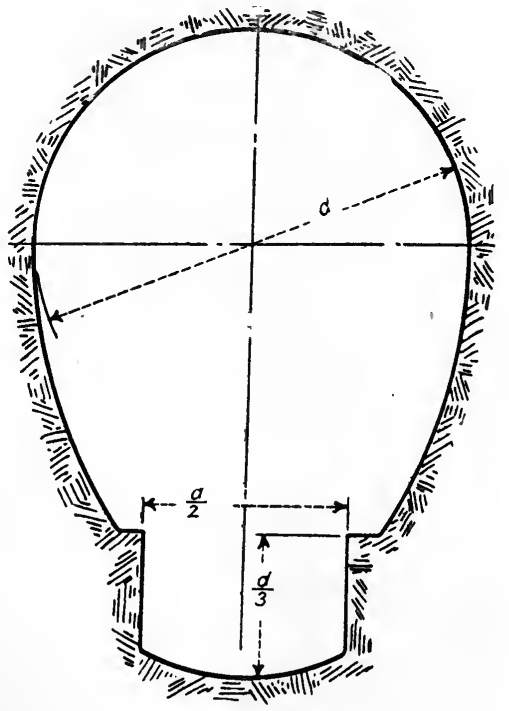

(b)

FIG. 112.

accompanying an increase in depth, any small increase in the cross sectional area produced by such a change in depth will be accompanied by 1.5 times this proportional increase in the discharge. 
Again assuming $A$ to be known and a small error to be made in the estimated value of $P$, we have

$$
\begin{gathered}
\frac{d Q}{d P}=C \sqrt{i}\left(-\frac{1}{2} \frac{A \frac{3}{2}}{P \frac{3}{2}}\right) \\
\therefore \frac{l Q}{Q}=-\frac{1}{2} \frac{d P}{P},
\end{gathered}
$$

so that this error leads to one-half the proportional error in the estimation of $Q$.

Since these errors should be severally small and may all occur in the same direction, the total possible error will be equal to their sum.

Thus if the probable error in the estimation of $i=p$ per cent.

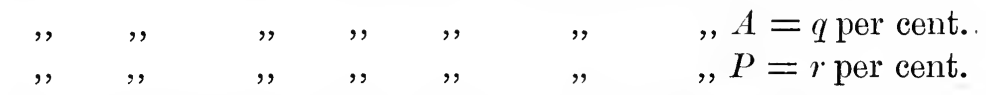

The possible area in the estimation of the discharge, assuming $C$ to have its correct value, will be given by

$$
\left\{\frac{p+r}{2}+1.5 q\right\} \text { per cent. }
$$

Art. 85.-General Equation of Flow in an Open Channel.

Consider a steady stream of cross-sectional area $A$, flowing over a bed having an inclination $\theta$ to the horizontal, where $\sin \theta=$ slope $=i$.

Let $A B$ (Fig. 113) be any stream tube, the vertical depths of

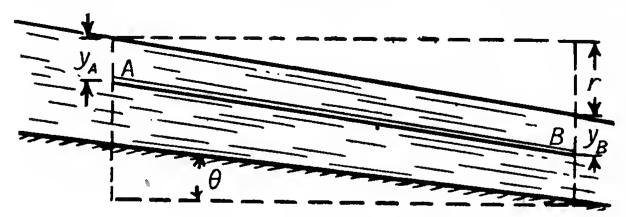

FIG. 113. $A$ and $B$ below the surface being $y_{A}$ and $y_{B}$.

Let ${ }_{A} h_{B}$ be the loss of head in this stream tube from $A$ to $B$, due to frictional resistances.

Then applying Bernoulli's equation of energy, we have

$$
\frac{p_{A}}{W^{\prime}}+\frac{r_{A}{ }^{2}}{2 g}+z_{A}=\frac{p_{B}}{W}+\frac{r_{B}^{2}}{2 g}+z_{B}+{ }_{A} h_{B} \text {. }
$$

Let the difference in the level of the water surface over the points $A$ and $B$ be $r$. 


$$
\begin{aligned}
& \text { Then } z_{A}+y_{A}=z_{B}+y_{B}+r \\
& \quad \therefore \quad z_{A}-z_{B}=r+y_{B}-y_{A} \\
& \quad \therefore \quad \frac{p_{A}}{W}+\frac{r_{A}^{2}}{2 !}+r+y_{B}-y_{A}=\frac{p_{B}}{W}+\frac{r_{B}^{2}}{2 g}+{ }_{A} h_{B} \cdot
\end{aligned}
$$

If the stream is sensibly parallel over the length $A B$, as will be the case if $\theta$ is not large, we have

$$
\begin{aligned}
& \frac{p_{A}}{\bar{W}^{2}}=y_{A} \text { and } \frac{p_{B}}{W}=y_{B} . \\
\therefore & \frac{v_{A}{ }^{2}}{2 g}+r=\frac{v_{B}{ }^{2}}{2 g}+{ }_{A} h_{B} \\
\therefore \quad & r=\frac{r_{B}{ }^{2}-v_{A}{ }^{2}}{2 g}+{ }_{A} h_{B} .
\end{aligned}
$$

If now we imagine the area $A$ divided into $n$ elementary sections, each equal to $\frac{A}{n}$ we get, for each stream tube of area $\frac{A}{n}:-$

$$
\frac{A}{n} \cdot r=\frac{A}{n} \cdot \frac{r_{B}^{2}-r_{A}{ }^{2}}{2 g}+\frac{A}{n} \cdot{ }_{A} h_{B}
$$

and, summing these over the whole section,

$$
\Sigma_{o}^{n}\left(\frac{A}{n} \cdot r\right)=\Sigma_{o}^{n}\left(\frac{A}{n} \cdot \frac{r^{2}-r_{A}^{2}}{2 g}\right)+\Sigma_{o}^{n}\left(\frac{A}{n} \cdot{ }_{A} h_{B}\right) .
$$

If we assume that the velocity is constant over any cross section, the above equation reduces to

$$
A r=A \frac{v_{B}^{2}-v_{A}^{2}}{2 g}+{ }_{A} H_{B}
$$

where ${ }_{A} H_{B}$ is the total frictional loss between the cross sections at $A$ and $B$.

This is still true of the whole mass of water in the stream if the velocity at a cross section is not uniform, provided that the distribution of velocity is such that the total kinetic energy at that section is equal to the mass of water multiplied by the square of the mean velocity at the section. In this case $v_{B}$ and $v_{A}$ become the mean velocities. Experiments by Messrs. Fteley and Stearns on the flow of water in the Sudbury conduit, 9 feet wide and 3 feet deep, in which the velocity was measured at 97 different points in a cross section, gave results showing that the error in assuming this to be true was less than 1 per cent. The error will be greater 
in a shallow channel having a rough bed, but in general the results calculated on this assumption may be taken as substantially correct.

If $P=$ wetted perimeter of section, and if $(\bar{r})^{2}=$ mean square of the velocity from $A$ to $B$ we have

$$
\begin{aligned}
{ }_{A} H_{B} & =\frac{f(\bar{c})^{2}}{2 ! !} \cdot P \cdot A B, \\
\therefore \quad r & =\frac{v_{B}^{2}-r_{A}{ }^{2}}{2}+\frac{f(\bar{v})^{2}}{2 !} \cdot \frac{P}{A} \cdot A B .
\end{aligned}
$$

If $A B=\delta l$ we have $r=\frac{d r}{d l} \cdot \delta l$, while if $v_{A}=v, v_{B}=$ $r+\frac{d v}{d l} \cdot \delta l$ and $(\bar{v})^{2}=\left(r+m \frac{d v}{d l} \delta l\right)^{2}$, where $m$ is less than unity.

So that, neglecting small quantities of the second order

$\mathrm{Ol}^{\circ}$

$$
\begin{gathered}
\frac{d r}{d l} \delta l=\frac{2 r \frac{d v}{d l} \delta l}{2 !}+\frac{f v^{2}}{2 g} \cdot \frac{P}{A} \delta l \\
\frac{d r}{d l}=\frac{r}{g} \cdot \frac{d v}{d l}+\frac{f v^{2}}{2 g} \cdot \frac{P}{A} .
\end{gathered}
$$

This is the general equation of flow in an open channel, $v$ being the mean velocity at a cross section, and though the assumptions made in its conception are not altogether justified by the result of experiment, yet it forms a useful guide and is capable of a wide range of application in the general problems of channel flow.

If $h$ is the depth of water at $A$ (measured vertically from the surface), the depth at $B$ is given by

$$
h-\frac{d r}{d l} . \delta l+i . \delta l .
$$

Again the depth at $B$ is given by

$$
\begin{gathered}
h+\frac{d h h}{d l} \cdot \delta l \\
\therefore \quad i \delta l=\frac{d r}{d l} \cdot \delta l+\frac{d h}{d l} \cdot \delta l \\
\therefore \quad \frac{d r}{d l}=i-\frac{d h}{d l} .
\end{gathered}
$$

H.A. 
Substituting this value in (1) we get

$$
i-\frac{l h}{l l}=\frac{r}{l} \frac{d v}{l l}+\frac{f v^{2}}{2 !} \cdot \frac{P}{A} .
$$

giving the general equation in terms of the slope of the bed.

The physical interpretation of this equation, is that the total loss of (potential and pressure) energy per unit length of the channel, due to the fall in the level of the bed and in the depth of the water, is equal to the increase of kinetic energy together with the loss in friction per unit length of channel.

For uniform flow such as occurs in a culvert with slope, velocity, and depth of water constant, we have $\frac{d h}{d l}=0, \frac{d r}{d l}=0$

$$
\therefore \quad i=\frac{f r^{2}}{2 !} \cdot \frac{P}{A}
$$

or the total potential energy is absorbed in overcoming frictional resistances. This gives the relation between the slope and the velocity, or the discharge $Q$, for since $r=\sqrt{\frac{2 g i A}{f P}}$

$$
\therefore \quad v A=Q=\sqrt{\frac{2 ! i A^{3}}{f \cdot P}}
$$

so that for a given slope we can find the section for $v$ to be a constant, and to give any required discharge.

With a rectangular section, breadth $b$, we get for either uniform or non-uniform flow, if $b$ is constant and if $Q$ is constant

$$
\begin{gathered}
Q=r b h=\text { const. } \\
\therefore \quad r h=\text { const. } \\
\therefore \quad h \frac{d r}{d l}+r \cdot \frac{d h}{d l}=0 \\
\therefore \quad \frac{d v}{d l}=-\frac{r}{h} \cdot \frac{d h}{d l} .
\end{gathered}
$$

Substituting this value, equation (2) becomes

$$
\begin{gathered}
i-\frac{d h}{d l}=-\frac{r^{2}}{g h} \frac{d h}{d l}+\frac{f v^{2}}{2 \eta} \cdot \frac{P}{A} \\
\therefore \frac{d h}{d l}=\frac{i-\frac{f \iota^{2}}{2 ! !} \cdot \frac{P}{A}}{1-\frac{v^{2}}{! h}}
\end{gathered}
$$


Here $-\frac{r^{2}}{g h} \cdot \frac{d h}{d l}$ still represents the rate of increase of kinetic energy with length, and shows that the K. E. increases when the depth diminishes, i.e., when $\frac{d h}{d l}$ is negative.

If $b$ be great in comparison with $h$, we may write $\frac{P}{A}=\frac{b+2 h}{b h}$ $=\frac{1}{h}$ (approximately), especially if, as is very usual in open channels, the bottom is rougher than the sides.

Substituting this value in equation (3) we have, for such a channel,

$$
\frac{d h}{d l}=\frac{i-\frac{f v^{2}}{2 g h}}{1-\frac{v^{2}}{g h}}=i\left\{\begin{array}{c}
1-\frac{f v^{2}}{2 g h i} \\
1-\frac{v^{2}}{g h}
\end{array}\right\}
$$

This gives the slope of the water surface, and from a solution of this equation, the profile of this surface may be determined.

Thus for steady uniform flow $\frac{d h}{d l}=0$, i.e., the depth is constant throughout.

or

$$
\begin{aligned}
\therefore \quad & 1-\frac{f^{2}}{2 g h i}=0 \\
& \varkappa=\frac{f^{2} r^{2}}{2 ! i}
\end{aligned}
$$

Art. 86.-Non-Uniforu Flow.

If, as is usual, the motion while being constant at a point, varies from point to point in the length of the stream, so that $\frac{d v}{d l}$ and therefore $\frac{d h}{d l}$, is not zero, let $H$ be the equivalent depth of an uniform stream of breadth $b$ which would give the same discharge as the variable stream in unit time.

Then

$$
H=\frac{f V^{2}}{2 g i}
$$

where

$$
\begin{gathered}
Q=V b H \text { or } V=\frac{Q}{b H} \\
\therefore \quad \frac{Q^{2}}{b^{2} H^{2}}=\frac{2 g i H}{f} \text { or } \frac{Q^{2}}{b^{2} h^{2}}=\frac{2 g i H^{3}}{h^{2} f}
\end{gathered}
$$


Substituting $\frac{Q^{2}}{b^{2} h^{2}}$ for $v^{2}$ in (4), this becomes

$$
\begin{array}{r}
\frac{d h}{d l}=i\left\{\begin{array}{c}
1-\frac{f Q^{2}}{2 g b^{2} h^{3} i} \\
1-\frac{Q^{2}}{2 g b^{2} h^{2}}
\end{array}\right\} \\
\therefore \quad \frac{d h}{d l}=i\left\{\begin{array}{c}
1-\frac{H^{3}}{h^{3}} \\
1-\frac{H^{3}}{h^{3}} \cdot \frac{2 \pi}{f}
\end{array}\right\}
\end{array}
$$

This is the differential equation to the curve forming the longitudinal profile of the surface.

Since the value of $\frac{d h}{d l}$ depends both on the ratio $\frac{l}{H}$ which may be artificially adjusted in any stream, and on the ratio $\frac{2 i}{f}$ which is fixed once the slope and physical condition of the bed is fixed, an investigation of any particular case of flow must take into account both these factors.

The surface curves corresponding to a few particular cases of flow will now be investigated.

First suppose $\frac{2 i}{f}$ less than unity, the state of affairs existing in a channel of slope less than $\frac{f^{\circ}}{2}$

The following may be taken as approximate values of $f^{\prime}$ at such velocities as are common in practice (Bazin), the foot

\begin{tabular}{|c|c|c|c|c|}
\hline & & \multirow{4}{*}{$\begin{array}{r}f=\cdot 00294 \\
=\cdot 00372 \\
=\cdot 00470 \\
=\cdot 00550\end{array}$} \\
\hline \multicolumn{3}{|c|}{ or brickwork } & & \\
\hline \multicolumn{4}{|c|}{$\begin{array}{l}\text { Ashlar or bric } \\
\text { Rubble mason }\end{array}$} & \\
\hline \multicolumn{4}{|c|}{$\begin{array}{lll}\text { Rubble masonry } & & \text {. } \\
\text { Earth } & . & \text {. }\end{array}$} & \\
\hline
\end{tabular}
being the unit of length.

so that for $\frac{2 i}{f}$ to be less than unity, with a rubble masonry channel the slope would not exceed 00235 feet per foot, or $1_{n}$ in 425. 
Case 1 (a). Let $\frac{2 i}{f}<1$ and also $h^{3}<\frac{2 i}{f} H^{3}$.

Both numerator and denominator of the right-hand side of equation (6) are now negative, so that $\frac{d h}{d l}$ is positive, i.e., the depth of water increases down stream.

Also as $h$ increases it finally reaches the critical value $\sqrt[3]{\frac{2 i}{f}} \cdot H$. Here the denominator becomes equal to zero, and in consequence the value of $\frac{d h}{d l}$ becomes $\infty$, or the surface curve at this point becomes

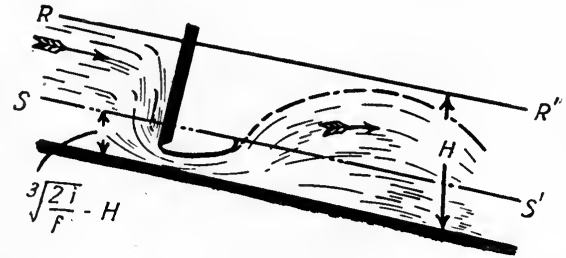

Fig. 114. vertical (Fig. 114), and the phenomenon known as the standing wave is produced.

In the figure, suppose the dotted line $S S^{\prime}$ to be drawn parallel to the bed, to represent a depth $\sqrt[3]{\frac{2 i}{f}}$. H. If by means of a sluice we can get the water surface below this level, then on passing the sluice the depth of water increases as shown. Finally, when $h=\sqrt[3]{\frac{2 i}{f}} . H$, the curve should be vertical where it intersects this line. Before this limit is reached, however, the hypothesis that the stream lines are sensibly parallel ceases to be even approximately true, and the curve becomes modified as shown in the dotted lines.

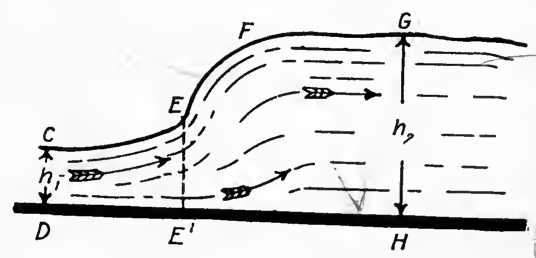

Fig. 115. This is exemplified in the case of a sluice fitted in a channel having a very small slope.

If $h_{1}$ and $h_{2}$ be the depths of the stream before and after its. sudden change of level, the value of $h_{2}$ may be calculated.

Let $v_{1}$ and $v_{2}$ be the velocities at sections $h_{1}$ and $h_{2}$. 
It is not now legitimate to assume that the loss due to shock at the sudden change of section is $\frac{\left(r_{1}-v_{2}\right)^{2}}{g}$ as in the case of pipe How, since the pressure over the area $E^{\prime} E$ (Fig. 115) is no longer uniform and equal to that from $E$ to $F$ but varies with the depth, and hence one of the fundamental assumptions made in deducing this formula is unjustified.

On applying the equation of momentum, however, to this particular case, we have

$\left.\begin{array}{l}\text { Difference of forces acting in the direction } \\ \text { of motion, on the faces } C D \text { and } G H\end{array}\right\}=p_{1} A_{1}-p_{2} A_{2}$, where $p_{1}$ and $p_{2}$ are the mean pressures over the areas $A_{1}$ and $A_{2}$.

Then

$$
p_{1}=\frac{W h_{1}}{2} \quad p_{2}=\frac{W h_{2}}{2} .
$$

The change of momentum per second, $\}=\frac{W}{g}\left\{A_{2} r_{2}{ }^{2}-A_{1} v_{1}{ }^{2}\right\}$
in passing the sections $C I$ and $G H\}$

Also $A_{1} v_{1}=A_{2} v_{2}$, and if the section is rectangular, $h_{1} v_{1}=$ $h_{2} r_{2}$, so that on equating the momentum per second to the force producing it, we have

$$
\begin{aligned}
& \frac{h_{2}}{2} \frac{r_{1}}{r_{2}}-\frac{h_{1}}{2}=\frac{r_{1}^{2}-r_{1} v_{2}}{g} \\
\therefore & \left.\frac{h_{2}}{2} \cdot \frac{h_{2}}{h_{1}}-\frac{h_{1}}{2}=\frac{r_{1}^{2}}{!} ! 1-\frac{h_{1}}{h_{2}}\right\} \\
\therefore \quad & \frac{r_{1}^{2}}{g}=\frac{\left(h_{2}^{2}-h_{1}^{2}\right)}{2 h_{1}} \cdot \frac{h_{2}}{h_{2}-h_{1}}=\frac{\left(h_{2}+h_{1}\right)}{2 h_{1}} h_{2} .
\end{aligned}
$$

If $h_{2}-h_{1}=x$ this reduces to

$$
\begin{aligned}
\frac{v_{1}^{2}}{y} & =\frac{\left(x+2 h_{1}\right)\left(x+h_{1}\right)}{2 h_{1}} \\
\therefore \quad x & =\sqrt{\frac{h_{1}^{2}}{4}+\frac{2 h_{1} r_{1}^{2}}{g}}-\frac{3}{2} h_{1} .
\end{aligned}
$$

This gives the height of the standing wave.

An explanation of the production of the standing wave may be found as follows. An examination of equation (3) shows that $\frac{d h}{d l}$ can only be infinite and a standing wave formed when $v^{2}=g h$, or when

$$
\frac{v^{2}}{g l}: \frac{l h}{d l}=\frac{d h}{d l}
$$


But

$$
\frac{v^{2}}{g h} \cdot \frac{d h}{d l}=\frac{v}{g} \cdot \frac{v}{h} \cdot \frac{d h}{d l}=-\frac{v}{g} \frac{d v}{d l}=-\frac{d}{d l}\left(\frac{v^{2}}{2 g}\right)
$$

so that the standing wave is produced when

$$
-\frac{l}{d l}\left(\frac{r^{2}}{2 g}\right)=\frac{d h}{d l}
$$

i.e., when the rate of decrease of kinetic energy is equal to the rate of increase of potential and pressure energy due to an increase in the depth, or vice versî.

Until this point is reached the rate of decrease of kinetic energy is greater than that of increase of pressure and potential energy, the difference being due to energy expended in eddy formation. Assuming for the moment that the surface curve could be continued through the point, we should have the rate of increase of potential and pressure energy greater than that of decrease of kinetic energy, and hence should have an actual increase in total energy, a state of affairs which is manifestly impossible.

This can only be overcome by a sudden change in the distribution of pressure over the section of the stream, the effect being almost identical with that produced by the introduction of a solid obstacle in the path of the stream. As a consequence of the shock thus produced there is a sudden loss of energy in eddy production, the velocity of flow of necessity falls, and a corresponding rise of surface ensues.

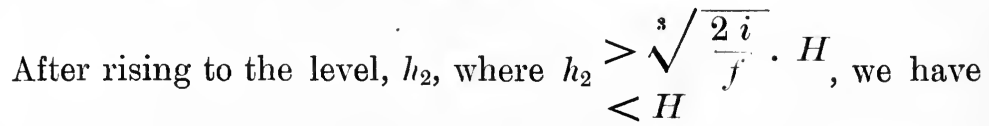
the state of affairs considered in Case $1(b)$.

Case $1(b)$. Here $\frac{2 i}{f}<1$, while $h$ is greater than $\sqrt[3]{\frac{2 i}{f}} \cdot H$ and is less than $H$.

In equation (6) the numerator is now negative, while the denominator is positive, so that $\frac{d l}{d l}$ is negative, or the depth $h$ diminishes down-stream.

As the velocity increases and $h$ diminishes, the denominator 
of the fraction $\frac{h^{3}-H^{3}}{h^{3}-\frac{2 i}{f} H^{3}}$ vanishes before the numerator, so that $\frac{d h}{d l}$ tends to a limiting value $-\infty$ where $h=\sqrt[3]{\frac{2}{f}} . H$.

At this point the surface curve becomes vertical as shown in Fig. 116. If produced by a sudden drop in the bed of a stream, as shown in this figure, $h$ increases up-stream, and approaches more nearly to $H$ as this distance increases, the surface curve being asymptotic to the line $R R^{\prime}$.

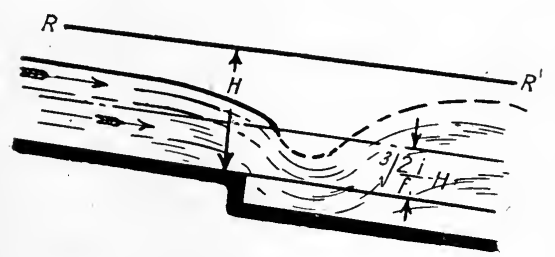

FIG. 116.

Such a drop in the bed may cause an appreciable increase in the velocity of flow for a considerable distance up-stream and may thus affect the foundations of structures (bridges, etc.) which may be placed upstream, besides causing serious erosion of the bed.

In the case of the sluice (Fig. 114), the water after rising to the height $h_{2}$ is governed by this second set of conditions, so that the level again falls until $h=\sqrt[3]{\frac{2 i}{f}} . H$. Inertia then causes the level to fall below this, when we have the conditions of Case 1 (a) repeated. Thus a series of stationary waves are produced, the level alternately rising and falling above and below that given by $h=\sqrt[3]{\frac{2 i}{f}} \cdot H$. At each successive jump a loss of energy occurs, and the velocity energy after the jump is therefore diminished. It follows that the value of $h_{2}$ must be greater: after each successive jump, and ultimately will become equal to $H$, after which steady flow occurs.

The same reasoning applies to the stream after passing the drop in the bed (Fig. 116) the depth ultimately settling down to $H$.

The state of affairs outlined in this second case may be met with where a flume having a slight inclination delivers water to 
a penstock from which one or more turbines or water wheels are fed. It then becomes important that the proportions of the flume should be such as to prevent the water level from falling below a certain minimum. This problem will afterwards be considered in detail (p. 305).

Case $1(c)$.

$$
\text { Let }\left\{\begin{array}{l}
\frac{2 i}{f^{\prime}}<1 \\
h>H .
\end{array}\right.
$$

Here the surface is everywhere above the line $R R^{\prime}$ (Fig. 117). Both the numerator and denominator in the fraction

$$
\begin{gathered}
i\left\{\frac{h^{3}-H^{3}}{h^{3}-\frac{2 i}{f} H^{3}}\right\} \text { (equation 6) are positive } \\
\therefore \quad \frac{d h}{d l} \text { is positive. }
\end{gathered}
$$

Down-stream then the depth increases, and $\frac{h^{3}-H^{3}}{h^{3}-\frac{2 i}{f} H^{3}}$ ultimately tends to the limit unity, i.e., $\frac{d h}{d l}$ tends to the limit $i$, the slope of the bed. It follows that the down - stream surface tends to become horizontal.

Up-stream $h$ tends to the limit $H$, and $\frac{d l}{d l}$ to the limit zero,

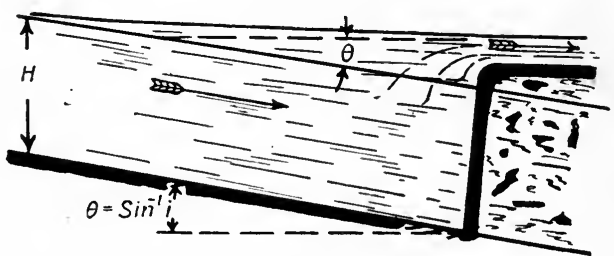

FIIG. 117. so that the curve tends to become asymptotic to the line $R R^{\prime}$.

This is the form of surface curve produced by a weir or dam in a stream of small slope, and is of importance since.the introduction of such a dam causes what may be a serious raising of the backwater level for some considerable distance up-stream.

Next let $\frac{2 i}{f}$ be greater than unity, the state of affairs usually existing in artificial water supply channels. 
Case $2(a)$.

$$
\text { Let }\left\{\begin{array}{l}
\frac{2 i}{f}>1 \\
h<H .
\end{array}\right.
$$

$\frac{d h}{d l}$ (equation 6) is now positive and the depth increases downstream. Since, as $h$ increases, the numerator vanishes before the

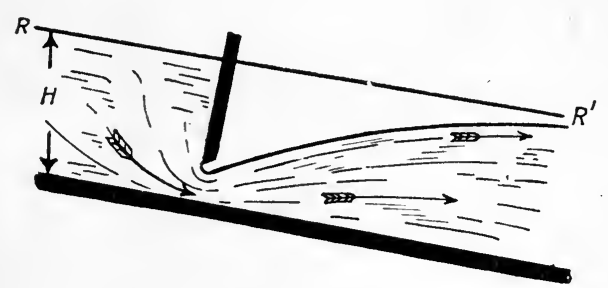

FIG. 118. denominator, $\frac{d h}{d l}=0$ in the limit, i.e., the surface curve tends to become asymptotic to the line $R R^{\prime}$.(Fig. 118).

This state of affairs is attained at a sluice in a

stream having a slope greater than $\frac{f}{2}$.

Case $2(b)$.

Let $\frac{2 i}{f^{\prime}}>1$, and let $h$ be greater than $H$ and less than $\sqrt[3]{\frac{2 i}{f}} \cdot H$

Here $\frac{d h}{d l}$ (equation 6 ) is negative and the depth diminishes

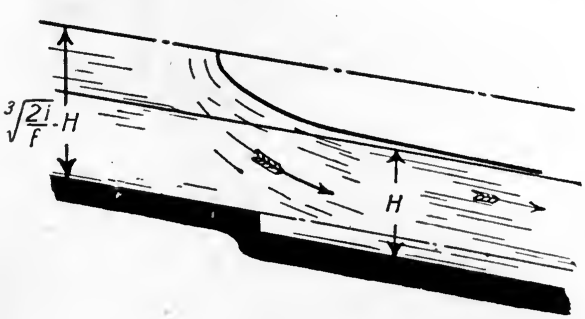

FIG. 119. down - stream. As $h$ diminishes the numerator vanishes before the denominator and in the limit $\frac{d h}{d l}=0$, or the curve becomes asymptotic to the line $R R^{\prime}$, and the stream settles down to the uniform depth $H$. This state of aftairs is realised where an obstacle in a river bed may have caused the level to rise to within the required limits. The surface curve is then as shown in Fig. 119. Up-stream, as $h$ increases it finally reaches the 
value $\sqrt[3]{\frac{2 i}{f}} \cdot H$. Here $\frac{d h}{d l}$ is $-\infty$, and the curve becomes perpendicular to the bed of the stream. As $h$ increases still further the state of affairs considered in Case $2(c)$ is attained. This vertical front is seen when a sudden rush of water, such as may be produced by the bursting of an embankment, is caused in a channel of fairly rapid slope. It is also seen in the bores which occur at certain states of the tide in the Seine between Havre and Rouen, and in various other rivers and contracted channels.

Case $2(c)$.

$$
\text { Let }\left\{\begin{array}{l}
\frac{2 i}{f}>1 \\
h>\sqrt[3]{\frac{2 i}{f}} H .
\end{array}\right.
$$

Here $\frac{d h}{d l}$ is positive $\therefore h$ increases down-stream. Down-stream $\frac{h^{3}-H^{3}}{i}$ tends to the limiting value unity, so that in this $l^{3}-\frac{2 i}{f} H^{3}$

direction the limiting value of $\frac{d h}{d l}$ is $i$, or the surface tends to become horizontal (Fig. 120).

Up-stream, as the depth diminishes, we reach a point where $l=\sqrt[3]{\frac{2 i}{f}} H$, and for this value of $l \cdot \frac{d l}{d l}=$ $\propto$ or the surface curve here becomes perpendicular to the bed of the stream, a standing wave being produced.

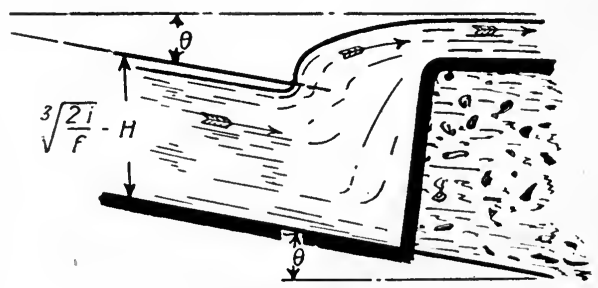

FIG. 120.

This is the curve obtained where an under-water obstruction such as a dam or broad-crested weir is placed across a stream of rapid slope. Since the possibility of $\frac{d l}{d l}$ becoming infinite, depends on $\frac{2 i}{f}$ being greater than unity, the production of a 
standing wave under these circumstances is only possible where this latter condition is satisfied.

In practice the two most important cases are those represented in $1(b)$ and $1(c)$. In the first of these, the effect of a sudden drop in the bed of a stream may, as already explained, be serious, while the case of the reduction in level in a fore-bay feeding a power plant, caused by the sudden demand for energy by the turbines also comes under this heading. In the second, the effect of a dam in increasing the surface elevation at points further up-stream is important. The investigation of each of these cases resolves itself into determining, from a solution of equation (6), the value of $h$ corresponding to any point at a distance $l$ from some datum, since when this is known, the rise or fall from normal, and consequently the change in velocity, can be determined.

To obtain a solution for the equation, we have

$$
\frac{d h}{d l}=i\left\{\frac{\frac{h^{3}}{H^{3}}-1}{\frac{h^{3}}{H^{3}}-\frac{2 i}{f}}\right\} .
$$

Writing $\frac{h}{H}=m$, so that $\frac{d h}{d \cdot m}=H$, this becomes

$$
\begin{gathered}
H \frac{d m}{d l}=i\left\{\frac{m^{3}-1}{m^{3}-\frac{2 i}{f}}\right\} \\
\therefore \quad d m\left\{1+\frac{1-\frac{2 i}{f}}{m^{3}-1}\right\}=\frac{i}{H} d l .
\end{gathered}
$$

Integrating this expression, we have

$$
\frac{i}{H} l=m+\left(1-\frac{2 i}{f}\right) \int \frac{d m}{m^{3}-1}+\text { const. }
$$

while

$$
\int \frac{d m}{m^{3}-1}=\frac{1}{2} \int\left\{\frac{m+1}{\left(m^{2}+m+1\right)(m-1)}-\frac{1}{m^{2}+m+1}\right\} d m
$$




$$
=-\frac{1}{2}\left\{\frac{1}{3} \log _{e} \frac{m^{2}+m+1}{\cdot(m-1)^{2}}+\frac{2}{\sqrt{3}} \tan ^{-1} \frac{2 m+1}{\sqrt{3}}\right\}
$$

$\therefore \quad \frac{i}{H} l=\left[m-\left(1-\frac{2 i}{f}\right)\right.$

$\left.\left\{\frac{1}{6} \log _{e} \frac{m^{2}+m+1}{(m-1)^{2}}+\frac{1}{\sqrt{3}} \tan ^{-1} \frac{2 m+1}{\sqrt{3}}\right\}\right]+C$.

The expression

$$
\left\{\frac{1}{6} \log _{e} \frac{m^{2}+m+1}{(m-1)^{2}}+\frac{1}{\sqrt{3}} \tan ^{-1} \frac{2 m+1}{\sqrt{3}}\right\}
$$

is often termed the "backwater function." Writing this as $\phi(m)$, the equation becomes

$$
\begin{gathered}
i \vec{H} l=m-\left(1-\frac{2 i}{f}\right) \phi(m)+C \\
\therefore \quad i l=h-H\left(1-\frac{2 i}{f}\right) \phi(m)+C \\
\therefore \quad h=i l+H\left(1-\frac{2 i}{f}\right) \phi(m)+C
\end{gathered}
$$

Thus if $h_{1}$ and $h_{2}$ are the depths at points distant $l_{1}$ and $l_{2}$ from the datum, we have

$$
h_{1}-h_{2}=i\left(l_{1}-l_{2}\right)+H\left(1-\frac{2 i}{f}\right)\left\{\phi\left(m_{1}\right)-\phi\left(m_{2}\right)\right\}
$$

\begin{tabular}{|c|c|c|c|c|c|c|c|}
\hline$\frac{b}{H}$ & $\phi(m)$ & $\frac{h}{H}$ & $\phi(m)$ & $\frac{h}{H}$ & $\Phi(m)$ & $\frac{h}{H}$ & $\phi(m)$ \\
\hline $1 \cdot 000$ & $\infty$ & 1.020 & 2.098 & $1 \cdot 10$ & $1: 587$ & $2 \cdot 20$ & 1.015 \\
\hline 1.001 & $3 \cdot 090$ & 1.025 & $2 \cdot 025$ & $1 \cdot 15$ & $1 \cdot 468$ & 2.50 & $\cdot 989$ \\
\hline 1.002 & 2.860 & 1.030 & $1.966 ;$ & $1 \cdot 20$ & $1 \cdot 387$ & $3 \cdot 0$ & $\cdot 963$ \\
\hline $1 \cdot 003$ & $2 \cdot 725$ & 1.036 & 1.908 & $1 \cdot 30$ & $1 \cdot 280$ & $4 \cdot 0$ & $\cdot 939$ \\
\hline $1.00 \mathrm{t}$ & $2 \cdot 629$ & 1.044 & $1 \cdot 843$ & $1 .+0$ & $1 \cdot 211$ & $5 \cdot 0$ & .927 \\
\hline 1.005 & 2555 & 1.050 & $1 \cdot 803$ & 1.50 & $1 \cdot 162$ & $7 \cdot 0$ & $\cdot 915$ \\
\hline 1.007 & $2 \cdot 45$ & $1 \cdot 056$ & $1 \cdot 763$ & $1 \cdot 60$ & $1 \cdot 125$ & $10 \cdot 0$ & $\cdot 911$ \\
\hline $1 \cdot 010$ & $2 \cdot 326$ & 1.060 & $1 \cdot 745$ & $1 \cdot 70$ & $1 \cdot 096$ & $15 \cdot()$ & $• 909$ \\
\hline 1.012 & $2 \cdot 266$ & 1.070 & $1 \cdot 697$ & $1 \cdot 80$ & 1.073 & $20 \cdot 0$ & .908 \\
\hline $1 \cdot 015$ & $2 \cdot 192$ & $1 \cdot 080$ & $1 \cdot 656$ & $2 \cdot 00$ & $1 \cdot 039$ & $50 \cdot 0$ & $\cdot 907$ \\
\hline
\end{tabular}

The following table gives values of $\phi(m)$ for different values of $\frac{h}{H}$ in the case of a dam, where $h$ is always greater than $H$.

From these values a curve may be constructed if required for use, and intermediate values obtained by interpolation. 
In the case of a fall down-stream, $\frac{h}{H}$ is always less than unity. The following table gives values of $\phi(m)$ for this case.

\begin{tabular}{|c|c|c|c|c|c|c|c|}
\hline$\frac{h}{\| t}$ & $\phi(m)$ & $\frac{h}{H}$ & $\phi(m)$ & $\frac{h}{H}$ & $\phi(m)$ & $\frac{h}{H}$ & $\phi(m)$ \\
\hline 1.000 & $\infty$ & 985 & $2 \cdot 183$ & 8.50 & $1 \cdot 367$ & t & · \\
\hline 99! & 3090 & 980 & $2 \cdot 085$ & .8001 & 1.2 .53 & 3.311 & $\cdot(0.5)(0)$ \\
\hline 899 & $2 \cdot 859$ & .975 & $2 \cdot 009$ & 7.50) & $1 \cdot 15 !$ & $\cdot 3011$ & $\cdot(01), 5$ \\
\hline ד999. & $2 \cdot 723$ & $\cdot 970$ & $1.9+6$ & $\cdot 7(1)$ & 1.078 & 2.2010 & 5.53 \\
\hline (9966 & $2 \cdot 628$ & 960 & $1.84 i$ & (65) & $1 \cdot(1) 6$ & $\cdot 2(1)$ & 3) \\
\hline (95) & 2552 & 950 & $1 \cdot 769$ & $\cdot 600)$ & ) & $\cdot 150$ & $\cdot .53$ \\
\hline 494 & $2+491$ & 940 & 1.705 & (5) & זהזי & $\cdot 1(10)$ & .402 \\
\hline$\cdot 99 ?$ & $2 \cdot 395$ & $\cdot 920$ & $1 \cdot 602$ & .500 & .81! & (1) & 3.52 \\
\hline •990) & $2 \cdot 319$ & $\cdot 900$ & 1.522 & 4511 & 763 & - & 3012 \\
\hline
\end{tabular}

As an example of the use of these tables, calculate the rise in level at a point $\frac{1}{2}$ mile up stream, produced by a dam arranged so as to raise the level at its crest through 8 feet. The original depth of the stream, supposed uniform, was 2 feet, the slope of the bed 1 in $500(=\cdot 002)$, and the value of $f=\cdot 006$.

The necessary height of the dam may be calculated by an application of equation (1), p. 160. Assuming this to be done, we have, in the preceding formulae

$$
H=2 . \quad 1-\frac{2 i}{f}=1-\frac{2}{3}=\frac{1}{3} .
$$

Let the suffix (1) refer to a point just above the dam, and the suffix (2) to the given point. Then since the positive direction of $l$ is down-stream, we have, in equation $(10), h_{1}=10 ; l_{1}=0$; $l_{2}=-2640$, and from the tables we get $\phi\left(m_{1}\right)=\phi\left(\frac{10}{2}\right)$ $=\cdot 927$, so that this equation becomes

$$
\begin{gathered}
10-h_{2}= \\
\quad 002(2640)+\cdot 66\left\{\cdot 927-\phi\left(\frac{h_{2}}{2}\right)\right\} \\
\therefore \quad h_{2}-\frac{2}{3} \phi\left(\frac{h_{2}}{2}\right)=4 \cdot 102 .
\end{gathered}
$$

This equation can only be solved by trial.

Let

$$
l_{2}-\frac{2}{3} \phi\left(\frac{h_{2}}{2}\right)-4 \cdot 102=?
$$

Then if $h_{2}=5, y=5-\cdot 6593-4 \cdot 102=+\cdot 2387$

$$
\text { , } \quad h_{2}=4, y=4-\cdot 6927-4 \cdot 102=-\cdot 7947
$$


For a solution of the equation, $y$ must equal 0 , and since the value of a continuous function such as $y$ cannot change from + to - without passing through the value zero, it follows that for some value of $h_{2}$ between 4 and $5, y=0$.

Evidently, too, the correct value of $h_{2}$ is nearer 5 than 4 . Try $h_{2}=4 \cdot 75$.

If $h_{2}=4 \cdot 75, y=4 \cdot 75-\cdot 6656-4 \cdot 102=-\cdot 0176$. The value of $h_{2}$ is then between $4 \cdot 75$ and $5 \cdot 0$.

A close approximation to the correct result can then be obtained by drawing a curve connecting those values of $y$ and of $h_{2}$ already found. Where this curve intersects the axis of $h_{2}$, we shall have the value of $h_{2}$ which makes $y=0$, and therefore which satisfies the equation. In the problem, $h_{2}=4 \cdot 78$ provides a very close

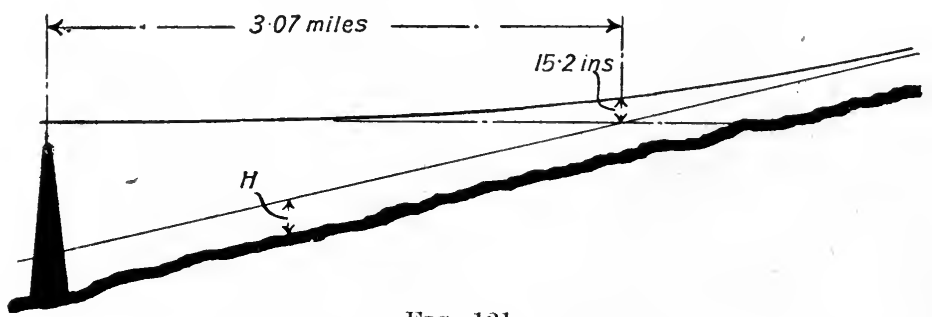

FIG. 121.

approximation to the correct value. At a distance up-stream equal to 4,000 feet the value of $h$, determined in the same way, is 2.34 feet. Since the slope is 002 the height of the bed at this latter point, above that at the dam, is $\cdot 002 \times 4,000=8 \cdot 0$ feet.

The surface at this latter point is therefore 34 feet higher than at the dam. With the dam removed and the flow per minute unchanged, the flow being uniform and the depth of channel equal to $H$, the difference of level instead of being $\cdot 34$ feet would be $8 \cdot 0$ feet.

Figure 121 illustrates the form of backwater curve observed by D'Aubuisson on the Wesser. Here the mean slope of the bed was $2 \cdot 33$. feet per mile $=000441$, and the depth before introducing the dam was 246 feet. The effect of the dam in raising the surface level was apparent for 4.33 miles up-stream.

In a second series of observations on the Werra, the following results were obtained. 
Mean depth $H=1.7$ feet; width $=80$ feet ; fall $=3.88$ feet per mile. A dam $15 \cdot 66$ feet in height was placed across the stream, and the height of water over the sill was found to be $1 \cdot 13$ feet.

The following table indicates the observed and calculated depths, and rises in surface level at points above the dam.

\begin{tabular}{|c|c|c|c|c|c|}
\hline \multicolumn{2}{|c|}{ Distance of observed point from Dam. } & 0. & 85 Miles. & 1.5 . Miles. & 3.5 Miles. \\
\hline \multirow{2}{*}{ Depth of water in feet } & observed & $16 \cdot 79$ & $12 \cdot 30$ & $11 \cdot 28$ & $3 \cdot 01$ \\
\hline & calculated & $\cdot-$ & $13 \cdot 50$ & $11 \cdot 35$ & $3 \cdot 20$ \\
\hline \multirow{2}{*}{ Rise of level in feet } & observed & $15 \cdot 09$ & $10 \cdot 60$ & $10 \cdot 58$ & $1 \cdot 31$ \\
\hline & calculated & - & $11 \cdot 80$ & $10 \cdot 65$ & $1 \cdot 50$ \\
\hline
\end{tabular}

In these calculations the value of $f$ has been taken as 002 . As an example of the effect of a drop in the bed of a stream in producing an increased velocity at points up-stream, consider the same stream as before to have a fall at some point in its length (Case $1(b)$ ), and suppose that this causes a lowering of the surface just above the fall through a depth of 6 inches.

Then as before $H=2 ; 1-\frac{2 i}{f}=\frac{1}{3}$.

Also in equation (10), we have

$$
h_{1}=1.5 ; l_{1}=0 ; \phi\left(m_{1}\right)=\phi\left(\frac{1.5}{2}\right)=1.159 \text { (from table). }
$$

To determine the depth at a point 50 feet up-stream we have $l_{2}=-50$ feet, so that the equation becomes

$$
\begin{aligned}
1.50-h_{2} & =\cdot 002(50)+\frac{2}{3}\left\{1 \cdot 159-\phi\left(\frac{h_{2}}{2}\right)\right\} \\
\therefore \quad h_{2}-\frac{2}{3} \phi\left(\frac{h_{2}}{2}\right) & =1.50-\cdot 10-\cdot 773 \\
& =\cdot 627 .
\end{aligned}
$$

Putting $h_{2}-\frac{2}{3} \phi\left(\frac{h_{2}}{2}\right)-\cdot 627=y$ we get

$$
\begin{aligned}
& \text { if } h_{2}=1 \cdot 8, y=1 \cdot 8-1 \cdot 0146-\cdot 627=+\cdot 1584 \\
& \text {, } h_{2}=1 \cdot 9, y=1 \cdot 9-1 \cdot 1793-\cdot 627=+\cdot 0937 \\
& \text {, } h_{2}=1 \cdot 95, y=1 \cdot 95-1 \cdot 3393-\cdot 627=-\cdot 0163
\end{aligned}
$$


On plotting values of $h_{2}$ and $y$, the curve shows that $y_{2}$ is zero when $h_{2}=1.946$ (approximately), and this, therefore, gives the depth at the given point.

At a point 20 feet up stream we have

$$
h_{2}-\frac{2}{3} \phi\left(\frac{h_{2}}{2}\right)=\cdot 667 .
$$

On solving this equation in the same way, we find that the depth here is $\mathbf{1 . 9 3 2}$ feet (approximately). Since the breadth is constant, the mean velucity at any point is inversely proportional to the depth, so that at the two points 20 and 50 feet up stream the velocities are increased by the fall in the ratios $\frac{2}{1 \cdot 932}$ and $\frac{2}{1 \cdot 946}$ respectively.

This action becomes increasingly important as the slope is diminished. For example, in the previous case, if the slope were diminished to 001 , the velocities at the same two points would be increased by the fall in the ratios $\frac{2}{1 \cdot 56}$ and $\frac{2}{1 \cdot 72}$ respectively.

As a further example of the use of these formulae, consider the case of a flume of rectangular section, suppling a forebay from which a turbine is to be driven.

The breadth of flume is 20 feet, the slope 1 in 1,000 , the length 1,000 yards, and the value of $f=\cdot 003$. The discharge required is 500 cubic feet per second.

The value of $H$, the uniform depth necessary to give this supply, is given by

$$
\begin{aligned}
H=\sqrt[3]{\frac{f^{\prime}()^{2}}{b^{2} 2 ! i}} & =\sqrt[3]{\frac{.003 \times 25 \times 10,000}{64.4 \times 400 \times 001}} \\
& =3.08 \text { feet. }
\end{aligned}
$$

If at the upper end the depth of water is greater than this, say 4 feet, we have (Case $1(c)$ ) and everywhere $h>H$. The depth of water now increases down stream.

Applying equation (10), we now have

$$
\left\{\begin{array}{l}
h_{1} \text { (at entrance to flume }=4 \cdot 0 ; l_{1}=0 \\
H=3 \cdot 08 ; l_{2}=3,000 \mathrm{ft} . ; \frac{2 i}{f}=\frac{002}{003}=\frac{2}{3} \\
\phi .\left(\frac{h_{1}}{H}\right)=\phi\left(\frac{4}{3 \cdot 08}\right)=\phi(1 \cdot 3)=1 \cdot 280 \text { (tables) }
\end{array}\right.
$$

H.A. 


$$
\begin{gathered}
\therefore \quad 4-h_{2}=-001(3,000)+\frac{3.08}{3}\left\{1.280-\phi\left(\frac{h_{2}}{H}\right)\right\} \\
\therefore \quad 7-1.315=h_{2}-1.027 \phi\left(\frac{h_{2}}{H}\right) \\
\therefore \quad h_{2}-1.027 \phi\left(\frac{h_{2}}{H}\right)-5.685=y . \\
\text { If } h_{2}=6.70 \quad y=1.015-1.027(1.017)=-.030 \\
y h_{2}=6.75 \quad y=1.065-1.027(1.015)=+.022 .
\end{gathered}
$$

The correct value of $h_{2}$, the depth in the forebay, is app:oximately $6 \cdot 73$ feet. The depth of the water in the flume at different points in its length can be calculated in the same way, and the necessary height of side ascertained.

If, at the upper end, the depth of water is equal to $H$, this will remain constant throughout, while if less than $H$ and greater than $\sqrt[3]{\frac{2 i}{f}} H$ or $912 H$, i.e., between 3.08 feet and 2.50 feet, we get Case $1(b)$. The height will now decrease down stream until it reaches the value $\sqrt{\frac{2 i}{f}}$, after which a series of waves will be produced, and the depth will, as explained in Case 1 (b), finally settle down to 3.08 feet.

The critical point is found by putting $h_{2}=\sqrt[3]{\frac{2 i}{f}} H=2.53$ in equation (10). Let $h_{1}=3.0$ feet.

Then we have at the critical point

$$
\begin{aligned}
3-2.50 & =.001\left(-l_{2}\right)+\frac{3 \cdot 08}{3}\left\{\phi\left(\frac{3 \cdot 0}{3 \cdot 08}\right)-\phi\left\{\frac{2.53}{3 \cdot 08}\right\}\right\} \\
\therefore \quad 50 & =.001\left(-l_{2}\right)+1.027\{1.996-1 \cdot 278\} \\
\therefore \quad \cdot 237 & =.001 l_{2} \\
\therefore \quad l_{2} & =237 \text { feet. }
\end{aligned}
$$

If, at the entrance, the depth is less than 2.50 feet, as might occur if this flume were fed from a sluice,. we have Case $1(a)$ repeated, and the depth would finally increase up to 3.08 feet. With an open channel leading directly out of a reservoir, the required discharge could only be obtained by having the depth at entrance equal to or greater than $H$. 
Art. 87.-Channel with Horizontal Bed.

Here $i$ is zero, so that equation (6) of the last article ceases to apply. Making $i=o$ in equation (3), we get

$$
\frac{d h}{d l}=\frac{\frac{f x^{2}}{2 g} \cdot \frac{P}{A}}{1-\frac{r^{2}}{g h}}
$$

Writing $r^{2}=\frac{Q^{2}}{b^{2} h^{2}}$ we have, if the channel is rectangular and broad, so that $\frac{P}{A}=\frac{1}{h}$ (sensibly),

$$
\begin{gathered}
d h=\frac{\frac{f Q^{2}}{2 g b^{2} h^{3}}}{1-\frac{Q^{2}}{g b^{2} h h^{3}}} \\
\therefore \quad l h\left\{\frac{2 g l^{2} l^{3}}{f Q^{2}}-\frac{2}{f}\right\}=d l .
\end{gathered}
$$

Integrating between the limits $l_{1}$ and $l_{2}$ we get

$$
l_{1}-l_{2}=\frac{1}{f}\left\{\frac{g l^{2}}{2 Q^{2}}\left(h_{1}^{4}-l_{2}^{4}\right)-2\left(h_{1}-l_{2}\right)\right\}
$$

from which the difference in level $\left(h_{1}-h_{2}\right)$ at any two points distant $l_{1}-l_{2}$ from each other, may be calculated when a given quantity $Q$ cubic feet per second is flowing along the channel.

Expressing (2) as

$$
\frac{d h}{d l}=\frac{1}{\frac{2}{f}\left\{\frac{g b^{2} h^{3}}{Q^{2}}-1\right\}}
$$

we have, for $\frac{d h}{d l}$ to be infinite, the condition

$$
\begin{gathered}
g b^{2} h^{3}=c^{2}=r^{2} b^{2} h^{2} \\
\therefore \quad r^{2}=g h,
\end{gathered}
$$

so that if by any means such as drawing off a considerable amount of water suddenly by opening a lock gate, the velocity can be made equal to $\sqrt{g h}$, a wave with vertical crest will be produced. 
Art. 88.-Change of Level in a Strean Produced bi Bridge, Piers, etc.

Where a series of piers are placed across the bed of a stream, the effect is to raise the up-stream level exactly as if a dam were placed in the stream, and the form of the surface curve will depend on whether the stream satisfies the conditions of Case $1(c)$ or 2 (c) (pp. 297 and 299).

The height will be a maximum at the up-stream end of the
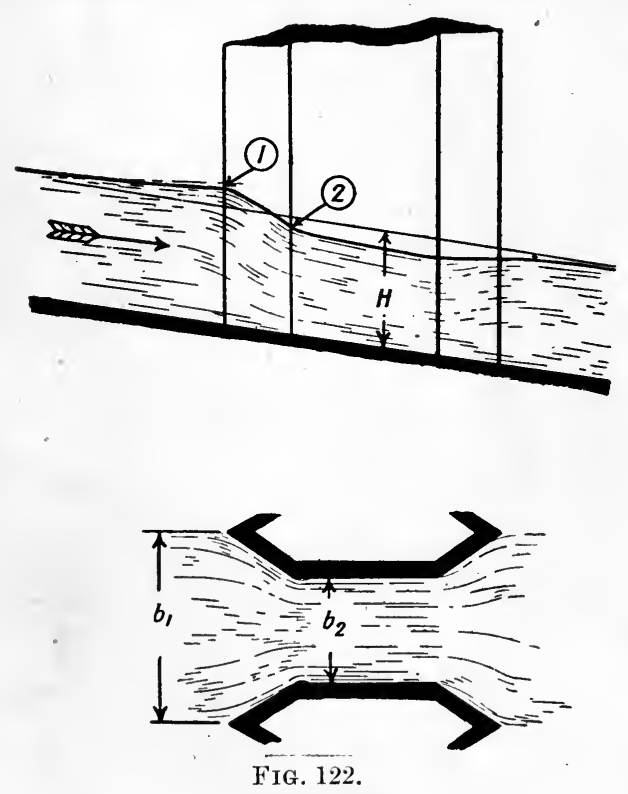
pier. On arriving at the contracted section of the stream, the velocity will be increased, the increase in kinetic energy necessitating a corresponding loss of potential energy, and the depth is diminished. On again arriving at the open channel the velocity diminishes and the depth increases (Fig. 122).

Neglecting losses by friction between the sections (1) and (2), if $b_{1}$ and $b_{2}$ are the effective breadths and $h_{1}$,

$h_{2}$ the depths at these points, we have

$$
h_{1}+\frac{v_{1}^{2}}{2 g}=h_{2}+\frac{v_{2}^{2}}{2 g}
$$

assuming the kinetic energy to be that given by $\frac{r_{1}^{2}}{2 g}$ and $\frac{r_{2}^{2}}{2 g}$ where $v_{1}$ and $v_{2}$ are the mean velocities at the two sections, an assumption which within narrow limits is justified by experiment.

$$
\therefore v_{2}{ }^{2}=\imath_{1}^{2}+2 g x, \text { where } x=h_{1}-h_{2} .
$$


If the discharge through the contracted area at (2) is given by $c b_{2} h_{2} v_{2}$, we have

$$
\begin{gathered}
\iota_{2}=\frac{Q}{c b_{2} h_{2}} \\
r_{1}=\frac{Q}{b_{1} h_{1}} . \\
\therefore \quad \frac{Q^{2}}{\left(c b_{2} h_{2}\right)^{2}}=\frac{Q^{2}}{\left(b_{1} h_{1}\right)^{2}}+2 g x \\
\therefore \quad x=\frac{Q^{2}}{2 g}\left\{\frac{1}{c^{2} b_{2}^{2} h_{2}^{2}}-\frac{1}{b_{1}^{2} h_{1}^{2}}\right\} \\
x=\frac{Q^{2}}{2 g}\left\{\frac{1}{c^{2} l_{2}{ }^{2}\left(h_{1}-x\right)^{2}}-\frac{1}{b_{1}^{2} h_{1}^{2}}\right\} .
\end{gathered}
$$

Also

The value of $c$ varies with the form of pier, but with pointed cutwaters is about 95 (Eytelwein), diminishing to 85 for a bridge having' square or rectangular piers. By considering the problem as one of flow through a weir or notch having a submerged crest (this crest being level with the bed of the stream), and under an effective head $x$, we may obtain a second expression for $Q$ in terms of $x$, and by equating these two expressions the value of $h_{1}$ may be obtained in terms of $Q$ and of $b_{1}$ and $b_{2}$. From this, an application of equation (9) (p. 301) will give the depths at any point up stream, and the entire up-stream profile may then be plotted. On the down-stream side of the obstacle there is a gradual rise of the surface level as the depth increases to a uniform value $H$.

Art. 89.

With radial outward flow over a horizontal bed, such as occurs when a vertical stream impinges on such a surface, we have, if $h$ is the depth at a radius $r, Q$ the quantity per second, and $v$ the velocity at radius $r$,

$$
\begin{gathered}
Q=v \times 2 \pi r h=\text { const. } \\
\therefore \quad v r \frac{d h}{d r}+r h \frac{d v}{d r}+v h=0 \\
\therefore \quad \frac{d v}{d r}=-\left\{\frac{v}{h} \frac{d h}{d r}+\frac{v}{r}\right\}
\end{gathered}
$$


Substituting this value in equation (2) (p. 290), we have

$$
i-\frac{d h}{d r}=-\left\{\frac{v^{2}}{g h} \frac{d h}{d l}+\frac{r^{2}}{g r}\right\}+\frac{f r^{2}}{2 g} \cdot \frac{P}{A} .
$$

But with a horizontal bed $i=o$.

Also

$$
\text { - } \frac{P}{A}=\frac{2 \pi r}{2 \pi r h}=\frac{1}{h}, \text { and } l=r
$$

$\therefore \quad$ (2) becomes $\frac{d h}{d r}=\frac{\frac{x^{2}}{g r}-\frac{f r^{2}}{2 g h}}{1-\frac{r^{2}}{g h}}=\frac{\frac{v^{2}}{g}\left\{\frac{1}{r}-\frac{f}{2 h}\right\}}{1-\frac{v^{2}}{g h}}$.

This becomes infinite if $r^{2}=q h$,

i.e., if

$$
\begin{aligned}
& \frac{Q^{2}}{4 \pi^{2} r^{2} h^{2}}=g h \\
& h^{3}=\frac{Q^{2}}{4 \pi^{2} g} \cdot \frac{1}{r^{2}} .
\end{aligned}
$$

At the radius at which this relation holds, a standing wave will be formed.

The height of this wave may be estimated, as in the case of the wave produced in a rectangular channel.

If $v_{1}$ and $v_{2}$ are the velocities and $h_{1}$ and $h_{2}$ the depths immediately before and after the rise, so that $h_{2}-h_{1}=x$, we have, as on p. 294 ,

from which

$$
\frac{r_{1}^{2}}{g}=\frac{\left(h_{2}+h_{1}\right) h_{2}}{2 h_{1}}
$$

$$
h_{2}-h_{1}=x=\sqrt{\frac{h_{1}^{2}}{4}+\frac{2 h_{1} \overline{r_{1}^{2}}}{g^{2}}}-\frac{3}{2} h_{1} .
$$

Art. 90. - Change of Level Produced bi the Passage of a Boat through a Narrow Caxal with Horizontal Bed.

Let $A=$ cross sectional area of canal.

, $a=$ sectional area of vessel amidships, beneath water line, by a plane perpendicular to its axis.

Let $v=$ velocity of vessel.

Here the state of affairs may be simplified if we first consider the water to be a perfect fluid. As the vessel moves along through this fluid, the volume displaced by the forepart passes along 
backwards between the vessel and the sides and bottom of the canal to fill the space vacated in the rear. In this case we get a backward current extending from the prow to the stern of the boat, its velocity increasing as the effective area of the channel diminishes, and having a maximum value $v\left(\frac{a}{A-a}\right)$ at the amidships section.

To produce this current a surface gradient is necessary, the surface falling from its normal level at the prow to a minimum at the amidships section, and from this point rising to its normal level at the stern.

Due to the adhesion and viscosity of the fluid, however, a mass of water is dragged along with the boat, forming a current confined mainly to the centre and surface of the canal.

Since, for permanence of the regime, the backward flow across any section of the canal must equal the corresponding forward
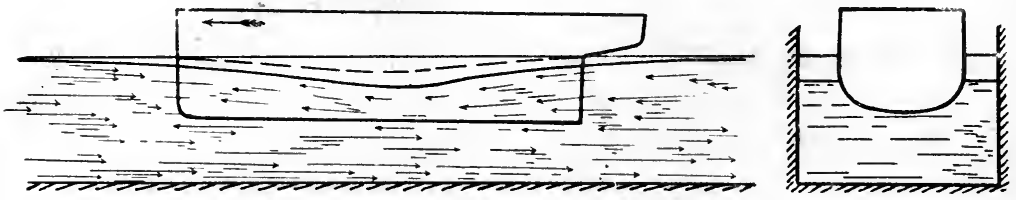

FIG. 123.

flow, the backward bottom current must now be sufficiently great to supply an additional mass of water equal to this, so that its velocity at the minimum section will be $\left(K+\frac{a^{\prime}}{A-a^{\prime}}\right) v$, its velocity at the bows being $K r$. Here $a^{\prime}=a+a_{1}$ where $a_{1}$ is the area of the channel occupied by the forward current at the amidships section. $A-a_{1}$ is then the effective area of the backward current.

But to produce a backward velocity of flow at the bows of the boat the surface level at the bows must be less than at some distance ahead, and will thus be below the normal. The result is that the water level in the canal falls as the boat approaches, has its minimum value near the amidships section, and then rises to attain its normal value. The effect of the bow waves in modifying the level at the bows is here neglected. 
By applying equation (3) (p. 307), the difference of level at any two points in advance of the boat may be deduced in terms of $K v$, since $Q=K v b h_{1}$, where $h_{1}$ is the depth of water at the bows.

Fig. 123 shows the surface curves for a perfect fluid (dotted lines) and for water. ${ }^{1}$

Art. 91.-Flow Round River Bends.

An interesting phenomenon in connection with the flow of rivers through alluvial plains is noted in the manner in which
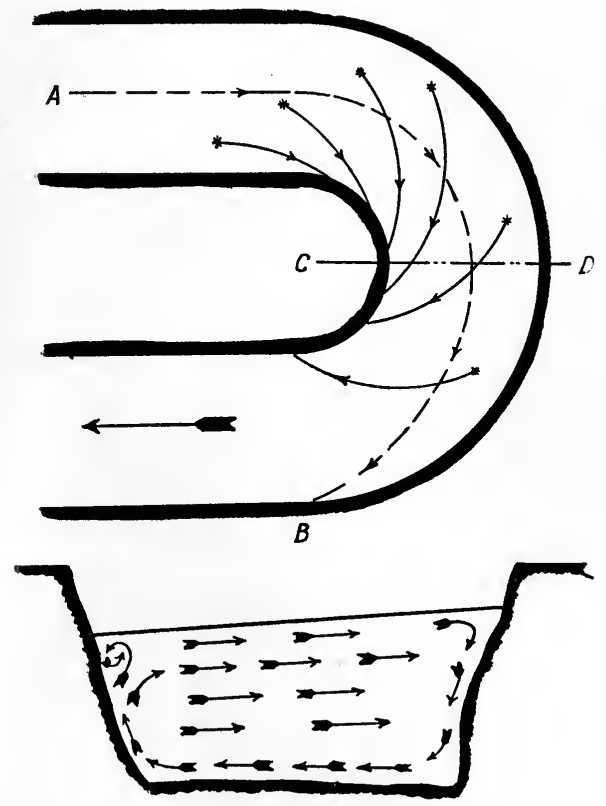

Section on $C-D$.

FIG. 124. such rivers tend to gradually increase any windings which may occur in their course, until finally a new channel is cut through the narrow neck of land thus formed. The following explanation of this scouring of the outer bank of a bend and the deposition of detritus on the inner bank has been given by Professor James Thomson. ${ }^{2}$ 'T'o understand this, it must be remembered that the motion of water flowing round a bend in an open channel is to some extent similar to that in a free vortex, the linear velocity being greatest at the inner bank. It would then appear that the greater scouring action should take place at this inner bank, and this would probably occur but for the modifying action of the bed and sides themselves.

1 For the further investigation of the change of level round a moving vessel, a paper in The Engineer, vol. 63, p. 252, may be consulted.

2 " Proceedings Royal Society," 1877, p. 356. 
In consequence of the centrifugal force, the pressure at any level in a transverse section of the stream increases outwards, so that the level of the free surface is highest near the outer bank. Near the bottom, however, the resistance of the bed reduces the velocity, and consequently the centrifugal force of the water, which now becomes insufficient to overcome the tendency to inward flow produced by the higher level of the free surface at the outside of the curve. The water near the bottom, then has a tendency to flow inwards and to carry with it gravel and other detritus which is left at the inner bank. Experiments in a model river bend, in which the direction of flow was indicated by coloured stream lines and by the behaviour of threads tied to pins fixed in the bed of the stream, as well as by floating particles of matter, indicated a state of affairs as represented in Fig. 124. Here the dotted line $A B$ indicates the path of a particle floating in the surface, while the curves shown in full represent the motion near the bed of the stream.

As indicated, a counter-current flows from inner to outer bank over the upper portion of the stream, but since the same volume of water is moved by the two currents, and since the sectional area of the outer current is comparatively very large, its effect in carrying suspended matter to the outer bank is negligible.

While this theory undoubtedly accounts for a portion of the erosion, and for the deposition of detritus at the inner bank, it is probable that the impact of the stream on the concave bank is a more potent factor in actually causing erosion, and more particularly is this the ease when the stream is in flood and when in consequence the erosive effects are most serious. Under such circumstances, moreover, observation shows that the surface velocity is a maximum near the outer, and not the inner, bank.

\section{Art. 92.-Distribution of Velocity in a Open Channel.}

As in the case of a closed pipe, the resistance introduced by the solid boundaries causes the velocity to diminish in the neighbourhood of the sides and bottom of an open channel, and from analogy with pipe flow it might be expected that the maximum velocity in any cross section would be found in the surface and at the centre of the stream. The presence of the surface film, 
however, appears to modify the distribution of velocity, this surface film having much the same retarding effect as a solid boundary, and the maximum velocity is usually found at some distance below the surface. The depth of the filament of maximum velocity varies with the direction of the wind and the depth of the stream, varying from $1 h$ to $4 h$ (where $h$ is the depth of stream), with a strong down-stream and up-stream wind respectively. Its average depth may be taken as from $\cdot 25$ to $\cdot 27 h$ in a shallow stream from 3 to 12 inches deep; from $\cdot 27$ to $\cdot 30 h$ in a stream from 1 to 3 feet deep; and from $\cdot 30$ to $\cdot 32$ in a stream from 3 to 6 feet deep.

Thus equal velocity contours in a cross section do not intersect the surface at right angles, and are in general inclined towards the centre, while near the centre they do not reach the surface. Fig. 125 indicates the general form adopted by such contours,

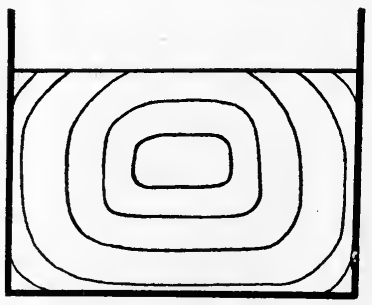

(a)

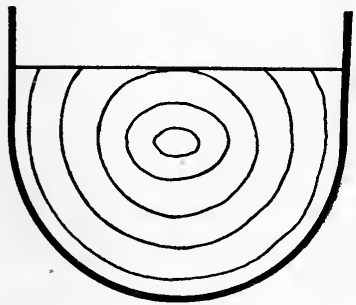

(b)

Fig. 125.

while in Fig. 126 the curves represent, by their horizontal abscissae, the velocities at different points in a vertical line through the centre of the stream.

Here the curve $a^{\prime} b c$ represents the distribution of velocity with a down-stream wind, while $a b c$ represents the distribution of velocity with no wind, and $a^{\prime \prime} b c$ represents the distribution of velocity with an up-stream wind.

The minimum velocity always occurs at the bed of the stream, while the depth of the filament whose velocity is the same as the mean velocity for the whole section varies (increases) with the depth of the stream, and with the ratio $\frac{\text { breadth }}{\text { depth }}$ of channel, increasing as the latter diminishes. 
As a result of many experiments carried out on streams and canals in the United States, ${ }^{1}$ the following conclusions were drawn.

(1) The depth of the filament of mean velocity varies from $\cdot 50$ to $\cdot 65 \mathrm{~h}$.

(2) In shallow streams, 3 to 12 inches deep, its depth varies from 50 to $55 h ; 1$ to 3 feet deep, its depth varies from $\cdot 55$ to $\cdot 60 h ; 3$ to 6 feet deep, its depth varies from $\cdot 60$ to $\cdot 62 h$.

In every case these streams had sandy or gravel beds in fairly good condition.

The depth of this filament is almost independent of the direction of the wind, and in general the velocity at a depth below the surface of $\cdot 6$ times the total depth of any vertical will give the mean velocity in that vertical within 5 per cent.

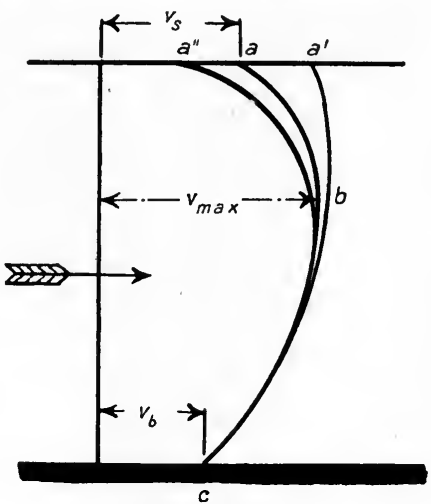

FIG. 126.

The mean velocity over the whole section is in general about 8 of the maximum surface velocity, the ratio varying, however, with the depth and with the state of the channel. The following values of this ratio are taken from Hanbury Brown's "Irrigation.,"

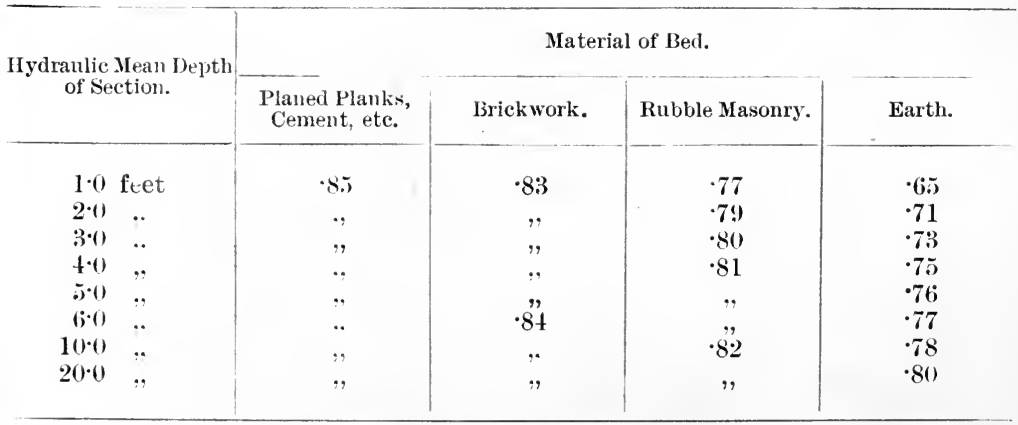

These values, however, vary considerably in any two streams, a more constant ratio being that of the mean to the surface

1 E. C. Murphy on "Accuracy of Stream Measurements," Water Supply and Irrigation Papers, U.S. Geol. Survey, No. 95, 1904.

2 Constable \& Co., London, 1907. 
velocity in any one vertical. This appears to range from 85 to $\cdot 91$, with an average value of $\cdot 88$.

An attempt to gauge the flow of a stream by any measurement of surface velocity can, however, only be expected to give a moderate approximation to the truth, so great is the influence of the wind on this velocity. The effect on the mean velocity is, however, very slight, and direct measurements of the latter may be relied on to give good results.

In spite of very many experiments which have been carried out to determine the distribution of velocity, and of many -attempts to formularise the results of such investigations, so many and so varied are the factors which influence this distribution, that, as might be expected from the nature of the case, the formulae so far collected can only be considered as giving useful approximations to the required result, and this is more particularly the case where the flow in a natural channel of irregular section is under consideration.

By making one or two assumptions as to the circumstances governing the flow in an open channel, a theoretical formula may be deduced which, while only applying so far as these assumptions are justified, may still serve as the rational basis of a more exact empirical formula, for giving the distribution of velocity. Such a formula will now be considered.

\section{Art. 93.-Distribution of Velocity along a Vertical} through the Centre of the Streali.

Suppose the stream to be sensibly parallel; of width which is

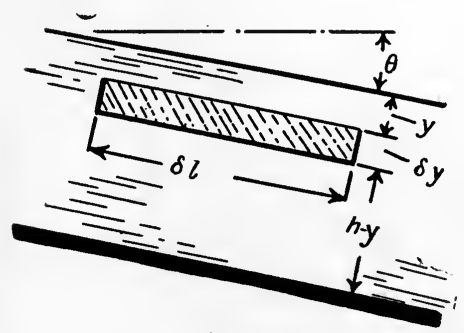

FIG. 127. great in comparison with its depth; flowing steadily; and that the resistance to flow is due entirely to viscosity, a state of affairs never exactly realised in practice.

Let $y$ be the vertical distance from the surface, of a stratum of the fluid, $\delta y$ the thickness, $\delta l$ the length, and $b$ the breadth of the stratum (Fig. 127). 
The weight of this element of fluid $=W b \delta y \delta l$. 'The resolved part of this weight
in the direction of motion $;=W i \delta y \delta l \cdot \sin \theta$.

The difference of tractive force on the upper and lower faces of the stratum $=\mu \frac{d^{2} r}{d y^{2}} \cdot b . \delta l . \delta y$, where $\mu$ is the coefficient of viscosity (p. 54) and where $v$ is the velocity of flow in the direction of the stream. The pressures on the two ends of the stratum are equal since these are at the same depth and are of the same area. Also since the stream is wide, the variation of shear on the two vertical sides of the stratum may be neglected, as explained on p. 54 .

$$
\begin{gathered}
\therefore \quad W b \delta y \delta l \sin \theta=-\mu \frac{d^{2} r}{d y^{2}} \cdot b \delta l . \delta y \\
\therefore \quad \frac{W \sin \theta}{\mu}=-\frac{d^{2} v}{d y^{2}},
\end{gathered}
$$

the negative sign denoting that the resultant shear force acting upon the element is in the opposite direction to the force $W \sin \theta$.

Integrating this expression twice, we get

$$
v=C+B y-\frac{W \sin \theta}{2 \mu} y^{2}
$$

If $r_{s}=$ surface velocity, i.e., where $y=0, C=r_{s}$

$$
\begin{aligned}
\therefore \quad r & =v_{s}+B y-\frac{W \sin \theta}{2 \mu} y^{2} \\
& =v_{s}-\frac{W \sin \theta}{2 \mu}\left(y-\frac{B \mu}{W \sin \theta}\right)^{2}+\frac{B^{2} \mu}{2 W \sin \theta} \\
\therefore \quad v & =v_{s}+2 \frac{B^{2} \mu}{2 W \sin \theta}-\frac{W^{2} \sin \theta}{2 \mu}\left(y-\frac{B \mu}{W \sin \theta}\right)^{2}
\end{aligned}
$$

the equation to a parabola having a horizontal axis at a depth

$$
\frac{B \mu}{W \sin \theta}=y_{1} \text {. }
$$

Since $\quad \frac{d v}{d y}=\frac{W \sin \theta}{\mu}\left\{y-\frac{B \mu}{W \sin \theta}\right\}$

we have $\frac{d r}{d y}=0$, i.e., the velocity is a maximum wher

$$
y=\frac{B \mu}{W \sin \theta} \text {, i.e., at a depth } y_{1}
$$




$$
\begin{aligned}
\therefore \quad v_{\text {max }} & =r_{s}+\frac{B^{2} \mu}{2 W \sin \theta} \\
& =r_{s}+\frac{W^{\prime} \sin \theta}{2 \mu} \cdot y_{1}{ }^{2}
\end{aligned}
$$

$\therefore$ from (2) and (4) we have $r_{\text {max }}-v=\frac{W \sin \theta}{2 \mu}\left(y_{1}-y\right)^{2}$.

If ${ }^{\circ} b=$ bottom velocity, where $y=h$ we have

$$
\begin{gathered}
r_{b}=r_{s}+B h-\frac{W^{r} \sin \theta}{2 \mu} h^{2} \text { from (2) } \\
\therefore \quad B=\frac{v_{b}-r_{s}}{h}+\frac{W^{r} \sin \theta}{2 \mu} h \\
\therefore \quad r=r_{s}+\frac{r_{b}-r_{s}}{h} \cdot y+\frac{W^{r} \sin \theta}{2 \mu}\left(h y-y^{2}\right) .
\end{gathered}
$$

Also

$$
\begin{aligned}
r-v_{b} & =B(y-h)+\frac{W \sin \theta}{2 \mu}\left(h^{2}-y^{2}\right) \\
& =\frac{W \sin \theta}{2 \mu}\left\{2 y_{1}(y-h)+\left(h^{2}-y^{2}\right)\right\} \\
\therefore \quad v_{\max }-v_{b} & =\frac{W \sin \theta}{2 \mu}\left\{h-y_{1}\right\}^{2} .
\end{aligned}
$$

Bazin, experimenting on a stream having the maximum velocity in the surface, obtained

the general equation being

$$
\begin{aligned}
& r_{s_{\text {(max })}}-r_{\text {mean }}=25.4 \sqrt{m \sin \theta} \\
& v_{\text {mean }}-r_{b}=10.87 \sqrt{m \sin \theta} \\
& r_{s \text { max })}-r_{b}=36.27 \sqrt{m \sin \theta}
\end{aligned}
$$

$$
v=\left(v_{s}\right)_{\max }-36 \cdot 27 \sqrt{m \sin \theta}\left(\frac{y}{h}\right)^{2} .
$$

Here $v_{\text {mean }}$ is the mean velocity over the whole section and $m$ is the hydraulic mean depth, the dimensions being taken in feet and the velocities in feet. per second. He also states that wherever the position of maximum velocity, the relation

$$
v_{\max }-v_{b}=36 \cdot 27 \sqrt{m \sin \theta}
$$

holds true. In the vertical plane containing the filament of maximum velocity, we have from equation (7)

$$
36 \cdot 27 \sqrt{m \sin \theta}=\frac{W \sin \theta}{2 \mu}\left\{h-y_{1}\right\}^{2}
$$

Substituting the value of $\frac{W \sin \theta}{2 \mu}$ thus found, in equation 
we get, when the maximum velocity is below the surface,

$$
v_{\max }-v=36.27 \sqrt{m \sin \theta}\left\{\frac{y-y_{1}}{h-y_{1}}\right\}^{2}
$$

a formula which gives fairly accurate resuits in practice.

Using Bazin's results and putting $m=h$ as would be approximately the case for a broad stream, we have

$$
\frac{v_{\text {max }}}{r_{\text {mean }}}=1+\frac{25.4}{r_{\text {mean }}} \sqrt{l \sin \theta} ; \quad \frac{r_{b}}{v_{\text {mean }}}=1-\frac{10.87}{r_{\text {mean }}} \sqrt{l_{\sin } \theta} .
$$

Writing $\bar{r}=v_{\text {mean }}$, we have, in such a stream, with uniform slope, $m=\frac{f \vec{r}^{2}}{2 g i}$ (Art. 85), and putting $m=l$ we get

$$
\begin{aligned}
\therefore \quad \frac{\left(v_{s}\right)_{\max }}{\bar{v}} & =1+25 \cdot 4 \sqrt{\frac{f}{2 !}} \\
& =1+3 \cdot 21 \sqrt{f .}
\end{aligned}
$$

Also

$$
\begin{aligned}
& \frac{v_{b}}{\vec{r}}=1-1.35 \sqrt{f} \\
\therefore \quad & \frac{\left(r_{8}\right)_{\max }}{v_{b}}=\frac{1+3 \cdot 21 f}{1-1 \cdot 35 f .} .
\end{aligned}
$$

Taking $f=\cdot 0075$ as a mean value for a fairly deep river bed, this gives

and

$$
\begin{gathered}
\frac{\left(v_{s}\right)_{\max }}{v_{b}}=1 \cdot 446 \\
\left(v_{s}\right)_{\max }: v^{\prime}: v_{b}:: 1 \cdot 446: 1 \cdot 278: 1 \cdot 00
\end{gathered}
$$

$$
:: 10: 9: 9 \text { (approx.). }
$$

This gives a ratio $\frac{\left(v_{8}\right)_{\text {max }}}{v_{\text {mean }}}=1 \cdot 13$.

Experiments on the Saone, Seine, Garonne, and Rhine gave values of this ratio varying from $1 \cdot 10$ to $1 \cdot 13$. In each case the maximum velocity was found to be in, or very near, the surface. On the Thames, Unwin found the ratio $\frac{\left(v_{s}\right)_{\max }}{v_{b}}$ to be $1 \cdot 34$. On the Mississippi, Messrs. Humphreys and Abbot found the maximum velocity at a depth approximately equal to $\frac{h}{3}$, and obtained values of the ratio $v_{\text {max }}: \bar{v}$, as low as $1 \cdot 02$.

Rankine states that the maximum, mean, and bottom velocities may be taken as being in the ratio $5: 4: 3$ in ordinary cases, 
and in the ratio $4: 3: 2$ in very slow currents, and these ratios may be taken as being approximately correct for streams and rivers of moderate size.

Velocity at Mid.depth.

From equation (5) of this article, we may obtain the mean velocity over any. vertical by integrating the sum of such terms as $v \delta y$ over the vertical, and by dividing this sum by its length $h$. Thus

$$
\begin{aligned}
r & =\frac{\int_{o}^{h}\left\{r_{\text {max }}-\frac{W \sin \theta}{2 \mu}\left(y_{1}-y\right)^{2}\right\} d y}{h} \\
& =r_{\text {max }}-\frac{W^{\prime} \sin \theta}{2 \mu}\left\{\frac{h^{2}}{3}-h y_{1}+y_{1}^{2}\right)
\end{aligned}
$$

while from (5) we may obtain the velocity at mid-depth, i.e., where $y=\frac{h}{2}$.

$$
\begin{aligned}
& \text { Thus } \quad v_{\frac{h}{2}}=v_{\max }-\frac{W \sin \theta}{2 \mu}\left\{\frac{h^{2}}{4}-h y_{1}+y_{1}^{2}\right\} \\
& \therefore \quad v_{\frac{h}{2}}-v=\frac{W \sin \theta h^{2}}{24 \mu} .
\end{aligned}
$$

From which, by determination of the mid-depth velocity, the mean velocity may be determined.

In general the mid-depth velocity is from 1.06 to 1.10 times the mean, and from $\cdot 94$ to $\cdot 98$ of the maximum.

If $\bar{y}$ be the depth at the point having a velocity equal to the mean, we have, from equations (5) and (9)

$$
\begin{aligned}
\frac{h^{2}}{3}-h y_{1}+y_{1}^{2} & =\left\{y_{1}-\bar{y}\right\}^{2} \\
\therefore \bar{y} & =y_{1} \pm \sqrt{\frac{h^{2}}{3}-h y_{1}+y_{1}^{2} .}
\end{aligned}
$$

Putting $y_{1}=\cdot 2 h$ this gives $\bar{y}=\cdot 62 h$.

$$
, \quad y_{1}=3 h, \quad, \quad \bar{y}=\cdot 65 h \text {. }
$$

Art. 94.-Velocity of Flow in Open Channels.

Water in motion exerts an erosive or scouring action on the bed and sides of the containing channel, and the maximum permissible velocity thus depends on the nature of the bed.

Ganguillet and Kütter give the following as the safe bottom 
and mean velocities, but state that these are probably too small rather than too large.

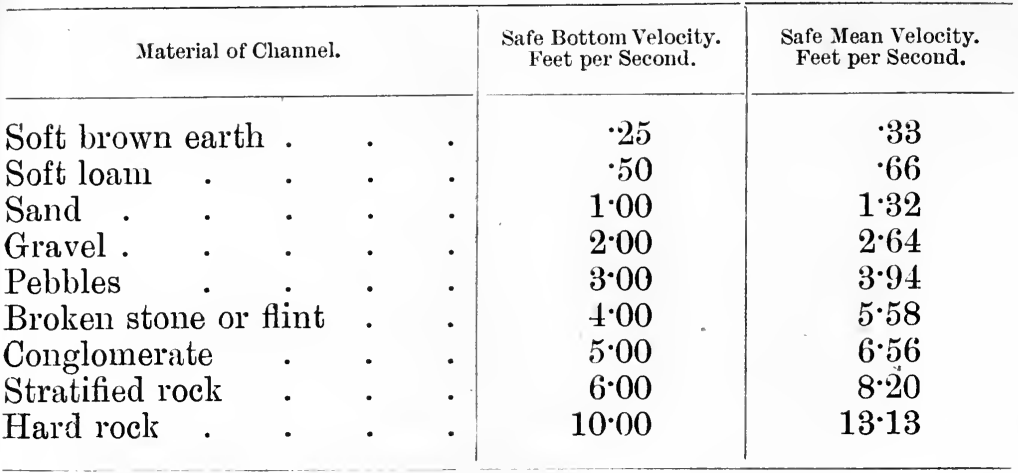

These values probably err considerably on the side of safety. The Cavour Canal over a gravel bed has a mean velocity of 5 feet per second. The Jamrao Canal, with a slope of 6 inches per mile, has a mean velocity of 3 feet per second over a sandy bed and works well-without scour or silting. The lower Ganges Canal has a velocity of 2.73 feet per second, while a canal for the plant of the St. Louis River Hydro-Electric Company, has a velocity through an earthen bed of 2.75 feet per second, and through a rock bed of $7 \cdot 5$ feet per second.

The minimum mean velocity required to prevent excessive deposit of silt, ${ }^{1}$ or growth of aquatic plants depends on the climatic conditions, but may be taken at about 1.25 feet per second. Any such growth has a protecting effect on the bed, and greatly diminishes the tendency to scour. In order to prevent deposit in small sewers or drains, a mean velocity of not less than 3 feet per second is necessary. For sewers from 12 to 24 inches diameter the velocity should not be less than 2.5 feet per second, while with larger sizes than this the velocity may be reduced to 2 feet per seciond.

1 The tendency to deposition of silt, however, depends more upon the changes of, velocity in the stream than upon the actual velocity. Thus, whatever the velocity, there is a tendency to such deposition at any enlargement of section where this velocity is temporarily checked.

H.A. 
While the erosive power of water varies as the square of its velocity, its transporting power, or the power to move boulders, etc., which may lie in its path, varies approximately as $v^{6}$. This may be seen if we consider that the force exerted by the stream on any body is equal to the change of momentum produced in the stream in passing the body, and since the area of that portion of the stream affected is proportional to the sectional area, $a^{2}$, of the body, this force will be equal to $K v^{2} a^{2}$ lbs. The force resisting motion is that of the friction of the body on the bottom of the stream and is proportional to its weight, and therefore to its volume $a^{3}$.

Equating these forces, we have $K r^{2} a^{2}=c a^{3}$

$$
\begin{aligned}
& \therefore a \propto v^{2} \\
& \therefore a^{3} \propto v^{6}
\end{aligned}
$$

i.e., the weight of the solid moved is proportional to the sixth power of the velocity. Obviously this only holds so long as the bodies are similar, the velocity necessary to move a sphere being much less than that to move a cubical block of the same weight.

\section{Art. 95.-Gauging of Flow in Streanis and Open} Channels.

Many methods are available for obtaining the discharge of a stream, these differing widely in the accuracy of their results and the cost and difficulty of their application. The method to be adopted in any case depends largely on the degree of accuracy required and on the size of the stream.

Where a high degree of accuracy is demanded, as may be required in determining the flow of compensation water from the supply reservoir of a waterworks, the best method is to catch the whole discharge for a given time in a graduated tank.

This can only be carried out in quite small streams where a measuring tank of sufficient capacity to hold the discharge for about two minutes is available. In this case the error should not exceed 1 per cent.

For larger streams, the most accurate method is that of gauging the flow by placing a weir across the stream and allowing the 
whole flow to take place over one or more triangular or rectangular notches, the former being used for small and the latter for fairly large streams. Where every precaution is taken as explained in Art. 56 this method gives results which may be relied upon as being accurate within 2 per cent. As a temporary measuring contrivance, however, the weir is too cumbrous and costly to be applied to a river of even moderate dimensions, and where the discharge is greater than about 250 cubic feet per second the only method of obtaining the discharge is to obtain as nearly as possible the mean velocity ( $v$ feet per second) of the stream, to multiply this by the cross sectional area ( $A$ square feet), and to get the discharge $Q$ by the relation

$(\mathcal{l}=v A$ cubic feet per second.

The value of $v$ may be approximated to in many ways.

(a) By using one or other of the formulae given in Art. 83, a suitable coefficient being applied to take into account the state of the channel. The slope of the bed of the stream must then be obtained by field observations. To do this a long straight reach of the river should be selected where

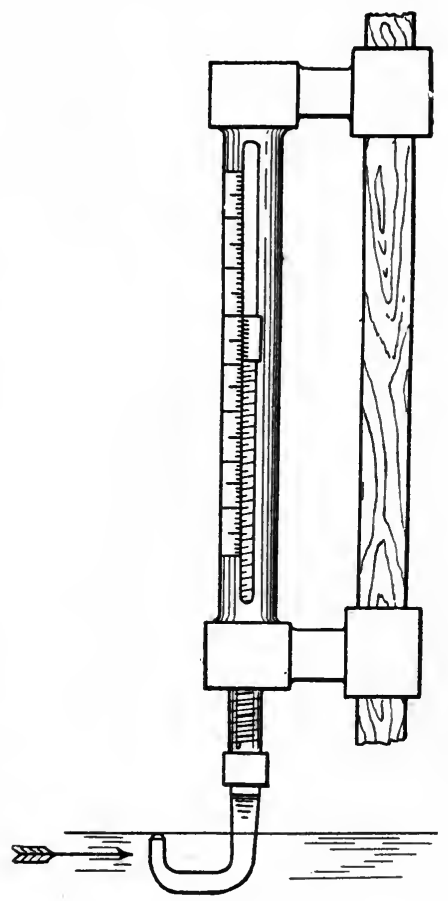

Fig. 128.-Hook Gauge. possible, and the reduced levels of bench marks placed at each end of the reach should be obtained by levelling. The level of each bench mark should then be transferred to a hook gauge (Fig. 128) or measuring staff placed vertically as nearly as possible in the centre of the stream, and away from any disturbing influences. likely to lead to eddy formation. The difference of surface level at each end of the reach can then be obtained. The distance: from end to end of the reach may be obtained by chaining a line running as nearly as possible parallel to the centre line of the 
river. The slope of the water surface is usually taken as giving the slope of the bed because of the difficulty of getting a mean level of the bed by observation. Soundings should then be taken at short distances apart at several cross sections of the stream, and these cross sections plotted. From these a mean value of the wetted perimeter and of the sectional area, and thus of the hydraulic mean depth, may be obtained, and the formula may then be applied. During the whole observation period the stream should be in a state of steady flow, and neither rising nor falling.

Under favourable circumstances, with a fairly shallow channel of uniform section and a judicious choice of the roughness coefficient, results correct to within 10 per cent. may be obtained, but in general a greater accuracy than 20 per cent. is not to be expected, and with any but the most skilful observers the error may increase to 50 per cent. Wherever possible, then, the mean velocity should be obtained in some other manner. This may be done

(b) By using current meters to give the velocity at a point or series of points in a cross section, and by deducing the mean velocity from such observations;

(c) By using one or other type of float, and by measuring the time necessary for a series of these to traverse a given length of the channel.

Before considering these methods in detail, a few general observations as to their relative advantages and disadvantages may be made. Experiment shows that the motion at any point in an open channel is never steady and uniform, but suffers a series of pulsations, the periodic time of which may vary from a few seconds to two or three minutes. These are due to a variety of causes. Eddies formed at the sides and bottom drift away to every portion of the stream; snags and hollows in the bed, bends, and falls, all produce some (irregular) disturbance of the How, with the result that the velocity at a point in the surface may vary by 20 per cent., and at a point near the bed by as much as 50 per cent. (Harlacher) in a short interval of time.

It follows that a float, measuring as it does the velocity due to a single pulsation, may give results which are greatly in error, 
and the only chance of obtaining a fair estimate of the mean velocity over a single section of the stream is to take the mean of a large number-40 or 50 - of the values given by floats. The complexity of the motion is very evident when floats are used. Of a series dropped into a stream at the same point, no two will trace out the same path, and as may be well understood when the multitude of observations necessary to give any pretensions to accuracy is remembered, this method though at first sight so simple, may easily prove the most expensive method of determining the discharge. With current meters, on the other hand, the mean velocity at any point may be obtained with great accuracy, provided the period of observation is sufficient to cover a series of the pulsations of velocity. Professor Unwin found that the

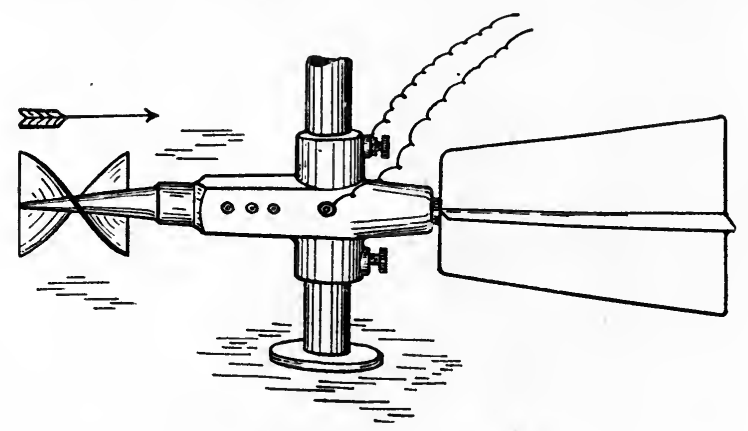

Fig. 129.-Amsler Current Meter.

mean time of successive 100 revolutions of such a meter in the Thames, when plotted, gave a very irregular curve, while the mean times of successive 500 revolutions gave an almost straight line. In general the time of a single observation should not be less than five minutes, a period of six to ten minutes being advisable.

Current Meters. - The current meter usually consists of a screw of the propeller type fixed on a horizontal axis, and either geared directly to a counter which registers the number of revolutions, or making and breaking the contact in an electrical circuit at the end of each ten revolutions, so that these may be directly indicated by a counter placed on shore or in a boat.

The advantages of the latter method in reducing the resistance to rotation and the tendency of the mechanism to clog are obvious. Fig. 129 illustrates a meter of this type. It is fitted 
with a guide vane which keeps the axis of the screw always perpendicular to the direction of the current. The water impinging on the blades of the screw causes it to rotate, the number of revolutions per minute depending on the velocity of the current. 'The instrument is previously calibrated by towing at known velocities through still water, the number of revolutions corresponding to these velocities being recorded. The meter may be used in either of two ways.

In the first, the "point" method, it is fixed at any point in the cross section either by clamping to a vertical post driven into the bed of the stream or is attached to a heavy sinker by means of a wire, and is suspended from a light framework placed across the stream above water level where possible, or from an outrigger fixed to an anchored boat where the width of the stream precludes this. In this way the velocity at a large number of points in the cross section may be obtained and the mean of these taken as giving the mean velocity over the section, or the velocity at some single point, where the filament of mean velocity is supposed to occur, may be taken as the mean. The former method is likely to give the best results. At the same time a series of soundings are taken over the cross section, from which the area may be obtained and the discharge computed. This is the most accurate method, the results obtained being comparable in accuracy with those obtained by weir measurements. Owing to the impossibility of fixing the meter very near to the sides and bottom of the stream where the velocities are least, the results tend to be too high. To obviate this, the centre of the meter should not be placed nearer to the surface than 1 foot. Also, because of this, the meter is only suitable for fairly deep streams where the proportion of dead water is comparatively small.

In the second, the "integration" method, the meter is moved from surface to bottom of the stream in a series of vertical lines, the velocity recorded being taken as the mean for the particular vertical, and the mean for the whole section is then deduced in the ordinary manner. While giving good results when used at a bend, or where the bed is choked with weeds or boulders, this is not in general so good as the point method, and usually gives higher values. 
In calibrating a current meter, at a velocity $v$, and when making $u$ revolutions per minute, it is found that at very low speeds the law obtained is

$$
v=a_{1} n+b_{1}
$$

where $a_{1}$ and $b_{1}$ are constants. Thus $b_{1}$ denotes the velocity at which the instrument begins to rotate. At a certain critical speed, depending on the instrument, the friction of repose of the instrument is overcome and takes a constant value, and the law becomes

$$
r=a_{2} n+b_{2}
$$

where $a_{2}$ and $b_{2}$ are different constants. Owing to the variability of the friction at very low speeds the meter should not be used to register speeds below the critical. This depends on the instrument, but in general varies from 3 to 6 inches per second. Above this velocity the meter may be relied upon to give results correct within about 2 per cent.

A form of current meter which is occasionally used consists of a flat circular plate which is rigidly attached by means of a horizontal arm to one end of an vertical wire, the other end of which is fixed. The wire is supported in bearings, and the free end carries a pointer which, working over a graduated disc, enables the angle of twist of the wire to be ascertained. In using the instrument, the pointer is adjusted to zero with the plate out of water and normal to the direction of flow of the stream. 'The plate is then submerged and the angle of twist necessary to bring it once more normal to the direction of flow is noted.

Then if $P=$ force on plate in lbs.

$l=$ length of arm from centre of plate to centre of wire.

$\boldsymbol{A}=$ area of plate in square feet.

$\theta=$ angle of twist of wire.

$v=$ velocity of flow of stream.

We have $P l=K \theta$, where $K$ is a constant for any instrument and depends solely on the material, length, and radius of the wire.

Also $P=1.15$ A $r^{2}$ (approx.) depending on the size of the plate (see Art. 105), from which $v^{2}=\frac{K}{1 \cdot 15 \mathrm{Al}} \cdot \theta$. 
Even with a constant velocity of flow, however, eddy formation at the rear of the plate causes the value of $P$ to undergo periodic fluctuations, and the difficulty in obtaining a true mean value for $\theta$, and in keeping the plate normal to the direction of the stream, prevent this method from having any pretensions to great accuracy. The instrument is, however, very seldom used.

Estimation of Telocity by Floats. - These are liberated at a series of points in a long straight reach (Captain Cunningham, from experiments on the Ganges Canal ${ }^{1}$ recommends that this length should not be less than 250 feet) and the time occupied in covering a measured distance is noted.

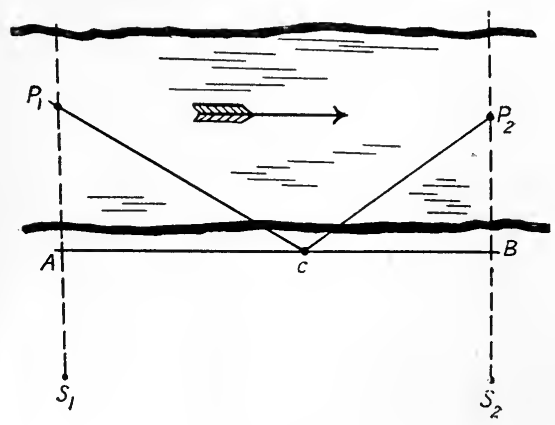

Fic. 130 .

Floats may be divided into four classes:-

(1) Surface floats.

(2) Sub-surface floats.

(3) Twin floats.

(4) Velocity rods.

(1) Surface Floats.These consist of any easily seen masses of light material, painted cork or discs of light wood for example, of small size so as to move along with, and register, the velocity of the surface filaments. A series of trials are necessary to get the maximum surface velocity $r_{s}$ of the stream, from which the mean velocity of flow may be estimated from Bazin's formula (p. 318). It is preferable however to deduce the mean velocity in each of a number of sections of the stream from repeated observations of the surface velocity in each of these sections. The sections may be marked in a stream of moderate dimensions by ropes hanging from a bridge or temporary support.

In a large river, observations with the theodolite are necessary to determine the track of the float. 'This may be satisfactorily carried out as follows. A base line $A B$ (Fig. 130) is chained out parallel to the river for a length of about 250 feet, depending on the width of the river. At the two ends, stakes are erected, while second stakes are erected in lines ranged perpendicularly to

1 "Proceedings Inst. Civil Engineers" 1882, vo]. 71. 
the base line, as at $S_{1}, S_{2}$. An observer with a theodolite is stationed near the centre of the base line at $C$, and an observer is stationed at each stake $S_{1}, S_{2}$. The float being liberated up stream, the theodolite observer keeps the line of collination of his instrument on this. As it passes the line of sight $S_{1} A$, the observer at $S_{1}$ gives an audible signal and the theodolite observer notes the angle $A C P_{1}$. On passing the line $S_{2} B$ a second signal is given and the angle $B C P_{2}$ noted. The line $P_{1} P_{2}$ can then be plotted. With a stream of moderate velocity the same observer may give the signals both at $A$ and $B$. The effect of wind on the surface velocity, however, together with the tendency of the floats to

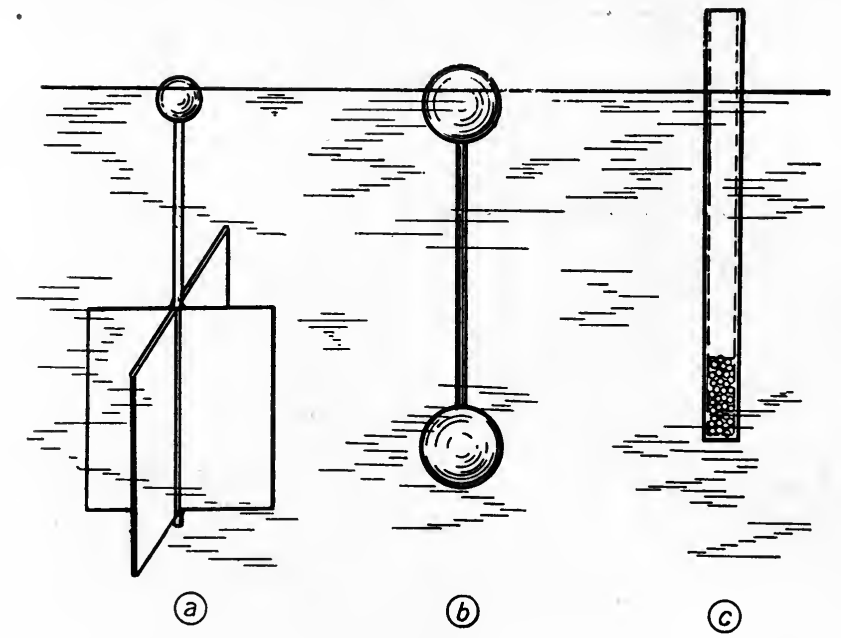

Fig. 131.

follow every variable cross current and to be affected by every surface eddy, renders the results obtained by this method unreliable except as approximations to the truth.

(2) Sub-surface Floats.-These consist of bodies having surfaces of large area as illustrated for example in Fig. $131 a$, attached to small surface floats for ease of observation, the length of connection being adjusted so as to allow the true float to remain at any given depth. The velocity of the float will then be approximately that of the current at the required depth. A series of such floats liberated at different points in the cross section of a stream and at different depths may be used to give by their mean velocity 
the mean of that of the stream, or by arranging a single row, the depth of each being $\frac{3}{5}$ that of the stream at the point of introduction, the mean of these velocities will be approximately the mean velocity of the stream. While more reliable than surface floats, the results here again, as wherever floats are used, can only be looked upon as useful approximations to the truth.

(3) Twin Floats.-These consist of two masses of equal size, usually spheres coupled together by means of a wire, the lower of which is weighted so as to remain vertically below the upper, which floats at the surface (Fig. 131 b). The velocity of the float then gives the mean of the velocities at the surface and at the depth of the lower mass. If this is adjusted so as to just clear the bottom, the velocity of the float will be approximately the mean velocity for the vertical in which the instrument floats.

(4) Velocity Rods. - The velocity rod, or rod float, consists of a light wooden rod or tin tube about 1 inch in diameter, and made in adjustable lengths. The lower end of the bottom length is weighted (Fig: $131 \mathrm{c}$ ), and the length adjusted until the rod floats vertically with its lower end clearing the bottom by a few inches. In a large river and where these are not likely to interfere with navigation, logs of wood about 12 inches in diameter, having their lower ends weighted with iron and their upper ends painted white, may be used.

'I'he velocity of the rod is approximately the same as the mean over its depth, and gives the mean velocity over the rertical in which it floats. The difficulty in using the rod, lies in its tendency to drag over shoals and weeds, and to obviate this its lower end may be arranged to float at a height $h^{1}$ above the bed of the stream.

For such a case Francis gives the empirical formula

$$
v_{m}=v_{r}\left(1 \cdot 012-\cdot 116 \sqrt{\frac{h^{1}}{h}}\right)
$$

giving the mean velocity in the vertical containing the rod in terms of the velocity of the rod $\left(v_{r}\right), l^{1}$, and $h$, the depth of the stream. Here $h^{1}$ should be less than $\cdot 25 h$.

Of all floats, the velocity rod gives the best results. In a series of experiments on the Loch Katrine Aqueduct; concreted 
surface; concave bottom; width $9^{\prime} 1^{\prime \prime}$; radius of curvature $20^{\prime} 10 \frac{1^{\prime \prime}}{}$; hydraulic mean depth $2 \cdot 87$ feet, velocity rods $2^{\prime} 2^{\prime \prime}$ long, gave results which agreed within $\frac{3}{4}$ of 1 per cent. with results as obtained by weir measurement, while the velocity as obtained from the maximum surface velocity, and an application of the formula $v=v_{8}-25.4 \sqrt{m i}$, was 18 per cent. too low.

Other Methods of Measuring the Velocity.

Ripple Formation. - An ingenious method of obtaining the surface velocity at various points in the cross section of a stream was described by Mr. E. C. Thrupp (“Proc. Inst. C.E.," vol. 167, 1907, p. 217). This depends upon the fact that if a small obstruction cut the surface of a stream, ripples are formed if the velocity exceeds about 9 inches per second, while the angle of divergence of these ripples appears to bear a definite relation to the surface velocity. To over come the difficulty of accurately measuring this angle Mr. Thrupp constructed a velocity meter consisting of two vertical pegs $\left(\frac{1}{8}\right.$ iron nails) at'a known distance $d$ inches

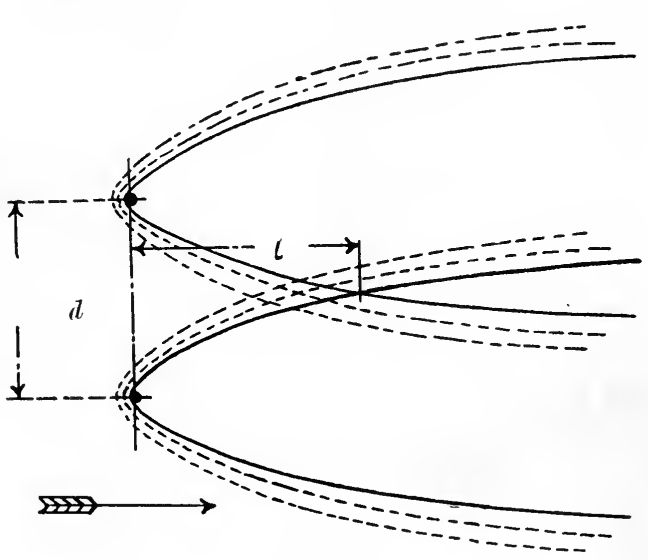

FIG. 132. apart, with a scale for measuring the distance from the base line of the point of intersection of the first ripples formed. Calling this distance $l$ (Fig. 132), the following equations were found to give the surface velocity in feet per second.

For $d=6^{\prime \prime}, v=\cdot 40+\cdot 206 l$.

$, d=4^{\prime \prime}, v=\cdot 40+\cdot 280 l$.

With $d=6^{\prime \prime}$ and with a velocity of 8 feet per second the value of $l$ is about 2 inches, while with a velocity of 3.5 feet per second, $l$ is 15 inches.

This method would appear to be capable of results at least as 
accurate as those obtained by the use of surface floats, probably more so, because of the greater possibilities of accuracy in the determination of the area of the stream at one definite cross section.

The Pitot Tube.-The velocity at any point in the cross section may also be estimated by means of the Pitot tube (p. 210).

This method is, however, not so well adapted for measuring. such low velocities as usually occur in open channels as for higher velocities such as are more common in pipe flow.

The Hydrometric Pendulum.- Where flow takes place through an uniform channel of small dimensions, and where the velocity can be initially determined by some accurate method, as by a. weir, the hydrometric pendulum may be calibrated so as to record this velocity at any future time. The instrument consists of a pendulum having a submerged spherical bob which is heavier than the water which it displaces, and which hangs vertically when the water is at rest. When in motion the pressure of the water causes the pendulum to take up an inclined position, the angle of inclination being a measure of the velocity, and from a previous calibration, enabling this to be read off directly.

Measurement of Flow in a Parallel Conduit. - A method recently devised ${ }^{1}$ for measuring the discharge from a parallel conduit consists in the provision of a light but rigid apron of canvas over a framework of angle iron, suspended vertically from a light carriage which runs on rails fixed on either side of the conduit. When lowered, the apron fills the conduit with very little clearance and is carried along with the same velocity as the stream. Its velocity is then measured by a chronometer and electrical contacts. This method necessitates a conduit of 80 to 100 feet in length, and has been applied to conduits up to 20 feet wide and 12 feet deep, for the measurement of the water supplied to turbines under test.

\section{Exayples.}

(1) A canal whose depth is 4 feet, having side slopes 2 to 1 ; has a bottom width of 10 feet. The bed is of earth (Kütter's

1 By Prof. E. Andersson of Stockholm. See Zeitschrift des Vereins Deutscher. Ingenieurs. April 20, 1907. 
$\mathrm{N}=\cdot 025$ ), and the gradient is 1 foot per mile. Determine the discharge in cubic feet per second.

$$
\text { Answer }\left\{\begin{array}{l}
\text { Hydraulic mean depth }=2 \cdot 58 \text { feet. } \\
C=68 \cdot 8 . \\
\text { Discharge }=109 \cdot 5 \text { cubic feet per second. }
\end{array}\right.
$$

(2) A rectangular flume 4 feet wide and 2 feet deep is roughly constructed of unplaned timber, and is required to deliver 80 cubic feet of water per second. Determine the necessary gradient, and assuming it to supply water to a power station distant 5 miles from the supply reservoir, determine the percentage loss in transmission if the difference of level between supply reservoir and tail race is 500 feet. $C=127 \cdot 6$.

\section{Answer. Gradient 1 in 1,629. \\ Loss of energy $=3 \cdot 24$ per cent.}

(3) A flat-bottomed channel is required to have a constant velocity of flow for all depths of water. The bottom breadth is 5 feet. Determine the depth at a section where the breadth is 20 feet, if the hydraulic mean depth is $1 \cdot 25$ feet. $C=60$.

\section{Answer. $4 \cdot 35$ feet.}

(4) The original depth of a wide stream is 3 feet, and the slope of its bed is 1 in 1,000, the value of $f$ being $0131, C=70$. A dam 10 feet high is erected across the stream. Determine the rise in the water level immediately behind the dam and at points $\frac{1}{2}$ and 1 mile up stream. (Assume the coefficient of discharge for the dam to be $\cdot 560$.)

Answer. Rise behind dam $=9 \cdot 28$ feet.

$$
\begin{aligned}
& , \quad \frac{1}{2} \text { mile up stream }=6.87 \text { feet. } \\
& , 1, ", \quad, \quad=4.04 \quad,
\end{aligned}
$$

(5) It is required to excavate a canal out of rock to be of rectangular section and to bring 500 cubic feet of water per second from a distance of 4 miles with a velocity of $7 \frac{1}{2}$ feet per second. Determine the gradient and the most suitable section for the canal. Take $C=150$.

Answer. Section 11.54 feet wide, $5 \cdot 77$ feet deep. Gradient 1 in 1,156. 
(6) In carrying out field operations to determine the discharge of a river, a straight reach 500 feet long is available. The slope is approximately $\overline{10000}$, and the levelling is possibly accurate within $\frac{1}{50}$ foot. The possible error in determining the wetted perimeter is 8 per cent., and in determining the mean sectional area is 5 per cent. To what degree of accuracy are the final results likely to approximate.

Answer. Within about $13 \frac{1}{2}$ per cent.

(7) The value of $f$ for a stream having a slope of $\frac{1}{1000}$ is $\cdot 0050$. Normally, the stream is of depth 4 feet and breadth 60 feet, but is passed through a sluice having an opening $2 \frac{1}{2}$ feet deep. Determine whether the conditions are such as to lead to the formation of a standing wave, and if so determine the probable height of the crest of this wave above the upper edge of the sluice.

Answer. Yes. 93 feet. 


\section{CHAPTER X.}

Impact of Jets on Stationary and Moving Vanes-Distribution of Pressure over Plate-Plate near to Orifice of Nozzle-Ball Nozzle-Graphical Construction to determine Pressure on a Vane--Centre of Pressure on Vane-Compounding of Jets-Jet Propulsion-Hydraulic Mining-Pressure of Water on Submerged Planes-With Normal Motion-With Oblique Motion-Rudder Action.

\section{Art. 96.-Inpact of Jets.}

When a steady jet of water impinges on any solid surface there is none of the rebound which follows the impact of two solid bodies. Instead of this, a thin stream is formed which glides along the surface until it reaches the boundaries, when it leaves approximately tangential to the surface.

In theoretical discussions it is usual to assume that the sheet of water leaves the surface tangentially. Actually the action is similar to that indicated in Fig. 133, where the dotted lines indicate the actual path of the sheet.

This digression from a straight path is due to the force exerted by the surface tension of the film of water clinging to the outer periphery of the plate. It may be reduced by making the edges of the plate extremely thin, and will be of less consequence as the velocity of the issuing stream is increased, and as the inertia of the water becomes, in consequence, of greater relative importance. In any case, however, it should not be lost sight of, and, as will be indicated later, may appreciably modify the results of calculations.

When the initial and final directions and velocities of an impinging jet are known, the pressure which it exerts on the surface in any direction may be calculated by equating this pressure to the total change of momentum of the jet per second in this direction.

It is important at this stage to differentiate between the "absolute" and "relative" velocities of a jet. Thus, if the jet 
be projected from a fixed nozzle and strike a moving surface, its velocity may be considered from the point of view of a person standing by the nozzle and who notes its relocity relatively to the earth, or from that of a person moving with the surface struck and who notes the motion relative to this moving surface. Since the earth itself is in motion, all motions of terrestrial bodies are merely relative. For simplicity, however, the earth is considered as being stationary, or rather velocities relative to the earth are taken as being absolute velocities. Thus the first person notes

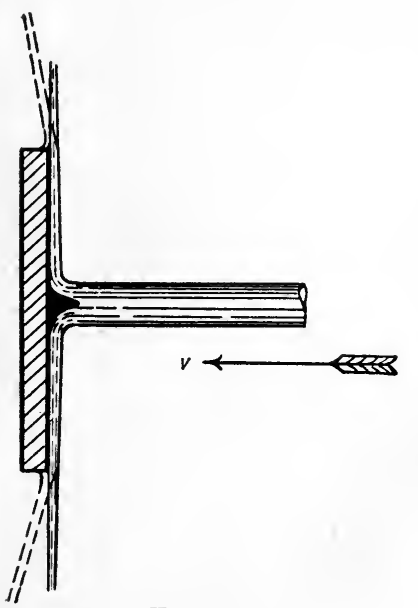

Fig. 133. the absolnte, and the second the relative velocity of the jet.

In the case of impact on a fixed surface the change of relative velocity at impact, will he identical with the change of absolute relocity, and in applying the equation of momentum either may be considered. In any other case, however, the change of monientum must be measured by the change of absolute and not of relative velocity.

If friction be neglected and also losses due to shock, the velocity relative to the surface will be unaffected by the impact. Also, the pressure exerted on the surface at any point will be in the direction of the normal at that point. On these assumptions we may consider the following cases :-

(1) Normal Impact on a Stationary Pline Surface (Fig. 133).

Let $A=$ sectional area of jet in square feet.

$v=$ velocity of jet in feet per second.

Then the weight of water impinging on the plane per second $=W A v$ lbs., where $W$ is the weight of 1 cub. ft. of water.

The initial momentum of this per second normal to the plane $=\frac{W A v^{2}}{g}$.

Since the final velocity is tangential to the plane, the final momentum normal to the plane $=0$. 
$\therefore$ Change of momentum per second normal to plane $=$ $\frac{W A r^{2}}{g}$

$\therefore$ Normal pressure on plane $=\frac{W A v^{2}}{g} \mathrm{lbs}$.

If $\theta$ be the angle which the sheet of water makes with the plane of the surface on leaving, the final velocity per second normal to the plane $=r \sin \theta$, and the momentum in this direction $=\frac{W^{Y} A r^{2}}{g} \sin \theta$.

The change of momentum per second and therefore the pressure normal to the plane is now $\frac{W A v^{2}}{!}(1-\sin \theta) \mathrm{lbs}$.

(2) Oblique Impact on a Stationary Plane Surface (Fig. 133 A).

Assuming the jet to be of the same width as the plane and to be prevented from spreading sideways by flanges, part of the stream will escape from each end of the plane. The sectional area of each of these streams can be calculated by expressing the fact that the change of momentum parallel to the plate is unchanged by impact if friction be neglected. Suppose the jet of unit width and of area $t$,

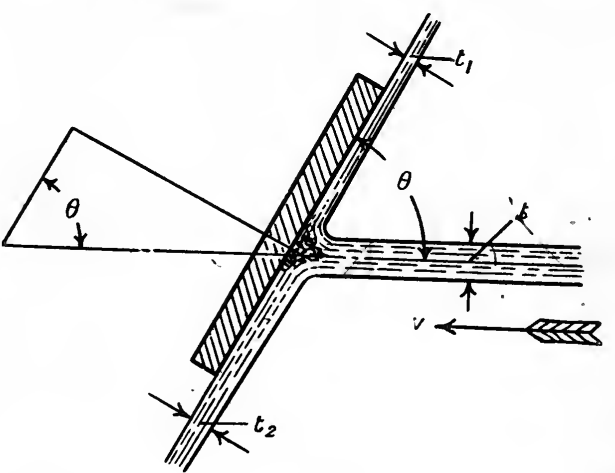

FIG. 133 A. the thickness of the discharge streams being $t_{1}$ and $t_{2}$.

Let $\theta$ be the (acute) angle between the direction of the jet and the plane, $v$ the velocity. of the jet.

Then initial momentum parallel to plane $=\frac{W t v^{2}}{g} \cos \theta$ and final momentum parallel to plane $=\frac{W v^{2}}{g}\left(t_{2}-t_{1}\right)$ $\therefore \quad t \cos \theta=t_{2}-t_{1}$. 
But

$$
\begin{array}{rl}
t & =t_{2}+t_{\mathrm{I}} \\
\therefore \quad t_{1} & =1-\cos \theta \\
t_{2} & 1+\cos \theta \\
t_{1} & =t\left(\frac{1-\cos \theta}{2}\right), t_{2}>t_{\mathrm{I}} . \\
t_{2} & =t\left(\frac{1+\cos \theta}{2}\right) .
\end{array}
$$

Also

Again, the initial momentum, in a direction perpendicular to the plane $=\frac{W t v^{2}}{g} \cdot \sin \theta$, and the final momentum in this direction $=0$.

$\therefore$ Normal pressure on plane $=\frac{\mathrm{I}^{r} t r^{2}}{g} \cdot \sin \theta \mathrm{lbs}$.

Pressure in direction of jet $=\frac{W^{r}+r^{2}}{!} \sin \theta \cdot \sin \theta^{\circ}$ lbs.

$$
=\frac{W^{\prime} t r^{2}}{g} \cdot \sin ^{2} \theta \mathrm{lbs} \text {. }
$$

Impact on Stationary Curved Tanes (Fig. 134).

If the inclined plane of the previous case be fitted with ends curved so as to deflect the escaping streams into directions

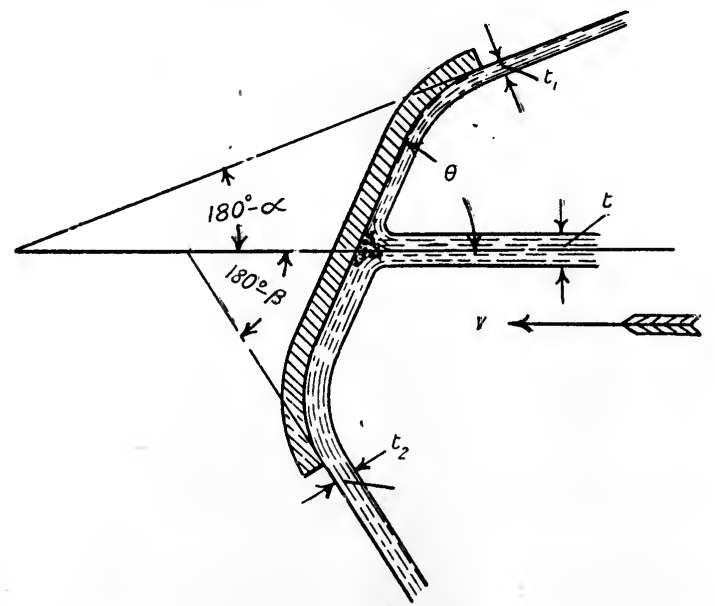

Fig. 134.

making angles $a$ and $\beta$ with that of the jet, the pressure on the vane in the direction of the jet will be increased or diminished according as $\alpha$ and $\beta$ are less or greater than $90^{\circ}$. 
As before

$$
\begin{aligned}
& t_{1}=t\left(\frac{1-\cos \theta}{2}\right) \\
& t_{2}=t\left(\frac{1+\cos \theta}{2}\right) .
\end{aligned}
$$

$\left.\begin{array}{l}\text { The final momentum per sec. } \\ \text { in the direction of the jet }\end{array}\right\}=\frac{W}{g} v\left\{t_{1} v \cos \alpha+t_{2} v \cos \beta\right\}$

$$
=\frac{W v^{2}}{g}\left\{t_{1} \cos a+t_{2} \cos \beta\right\}
$$

$\therefore$ Change of momentum per sec. $\quad=\frac{W v^{2}}{g}\left\{t-t_{1} \cos \alpha-t_{2} \cos \beta\right\}$

$\therefore$ Pressure on vane in direction of jet

$=\frac{W}{g} r^{2} t\left\{1-\frac{\cos \alpha+\cos \beta}{2}-\frac{\cos \theta(\cos \beta-\cos \alpha)}{2}\right\}$ lbs.

This is a maximum when $a=180^{\circ}$ and $\beta$ $=180^{\circ}$, i.e., when the discharge is returned parallel to the.jet, and then has the value. $2 W^{r} r^{2} t$ lbs. In this particular case, as whenever $a=\beta$, the pressure is independent of $\theta$, the angle of impact.

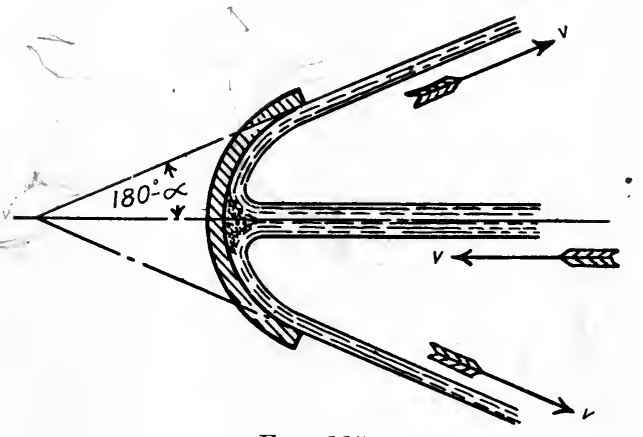

FIG. 135.

(3) Impact on a Surface of Revolution Symmetrical with respect to the Jet (Fig. 135).

Here let $a=$ angle of deflection of jet. $\left.\begin{array}{c}\text { Then the initial momentum per sec. } \\ \text { in direction of jet }\end{array}\right\}=\frac{W A v^{2}}{g}$ $\begin{gathered}\text { Final momentum per sec. in this } \\ \text { direction }\end{gathered}=\frac{W A v^{2}}{g} \cdot \cos \alpha$ $\left.\begin{array}{rl}\therefore & \text { Change of momentum per sec. }= \\ \text { pressure on surface in this direction }\end{array}\right\}=\frac{W A r^{2}}{g}(1-\cos a)$ lbs. This is a maximum when $\cos a$ is a minimum, i.e., when $a=180^{\circ}$, as in the case in a hemispherical cup.

Here the pressure $=\frac{2 W A v^{2}}{g}$ lbs. 
Art. 97.-Actual Force of Impact.

In practice, with flat or recurved vanes, the pressure actually obtained is always slightly less than that given by the above formulae. With normal incidence on a flat plane, as previously explained the discharging stream is always slightly inclined to the plane and possesses some undestroyed momentum in that direction. This, too, however large the plane (within practical limits). If the plane is too small so that the direction of the whole mass of water has not been completely changed before discharge, a further loss will occur. To obviate this the diameter of plate should be not less than three times the diameter of the jet.

In the case of a curved vane or of a plane with oblique impact, any change in the velocity of discharge affects the change of momentum and hence the force of impact. In every case the final velocity is reduced by surface friction and generally also by loss of energy due to eddy formation in the mass of dead water at the point of impact. This latter loss is obviated when the jet strikes the plane tangentially. The total effect therefore depends on the size and form of the vanes. Where these are arranged to deflect the jet through less than $90^{\circ}$ the actual should exceed the theoretical pressure, these losses reducing the final momentum and thus increasing the change of momentum. Where the jet is deflected through more than $90^{\circ}$ the actual pressure is less than the theoretical, while with normal incidence the velocity of discharge only affects the force of impact in that the effect of surface tension in affecting the angle of discharge, is more marked as this velocity diminishes. This latter effect is, of course, common to every form of vane, but becomes of less importance as the angle of deflection increases or decreases from $90^{\circ}$.

On the whole, the ratio $\frac{\text { actual }}{\text { theoretical }}$ pressure may be expected to become less as the angle of deflection is increased from $90^{\circ}$ to $180^{\circ}$ and to have its minimum value for the latter angle, while its value may be expected to become more nearly equal to unity as the velocity of impact increases. For let $v_{1}$ and $v_{2}$ be the initial and final velocities of the jet relative to the vane. Then 
neglecting changes of level at impact and losses due to eddy formation, we have, per lb. of the water,

$$
\frac{r_{1}^{2}}{2 g}=\frac{r_{2}^{2}}{2 g}+\frac{f l v^{n}}{2 g m} \text {. }
$$

Where $f=$ coefficient of friction between water and vane.

$l=$ length of path of contact of jet in feet.

$m=$ hydraulic mean depth of stream in contact with vane $=$ thickness of stream,

and where $n$ is less than 2 for any but very rough surfaces. For such surfaces as are commonly met with forming the vanes of impulse turbines, $n$ may be taken as 1.83 and $f$ as ${ }^{\circ} 005$.

Also if $v_{1}-v_{2}$ is small, as is usually the case, $v$ may be taken equal to $v_{1}$ without sensible error

$$
\therefore \quad r_{1}^{2}\left\{1-\frac{f l}{m v_{1}^{2-n}}\right\}=\dot{i}_{2}^{2}
$$

and since the ratio $\frac{\text { actual pressure }}{\text { theoretical pressure }}=\frac{v_{1}-v_{2} \cos a}{v_{1}-v_{1} \cos a}$, where $a=$ angle of deflection

this equals

$$
\begin{aligned}
& \frac{1-\cos a \sqrt{\left(1-\frac{f l}{m r^{2-n}}\right)}}{1-\cos a} \text { (approximately) } \\
& =1+\frac{\cos a}{1-\cos a}\left(\frac{f l}{2 m v^{2-n}}\right) \text { (approximately) }
\end{aligned}
$$

an expression which diminishes as a (Fig. 135) increases from $90^{\circ}$ to $180^{\circ}$, and which also diminishes as $v$ increases.

\section{Example.}

A 1 inch circular jet strikes the bucket of a Pelton wheel with a relative velocity of 50 feet per second. The wetted surface is 20 square inches, the bucket being 4 inches wide and 3 inches broad, so that the escaping streams are each 3 inches wide. The length of path of contact is 3.33 inches $=\cdot 277$ feet.

$$
m=\frac{\frac{\cdot 7854}{144}}{\frac{6}{12}} \text { feet }=\frac{\text { sectional area of jet }}{\text { width of streams }}=\cdot 0109 \text { feet }
$$




$$
\begin{gathered}
\therefore \quad \frac{f l}{m v^{2-n}}=\frac{.005 \times \cdot 277}{0109 \times 50^{17}}=\frac{.005 \times \cdot 277}{.0109 \times 1.945}=\cdot 0653 \\
\therefore \quad v_{2}=v_{1} \sqrt{1-.0653}=.967 v_{1} .
\end{gathered}
$$

Also $\frac{\text { actual pressure }}{\text { theoretical pressure }}=\frac{1-\cdot 967 \cos a}{1-\cos a}$.

If $a=20^{\circ}(\cos a=\cdot 9397)$ the ratio becomes 984 , a value which would diminish as the velocity diminished. This demonstration has neglected any loss directly due to eddy formation. The actual ratio in the case of a Pelton wheel bucket, where such loss is small, would probably be about $\cdot 97$, and would be less than this in the case of a jet impinging normally at the centre of a hemispherical cup.

The following are the results of a series of experiments carried out by the author on an apparatus designed by Professor Osborne

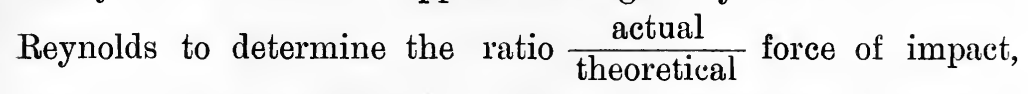
under varying conditions. In each case the surface to be acted upon was rigidly fixed to the end of a horizontal lever, which was then accurately balanced. A vertical jet was allowed to impinge from below on this surface, and the flow was adjusted until the force of impact was sufficient to overcome a known weight resting on the surface and vertically above the axis of the jet, and to cause the lever to float in its equilibrium position. The velocity of impact was measured by measuring the area of the discharge orifice and the weight of water discharged in a given time, and by taking into account the height $h$ (small) between the orifice and the surface. Thus if $v=$ velocity of impact and $v_{0}=$ velocity at orifice, we have $v=\sqrt{r_{0}^{2}-2 g h}$.

The surfaces experimented upon consisted of three flat circular brass plates of diameters respectively $\cdot 54$ inch, $1 \cdot 15$ inches, and $2 \cdot 0$ inches, each $\frac{1}{16}$ inch thick and having edges perpendicular to the plane of the plate; a similar flat plate of 2 inches diameter, having its rear face ground down until it formed a knife edge around the discharging periphery; and a plain hemispherical cup of 1 inch diameter. The diameter of the orifice was accurately $\cdot 200$ inches. 
The results of these experiments are tabulated below.

(1) Flat Plate ·54 in. diameter, $\frac{1}{16}$ in. thick.

\begin{tabular}{ll|c|c|c|c|c|c}
\hline Velocity $v$ ft. per sec. . &. & $25 \cdot 60$ & $32 \cdot 61$ & $36 \cdot 70$ & $49 \cdot 10$ & $50 \cdot 73$ & $58 \cdot 95$ \\
\hline Ratio $\frac{\text { actual }}{\text { calculated }}$ pressures. & $\cdot$ & $\cdot 891$ & $\cdot 940$ & $\cdot 874$ & $\cdot 858$ & $\cdot 914$ & $\cdot 917$ \\
\hline
\end{tabular}

(2) Flat Plate 1·15 ins. diameter, $\frac{1}{16}$ in. thick.

\begin{tabular}{l|c|c|c|c|c}
\hline Velocity . & $25 \cdot 82$ & $32 \cdot 21$ & $36 \cdot 90$ & $48 \cdot 05$ & $54 \cdot 70$ \\
\hline Ratio. & $\cdot 874$ & .962 & $\cdot 865$ & $\cdot 897$ & $\cdot 877$ \\
\hline
\end{tabular}

(3) Flat Plate $2 \cdot 0$ ins. diameter, $\frac{1}{16}$ in. thick.

\begin{tabular}{l|c|c|c|c|c|c}
\hline Velocity · & $25 \cdot 94$ & $31 \cdot 92$ & $37 \cdot 85$ & $48 \cdot 05$ & $50 \cdot 78$ & $57 \cdot 15$ \\
\hline Ratio & $\cdot 870$ & $\cdot 978$ & $\cdot 831$ & $\cdot 897$ & .914 & $\cdot 95 \tilde{5}$ \\
\hline
\end{tabular}

(4) Flat Plate 2.0 ins. diameter, ground to knife edge.

\begin{tabular}{l|c|c|c|c|c|c|c}
\hline Velocity & $25 \cdot 8$ & $33 \cdot 6$ & $36 \cdot 15$ & $41 \cdot 90$ & $46 \cdot 55$ & $52 \cdot 50$ & $57 \cdot 80$ \\
\hline Ratio & $\cdot 901$ & .903 & $\cdot 906$ & .925 & .944 & .956 & .962 \\
\hline
\end{tabular}

(5) Hemispherical cup, 1 in. diameter.

\begin{tabular}{l|c|c|c|c|c|c}
\hline Velocity . & $18 \cdot 19$ & $22 \cdot 66$ & $33 \cdot 45$ & $43 \cdot 15$ & $51 \cdot 15$ & $54 \cdot 50$ \\
\hline Ratio $\cdot$ & $\cdot 890$ & $\cdot 963$ & $\cdot 890$ & $\cdot 853$ & $\cdot 833$ & $\cdot 834$ \\
\hline
\end{tabular}

In the first three of the flat-plate experiments, the most noteworthy features are the remarkable increase in efficiency in each 
case with a velocity of about 32 feet per second (an effect which is also marked in the case of the hemispherical cup at about 22 feet per second), and the disturbing effect of the capillary action at the periphery. This deflected the escaping stream upward through an angle varying from $3^{\circ}$ to $6^{\circ}$, the value of $1-\sin \theta$ varying between 9477 and 8955 . With a sharpedged periphery the effect of surface tension was less marked, particularly at low velocities, the angle apparently varying steadily from about $5^{\circ}$ to $2^{\circ}((1-\sin \theta)$ from $\cdot 913$ to $\cdot 965)$ as the velocity increased.

It will be noticed that, except at very high velocities and at the " critical " velocity, the smallest was quite as efficient as the two larger plates.

In the case of the hemispherical cup, the escaping stream, which theoretically should have been vertical, showed the effect of capillary attraction by being inclined at $13^{\circ}$ to the vertical.

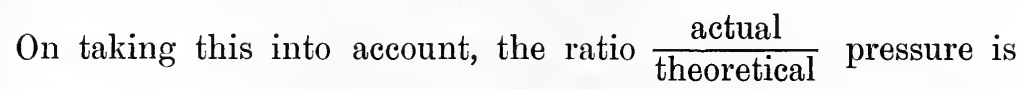
increased by about 1 per cent. in each case. The reduction of efficiency at high as compared with low velocities, is here to be accounted for by the unsteadiness of the descending stream and by its consequent interference with the ascending jet.

\section{Art. 98.-Distribution of Pressure over Plate at InpaCt.}

In general, this is unimportant, the total pressure only being needed. Owing to the curvature of the stream in the neighbourhood of the plate (Fig. 136), and to the centrifugal force caused thereby, the pressure will increase along the radius of curvature towards the surface of the plate and the centre of the jet, the velocity suffering a corresponding diminution.

Assuming no loss of energy before impact, the energy per lb. of water will be $\frac{r^{2}}{2 g}=h \mathrm{ft}$. lbs., where in the case of a vertical jet $h$ is the height of the free surface of the supply reservoir 
above the plate. Thus, at the centre of the jet at the plate where the velocity is zero, the pressure intensity will have its maximum value $W h=\frac{W r^{2}}{2 g}$ lbs. per square foot. From this point the pressure intensity falls off radially as indicated in the figure, the pressure curve being approximately as shown.

Since the total pressure on the plate $=\frac{W r^{2}}{g} \cdot A$ lbs., the area of the plate must be greater than twice that of the jet in order to entirely destroy its momentum. In general, with a

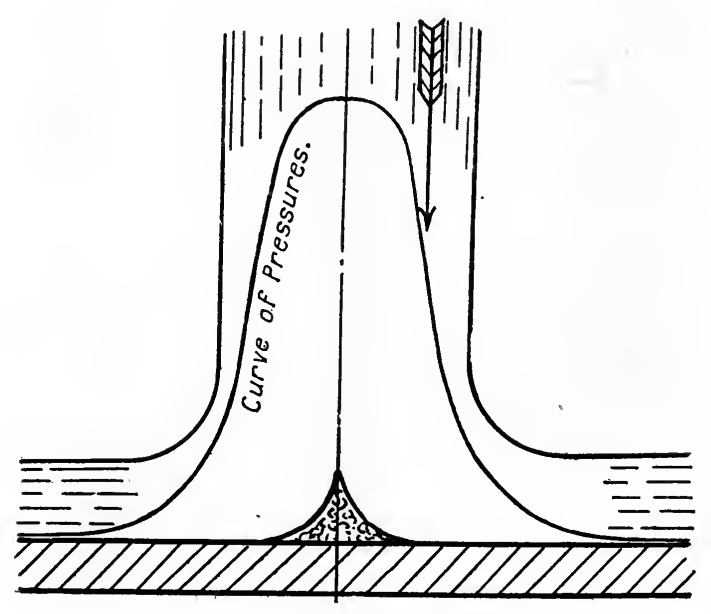

FIG. 136.

plate having a diameter from two to three times that of the jet, the total pressure exerted will exceed 90 per cent. of the theoretical.

Experiments show that, probably due to the formation of dead water at the centre of the jet as indicated in black in the figure, the pressure at the centre of the jet is slightly less than that corresponding to $\frac{r^{2}}{2 g}$ feet of water.

The following table, taken in abstract from the Roorkee Papers on Indian Engineering ("Proc. Inst. C.E.," vol. 60, p. 436), shows 
the pressure exerted at different radii by a vertical jet $1 \frac{1}{2} 9^{\prime \prime}$ diameter, when impinging normally on a fixed plate.

\begin{tabular}{c|c|c}
\hline $\begin{array}{c}\text { Height from free surface in } \\
\text { containing vessel to surface of } \\
\text { plate in inches. }\end{array}$ & $\begin{array}{c}\text { Radial distance from axis } \\
\text { of jet in inches. }\end{array}$ & $\begin{array}{c}\text { Pressure intensity on plate is } \\
\text { inches of water. }\end{array}$ \\
\hline $27 \cdot 125$ & $\cdot 000$ & $26 \cdot 875$ \\
,$"$ & $\cdot 125$ & $26 \cdot 75$ \\
,$"$ & $\cdot 225$ & $26 \cdot 375$ \\
27 & $\cdot 325$ & $26 \cdot 125$ \\
, & $\cdot 425$ & $25 \cdot 50$ \\
, & $\cdot 525$ & $24 \cdot 50$ \\
, & $\cdot 625$ & $23 \cdot 25$ \\
, & $\cdot 725$ & $21 \cdot 75$ \\
$26 \cdot 5$ & $\cdot 825$ & $20 \cdot 25$ \\
,, & $\cdot 925$ & $18 \cdot 00$ \\
, & $1 \cdot 125$ & $13 \cdot 50$ \\
, & $1 \cdot 225$ & $10 \cdot 75$ \\
,, & $1 \cdot 325$ & $8 \cdot 00$ \\
, & $1 \cdot 425$ & $6 \cdot 25$ \\
, & $1 \cdot 525$ & $4 \cdot 50$ \\
\hline
\end{tabular}

The dead water at the centre of a jet is productive of eddies, and to obviate this in the Pelton wheel (Fig. 159), the jet is received on a knife edge which splits and guides it without shock into the two halves of the bucket.

Art. 99.-Plate near to Orifice of Nozzle.

If a plate on which a jet is impinging normally be brought gradually nearer to the orifice, a point is reached where the escaping stream touches both plate and nozzle (Fig. 137), and here the state of affairs existing is entirely different to that previously outlined.

Let $r_{1}, p_{1}, v_{1}, a_{1}$, be the radius, pressure, velocity, and area of the issuing stream at the exit from the orifice and at the commencement of its flow between the plate and the nozzle. 
Let $r_{0}, p_{0}, v_{0}$, and $a_{0}$ represent similar quantities at the point of escape of the stream into the atmosphere.

Then

$$
\left\{\begin{array}{l}
a_{1}=2 \pi r_{1} t \text { where } t=\text { thickness of the escaping stream } \\
a_{0}=2 \pi r_{0} t \\
v_{0}=v_{1} \frac{a_{1}}{a_{0}}=\frac{r_{1} r_{1}}{r_{0}} \\
p_{0}=\text { atmospheric pressure. }
\end{array}\right.
$$

Neglecting frictional and eddy losses between (1) and (0), we have

$$
\begin{gathered}
\frac{p_{1}}{W}+\frac{r_{1}^{2}}{2 g}=\frac{p_{0}}{W}+\frac{r_{0}^{2}}{2 g}=\frac{p_{0}}{W}+\frac{v_{1}^{2}}{2 g} \cdot \frac{r_{1}^{2}}{r_{0}^{2}} \\
\therefore \quad \frac{p_{0}-p_{1}}{W}=\frac{v_{1}^{2}}{2 g}\left\{1-\left(\frac{r_{1}}{r_{0}}\right)^{2}\right\}
\end{gathered}
$$

Since $r_{1}$ is less than $r_{0}, p_{0}-p_{1}$ is positive, and the pressure at (1) is less than atmospheric. It follows that since the pressure between (1) and (0) is everywhere less than atmospheric, while that on the corresponding portion of the outer face of the plate is atmospheric, there will be a

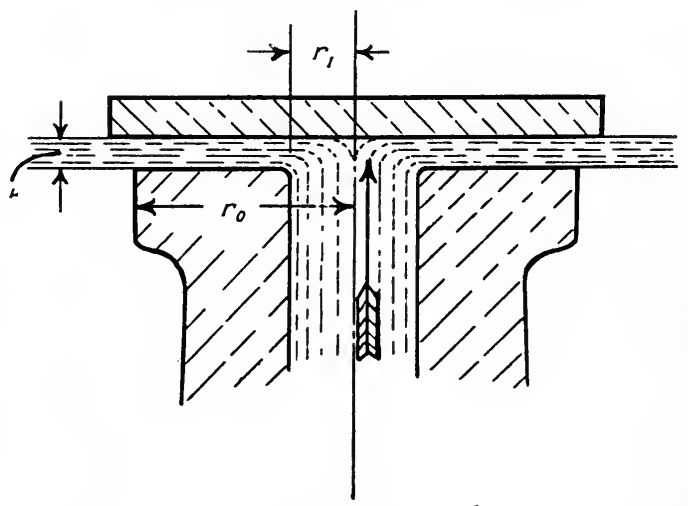

FIG. 137. resultant force tending to keep the plate up to the orifice. The magnitude of this may be calculated, for writing(1) as

$$
\frac{p_{0}-p_{1}}{W}=\frac{v_{0}^{2}}{2 g}\left\{\frac{r_{0}^{2}}{r_{1}^{2}}-1\right\} \text {. }
$$

we have the difference of pressure intensity on the two faces of the plate at any radius $r$ given by

$$
p_{0}-p=W\left\{\frac{v_{0}^{2}}{2 g}\left(\frac{r_{0}^{2}}{r^{2}}-1\right)\right\} \text { lbs. per square foot. }
$$


The difference of the force acting on corresponding elementary rings of width $\delta r$, at this radius then equals

$$
\left(p_{0}-p\right) 2 \pi r \delta r=\frac{2 \pi W v_{0}^{2}}{2 g}\left\{\frac{r_{0}^{2}}{r}-r\right\} \delta r
$$

And the total difference of pressure on the whole areas between the radii $r_{0}$ and $r$, is given by

$$
\begin{aligned}
& \frac{2 \pi W r_{0}^{2}}{2 g} \int_{r_{1}}^{r_{0}}\left(\frac{r_{0}^{2}}{r}-r\right) d r \cdot \mathrm{lbs} . \\
= & \frac{\pi W v_{0}^{2}}{g}\left\{r_{0}^{2} \log _{e} \frac{r_{0}}{r_{1}}-\frac{1}{2}\left(r_{0}^{2}-r_{1}^{2}\right)\right\} \mathrm{lbs} .
\end{aligned}
$$

The pressure on the circular area of the plate opposite the orifice may be taken as that corresponding to the pressure intensity $p_{1}$, together with that necessary to cause the required change of momentum normal to the plate, i.e.,

$$
\frac{W \pi r_{1}^{2} v_{1}^{2}}{g}=\frac{W^{r} \pi r_{0}^{2} v_{0}^{2}}{g} \mathrm{lbs} .
$$

$\therefore$ Outward pressure on this portion of the plate is given by $p_{1} \pi r_{1}^{2}+\frac{W \pi r_{0}^{2} v_{0}^{2}}{g}=\frac{\pi V_{0}^{2}}{g}\left\{\frac{r_{1}^{2}-r_{0}^{2}}{2}+r_{0}^{2}\right\}+\pi r_{1}^{2} p_{0}$

The inward pressure on the corresponding outer face $=$ $\pi r_{1}^{2} p_{0}$ lbs.

$\therefore$ Resultant outward pressure on this area

$$
=\frac{W \pi v_{0}^{2}}{g}\left\{\frac{r_{1}^{2}+r_{0}^{2}}{2}\right\} \text { lbs. }
$$

The resultant inward force on the whole plate is then given by the difference of (3) and (4), i.e., by

$$
\begin{aligned}
& \frac{W \pi r_{0}^{2}}{g}\left\{r_{0}^{2} \log _{e} \frac{r_{0}}{r_{1}}-r_{0}^{2}\right\} \text { lbs. } \\
= & \frac{W \pi v_{0}^{2} r_{0}^{2}}{g}\left\{\log _{e} \frac{r_{0}}{r_{1}}-1\right\} \text { lbs. }
\end{aligned}
$$

an expression which is positive so long as $\frac{r_{0}}{r_{1}}$ is greater than $2 \cdot 718$. 
When the distance $t$ is very small, the pressure over the area $\pi r_{1}^{2}$ approximates to the static head and the plate is forced away from the orifice, so that an intermittent action is set up, the plate vibrating to and from the orifice, but never being driven far away.

The ball-nozzle (Fig. 138), designed to give a thin evenly distributed discharge from a fire hydrant, acts on exactly the same principle.

Here a cage is fitted to prevent the loss of the ball on the first impact of the jet.

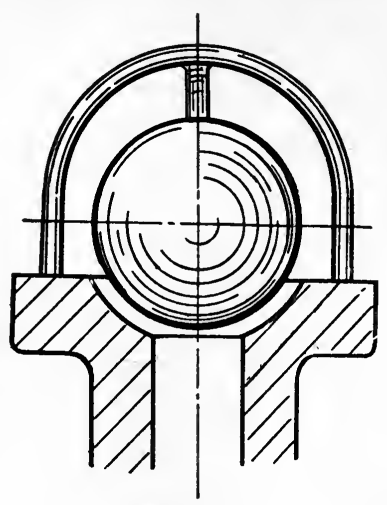

FI\%. 138.

\section{Art. 100.-Impact on Moving Vanes.}

Let $v$ be the velocity of the jet in feet per second.

,,$u$ be the velocity of the vane in the direction of motion of the jet.

Then the relative velocity of jet and vane $=v-u$.

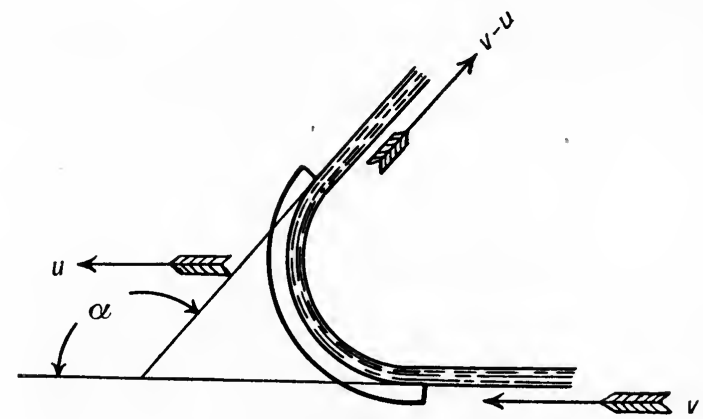

FIG. 139 .

(1) Impact on a single vane curred through an angle a, and moving in the direction of the jet (Fig. 139).

Here the weight of water ) striking the vane per sec. $)=W A(v-u)$ lbs.

$\left.\therefore \begin{array}{l}\text { Initial momentum in } \\ \text { direction of motion }\end{array}\right\}=\frac{W A(v-u) v}{g}$ 
Final velocity in direc-) tion of motion

$$
=u+(v-u) \cos a
$$

$\therefore \begin{aligned} & \text { Final momentum in } \\ & \text { direction of motion }\end{aligned}=\frac{W A}{g}\left\{(v-u) u+(v-u)^{2} \cos \alpha\right\}$

$\therefore$ Change of momentum) in this direction

$$
\begin{aligned}
& =\frac{W A}{g}\{(v-u)[v-u-(v-u) \cos a]\} \\
& =\frac{W A}{g}(v-u)^{2}(1-\cos a)
\end{aligned}
$$

$\therefore \quad$ Work done on vane $=\frac{W A}{g}(v-u)^{2}(1-\cos a) u\left\{\begin{array}{l}\text { ft. lbs. } \\ \text { per sec. }\end{array}\right.$ $\begin{gathered}\text { Initial kinetic energy } \\ \text { of jet }\end{gathered}=\frac{W A v^{3}}{2 g} \mathrm{ft}$. lbs.

$$
\therefore \quad \text { Efficiency }=\frac{2(v-u)^{2}(1-\cos a) u}{v^{3}}=\eta .
$$

For maximum efficiency

$$
\frac{d \eta}{d u}=0
$$

$$
\begin{aligned}
\therefore \quad 2(v-u)^{2}-4(v-u) u & =0 \\
\therefore \quad \text { either } v=u \quad \text { or } v-u & =2 u \\
v & =3 u .
\end{aligned}
$$

so that

In the first case the velocity of the jet is unchanged and the work done on the vane is zero. In the second case we have :Work done on vane $=\frac{W A}{g}\left(\frac{2}{3} v\right)^{2} \cdot \frac{v}{3}(1-\cos a)$ ft. lbs. per sec.

$$
=\frac{4}{27} \frac{W^{Y} A}{g} \cdot v^{3}(1-\cos a) \mathrm{ft} . \text { lbs. per sec. }
$$

$\therefore$ Efficiency $(\max )=\frac{8}{27}(1-\cos a)$.

This has its greatest value when $a=180^{\circ}$, and then equals $\frac{16}{27}$.

When $a=90^{\circ}$, i.e., in the case of normal impact on a single flat plate, we have, as before, the maximum efficiency when $u=\frac{v}{3}$, and then efficiency $(\max )=\frac{8}{27}$.

(2) Impact on a Series of Moving Vanes.

If, instead of a single vane, we have a series of vanes successively placed in the path of the jet at frequent intervals, the weight of water striking these vanes per second becomes $W A v$ lbs. 
If the vanes form surfaces of revolution having their axes in the line of action of the jet, neglecting losses due to splash we have, if $a$ be the angle through which the stream is deflected:Change of momentum ) $\left.\begin{array}{c}\text { per second in direction } \\ \text { of jet }\end{array}\right\}=\frac{I r A v}{g}(r-u)(1-\cos a)$ $\therefore$ Total pressure on vanes $=\frac{\Pi^{r} A r}{! !}(v-u)(1-\cos a)$ lbs. $\left.\begin{array}{l}\text { Work done on vanes } \\ \text { per second }\end{array}\right\}=\frac{W^{r} A v u}{g}(v-u)(1-\cos \alpha) \mathrm{ft.}$ lbs. $\therefore \quad$ Efficiency $=\frac{2(r u)(r-u)(1-\cos a)}{\imath^{3}}=\eta$ For maximum efficiency $\frac{d \eta}{d u}=0$

Or

$$
\begin{aligned}
\therefore \quad v(r-u)-v u & =0 \\
v & =2 u \\
\therefore \quad \text { Efficiency (max) } & =\frac{v^{2} \times \frac{r}{2}(1-\cos a)}{v^{3}} \\
& =\frac{1-\cos a}{2}
\end{aligned}
$$

This is a maximum for $a=180^{\circ}$, i.e., with a series of hemispherical cups and then equals unity. With a series of flat plates having normal incidence $a=90^{\circ}$ and the efficiency equals $\cdot 5$.

Art. 101.-Graphical Construction to Deterinine the

Pressure Exerted on a Vane (Fig 140).

(1) Impact on a Fixed Tane.

Let the vector $\overline{B A}$ represent the initial velocity of the jet in feet per second.

, , , $\quad \overline{B C}$ represent its final velocity on leaving the vane.

Then , , $\overline{A C}$ represents the change of velocity, and therefore gives a measure of the resultant pressure on the vane, for if $W^{\prime} Q$ lbs. of water strike the vane per second, the change of momentum in the direction $A C$ per second $=W Q . \overline{A C}$. 
$\therefore$ Resultant pressure on vane acts in the direction $C A$ and is of magnitude $\frac{W Q}{q} \cdot \overline{C A}$ lbs.

(2) Impact on One of a Series of Moving Vanes.

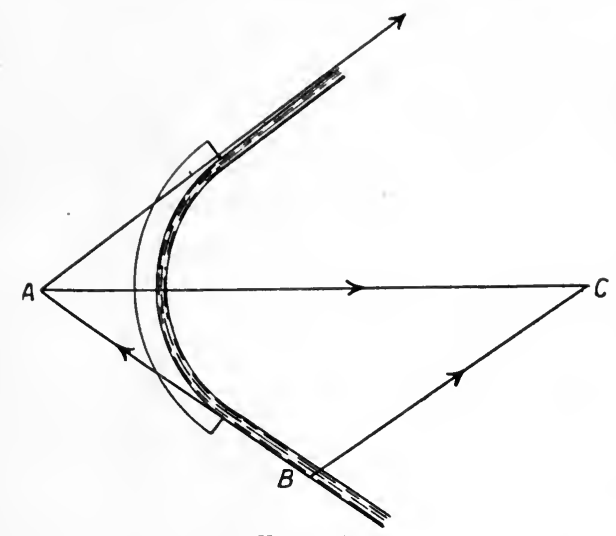

FIG. 140 .

Let $\overline{B A}$ (Fig. $141 a$ and b) represent the initial velocity of the jet in magnitude and direction.

Let $\overline{B C}$ represent the velocity of the vane.

Then $\overline{C A}$ represents the relative velocity of the water over the surface of the vane.

Draw $E F=C A$ tangential to the vane at exit and make

If $G$ equal and parallel to $B C$. The vector $\overline{E G}$, which represents the velocity compounded of the velocity of the vane and of

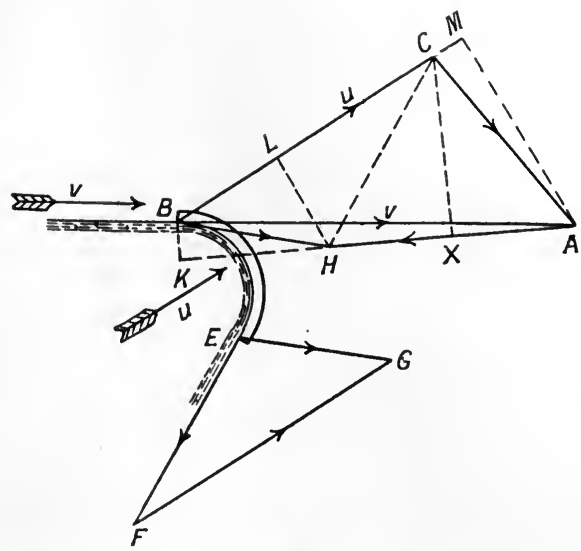

(a)

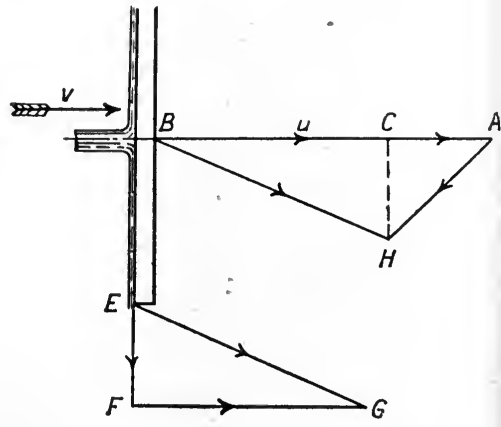

(b)

FIG. 141 .

that of the water relative to the vane, now gives the absolute velocity of the discharge stream. 
Drawing $B H$ equal and parallel to $E G$, the acceleration which is given to the water by the vane is represented by $\overline{A H}$, and the resultant pressure on the vane $I^{\prime}=\frac{W \cdot Q}{g}: H A$ lbs. The line of action of this pressure is parallel to $H A$.

The effective pressure is the component of this in the direction of motion of the vane. Thus in Fig. $141 a$ this is given by $\frac{W \cdot Q}{g} \cdot \overline{L M}$ lbs., and the work done per second by $\frac{W \cdot Q}{g} \cdot \overline{L M} \cdot \overline{B C}$ ft. llis. In Fig. $141 l$, the effective pressure $=\frac{W \cdot Q}{g} \cdot \overline{C A}$ lbs. and the work per second $={ }^{W \cdot(} \cdot C^{\prime} A \cdot B C$ ft. lbs.

By producing $A H$ to $K$ (Fig. $141 a$ ) and dropping perpendiculars $B K, C X$, on to $A H$, we can prove that

$$
B A^{2}-\overline{B H^{2}}=\overline{H A}(\overline{H A}+2 \overline{K H}) .
$$

Also, since $\overline{C A}$ represents the initial relative velocity of jet and vane and $\overline{C H}$ their final relative velocity, we haveneglecting friction- $\overline{C^{\prime} I}=\overline{C^{\prime} A} . \quad \therefore \quad \overline{H A}=2 \overline{I X}$

$$
\therefore K A=K H+2 \dot{H} X
$$

$$
\therefore \quad K A+K H=H A+2 K H=2(K H+H X)
$$

$$
\therefore \quad H A+2 K H=2 K X
$$

$$
\text { Also } \frac{2 K X}{2 B C}=\frac{L M}{A H}
$$

$$
\therefore \quad H A(H A+2 K H)=2(I M . B C)
$$

$$
\begin{aligned}
\therefore \text { Work done } & =\frac{W \cdot Q}{2 !}\left(\overline{B A}^{2}-B \bar{H}^{2}\right) \\
& =\frac{W \cdot Q}{2} \cdot\left(r_{1}{ }^{2}-r_{2}{ }^{2}\right)
\end{aligned}
$$

where $v_{1}$ and $v_{2}$ are the initial and final absolute velocities of the water.

H.A.

$$
\begin{aligned}
\therefore \quad \text { Efficiency } & =\frac{\text { Work done on vane }}{\text { Total energy of jet per second }} \\
& =\frac{\frac{W \cdot Q}{2 ! g}\left(r_{1}{ }^{2}-v_{2}{ }^{2}\right)}{\frac{W \cdot Q}{2 !} r_{1}{ }^{2}} \\
& =1-\left(\frac{v_{2}}{v_{1}}\right)^{2}
\end{aligned}
$$


Centre of Pressure on Vane.-The position of the centre of pressure on any vane receiving a jet tangentially may be determined as follows. Consider any small arc $P^{\prime} Q$ (Fig. $142 a$ ) of the vane. If the velocity is supposed unaltered by friction, $P A$ and $A Q$, tangents at $P$ and $Q$ represent to some scale the (equal) velocities at $P$ and $Q$, while $R Q$, perpendicular to the chord $P Q$, represents to the same scale the change of velocity between $P$ and $Q$. Normals $P C$ and $Q C$ to the curve intersect in $C$, the centre of curvature of the arc, and $\frac{R Q}{A Q}=\frac{P Q}{P C}$, so that
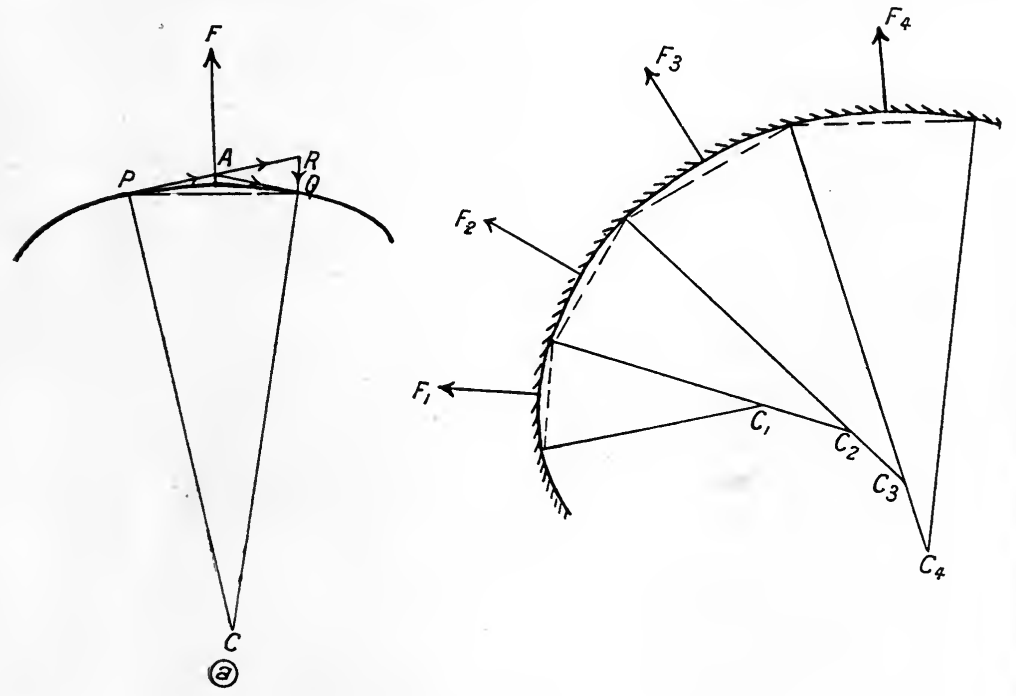

(2)

(b)

Fig. 142.

if $P C$ represent the velocity $v$ at $P$, the chord $P^{\prime} Q$ represents the change of velocity between $P$ and $Q$ and the resultant pressure on the $\operatorname{arc} P Q$ is given by $\frac{W \cdot Q}{g} . \overline{P Q}$ lbs., if $Q$ is the volume in eubic feet striking the vane per second.

Thus if a series of normals to the curve are drawn intersecting at $C_{1}, C_{2}, C_{3}$, etc. (Fig. $142 \mathrm{~b}$ ), the pressure on the corresponding arcs will be given by $\frac{W \cdot Q}{g} \cdot\left\{\frac{\text { arc }}{\text { radius }}\right\} v$ lbs., and these pressures act outwards through the middle points of their respective 
arcs. Drawing in the lines representing these pressures $F_{1}, F_{2}, F_{3}$, etc., the funicular polygon for these forces may be drawn, and the line of action of their resultant obtained as explained in any work on applied mechanics. The point of intersection of this line with the surface of the vane gives the centre of pressure on the vane.

If the velocity varies from point to point of the vane the same general principles apply except that now the velocity $r$ in the expression-pressure $\left.=\frac{W \cdot Q}{g} ; \frac{\text { arc }}{\text { radius }}\right\} r$ lbs.-varies from point to point. Where the law of its variation is known, the problem becomes perfectly determinate.

Arit. 102.-Conipounding of Jets.

If two steady streams are allowed to impinge on each other under atmospheric pressure, they combine to form a single stream.

Let streams (1) and (2) combine to form stream (3) (Fig. 143). Then if $a, v$, and $Q$ represent the sectional areas, velocities of flow, and quantity carried by

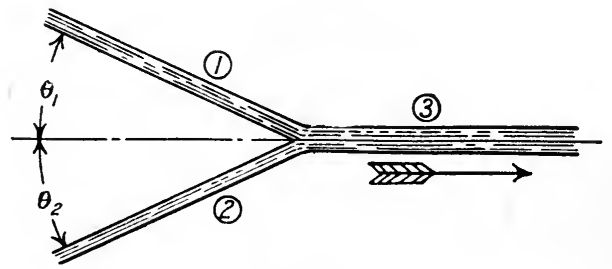

Fig. 143. each stream, we have, if $\theta$ be the angle made by an impinging stream with the direction of the resultant stream :-

For continuity of flow, $v_{1} a_{1}+v_{2} a_{2}=v_{3} a_{3}$

The equations of momentum give :-

$$
\begin{gathered}
Q_{1} v_{1} \sin \theta_{1}-Q_{2} v_{2} \sin \theta_{2}=0 \\
Q_{1} v_{1} \cos \theta_{1}+Q_{2} v_{2} \cos \theta_{2}=Q_{3} r_{3}
\end{gathered}
$$

while the equation of energy gives:-

$$
\begin{aligned}
\frac{W Q_{1} v_{1}^{2}}{2 g}+\frac{W Q_{2} v_{2}^{2}}{2 g} & =\frac{W Q_{3} v_{3}^{2}}{2 g}+\text { loss at impact. } \\
& =\frac{W\left(Q_{1}+Q_{2}\right) r_{3}^{2}}{2 g}+H^{\prime}
\end{aligned}
$$




$$
\begin{aligned}
\therefore \quad H^{\prime} & =\frac{W}{2 g}\left\{Q_{1} v_{1}^{2}+Q_{2} r_{2}^{2}-\left(Q_{1}+Q_{2}\right) r_{3}^{2}\right\} \\
& =\frac{W}{2 g}\left\{Q_{1}\left(v_{1}^{2}-v_{3}^{2}\right)+Q_{2}\left(v_{2}^{2}-v_{3}^{2}\right)\right\}
\end{aligned}
$$

By substitution from equations (2) and (3) above, this loss may be determined in terms of $v_{1}, v_{2}, \theta_{1}$ and $\theta_{2}$.

In two particular cases $H^{\prime}$ may be zero. In each of these it is necessary that $\left.\begin{array}{l}v_{1}{ }^{2}-r_{3}{ }^{2}=0 \\ r_{2}{ }^{2}-r_{3}{ }^{2}=0\end{array}\right\}$ simultaneously, and therefore that $\pm v_{1}= \pm v_{2}= \pm r_{3}$.

The first case is found where $v_{1}=r_{2}=v_{3}$, i.e., where two parallel streams moving with the same velocity combine to form

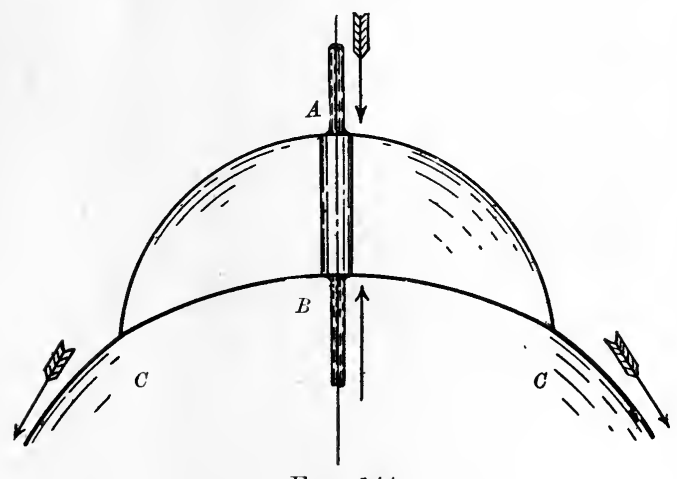

FIG. 144 . a single stream, with an area equal to the combined area of the two. The second case is found where $v_{1}=$ $-v_{2}$, each of these being numerically equal to $v_{3}$, and corresponds to the direct impact of two streams of equal velocity. A film of water is then formed, the velocity of whose mass centre $\left(v_{3}\right.$ in the equation of momentum) is zero, but which has a velocity of outward flow equal to $v_{1}$ or $v_{2}$. Professor Osborne Reynolds illustrated this by allowing two streams of equal velocity to meet by direct impact, and noting the clear and glassy appearance of the resultant film. If a cylindrical prism having plane and parallel ends be placed in the path of the stream (Fig. 144), the films from $A$ and $B$ to the point of contact $C$, are still perfectly clear. After $C$, however, the frosted appearance of the film indicates eddy formation and the institution of sinuous motion. 


\section{Art. 103.-Jet Propulsion.}

When a jet of water escapes from an orifice in the side of a vessel, the force necessary to produce this outflow is equal in magnitude to the flux of momentum per second across the vena contracta of the jet, and acts in the direction of flow of the jet. This force necessitates an otherwise unbalanced pressure or reaction on the side of the vessel opposite to the orifice (Art. 39), which reaction will tend to move the vessel in the opposite direction to that of the jet.

Advantage has been taken of this in a system which has been applied (though with only moderate success) to the propulsion of large vessels. Here a supply of water is drawn into the boat, usually through a vertical pipe opening amidships, by a centrifugal pump driven by the main engines, and is then discharged directly astern. The escaping stream, in virtue of its reaction on the vessel, does work in propulsion, while the theoretical efficiency of the system may be calculated as follows:-

Let $A=$ sectional area of discharge orifices in square feet.

$u=$ velocity of vessel in feet per second.

$v$ = velocity of efflux of stream relative to boat.

Then $v-u=$ absolute velocity of efflux of stream, i.e., its velocity relative to the surrounding water.

The initial velocity of ) the water before being $=0$

drawn into the vessel

Its final velocity in the

$\left.\begin{array}{c}\text { direction of motion of } \\ \text { the vessel }\end{array}\right\}=u-v \mathrm{ft}$. per sec.

The weight of water discharged per second $j=W A v$ lbs.

$\therefore$ The change of momentum of the water in the opposite direction to that of motion of the vessel

$\therefore$ Propelling force on $;=\frac{W A v}{g}(v-u)$ lbs. 
$\left.\begin{array}{l}\text { Work donein propulsion } \\ \text { per sec. }\end{array}\right\}=\frac{W A v u}{g}(v-u) \mathrm{ft}$. lbs.

Kinetic energy rejected) per sec. in the discharge $=\frac{1}{2 g}(r-u)^{2} \mathrm{ft} . \mathrm{lbs}$.

$\therefore$ Total energy given to $\left.\begin{array}{l}\therefore \text { Tater per sec. neglecting } \\ \text { eddy and frictionallosses }\end{array}\right)=\frac{W A r}{2 !}\left((v-u)^{2}+2 u(v-u)\right\}$ ft.lbs.

$$
=\frac{W A v}{2 g}\left(v^{2}-u^{2}\right) \mathrm{ft} . \mathrm{lbs} .
$$

$$
\begin{aligned}
\therefore \begin{array}{c}
\text { Theoretical efficiency } \\
\text { of jet }
\end{array} & =\frac{\text { useful work done by jet }}{\text { energy given to jet }}=\frac{2 u(v-u)}{v^{2}-u^{2}} \\
& =\frac{2 u}{v+u} .
\end{aligned}
$$

This has its maximum value, unity, when $v=u$.

An examination of equation (1) will, however, show that under these circumstances the propelling force is zero, and that with a large propelling force it is impossible to work under conditions which conduce to high efficiency.

Water may be taken into the pumps either through one or more vertical or horizontal pipes at right angles to the axis of the vessel, through pipes facing forwards in the direction of the axis, or through vertical or horizontal pipes fitted with a scoop facing in the direction of motion of the vessel. In the latter case the effect is the same as with the inlet pipes facing forward except that the scoop introduces a slight additional resistance to motion. If $h$ be the depth of the inlet, the head producing flow along the inlet pipe is given by $\iota+\frac{u^{2}}{g}$ feet in the two latter cases, and by $\iota$ feet if the inlet opens flush with the sides or bottom of the boat, and since in every case the delivery pressure is that corresponding to the depth of immersion of the outlet, less energy is required of the pump per lb. of water to discharge at a given velocity with the inlet facing forward than with a plain side or bottom inlet. In any case the total change of momentum per second is the same provided the discharge velocities are the same. 
Variation of Efficiency with Size of Orifices.

Assuming the energy of propulsion to be given by $k u^{3} \mathrm{ft}$. lbs. per second (p. 182), where $k$ is constant for a given vessel, we have

$\mathrm{Ol}^{\circ}$

$$
\begin{gathered}
k u^{3}=\frac{A W}{g} u v(v-u) \\
\therefore k u^{2}+u \cdot \frac{A W}{g} r-\frac{A W}{g} r^{2}=0 \\
u=-\frac{A W}{2 g k} v ! 1-\sqrt{\left.1+\frac{4 g k}{A W}\right)} .
\end{gathered}
$$

Substituting this value of $u$ in equation (5), we have

$$
\text { Efficiency }=\frac{2 u}{u+r}=\frac{2\left\{1-\sqrt{\left.1+\frac{4 g k}{A W}\right\}}\right.}{1-\sqrt{1+\frac{4 g k}{A W}-\frac{2 g k}{A W}}} .
$$

Multiplying numerator and denominator by $1+\sqrt{1+\frac{4 g l}{A W}}$ this finally gives

$$
\text { Efficiency }=\frac{4}{3+\sqrt{1+\frac{4 g k}{A W}}},
$$

so that the efficiency of propulsion increases as the ratio $\frac{A}{k}$ increases, i.e., in the case of any given boat, as the area of the orifices increases. But as the quantity discharged per minute, and also the size of pump necessary to give this discharge increase with $A$, the limit of efficiency in this direction is soon reached.

\section{Example.}

If $l=2 \cdot 4$ and if $A=2$ square feet, we have efficiency of jet

$$
=\frac{4}{3+\sqrt{1+\frac{4 \cdot 8}{2}}}=\underset{4 \cdot 85}{4}=82.5 \text { per cent. }
$$

Suppose the boat to travel at 15 miles per hour, $u=22$ feet per second. 


$$
\begin{aligned}
\text { Then efficiency } & =\frac{2 u}{u+v}=\cdot 825 \\
\therefore \quad 1.175 u & =825 v \\
\therefore \quad v & =\frac{1 \cdot 175 \times 22}{\cdot 825}=31.33 \text { feet per second. } \\
Q=31.33 \times 2 & =62.66 \text { cubic feet per second. }
\end{aligned}
$$

Assuming the combined efficiency of engine, pump, and jet to be $80 \times \cdot 60 \times \cdot 825=\cdot 396$, we have :-

$$
\begin{aligned}
\text { I.H.P. of engine } & =\frac{k u^{3}}{550} \times \frac{1}{.396} \\
& =\frac{2.4 \times 22^{3}}{550 \times \cdot 396}=117 \text { I.H.P. }
\end{aligned}
$$

The most noteworthy experiments on hydraulic propulsion have been carried out by the British Government. In 1866 the Admiralty built two almost similar gunboats, the Wateruitch and the Viper, the former being fitted with jet propulsion and the latter with a screw propeller. Their displacements were, Waterwitch 1,161 tons, Viper 1,180 tons. The Waterwitch took water in through a vertical opening amidships, passed it through a 14-foot centrifugal pump, and discharged astern through two 24 -inch nozzles. When discharging $5 \cdot 2$ tons of water per second, with a relative velocity of 29 feet per second, the engines indicated 760 I.H.P., and gave a speed of $9 \cdot 3$ knots (15.71 feet per second) with a jet efficiency of 70 per cent. The Viper, with 696 1.H.P., gave 9.58 knots. This comparison is, however, rather unfair to the Viper, as its speed suffered from the provision of a double bilge keel, and from its slightly fuller run.

In 1878, the Swedish Government built two torpedo boats, 58 feet long-10' $9^{\prime \prime}$ beam-and with 20 and 21 tons displacement, the heavier machinery of the hydraulic boat necessitating the larger displacement. The latter boat took in water vertically and with 78 I.H.P. gave a speed of 8.12 knots. The screw boat with 90 I.H.P. gave 10 knots.

In 1882, the Admiralty had one of a batch of torpedo boats fitted with hydraulic propulsion. 
The details of the boats were as follows :-

\begin{tabular}{l|c|c|c|c|c|c}
\hline & Length. & Beam. & Draught. & Displacement. & I.H.P. & Speed. \\
\hline Screw & $63 \cdot 0 \mathrm{ft}$ & $7^{\prime} 6^{\prime \prime}$ & $3^{\prime} 8 \frac{1}{2}^{\prime \prime}$ & $12 \cdot 89$ tons & 170 & $17 \cdot 3 \mathrm{knots}$ \\
\hline Hydraulic & $66 \cdot 4 \mathrm{ft}$ & $\bar{\sigma}^{\prime} 6^{\prime \prime}$ & $2^{\prime} 6^{\prime \prime}$ & $14 \cdot 4$ tons & 167 & $12 \cdot 6$ knots \\
\hline
\end{tabular}

The hydraulic boat was fitted with a centrifugal pump 2' $6^{\prime \prime}$ diameter, running at 428 revolutions per minute, and discharged astern through two 9-inch outlets. The pump delivered approximately one ton of water per second at a velocity of $37 \cdot 25$ feet per second (as against $21 \cdot 28$ feet per second of the boat), and was fitted with a vertical inlet pipe, provided with a scoop facing forward.

In each case the efficiency of the pump was approximately 48 per cent. The last-mentioned torpedo boats gave the highest jet efficiency, viz., 72.8 per cent. The combined efficiency of jet and pump was then 34.9 per cent. as compared with an average efficiency of about 65 per cent. in the case of a screw propeller. Taking an efficiency of 80 per cent. for the engine and shafting in each case, though this would be slightly greater in the case of the pump, we have the combined efficiency of propelling mechanism

\begin{tabular}{c|c}
\hline Hydraulic. & Serew. \\
\hline $27 \cdot 9 \%$ & $52.0 \%$ \\
\hline
\end{tabular}

The low efficiency of the centrifugal pump effectively militates against the adoption of the system of jet propulsion on any large scale. As a propeller, the jet itself is quite as efficient as the screw, the chief losses occurring in the production of the jet.

It should be noted that the system works equally well with the discharge either above or below water level, except that the former case necessitates work being wasted in lifting the water 
to the level of the discharge orifice. The advantages of the system are

(1) No racing of propellers in rough weather ;

(2) No under water obstacles to become entangled in wreckage ;

(3) Ease of control from some central station on the bridge;

(4) Great facilities for manœuvring;

and in view of these its adoption for the propulsion of steam lifeboats is likely to become more general.

\section{Art. 104.-Hydraulic Mining.}

The fact that a high velocity jet of water carries an immense amount of destructive energy, was first taken advantage of to any large extent on the Pacific Coast of North America. Here, owing to the configuration of the country, the only available water supply is to be found in comparatively small streams, at considerable elevations. The construction of a small dam and a flume or ditch to bring the supply to the nearest suitable point on the mountain side, and the further construction of a pipe line leading the supply under pressure down the mountain side, renders available a comparatively small quantity of water, under great pressure. In the process known as Hydraulicing, chiefly applied to gold mining, this supply is led into a pressure box securely bolted to the ground at a distance of from 100 to 200 feet from the face of the cliff to be mined. From the pressure box one or two jets, of diameters ranging from 4 inches to 11 inches, are led by means of nozzles mounted on ball-and-socket joints, and are played on to the face of the cliff, the débris being carried away by sluices on which the process of washing and amalgamating is completed.

Art. 105.-Pressure on Subuerged Plane Surfaces.

If an entirely submerged plane surface be moved normally or obliquely with uniform velocity through still water, the resistance to motion will depend slightly on frictional resistances, but to a much larger extent on the change of momentum produced in the surrounding water during the passage of the plane, and on the eddy production in the rear of the plane. 


\section{Normal Motion of a Plane through Still Water.}

Here, in front of the plane, stream line motion is set up, and if $a$ be the area of the plate, and $v$ its velocity, a column of water of sectional area $a$ and of length $r$ feet, is transferred from front to rear of the plane per second. The effect of this in producing resistance, may be seen by considering the flow of a steady stream past such a submerged plane. As before, up to the plane the motion is steady, and the stream line formation is as indicated in Fig. 145. ${ }^{1}$ The plane, therefore, affects the momentum in a direction normal to its plane, of a mass of water of volume $a r$ cubic feet, per second. Since the velocity of the mass in this direction is initially $v$ feet per second, if it were possible to destroy the whole of its momentum the total pressure on the front of the

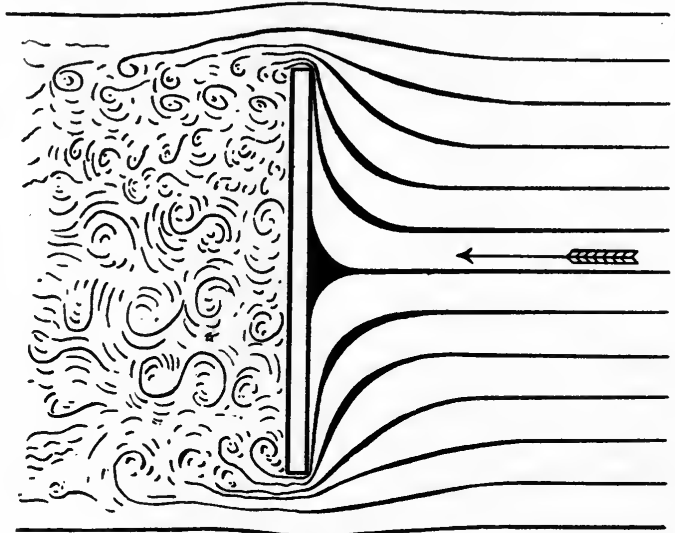

Fig. 145 .

plane would be $\frac{W a r^{2}}{g}$ lbs. From a consideration of the stream line formation, it is, however, clear that it is only those stream lines at and near the centre of the plane which are actually diverted at right angles to their normal direction, the outer layers being diverted through a smaller angle depending on their distance from the centre, so that the change of momentum in the direction of motion, and hence the pressure on the front of the plane, is less than $\frac{W a r^{2}}{g}$. At only one point, i.e., the centre of the plane, is the velocity actually destroyed, and here the pressure intensity amounts to $\frac{W r^{2}}{2 g}$, the head corresponding to the velocity

1 From a paper by Professor Hele Shaw, "Trans. Inst. Naval Architects," 1898, vol. 85 . 
of flow. Immediately after passing the plane the motion becomes sinuous, eddies are formed, and the effect of this formation on the resistance will now be considered. These eddies, which are formed behind the edge of the plate, and which consist of water partly from the front and partly drawn from behind the plate, are not suddenly developed but grow until the whole space behind the plate is filled, when they break away and drift in the wake, other eddies then being formed. Since the energy of eddy production has to be supplied in the form of extra work done on the plate, this directly increases the resistance to motion. In other words, while the pressure on the front of the plane is unaffected, that on the rear face is reduced by this eddy production, and since the resistance to motion is equal to the difference of pressure on the two faces, this is increased. Plates of different shapes have different effects as regards eddy production, the circular shape giving least resistance for a given area. Also it would appear that as the size of plate increases, the proportional effect of the eddy production increases slightly. Experiments show that for the normal motion of a submerged plane through still water, where the boundaries are so remote as not to affect the resistance, this is given by $k \frac{W a v^{2}}{g} \mathrm{lbs}$., where $k$ is a coefficient depending on the size and shape of the plate.

With a circular plate, $k$ varies from about 560 in a plate of 1 inch diameter to 650 with a diameter of 3 inches and 720 with a diameter of 6 inches, afterwards increasing slightly with the diameter. Experiment also tends to show that the resistance is less where the plate is stationary in a moving current than where the plate is moved through still water. This is probably due to the fact that eddy production is unable to proceed to such an extent where the passing current tends to remove the eddies while in process of formation.

Owing to this process of formation the pressure on the plate, even in a steady stream, is not uniform but periodic, the period being determined by the time of formation of an eddy behind the plate, and consequently by the shape and size of the latter.

This action may often be seen where a flexible bough dips into 
a steady stream of water. Here, when the period of eddy formation synchronizes, or nearly so, with that of the natural vibration of the bough, violent vibration is set up which may, in an extreme case, be sufficient to break the bough.

On account of this action, the use of velocity meters in which the pressure on a flat plate is taken as a measure of the velocity of a stream is not to be recommended (p. 327).

Submerged Plane in Pipe of small Cross-Sectional Area.

The effect of placing the plane in a pipe of restricted area, is to increase the velocity with which the water escapes over the edge of the plane. This causes an increased eddy production, a consequent reduction of pressure on the rear of the plane, and hence an increased resistance.

Oblique Motion of Submerged Planes.

Where an oblique plane makes an angle $\theta$, with its own direction of motion (Fig. 146), the velocity of the water normal to the plane $=r \sin \theta$.

The column of water whose momentum is affected, is now

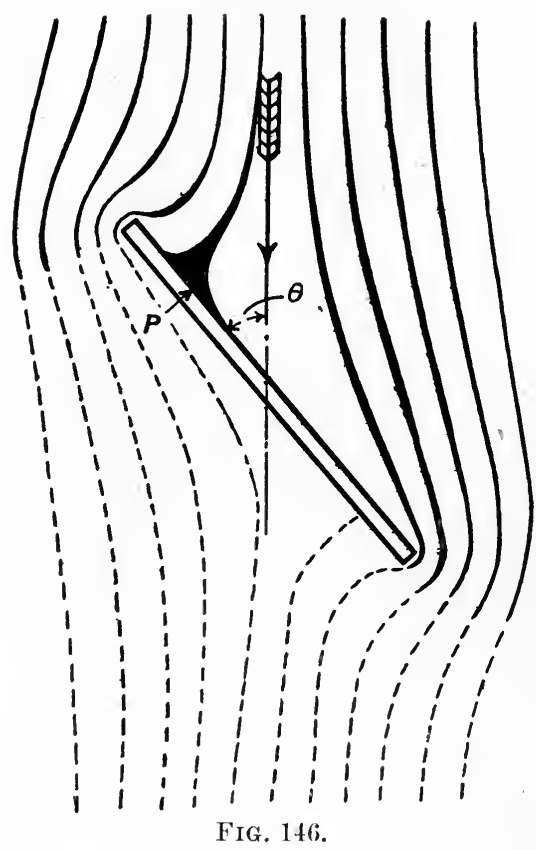
of sectional area $a \sin \theta$, so that the change of momentum produced by the passage of the plane is proportional to

$$
\frac{W v a \sin \theta v \sin \theta}{g}, \text { i.e., to } \frac{W a}{g} v^{2} \sin ^{2} \theta \text {. }
$$

It might then be expected that the resistance to the oblique motion of a plane would equal

Direct resistance $\times \sin ^{2} \theta$.

Due, however, to the unequal eddy formation at the rear of the plate with the two kinds of motion, and to the fact that as $\theta$ 
is diminished, frictional resistances form an increasingly large proportion of the whole, this law. does not hold. Lord Rayleigh, ${ }^{1}$ indeed, showed that a more rational expression for the pressure on the front face of the plane when moving through an infinite mass of fluid is given by

$$
P=\frac{W a}{l} \cdot \frac{\pi \sin \theta}{4+\pi \sin \theta} \cdot r^{2} \mathrm{lbs} .
$$

this neglecting the effect of eddy formation.

From this we have, if $P^{1}=$ normal pressure on oblique plane, and if $P=$ corresponding resistance of normal plane,

$$
P^{1}=P \cdot \frac{(4+\pi) \sin \theta}{4+\pi \sin \theta}=P \cdot \frac{\sin \theta}{56+\cdot 44 \sin \theta} .
$$

As the result of experiment M. Joessels, of the French Navy, deduced an empirical formula of this type, making

$$
P^{1}=P \frac{\sin \theta}{\cdot 39+\cdot 61 \sin \theta} .
$$

The following table shows results experimentally obtained by Colonel Beaufoy, and also some results of experiments by Vince quoted by Rayleigh, together with corresponding values as

\begin{tabular}{|c|c|c|c|c|c|c|c|c|c|}
\hline $\begin{array}{l}\text { Angle made by plane with } \\
\text { direction of motion }\end{array}$ & $90^{\circ}$ & $80^{\circ}$ & $70^{\circ}$ & $60^{\circ}$ & $50^{\circ}$ & $40^{\circ}$ & $30^{\circ}$ & $20^{\circ}$ & $10^{\circ}$ \\
\hline Ratio $\frac{P_{1}}{P}$ experi- $\left\{\begin{array}{l}\text { Vince } . \\
\text { mentally obtained }\end{array}\right.$ & $\begin{array}{l}1 \cdot 00 \\
1 \cdot 00\end{array}$ & - & $\begin{array}{l}\cdot 974 \\
\cdot 845\end{array}$ & $\cdot 828$ & $\begin{array}{l}\cdot 873 \\
\cdot 722\end{array}$ & -5 & $\begin{array}{l}663 \\
-\end{array}$ & $\begin{array}{l}\cdot 458 \\
\cdot 321\end{array}$ & $\begin{array}{l}\cdot 278 \\
\cdot 272\end{array}$ \\
\hline $\sin ^{2} \theta . \quad . \quad . \quad$. & $1 \cdot 00$ & 97 & $\cdot 88$ & $\cdot 75$ & $\cdot 587$ & 413 & $\cdot 250$ & $\cdot 117$ & .030 \\
\hline$\frac{\sin \theta}{56+\cdot 44 \sin \theta}($ Rayleigh $)$. & $1 \cdot 00$ & $\cdot 990$ & 965 & $\cdot 919$ & $\cdot 854$ & $\cdot 751$ & $\cdot 641$ & $\cdot 481$ & $\cdot 273$ \\
\hline$\frac{\sin \theta}{39+61 \sin \theta}$ (Joessels) & $1 \cdot 00$ & 994 & 975 & 944 & $\cdot 894$ & $\cdot 821$ & $\cdot 715$ & 17\% & 3.51 \\
\hline
\end{tabular}
calculated by the $\sin ^{2} \theta$ formula, and by those of Rayleigh and of Joessels:-

The resistance in the direction of motion is given by $P^{1} \sin \theta$. As in the case of the normal plane, there is only one point $I$,

1 "Scientific Papers," I., p. 287, and III., p. 491 ; or see Lamb's "Hydrodynamics," p. 93. 
this in the median plane of the plate, at which the velocity is zero and at which the maximum pressure $\frac{\mathrm{W}^{r} r^{2}}{2 !}$ is therefore attained. This is now in advance of the centre of the plane, its distance from the centre in terms of the length $l$ of the plane as obtained theoretically being given in the following table. ${ }^{1}$ The distance $\bar{x}$ of the centre of pressure from the centre of the plane, is theoretically given by the formula $\bar{x}=\frac{3}{4} \cdot \frac{\cos \theta}{4+\pi \sin \theta} \cdot l,{ }^{1}$ and values of $\bar{x}$ are also tabulated below.

\begin{tabular}{|c|c|c|c|c|c|c|c|c|c|c|c|c|}
\hline$\theta$ & . & . &. & . & . & . & $90^{\circ}$ & $70^{\circ}$ & $50^{\circ}$ & $30^{\circ}$ & $20^{\circ}$ & $10^{\circ}$ \\
\hline $\bar{x}$ & . & . & . & . & . & . & $\cdot 000$ & $\cdot 037 l$ & .0757 & $\cdot 117 l$ & $\cdot 139 l$ & $\cdot 163 l$ \\
\hline & & & & & . & livides, & $\cdot 000$ & $\cdot 232 l$ & 4027 & $\cdot 4837$ & $496 ; 7$ & $500 l$ \\
\hline
\end{tabular}

It is found, however, that with a rectangular oblique plane the total pressure for a given value of $\theta$ depends largely on whether the long or short edges of the plane are perpendicular to the stream, the resistance being greatest when the long edges are so placed. The reason for this is explained by Lord Rayleigh as follows. Although there is only one point of maximum pressure whatever the manner of presentation of the plane, yet with the long edges perpendicular to the stream the motion is approximately in two dimensions, and a region of almost maximum pressure extends over the greater part of the length. The case is very different, however, when the short dimension is perpendicular to the stream, for then, along the greater part of the length the flow is rapid and the pressure in consequence low.

This is of importance in the design of oar blades, the floats of paddle-wheels, and the gliders of aeroplanes, since, area for area, a short (in the direction of motion) wide surface is considerably more efficient than a long narrow one, the total pressure

1 From Lamb's "Hydrodynamics," p. 94. 
approximating more nearly to $\frac{W a v^{2}}{2 g}$ as the surface is made shorter and wider. ${ }^{1}$

As applied to the design of rudders it explains why a deep narrow rudder gives better results than one which, having the same area, is shallower and wider.

Art. 106.-Rudder Action.

The normal pressure which is produced on an oblique plane by its motion through water is taken advantage of in the ordinary
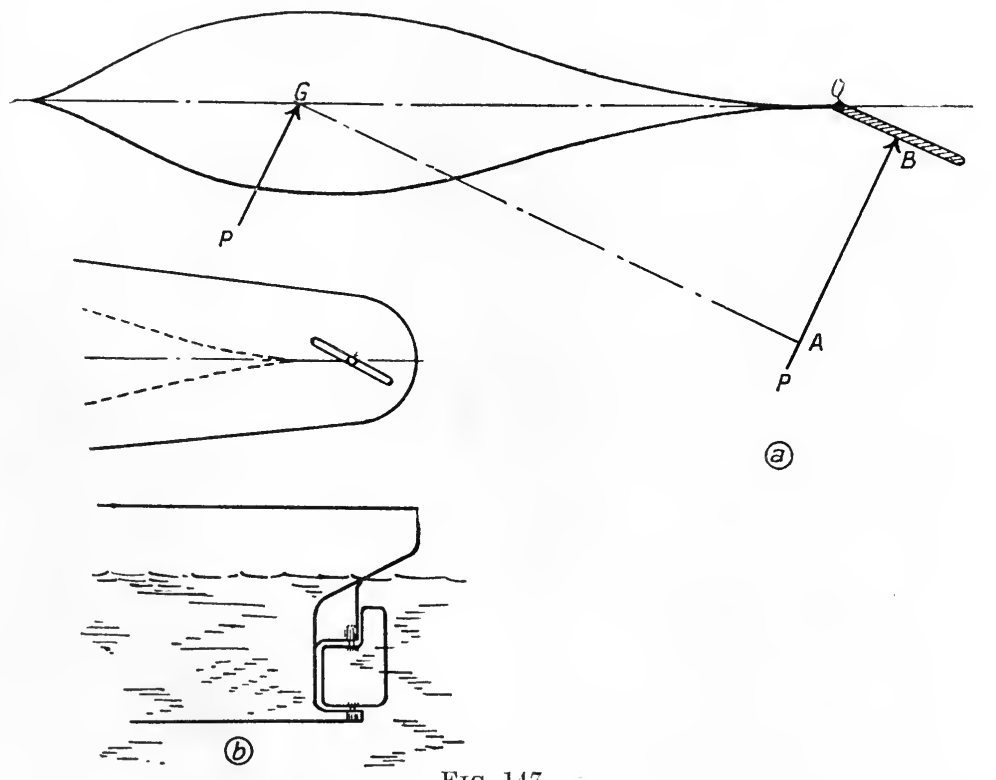

FIg. 147 .

rudder. Since the flow of water to the rudder is guided by the form of the stern of the vessel, the distribution of pressure is entirely different to that occurring in the cases previously considered. In any case, however, the effect of the motion is to produce a distribution of normal pressure over the rudder which has a single resultant tending to turn it about its point of attachment

1 For curves showing the effect of varying the manner of presentation of glider surfaces for aeroplanes, see Lord Rayleigh's paper, ante loq. 
to the stern post. This action being resisted by the rudder chains, the nett effect is that of a single force $P$ acting on the vessel at $B$ in the direction $A B$ (Fig. 147 a).

This is equivalent to an equal and parallel force applied at the centre of gravity $G$ of the vessel, together with a couple of moment $P \times A G$ tending to rotate the vessel about $G$. It is this moment which tends to turn the vessel. The single force simply tends to produce a bodily drift of the vessel in a direction parallel to $A B$. This explains why a constant rudder angle will not enable a vessel to describe a circle.

The point in the rudder body, at which it is pivoted, does not affect this force or the couple produced, and advantage has been taken of this to mount the rudder on pivots near its centre. Being then approximately in balance, the moment necessary to rotate it is considerably reduced, and a much greater proportion of the steering force is directly transmitted through the pivots (Fig. 147 b), instead of through the rudder chains.

\section{Art. 107.-Resistance to Motion of Subuerged Bodies.}

If the plane already considered forms the end of a solid prism whose axis is parallel to the direction of motion and whose length

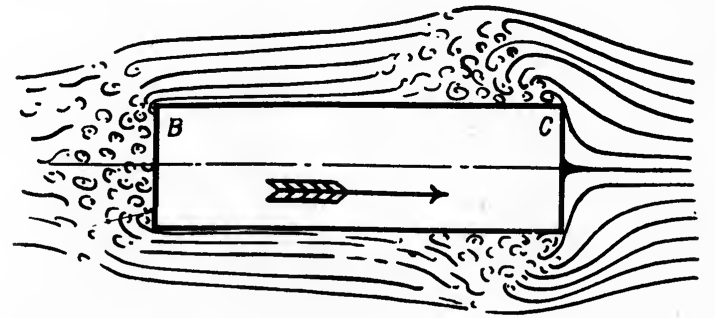

FIG. 148.

is more than three times the diameter of the plane, the directions of the stream lines are modified as shown in Fig. 148. Eddies are still formed at $C$, the direction of motion then settling down parallel to the axis of the prism, until at $B$ a second formation of eddies takes place.

But the total eddy formation at $B$ and $C$ is now less than at $B$ alone with a plane surface, and thus we get a higher pressure on

H.A. 
the rear of the prism than on the plane and a consequent smaller resistance to motion. Even including skin friction, with a moderate ratio of length to diameter the resistance of the prism is less than that of the plane. Putting the resistance equal to $k \frac{W a}{g} r^{2}$, we have $k=\cdot 55$ with a prism having plane ends and a length of about three times its diameter. If fitted with a tapering cutwater the production of eddies at $C$ is obviated and the value of $k$ becomes about 40 . Fitted also with a tapering stern eddy formation is largely prevented at $B$, and $k$ is reduced to about $\cdot 125$. The resistance is now largely due to skin friction and should be calculated on that assumption (p. 169).

\section{Examples.}

(1) A horizontal jet issuing from an orifice 1 inch in diameter discharges 2 gallons per second and impinges normally on a large fixed plane. Determine the force exerted on the plane and also, neglecting losses, the maximum pressure intensity likely to be attained.

$$
\text { Answer: }\left\{\begin{array}{l}
36 \cdot 5 \text { lbs. } \\
23 \cdot 25 \text { lbs. per square inch. }
\end{array}\right.
$$

(2) If the above plate has edges curved so as to deflect the stream through a total angle of $150^{\circ}$, determine the pressure on the plate.

\section{Answer. $68 \mathrm{lbs}$.}

(3) A jet from a rectangular orifice $6^{\prime \prime}$ wide $\times 1^{\prime \prime}$ deep impinges on a curved vane $6^{\prime \prime}$ wide, with a velocity of 60 feet per second. On incidence the jet makes an angle of $30^{\circ}$ with the normal to the vane, and the portions into which it is divided are deflected through total angles of $120^{\circ}$ (the smaller portion) and $90^{\circ}$ (the larger portion). Determine the pressure on the plane in the direction of the jet.

\section{Answer. 300 lbs.}

(4) Assuming a series of vanes similar to that in question (3) to move in the direction of the jet with a velocity of 30 feet per second, determine the efficiency of the arrangement. 
(5) 'Two jets of sectional area 2 square inches and 1 square inch, and of velocities 20 f.s. and 30 f.s., are inclined to each other at an angle of $60^{\circ}$, and combine to form a single stream. Determine the direction and velocity of this stream and the loss of head at impact.

Answer. $\left\{\begin{array}{l}\text { Resultant stream makes an angle of } 31^{\circ} 58^{\prime} \\ \text { with direction of first stream. } \\ \text { Velocity }=21.06 \text { feet per second. } \\ \text { Loss of head }=8.03 \text { feet. }\end{array}\right.$

(6) A jet propelled vessel has a wetted surface area of 800 square feet, and a coefficient of resistance of 004 . If the area of the nozzle openings is 3.0 square feet, determine the maximum theoretical efficiency of the jet considered as a propeller. Also, assuming the pump efficiency to be 50 per cent. and the mechanical efficiency of the engines to be 85 per cent., determine the efficiency of propulsion.

$$
\text { Answer. }\left\{\begin{array}{l}
\text { Efficiency }=\cdot 836 . \\
\text { Total efficiency }=\cdot 355 .
\end{array}\right.
$$

(7) If the boat of the preceding example is travelling at 12 miles per hour, determine the necessary jet velocity and the discharge per second, for maximum efficiency.

$$
\text { Answer. }\left\{\begin{array}{l}
\text { Velocity }=24.56 \text { feet per second. } \\
\text { Discharge }=2.05 \text { tons per second. }
\end{array}\right.
$$

(8) Water flows at 20 feet per second through a 6 " pipe forming a right-angled bend. Find the magnitude of the resultant force tending to move the pipe.

Answer. $215 \mathrm{lbs}$. 


\section{CHAPTER XI.}

Hydraulic Prime Movers-Water-Wheels-The Overshot-Breast-Sicle-Undershot and Poncelet Wheels-The Pelton Wheel-Losses-Form and Number of Buckets-Speed Regulation-Typical Installations-Summary.

\section{Art. 108.-Hydraulic Prine Movers, Water-IVheels.}

ALthough the question of the utilization of natural water powers has always been one of great economic importance, the introduction and perfection of electrical manufacturing processes, and the possibility of transmitting electrical energy without great loss or expense to a great distance from the place of its generation, has of recent years made it practicable to take advantage of many water powers far remote from large centres of industry and has raised the whole question to an altogether higher plane of importance, while the consequent demand for hydraulic prime movers capable of developing large powers in single units, and of satisfying the exhaustive demands of such installations in the way of speed, regulation and efficiency, has led to a great transformation in the design of such motors.

The first hydraulic prime mover consisted of a wooden paddle water-wheel dipping into the current of a stream, and as such a motor was only required to do the work previously performed by an animate agency, the power required was small and the efficiency of only secondary importance.

The construction was at first of the most primitive type, but was gradually improved; iron took the place of wood; improvements in design led to increased efficiency; the demand for greater powers led to the necessity for utilizing larger falls and the consequent development of the breast and overshot wheels, until a type of wheel was evolved, which within its limitations was as efficient as the most modern of turbines. Its chief disadvantages lay in its slow speed of rotation, the impossibility of 
close speed regulation, and in the large size of wheel required for even small powers; and while for such purposes as the motor was first required these were not serious, the introduction of more modern machinery, more particularly for textile purposes, involved the necessity for a motor which, having a fairly high speed of rotation in order to avoid excessive loss in gearing, should be capable of close speed regulation and of taking advantage of higher falls and of large quantities of water, and for such purposes the water-wheel was almost entirely superseded by one or other type of turbine.

The introduction of electric driving with its large and almost instantaneous changes of load, while giving an additional fillip to the manufacture of high-speed turbines, had its greatest effect in modifying and perfeeting the methods of speed regulation, and in increasing the size of the unit, while at the same time rendering it imperative to design a motor which should be highly efficient under a wide range of loads.

The success which has attended the attempt to satisfy these onerous conditions may be inferred when it is remembered that many manufacturers will now guarantee to construct a turbine which shall give an efficiency of over 80 per cent. over a range of loads of 50 per cent., and which shall respond to an increased demand for power of 33 per cent. with less than 3.5 per cent. variation in speed. With smaller load variations the speed variation is almost infinitesimal, and it becomes easy to run a series of alternating current machines in step with such motors.

The design of hydraulic motors has thus proceeded by welldefined stages, the size and efficiency steadily increasing until at the present time a single unit delevoping 10,000 H.P. and giving an efficiency of 85 per cent. is not at all uncommon, while further development promises to proceed in the direction of still larger units. So far, indeed, as mechanical difficulties affect the question, there appears to be no reason why units developing up to at least 25,000 H.P. should not be constructed, directly the demand arises.

\section{Art. 109.}

Wherever a continuous supply of water at a sufficient elevation, or in motion as in a stream, is available, the potential 
or kinetic energy which this possesses may be turned into useful work.

Before embarking on any power scheme for utilizing such energy, it is however of the highest importance that the true possibilities, of the scheme should be ascertained, for as the usefulness of the supply depends in most cases on its uniformity over long periods of time, the maximum available power is strictly regulated by the least power which is available after the longest probable period of drought.

This minimum supply can only be satisfactorily ascertained by investigation of past records extending over many years. Where such records are not available, every attempt should be made by a close investigation of the rainfall records for the particular districts over a long period of years, and of the character, condition, and area of the gathering ground, to estimate the minimum supply likely to be available under the worst probable combination of circumstances, and the scheme may then, and not until then, be developed.

The method of utilizing the supply depends largely on its magnitude, form, and locality. Where, though comparatively small, it is continuous, the available horse-power may be largely increased by the formation of a storage reservoir capable of impounding at least a 24 hour's' inflow. By this means energy may be utilized for the eight hours or so comprising a working day at a rate greatly in excess of the mean rate of inflow.

Where the natural configuration of the country necessitates the power plant being placed at some distance from the storage reservoir, the supply is usually led though an open canal or ditch having a slight gradient, into a smaller storage reservoir termed the forebay, which is placed as near to the power plant as possible. From the forebay the supply is then taken to the prime mover by means of a closed pipe termed the penstock.

In the case of a water-wheel installation the penstock may consist of an open channel.

The supply of water to the prime mover is regulated by means of sluices or gates, which may either form an integral part of the machine, as in the case of most turbines, or may be fitted in the supply pipe or channel. 
After doing work, the water is rejected into a discharge channel termed the tail-race.

The most suitable type of prime mover for any particular case depends on-

(1) The quantity of water available.

(2) The supply head.

(3) The regularity of flow.

(4) The possibility of floods.

(5) The purpose for which power is required.

Those types in general use consist of-

(1) Water-wheels.

(2) Turbines.

(3) Piston engines.

Each has its own sphere of usefulness, and in determining the type to be adopted each installation demands special consideration, guided by the circumstances peculiar to the case.

In general, the water-wheel is only suitable for small powers and for comparatively low heads and where close speed regulation is not essential. Its efficiency is greatly affected by a variation in the supply and in the head or tail-race levels. It is of great size and weight in proportion to the power developed, and has a low rotative speed. On the other hand, its construction is simple, its repair inexpensive and easy, and the construction of the supply channel, tail-race, and housing in general inexpensive, while for heads of less than 1 foot it forms the only suitable type of motor.

For all heads above 1 foot, where large power is desired, one or other type of turbine becomes suitable, while in certain cases for comparatively small powers, and where a high head is available and a slow rate of rotation is required, the piston engine is most satisfactory.

In passing through a prime mover, water may do work either in virtue of its weight; by changing potential energy into useful work in virtue of its motion; by changing kinetic energy into work; by a combination of these processes; or in virtue of its pressure energy, which is simply potential energy in another form. 
In the overshot water-wheel, for example (Art. 110), rotation is produced almost entirely by the weight of the water; in impulse wheels deriving their motive force from the impact of a high velocity jet of water, work is done solely in virtue of the kinetic energy of the jet; in turbines of the reaction type the pressure energy of the water is partly changed into kinetic energy in the wheel itself, this being absorbed in producing rotation of the wheel; while in a piston engine the water does work in virtue of its pressure, its velocity being so small as to be negligible.

In designing any type of hydraulic prime mover, certain general principles should be borne in mind.

(a) All shock, whether of water on moving or stationary surfaces, or on water moving with a lower velocity, should be avoided as being productive of loss of energy in eddy formation.

This may be prevented by arranging that as far as possible any stream of water on meeting a solid surface is moving tangentially to the surface, and that passages conveying the working fluid are not subject to abrupt changes of sectional area or of form.

(b) Abrupt changes in the direction of motion are productive of eddy formation and should be avoided by designing passages and channels with as far as possible an uniform or gradually changing curvature.

(c) Frictional losses should be reduced to a minimum by reducing the area of the wetted surface to a minimum compatible with giving easy curves of flow, and by reducing the relative velocity of flow over such surfaces.

(d) As far as possible, the motive fluid should be rejected devoid of energy, and therefore moving with as low an absolute velocity as will suffice to carry it out of the motor.

The possibility of conforming to these general principles varies with the type of motor. In general, apart from mechanical friction, water-wheels suffer chiefly from the causes outlined in sections $(a),(b)$, and $(d)$; turbines from those in sections $(a),(c)$, and $(d)$; while losses due to shock and eddy production, $(a)$ and (b), are all-important in piston engines.

These types of prime mover will now be considered somewhat in detail. 
Art. 110.-The Overshot Water-Wheel (Fig. 149).

In this type of wheel water is supplied rear the highest point of the circumference to a series of buckets formed by vanes connected at each end to circular shrouds, the bottoms of the buckets being formed by the inner circumference of the wheel. For

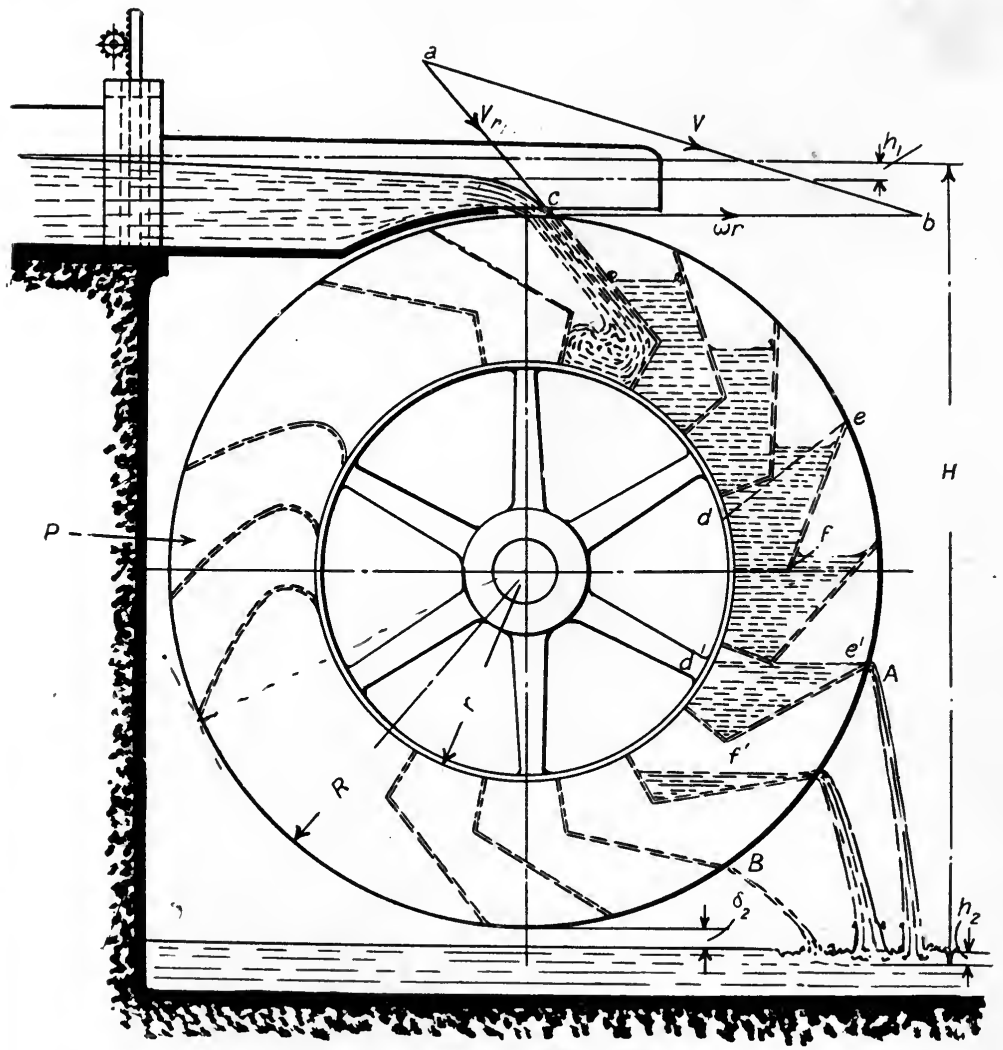

Fig. 149.-Overshot Water-Wheel.

convenience of construction the vanes are often made of wood and in two parts, the inner part being radial and the outer inclined to this at an angle depending on the speed of the wheel and the velocity of the supply stream.

A preferable form of bucket is that indicated at $P$ (Fig. 149). Here the vanes, usually of metal, are made in a single piece, and have a continuous curvature throughout. 
Theory of Action.-Let $H$ be the total head available. A certain proportion of this head must first be utilized in giving the supply water sufficient velocity to carry it into the wheel buckets. If $h_{1}$ is this head, the velocity will then be given by $v=\sqrt{2 g h_{1}}$.

A small clearance must be allowed between the highest point of the wheel and the bottom of the inlet channel, this clearance $\delta_{1}$ being usually about 1 inch.

A rather larger clearance $\delta_{2}$ must in general be allowed between the lowest point of the wheel and the level in the tail-race, so as to prevent submergence of the wheel buckets in time of flood.

In general $\delta_{2}$ is about 6 inches, but depends largely on the special circumstances of the plant.

Finally, a certain proportion $h_{2}$ of the head must be devoted to producing velocity of flow along the tail-race, so as to give a free discharge from the wheel.

The outer diameter of the wheel buckets is thus limited to $H-\left(h_{1}+h_{2}\right)-\left(\delta_{1}+\hat{o}_{2}\right)$.

Let $R=$ outer radius of wheel.

, $r=$ inner , ,

Then $2 R=H-\left(h_{1}+h_{2}\right)-\left(\delta_{1}+\delta_{2}\right)$.

The depth of buckets depends on the diameter, breadth and velocity of wheel, and on the quantity of water to be utilized.

Let $Q=$ quantity of water per second in cubic feet.

,$\omega=$ angular velocity of wheel in radians per second.

,$b=$ breadth of wheel,

and since the buckets are never completely filled with water, let $x=$ fraction of bucket volume occupied by water. Generally $x$ lies between $\frac{1}{3}$ and $\frac{2}{3}$.

Then we have, neglecting the volume occupied by the wheel vanes,

$\left.\begin{array}{c}\text { Bucket volume passing } \\ \text { inlet per second }\end{array}\right\}=\pi\left(R^{2}-r^{2}\right) \times \frac{\omega}{2 \pi} \times b$ cubic feet.

$\therefore \quad Q=\frac{x\left(R^{2}-r^{2}\right) \leftrightarrow b}{2}$ cubic feet per second.

$$
\therefore \quad r=\sqrt{R^{2}-\frac{2()}{\omega b x}} \text {. }
$$

giving $r$, and therefore $R-r$, the depth of the buckets. 
To avoid excessive loss under low heads this should be as small as practicable.

Efficiency of Wheel.-Soon after the buckets pass the centre line of the wheel they begin to empty, at $A$ (Fig. 149), while they are completely emptied by the time they reach the position $B$, where the outer part of the bucket is horizontal. If then $h$ is the mean vertical distance through which the water is carried before being discharged, the work done in virtue of its weight $=W Q h$ foot lbs. per second.

If $v=$ velocity of supply stream, and if $a=$ angle which this stream makes with the tangent to the wheel at the point of impact, the initial relative velocity of stream and $=v \cos a-\omega R$. bucket in the direction of motion of the bucket

The final relative velocity after impact is zero.

$\therefore$ Change of momentum?

in this direction per sec.)

$=\frac{W Q}{g}\{r \cos a-\omega R\}$

$\therefore$ Work done on wheel )

$\left.\begin{array}{c}\text { per sec. in virtue of this } \\ \text { change of momentum }\end{array}\right\}=\frac{W Q}{g}\{r \cos a-\omega R\} \omega R$ ft. lbs. The total work done

$\left.\begin{array}{c}\text { on the wheel per sec. } \\ \text { is then given by }\end{array}\right\} W Q !(r \cos a-\omega R) \frac{\omega R}{g}+h ! \mathrm{ft}$. lbs.

$$
\therefore \quad \text { Efficiency }=\frac{\frac{(c \cos a-\omega R) \omega R}{!}+h}{H} \text {. }
$$

Since $h$ is approximately independent of the speed at low speeds, the efficiency is a maximum when $(v \cos \alpha-\omega R) \omega R$ is a maximum, i.e., when $v \cos a=2 \omega r$, or since $a$ is usually small, when $r=2 \omega r$, or when the peripheral velocity is one half that of the inflowing stream. The momentum term in the above expression is, however, usually so small compared with the term $W Q h$, that a considerable increase in speed has very little effect on the efficiency. The most important effect of such an increase is due to the tendency to a premature emptying of the buckets by increased centrifugal action.

To prevent loss by splash and shock at entrance to the buckets, the vane angles should be so arranged that the relative motion of water and vane at entrance is parallel to the tip of the vane. 
Thus if $a b$ (Fig. 149) represents the velocity $v$ in direction and magnitude, and if $c b$ represents the linear velocity, $\omega R$ of the vane, then $a c$ represents the velocity of the water relative to the vane, and for the water to enter by sliding along the vane this should be parallel to $a c$ at entrance.

In addition to those already considered, further losses occur in the wheel owing to

(1) Rejection of water before reaching the bottom of the wheel.

(2) Rejection of water while possessing kinetic energy.

(3) Frictional losses in wheel.

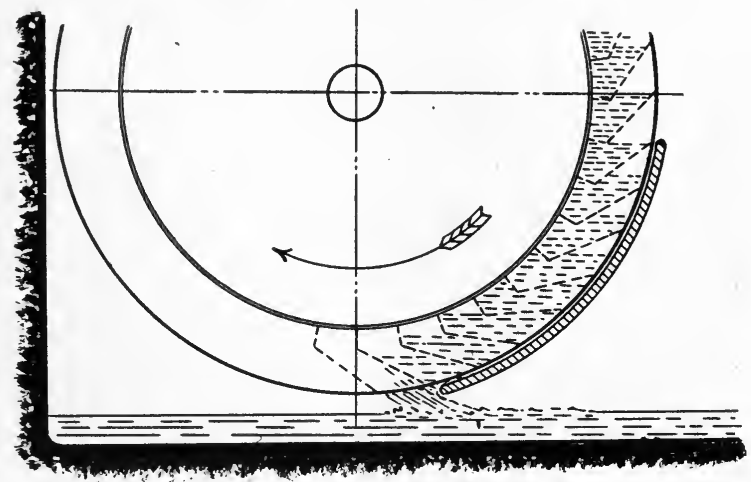

FIG. 150.

(1) This loss depends largely on the form of the buckets.

The general practice is to form the bucket tips so as to make an angle of $25^{\circ}$ to $30^{\circ}$ with the tangent to the circumference at the tip. This gives a bucket which retains the water for a vertical distance equal to about 8 of the wheel diameter.

If this angle be $\beta$, the bucket is not completely emptied until the wheel has turned through $180^{\circ}-\beta$.

Having drawn in the profile of the buckets, if a straight line d $e$ (Fig. 149) be drawn through the bucket tip so as to enclose an area $d$ e $f=(x \times$ bucket area $)$, the water will begin to escape from the bucket when $e d$ becomes horizontal. When $f e$ becomes horizontal the bucket is completely emptied. Thus with radial vanes the water is entirely emptied from a bucket by the time it has fallen to the level of the wheel axle. 
Water may be retained in the buckets until these are near the lowest point of the wheel, by enclosing the discharge side of the wheel below $A$ in a closely fitting shrouding, with as small a clearance as possible (Fig. 150), and by this means the efficiency may be increased. This method has the further advantage that the velocity of discharge is now increased by the head of water

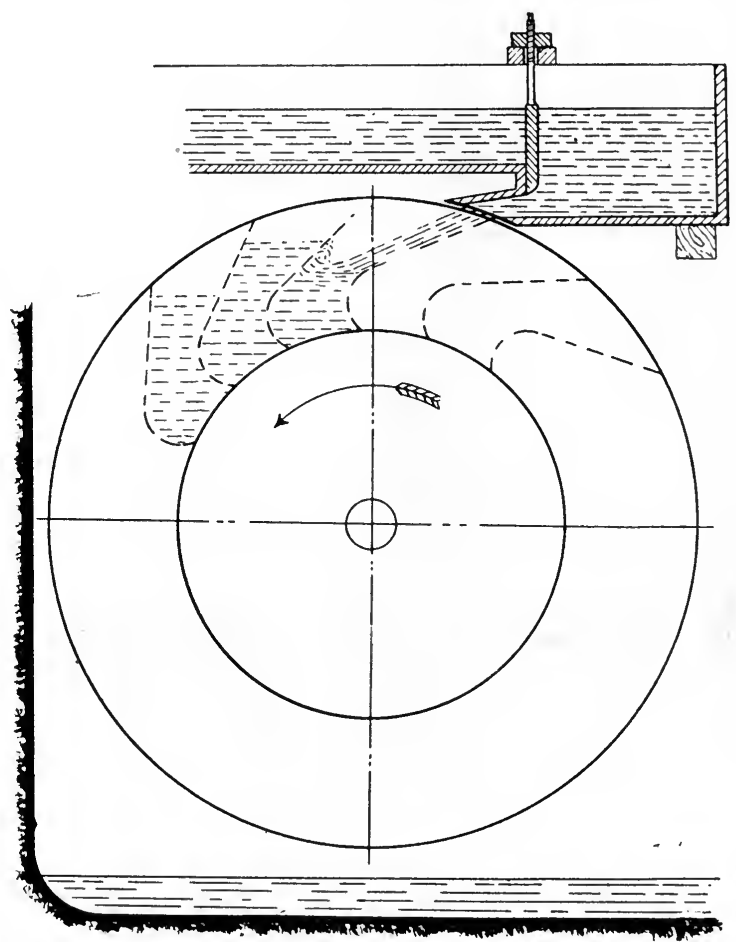

FIG. 151.-Overshot Wheel with Reversed Inflow.

in the buckets, and as the direction of this motion is opposite to that of the wheel, the absolute velocity of discharge, and therefore the kinetic energy rejected, is reduced.

(2) In the ordinary type of wheel the water when rejected has the velocity of the wheel rim and hence carries away an amount of energy per pound, given by $\frac{\omega^{2} R^{2}}{2 g} \mathrm{ft}$. lbs.

(3) The hydraulic frictional losses in the wheel are unimportant 
because of the low relative velocity of water and buckets. Where a shrouding is used to confine the water on the discharge side the additional frictional resistance largely counterbalances the additional work done by the water.

Effect of Varying the Tail-Race Level.-If the tail-race level rises so that the wheel is partially submerged, considerable resistance to rotation is caused if the vanes move in the opposite direction to that of flow in the tail-race. By reversing the direction of inflow, however (Fig. 151), the direction of the tail-race flow

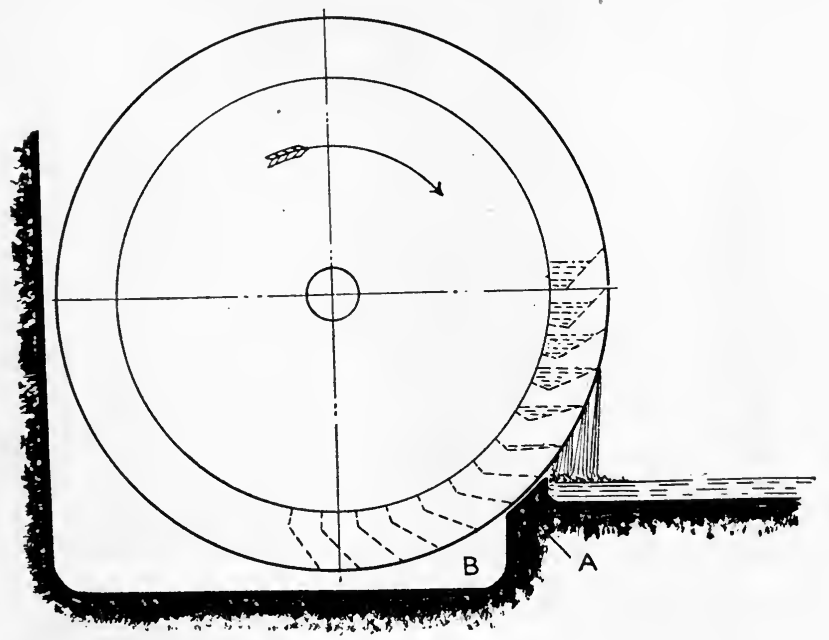

FIG. 152.

becomes the same as that of the buckets, and these may now become partially submerged without serious loss of efficiency.

Further advantage may be taken of the fall by arranging a masonry breastwork $A$ (Fig. 152) to closely fit the wheel, the tail-race level being now slightly below the level at which each bucket finally empties itself. The compartment $B$ is kept dry either by draining into a sump where practicable, or by means of a small pump driven by the wheel.

Where the supply to the wheel is delivered by means of an open channel discharging directly on to the wheel, if $h_{1}$ is the head producing flow, and if $b$ is the breadth of the channel, we have 


$$
Q=\frac{2}{3} C b \sqrt{2 g} \cdot h_{1}^{\frac{3}{2}}
$$

where $h_{1}$ and $b$ are measured in feet.

Also, since the area of the stream at discharge is given by $C l h_{1}$, we have, if $r$ be the mean velocity on entering the wheel,

$$
\begin{aligned}
Q & =C b h_{1} v \\
\therefore \quad v & =\frac{2}{3} \sqrt{2 g h_{1} .}
\end{aligned}
$$

If $\delta_{1}$ is the vertical clearance between channel and wheel, the velocity is increased by falling through this space, and now becomes $\bar{r}$ where

$$
\begin{aligned}
\bar{v} & =\sqrt{\frac{4}{9} \times 2 g h_{1}+2 g \delta_{1}} \\
& =\sqrt{2 g\left\{\frac{4}{9} h_{1}+\delta_{1}\right\}}
\end{aligned}
$$

the angle $a$, if the supply channel is horizontal, being given by

$$
\tan a=\frac{\sqrt{2 g \delta_{1}}}{\frac{2}{3} \sqrt{2 g h_{1}}}=\frac{3 \sqrt{\delta_{1}}}{2 \sqrt{h_{1}}} .
$$

The thickness of the stream $=C h_{1}=62 h_{1}$ (approximately) and to prevent this becoming excessive $h_{1}$ should not exceed 1 foot. For falls between 15 and 40 feet $h_{1}$ may be taken at $\frac{H}{40}$. Obviously this gives a very low value to $i$. For example, where $k_{1}=1$ foot and $\delta_{1}=\frac{1}{12}$ foot, $v=5.80$ feet per second.

For maximum efficiency, then, the peripheral speed of the rim should be 2.90 f.s., and with a slowly running wheel of this type efficiencies as high as 80 per cent. may be obtained.

To avoid running at such inconveniently low speeds either of two expedients may be adopted. The first is to run the wheel at a speed from 50 to 100 per cent. in excess of that giving maximum efficiency. To avoid shock at this speed the bucket tips would need to be approximately radial, but this would give a bucket which would empty itself before reaching the level of the axle. In practice, then, the loss due to shock is considered unavoidable, the vane angles are made about $25^{\circ}$, and since velocities are small, the amount of energy wasted in this way is not considerable. 
The second expedient consists in giving $h_{1}$ a larger value, and in taking the supply by means of a closed guide passage of suitable depth out of the bottom of an open forebay (Fig. 153).

The velocity of efflux is then increased, and the velocity of rotation may be increased proportionately. The diameter of the wheel is reduced, and also its width for a given volume of water. A cheaper wheel, and one rotating at a more convenient speed for transmission purposes is thus obtained. The efficiency is,

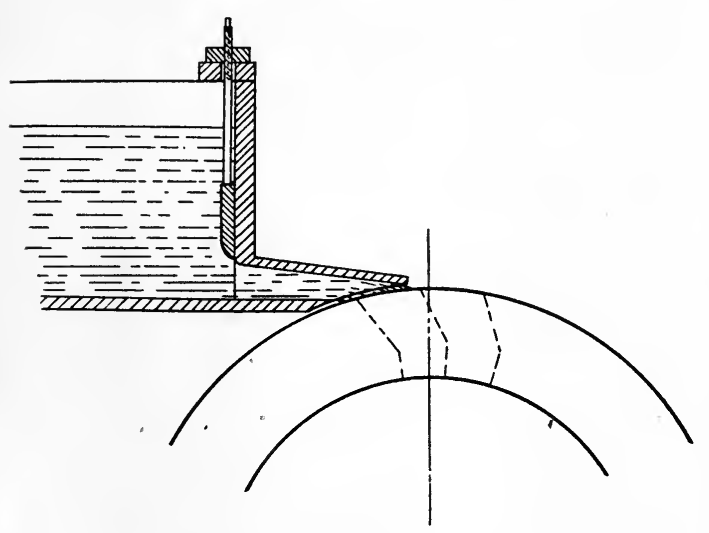

FIg. 153. however, slightly reduced, since losses due to shock and friction are increased by the higher velocities, as are the losses due to rejection of kinetic energy in the tail water, while in addition, the increased centrifugal action tends to empty the

buckets sooner. The latter action, however, while reducing the efficiency of the wheel, tends to make it, to a certain extent, selfgoverning under variable loads, since a diminution in load, followed by an increase in speed, tends to empty the buckets.

The following results of experiments by Smeaton indicate to what extent the efficiency is lowered by increasing the proportion of the total head absorbed in giving velocity energy to the supply water.

\begin{tabular}{c|c|c}
\hline \begin{tabular}{c|c} 
Diameter of wheel \\
Total Head $H$.
\end{tabular} & $\begin{array}{c}\text { Proportion of Total Head absorbed in } \\
\text { giving Velocity to Supply Water. }\end{array}$ & Efficiency. \\
\hline$\cdot 90$ & $\cdot 10$ & $\cdot 73$ \\
$\cdot 84$ & $\cdot 16$ & $\cdot 69$ \\
$\cdot 80$ & $\cdot 20$ & $\cdot 66$ \\
$\cdot 73$ & $\cdot 27$ & $\cdot 62$ \\
$\cdot 68$ & $\cdot 32$ & $\cdot 59$ \\
\hline
\end{tabular}


Regulation is usually performed by a sluice governing the discharge from the penstock.

The overshot wheel is well suited for small powers and heads ranging from about 15 to 50 feet, and when working under suitable conditions gives efficiencies up to about 80 per cent. As the head diminishes, the larger proportional loss of head necessitated by the depth of the buckets and by the clearances $\delta_{1}$ and $\delta_{2}$ renders the wheel less efficient, and for heads between 15 feet and 6 feet the breast wheel becomes more suitable.

\section{Art. 111. - The Breast Wheel (Fig. 154).}

Here the wheel itself is almost identical with the overshot. The principles of its construction and of the design of its buckets

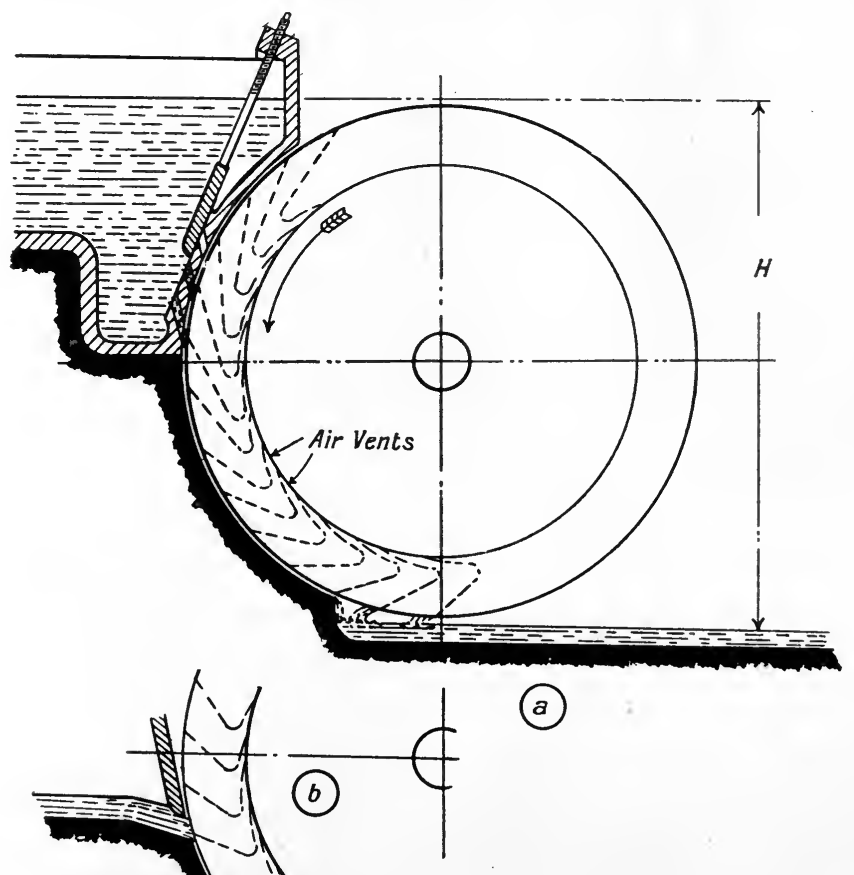

Fig. 154.-Breast Wheel.

are the same, but water is now admitted to the buckets at some point in the breast of the wheel.

H.A. 
As before, the supply may be brought to the wheel either in an open supply channel under a comparatively low head not exceeding 1 foot, in which case the supply is led on below the wheel centre, or by means of a closed supply pipe under a greater head, when the supply is led on above the centre. The general arrangement in each case is indicated in Fig. $154 a$ and $b$. The water is prevented from escaping from the buckets before reaching the bottom of the wheel by means of a breastwork of masonry, the clearance between the wheel and masonry being reduced to the minimum possible, usually about $\frac{3}{16} \mathrm{inch}$. The necessity for this breast-work renders the wheel more expensive than the overshot. Regulation is performed by throttling the supply by means of sluices arranged as indicated in Figs. $154 a$ and $b$.

As in the case of the overshot wheel, the maximum efficiency is obtained when the bucket angles are arranged so as to give entry without shock and when the peripheral speed of the wheel is one half $v \cos a$. Here $a$ is the angle which the direction of the inflowing stream makes with the tangent to the wheel at the point of entry.

The efficiency is then given by $\frac{\frac{(v \cos a-\omega R) \omega R}{g}+h}{H}$ where $H$ is the total fall.

, $\quad h$ is the distance through which the water falls while doing work on the wheel.

-For maximum efficiency the level in the tail-race should be the same as that in the buckets at discharge. In this type of wheel, on account of the manner of filling the buckets, special provision must be made for letting air out as the water rushes in. To this end air vents are usually formed in the inner circumference of the buckets. The wheel is capable of more accurate speed regulation under varying heads than is the overshot, and its efficiency under favourable circumstances may be as high as 65 per cent.

\section{Art. 112.-The Side Wheel.}

Where the fall is between 3 feet and 6 feet, the breast wheel becomes unsuitable because of the smallness of its diameter, 
and in such a case the Sagebien wheel may be adopted. This wheel (Fig. 155) has buckets formed by a series of flat vanes, which are tangential to a circle concentric with the wheel itself. The buckets are open top and bottom, and are of comparatively great depth. The water enters the buckets with a velocity sensibly the same as that in the approach channel, the vane angles being determined as indicated in the figure, so that the vanes enter the water without shock.

A circular casing is provided in which the wheel works with little clearance, and which connects the head and tailrace. Then, neglecting leakage between this easing and the

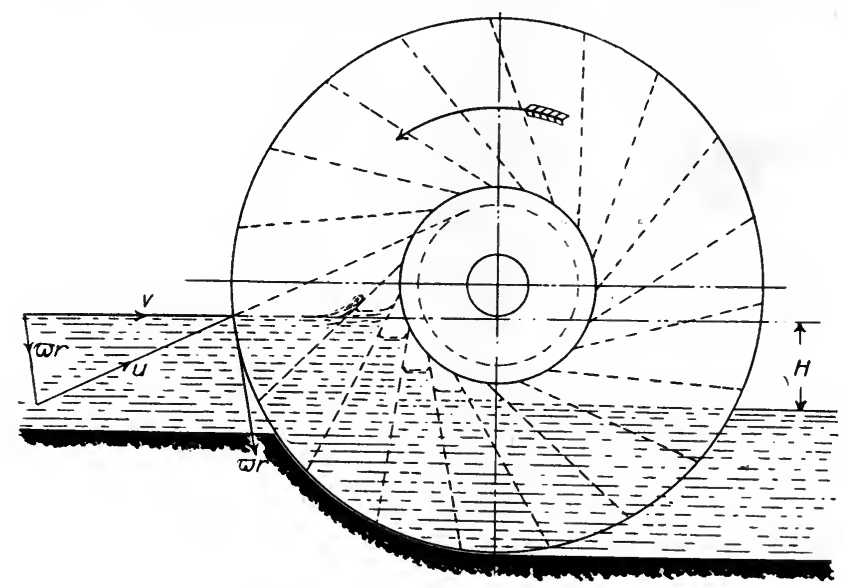

Fig. 155.-Sagebien Wheel.

vanes, each bucket retains its supply until it passes the lowest point of the wheel, after which communication is made with the tail-race, and the level in the bucket falls to that of the tail-race water. For maximum efficiency the wheel should be designed so that the level in the bucket on reaching the bottom of the wheel is the same as that in the tail-race.

If $u$ represents the relative velocity of water and bucket at entrance, the water will rise initially to a height in the bucket above that in the head-race, this height being given by $\frac{u^{2}}{2 g}$, and the depth of bucket should be such that in time of flood this does not cause flow to take place over the inner end of the buckets. 
In this wheel the velocity of rotation is proportional to the flow, and the wheel is thus capable of dealing with large quantities of water. It is, however, unfitted for driving a variable load, since an increase in load, by causing a diminution in speed, reduces the flow through the wheel, and thus reduces the energy supply when it is most needed. Its peripheral velocity is low, and on that account it is well adapted for driving a pumping plant or milling machinery where the load is uniform and where high velocities are not required.

The chief losses occur during the emergence of the vanes from the tail-race. Owing, however, to the slow speed of the wheel, all hydraulic losses are low and an efficiency of up to 80 per cent. may be obtained under favourable circumstances, though the large size of the wheel and its comparatively costly construction very largely counterbalance the advantages of high efficiency.

\section{Art. 113.-The Undershot Wheel.}

For heads below 3 feet the undershot wheel is preferable. In its simplest form this consists of a wheel carrying a series of flat
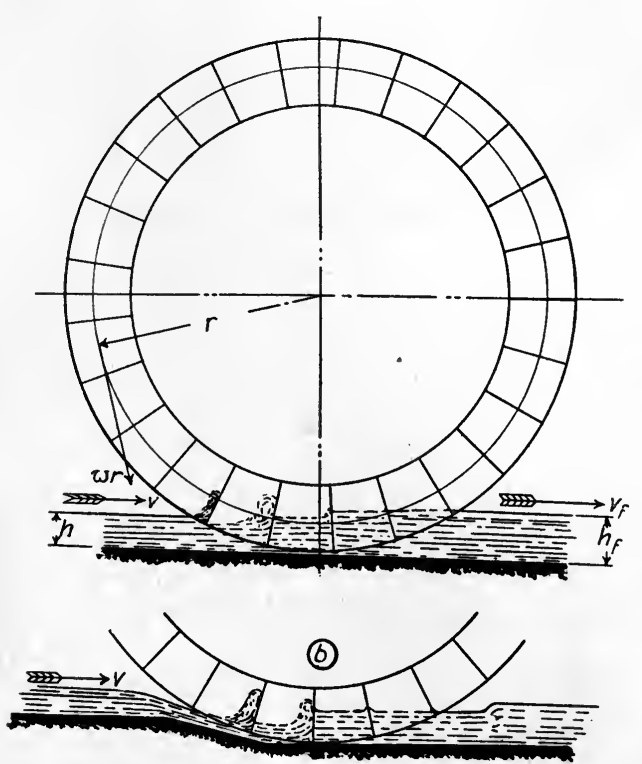

FIG. 156.-The Undershot Wheel. radial vanes around its circumference (Fig. 156). These dip into the water flowing either through an open channel or through a penstock of slightly greater width than the wheel, and are arranged so as to clear the bottom of the penstock by about $\frac{1}{4}$ inch. In some cases the penstock itself is curved to fit the wheel, and leakage past the buckets, in the interval between successive buckets 
arriving at the lowest point of their path, is then largely prevented.

In this wheel, work is done solely in virtue of the kinetic energy of the moving stream, the force on the moving vanes being due to the change of momentum produced in the stream by their presence.

Let $a=$ inclination to horizontal of stream when meeting the wheel.

,$\quad \beta=$ inclination of stream when leaving wheel.

,$r=$ initial velocity of stream.

,$v_{f}=$ final velocity on leaving wheel.

,$\quad \omega=$ angular velocity of wheel.

,$r=$ mean radius of wheel vanes.

,,$\quad b=$ breadth of wheel vanes.

,$h=$ initial depth of stream on meeting the wheel.

, $h_{f}=$ final $, \quad, \quad$,, leaving wheel.

Then the change of momentum of the stream per second in its initial direction of motion $=\frac{W Q}{g}\left\{v_{f} \cos (a-\beta)-v\right\}$

$\therefore \quad$ Force in the opposite direction to produce this

$$
=-\frac{W Q}{g}\left\{v_{f} \cos (\alpha-\beta)-v\right\} \text { lbs. }
$$

If, as is usual, $a=\beta$, this becomes :-

$$
\frac{W Q}{g}\left\{x-r_{f}\right\} \text { lbs. }
$$

This force is produced mainly by the reaction of the wheel vanes, but partly by an increase in statical pressure over the section of the stream produced by an increase in depth from $h$ to $h_{f}$, in passing through the wheel.

This increase in depth can only take place in a confined channel, where the final velocity of the water is the same as that of the wheel vanes, and in such a case we have, for continuity of flow, $b v h=b v_{f} h_{f}=Q$.

$$
\therefore \quad h_{f}=\frac{h v}{v_{f}} \text {. }
$$


If the effect of this increase in depth be neglected, we have

Work done on vanes $=\frac{W Q}{g}\left\{v-v_{f}\right\} \omega r$ ft. lbs. per sec., or, writing $o r=v_{f}$ (with radial vaneṣ)

$$
U=\frac{W Q}{g}\left\{v-v_{f}\right\} v_{f} \text { ft. lbs. per sec. }
$$

For a maximum value of $U$, $r_{f}$ must equal $5 r$, or the peripheral speed of the wheel should equal one half that of the oncoming stream.

The maximum efficiency is then given by

$$
\begin{gathered}
\eta=\frac{\left(v-v_{f}\right) v_{f}}{\frac{v^{2}}{2}}, \text { where } v_{f}=\cdot 5 v \\
\therefore \quad \eta_{(m(x)}=\cdot 5 .
\end{gathered}
$$

Owing to mechanical friction and hydraulic losses this efficiency is, however, reduced to about 35 per cent., and the maximum efficiency is obtained with a value of $v_{f}$, slightly less than $\frac{1}{2} r$.

These hydraulic losses are :-

(1) Loss due to shock because of incorrect vane angles at entrance (proportional to the square of the relative velocity at impact, and comparatively large).

(2) Frictional losses in wheel (small, because of small relative velocities - proportional to the mean square of relative velocities).

(3) Loss of head due to eddy production at enlargement- of section of stream. This is given approximately by $\frac{W Q}{2 g}\left(v-v_{f}\right)^{2}$ foot lbs. per second.

(4) Loss by leakage between buckets and wheel-race (proportional to $\sqrt{ } \bar{h}$.

(5) Kinetic energy rejected in tail-race. This equals $\frac{W Q}{2 g} \cdot v_{f}^{2}$ foot lbs. per second.

The following are results of tests made by L'Abbe Bossut and by Smeaton, on water wheels of this type. ${ }^{1}$

(a) Wheel working in open canal, 12 feet wide and 8 inches deep. Width of wheel 5 inches; diameter of wheel $3^{\prime} 6^{\prime \prime}$; depth

1 "Tracts on Hydraulics," Tredgold, London, 1826. 
of immersion of vanes $=4^{\prime \prime} ; v=5.71$ feet per second $; \frac{v_{f}}{v}$ (for maximum efficiency) $=\cdot 435$.

On varying the number of floats in this wheel it was found that the efficiency was the same with twenty-four as with forty-eight floats. On reducing the number to twelve, however, the efficiency was considerably reduced.

(b) When working in a closed channel with small clearance, the above wheel gave the best results with not less than forty-eight floats. In this series of experiments $v=9 \cdot 1$ foot per second and $\frac{v_{f}}{v}$ (for maximum efficiency) $=\cdot 41$.

(c) In a third series of experiments on a second wheel working in a closed channel, the following results are recorded :-

\begin{tabular}{|c|c|c|}
\hline Head. & $\frac{v_{f}}{v}$ & Efficiency. \\
\hline $\begin{array}{l}h_{1} \\
, " \\
,\end{array}$ & $\begin{array}{l}\cdot 34 \\
\cdot 355 \\
\cdot 377 \\
\cdot 380\end{array}$ & $\begin{array}{l}\cdot 324 \\
\cdot 312 \\
\cdot 280 \\
\cdot 282\end{array}$ \\
\hline $\begin{array}{l}h_{2} \\
" \\
"\end{array}$ & $\begin{array}{l}\cdot 366 \\
\cdot 397 \\
\cdot 455\end{array}$ & $\begin{array}{l}\cdot 307 \\
\cdot 294 \\
\cdot 298\end{array}$ \\
\hline $\begin{array}{l}h_{3} \\
"\end{array}$ & $\begin{array}{l}\cdot 405 \\
\cdot 490\end{array}$ & $\begin{array}{l}\cdot 305 \\
\cdot 299\end{array}$ \\
\hline$h_{4}$ & $\cdot 49$ & $\cdot 292$ \\
\hline$h_{5}$ & $·_{52}$ & $\cdot 295$ \\
\hline
\end{tabular}

The heads $h_{1}, h_{2}$, etc., are unfortunately not recorded.

The effective head on such a wheel working in a closed channel may be slightly increased by a device which was probably first described by Venturi. Here a sudden drop is given to the bed of the channel immediately before the wheel, and the channel 
is continued at this level for some distance past the wheel (Fig. $156 b$ ).

Owing to this drop, a standing wave (Art. 86) is produced immediately the wheel is passed, the level of the crest of this wave marking that of the tail-race. The effective head is then increased by the height of this wave. This device has the further advantage that the effect of a flood in raising the tail-race level is rendered much less serious.

\section{Art. 114.-The Poncelet Wheel.}

If the vanes of an undershot wheel, instead of being radial are inclined backward so as to make an angle $\beta$ at the tips with the

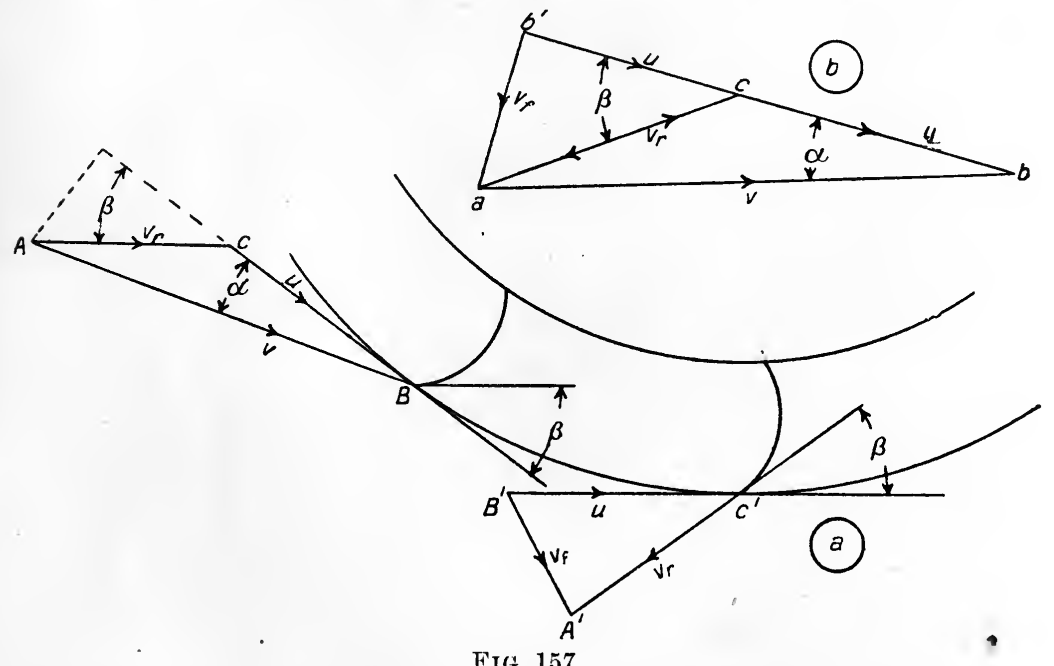

FIG. 157.

tangent to the circumference, and if $a$ be the angle between the direction of the approach stream and this tangent, then by suitable adjustment of $a, \beta$, and the speed of rotation, the loss due to shock at entrance may be prevented, and at the same time the discharge water may be given a backward velocity relative to the wheel, thus reducing the absolute velocity of discharge and the loss by rejection of kinetic energy to the tail-race. .

With flat vanes the best results are obtained when $\beta$ is about $30^{\circ}$, and efficiencies of up to 55 per cent. may then be obtained. 
In the Poncelet wheel (Fig. 158) these vanes, instead of being flat, form ares of circles, and with this type of wheel efficiencies of from 60 to 70 per cent. are usual.

To determine the correct vane angles, let

$A B$ (Fig. $157 a$ ) represent the absolute velocity of water at entrance.

$C B$ represent the absolute velocity of vanes.

Then $A C$ represents the velocity of water relative to vanes, and to avoid shock, the vane tips should be parallel to $A C$.

Again, at the discharge point, let

$B^{\prime} C^{\prime}=$ velocity of vanes.

$C^{\prime} A^{\prime}=$ velocity of water relative to the vanes.

Then $B^{\prime} A^{\prime}=$ absolute velocity of discharge.

Let $r=$ initial velocity of water.

$" v_{f}=$ final $, ", \quad$,

$" v_{r}=$ relative $, \quad, \quad,$, and wheel.

,$u=$ velocity of buckets.

On entering the buckets the relative velocity is $v_{r}$, and in virtue of this the water will rise to a height approximately equal to $\frac{v_{r}{ }^{2}}{2 g}$ feet above its normal level.

It then falls through this height relative to the wheel under the action of gravity, so that on leaving the wheel its relative velocity will again be approximately equal to $v_{r}$. This assumption neglects the effect of friction and of eddy formation in the buckets, both of which tend to make the final less than the initial relative velocity. Assuming, however, for simplicity that $v_{r}$ is the same at inlet and at discharge, we have

$$
\begin{aligned}
& A C=C^{\prime} A^{\prime}=v_{r} \\
& C B=B^{\prime} C^{\prime}=u .
\end{aligned}
$$

For $r$, to be as small as possible with a given value of $v_{r}$, evidently $A^{\prime} B^{\prime}$ should be perpendicular to $B^{\prime} C^{\prime}$.

The two diagrams may now be combined so as to give graphically the most suitable angles $a$ and $\beta$ by making $A^{\prime} C^{\prime}$ coincide with $A C$.

Thus, draw $c b$ (Fig. 157 $b)=u$ and produce $b c$ to $b^{\prime}$, making $b^{\prime} c=c b$. From $b^{\prime}$ draw a perpendicular to $b b^{\prime}$, and with $b$ as 
centre describe an arc of a circle with $v$ as radius to cut this at $a$. Join $a c$. Then $a c=v_{r} ; \widehat{a b c}=a ; \widehat{a c b^{\prime}}=\beta$.

From the figure it is evident that for minimum loss of kinetic energy at discharge we must have $v \cos a=2 u$.

$$
\therefore u=\frac{v}{2} \cdot \cos a \text {. }
$$

In practice $a$ is usually made about $15^{\circ}$.

$$
\therefore \quad \cos a=\cdot 966 . \quad \therefore \quad u=\cdot 488 v .
$$

The theoretical'efficiency is given by

$$
\begin{gathered}
\eta=\frac{\frac{W Q}{g}\left\{r^{2}-r_{f}^{2}\right\}}{\frac{W Q}{g}\left\{r^{2}\right\}} \\
\therefore \quad \eta=\frac{v^{2}-r_{f}^{2}}{v^{2}}
\end{gathered}
$$

But from the figure we have

$$
\begin{aligned}
v^{2}-v_{f}^{2} & =4 u^{2} \\
\therefore \quad \eta & =\frac{4 u^{2}}{\iota^{2}}=\cos ^{2} a
\end{aligned}
$$

$\therefore$ With $a=15^{\circ}$, the maximum theoretical efficiency is 93 .

Again (Fig. 157), we have

$$
\begin{aligned}
& u \sin \beta=v \sin (\beta-\alpha) \\
& \therefore \quad \frac{v}{2} \cos a \sin \beta=v \sin (\beta-\alpha) \\
& \therefore \quad \cot \beta \tan a=\frac{1}{2} \\
& \therefore \quad \tan \beta=2 \tan \alpha \\
& \alpha=15^{\circ}, \tan \beta= 5358 \\
& \therefore \quad \beta=28 \cdot 2^{\circ} .
\end{aligned}
$$

If

Actually, because of hydraulic losses caused by relative motion of the mass of water in the buckets, and which are proportional to $v_{r}^{2}$, it is found advisable to slightly diminish the relative velocity by giving the vanes a velocity varying from 5 to $6 x$. Under these circumstances the maximum working efficiency is obtained.

The construction of the wheel is substantially as shown (Fig. 158). The buckets are open both at their inner and outer circumferences, and to prevent water at impact from flowing 
into the inner part of the wheel, the depth of buckets should be not less than $h+\frac{v_{r}{ }^{2}}{2 g}$, where $h=$ thickness of stream. In practice $h$ should not exceed 9 inches.

But

$$
v_{r}=\frac{u \sin a}{\sin (\beta-\alpha)}
$$

$\therefore$ taking $a=15^{\circ}, \beta=30^{\circ}$ (approx.), we have $v_{r}=u=\cdot 55 v$ (approx.)

$\therefore$ taking $r^{2}=2 y H$, where $H$ is the supply head, we have

$$
\begin{aligned}
\text { Depth of buckets } & =\cdot 3 \frac{v_{r}^{2}}{2 g}+h \\
& =\cdot 3 H+h .
\end{aligned}
$$

In practice the depth is usually taken as $\frac{H}{3}+h$.

The spacing varies so as to give about forty-eight buckets in the circumference, the maximum spacing being about 16 inches. The most suitable arc of water contact is about $30^{\circ}$. Since at all points of this arc the direction of the approach stream should make an angle $\beta$ with the tangent to the circumference, the approach channel is not straight but has a bed curved so as to fulfil this condition.

To draw this curve, let $K$ and $M$ (Fig. 158) be the extremities of the are of contact, and from $K$ and $M$ draw $K O$ and $M O$ perpendicular to the required directions of the stream, i.e., making an angle $a$ with the normals at $K$ and $M$, and intersecting in $O$. Then an approach bed, having as profile the arc described with centre $O$, and passing through $M$, will give the required inclination at $K$ and $M$, and approximately for all points between.

Regulation is performed by an adjustable sluice $S$, whose position is regulated by some form of centrifugal or float governor. In order to prevent loss by leakage past the vane tips the supply bed is extended to form a circular lip closely fitting the wheel. This should be no longer than is necessary to give contact with two vanes at once. If made too long, the water on flowing down the vanes, instead of freely escaping with relative velocity $v_{r}$, meets this lip, its relative velocity is destroyed, 
and on emerging from the lip it escapes with the forward velocity of the vane as in the ordinary type of undershot wheel.

In common with all impulse wheels, for efficient working the vanes must not be submerged, and the level of the supply bed must be sufficiently above that of the tail-race to obviate any flooding. 'This wheel is well adapted for heads between 4 and 7 feet. Its speed is fairly high, and the consequent fly-wheel effect renders it easy of regulation under variable loads.

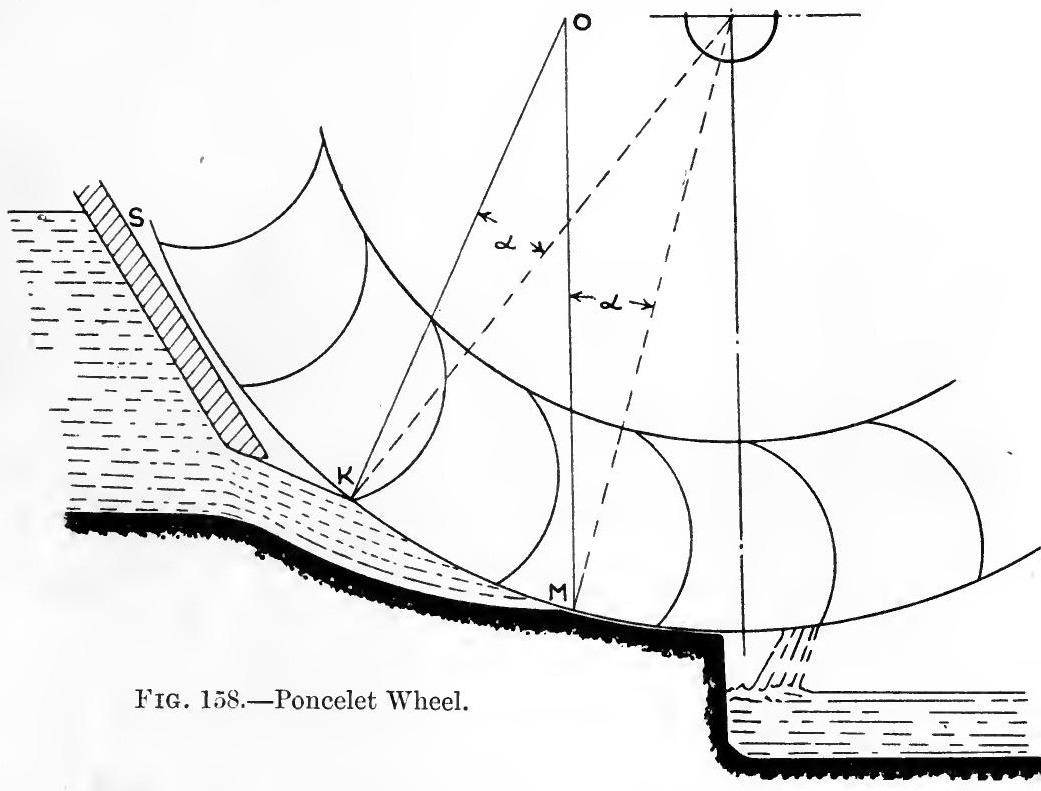

Its part-gate efficiency is high if the head be kept fairly constant, but with very variable heads, owing to the variation in velocity of approach, its efficiency is not so good.

\section{Art. 115.-The Pelton Wheel.}

The Pelton wheel (Fig. 159 ${ }^{1}$ ) is the only form of water-wheel which is adapted for use with high heads, and where a limited supply of water under such a head is available it often forms the most suitable type of prime mover. In such a case the turbine 
proper, with the exception of the Girard type (Art. 118), is unsuitable, as will be seen later (Art. 117), while where the supply water is charged with sand or similar matter in suspension, as is not unusual, the Pelton wheel, on account of the simplicity of its construction and of the ease with which its buckets can be renewed, has manifest advantages over the Girard turbine. The pressure supply is now led through a pipe line terminating in one or more nozzles which play on to a series of buckets fixed around the periphery of the wheel.

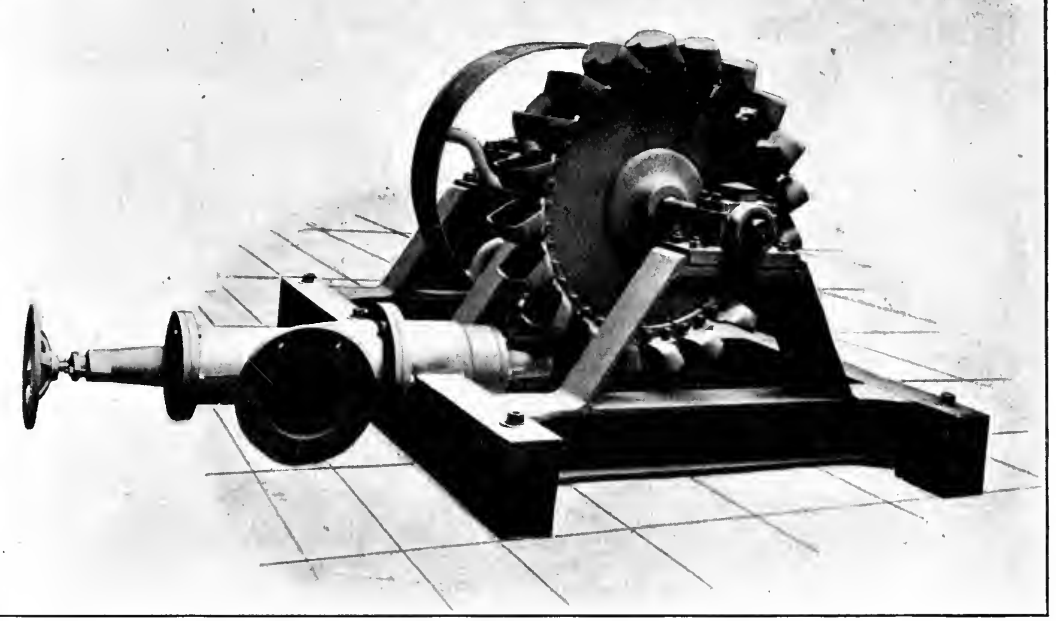

FIG. 159.- - Pelton Wheel.

The latter is a development of the old hurdy-gurdy of the Pacific Slope. This consisted of a wheel having a rim to which a series of flat plates were fixed radially, the jets from one or more nozzles impinging freely on these and causing rotation. Under these conditions, the theoretical efficiency cannot exceed 50 per cent. (p. 351), while in practice loss by splashing, friction, etc., reduces this to about 30 per cent.

The first important step towards the improvement of the machine was made about 1870, when the flat plates were replaced by hemispherical cups, fixed alternately on each side of the centre line of the wheel with their concave 
sides to the jet. This was known as the Cascade wheel (Fig. 160). By this means the jet was deflected backwards, and the theoretical possibilities of the wheel were at once doubled, the conditions now approximating to those of p. 351,

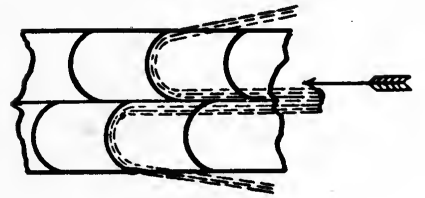

FIG. 160. and the maximum theoretical efficiency becoming unity. The next step was to replace these cups by a series of concave buckets mounted on the centre line of the wheel, fitted with knife-edged ridges to split the jet, and having surfaces curved so as to give the jet its backward deflection as smoothly and uniformly as possible. Several types of bucket have been designed with this end in
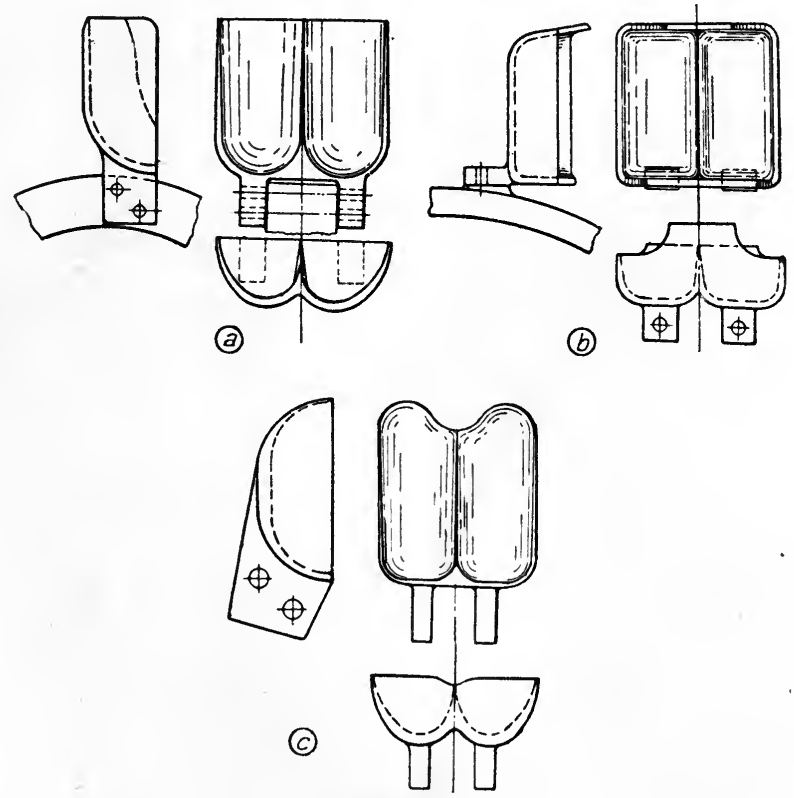

FIG. 161.

view and some of the more successful are illustrated in Fig. 161 $(a, b$, and $c)$.

In practice an efficiency of unity is impossible of attainment for several reasons.

(1) In order that the discharge from one shall clear the back 
of the following bucket, the jet cannot be deflected through the full $180^{\circ}$, the actual deflection usually being $160^{\circ}$. Thus kinetic energy is rejected in virtue of the motion of the water parallel to the axis of the wheel at discharge. To prevent this loss becoming large, the buckets should not be spaced too closely together.

(2) The relative velocity of water and bucket at discharge is less than at the point of impact because of skin friction, while windage causes the actual velocity of impact to be less than that theoretically equivalent to the head at the nozzle. Both these causes have the effect of reducing the pressure on the bucket, and to obviate this loss as far as possible the wetted surface of the buckets should be a minimum, and therefore the number of buckets should be as small as is consistent with continuous impact, while they should be made no larger than is necessary to give the required change of direction with easy curves and without shock. Also the surface should be as smooth and well finished as possible.

'To reduce windage the jet should be circular in section, since this gives the minimum perimeter per unit area of cross section, while it has the further advantage of being the most stable form of jet. Other forms ultimately tend to the circular, and in doing. so tend to become unsteady.

(3) Sharp corners and uneven curves in the buckets cause loss of energy by eddy formation.

(4) Splash on entering buckets, if unsuitably designed (reduced by reducing the number of buckets).

(5) Loss of energy due to impact on dead water in bucket if unsuitably designed.

(6) The jet, being placed tangential to the pitch circle of the buckets, meets and remains in contact with each bucket in turn through some appreciable angle of rotation. The angle at which the jet meets the bucket, and also the angle of discharge, will in consequence vary as the wheel rotates, and it follows that unless the buckets are designed so as to give normal impact on the ridge and a discharge which is tangential to the wheel, and unless the speed of the wheel is regulated so that the backward velocity of discharge is equal to the forward velocity of the 
buckets, an excess of kinetic energy will be rejected to the tail-race.

Theoretically, assuming the angle of deflection to be $180^{\circ}$ and neglecting the effect of friction, the most efficient speed of the buckets is one half that of the jet (p.351). When allowance is made for the effect of friction, as on p. 341, the most efficient bucket speed is seen to be slightly less than this. Thus, with a jet velocity of 100 feet per second, assuming the data of p. 341 . to hold, the final relative velocity will be $\cdot 967$ of the initial, and the velocity of the buckets for zero absolute velocity of the discharge will be 483 of the initial jet velocity.

Also, under these circumstances, taking the angle of deflection to be $160^{\circ}(\cos a=-9397)$, the ratio,

$\frac{\text { Actual }}{\text { Theoretical }}$ pressure of jet $=\frac{1+\cdot 967 \times \cdot 9397}{1.9397}=\cdot 984$

$$
\therefore \quad \frac{\text { Actual }}{\text { Theoretical }} \text { efficiency }=984 \times 967=\cdot 95 .
$$

Neglecting all other losses, the maximum possible efficiency of the wheel could not exceed 95 per cent., and in general these combine to bring down the efficiency to about 85 per cent. in the case of a well-designed wheel working under perfect conditions. In practice the most efficient bucket speed is found to be from 46 to 475 times that of the jet.

\section{Efficiency of Pelton Wheel.}

Let $u=$ peripheral speed of buckets at pitch circle.

$=\frac{2 \pi r N}{60}$ where $r=$ perpendicular distance from axis

of jet to centre of wheel; $N=$ revs. per minute.

Let $v=$ initial velocity of jet.

Let $k=$ ratio of relative velocity on leaving, to that on striking buckets.

Let $a=$ total angle of deflection of jet.

The mean initial relative velo-

city of jet and bucket $\}=v-u$.

$\left.\begin{array}{c}\text { The final relative velocity of } \\ \text { jet and bucket }\end{array}\right\}=k(v-u)$.

$\left.\begin{array}{l}\text { The final absolute velocity of jet } \\ \text { in direction of tangent to wheel }\end{array}\right)=u+k(v-u) \cos a \ldots$ 


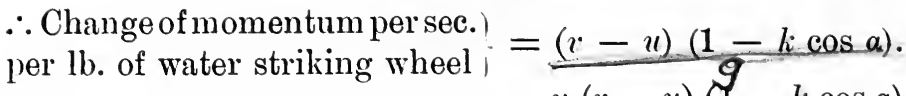

$\therefore \quad$ Work done per lb. $=\frac{u(v-u)(1-k \cos a)}{g} \mathrm{ft} . \mathrm{lbs}$.

$$
\therefore \quad \text { Efficiency } \eta=\frac{u}{g h}\{v-u\}\{1-k \cos a\}
$$

Form of Buckets. - Where the dividing ridges of the buckets are straight in profile, these are not fixed radially but are inclined backwards from the direction of rotation at such an

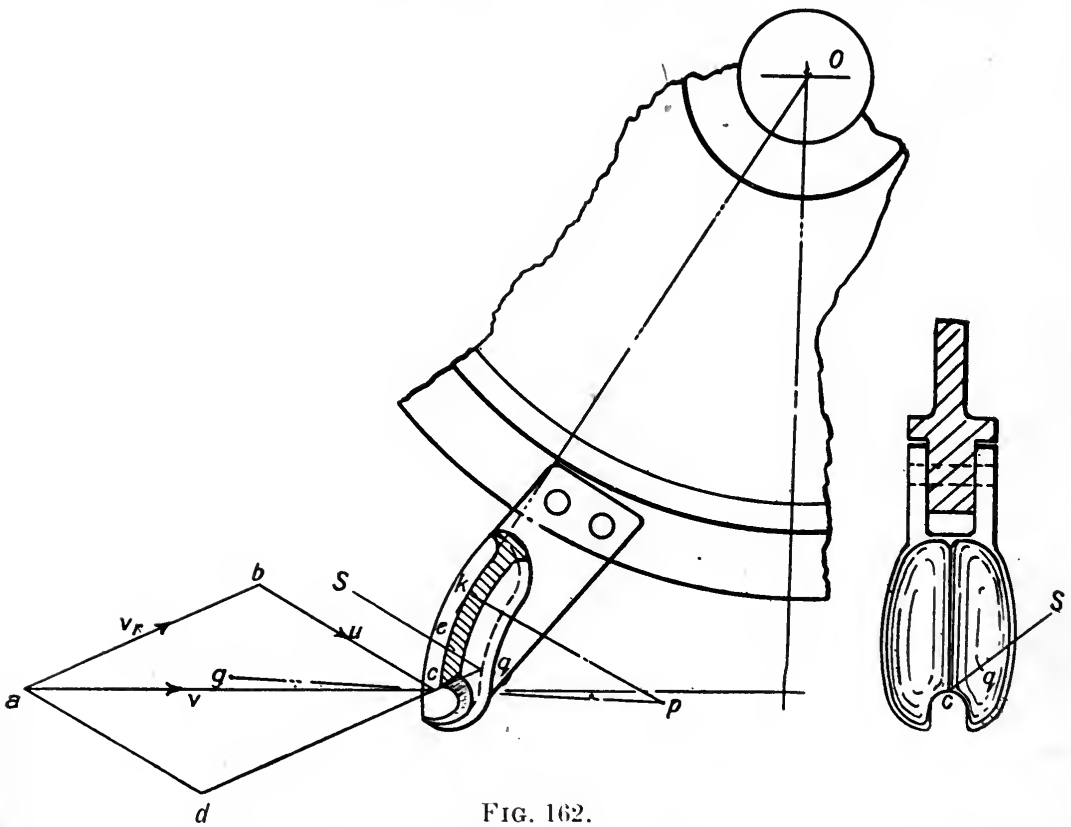

angle as to give normal incidence on the first impact of the jet. If placed radially the jet would be deflected into the rim of the wheel during the first half of the period of impact and would tend to produce serious inefficiency.

A type of construction which is found to give better results in practice, and which is more theoretically correct, is indicated in Fig. 162.

Let $\overline{a c}=v=$ velocity of jet.

Let $\overline{b c}=u=$ velocity of bucket.

Then $\bar{a} b=$ relative velocity of jet and bucket.

H.A. 
If $\omega=$ angular velocity of wheel, and if $r=$ radius at the point of impact, we have $u=\omega r$ and $\overline{b c}$ is perpendicular to $\overline{o c}$.

Draw $d c$ parallel to $a b$. Then $\overline{d c}$ represents the direction of the jet relative to the moving bucket.

For the jet to leave the bucket with zero absolute tangential velocity, its final direction must be parallel to, and the component parallel to the plane of the wheel of its final relative velocity must be equal to $\bar{c} \vec{b}$. If then $\overline{g c}$, the bisector of the angle $\bar{b} c d$, be a normal to the surface of impact at $c$, the required conditions will be fulfilled, the jet striking the vane in the direction $\overline{d c}$ and leaving (relatively to the wheel) in the direction $\overline{c b}$. The direction and magnitude of $\overline{a c}$, and the direction of $\overrightarrow{b c}$ being fixed, if the final relative velocity be calculated as indicated on p. 341, the magnitude of $\widetilde{b c}$ and therefore the most efficient speed of rotation may be determined. In general the final relative velocity may be taken as 96 of the initial. If $c$ and $k$ be the first and last points at which the jet impinges on the ridge and if a third point $l$ be taken midway between $c$ and $k$, the directions of such normals as $c g$ may be determined for these three points, and a smooth curve drawn through these points and having the required normals will give the correct curve for a longitudinal section of the receiving edge of the bucket. In general, a circular arc with centre at $p$, the intersection of the normals through $c$ and $k$ will give a very close approximation to the curve.

Strictly, since the path of the mid particles of the jet relative to the bucket is given by $c q s$, the normal at $q$ should bisect this angle. If, however, the curve through $q$ be made parallel to $c l k$, the approximation to the correct curve will be sufficiently near.

A close approximation may also be obtained by determining graphically the points of intersection of the bucket with the axis of the jet for different positions of the bucket, and by drawing a smooth curve such that the axis of the jet is normal to the curve in every position of the bucket.

To prevent the jet striking the back of the bucket, this should be everywhere above the line $d c$, while to reduce splash on passing through the jet the edge at $c$ should be as sharp as possible. 
Of the types of bucket shown in Fig. 161 it will be noted that ( $a$ ) conforms more nearly to the theoretical construction.

In modern practice the width of the buckets is between three and five times the diameter of the jet, the ratio diminishing as the size of jet increases.

Number of Buckets.-For minimum loss these must be as few as is consistent with the jet being wholly intercepted for all bucket positions, so that the entering bucket must entirely intercept the jet before the leaving bucket begins to free itself. From this consideration, a simple geometrical construction shows that if $n$ be the minimum possible number of buckets, $R$ the extreme outer radius over the receiving edges of the buckets, $r$ the pitch circle radius, and $t$ the thickness or diameter of the jet, $n$ is very approximately given by the relationship

$$
n=\frac{\pi}{\sqrt{1-\frac{\left(v+\frac{t}{2}\right)^{2}}{R^{2}}}}
$$

If $R=r+s$, so that $s$ is that portion of the bucket projecting beyond the pitch circle, we have

$$
n=\frac{\pi}{\sqrt{1-\left(\frac{r+\frac{t}{2}}{r+s}\right)^{2}}}
$$

Giving $s$ a value ranging from $60 t$ in the case of a wheel of less than 3 feet diameter to $565 t$ where the wheel is upwards of 6 feet in diameter, values of $n$ in close accord with modern practice are obtained. Generally, values of $n$ given by the formula $n=k \sqrt{\frac{r}{t}}$, where $k$ ranges from $7 \cdot 0$ to $8 \cdot 0$ as the wheel diameter decreases from 6 feet to 3 feet, will be found to give results which are sufficiently near for all practical purposes. The theoretical value of $s$ thus being obtained, a little additional overlap is usually given to allow, for any slight variation in the axial position of the jet.

Speed Regulation.--Since the efficiency of a Pelton wheel, or other impulse wheel, depends on the maintenance of the correct 
ratio of peripheral velocity of bucket and velocity of jet, if high efficiency is to be expected at all loads the method of governing must be such as to keep the latter velocity as nearly as possible constant. Where this is the case, there is no reason, except for the greater proportional effect of windage and mechanical friction at part loads, why the efficiency should not be independent of the load. Where, however, the jet velocity is variable, the efficiency falls off considerably as this departs from its theoretically correct value, and for this reason the impulse wheel, while giving excellent part-load efficiencies under a constant supply head, is unfitted for situations in which the percentage variation of head is likely to be great. Since this is more likely to be the case under a low supply head, it affords one reason why the impulse wheel is not in general advisable under such conditions.

The Speed Regulation of a Pelton wheel is usually performed in one of four ways.

(1) The stream may be deflected so as to partially miss the buckets at part load, either by swivelling the nozzle, which is then carried on a ball-and-socket joint, or by a stream deflector placed between the nozzle and the wheel. Owing to the friction at the swivelling joint a considerable force is required to deflect the nozzle, and in consequence the governor must be fitted with a hydraulic relay cylinder, as at $C$ (Fig. 167). The piston rod of this cylinder carries the nozzle, and the governor by regulating rhe supply of pressure water to one side or other of this piston, also regulates the position of the nozzle.

This method of regulation has the disadvantage of being wasteful of energy at part load, while the nuisance caused by the discharge of the jet directly into the wheel pit may be very great.

On the Pacific slopes, however, many of the water companies require that a constant flow through the pipes be maintained, in order that a constant supply may be delivered over a weir to a ditch of lower level, and in this case the deflecting nozzle affords the most suitable means of speed regulation. The method possesses a further advantage in that it avoids all action of the nature of water ram in the pipes.

(2) The velocity of the jet may be reduced by means of a 
throttle valve placed behind the nozzle. This is not to be recommended, since the contraction and subsequent enlargement of the stream which occurs at the valve is wasteful of energy, while the variation in the velocity of the jet tends to inefficiency in working.

-Further, since the sudden closing of the valve causes a corresponding increase of pressure throughout the pipe due to water ram (p. 217), this method of governing should never be adopted without the addition of some suitable protecting device such as a stand pipe, relief valve, or pressure regulator, to the pipe line, this being placed as near to the valve as possible.

(3) A portion of the jet may be cut off at the nozzle by means of a sharp-edged sluice sliding across the orifice, or the section of the jet may be reduced by means of a needle regulator (Fig. 163). This consists of a cylindrical needle of tapering section fitted inside the nozzle axially with the jet. The water flows through the annulus between the needle and the nozzle, forming a solid cylindrical jet on leaving the needle.

By axial regulation, the latter may be adjusted so as to partially or wholly fill the oritice.

While giving a slightly greater loss by friction than the sluice regulation, a more stable jet is obtained, and on the whole needle regulation is to be preferred.

By either method, the velocity of efflux is maintained approximately constant, and the efficiency is therefore only slightly affected at low loads, the quantity of water used being approximately proportional to the load. ${ }^{1}$

On the other hand, the inertia of the supply eolumn tends to prevent close governing unless a relief valve, or some such device, is fitted near to the nozzle, while care should always be taken, as explained on p. 258, that the closing of the nozzle actually does diminish the supply of energy to the wheel.

One device which prevents a rise in pressure following any

] Experiments by H. C. Crowell and G. C. Lenthe (Massachusetts Institute of Technology, 1903), on a needle regulating nozzle, showed that at mid-opening the coefficient of velocity diminishes as the head increases, from about .98 with $120 \mathrm{ft}$, head to 996 with $23 \mathrm{ft}$. head. With a given head the velocity was slightly the greatest with the nozzle half open. The area with mid-opening was $1 \cdot 227$ square inches, as against 1.767 square inches with the nozzle full open. 
sudden closing of the regulating nozzle is shown in Fig. 163. Here the relay eylinder $C$ is supplied with oil or water under pressure, this supply being regulated by a valve operated by the governor link. Any increase in speed is then accompanied by the admission of pressure water to the right-hand side of the piston. 'This tends to force the needle over to the left, and at the same time to move the cylinder itself to the right against the resistance of the springs at $\dot{S}$, and thus to open the byepass valve $r$. An increase in pressure at the nozzle also directly tends to

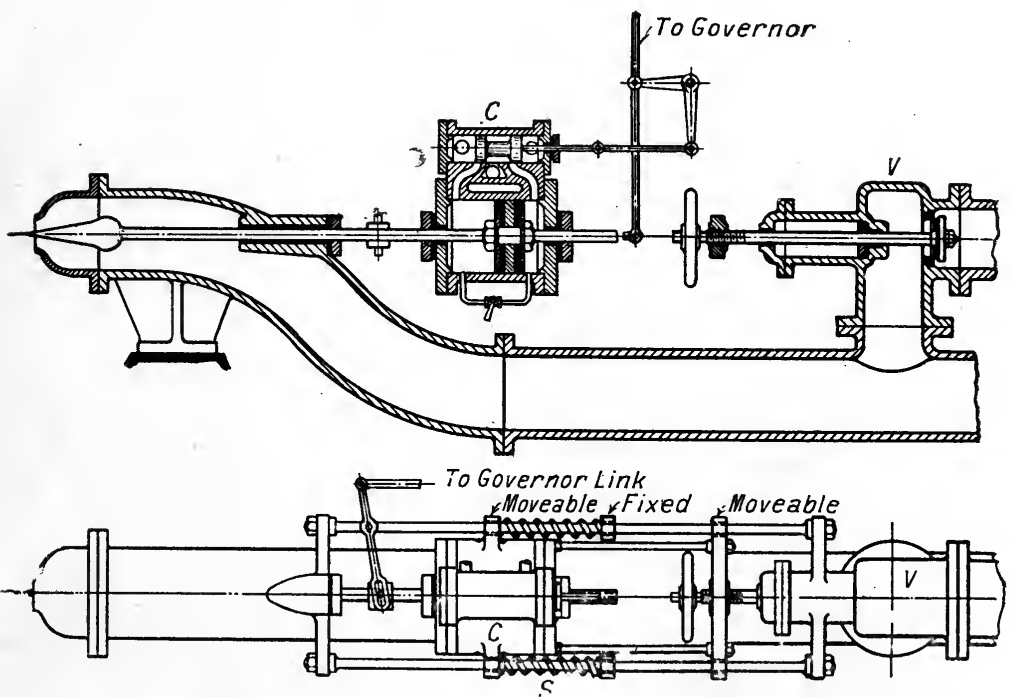

FIa. 163.- Needle Regulator and Pressure Regulating Device for Pelton Wheel.

open this valve against the resistance of its springs, so that the pressure is quickly relieved. 'I'he motion of the cylinder relatively to its valve moreover tends to cut off the supply of pressure water to the right-hand side of the piston, while the motion of the needle is utilized to bring the governor link back into its central position. This equalizes the pressure on the two sides of the piston, and the cylinder itself under the action of the side springs $S$, returns to its central position, at the same time closing the byepass valve. 'l'he whole apparatus is now ready to respond to a further change of speed in either direction. Some relay 


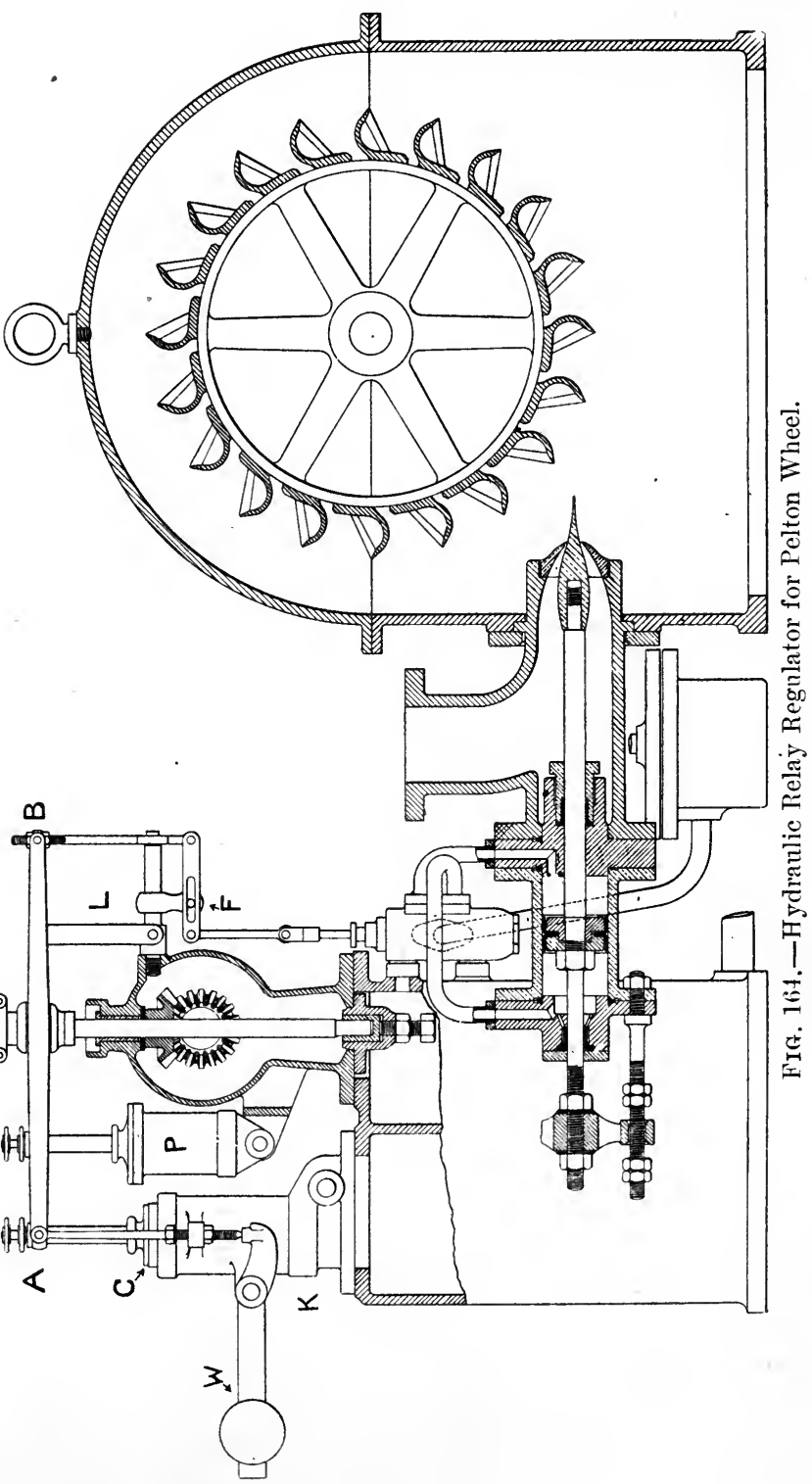


returning device of this nature is indispensable if hunting is to be prevented (Art. 128).

An extremely neat device for the same purpose is illustrated in Fig. $164,^{1}$ and is shown in Fig. $165^{1}$ as fitted to a twin Pelton wheel.

Here the horizontal governor lever $A B$ is not connected to any fixed fulcrum, but is pivoted at $A$ on the end of a plunger working in the dashpot $C$. At $B$ it is connected to the spindle

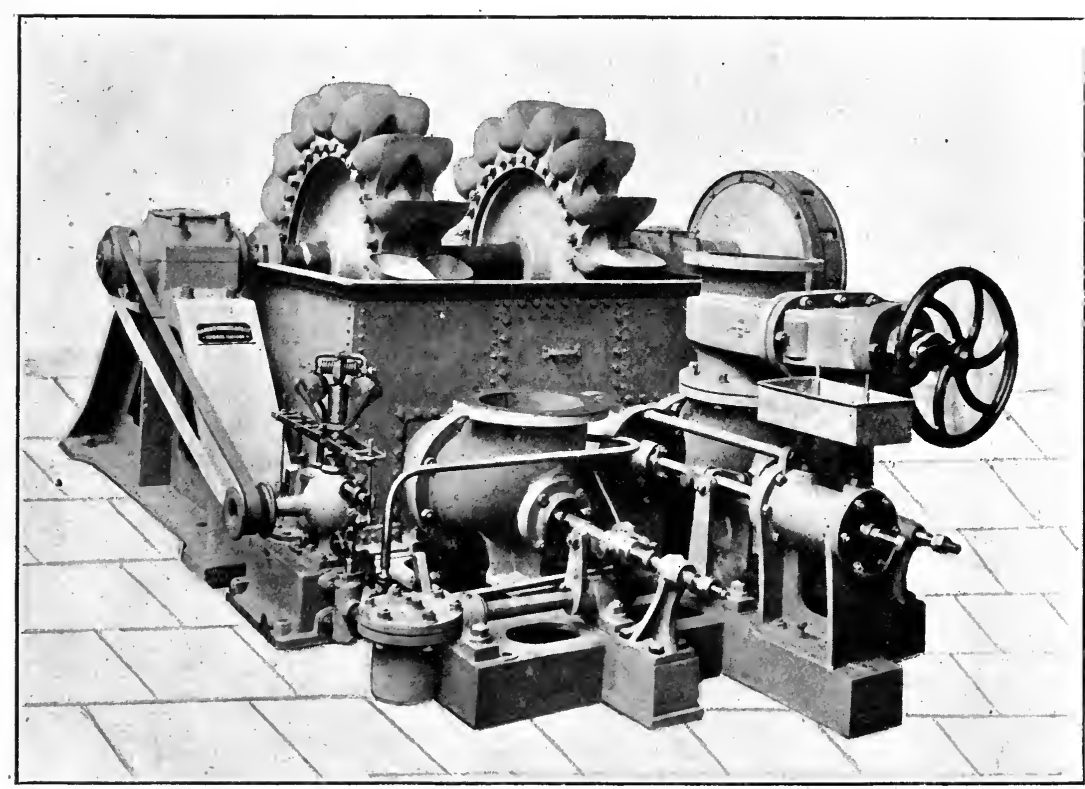

FIG. 165.-Twin Pelton Wheel with Hydraulic Relay Governor,

of the regulating valve, $F$ being a fixed fulcrum. A subsidiary lever connects the end of the plunger working in the dashpot $I^{j}$, with the anchor link $L$ and with the governor collar, this being solely for the purpose of steadying the motion of the governor.

On a sudden increase in speed, following a reduction in load, the governor collar lifts and the valve spindle is depressed, admitting water behind the relay piston and forcing the spear rod into the nozzle. This spear rod is connected with the dash-

1 By courtesy of Messrs. Gilkert Gilkes \& Co., Ltd., Kendal. 
pot $C$, which itself works in the outer fixed casing $K$, by a series of links and a bell-crank lever not shown in the sketch, and as the spear rod moves to the right the dashpot is lifted, raising at the same time the fulcrum $A$. During this portion of the motion, there is a slight downward motion of the dashpot plunger and fulcrum relative to the cylinder. As the motion of the latter ceases, however, the plunger is gradually lifted by the weighted lever $W$, bringing down the pin at $B$, and returning the valve

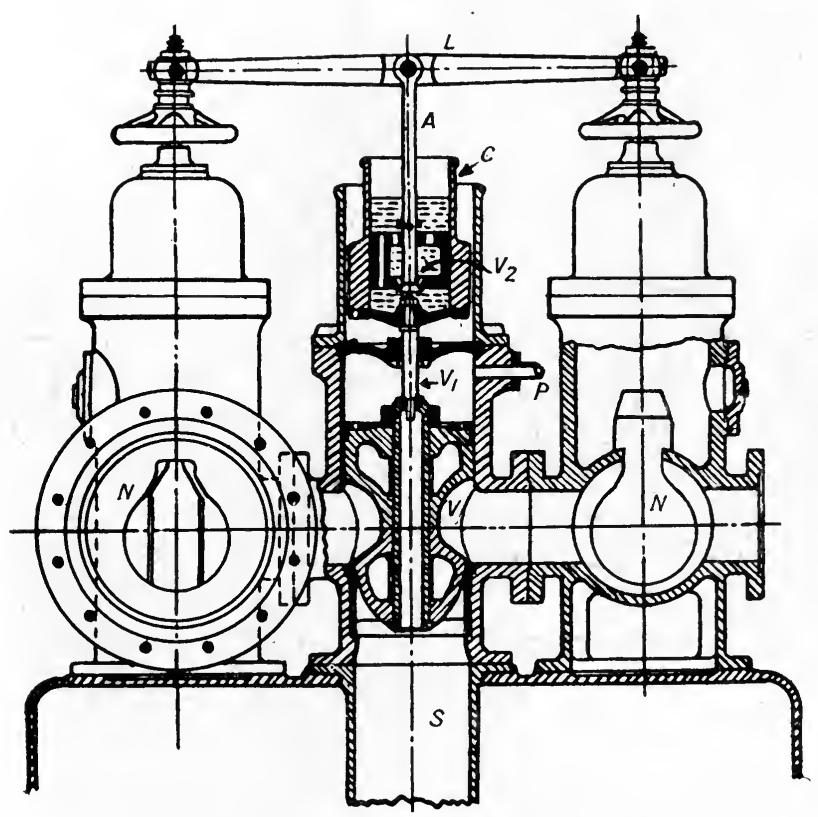

Fig. 166.-Pressure Regulator for Pelton Wheel.

to its central position with the governor lever also in its central position.

Any further motion of the spear rod is thus stopped until the wheel has had time to readjust itself to the changed conditions, when the whole arrangement is again ready to adjust itself to any fresh change of speed.

Water ram on closing the nozzle may, if necessary, be prevented by a special automatic device of the makers. In this the spear rod is directly connected through a link with 
a dashpot plunger, the cylinder of which is vertical, is capable of axial movement, and which is itself connected to a small needle valve which is opened by any upward motion of the cylinder. The main relief valve is slightly overbalanced hydraulically so as to remain closed whatever the pressure in the main. If, however, the spear rod closes the nozzle rapidly, the sudden motion of the

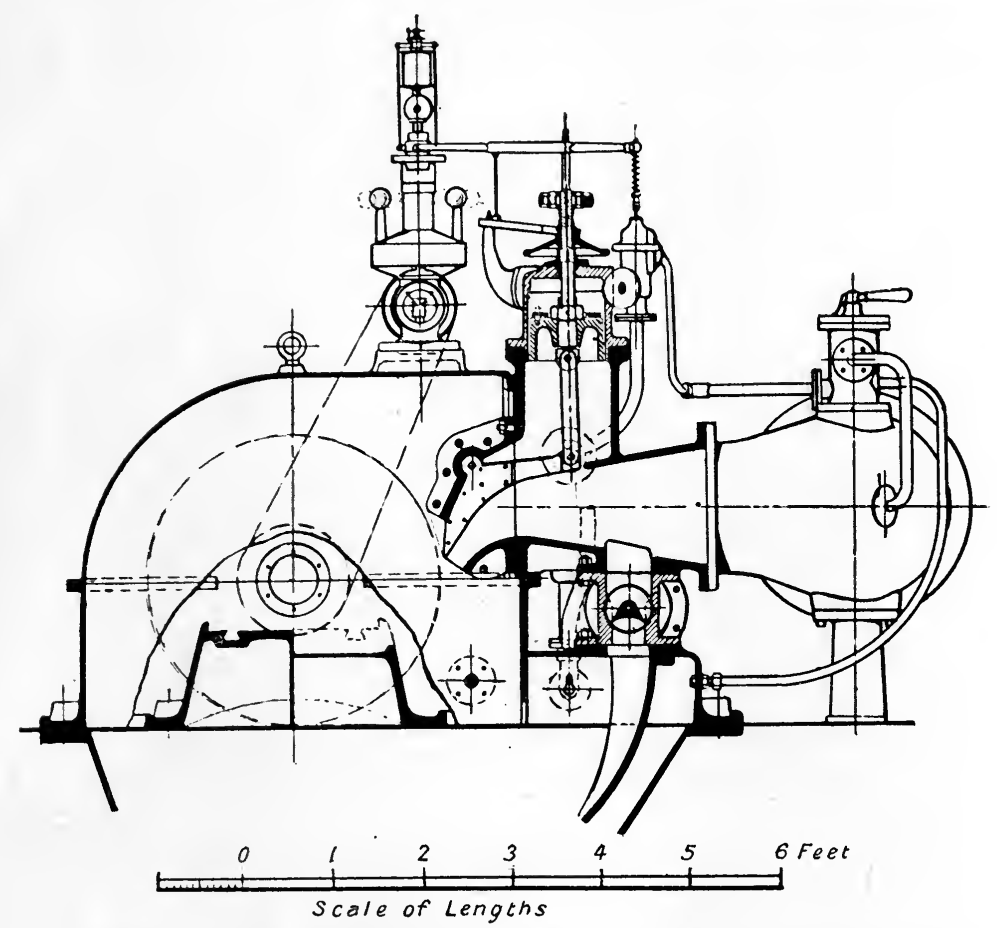

Fig. 166 A.-Double Tangential Impulse Wheel, 500 H.P. at 375 Revolutions under 262 feet head; wheel diameter $3 \cdot 28$ feet. The Kubel Electric Power Plant, St. Gall.

dashpot plunger sucks up the dashpot cylinder and with it the small needle valve. This allows water to escape from above the main relief valve, which is then lifted by the excess pressure on its under side and permits of free discharge from the body of the nozzle.

The dashpot cylinder now begins to fall by its own weight, closing the needle valve and thus the relief valve, the time of 
closing being adjusted by regulation of the dashpot orifices to suit the length of the supply pipe line.

This system has the great advantage that its working is quite independent of any rise in pressure in the main, but rather anticipates any such possible rise.

The whole arrangement is shown in Fig. 202, as applied to the regulation of a Thomson Vortex Turbine, while Fig. 166 shows details of a device working on exactly the same principle, and applied in this case to a twin Pelton wheel. ${ }^{1}$ Here the cross lever $L$ is connected to the piston rods of the two relay cylinders, and carries the dashpot rod $A$. Its connected plunger works in the

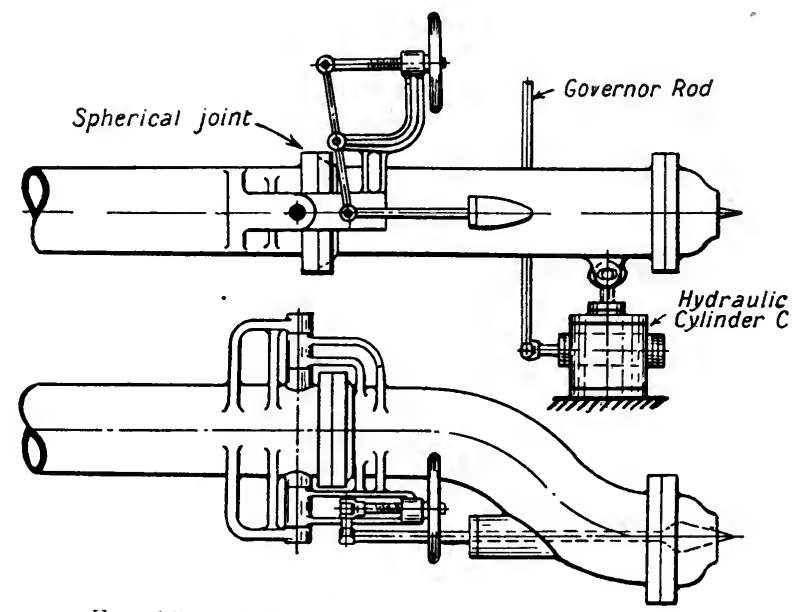

FIG. 167.-Combined Needle and Deflecting Nozzle.

weighted dashpot $C$, which itself carries the needle valve $I_{1}$. Pressure water supplied through the small pipe $P$ keeps the main escape valve $I^{\prime}$ closed so long as the valve $V_{1}$ is closed. If this valve is opened, however, by a sudden upward motion of $L$, the pressure above the main valve is relieved, and the valve opens, relieving the pressure at the nozzle $N N$.

The valve $I_{2}$ permits of a sudden depression of $L$, without unduly stressing the dashpot $\operatorname{rod} A$.

A modification of the needle method of regulation is indicated in Fig. 166A, ${ }^{1}$ which shows a section of a double tangential wheel

1 By courtesy of Messrs. Escher, Wyss d_Cie., Zurich. 
of 3.28 feet diameter developing 500 H.P. under a head of 262 feet. Here the nozzle is rectangular in section, while its upper side is formed by a pivoted flap whose position is regulated by that of the piston of a relay cylinder actuated by the governor. Any variation in the position of this piston thus produces a corresponding variation in the sectional area of the nozzle. A striking feature of this installation is that it is fitted with a draught tube (Art. 123), and works under a suction head of 22 feet.

(4) A modern and very common method of regulation is illustrated in Fig. 167. Here a combination needle and deflecting nozzle is used, the needle being set by hand, so as to take the maximum load likely to occur during any hour, while the deflection takes care of any variation of load up to this peak. With a very variable load such as occurs in electric lighting plants considerable economy may thus be effected, while the possibility of water ram is eliminated.

A self-regulating wheel which has been tried with good results as regards speed regulation, consists of two discs mounted side by side on the same shaft and capable of relative sidelong motion. These are kept in position by springs, and each carries a series of half buckets which fit together when the discs are close together and thei form ordinary Pelton buckets. An increase in speed, by the consequent increased centrifugal force on masses mounted on bell-crank levers connected to the wheel, produces a relative sidelong motion of the discs, which part in the middle and allow a portion of the jet to pass through to waste.

The complication introduced by this device, together with the waste of energy common to any such method of governing, form the chief drawbacks to the scheme.

The table on the opposite page gives some details of typical Pelton wheel installations of comparatively recent date.

If desired, two or three jets may be arranged so as to play on a single wheel and the power obtained is then practically proportional to the number of jets. In such a case the sliding hood provides the most suitable method of speed regulation.

For heads above 300 feet, and for powers in single units up to about 2,000 B.H.P., the Pelton wheel is by far the most suitable 


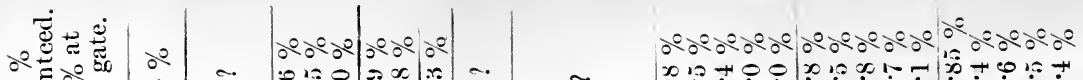

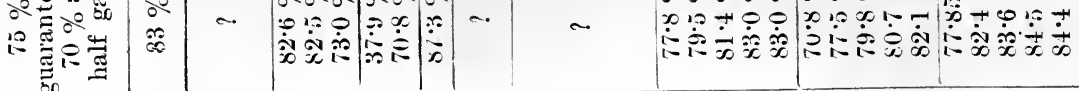

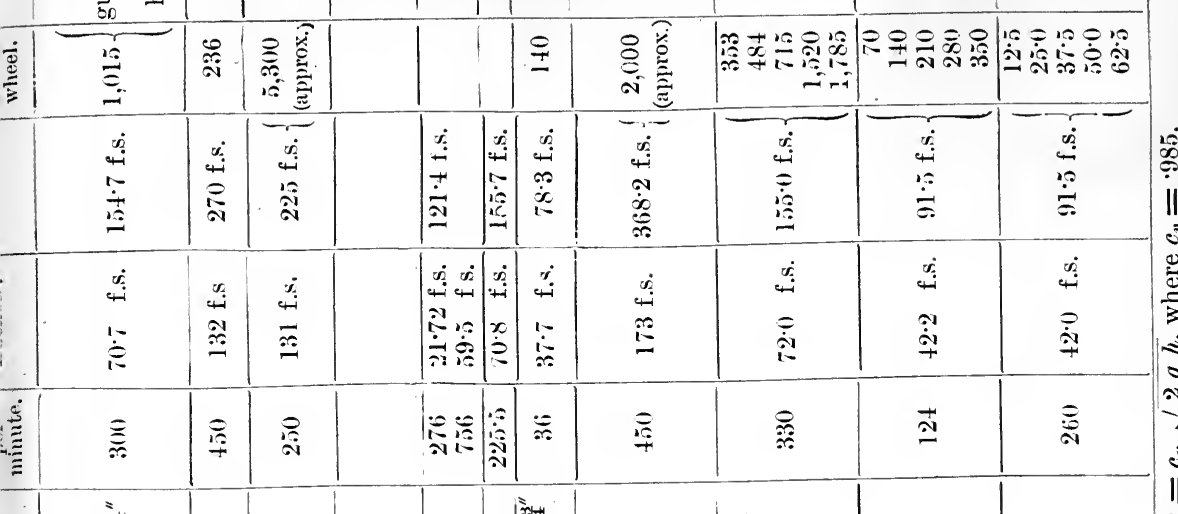

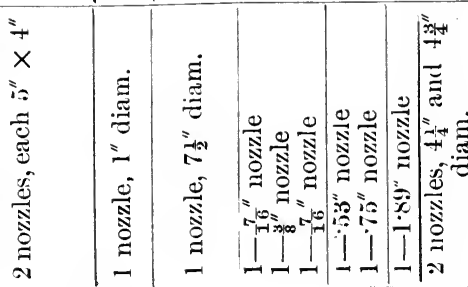

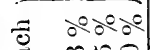

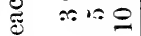

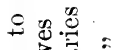
$\therefore \frac{1}{2}$

So ق气.

$+\stackrel{+}{\circ} \overline{0}$

政 =:

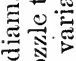

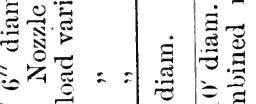

-

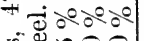

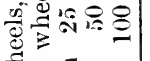
焉: $=$ $0 \geqslant: \div$
施.

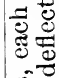

焉

כ

$\therefore \stackrel{0}{\square}$

$\ldots$

की

Ð..

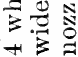

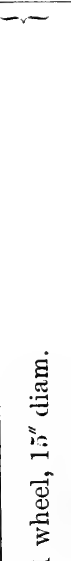

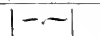

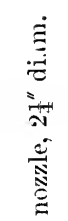

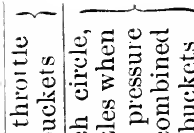
늘 ठำ - + ङ

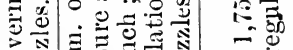

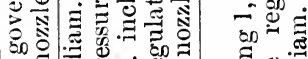

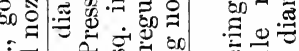

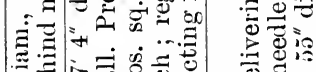

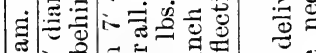

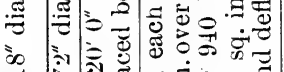

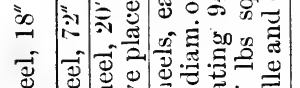
焉

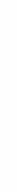

突

\section{$\frac{10}{0}$}

$\mathrm{N}$

II

要

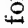

$\stackrel{0}{ \pm}$

छ्ष

这

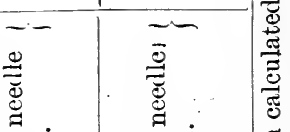

$\dot{10}$

so $\approx$ 임

응.

용

응.

.

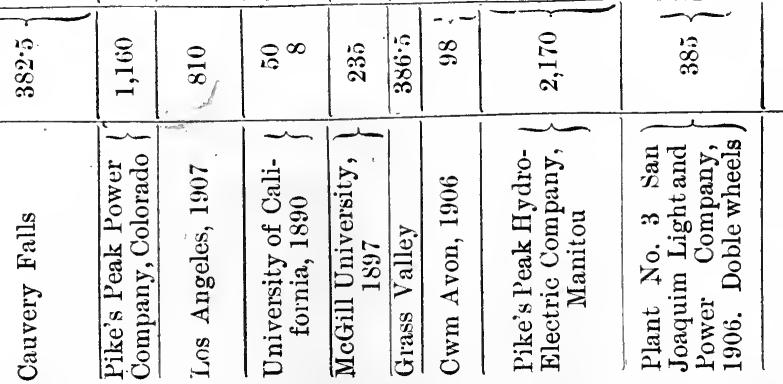

衰

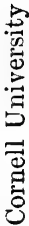


type of prime mover, while for units up to 10,000 H.P. and with heads ranging from 100 to 300 feet, it is for many purposes to be preferred to its only serious rival, the inward radial flow or Francis turbine. In view of its combined simplicity, efficiency, and ease of regulation, it is probably the most perfect of all hydraulic prime movers, and this may be the more readily granted when the difficult conditions under which it works are remembered. Taking a jet of water to all intents and appearances as rigid as a rod of glass, and, in virtue of its enormous velocity possessing almost infinite destructive possibilities; dropping it almost without splash into the tail-race divested of practically the whole of its kinetic energy; the whole affords an unique example of the possibilities of engineering science.

\section{Design of Pelton Wheel.}

\section{Example.}

To design a Pelton wheel to work under an effective head of 500 feet and to develop 800 H.P. at 360 revolutions per minute.

Assuming a coefficient of velocity $=\cdot 985$, the velocity of efflux of the jet $=\cdot 985 \times \sqrt{500 \times 64 \cdot 4}$

\section{$\doteq 177$ feet per second.}

Taking the velocity of the pitch circle of the wheel as $\cdot 46$ times that of the jet, we have

Peripheral velocity of wheel $=81.3$ feet per second.

$\therefore$ Radius of pitch circle $=\frac{81.3 \times 60}{2 \pi \times 360}=2.158$ feet.

$\therefore \quad$ Diameter of pitch circle $=4$ feet $3 \frac{3}{4}$ inches.

Next assuming an efficiency of 85 per cent., we have the energy passing the nozzle per second given by $\frac{800 \times 550}{.85} \mathrm{ft}$. lbs. $=518,000 \mathrm{ft} . \mathrm{lbs}$., and since each cubic foot of water contains $\frac{62.4 \times(177)^{2}}{2 g}-\mathrm{ft} . \mathrm{lbs} .=30,380 \mathrm{ft}$. lbs. in the form of kinetic energy, this requires $\frac{518,000}{30,380}=17: 06$ cubic feet per second. 
The required area of the nozzle is thus $\frac{17 \cdot 06}{177}=\cdot 0964 \mathrm{sq} . \mathrm{ft}$. $=13.89 \mathrm{sq}$. ins.

giving a jet diameter of $4 \cdot 22$ inches.

Taking $n=7 \cdot 5 \sqrt{\frac{r}{t}}$, this gives the number of buckets as equal to $7 \cdot 5 \sqrt{\frac{25 \cdot 9}{4 \cdot 2 \cdot 2}}=18 \cdot 6$, or say 20 for convenience in balancing.

Next applying the formula $n=\frac{\pi}{\sqrt{1-\left(\frac{r+\frac{t}{2}}{r+s}\right)^{2}}}$ we get, on substituting for $n, r$ and $t$, on reduction $s=2.5$ inches, giving the amount by which the buckets must project beyond the pitch circle for continuous impact. For safety it is usual to increase this slightly, say to 2.75 inches, giving an extreme wheel diameter of 4 feet $9 \frac{1}{4}$ inches.

The buckets would in this case be about 21 inches wide.

\section{Examites.}

(1) A Pelton wheel working under an effective head of 2,100 feet, is $36^{\prime \prime}$ diameter and is supplied through a single $\frac{1}{2}$ " nozzle. Determine the necessary number of revolutions of the wheel for approximately maximum efficiency and the probable horse-power, assuming an efficiency of 83 per cent.

\section{Answer. $\quad\left\{\begin{array}{l}1,060 \text { revolutions per minute. } \\ 97.5 \text { H.P. }\end{array}\right.$}

(2) A Pelton wheel develops 140 B.H.P. under a head of 98 feet. The wheel is $20^{\prime} 0^{\prime \prime}$ in diameter, and is supplied by two nozzles. Determine the number of revolutions per minute and the necessary nozzle diameter, if the efficiency is 80 per cent.

$$
\text { Answer. } \quad \begin{aligned}
& 35 \text { revolutions per minute. } \\
& \text { Diam. }=4 \cdot 3 \text { ins. }
\end{aligned}
$$


(3) Show that the efficiency of a Pelton wheel is theoretically equal to

$$
\frac{2 \pi \cdot N}{60 g h}\left\{C_{v} \sqrt{2 g h}-\frac{2 \pi r N}{60}\right\}\{1-k \cos a\}
$$

Where $r=$ mean radius of bucket circle.

$N=$ revolutions per minute.

$h=$ effective head at nozzle.

$C_{v}=$ coefficient of velocity at nozzle.

$k=$ ratio of relative velocity at exit from and entrance to buckets.

$a=$ total angle through which jet is deflected.

(4) Explain why experimental results usually show a slight disagreement with the theoretical result of the preceding example. 


\section{CHAPTER XII.}

Turbines-Types-Impulse-Pressure-Girard-Haenel-Barker's Mill-Fourneyron-Jonval-The Suction Tube-Francis-Thomson-Compound TurbinesAmerican Mixed Flow Type-Governing of Turbines-Head dnd Tail Races.

\section{Art. 116.-Turbines.}

Is general, by a turbine, is meant a water-wheel which is so arranged as to allow of water being admitted simultaneously at all points on its circumference, thus enabling a greatly increased power to be obtained with the same wheel diameter.

Turbines may be divided into two main classes, known respectively as Impulse turbines and as Pressure or Reaction turbines, according to the manner in which they abstract energy from the supply water.

In an impulse turbine, the whole head of the supply water is converted into kinetic energy before the wheel is reached, the water issuing from the nozzles or guide passages in a series of streams or jets moving with high velocity and exposed to the pressure (usually atmospheric), obtaining in the turbine casing. It then enters a series of buckets formed by.curved vanes in the turbine wheel and in virtue of the change of direction, and hence of tangential momentum produced by these vanes, exerts a driving force, and so does work on the turbine shaft. Its surface pressure remains uniform throughout the turbine if this is correctly designed, and its direction is freely deviated by the vanes. For this reason, this is sometimes termed a turbine of free deviation.

For the pressure to remain uniform throughout the wheel it is essential that the stream should not fill the space between any two moving vanes, and to prevent this occurring the buckets are usually ventilated as shown in Fig. 168, which represents a part section through the wheel and guides of a Girard turbine. 
In a Pressure or Reaction turbine, the water on leaving the guide vanes and entering the wheel is under pressure, and thus supplies energy partly in the kinetic and partly in the pressure form. In its passage through the wheel this pressure energy is gradually converted into kinetic energy, and the water finally leaves the wheel at a pressure not sensibly greater than that of the atmosphere. The change of momentum accompanying this change from pressure head to velocity head necessitates an equivalent reaction on the moving vanes, and work is thus done on the turbine shaft. This turbine, as well as the impulse
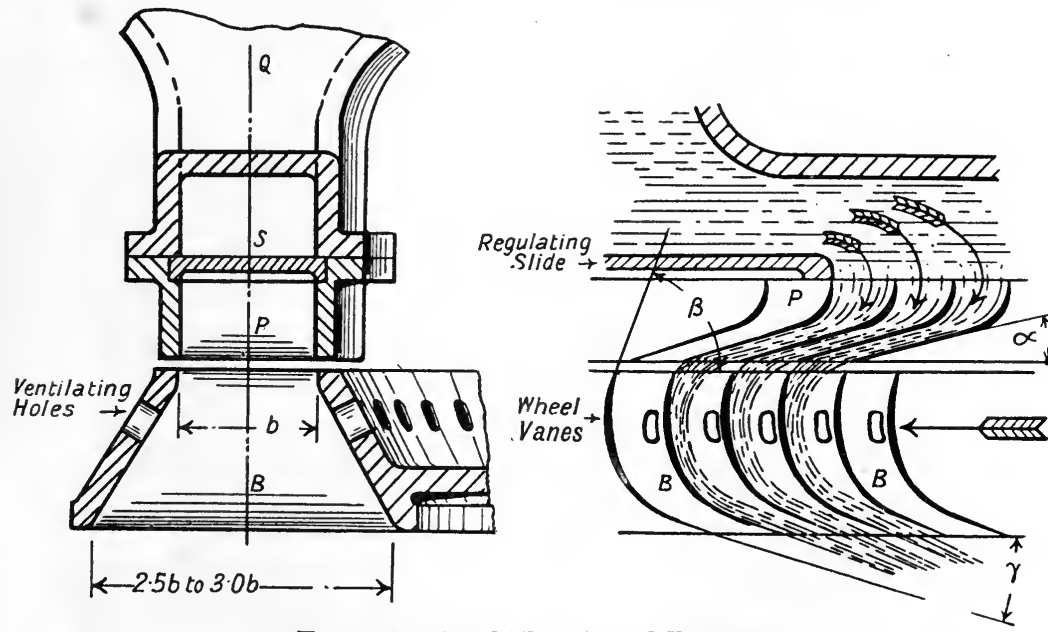

FIG. 168.-Axial Flow Girard Turbine.

type, therefore owes its propelling force not to statical pressure, but to changes of momentum, the pressure difference over the concave and convex faces of a bucket being produced, as in the impulse wheel, by the change of momentum in the stream passing the bucket. In the case of a wheel having vertical downward flow the weight of the water also adds to this propelling force.

Since the turbine works under pressure the buckets should always remain full of water, and to this end admission should take place continuously all around the circumference of the wheel. If not, those buckets which happen to be idle will either be empty, having discharged their contents into the discharge pipe, or will contain dead water. In the first case the 
buckets must be refilled before the pressure at the circumference can be utilized, while in the second case the necessity for imparting momentum to this still water causes loss of energy by impact. In either case the loss of energy may be considerable. In the impulse wheel, on the other hand, the supply may be admitted either wholly or partially around the circumference without loss of energy.

These two main types of turbine may be subdivided, according as the general direction of flow through the wheel is radial and perpendicular to the axis, parallel to the axis, or is a combination of these, these sub-types being designated respectively as

$\left.\begin{array}{l}\text { Radial flow } \\ \text { Axial or parallel flow } \\ \text { Mixed flow }\end{array}\right\}$ turbines.

Radial flow turbines may again be subdivided into inward flow machines, when flow takes place from the circumference to the centre of the wheel, and outward flow when the flow is in the opposite direction.

The chief turbines in the various classes are :-

(1) Impulse Turbines.

Axial or radial flow . . Girard turbine.

Mixed flow . . . . Pelton wheel.

(2) Pressure or Reaction 'Turbines. Radial flow $\left\{\begin{array}{l}(a) \text { Inward.-Whomson vortex turbine and the } \\ \text { original Francis turbine. }\end{array}\right.$
(b) Outward.-Fourneyron turbine.

Axial flow . . Borda turbine, Henschel-Jonval turbine.

Mixed flow . . Hercules, Victor, and most turbines of the American type. All modern Francis turbines are to a certain extent of the mixed flow type.

One special type of turbine, the Haenel, may work either as an impulse or pressure wheel.

\section{Art. 117.-The Inpulse Turbine.}

All impulse turbines may be considered as modifications of the tangential or Pelton wheel, in which the jet is unconfined laterally. 
Where the supply head is very great the necessary peripheral speed of a turbine to take full advantage of this is also great, while, as will be shown later, the necessary speed is greater in the case of a pressure turbine than with one of the impulse type. This peripheral speed may be obtained either by having $\dot{a}$ wheel of large diameter with low angular velocity, or with a small wheel making-a large number of revolutions per minute, and for a pressure turbine, where it is necessary to admit water all around the circumference, it is imperative that the periphery, and therefore the diameter, be comparatively small, and the angular velocity in consequence high. For many purposes the necessary speed of rotation under very high heads then becomes too great, while the hydraulic resistances inside the turbine casing become excessive at such high speeds.

In such a case the pressure turbine suffers from the further disadvantage that the ports and passages are of necessity small and constricted in area, and are in consequence liable to be choked by any floating matter which may escape the strainers in the head-race.

For very high falls, then, the most suitable turbine is one in which the wheel itself is not submerged; in which the supply may be admitted to as much of the circumference as is necessary to develop the required power; and such that any particular diameter may be adopted which-will best suit the desired speed of rotation.

The impulse turbine in the form of the tangential water-wheel or of the Girard turbine satisfies these conditions within wide limits, and for heads between 100 and' 300 feet will often, and for heads above 300 feet will generally be the most suitable type of prime mover for all but the largest powers. Since with suitable means of regulation the jet velocity is constant under a constant supply head for all loads, under these circumstances the efficiency of an impulse turbine is approximately independent of the load, the variation in efficiency being chiefly due to the proportionately greater effect of mechanical friction and of air resistance or windage at low loads. 
Art. 118.-The Girard Turbine.

This turbine may be constructed either as an axial flow machine (Fig. 168), in which case the axis is usually vertical, or with inward or more commonly outward radial flow (Figs. 169 and $169 \mathrm{~A}^{1}$ ). With radial flow the axis may be either vertical or horizontal. The axial flow type is more suitable for large powers under comparatively low heads, where full circumferential injection is required.

Pressure water supplied through the pipe $Q$ is guided by the converging passages $P P$, its pressure diminishing as its velocity increases, and is discharged at atmospheric pressure into the
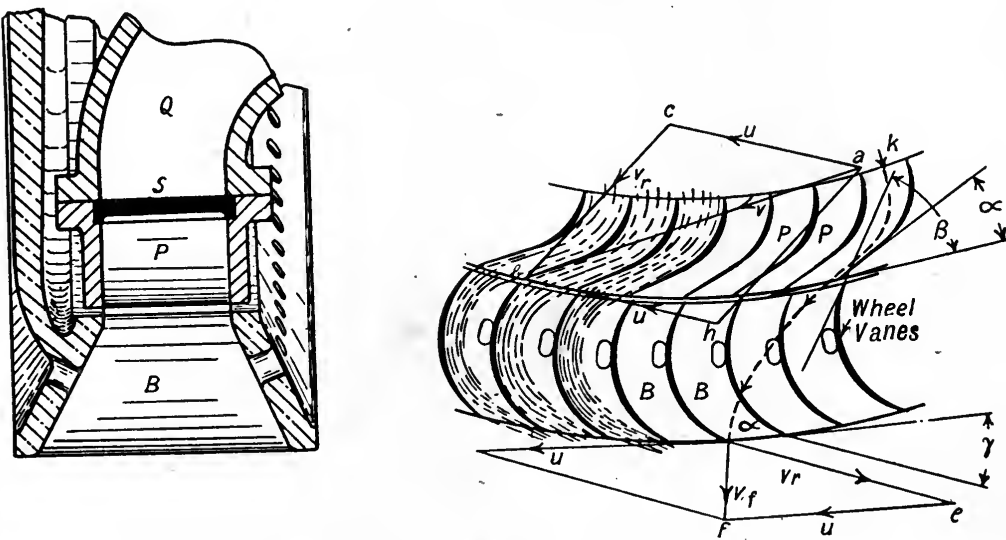

FIG. 169.-Girard Turbine with Outward Radial Flow.

moving buckets $B B$. These are ventilated to prevent the jet ex panding to touch the rear vane, and since the width of bucket di minishes with the angle of inclination of the vanes, they are al so splayed out from the inlet to the discharge side, the breadth at discharge usually varying from 2.5 to 3 times that at inlet. The outward deviation produced by this splaying of the buckets, while tending to slightly diminish the efficiency of working, cannot be avoided. The guide and vane angles at entry and exit are so designed that water enters the buckets without shock and is discharged with an absolute velocity which is only sufficiently great to ensure its ready removal from the wheel.

1 By courtesy of Messrs. Gilbert Gilkes \& Co., Ltd., Kendal. 
When less power is required one or more of the inlet passages may be cut off by means of the slide $S$, the motion being regulated either by hand or by a governor. By the provision of a series of supply ports which may be completely cut out of action one by one, the loss of energy which is inevitable through contraction and re-enlargement of section whenever a stream is throttled, is reduced to a minimum. Where only one admission port is used, the supply should be regulated by means of a sluice or hood working between the port and the entrance to the buckets. By this

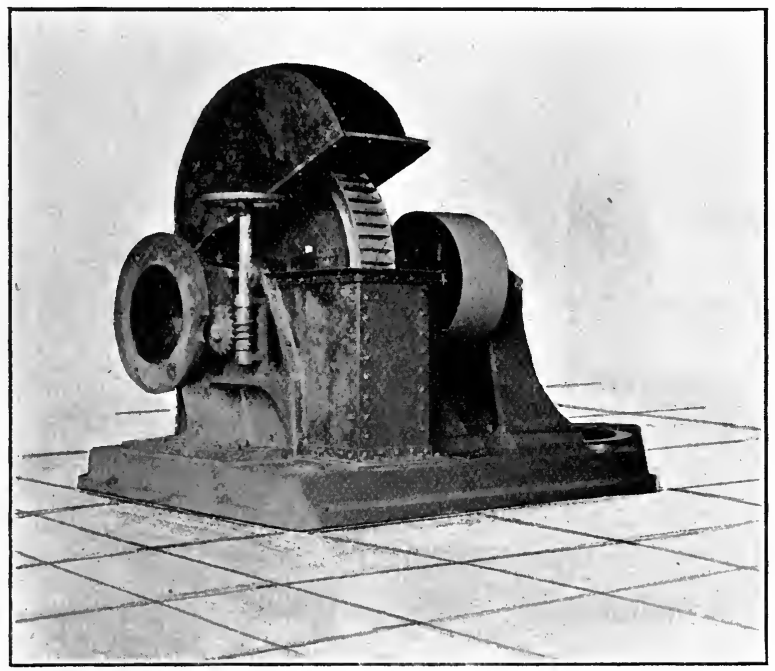

Fig. 169 A.-Outward Flow Girard Turbine.

means the velocity of efflux is unaltered, and the only loss introduced is that which may be caused by any alteration in the angle of impact of the jet.

In a radial flow machine having full circumferential admission, a cylindrical sluice or gate is often used, this partially cutting off the supply to each bucket.

While specially well fitted for heads of 100 feet and upwards, this turbine, with full circumferential injection, gives good results with heads from 10 to 50 feet, and has indeed been used with a head as low as $16 \frac{1}{2}$ inches. Under the latter head an efficiency of about 55 per cent. may be attained. With low heads and full 
injection the wheel must be horizontal, since with a vertical wheel the jet velocity at the highest and lowest points would be very different, while at the same time the loss of head due to the difference in level of the guide vanes at exit and the tail-race would become serious. To avoid the latter loss as far as possible in the case of a vertical wheel with partial injection, the guides are placed as near the bottom of the wheel as practicable. The horizontal wheel offers the further advantage for large volumes of water, in that it affords greater facilities for getting rid of a complete circumferential discharge.

In common with all impulse turbines, the part gate efficiency is high, while under suitable conditions the full load efficiency may amount to 80 per cent. In the case of an outward flow Girard turbine described in the "Proceedings of the Institution of Civil Engineers," ${ }^{1}$ and giving 400 H.P. under 594 feet head, the outer diameter of the wheel was $8^{\prime} 11^{\prime \prime}$, inside diameter $7^{\prime} 10 \frac{1}{4}^{\prime \prime}$, the vanes, 110 in number, were 4.7 inches wide at the entrance, and were splayed out to $15 \frac{3}{4}$ inches. A single inlet passage was used $4: 31$ inches in width. Under this head the jet velocity was $181^{\circ} 6$ feet per second, and the efficiencies were as follows :-

\begin{tabular}{c|c|c}
\hline B. H. P. & Revolutions. & Efficiency. \\
\hline 82.5 & 211 & $59 \cdot 5 \%$ \\
341 & 210 & $76.4 \%$ \\
400 & 209 & $79.0 \%$ \\
\hline
\end{tabular}

In this type of impulse wheel the impossibility of making the jet tangential to the wheel at entrance, and the necessity for the escaping stream to clear the discharge from the following bucket render it impossible to deflect the jet through $180^{\circ}$. If $a$ is the angie which the incoming jet makes with the plane of the wheel in an axial flow turbine and with the tangent plane to the wheel at the point of impact in a radial flow machine, and if $\gamma$ be the angle which the discharge tips of the vanes make with the same planes $F$ (Figs. 168 and 169), $a$ is generally made about $24^{\circ}$ and 
$\gamma$ about $21^{\circ}$. These values, however, depend upon the head, increasing from about $12^{\circ}$ and $13^{\circ}$ respectively with large heads and small volumes of water, to about $30^{\circ}$ and $28^{\circ}$ with low heads and large volumes. $\beta$, the angle of inclination of the vanes at entrance is then made so that the entering stream slides along the vanes without shock.

The construction for determining the vane angles is shown in Fig. 169. Here $a b$ represents $r$, the velocity of the stream leaving the guide vanes, in magnitude and direction, while $h b=a c$ represents $u$, the velocity of the wheel buckets at the point of entry. $c b=a h$ then represents the relative velocity at entry of the

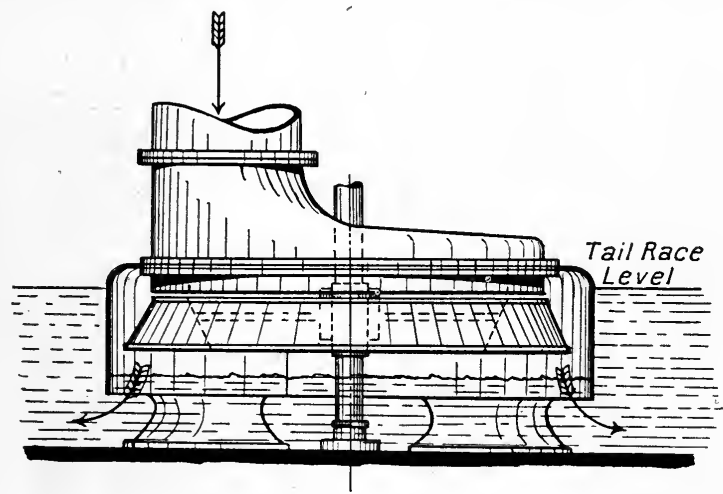

FIg. 170.-Axial Flow Girard Turbine with Full Circumferential Injection and with Girard's System of Hydro-pneumatization.

stream and the buckets, and marks the correct inclination of the bucket tips.

In order to take advantage of the full head any impulse turbine working under atmospheric pressure should be located at as small an elevation above the tail-race as is possible. While keeping this in view, however, it is highly important that the situation of the wheel be such that it is not liable to become submerged by a rising of the tail-race level in time of flood. If this should occur, the buckets run full, the wheel works as a pressure turbine, the conditions for which it is designed are entirely violated and efficient working becomes impossible.

This often necessitates the sacrificing of a portion of the available head, and with a low fall renders the use of such a turbine 
inadvisable. With a high fall this proportional loss is in general so small as to be negligible.

Attempts have been made to remove this drawback to the impulse turbine. Thus Girard, in his system of Hydro-pneumatization, placed the whole turbine in an air-tight casing, the lower end of which opened out beneath the surface in the tailrace (Fig. 170). By means of an air pump driven by the turbine the air in this casing was maintained at such a pressure as to keep the water level inside the casing below that of the turbine wheel, whatever the tail-race level. The complication and expense thus introduced, however, together with the fact that power is required to work the air pump, prevented the general adoption of this idea.

By mounting the wheel in an air-tight casing at some distance above the tail-race and coupling this to a discharge pipe or draught tube (Art. 123), opening out below the surface of the tail-race, the difficulty may be overcome. On starting up the turbine the escaping water now ejects the air from the casing and creates a partial vacuum. An air valve worked by a float in a chamber connected with the casing and the draught tube, then admits sufficient air to prevent the water level from rising as high as the wheel. Fig. 166 shows one of a series of double Pelton wheels of 500 H.P. which work perfectly well with a suction head of 20 feet.

An older device, due to Meunier, consisted in regulating the discharge by means of a sluice automatically regulated by a float. The required level in the turbine casing was thus maintained, and the addition of a draught tube rendered possible.

In the Haenel "limit" turbine, which is essentially the same as the Girard, the buckets are so designed that they run full when working as an impulse turbine, the areas of the wheel passages being approximately the same throughout. The flooding of the turbine does not then affect its efficiency except in so far as it affects the available head, since the machine now acts as a pressure turbine. It is fairly good for low falls with a head which does not vary greatly, while its efficiency varies from about 60 per cent. at half-gate to a maximum of about 72 per cent. It is, however, seldom met with in modern practice. 
Whether a horizontal or a vertical shaft machine is to be preferred depends largely on the location of the plant.

The horizontal shaft design gives a motor which is very accessible, and which is conveniently situated for gearing by means of belting to other machinery, and where the power-house is situated near the tail-race level, the supply being conveyed from the head-race by pipes, this design will in general be adopted, the turbines being placed directly on the floor of the power-house. Where the more convenient site for the power-house is near the head-race, a well must be sunk down to tail-race level, connected to the tail-race by means of a tunnel or pipe, and the turbines erected at the bottom of this well. A vertical shaft machine is now almost essential, this shaft being carried vertically upwards into the power-house and supported at intervals by suitable bearings. When driving an electrical generator this forms a convenient arrangement. The armature is mounted directly on the rotating shaft, and the arrangement has the further advantage that since all the electrical machinery may be placed well above the head-race level it will not be likely to be affected by floods. This general arrangement is, however, in general not to be recommended where it is possible to place the power station near the tail-race level. Not only is the turbine well costly to construct, but the cost of construction of the discharge tunnel or pipe line is much greater than that of the corresponding length of the supply pipe line in the latter scheme. In fact, in all turbine schemes, it may be taken as a general principle that the cost per unit length of tail-race is greater than that of head-race or supply pipe. Also the necessity for a long and heavy vertical shaft increases the first cost of the turbine, renders it less accessible, and involves the use of expensive and complicated bearings for dealing with the end thrust thus set up. ${ }^{1}$.

Art. 119.--Pressure or Reaction Turbines.

All pressure turbines of the radial outward flow type may be considered as modifications of the old Scotch turbine-

1 For a description of such bearings see Arts. 122 and 124, and Figs. 182 and $191 \mathrm{D}$. 
Reaction wheel, Barker's mill, or Segner's turbine, as it is variously called. In this turbine (Figs. 171 and 172), water is admitted through a vertical supply pipe, flows outwards through straight or curved horizontal arms, and escapes through orifices so placed in these arms as to give a series of horizontal jets perpendicular to the diameters containing the orifices. The reaction of the jets then produces rotation of the wheel. This is, however, only made in small sizes and is not of great practical importance.

Theory of the Barker's Iill.

Let $v=$ velocity of water issuing from each nozzle relatively to the nozzle, in feet per second.

Let $u=$ velocity of nozzle relatively to the ground.

Then $v-u=$ absolute velocity of discharge.

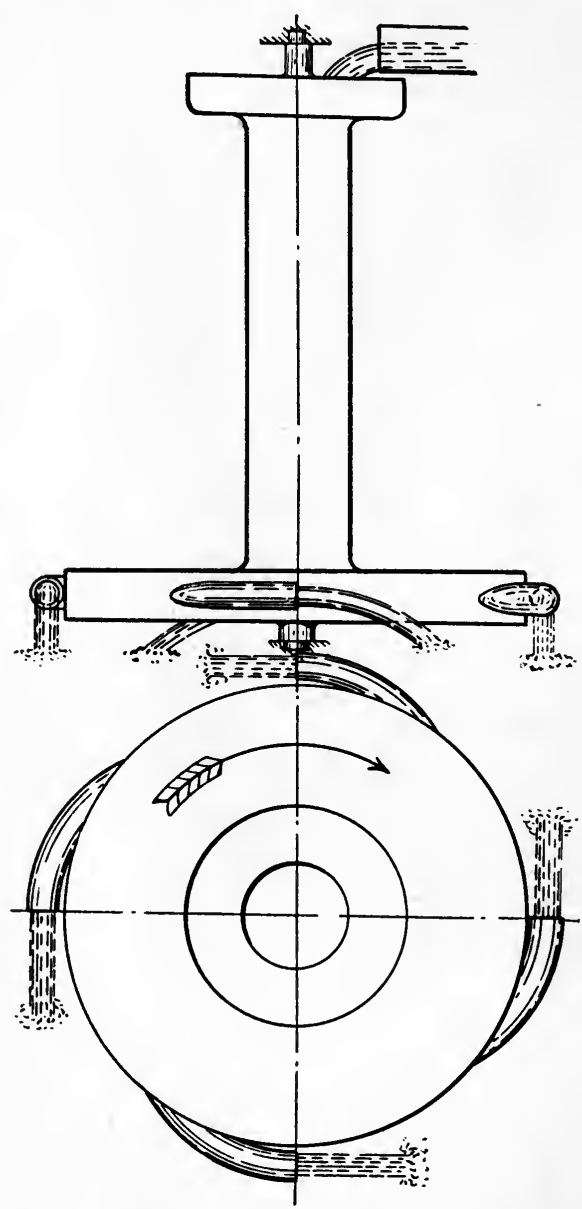

FIg. 171.-Barker's Mill.

Let $Q=$ volume of water discharged per second in cubic feet.

$h=$ head of water above the orifice in feet.

Then the horizontal reaction of $\left.\begin{array}{c}\text { the jets, i.e., the momentum } \\ \text { generated per second }\end{array}\right\}=\frac{Q W}{g}(v-u)$ lbs. 
$\left.\therefore \quad \begin{array}{c}\text { Work done by this reaction } \\ \text { per second }\end{array}\right\}=U=\frac{Q W}{g}(v-u) u \mathrm{ft}$. lbs. $\left.\begin{array}{c}\text { The energy given to the wheel } \\ \text { per second }\end{array}\right\}=Q W h$.

$$
\therefore \text { Efficiency }=\frac{(v-u) u}{g h}=\eta .
$$

Again, the total head at the orifice is the sum of the pressure head, and the velocity head produced by the velocity of whirl $u$, so that, neglecting friction, we have

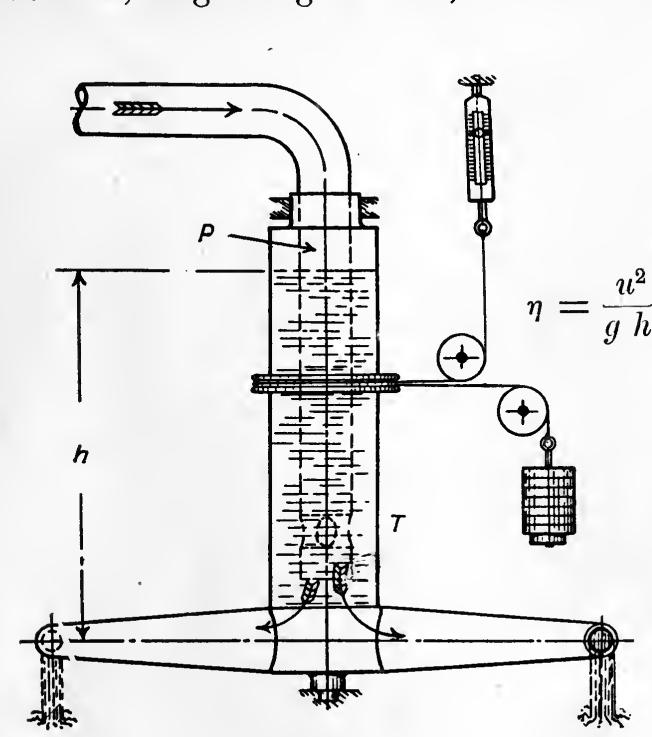

$$
\frac{v^{2}}{2 g}=h+\frac{u^{2}}{2 g}
$$

Substituting this value of $v$ in the expression for the efficiency, we get

$\left\{\sqrt{1+\frac{2 g h}{u^{2}}}-1\right\}$

Differentiating this with respect to $u$ and equating the result to zero, we finally get for maximum efficiency $\frac{g h}{u^{2}}=0$, a result which can only be true when $u$ is infinitely large. It follows that with a frictionless wheel the efficiency would increase with the speed and would become unity when the speed was infinite. Actually, however, frictional lọsses, which increase with the speed, cause

Fı̣̆. 172-Barker's Mill. a maximum efficiency to be obtained at some definite speed with any given wheel. 
Taking frictional resistances inside the wheel into account, and assuming these to be proportional to $v^{2}$ and to equal $F^{r} \frac{v^{2}}{2 g}$ we have:-

Total head at orifice $=h+\frac{u^{2}}{2 g}$.

This must equal the kinetic energy at the orifice together with the loss by friction.

$$
\begin{aligned}
\therefore \quad h+\frac{u^{2}}{2 g}=\frac{v^{2}}{2 g}+F \frac{v^{2}}{2 g} & =\frac{v^{2}}{2 g}(1+F) \\
\therefore \quad v^{2}(1+F)-u^{2} & =2 g h .
\end{aligned}
$$

Substituting this value of $v$ in the expression for the efficiency, this now becomes

$$
\eta=\frac{u}{g l}\left\{\sqrt{\frac{2 g h+u^{2}}{1+F}}-u\right\}
$$

And on differentiating and equating the result to zero we. get, for maximum efficiency

$$
u^{2}=\left\{\sqrt{1+\frac{1}{F}}-1\right\} g h .
$$

In general the maximum hydraulic efficiency of the wheel does not exceed $66^{\circ} 0$ per cent., while the mechanical friction losses bring this down to about 60 per cent.

If the wheel is loaded by means of a brake, the magnitude of the various transformations of energy may be determined as follows :-

Let $L=$ nett brake moment in foot pounds.

$N=$ number of revolutions of wheel per second.

Then useful work per second $=2 \pi L N \mathrm{ft}$. lbs. $=U$, while the energy rejected in the discharge

$$
=\frac{Q W(v-u)^{2}}{2 g} \mathrm{ft} \text {. lbs. }=E_{W} \text {. }
$$

From this we have:-

Energy، lost inside the wheel by hydraulic friction, etc. $=$ Energy given to wheel, - Energy accounted for per second

$$
=Q W\left[\iota-\frac{(v-u)^{2}}{2 g}-\frac{(v-u)}{g} u\right]=E_{F} .
$$

Energy lost per second in overcoming friction of bearings 
$=E_{B}=\frac{Q W(v-u) u}{g}-2 \pi L N$, giving $E_{F}+E_{B}+E_{W}+U$ $=Q W h$.

The distribution of energy may be shown graphically as in Fig. 173, which records the results of a series of trials by the

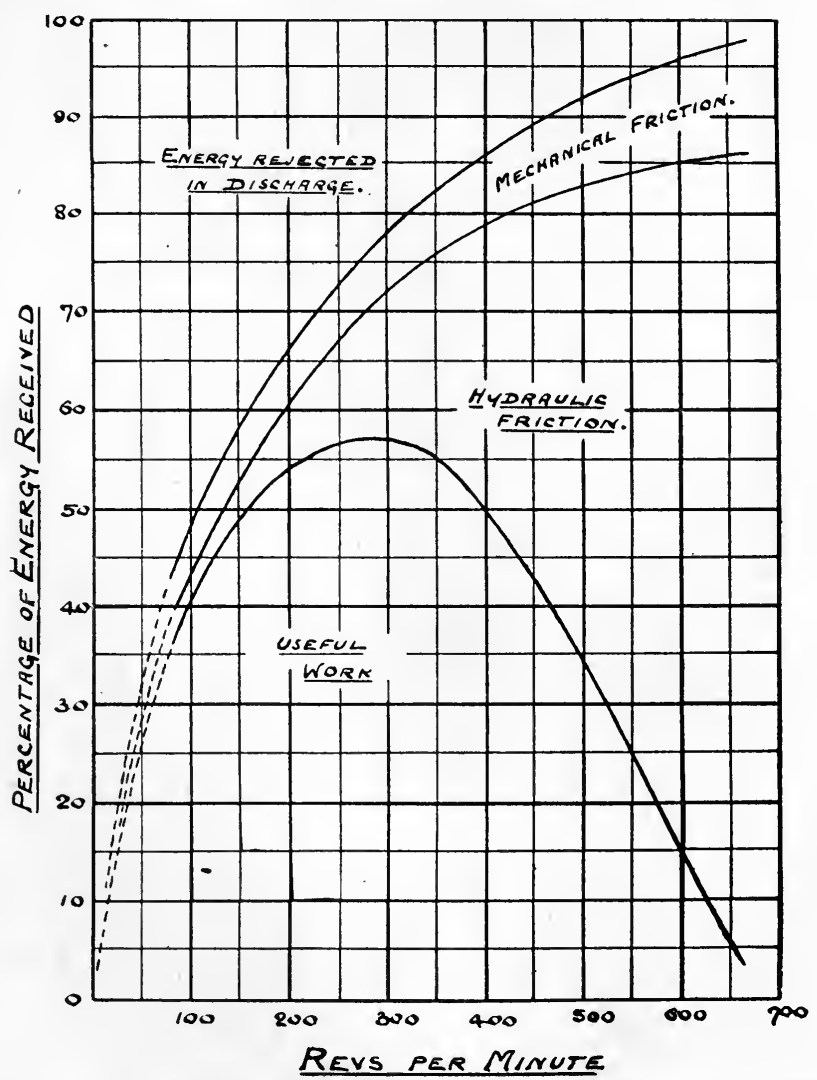

Fig. 173.-Curves showing Distribution of Energy in a Barker's Mill. author on such a wheel. The details of the wheel were as follow :-
Diameter of nozzles . . . 253 inches.
Number of nozzles . $\quad . \quad$. $\quad .2$.
Radius of nozzle path . . . 5.875 inches.
Head of water above nozzles . . 3.20 feet. 
In these trials the power was absorbed by means of a Prony brake applied to a horizontal drum. Water is admitted by means of a vertical down pipe $P$ (Fig. 172) open at the bottom, and concentric with the rotating tube $T$, the trouble caused by entrainment of air bubbles in the down-coming stream thus being avoided. The maximum brake efficiency of this machine, as determined from the results of a large number of tests is 60.6 per cent.

The maximum efficiency was obtained with a speed of 295 revolutions per minute, giving a nozzle velocity of 14.75 f.s.

In this case, for maximum efficiency $u=1 \cdot 49 \sqrt{g} h$

$$
=1.052 \sqrt{2 g h} \text {. }
$$

From this we have $\sqrt{1+\frac{1}{F}}-1=1 \cdot 49^{2}=2 \cdot 22$.

$$
\therefore \quad F^{\prime}=\frac{1}{9 \cdot 38}=\cdot 1065 \text {. }
$$

Substituting these values in the expression for the theoretical hydraulic efficiency we get $\eta=$ $69 \cdot 2$ per cent., as against the value, 64 per cent., actually obtained.

One form of this wheel, Whitelaw's mill, was constructed with arms in the form of an equiangular spiral (Fig. 174), the idea being that when in motion the water would flow outwards from the centre to the jet in radial lines, and that

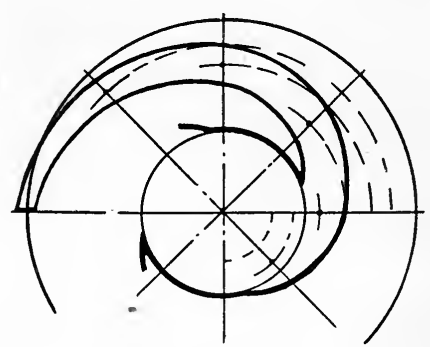

FIG. $17 t$. any loss due to eddy formation would be avoided. A little consideration will, however, show that this can only be the case when $v=u$, and when in consequence no work is being done by the wheel.

\section{ART. 120.}

The Borda wheel was in all probability the first practical pressure turbine to be constructed. This consists of a couple of concentric cylindrical casings (Fig. 175) mounted on a vertical shaft, the space between these being provided with a series of inclined 
vanes. The casing is usually of some considerable depth and the mean radius of the vanes large. Water is led into the casing in a direction almost normal to the vanes and acts partly by

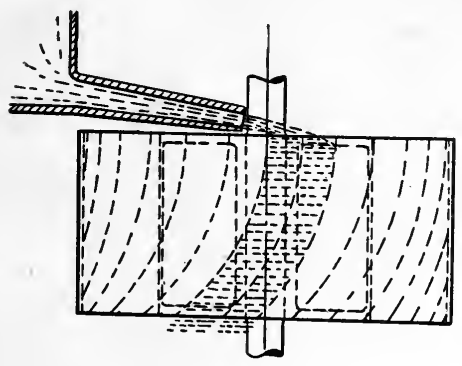
impact. The pressure produced by its weight is, however, the chief factor in producing rotation. Strictly speaking, the wheel is a pressure or impulse turbine according as the buckets run wholly or partially full, this depending on the distance between consecutive vanes. In the Borda, in common with all

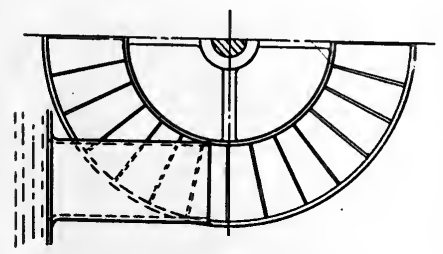

FIG. 175.-Borda Turbine. other axial flow turbines, since the direction of flow is parallel to the axis, the effect of centrifugal force on the flow may be neglected. In spite of its crudity, efficiencies of up to 70 per cent. have been obtained with this, which is the origin of all modern turbines of the parallel flow type.

\section{Art. 121.-The Fourneyron Turbine.}

The first highly efficient pressure turbine was, however, a development of the reaction wheel by Fourneyron (1827). This consists of an outward radial flow reaction wheel (Fig. 176). Water is supplied through the pipe $S$ which is closed at the bottom and terminates in an inverted cone, the outside of the pipe forming the entrance to a series of guide passages $P$. Guided by these passages, the water enters the wheel moving in the same direction as the wheel buckets, impinges on the curved vanes $B$, has its direction of motion changed, and finally escapes around the periphery of the wheel.

This type of turbine has been used with heads from 1 foot to 350 feet, and with moderate heads is capable of an efficiency of about 75 per cent. at full power. With high heads the speed is, 
however, inconveniently high, and the size of buckets and guide passages consequently small, rendering these very apt to be choked. Speed regulation is usually performed either by throttling the supply at its entrance to the wheel by means of a sliding cylindrical or ring gate, or sluice, fitting between the fixed and moving vanes and actuated by means of a governor (Fig. 177), or by throttling the discharge by means of a similar ring gate fitting outside the moving vanes (Fig. 178). This, by

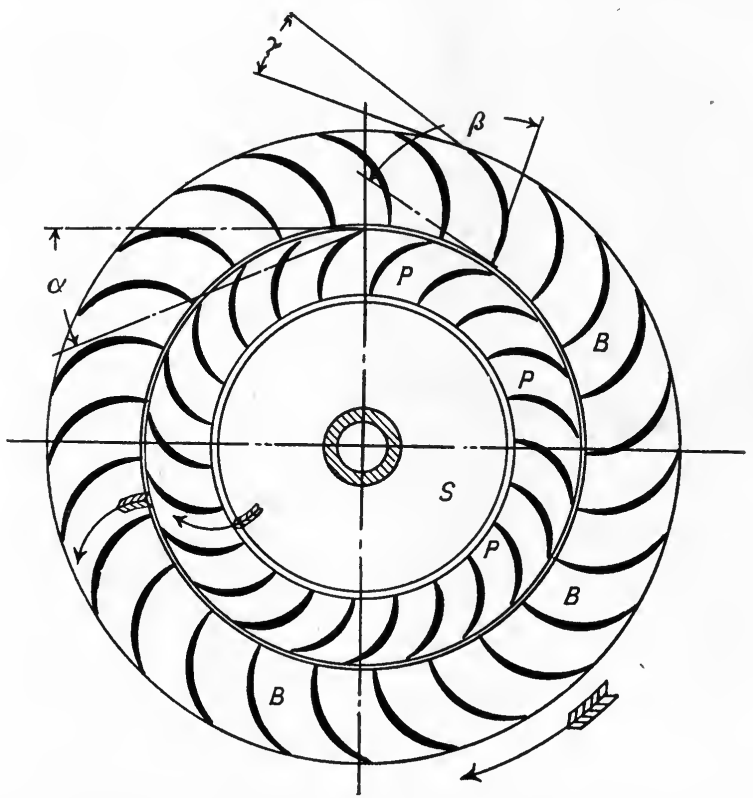

FIG. 176.-Section through Guides and Runner of Fourneyron Turbine.

increasing the pressure in the wheel, reduces the effective head producing rotation.

The former method has the disadvantage that the entering streams of water, after their contraction in escaping past the edge of the ring gate, re-expand to fill the moving buckets with consequent loss of energy. On account of this the efficiency at part gate is low. It may be improved if the turbine wheel be divided by parallel diaphragms as indicated in Figs. 177 and 178 , into what is in effect a series of wheels in parallel.

H.A. 
In such a wheel this enlargement of section after cut off can only affect one chamber, and the part gate efficiency is in consequence increased. The method has the drawback, however, that the areas of the apertures are reduced and also that frictional

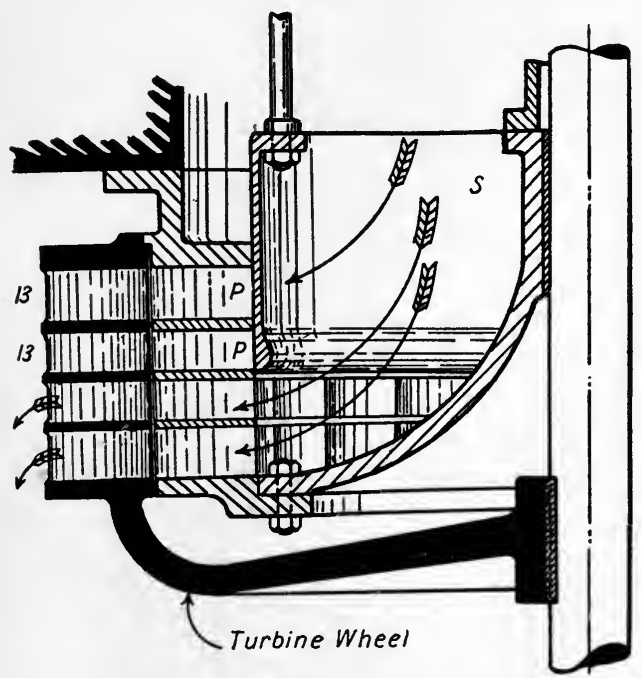

FIG. 177.--Section of Multiple Chamber Fourneyron Turbine Runner and Guides, with Inside Cylinder Gate. resistances to flow are increased by the diaphragms, so that the full gate efficiency suffers. In spite of this, it forms the most general and satisfactory method of governing turbines of this type.

The second method, by increasing the pressure at exit, diminishes the effective head and increases the loss of kinetic energy at exit, and is hence very inefficient at part gate.

In the first turbines erected at Niagara Falls, which were of the Fourneyron type and of 5,500 H.P., this method of governing was adopted with satisfactory results as regards speed regulation. ${ }^{1}$ Outward flow turbines are in general difficult to govern,

1 These turbines were designed by Messrs. Faesch and Piccard (1895), and work under 132 feet head. A full load efficiency of 82.5 per cent. is said to have been obtained. In this first series of turbines the ring gates are arranged to open downwards, the idea being that it is safer to allow the gates to open suddenly in case of accident to the coupling rods than to close suddenly, because of the probable effect of water ram in the latter case. The fact that the lower section of both wheels is not opened for the escape of water except at full load, and the possibility of a dangerous accumulation of detritus taking place in these sections, along with the nuisance caused by the violent upward escape of water against the turbine deck, led to the gates in the second series of turbines being designed to shut downwards. The gates are regulated by a mechanical relay governor having the gearing, which moves the gates, operated by clutches put in motion by changes of speed, the motion of the governor balls allowing one or another pawl to gear 
because an increase in speed caused by a reduction in load increases the centrifugal pressure of the water in the turbine wheel, and thus causes an increased outward flow which tends to increase the speed still further. Governing by throttling the discharge has the advantage that it does to some extent tend to counterbalance this action.

The fact that centrifugal force tends to increase the velocity of flow through the wheel also explains to some extent why the speed of the wheel is of necessity so high under high heads.

The efficiency of the Fourneyron turbine may be increased by

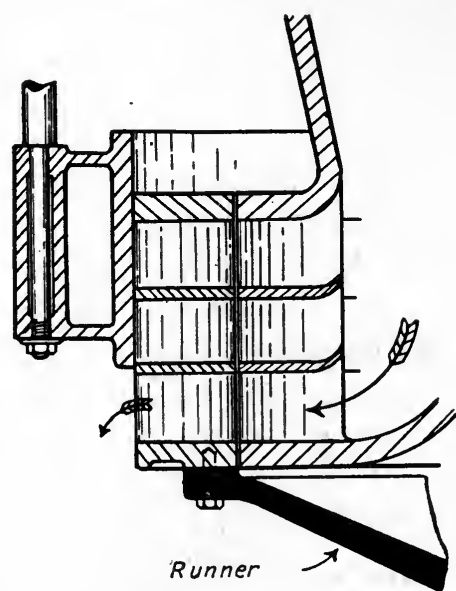

Frig. 178.-Outside Cylinder Gate. the addition of the diffuser, probably invented by Boyden (1844).

This consists of a fixed annular casing (Fig. 179), surrounding the wheel and fitting closely to the outer periphery of the moving vanes. The sides of this casing diverge gradually, its

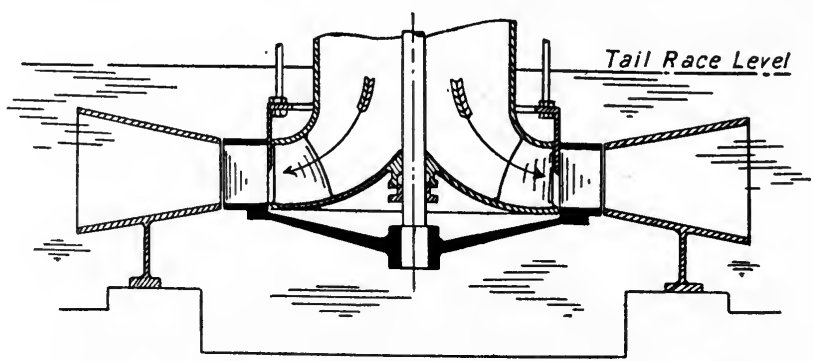

FIg. 179.-Outward Radial Flow Turbine with Diffuser.

depth increasing from that of the buckets to about twice this, and its width being about four times that of the wheel. By with a ratchet wheel, which gives motion through the clutches and gearing to a rack coupled to a lever on the lay shaft from which the gates are directly worked.

A counterbalance weight is used to balance the weight of the gates and coupling rods.

While these turbines have given satisfactory results, the whole of the later series of 5,000 H.P. and 10,000 H.P. turbines are of the inward radial flow type. 
this means, part of the kinetic energy of the discharge is converted into pressure energy, and since the pressure at the outside of the diffuser is that corresponding to the depth of immersion, the pressure at the inside and at the exit from the turbine wheel is less than this, so that the effective head is increased. In this manner the efficiency may be increased by about 6 per cent. in a well-designed turbine under moderate head, the proportional increase being less as the head increases. This device is, however, now practically obsolete.

The outward flow turbine suffers in efficiency from the fact that its passages are of necessity divergent and that flow through these diverging passages is always accompanied by loss of energy in eddy production. It is, moreover, an expensive machine, not easy to govern well, and has been generally replaced by turbines of the Francis or some other more modern type.

The following results of tests on a Fourneyron turbine fitted with an internal cylinder gate, are given by Unwin :-

\begin{tabular}{lll|l|l|l|l}
\hline Gate opening & $\cdot$ & $1 \cdot 0$ & $\cdot 875$ & 625 & $\cdot 375$ \\
\hline Efficiency & $\cdot$ & $\cdot$ & $\cdot 62$ & $\cdot 60$ & $\cdot 43$ & $\cdot 30$ \\
\hline
\end{tabular}

Art. 122.-The Jonval Turbine.

By shortening the casing of the Borda turbine, and adding suitable guide vanes to direct the water under pressure into the wheel, Jonval (1843) devised a form of turbine which had many advantages over and largely displaced the Fourneyron turbines then in use. In this, which is an axial flow turbine, water is directed by means of radial guides into a series of radial buckets (Fig. 180). Since each particle of water in its motion through the wheel remains at approximately the same distance from the axis, the effect of centrifugal force on the flow becomes negligible, and the difficulty in governing which is so pronounced in the Fourneyron turbine is thus removed.

In all axial flow turbines, however, whether of the pressure or impulse type, the linear velocity of points at the entrance to the. 
buckets varies with the radius, and as the velocity of efflux from the guide passages is approximately uniform, there is, with radial vanes, only one particular radius at which the ratio between the velocities of the wheel and of efflux is suited to any given blade angles. For efficient working, and to avoid undue shock at entrance, the radial width of the buckets must then be small
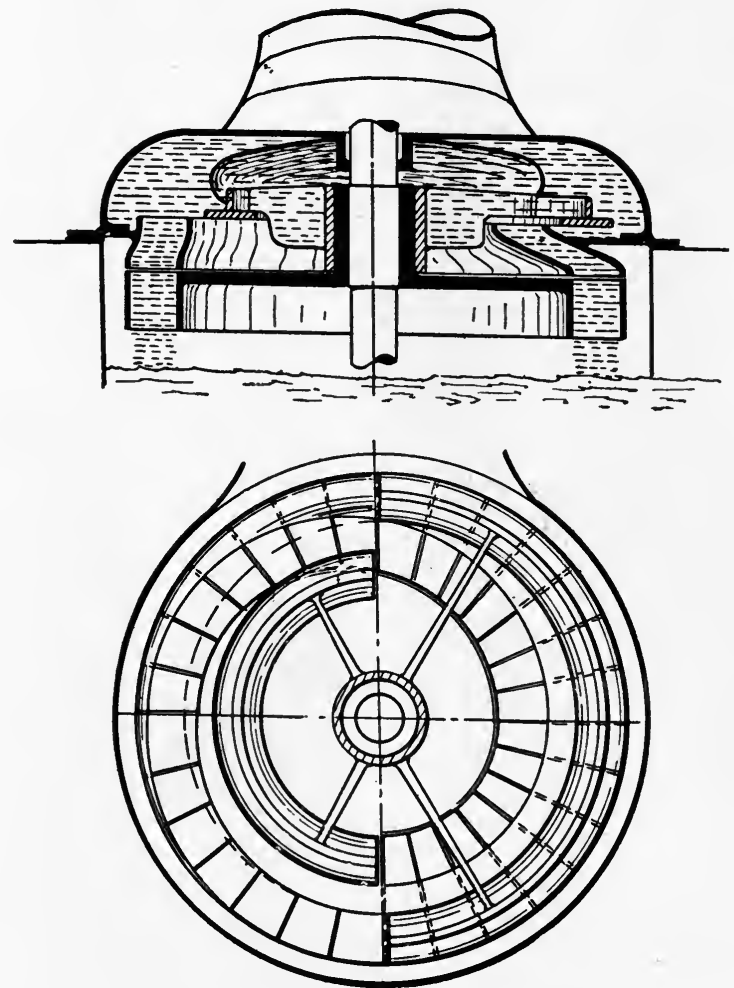

Fig. 180.-Jonval Turbine.

compared with the radius of the wheel, and in general should not exceed $\frac{1}{5}$ of the latter.

Governing is usually performed by cutting off the supply to one or more guide passages by a circular slide (Fig. 181), or by a scroll gate, and this greatly reduces the efficiency at part gate. Thus a turbine which has an efficiency of, say, 82 per cent. at.full gate, will probably not.exceed 66 per cent. at half gate. 
The most important improvement in the design of the Jonval turbine consisted in the subdivision of the wheel into concentric compartments, each forming a complete turbine.

Then by regulating the water supply to each compartment in turn, and, if necessary, completely cutting off the supply to one or more compartments, the wheel may be utilized with fairly high efficiencies, and, by supplying compartments at different distances from the axis, may be given a constant speed under varying heads. Thus with a double turbine the outer compartment, having a

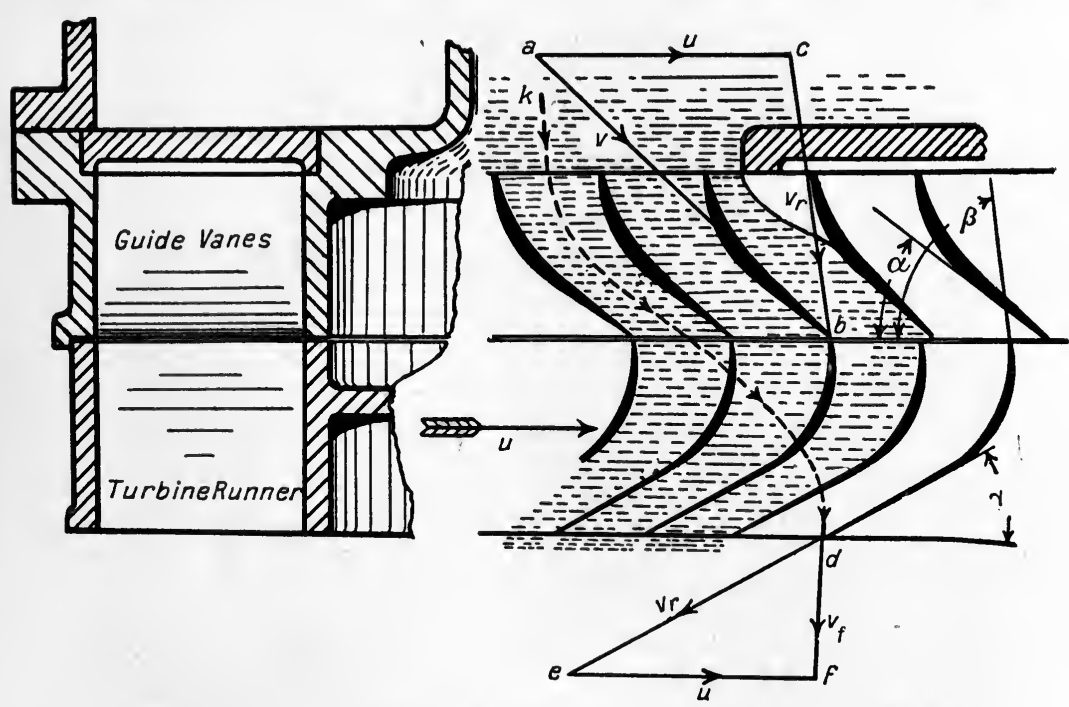

Fig. 181.-Section through Runner and Guides of Downward Flow Jonval Turbine.

higher velocity, would be used with a minimum supply and a maximum head, while the outer and inner compartments together would be designed to give the same power with a reduced head and an increased supply. The vane angles should be different in the two compartments.

In practice, the turbine is regulated by opening the necessary number of guide buckets in one compartment after another, and while causing loss by shock, this is probably the best method of regulation.

In the case of a Jonval turbine, built for the Zurich Water- 
works, to work under a head which varies from $4^{\prime} 9^{\prime \prime}$ to $10^{\prime} 6^{\prime \prime}$, the number of compartments was three. The turbine was designed for 90 H.P., and the outer compartment alone gave this power under the full head, when using 6,300 cubic feet per minute. The outer and middle compartments together gave 90 H.P., under a head of $7^{\prime} 10^{\prime \prime}$, using 8,400 cubic feet per minute, while the three together gave 90 H.P. with a head of $4^{\prime} 9^{\prime \prime}$ using 10,000 cubic ft. per minute. The speed throughout was 25 revolutions, and official tests gave the following results :-

Head.

$10^{\prime} 6^{\prime \prime}$

Outer compartment alone Outer and middle together All three compartments $7^{\prime} 0^{\prime \prime}$

$4^{\prime} 9^{\prime \prime}$
Efficiency.

$73 \cdot 71$ per cent.

$75 \cdot 39$ per cent.

$80 \cdot 70$ per cent.

'This type of turbine combines the advantages of fairly high efficiency at part gate and of constant speed under variable head in a manner which is unequalled by any but the radial inward flow or Francis turbine, and is therefore suitable for variable conditions of working. It may be applied to heads from 2 feet to 180 feet, and for speeds from 20 to 400 revolutions per minute, but is more particularly fitted for low and medium heads with large quantities of water, the large area of the water passages enabling it to pass large volumes of water. Since European users are in general compelled to take advantage of such sources of power, this largely accounts for the favour with which the Jonval turbine was received and for its large development in Europe. Of late years, however, it has been largely displaced by the inward radial flow turbine, and is not at the present time manufactured to any very large extent.

One of its chief disadvantages is, that with a downward flow machine the whole water pressure is transmitted to the step bearing unless preverted by some special device. One method of preventing this is to allow the water to flow upwards through the wheel, while a second method consists in the addition of a balance piston to the turbine shaft as shown applied in Fig. 183, one side of this piston being exposed to the supply pressure, and any leakage past it being removed to the tail-race by drain pipes. 
By suitably proportioning the area of this piston, it then becomes possible to balance not only the water pressure on the runner but also the weight of the rotating parts.

In any case a thrust bearing must be provided to take care of any unbalanced pressure, this consisting either of an ordinary submerged step bearing with the shaft running in a lignum-vitæ bush, or preferably of a suspension bearing placed above tail-water level. The latter method provides obvious

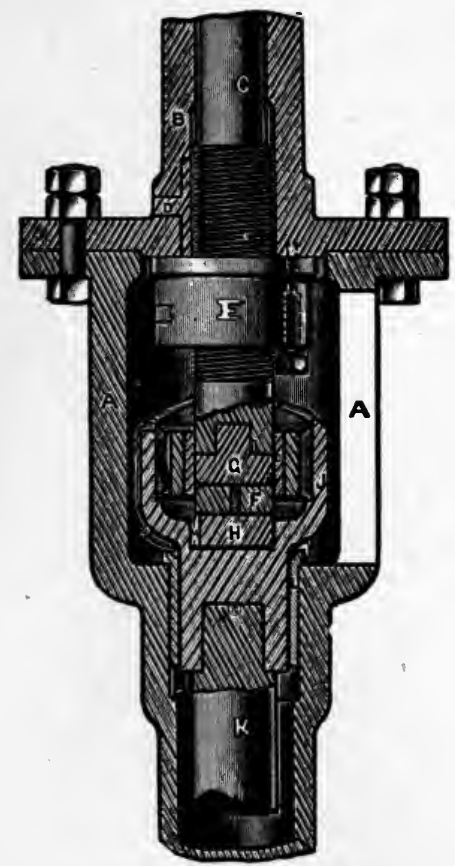

Fig. 182.--Suspension Bearing for Vertical Turbine Shaft. advantages in the way of accessibility, ease of examination, and freedom from grit, and enables forced lubrication to be applied. One type of such a bearing is illustrated in Fig. 182. ${ }^{1}$ Here the turbine wheel is keyed to a hollow shaft which terminates at its upper end in the lantern $A$. This lantern is connected by the feather key $D$ to the steel shaft $C$, which is fitted with a locknut $E$, by which the vertical adjustment of the wheel may be altered.

A gun-metal washer $F$ works between the hardened steel dises $G$ and $H$, of which $G$ is rigidly fixed to the bottom of the shaft $C$, and $H$ to the oil cup $J$, which in its turn is cottered on to the shaft $K$. This latter shaft is continued downwards and is firmly fixed in a cast-iron socket on the tail-race floor. The bottom end of the shaft $C$ is prevented from moving laterally by the gunmetal bush $O$, and a series of radial grooves on each face of the washer $F$ enable oil to reach every part of the bearing surfaces.

The comparative ease of regulation by cylindrical gates, together with the large passage areas possible with the axial

1 By kind permission of Messrs. Gilbert Gilkes \& Co., Ltd., Kendal. 
flow type of wheel, led to the design, for fairly large powers under low heads, of the cone turbine.

Here, as indicated in the sketch (Fig. 183), ${ }^{1}$ the flow is diagonal

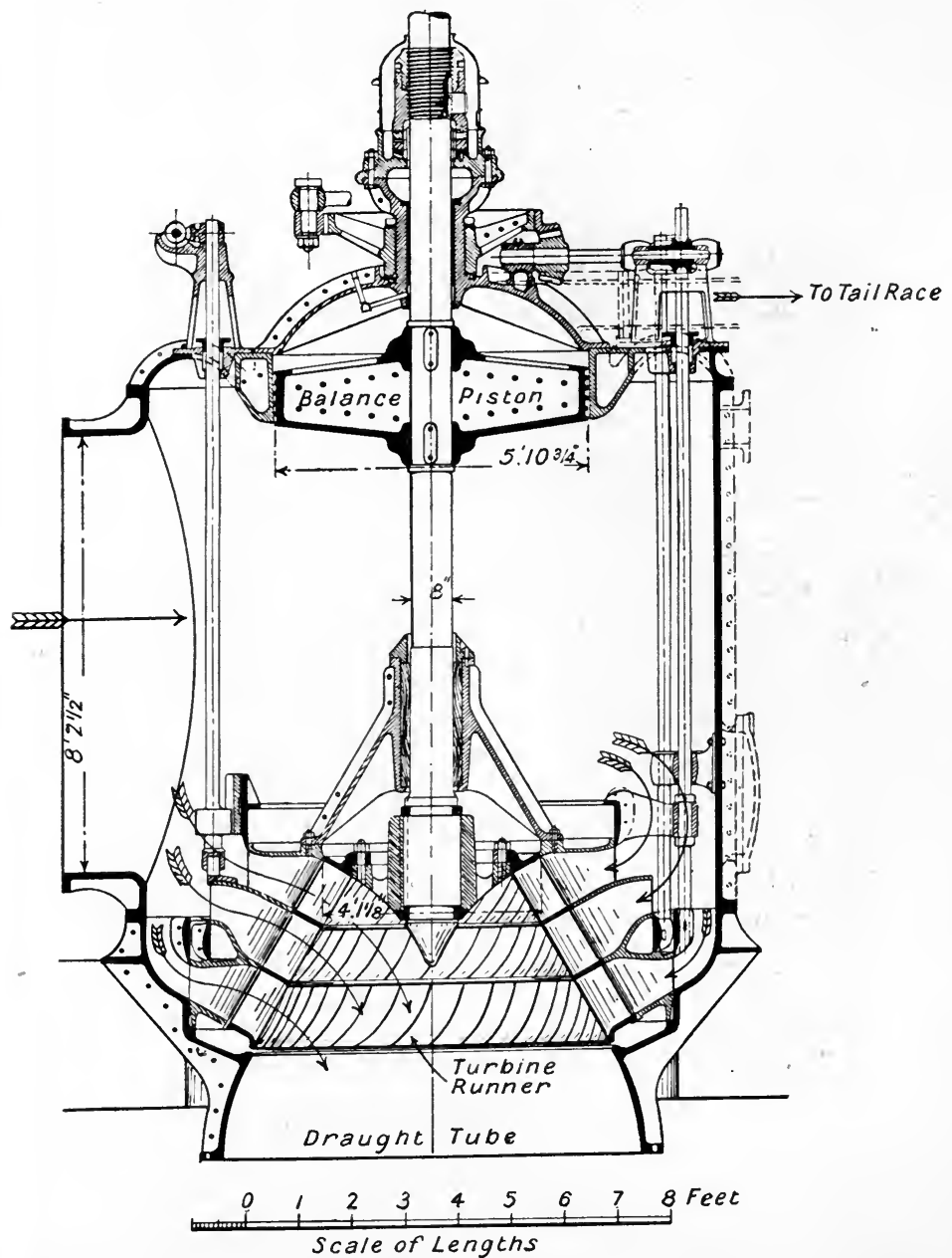

FIG. 183.-Cone Turbine, 1,250 H.P., Head $26 \mathrm{ft}$. to $33 \mathrm{ft.} .120$ Revolutions, with Water Balance Piston.

from its entrance to its exit from the wheel, and the turbine becomes intermediate between the inward radial and the axial flow

By permission of Messrs. Escher, Wyss \& Cie., Zurich. 
type. As shown, the wheel may be subdivided into several complete wheels of different diameters, each of which may be regulated by cylindrical gates. This type, which has only been manufactured to a limited extent, is thus very suitable where a constant speed is required under a low head which may suffer considerable percentage variation.

\section{Art. 123.-The Suction Tube.}

An invention of Jonval (1843) greatly increased the possibility of adapting the pressure turbine to suit local conditions. This

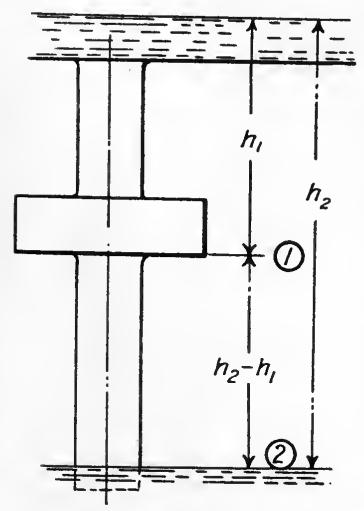

FIG. 184. consists in lengthening the vertical discharge pipe until its lower end always discharges below the surface level in the tail-race, thus forming what is termed a Suction or Draught tube. By this means the turbine may be placed at any level, up to about 25 feet $^{1}$ above the tail-race without any loss of head. 'The truth of this statement may be seen if we consider that since the pressure in the draught tube at the tail-race level is approximately equal to that of the atmosphere (neglecting the kinetic head in the tube), the pressure at the turbine will be less than this by an amount equivalent to the difference of statical head at turbine and tail-race, so that the available head at the turbine is equal to the difference of level between turbine and head-race, together with the difference between turbine and tail-race, i.e., to the

1 The maximum elevation depends largely on the diameter of the draught tube, the following values, adapted from those given by Meissner, showing the maximum values to be used with a given diameter of tube.

\begin{tabular}{|c|c|c|c|c|c|c|c|c|c|c|}
\hline Diameter of suction tube in feet & $\cdot 5$ & $1 \cdot 0$ & $1 \cdot 5$ & $2 \cdot 0$ & $2 \cdot 5$ & $3 \cdot 0$ & $4 \cdot 0$ & $6 \cdot 0$ & $8 \cdot 0$ & $10^{\circ} 0$ \\
\hline $\begin{array}{l}\text { Maximum possible elevation in } \\
\text { feet. }\end{array}$ & $31 \cdot 1$ & $29^{\circ} \mathrm{S}$ & 28.0 & $26^{\circ} 4$ & $24 \cdot 8$ & $23 \cdot 4$ & 21.0 & $17 \cdot 0$ & $14 \cdot 0$ & $12 \cdot 2$ \\
\hline
\end{tabular}

From these values, however, should be subtracted the head $\frac{v^{2}}{2 g}$, corresponding to the velocity of flow $v$ feet per second down the tube. 
difference between head-race and tail-race. Expressed symbolically, we have, if suffixes (1) and (2) refer respectively to turbine and tail-race (Fig. 184),

$\Varangle \frac{p_{1}}{W}=\frac{p_{2}}{W}-\left(h_{2}-h_{1}\right)$ feet.

$\therefore$ (Pressure 7 potential) head at exit from turbine, which equals $\frac{p_{1}}{W}+h_{2}-h_{1}$ feet.

$$
=\frac{p_{2}}{W}-\left(h_{2}-h_{1}\right)+h_{2}-h_{1}=\frac{p_{2}}{W}=0 .
$$

$\therefore$ Available head for driving turbine $=h_{2}-\frac{p_{2}}{W}$ feet.

$$
=h_{2} \text { feet. }
$$

In order that on starting a turbine the air may be carried out of the draught tube, the velocity of flow through the tube should be greater than 2 feet per second. Also the area of the tube at its point of connection to the turbine, should be as nearly. as possible equal to the discharge area of the runner so as to avoid loss by shock at the sudden change of section. The area should then increase gradually to the open end of the tube, the angle of flare not exceeding about $15^{\circ}$ and decreasing as the length of tube increases. This serves two useful purposes, since, in addition to changing part of the kinetic energy of discharge into useful pressure energy, it usually improves the speed regulation of the plant. With quick regulation and a sudden closing of the turbine gates, the momentum of the suction column may break the column and cause a vacuum at the turbine. ${ }^{1}$ Immediately this action is

1 Let the suction tube be parallel ; $l$ feet long; dipping $l d$ feet below the surface in the tail-race, and suppose air leakage increases the pressure at the top of the tube by the equivalent of $h_{a}$ feet of water.

Thus for separation due to downward momentum we must have

$$
\begin{gathered}
3 t-\left(l-h_{d}\right)-h_{a}=\frac{l}{g} a \\
\therefore \quad a=\frac{32}{l}\left(34+h_{d}-l_{a}\right)-32 \text { feet per second per second. }
\end{gathered}
$$

EXAMPLE.

$p_{a}=2$ lbs. per square inch, $h_{a}=4 \cdot 6$ feet, $l=28$ feet, $h_{d}=3$ feet.

Thus $\alpha=\frac{32}{28}(3 \tau-4 \cdot 6)-32=5$ feet per second per second.

If $v=8$ feet per second, separation would take place if the gates were shut in less time than 1.6 seconds. Actually since the retardation is not uniform during a 
orercome, atmospheric pressure forces water up the tube again, this striking the runner with great force. Even a small change of load may set up such pulsations, which are detrimental to steady running and are reduced by the use of a conical draught tube.

When fitted to a pressure turbine, water will, in general, enter the draught tube with a not inconsiderable velocity of whirl, and with a tube of large diameter, when working at part gate, an air core may be formed in the tube when starting up the plant, and may exist for some considerable time before being expelled, the turbine then partially losing the advantage of the tube. To obviate this, gates for throttling the lower end of the tube have sometimes been used. While advantageous when starting the turbine, they are, however, not often fitted on account of the expense.

The lower end of a draught tube should always be bell-mouthed to facilitate the escape of water.

The draught tube is applicable to any type of pressure turbine, but more particularly, for mechanical reasons, to the inward flow type. It is not commonly used with impulse turbines, though it may be fitted if desired.

The pressure turbine thus fitted possesses many advantages over the submerged type. The turbine runs dry when the head water is cut off, and is easily accessible: a horizontal shaft machine may frequently be adopted where otherwise this would be impossible, and the general accessibility for examination and repairs is greatly increased, while the risk of damage to machinery by floods is diminished.

With a single horizontal shaft turbine, or with two horizontal turbines on the same shaft if these discharge outwards, it is necessary for the wheel shaft to pass through the draught tube as in Fig. 185, and a stuffing box becomes necessary to prevent leakage of air into the tube. Tightness is commonly assured by means of a water seal, consisting of a chamber $C$ surrounding the shaft (Fig. 186), and supplied with pressure water from the

uniform closing of the gates, but increases to a maximum at the instant of closing, this retardation would probably be attained if the time of closing were less than three seconds. 


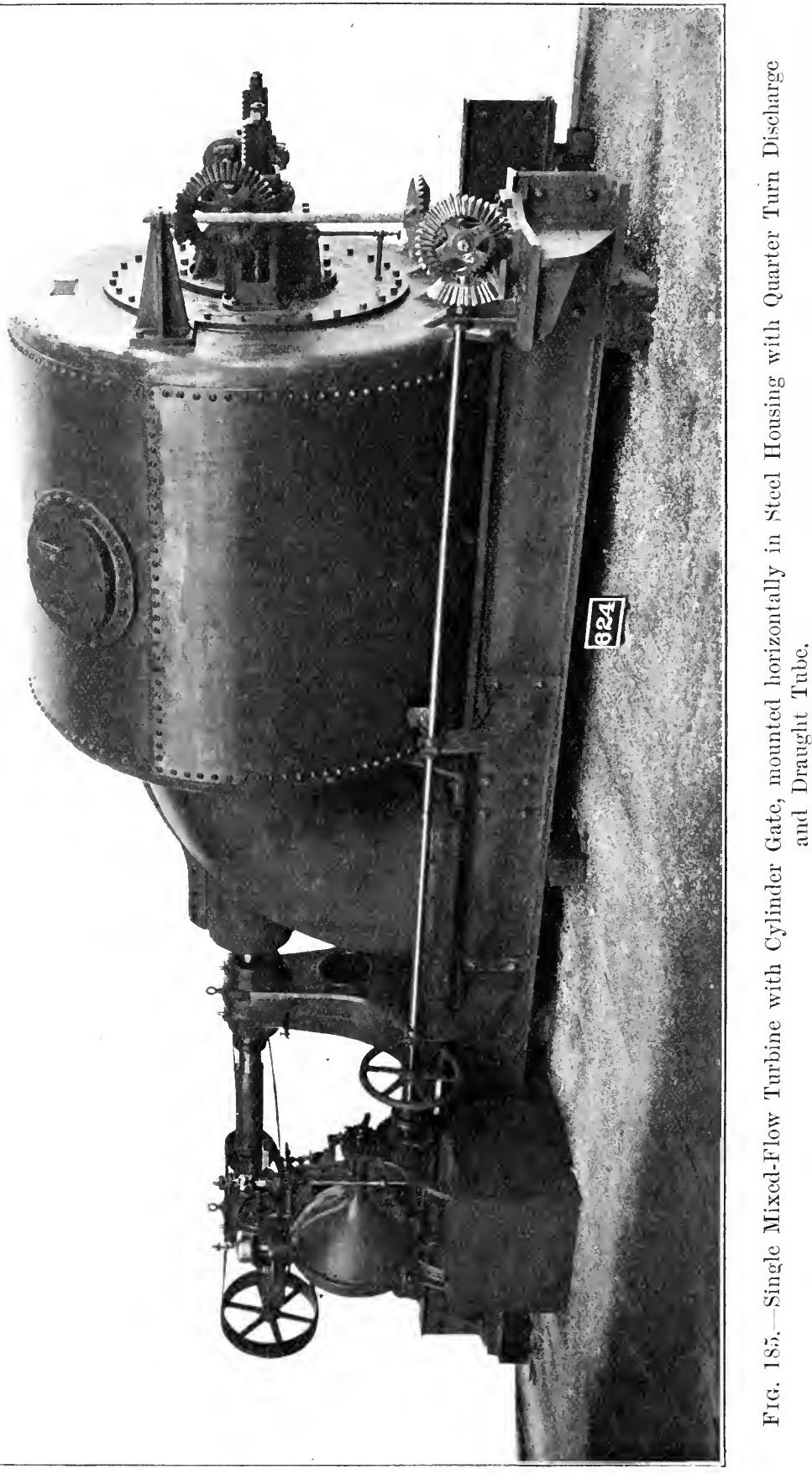


penstock by means of a small pipe $P$. Any slackness at the gland then allows this pressure water to escape outwards, or into the draught tube, and does not lead to air

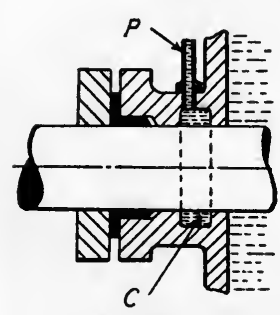

Fig. 186.-Water Seal for Draught Tube Stuffing Box. leakage.

While the draught tube usually discharges vertically into the tail-race, it is advantageous to fit a right-angled bend beneath the water level at exit, and to discharge in the direction of flow of the tail-race. By so doing the kinetic energy of discharge is not entirely wasted, a fair proportion being utilized in producing this flow. A still further proportion may be utilized if the suction tube be made of a gradually increasing section towards its exit.

\section{Art. 124.-The Francis Turbine.}

The next important step in turbine development was due to J. B. Francis (1849), who, placing the guide vanes outside the wheel, and reversing the direction of flow of the Fourneyron turbine so as to discharge at the centre, obtained the inward radial flow turbine bearing his name (Fig. 187).

Several important advantages accompanied this change in design.

(1) The inlet ports and wheel passages now being convergent, steady flow became possible throughout the wheel.

(2) The increased accessibility of the guide passages and vanes made it possible to use improved methods of regulating the flow of water to the wheel.

(3) Any increase in the speed of rotation, by increasing the centrifugal pressure at the outside of the wheel and at the outlet from the guide passages, tends to check the flow through and the supply of energy to the wheel, which now becomes to a certain extent self-regulating.

(4) 'The centrifugal pressure of the water also balances the pressure due to a portion - in practice generally about one-halfof the supply head, so that only one half of the pressure head at entrance is absorbed in producing velocity of influx, the other 
half remaining as pressure head and being gradually absorbed in its passage through the wheel. The velocity of influx is thus never greater than about $\sqrt{g h}$, and hydraulic friction losses are thereby much reduced, while the corresponding reduction in the peripheral speed of the wheel enables this to be applied successfully to very high heads, up to about 350 feet. Since the mean velocity of flow in an inward flow turbine is less under a given head than in an outward flow, or axial flow machine, the size of wheel is greater for a given power. As the size is usually increased axially, the extra cost is, however, only small.

(5) In this type of turbine the supply water when moving with its maximum velocity (at entrance), is admitted to the wheel at its outer circumference, and consequently at its most rapidly moving part. It thus becomes practicable to design the guide passages and inlet vanes so that even for the highest heads water may be admitted to the wheel without shock, while since the water leaves at the

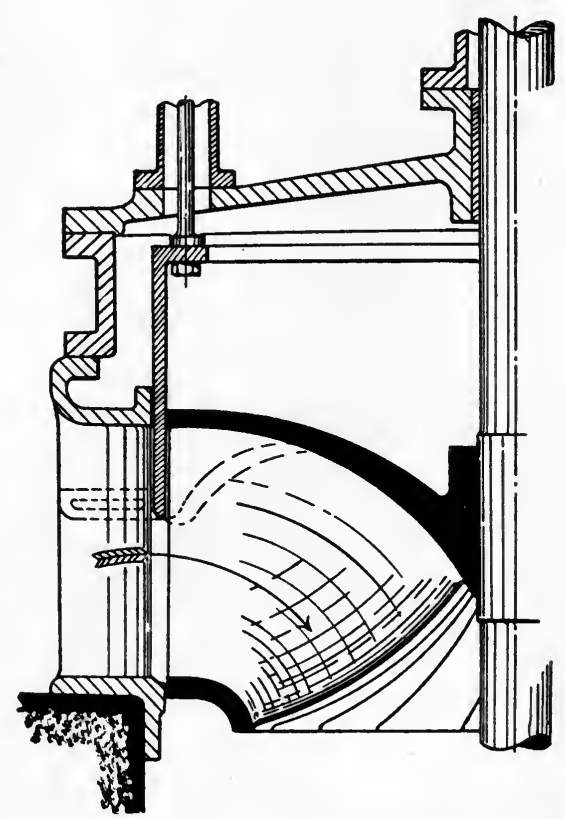

FIG. 187.-Francis Turbine with Inside Cylinder Gate Regulation. centre, which is the most slowly moving part of the wheel, it is more easily discharged without excessive loss of kinetic energy.

The wheel was improved by constructing its vanes so as to give a combined radial and axial discharge, and to this end these vanes at exit are given a curvature in a direction parallel to that of the dise (see Fig. 187). This largely increases the available discharge area and enables a greater volume of water to be handled with a given size of wheel.

Regulation is usually performed by throttling the supply of 
water to the wheel either by sliding cylindrical gates, register gates, or wicket gates. The cylindrical gate usually consists of a plain cylinder throttling the supply at the entrance to the wheel buckets (Fig. 187). In some instances these gates have been fitted with fingers fitting between the guide vanes, as indicated in dotted lines in Fig. 187, with the idea of diminishing the contraction in section of the entrant stream, and thus the loss by shock at part gate. While this object is realized to a limited extent, yet the introduction of the fingers, as will be explained later (Art. 128), renders close speed regulation almost impossible.

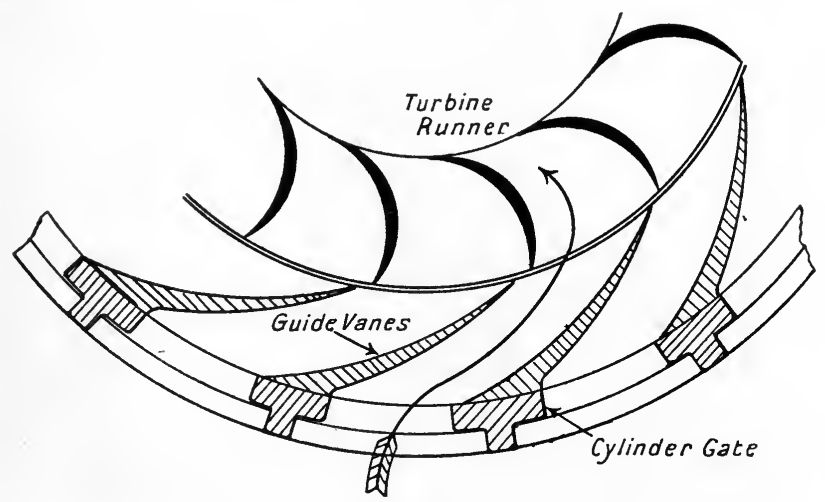

FIG. 188.-Outside Register Gate.

The register gate, which consists of a rotating cylinder gate, and of which two types are illustrated in Figs. 188 and 189, has the advantage that the travel necessary to cut off the supply is small, and therefore tends to more rapid regulation. On the other hand, it is very liable to get out of order where floating material in the water may jam between gate and guide or wheel vanes, and is less efficient than the plain cylinder gate at part load. In view of these disadvantages, these gates are now practically obsolete. The latter remark also applies to the original form of wicket gate, which, as its name suggests, consisted of a series of plates pivoted either at their ends or preferably at their centres, and which throttled the supply by closing the guide passages as the plates were rotated. As modified by Professor Thomson and by Fink and others, the wicket gate, however, gives very close 
and efficient regulation under widely varying conditions. It will be considered in further detail in Art. 125.

Where a draught tube is fitted, regulation may be performed by means of a butterfly valve placed in the tube so as to throttle the discharge. The drawback to this system is that the valves are

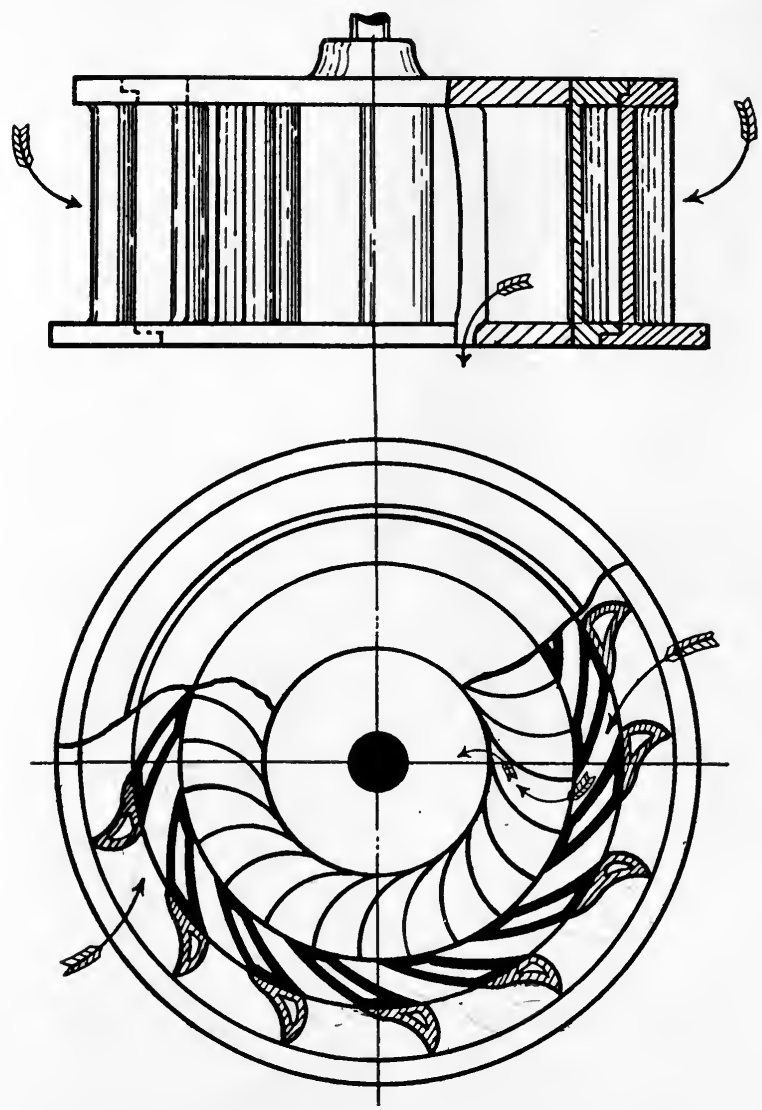

FIG. 189.-Inside Register Gate for Francis Turbine.

large and heavy, possess considerable inertia, and require a large force to move them, while part-gate efficiency is low. This method of governing has been adopted with success as regards speed regulation in the case of Francis turbines of 6,000 H.P. working under 135 feet head at the Shawinigan Falls. ${ }^{1}$ Here

1 For a description of the plant see Cassier's Magazine, June, 1904. 
the penstocks are of considerable length, and the method promised to give better results than gates on the pressure side of the turbine runner.

Figs. 190 and 191 illustrate noteworthy examples of Francis turbines with cylinder gate regulation. Fig. 190 shows one of a series of turbines installed in 1900 in Power-house No. 2 of the Niagara Falls Power Company. ${ }^{1}$ 'These are single Francis tur-

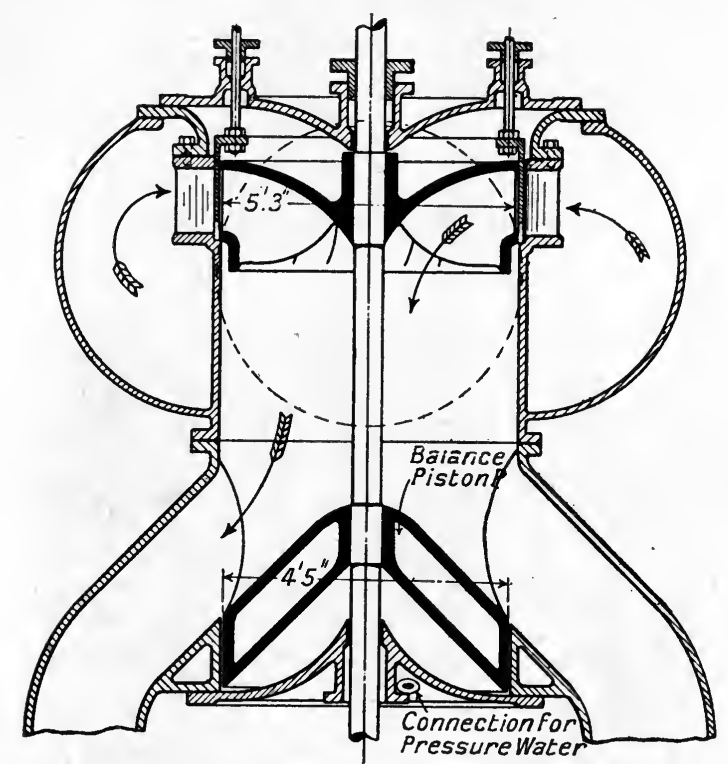

FIG. 190.-Francis Turbine with Cylinder Gate Regulation developing 5,500 H.P. at 250 revs. per min. under $146 \mathrm{ft}$. head. Penstock $7^{\prime} 6^{\prime \prime}$ diam.

bines with vertical shaft direct-coupled to a dynamo, and developing 5,550 H.P. at 250 revolutions per minute under a head of 146 feet. The turbine runner is $5^{\prime} 3^{\prime \prime}$ outside diameter, and regulation is performed by an annular bronze ring operated by an oil pressure relay governor (see Art. 128), and arranged so as to close by its own weight.

Power is transmitted through a tubular steel shaft 38 inches in diameter, the weight of this shaft, which is $\mathbf{1 2 0}$ feet long and weighs 71 tons, being balanced by the upward pressure on the

1 By Messrs. Escher, Wyss \& Cie., Zurich. 

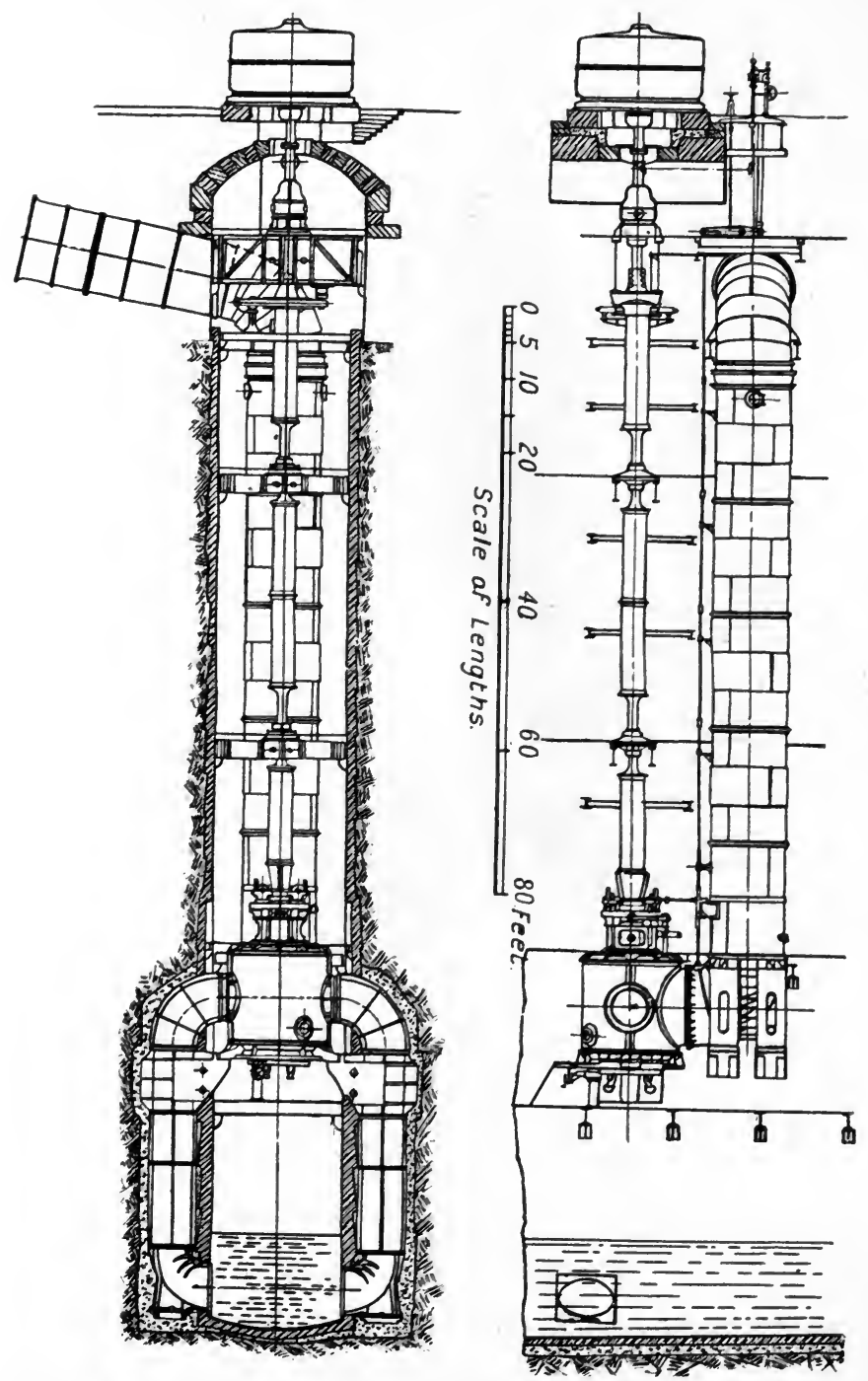

FIG. 191 A.-General arrangement of Double Francis Turbine developing 10.2.0 H.P. at 250 revs. per min. under $133 \mathrm{ft}$. head. Canadian: Niagara P'ower Co. 
balance piston $P$, which is $4^{\prime} 5^{\prime \prime}$ diameter. The under side of this piston is exposed to the full supply head by means' of a pipe not shown in the sketch, and any leakage past the piston escapes directly into the tail-race. Any unbalanced end thrust on the

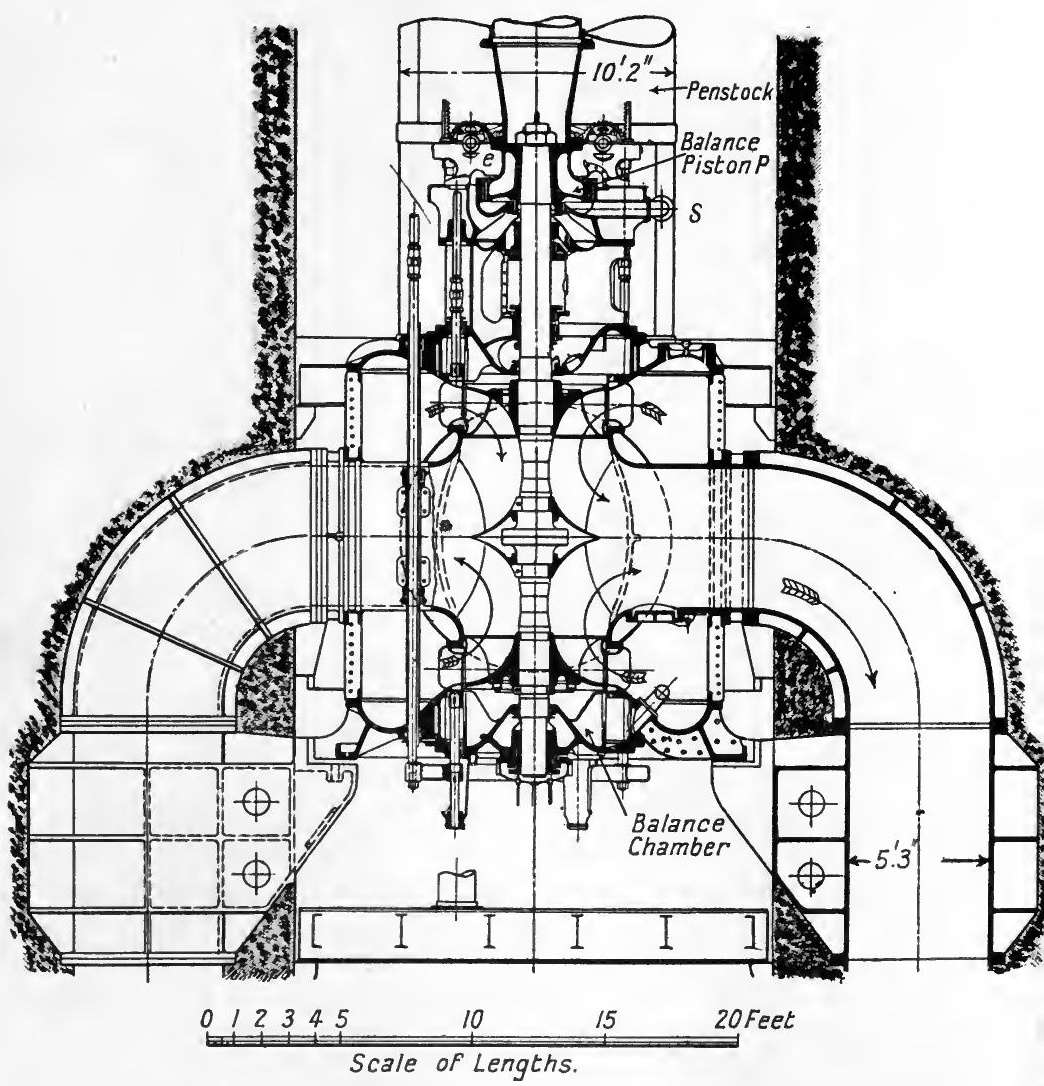

Fuc. 191 B.--Sectional Elevation of Double Francis Turbine for 10,250 H.P. Canadian Niagara Power Co.

shaft is taken up by means of a thrust bearing on the top deck. The draught tube is forked to keep the tail-race free, and is arranged so as to give 22 feet of suction head.

Figs. 191 A, B, C, D and E, illustrate one of a series of double Francis turbines installed $(1903)^{1}$ in the power-house of the 1 By courtesy of Messrs. Escher, Wyss at Cie. 
Canadian Niagara Power Company. These are vertical shaft machines, each direct-coupled to a generator and developing 10,250 H.P. at 250 revolutions under a head of 133 feet.

Power is transmitted through a tubular steel shaft 40 inches diameter and 582 inches thick. The weight of the rotating parts is about 120 tons and is balanced, partly by the upward pressure on the bottom face of the lower runner, water under the full pressure of the supply head being admitted to a balance

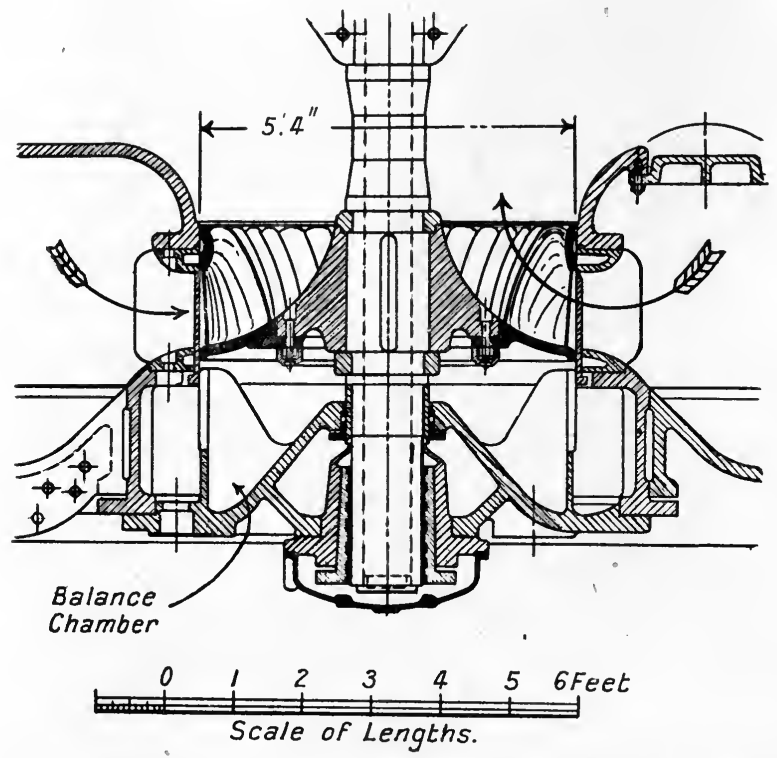

Fig. 191 c.-Section through Lower Runner and Balance Chamber of 10,250 H.P. Turbine.

chamber (Fig. $191 \mathrm{c}$ ), beneath this runner, and partly by the upward pressure on the rotating balance piston $P$ (Fig. $191 \mathrm{~B}$ ), which is mounted on the turbine shaft, and the under side of which is exposed to the full pressure of the supply head. Leakage past this piston is drained away to the tail-race, and by adjusting the valve on the pipe $S$ which supplies pressure water, the upward pressure on the piston may be regulated with great nicety. Any unbalanced load is supported by the suspension bearing (Fig. $191 \mathrm{D}$ ), which is placed on the upper deck. .In this bearing, oil under a pressure of $375 \mathrm{lbs}$. per square inch is 
supplied to the annular chamber $C$ surrounding the bush $B$, and

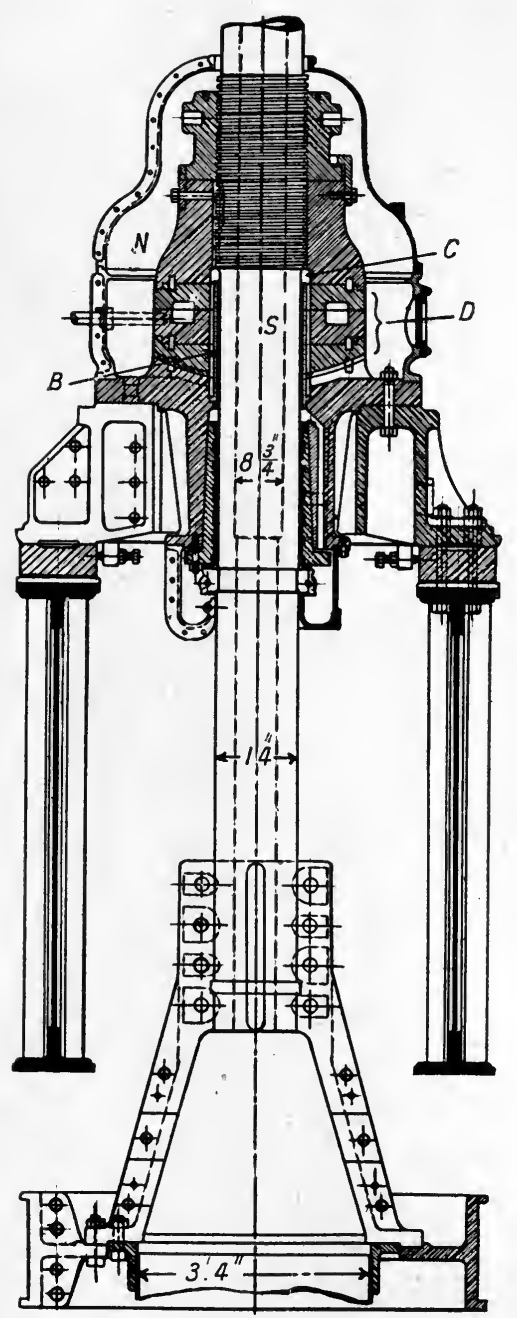

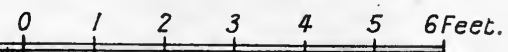

Scale of Lengths.

FIG. 191 D.-Main Footstep Eearing for 10,250 H.P. Turbine.

escapes outwards between the fixed and rotating discs at $D$. These discs have an outside diameter of 36 inches and a bearing area of about 780 square inches, the upper thus floating on a film of oil and giving a very frictionless bearing. The hollow screwed spindle $S$ is $14^{\prime \prime}$ outside and $83^{3 \prime \prime}$ inside diameter, and is provided with two locknuts at $N$,- by means of which the turbine shaft may be adjusted vertically. Any slight swing or lateral wear of the shaft is permitted by the spherical bearing surface of the lower disc.

Speed regulation is performed by a centrifugal governor (Fig. 191 E), operating a regulating valve which regulates the supply of oil (maintained at a pressure of 1,120 lbs. per square inch) to the upper side of a relay cylinder $C$, the downward pressure on this piston overbalancing the weight of the gate mechanism and thus operating the cylindrical speed gates. Adjustment of the speed while the turbines are running may be performed by the hand regulating wheel $H$. 
The turbine runners have a diameter of 64 inches and a blade depth of 11.8 inches, while each carries twenty-one vanes. The guide vanes are twenty-two in number. 'The tail-race which takes

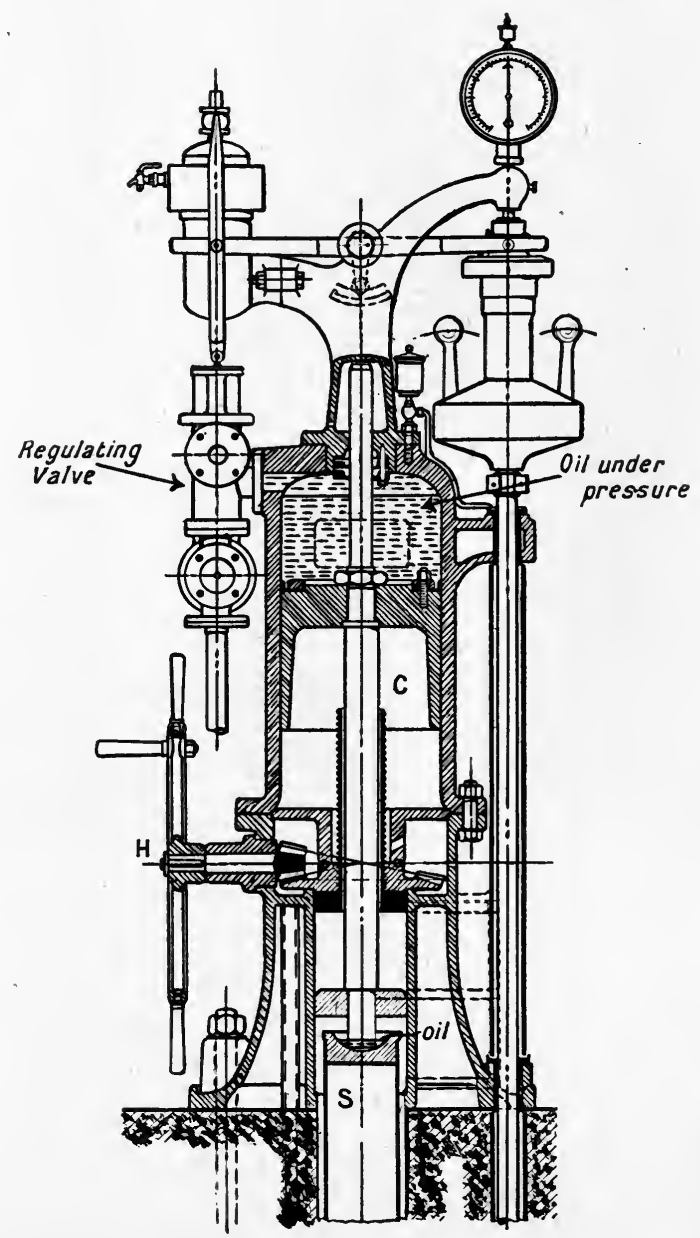

FIG. 191 E.- Oil Pressure Relay Governor for 10,250 H.P. Turbine.

the discharge from the whole battery of turbines is 2,590 feet in length, and has a gradient of 7 in 100 , being designed to discharge, at full load, 8,800 cubic feet of water per second. As the 
sectional area of the tail-race tunnel is 366 square feet, this gives a velocity of efflux of 24 feet per second.

With the Francis turbine and cylindrical gate regulation, the full-load efficiency may be as high as 86 per cent., but this efficiency falls rather rapidly as the gate opening is diminished, and will not in general exceed 75 per cent. at half gate. Two Francis turbines installed at Schaffhausen in an open water chamber, and developing at full load 434 H.P. at 169 revolutions and under 13.6 feet head, gave the following efficiencies :-

\begin{tabular}{lr|c|c|c}
\hline Gate opening · & $\cdot$ & $\frac{1}{2}$ & $\frac{3}{4}$ & Full. \\
\hline Efficiency & $\cdot$ & $77 \%$ & $82.6 \%$ & $86 \cdot 6 \%$ \\
\hline
\end{tabular}

Where an ample water supply is available this falling off in efficiency is unimportant, and as this turbine admits of a simple and fairly cheap construction, and is capable of close speed regulation, it often forms the most suitable type to use. Where, however, high part-gate efficiency is more important than low first cost, one form or other of the turbine invented by Professor James Thomson becomes advisable.

Art. 125.-The Thonson Vortex 'T'urbine (Fig. 192). ${ }^{1}$

The special features of this turbine, which is an inward radial flow machine, consist in the form of chamber in which the runner is mounted, called the vortex chamber, and in the type of guide vane adopted. The runner, which is similar to that of the Francis turbine, is mounted inside a spiral casing. Water enters tangentially at the largest part of the spiral (as shown in Fig. 193), and sweeps around the casing, the area of which is so arranged that the linear velocity of the supply water is the same at all points of the circumference. A common, but less correct type of construction, is that indicated in Figs. 192 and 194, in which the water enters the casing radially and divides into two streams flowing along either side of the wheel. In either case the entering water is directed into the wheel

1 By courtesy of Messrs. G. Gilkes d Co., Ltd., Kendal. 
buckets by a series of guide vanes placed around the outer circumference, these being designed so as to follow the lines of flow in a spiral vortex. 'These guides vary in number from four'

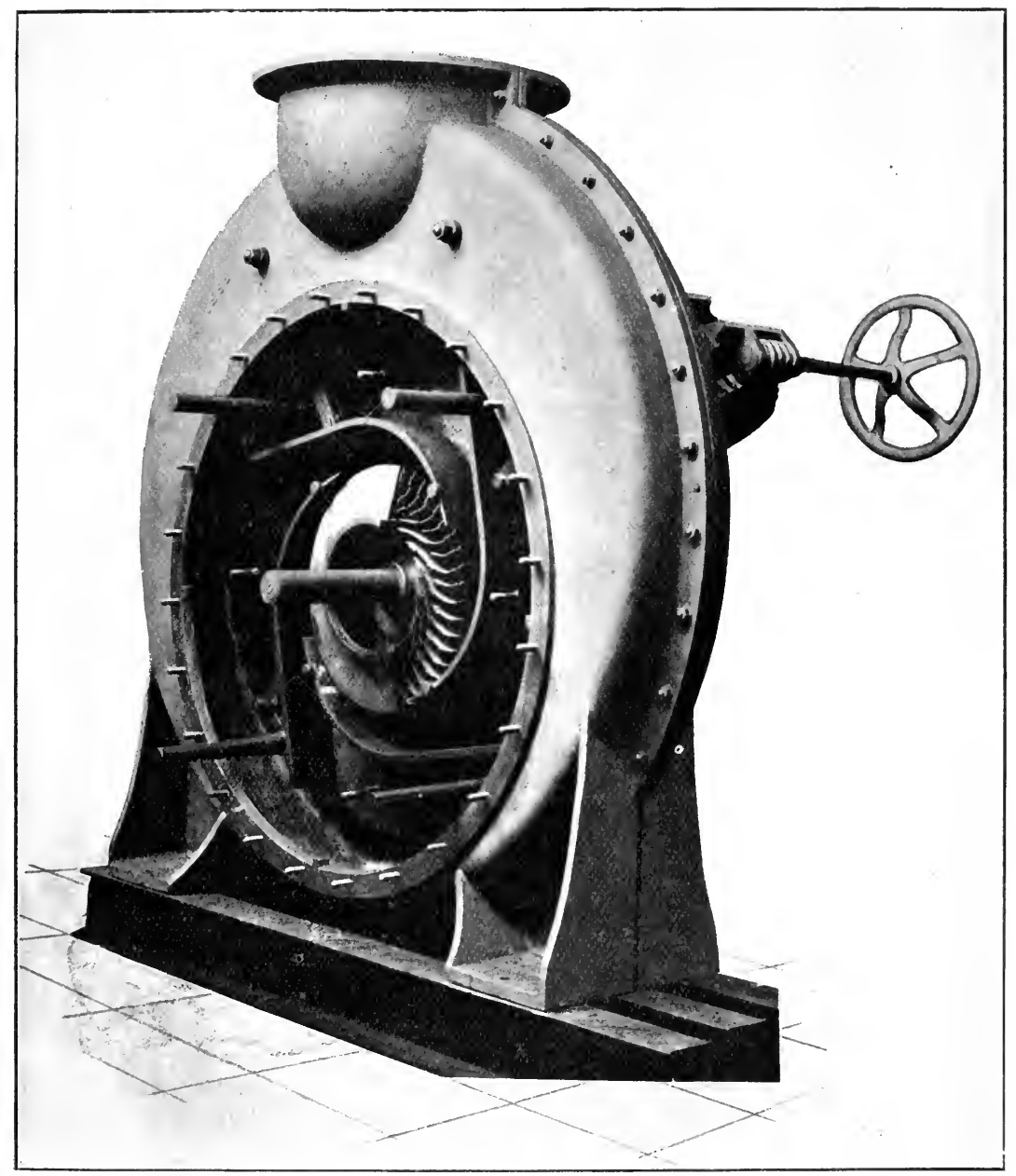

FIG. 192.-Thomson V'ortex 'I'urbine.

to eight and are movable, being pivoted near their inner ends, so as to be approximately in balance. They are coupled together by a series of bell-crank levers and links as indicated in 
Fig. $193,{ }^{1}$ so as to rotate together and shut off water equally from all parts of the wheel. Motion is given to these guide vanes either

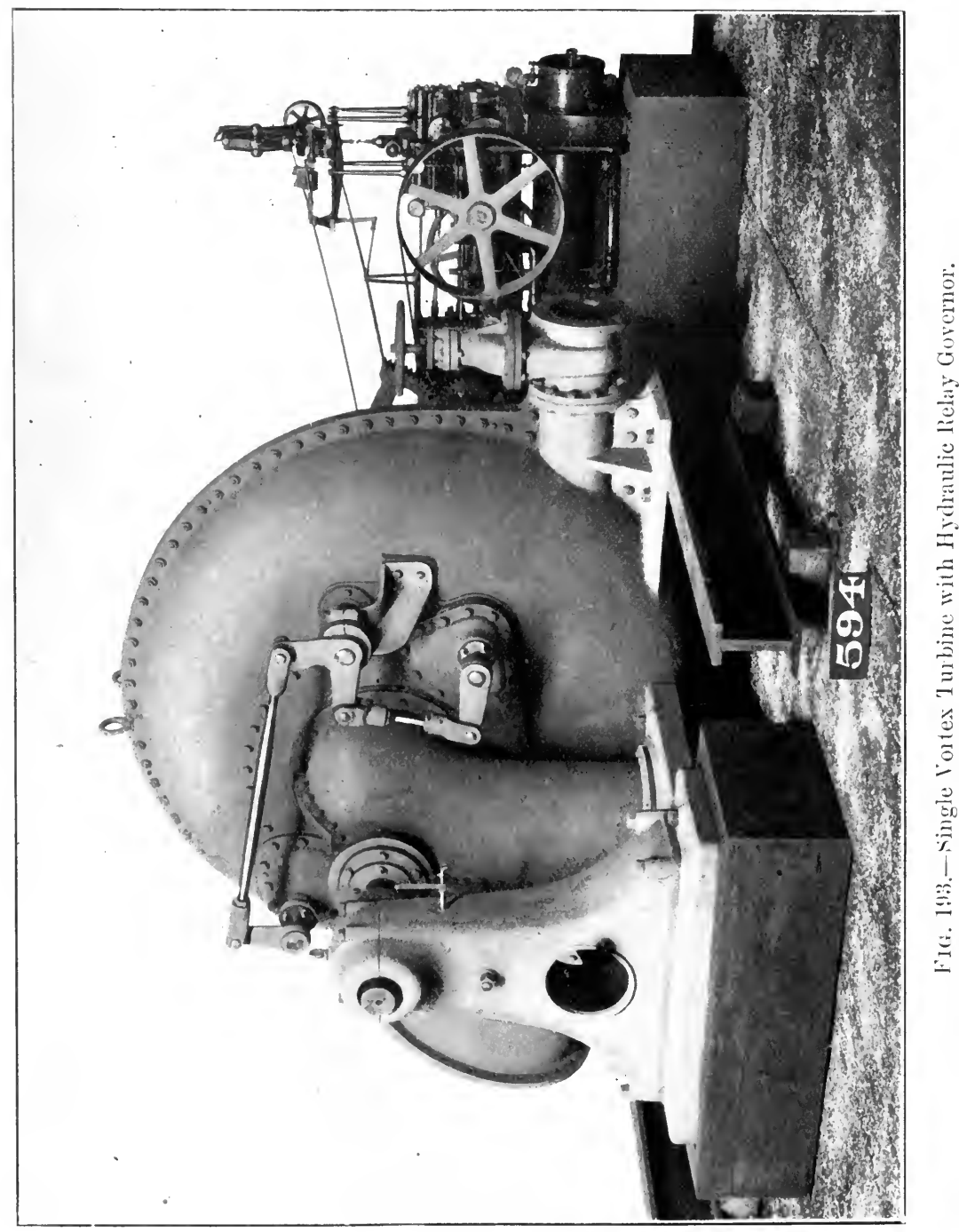

by hand or by an automatic governor. Where the load and the supply head are constant the guide blades may be fixed, the ports By courtesy of the Platt Iron Works, Dayton, Ohio. 
then being designed so as to give the full-power flow. This gives a much cheaper machine, and where a battery of turbines is in use, it is in general preferable to give fixed blades to all but one or two, regulation for small load variations being performed by those machines having movable guides, and for large variations by cutting out one or more of the machines with fixed guides.

This turbine may be constructed either as a double or single vortex wheel. In the double wheel a series of vanes is fixed on either side of the runner disc, which is leyed to the turbine shaft. Discharge takes place radially at the centre, and the water is then diverted axially through two discharge pipes, or draught tubes, placed one on each side of the wheel. This gives a wheel which is perfectly in balance as regards end thrust. To facilitate the discharge, the wheel vanes are usually curved at their outlet edges so as to direct the discharge water in the direction of the axis, while for the same reason only alternate wheel vanes are carried to the centre. This has the further advantage of reducing friction losses. This double vortex wheel with pivoted guide vanes is very suitable for medium and fairly high falls where the load is very variable, and where an economical use of the supply water at all loads is essential. The full-load efficiency is high, up to 87 per cent. under favourable conditions, while the part-load efficiency is also high, half-load efficiencies of as much as 82 per cent. being on record. The turbine may be constructed with either horizontal or vertical shaft, mechanical considerations rendering the former construction preferable.

In the single vortex wheel the vanes are fixed on one side of the rotating disc, and discharge takes place on one side only. The disc is therefore subject to end thrust, which must either be hydraulically balanced or taken up by means of a suitable thrust bearing. This disadvantage, which is common to all single discharge pressure turbines, may be overcome by mounting a pair of similar wheels on the same shaft, the flow being in opposite directions through the two wheels. The two end thrusts thus balance each other, while the arrangement has the further advantage for electric driving, that with a given head, since two smaller wheels are used instead by a single larger one, and since the peripheral speed of the wheel depends solely on the head, the 
speed of rotation is higher with the twin wheel than with the single wheel machine. The single vortex wheel with vertical shaft is well fitted for medium and low falls where the quantity of water available is large and where the head is variable, and its efficiency is practically the same as that of the double wheel.

The method of regulation by pivoted guides has many advantages. Of these the following are the more important:-

(1) At all gates the guide passages are of a gradually convergent form and offer easy curves to the supply water.

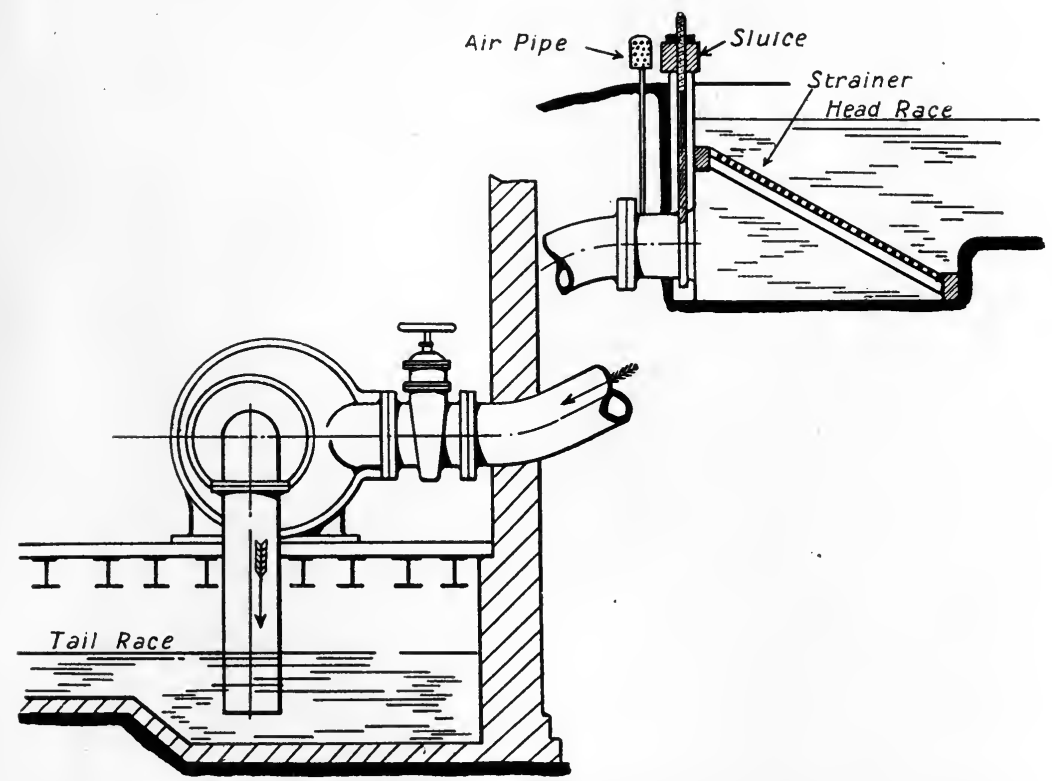

FIG. 194.-Arrangement of Vortex Turbine with Horizontal Shaft and Draught Tube.

(2) Water is admitted (with the spiral casing) evenly all around the circumference.

(3) The guide' vanes are easily moved, and an easy and rapid regulation of speed under sudden load variations is possible (specially important in electric driving).

(4) The guides may be arranged to give a small difference in the inclination of the stream entering the wheel buckets, together with a large difference in flow, and therefore to give 
a constant speed with a constant head and under a variable load, or may be so arranged as to give a greater change in inclination of the guides with a medium change in the flow, and thus to give a fairly constant speed under a variable head and variable load.

(5) The efficiency at part gate is high. The following test results have been chosen as showing how the efficiency varies with gate opening in this type of machine.

\begin{tabular}{|c|c|c|c|c|c|c|}
\hline \multirow{4}{*}{$\begin{array}{c}(a)^{1} 45^{\prime \prime} \text { diameter, single } \\
\text { wheel. } \\
198 \frac{1}{2}^{\prime} \text { head, } 1,000 \text { H.P., } \\
\text { at } 286 \text { revolutions. }\end{array}$} & Gate opening . & $\cdot 2$ & $\cdot 4$ & $\cdot 6$ & $\cdot 8$ & $\cdot 9$ \\
\hline & Efficiency & $70 \cdot 9$ & $77 \cdot 4$ & $78 \cdot 4$ & $78 \cdot 4$ & $78 \cdot 3$ \\
\hline & H.P. . & 209 & 469 & 712 & 950 & 1,066 \\
\hline & $\begin{array}{l}\text { Quantity of water, } \\
\text { cubic feet per minute. }\end{array}$ & $78: 3$ & 1,615 & 2,424 & $3,19 \pi$ & 3,600 \\
\hline
\end{tabular}

\begin{tabular}{|c|c|c|c|c|c|}
\hline & H.P. & Head feet. & Revolutions. & \multicolumn{2}{|c|}{ Efficiency. } \\
\hline$(b)^{2}$ & 200 & $21 \cdot 9$ & 136 & $\begin{array}{c}\frac{1}{2} \text { gate } \\
\frac{3}{4},, \\
\text { full, }\end{array}$ & $\begin{array}{l}86 \cdot 0 \\
85 \cdot 2 \\
85 \cdot 4\end{array}$ \\
\hline$(c)^{3}$ & 1,050 & 322 & 500 & $\begin{array}{l}\frac{1}{2} \text { gate } \\
\text { 歨 ", } \\
\text { full,", }\end{array}$ & $\begin{array}{l}80 \cdot 0 \\
85 \cdot 0 \\
79 \cdot 0\end{array}$ \\
\hline
\end{tabular}

Strictly speaking, the spiral casing forms an essential part of the Thomson wheel, though this may be replaced by an ordinary cylindrical casing (Fig. 194), or the turbine be submerged in the open penstock with free approach from all sides (Fig. 195), without seriously affecting the efficiency.

Where, however, it is required to take off power below headwater level a casing is essential, while in any case it is advisable for all heads exceeding 10 feet. For medium falls above 10 feet, the horizontal shaft machine is, in general, to be preferred.

As modified by Fink and others, the pivoted guide vane devised by Thomson is largely fitted to modern turbines of the Francis

1 By the Platt Iron Works, Dayton, Ohio, at the Quebec Railway Light and Power Company's Plant, Montmorency, Quebec.

${ }_{2}$ By Messrs. Escher, Wyss \& Cie., at Sihl Paper Mill, Zurich.

${ }^{3}$ By Messrs. Escher, Wyss \& Cie., at Reutte, Austria. 
type, these turbines only differing from the former type in that the guide vanes are shorter, while their number is increased until approximately equal to the number of wheel vanes.

The machine as thus constructed is better fitted for dealing with large volumes of water, and may be taken as marking what is, so far, the highest development of the pressure turbine.

Figs. 196 A, B, C, D, E, ${ }^{1}$ illustrate with some detail what is one of the largest single-wheel turbines of this type yet built. This wheel, which has inward radial flow and combined radial and axial discharge, is designed to give 10,000 B.H.P. at 300 revolu-

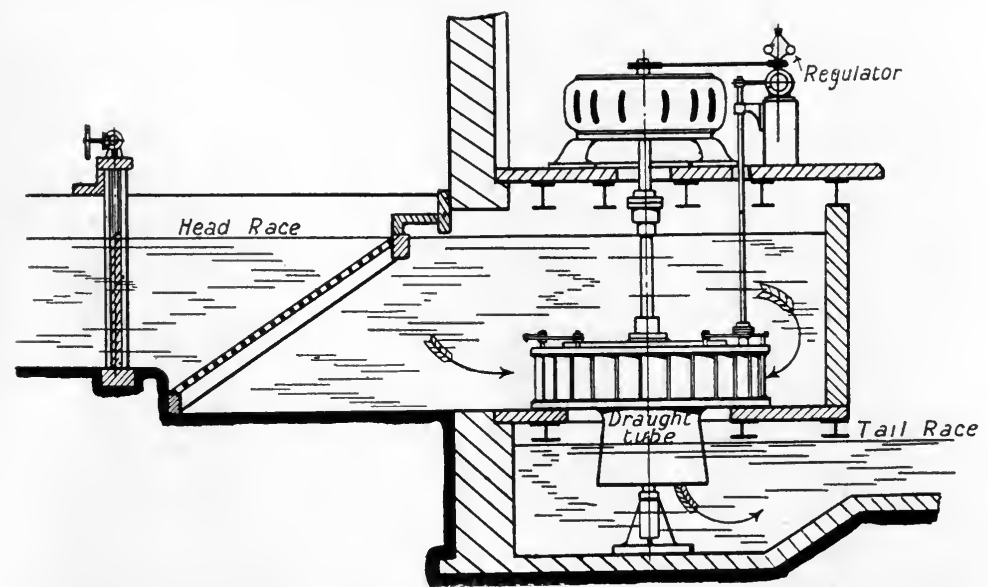

FiG. 195.-Arrangement of Vertical Shaft Single Vortex or Combined Flow Turbine working under Low Head in Open Forebay.

tions per minute, under an effective head of 260 feet. The runner is mounted in a spiral casing and is 66 inches outside diameter, and $9 \frac{1}{2}$ inches wide through the vanes, which are thirty-four in number. The guide vanes, thirty-two in number, are of the pivoted type, and are connected by means of arms projecting radially inward, as shown in Fig. $196 \mathrm{~B}$, to a movable ring concentric with the turbine shaft. This ring is rotated by means of a pinion actuated by the governor, and which gears into a rack mounted at the extremity of a rocking lever. This lever transmits its motion to the ring by means of two links

1 At Snoqualmie Falls; reproduced by courtesy of the makers, the Platt Iron Works, Dayton, Ohio. 
so formed as to act as preliminary guides. The turbine proper has only one bearing, the direct-coupled generator having two

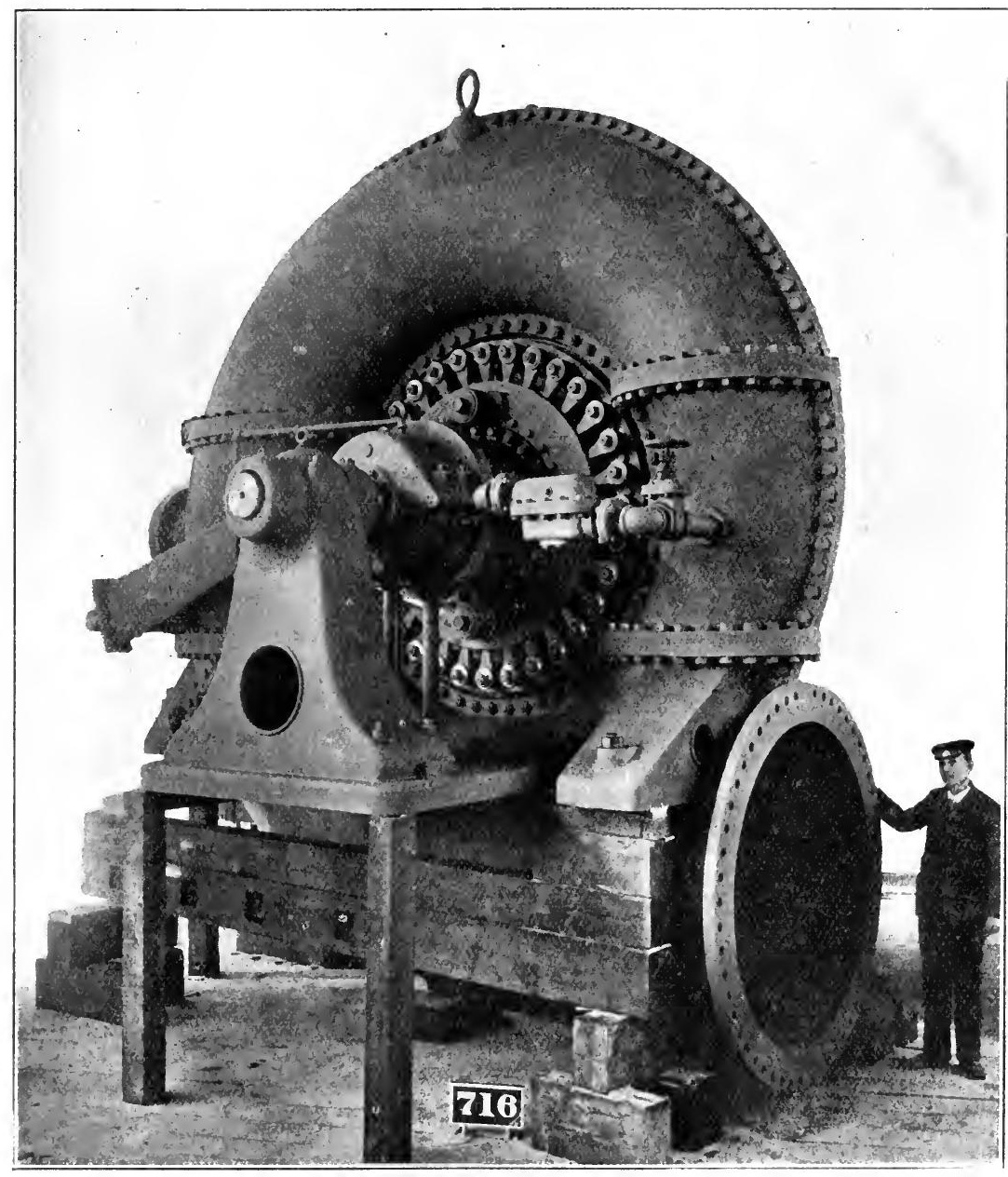

FIG. 196 B.-liear Elevation of Francis Turbine for Snofualmie Falls.

bearings, making the whole unit a three-bearing machine. The turbine runner is a steel casting whose radial depth is very slightly greater than the vanes, the shaft being enlarged into a 
dise of sufficient diameter to permit of bolting the vane ring directly to it. The vanes are finished by filing smooth.

Owing to the large diameter of the wheel and the high statical pressure at entrance, special means were necessary to balance the considerable end thrust on the shaft. Owing to the leakage of pressure water into the space behind the wheel, the rear face

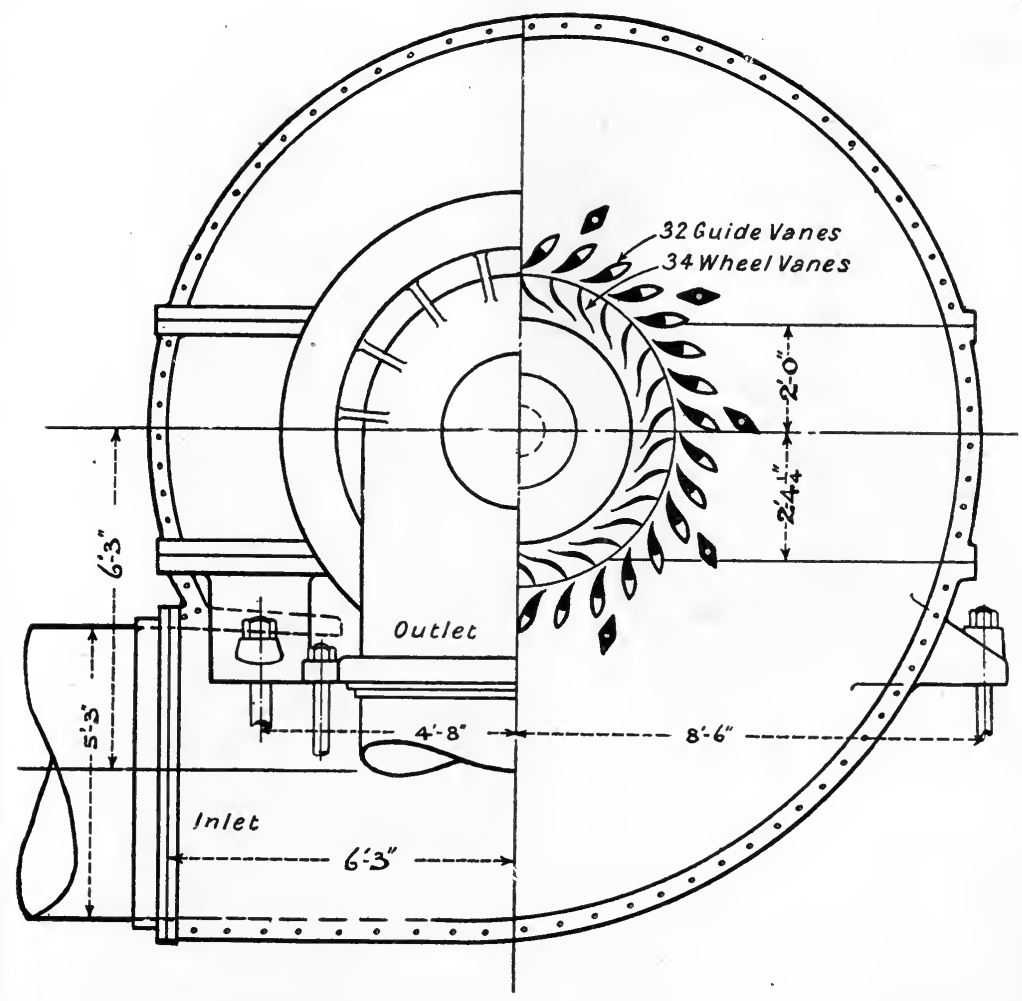

Fig. 196 c.-Cross Section and End Elevation of Turbine for Snoqualmie Falls.

is subject to a pressure substantially equal to that at entrance, while the front face is subject to a pressure varying from that at entrance to that in the draught tube. This produces a large excess of pressure towards the draught tube, and although the axial discharge, by producing a change of momentum in an axial direction calls for a reaction on the wheel in the opposite H.A. 
direction, yet the effect of this is small in comparison with that previously considered.

The greater part of the pressure thrust is eliminated by venting the space behind the wheel into the draught tube through six holes in the wheel disc, while a series of radial vanes cast on

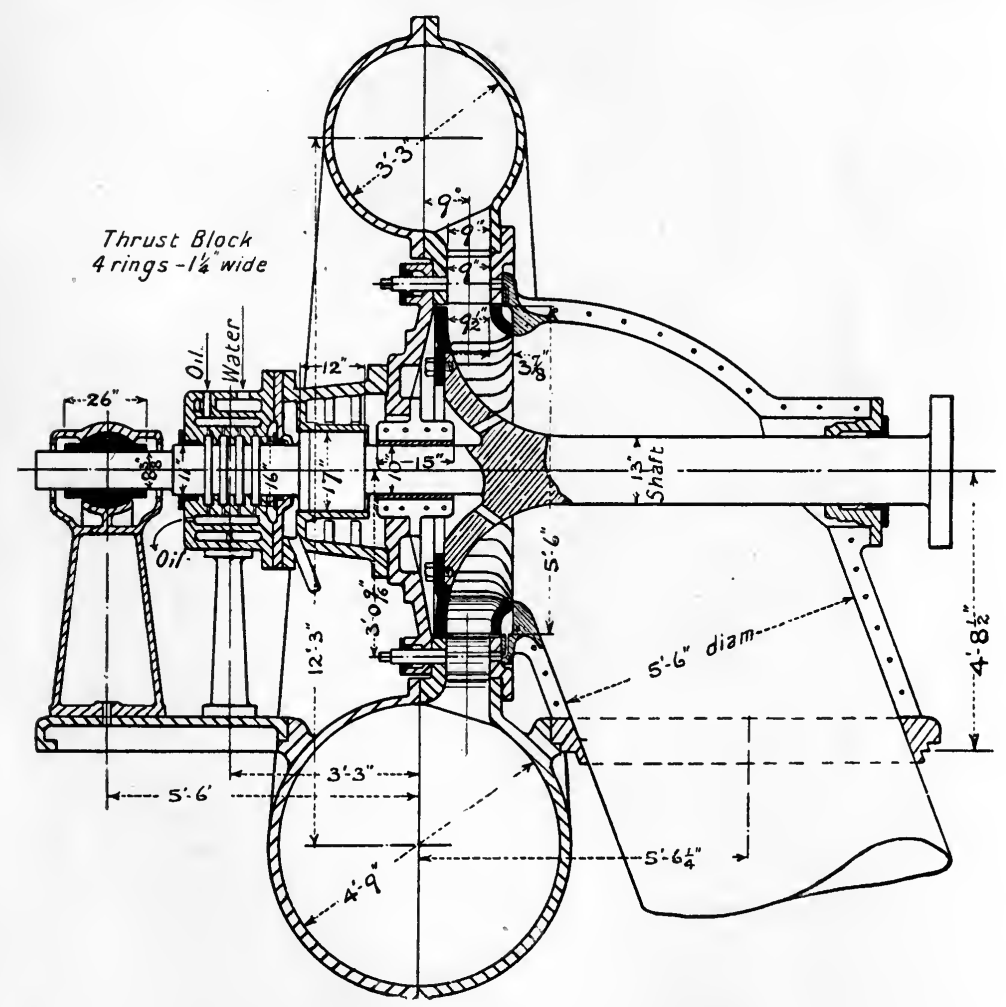

FrG. 196 D.-Longitudinal Section of Single Wheel Horizontal Shaft Francis Turbine, developing 10,000 H.P. at 300 revs, per min. under $260 \mathrm{ft}$. effective head. Outer diameter of runner 66 ins.

the back head of the wheel casing and almost touching the wheel prevent the formation of a forced vortex behind the wheel. The increase in pressure outwards, due to centrifugal action, is thus prevented to a large extent, and the mean pressure reduced. 'There is, however, a resultant thrust towards the draught tube, which increases with the gate opening, and a 
closer balance is obtained by means of a balancing piston behind the back head of the wheel casing. This is a forged enlargement of the shaft, 17 inches in diameter, and works in a water-packed brass sleeve as shown in Fig. $196 \mathrm{D}$.

The chamber in front of the piston is supplied with pressure

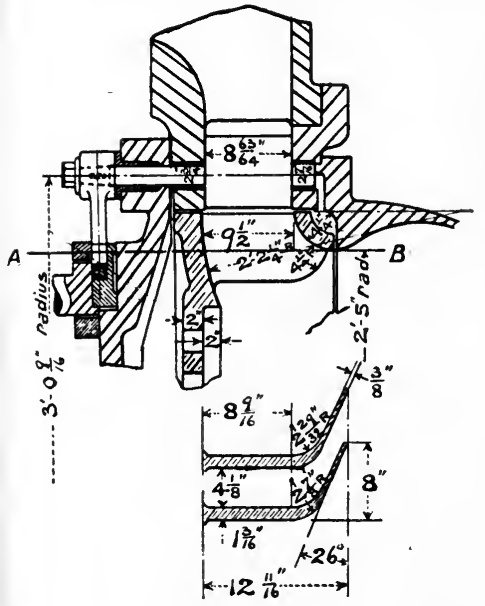

Section on $A B$.

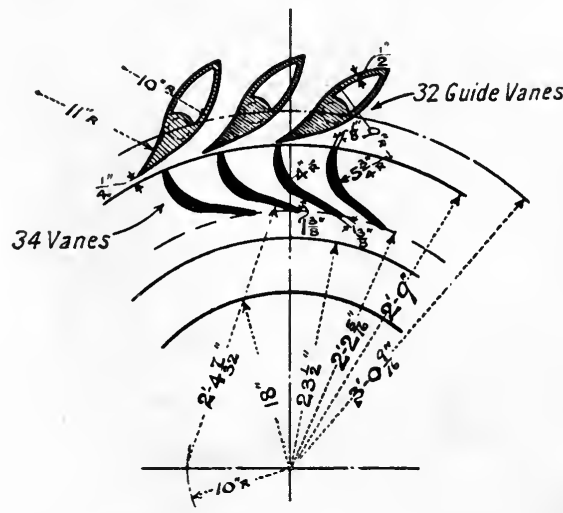

Details of Runner and Guide Vanes.

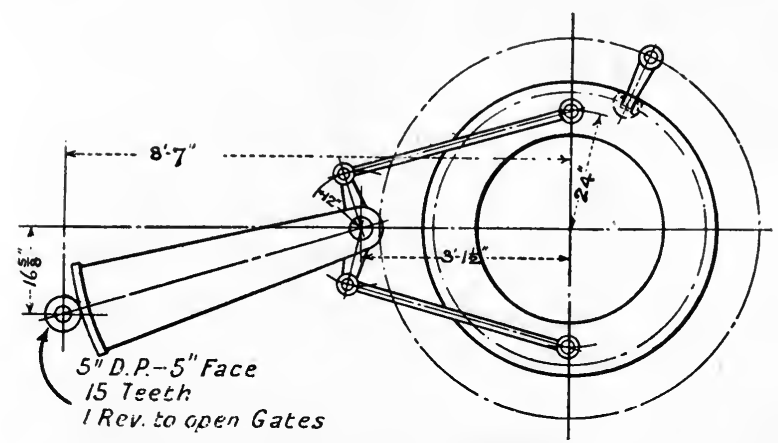

Details of Gate Connections.

FIG. 196 E.-Details of Turbine for Sno łualmie Falls.

water from the supply pipe, through a strainer, while the space behind the piston is drained into the draught tube. A constant thrust towards the left is thus produced, and since there is a small leakage past the piston, this thrust may be very accurately adjusted to suit the conditions of running, by adjustment of 
the supply valve. Any remaining thrust due to change of load is taken up by a collar thrust-bearing situated behind the balance piston. An adjustment of the balance piston supply valve to give balance at $\frac{5}{8}$ full load, renders the thrust bearing liable to a possible thrust of about $25,000 \mathrm{lbs}$. at maximum, or very low loads. The collars, four in number and of $13 \frac{1}{2}$ inches mean diameter, have a total bearing area of 418 square inches. All bearing surfaces are babbited. The supply

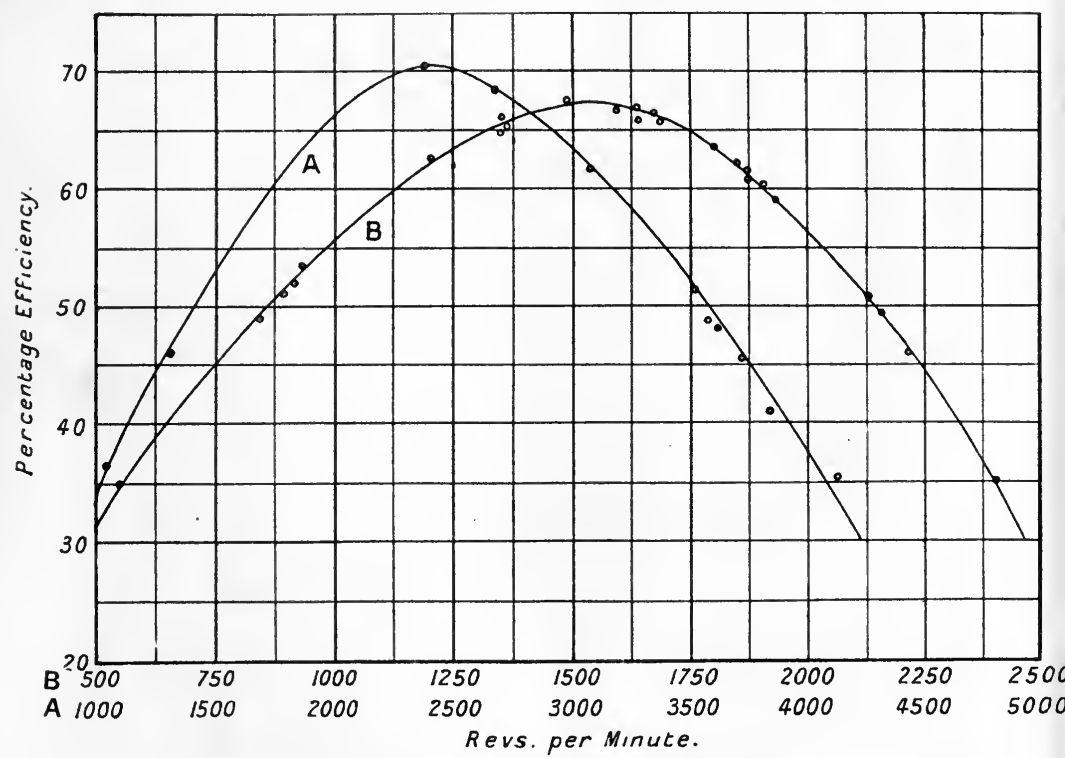

FIg. 197.-Efficiency Curves for Single and for Quadruple Compound Turbine.

pipe is 5 feet 3 inches and the discharge 5 feet 6 inches diameter. A test of this wheel when driving 10,000 H.P. is stated to have given 84 per cent. efficiency, though this figure is subject to the inaccuracy of the method used for measuring the water consumption, this being deduced from the fall in pressure across the head gate, whose opening was accurately known.

\section{Art. 126.-Conpound Turbines.}

One possible method of getting over the difficulty of adapting pressure turbines of small power to very high heads consists in 
compounding two or more runners in series on the same shaft, the fall in pressure and the work done then taking place in stages. As will be shown later, the peripheral speed of a pressure turbine for maximum efficiency is proportional to $\sqrt{H}$, where $H$ is the working head, so that by doubling the number of runners the rotative speed is reduced in the ratio $1: \sqrt{2}=$ $\cdot 707$.

This reduction in speed has the effect of reducing dise friction, and the hydraulic resistances in each chamber will be less than in the single chamber.

Since, however, these losses are duplicated in each successive chamber, and since in addition there is a loss of head due to the resistance of the connecting passages, it is not to be expected that the efficiency of the compound will be so high as that of the single chamber turbine.

Experiments carried out by the author on a small inward radial flow and axial discharge turbine fitted with four similar runners in series, and which could also be worked as a single turbine, showed that for this machine at all events this conclusion is correct. Fig. 197 shows the efficiency curves obtained from the two turbines; $A$ when working with a single runner; $B$ with four runners in series, under approximately the same head.

Here the outer diameter of the runner $=3.35$ inches.

$$
\begin{aligned}
& \text { ", , inner ", (mean) , , }=1.675, \\
& \text { Vanes radial at inlet. Head }=\text { (approx.) } 85 \text { feet. }
\end{aligned}
$$

Art. 127.-The "Ayerican" T'type or "Mixed Flow" 'Turbine.

While the improved Francis or Thomson turbine is undoubtedly the most efficient and offers advantages in respect of ease of regulation and high part-gate efficiency which are unequalled ly any other type, the high first cost prohibits its use in many instances. The demand, particularly in the United States, for a turbine suitable for low and medium falls, which should, above all things be cheap, and in which the efficiency need not necessarily be very high, has led to the development of a type of machine which, although for many years confined to the United States, is at present being manufactured in some 
numbers in Great Britain and to less extent on the Continent. The Hercules and Victor low-pressure turbines may be taken as representative of this class.

- These machines, which are almost invariably fitted with fixed guide vanes and regulated by means of the cylindrical gate or ring:

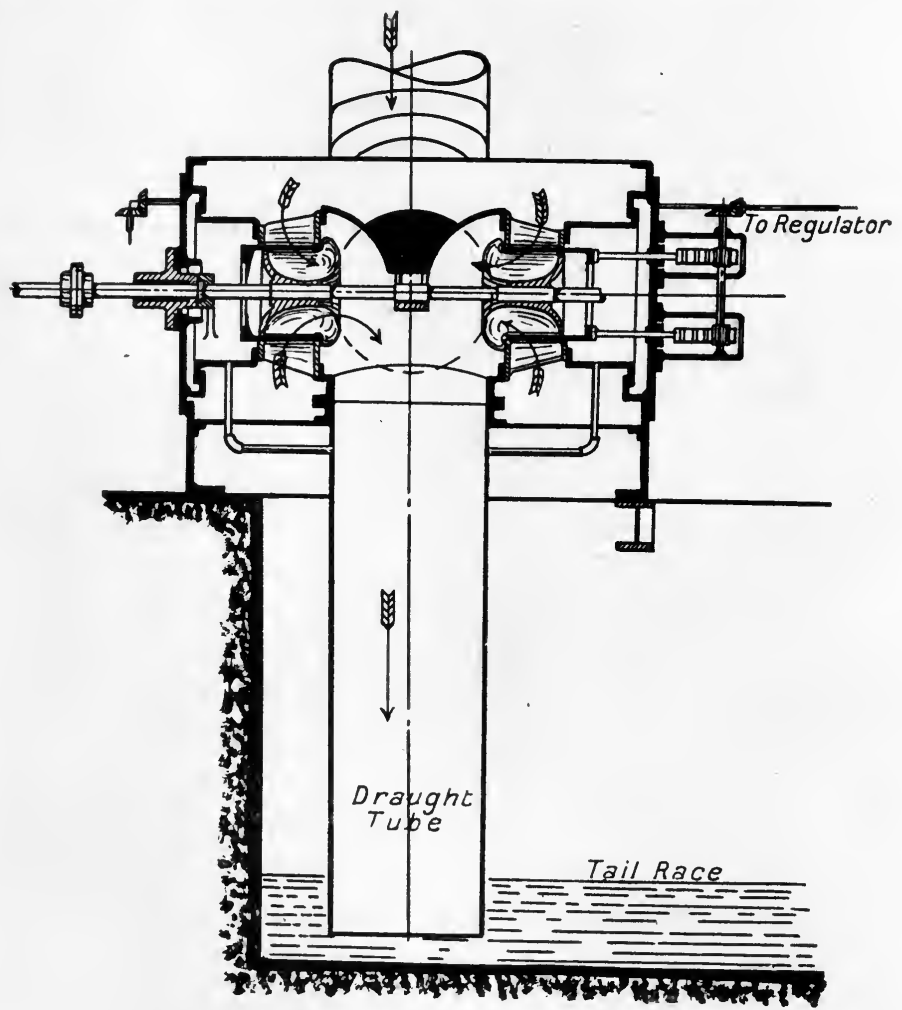

Fig. 198.-General Arrangement of Twin Mixed-Flow Turbine with Horizontal shaft.

sluice, have inward radial flow as in the Francis turbine. After the inlet the wheel buckets are curved both laterally and vertically, the water in its passage through the wheel tracing out a path which is approximately a quadrant of a circle, and being finally discharged partly in an axial and partly in an outward radial direction.

Fig. 198 shows the general arrangement of an enclosed double 


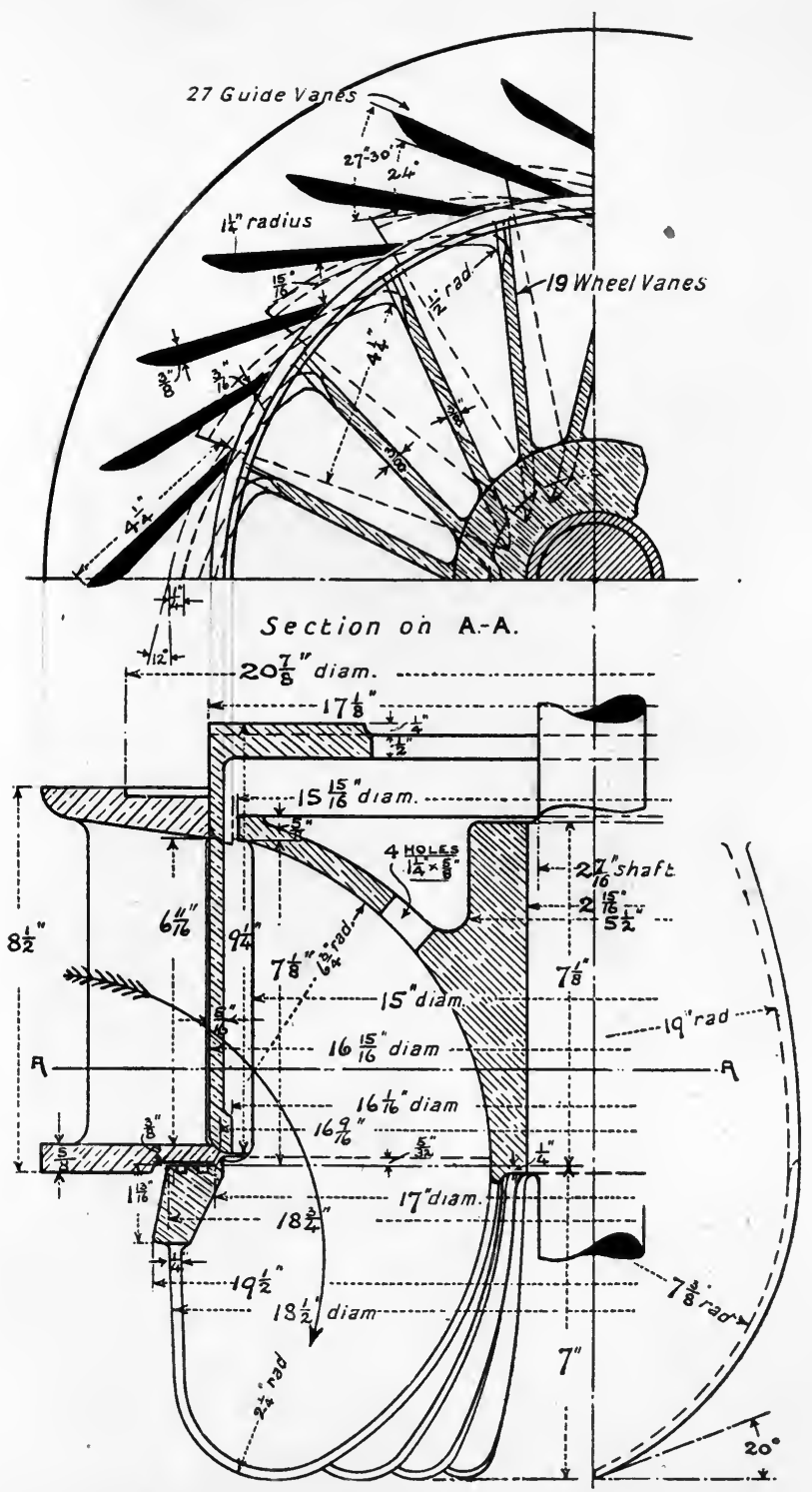

FIG. 199.--Section through Guides and Runners of Victor MixedFlow Turbine with Cylinder Gate; 179 H.P. at $66+$ revs. under $50 \mathrm{ft}$. head. 
horizontal shaft turbine of this type, while Figs. 199 and 200 show details of the guide vanes and runner of a Victor turbine, ${ }^{1}$ as designed to develop 180 H.P. when running at 665 revolutions per minute under a head of 50 feet. The vanes at the outlet are spoon-shaped. By this type of construction an extremely large discharge area is ensured, while by making the wheel deep at inlet, the inlet area is correspondingly increased. The wheel thus becomes of very compact construction and is capable of

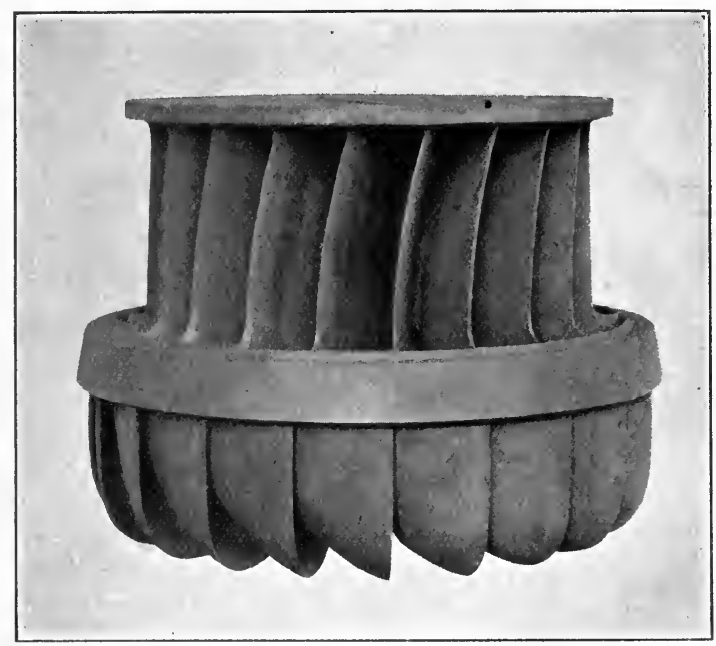

FIG. 200.-Runner for 15 in. Mixed-Flow Victor Turbine.

dealing with a large volume of water, but has the grave defect that in virtue of this great depth its efficiency at part gate is comparatively low.

In the turbine illustrated, the wheel diameter is $\mathbf{1 5}$ inches and the depth $7 \frac{1}{8}$ inches at entrance, while at exit the diameter is $18 \frac{1}{2}$ inches, the over-all depth of the vanes being $14 \frac{1}{8}$ inches. The guide vanes, twenty-seven in number, are fixed, and give a mean inlet angle of about $26^{\circ}$. The wheel vanes, nineteen in number, are flat at entrance, and are inclined forwards towards the direction of rotation, making an angle of about $110^{\circ}$ with the tangent to the inlet circle.

1 By courtesy of the makers, the Platt Iron Works, Dayton, Ohio. 
The vane angle at exit varies from $12^{\circ}$ to $20^{\circ}$, the minimum value being that affecting the radial outward discharge. As may be readily understood, the difficulty of designing these exit edges so as to give the correct inclination at each radius is almost insuperable, and experiment proves the only safe guide as to the precise curvature to give to the vanes. Governing is performed by means of a cylinder gate set between guide vanes and wheel, sliding axially and receiving its motion from two drawbars which carry racks gearing with pinions which are actuated either by an automatic governor or by hand (Figs. 185 and 198).

As thus constructed the turbine is capable of a full-load efficiency of about 82 per cent., falling to about 65 per cent. at half gate.

In the Hercules turbine the wheel vanes are provided with a series of horizontal wings or projections, which to some extent serve the purpose of separate compartments in confining the effect of throttiing to one portion of the wheel and thus increasing the part-gate efficiency, at the expense, however, of that at full gate.

If specially designed for the particular head, flow, and speed of rotation, this wheel is well adapted for moderate powers and for medium heads up to about 35 feet where the head is fairly constant, and where part-gate efficiency is unimportant as compared with low first cost. The general practice of manufacturing this type of turbine in stock sizes, and of supplying that size which most nearly meets the requirements of the purchaser instead of designing the machine to suit its location, together with the fact that such American machinery has in the past been characterized by a flimsiness of construction unusual in English and Continental practice, has, however, had the effect of discrediting this class of turbine among European engineers to a greater extent than its inherent disadvantages deserve. Evolution in this type of machine would appear to be tending in the direction of fewer and deeper buckets with wider openings to avoid obstruction.

\section{Art. 128.-Governing of 'T'urbine Plants.}

The difficulties in the way of the efficient speed regulation of a water wheel or turbine are many and peculiar to this form of 
motor. In the case of a steam engine or turbine the problem is, however, comparatively simple. Now in either type of motor, when running at a uniform speed, there is an exact balance between the energy given up by the motive fluid per unit time in its passage through the motor and the energy absorbed in useful work and in overcoming friction. If more load is thrown on, in either case the speed diminishes until the work done against the increased resistance is again equal to the energy given up by the fluid.

In the case of a steam engine or turbine, directly the speed diminishes the governor alters the admission valves, admits more steam to the cylinders, and in a very short interval of time an exact balance is again set up between the supply of and the demand for energy, so that the engine again runs at a uniform, though slightly lower speed. The admission valves being light and easily moved, the governor itself is in general quite capable of adjusting these rapidly and accurately, while since the steam is an elastic fluid and in a state of high compression, any slight opening of the valves is accompanied by an instantaneous rush of steam at high velocity.

Also, since the amount of available energy per pound of high pressure steam is very great, the mass and inertia of the column of motive fluid are comparatively small, so that its velocity may be rapidly changed without any appreciable change in the pressure in the steam chest. Thus, in an efficiently governed steam engine, fitted with a flywheel of fair size, it is possible to throw the whole external load off with an instantaneous increase in speed not exceeding 2 per cent. of the normal and with a final increase of less than 1 per cent., the time to attain this normal speed not exceeding 5 seconds. 'I'his, then, is the ideal to be aimed at in the governing of a water-power plant, and while such close regulation as this is practically impossible, an examination of the special difficulties to be overcome in this case will indicate in what direction their most satisfactory solution is to be found.

In the first place, the motive fluid (water) is almost incompressible, and contains a much smaller store of energy per unit weight than in the case of steam, so that a similar 
demand for energy must be followed by a largely increased mass flow. The only force available to give the water this increased velocity is that of gravity, and it follows that even in the most favourable circumstances, i.e., when the turbine is set directly in an open forebay of ample dimensions, this velocity cannot exceed that due to the supply head. Where the turbine is supplied through a long pipe line of small slope the state of affairs is much worse, since the force of gravity has now not only to produce increased mass flow and hence to give increased kinetic energy to the moving column of water, but has also to overcome the pipe resistance, with the result that the possible acceleration of the supply column is reduced.

Next consider the action of the governor. In reply to a demand for energy, denoted by a reduction in the speed of rotation, the governor attempts to open the turbine gates. These may be very massive, possessing considerable inertia, and may in addition, through working submerged in water containing solid matter in suspension, offer considerable frictional resistance to rotation.

The governor itself is thus quite incapable of giving the required motion, and some form of relay becomes necessary. A device which was often adopted in the earlier days, but which is now practically obsolete, consisted in a system of fast and loose pulleys, mounted on one shaft and driven from a countershaft by means of two belts, one open and the other crossed.

The position of these belts, one of which is always riding on the loose pulley, is regulated by the governor, and as in consequence the open or crossed belt comes to ride on one of the fixed pulleys, the direction of rotation of this shaft changes. Its rotation causes the turbine gates to open or close and thus regulates the speed of the wheel.

Two types of a more modern mechanical relay device are shown in Figs. 185 and 201. In the latter, ${ }^{1}$ a double ratchet is worked by a link as shown, from a lay shaft belt-driven from the turbine shaft. A ratchet wheel, which is mounted on a second lay shaft by which the turbine gate is directly operated, is wholly or partially masked by a plate whose position depends on the height

1 By courtesy of Messrs. Gilbert Gilkes \& Co., Itd.. Kendal. 
of the governor. Thus, under normal conditions of working, the plate covers the wheel so as to put both pawls out of gear. Any increase in speed then raises the governor, rotates the guard plate, and allows one pawl to gear with the ratchet wheel, rotating the lay shaft and closing the turbine gates. A decrease in speed causes the second pawl to be put into gear, and thus produces an opposite rotation of the lay shaft and an opening of the gates.

With any such type of mechanical device, however, some

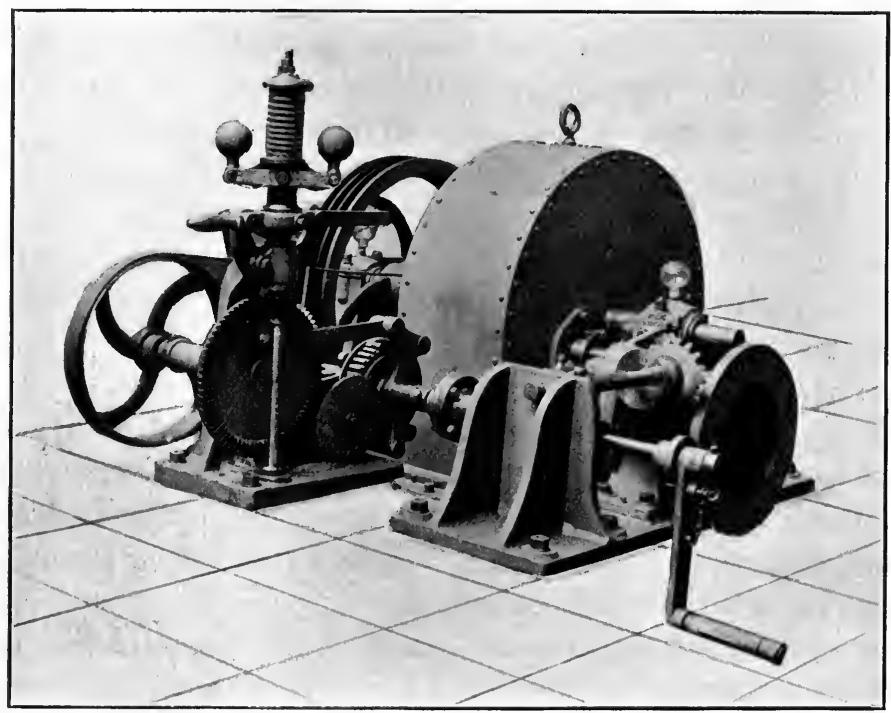

FIG. 201.-Governor with Mechanical Relay as fitted to Girard Turbine.

considerable time is required to entirely open a sluice gate, and while for such a purpose as driving textile machinery, where the changes of load are relatively small, this type fulfills the requirements, yet for electric driving for lighting and power transmission, where a constant speed is required with very large and sudden variations in load, the mechanical relay, except in combination with a large and costly flywheel, is unsatisfactory.

In such a case the hydraulic relay provides the only satisfactory solution. Here the centrifugal governor operates a 
regulating valve which admits either water or oil under pressure to one side or other of a piston in a relay cylinder, this piston being connected with, and operating the turbine gate mechanism. Types of this relay mechanism are illustrated in Figs. 164 to 167, $191 \mathrm{e}, 193,196 \mathrm{~A}, 202$ and 203.

Even with this relay accurate speed regulation under difficult circumstances cannot be obtained without the provision of what is termed a relay return or compensating device. The reason for this is evident if we consider that as the speed falls, the gates are opened and the supply column is accelerated, this opening going on until the supply of energy per unit time is equal to the demand. But the acceleration of the water column goes on for an appreciable time after the gate opening has ceased, and in consequence the supply now becomes too great for the requirements of the wheel, the speed rises, and the governor commences to close the gates. This suddenly checks the motion of the supply column, and in virtue of its inertia produces an increased pressure at the valve and a temporarily increased velocity of flow through the gates. The speed of the wheel thus increases still further, and the gates are closed until an instantaneous balance is set up between supply and demand. As the inertia pressure falls the supply now becomes less than the demand, the speed falls, the gates commence to reopen, and the state of hunting, which is here outlined, may not die out for some considerable time. To prevent this some form of relay return device should be fitted, this being so arranged that all parts of the governor connections, including the regulating valve, return to a normal mid position as soon as the action of the governor ceases. This tends to prevent over regulation and hunting. Where, in the case of a long penstock, the quick closing of the turbine gates may lead to excessive water hammer, a pressure regulator should also be fitted, this consisting of an automatic relief valve which is thrown open by the closing of the turbine gates, and which is then slowly returned to its seat. One such pressure regulator is shown in Fig. 163, while a second type, as described on page 410, is shown applied at $I^{\prime}$ to a Thomson vortex turbine in Fig. 202 .

Types of relay return devices are shown in Figs. 163 and 164, 
and a further type, similar in general principle to those fitted to the Niagara turbines (Art. 124), is illustrated in Fig. 203. Here a force of 50 tons is available on the relay piston, for operating the governing mechanism and gates. Hunting is prevented by the wedge $I T$, which, as the piston moves out, lowers the fulcrum of the governor lever, and thus closes the relay valve until a fresh movement of the governor reopens it.

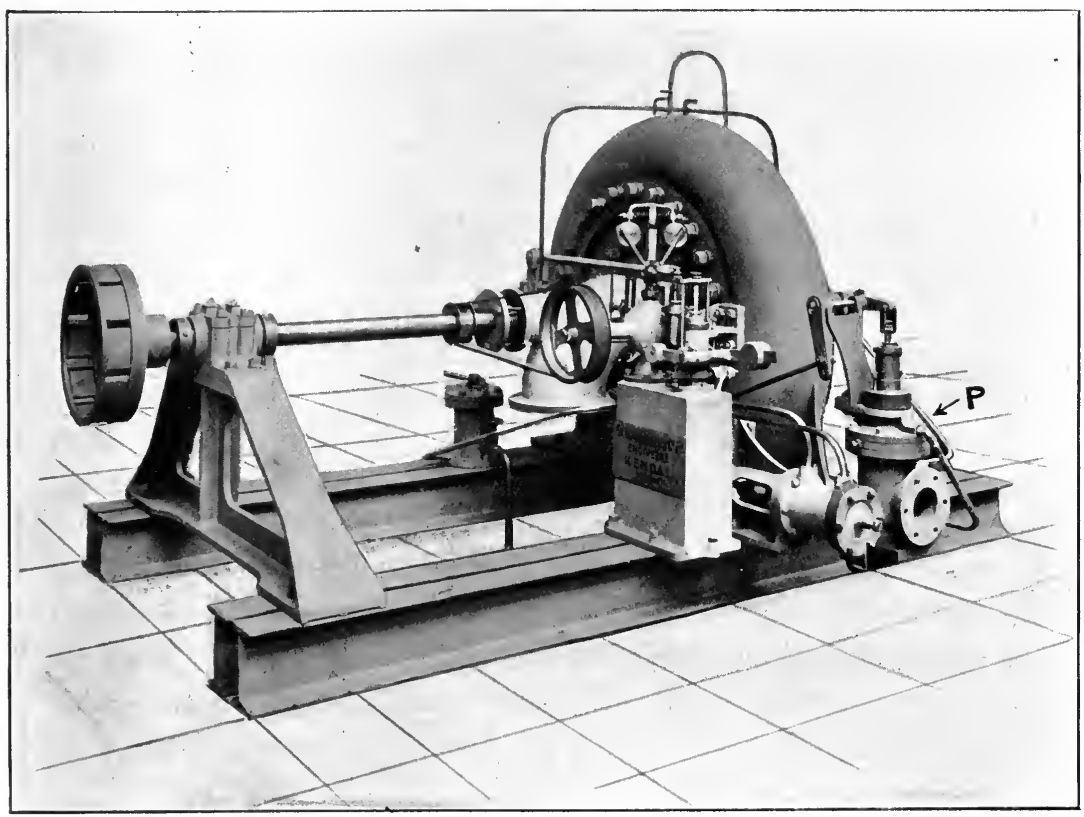

FIG. 202.--Thomson Vortex Turbine fitted with Hydraulic Relay Governor, Compensating Device, and Automatic Pressure Regulator.

Two other devices which also give considerable assistance in special cases are the stand pipe and the relief valve.

The stand pipe consists of a vertical open pipe, its lower end being connected to the penstock near to its connection with the turbines, and of such a height that when exposed to the statical head in the supply reservoir the water level is within a short distance of the top. Any increase in pressure at the turbine, due to a sudden closing of the gates, thus produces a flow up the 


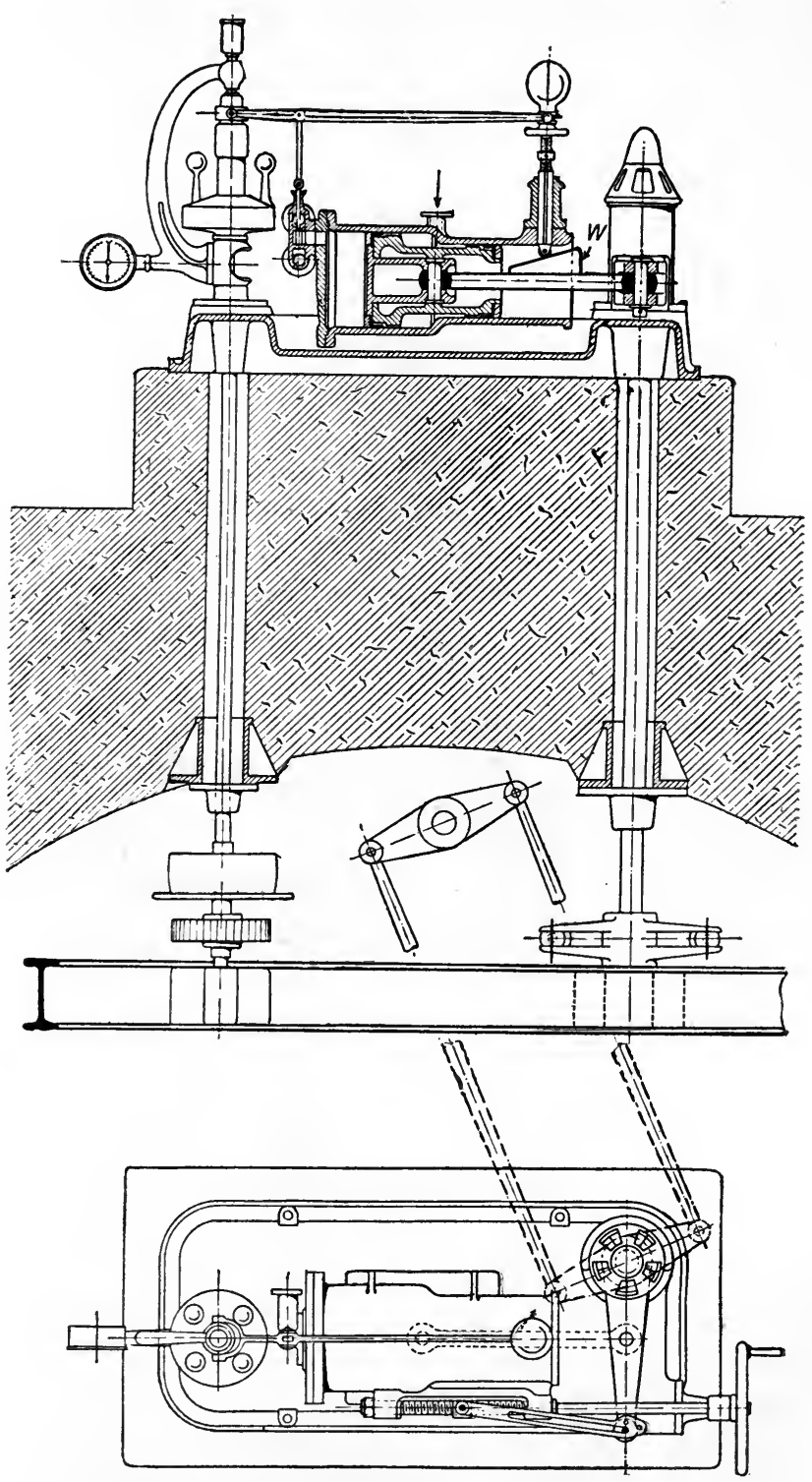

FIG. 203.-Hydraulic Relay and Compensating Device. 
stand pipe, the water escaping at the top, and the maximum possible pressure in the penstock becomes that due to the statical head in the supply reservoir together with that necessary to produce flow up the stand pipe. Obviously the stand pipe cannot be applied where the supply head is very great, although in one modern plant ${ }^{1}$ (Art. 141), such an open pipe 235 feet in height has been fitted.

A sudden demand for power is also responded to more easily where a stand pipe is provided, the level in the pipe falling, and energy thus being supplied to the wheel while the supply column is being accelerated (Art. 141).

The relief ralce, as its name implies, consists simply of a valve placed on the penstock near to the turbine casing and arranged so as to open outwards. This is adjusted so as to open directly the pressure exceeds the normal by a few pounds per square inch, and its area should be such that, if the gates are closed suddenly due to a throwing off of the entire load, it is capable of taking the whole discharge. If $A$ be the minimum total cross sectional area of the guide passages when these are wide open, in order to allow for exceptional circumstances the total relief valve area should not be less than $\cdot 7 \mathrm{~A}$.

Special consideration of the circumstances of each installation is necessary to determine which of these devices is likely to give the best results.

Dealing firstly with pressure turbines, the easiest type of plant to govern is one in which the turbine is placed in a forebay of ample dimensions fed directly from the supply canal (Fig. 195). Here a demand for power is instantly met by an increased flow, at the velocity corresponding to the supply head, while when the gates are closed inertia effects are unimportant. Under these circumstances the speed may be regulated with great nicety, and in general the more nearly the general arrangement of any power plant approximates to this the easier does it become to get good regulation. Both head and tail-race should be of ample size, so that any fluctuation in flow may not cause an appreciable difference in either level, while all approach channels and passages should have easy curves and well-finished surfaces

1 At the St. Louis Hydro-Electric Plant. See The Lingineer, Fébruary 15, 1907. 
so that the production of any periodic wave motion may be prevented.

Wherever possible the use of a long penstock should be avoided, and the water brought as near as possible to its work in an open channel of ample area, for it may be laid down as a general principle that the easiest plants to govern are those in which the slope from open head-water to open tail-water is as steep as possible, and in which the ratio of kinetic energy to total energy is as small as possible.

A few specific types of plant will now be considered in more detail, with respect to their possibilities in the way of speed regulation.

(a) Turbine fed by a Supply Pipe which is almost vertical, i.e., with a slope of $60^{\circ}$ or over.

Here a demand for energy on an increasing load receives an immediate response, and speed regulation in this direction, with a supply pipe of ample area, is comparatively easy. The increase of pressure caused by closing the gates on a diminishing load must, however, be guarded against by the provision of a stand pipe, pressure regulator, or relief valve.

(b) Turbine fed by a Supply Pipe which is of considerable length or of slight inclination.

Here regulation on a diminishing load is easy, the inertia effect on closing the gates being taken care of as in the previous case. Where the statical head is not very great a stand pipe is preferable, since on an increasing load this responds more quickly to an increased demand for energy.

Where the head is so great as to preclude the possibility of a stand pipe, and where the pipe line is very long, the response to such a demand is slow, this type of plant providing one of the most difficult problems in governing. In such a situation, indeed, the only motor to give satisfactory results is the Pelton wheel fitted with combined hand regulation and deflecting nozzle, and with relief valve or pressure regulator.

(c) Turbine fed by a long Horizontal Pipe.

(1) Turbine discharging divectly into tail-race without draught tube.-As in the previous cases, the difficulty in governing occurs 
on an increasing load and may be met by the provision of a stand pipe near the turbine. The efficiency of this stand pipe becomes greater as its area, and in consequence the reserve of pressure water, is increased. In the limit the stand pipe becomes an open forebay in which the turbine works with free access, or to which it is connected by short pipes of ample area. With this arrangement governing is easy, but without it close regulation becomes impossible.

(2) Turbine fitted with draught tube.-Where circumstances necessitate the placing of a turbine which works under a low head, at a considerable distance from the supply reservoir and at the same time at some distance above the tail-race level, a suction tube must be used for the discharge, and the problem of successful regulation becomes still more complicated. In such a case, with a very small head above the turbine, if this is installed in an open forebay, a sudden demand for water may result in this head being reduced until air is drawn into the turbine. The vacuum in the suction tube is then destroyed, the suction tube emptied and the turbine at once stops. To prevent this occurring a minimum depth of water of from 3.5 to 4 feet should be allowed above the turbine, this being increased if the suction head is considerable. Where this is impracticable the turbine must be mounted in a closed casing (Fig. 198), and be supplied through a pipe line. The provision of a stand pipe is here out of the question, inertia effects being guarded against by the provision of a relief valve placed near the turbine casing. A sudden demand for water is met by the accelerating effect of the suction tube, and under these circumstances it is possible to get fairly accurate governing.

Where a stand pipe is adopted in any plant with a view to preventing inertia effects, the freezing of the water which is spilt over the top of the pipe may prove detrimental to the working of the plant. To avoid this it becomes preferable to use the stand pipe in connection with a pressure regulator or relief valve, the latter taking charge of any rise in pressure, and venting the supply pipe, while the sole function of the stand pipe is to take charge of any fall in pressure on an increasing load.

Effect of Gates and Governor Connections.-Whatever the type 
of plant, a well-designed system of gates and gate connections is essential for close speed regulation.

In all connections simplicity, directness, and freedom from backlash are essential, while close regulation is not to be expected from any multiplying gear which needs several turns of a worm or spur wheel to close the turbine gates.

The gates themselves should be as light, well balanced, and frictionless as possible. Of the two types of gate in common use, viz., the sliding cylinder gate and that consisting of a system of pivoted guide vanes, the latter conforms most nearly to these requirements. To give good results these guides should be pivoted near their centres, so as to be approximately in balance under the action of the supply water. If this condition is not satisfied, a large force is required to cause motion, while the gate connections need to be of massive proportions, and the consequent gain in weight and inertia of these connections makes quick regulation difficult of attainment.

In the enclosed 'Thomson turbine, and in enclosed turbines of the Francis type fitted with this type of guide, the guide spindles are passed through stuffing boxes in the turbine casing, and the whole governing mechanism, with the exception of the guides and their pivot bearings, is removed from the action of the water. In the American type of mixed flow turbine, when fitted with pivoted guides, these are usually rotated either by means of an annular gear wheel, which gears with pinions mounted on the guide-spindles, and which is rotated by means of a link coupled to an eccentric which receives its own motion from the relay mechanism, or a series of links mounted on the guide spindles are connected by levers to a central ring, which is rotated by means of the relay. In either case the gearing is usually placed inside the casing, and while accurate speed regulation is possible with either design, the submerged gearing needs to be designed on more substantial lines to compensate for its inaccessibility for examination.

Sliding cylinder gates are commonly moved through a couple of parallel drawbars terminating in racks, which gear with pinions on a shaft perpendicular to the wheel axis, this latter shaft deriving its motion from the relay mechanism by one or 
other of the devices shown in Figs. 185, 191 A, and 203. Where this type of gate is fitted to a vertical shaft machine, it often becomes advisable to balance the weight of the gate and its drawbars by means of a counterbalance weight, in which case a suitable type of connection is shown in Fig. 204.

For successful governing, the gate should consist of a plain cylinder. If provided with fingers, as indicated by the dotted lines in Fig. 187, the water pressure on the upper side of these is largely in excess of that on their lower face. This necessitates a very heavy counterbalance weight, possessing considerable

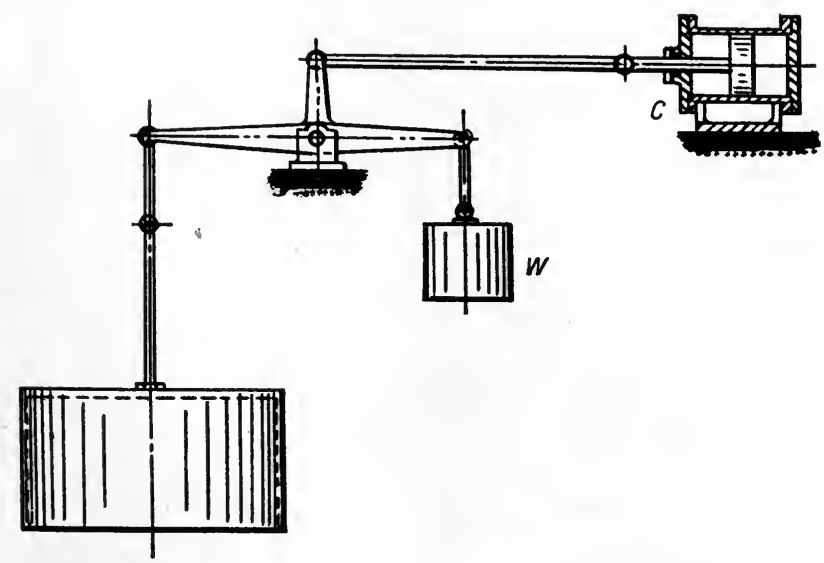

FIG. 204.-Direct Relay Governing Mechanism for Sliding Cylinder Gate.

inertia, and increases the force necessary to start and stop the motion of the gate, while the weight and cost of the connecting links necessary to withstand the stress become excessive. Without the balance weight the steady resistance produced by the unbalanced pressure produces an excessive stress on the gate connections. In either case the very slight addition to part-gate efficiency is totally insufficient to compensate for the increased difficulty in governing.

Governing of Impulse Wheels.-The preceding considerations also apply, so far as cases $a$ and $b$ are concerned, to the governing of impulse turbines, except where, as in the case of a Pelton wheel fitted with deflecting nozzle, the velocity of flow through 
the supply main is maintained uniform for all loads. Although in this case inertia effects are not to be feared in the governing of the turbine, yet the occasional necessity for quickly shutting off the supply renders the provision of a relief valve near the lower end of the pipe essential.

Owing to the absence of any cyclical variation of turning moment in a pressure turbine, a special flywheel is not usually necessary for close governing, sufficient flywheel effect in general being obtained from the turbine runner and shaft itself, and from the electric motor when such is used.

In the Pelton wheel, however, the turning moment undergoes a slight periodic variation, the period depending on the number of buckets and on the speed of the wheel. On account of this and on account of the comparatively small weight of the wheel for a given power, a flywheel is in general essential for such close governing as is necessary for the driving of alternators in parallel.

\section{Art. 129.-Design of Head and Tail-Races.}

The construction of the head and tail-races for a turbine plant requires careful consideration. The forebay should be free from sharp turns and sudden changes of section, the velocity of flow not exceeding from 2 to $2 \frac{1}{2}$ feet per second. The tail-race should be of the same capacity and should be formed with a well under the turbine outlet, which should contain from 2 to 4 feet of dead water when the turbines are idle. If this be not attended to, a serious loss of head may be caused.

Where a draught tube is fitted this should be submerged from 3 to 6 inches in the standing tail-water, while it is preferably bent at its lower end, so as to discharge in the direction of flow in the tail-race.

Where a long penstock is installed, this should be supplied with an air pipe (Fig. 194) at its highest point and also at the highest point of any vertical bends in its length, to permit the escape of accumulated air.

A strainer is always fitted at the exit from the forebay, or supply canal, and consists of a series of wooden or metal grids. 
These are usually spaced about 1 inch apart, and in order to reduce the loss due to the contraction and subsequent expansion of section of the stream, should be as narrow as is consistent with strength. The angle of inclination of the strainer should be not less than $45^{\circ}$, in order to give a large area of waterway.

Effect of Ice Formation.-Although the hydraulic power plant is . not greatly hampered by ice formation in Great Britain and the western portion of the continent of Europe, ice troubles may become serious in such climates as are found in Canada and the northern portion of the United States, and may necessitate some modification in the design of the head-race.

In addition to existing in the ordinary surface form, ice is found in the form of anchor ice and of frazil. Anchor ice consists of an agglomerated mass of coarse crystals clinging to the bed of the channel and formed there by loss of heat by radiation, the cooling of the bottom thus produced causing ice to form. This can only occur with a clear sky and where the surface ice-if any-is transparent. Surface ice will in general effectually prevent its formation. It is usually found in rapid streams, where surface ice cannot form.

Frazil consists of fine spicular ice crystals floating in the water and formed by slight supercooling below $32^{\circ}$ (probably about $\cdot 001^{\circ}$ being, sufficient). This adheres to the surface ice and to the anchor ice, and may finally choke up the stream. In engineering work frazil ice is particularly objectionable, as it adheres to the racks and strainers and to the gates of a turbine, and if, due to exposure to air, these are slightly supercooled, freezes into one solid mass.

This can, however, be prevented by a slight heating of the racks or turbine gates. At the Ottawa Electric Company's Power House No. 1 a line of steam pipes laid above water level and against the face of the rack was found to answer perfectly. In the same installation, consisting of three 39-inch wheels, 30 feet head using 100,000 cubic feet of water per minute, steam was supplied by a small pipe to each of the wheel housings when the unit began to loose capacity. To supply this, 20 tons of coal were used during four months of winter, with eleven days on which frazil was bad, only occasional injection of steam being 
found to be necessary. Electric heating of the same racks has also been tried successfully, 600 amperes at 3 volts removing the ice at once from a single rack bar with the air temperature at $15^{\circ}$ Fahr. ${ }^{1}$ These bars were $\frac{5}{16}$ inches thick and 18 feet long.

In a power plant it is usually advisable that the water at the intake should be covered with surface ice, as being the most effective preventative of the formation of both frazil and anchor ice. If there are large stretches of open water above the surface ice, however, frazil is formed and adheres to the lower surface, resulting in a possible stoppage of the channel. In such a case, or when located at the foot of rapids, it is better to construct a head-race of sufficient size to serve as a settling basin for the ice drawn in. Even then it may be necessary to blast a channel in the surface sheet. Where a long narrow canal is fed from a stretch of open water the ice difficulty becomes very great. A surface covering is then harmful, as encouraging the adherence of frazil.

\section{Exairples.}

1. Discuss the relative advantages and disadvantages of the impulse and pressure turbine as regards-

(a) Applicability to high, medium, or low heads.

(b) Applicability to suit local conditions.

(c) Efficiency.

(d) Speed regulation,

(e) Speed of rotation.

2. Sketch any device suitable for the speed regulation of(a) A Pelton wheel.

(b) A Girard turbine.

(c) An inward radial flow turbine.

(d) An outward radial flow turbine.

3. The nozzle circle of a Barker's mill has a radius of 2 feet. The nozzles, two in number, are $\frac{3 \prime 4}{4}$ diameter, and when working

1 These results have been taken from "Ice Formation," by Barnes, Wiley \& Son, N.Y., 1907. Here the whole subject is considered in detail. 
under a head of 1 foot the wheel makes 240 revolutions per minute. Determine its hydraulic efficiency, neglecting the effect of friction.

\section{Answer • 882 .}

4. In the wheel of the previous example, the weight of water used at 240 revolutions per minute, working head 6 feet, was 335 lbs. The brake horse power was 056 .

Determine the coefficient of frictional resistance inside the wheel; the hydraulic efficiency, the total efficiency, the speed for maximum possible hydraulic efficiency; and this efficiency.

Answer :-

$\begin{array}{lrl}F & =\cdot 2 . \\ \text { Hydraulic efficiency } & =\cdot 522 . \\ \text { Total efficiency } & =\cdot 46 . \\ \text { Speed for maximum efficiency } & =160 \text { rerolutions. } \\ \text { Maximum efficiency } & =\cdot 593 .\end{array}$




\section{CHAPTER XIII.}

Theory of Turbine Design-Design of Stand Pipes.

\section{Art. 130.-Theory of Turbine Design.}

The turbine system, as a whole, may be divided into the supply pipe, the turbine itself, and the discharge pipe. Losses of head are experienced in each branch of the system, so that if $H$ be the total difference of level between open head and tail-race, the head available for doing work on the turbine will be less than $H$ by the amount necessary to overcome the frictional and other losses in the supply and discharge pipes.

The loss due to this cause is approximately proportional to the lengths of the pipes and to the square of the velocity of flow, and its relative importance diminishes as the gradient of the pipe line and its sectional area are increased. Theoretically, by making the pipes of sufficiently large area the loss may be made quite negligible. This increase in area is, however, accompanied by a corresponding increase in the first cost, and it appears to be fairly well agreed that in practice it does not pay to reduce the pipe line velocity below from 4 to 7 feet per second, the value to be adopted increasing with the gradient and with the size of pipe.

In a large power plant where the head is high and the penstock direct and of large diameter, this velocity may be increased without serious proportional loss of head up to a maximum of about 12 feet per second.

If, in any type of turbine, $v$ be the velocity of the supply water at its exit from the guide vanes, and if $a$ be the angle between the guide vanes and the direction of motion of the wheel at the point of entry, $v$ may be resolved into two components; $v$ sin $a$, perpendicular to the direction of motion of the vanes, which is usually termed the velocity of flow, and $v \cos$ a parallel to this 
direction and which is termed the velocity of whirl. Throughout the following discussion the velocity of flow will be denoted by $f$; and that of whirl by 'w. 'Thus, in a radial-flow turbine $f$ is radial and $w$ tangential to the wheel, while in an axial-flow machine $f$ is parallel to the turbine axis and $w$ is in the plane of the wheel.

In virtue of the velocity of whirl, the supply water possesses momentum in the plane of the wheel, and it is the change in the moment of this momentum about the axis of the wheel during the passage of the water through the wheel buckets which provides the turning moment on the shaft. For this turning moment to be a maximum with a given value of $v$ and a given quantity of water, it follows that the value of $u$ should be a maximum, and therefore that $a$ should be as nearly as possible zero.

On the other hand, the volume of water which a given wheel is eapable of handling per second depends on $f$, since it is in virtue of this component of velocity that water is carried into the turbine buckets. As $a$ is diminished, therefore, the size of turbine for a given power, and the first cost, increases, and the problem before the designer is to determine at what point the diminution in efficiency due to an increase in a becomes of more importance than the corresponding diminution in first cost. A similar state of affairs holds at exit. For maximum hydraulic efficiency the energy rejected in the discharge should be reduced to a minimum. This requires the absolute velocity of discharge from the wheel to be as small as possible. The minimum permissible velocity of discharge is, however, governed by the necessity for getting a given volume of water per second through the limited sectional area of the buckets at this point, and here also an increase in the velocity of flow at the expense of the velocity of whirl will enable a larger volume of water to be dealt with and a greater power to be obtained, but with a sacrifice of the hydraulic, though possibly not of the economic, efficiency.

Where an abundant supply of water is available at all times, the efficiency of the turbine may become quite a secondary consideration as compared with its prime cost, and a cheap but comparatively inefficient turbine may be preferable on all counts. 
Here, however, it must be remembered that where a long supply pipe or channel is necessary, a decrease in the efficiency of the turbines, particularly at full load, necessitating as it does an increased water supply, may cause the initial cost of construction of such channels or pipes to more than counterbalance the decreased prime cost of the motor. In such a case high efficiency at part loads is a minor consideration and may be entirely subordinated to consideration of cheapness and of ease of governing.

Again, where the supply is variable and where in times of drought barely sufficient water is available, it is highly important that high efficiency at all gates be the first consideration.

Thus the turbine designer must keep many conflicting possibilities in view, each of which has its own effect on the most suitable design to be adopted, and no hard and fast rules can be formulated for the design of any one type of machine. Certain broad principles may, however, be laid down, to which the design must conform if the turbine is to be efficient, and these will now be briefly indicated.

In the following demonstrations :-

Let $\omega=$ angular velocity of the wheel in radians per second.

$r=$ radius in feet at any point indicated by a suffix.

$v=$ velocity of supply stream at its exit from the guide vanes, in feet per second.

$u=\omega r=$ velocity of wheel at point indicated by a suffix.

$f=$ velocity of flow.

$w=$ velocity of whirl.

$a=$ angle between guide vanes and direction of motion of wheel at entrance.

$\beta=$ angle between lip of moving vanes and direction of motion of wheel.

$\gamma=$ angle between discharge edges of moving vanes and direction of motion of wheel.

$Q=$ flow in cubic feet per second.

$W=$ weight of 1 cubic foot of water $=62 \cdot 4 \mathrm{lbs}$. 
Art. 131.-General Case of Inward Radial-flow Turbine (Fig. 205).

Let the suffix $(a)$ refer to the state of affairs in the supply pipe.

(b) refer to the state of affairs in the discharge pipe.

(1) refer to the state of affairs at the exit from guide vanes.

(2) refer to the state of affairs at the inlet to wheel vanes.

(3) refer to the state of affairs at the exit from wheel vanes.

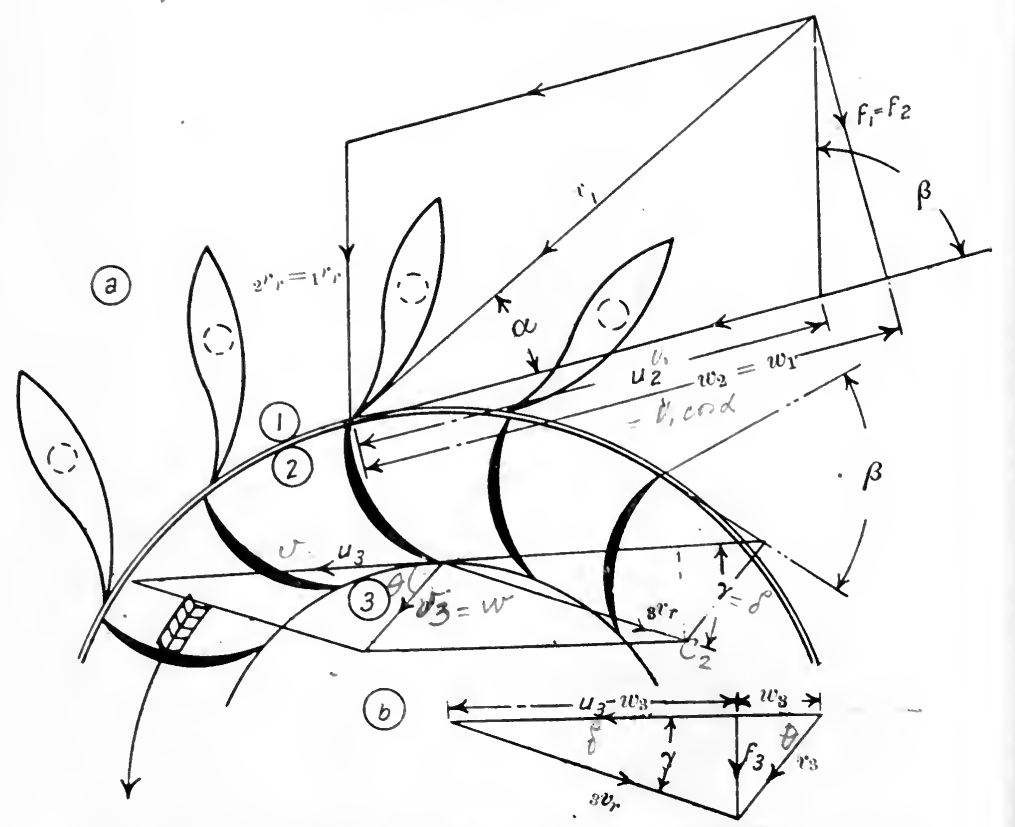

Fig. 205.-Guide and Vane Angles for Inward Radial-Flow Turbine.

Then a consideration of the diagram shows that

$\left\{f_{2}=u_{2} \tan a=\left(u_{2}-u_{2}\right) \tan \beta, \therefore u_{2}=u_{2}\left(1-\frac{\tan a}{\tan \beta}\right)\right.$;

$\left\{f_{3}=\left(u_{3}-w_{3}\right) \tan \gamma ;{ }_{2} r_{r}=f_{2} \operatorname{cosec} \beta ;{ }_{3} r_{r_{0}}=f_{3} \operatorname{cosec} \gamma\right.$. 


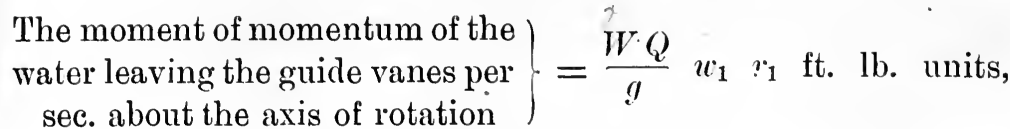
and since, if losses at the exit from the guide vanes are neglected, $w_{1}=w_{2}$, while, neglecting clearance, $r_{1}=r_{2}$, this becomes $\frac{W Q}{g} u_{2} r_{2}$ ft. lb. units.

The final moment of momentum $=\frac{W Q}{g} u_{3} r_{3}$ ft. lb. units. $\therefore \quad$ Change of moment of mo- $\}=\frac{W Q}{g}\left\{u_{2} r_{2}-v_{0} r_{3}\right\}$ ft. lbs. $\left.\therefore \quad \begin{array}{l}\text { Work done by this moment } \\ \text { per second }\end{array}\right\}=\frac{W Q}{g}\left\{u_{2} r_{2}-u_{3} r_{3}\right\} \omega \mathrm{ft} .1 \mathrm{bs}$. $=\frac{W Q}{g}\left\{w_{2} u_{2}-u_{3} u_{3}\right\}$ ft.lbs. (1)

Evidently this has its maximum value when $u_{3}=0$, and then the work done per second $=U=\frac{W Q}{g}\left\{u_{2} u_{2}\right\}$ ft. lbs.

If $u_{3}$ is not zero, we have, on substituting its value $u_{3}=$ $u_{3}-f_{3} \cot \gamma$ in $(1):-$

$U=\frac{W Q}{g}\left\{u_{2} u_{2}-u_{3}^{2}+u_{3} f_{3} \cot \gamma\right\}$ ft. lbs. per second. (2a)

If the vane angles are correctly proportioned as indicated in Fig. 205 so as to avoid shock at entrance, we have:-

$$
u_{2}=w_{2}\left(1-\frac{\tan a}{\tan \beta}\right) ;
$$

while if $b_{2}$ and $b_{3}$ are the effective breadths of the wheel passages at entrance and exit so that $2 \pi l_{2} r_{2}$ and $2 \pi l_{3} r_{3}$ are the effective passage areas, we have, for continuity of flow, $f_{3}=f_{2} \frac{b_{2} r_{2}}{b_{3} r_{3}}$. Also $\frac{u_{3}}{u_{2}}=\frac{r_{3}}{r_{2}}$ so that

$$
J=\frac{W Q}{g}\left[u_{2}^{2}\left(1-\frac{\tan a}{\tan \beta}\right)\left\{\begin{array}{c}
1-\left(\frac{r_{3}}{r_{2}}\right)^{2}\left(1-\frac{\tan a}{\tan \beta}\right) \\
+\frac{l_{2}}{b_{3}} \frac{\tan a}{\tan \gamma}
\end{array}\right\}\right.
$$


Assuming the turbine to be designed however, so that $w_{3}=0$, we get, from (2)

$$
U=\frac{W Q}{g} w_{2}^{2}\left(1-\frac{\tan a}{\tan \beta}\right) .
$$

If the wheel is horizontal, or if in a vertical wheel we neglect the differences of level at the highest and lowest points and also neglect losses at entrance, we have:-

$$
\frac{p_{2}}{W}+\frac{v_{2}^{2}}{2 g}=\frac{p_{3}}{W}+\frac{v_{3}^{2}}{2 g}+\underset{\text { water per lb. }}{\text { work done by }}+\begin{aligned}
& \text { losses per lb. be- } \\
& \text { tween(2) and (3). }
\end{aligned}
$$

If then $H^{\prime}=H-\frac{F L \ddot{v}^{2}}{2 g m}=$ head available for producing flow through the wheel, i.e., the total head minus that necessary to overcome pipe line losses, we must have :-

$$
H^{\prime}=\frac{p_{2}}{W^{r}}+\frac{r_{2}^{2}}{2 !}-\frac{p_{3}}{W^{r}}
$$

so that, substituting in (4) and putting $\left\{\begin{aligned} r_{2}^{2} & =u_{2}{ }^{2}+f_{2}{ }^{2}, \\ r_{3}{ }^{2} & =w_{3}{ }^{2}+f_{3}^{2}, \\ & =f_{3}{ }^{2} \text { if } u_{3}=0 \text {, }\end{aligned}\right.$ we have

$$
H^{\prime}=\frac{f_{3}^{2}}{2 !}+\frac{w_{2}^{2}}{g}\left(1-\frac{\tan a}{\tan \beta}\right)
$$

from which, writing $f_{3}=f_{2} \frac{b_{2} r_{2}}{b_{3} r_{3}}=u_{2}$ tan $a \frac{b_{2} r_{2}}{b_{3} r_{3}}$ we geít-

$$
\begin{gathered}
\left.H^{\prime}=\frac{w_{2}^{2}}{2 g} i^{2}+\left(\frac{l_{2} r_{2}}{l_{3} r_{3}} \tan a\right)^{2}-2 \frac{\tan a}{\tan \beta}\right) . \\
\therefore \quad w_{2}=\sqrt{\frac{2 g H^{\prime}}{2+\left(\frac{b_{2} r_{2}}{b_{3} r_{3}} \tan a\right)^{2}-2 \frac{\tan a}{\tan \beta}}} \\
\therefore \quad r_{2}=\left(1-\frac{\tan a}{\tan \beta}\right) \sqrt{\frac{2 g H^{\prime}}{2+\left(\frac{b_{2} r_{2}}{l_{3} r_{3}} \tan a\right)^{2}-2 \frac{\tan a}{\tan \beta}}}
\end{gathered}
$$

This gives the velocity of whirl, and the peripheral speed of the wheel for maximum efficiency, in terms of the available head $H$ ' $^{\prime}$

The effect of any variation in $a$ or $\beta$, on the peripheral speed for maximum efficiency is shown in the following table, which 
gives the theoretical values of $k$ (where $u_{2}=k \sqrt{2 g H^{\prime} \text { ) }}$ in the case where $f_{2}=f_{3}$.

\begin{tabular}{l|c|c|c|c|c|c|c|c}
\hline \multirow{2}{*}{$\begin{array}{c}\text { Values of } \\
\beta .\end{array}$} & \multicolumn{7}{|c}{ Values of $a}$. \\
\cline { 2 - 7 } & $0^{\circ}$ & $5^{\circ}$ & $10^{\circ}$ & $12 \frac{1}{2}$ & $15^{\circ}$ & $20^{\circ}$ & $25^{\circ}$ & $30^{\circ}$ \\
\hline $60^{\circ}$ & & & $\cdot 658$ & & $\cdot 636$ & $\cdot 604$ & $\cdot 564$ & $\cdot 516$ \\
\hline $75^{\circ}$ & & & $\cdot 685$ & & $\cdot 669$ & $\cdot 648$ & $\cdot 625$ & $\cdot 596$ \\
\hline $90^{\circ}$ & $\cdot 707$ & $\cdot 705$ & $\cdot 702$ & $\cdot 698$ & $\cdot 695$ & $\cdot 685$ & $\cdot 672$ & $\cdot 655$ \\
\hline $105^{\circ}$ & & & $\cdot 724$ & & $\cdot 729$ & $\cdot 732$ & $\cdot 733$ & $\cdot 730$ \\
\hline $120^{\circ}$ & & & $\cdot 741$ & & $\cdot 748$ & $\cdot 756$ & $\cdot 764$ & $\cdot 770$ \\
\hline
\end{tabular}

On the assumption that $w_{3}=o$ and that the loss at entrance is zero, the maximum theoretical efficiency $\eta$ of a turbine is given by $\eta=\frac{\text { work done per second by water }}{\text { energy supplied per second }}=\frac{\frac{W Q}{g} \cdot w_{2}^{2}\left(1-\frac{\tan \alpha}{\tan \beta}\right)}{W Q H^{\prime}}$

$$
\begin{aligned}
& =\frac{2\left(1-\frac{\tan a}{\tan \beta}\right)}{2\left(1-\frac{\tan a}{\tan \beta}\right)+\frac{b_{2}^{2} r_{2}^{2}{ }^{2}{ }_{3}^{2} r_{3}^{2}}{\tan ^{2} a}} \\
& =\frac{1}{1+\frac{1}{2}\left(\frac{b_{2} r_{2}}{b_{3} r_{3}}\right)^{2}-\frac{\tan ^{2} a}{1-\frac{\tan a}{\tan \beta}}}
\end{aligned}
$$

This gives the hydraulic efficiency of the machine, and does not take into account losses due to mechanical friction in the turbine. The actual efficiency of the machine is the ratio of the useful work delivered at the turbine shaft to the energy supplied at the wheel inlet, and is given by the ratio

$2 \pi N M$ where $N=$ revolutions per second

$\overline{W Q H^{\prime}} \quad M=$ turning moment on shaft in foot lbs.

Here $M$ is less than the hydraulic turning moment $\frac{W Q}{g}\left(w_{2} r_{2}-w_{3} r_{3}\right)$ foot lbs. by an amount depending on the 
frictional resistances of the turbine bearings and on the hydraulic frictional losses in the wheel itself.

From (10) it appears that the efficiency should theoretically increase as the ratio $\frac{b_{2} r_{2}}{b_{3} r_{3}}$ diminishes, i.e., as the breadth of the turbine is more rapidly increased towards the centre. Even theoretically, however, this possible increase is only small because of the smallness of $\tan ^{2} a$, while such a construction, by giving wheel passages which have a sectional area increasing in the direction of flow, tends to produce unsteady motion with a consèquent loss of energy in eddy production.

Again from equation (10) it appears that the efficiency increases as $\beta$ increases, and diminishes with an increase in $a$, the following table giving values of $\eta$ corresponding to different values of these angles, in the case where $\frac{b_{2} r_{2}}{b_{3} r_{3}}=1$, and where in consequence $f_{2}=f_{3}$.

\begin{tabular}{|c|c|c|c|c|c|}
\hline \multirow{2}{*}{ Values of $\beta$. } & \multicolumn{5}{|c|}{ Values of $\alpha$. } \\
\hline & $10^{\circ}$ & $15^{\circ}$ & $20^{\circ}$ & $25^{\circ}$ & $30^{\circ}$ \\
\hline $60^{\circ}$ & $\cdot 981$ & $\cdot 959$ & $\cdot 922$ & .871 & $\cdot 800$ \\
\hline $75^{\circ}$ & $\cdot 983$ & $\cdot 962$ & $\cdot 931$ & $\cdot 888$ & $\cdot 835$ \\
\hline $90^{\circ}$ & $\cdot 984$ & $\cdot 964$ & $\cdot 936$ & $\cdot 902$ & $\cdot 857$ \\
\hline $105^{\circ}$ & $\cdot 985$ & $\cdot 966$ & $\cdot 941$ & $\cdot 910$ & .874 \\
\hline $120^{\circ}$ & $\cdot 986$ & $\cdot 968$ & $\cdot 946$ & $\cdot 920$ & $\cdot 889$ \\
\hline
\end{tabular}

From this it would appear that it is of advantage to make $\beta$ as large as possible. But since we have $f_{2}=w_{2}$ tan $a=$ $u_{2}\left\{\frac{\tan a}{1-\frac{\tan a}{\tan \beta}}\right\}$, the velocity of flow for a given peripheral wheel speed diminishes as $\beta$ increases, so that an increase in $\beta$ necessitates in general a larger and more expensive turbine for a 
given power. In view of this, and of the fact that with the higher peripheral speed necessitated for efficient working with an increased value of $\beta$, frictional losses are increased, it has become usual to make $\beta=90^{\circ}$, i.e., to make the wheel vanes radial at their tips.

In this case the hydraulic efficiency is given by

$$
\eta=\frac{2}{2+\left(\frac{b_{2} r_{2}}{b_{3} r_{3}} \tan a\right)^{2}} .
$$

Similarly, although the hydraulic efficiency decreases as a increases, yet the volume passing through the turbine, and consequently its horse power, also increases, the maximum power being obtained when the product of $Q$ and $\eta$ is a maximum. It follows that the most satisfactory value of $a$ depends on the purpose for which the turbine is desired. In a high class turbine, $a$ will be as small as mechanical considerations permit - generally between $10^{\circ}$ and $15^{\circ}$, and the turbine will gain in efficiency at the expense of a higher prime cost. Where a cheap turbine is required a may have any value up to about $30^{\circ}$.

The volume of water passing through the turbine per second

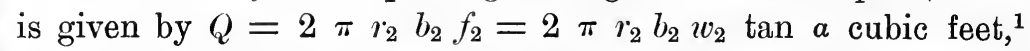
and when the turbine is working under conditions of maximum efficiency this becomes

$$
Q=2 \pi r_{2} b_{2} \tan a \sqrt{\frac{2 g H^{\prime}}{2+\left(\frac{b_{2} r_{2}}{b_{3} r_{3}} \tan a\right)^{2}-2 \frac{\tan a}{\tan \beta}}}
$$

so that both the velocity of the turbine for maximum efficiency and the volume of water passed through the wheel vary as $\sqrt{H^{\prime}}$.

1 This assumes that the passages run full, and neglects the effect of the thickness of the vanes. The latter factor may readily be allowed for, and is considered later (Art. 133). The construction of the vanes may, however (p. 504), cause some contraction of the stream as indicated in Fig. $207 c$, in which case the actual area over which flow takes place is less than that of the passages. We then get

$$
Q=k \times 2 \pi r_{2} b_{2} f_{2}=k \times 2 \pi r_{2} b_{2} w_{2} \tan \alpha \text { cubic feet, }
$$

where $k$ equals the coefficient of discharge of the passages. This, which includes both the coefficient of velocity and of contraction, is usually taken as about $\mathbf{9 5}$ and must be introduced in the application of these formulae to any specific example.

H.A. 
Since for maximum efficiency, the work done

$$
\begin{gathered}
U=\frac{W Q}{g} v_{2}^{2}\left(1-\frac{\tan a}{\tan \beta}\right) \text { foot lbs. per second. } \\
\therefore \quad U=\frac{W \times 2 \pi r_{2} b_{2} \tan a}{g}\left(1-\frac{\tan a}{\tan \beta}\right) \times \\
\left\{\frac{2 g H^{\prime}}{\left.2+\left(\frac{b_{2} r_{2}}{b_{3} r_{3}} \tan a\right)^{2}-2 \frac{\tan a}{\tan \beta}\right\}^{\frac{3}{2}} \text { foot lbs. per second. }}\right.
\end{gathered}
$$

$\therefore$ the work done is proportional to $H^{\prime \frac{3}{2}}$.

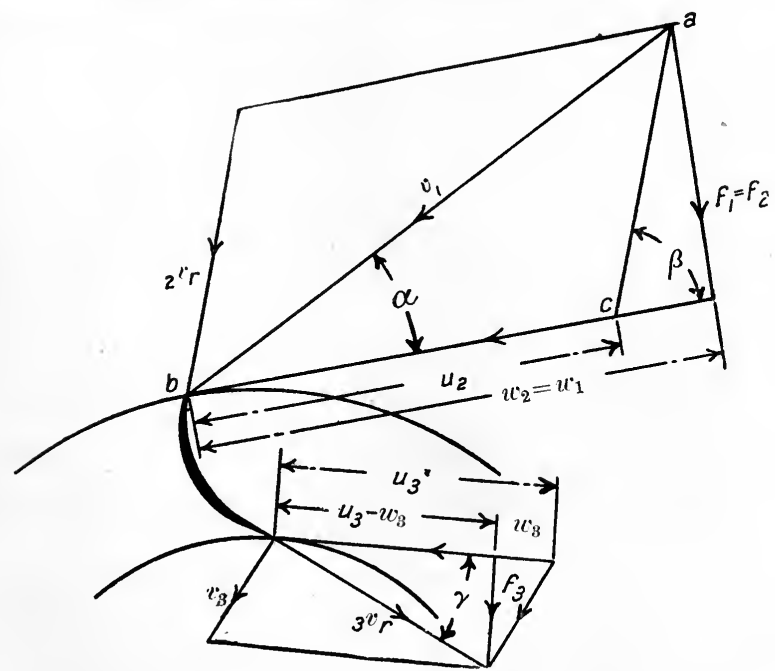

FIG. 206.-Vane Angles for Inward Radial Flow T'urbine.

An examination of equation (10) shows that the hydraulic efficiency diminishes with an increase in the ratio $\frac{r_{2}}{r_{3}}=n$, except where $b_{2} r_{2}=b_{3} r_{3}$, and therefore where the velocity of flow is the same at inlet and outlet. Apart from this, an increase in $n$, by increasing the length of wheel passages, reduces the efficiency by increasing frictional losses. This disadvantage is, however, counterbalanced by the increased regulating effect of the centrifugal pressure. It follows that as the efficiency of the governing mechanism is improved, it becomes advisable to reduce the value of $n$, and this explains why the value adopted in practice 
has been gradually reduced of recent years from 2 to as low as $1 \cdot 25$. Its usual value is about $1 \cdot 5$.

The discharge angle $\gamma$ of the vanes (Fig. 206) may be determined from the consideration that, if $w_{3}=0$,

$$
\begin{aligned}
\tan \gamma & =\frac{f_{3}}{u_{3}}=\frac{f_{2} b_{2} r_{2}}{u_{3} b_{3} r_{3}} \\
& =\frac{w_{2}}{u_{3}} \tan a \frac{b_{2} r_{2}}{b_{3} r_{3}}
\end{aligned}
$$

If $\beta=90^{\circ}, u_{2}=u_{2}$, and since $\frac{u_{2}}{u_{3}}=\frac{r_{2}}{r_{3}}$

we have:- $\quad \tan \gamma=\frac{b_{2} r_{2}^{2}}{b_{3} r_{3}^{2}} \tan a$.

If $f$ is constant this gives us :-

$$
\tan \gamma=\frac{r_{2}}{r_{3}} \tan a .
$$

And if $\frac{r_{2}}{r_{3}}=n$ we have :-

$$
\tan \gamma=n \tan a .
$$

Change of Pressure through Wheel.--Since the motion of a particle of water at any point in the wheel may be compounded of its motion in a forced vortex with angular velocity $\omega$, and of its motion parallel to the wheel vanes with (variable) velocity $v_{r}$, the difference of pressure at any two points in the wheel will be the algebraic sum of the differences necessary to produce these motions.

Thus, due to the vortex motion we have

$$
\frac{p_{2}-p_{3}}{W}=\frac{u_{2}^{2}-u_{3}^{2}}{2 g} \text { feet, }
$$

while due to the flow between the vanes we have

Summing these we have:-

$$
\frac{p_{2}-p_{3}}{W}=\frac{3 v_{r}^{2}-{ }_{2} v_{r}^{2}}{2 g} \text { feet. }
$$

$\left.\begin{array}{c}\text { Total difference of pressure } \\ \text { at inlet and outlet }\end{array}\right\}=\frac{p_{2}-p_{3}}{W}=\frac{u_{2}{ }^{2}-u_{3}{ }^{2}}{2 g}+\frac{{ }_{3} v_{r}{ }^{2}-{ }_{2} v_{r}{ }^{2}}{2 g}$.

But ${ }_{3} v_{r}^{2}=f_{3} \operatorname{cosec} \gamma$, and ${ }_{2} v_{r}=f_{2} \operatorname{cosec} \beta$, while $u_{3}=\frac{u_{2}}{n}$,

$\therefore \frac{p_{2}-p_{3}}{W}=\frac{u_{2}^{2}}{2 g}\left(1-\frac{1}{n^{2}}\right)+\frac{f_{3}^{2} \operatorname{cosec}^{2} \gamma-f_{2}^{2} \operatorname{cosec}^{2} \beta}{2 g}$. 
The pressure $p$, at any radius $r$ between inlet and outlet may be obtained if the angle $\theta$ made by the vanes at this radius with the tangent to the corresponding circle, and if the velocity of flow, $f$, be known.

Here, if $u$ be the velocity of the wheel at $r$, we have

$$
\begin{gathered}
u=\frac{u_{2} v}{r_{2}}, \text { while } r_{r}=f \operatorname{cosec} \theta, \\
\therefore \frac{p_{2}-p}{W}=\frac{u_{2}^{2}}{2 g}\left\{1-\left(\frac{r}{r_{2}}\right)^{2}\right\}+\frac{f^{2} \operatorname{cosec}{ }^{2} \theta-f_{2}^{2} \operatorname{cosec}{ }^{2} \beta}{2 g} \\
\text { or } p=p_{2}-W\left[\frac { u _ { 2 } ^ { 2 } } { 2 g } \left\{\begin{array}{c}
\left.\left.1-\left(\frac{r}{r_{2}}\right)^{2}\right\}+\frac{f^{2} \operatorname{cosec}^{2} \theta-f_{2}^{2} \operatorname{cosec}^{2} \beta}{2}\right] \\
\text { libs. per square foot. }
\end{array}\right.\right.
\end{gathered}
$$

If the velocity of flow is constant $f_{2}=f$, and this expression simplifies to :-

$$
p=p_{2}-\frac{W}{2 g}\left[u_{2}^{2}\left\{1-\left(\frac{r}{r_{2}}\right)^{2}\right\}+f_{2}^{2}\left(\operatorname{cosec}^{2} \theta-\operatorname{cosec}{ }^{2} \beta\right)\right] .
$$

As every term in this expression is known, the pressure at any point, and therefore the whole pressure on that portion of the runner which carries the vanes, may be determined.

An easier graphical method is, however, indicated in Art. 134.

The pressure over the face of the runner inside the vanes $=p_{3}$ lbs. per square foot, so that the total pressure over this face may be determined. Owing to leakage past the outer periphery of the runner the pressure on the rear face may, however, amount to as much as $p_{2}$ lbs. per square foot, and owing to the large difference of pressure thus produced on the two faces the end thrust on the shaft may become excessive. Various methods of balancing this end thrust have been adopted, and have been illustrated in the preceding chapter.

Further consideration of these methods will be postponed to Art. 173, where the similar problem of balancing the end thrust on the spindle of a centrifugal pump is considered in some detail.

Summary. - Collecting the more important of the results so far obtained we have, in the case of an inward radial flow turbine, working without shock at entrance and rejecting its discharge 
water without any tangential velocity, and therefore (neglecting the effect of other losses) working at its maximum efficiency,

$$
\begin{gathered}
{\psi_{2}}_{2}=\left(1-\frac{\tan a}{\tan \beta}\right) w_{2}= \\
\left(1-\frac{\tan a}{\tan \beta}\right) \sqrt{\frac{2 g H^{\prime}}{2-2 \frac{\tan a}{\tan \beta}+\left(\frac{n b_{2}}{b_{3}} \tan \sigma\right)^{2}}}
\end{gathered}
$$

$(3)_{a}$ $f_{2}=w_{2} \tan a$ feet per second. $\tan \gamma=n \frac{w_{2} b_{2}}{u_{3} b_{3}} \tan a$ $f_{3}=n \frac{b_{2}}{b_{3}} f_{2}$ feet per second.

$(5)_{a} p=p_{2}-\frac{W}{2 g}\left[u_{2}^{2}\left\{1-\left(\frac{r}{r_{2}}\right)^{2}\right\}+f^{2} \operatorname{cosec}^{2} \theta-f_{2}^{2} \operatorname{cosec}^{2} \beta\right]$ lbs. per square foot.

(6) Hydraulic efficiency $\eta=\frac{2}{2+\left(\frac{b_{2}}{b_{3}} n \tan a\right)^{2} \times \frac{1}{1-\frac{\tan a}{\tan \beta}}}$

$$
\text { Revolutions }=\frac{u_{2} \times 60}{2 \pi r_{2}} \text { per minute. }
$$

Radial Inward Flow Turbine with Radial Vane Tips.

In practice the vanes are very commonly made radial at the tips. In this case, putting $\beta=90^{\circ}, \tan \beta=\propto$, we now have, for no shock at entrance, the velocity of whirl $u_{2}$ equal to the peripheral velocity $u_{2}$ of the wheel. Making these substitutions in the general expressions already obtained we get:-

(1) $)_{b} \quad u_{2}=u_{2}=$

$$
\sqrt{\frac{2 g H^{\prime}}{2+\left(n \frac{b_{2}}{b_{3}} \tan a\right)^{2}}} \text { feet per second. }
$$

$(3)_{b}$ $\dot{t_{2}}=w_{2} \tan a$ feet per second.

$$
\tan \gamma=n^{2} \frac{b_{2}}{b_{3}} \tan a
$$

$$
f_{3}=n \frac{b_{2}}{b_{3}} t_{2} \text { feet per second. }
$$


$(5)_{l} \quad p=p_{2}-\frac{W}{2 g}\left[u_{2}^{2}\left\{1-\left(\frac{r}{r_{2}}\right)^{2}\right\}+f^{2} \operatorname{cosec}^{2} \theta-f_{2}^{2}\right]$ lbs. per square foot.

$$
\eta=\frac{2}{2+\left(n \frac{b_{2}}{b_{3}} \tan a\right)^{2}}
$$

In some cases the velocity of flow is kept constant by increasing the breadth of the turbine as the radius diminishes so as to keep $b r$ constant. In this case $f_{2}=f_{3}, b_{2} r_{2}=b_{3} r_{3}$, and therefore $n \frac{l_{2}}{l_{3}}=1$, and the foregoing results are modified as follows :-

$$
u_{2}=u_{2}=\sqrt{\frac{2 g H^{\prime}}{2+\tan ^{2} \alpha}} \text { feet per second. }
$$

$$
\begin{gathered}
\tan \gamma=n \tan a \\
f_{3}=f_{2} \text { feet per second. } \\
\left.p=p_{2}-\frac{W}{2 g}\left[u_{2}^{2} ! 1-\left(\frac{r}{r_{2}}\right)^{2}\right\}+f_{2}^{2}\left(\operatorname{cosec}^{2} \theta-1\right)\right]
\end{gathered}
$$
lbs. per square foot.

$$
\eta=\frac{2}{2+\tan ^{2} a}
$$

$$
\text { Revolutions }=\frac{u_{2} \times 60}{2 \pi r_{2}} \text { per minute. }
$$

Since $a$ is always small, we see from $(1)^{\prime}$ that the peripheral velocity of the wheel for maximum velocity is very approximately given by

$$
u_{2}=w_{2}=\sqrt{\frac{2 g H^{\prime}}{2}}=\cdot 707 \sqrt{2 g H^{\prime}},
$$

so that with radial tips the peripheral velocity at entrance is nearly that due to a fall through a height $\frac{H^{\prime}}{2}$, while the supply water at this point has changed only approximately one half of its total store into kinetic energy.

From the foregoing analysis, or from a consideration of the triangle of velocities $a \cdot b c$ of Fig. 206, it is evident that the speed of the wheel is a function solely of the velocity due to the head, and of the angle $a$.

This being so, it remains to decide upon some relation between 
the velocities of flow and of whirl before this angle, and the wheel speed, can be settled. The precise relation between these two velocities, which shall conduce to the highest economic efficiency,

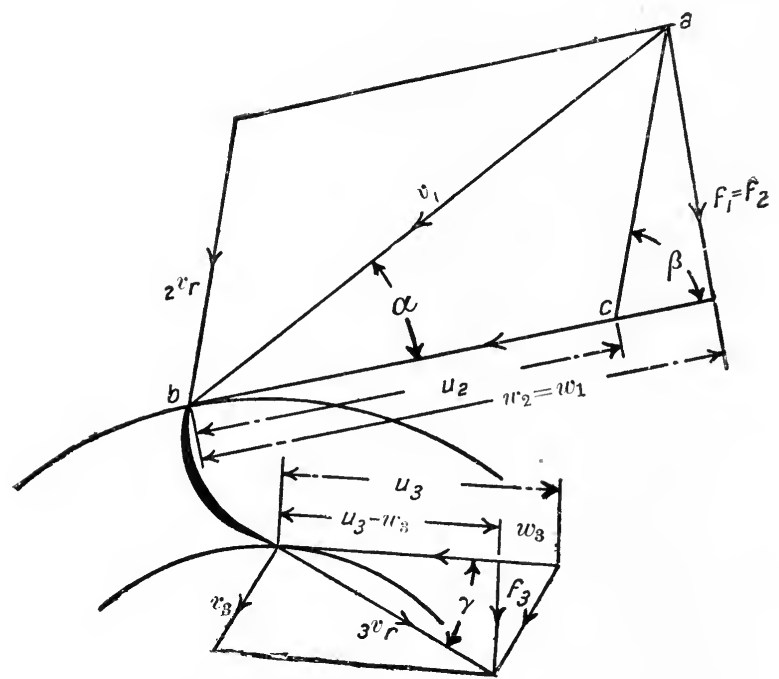

Fig. 206.-Wane Angles for Inward Radial Flow Turbine.

can only be deduced from experiment, and the following table shows the relationships which modern practice has shown to be most successful :-

\begin{tabular}{|c|c|c|c|c|}
\hline & $f_{2 .}$. & $u_{2}$. & $\alpha$. & $n=\frac{r_{2}}{r_{3}}$ \\
\hline $\begin{array}{l}\text { Inward radial flow turbine } \\
\text { Thomson type } \\
\text { Francis type }\end{array}$ & 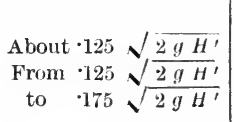 & $\begin{array}{l}\text { About } 66 \sqrt{\frac{2 g H^{\prime}}{2 g H^{\prime}}} \\
\text { From } \cdot 66 \sqrt{\frac{2 g H^{\prime}}{}} \\
\text { to } 72 \sqrt{n^{2}}\end{array}$ & $\begin{array}{r}\text { About } 11^{\circ} \\
\text { From } 11^{\circ} \\
\text { to } 14^{\circ}\end{array}$ & $\begin{array}{c}2 \\
\text { From } 1 \cdot 25 \\
\text { to } 1 \cdot 50\end{array}$ \\
\hline $\begin{array}{l}\text { Mixed flow turbine of the } \\
\text { American type }\end{array}$ & $\begin{array}{c}\text { From } \cdot 3 \pi \sqrt{\frac{2 g H^{\prime}}{2 g H^{\prime}}} \\
\text { to } 40 \sqrt{2}\end{array}$ & 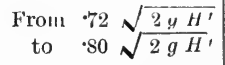 & $\begin{array}{l}\text { From } 22^{\circ} \\
\text { to } 28^{\circ}\end{array}$ & \\
\hline Outward radial flow turbine & About $\cdot 25 \sqrt{2 g H^{\prime}}$ & $\begin{array}{c}\text { From } 56 \sqrt{\frac{2 g H^{\prime}}{2 y H^{\prime}}} \\
\text { to } \cdot 62 \sqrt{2}\end{array}$ & $\begin{array}{l}\text { From } 20^{\circ} \\
\text { to } 25^{\circ}\end{array}$ & $\begin{array}{c}\text { From } \cdot 80 \\
\text { to } \cdot 85\end{array}$ \\
\hline Axial flow turbine & $\begin{array}{l}\text { From } \cdot 175 \sqrt{\frac{2 g H}{2 g H}} \\
\quad \text { to }-225 \sqrt{2 g H^{\prime}}\end{array}$ & $\begin{array}{c}\text { From } 62 \sqrt{2 g H^{\prime}} \\
\text { to } 68 \sqrt{2 g H^{\prime}}\end{array}$ & $\begin{array}{l}\text { From } 16^{\circ} \\
\text { to } 22^{\circ}\end{array}$ & $1 \cdot 0$ \\
\hline
\end{tabular}




\section{Remarks on the Theory of Turbine Design.}

Although the preceding theory is extremely valuable, its limitations are important and must be kept in view. In the first place, even with vanes designed so as (theoretically) to prevent shock at entrance, experiment shows that a certain contraction of section of the stream, as indicated in Fig. $207 a$ and $b$, takes place, and also indicates that this contraction and the subsequent re-expansion is greater with the theoretically correct entrant angle than with one slightly different. When working at part gate the ingoing stream may, if sufficiently reduced in width, not fill the buckets at all, in which case the wheel runs as an impulse turbine, the pressure at the exit from the guides falls to that in the discharge pipe, and the outflow per unit area is largely increased. Since the wheel speed is now altogether unsuited to the velocity of influx, the efficiency is low. Even though the stream may re-expand to fill the buckets and the wheel act as a pressure turbine, yet with the same pressure at exit from the wheel, the pressure at the exit from the guides will be greater than when running full, though not to the same extent as before, so that in either case the volume of water passing the wheel is larger than might be expected from a consideration of the gate opening.

This action is well illustrated by the following results of tests carried out by Mr. J. B. Francis on a mixed-flow turbine, of which the following are the leading dimensions ${ }^{1}:-$

Outer diameter of runner, 6 feet.

Least diameter of runner, 2 feet 8 inches.

Width of guide passages, $13 \cdot 1$ inches.

Width of buckets, $b_{2}, 13 \cdot 3$ inches.

Measured area of outflow from guides,

$9 \cdot 88$ square feet.

Measured area of outflow from buckets, $9 \cdot 56$ square feet.

\begin{tabular}{c|c|c|c|c|c}
\hline$H$ feet. & $\begin{array}{c}\text { Gate } \\
\text { Opening. }\end{array}$ & $\begin{array}{c}Q \text { cubic feet } \\
\text { per second. }\end{array}$ & $\begin{array}{c}\text { Percentage of } \\
\text { Flow. }\end{array}$ & Revolutions. & Efficiency. \\
\hline $14 \cdot 3$ & $\cdot 153$ & $51 \cdot 2$ & $31 \cdot 0$ & $60 \cdot 5$ & $47 \cdot 4 \%$ \\
$13 \cdot 7$ & $\cdot 306$ & $83 \cdot 9$ & $50 \cdot 8$ & $60 \cdot 1$ & $66 \cdot 6 \%$ \\
$13 \cdot 1$ & $\cdot 459$ & $110 \cdot 1$ & $66 \cdot 6$ & $66 \cdot 4$ & $76 \cdot 1 \%$ \\
$13 \cdot 0$ & $\cdot 612$ & $130 \cdot 2$ & $78 \cdot 9$ & $64 \cdot 0$ & $80 \cdot 1 \%$ \\
$12 \cdot 7$ & $\cdot 765$ & $144 \cdot 8$ & $87 \cdot 5$ & $62 \cdot 7$ & $83 \cdot 6 \%$ \\
$12 \cdot 7$ & $1 \cdot 00$ & $165 \cdot 1$ & $100 \cdot 0$ & $66 \cdot 6$ & $82 \cdot 8 \%$ \\
\hline
\end{tabular}

: Journal Franklin Institute, Vol. 99 (p. 249). 
Again, friction losses in the wheel increase continuously with the speed, and do not attain a minimum value simultaneously with the minimum values of the losses by shock and by rejection of kinetic energy, so that, although the expressions previously obtained for the efficiency by neglecting these losses,
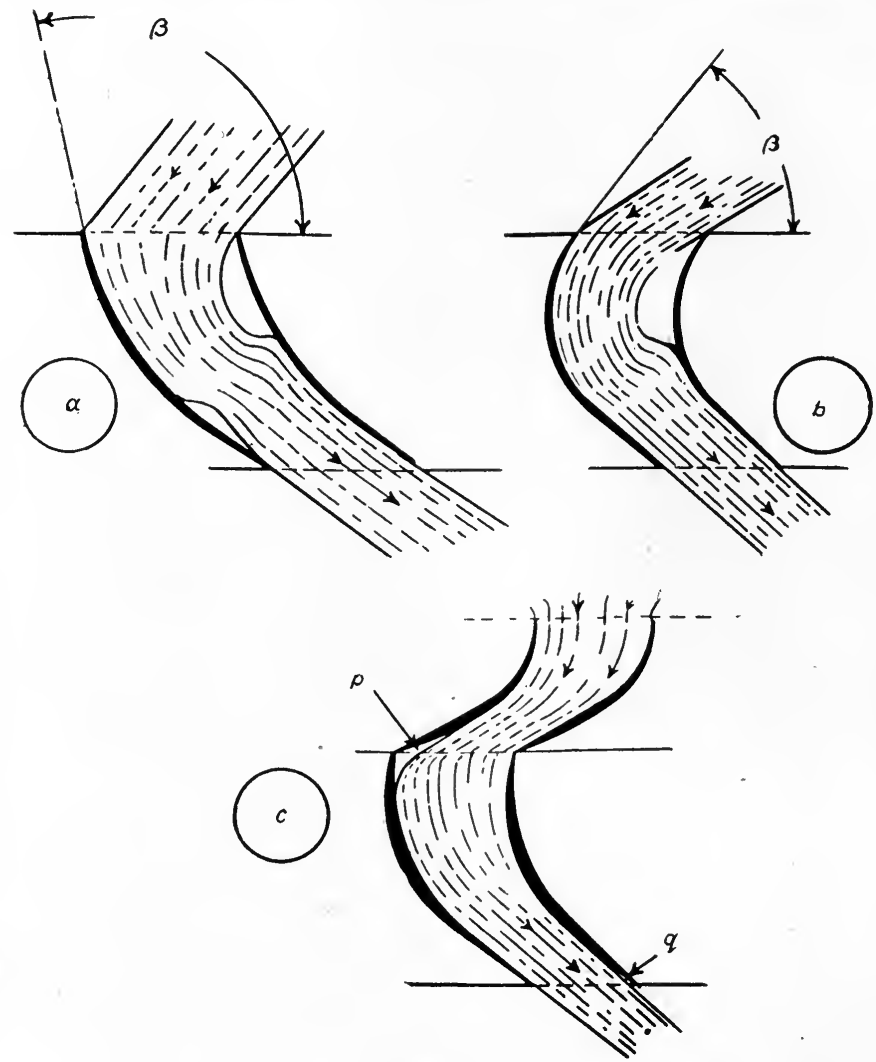

Fig. 207.

indicate that this is independent of the speed of rotation, there will, in actual working, be one particular speed for any turbine working under a given head-that speed at which the 'sum of the various losses forms the least proportion of the work done by the water-for which the efficiency will be a maximum.

Experiments by Professor Fliegner, of Zurich, ${ }^{\mathbf{1}}$ indicate that the

1 Zeitschrift des Vereines Deutscher Ingenieure, vol. 23, p. 459. 
loss at entrance diminishes as the working head increases, and that its minimum value does not occur for inflow without shock, but when the angle $\beta$ has a value differing from the theoretical, the best angle being greater than the theoretical for values of $\beta$ less than about $105^{\circ}$, and less than the theoretical for values of $\beta$ greater than this.

The following table gives some of Fliegner's results :-

\begin{tabular}{l|c|c|c|c|c|c|c}
\hline Value of discharge angle $\gamma$. & $15^{\circ}$ & $30^{\circ}$ & $15^{\circ}$ & $30^{\circ}$ & $15^{\circ}$ & $15^{\circ}$ \\
\hline $\begin{array}{l}\text { Value of } \beta \text { for no shock at entrance } \\
\begin{array}{l}\text { Experimental value of } \beta \text { for maximum } \\
\text { efficiency }\end{array}\end{array}$ & $120^{\circ}$ & $120^{\circ}$ & $90^{\circ}$ & $90^{\circ}$ & $60^{\circ}$ & $60^{\circ}$ \\
\hline & $\cdot$. & $111^{\circ}$ & $109^{\circ}$ & $10 \tilde{5}^{\circ}$ & $102^{\circ}$ & $98 \frac{1}{2}^{\circ}$ & $85^{\circ}$ \\
\hline
\end{tabular}

It follows that with angles designed for entry without shock, the most efficient speed will be less than the theoretical for values of $\beta$ less than $105^{\circ}$, and will be greater for greater values of $\beta$, a conclusion which is verified by the results of experiment, and which is indicated by the values of $u_{2}$ given in the table on p. 503.

Fliegner's results led him to the conclusion that the best value for $\beta$ is given by the formula $\beta^{\prime}=90^{\circ}+\frac{\beta-\gamma}{5}-30 \frac{b_{3}-b_{2}}{b_{2}}$, where $\beta$ and $\gamma$ are the values of inlet and discharge angles calculated in the usual way for maximum efficiency, and where $b_{2}$ and $b_{3}$ are the bucket widths at entrance and exit.

Again, in the pressure turbine, the assumption that the water is completely guided by the vanes is probably hardly even approximately true, only a small proportion of the water being directly guided, and that only on one side of the stream. Other parts of the stream may follow very different paths. Moreover, unless the guide passages are parallel for some short distance before the point of exit, the issuing stream may (as at $p$ in Fig. $207 c$ ) fail to occupy the total exit area, so that, even apart from the effect of the vane thickness, which will be considered later, the effective area of flow is less than $2 \pi r_{2} l_{2}$.

It is usual to allow for this effect approximately by making the calculations on the assumption that the co-efficient of contraction is 95 . The same reasoning applies at the point of discharge. 
Further, we have the impossibility of taking full account of the various frictional and eddy formation losses in the turbine except when guided by the results of experiment on the particular type of wheel under consideration.

The true value of the theory thus lies in its power of indicating the relative influence of the different details of design on the efficiency, and in its possibility of giving a preliminary design which may afterwards be slightly modified by the results of experiment on some similar type of machine, so as to approximate more nearly to that of the perfect turbine.

As an application of the results of the foregoing analysis, consider the design of an inward radial flow turbine of the Francis swivelling gate type, to give 10,000 H.P. at 300 revolutions per minute under a total head of 260 feet. The wheel vanes to have radial tips, the velocity of flow to be kept constant, and the wheel to be supplied through a steel penstock whose length is 450 feet.

Assuming a probable full-load efficiency of 84 per cent., the capacity of the penstock must be sufficient to allow of a supply of $\frac{10,000 \times 550}{.84 \times 260 \times 62.4}=404$ cubic feet per second.

Allowing a mean velocity under maximum load of 12 feet per second in the penstock, the area of this becomes $33 \frac{2}{3}$ square feet, corresponding to a diameter of approximately 6 feet 6 inches.

In such a turbine it is usual to arrange the design so as to give a maximum efficiency at about $\frac{3}{4}$ full-load. At this load the velocity of pipe flow is approximately 8 feet per second, and if the coefficient of friction be taken as $\cdot 005$, the loss of head due to friction and to the velocity of flow (assuming the kinetic energy due to the latter to be entirely lost) may be written as equal to

$$
\begin{aligned}
\frac{v^{2}}{2 g}\left(\frac{f l}{m}+1\right) \text { feet }= & \left.\frac{64}{64 \cdot 4} ; \frac{005 \times 450 \times 4}{6.5}+1\right) \text { feet } \\
& =2.37 \text { feet. }
\end{aligned}
$$

The effective head $H^{\prime}$ is thus $257 \cdot 6$ feet, so that $\sqrt{2 g H^{\prime}}=$ 129 ft. per sec.

Taking $a=13^{\circ}, \tan a=\cdot 231, \tan ^{2} a=\cdot 053$, while from (1) we 
have $w_{2}=u_{2}=\frac{129}{\sqrt{2 \cdot 053}}=90 \cdot 1$ feet per second.

The outer radius of the rumner is then given by the relation

$$
\frac{2 \pi r_{2} N}{60}=90 \cdot 1 . \quad \therefore r_{2}=\frac{60 \times 90 \cdot 1}{2 \pi \times 300}=2.87 \text { feet. }
$$

$\therefore$ Outer diameter of runner $=5 \cdot 74$ feet $=5^{\prime} 9^{\prime \prime}$.

Assuming an efficiency of 86 per cent. at $\frac{3}{4}$ load, we have :-

$Q=296$ cubic feet per second, so that from the expression

$Q=2 \pi r_{2} b_{2} \tan a w_{2}$, we get :-

$$
\begin{aligned}
b_{2} & =\frac{Q}{2 \pi r_{2} w_{2} \tan a}=\frac{296}{2 \pi \times 2.87 \times 90 \cdot 1 \times \cdot 231} \text { feet } \\
& =\cdot 79 \text { feet }=9 \cdot 5 \text { inches (approximately). }
\end{aligned}
$$

Taking $n=1 \cdot 30$, we have $r_{3}=\frac{2 \cdot 87}{1 \cdot 30}=2 \cdot 21$ feet, so that the inner diameter of the runner is $4^{\prime} 5 \frac{1}{4}{ }^{\prime \prime}$, while $b_{3}=\cdot 79 \times 1 \cdot 30=$ $12 \frac{5}{16}$ inches.

Also, since $\tan \gamma=n \tan a$, this makes $\tan \gamma=\cdot 300$, giving $\gamma$ the value $17^{\circ} 30^{\prime}$.

The theoretical hydraulic efficiency $\frac{2}{2+\tan { }^{2} a}$ now equals $\frac{2}{2 \cdot 053}=97 \cdot 4$ per cent.

Applying Fliegner's correction, it appears that for maximum efficiency at this speed of rotation $\beta$ should be increased from $90^{\circ}$ to a value $\beta^{\prime}$, where

$$
\begin{aligned}
\beta^{\prime} & =90^{\circ}+\frac{90^{\circ}-17 \cdot 5^{\circ}}{5}-30 \frac{\cdot 79(1 \cdot 30-1)^{\circ}}{\cdot 79} \\
& =90^{\circ}+14 \cdot 5^{\circ}-9^{\circ} \\
& =95 \cdot 5^{\circ} .
\end{aligned}
$$

As will be seen later, these results, however, need further correction for the thickness of the vanes at their inlet and outlet edges.

Art. 132.-Sources and Magnitude of Losses in the Pressure Turbine.

So far the various losses in the turbine and its appendages have been neglected, and while it is impossible to take these 
fully into account in a theoretical discussion, yet a more detailed examination will be of value as indicating their relative importance. In general, these losses consist of :-

(1) Frictional losses in supply and discharge pipes.

(2) Loss by leakage between guides and wheel.

(3) Losses due to shock at entrance if the vane angles are not adapted to the speed of the wheel, and to contraction and subsequent expansion of section of the stream.

(4) Frictional losses due to motion of the water over the vanes and crown of the wheel.

(5) Eddy losses caused by any sudden curvature of the vanes by sudden changes of section or divergence of the passages.

(6) Losses due to shock caused by sudden enlargement of the stream section on entering the buckets at part gate.

(7) Loss due to rejection of kinetic energy in the discharge.

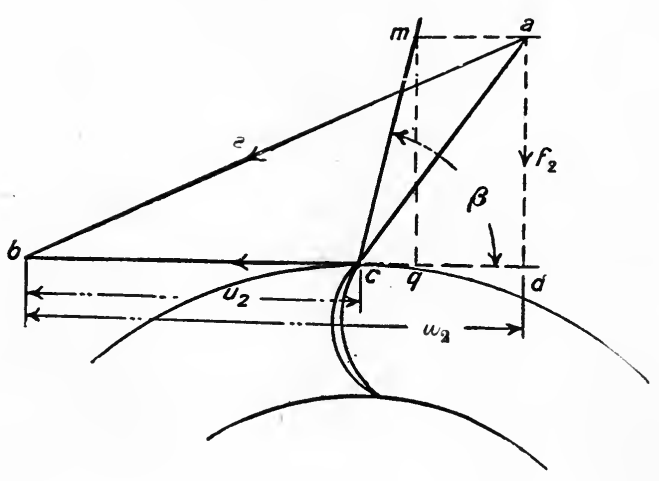

Fig. 208.

(8) Loss due to mechanical friction at bearings.

Of these losses, that due to the friction of the pipe line may be readily estimated, and depends on the length, diameter and velocity of flow through the pipes.

(2) Loss by Leakage, $H_{L}$.- This loss being proportional to the possible area through which leakage may take place and to the velocity of efflux over this area, is proportional to the periphery of the wheel $x$ clearance at entrance, and to the square root of the head at entrance. Its magnitude is greatest in turbines of the inward flow type, and may amount to from 2 to 4 per cent. of the total energy supplied.

(3) Loss due to Shock at Entrance. $-H_{E}$, if angle $\beta$ is not correctly proportioned. 
Let $\overline{a b}=v$ (Fig. 208) represent the velocity at the exit from guides.

Let $\overline{c b}=n_{2}$ represent the velocity of vane tips.

Then before entering the buckets the relative velocity of water and of wheel in the direction of the tangent at $c=\overrightarrow{c d}$.

Also if $\widetilde{m q}=\beta$, the relative velocity in the same direction on entering the buckets $=\overrightarrow{c q}$.

$\therefore$ Loss of head due to shock at entrance $=\frac{(\overline{c d}-\overline{c q})^{2}}{2 g}$ feet of water.

But $\overline{c d}=w_{2}-u_{2}$

And $\overline{c q}=\overline{m q} \cot \beta=f_{2} \cot \beta$.

$\therefore$ Loss of head $H_{E}=\frac{\left\{w_{2}-u_{2}-f_{2} \cot \beta\right\}^{2}}{2 g}$ feet

$$
=\frac{w_{2}^{2}}{2 g}\left\{1-\frac{u_{2}}{u_{2}}-\frac{\tan a}{\tan \beta}\right\}^{2} \text { feet. }
$$

The actual loss due to shock is in general less than that calculated, since only a portion of the whole supply stream suffers the extreme change of velocity.

(4) Frictional Losses in the Wheel, $H_{F}$ - - These may be divided into the losses caused by (a) Flow over the surfaces of the vanes and crowns; (b) Disc friction due to rotation of the turbine crown or crowns through the surrounding water.

(a) If $v_{r}=$ mean relative velocity of flow through turbine, $m$ the hydraulic mean depth of turbine passages, $l$ the effective length of passages, $F$ the coefficient of friction, this loss is given by

$$
H_{F}=\frac{F l r_{r}^{2}}{2 g m} \text { feet of water. }
$$

This loss will obviously be increased by the presence of dividing diaphragms, and by any increase in the width (in the direction of flow) of the wheel.

(b) The disc friction due to rotation of the turbine crowns may be calculated as follows :-

Consider an element of the area of the crown forming a ring of inner and outer radii $r$ and $r+\delta r$, concentric with the wheel axis, and rotating with an angular velocity $\omega$. 
The moment of resistance to
rotation of this element $;=\frac{F W \omega^{2} r^{3} 2 \pi r \delta r}{2 g}$ foot lbs.

$\therefore \quad$ Work done per second $\}=\frac{F W \omega^{3} r^{4} 2 \pi \delta r}{2 g}$ foot lbs.

$\therefore$ Work done against the resistance of a single disc of inner and outer radii $r_{1}$ and $r_{0}$, rubbing on one side only

$$
\begin{aligned}
& =\frac{F W \omega^{3} \pi}{g} \int_{r_{0}}^{r_{1}} r^{4} d r \\
& =\frac{F W \omega^{3} \pi}{5 g}\left\{r_{1}^{5}-r_{0}^{5} ; \begin{array}{l}
\text { foot lbs. } \\
\text { per sec. }
\end{array}\right.
\end{aligned}
$$

Where for smooth metal surfaces $\frac{F W}{g}=F^{\prime}=$ about $\cdot 0025 .^{1}$ The magnitude of this loss depends considerably on the type of turbine. In a single wheel radial flow turbine the whole of the rear face of the runner will, in general, be subject to this resistance, and that portion of the front face which lies between the shaft and the inner tips of the vanes, while in the case of a double discharge turbine of the Thomson vortex type, this rubbing area is almost doubled. A large increase in disc friction is also experienced in the case of a turbine balanced by the addition of a rotating balance piston.

In a wheel of the parallel flow type the design may be such as to cause a similar resistance at the outer circumference whose radius is $r_{2}$ and breadth $b_{2}$, the loss of energy in this case being given by $\frac{F^{\prime} W \omega^{3} b_{2} r_{2}^{4}}{2 g}$ foot lbs. per second.

In any case this loss of energy per second is proportional to $\omega^{3}$, and the loss per $\mathrm{lb}$. to $\omega^{2}$, since $(\mathcal{Q}$ is proportional to $\omega$. The magnitude of the loss may be from 3 to 6 per cent.

(5) and (6), $H_{G}$. - These losses due to eddy formation at changes of curvature and to shock at entrance at part gate do not admit of even approximate calculation. They may, however, be minimised by designing all passages to have as easy a curvature as

1 Experiments by Unwin, "Hydraulics," p. 136, show that the loss of energy in disc friction depends not only on the disc, but also on the roughness of the surface of the containing chamber and on the distance between its walls and the disc. The resistance was found to diminish slightly as the clearance was decreased from 6 inches to $1 \frac{1}{2}$ inches. 
possible, and by the adoption of gates of the swivel type. With this type of gate these losses may account for between 1 and 8 per cent. of the total head, while with cylinder regulation they may amount to as much as 20 per cent. at half gate.

(7) Loss due to Rejection of Kinetic Energy in the Discharge, $H_{K^{*}}$ - - Assuming the whole of the kinetic energy of discharge from the buckets to be lost, this loss is given by-

$$
\frac{f_{3}^{2}+w_{3}^{2}}{2 g} \text { foot lbs. per lb. }
$$

In an inward radial flow turbine (Fig. 204), where $n=\frac{r_{2}}{r_{3}}$, we have $w_{3}=u_{3}-f_{3} \cot \gamma=\frac{u_{2}}{n}-f_{3} \cot \gamma$, and if $f_{3}=f_{2}$, the loss becomes-

$$
\frac{f_{2}^{2}+\left(\frac{u_{2}}{n}-f_{2} \cot \gamma\right)^{2}}{2 g} \text { foot lbs. per lb. }
$$

Expressed in terms of $w_{2}$, this becomes-

$$
\frac{w_{2}^{2}}{2 g}\left[\tan ^{2} a+\left\{n\left(1-\frac{\tan a}{\tan \beta}\right)-\frac{\tan a}{\tan \gamma}\right\}^{2}\right] \text { foot lbs. }
$$

This loss becomes a minimum when $w_{3}$, i.e., the velocity of whirl at exit $=0$, and then has the value $\frac{f_{3}^{2}}{2 j}$.

Practically the whole of this energy is lost where the turbine discharges either directly into the tail-race or into a parallel suction tube whose area is equal to that of the vanes at discharge. If, however, the suction tube has a gradually increasing area towards its outlet, a portion, up to about 25 per cent., of this may be converted into pressure energy, with a consequent gain of efficiency by the wheel.

In general this source of loss accounts for between 3 and 8 per cent. of the total energy supplied, being greatest in turbines of the outward flow type and least in those of the axial flow type.

(8) Mechanical Friction, $H_{b}$ - This generally accounts for between 2 and 4 per cent. of the total energy, its magnitude depending largely on the type of machine (whether hydraulically balanced or not). 
Taking these losses into account, we have, if $H$ is the total supply head, $H=H^{\prime}+$ loss in pipe friction.

The hydraulic efficiency $\eta$ of

the turbine given by

$\frac{U}{H-\text { loss in pipe friction }}=\frac{U}{H^{\prime}}$

The work done on the !

turbine shaft

$=U-H_{L+E+F^{+}{ }_{G}}$ ft. lbs. per sec.

$\therefore \begin{gathered}\text { Gross efficiency of } \\ \text { turbine wheel }\end{gathered}=\frac{U-H_{L+E+F^{\prime}+G}}{H^{\prime}}$

$\begin{aligned} & \text { Useful work delivered at } \\ & \text { turbine shaft }\end{aligned}=U-H_{L+E+F^{F}+G^{*}+B}$ ft. lbs. per sec.

$\therefore$ Nett efficiency of $\begin{gathered}\text { turbine } \\ \text { turbing }\end{gathered}$

While the gross efficiency ) $\left.\begin{array}{l}\text { of the plant, including } \\ \text { supplyand dischargepipes }\end{array}\right\}=\frac{U-H_{L+E+F+G+B}}{H}$.

\section{Art. 133.-Thickness of Vanes.}

So far no account has been taken of the fact that the vanes must be made of a certain thickness, and therefore reduce the effective area of the guide and wheel passages. The necessary corrections for this in the case of an inward flow turbine, may be made as follows :-

Let $t$ be the vane thickness, and $n$ the number of vanes which eut the circumference under consideration.

At inlet, the area of guide vane circle occupied by these vanes $=b_{1} n_{1} t_{1} \operatorname{cosec} a$ (approx.), while the area of bucket vane circle $=b_{2} n_{2} t_{2} \operatorname{cosec} \beta$ (approx.).

At outlet the area of bucket vane circle $=b_{3} n_{2} t_{3} \operatorname{cosec} \gamma$ (approx.), where $a, \beta$, and $\gamma$, are the angles as calculated when neglecting the blade thickness.

Thus the outlet area is reduced in the ratio

$$
\begin{gathered}
\frac{2 \pi r_{3} b_{3}-n_{2} t_{3} b_{3} \operatorname{cosec} \gamma}{2 \pi r_{3} b_{3}} \text {, so that- } \\
f_{3} \text { (true) }=f_{3} \text { (approx.) }\left\{1-\frac{n_{2} t_{3} \operatorname{cosec} \gamma}{2 \pi r_{3}}\right\} .
\end{gathered}
$$

For $f_{3}$ to be kept the same we must then either have H.A. 
$\frac{\tan \gamma(\text { true })}{\tan \gamma(\text { approx. })}=\frac{1}{1-\frac{n_{2} t_{3} \operatorname{cosec} \gamma}{2 \pi r_{3}}}$, or the breadth $b_{3}$ must ba increased in the same ratio.

Similar corrections may be applied at the inlet to the buckets and the outlet from the guides.

Allowance should be made for the difference in radii between the guide and wheel vane circles, and in order to keep the velocity of flow constant where the water leaves the guides and enters the wheel, the breadth $b$ of the guides may be made slightly different to that of the vanes, so as to keep the area through which radial flow takes place, constant. We then have-

$$
b_{1}=\left\{\frac{2 \pi r_{2}-n_{2} t_{2} \operatorname{cosec} \beta}{2 \pi r_{1}-n_{1} t_{1} \operatorname{cosec} a}\right\} b_{2} .
$$

This is only approximate since the passage of the wheel vanes before the guide passages tends to diminish the effective area of the latter, while the effective area of the wheel at inlet is similarly diminished by the presence of the guide vanes. Sufficient data are not available to fix the best number of wheel vanes in any particular case. The greater the number, the more perfect is the guidance given to the water, although at the same time frictional losses are increased. The longer the water passages, and the fewer vanes are necessary to give sufficient guidance In the case of a number of modern inward radial flow turbines examined by the author. and of sizes ranging from 3 inches diameter to 66 inches diameter, the number of vanes was given with fair accuracy by the relation $n=k \sqrt[3]{d}$, where $d=$ diameter in inches and where $k$ is a coefficient varying from about $7 \cdot 7$ in the smaller to 8.4 in the larger wheels and having a mean value $=8$.

Opinions appear to differ widely as to whether the number of guide vanes should be greater or less than that of the wheel vanes. In general, with swivelling vanes, the number of guide vanes is made slightly less than the number of wheel vanes, while with stationary guides the number is up to about 50 per cent. greater. In high class turbines the vanes have rounded edges at entrance and exit, so that the value of $t$ 
generally varies from $\frac{1}{16}$ inches to $\frac{3}{8}$ inches, having its maximum value in a large turbine with cast-iron blades.

\section{Art. 134.-Curvature of Vanes.}

So long as the inlet and outlet angles of the vanes are correctly proportioned, the shape of the vane between these points only

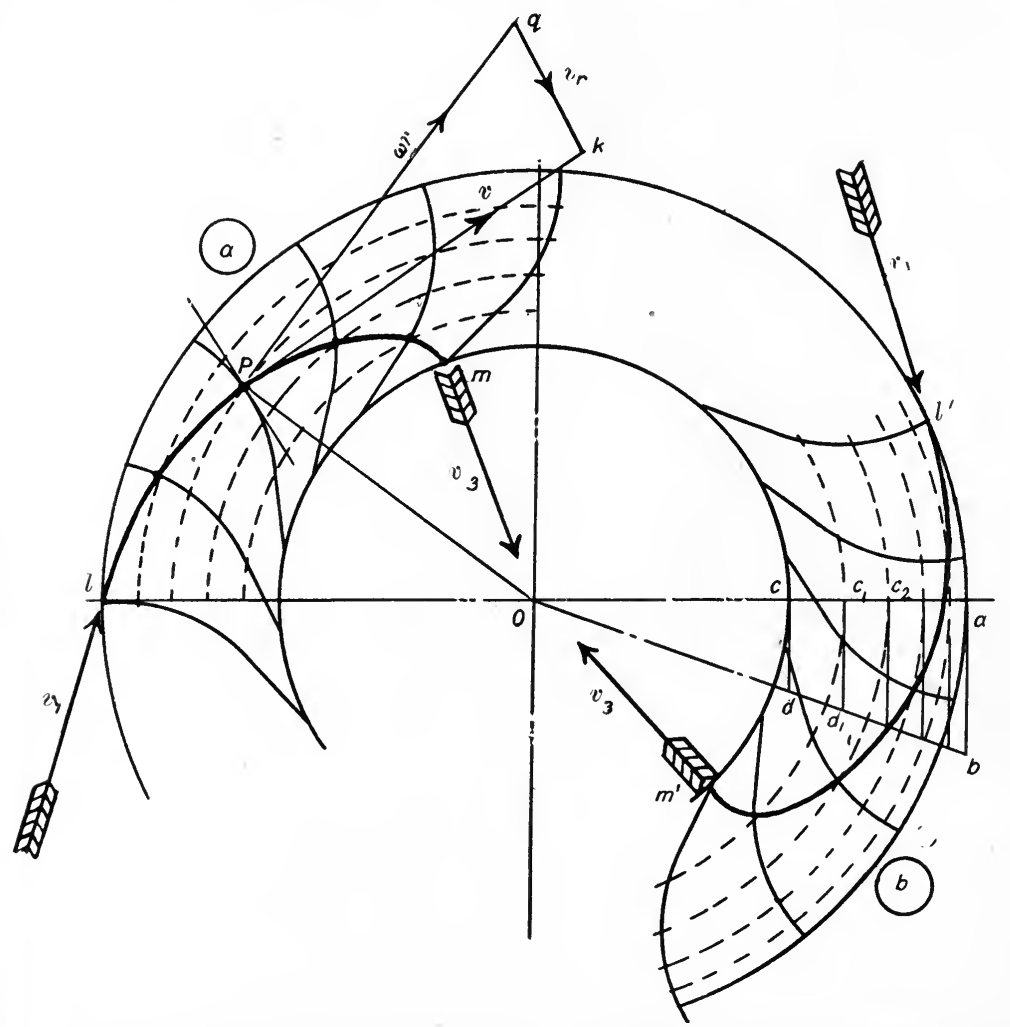

Frg. 209.-Sketch showing actual path of particles of water through an Inward Radial Flow Turbine.

affects the efficiency in so far as it tends to give steady or unsteady motion in the stream. To this end the design should be such that the passages are nowhere divergent, and that any changes of curvature are as gradual as possible. The first of these requirements is more easily satisfied in the inward flow than in the outward flow turbine. With this requirement fulfilled, 
the most efficient vane curve will be that with which the change in curvature of the path of the stream is most gradual, and to determine this it is advisable to set out a diagram showing for any proposed vane the true path of the particles of water in passing through the wheel. Two such diagrams are shown in Fig. 209, in which (a) shows the true path where the velocity of flow is uniform, and $(b)$ where the velocity of flow varies inversely as the radius. To construct diagram $(a)$, a series of equidistant circles are set out between the inner and outer vane circles. If $s$ be the radial distance between each pair of these, the time for a particle to pass from one to the other $=\frac{s}{f}$ seconds. Next set out the same number of equidistant vanes, the distance apart on the outer vane circle being $\omega r_{2} \frac{s}{f}$ feet. A particle leaving the intersection of the first circle and first vane will then be at the intersection of the second circle and vane after an interval of time $\frac{s}{f}$. seconds and so on, so that the path of the particle can be sketched in, as at $l m$.

Where the velocity of flow is not uniform, the only difference in the construction is that the intermediate circles are not equidistant but are so spaced that the particle travels radially from one to the other in equal intervals of time. Thus in case $(b)$ a radius $o$ a being drawn, a right-angled triangle $o a b$ is constructed on this and lines $c d, c_{1} d_{1}$, etc., drawn, cutting off equal areas $c_{2} d_{1}, c_{1} d$, etc., from the triangle. Circles drawn through $c_{1}, c_{2}$, etc., will now, by their intersections with the equidistant vanes, give points on the path $l^{\prime} m^{\prime}$ of a particle.

These curves may be utilized to obtain the absolute velocity of the water and the relative velocity of water and vane at any radius, for if at any point $P$ of radius $r, P q$ be drawn perpendicular to $O P$ and equal to $\omega r$, and if $q k$ be drawn parallel to the tangent to the vane at $P$ and $P k$ be drawn tangential to the curve at $P$, then since the actual velocity of the particle at $P$ is compounded of the velocity of the vane at $P$, i.e., of $\omega r$, and of its velocity relative to the vane, and since its actual velocity is tangential to the curve at $P$ and its relative 
velocity is parallel to the vane, $P q k$ serves as the triangle of velocities at $P$, and $\overline{P k}=$ the absolute velocity $v$, while $\overline{q k}=$ the relative velocity $v_{r}$ of water and vane.

By obtaining $v_{r}$ in this way the pressure " $p$ " at any radius $r$ may be easily obtained by an application of the formula

$$
p=p_{2}-\frac{W}{2 g}\left\{u_{2}^{2}-u^{2}+v_{r}{ }^{2}-{ }_{2} v_{r}{ }^{2}\right\} \text { lbs. per square foot, }
$$

where $u$ and $v_{r}$ refer to a point at radius $r$.

\section{Example.}

A turbine runner has an outer diameter of 5 feet 6 inches and an inner diameter 4 feet 3 inches. It makes 300 revolutions per minute. The space occupied by the blades being divided into five concentric strips, each $1 \frac{1}{2}$ inches wide, it is found graphically that the relative velocities at the centres of these strips, commencing at the outside are,

$$
26 ; 24 ; 26 ; 40 ; 65 \text { feet per second. }
$$

The relative velocity at entrance is $27 \mathrm{ft}$. per sec. and at exit is $76 \mathrm{ft}$. per sec. The pressure head at inlet is 134 feet.

Denoting these strips by $a, b, c, d, e$, we have :-

$$
\begin{aligned}
p_{a} & =p_{2}-\frac{62 \cdot 4}{64 \cdot 4}\left\{(89 \cdot 5)^{2}-(84 \cdot 5)^{2}+(26)^{2}-(27)^{2}\right\} \\
& =p_{2}-791 . \\
p_{b} & =p_{2}-\frac{62 \cdot 4}{6 \cdot 4}\left\{(89 \cdot 5)^{2}-(80 \cdot 5)^{2}+(24)^{2}-(27)^{2}\right\} \\
& =p_{2}-1,335 . \\
p_{e} & =p_{2}-\frac{62 \cdot 4}{64 \cdot 4}\left\{(89 \cdot 5)^{2}-(76 \cdot 5)^{2}+(26)^{2}-(27)^{2}\right\} \\
& =p_{2}-2,021 . \\
p_{d} & =p_{2}-\frac{62 \cdot 4}{64 \cdot 4}\left\{(89 \cdot 5)^{2}-(7 \cdot 6 \cdot 6)^{2}+(40)^{2}-(27)^{2}\right\} \\
& =p_{2}-3,520 . \\
p_{e} & =p_{2}-\frac{62 \cdot 4}{64 \cdot 4}\left\{(89 \cdot 5)^{2}-(68 \cdot 8)^{2}+(65)^{2}-(27)^{2}\right\} \\
& =p_{2}-6,550 .
\end{aligned}
$$




$$
\begin{aligned}
& \text { At exit } \begin{aligned}
p_{3} & =p_{2}-\frac{62 \cdot 4}{64 \cdot 4}\left\{(89 \cdot 5)^{2}-(66 \cdot 8)^{2}+(76)^{2}-(27)^{2}\right\} \\
& =p_{2}-8,320 \\
& \quad \frac{p_{2}-p_{3}}{W}=\frac{8,320}{62 \cdot 4}=133.5 \text { feet. }
\end{aligned}
\end{aligned}
$$

This affords a check as to the accuracy of the construction. Assuming the pressure over the corresponding portion of the back face of the disc to be $p_{2}$ lbs. per square foot, the total unbalanced pressure over this portion in the direction of the suction tube is equal to

$$
\begin{aligned}
\frac{2 \pi \times 1 \cdot 5}{12} & {\left[\left(\begin{array}{c}
32 \cdot 25 \\
12
\end{array} \times 791\right)+\left(\begin{array}{c}
30 \cdot 75 \\
12
\end{array} \times 1,335\right)+\left(\frac{29 \cdot 25}{12} \times 2,021\right)\right.} \\
& \left.+\left(\begin{array}{c}
27 \cdot 75 \\
12
\end{array} \times 3,520\right)+\left(\begin{array}{c}
26 \cdot 25 \\
12
\end{array} \times 8,320\right)\right] \mathrm{lbs} . \\
= & \cdot 7854\{2,130+3,420+4,935+8,125+18,210\} \\
= & 29,000 \mathrm{lbs} .
\end{aligned}
$$

Art. 135.-The Outward Radial Flow Pressure Turbine.

The general considerations of design of the outward flow turbine are exactly the same as for the inward flow type, and the same symbols and equations apply throughout.

Now, however, $n=\frac{r_{2}}{r_{3}}$ is less thán unity, so that in equation (16) of article 131

$$
\frac{p_{2}-p_{3}}{W}=\frac{u_{2}^{2}}{2 g}\left(1-\frac{1}{n^{2}}\right)+\frac{f_{3}^{2} \operatorname{cosec}{ }^{2} \gamma-t_{2}^{2} \operatorname{cosec}^{2} \beta}{2 g}
$$

the term $\frac{u_{2}^{2}}{2 g}\left(1-\frac{1}{n^{2}}\right)$ is negative, while, strictly speaking, the second term of the equation ceases to apply, since the flow now takes place through a series of diverging channels, and eddy formation is in consequence set up.

Centrifugal force now aids the flow through the turbine, and an increase in speed, by increasing the term $\frac{u_{2}{ }^{2}\left(\frac{1}{n^{2}}-1\right)}{2 g}$, decreases the inlet pressure, and causes an increased flow 
through the wheel. This turbine is in consequence difficult to govern satisfactorily. To reduce this effect as far as possible the term $\left(\frac{1}{n^{2}}-1\right)$ should be small. This necessitates $n$ or $\frac{r_{2}}{r_{3}}$ being made as nearly unity as practicable, and necessitates a bucket depth as small as is compatible with easy curves connecting inlet and outlet lips. In general $r_{3}$ is made from $1 \cdot 20$ to $1 \cdot 25$ times $r_{2}$.

Owing to the high peripheral velocity at exit, it is now impracticable to design the exit angles so as to make the velocity of whirl at exit equal to zero. The losses due to rejection of kinetic energy are thus in general higher with this than with the inward flow type.

In this type of turbine $f_{2}$ is generally given a value about $.25 \sqrt{2 g H^{\prime}}$, while $u_{2}$ varies from $[\cdot 56$ to $\cdot 62] \sqrt{2 g H^{\prime}}$. In general $\gamma$ lies between $20^{\circ}$ and $30^{\circ}$, and in high-class turbines $a$ lies between $20^{\circ}$ and $25^{\circ}$.

\section{Art. 136.-Axial Flow Pressure Turbine.}

Here the general theory is the same as for the inward and outward radial flow types of turbine, and the same demonstrations hold good if it be remembered that $r_{2}=r_{3}=r$; $u_{2}=u_{3}=\omega r ; n=1$.

If, as assumed in the previous cases, the plane of the wheel is horizontal, the equation of energy now becomes:$\frac{p_{2}}{W}+\frac{r_{2}^{2}}{2 g}=\frac{p_{3}}{W}+\frac{f_{3}^{2}}{2 g}+\{$ work done in wheel + losses from (2) to (3) $-h ;$ per $1 b$.

where $h=$ depth of wheel.

This assumes no velocity of whirl at exit, so that $f_{3}=v_{3}$.

We now have

$$
\frac{p_{2}-p_{3}}{W}=\frac{f_{3}^{2} \operatorname{cosec}^{2} \gamma-f_{2}{ }^{2} \operatorname{cosec}^{2} \beta}{2 g}-h
$$

from equation (16) p. 499 , the term $\frac{u_{2}^{2}}{2 g}\left(1-\frac{1}{n^{2}}\right)$ expressing the centrifugal effect, now vanishing. 
Here $f_{2}$ generally equals from $[\cdot 175$ to $\cdot 225] \sqrt{2 g\left(H^{\prime}-h\right)}$, while $u_{2}$ varies from $[\cdot 62$ to $\cdot 68] \sqrt{2 g\left(H^{1}-h\right)}$. $a$ varies from $16^{\circ}$ to $22^{\circ}$, while $\gamma$ varies from $15^{\circ}$ to $25^{\circ}$.

Evidently with a given value of $\alpha$ and of $\beta$, at only one point in the radius of the wheel for any given speed, will the conditions be suitable for entrance without shock. For entrance without shock at all radii, the value of $\beta$ should change continuously, so as to suit the corresponding peripheral speed. This construction is seldom carried out in practice, it being usual to have helical vanes, with radial inlet and outlet edges, both for guides and wheel, and to give $a$ and $\beta$ their correct values at the mean radius. With such vanes, having a constant pitch, the angle of inclination diminishes as the radius increases, and while this is an advantage in the case of the guides, it is the reverse .in the case of the wheel, where the value of $\beta$ for no shock should increase with the peripheral velocity and therefore with the radius. Better results would be obtained by giving the wheel vanes the same angle at all radii, although if the width of the buckets is small-not exceeding $\frac{1}{10}$ the wheel diameter-the loss from shock is trifling.

Art. 137.-Ayrerican Type: Combined Radial Inward Flow and Axial Discharge Pressure Turbines.

Here, as in the inward flow type of wheel, the turbine is to a certain extent self-regulating. The design of the vanes at inlet is regulated by the laws governing the design of the inward flow wheel and at outlet by the laws governing that of the axial flow type. In general, a higher peripheral velocity is adopted for this type, this varying from $\cdot 7$ to $\cdot 75 \sqrt{2 g h}$ for maximum efficiency at full gate. Where regulated by cylinder gates, the speed for maximum efficiency falls to from 57 to $61 \sqrt{2 g H}$ at half gate, the efficiency under these conditions varying from about 80 per cent. at full gate to 65 per cent. at half gate.

With the better makes of this class of machine, having inlet angles of about the same magnitude as those of the Francis and Thomson turbines, the full load efficiency would appear to be almost, if not quite, as high as is attainable with 
either of the latter types. The exaggerated claims of efficiencies of over 87 per cent.- 90 per cent. in the case of one well-known American machine-which have been made by the respective makers, may, however, be rejected without hesitation, and anything over 82 per cent. with this type of turbine may be considered as extremely good.

The correct inclination of the tangent plane to the vane at discharge, at any radius, may be determined on the assumption that when the wheel runs full there is no relative radial interchange of the particles of water. The turbine may then be imagined as subdivided into a series of " elements each having a depth at inlet $=b \div n$, and a width at outlet $=l \div n$, where $b$ is the depth of the wheel at inlet and $l$ is the length of the discharging periphery of each bucket (Fig. 210). It then becomes easy to calculate the respective

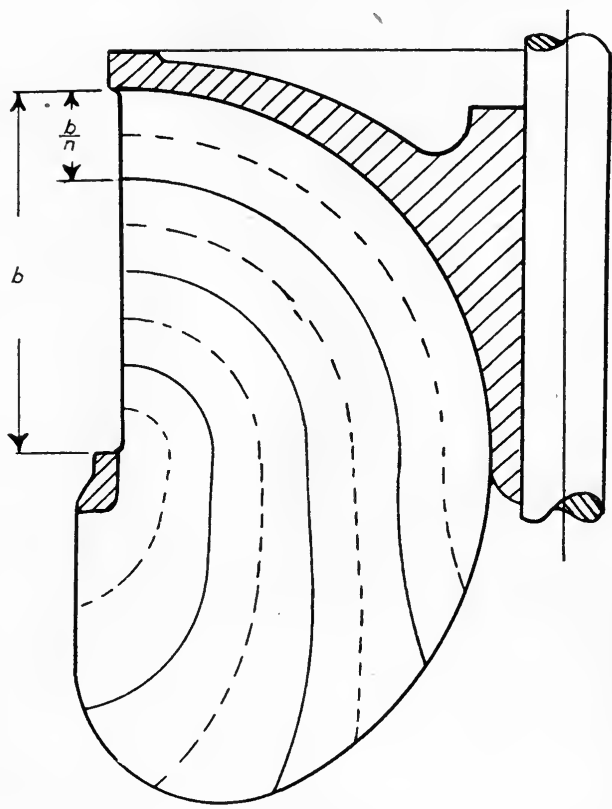

Fig. 210. angles of inclination to the horizontal and the vertical at the mid point of each element at outlet in order that the discharge may be as nearly as possible axial, and so to obtain the contours of the vane.

This method cannot, however, be relied upon to give very accurate results, since the velocity of flow across the whole outlet area will not be equal. 'This follows because of the reduced resistance to flow through those elements which offer the shorter path to the water, and in which at the same time, because of their shorter radial length, the resistance to inward flow caused 
by the centrifugal action of the water, is least. Thus the velocity of flow will be greater in those elements of the discharge area which are at a greater distance from the centre.

It thus becomes necessary for accurate results to treat each section as a separate turbine with given inlet and outlet pressures, and so to calculate the relative flow per unit area across each section.

This involves very elaborate calculations, ${ }^{1}$ and the more usual method in practice is to determine the correct mean angle for the outflow at the point of mean radius on the assumption that this outflow is uniform over the whole section.

\section{Art. 138.- Impulse Turbine of the Girard Type.}

Here the pressure remains constant throughout the turbine, being either atmospheric or that corresponding to the air pressure inside the turbine casing, so that $p_{2}=p_{3}$.

As in the pressure turbine, the work done by the water, assuming the vanes designed to give no velocity of whirl at exit, is given by

$$
U=\frac{U^{r} Q}{g} u_{2} u_{2} \text { foot lbs. per second. }
$$

The equation of energy-

now simplifies to

$$
\frac{p_{2}}{W}+\frac{w_{2}^{2}+f_{2}^{2}}{2 g}=\frac{p_{3}}{W}+\frac{f_{3}^{2}}{2 g}+\frac{w_{2} u_{2}}{!}
$$

$$
w_{2}^{2}+f_{2}^{2}-f_{3}^{2}=2 u_{2} u_{2} \text {. }
$$

Writing $f_{3}=k f_{2}$, this becomes-

$$
w_{2}^{2}+\left(1-k^{2}\right) f_{2}^{2}=2 w_{2} u_{2} \text {. }
$$

If $f_{2}=f_{3}$, i.e., $k=1$, this reduces to $u_{2}=\frac{w_{2}}{2}$, and since $\sqrt{w_{2}^{2}+f_{2}^{2}}=C_{v} \sqrt{2 g H^{\prime}}$, where $C_{v}=\cdot 97$ (approximately) we have

$$
\begin{aligned}
u_{2} & =\frac{\sqrt{{C_{v}{ }^{2} \times 2 g H^{\prime}-{\dot{f_{2}}}^{2}}_{2}}}{} \\
& =\frac{C_{v} \sqrt{2 g H^{\prime}}}{2} \text { (approximately). }
\end{aligned}
$$

1 For a mathematical investigation into this matter the reader may consult an article by Professor Lorenz, Zeitschrift des Vereines Deutscher Ingenieure, October, 1905 (p. 1670). 
So that the peripheral speed for maximum efficiency is approximately half that due to the supply head.

Since the kinetic energy rejected at discharge, however, increases with an increase in $f_{3}$, it is necessary to keep this as small as possible, so that in practice the vanes are usually designed to give $k$ a value between $\frac{1}{2}$ and $\frac{1}{3}$, while $f_{2}$ is given a value about $\cdot 40 \sqrt{2 g H^{\prime}}$. On this assumption, we have-

$$
\begin{aligned}
w_{2}^{2}= & C_{v}^{2} \times 2 g H^{\prime}-f_{2}^{2} \\
= & 2 g H^{\prime}\left\{C_{v}{ }^{2}-\cdot 16\right\} \\
= & 781 \times 2 g H^{\prime} \\
\therefore \quad u_{2}= & 884 \sqrt{ } \frac{\sqrt{2 g H^{\prime}} .}{w_{2}}=\tan a \\
\therefore \quad \tan a & =\frac{\cdot 40}{884}=\cdot 453 \\
& a=24^{\circ} 24^{\prime} \text { (approximately). }
\end{aligned}
$$

Again,

Assuming $k=\frac{1}{2}$, and writing (2) in the form

this becomes on substituting

$$
u_{2}=\frac{w_{2}}{2}+\frac{\left(1-k^{2}\right) f_{2}^{2}}{2 w_{2}}
$$

$$
\begin{aligned}
u_{2} & =\frac{w_{2}}{2}\left\{1+\frac{3}{4} \tan ^{2} a\right\} \\
& =577 w_{2}=\cdot 525 C_{v} \sqrt{2 g H^{\prime}} ;
\end{aligned}
$$

so that the peripheral speed of the wheel is slightly greater than half that corresponding to the supply head.

$$
\text { Again, since } \quad \begin{aligned}
f_{2} \cot \beta & =u_{2}-u_{2} \\
& =u_{2}\left(1-{ }^{2} 577\right)
\end{aligned}
$$

we have

$\cot \beta=\cdot 423 \cot a$

$$
=\frac{\cdot 423}{\cdot 453}=\cdot 934 \text {. }
$$

$$
\therefore \beta=47^{\circ} \text { (approximately). }
$$

Again,

$$
\begin{aligned}
f_{3} & =u_{3} \tan \gamma \\
& =u_{2} \cdot \frac{r_{3}}{r_{2}} \tan \gamma .
\end{aligned}
$$


In the case of an axial flow turbine $r_{3}=r_{2}$, so that

$$
\begin{aligned}
f_{3} & =u_{2} \tan \gamma \\
\therefore \quad \tan \gamma & =\frac{k f_{2}}{u_{2}}=\frac{k w_{2} \tan a}{\cdot 577 w_{2}} \\
& =\frac{\cdot 5}{\cdot 577} \tan a=\cdot 393 \\
\therefore \quad \gamma & =21^{\circ} 30^{\prime} \text { (approximately). }
\end{aligned}
$$

Evidently the whole series of angles depends entirely on the value to be given to $f_{2}$.

If the relative velocity of water and vane is unaltered by its
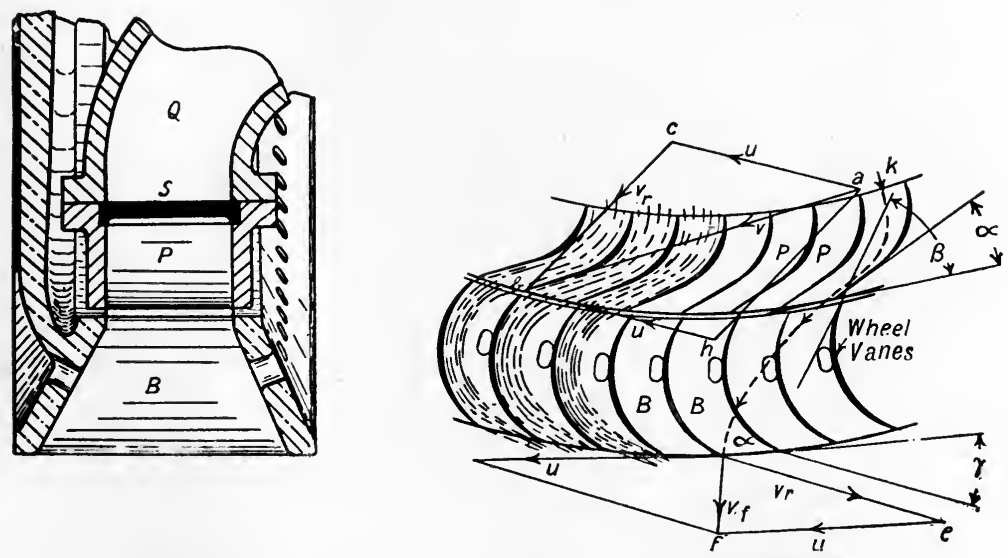

FIG. 210A.-Girard Turbine with Outward Radial Flow.

passage through the wheel, we have

$$
f_{2} \operatorname{cosec} \beta=f_{3} \operatorname{cosec} \gamma \quad \therefore \frac{\sin \beta}{\sin \gamma}=\frac{f_{2}}{f_{3}}=\frac{1}{k} \text {. }
$$

Again, the effective sectional area of the wheel passages at inlet $=2 \pi r_{2} b_{2} \sin \beta$, and at outlet $=2 \pi r_{3} b_{3} \sin \gamma$ (neglecting the thickness of the vanes), and since the effective area should increase rather than diminish towards the outlet, in order to give free deviation of the jet, we must have

$$
\frac{2 \pi r_{3} b_{3} \sin \gamma}{2 \pi r_{2} b_{2} \sin \beta}=1
$$

In an axial flow machine this makes $\frac{b_{3} \sin \gamma}{b_{2} \sin \beta}\left(=k \frac{b_{3}}{b_{2}}\right)$ equal to or greater than 1 , so that $b_{3}$ must be equal to or greater than $\frac{b_{2}}{k}$. 
This necessitates the buckets being splayed out towards the exit as illustrated in Fig. 210A.

The buckets should not be more than 9 full at entrance, and $\cdot 75$ at outlet. The pitch of the guides should then be less than that of the wheel vanes, the breadth $b$ being calculated to give the necessary proportion full at entrance.

The pitch of the vanes should be small to avoid excessive loss by shock at entrance when the tip of the wheel vanes has passed the corresponding guide vane.

The actual path, $k d$, of a particle through the wheel may be drawn as in the case of the pressure turbine, except that now, in the case of an axial flow turbine, the relative velocity of water and vane is fixed, ${ }^{1}$ while the velocity of flow varies. In this case,

1 Except as modified by the influence of gravity. Neglecting frictional resistances and windage, this is true so long as every portion of the vane over which a given filament passes is moving at the same speed, as is the case in an axial flow machine where the path of each particle is presumably parallel to the axis. If, however, different portions of the surface with which a particle comes in contact have different velocities, as in the case of a radial flow turbine, the relative velocity is no longer constant. It may, however, be determined graphically, since it will be the resultant of the relative velocity at inlet, and of the component in the direction of the vane at the required point of the relative velocity of the vane at that point and at inlet. Thus, if in Fig. 211,

$$
\left\{\begin{aligned}
n a & =\text { relative velocity at inlet } \\
a b & =\text { velocity of vane at inlet } \\
c d & =\text { velocity of vane at outlet } \\
\pi c p & =\gamma .
\end{aligned}\right.
$$

Then $d e=$ relative velocity of vane at outlet and at inlet, and $e f$, drawn parallel

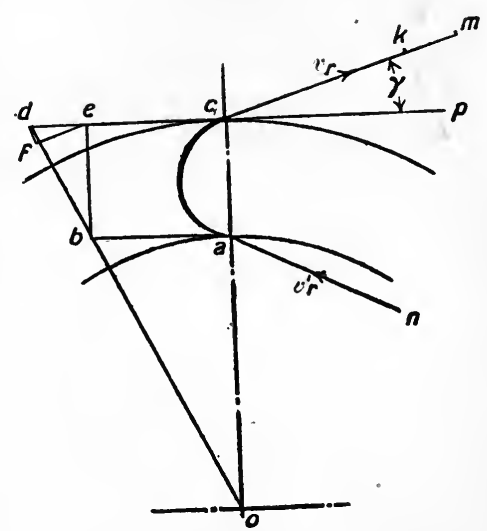

FIG. 211. to $c . m$, represents the component of this in the direction of the vane at outlet. If then $c k=n a$, and if $c k$ be produced to $m$ where $k m=e f$, the relative velocity at outlet is represented by $c \mathrm{~m}$. Where, in the case of a Girard turbine, the ratio of outer and inner radii $=1 \cdot 25$, with a value of $\gamma=21^{\circ}$, the actual relative velocity at outlet is approximately 1.23 times that at inlet. Frictional resistance will, however, reduce this by some unknown amount, and will probably bring the ratio down to about $1 \cdot 10$. In any case, the effect on the value of $\gamma$ for maximum efficiency will be slight, the effect being to reduce this value, and this should be taken into account in arranging theldesign (see Fig. 210A). 
then, a series of equidistant points on the curve of the vane being taken from entrance to exit, the points in which concentric circles through these points cut corresponding equidistant vane positions will give points on the true path of the particle, the time required for the particle to travel from point to point along

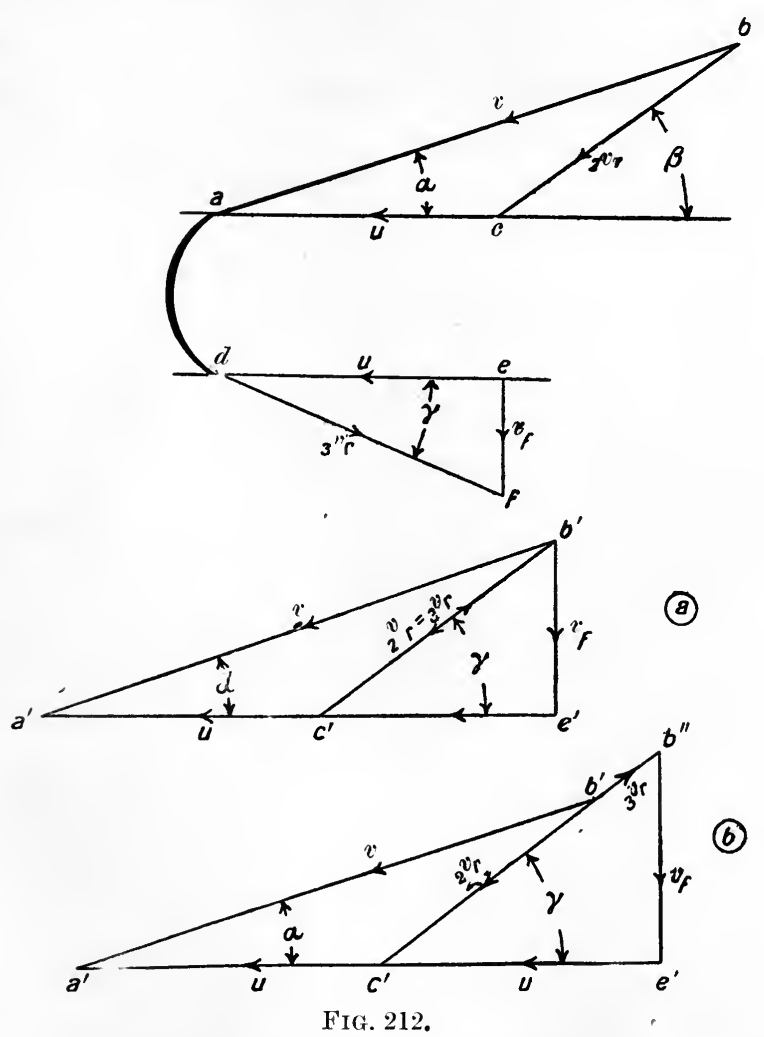

the vane being equal to the time required for the vane to travel from one position to the next.

Once $a$ and $\gamma$ have been determined, the peripheral speed for maximum efficiency may readily be obtained graphically.

Thus, with the usual notation, $a b c$ and $d$ e $f$ (Fig. 212) represent the triangles of velocity for the inlet and outlet edges of an impulse turbine. In an axial flow turbine the relative velocity $d f$ at exit will be equal to that of $a c$ at inlet. In an outward 
flow turbine $d f$ will be greater, but, as previously explained, may be determined graphically.

Also for maximum efficiency the velocity of the water on leaving the buckets must have a minimum value so that $e f$ must be perpendicular to $d e$.

If then a straight line, $b^{\prime} a^{\prime}$, be drawn to represent $v$, and if a right-angled triangle, $b^{\prime} \quad a^{\prime} e^{\prime}$, be described on $b^{\prime} a^{\prime}$, the angle $\overline{b^{\prime}} a^{\prime} \bar{e}^{\prime}$ being equal to $a$, the side $a e^{\prime}$ will represent $2 u$. In the case of a radial flow turbine the construction is modified as shown in Fig. $212 b$, where $c^{\prime} b^{\prime \prime}$ represents the relative velocity at exit.

\section{Art. 139.-Effect of Centrifugal Action.}

In an axial flow impulse turbine with radial vanes, centrifugal action tends to heap up the water towards the outside of the buckets and so to cause an unevenness of flow which militates against efficient working.

Thus in Fig. 213 a particle of water entering at $P$ (in plan) tends to follow a path $P A$ instead of its actual path $P B$. The outer particles are prevented from following the path $P A$ by the action of the outer walls of the bucket, but at points nearer the centre of rotation this constraint is absent and the particles tend to follow

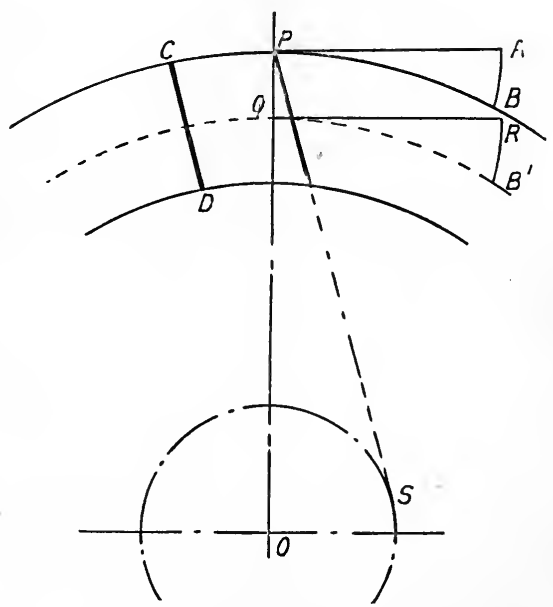

FIG. 213. their natural paths. The relative motion which then takes place may be prevented by designing the buckets so that the actual path of each particle, in plan, is a straight line perpendicular to the radius. In this case, if $Q$ is the middle point of the bucket at inlet, $R$ will be its middle point at outlet, and the bucket will be splayed out symmetrically about $R$ instead of $B^{\prime}$. 
A second method which has been adopted consists in making the outlet edge at $P$, instead of being radial, parallel to $P S$, where $\widehat{S P O}=\widehat{A P B}$. The inlet edge $C D$ is then made parallel to this, with the result that the length of path traversed by the various particles becomes more nearly equal and the relative motion is largely prevented.

Art. 140.-General Comparison of Impulse and Pressure Turbines.

The impulse wheel, having a peripheral velocity of approximately $\frac{1}{2} \sqrt{2 g H}$, as against about $\cdot 7 \sqrt{2 g H}$ for the pressure turbine, is well fitted for very high falls. For the same reason its velocity becomes low under low heads, and this renders it unsuitable for driving electric generating machinery under such conditions. Further, with low falls the percentage variation in head is generally comparatively large, and the difficulty of maintaining the speed approximately constant, and of maintaining the efficiency, under such head variations is great. On the other hand, the part gate efficiency is high where the head is constant and where the load or supply is variable. Either type may be used for any head up to 300 feet, though the impulse type is preferable for heads above about 200 feet, except for very large powers. Also with either type full load efficiencies of slightly over 80 per cent. may be obtained under favourable circumstances, the more modern type of Francis turbines with swivel guides having a slight advantage in this respect. Either type may be used in connection with a suction tube, though the pressure turbine lends itself more readily to this construction and has the further advantage that it may be drowned without loss of efficiency. The efficiency of the pressure wheel is not so sensitive to changes of supply pressure as that of the impulse wheel, and therefore this type is better fitted for work under a variable head. On the other hand, except when fitted with swivelling guide blades, its part gate efficiency is low. Apart from the conditions outlined, the possibilities of accurate speed regulation are about equal in the two types.

While the cost of the machine depends entirely on the type and form of construction, the relative cost of a pressure and an 
impulse turbine to give equally good results as regards tfficiency and speed regulation will be almost equal for a head of about 175 feet. For greater heads the pressure turbine, and for lower heads the impulse turbine, becomes the more expensive.

\section{Art. 141.-The Stand Pipe.}

'The advantages of a stand pipe in increasing the possibilities of accurate speed regulation on an increasing load have already been indicated, and, as will be readily understood, the larger the area of this pipe the more satisfactory the results are likely to be. Mechanical difficulties, as well as considerations of first cost, however, limit the maximum permissible size, and it becomes important to determine what minimum size of pipe will enable satisfactory speed regulation to be performed.

The following investigation, though only approximate, gives results which are sufficiently near to enable the necessary size to be estimated with fair accuracy.

In this investigation, which will take the form of a specific example, the horse power of the turbine, its efficiency, the diameter of the penstock, its length, and the working head, are assumed as being known, as is the maximum increase in load likely to occur at any one time. This enables the necessary velocity of flow along the penstock to be determined both before the increase in load and after the velocity has again become constant after this increase. By applying the equation of energy in the two cases, the pressure at the stand pipe, and thus the free level may be obtained, from which the fall in level, and hence the volume of water leaving the stand pipe during the change, may be obtained in terms of the area. The energy entering the wheel from the stand pipe may then be calculated, and, if it be assumed that the acceleration in the penstock is sensibly uniform, the energy entering the wheel from the supply reservoir during the change may also be calculated, so that the total energy supplied to the wheel during the period of transition may be obtained. Equating this to the energy required to develop the required horse power, the time necessary to produce the required change in the velocity of flow, and hence the acceleration may be calculated 
in terms of the stand pipe area. Having obtained this, it only remains to equate it to the maximum acceleration consistent with good speed regulation.

The author regrets that his opportunities of determining this latter factor from plants in actual operation have been limited, but so far as the data at his disposal go, these tend to show that this acceleration should not exceed that given by the formula

$$
\begin{aligned}
a & =\frac{.075 g h}{l} \mathrm{ft} . \text { per sec. per sec. } \\
& =2 \cdot 4 \frac{h}{l} \mathrm{ft} . \text { per sec. per sec. }
\end{aligned}
$$

\section{Exayple.}

Consider a turbine, supplied under a head of 60 feet, through a penstock 4 feet diameter and 200 feet long, and working under a normal load of 300 B.H.P. Assuming the efficiency of the turbine to be 80 , this necessitates a supply of energy $=\frac{300 \times 550}{\cdot 8}=206,000$ foot lbs. per sec.

$\left.\begin{array}{c}\text { And since the energy entering } \\ \text { the wheel casing per second }\end{array}\right\}=62.5$ a $v\left\{h-\frac{v^{2}}{2 g} \frac{f l}{m}\right\}$

Where $a=$ area of penstock $\quad=12.57$ square feet.

$$
\begin{aligned}
& h=\text { supply head }=60 \text { feet. } \\
& f=\text { coefficient of friction }=.005 \text { (say). }
\end{aligned}
$$

This gives us on substitution and reduction-

$$
v=4.4 \text { feet per second }
$$

as the velocity of flow along the penstock.

Similarly, if the maximum increase in load is one of $50 \mathrm{per}$ cent. up to 450 H.P., the new velocity, when steady flow is once more attained, will be $6 \cdot 64$ feet per second.

Now, if $h_{j}$ is the head at the entrance to the turbine casing, and therefore at the stand pipe, we have, in the first case-

$$
\begin{aligned}
h_{j} & =h-\frac{v^{2}}{2 g}\left(1+\frac{f l}{m}\right) \\
& =60-\cdot 3(1+1) \\
& =59.4 \text { feet. }
\end{aligned}
$$


While in the second case, when $v=6 \cdot 64$, we have-

$$
\begin{aligned}
h_{j} & =60-\cdot 706 \times 2 \\
& =58.59 \text { feet. }
\end{aligned}
$$

$\therefore$ Fall in level at stand pipe $=\cdot 81$ feet.

The mean height in stand pipe $=59.0$ feet, so that if $A$ is its area, the energy leaving during the transition

$$
\begin{aligned}
& =81 \times 59 \times 62.4 \times A \text { foot lbs. } \\
& =2,980 \mathrm{~A} \text { foot lbs. }
\end{aligned}
$$

The energy entering the casing from the penstock in the same interval of time, $t_{1}$ seconds, is given by

$$
\int_{0}^{t_{1}} 62 \cdot 4 a v\left\{h-\frac{v^{2}}{2 g} \frac{f l}{m}\right\}
$$

and, assuming uniform acceleration so that $\frac{6 \cdot 64-4 \cdot 4}{t_{1}}=a$, and writing $v=v_{1}+a t=4 \cdot 4+\frac{2 \cdot 24}{t_{1}} \cdot t$, this becomes

$$
\begin{aligned}
62 \cdot 4 a \int_{o} & \left.t_{1} h\left(v_{1}+a t\right)-\frac{f l}{2 g m}\left(v_{1}+a t\right)^{3}\right\} d t \\
= & 784 t_{1}[(60 \times 4 \cdot 4)+30 \times 2 \cdot 24) \\
-\frac{1}{64 \cdot 4}\left\{\left(4 \cdot 4^{3}\right)+\right. & \left.\left.\left(\frac{3}{2} \times 4 \cdot 4^{2} \times 2 \cdot 24\right)+\left(4 \cdot 4 \times 2 \cdot 24^{2}\right)+\frac{2 \cdot 24^{3}}{4}\right\}\right] \\
& =784 t_{1}\{264+67 \cdot 2-2 \cdot 7\} \\
& =257,500 t_{1} \text { foot lbs. }
\end{aligned}
$$

$\therefore$ For speed to be maintained with the increased load, we must have :-

$$
\begin{gathered}
2,980 A+257,500 t_{1}=\frac{450 \times 550}{\cdot 8} \times t_{1} \\
=309,000 t_{1} \\
\therefore \quad A=\frac{51,500}{2,980} t_{1}=17 \cdot 26 t_{1} \\
\text { or } t_{1}=\cdot 058 A .
\end{gathered}
$$

Thus the acceleration of the supply column, corresponding to any increase in load, varies inversely as the area of the stand pipe. 
With a 4 foot stand pipe $a=\frac{2 \cdot 24}{t_{1}}=\frac{2 \cdot 24}{12 \cdot 87 \times 058}$

$$
=3.01 \text { f.s.s. }
$$

Applying the rule $a=2 \cdot 4 \frac{h}{l}=\frac{2 \cdot 4 \times 60}{200}=7.2$ f.s.s.. this gives us $\cdot 72=\frac{2 \cdot 24}{.058 \mathrm{~A}}$

$$
\therefore A=53 \cdot 6 \text { square feet, }
$$

corresponding to a diameter at the top of $8 \cdot 26$ feet.

The stand pipe would in this case take the form of a vertical pipe about 3 feet in diameter and carrying a circular cistern 8 feet 3 inches in diameter at the top, the top of this cistern being about 62 feet above the centre line of the turbine and its depth about 3 feet 3 inches, thus leaving a depth of water equal to $3 \cdot 25-2 \cdot 0-\cdot 81=\cdot 44$ feet; when working under full over-load.

The stand pipe is not usually fitted where the supply head is above 150 feet. The largest pipe of which the author has cognizance is to be fitted to the power plant of the St. Louis Hydro-Electric Company. ${ }^{1}$

Here the total head is 380 feet, developed on a pipe line about 5,000 feet long. When finally completed there are to be eight parallel pipes, each 7 feet in diameter, and coupled to a transverse receiver 500 feet back from the power house, which receiver is itself at an elevation of 145 feet. From the receiver, an open stand pipe 235 feet long and 6 feet diameter, carrying at the top a circular tank 30 feet in diameter, is erected.

The whole plant is intended to consist of eight units of 13,000 B.H.P. each, the stand pipe being designed to supply sufficient energy for an additional sudden demand of 10,000 B.H.P.

\section{ARt. 142.-F Lywheel ÉfFect.}

So far, the effect of any flywheel which may be fitted to the turbine shaft has been neglected, the rules already given applying where no special flywheel is fitted.

To consider the effect of such a wheel it must be remembered that the total store of kinetic energy in a wheel of weight $W$ lbs.

\footnotetext{
1 The Engineen, February 15, 1907, p. 15i.
} 
and of effective radius $r$ feet when rotating at a speed of $\omega$ radians per second $\left(N\right.$ revolutions per minute, where $\left.\omega=\frac{2 \pi N}{60}\right)$, is equal to $\frac{1}{2} \frac{W r^{2}}{g} \omega^{2}$ foot lbs. $=\frac{1}{2} 1 \omega^{2}$, where $1=$ moment of inertia of wheel. If then the speed of such a wheel is reduced from $\omega_{1}$ to $\omega_{2}$, the store of energy given out

$$
=\frac{1}{2} 1\left(\omega_{1}^{2}-\omega_{2}^{2}\right)=\frac{1}{2} 1\left(\omega_{1}+\omega_{2}\right)\left(\omega_{1}-\omega_{2}\right)=\delta \mathrm{E},
$$

and if $\omega$ is the mean angular velocity, this may be written :-

$$
\begin{aligned}
\frac{1}{2} 1 \omega \delta \omega & =\frac{\delta E}{2} \\
\therefore \quad \frac{1}{2} 1 \omega^{2} \frac{\delta \omega}{\omega} & =\frac{\delta E}{2}
\end{aligned}
$$

Putting $\frac{1}{2} 1 \omega^{2}=E$, this becomes

$$
\frac{\delta \omega}{\omega}=\frac{\delta E}{2 E}
$$

i.e., the proportional change in the store of energy in the wheel is twice as great as the proportional change in velocity.

Suppose, for example, in the num erical example just considered (p. 530), the turbine, rotating at a mean speed of 240 revolutions per minute $(\omega=8 \pi)$, is fitted with a flywheel weighing 5 tons and having an effective radius of 2.5 feet. The value of $E=$ 1

$1 \omega^{2}$ now becomes

$$
\begin{gathered}
\frac{1}{2} \cdot \frac{5 \times 2,240}{32 \cdot 2} \times \frac{25}{4} \times 64 \pi^{2} \\
=68,700 \text { foot lbs. }
\end{gathered}
$$

If now the maximum speed variation on throwing on the excess load is to be 4 per cent. of the mean, so that $\frac{\delta \omega}{\omega}=\cdot 04$, we have $\delta E$, the energy given out by the wheel during its retardation, given by

$$
\begin{aligned}
\delta E & =2 \times \cdot 04 \times 68,700 \text { foot lbs. } \\
& =55,000 \text { foot lbs. }
\end{aligned}
$$

If the same acceleration in the penstock be assumed, the amount of energy required from the stand pipe during the 
transition may be reduced by this amount, and since each square foot of stand pipe area gives up 2,980 font lbs. of energy, this area may be reduced by $\frac{55,000}{2,980}$ square feet $=18 \cdot 4$ square feet.

This gives an area $=53 \cdot 6-18 \cdot 4=35 \cdot 2$ square feet, and 'a diameter of 6 feet $8 \frac{1}{2}$ inches, as against 8 feet 3 inches without the wheel.

Evidently with a sufficiently heavy flywheel it would be possible to eliminate the upper cistern altogether.

\section{Exanples.}

(1) Show that in a reaction turbine working under conditions of maximum efficiency, the efficiency is given by

$$
\eta=2(1-e) \cos ^{2} a\left(1-\frac{\tan a}{\tan a}\right)
$$

where $e$ measures the proportion of energy existing in the form of pressure energy, in the water at entrance to the wheel.

(2) The angle of the guide blades in an I.F. reaction turbine is $12^{\circ}$, the peripheral speed is 32 feet per second, and the velocity of the water at inlet is 36 feet per second. Determine suitable vane angles at inlet and outlet, the inner being $\frac{2}{3}$ the outer diameter and the velocity of flow constant.

$$
\text { Answer. }\left\{\begin{array}{l}
\text { Inlet angle }=68^{\circ} . \\
\text { Outlet angle }=17^{\circ} \cdot 20^{\prime} .
\end{array}\right.
$$

(3) If in the previous example the head is 36 feet, the B.H.P. 50 , the gross efficiency 82 per cent., what must be the effective area of the inlet surface, assuming a coefficient of contraction of $\cdot 95$.

Answer. $2 \cdot 10$ square feet.

(4) The external and internal diameters of an I.F. reaction turbine are 4 feet and 2 feet 3 inches, the vanes are radial at inlet, the velocity of flow through the wheel is $=\frac{1}{8} \sqrt{2 g H^{\prime}}$, and the peripheral velocity is that due to a fall through $\frac{H^{\prime}}{2}$. Determine the 
vane angle at outlet for the water to be discharged without any tangential velocity.

\section{Answer. $17^{\circ} \cdot 44^{\prime}$.}

(5) If in the turbine of the preceding question the vanes at outlet make an angle of $20^{\circ}$ with the circumference, determine the speed at which the wheel should run; the velocity of flow; and the pressure and kinetic heads in the supply chamber, in terms of the available head $H^{\prime}$, and also determine the angle which the guide blades make with the circumference.

Answer.

$\begin{array}{ll}\text { Revolutions per minute } & =4.71 \sqrt{g H^{\prime}} \quad a=10^{\circ} 11^{1} . \\ \text { Velocity of flow, } f & =.7 \sqrt{g H^{\prime}} \\ \text { Pressure head } & =.492 H^{\prime} \\ \text { Kinetic head } & =.508 H^{\prime} .\end{array}$

(6) A Jonval turbine works under a head of 13.5 feet. The mean diameter is 8 feet, the width of buckets $=17 \frac{3}{4}$ inches, the number of revolutions 46 per minute, and the turbine develops 266 horse power with an efficiency of 86 per cent. 'The number of vanes is thirty-eight, these being $\frac{3}{16}$ inch thick. Assuming guide angles of $18^{\circ}$, and a coefficient of discharge of 9 , determine the velocity of exit from the guides, and the inlet and outlet angles of the wheel vanes for entry without shock and for rejection of water without velocity of whirl. Assume a coefficient of discharge of 9 for the discharge orifices.

(7) The following are details of an outward flow reaction turbine :-

$$
\begin{aligned}
& a=28^{\circ} ; \beta=90^{\circ} ; \gamma=22^{\circ} ; \\
& r_{2}=3.875 \text { feet } ; r_{3}=4 \cdot 146 \text { feet } \\
& b_{1}=\cdot 971 \text { feet } ; b_{2}=\cdot 937 \text { feet } ; b_{3}=\cdot 932 \text { feet } ; \\
& n_{1}=33 ; n_{2}=44 ; t_{1}=\cdot 0083 \text { feet } ; t_{2}=\cdot 0117 \text { feet. }
\end{aligned}
$$

Measured outflow area of guide passages $=6.537$ square feet.

$$
\text { , , , }, \quad \text { buckets . }=7 \cdot 687 \quad \text {, }
$$

The available head is 13 feet. Determine the number of revolutions for maximum efficiency, and determine the hydraulic efficiency under such conditions. 
(8) The following are details of a 800 H.P. outward radial flow Girard turbine at Terni (Italy) :-

$$
a=20^{\circ} ; \beta=36^{\circ} ; \gamma=30^{\circ} ; r_{2}=4.1 \text { feet } r_{3}=4.71 \text { feet; }
$$
$b_{1}=b_{2}=4 \cdot 91$ inches $; b_{3}=16 \cdot 14$ inches. The effective head is 595.5 feet, and the coefficient of discharge from the guide passages is found to be $\cdot 85$, the coefficient of velocity being $\cdot 92$. Calculate the speed of rotation (actually 200 per minute) for maximum efficiency. 


\section{CHAP'TER XIV.}

The Hydraulic Engine-Brotherhood-Rigg-Theory of Action-Losses-Port Areas.

Art. 143.-The Hydraulic Engine.

Where a supply of high pressure water is available, and where intermittent rotary motion at a moderate speed is desired, the reciprocating piston engine has certain advantages, particularly where it is able to work at or near full load, and where the speed variation may be excessive, as occurs, for example, in the working of a capstan. For such work the rotary motor is out of the question, both on account of the necessity for gearing to reduce its necessarily high speed, and of the great reduction in its efficiency under variable speed conditions. The reciprocating engine, however, having an efficiency which is approximately independent of its speed, and being compact, is particularly well adapted for such work. For small powers, too, where the load is fairly constant, such as for driving ventilating fans, organ bellows, etc., its high efficiency and the absence of noise connected with its use often render it, in the absence of an electric supply, the most suitable motor to use.

One of the most widely used types of engine is the Brotherhood (Fig. 214), which is designed to work with pressures from $60 \mathrm{lbs}$. to 1,050 lbs. per square inch.

Here three single acting cylinders fitted with trunk pistons are fixed radially at $120^{\circ}$ to an external cylindrical casing, the three connecting rods working on a single crank pin. Each cylinder is fitted with a single inlet and outlet port, the opening of this to supply and exhaust being regulated by the rotary valve shown at $D$ in Fig. 214.

This valve is rotated by the crank shaft, and carries passages connecting with the pressure supply and the exhaust which are 


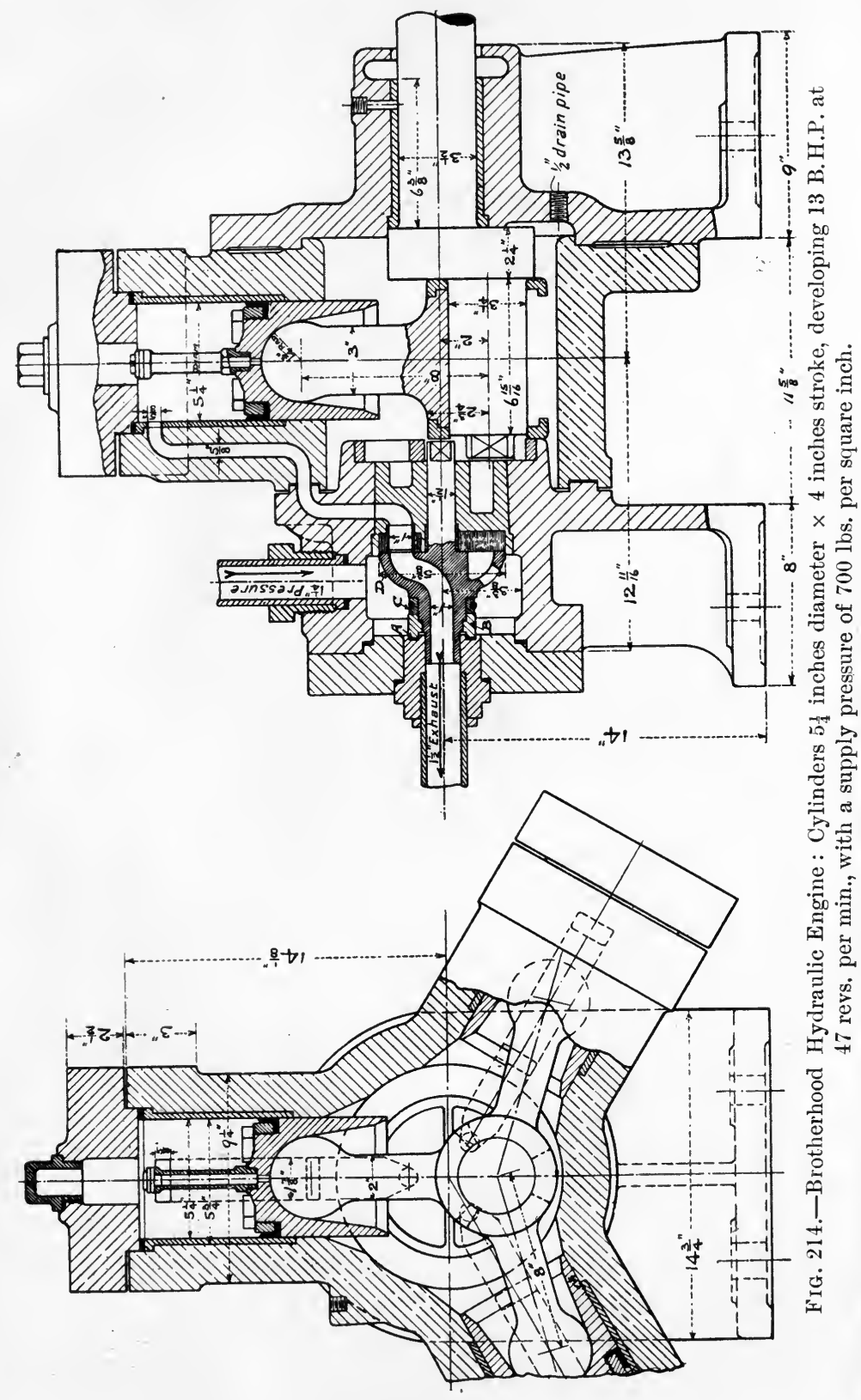


alternately presented before the port of each cylinder in turn. The method of keeping the rotating joints tight against such high pressures is indicated in the sketch. Here $A$ is a leather joint washer, on which the back nut $B$ rotates, the play due to wear on the joint being taken up by the expansion of the rubber ring $C$, which rotates with the valve and back nut. The valve itself bears against a lignum-vitæ seat. All pistons are packed with " $\mathrm{L}$ " leathers, and the cylinders and all working parts are lined with brass.

The engine as thus constructed forms a compact and serviceable motor, has no dead centre, and is built in sizes up to about 30 B.H.P. The piston speed is about 30 feet per minute for all sizes, the engine shown in Fig. 214 having cylinders $5 \frac{1}{4}$ inches diameter $\times 4$-inch stroke and developing 13 B.H.P. at forty-seven revolutions per minute when supplied with water at $700 \mathrm{lbs}$. pressure.

The full power efficiency, as measured on the brake horse power, varies with the working pressure from about 52 per cent. with $60 \mathrm{lbs}$. pressure, to 60 per cent. at 1,050 lbs. This is equivalent to an hydraulic efficiency of from 58 per cent. to 67 per cent. These values are probably too low rather than too high.

All constant stroke engines, however, suffer from the fact that water cannot be used expansively, and since, to avoid shock, it is necessary to keep the cylinders full of water, the same energy is used whatever the load on the engine, causing the efficiency to be low at light or variable loads. It has been attempted to overcome this difficulty, but without great success, by cutting off the pressure supply before the end of the stroke and admitting low pressure water from an auxiliary supply for the rest of the stroke.

A device due to Meyer consists in providing an air chamber at each end of the (double-acting) cylinder. Cut-off may then take place before the end of the stroke, the air afterwards expanding and driving the piston. No gain in efficiency is to be anticipated from this method of working.

The more usual device is to reduce the stroke and piston displacement to suit the varying demands for power. In the 
Brotherhood engine this has been accomplished by the Hastie regulating device.

As thus constructed, power is transmitted through a hollow crank shaft to the driving pulley by means of a volute spring, an increase in the load producing a further coiling of this spring. The inner end of the spring is fixed to the hollow crank shaft, while the driving pulley carries a disc keyed to a cam shaft working inside the crank shaft. Any variation in the load then produces a relative rotation of the crank and cam shaft. A cam keyed to the latter shaft utilizes this relative rotation to increase or diminish the crank radius, and thus to adapt the volume of piston displacement to the demand for power. This device is, however, seldom now fitted to the engine.

Of all variable power engines, that of $\mathrm{Mr}$. R. Rigg has achieved the greatest success in practice.

This engine is provided with three cylinders, fitted with plungers which are pivoted at their outer ends to an external cylindrical ring, which is itself keyed to the driving shaft. The cylinders face radially outwards at $120^{\circ}$, are single acting, and are rigidly fixed at their inner ends to a ring which rotates on a fixed hollow shaft forming the supply pipe, eccentric with the external casing, and which carries the inlet and outlet ports. The rotation of the outer ring is thus accompanied by a reciprocating motion of the pistons in their cylinders, the stroke of each being twice the eccentricity of the two rings. There are three ports, one to each cylinder, and as these rotate each in turn is presented before an inlet and an outlet port in the central hollow shaft. Regulation of power at constant speed is obtained by altering the eccentricity, and therefore the stroke of the engine, by means of an hydraulic relay governed by a centrifugal governor. The reduction in water consumption is thus proportional to the reduction in the stroke. When regulating the eccentricity by hand, at constant power, a decrease in stroke will be accompanied by a corresponding increase in speed, the work done per second then being approximately the same. This method of working may be adopted for capstans and the like, where when hauling in slack, etc., a rapid rotation with very short stroke is required, while when the full pull is to be exerted by the rope, a long 
stroke and slow rate of rotation is essential. By reversing the relative position of the centre the direction of rotation is reversed.

The speed of the engine may be anything up to about 500 revolutions per minute, though probably 250 revolutions marks the limit of its really efficient performance. At the latter speed the inventor claims to obtain efficiencies up to 80 per cent., though this value would appear to be improbably high.

In the Armstrong type of engine, oscillating cylinders mounted side by side and driving on to a common crank shaft are adopted. Three cylinders driving cranks at $120^{\circ}$ are usual, and are fitted, either with externally packed rams forming a single acting engine, or with differential rams having areas in the ratio $2: 1$. The smaller of these is exposed to a constant pressure head, and the engine becomes double acting with equal efforts on each stroke. The single acting engine is more commonly used. Admission and discharge take place through a circular slide valve working within the hollow trunnions which carry the cylinders. This engine is made to develop up to about 70 B.H.P.

Art. 144.-'Theory of the Hydraulic Engine.-Horse Power.

If $K=$ number of cylinders-supposed single acting.

$p=$ mean effective pressure in lbs. per square inch in each cylinder.

$N=$ number of revolutions per minute.

$l=$ length of stroke in feet.

$A=$ ram area in square inches.

Then I.H.P. $=K \cdot \frac{p l A N}{3,3000}$.

The mechanical efficiency varies with the size and type of engine, and with its condition, but will be generally about 90 per cent.

If $Q=$ volume of water used per minute in cubic feet.

$H=$ supply head in feet. 
The hydraulic efficiency

or writing

$$
=\frac{\text { indicated work on piston }}{\text { energy in supply water }}=\frac{K \cdot p l A N}{62 \cdot 4 Q H},
$$

we have the hydraulic efficiency $=\frac{144 p}{62 \cdot 4 H}=\frac{2 \cdot 31 p}{H}$.

'I'he total efficiency of the system $=\frac{33,000 \text { (B.H.P.) }}{62 \cdot 4 Q H}$

$=$ hydraulic efficiency $\times$ mechanical efficiency.

Pressure on Piston.-As in the reciprocating pump, the pressure on the piston at any point of the stroke is affected by the inertia of the supply column. The engine may in fact be considered as a reversed pump with mechanically operated valves, and the investigation into pressure conditions in the cylinder proceeds on exactly similar lines to that in the case of the pump. For a further investigation of these pressure effects the reader is referred to Art. 151. It may be noted, however, that at any point of the stroke, where $a$ is the piston acceleration and $v$ its velocity, if $a_{s}$ is the area and $l_{s}$ the length of the supply pipe, $m$ being its hydraulic mean depth, we have :-

$\left.\begin{array}{c}\text { Head on piston } \\ \text { in feet }\end{array}\right\}=\left[\begin{array}{c}\text { supply head } \\ \text { in feet }\end{array}-\frac{A}{a_{s}} \cdot \frac{l_{s}}{g} \cdot\left\{a+\frac{f v^{2} A}{2 m a_{s}}\right\}\right.$ ft. $]$.

Here $a$ is positive during the first, and negative during the last part of the stroke.

A weighted accumulator feeding the supply pipe line has the effect of increasing acceleration pressures as explained in Art. 181. The provision of an air vessel on the supply side of the engine materially improves the smoothness of running, and by maintaining an approximately uniform flow in the supply main, reduces frictional and shock losses and so increases the efficiency of the motor. The necessary dimensions of this air vessel depend on the number and arrangement of the cylinders; on the speed and dimensions of the engine; and on the length of supply pipe. If more than one cylinder is used, the cranks being placed at equal angles round the shaft, the velocity of flow through the supply pipe is considerably steadied, and indeed with the usual type of three-cylinder engine having cranks at $120^{\circ}$, becomes so 
nearly uniform that it becomes possible to dispense with the air vessel.

For a further consideration of the questions deciding the size of the air véssel in any particular case reference should be made to Art. 154. With the high pressures usually adopted in these engines it becomes imperative either to provide some mechanical device for maintaining the charge of air in the vessel, or to make the vessel of ample area to maintain its mean working level approximately constant over long periods of working. Thus the necessary size of air vessel for the engine will in general be slightly greater than that for the corresponding pump.

Losses in the Hydraulic Engine.-Port Areas.-These losses are due, partly to friction but more particularly to shock produced at sudden changes of section, and are therefore approximately proportional to the square of the velocity. To reduce these, all throttling is to be prevented as far as possible, inlet and outlet ports are to be short, direct, and of ample area, with easy curves and with few changes in sectional area or shape.

Where, as in some instances, the inlet ports are emptied at the end of each exhaust stroke, to be refilled before the commencement of the next working stroke, this is productive of a direct loss of energy, since, owing to the fact that the fluid is inexpansive, no work is done on the piston until these ports are completely filled. This loss increases with the area of the ports, while friction losses and those due to the loss of the kinetic energy of entrance decrease as this area increases, so that in any particular case there will be some one port area for which the total loss is a minimum. Consider a single acting engine.

Then the loss of energy per revolution due to water necessary to fill inlet ports of area $a\}=2 \cdot 31 p . l . a$ foot lbs. square feet and length $l$ feet, at a pressure $p$ lbs. per square inch

Loss by friction per stroke $\left.\begin{array}{c}\text { Loss of kinetic energy per stroke } \\ \quad \text { (assuming all this to be lost) }\end{array}\right\}=62 \cdot 4 L A \frac{\bar{v}^{2}}{2 g}$ foot lbs. where $L=$ length of stroke of piston. 
$D=$ diameter, $A=$ area of piston in square feet.

$\bar{v}^{2}=$ mean square of velocity of flow through ports.

$=v^{2} \frac{A^{2}}{a^{2}}$, where $v^{2}=$ mean square of piston velocities throughout a stroke. In general $v$ may be taken as 1.25 times the mean piston velocity $r_{m}$ with sufficient accuracy for practical calculations. ${ }^{1}$

Since the losses of head included under the two latter headings serve both to diminish the effective pressure on the working stroke and to increase the back pressure on the discharge, we have the total loss of energy per working stroke

$$
=2.31 p l a+195 \frac{L A^{3}}{a^{2}} \cdot \frac{v_{m}^{2}}{2 g}\left\{\frac{f l}{m}+1\right\} \text { foot lbs. }
$$

Substituting for $A$ and $a$ in terms of $D$ and $d$, and writing $m=\frac{d}{4}$, this becomes

$$
\frac{\pi}{4}\left[2 \cdot 31 p l d^{2}+\frac{195 D^{6}}{d^{4}} L \frac{v_{m}^{2}}{2 g}\left\{\frac{4 f l}{d}+1\right\}\right] .
$$

Differentiating this with respect to $d$ and equating the result to zero, we have :-

$$
d^{6}=D^{6} \times \frac{2 \cdot 62 L v^{2}{ }_{m}\left\{1+\frac{5 f l}{d}\right\}}{p l},
$$

from which, by successive approximations, or by graphical solution, we can find $d$ the diameter of inlet ports for the total loss to be a minimum. If the inlet passages are rectangular in section it is sufficiently near for practical purposes to make their sectional area equal to that of this circle.

1 Here $v_{m}=L \div$ time to complete one stroke.

If a curve be plotted having the squares of the piston velocities as ordinates on a displacement base, the mean height of the curve will equal $\bar{v}^{2}\left(\frac{a}{\bar{A}}\right)^{2}$. In the case of an oscillating cylinder with a connecting rod equal to three cranks in length, the ratio $\frac{v}{r_{m}}$ is approximately equal to $1 \cdot 27$, so that $\left(\frac{\bar{v}}{v_{m}}\right)^{2}=1 \cdot 61$. With a longer connecting rod this will be reduced, so that in general $\left(\frac{\bar{\tau}}{v_{m}}\right)^{2}$ will approximately equal. $1 \cdot 56=(1 \cdot 25)^{2}$. 


\section{Example.}

Hydraulic engine, 12 inches diameter, 12 inches stroke.

$p=750 \mathrm{lbs}$. per square inch, mean piston velocity 3 feet per second, length of ports 2 feet; $f=\cdot 01$. Here $L=1$; $D=1 ; l=2$ :

We now have $\quad d^{6}=\frac{2 \cdot 62 \times 9}{750 \times 2} \times\left\{1+\frac{\cdot 1}{d}\right\}$

Neglecting the $\frac{\cdot 1}{d}$ in the last factor, we get $d^{6}=\cdot 0157$ as a first approximation, from which $d=\cdot 50$ feet.

Substituting this value of $d$ in the factor $1+\frac{\cdot 1}{d}$, we now get as a second approximation-

$$
\begin{aligned}
d^{6} & =\cdot 0157 \times 1 \cdot 2=\cdot 0189, \\
\text { giving } d & =\cdot 515 \text { feet }=6 \frac{3}{16} \text { inches. }
\end{aligned}
$$

If this value were adopted for $d$ in the last factor of the above equation, and a solution obtained as before, a still closer approximation to the true value would be obtained. Generally, however, a second approximation gives results which are sufficiently close for any practical purpose.

If this method be applied to the design of the Brotherhood engine (Fig. 214), having a $5 \frac{1}{4}$-inch cylinder with 4 -inch stroke, working at forty-seven revolutions per minute $\left(v_{\text {(mean })}=.52\right.$ feet per second) and having a port length of 18 inches, taking $f=\cdot 010$ we have :-

$$
d^{6}=\frac{2 \cdot 62 \times(\cdot 52)^{2} \times 7^{6}}{1.5 \times 3 \times 16^{6} \times 750}\left\{1+\frac{\cdot 075}{d}\right\}
$$

From which we finally get $d=\cdot 116$ feet $=1 \cdot 39$ inches.

The area of this corresponds to that of a rectangular port $1 \frac{3}{4}$ inches wide by $\frac{25}{3} \frac{5}{2}$ inch deep, as compared with the actual dimensions, $1 \frac{3}{4}$ inches $\times \frac{5}{8}$ inch.

This investigation shows the importance of having as far as possible separate inlet and outlet ports for these engines, or, where using a single port, of designing this so as to remain full until readmission takes place. Where this design is carried out the port area may be arranged to give a mean velocity of flow of from 2.5 to 3.5 feet per second. In the alternative case a 
smaller port area will in general give better results, the velocity being increased up to a maximum of about 8 feet per second.

\section{Examples.}

(1) A three-cylinder single acting hydraulic engine is required to develop 75 B.H.P., using water at a pressure of $1,120 \mathrm{lbs}$. per square inch. Assuming an efficiency of 65 per cent., and designing the engine so that it has a mean piston speed of 2 feet per second, at sixty revolutions per minute, determine the diameter of the cylinders and the length of stroke.

Answer. Cylinder diameter $=5$ inches (approx.). stroke $=12$ inches. 


\section{CHAPTER XV.}

Pumping Machinery-The Scoop Wheel-The Archimedean Screw-The Reciprocating Pump - Types - Valves-Theory of Action-Air Vessels - Positive Rotary Pump.

\section{Art. 145.-Punips.}

Most hydraulic prime movers are, with slight modifications, capable of being reversed, and when driven by an external agency provide a means of raising water from a lower to a higher level.

Thus the overshot water wheel corresponds to the chain and bucket pump ; the breast or Sagebien wheel to the scoop wheel ; the reversed pressure turbine gives the centrifugal pump, and the reversed reciprocating engine the reciprocating pump. The capacity of each of these machines as a prime mover also to a large extent indicates its capacity, when reversed, as a pump, and just as the reciprocating engine and turbine are the most important types of prime mover, so the reciprocating piston pump and the centrifugal pump are the most important types of pump.

In addition to those already mentioned, we have a series of special types of pump, each of which is specially adapted to some particular combination of circumstances. Of these, the Archimedean screw, the jet pump, the positive rotary pump, the hydraulic ram, and the air lift pump, are interesting examples and will be considered in due course.

\section{Art. 146.-The Scoop Wheel.}

This has in the past been largely applied to the drainage of fen districts, and simply consists of a Sagebien wheel reversed and driven usually by steam power, the water then being lifted in the buckets from the lower to the higher level. It is fitted for lifts up to about 6 feet and may be constructed to deliver up to about 300 tons per minute, giving an efficiency under 
favourable conditions of upwards of 75 per cent. Its chief advantages appear in cases where a large quantity of débris is encountered. As in the Sagebien wheel, the inclination of the floats should be such as to prevent shock at entrance and losses at exit as far as possible. It is found that the best results are obtained when the angles which the vanes make with the water surface at entrance and exit are equal. If any variation is made from this design, it is better to increase the angle of egress, since this reduces the loss due to lifting the water above head-race level. While increasing the loss at entrance due to shock, and to driving the water back from the floats, this is in general less serious than the former source of loss. In some cases the angle of egress is made equal to twice the angle of ingress. The floats are found to give better results when flat than when curved, and are arranged to make an angle of from $20^{\circ}$ to $40^{\circ}$ with the radial line. Since any variation in the head or tail-race level militates against efficient working, it is advisable to regulate the supply level at the wheel by means of an adjustable sluice, so as to keep this constant. As usually constructed, the diameter of the wheel lies between $9 \sqrt{ } \bar{H}$ and $10 \sqrt{H}$, where $H$ is the lift in feet. The peripheral speed is usually about 8 feet per second.

\section{Art. 147.-The Screw Pump or Archimedean Screw}

has in the past been largely used in Holland, and works with advantage against heads not exceeding 10 feet, having an efficiency equal to that of the scoop wheel. The pump consists of an inclined shaft carrying one or more helices of considerable diameter, which rotate with small clearance-in a closely-fitting tube or open semicircular channel connecting head and tail-water.

The angle of inclination of the shaft to the horizontal is so arranged as to be less than that of the helical surface, so that water on being admitted to the bottom of the tube always tends to run down this surface. Thus (Fig. 215), a particle of water admitted at $P$ tends to flow to the point $Q$. By a rotation of the axis portions of the helix from $Q$ to $R$ successively adopt the same position as $Q$ relative to the axis. As they do so the water 
tends to flow into these positions and is thus passed along the

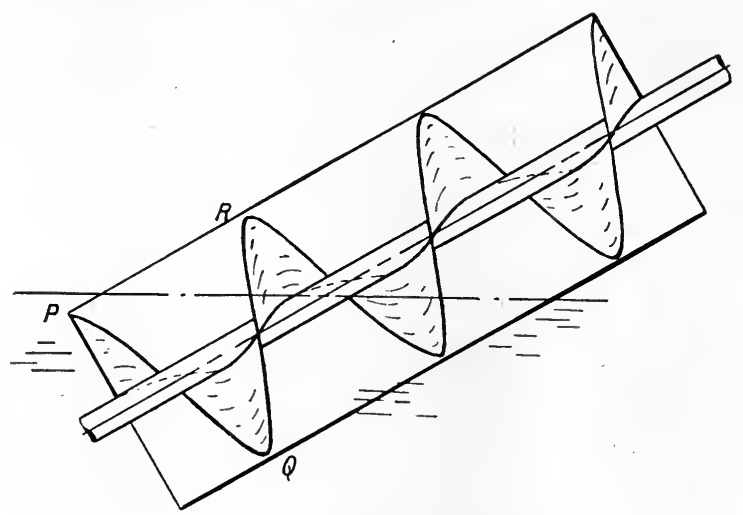

FIG. 215.-Archimedean Screw.

screw, finally emerging at the top into the head-water. The most advantageous angle for the helix is found to be between $30^{\circ}$ and $40^{\circ}$.

Although both screw pumps and scoop wheels are capable of high efficiencies they are cumbrous, and run at inconveniently low speeds, and are in almost every case being replaced by the centrifugal pump.

Art. 148.-The Reciprocating Pump.

The oldest type of reciprocating pump is the bucket pump illustrated diagrammatically in Fig. 216. Originally devised as a lift pump, it was fitted with a hollow bucket or piston surmounted by a valve, and with a foot valve to prevent escape of water on the down stroke.

On the up stroke of the pump a partial vacuum is produced below the bucket, and the pressure of the atmosphere acting on the free surface in the supply reservoir produces a flow up the suction tube in virtue of this difference of pressure.

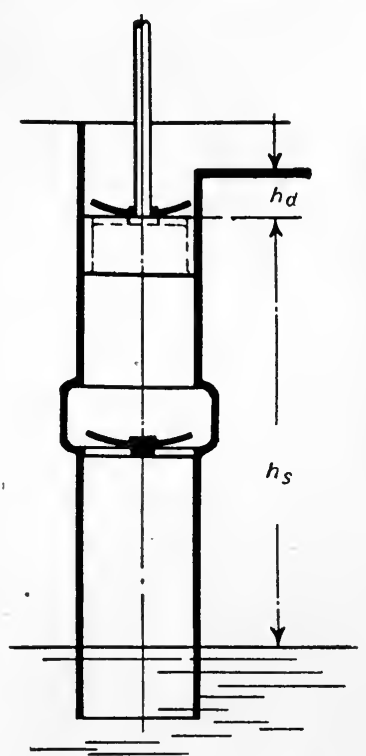

FIG. 216.-Bucket or Lift Pump. 
If $h_{s}=$ suction head in feet of water for any given position of the bucket.

$\pi_{a}=$ atmospheric pressure (in feet of water).

Then, neglecting frictional losses and the effect of acceleration, the pressure head on the under side of bucket $=\pi_{a}-h_{s}$ feet. This has its minimum theoretical value when $\pi_{a}-h_{s}=0$, i.e., when an absolute vacuum is produced below the bucket, and the

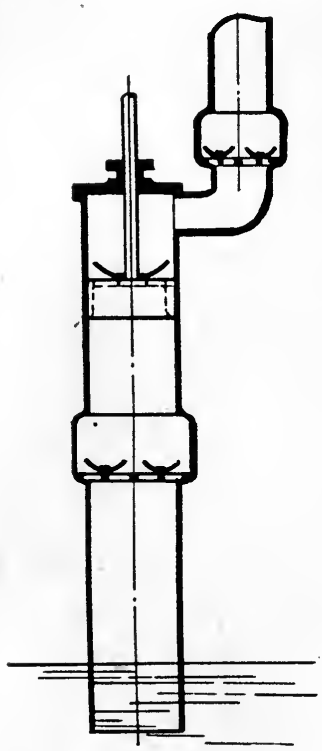

Fig. 217.--Single-acting

Force Pump. maximum possible suction head is thus equal to $\pi_{a}$ or $3 \pm$ feet approximately. Practically, owing to the resistance of the suction valve, leakage at joints and past the bucket, and to the liberation of dissolved air at low pressures, this head is impossible of attainment, a suction head of 24 feet being only maintained with difficulty, and the maximum value of the suction head, i.e., the distance from supply level to level of bucket valve with the bucket in its highest position must not exceed this value.

If $h_{d}=$ delivery head on pump in feet, i.e., height between bucket and outlet, the pressure head on upper side of bucket $=\pi_{a}+h_{d}$ feet.

$\therefore$ Total head on bucket

$$
\begin{aligned}
& =\pi_{a}+h_{d}-\left(\pi_{a}-h_{s}\right) \text { feet. } \\
& =h_{d}+h_{s}=H \text { feet. }
\end{aligned}
$$

Where $H=$ total lift of pump.

On the up stroke, then, water is lifted out at the top of the pump and the tension of the pump rod is given by $W H A$ lbs., where

$A=$ area of bucket in square feet.

$W=$ weight of 1 cubic foot of water $=62.4 \mathrm{lbs}$.

(This neglects the resistance of valves and passages.) On the down stroke water escapes through the valve, from the under to the upper side of the bucket, so that all the work is done on the up stroke.

By the addition of a closed top fitted with a stuffing box through which the bucket rod works, the machine becomes a 
force pump for delivering water under pressure (Fig. 217). The provision of a back pressure valve on the delivery side of the pump is now necessary to prevent the backward escape of the water on the down or idle stroke. As thus arranged the pump may be either vertical or horizontal.

As pressures increase, the bucket type of pump is found to be unsatisfactory on account of the difficulty in keeping the bucket and its valves tight and of detecting any leakage, and this led to the adoption of the Plunger Pump (Fig. 218). Here the bucket is replaced by a plunger of uniform section, all the packing being supplied from the outside and being easily replaced and examined. In this type of pump, which may be either vertical or horizontal, work is done on both strokes, suction taking place on the out-stroke of the plunger, and delivery by plunger

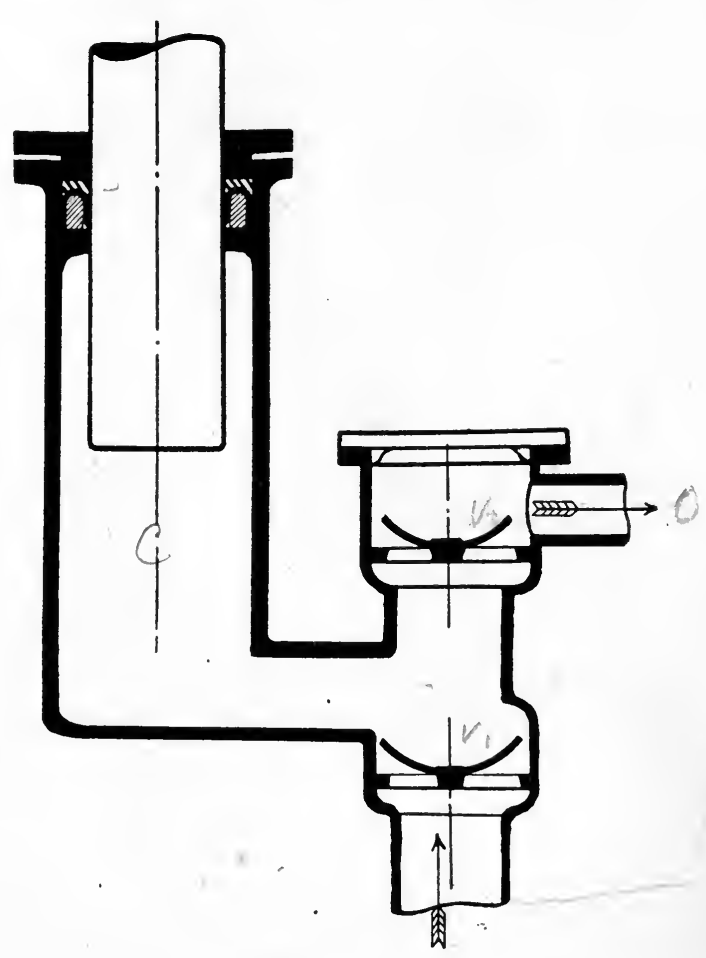

FIG. 218. - Single-acting Plunger Pump. displacement on the in-stroke. The latter in general necessitates the greater work, and as will be noted the ram or plunger is in compression during this stroke.

If $L=$ length of stroke we have :Work done on suction stroke $=A\left\{\pi_{a}-\left(\pi_{a}-h_{8}\right)\right\} W L$ foot lbs. $=W h_{8} A L$ foot lbs.

Work done on delivery stroke $=W h_{d} A L$ foot libs. 
A separate valve box may now contain both suction and delivery valves, and these become more accessible for examination and repairs.

So far, each type considered has suffered from the disadvantage that it only delivers water on alternate strokes. This difficulty is, however, overcome in the "Bucket and Plunger" pump (Fig. 219). Here the piston rod of the ordinary bucket pump is

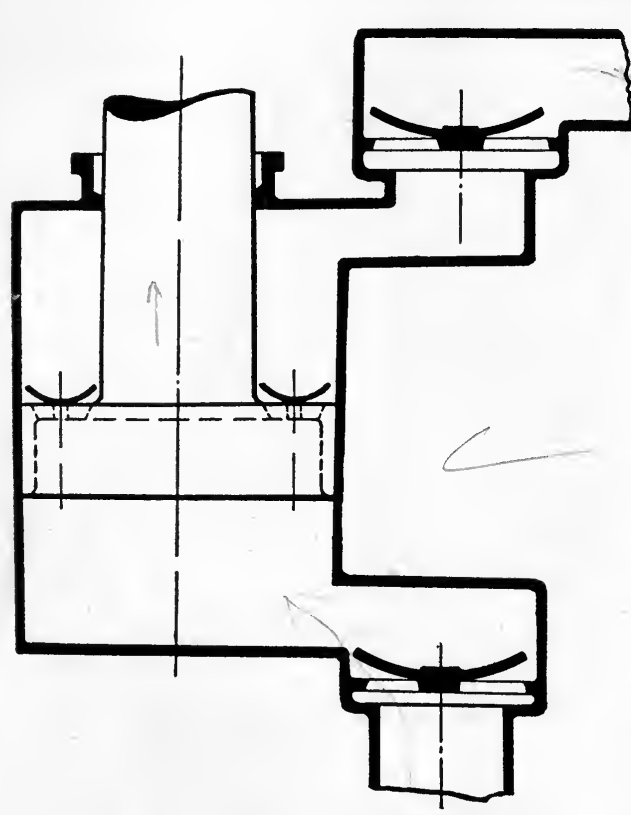

Fig. 219.-Bucket and Plunger Pump.

enlarged to form a plunger of about half the area of the bucket which it carries.

Suction now takes place on the up stroke, and, if $a$ and $A$ are the areas of plunger and bucket, a volume of water $=A L$ is drawn into the pump, while a volume $(A-a)$ $L$ is displaced through the delivery valves. On the down stroke the plunger displaces a volume $A L$, and in consequence this volume passes to the upper side of the bucket. Of this the volume $(A-a) L$ remains in the annular space between the barrel and the plunger, while the remaining volume $a L$ is displaced through the delivery valves.

Thus for equal deliveries on the two strokes we have :-

$$
\begin{gathered}
(A-a) L=a L \\
\therefore A=2 a .
\end{gathered}
$$

But if $h_{s}$ and $h_{d}$ are the mean suction and delivery heads, we have:-

Work on up stroke $=W\left\{(A-a) h_{d}+A h_{s}\right\} L$ foot lbs.

Work on down stroke $=W^{r}\left\{a h_{d}\right\} L$ foot lbs. 
$\therefore$ For equality we have :-

$$
\begin{aligned}
(A-a) h_{d}+A h_{s} & =a h_{d} \\
\therefore \quad A\left(h_{d}+h_{s}\right) & =2 a h_{d} \\
\therefore \quad \frac{a}{A} & =\frac{h_{d}+h_{s}}{2 h_{d}},
\end{aligned}
$$

which approximates to $\frac{1}{2}$ as $h_{d}$ becomes large compared with $h_{s}$.

Where the pump is vertical, and the weight of plunger line great, this requires modification if the forces acting at the upper end of the plunger are to be equal on both strokes. In this case, if $W_{P}=$ weight of plunger line, we have :-

$\left.\begin{array}{c}\text { Force to be exerted } \\ \text { on up stroke }\end{array}\right\}=W\left\{(A-a) h_{d}+A h_{s}\right\}+W_{P}$ lbs. Force to be exerted

on down stroke $\}=W\left\{a h_{d}\right\}-W_{P}$ lbs.

$\therefore$ For equality, $2 W_{P}=W\left\{(2 a-A) h_{d}-A h_{s}\right\}$

$$
\begin{aligned}
& =W\left\{2 a h_{d}-A\left(h_{d}+h_{s}\right)\right\} \\
\therefore \quad a & =\frac{W_{P}}{W h_{d}}+\frac{A\left(h_{d}+h_{s}\right)}{2 h_{d}} .
\end{aligned}
$$

The bucket valves may be done away with, while retaining the advantage of uniform delivery or uniform work on both strokes;

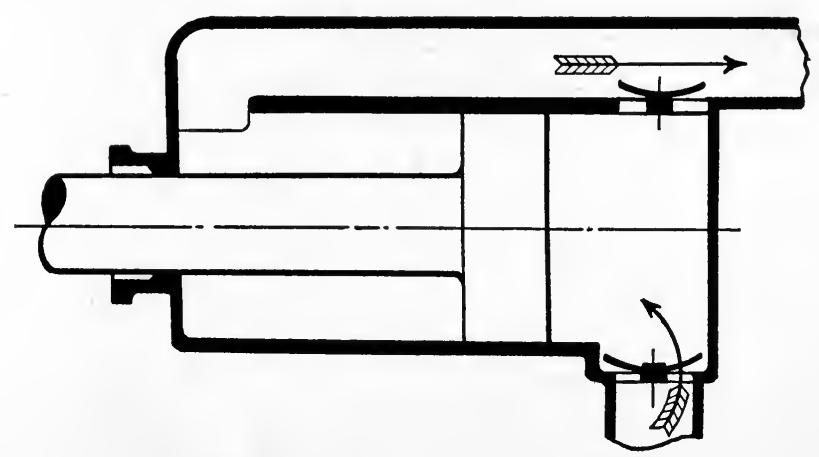

Fig. 220.-Piston and Plunger Pump.

by the type of construction indicated in Fig. 220 and known as the Piston and Plunger pump. Here a solid piston is used instead of the hollow bucket. Suction takes place on the outstroke, while at the same time a volume $(A-a) L$ is delivered. On the in-stroke, a volume $L A$ is discharged through the delivery 
valve, but of this a volume $=(A-a) L$ finds its way to the other side of the piston, and the volume actually delivered $=a L$. As before, for equality of delivery on the two strokes, $\frac{A}{a}=2$. In addition to an equal delivery on each stroke, the further advantage of an equal suction on each stroke may be obtained

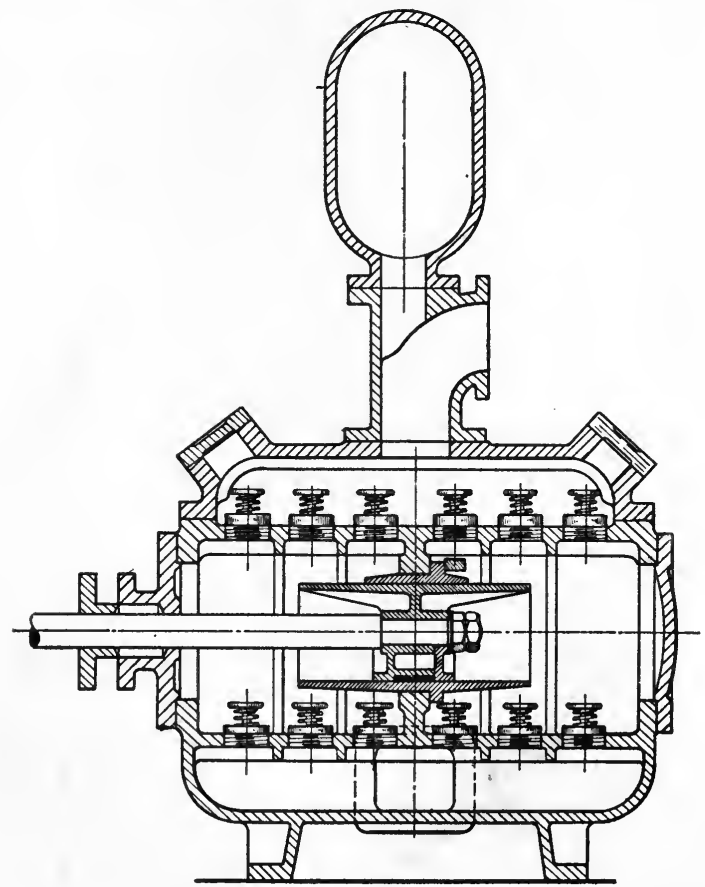

FIG. 221.-Section of 12-inch Duplex Plunger and Ring, or Piston Pump: 10-inch suction ; 8-inch delivery; 12-inch stroke. For pressures up to $100 \mathrm{lbs}$. per square inch.

by duplicating the suction and delivery valves at each end of the barrel as indicated in Fig. 221, each end becoming in effect a separate displacement pump.

This, the Double Acting type, may be fitted either as a piston pump, having a packed piston and sleeve, or with a turned plunger working in an unpacked ring, both types being shown in Fig. 221. 
For fairly low pressures, up to about $75 \mathrm{lbs}$. per square inch, either type is good, the piston pump giving a slightly larger displacement for the same floor space. Where clean water is to

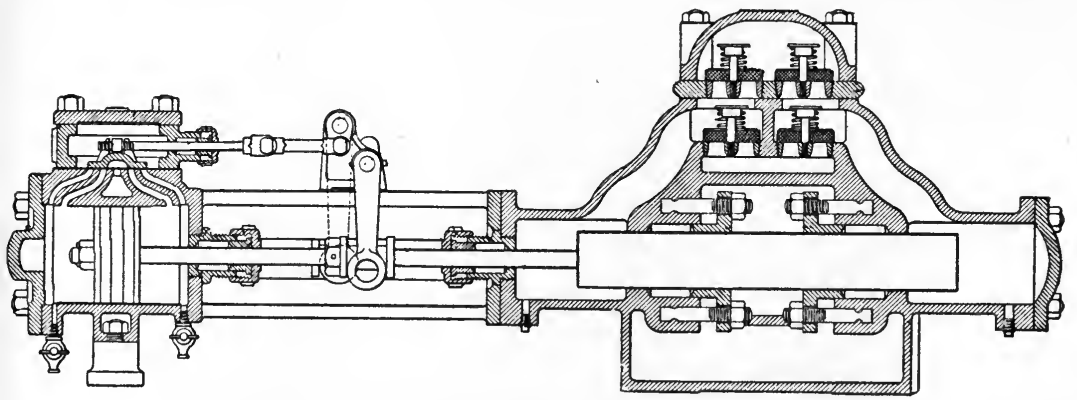

Fig. 222.-Central-Packed Double-Acting Plunger Pump.

be pumped, the unpacked plunger type, however, is advantageous on the ground of its reduced friction.

A short fitting ring is used, easily renewable, and the ease of refitting such a ring compared with that of re-boring a piston barrel renders its use advisable, where the condition of the

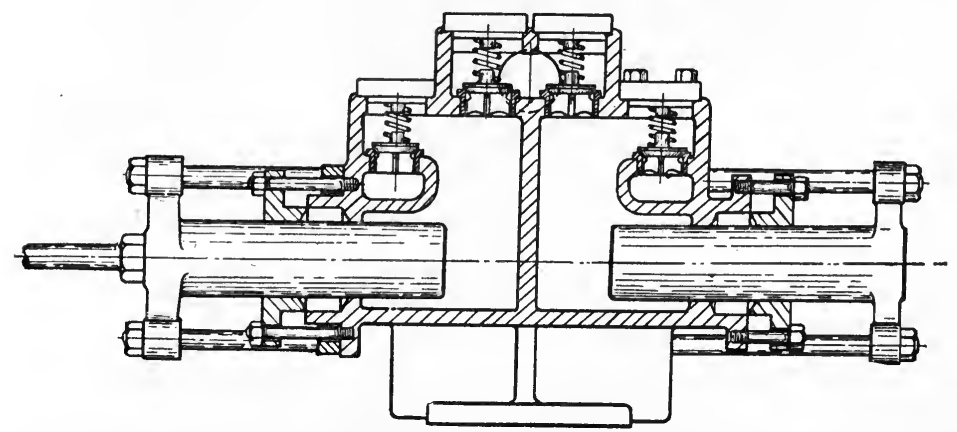

FIG. 223.-Double-Acting Duplex Outside End-packed Plunger Pump, 6-inch plunger; 12-inch steam cylinder; 12-inch stroke; 5-inch suction; 4 -inch delivery; 80 strokes per min.; up to $300 \mathrm{lbs}$. per square inch.

liquid to be pumped permits. In either case the work done on the two strokes is practically the same.

For very high pressures, or where the water contains gritty matter in suspension, the piston, bucket, or internally packed 
plunger pump is unsatisfactory, and the type known as the Double Acting Outside-Packed Plinger pump becomes more suitable.

Here again the pump discharges a volume $A L$ from one end of the barrel and admits an equal volume (neglecting the volume of the plunger rod if this is used) at the other end at each stroke. Fig. 222 shows a direct steam-driven pump of this type having centre-packed plungers, ${ }^{1}$ and designed for eighty strokes per

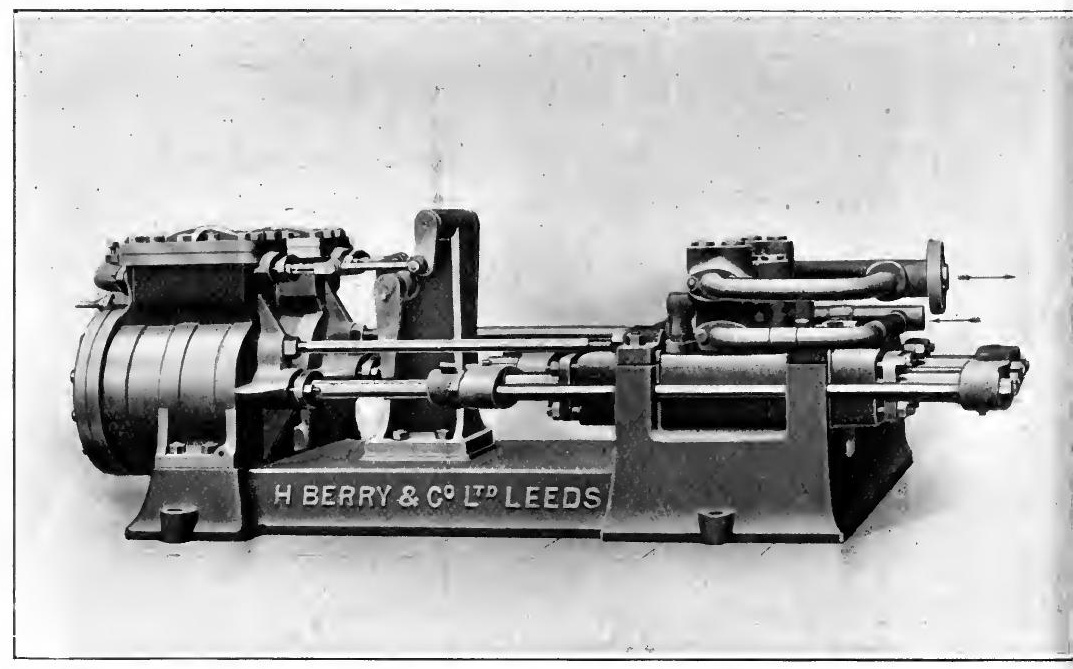

FIG. 224.-Outside End-packed Plunger Pump for pressures up to 1,120 lbs. per square inch.

minute, with 10-inch plunger; 12-inch stroke; 18-inch steam cylinder; 10-inch suction and 7-inch discharge pipes.

Fig. 223 shows an outside end-packed plunger pump of this type, suitable for moderately high pressures, the two plungers carrying crossheads which are connected by side rods, while Fig. 224 shows a similar pump as constructed for the highest pressures. This latter pump has a separate valve box for each end of the cylinder, the construction of the valves (which are 1 inch in diameter) and of the valve box, being shown in Fig. $225{ }^{2}$

1 By courtesy of the Buffalo Steam Pump Company.

2 Figs. 224 and 225 are inserted by courtesy of the makers Messrs. Henry Berry \& Co., Leeds. 
This type of pump has the advantage that only two glands need packing as against three in the centre-packed type, although the latter type lends itself to a more compact construction.

\section{Art. 149.-Pump Valves.}

The valve area should be so designed as to allow of a mean velocity not exceeding 4 feet per second. For low pressures
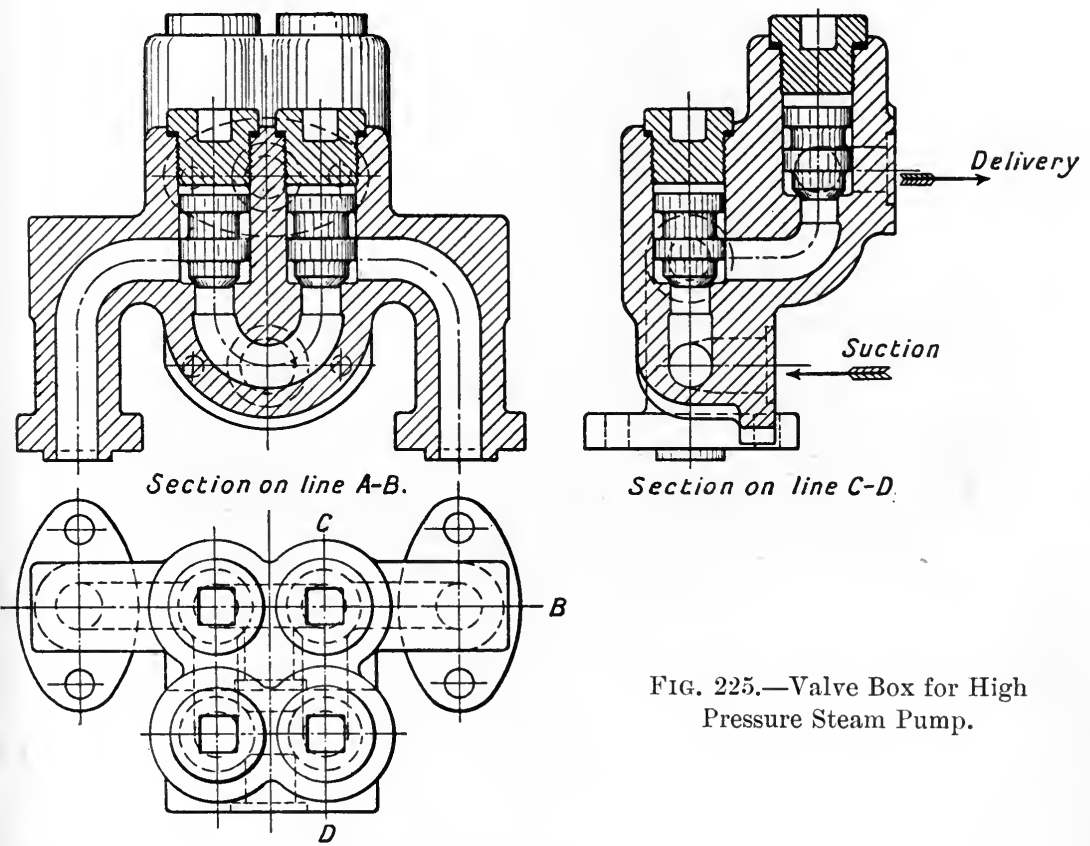

these valves usually consist of rubber or composition dises (Fig. $226 a$ and $b$ ), working against a perforated grid, and are either spring loaded or automatically return to their seats in virtue of their own elasticity, together with the pressure of water above them. For moderate speeds and pressures these are very satisfactory.

At high pressures, however, the discs quickly become seriously indented, and some form of metallic valve becomes essential. Fig. $226 c$ shows a double-beat valve of this type. 
These valves may be spring loaded, or may return to their seats by their own weight, and so long as the speed is moderate are satisfactory. For very high speeds some form of mechanically operated valve, opened and closed by tappets and levers worked from the pump piston, is necessary if excessive shock and leakage is to be avoided. Such valves offer many advantages over the self-closing or automatic type.

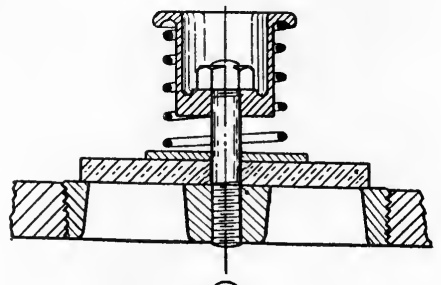

(a)

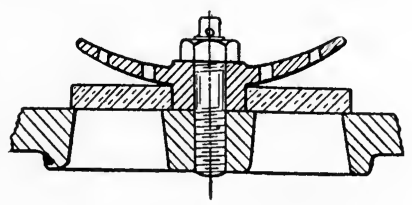

(b)

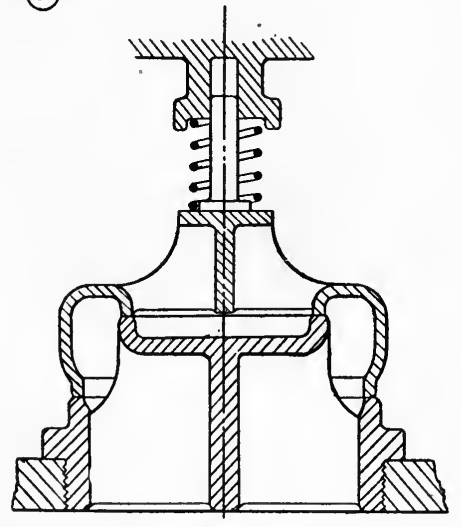

(c)

Frg. 226.-Types of Pump Valve.

In the first place, with automatic suction valves, the difference of pressure below and above must be sufficient to lift the valve, and with a heavy valve this appreciably diminishes the possible suction head. A light valve, by coming slowly to its seat at the end of the stroke enables water to leak back into the suction pipe, since, although theoretically the valve is on its seat when the piston is at rest, actually this is not by any means the case except at slow speeds. The same thing applies on the delivery 
side. Also considerable shock takes place when these valves close, this shock being due, not only to the valve itself dropping suddenly to its seat, but also to the fact that a large mass of water partakes of the return motion.

The violence of the shock depends on the kinetic energy possessed by the valve and accompanying water at the instant of closing, and will therefore increase with the weight of the valve, with the mass of the accompanying water, and with the maximum distance of the valve from its seat, since the latter factor will determine the velocity of closing.

The mass of water accompanying the valve is found to be proportional to the mass included between the valve and the water level in the corresponding air vessel, so that to minimise this effect, the difference in level between valve and air vessel should be reduced as far as possible.

Also the lift of the valve should be as small as is consistent with giving ample inlet and outlet areas.

With self-closing valves, the lift may be reduced either--

(1) by increasing the weight of the valve;

(2) by using a light spring-loaded valve ;

(3) by limiting the lift by stops;

(4) by increasing the number or diameter of the valves.

The first method, while reducing the lift and giving a quicker closing effect, increases the hydraulic resistance; while the increased weight of the valve is in itself productive of shock. On the other hand, a light valve is more subject to vibration while opening or closing, and this may cause large oscillations of pressure in the pipe line. This tendency to vibration increases with the speed of the pump, and diminishes with an increase in the delivery pressure and in the weight of the valve. On the whole, however, the balance of advantage lies with the fairly light spring-loaded valve.

The method of limiting the lift by stops is decidedly unsatisfactory, as giving rise to oscillations of the valve, and hence of the pressure in the pipe line.

The best method is to increase the effective valve area by an 
increase in the number and diameter of the valves., In the ordinary disc valve (Fig. $226 a$ and $b$ ),

$$
\text { If } r=\text { radius and } l=\text { lift of valve, }
$$

the area of valve seat $=\pi r^{2}$.

But the effective valve area is the area of the cylindrical surface generated by the perimeter of the valve during its lift $=2 \pi r l$.

The most effective lift is thus obtained when these values are identical, i.e., when-

$$
\begin{aligned}
2 \pi r l & =\pi r^{2} \\
\therefore l & =\frac{r}{2} .
\end{aligned}
$$

It follows that, in order to take arvantage of the available valve seat area, it is impracticable to use very large values, since for a given lift, the effective discharge area varies as the diameter of the valves, while the valve weight varies more nearly as $d^{\frac{5}{2}}$, while if the lift were given the value $\frac{r}{\overline{2}}$, this would soon become excessive. Because of this, small valves should be used, with a lift giving a discharge area approaching that of the valve seat, and for high speed pumps modern practice is opposed to the use of valve seats of more than about 3 inches diameter. Where double beat valves (Fig. 224c) are used this may be increased, but in no case should the valve have a lift exceeding $\frac{3}{4}$ inch.

Valve Leakage and Slip.-A further point to be noted is that slip, or leakage past the valves while closing, is proportional to the mean effective opening of the valve : to $\sqrt{h}$, where $h$ is the head on the valve, and to the time of closing, $t$.

Where a valve of specific gravity $\rho$ is submerged in water and falls by its own weight, its vertical acceleration is equal to $\rho g$ feet per second per second (neglecting the resistance of the water), so that the time to fall through a distance $l$ is given approximately by $t=\sqrt{\frac{2 l}{\rho g}}$ seconds.

With a spring-loaded valve this time will, of course, be reduced, but in any case we may take $t$ as proportional to $\sqrt{l}$. 
If, then, a number of small valves replace a single large valve of the same effective discharge area, this slip will be reduced, since to get the maximum discharge effect the lifts of the valves must in every case be proportional to their diameters and this lift, and therefore the time of closing, will be greater with the larger single valve.

Thus, whereas the slip past $n$ valves of diameter $d$ is proportional to $n h^{\frac{1}{2}} d^{\frac{5}{2}}$, that past a single valve of the same effective discharge area, and, therefore, of diameter $D$ where $D=d \sqrt{n}$ is proportional to $h^{\frac{1}{2}} d^{\frac{5}{2}} n^{\frac{5}{4}}$, so that by increasing the number of valves to $n$, the leakage is reduced in the ratio $\frac{n}{n^{\frac{5}{4}}}=\frac{1}{n^{\frac{1}{4}}}$.

E.g., where $n=4$, the leakage is equal to $\frac{1}{4^{\frac{1}{4}}}=\cdot 707$ times that found with a single large valve.

Owing to the length of time necessary for an automatic valve to close, and to the irregularities in its action produced by inertia effects of the water in the supply and delivery pipes, a high rotative speed is impossible with a reciprocating pump fitted with this type of valve, and it becomes necessary to use a long stroke, slow rotation pump, giving ample time for the valves to come to rest at the end of each stroke. With this type of pump, and until comparatively recently, the maximum attainable piston speed was about 100 feet per minute at about sixty revolutions per minute.

By the substitution of mechanically operated valves, however, uncertainty as to the exact time of closing is avoided, more uniform closing is effected with less accompanying shock, and by this means, and by careful design of the valves, the speed has been increased until piston velocities of 600 feet per minute at 300 revolutions per minute are now easily obtained with almost entire absence of shock. This increase in speed permits of the pump dimensions being reduced for the same duty; gives a less delivery per stroke, and hence reduces the liability to shock; while the possibility of a more uniform flow in both supply and discharge pipes tends to the same end. 
For the improvement of the high-speed reciprocating pump, investigations by Professor Reidler ${ }^{1}$ have been of great importance. Among other things, these experiments go to show that:-

(1) Shock is produced more particularly at the suction valves and increases with the weight of the valve and with the mass of water between the valve and the water level in the suction air vessel.

(2) The amount of throttling at valve seats is very slight indeed with well-designed valves, having a value largely below that indicated by theory.

Fig. 227 shows the construction of the pump barrels, rams,

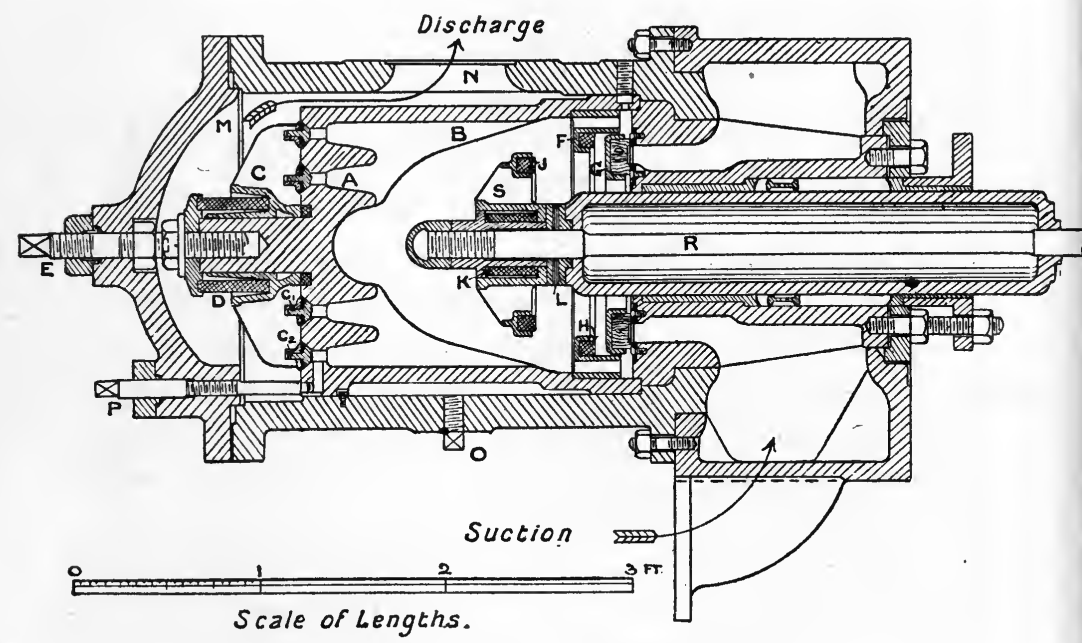

Fig. 227.-Reidler Express Pump.

and valves of a type of high-speed pump designed by Professor Reidler, this particular pump being designed to deliver 375 gallons per minute against a head of 500 feet. It is a two-throw pump having rams $6 \frac{3}{8}$ inches diameter by 9 inches stroke; running at 200 revolutions per minute and driven by an electric motor of 75 B.H.P. running at 500 revolutions per minute.

The outer end $A$ of the working cylinder $B$ forms the seating of the delivery valve, which consists of two annular brass rings, $C_{1}$ and $C_{2}$, let into a gun-metal frame $C$. This valve is kept up to

1 Revue de Méchanique, November, 1902 (p. 429). 
its seat by means of the cylindrical indiarubber spring $D$, the compression of which may be adjusted by means of the bolt $E$, while leather sealing rings are also provided to ensure its efficient action.

The inner end of the working cylinder carries a gun-metal guide ring $F$, in which works the annular suction valve $G$. This valve is constructed with a wooden face let into a channel section annular brass ring, and its range of opening is limited by the rubber ring $H$, let into the guide ring $F$.

The ram $R$ passes through the suction valve, and at its inner end carries a buffer stop $S$, which mechanically closes the suction valve at the end of the outer or suction stroke. Shock at impact is minimised by the provision of cylindrical rubber springs at $J$ and $K$. A series of removable brass liners $L$ between the ram and the buffer permit of the latter being adjusted so as to take up any wear in the buffer itself or in the suction valve.

The delivery valve is thus automatically regulated, while the suction valve is mechanically operated only so far as affects its closing at the end of the suction stroke, this valve being both opened and closed on the out-stroke of the ram. On the in-stroke, water is delivered against the resistance of the delivery valve into the annular chamber $M$, from which it flows away through the pipe $N$ into an air vessel and away to the discharge. The chamber $M$ is fitted with a starting valve at $O, 2$ inches in diameter, so that in starting the pump the pressure may be relieved and the pump started light. The small spindle valve $P$ allows of water passing from the chamber $M$ into the working barrel, so that if for any cause sufficient water cannot enter through the suction valves, more water may be allowed to enter through the valve $P$, so as to enable the pump to run without shock.

A small compound air pump, having a high-pressure plunger $1 \frac{1}{2}$ inches diameter by 1.8 inches stroke and a low-pressure plunger 3 inches diameter by 2.7 inches stroke, is provided, and is worked directly from the crank shaft of the main pump, exhausting air from the suction air vessel and discharging it into the delivery air vessel. 
Should the air supply in the suction air vessel be insufficient, air may be admitted through a small valve; while should the air
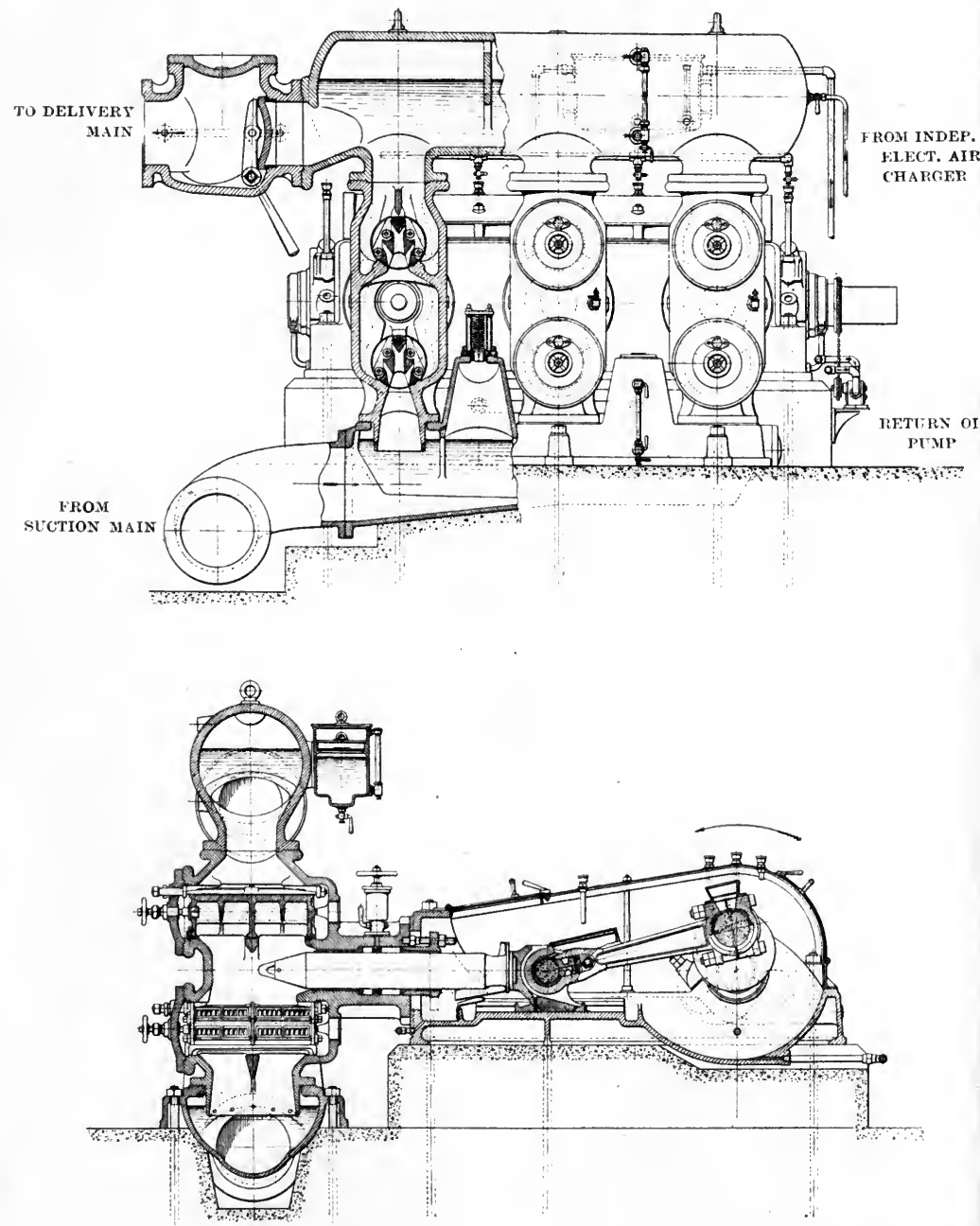

Fig. 228.-Gutermuth 3-Throw Pump : $6 \frac{3}{4}$-inch diameter $\times 16$-inch stroke: $\times 180$ revs. per min. ; 1,000 gallons per $\min : 7 \tilde{0}) \mathrm{ft}$. head.

pump deliver too great a supply, air can be let out of the delivery air vessel. 
Tests carried out on such a pump, as described ${ }^{1}$ showed a combined efficiency of pump and motor of about 85 per cent., with a discharge coefficient of $\cdot 92$.

For still higher speeds, the pump is designed with suction and delivery valves mechanically operated from a wrist-plate driven by an eccentric on the main shaft.

Another modern type of high-speed pump which has proved itself capable of excellent results is the Gutermuth (Fig. 228). ${ }^{2}$ The illustrations show a sectional plan and elevation of one of a pair of three-throw pumps of this type, having plungers $6 \frac{3}{4}$ inches diameter by 16 inches stroke and delivering 1,000 gallons per minute against 750 feet head at 180 revolutions per minute. Each pump is driven frorn a 275 H.P. three-phase motor through a flexible coupling. The design is very compact, the pump bodies being mounted on a suction air vessel of cylindrical form, which also acts as a bedplate. On this air vessel is cast a branch flange for connecting up to the suction main. On top of one of the pump bodies is bolted a delivery air vessel, having flanged branches to connect to the pump bodies, and with the end flanged to connect to the retaining valve on the delivery main.

The bodies themselves are made separately and are connected to the guides of the main bedplate, the latter having machined circular flanges to receive these. The bedplate is cast in one piece, the main bearings being of the two-part type set at an angle of $45^{\circ}$ and having cast-iron babbited boxes.

T'he connecting rods are of cast steel with marine type babbited ends for the crank pin, and gun-metal wedge adjustment boxes for the crosshead end.

The plungers are of gun-metal, and are supported in gun-metal bushed glands and neck rings.

Forced lubrication is fitted throughout, while the two delivery air vessels are supplied by an independent electrically-driven two-stage air charger of the Reavel type, the compressor being

1 By Mr. John Morris. See a paper on the "Unwatering of the Achddu Colliery." Trans. Inst. of Mining Engineers, Vol. 30, part 2, p. 131.

2 Figs. 228 and $228 \mathrm{~A}$, as well as the accompanying description, are by courtesy of the manufacturers, Messrs. Fraser \& Chalmers, Itd. 
capable of compressing 5 cubic feet of free air per minute to a pressure of $350 \mathrm{lbs}$. per square inch.

The valves, which are perhaps the most interesting part of these pumps, are contained in cylindrical bronze valve seats, each pump having one set for suction and one for delivery. These are held in place and tightened by means of wedges which are readily accessible when the valve covers are removed.

The valves themselves, which are of the Gutermuth type (Fig. $228 \mathrm{~A}$ ), and are automatic in action, are formed from a single sheet of special bronze, either of the same thickness throughout or, in large valves, having the end forming the valve thickened. The sheets are slightly wider at the coiled end to prevent fouling, and are simply slipped on to a grooved spindle and clamped to the valve seat cones at the required tension.
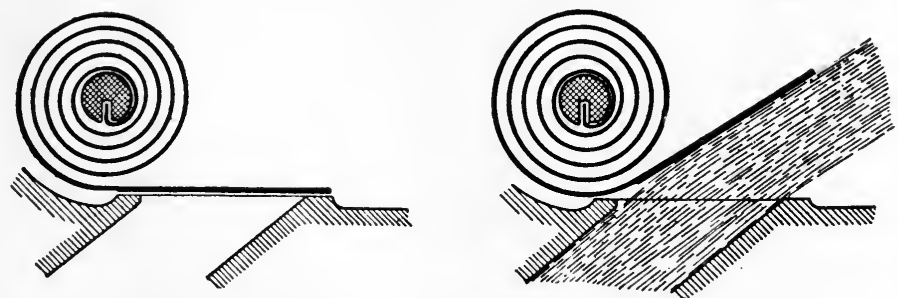

Fig. 228 A.-The Gutermuth Valve.

The valve is always placed at an angle with the port opening, and the latter thus becomes uncovered with a very small movement of the valve itself, while no such abrupt changes in the direction of the escaping stream are necessitated as in the case of disc or mushroom valves. The sectional end view of Fig. 228 shows very clearly the straight and unobstructed passages offered to the water in this type of pump.

Frictional losses are thus low; the lightness of the valve and its small opening tend to reduce shock on closing; and the possible speed of rotation is correspondingly increased.

The high speed at which these pumps can be run, as a direct result of the valve action, permits of a design which takes up considerably less floor space than the ordinary slow running pump, the actual overall length being, in this case, 11 feet 8 inches $\times 8$ feet 6 inches. It, also allows the pump to be direct 
connected to the motor, which, though adding somewhat to the expense of the latter, does away with transmission gears which are usually noisy and troublesome, and which, under the most favourable circumstances, in a pump of this size, would reduce the efficiency by from 3 per cent. to 4 per cent.

Discharge Coefficient.-In the theoretically perfect pump the action would be simple. On the suction stroke the delivery valves would be tightly closed while the suction valves would open, admitting sufficient water to entirely fill. the barrel. At the commencement of the delivery stroke these valves would immediately close, while the delivery valves would open, and a volume of water equal to the plunger displacement would be discharged. In practice, however, owing to the fact that the valves do not instantaneously close, and because of leakage past the plunger, etc., the volume delivered is not in general equal to the plunger displacement. The ratio $\frac{\text { actual }}{\text { theoretical }}$ discharge, termed the discharge coefficient, varies with the type, speed and condition of the pump, but with moderate speed pumps in good condition and of good design lies between .94 and 99 . With high-speed pumps the modifying effects of the inertia of the suction column may be such as to give a discharge greater in volume than the piston displacement, the excess amounting in exceptional cases to as much as 50 per cent. This effect will be considered in further detail at a later stage.

\section{Art. 150.-Displacement Curves.}

If a curve, having piston velocities as ordinates be plotted on a time base, the area included between the curve, the base line, and any two ordinates, will measure to some scale the volume displaced by the piston in the corresponding interval of time. The curve so obtained is termed a displacement curve. Thus, if $O Q$ and $Q P$ represent the crank and connecting rod of a pump for a given piston position, and if $P Q$ be produced to meet the perpendicular $O C$ to the line of centres in $C$, then $O C$ represents the corresponding piston velocity to the scale on which $O Q$ represents $\omega r$ the velocity of 
the crank pin. If a series of such points as $Q$ are taken at equidistant intervals on the crank circle, and the corresponding values of $O C$ found, the displacement curve may then be plotted as in Figs. $229_{A-D}$. Here Fig. $229_{\mathrm{A}}$ represents the curve for a

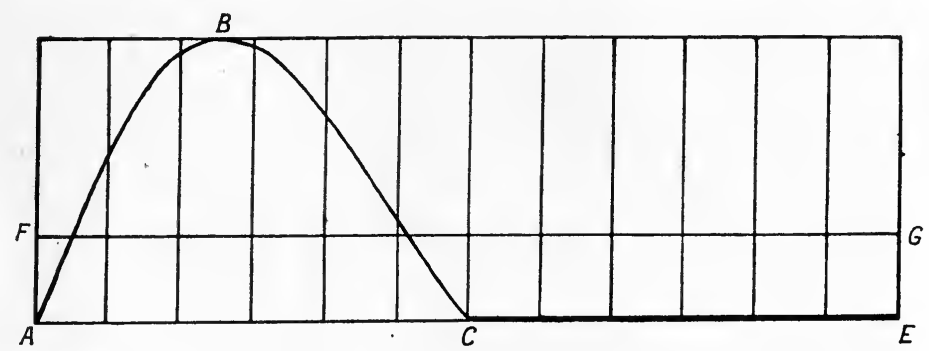

FIG. 229 A. - Displacement Curve for Single Cylinder Single-acting Pump.

single-barrel single-acting pump having a connecting rod four cranks in length, $A C$ representing the time to perform half, and $A E$ to complete a revolution.

The area $A B C$ now represents the displacement of the pump per revolution, while $C E$ represents the idle stroke.

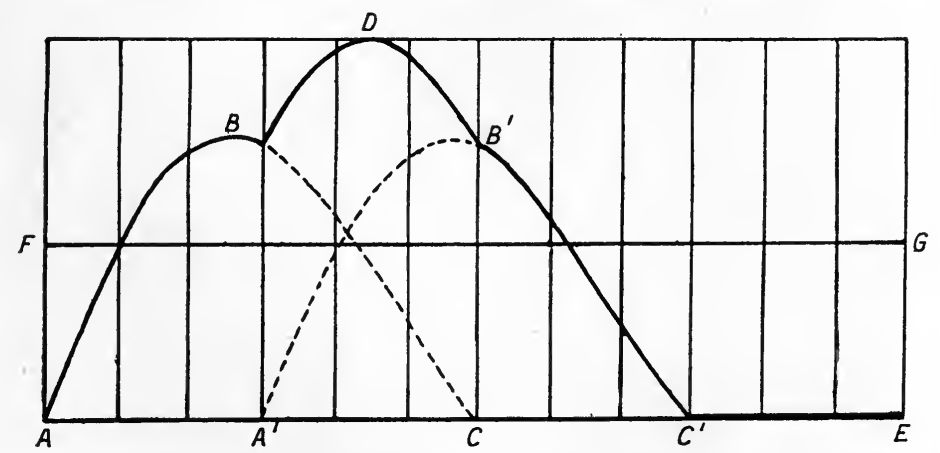

FIG. 229 B. - Curve for Pair of Single-acting Cylinders with Cranks at Right Angles.

If $F G$ be drawn parallel to $A E$, so that $A F$ represents the mean velocity of the piston, the area $A F G E=$ area $A B C$.

Similarly, Fig. 229 в represents the displacement curve $A B D B^{\prime} C^{\prime} E$, for a pair of single-acting pumps having cranks at right angles and drawing from and delivering into a common main. Here $A B C$ and $A^{\prime} B^{\prime} C^{\prime}$ are the respective curves for 
each piston, and these are compounded by adding ordinates so as to give the displacement curve. The area $A F G E$ is now equal to the sum of the areas $A B C$ and $A^{\prime} B^{\prime} C^{\prime}$, or to the whole area $A B D B^{\prime} C^{\prime}$.

Again, Fig. $229 \mathrm{c}$, obtained in a similar manner, shows the

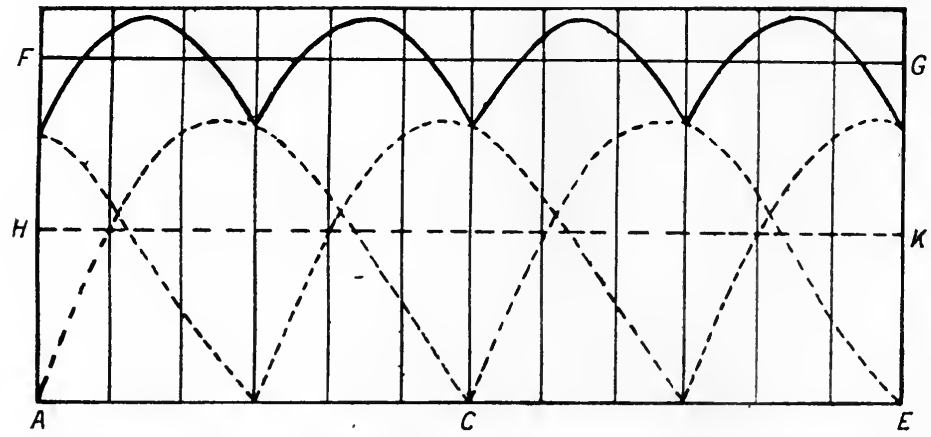

Frg. 229c.-Curve for Pair of Double-Acting Pumps with Cranks at Right Angles.

curve for a pair of double-acting pumps having cranks at right angles, $F G$ again representing the mean velocity line. Here

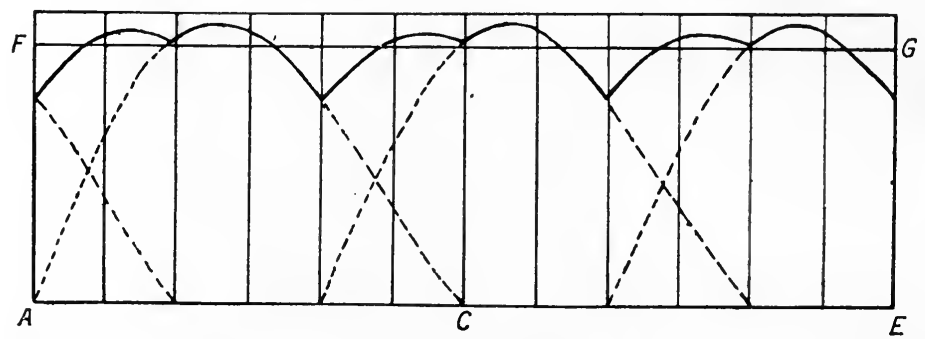

Frg. 229 D.-Displacement Curve for Three-Throw Single-Acting Pump with Cranks at $120^{\circ}$

$H K$ represents what would be the mean velocity line for a single double-acting pump.

Similarly, Fig. 229 D represents the curve for a three-throw pump having cranks at intervals of $120^{\circ}$.

It will be noted that as the frequency with which the pumps discharge per revolution increases, the range of velocities in the discharge and suction pipes decreases, and also the rate of change 
of this velocity, i.e., the acceleration, and the smoothness of working in consequence increases.

The following table indicates how this velocity changes:-

Type of Pump.

Single cylinder, single-acting

Two single-acting cylinders, cranks at right angles Single cylinder, double-acting

Two double-acting cylinders, cranks at right angles Three-throw pump, crank at $120^{\circ}\left\{\begin{array}{l}\text { single-acting } \\ \text { double-acting }\end{array}\right.$
Ratio of Maximum to Mean Velocity in Discharge Pipes.

As will be readily understood, the varying velocity and acceleration in the supply and discharge pipes-particularly in the supply pipes-produce a tendency to water hammer.

So long as this hammer is prevented, either by suitably enlarging the pipes; by reducing the maximum piston velocity or acceleration; or by the provision of air vessels on suction and delivery pipes, the action of a pump may be predicted very accurately from theoretical considerations. Once water hammer is set up so many factors combine to influence the result, and the subject presents such difficulties of treatment that, except in simple cases, no satisfactory attempt can be made to deal with the matter analytically. This is, however, less important, in that water hammer is not, under any conditions, admissible in a pump, and while its effect may be impossible to predict with any degree of accuracy, save in the most simple cases, the steps necessary to prevent its occurrence are well understood.

The following demonstration, may be relied upon as giving results which are substantially correct so long as the pump is working without shock.

Art. 151.-Variations of Pressure in the Cylinder of a Single Cylinder Pump without Air Vessels.

For the water in the suction pipe to follow and maintain contact with the piston throughout the suction stroke, this column 
of water must receive an acceleration at the commencement of the stroke, the magnitude of the acceleration being given by-

$$
a \frac{A}{a_{s}} \text { f.s.s. }
$$

where $\quad a=$ piston acceleration in f.s.s.

$A=$ piston area in square feet.

$a_{s}=$ area of suction pipe in square feet.

The force necessary to give this acceleration can only be produced by a difference in pressure at the two ends of the suction pipe, and varies as the mass of water in the pipe and as its acceleration. Expressed symbolically we have-

Force necessary to produce acceleration $=\frac{W}{g} l_{s} a_{s} \cdot \frac{A}{a_{s}} a \mathrm{lbs}$.

If the pressure difference at the two ends of the pipe corresponding to this force is $p^{\prime}$ lbs. per square foot, we have-

$$
\begin{aligned}
p^{\prime} a_{s} & =\frac{W}{g} l_{s} A \text { a lbs. } \\
p^{\prime} & =\frac{W}{g} l_{s} \frac{A}{a_{s}} a \text { lbs. per square foot. }
\end{aligned}
$$

If at the same instant the piston velocity is $v$ f.s., for continuity of flow we have $r_{s}=r \frac{A}{a_{s}}$; and the loss of pressure due to friction in the supply pipe corresponding to this is given by:-

$$
p^{\prime \prime}=\frac{W f l_{s} r_{s}^{2}}{2 g m}=\frac{W f l_{s} A^{2}}{2 g m a_{s}^{2}} v^{2} \text { lbs. per square foot. }
$$

$\therefore$ Total difference of pressure at the inlet and outlet of the suction pipe is given by :-

$$
p^{\prime}+p^{\prime \prime}=p=\frac{W}{g} \frac{A}{a_{s}} l_{s}\left\{a+\frac{f A}{2 m a_{s}} v^{2}\right\} \text { lbs. per square foot. }
$$

Or, expressed as a head " $h$ " in feet of water,

$$
h=\frac{A l_{s}}{g a_{s}}\left\{a+\frac{f A}{2 m a_{s}} \cdot v^{2}\right\} \text { feet, }
$$

where $a$ is positive or negative according as the piston is being accelerated or retarded.

Now the head actually available to produce this flow is strictly limited, the higher pressure-at the pipe inlet-being that corresponding to its depth of immersion together with that of 
the atmosphere, while the lower limit of pressure is theoretically that corresponding to an absolute vacuum, although in practice it is impossible to obtain this degree of exhaustion in the pump. If the level of the pump is above that of the suction reservoir so as to give a suction lift of $h_{s}$ feet (Fig. 230), the available head is correspondingly reduced, and has a maximum theoretical value of $\left(\pi_{a}-h_{s}\right)$ feet, where $\pi_{a}$ is the height of the water barometer.

Thus, for continuity of contact between piston and water on the suction stroke, we must have :-

$$
\pi_{a}-h_{s}>\frac{A l_{s}}{g a_{s}}\left\{a+\frac{f A}{2 m a_{s}} v^{2}\right\} \text { feet. }
$$

Should this condition not be satisfied the piston leaves the

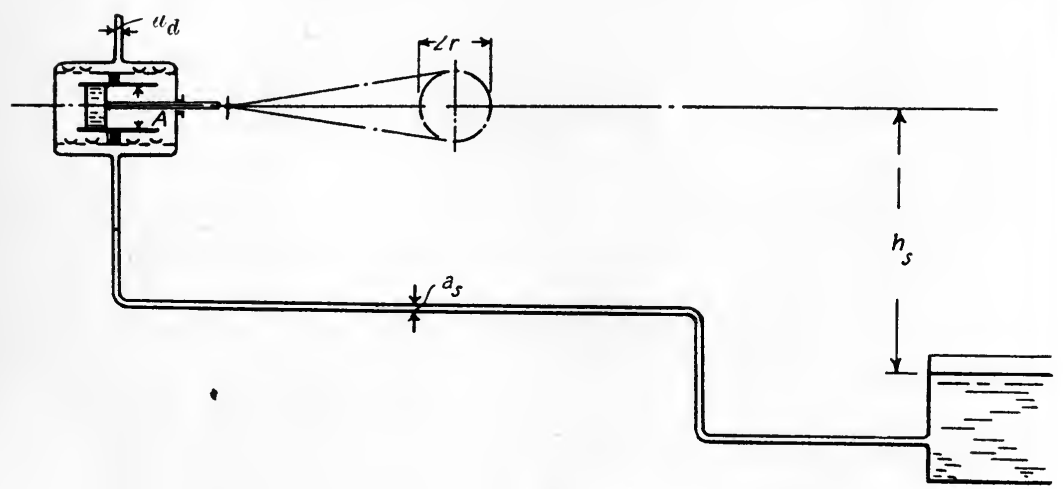

Fig. 2:30.

water at some point-usually at the beginning of the stroke, since here $a$ has its maximum value-the action being termed Separation. So long as this state of affairs exists, the pressure behind the piston is uniform, this pressure being that of water vapour at suction temperature, and in consequence the water flows along the pipe with an acceleration which is uniform, except for the increasing effect of friction as its velocity increases. The piston acceleration is, however, continuously diminishing, so that the water overtakes the latter at some point before the completion of the stroke, the meeting being usually accompanied by considerable shock, while pressures considerably in excess of those corresponding to the statical head are produced.

Separation may also occur between the delivery column and 
the piston during the second half of the delivery stroke, when the piston is being retarded. Thus if $-a$ is the magnitude of this retardation, the head necessary to produce the retardation in the delivery column is given by $-\frac{A}{a_{d}} \frac{l_{d}}{g}$ a feet, so that to prevent separation during this stroke we must have:-

$$
\pi_{a}+h_{d}>\frac{A}{a_{d}} \frac{l_{d}}{g}\left\{-a-\frac{f A}{2 m a_{d}} v^{2}\right\}
$$

Note.-Since during the latter half of the stroke the piston is being retarded $(-a)$ is a positive quantity.

Where the pump is driven from a shaft rotating with uniform angular velocity $\omega$ radians per second, by means of a crank of radius $r$, we have, assuming the connecting rod to be of infinite length :-

$$
\begin{cases}a=\omega^{2} r \cos \theta & \text { f.s.s. } \\ v=\omega & r \sin \theta\end{cases}
$$

where $\theta$ is the crank angle, measured from the inner dead centre, while with a connecting rod of length $l$ these expressions become :-

$$
\left\{\begin{array}{l}
a=\omega^{2} r\left\{\cos \theta+\frac{r l^{2} \cos 2 \theta+r^{3} \sin ^{4} \theta}{\left(l^{2}-r^{2} \sin ^{2} \theta\right)^{\frac{3}{2}}}\right\} \text { f.s.s. } \\
v=\omega r \cdot\left\{\sin \theta+\frac{r \sin 2 \theta}{2 \sqrt{l^{2}-r^{2} \sin ^{2} \theta}}\right\} \text { f.s. }
\end{array}\right.
$$

giving $a$ the maximum value $\omega^{2} r\left\{1 \pm \frac{r}{l}\right\}$ according as the crank is on the inner or outer dead centres respectively (i.e. according as $\theta=0^{\circ}$ or $180^{\circ}$ ).

Neglecting, for simplicity, the effect of the obliquity of the connecting rod, and therefore assuming the piston to have simple harmonic motion, the maximum velocity is given by $\omega r$, while the maximum acceleration $=\omega^{2} r$, and therefore for a given displacement the acceleration is comparatively reduced by making the stroke of the pump large and the angular velocity of the crank, or the number of strokes per minute, low. This enables a higher piston speed to be adopted, since frictional effects are usually small compared with those due to acceleration. 
Substituting for $a$ and $v$ in terms of the crank angle $\theta$, equation (1) becomes:-

$$
\begin{aligned}
\pi_{a}-l_{s} & >\frac{A}{a_{s}} \frac{l_{s}}{g}\left\{\omega^{2} r \cos \theta+\frac{f \omega^{2} r^{2} \sin ^{2} \theta A}{2 m a_{s}}\right\} \\
& >\omega^{2} r \frac{A l_{s}}{a_{s} g}\left\{\cos \theta+\frac{f r \sin ^{2} \theta A}{2 m a_{s}}\right\} \\
\omega & =\sqrt{\frac{\frac{g a_{s}}{r A l_{s}}\left\{\pi_{a}-h_{s}\right\}}{\cos \theta+\frac{f r \sin { }^{2} \theta A}{2 m a_{s}}}}
\end{aligned}
$$

giving

as the critical velocity, above which separation will occur on the suction stroke.

Similarly, substituting in equation (2) we obtain :-

$$
\omega=\sqrt{\frac{\frac{g a_{d}}{r A l_{d}}\left\{\pi_{a}+h_{d}\right\}}{-\cos \theta-\frac{f r \sin { }^{2} \theta A}{2 m a_{d}}}}
$$

as the velocity, above which separation occurs on the delivery stroke.

Here $\theta$ is in every case measured from the beginning of the stroke, so that $\cos \theta$ in the latter expression is negative.

Putting $\pi_{a}=34$ feet, and writing $\theta=0$ in equation (3), we get :-

$$
\omega=\sqrt{\frac{g a_{s}}{r A l_{s}}\left\{34-h_{s}\right\}}
$$

as the limiting speed at which separation will occur at the commencement of the suction stroke. Since $\omega=\frac{2 \pi N}{60}$ (where $N=$ revolutions per minute), this becomes :-

$$
N=\frac{30}{\pi} \sqrt{\frac{g a_{s}}{r A l_{s}}\left(34-h_{s}\right)} .
$$

With a finite connecting rod of length $l$, we have :-

$$
N=\frac{30}{\pi} \sqrt{\frac{g a_{s}}{r A l_{s}} \cdot \frac{\left\{34-h_{s}\right\}}{\left\{1+\frac{r}{l}\right\}}} .
$$


The action may be shown graphically as follows:-

In Fig. 231, $O O^{\prime}$ represents the atmospheric pressure line, and, assuming simple harmonic motion, ordinates drawn to the straight line $A H A^{\prime}$ represent the head necessary to accelerate the water column in the suction pipe. Then $O A=O^{\prime} A^{\prime}=$ $\frac{\omega^{2} r l_{s}}{g} \cdot \frac{A}{a^{8}}$. Vertical ordinates, set off from $A H A^{\prime}$ as base line, to the curve $A B A^{\prime}$, represent the heads necessary to overcome

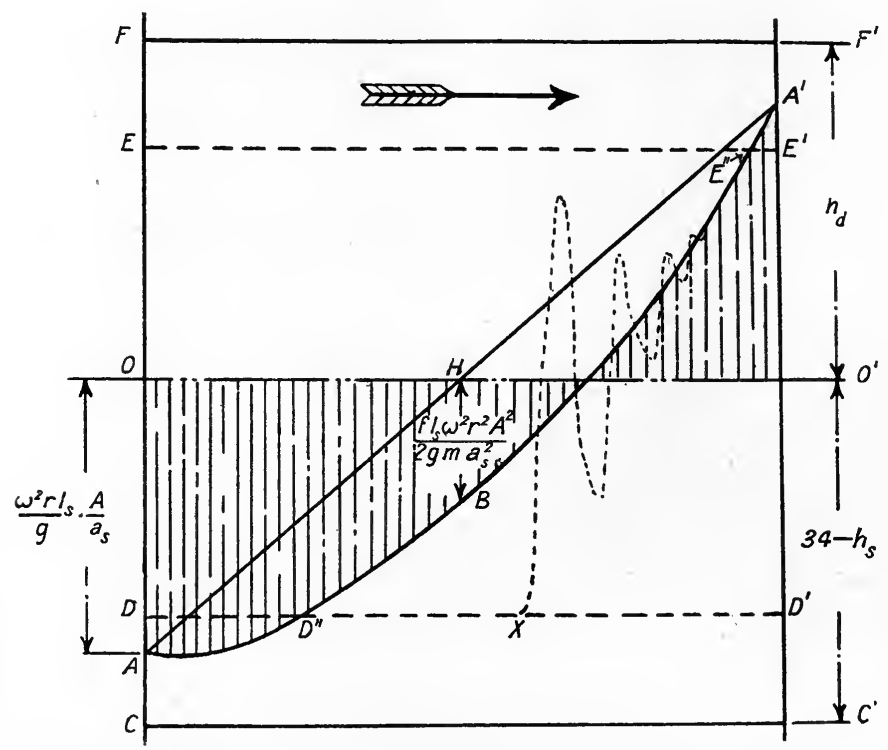

FIG. 231. - Theoretical Diagram of Piston Pressure during Suction Stroke of Reciprocating Pump.

frictional resistance, zero at the ends, and having a maximum value $=\frac{f l_{s} A^{2}}{2 g a_{s}^{2}} \cdot \frac{\omega^{2} r^{2}}{m}$, at the middle of the stroke.

The vertical ordinates of the shaded area then give the differences of head between the two ends of the suction pipe, these being negative or positive, according as the ordinates are measured below or above $O O^{\prime}$.

If now $C C^{\prime}$ be drawn at a distance below $O O^{\prime}$, representing the available head $\left(34-h_{s}\right)$ feet, the ordinates of the curve 
$A B A^{\prime}$, measured from $C C^{\prime}$ as base line, give the effective pressures in the suction chamber, expressed in feet of water.

If the suction head be increased so that $O D$ represents $34-h_{8}$ feet, separation will occur at the commencement of the stroke, since the available head is now insufficient to give the necessary acceleration. At $D^{\prime \prime}$ the head becomes sufficient both for this purpose and to overcome frictional losses, while at some point $X$, the acceleration of the water remaining approximately constant while that of the piston is continually diminishing, the water overtakes the piston and knocking occurs. Up to this point the pressure behind the piston is constant, and has a negative value equal to $O D$ in feet of water. At the shock, violent oscillations of pressure are set up as represented by the dotted lines and the most that theory can do in this case is to indicate the maximum pressure to be expected and the means of reducing this.

Under normal conditions of working, the maximum pressure attained on the suction stroke is less than the delivery pressure $O F$.

If, however, this latter pressure be reduced, as for example to $O E$, the suction pressure becomes equal to this at the point $E^{\prime \prime}$, and for the remainder of the stroke discharge takes place through the delivery valves, the pressure remaining constant and following the line $E^{\prime \prime} E^{\prime}$.

The coefficient of discharge now becomes greater than unity. Its value may be deduced since the area $A^{\prime} E^{\prime \prime} E^{\prime}$ represents the work done in pumping a volume of water $v$ cubic feet against a head $\left(h_{d}=O E\right)$ feet.

$\therefore$ Area $A E^{\prime \prime} E^{\prime}$ represents $62.4 v h_{d}$ foot lbs. The scale to which this area is to be measured is given by the product of the scales to which ordinates of the diagram represent heads in feet and abscissæ represent weights $(62 \cdot 4 \times$ volume in cubic feet).

Taking the work normally done by the piston per stroke (neglecting slip) as $62 \cdot 4 V h_{d}$ where $V$ is the piston displacement, we have:-

$$
\frac{v}{V}=\frac{62.4 v h_{d}}{62 \cdot 4 V h_{d}}=\frac{\operatorname{area} A^{\prime} E^{\prime \prime} E^{\prime}}{\text { area } O O^{\prime} E^{\prime} E}
$$

$\therefore$ Coefficient of discharge $C_{d}=1+\frac{v}{V}=\frac{\operatorname{area} O E^{\prime} L^{\prime \prime} A^{\prime} E^{\prime} O^{\prime}}{\text { area } O E^{\prime} E^{\prime} O^{\prime}}$. 
Assuming simple harmonic motion, the area $A^{\prime} E^{\prime \prime} E^{\prime}$ may be calculated analytically. In more complicated cases, it is preferable to measure it by planimeter.

Where an actual diagram is to be estimated (Fig. 232), the suction head curve $A B E^{\prime \prime}$ may be produced by hand with fair accuracy to $A^{\prime}$, and the area then measured.

Since, at the speed at which separation occurs, water hammer raises the pressure towards the end of the suction stroke by an abnormal amount, it is to be inferred that about this speed a

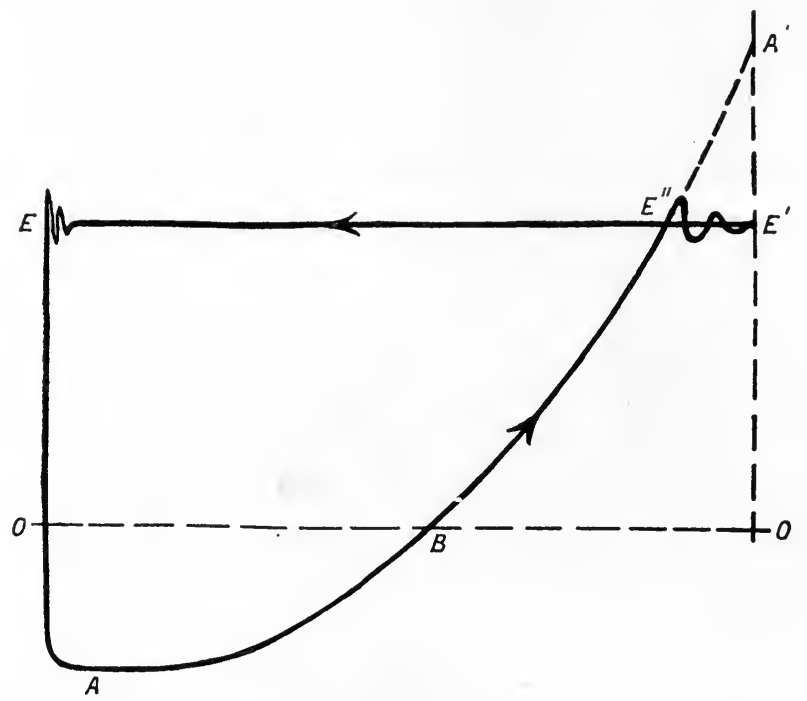

FIG. 232.-Pump Diagram showing delivery during suction stroke.

sudden increase in the discharge coefficient will take place. This inference is justified by the results of experiments by Professor John Goodman, the increase in this coefficient at the knocking speed in his pump, varying from 58 per cent. with a delivery pressure of $10 \mathrm{lbs}$. per square inch to 5 per cent. with a pressure of 70 lbs. per square inch.

Although the capacity of the pump is increased by this increase in the coefficient of discharge, yet, as might be expected, its efficiency is lowered, due to the increased losses by shock. These experiments of Professor Goodman showed that in this particular pump an increase in the discharge coefficient from 1.059 to

H.A. 
$1 \cdot 517$, was accompanied by a decrease of 10 per cent. in the efficiency.

A theoretical pressure diagram for the delivery side of the pump is shown in Fig. 233.

Here, however, the line $A^{\prime} H A$, representing the acceleration

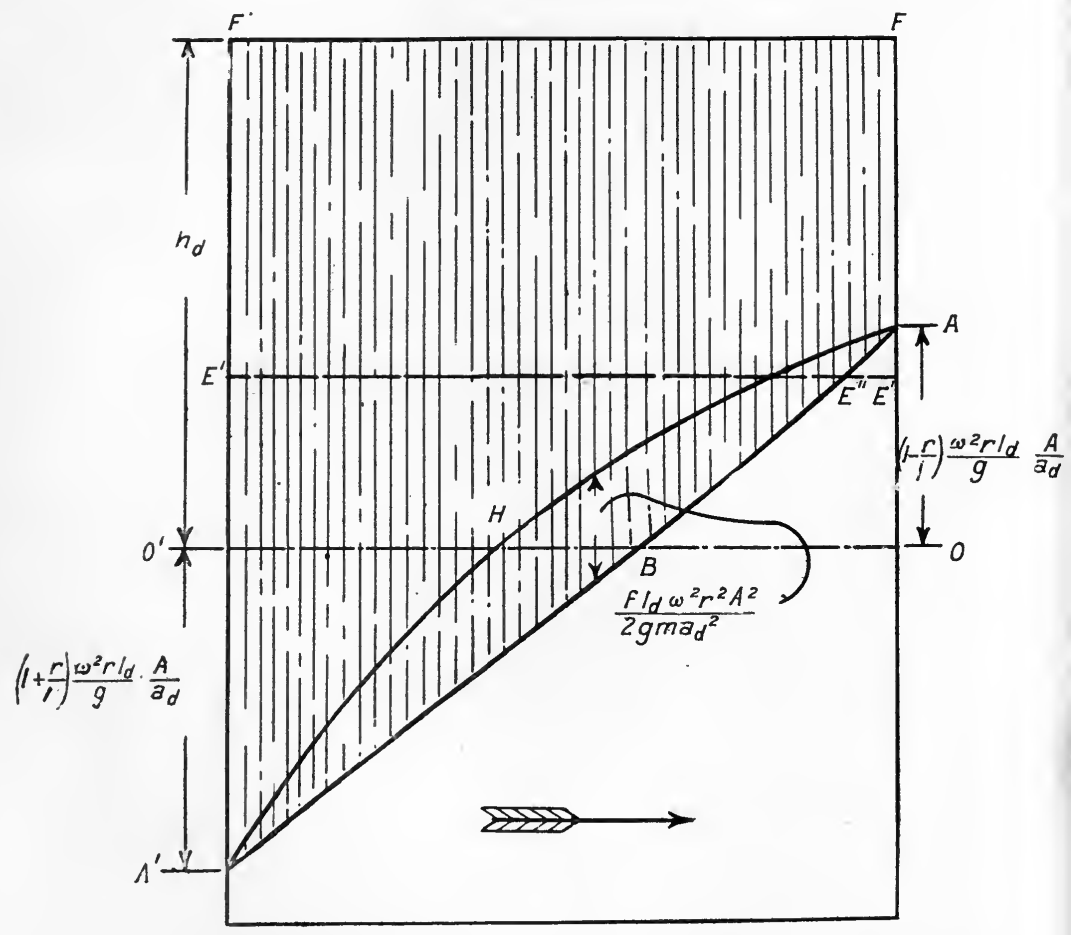

FIG. 233.--Theoretical Diagram of Piston Pressures during Delivery Stroke of Reciprocating Pump.

pressure, has been drawn to take into account the effect of the obliquity of the connecting rod.

Also, since frictional resistances now increase the head on the piston, the friction line $A^{\prime} B A$ is drawn below the acceleration line.

If $O F$ represents the delivery head $h_{d}$, the total head on the piston is given by the ordinates of the curve $A E^{\prime \prime} B A^{\prime}$, measured from $F F^{\prime}$ as datum. 
If $O E$ were to represent the delivery head, the pressure would become negative on passing the point $E^{\prime \prime}$ and the suction valve would open, giving suction on the delivery side of the pump. At high speeds, and with self-closing valves, this valve action becomes very irregular, delivery often occurring, during the major part of the suction stroke and suction during the delivery stroke. $^{1}$

Art. 152.-Rise in Pressure following Separation in a Pump.

During separation the accelerating force on the suction column $=W a_{s}\left\{\pi_{a}-h_{s}-h_{f}-h_{t}\right\}$ lbs., where $h_{f}$ is the head necessary to overcome friction, and therefore depends on the velocity, and $h_{t}$ is the pressure in the cylinder due to vapour tension and to air leakage.

Neglecting the effect of friction, we have :-

Acceleration in pipe line

$$
=\frac{g W a_{s}\left\{\pi-h_{s}-h_{t}\right\}}{W l_{s} a_{s}}=\frac{g\left(\pi_{a}-h_{s}-h_{t}\right)}{l_{s}} \text { f.s.s. }
$$

$\left.\therefore \begin{array}{c}\text { Acceleration of water } \\ \text { surface in cylinder }\end{array}\right)=g \frac{\left(\pi_{a}-h_{s}-h_{t}\right)}{l_{s}} \cdot \frac{a_{s}}{A}$ f.s.s.

If the water overtakes the piston at a distance $x$ feet from the beginning of the stroke, the time taken by the water surface to cover this distance under its constant accelerating force must equal that taken by the piston to cover the same space.

$$
\begin{aligned}
\text { The former of these times is given by } T=\sqrt{\frac{2 x l_{s} A}{g\left\{\pi_{a}-h_{s}-h_{t}\right\} a_{s}}} \\
\text { since, with uniform acceleration. } T=\sqrt{\frac{2 \text { space }}{\text { acceleration }}} .
\end{aligned}
$$

Assuming S.H. motion, the time occupied by the piston to cover the same space is given by $\frac{\theta}{\omega}$ seconds,

where

$$
\begin{gathered}
x=r(1-\cos \theta) \text { (Fig. 234) } \\
\theta=\cos ^{-1}\left(1-\frac{x}{r}\right) .
\end{gathered}
$$

1 For a series of diagrams showing this action reference may be made to Professor Goodman's paper. Proc. Inst. Mech. Engineers, 1903. 
$\therefore$ Equating these times, we have:-

$$
\begin{gathered}
\frac{1}{\omega} \cos ^{-1}\left(1-\frac{x}{r}\right)=\sqrt{\frac{2 x l_{s} A}{g\left\{\pi_{a}-h_{s}-h_{t}\right\} a_{s}}} \\
\therefore 1-\frac{x}{r}=\cos \omega \sqrt{\frac{2 x l_{s} A}{g\left(\pi_{a}-h_{s}-h_{t}\right) a_{s}}},
\end{gathered}
$$

an equation from which $x$ may be obtained by trial. For purposes of calculation $h_{t}$ may be taken as zero. An attempt

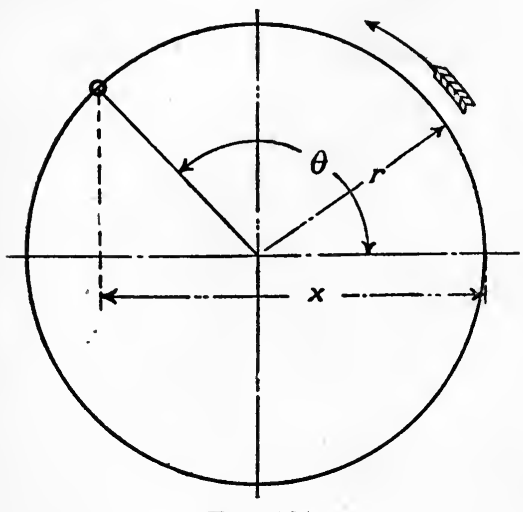

FIG. 234. to include the effects of friction and of a finite connecting rod greatly complieates the result unless $h_{f}$ be taken to be constant and to have a value corresponding to the mean velocity during the period of separation. By this means a close agreement with experimental results is obtained.

The above formula has been applied to the case of a pump described by Professor John Goodman ${ }^{1}$ and having the following dimensions :-

$$
\frac{A}{a_{s}}=1.83 ; l_{s}=63 \text { feet } r=\cdot 25 \text { feet. }
$$

In this particular experiment the pressure in the suction valve chamber was measured, the loss of head due to height of suction, to friction, and to air tension, being approximately 8.0 feet, making-

$$
\left(\pi_{a}-h_{s}-h_{f}-h_{t}\right)=26 \cdot 0 \text {. }
$$

The revolutions per minute were 70 , making $\omega=\frac{2 \pi \times 70}{60}$

$$
=7 \cdot 33 \text {. }
$$

The equation then becomes :-

$$
\cos 7 \cdot 33 \sqrt{\frac{126 \times 1.83}{32 \times 26} \cdot x}=1-4 x
$$

1 Proc. Inst. Mech. Engineers, 1903, part 1 (p. 143). 
or

$\cos 3 \cdot 86 \sqrt{x}=1-4 x$

giving a value of $x=\cdot 47$ feet;

i.e., knock takes place at $\frac{\cdot 47}{\cdot 50}=\cdot 94$ of the stroke.

The indicator diagram, of which Fig. 235 is a copy, show this to occur at about 95 of the stroke.

The velocity of the water column immediately before impact may be deduced from the equation

$$
\text { velocity }=\sqrt{2 \times \text { acceleration } \times \text { space covered under }} \begin{gathered}
\text { f.s. } \\
\text { this acceleration. }
\end{gathered}
$$

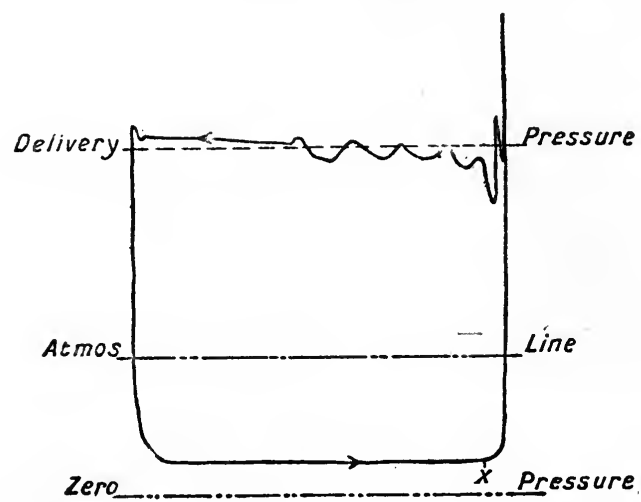

FIG. 23\%.

And since this effective space $=x \cdot \frac{A}{a_{8}}$, this gives us velocity $=\sqrt{\frac{2 g\left(\pi_{i}-h_{s}-h_{f}-h_{t}\right)}{l_{s}} \cdot x \frac{A}{a_{s}}}$ feet per second.

While the piston velocity $=\omega r \sin \theta$

$$
\begin{aligned}
& =\omega r \sqrt{1-\cos ^{2} \theta} \\
& =\omega \sqrt{\left(2-\frac{x}{r}\right) x r} \text { feet per second. }
\end{aligned}
$$

After impact the mean velocity of the supply column becomes equal to that of the piston multiplied by $\frac{A}{a}$. 
$\left.\therefore \begin{array}{c}\text { Change of velocity } \\ \text { at impact }\end{array}\right\}=\left\{\sqrt{\frac{2 g\left(\pi_{a}-h_{s}-h_{f}-h_{t}\right)}{l_{s}} \cdot \frac{x A}{a_{s}}}\right.$

$$
\left.-\frac{A}{a_{s}} \omega \sqrt{x v\left(2-\frac{x}{r}\right)}\right\}
$$

$=r_{x}$ feet per second.

If this change of velocity be assumed to take place instantaneously, the increase of pressure due to water hammer is given by $63 \cdot 7 v_{x}$ lbs. per square inch (p. 214). In the example previously considered, taking $x=\cdot 47$, we have:-

Change of velocity at impact

$$
\begin{aligned}
& =\sqrt{\frac{64 \times 26 \times \cdot 86}{63}}-1.83 \times 7.33 \times \sqrt{\cdot 12 \times \cdot 12} \\
& =4.76-1.61 \\
& =3.15 \text { feet per second. }
\end{aligned}
$$

$\therefore \quad$ Water hammer pressure $=3.15 \times 63.7 \mathrm{lbs}$. per square ir.ch. $=201$ lbs. per square inch.

In addition to this we have the pressure necessary to produce a retardation $\omega^{2} v \cos \theta \frac{A}{a_{s}}$ feet per second per second in the supply column.

This pressure $=-\frac{\omega^{2} v A\left(1-\frac{x}{v}\right) \times W a_{s} L}{a_{s} \times(x)}$

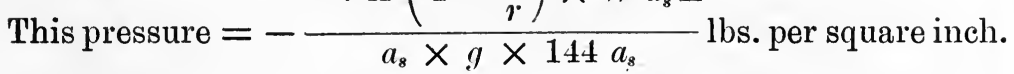

$$
=\frac{(7 \cdot 33)^{2} \times 25 \times 1.83 \times .88 \times 62.4 \times 63}{32 \times 144} .
$$

$=18.5 \mathrm{lbs}$. per square inch.

On taking into account the obliquity of the connecting rod, this becomes $23 \mathrm{lbs}$. per square inch.

The total pressure which may be attained at impact (provided this pressure is not sufficiently great to lift the delivery valve), is then given by the sum of the water hammer and the retardation pressures, and is $219 \mathrm{lbs}$. per square inch.

By exterpolation from the curves showing the results of the experiments under consideration, the actual pressure at this speed would apparently vary from about $115 \mathrm{lbs}$. per square inch with a delivery pressure of $20 \mathrm{lbs}$. per square inch, to about $195 \mathrm{lbs}$. per square inch with a delivery pressure of $60 \mathrm{lbs}$. per 
square inch. This is a result which might have been inferred, since directly the delivery pressure is exceeded, the delivery valve opens, and the maximum pressure is reduced by an amount which cannot be deduced quantitatively.

It is, however, to be expected-and this is verified in practicethat this reduction will be greater as the delivery valve area is increased, and as the delivery pressure is reduced, since the latter reduces the pressure necessary to produce flow through the valves, while the former ensures a more easy delivery.

Art. 153.-EFfect of the Elasticity of the Suction Colums.

While the formulae already obtained enable the pressure on the piston to be calculated with fair accuracy for the greater part of the stroke, it is found that the pressures actually obtained towards the end of the suction stroke, even when separation does not take place, are usually much greater than those calculated in this manner. So far, it will be noted, any effect which the elasticity of the water columns may have in modifying this pressure has been neglected. Because of this elasticity, however, the change of velocity and of pressure at the open end of the pipe will lag behind that at the piston by the time necessary for a pressure wave to traverse the pipe, i.e., by a time $\frac{l}{4,700}$ seconds, and if the acceleration at the plunger end at a given instant be $a$, the difference in velocity at the two ends will be $a \times \frac{l}{4,700}$ f.s.

Thus, assuming S.H. motion, when the piston comes to rest the velocity at the open end will still be $\frac{\omega^{2} v l}{4,700} \cdot \frac{A}{a_{s}}$ f.s., while the mean velocity in the pipe will be half this. In virtue of this velocity the columu possesses a store of kinetic energy which, on stoppage of the piston, is transformed into energy of strain, and which in consequence gives rise to a rapid rise in pressure, of the nature of water hammer.

The action is similar to that which takes place during the closing of a valve at the outlet to a water main, so that (p.220) this rise 
in pressure will occur slightly before the end of the stroke, at an interval of time $\frac{2 l}{4,700}$ seconds, before the piston actually comes to rest. If then $v^{\prime}$ be the velocity and $a^{\prime}$ the acceleration of the plunger at this point, the velocity at the open end of the suction pipe is given by $v^{\prime}+\frac{a^{\prime} l}{4,700}$, and the mean velocity in the pipe by $v^{\prime}+\frac{a^{\prime} l}{9,400}$ f.s. $=\bar{\imath}$. The rise in pressure after this point of the stroke-to be added to the normal retardation pressure-is then given by $63 \cdot 7 \bar{v}$ lbs. per square inch.

For example, in the pump previously considered,

$$
\text { where }\left\{\begin{array}{l}
\omega=4 \cdot 19, \text { i.e., } 40 \text { revolutions per minute, } \\
l=63 \text { feet } ; r=\frac{1}{4} ; \frac{A}{a_{s}}=1 \cdot 83,
\end{array}\right.
$$

the angle which the crank makes with the dead centre position at a time $\frac{2 l}{4,700}$ seconds before the end of the stroke is given by $\theta=\frac{126}{4,700} \times \frac{360^{\circ} \times 40}{60}=6^{\circ} 26^{\prime}$.

In this position the velocity of the piston is $\mathbf{1 1 7 4} \mathrm{f} . \mathrm{s}$., while the acceleration is 4.35 f.s.s. Thus the velocity at the plunger end of the suction line is $1174 \times 1 \cdot 83=\cdot 215 \mathrm{f}$.s., while the acceleration at this point is $4.35 \times 1 \cdot 83=7 \cdot 95$ f.s.s. The mean velocity in the suction pipe is then given by

$$
\cdot 215+\frac{63}{9,400} \times 7 \cdot 95=\cdot 268 \text { f.s. }
$$

and the consequent hammer pressure by

$$
63 \cdot 7 \times \cdot 268=17 \cdot 1 \mathrm{lbs} \text {. per square inch. }
$$

The acceleration pressure as calculated in the ordinary way is equal to $8 \cdot 6 \mathrm{lbs}$. per square inch, so that the total pressure on this assumption amounts to about $25.7 \mathrm{lbs}$. per square inch.

From Fig. 35 of Professor Goodman's paper it appears that the maximum pressure attained increases with the delivery pressure, i.e., with the resistance to opening of the outlet valve, and with the highest delivery pressure there adopted-viz. $60 \mathrm{lbs}$. per square inch-attained a value of $26 \cdot 2 \mathrm{lbs}$. per square inch. This is in close agreement with the result as obtained above. 
With lower delivery pressures, and at the same speed, the total pressure at the end of the stroke had a minimum value of $13.4 \mathrm{lbs}$. per square inch, with a delivery pressure of $14 \mathrm{lbs}$. per square inch.

The effect of the elasticity of the suction column is therefore to modify the shape of the diagram as shown in Fig. 236, where

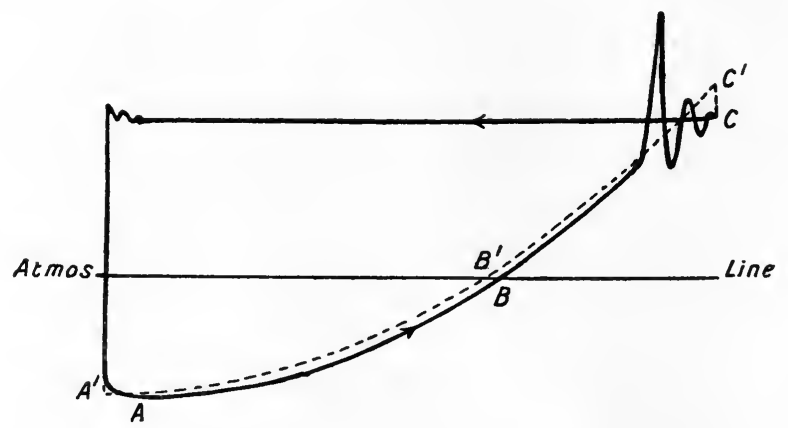

FIG. 236. - Pump Diagram showing the effect of the elasticity of suction Column. Air vessel or Delivery side only.

the dotted line $A^{\prime} B^{\prime} C^{\prime}$ represents the theoretical curve, neglecting the effect of elasticity, and $A B C$ is the actual curve.

In the preceding investigations, the effect of loss of energy due to the sudden enlargement of section of the stream on entering the pump barrel, and to valve resistances, has been neglected. In general, however, this will be comparatively small, except where

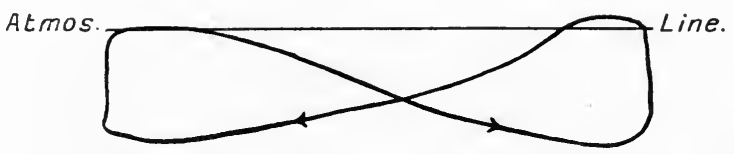

FIG. 23\%. Pressure Diagram from Suction Air Vessel of Reciprocating Pump.

the suction pipe is very short, in which case the difficulties already outlined cease to exist.

Even with a long suction pipe these may be considerably minimised, if not entirely removed, by the provision of an air vessel-or vacuum vessel as it is sometimes termed, since the pressure of the contained air is less than that of the atmosphere 
-on the suction side of the pump. The effect of such an air vessel will now be considered. Its action is as follows :-During the first part of the stroke the pressure behind the piston is reduced and water flows out of the air vessel. The flow along the suction pipe is thus reduced, as is the acceleration of the whole mass of water. This reduces the frictional resistances in the suction pipe, while the pressure behind the piston is increased both on this account and because of the reduced acceleration. At the same time, tendency to separation and to water hammer at the end of the stroke is reduced, if not entirely eliminated; the discharge coefficient becomes unity, or slightly less than unity; and the pump diagram approximates more and more nearly to the rectangle given by a perfect pump, as the sizes of the air vessels are increased.

A typical indicator diagram taken from the suction air vessel of a double-acting pump is shown in Fig. 237.

Art. 154.-Air Vessel applied to a Single or DoubleActing Single Cylinder Pump.

Effect of Air Vessel placed on Suction Side of Pump.

Let $a_{s}, v_{s}$ and $a_{s}$ represent the area of suction pipe between supply reservoir and air vessel, the velocity, and the acceleration in this pipe respectively.

$a_{v}, v_{v}, a_{v}$ represent ditto, in the air vessel itself.

$A, V$, a represent ditto, in the pump cylinder.

$h_{a}$ represent the pressure of air in air vessel, in feet of water.

$\pi$ represent the atmospheric pressure, in feet of water.

$h_{v}=$ height in feet, of water level in air vessel above centre line of pump.

During the first part of suction stroke water will flow out of air vessel into pump, and for continuity of flow we have :-

$$
r_{s} a_{8}+r^{\prime \prime} a_{v}=\mathrm{VA}
$$

$\therefore$ differentiating, we have $a_{s} a_{s}+a_{v} a_{v}=A a$

If, as is usual, the air vessel is placed near to the pump, so that the pressure at the junction of air vessel and suction pipe may be taken as substantially equal to that in the suction 
chamber we have, denoting this pressure by $h_{j}$ feet of water, neglecting frictional losses in the supply pipe, and simply considering the acceleration effect :-

$$
\begin{gathered}
\left(\pi-h_{s}-h_{j}\right) a_{s} W=\frac{a_{s} W l_{s} a_{s}}{g} \\
\therefore \quad h_{j}=\pi-h_{s}-\frac{l_{8} a_{s}}{g}
\end{gathered}
$$

Also, considering the flow down the air vessel, we have:-

$$
\begin{aligned}
& h_{a}+h_{v}-h_{j}=\frac{h_{v} a_{v}^{1}}{g} \\
& \therefore \quad h_{j}=h_{a}+h_{v}-\frac{h_{v} a_{i}}{g}
\end{aligned}
$$

substituting for $a_{v}$ in terms of $a_{s}$ from (1) we get-

$$
h_{i}=h_{a}+h_{v}-\frac{h_{v}}{g}\left\{\frac{A a-a_{8} a_{8}}{a_{v}}\right\}
$$

substituting for $h_{j}$ from (2), this becomes :-

$$
\begin{gathered}
\pi-h_{s}-\frac{l_{s} a_{s}}{g}=h_{a}+h_{v}-\frac{h_{v}}{g}\left\{\frac{A a-a_{s} a_{s}}{a_{v}}\right\} \\
\therefore \quad a_{s}\left\{\frac{a_{s}}{a_{v}} \cdot \frac{h_{v}}{g}+\frac{l_{s}}{g}\right\}=\pi-h_{s}-h_{a}-h_{v}+\frac{h_{v}}{g} \frac{A a}{a_{v}} \\
\therefore \quad a_{s}=g \frac{\left\{\pi-\left(h_{s}+h_{a}\right)+h_{v}\left(\frac{A a}{g a_{v}}-1\right)\right\}}{\frac{a_{s} h_{v}}{a_{v}}+l_{s}}
\end{gathered}
$$

as compared with its value $\frac{A a}{a_{s}}$, without air vessel.

Neglecting, for the time being, the variation in $h_{a}$ and $h_{v}$ with a variation in the piston acceleration $\alpha$, it is evident from (6) that for $a_{s}$ to be affected as little as possible by a variation in $u$, the term involving $a$ must be as small as possible. This indicates that $a_{v}$, the sectional area of the vessel, should be as large, and $h_{v}$ as small as possible-a deduction which is verified in practice.

When the pump is working, the water surface $h_{v}$ undergoes cyclical variations in height, $h_{a}$ assuming corresponding values.

1 This assumes the air vessel to be of uniform diameter to its junction with the suction pipe. If, as is more usual, the junction is made through a smaller pipe of area $a_{1}$, this formula beeomes $h_{a}+h_{v}-h_{j}=\frac{h_{v} \alpha_{1}}{g}$, where $\alpha_{1}=a_{v} \frac{a_{v}}{a_{1}}$. 
The connection between the two may be obtained on the assumption that the air follows the law: pressure $\times$ volume $=$ constant, for if $H_{v}=$ height of top of air vessel above centre line of pump, we have:-

$$
\begin{aligned}
h_{a}\left\{H_{v}-h_{v}\right\} & =\text { constant }=K \\
\therefore \quad h_{v} & =H_{v}-\frac{K}{h_{a}} \\
\text { or } h_{a} & =\frac{K}{H_{v}-h_{v}}
\end{aligned}
$$

The mean height $h_{v}$ may be adjusted by admitting more or less air into the air vessel, and this adjustment should be made until $h_{v}$ is as low as is consistent with no air being drawn over into the suction chamber as the level varies.

With a single-acting pump, the total cyclical variation in volume in the air chamber is about 56 of the piston displacement per stroke.

Since this fluctuation in level diminishes with an increase in cross-sectional area, an increase in $a_{v}$ has the further advantage that it permits the mean working value of $h_{v}$ to be reduced.

Again, substituting in (6) from (8), it appears that while the fluctuation in $a_{s}$ corresponding to a given variation in $l_{v}$, diminishes as $H_{v}$ increases, yet this effect is small compared with that of an increase in $a_{v}$. In effect, then, the area of the vessel is of much greater importance than its length, and for a given volume, the ratio $\frac{\text { area }}{\text { length }}$ should be as large as possible.

Example.

$$
\begin{array}{llrl}
A & =1 \cdot 0 \text { square foot. } & & l_{s}=30 \text { feet. } \\
a_{s}=25 \text { square foot. } & h_{s}=6 \text { feet. } \\
a_{v}=1.00 \text { square foot. } & H_{v}=4 \text { feet. }
\end{array}
$$

Length of stroke $=1 \cdot 0$ foot. $\quad$ No. of revolutions $=100$ per min.

When the pump is standing let $h_{v}=6$ inches. We then have $h_{a}=(34-10)=24$ feet absolute head,

$$
\begin{gathered}
\text { so that } 24\left\{4-\frac{1}{2}\right\}=\text { constant }=K \\
\therefore \quad K=84 .
\end{gathered}
$$

Assuming the total fluctuation of volume in the air vessel to 
be equal to 56 times the delivery per stroke, this gives a total fluctuation equal to 56 cubic feet, and therefore a fluctuation in level of 56 feet.

The acceleration in the supply pipe corresponding to any value of the piston acceleration may now be obtained from equation (6).

$$
\text { Thus } a_{s}=\frac{32 \cdot 2\left\{34-6-\frac{84}{4-h_{v}}+h_{v}\left(\frac{a}{32 \cdot 2}-1\right)\right\}}{\cdot 25 h_{v}+30} \text { f.s.s. }
$$

It only remains to substitute for $h_{v}$, and if, as indicator diagrams show to be usually the case, we assume that this has its minimum value shortly after the piston begins its suction stroke, we may obtain the acceleration at this point by putting $h_{v}=[\cdot 5-\cdot 28]=\cdot 22$ feet in the above expression, and by writing

$$
\begin{aligned}
a & =\frac{4 \pi^{2} \times 100^{2}}{3,600} \times \frac{1}{2}=54 \cdot 8 \text { f.s.s. } \\
\text { Then } a_{s} & =\frac{32 \cdot 2\left\{28-\frac{84}{3 \cdot 78}+\cdot 22\left(\frac{54 \cdot 8}{32 \cdot 2}-1\right)\right\}}{30 \cdot 055} \\
& =6 \cdot 35 \text { f.s.s. }
\end{aligned}
$$

as compared with its value $\omega^{2} r \times 4=219 \cdot 3$ f.s.s. without air vessel.

The maximum acceleration will in general be found to occur at about $\cdot 2$ of the suction stroke.

Evidently a further increase in the size of air vessel, or an increase in the length of suction pipe will reduce the value of $a_{s}$ still further, and with a suction pipe of any considerable length its value approximates very sensibly to zero. In such a case the flow along the pipe is sensibly constant, and the velocity is equal to the discharge in cubic feet per second divided by the area of the pipe. If this assumption be made, calculations relating to the necessary size, etc., of the air vessel are considerably simplified, as willı be shown later.

Modifying Effect of Friction and Kinetic Losses in Suction Pipe.-Taking the total difference of head between supply reservoir and piston as being given by

$$
\frac{v_{s}{ }^{2}}{2 g}\left(1+\frac{f l_{s}}{m}\right)+\frac{l_{s} a_{s}}{g} \text { feet, }
$$


this may be written as :-

$$
\begin{gathered}
\frac{a_{s}}{g}\left\{\frac{r}{2} \frac{\sin 2 \theta}{\cos \theta} \cdot \frac{A}{a_{s}}\left(1+\frac{f^{\prime} l_{s}}{m}\right)+l_{s}\right\} \text { feet. } \\
\text { Since } a_{s}=\omega^{2} r \cos \theta \frac{A}{a_{s}} \\
\text { and } \left.v_{s}^{2}=\omega^{2} r^{2} \sin ^{2} \theta \frac{A^{2}}{a_{s}^{2}}=a_{s} r \frac{\sin ^{2} \theta}{\cos \theta} \cdot \frac{A}{a_{s}}\right) \\
\therefore \pi-h_{s}-h_{j}=\frac{a_{s}}{g}\left\{l_{s}+B \frac{\sin ^{2} \theta}{\cos \theta}\right\} \text { feet. }
\end{gathered}
$$

The preceding equations now become :-

$$
\begin{aligned}
& h_{j}=\pi-h_{s}-\frac{a_{s}}{g}\left\{l_{s}+B \frac{\sin ^{2} \theta}{\cos \theta}\right\} \\
& h_{j}=h_{a}+h_{r}-\frac{h_{v} a_{v}}{g}
\end{aligned}
$$

(neglecting frictional losses in air vessel as being comparatively small).

On substituting for $\alpha_{v}$ in ( $\left.3^{\prime}\right)$ in terms of $\alpha_{s}$ from (1), as before, we have, equation (4) :-

$$
h_{j}=h_{a}+h_{v}-\frac{h_{v}}{g}\left\{\frac{A a-a_{s} a_{s}}{a_{v}}\right\}
$$

and on substituting in this equation for $h_{j}$ from $\left(2^{\prime}\right)$ we get, on reduction :-

$$
a_{s}=\frac{g\left\{\pi-\left(h_{s}+h_{a}\right)+h_{v}\left(\frac{A a}{g a_{v}}-1\right)\right\}}{l_{s}+\frac{a_{s} h_{v}}{a_{v}}+B \frac{\sin ^{2} \theta}{\cos \theta}}
$$

By substituting in equation $\left(4^{\prime}\right)$ for $a_{s}$ from $\left(2^{\prime}\right)$ we get, on reduction :-

$$
h_{j}=\frac{\pi-h_{s}+\frac{a_{v}\left(l_{s}+B \frac{\sin ^{2} \theta}{\cos \theta}\right)}{h_{v} a_{s}}\left[h_{c}+h_{v}-\frac{h_{v} A a}{a_{v} g}\right]}{1+\frac{a_{v}}{h_{v} a_{s}}\left\{l_{s}+B \frac{\sin ^{2} \theta}{\cos \theta}\right\}} \text { feet. }
$$

from which, on assuming values for $h_{v}$, a curve similar to Fig. 231, showing the pressure on the piston for a series of values of $\theta$ may be plotted.

In practice, with single-barrel double-acting pumps, suction 
air vessels of from 1.0 to 3.0 times the capacity of the pump per revolution are found to give good results, the necessary volume increasing with the rotative speed of the pump and with the length of suction pipe.

\section{Art. 155.-Air Vessei on Delivery Side of Pump.}

Here exactly the same reasoning applies, except that now flow into the air vessel will take place during the first half of the delivery stroke, and flow out of the vessel during the second half when the water column in the delivery pipe is being retarded.

Considering the latter part of the stroke we have, for continuity of flow :-

$$
\begin{aligned}
a_{d} r_{d}-a_{v} r_{r}=A V_{r} \\
\therefore \quad a_{d} a_{d}-a_{v} a_{v}=A \quad a .
\end{aligned}
$$

Also $h_{d}$ now corresponds to $\left(h_{s}-\pi\right)$ in the case of the suction vessel.

$\therefore$ equation (2) becomes :-

while (3) becomes :-

$$
h_{j}=h_{d}+\frac{l_{d} a_{d}}{!}(\text { neglecting friction }) ;
$$

$$
\begin{gathered}
h_{j}-h_{c}-h_{a}=\frac{h_{v} a_{r}}{!} \\
\therefore \quad h_{d}+\frac{l_{d} a_{d}}{g}=h_{v}+h_{a}+\frac{h_{v} a_{v}}{g} \\
=h_{r}+h_{a}+\frac{h_{v}}{g}\left\{\frac{A a-a_{d} a_{d}}{a_{v}}\right\} \\
\therefore \quad a_{d}=\frac{g\left\{h_{v}+h_{a}-h_{d}+\frac{h_{v}}{g} \frac{A a}{a_{v}}\right\}}{\left\{l_{d}+\frac{a_{d} h_{v}}{a_{v}}\right\}}
\end{gathered}
$$

But without air vessel, so long as separation does not occur' we have :-

$$
\begin{aligned}
a_{d}^{\prime} & =a \frac{A}{a_{d}} \\
\therefore \quad a_{d}^{\prime}-a_{d} & =\frac{1}{l_{d}+\frac{a_{d}}{a} h_{v}}\left\{\frac{A a l_{d}}{a_{d}}-g\left(h_{d}-h_{a}-h_{v}\right)\right\}
\end{aligned}
$$


An examination of this equation indicates that the change in the retardation in the delivery pipe, and therefore in the pressure in the pump, due to the provision of the chamber, increases with an increase in $a_{v}$, and since $h_{v}$ is in general small compared with $h_{d}$, diminishes with an increase in $h_{r}$. It follows that the mean level in the air vessel should be reduced to the lowest practicable limit, and that $h_{a}$ should be as large as practicable, i.e., the air supply should be maintained so as to keep the water-level as low as is consistent with the air vessel still containing some water, at the end of the delivery stroke.

The modifying effect of frictional resistances may be examined as in the case of the suction air vessel. In general, owing to the fact that the delivery head is much greater than the suction head, and that the water at high pressure is able to dissolve an increased volume of air, the air in the vessel is gradually absorbed, so that either some device must be fitted for renewing the supply, or an air vessel of such dimensions must be fitted as will enable the mean level to be maintained fairly constarit over long periods of working. In practice, with a single-barrel doubleacting pump, the delivery air vessel has usually a volume equal to from six to nine times the pump displacement per revolution, depending on (increasing with) the speed of rotation and the length of delivery pipe. Here, again, an increase in the area of the vessel is of greater advantage than an increase in its length.

\section{ART. 156.}

If it be assumed that the velocity of flow along the suction and delivery pipes is sensibly uniform, and equal to the mean velocity of the pump plunger, multiplied by the ratio of areas of plunger and pipe, the volume of water entering the air chamber per stroke may be determined with fair accuracy. Thus, if the ordinates of the displacement curves $A B C D E$ (Fig. 238) represent piston velocities, and if $A F$ represent the mean piston velocity, on multiplying the vertical scale by $\frac{A}{a_{d}}$, the curve would give the velocities of flow along the delivery pipe if no air vessel were fitted, $A F^{\prime}$ measuring the mean velocity. 
Thus the ratio of each of the sectioned areas to the whole area $A F G E$ gives the ratio of the volume entering the air vessel per cycle to the total discharge per revolution. 'This applies to a double-acting pump. In a single-acting pump the volume

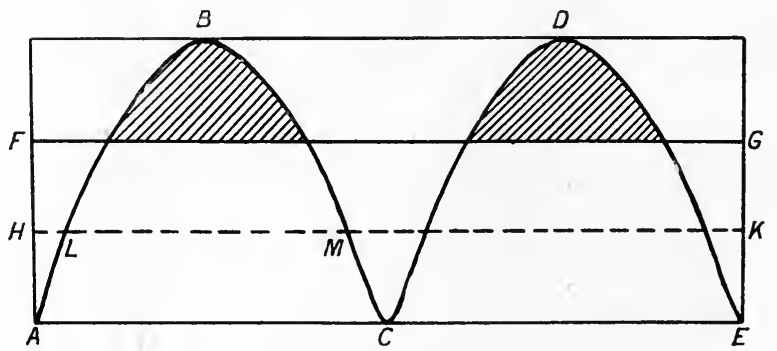

FIG 238.

discharged will be given by the area $A H K E$, where $A H=\frac{1}{2} A F$, while the volume entering the air vessel will be given by the area $L B M$. In the case of a double-acting duplex pump, having cranks at right angles, Fig. 239 represents the state of affairs. Here the curve $D^{\prime} K B L B^{\prime}$, etc., formed

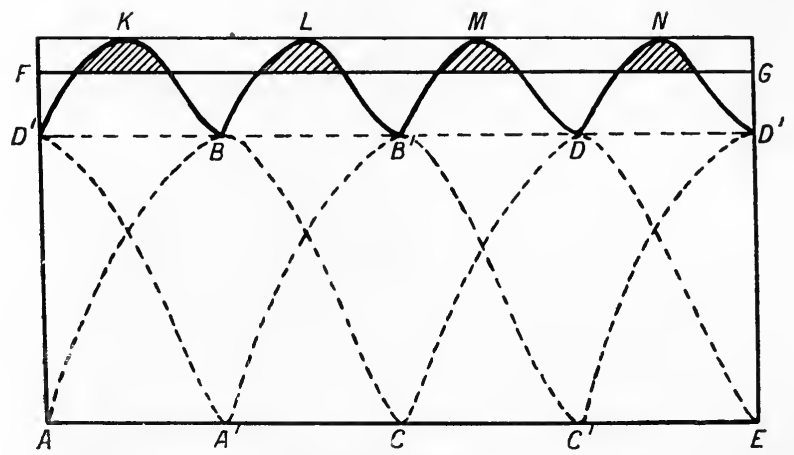

FIG 239.

by adding the ordinates of the two single velocity curves $A B C, A^{\prime} B^{\prime} C^{\prime}$, etc., gives the velocity of the combined discharge, without air vessel. The area $A E G F$ represents the discharge per revolution, and each of the sectioned areas represents to the same scale the volume entering and leaving the air vessel per cycle. 
These areas may be calculated or measured by planimeter. The following table gives the proportion of the whole discharge per revolution which enters and leaves the air vessel per cycle.

\begin{tabular}{|c|c|c|}
\hline \multirow{2}{*}{ Type of Pump. } & \multicolumn{2}{|c|}{$\begin{array}{c}\text { Proportion of Water entering the Air Vessel } \\
\text { per Cycle. }\end{array}$} \\
\hline & $\begin{array}{l}\text { Assuming an infinitely } \\
\text { Long Connecting Rod. }\end{array}$ & 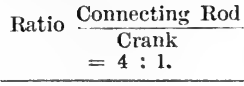 \\
\hline Single-acting pump & $• \check{\jmath}$ & $\cdot 562$ \\
\hline $\begin{array}{l}\text { Two single - acting pumps with } \\
\text { cranks at right angles. }\end{array}$ & $3 e^{\circ}$ & $\cdot 365$ \\
\hline Double-acting pump & $\cdot 10 \check{z}$ & $\cdot 115$ \\
\hline $\begin{array}{l}\text { Two double - acting pumps with } \\
\text { cranks at right angles. }\end{array}$ & $\cdot 0105$ & .0106 \\
\hline Three-throw pump with $\left\{\begin{array}{c}\text { Single- } \\
\text { acting }\end{array}\right\}$ & $\cdot 0109$ & $\cdot 0111$ \\
\hline cranks at $1 \because 0^{\circ} \quad\left\{\begin{array}{c}\text { Double- } \\
\text { acting }\end{array}\right\}$ & $\cdot 0029$ & $\cdot 003$ \\
\hline
\end{tabular}

For satisfactory working the volume of the delivery air vessel should vary from forty to sixty times the volume of water entering it per cycle, this proportion increasing with the speed of rotation and the length of delivery pipe.

On the suction side the volume should be from ten to thirty times the volume entering per cycle, this proportion also increasing with the speed and with the length of suction pipe.

To summarise: An air vessel on the suction side of a pump reduces the maximum acceleration and the range of velocities in the supply pipe, and thus, besides reducing friction losses, reduces the fluctuations of pressure in the suction chamber, and therefore the liability to water-hammer or separation. The steadying effect becomes more pronounced as $A, a, l_{s}$ are increased and less pronounced as $a_{s}$ is increased. The larger the volume of the chamber and the greater its effect, an increase of sectional area being of more value than an increase in length. Also, for efficient working, the water-level in the air vessel should be adjusted by adjusting the air pressure, until as low as is consistent with water remaining in the vessel during the first part of the suction stroke. For the air vessel to be as effective as possible in preventing shock it should be so situated as to provide an effective air 
cushion to the entrant water. Fig. $241 a, b$, and $c$, shows various methods of applying the air vessel. Of these (1) is very little good in preventing shock. (b) is good except that the provision of a right-angled bend at the entry to the suction chamber is inadvisable. The best method is that illustrated at $(c)$, since here the air vessel is in a direct line with the suction

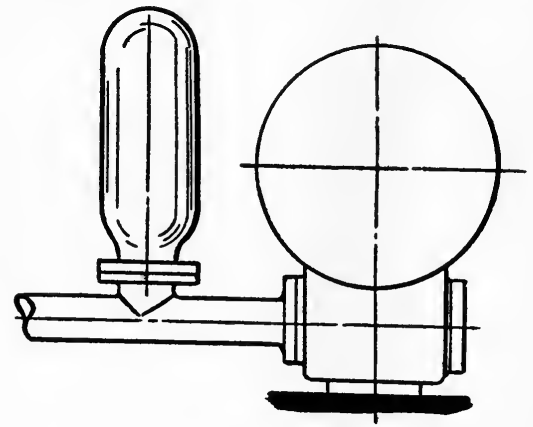

(a)

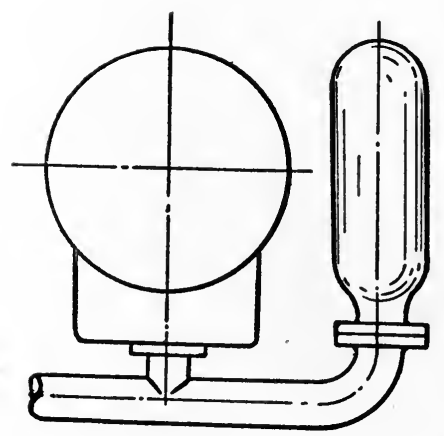

(b)

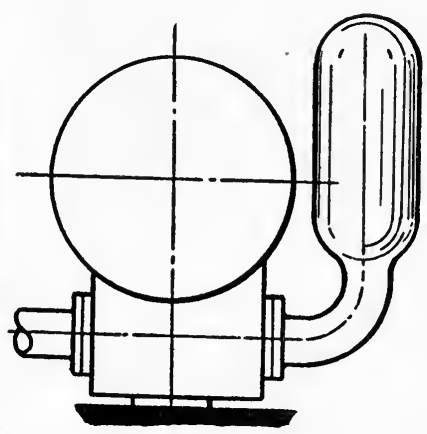

(c)

Fig. 240.-Suction Air Vessels for Reciprocating Pumps.

pipe, while discharge either from the air vessel or supply pipe takes place directly into the suction chamber without the intervention of bends or elbows of any description. It may be premised that the air vessel should always be placed so that a sudden retardation of the suction column may produce a direct flow into the vessel, without the intervention of any acute bends or contracted passages. 
Precisely the same reasoning applies to the delivery air vessel, and here again, for efficient working, the water-level should be adjusted by adjusting the air pressure until as low as is consistent with water remaining in the vessel throughout the whole cycle.

\section{Art. 157.-Air-chaliging Devices.}

One of the simplest devices for maintaining the level in an air vessel is the air injector of Messrs. Wippermann and Lewis.

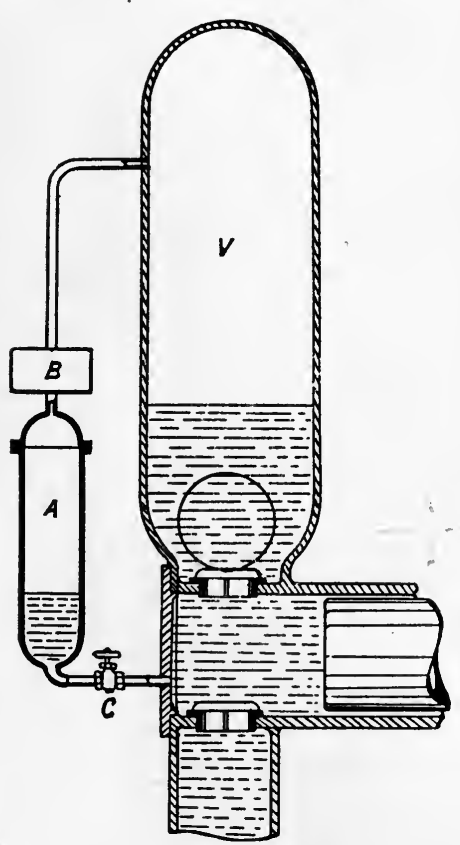

FIG. 241.-Wippermann Air Injector.

In this apparatus, which is represented diagrammatically in Fig. 241, the small cylindrical chamber $A$ is connected to the pump barrel by means of a small pipe with regulating cock, and at its upper end carries a valve box $B$ containing air inlet and outlet valves, which is in turn connected to the air space of the air vessel $V$.

On the suction stroke in the main pump this chamber is partially emptied, and air is drawn in through the inlet valve. On the delivery stroke of the pump, water is forced into the chamber $A$ and its contained air is driven through its outlet valve into the air vessel $V$.

The amount of water entering and leaving $A$ per cycle, and therefore the air entering $V$, may be accurately adjusted by means of the regulating cock $C$.

Another simple device consists of a small compound air pump, worked by the main pump shaft (p. 563), which draws air out of the suction chamber, where it tends to accumulate, and pumps it into the delivery chamber. 
Art. 158.-Efficiency of the Reciprocating Pump.

When dealing with considerable pressures, the reciprocating pump if well designed and working at a fairly slow speed is capable of an efficiency of up to about 90 per cent.

Since a large proportion of the loss is due to mechanical friction and is therefore approximately independent of the pressure, this efficiency falls off rapidly as the working head is reduced, so that in general, for heads below about 40 feet, the centrifugal pump becomes the more efficient. The piston pump, however, has the advantage of being positive in action, is not so liable as the centrifugal pump to lose its water, and has an efficiency which, to a larger extent than in the ease of the latter type, is independent of speed.

\section{Art. 159.-Positive Rotary Pumps.}

This type of pump forms the connecting link between the centrifugal and the reciprocating piston pump. Like the former,

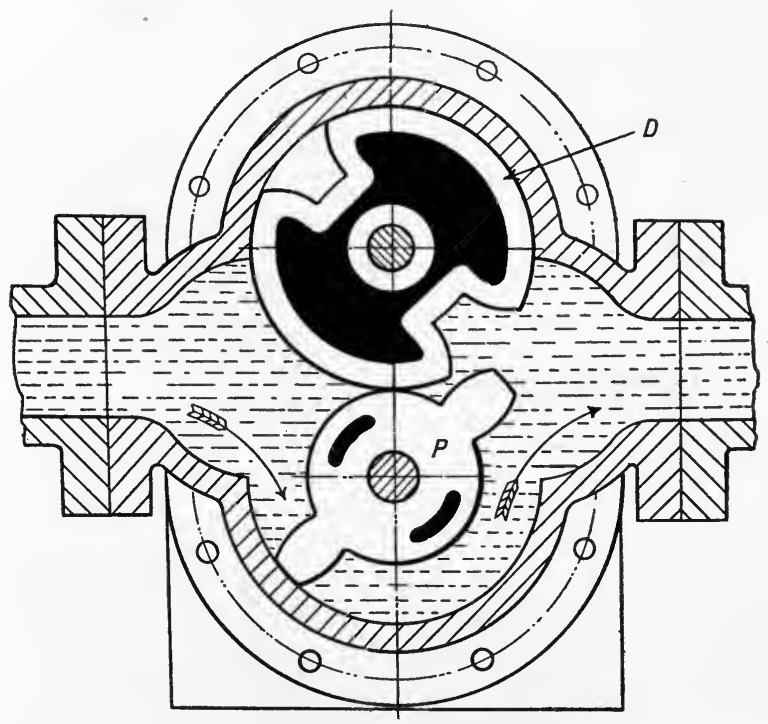

Fig. 242,-Drum Pump.

its motion is rotary and its delivery practically continuous and free from vibration, while, like the latter, its action is positive and it 
will work well over a large range of speeds. Its great drawback lies in the difficulty of keeping the rotating pistons tight against each other and against the pump casing, the wear which invariably occurs leading to considerable leakage and loss of efficiency.

In spite of this, the convenience of the method of driving, the fact that no valves are required, and the steadiness of working, render the pump valuable in many instances, and more particularly where viscid liquid is to be handled. One of the best of this type, the Drum Pump, is illustrated in section in Fig. 242. ${ }^{1}$ Here $P$ is the revolving piston, which carries two projecting ribs, these gearing into suitable slots on the revolving drum $D$. The piston and drum are geared together by external gear wheels, and at each rotation of the piston a volume of liquid $=2 \pi R A$ cubic feet is delivered, where $R=$ mean radius of the projecting rib and $A=$ its projected area on a diametrical plane. The pump is built in sizes to deliver up to about 700 gallons per minute.

\section{Exanples.}

(1) Sketch the displacement curve for a five-cylinder singleacting pump having cranks at $72^{\circ}$ and, assuming zero acceleration in the pipe line, determine the proportion of the delivery per revolution which enters the air vessel per cycle.

Answer. $\cdot 0013$.

(2) Assuming no air vessels, determine the critical speeds at which separation will occur on the suction and delivery sides respectively of the following pump :-

$\left\{\begin{array}{l}\text { Cylinder diameter } \\ \text { Cylinder stroke } \\ \text { Diameter of suction pipe }=18 \text { inches. } \\ \text { Diameter of delivery pipe }=4 \text { inches. }\end{array} \quad\left\{\begin{array}{l}\text { Length of connecting rod }=3 \text { feet. } \\ \text { Length of suction pipe }=40 \text { feet. } \\ \text { Length of delivery pipe }=250 \text { feet. } \\ \text { Suction lift } \\ \text { Delivery lift }\end{array}\right.\right.$

Answer. $\left\{\begin{array}{l}\text { On suction stroke } 21 \cdot 7 \text { revolutions per minute. } \\ \text { On delivery stroke } 20 \cdot 7 \text { revolutions per minute. }\end{array}\right.$

(3) Assuming the pump of example (2) to run at forty revolutions per minute, determine at what point in the stroke shock will take place, and assuming all connections, etc., to be rigid, and

1 By courtesy of the Drum Engineering Company, Bradford. 
neglecting the effect of the opening of the delivery valves, determine the maximum hammer pressure then produced

Answer. $99 \cdot 3$ per cent. of stroke.

Hammer pressure 524 lbs. per square inch.

(4) Determine the discharge coefficients for a pump of 6-inch stroke, having a ratio $\frac{\text { connecting rod }}{\text { crank length }}=\frac{4}{1}$; a ratio $\frac{\text { piston area }}{\text { suction pipe area }}=1.83$; length of suction pipe $=63$ feet, when running at fifty revolutions per minute, and when the delivery pressure is respectively

(a) 5 lbs. per square inch.

(b) $10 \mathrm{lbs}$. per square inch.

Also $(c)$ for the same pump when fitted with a suction pipe 36 feet long and when running at sixty revolutions per minute against a head of 11.5 feet.

$$
\begin{aligned}
& \text { Answer. (a) • . 1.15 } \\
& \text { (b) . . 1.01 } \\
& \text { (c) . . 1'10. }
\end{aligned}
$$

(5) A double-acting pump, cylinder diameter 10 inches, stroke 18 inches, is fitted with an air vessel on the suction side 15 inches diameter, and 4 feet 6 inches long measured from its point of junction with the suction chamber. The length of suction pipe is $\mathbf{1 5 0}$ feet, its diameter is 5 inches, the suction lift, measured to the level of the suction chamber, is 10 feet, and air is admitted to the vessel until the mean working level is 4 feet from the top, the fluctuation in level being 2 inches. Determine the acceleration in the suction column at the beginning of the outstroke-ratio of connecting rod to erank length being $4: 1$ when running at eighty revolutions per minute.

$$
\text { Answer }\left\{\begin{array}{l}
h_{a}=23 \cdot 5 \text { feet. } \\
d_{s}=\cdot 32 \text { f.s.s. }
\end{array}\right.
$$




\section{CHAPTER XVI.}

Centrifugal Pumps-Theory-Types and Construction-Balancing of End Thrust.

\section{Art. 160.-Centrifugal Pumps.}

UNDER low heads the efficiency of the reciprocating pump falls off considerably, and when in addition very large quantities of water are to be handled its excessive dimensions render it expensive to construct and to instal. When working against a variable head, it also suffers from the disadvantage that its speed cannot be increased to any large extent to enable it to deliver a larger quantity of water as the head is reducedd.

Under such conditions, with heads ranging from about 6 feet to 45 feet, the centrifugal pump having a single impeller is on all accounts most suitable, giving as it does a good efficiency-up to about 75 per cent. in the modern type of pump-along with moderate dimensions, simple construction, ease of installation and maintained high efficiency under continuous working conditions.

In the latter respect its freedom from valves gives it an advantage over the reciprocator, whose valves, glands, and packing rings need to be frequently overhauled if the efficiency is to be maintained, this advantage being still more pronounced where the liquid pumped contains gritty matter in suspension.

A further advantage is involved in its continuous and even discharge and the consequent freedom from shock in the delivery pipe line.

The invention, in 1875, by Professor Osborne Reynolds, of the modern form of high lift pump, having an efficiency equal to that of the old type of low lift pump, opened out a new field of application for the centrifugal pump, and of recent years this has been to an increasing extent invading the province of the reciprocator, its efficiency increasing with improvements in design, 
until at the present time heads of upwards of 1,500 feet may be overcome with efficiencies of from 75 to 80 per cent. In a pump designed for such work the water passes through a sequence of impellers mounted in series on the same shaft, the increase in head taking place in stages.

A further advantage of the centrifugal pump consists in the possibility of adapting it to the high speeds of rotation common in the case of the electric motor or steam turbine, its even torque rendering it particularly well fitted for such a purpose, and the general tendency towards the extended use of such motors, has of recent years given a great impetus to the development of the pump.

The extent of this adaptability may be realised from experiments by M. Ratean, ${ }^{1}$ who, using a pump having a single impeller of $3 \cdot 15$ inches diameter, and rotating at 18,000 revolutions per minute, obtained an efficiency of approximately 60 per cent. when pumping against 863 feet head.

Indeed, to such an extent have the possibilities in design responded to the demands made upon them in recent years, that the centrifugal pump promises to replace the reciprocator to an ever-increasing extent in every class of work, except where the volume of water to be handled is very small in comparison with the working head.

For mine drainage, the motor-driven centrifugal has the advantage of requiring no expensive emplacements; while for elevator work this motor-driven pump, with an automatically controlled rheostat to regulate the discharge, has an advantage in that since the delivery pressure cannot increase to any large extent, no bye-pass is required. With a reciprocating pump this bye-pass is essential and involves a loss of energy by leakage.

Applied to dredging operations, the centrifugal pump is capable of removing sand, gravel, or clay broken up by a water jet or mechanical agitator, the amount of solid varying up to $30 \mathrm{per}$ cent. of the volume handled. With a mixture containing 15 per cent. of gravel, efficiencies of about 45 per cent. may be attained.

In its essentials the pump may be looked upon as a reversed

1 Engineer, March 7th, 1902 (p. 231). 
inward radial or mixed flow turbine, having the wheel vanes driven in the opposite direction to that of rotation in the case of the turbine, and discharging outwards.

Supposing the pump to be filled, as the wheel rotates a forced vortex is produced in the contained water, with a consequent increase of pressure in an outward radial direction and a tendency to outward flow. If the speed of rotation is sufficiently high, this increase in pressure becomes more than sufficient to balance the statical pressure of the delivery head and flow takes place. A partial vacuum is thus produced at the centre of the wheel and water is forced up through the supply pipe by atmospheric pressure to take the place of that discharged outwards by centrifugal action. The pump will, however, not begin to lift unless first filled with water, so that where placed above supply water level, provision must be made for priming or flooding the pump casing from an auxiliary pressure supply.

\section{Art. 161.-Losses in the Centrifugal Pump.}

The losses of head in the pump, while being similar in nature to those occurring in a turbine, are very different in relative magnitude. As in the turbine, we have losses due to shock on entering the vanes, to frictional resistances in the wheel itself, and to rejection of kinetic energy in the discharge. 'The latter source of loss, while being comparatively unimportant in the turbine, becomes of the greatest importance in the pump, since it is when leaving the wheel that the water is moving with its maximum velocity. Various devices are adopted to reduce this loss. In the first place, the absolute velocity of discharge may be reduced by curving the vanes backward at the tips, so as to discharge the water as nearly as possible in the opposite direction to that of the wheel's rotation. In Fig. 243 the triangles of velocity are drawn for the cases in which the vanes are respectively radial, curved forward at exit, and curved backward at exit. In each case the same value has been adopted for $f_{3}$, the velocity of flow at exit, and for $u_{3}$ the peripheral velocity of the vanes, while $\overline{a c}$ represents the direction and the absolute velocity of discharge.

A comparison of the diagrams will indicate how this diminishes as the angle of backward inclination of the vane tips increases. 
As will be seen later, however, the necessary peripheral speed of the wheel for pumping against a given head increases with the backward inclination of the vanes, and this causes the frictional losses, which vary approximately as $\omega^{3}$, to increase.

This backward curvature of the vanes offers a further advantage in that it gives passages of more uniform cross section. This reduces the divergence, and thus the tendency to instability of flow, which is one of the most important factors in reducing

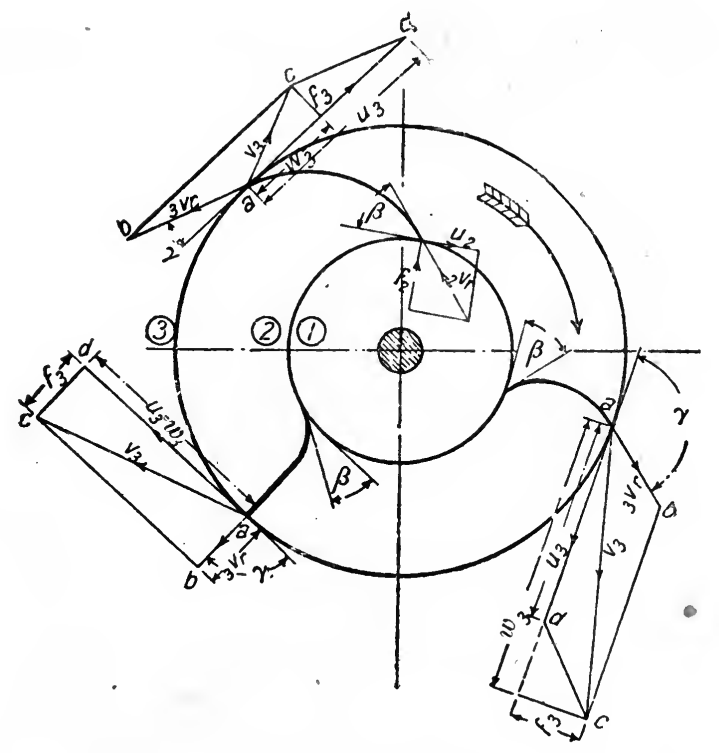

Fig. 243.-Velocity Diagram for Vanes of a Centrifugal Pump.

the efficiency of the pump as compared with the invard flow turbine. Since the relative velocity of flow increases outwards, the passages would in all probability be most effective if designed so as to be convergent outwards to suit this increased relative velocity. This view is borne out by the results of a series of experiments carried out by $\mathrm{Mr}$. J. A. Smith, of Melbourne, ${ }^{1}$ in which instantaneous photographs of the flow through the impeller of a pump which was freely discharging into the atmosphere, indicate that at a certain velocity the water tends to leave the 
leading face of a passage as indicated in Fig. 244, which is reproduced from his paper. If discharging under pressure it is evident that the empty space shown in these passages would be occupied by dead water and would be the source of considerable loss in eddy production. The successive curves 1,2 , and 3 , mark the boundaries of the stream with increasing velocities.

In practice, the purpose for which the pump is designed determines the amount of the backward curvature, which may have any value from $0^{\circ}$ to $75^{\circ}$, so that in general the water on leaving the vanes has a comparatively high absolute velocity.

The kinetic energy which the water possesses in virtue of this velocity may be entirely wasted, or may be partially converted
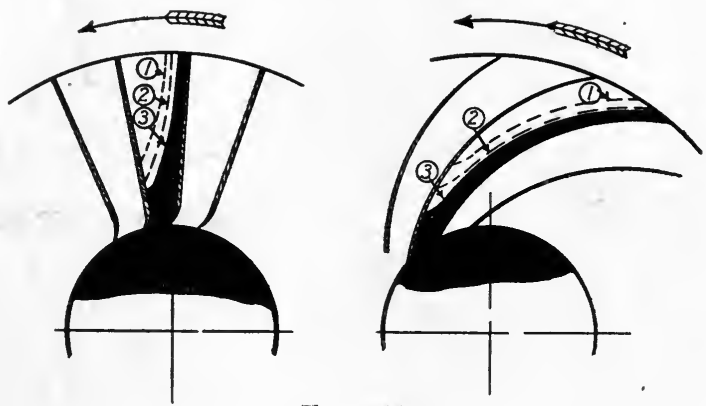

FIG. 244.

into pressure energy (the only form in which it is useful in a pump) according as to how the discharge is collected and led into the discharge pipe.

The most unsatisfactory manner of doing this is to allow the water to discharge into a small chamber surrounding, and concentric with the wheel, out of which chamber the discharge pipe is led (Fig. 245). Since each of the vanes is continuously discharging, the quantity passing a section of the collecting chamber, will increase continuously from a section at $A$ just past the discharge pipe, to be a maximum at the section $B$ just before reaching this pipe. The velocity of whirl in this chamber will thus vary from $A$ to $B$, and can only at one particular section correspond with that at discharge. The result is, that practically the whole of the kinetic energy at discharge is dissipated in shock and eddy production. 
This may be avoided to a certain extent by designing the chamber so as to have a sectional area which increases uniformly from $A$ to $B$, a cutwater being placed as shown at $A$ (Fig. 246), so as to ensure the whole flow being at once discharged from the chamber. ${ }^{1}$ This is termed a volute chamber and is usually so designed as to give a uniform velocity of whirl in the chamber, equal to that of flow in the discharge pipe.

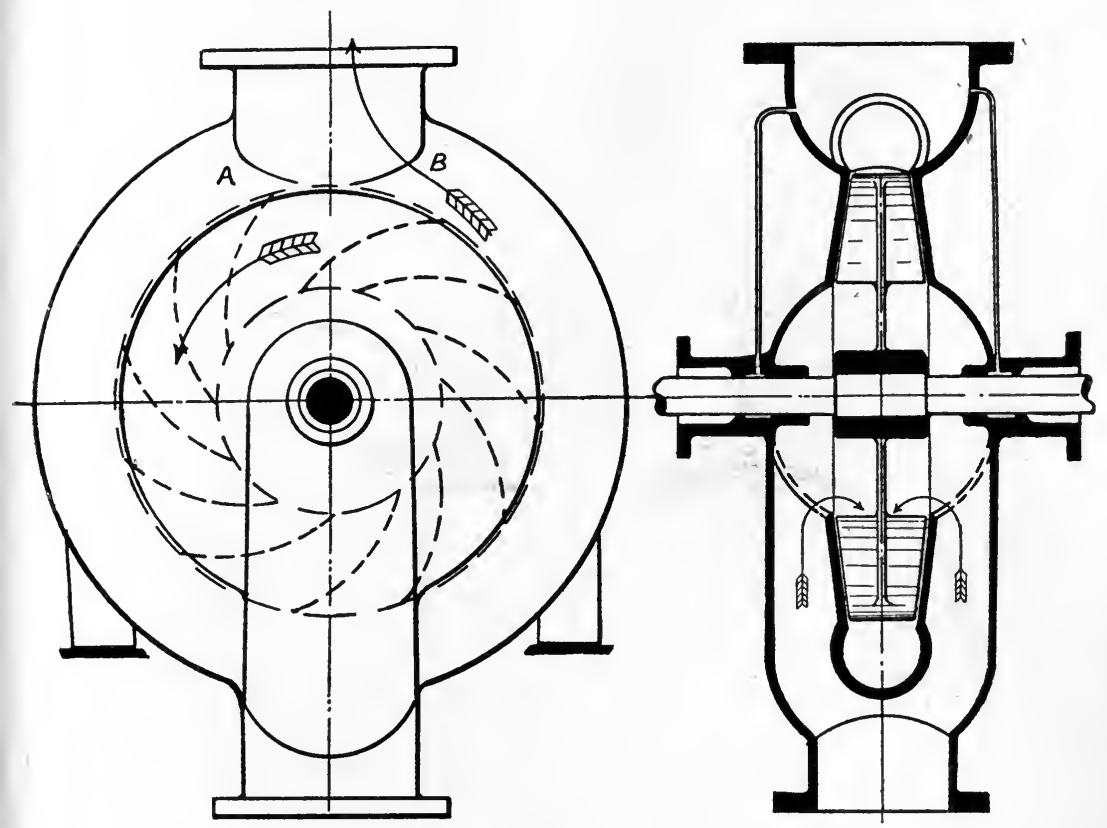

FIG. 245.

Here again, however, the velocity of whirl on leaving the wheel is in general much greater than that of flow in the discharge pipe, so that there is still a loss by shock due to the impact of the high velocity water leaving the vanes, on the more slowly moving water in the volute chamber. Also, there is an entire loss of the kinetic energy possessed by the water in virtue of

1 This cutwater, if designed so as almost to touch the impeller, tends to cause considerable vibration when the pump is working. Generally the working is found to improve, and the efficiency not to suffer unduly, by the provision of a generous amount of clearance at this point. 
its radial velocity at discharge. Neglecting this, if $w_{3}$ represents the velocity of whirl at discharge, and if $\bar{v}$ represents the tangential velocity in the volute chamber, we have the loss of hear in shock $=\frac{\left(u_{3}-\bar{v}\right)^{2}}{2 g}$ feet.

$\therefore$ Neglecting losses in eddy production caused by divergent flow, we have :-

Gain of pressure head in volute chamber

$$
\begin{aligned}
& =\frac{u_{3}^{2}-\bar{v}^{2}-\left(u_{3}-\bar{v}\right)^{2}}{2 g} \text { feet. } \\
& =\frac{\bar{v}\left(u_{3}-\bar{v}\right)}{g} \text { feet. }
\end{aligned}
$$

This is a maximum when $\bar{x}=\frac{u_{3}}{2}$ and then equals $\frac{v_{3}^{2}}{4 g}$ $=\frac{1}{2}\left(\frac{u_{3}^{2}}{2 !}\right)$ feet. Thus the volute chamber as an energy conversion device cannot have an efficiency so great as 50 per cent.

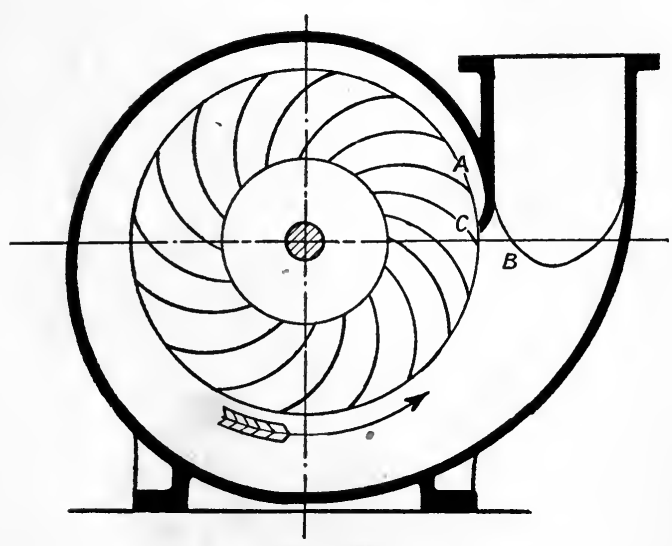

Fig. 246.

Piactically the efficiency is considerably below this, experiments by Dr. Stanton ${ }^{1}$ indicating that it does not exceed about 10 per cent., and that the volute chamber in itself is only slightly more efficient than the concentric chamber.

In the arrangement of collecting chamber designed by Professor James Thomson and known as the vortex or whirlpool chamber, the impeller is surrounded by a casing which may be looked upon as a volute chamber of uniformly increasing area superposed upon a circular chamber concentric with and of considerably larger diameter than the

1 " Proceedings Institute Mechanical Engineers," 1903 (p. 715). 
wheel. Fig. 247 shows such a vortex chamber. In the concentric portion of the casing, the water on leaving the wheel is free to adopt its own manner of motion which approximates to that of a free vortex. In this vortex the pressure increases outwards, theoretically following the ordinary free vortex law. Uniform discharge then takes place around the circumference of the vortex chamber, through the gradually increasing volute passage. The great drawback to this device is that to get a very efficient chamber, the dimensions become

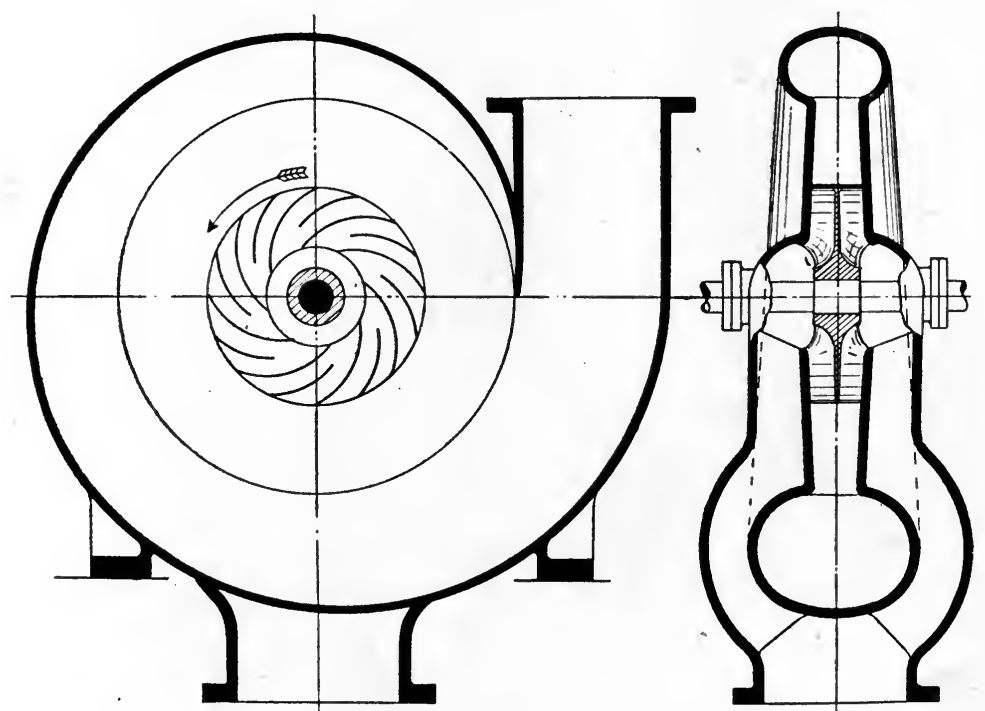

FIG. 247.

excessive-the efficiency increasing with the radius-and, in consequence, it is seldom adopted save in a modified form. In this form it is very general.

Even with this chamber, however, the efficiency of transformation is greatly diminished owing to the instability of diverging motion and the consequent loss of head in eddy formation, and the efficiency actually obtained does not in general exceed about 40 per cent. of the theoretical. To prevent this loss as far as possible the concentric portions of the vortex chamiver-as well as the wheel itself-should be coned, the breadth diminishing as the radius increases. 
Guide Vanes.-The tendency to instability of motion and the heavy losses due to shock may be largely prevented by the introduction of fixed guide vanes around the impeller, as shown in Fig. $248,{ }^{1}$ having angles so designed as to receive the water without shock on leaving the wheel and to direct this by gradually diverging passages, either into a vortex chamber, or, directly into the collecting volute from which it is taken by the discharge pipe. The pressure change then takes place in

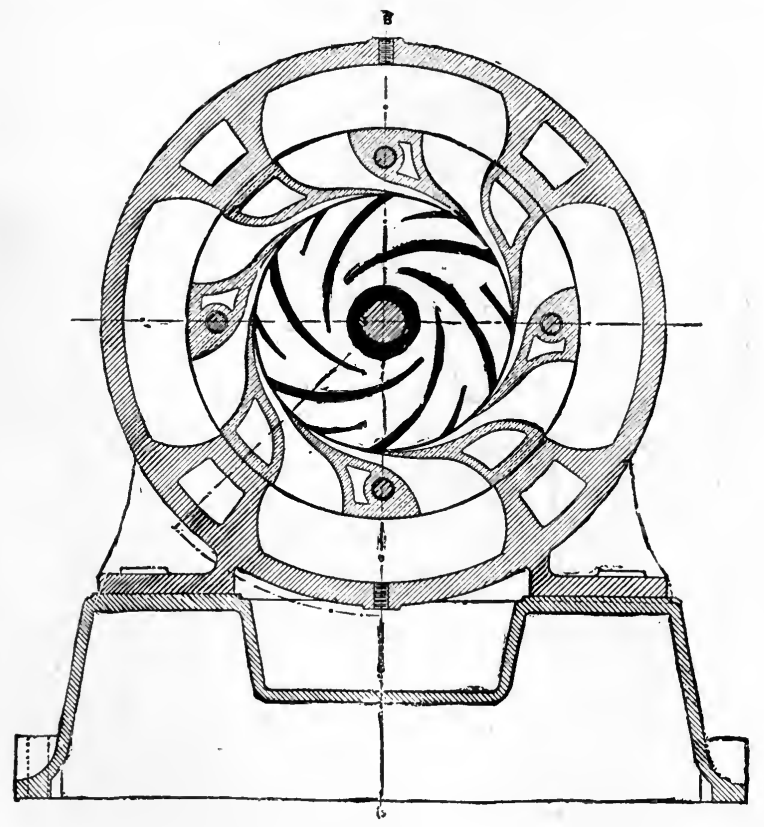

Fig. 248 .

the guide passages themselves. The angle $a$ which the guide vanes make with the circumference of the discharge circle is calculated exactly as in the case of the inlet vanes of a turbine, and, where the pump is required to work under variable conditions, should be suited to the discharge at which the maximum efficiency is desired. Thus fitted, the pump becomes in every essential a reversed turbine. Where the conditions are very variable, however, the guide-or diffuser ring-may easily prove

1 By courtesy of the Buffalo Steam Pump Company. 
an actual source of loss by shock rather than of efficiency. Under such circumstances the guides are preferably omitted, the most suitable type of pump being one fitted with a moderate vortex chamber, this both on account of its greater adaptability to varying circumstances, and of its cheaper construction. Under steady working conditions the efficiency of the ordinary volute chamber is largely inereased by the addition of guides, becoming greater than that of the vortex chamber without guides, while the most efficient arrangement of all, that of the vortex chamber with guides arranged so as to give the issuing streams the direction natural to a free vortex, is capable of converting up to

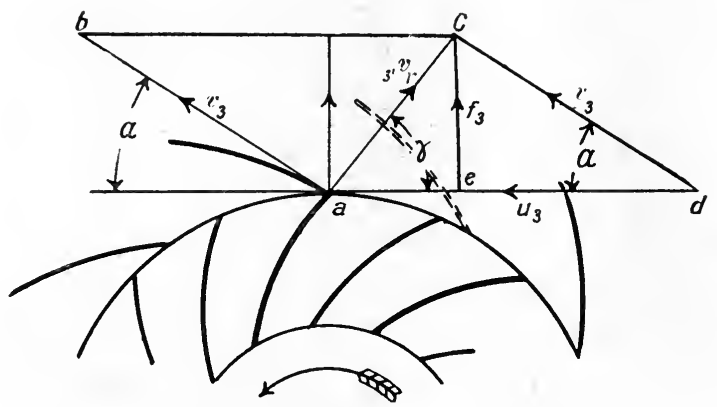

FIG. 249.

75 per cent. of the kinetic energy at discharge into pressure energy.

In Dr. Stanton's paper, ${ }^{1}$ experiments on a pump having a vortex chamber 18 inches diameter showed a chamber efficiency of about 39 per cent. with either a 7 -inch or an 11-inch wheel, the head throughout the trials being about 29 feet. With guides and radial vanes, the guide efficiency varied from 59 per cent. to 70 per cent., while with back curved vanes this efficiency varied from 47 per cent. to 52 per cent. With a third wheel of 12 inches diameter having curved vanes and working under a head of 65 feet, the guide passages had an efficiency of 75 per cent.

In these wheels, which were not designed with a view to high 
total efficiency, the maximum pump efficiencies were as follow :-

Curved vanes with free vortex . Efficiency.

Curved vanes with guide passages Radial vanes with free vortex . 49 per cent.

Radial vanes with guide passages . 56 per cent.

In each case, with curved vanes, the vane angle at inlet was $15^{\circ}$, at outlet $30^{\circ}$, guide angle $a=3^{\circ}$. Vane thickness $\cdot 05$ inch. Four guides were fitted, and the number of impeller vanes was varied from twelve to twenty-two without any appreciable effect on the efficiency. ${ }^{1}$

An examination of Fig. 249 shows that the value of $a$ is given by-

$$
\begin{aligned}
\tan a & =\frac{c e}{e d}=\frac{c e}{a d-a e} \\
& =\frac{f_{3}}{u_{3}-f_{3} \cot \gamma} .
\end{aligned}
$$

Art. 162.-Theori of the Centrifugal Pünp.

In the following theoretical discussion of the design of the centrifugal pump, it is assumed throughout that the pump runs full at all speeds within its working limits, the theory ceasing to apply if any action of the nature of cavitation take place. The further assumption is made that each particle of water, immediately before entering the wheel is moving radially, and that its initial velocity of whirl $w_{2}$ is zero.

On leaving the wheel the water has both radial and tangential velocity, and it is the whole object of pump design to reduce this velocity to the lowest possible amount, and to convert the corresponding kinetic energy into pressure energy before entering the discharge pipe. "Any unconverted energy, except in so far'

1 The following papers may also be consulted as giving the detailed results of experiments on centrifugal pumps :-

Professor Unwin, "Proceedings Institute Civil Engineers," vol. 53, 1877-8, p. 249.

Hon. R. C. Parsons, " Proceedings Institute Civil Engineers," vol. 47, 1876-7, p. 267.

Dr. Stanton, "Proceedings Institute Mcchanical Engineers," 1903, p. 715.

Gerard Lavergue, "La Génie Civil," April 21, 1894. 
as is necessary to give flow up the discharge pipe, is entirely a source of loss.

In this discussion the symbols used are the same and have the same meaning as in the case of the turbine (pp. 491 and 492). Thus the suffix (1) refers to the water just before entering the impeller.

(2) refers to the water just after entering the impeller.

(3) refers to the water discharging from the impeller.

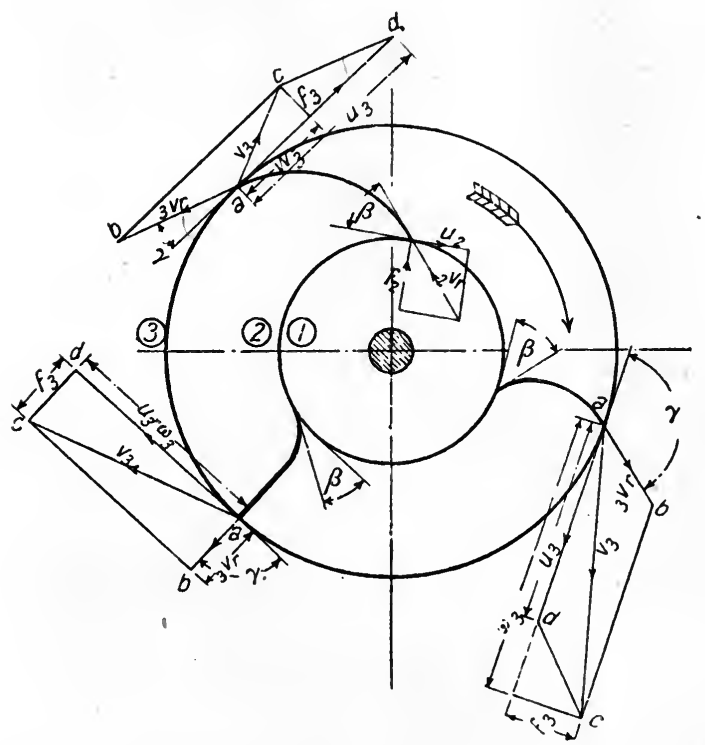

FIG. 249A.-Velocity Diagram for Vanes of a Centrifugal Pump.

while $u_{2}=$ peripheral velocity of impeller at entrance.

$u_{3}=$ peripheral velocity of impeller at exit.

$\beta=$ vane angle at entrance.

$\gamma=$ vane angle at exit. (Fig. 249A.)

Then $f_{2}=u_{2} \tan \beta$, and $f_{3} \cot \gamma=u_{3}-u_{3}$;

while the relative velocity at entrance $={ }_{2} v_{r}=f_{2} \operatorname{cosec} \beta$.

and the relative velocity at exit

$$
\begin{aligned}
& =\sqrt{f_{2}^{2}+u_{2}^{2}}, \\
& ={ }_{3} v_{r}=f_{3} \operatorname{cosec} \gamma .
\end{aligned}
$$


Art. 163.-Pump with inefficient Collecting Chamber.

(a) Change of Pressure in Passing through the Wheel.-The absolute velocity of a particle of water at any point in the wheel may be resolved into two components, one of whirl with the wheel and with a velocity $\omega r$, and the second of flow parallel to the vanes with relative velocity $v_{r}$. This latter velocity is evidently that which the water would have if the same volume were passing with the wheel at rest. The total difference of pressure at any two different radii is thus compounded of the differences due to--

(1) Rotation in a forced vortex with angular velocity $\omega$.

(2) Outward flow parallel to the vanes with velocity $v_{r}$.

In the forced vortex we have, considering the points and $(3)$ :-

$$
\begin{aligned}
& \frac{p_{2}^{\prime}}{W}-\frac{\omega^{2} r_{2}^{2}}{2 g}=\frac{p_{3}^{\prime}}{W}-\frac{\omega^{2} r_{3}^{2}}{2 g} \\
\therefore \quad & \frac{p_{3}^{\prime}-p_{2}^{\prime}}{W}=\frac{\omega^{2}\left(r_{3}^{2}-r_{2}^{2}\right)}{2 g}=\frac{u_{3}^{2}-u_{2}^{2}}{2 g} ;
\end{aligned}
$$

while due to relative outward flow we have-

$$
\begin{aligned}
\frac{p_{2}{ }_{2}}{W}+\frac{{ }_{2} v_{r}^{2}}{2 g} & =\frac{p_{3}^{\prime \prime}}{W}+\frac{{ }_{3} v_{r}^{2}}{2 g} \\
\therefore \quad \frac{p_{3}^{\prime \prime}-p_{2}{ }_{2}}{W} & =\frac{{ }_{2} v_{r}^{2}-{ }_{3} v_{r}^{2}}{2 g} \\
& =\frac{f_{2}^{2}+u_{2}^{2}-f_{3}^{2} \operatorname{cosec}^{2} \gamma}{2 g} .
\end{aligned}
$$

Summing these and writing $p_{3}=p^{\prime \prime}{ }_{3}+p^{\prime \prime}{ }_{3}$, etc., we get the total difference in pressure between the inlet and discharge edges of the vanes, i.e.,

$$
\frac{p_{3}-p_{2}}{W}=\frac{u_{3}^{2}+f_{2}^{2}-f_{3}^{2} \operatorname{cosec}^{2} \gamma}{2 g} .
$$

If, as in the particular case assumed, the water on leaving the vanes is allowed to make the best of its way to the discharge pipe without the provision of a vortex chamber or fixed guides, the kinetic energy of discharge is entirely dissipated in shock, and no further gain of pressure head is obtainable.

\section{Exanple.}

A pump, 1 foot diameter at inlet, 2 feet diameter at outlet, 6 inches broad at inlet, 4 inches at outlet, discharges $5 \cdot 0$ cubic feet 
per second, when making 200 revolutions per minute. Determine the rise in pressure in passing through the wheel, and hence, neglecting all frictional losses, the head pumped against. Assume $\gamma=25^{\circ}$ and neglect the effect of the vane thickness.

Here $Q=f_{2} \times \pi \times \frac{1}{2}=f_{3} \times 2 \pi \times \frac{1}{3}$

$$
\therefore f_{2}=\frac{10}{\pi}=3 \cdot 18 \text { f.s. } \quad f_{3}=\frac{15}{2 \pi}=2 \cdot 386 \text { f.s. }
$$

Again, $u_{3}=\frac{2 \pi \times 1 \times 200}{60}=20.96$ f.s.

and $\operatorname{cosec}^{2} \gamma=5 \cdot 6$

$$
\therefore \quad \frac{p_{2}-p_{3}}{W}=\frac{(20 \cdot 96)^{2}+(3 \cdot 1 s)^{2}-(2 \cdot 386)^{2} \times 5 \cdot 6}{64.4}=6.48 \mathrm{ft} \text {. }
$$

$\therefore \quad$ rise in pressure, $p_{2}-p_{3}=6.48 \times 62.4=404$ lbs. per square foot.

Head pumped against $=6 \cdot 48$ feet.

(b) Energy obtained from Pump.-The useful work done by the pump, if a lifting pump, is the lifting of a quantity of water $Q$ cubic feet per second, through a height $H$, where $H$ is the difference of level between supply and discharge reservoirs. In addition to this, the pump does work against the frictional resistances of the supply and discharge pipes and also delivers a certain amount of kinetic energy due to the velocity of the flow along the discharge pipe. In a pump fitted solely for circulating purposes, the whole or a greater portion of the work will be absorbed in overcoming frictional resistances. In either case, if the motion be steady, the gain of pressure through the wheel must be such as to exactly balance the statical head, together with the head necessary to overcome frictional resistances and that equivalent to the kinetic energy of flow along the suction or discharge pipes.

If then $L$ be total length of supply and discharge pipes, $v$ be velocity through pipes (supposed uniform), we have :-

Gain of pressure through wheel $=H+\frac{F \cdot L \bar{v}^{2}}{2 g m}+\frac{\bar{v}^{2}}{2 g}$ feet.

Equating this to the expression previously obtained, we get :-

$$
u_{3}^{2}+f_{2}^{2}-f_{3}^{2} \operatorname{cosec}^{2} \gamma=2 g I H+\frac{F L \tilde{v}^{2}}{m}+\bar{v}^{2},
$$


and as in general $v$ is approximately equal to $f_{2}$, on making this assumption we get:-

or $\quad f_{3}=\sqrt{\frac{u_{3}^{2}-2 a H}{\operatorname{cosec}^{2} \gamma+\frac{F L_{1} r^{2}}{f_{3}^{2} m}}}$ feet per second.

If $A=$ area of discharge pipe, and if $A_{3}=$ peripheral area of wheel at discharge, we have :-

$$
\bar{r}=\frac{2 \pi r_{3} b_{3}}{A} \cdot \dot{f_{3}}=\frac{A_{3}}{A} f_{3}
$$

(neglecting the vane thickness), while

$$
\begin{aligned}
Q & =2 \pi r_{3} b_{3} f_{3} \text { cubic feet per second, } \\
& =A_{3} f_{3} .
\end{aligned}
$$

$\therefore \quad$ Substituting in equation (6), we have :-

$$
f_{3}=\sqrt{\frac{u_{3}^{2}-2 ! H H}{\operatorname{cosec}^{2} \gamma+\frac{F I}{m} \frac{A_{3}{ }^{2}}{A^{2}}}} \text { feet per second, }
$$

while $Q=\sqrt{\frac{1_{3}^{2}-2, H}{\frac{\operatorname{cosec}^{2} \gamma}{A_{3}{ }^{2}}+\frac{F L}{A^{2} m}}}$ cubic feet per second,

giving the velocity of flow through the pump and the discharge when running at a speed $\omega=\frac{l_{3}}{r_{3}}$ and lifting through a head $H$ feet. From these results it follows that with a constant speed of rotation $Q$ diminishes as $H$ increases and will be zero if $u_{3}^{2}=$ $2 g H$. Also, since any increase in the value of $\operatorname{cosec}^{2} \gamma$ increases the denominator of equation (8), the peripheral speed necessary for pumping against a given head for a given discharge increases as $\gamma$ diminishes, and has its minimum value when $\gamma=90^{\circ}$.

If $H^{\prime}$ measures the difference of head in the suction and delivery chambers, so that $H^{\prime}=H+\frac{F^{\prime} l r^{2}}{2 g m}$, the preceding equations

$$
\begin{aligned}
& \text { become :- } \quad{ }_{1}^{2}+f_{2}{ }^{2}-f_{3}{ }^{2} \operatorname{cosec}^{2} \gamma=2 g H^{\prime}+i^{2} \\
& \text { I or } 2 \text {. } H^{\prime}=1{ }_{3}^{2}-._{3}^{2} \operatorname{cosec}^{2} \gamma \text { approx. } \\
& \therefore f_{3}=\sqrt{\frac{11_{3}^{2}-2 g H^{\prime}}{\operatorname{cosec}^{2} \gamma}}=\sin \gamma \sqrt{{13^{2}}^{2}-2 ! H^{\prime}} \\
& Q=A_{3} \sin \gamma \sqrt{1_{3}^{2}-2 ! H^{\prime}} \text {. }
\end{aligned}
$$


Work done on Pump.-The hydraulic turning moment on the shaft, equivalent to the change per second in angular momentum of the water in passing through the wheel

$$
=\frac{W Q}{g}\left\{u_{3} r_{3}-u_{2} r_{2}\right\} \text { foot lbs. }
$$

$\left.\therefore \quad \begin{array}{l}\text { Work done on water } \\ \text { per second }\end{array}\right\}=\frac{W Q}{g}\left\{w_{3} r_{3}-w_{2} r_{2}\right\} \omega$ foot lbs. (9) and since $w_{2}=0$, this becomes :-

$$
\begin{gathered}
U=\frac{W Q}{g} u_{3} r_{3} \omega=\frac{W^{r} Q}{g} u_{3} u_{3} \text { foot lbs. per second, } \\
\frac{u_{3} \|_{3}}{!} \text { foot lbs. per lb. }
\end{gathered}
$$

or

So that the power required to drive the pump-neglecting friction-is given by $\frac{w_{3} \|_{3}}{!} \cdot \frac{W Q}{550}$ horse power.

Actually the turning moment on the shaft is greater than $W Q_{Q} \frac{u_{3} r_{3}}{g}$ by the moment necessary to overcome hydraulic and mechanical frictional resistances, but neglecting this, the ratio-

Gain in head in pump as measured from pressure differences at entrance and exit

Head equivalent of energy expended on pump spindle per lb. of water,

is termed the Hydranlic Efficiency $\eta$,

$$
\therefore \quad \eta=\frac{I I+\frac{F^{\prime} L T^{2}}{2 ! ! m}}{\frac{\left\|_{3}\right\|_{3}}{!}}=\frac{g H^{\prime}}{\|_{3}\left(\|_{3}-f_{3} \cot \gamma\right)} ;
$$

while on putting $H^{\prime}=I I+\frac{F^{\prime} L \bar{T}^{2}}{2 ! m}=\frac{1}{2 !}\left\{n_{3}^{2}-f_{3} \operatorname{cosec}{ }^{2} \gamma\right\}$ from equation (5), this hecomes :-

$$
\eta=\frac{1}{2} \frac{u_{3}^{2}-f_{3}^{2} \operatorname{cosec}{ }^{2} \gamma}{u_{3}\left(u_{3}-f_{3} \cot \gamma\right)}
$$

Thus, if $\gamma=90^{\circ}$, i.e., with radial vane tips,

$$
\eta=\cdot 5-\frac{{\dot{s_{3}}}^{2}}{2 u_{3}^{2}},
$$


and the efficiency in such a case cannot amount to so much as 50 per cent.

Equation (12) indicates that as $\gamma$ is diminished the efficiency is increased, and this is in general borne out by the results of experiment. As previously pointed out, however, this gain in theoretical efficiency is to a certain extent counterbalanced by the fact that since a diminution in $\gamma$ necessitates an increased speed of rotation for pumping against a given head, this involves increased frictional losses.

Again, since :-

$$
H^{\prime}=\frac{1}{2 g}\left\{u_{3}^{2}-f_{3}^{2} \operatorname{cosec}^{2} \gamma\right\}
$$

it follows that the necessary peripheral speed increases with the working head, so that, because of increased frictional losses at these high speeds, it might be inferred that the higher efficiencies are to be expected with comparatively low working heads - a view which, in the ordinary type of single impeller pump, is borne out in practice. The minimum permissible value of $\gamma$ thus increases with the working head, and while for heads of about 10 feet this may be as low of $15^{\circ}$, it increases to about $25^{\circ}$ with 30 feet head, and for heads of upwards of 60 feet is not generally less than $75^{\circ}$.

Effect of a V'ariation in $\gamma$--Although a high efficiency is to be aimed at in the design of a pump, this is not the only factor which may affect the most suitable value of $\gamma$.

On differentiating equation $\left(8^{\prime}\right)$ with respect to $H^{\prime}$, we finally get :-

$$
\frac{d Q}{d H^{\prime}}=-\frac{g}{Q} A_{3}^{2} \operatorname{Sin}^{2} \gamma
$$

and, therefore, other things being equal, $\frac{d H^{\prime}}{d Q}$ will have its least value when $\sin \gamma$ has its greatest value, i.e., when $\gamma=90^{\circ}$; that is to say, the change in delivery head corresponding to a change in the volume delivered by the pump, will be least when radial tipped vanes are used.

This is of importance in the case of a high lift pump used for boiler feed purposes where the quantity pumped may be varied, but where the delivery pressure is required to remain 
approximately constant, and for this purpose radial vanes are most suitable. The same reasoning applies to the case of pumps for elevator work, where the delivery pressure is to be approximately constant.

Also, where a pump is required for dry dock or similar work, the possibility of obtaining a large increase in $Q$ as $H$ diminishes with the emptying of the dock, renders the radial vane type most suitable, for although the increased volume necessitates a largely increased power, this is in general unimportant as compared with the reduction in the time necessary to empty the dock.

Where a pump is electrically driven, however, this large increase in power with a reduced head involves the danger of overloading the motor, and this is more particularly the case when induction motors are used and where speed variation is in consequence impossible. In such a case backward curved vanes are essential, for, since the horse power is proportional to, $H^{\prime} Q$ and therefore to

$$
H^{\prime} A_{3} \operatorname{Sin} \gamma \sqrt{u_{3}^{2}-2 g H^{\prime}}
$$

we get, on differentiating,

$$
\frac{d\left(H^{\prime} Q\right)}{d H^{\prime}}=A_{3} \operatorname{Sin} \gamma\left\{\frac{u_{3}^{2}-g H^{\prime}}{\sqrt{u_{3}^{2}-2 g H^{\prime}}}\right\}
$$

an expression which diminishes with $\gamma$.

Again, with a pump initially designed to work against a certain head, if the vanes are radial, the possible diminution in speed is very small, and increases as the backward curvature of the vanes increases. With radial vanes, indeed, the pump ceases to lift altogether when the speed falls slightly below that corresponding to normal working. It follows that where the working head cannot be accurately predetermined, the pump with recurved vanes has a great advantage in virtue of its greater adaptability to suit varying conditions without serious loss of efficiency.

If, on installing a pump, the head is greater than that for which the vanes were designed, less than the rated quantity will be delivered, or in an extreme case the pump will not lift at all. Generally a slight increase in speed will rectify this.

If, on the other hand, the designed head is greater than the actual, the delivery will be increased, and the engine or motor 
may be overloaded. This may be rectified either by reducing the speed or, and in general preferably, by slightly throttling the discharge, so as to artificially increase the head. In either case, the possibilities of perfect adjustment are much greater with recurved vanes.

These points are well brought out by a comparison of Figs. $250^{1}$ and $251,{ }^{1}$ which show the characteristic curves from a low lift pump having recurved vanes with an angle $\gamma$ approximately $30^{\circ}$

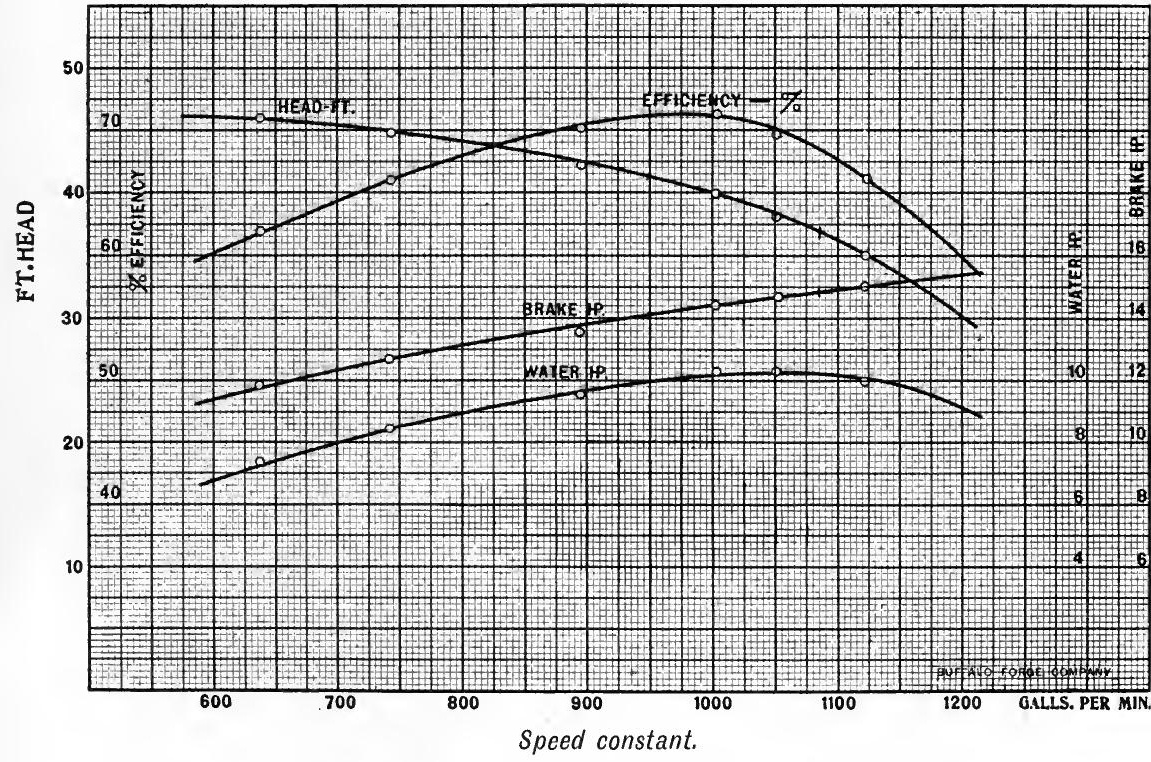

FIG. 250.-Characteristic Curves for Single Low-Lift Centrifugal Pump with Re-curved Vanes.

and a high lift quadruple pump with radial vanes, the speed being constant in both cases. Thus, in the pump with radial vanes, a 10 per cent. diminution in head about the point of maximum efficiency is accompanied by a 23 per cent. increase in B.H.P., while the same percentage decrease in the second pump only involves a $7 \frac{1}{2}$ per cent. increase in B.H.P.

On the other hand, the corresponding increase in the volume delivered is 31.3 per cent. in the case of the radial vaned as

1 By courtesy of the Buffalo Forge Company. 
against only 11.7 per cent. with the other pump. A glance at the two curves shows that the efficiency falls off much more rapidly with varying conditions when the vanes are radial.

Fig. 252 indicates approximately the effect of a variation in $\gamma$ on the speed (angular velocity) required to deliver a given volume of water, where the head is constant. Here the lowest points $A$

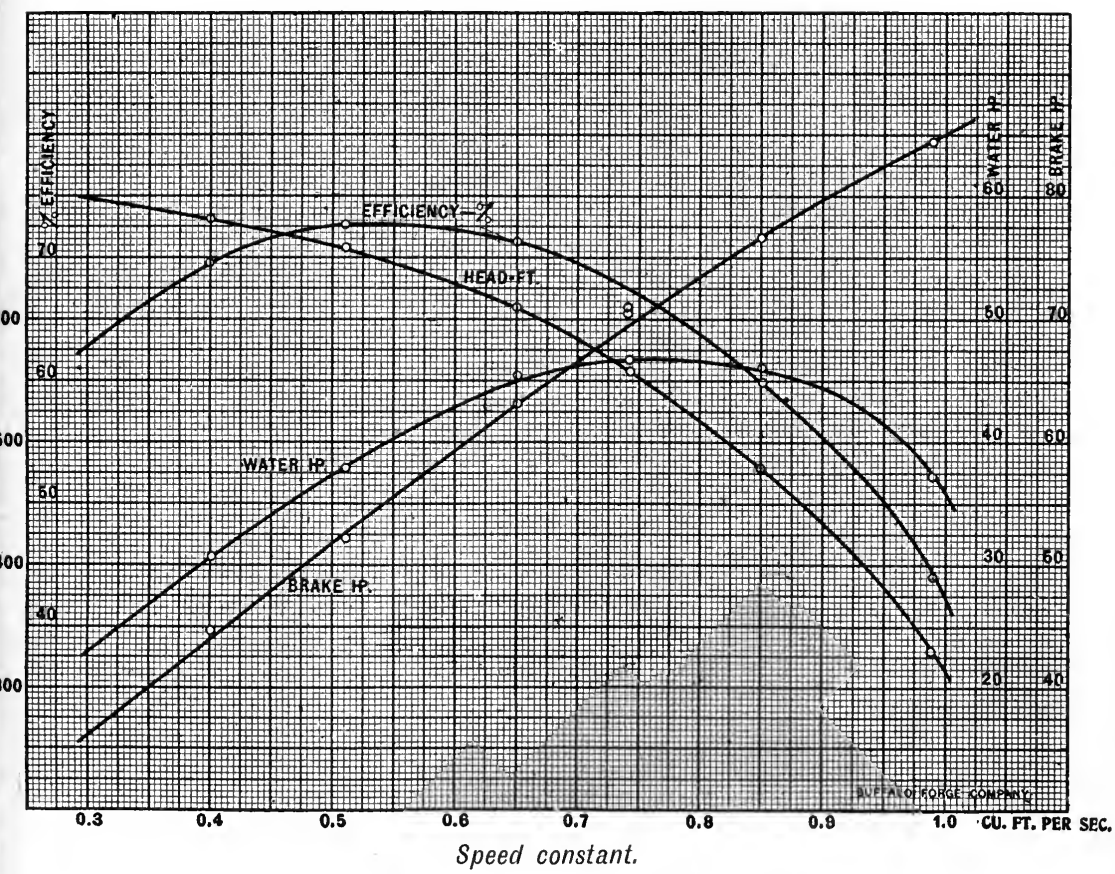

Fig. 2:51.-Characteristic Curves from 4-Stage Quadruple High-Lift Pump with Radial Vanes.

and $A^{\prime}$ of each curve indicate the minimum volume the pump will lift, and the speed below which pumping will not take place. Obviously the minimum speed is increased and the minimum quantity diminished by any recurvature of the vanes.

Pump used for Circulating Purposes.-Where a centrifugal pump is used for circulating water through the tubes of a surface condenser or of a cooler, and where the actual height of lift is small, the resistance to flow, and therefore the head against 
which the pump works, varies approximately as the square of the velocity of flow. In such a case the suction and delivery pipe line is often arranged so as to form a syphon, in which case the

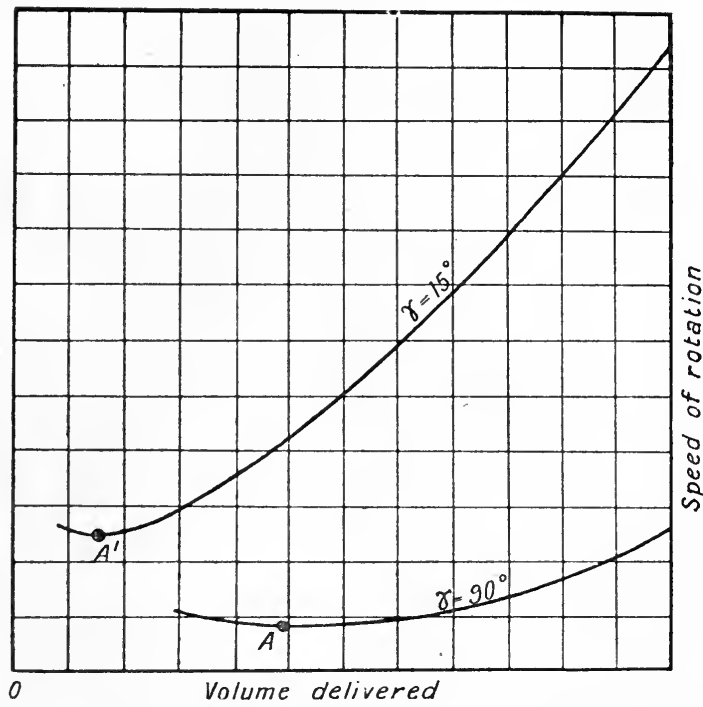

FIG. 252.-Curves showing the influence of the discharge angle $\boldsymbol{\gamma}$ on the volume delivered under constant head.

whole work of the pump consists in overcoming frictional resistances.

Here, putting $H=0$ in equation $(7)$, p. 614 , we get-

$$
\begin{gathered}
f_{3}=\sqrt{\frac{u_{3}{ }^{2}}{\operatorname{cosec}^{2} \gamma+\frac{F \cdot A_{3}{ }^{2}}{m A^{2}}}}=B u_{3}, \\
\therefore f_{3} \propto u_{3} \text { for all speeds. }
\end{gathered}
$$

Equation (13) now becomes :-

$$
\eta=\frac{1-B^{2} \operatorname{cosec}^{2} \gamma}{2(1-B \cot \gamma)} .
$$

So that the hydraulic efficiency is independent of the speed of rotation. Since this discussion neglects frictional losses in the wheel which increase with the speed, the actual efficiency will then diminish as the speed increases. 
Art. 164.-Punp with Whirlpool or Vortex Chamber.

Here the water on leaving the vanes forms approximately a free vortex; the pressure increases as the velocity diminishes towards the outside of the chamber, and part of the kinetic energy of discharge may thus be recovered as pressure energy.

If the suffix $\left(3^{\prime}\right)$ now refers to the outside of the vortex, we have, neglecting changes of level between (3) and $\left(3^{\prime}\right)$ :-

$$
\frac{p_{3^{\prime}}-p_{3}}{W}=\frac{r_{3}{ }^{2}-v_{3^{\prime}}{ }^{2}}{2 !},
$$

giving the gain of pressure head in the chamber on the assumption of true vortex motion, with no eddy losses.

But if $r_{3}$ and $r_{3}^{\prime}$ are the inner and onter radii of the chamber we have $\frac{v_{3^{\prime}}}{r_{3}}=\frac{r_{3}}{r_{3^{\prime}}}=c$ (say)

$\therefore \frac{p_{3^{\prime}}-p_{3}}{W}=\frac{r_{3}^{2}\left(1-c^{2}\right)}{2 g}=\frac{\left(w_{3}^{2}+f_{3}^{2}\right)\left(1-c^{2}\right)}{2 !}$ feet of water.

The theoretical efficiency of the vortex chamber may be taken as $\left(1-c^{2}\right)$, the following table indicating how this varies with an increase in the radius of the chamber :-

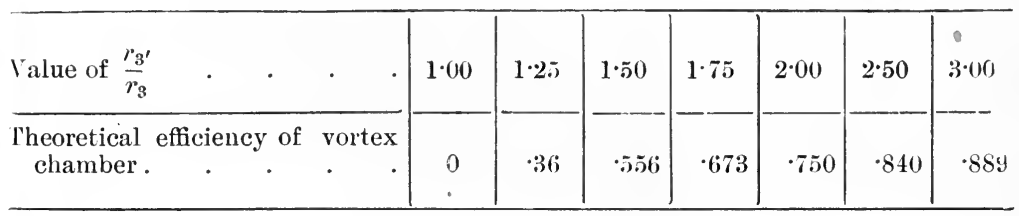

As previously indicated, the actual gain in pressure is considerably less than that obtained in the ideal case, and is given inore nearly by-

$$
\frac{K\left(w_{3}{ }^{2}+f_{3}{ }^{2}\right)\left(1-c^{2}\right)}{2 g} \text { feet, }
$$

where $K$ varies probably from $\cdot 4$ to 55 , depending on the form of the vortex chamber. On adding this gain of head to that 
previously obtained in passing through the wheel (equation 3), we have :-

$$
\begin{array}{r}
\frac{p_{3^{\prime}}-p_{3}+p_{3}-p_{2}}{W}=\frac{K\left(u_{3}^{2}+f_{3}^{2}\right)\left(1-c^{2}\right)}{2 !} \\
+\frac{u_{3}^{2}+f_{2}^{2}-f_{3}^{2} \operatorname{cosec}^{2} \gamma}{2 !}
\end{array}
$$

$=$ total gain of pressure head in pump.

But, as before, if the pump is running steadily, this is equivalent to the statical head $H$, pumped against, together with the head necessary to overcome the frictional resistances in the supply and delivery pipes, and the kinetic energy rejected in the discharge pipe. Equating these we have :-

$$
\begin{gathered}
\frac{K\left(u_{3}^{2}+f_{3}^{2}\right)\left(1-c^{2}\right)}{2 !}+\frac{u_{3}^{2}+f_{2}^{2}-J_{3}^{2} \operatorname{cosec}^{2} \gamma}{2 !} \\
=H+\frac{F L r^{2}}{2 g m}+\frac{\vec{r}^{2}}{2 g} ;
\end{gathered}
$$

or, assuming $f_{2}=\bar{\tau}$ (approx.)

$$
\begin{aligned}
K\left(w_{3}{ }^{2}+f_{3}^{2}\right)\left(1-c^{2}\right)+u_{3}^{2}-{f_{3}^{2}}^{2} \operatorname{cosec}^{2} \gamma & =2 g H+\frac{F^{\prime} L \bar{v}^{2}}{m} \\
& =2 g H^{\prime}
\end{aligned}
$$

Putting $u_{3}=u_{3}-f_{3} \cot \gamma$, we get

$K\left(u_{3}-f_{3} \cot \gamma\right)^{2}\left(1-c^{2}\right)+u_{3}^{2}-f_{3}^{2} \operatorname{cosec}^{2} \gamma+K f_{3}^{2}\left(1-c^{2}\right)$

$$
=2 ! H^{\prime} \text {, }
$$

from which $f_{3}$ may be found in terms of the peripheral speed and the head.

The efficiency $\eta$ is thus equal to-

$$
\frac{1}{2 g} \cdot \frac{\left[\begin{array}{c}
u_{3}^{2}\left\{K\left(1-c^{2}\right)+1\right\}+f_{3}^{2} \operatorname{cosec}^{2} \gamma\left\{K\left(1-c^{2}\right)-1\right\} \\
-2 K u_{3} f_{3} \cot \gamma\left(1-c^{2}\right)
\end{array}\right]}{\frac{u_{3}}{g}\left(u_{3}-f_{3} \cot \gamma\right)}
$$

Putting $K=1$, i.e., neglecting losses in the whirlpool chamber, this reduces to-

$$
\eta=\frac{u_{3}^{2}\left(2-c^{2}\right)-f_{3}{ }^{2} c^{2} \operatorname{cosec}^{2} \gamma-2 u_{3} f_{3} \cot \gamma\left(1-c^{2}\right)}{2\left(u_{3}^{2}-u_{3} f_{3} \cot \gamma\right)} .
$$

Assuming $f_{3}=\frac{1}{4} u_{3}$, the following table traces the variation 
in hydraulic efficiency with a change in $\gamma$ in the ideal case when $K=1$, and when $K=\cdot 5$.

\begin{tabular}{|c|c|c|c|c|c|c|c|c|}
\hline \multirow{3}{*}{$\frac{r_{3^{\prime}}}{r_{3}}$} & \multicolumn{8}{|c|}{ - Efficiency. } \\
\hline & \multicolumn{2}{|c|}{$\gamma=90^{\circ}$} & \multicolumn{2}{|c|}{$\gamma=600^{\circ}$} & \multicolumn{2}{|c|}{$\gamma=45^{\circ}$} & \multicolumn{2}{|c|}{$\gamma=30^{\circ}$} \\
\hline & $K=1$ & $K=\check{r}$ & $K=1$. & $K=\because$. & $\kappa=1$. & $K=\sigma$ & $K=1$ & $K=\therefore$. \\
\hline$* 1 \cdot() 0$ & $\cdot 469$ & $\cdot 469$ & (535) & (53) & $\because 594$ & 584 & .661 & $\cdot 661^{*}$ \\
\hline $1 \cdot 2 \pi$ & $\cdot 660$ & $56 t$ & $\cdot 695$ & 618 & $\cdot 734$ & 658 & $\cdot 78: 3$ & $\cdot 737$ \\
\hline 1.50 & $\cdot 764$ & 61. & .793 & $\cdot 66 ; 3$ & .815 & - & .8 .52 & $\cdot 772$ \\
\hline $1 \cdot 75$ & .826 & $\cdot 6+i$ & .848 & 6994 & - 663 & .724 & .890 & $\cdot 795$ \\
\hline $2 \cdot 00$ & .867 & 670 & 583 & $\cdot 710$ & .89 .5 & .7333 & .915 & .806 \\
\hline $3 \cdot 00$ & $9+1$ & 700 & -!4!) & $7+5$ & 95 & 769 & .963 & .830 \\
\hline
\end{tabular}

* No whirlpool chamber.

From these results it is evident that with an efficient whirlpool chamber there is very little advantage to be gained by giving the vanes any considerable backward curvature. Moreover, as these efficiencies do not take into account friction losses which increase as $\gamma$ diminishes, the actual advantage is less than appears from the table.

Art. 165.-Punp with Guide Vanes or Diffuser Ring.

As previously mentioned, experiments (p. 609) tend to show that with well designed guide vanes on the discharge side of the wheel, up to 75 per cent. of the kinetic energy of discharge may be converted into pressure energy, so that the gain of energy per pound in the guide passages will be given by $\frac{K\left(u_{3}^{2}+f_{3}^{2}\right)}{2 g}$ where $K$ has a maximum value of about $\cdot 75$.

The total gain of pressure head in the pump is now equal to

$$
\frac{K\left(w_{3}{ }^{2}+f_{3}{ }^{2}\right)}{2 g}+\frac{u_{3}{ }^{2}+f_{2}{ }^{2}-f_{3}{ }^{2} \operatorname{cosec}{ }^{2} \gamma}{2 g}=H^{\prime}+\frac{\bar{v}^{2}}{2 g} \text {. }
$$

Assuming $\bar{v}=f_{2}$ and putting $\|_{3}=\omega_{3}-f_{3} \cot \gamma$, we get :$K\left(u_{3}-f_{3} \cot \gamma\right)^{2}+u_{3}^{2}-f_{3}^{2} \operatorname{cosec}^{2} \gamma+K f_{3}^{2}=2 g H^{\prime}$ or $2 g H^{\prime}=u_{3}^{2}(K+1)+f_{3}^{2} \operatorname{cosec}^{2} \gamma(K-1)-2 K u_{3} f_{3} \cot \gamma$.

The efficiency $\eta$ of the pump is thus given by -

$$
\eta=\frac{u_{3}^{2}(K+1)+f_{3}^{2} \operatorname{cosec}^{2} \gamma(K-1)-2 K u_{3} f_{3} \cot \gamma}{2 u_{3}\left(u_{3}-f_{3} \cot \gamma\right)}
$$


Giving $K$ the value $\cdot 75$ and taking $f_{3}=\frac{1}{4} u_{3}$, we get the following values of $\eta$.

\begin{tabular}{c|c|c|c|c|c}
\hline$\gamma$ & $90^{\circ}$ & $75^{\circ}$ & $60^{\circ}$ & $45^{\circ}$ & $30^{\circ}$ \\
\hline$\eta$ & .867 & .875 & .884 & .896 & $\cdot 915$ \\
\hline
\end{tabular}

Here again, any large reduction in the value of $\gamma$, by necessitating an increased speed of rotation, is likely to give diminished rather than increased efficiency.

For the guides to be efficient it is essential that their angle of divergence be small-not exceeding $6^{\circ}$ where possible; that the blade angle $a$ be so designed as to take the water without shock, on leaving the wheel; that a sufficient number of guide vanes be used to efficiently direct the mass of water, four being about the minimum; that the areas of the guide passages at the exit from the wheel should be proportioned so as to keep the velocity at entrance, the same as on leaving the wheel, and that all guide curves should be smooth and gradual.

Since the whole object of the guides is to neutralise the evil effects of a high velocity of discharge, their relative effect will be greatest where this velocity is greatest, i.e., with radial pump vanes when delivering against a high head, and will be least with recurved vanes and a low head. For this reason, and since large percentage variations of head are more likely to occur where this is low, the diffuser ring is seldom fitted for heads under about 60 feet.

The gain in efficiency attainable by its use naturally varies considerably, but may be taken as between 10 per cent. and . 20 per cent.

Art. 166.--Peripheral Sipeed of a Pump.

If $H^{\prime}=$ total head pumped against, including friction head, we have (equation 12):-

$$
\eta=\frac{g H^{\prime}}{u_{3}\left(u_{3}-j_{3} \cot \gamma\right)},
$$

so that with a perfect pump in which all losses were negligible, 
the peripheral velocity of the vanes at discharge would be given by

$$
u_{3}\left(u_{3}-f_{3} \cot \gamma\right)=g H^{\prime} .
$$

so that with radial vanes we should have $u_{3}=\sqrt{ } \overline{g H^{\prime}}$. As $\gamma$ diminishes the peripheral speed increases, while any diminution in efficiency naturally necessitates a higher peripheral speed again, so that actually we have $u_{3}=k \sqrt{g H^{\prime}}$, where $k$ depends upon $\gamma$; upon the value adopted for $f_{3}$; and upon the type of pump. In practice it is usual to make $f_{3}$ from $[\cdot 2$ to $\cdot 3] u_{3}$, the co-efficient increasing from about $\cdot 21$ when $\gamma=15^{\circ}$ to $\cdot 29$ when $\gamma=90^{\circ}$, while $k$ is given a value between $1 \cdot 2$ and $1 \cdot 8$, increasing from about 1.2 in the case of a well designed pump with whirlpool chamber or guides and with a value of $\gamma=90^{\circ}$, to about 1.8 in the case of a pump having a volute chamber only, and a value of $\gamma=15^{\circ}$.

This makes $f_{3}=\frac{1}{4} \sqrt{2 g H^{\prime}}$ (approx.). In the case of a pump dealing with a large head in a single chamber this, however, gives an excessive value of $f_{3}$, and in such a case the value may be reduced to as little as $\frac{1}{8} \sqrt{2 g H^{\prime}}$.

\section{Art. 167.-Compound Multiple Chamber Pump.}

Where a number " $n$ " of impellers mounted on the same shaft are used in series so as to form a compound high-lift pump, the impeller, diameters and vane angles are made the same for each chamber, so that each impeller gives a total head $=\frac{H^{\prime}}{n}$ feet. If, in the foregoing theory then, $H^{\prime}$ be replaced by $\frac{H^{\prime}}{n}$, the deductions also apply to the compound pump. The work done on the shaft per lb. of water,
neglecting frictional losses, is now given by $\frac{n w_{3} u_{3}}{g}$ foot lbs. and the hydraulic efficiency $\eta$ by $\frac{g H^{\prime}}{n w_{3} u_{3}}$. $\left.\begin{array}{l}\text { while neglecting friction the power } \\ \text { required to drive the pump }\end{array}\right\}=\frac{W Q}{550} \frac{H^{\prime}}{\eta}$ B.H.P. $=\frac{W Q}{550 g} \cdot n w_{3} u_{3}$ B.H.P. 
Art. 168.-Speed at which Pumping Commences.

Even if a centrifugal pump be primed, there will be no flow through the delivery pipe until the pressure difference through the wheel, which depends entirely on the speed of rotation, is sufficiently great to overcome the total head of lift, the pump until then merely sustaining a stationary column of water in this pipe.

If the suction lift is $H_{s}$ feet, the pump will not maintain its charge without a foot valve on the suction pipe, unless the speed is sufficiently great to cause a pressure difference of more than $H_{s}$ feet, between the pump inlet and outlet.

Assuming uniform rotation with no flow through the pump, the pressure difference in the forced vortex becomes-

$$
\frac{p_{3}-p_{2}}{W}=\frac{u_{3}^{2}-u_{2}^{2}}{2 g} \text { feet, }
$$

$f_{2}$ and $f_{3}$ being zero.

$\therefore$ For the pump to remain charged when once filled we must have-

$$
\frac{\omega^{2}\left(r_{3}^{2}-r_{2}^{2}\right)}{2 g}>H_{s} \text { feet, }
$$

while for delivery to take place we must have-

$$
\frac{\omega^{2}\left(r_{3}^{2}-r_{2}^{2}\right)}{2 g}>H \text { feet. }
$$

Once free flow is established, the state of affairs changes. A further increase of pressure is now necessary to overcome frictional resistances, and in the case of a pump not fitted with vortex chamber or guides this necessitates the speed for free delivery being slightly greater than is given above. A reduction of the speed below this limit will cause pumping to cease.

With a whirlpool chamber, or guide ring, however, once flow is instituted, part of the kinetic energy of flow through the wheel is converted into pressure energy, so that the speed may be reduced below that necessary to initiate flow, without pumping being stopped. 
Art. 169.-Size of Pump for a Given Discharge--Sinilar Punps-Proportions of Punps.

For a given sped of rotation and a given radius $r_{3}$, the difference in pressure between inlet and outlet, and therefore the head pumped against, increases with the difference between the inner and outer radii of the impeller $r_{2}$ and $r_{3}$. Since, too, the proportional effect of disc friction $\left(\propto r^{2}\right.$, see p. 511), diminishes rapidly as the radius diminishes, the inner radius should, for efficiency, be made small. This may be accomplished, keeping the discharge constant, by increasing the velocity of flow $f_{2}$, but with a large suction head a limit to this maximum velocity is soon reached, and in practice it is usual to make the inner radius from $\frac{1}{3}$ to $\frac{1}{2}$ the outer $\left(r_{3}=3 r_{2}\right.$ to $\left.2 r_{2}\right)$, the former value being preferable.

Again, since the head pumped against is approximately proportional to $u_{3}^{2}$, i.e., to $\omega^{2} r_{3}^{2}$, a given head may be obtained either by an increase in $\omega$ or in $r$. But $Q \propto f_{3} r_{3} b_{3}$, and assuming $f_{3}$ to be proportional to $u_{3}$, we have :-

$$
\begin{aligned}
& Q \propto u_{3} r_{3} b_{3}, \\
& \propto \omega r_{3}^{2} b_{3}, \\
& \therefore \frac{H^{\prime}}{Q} \propto \frac{\omega}{b_{3}} .
\end{aligned}
$$

It follows that for large values of $H^{\prime}$, in order to avoid excessive values of $\omega$, the value of $b_{3}$ should be comparatively small. This is borne out in practice, where the breadth $b_{3}$ of the impeller at the rim is usually proportional to the radius and has a value ranging from $\frac{1}{5} r_{3}$ to $\frac{1}{10} r_{3}$, the former value applying to low lift, and the latter to high lift pumps.

Putting $b_{3} \propto r_{3}$, we have $Q \propto \omega r_{3}{ }^{3}$

$$
\therefore \frac{H^{\prime}}{Q} \propto \frac{\omega}{r_{3}} \propto \frac{u_{3}}{r_{3}{ }^{2}} \propto \frac{u_{3}^{2}}{r_{3}^{2} u_{3}} .
$$

While this is not strictly true in the case of an actual pump, yet it shows that every case for a given value of $H^{\prime}$ and of $Q$, there is a definite value of $r$ for maximum efficiency, this value, however, depending on the relative magnitude of the frictional losses. 
Since, in a perfect pump, with radial vane tips, we have g $H^{\prime}=u_{3}^{2}$, while $Q \propto u_{3} r_{3}^{2}$, if the ratios $\frac{g H^{\prime}}{u_{3}{ }^{2}}$, and $\frac{Q}{u_{3} r_{3}{ }^{2}}$ be plotted this enables a comparison to be made between the performances of different pumps.

Professor Rateau terms the ratio $\frac{g H^{\prime}}{u_{3}{ }^{2}}$ the manometric efficiency; the ratio $\frac{Q}{u_{3} r_{3}^{2}}$ the volumetric efficiency; and the ratio of the product of these, viz., $\frac{\text { ! } H^{\prime}}{u_{3}{ }^{2}} \cdot \frac{Q}{u_{3} r_{3}{ }^{2}}$ to the mechanical efficiency $\frac{W Q H^{\prime}}{U}$, the co-efficient of transmission to the pump shaft. The latter thus equals-

$$
\frac{g H^{\prime}}{u_{3}{ }^{2}} \cdot \frac{Q}{u_{3} r_{3}^{2}} \cdot \frac{U}{W^{\prime} Q H^{\prime}}=\frac{g L^{\prime}}{W u_{3}^{3} r_{3}^{2}} .
$$

The three curves showing the mechanical efficiency, the manometric efficiency, and the co-efficient of transmission, on a base of volumetric efficiencies, are then termed the characteristics of the pump, and if drawn for a given pump apply to any similar pump of different size, so long as the previous relationships hold, and therefore so long as

(1) The peripheral speeds are proportional to $\sqrt{ } \overline{H^{\prime}}$;

(2) The revolutions are proportional to $\frac{\sqrt{H^{\prime}}}{S^{\prime}}$

(3) The quantity discharged is proportional to $S^{2} \sqrt{ } \overline{H^{\prime}}$; where $S$ measures the ratio of similar linear dimensions of the two pumps.

The same conditions hold for identical results to be obtainer from two similar pressure turbines.

\section{Art. 170.-Suction and Delivery Pipes.}

The area of the suction pipe is commonly made equal to the discharge area of the pump vanes. A foot valve and strainer should always be fitted to this pipe, the area throngh the valve being not less than $\frac{1}{2}$ that of the pipe. No part of the pipe should be above the pump inlet in order that the formation of 
air pockets may be prevented, while when the pump is circulating water through a pipe line forming a syphon, an ejector or air pump should be fitted to the highest point of the pipe, for priming the pump and for the removal of any accumulation of air.

The suction lift should be as small and as direct as practicable, and should never, if possible, exceed 20 feet. Where hot liquids are to be handled, the vapour pressure reduces the possible suction lift, the effect of temperature in the case of water being as follows:-

\begin{tabular}{l|c|c|c|c|c}
\hline I'emperature of water, degrees Fahr. & $60^{\circ}$ & $100^{\circ}$ & $140^{\circ}$ & $180^{\circ}$ & $212^{\circ}$ \\
\hline Maximum theoretical suction lift & $33 \cdot 0 \mathrm{ft}$. & $3 \mathrm{i} \cdot \mathrm{ft}$. & $26 \cdot 7 \mathrm{ft}$. & $16 \cdot 7 \mathrm{ft}$. & 0 \\
\hline
\end{tabular}

Practically the limit is reached much before this because of the liberation of air when the pressure is reduced nearly to that corresponding to the saturation temperature, and because of the increasing importance of slight air leakage at joints, and at the stuffing boxes of the pump spindle at these low pressures. In no case should the suction lift exceed $\frac{2}{3}$ of the values given above, while where hot liquids are to be lifted it is advisable to place the pump below the supply level.

The above remarks may be taken as applying with equal force to the reciprocating pump.

When parallel, the area of the discharge pipe should, in general, be not less than that of the suction pipe, and may with advantage be increased to three times this area where the working head is high. It is, however, advantageous to have i main discharge pipe of greater area than the outlet from the collecting chamber of the pump, the connecting pipe having a gradual divergence. By this means the diameter of the vortex chamber may be reduced, the velocity of discharge being increased up to about 10 feet per second, and a partial conversion of kinetic into pressure energy taking place in this comnecting pipe.

With heads exceeding about 100 feet, a non-return valve 
should be fitted on the delivery side, while when delivering into a rising main of great length an air vessel should be fitted on

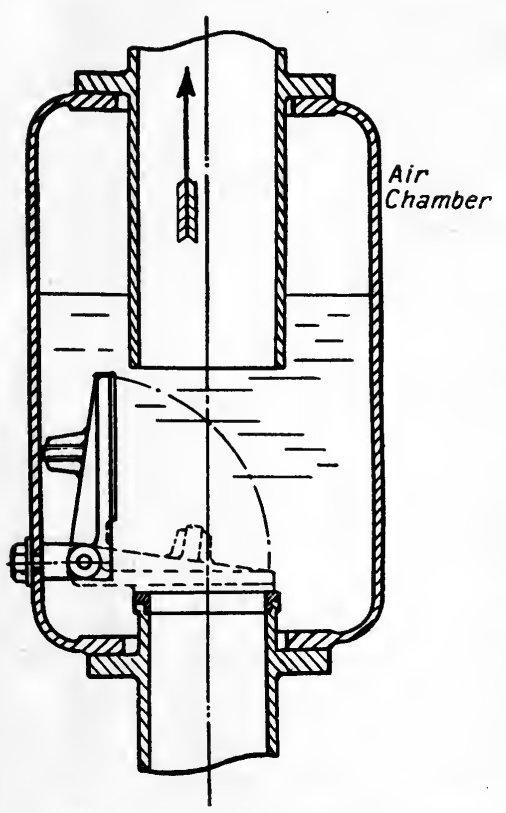

Fig. 253.-Delivery Valve with Air Vessel for High-Lift Centrifugal Pump. the same side of the pump to avoid shock on starting and stopping.

This is specially advisable with a belt-driven pump, where the vibration of the belting may produce variations of considerable magnitude in the angular velocity of the shaft under normal working conditions. In a belt-driven quadruple high-lift pump under the author's observation, making 1,400 revolutions per minute and discharging through 600 feet of 4 -inch piping, against a total head $H^{\prime}$ of 143 feet, the pressure in the last collecting chamber varied from 122 to 164 feet. One successful combination of non-return valve and air vessel is illustrated in Fig. 253.

Art. 171.-Examples of the Design of Centrifugal Pumps.

As an example of the application of the foregoing formulæ, the main points in the design of one or two types of pump will now be considered.

(1) Low-lift pump-12 feet working head-to deliver 6,000 gallons per minute at 220 revolutions.

$$
\text { Assume }\left\{\begin{array}{l}
f_{3}=\frac{1}{4} \sqrt{2 g H^{\prime}}=\frac{8.02}{4} \sqrt{12}=6.95 \text { f.s. } \\
\gamma=30^{\circ} . \\
\iota_{3}=1 \cdot 6 \sqrt{g H^{\prime}}=31.5 \text { f.s. }
\end{array}\right.
$$

Then $\quad A_{3}=\frac{Q}{f_{3}}=\frac{1,000}{62.4 \times 6.95}=2.31$ square feet.

$$
=332 \text { square inches. }
$$


while $\quad r_{3}=\frac{31.5 \times 60}{2 \pi \times 220}=1.367$ feet $=16.4$ inches.

$$
\therefore \quad b_{3}=\frac{A_{3}}{2 \pi r_{3}}=\frac{332}{2 \pi \times 16.4}=3.22 \text { inches. }
$$

Assuming a ratio $\frac{r_{3}}{r_{2}}=\frac{2 \cdot 25}{1}$, this makes $r_{2}=7 \cdot 3$ inches, and if the vane breadth at inlet be increased to 4.5 inches so as to reduce the velocity of flow at this point, this makes:-

$$
f_{2}=f_{3} \times \frac{3 \cdot 22}{4 \cdot 5} \times 2 \cdot 25=\frac{6 \cdot 95 \times 3 \cdot 22 \times 2 \cdot 25}{4 \cdot 5}=11 \cdot 20 \text { f.s. }
$$

Since $f_{2}=u_{2} \tan \beta$, we have :-

$$
\begin{aligned}
\tan \beta & =\frac{f_{2}}{u_{2}}=\frac{11 \cdot 20}{\frac{31 \cdot 5}{2 \cdot 25}}=\frac{11 \cdot 20 \times 2 \cdot 25}{31 \cdot 5}=\cdot 800, \\
\beta & =38^{\circ} \cdot 37^{\prime}
\end{aligned}
$$

giving the inlet angle for the vanes.

(2) A pump-50 feet working head-is fitted with a whirlpool chamber whose radius is twice that of the impeller, and whose efficiency is 50 per cent. The pump is to deliver 5 cubic feet per second. Determine its leading dimensions-its speed of rotation -its hydraulic efficiency, and the probable H.P. necessary to drive it.

To begin with, we will assume that since the whirlpool chamber is fairly efficient and the head high, the value of $\gamma$ may be taken as fairly high - say $60^{\circ}$ - so as to keep down the speed and friction losses. Also assume $f_{3}=\frac{1}{8} \sqrt{2 g H^{\prime}}$.

Then $f_{3}=\frac{8.02}{8} \sqrt{50}=7 \cdot 09$ f.s.

Now substituting 55 for $c$ and $\cdot 5$ for $k$ in equation (18), (p. 622), we get-

$\cdot 5\left(u_{3}-7 \cdot 09 \cot 60^{\circ}\right)^{2} \times \frac{3}{4}+u_{3}{ }^{2}-50 \cdot 2 \operatorname{cosec}{ }^{2} 60^{\circ}+25 \cdot 1 \times \frac{3}{4}$

$$
=100 \mathrm{~g}=3,220
$$

$$
\therefore \quad u_{3}^{2}-2 \cdot 232 u_{3}-2,378=0 \text {, }
$$

$$
\text { or } u_{3}=49 \cdot 9 \text { feet per second. }
$$

Since $\quad \sqrt{g H^{\prime}}=40 \cdot 1$, this makes $u_{3}=1 \cdot 245 \sqrt{g H^{\prime}}$.

Next taking $\quad b_{3}=\frac{r_{3}}{10}$ we have $A_{3}=2 \pi r_{3} b_{3}=\frac{\pi r_{3}^{2}}{5}$, 
and since

$$
\begin{aligned}
Q & =A_{3} f_{3}=\frac{\pi r_{3}^{2}}{5} \times 7 \cdot 09, \\
r_{3}^{2} & =\frac{25}{7 \cdot 09 \pi}=1.122 . \\
\therefore \quad r_{3} & =1.06 \text { feet }=12 \frac{3}{4} \text { inches. } \\
\therefore \quad b_{3} & =1.275 \text { inches. }
\end{aligned}
$$

we have

This neglects the effect of the vane thickness. Since the vanes reduce the effective discharge area by $b_{3} n t_{3} \operatorname{cosec} \gamma$, when the number and thickness of vanes has been decided upon the above value of $b_{3}$ must be increased in the ratio

$$
\frac{2 \pi r_{3}}{2 \pi r_{3}-n t_{3} \operatorname{cosec} 60^{\circ}} \text {. }
$$

Thus, taking eighteen vanes, each having a thickness at the tips of $\frac{1}{4}$ inch, this ratio becomes

$$
\frac{2 \pi \times 12.75}{2 \pi \times 12.75-\frac{18}{4 \times \cdot 8660}}=\frac{80.1}{74.9}=1.207,
$$

and the true breadth $b_{3}=1.275 \times 1.207=1.365$ inches.

$$
\begin{aligned}
\text { Again } \omega & =\frac{49 \cdot 9}{1 \cdot 06}=47 \cdot 0 \text { radians per second. } \\
\therefore \quad N & =\frac{60 \omega}{2 \pi}=449 \text { revolutions per minute. }
\end{aligned}
$$

Assuming $r_{3}=2.5 r_{2}$, this makes $u_{2}=\frac{49 \cdot 9}{2 \cdot 5}=19 \cdot 95$ f.s., while if the vanes be. broadened out towards the centre so as to keep the velocity of flow down to say 10 feet per second, this makes $\tan \beta=\frac{f_{2}}{u_{2}}=\frac{10}{19 \cdot 95}=\cdot 5012$.

$$
\therefore \quad \beta=26^{\circ} 37^{\prime} \text {. }
$$

The hydraulic efficiency $\eta=\frac{g H^{\prime}}{u_{3} u_{3}}=\frac{g H^{\prime}}{u_{3}\left(u_{3}-f_{3} \cot \gamma\right)}$ is equal to

$$
\frac{1,610}{49 \cdot 9(49 \cdot 9-7 \cdot 09 \times \cdot 5774)}=\frac{1,610}{2,282}=\cdot 705 .
$$

Probably mechanical and hydraulic frictional resistances will combine to reduce this efficiency to about 65 . 


\section{DESIGN OF CENTRIFUGAL PUMPS.}

Taking this value, the work done on the pump shaft per second

$$
\begin{aligned}
& =\frac{62 \cdot 4 Q H^{\prime}}{65} \text { foot lbs. } \\
\therefore \quad \text { H.P. } & =\frac{62 \cdot 4 Q H^{\prime}}{550 \times \cdot 65}=\frac{62 \cdot 4 \times 5 \times 50}{550 \times \cdot 65}=43 \cdot 6 .
\end{aligned}
$$

The speed at which the pump begins to lift is given by

or

$$
\begin{gathered}
u_{3}{ }^{2}-u_{2}{ }^{2}=2 ! I I=3,220 \\
\omega^{2}\left(r_{3}^{2}-r_{2}^{2}\right)=3,220 \\
\therefore \quad \omega^{2}=\frac{3,220}{1 \cdot 06^{2}-\cdot 425^{2}}=3,420 \\
\therefore \quad \omega=58 \cdot 5 \\
\therefore \quad N=\frac{60 \times 58 \cdot 5}{2 \pi}=558 .
\end{gathered}
$$

Thus the speed necessary to institute pumping is considerably higher than that necessary for working once delivery has commenced. This assumes that until flow takes place the whirlpool chamber has no effect in converting kinetic into pressure energy. Actually, however, owing to viscosity, the water in this chamber will receive a rotary motion with the greatest velocity near the centre, and with a consequent increase of pressure outwards, the result being that the actual speed for lifting to commence is somewhat less than that indicated above.

(3) Compound high-lift pump, fitted with guide vanes and vortex chambers having a pressure conversion efficiency of 75 per cent., to deliver 5 cubic feet per second under 300 feet head. Assuming a lift of 50 feet in each of six chambers; $\gamma=60^{\circ}$, $f_{3}=\frac{1}{8} \sqrt{2 g H^{\prime}}$; and considering each chamber as a separate pump, we have, as in example (2), $f_{3}=7 \cdot 09$ f.s. Then, taking $b_{3}=\frac{r_{3}}{10}$, we get, as before, $b_{3}=1 \cdot 275$ inches, or, allowing for the same vanes, $b_{3}=1.365$ inches, while $r_{3}=1 \cdot 06$ feet. We now have the factor $k$ of equation (19) (p. 623) equal to 75 , and this equation becomes :-

$$
\begin{gathered}
\cdot 75\left(u_{3}-4 \cdot 09\right)^{2}+u_{3}^{2}-66 \cdot 95+37 \cdot 6=3,220 . \\
\therefore \quad u_{3}^{2}-3 \cdot 51 u_{3}-1,850=0 . \\
\therefore \quad u_{3}=44 \cdot 76 \text { f.s. }
\end{gathered}
$$


This makes $u_{3}=1 \cdot 116 \sqrt{g H^{\prime}}$.

Also $N=\frac{60 u_{3}}{2 \pi r_{3}}=\frac{60 \times 44.76}{2 \pi \times 1 \cdot 06}=403$ revolutions per minute, while since $\tan a=\frac{f_{3}}{u_{3}-f_{3} \cot \gamma}=\frac{7 \cdot 09}{44 \cdot 76-\dot{t}^{\circ} 09}=\cdot 1744$. 'I'his gives a guide vane angle $a=9^{\circ} 54^{\prime}$.

In this case the hydraulic efficiency $=\frac{g H^{\prime}}{u_{3}\left(u_{3}-f_{3} \cot \gamma\right)}$

$$
=\frac{1,610}{44 \cdot 76 \times 40 \cdot 67}=\frac{1,610}{1,822}=\cdot 884 .
$$

Frictional losses would probably reduce this to about 80 , so that the H.P. would be equal to

$$
\frac{62.4 \times Q \times n H^{\prime}}{550 \times \cdot 80}=\frac{62.4 \times 5 \times 300}{550 \times \cdot 80}=213 .
$$

\section{Art. 172.-Types of Centrifugal Pump.}

These may be divided into three classes :-

(1) Pumps having a single impeller with open vanes, and discharging directly into a volute casing or vortex chamber.

(2) Pumps having a single impeller with encased vanes, and either discharging as above, or fitted with a diffuser ring between impeller and vortex chamber.

(3) Compound pumps, which are invariably fitted with encased vanes and with diffuser rings with or without the addition of a vortex chamber.

Fig. 254 shows an example of the first type, having an axial inlet on one side only of the impeller. Here the water is deflected into a radial direction by the conical disc on which the vanes are formed.

Figs. 245 and 247 illustrate pumps having vanes open on both sides, with a suction inlet on each side of the wheel. This type has the advantage of being perfectly in balance as regards axial thrust on the shaft, while the single inlet type necessitates provision being made for balancing any such thrust. On the other hand, the single inlet pump is particularly convenient.for situations where a vertical shaft is permissible, and, as denoted in the figure, lends itself to a very compact and simple type of construction. 
The open vane pump is, however, subject to considerable and incalculable loss by slip or leakage of water between the pump casing and impeller blades, and while this may be reduced by making the clearance at these points as small as possible, the presence of gritty matter in suspension in the water causes rapid wear, and the slip may then become excessive. Under such circumstances the encased type of pump (Fig. $255 a$ and $b$ ), in

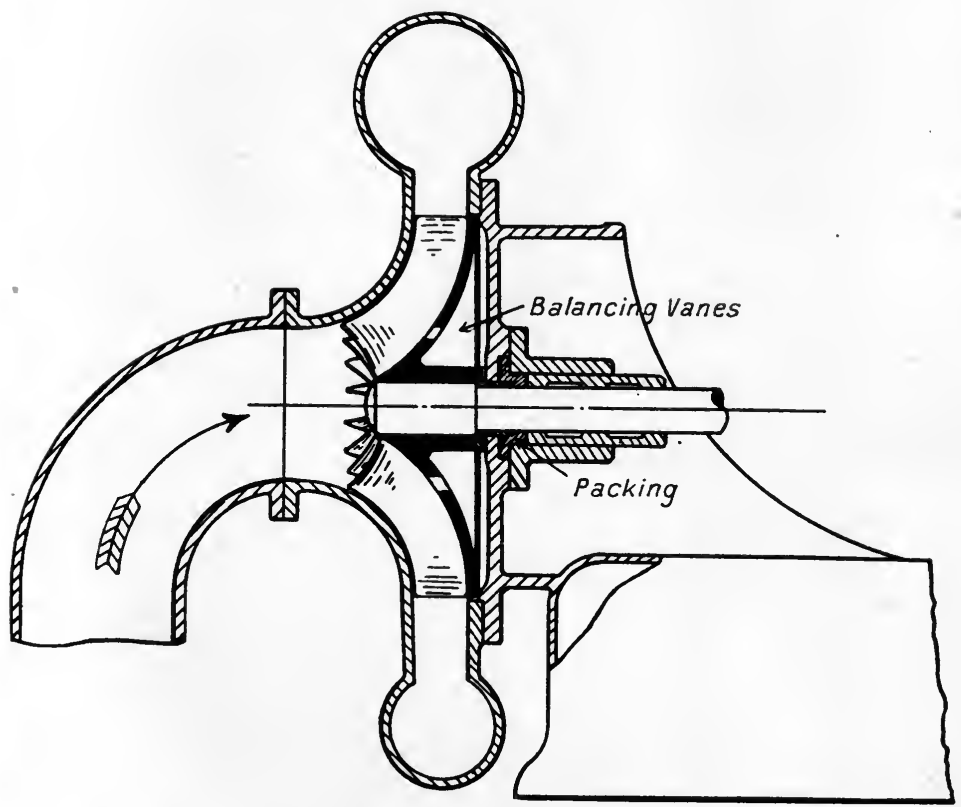

FIG. 254.-Open Vaned Centrifugal Pump with Balancing Vanes.

which the vanes are enclosed at the sides by dises or shroudings rotating with the shaft, becomes preferable. Slip is eliminated, and although disc friction is increased by the provision of the shroudings, yet the loss due to this cause is much less than that due to slip, and is, moreover, calculable with a fair degree of accuracy. It may be reduced within limits by diminishing the clearance between casing and shrouding (p. 511, footnote).

While the open-vaned pump gives good results and is capable of efficiencies up to about 70 per cent. with lifts of up to about 
30 feet, it is in any case preferably replaced by the encased type for higher lifts.

The latter may be used with either single or double suction inlet, and will thus require balancing for end thrust or not, just as does the open vaned pump. The possibilities in this direction are somewhat greater than with the latter type.

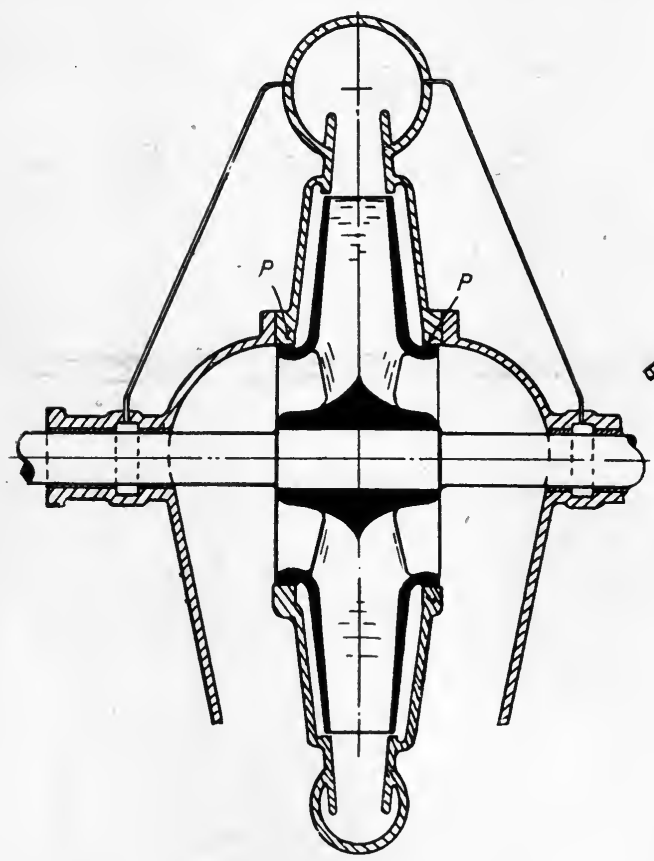

(a)

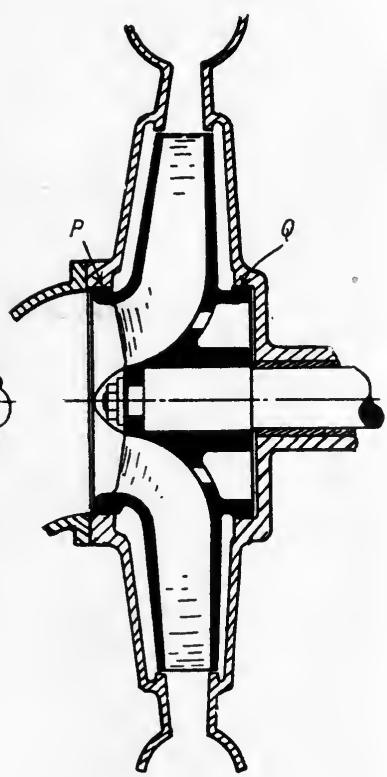

(b)

FIG. 25i.-Double and Single Inlet Encased Inpeller Pump with Vortex Chamber and Volute.

It is very suitable for heads between 30 and 80 feet, but must, for high efficiency under high heads, be fitted with guide vanes outside the impeller ring. As thus constructed, efficiencies up to about $80 \mathrm{per}$ cent. may be obtained. Free circulation of water behind the impeller and into the suction space is usually prevented by the introduction of a brass packing ring at $P$ (Fig. 255).

Where the working head exceeds about 60 feet, the single 
impeller pump rapidly falls off in efficiency owing to the necessary high speed of rotation and the consequent excessive frictional and eddy losses. It may be compounded and so made suitable for such work by mounting a number of impellers in series in

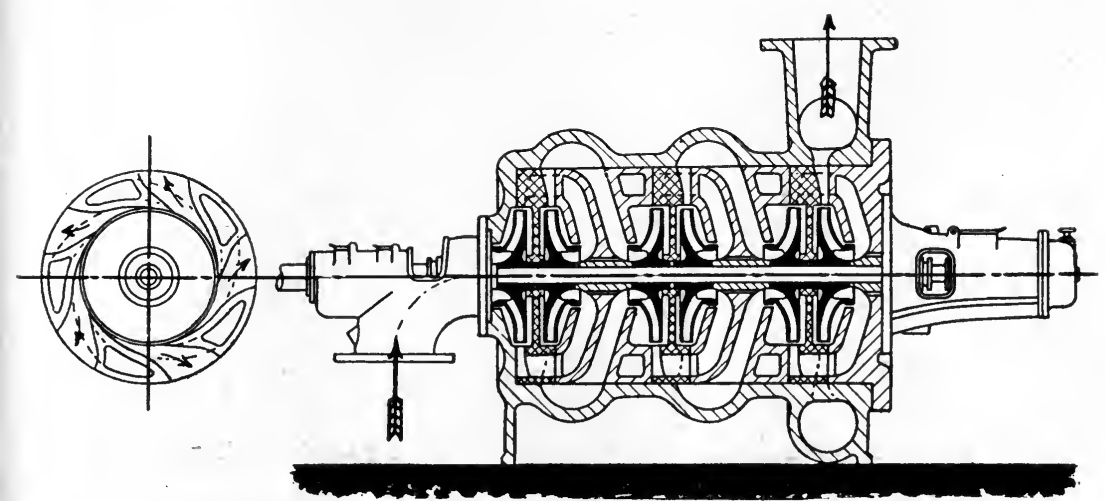

F'IG. 256.-Sulzer Sextuple Compound High-Lift Pump.

separate chambers and on the same shaft, each taking the discharge from its predecessor and raising it through a fraction of the whole head depending on the number of impellers used. For this to be done with fair efficiency, it is essential that as far as possible the kinetic energy of discharge from each wheel be converted into pressure energy before entering the next chamber, and this renders the use of guide or diffuser vanes on the discharge side essential.

Some modern types of construction of the compound high-lift pump are indicated in Figs. 256 to 261.

Fig. 256 shows the construction adopted by Messrs. Sulzer Bros. Here the impellers are mounted in

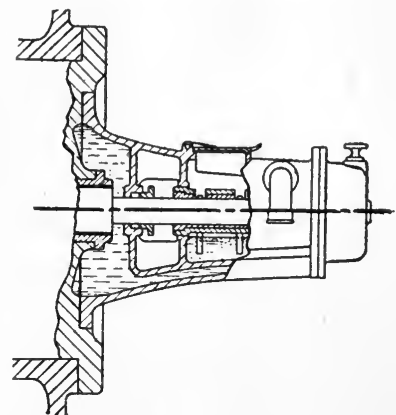

Fig. 25\%.-Water-Sealed stuffing-box for Sulzer Pump. pairs back to back, the flow through these being in opposite directions. By this construction each pair of impellers is in balance as regards end thrust. The pump is fitted with diverging guide passages, curved vanes, and a vortex chamber, 
while Fig. 257 shows the type of water-sealed stuffing box and water-cooled bearing adopted by the makers.

The Buffalo high-lift pump (Figs. 258 and 259) is built on somewhat similar lines, also having impellers mounted in pairs, while in its most modern form the Mather-Reynolds pump is constructed with a double inlet to each propeller, the guide pass sages and curved vanes being retained, while the vortex chamber is omitted. The construction is thus simplified without seriously

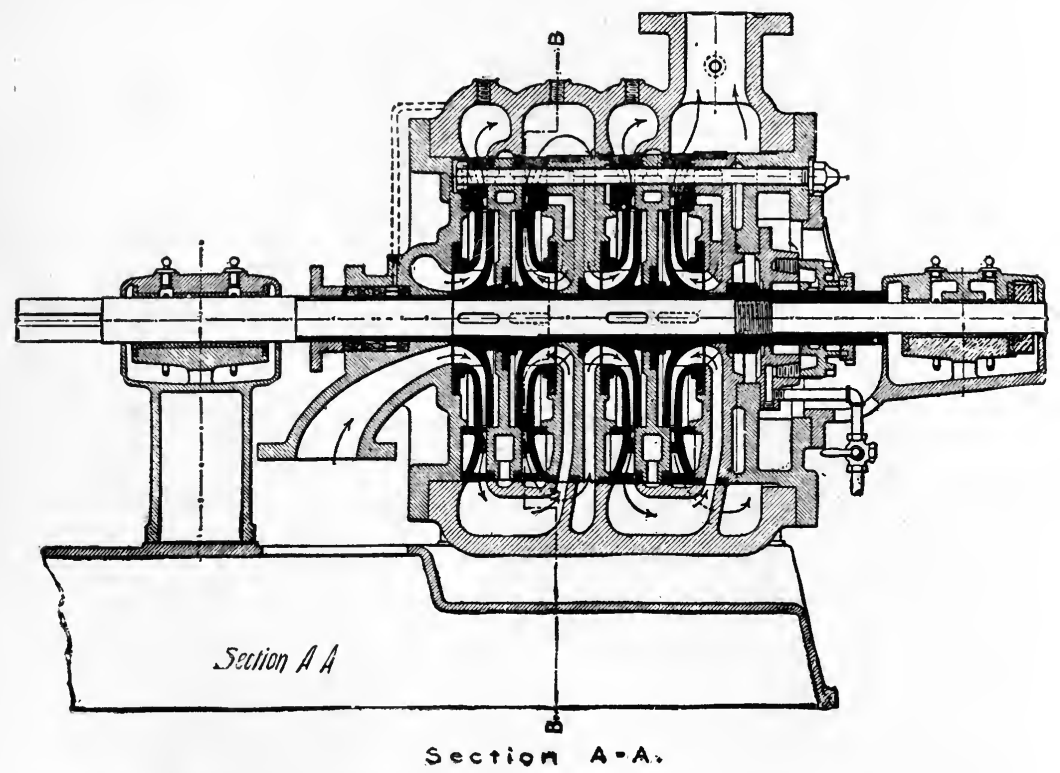

FIG. 258.- Sectional Elevation of Quadruple Compound Buffalo High-Lift l'ump.

affecting the efficiency, a quadruple pump of this type giving efficiencies up to 75 per cent. when delivering 1,000 gallons per minute against 320 feet head.

The foregoing arrangement of impeller, however, necessitates complicated and tortuous connecting passages, and a simpler construction is obtained where, as shown in Figs. 260 and 261, representing respectively the Rateau and the Worthington highlift pumps, impellers, each having a single inlet, are used. This system has the further advantage that any odd number of impellers may be used. On the other hand, with the single 
inlet, end thrust becomes serious and special devices must be adopted to overcome this difficulty. These will be considered in detail in the next article.

With any of these types of pump, efficiencies of between 72 and 80 per cent. may be obtained, while they are all capable of being constructed so as to deal with heads up to about 600 feet, the head in each chamber usually varying from 60 to 100 feet.

The maximum speed of the impeller is limited by the fact that at extreme speeds cavitation is set up, and the pump will not fill

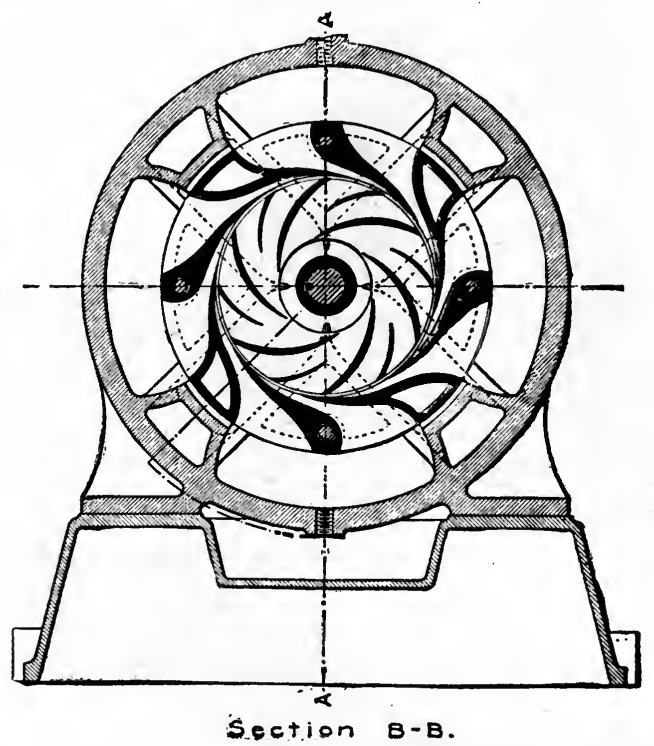

Fig. 259.- Sectional End Elevation of Buffalo High-Lift Pump.

itself. In such cases, as for example when directly driven by a steam turbine of the De Laval type, an auxiliary pump having a lower peripheral speed may be used to supply water under pressure to the main pump.

Leakage from stage to stage of a high-lift pump is prevented by brass packing rings surrounding the shaft between each pair of chambers, while to prevent corrosion of the pump spindle this is usually protected by a brass sleeve.

Admission of air on the suction side of such a pump is to be 
guarded against with the greatest care, as being productive of inefficiency and of considerable shock in the pump casing and delivery pipes.

\section{Art. 173.-Balancing of Exd Thrust.}

End thrust on a pump shaft having one or more impellers with single axial inlets is due to a number of causes.

(a) To the difference of pressure at a given radius on the two sides of the vane shrouding, due to the fact that while the water

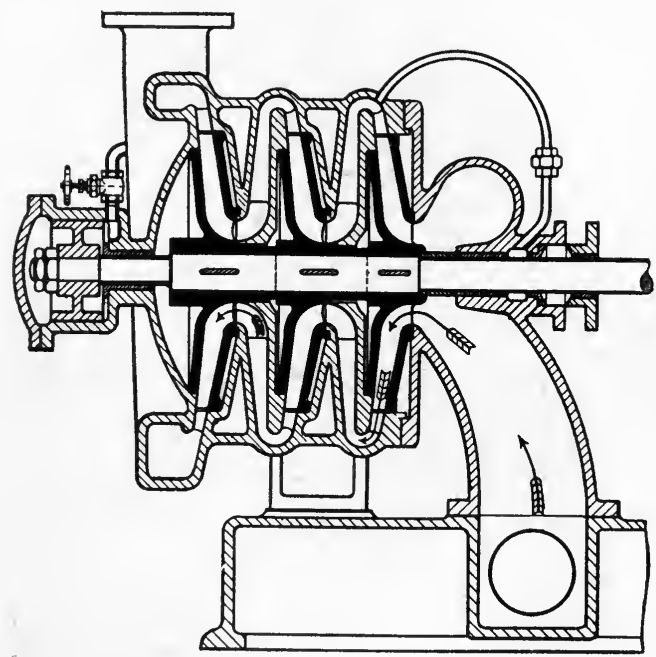

FIG. 260.-Rateau Triple High-Lift Pump.

in the pump is rotating in a forced vortex, that outside the shrouding is in a state of comparative rest, and therefore exists under different pressure conditions.

This is by far the most important factor in producing end thrust.

(b) To the fact that if the discharge diameters of the shroudings in a double cased pump are equal, there is an unbalanced pressure on the portion of the shrouding opposite to the inlet opening. This produces a thrust in the direction of inflow and so tends to balance $(a)$.

(c) Since the water is taken in axially and diverted radially, it 
suffers a change of momentum in an axial direction, and this change of momentum can only be produced by an axial force transmitted through the shaft to the impellers.

Thus if $\bar{v}$ is the velocity of axial flow through the supply passages; $Q$ the discharge per second; and $n$ the number of impellers, this change of momentum takes place $n$ times in the pump, and the total end thrust on the shaft due to this cause is given by-

$$
n \cdot \frac{62 \cdot 4 Q}{g} \cdot \bar{v} \text { lbs. }
$$

This thrust also acts in the direction of inflow.

Apart from the system of arranging the impellers in pairs

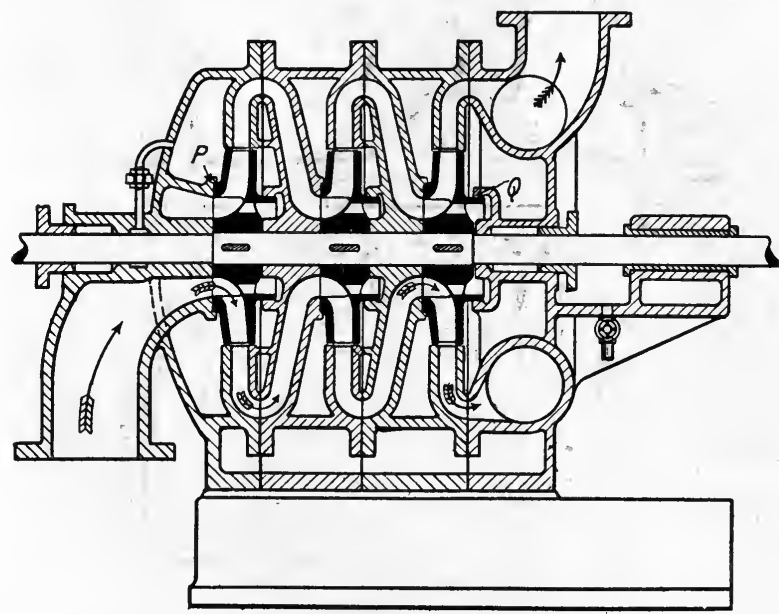

FIG. 261.-Worthington Triple High-Lift Pump.

placed back to back, each pair thus being in balance, four methods of balancing are in general use :-

(a) By radial balancing vanes mounted on the rear face of the shrouding (Fig. 254).

(b) By making the diameter of shrouding on the entrant side greater than that on the exit side, the difference of area being just sufficient to balance the pressure difference (Fig. 260).

(c) By a rotary balance piston lieyed to the pump shaft, one side being exposed to delivery pressure, and the other to the pressure on the suction side of the pump (Fig. 260).

H.A. 
(d) By relieving the pressure over a certain area behind the shrouding by means of a rotating balance ring (Fig. 261 and Fig. $255 b$ ).

Whichever of these methods is adopted, with the possible exception of $(c)$, the wheel will only be in perfect balance at one speed and with one rate of delivery, and it is usual to

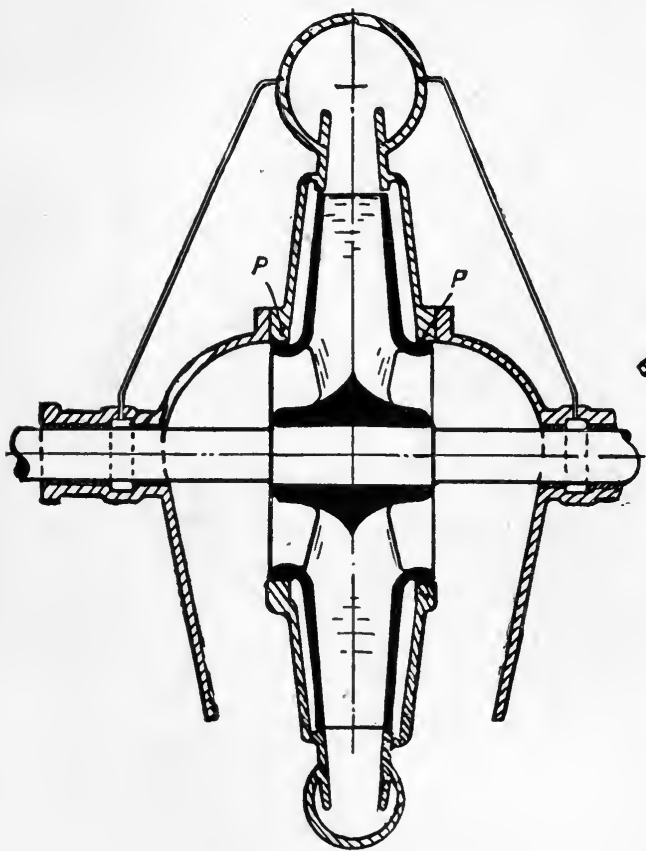

(a)

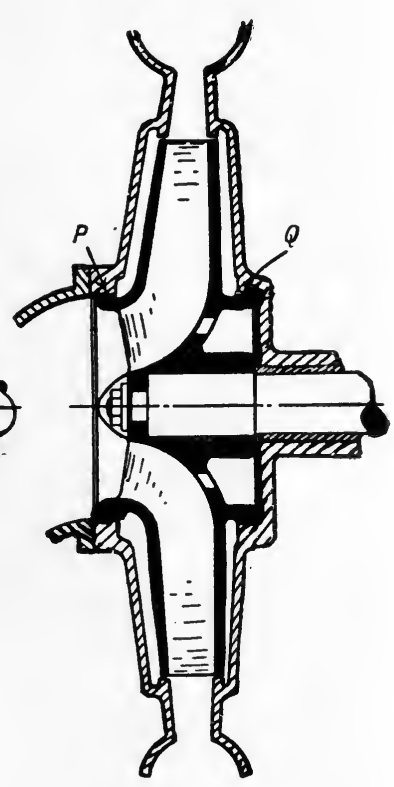

(b)

FIG. 262.-Double and Single Inlet Encased Impeller Pump with Vortex Chamber and Volute.

arrange a thrust block to take the end thrust caused at starting or stopping and in the case of a variable load.

These methods of balancing will now be considered in further detail :-

(a) Radial Balancing Vanes.-Since it is impossible to keep the joint between the outer periphery of the shrouding and casing tight, the space behind the shrouding will be full of water normally at delivery pressure, and since the mean pressure 
in the wheel is considerably less than this, the resultant effect in a single shrouded wheel (Fig. 254) will be an axial thrust of considerable magnitude, in the opposite direction to that of axial flow.

If a series of holes be made through the disc, as indicated in Fig. 262, the pressure at this point on both sides of the shrouding will be equalised, and there will be a constant circulation of water past the rim of the impeller, behind the shrouding to the zone of low pressure, and out to the working side of the shrouding.

While the axial thrust will thus be to a considerable extent balanced, this leakage is productive of inefficient working, and the method, though often adopted in turbine practice, is inadvisable.

If, however, shallow radial vanes are fitted to the rear face of the shrouding, the water in the clearance space is forced to rotate with vortex motion, and in consequence its pressure diminishes from the outside, where it has a definite value, to the inside, following approximately the forced vortex law. The degree to which it deviates from this law will depend on the amount of side clearance between the balance vanes and the pump casing, any increase in this clearance, by reducing the mean angular velocity, tending to increase the mean pressure behind the shrouding. By suitably regulating the clearance and the radial length of the balance vanes, the whole, or any portion of the axial thrust, may thus be balanced.

For example, in the wheel shown in Fig. 254, the pressure in the wheel itself varies at different radii owing, firstly, to the production of a forced vortex, and secondly, to the necessity of maintaining an outward flow. In the clearance space to the right this outward flow is absent, so that the difference of pressure on the two sides of the shrouding at a radius $r$ is given by the pressure necessary to maintain this flow, and if $p_{r}$ represent this pressure intensity in pounds per square foot we have

$$
\frac{p_{r}}{W}=\frac{p_{2}}{W}+\frac{{ }^{2} v_{r}{ }^{2}-{ }_{r} v_{r}{ }^{2}}{2 g} .
$$

This assumes the law of pressure variation in outward flow to be the same as in inward flow, i.e., neglects losses of energy 
due to eddy formation, and since this loss varies with the forms and number of the blades (i.e., with the rate of their divergence), becoming less as the number and curvature of the vanes is increased, the theory is to this extent unsatisfactory. Allowing for this, we have:-

$$
\frac{p_{r}}{W}=K \frac{p_{2}}{W}+\frac{K_{2} v_{r}{ }^{2}-{ }_{r} v_{r}^{2}}{2 g},
$$

where $K$ is a co-efficient, varying probably from about $\cdot 7$ in the case of radial vanes to 85 with vanes having a delivery angle of $30^{\circ}$.

Now

$$
{ }_{2} v_{r}=\sqrt{{f_{2}^{2}}^{2}+u_{2}^{2}}
$$

And ${ }_{r} v_{r}=f_{r} \operatorname{cosec} \theta$, where $\theta=$ angle made by the vanes at this radius with the tangent to the corresponding circle.

$$
\therefore \quad \frac{p_{r}}{W}=K\left\{\frac{p_{2}}{W}+\frac{f_{2}^{2}+u_{2}^{2}}{2 g}\right\}-\frac{f_{r}^{2} \operatorname{cosec}^{2} \theta}{2 g} .
$$

If $\theta=90^{\circ}, i . e$. , with vanes radial throughout, we have :-

$$
\begin{aligned}
\frac{p_{r}}{W} & =K\left\{\frac{p_{2}}{W}+\frac{f_{2}^{2}+u_{2}^{2}}{2 g}\right\}-\frac{f_{r}^{2}}{2 g} \\
& =K\left\{\frac{p_{2}}{W}+\frac{u_{2}^{2}}{2 g}+\frac{f_{2}^{2}}{2 g}\left(1-\frac{b_{2}^{2} r_{2}^{2}}{K b^{2} r^{2}}\right)\right\} .
\end{aligned}
$$

So that if the balance vanes have internal and external radii $R_{1}$ and $R_{0}$, we have the unbalanced pressure $F$, due to these given by

$$
\begin{aligned}
& F=\int_{R_{1}}^{R_{0}} 2 \pi r p_{r} d r \text { lbs. } \\
& =2 \pi K \int_{R_{1}}^{R_{0}}\left\{p_{2}+\frac{u_{2}^{2} W}{2 g}+\frac{{f_{2}}^{2}}{2 g}\left(1-\frac{b_{2}^{2} r_{2}^{2}}{K b^{2} r^{2}}\right) W\right\} r d r \text { lbs. } \\
& =2 \pi K\left[\left\{p_{2}+\frac{\left(u_{2}^{2}+f_{2}^{2}\right)}{2 g} W\right\}\left\{\frac{R_{0}^{2}-R_{1}^{2}}{2}\right\}\right. \\
& \left.-\frac{f_{2}^{2} b_{2}^{2} r_{2}^{2}}{2 g K l^{2}} \log _{e} \frac{R_{0}}{R_{1}}\right] \mathrm{lbs} .
\end{aligned}
$$

Equating this to the unbalanced pressure on the inlet side of the shrouding produced by the axial change of momentum of the water, and to the unbalanced pressure on the remaining annulus of width $r_{3}-R_{0}$, on fixing $R_{1}$ the required outer radius $R_{0}$ may be determined. 
The force $F$ may, however, be calculated more simply by dividing the dise into a series of concentric elements, and by obtaining the relative velocity at the mean radius of each element by the graphical construction of Art. 134.

The unbalanced force on each element may then be calculated by an application of equation (2) above, as shown on p. 517, and the sum of these gives the force $F$.

(b) In this method (Fig. 260) the diameter of the shrouding on the exit side is reduced so as to make the total area of the shroudings approximately equal.

The clearance space pressures are thus balanced, and the excess of pressure on the inlet shrouding due to the greater pressure at the outer radius of the runner tends to balance the pressure in the opposite direction due to momentum changes. Each of these methods $(a)$ and $(b)$ is extremely sensitive; is impossible of exact calculation; and is therefore only suitable when used in conjunction with a thrust block or balance piston to take up any minor unbalanced thrust.

(c) Where a balance piston is used (Fig. 260), leakage past this piston is slight, and by adjustment of a valve on the pressure pipe $P$, which adjustment may be automatic, the pressure may be

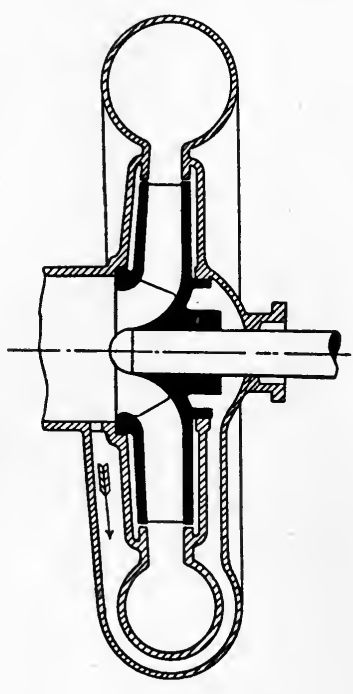

FIG. 263. very accurately adjusted while running, to suit any condition of working.

(d) In this method of balancing (Figs. 261 and $262 b$ ) the outer diameter of the balance ring is made slightly less than that of the impeller at the packing ring $P$, while a series of holes through the shrouding equalises the pressure in the interior of the balance ring and of the impeller.

This is then approximately in balance, the slight reduction in the diameter of the balance ring creating an excess pressure towards the inlet, which counterbalances the change of axial momentum of the supply water. 
Brass packing rings are fitted at $Q$ to prevent leakage of pressure water from the clearance space into the balance ring.

A somewhat analogous method of balancing a single impeller is shown in Fig. 263.

Where a centrifugal pump is fitted with a vertical shaft, the whole weight of the pump and driving shaft may usually be balanced by an application of the preceding principles; while exactly the same reasoning applies to the balancing of the axial thrust on a turbine shaft.

\section{Examples.}

(1) A centrifugal pump is 4 feet in diameter and makes 200 revolutions per minute, delivering 64.8 cubic feet of sea water per second, against a head of 20 feet. The discharge area $=$ 8.33 square feet, and the discharge angle $\gamma=26^{\circ}$. The ratio $\frac{r_{3}}{r_{2}}=\frac{2}{1}$.

Determine the hydraulic efficiency and, assuming a loss of 10 per cent. in friction, the H.P. to drive the pump, and the speed at which lifting commences.

$$
\text { Answer. }\left\{\begin{array}{l}
\text { Hydraulic efficiency }=\cdot 592 . \\
\text { Mechanical efficiency }=\cdot 492 . \\
\text { H.P. }=306 . \\
\text { Lifting commences at } 198 \text { revolutions. }
\end{array}\right.
$$

(2) The following are results obtained from tests of a centrifugal pump, having the following dimensions : $r_{3}=15 \cdot 25$ inches ; $r_{2}=7 \cdot 675$ inches; $b_{3}=3 \cdot 0$ inches $; b_{2}=4 \cdot 0$ inches $; \gamma=30^{\circ}$.

\begin{tabular}{|c|c|c|c|c|}
\hline \multicolumn{2}{|c|}{ Revolutions per minute } & $188 \cdot 3$ & $202 \cdot 7$ & $213 \cdot 7$ \\
\hline Gallons per minut & - & 1,395 & 1,705 & 1,976 \\
\hline Lift in feet, $H^{\prime}$ & & $12 \cdot 33$ & $12 \cdot 58$ & $13 \cdot 0$ \\
\hline Water H.P. . & & $5 \cdot 22$ & $6 \cdot 51$ & $7 \cdot 81$ \\
\hline Dynamometric $\mathrm{H}$. & & $8 \cdot 11$ & $10 \cdot 74$ & $14 \cdot 02$ \\
\hline Efficiency & & $64 \cdot 5$ & $60 \cdot 74$ & $55 \cdot 72$ \\
\hline
\end{tabular}


Determine from this the value of $u_{3}$ for maximum efficiency in terms of $\sqrt{g H^{\prime}}$, and determine the hydraulic efficiency in each case.

(3) A pump delivers 3 cubic feet per second against 60 feet head, and is required to rotate at 500 revolutions per minute. Making $f=\frac{1}{6} \sqrt{2 g H^{\prime}}$, and giving the vanes radial tips, settle the leading features of the design, on the assumption that guides are fitted with 'a conversion efficiency of 65 per cent.

(4) A pump has an inner radius of 1 foot, and an outer radius of 2 feet. It is not fitted with vortex chamber or guide vanes.

Determine the speed at which lifting will commence against heads of 10, 20, 40 and 60 feet respectively.

Answer. $99 ; 140 ; 198 ; 242$ revolutions per minute.

(5) A pump is intended to lift 25 cubic feet per second against a head of 16 feet. A model is made and delivers 1 cubic foot per second àgainst 16 feet head, when making 1,450 revolutions per minute, then giving its maximum efficiency. The radius of the model impeller is 4.5 inches. Determine the speed and radius of the large pump for maximum efficiency.

$$
\text { Answer. }\left\{\begin{array}{l}
\text { Radius }=22.5 \text { inches. } \\
\text { Speed }=290 \text { revolutions. }
\end{array}\right.
$$

(6) The peripheral speed of a centrifugal pump $=30 \mathrm{f} . \mathrm{s}$. The vanes are curved backward so that the discharge angle. $\gamma=$ $35^{\circ}$, while the water leaves the wheel with a radial velocity of 5 f.s. If 120 eubic feet of water pass through the pump per minute, determine the hydraulic turning moment on the shaft if the radius of the wheel is 2 feet.

Answer. 177 foot pounds. 


\section{CHAPTER XVII.}

Other Types of Pumping Machinery-Water Hoisting-The Hydraulic RamHydraulic Air Compressor-The Jet Pump-The Injector Hydrant-The Air Lift Pump-Reversed Air Lift Pump as Air Compressor.

\section{Art. 174.-Water Hoisting from Mines. ${ }^{1}$}

THE method of direct hoisting of water in large tanks has come very rapidly into favour of recent years in the anthracite region of Pennsylvania for mine drainage purposes.

The system has the advantages that in general, with the

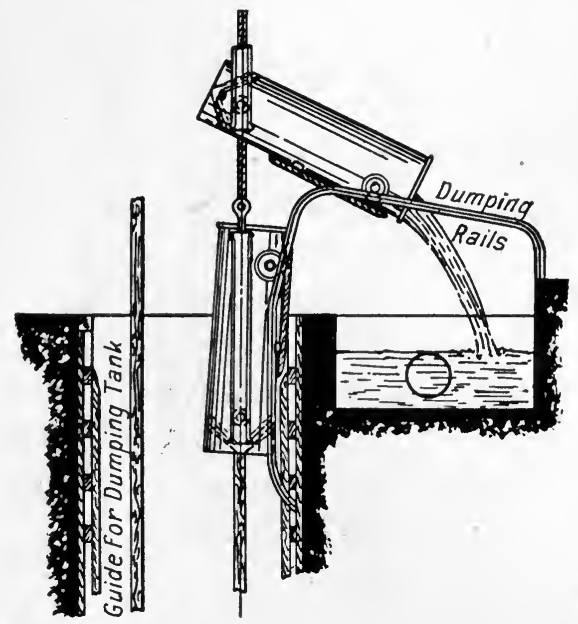

Fig. 264.-Method of End Dumping in direct Water-hoisting Plant. exception of the tanks, and the guides used for keeping these vertical while hoisting, no new machinery is needed, the only cost being that due to extra wear and tear of the hoisting engines and to the steam used while hoisting. Further, the whole of the operating machinery is on the surface and free from the danger of being flooded, while no underground steam pipes, with the accompanying losses by condensation and the danger of damage by a slip of the roof, are necessary.

1 For a descriptive article on this method of mine drainage, see a paper by R. V. Norris, before the American Inst. of Mining Engineers, 1903, or an abstract of this paper in "Cassier's Magazine," for May, 1904. 
Cylindrical hoisting tanks are now general, these having a couple of butterfly valves in the bottom placed at an angle of $45^{\circ}$ (Fig. 264), and which enter the sump without shock.

The tanks may discharge at the top of the shaft either by overturning or by automatic opening of the bottom valves. The former method is preferable as rendering more rapid manipulation possible. With tanks of 1,500 gallons capacity, the capacity of such a plant ranges up to about 750,000 gallons per day of twelve hours.

\section{Art. 175.-The Hydraulic Rav.}

The hydraulic ram, which owes its conception as a practical machine to Montgolfier (about the end of the eighteenth century),

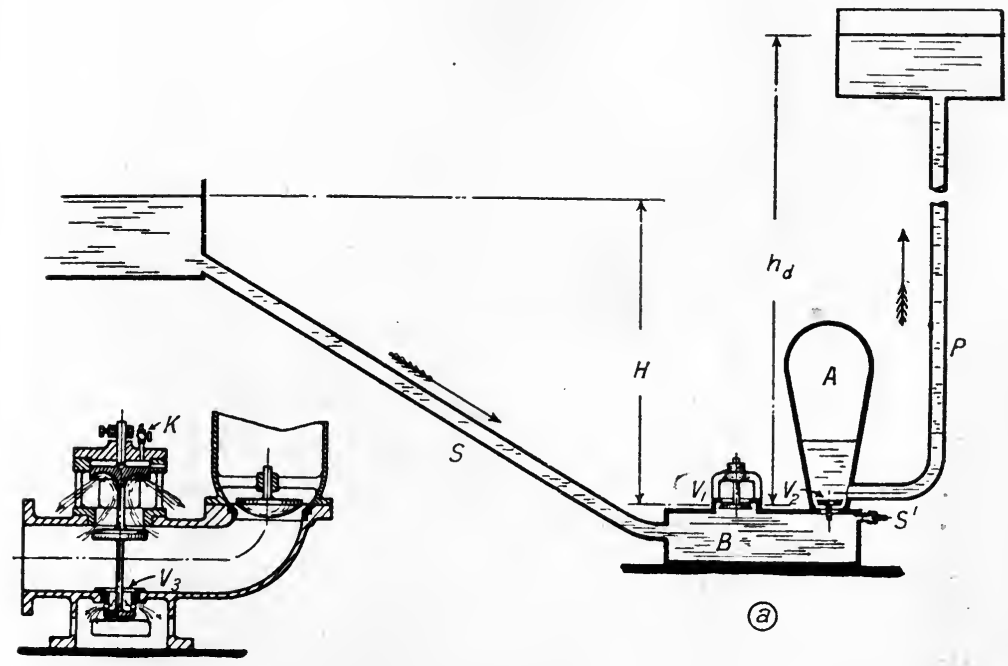

(b)

Fig. 265.-Types of Hydraulic Ram.

is an apparatus devised to utilize the kinetic energy of a moving column of water to pump up part of this water to a height greater than that of the supply head. In its simplest form, the ram consists of an inclined supply pipe $S$ (Fig. 265 a), terminating in a valve box $B$. This valve box is fitted with a waste valve $V_{1}$, opening inwards, and a discharge valve $V_{2}$ opening outwards and 
delivering pressure water into an air vessel $A$, from which it is delivered in a steady stream by the discharge pipe $P$.

The action of the ram is as follows: The waste valve being opened, water is free to escape, and flow is set up along the supply pipe. 'I'he velocity of flow increases under the influence of the supply head until the dynamic pressure on the under side of the valve becomes sufficiently great to overcome its weight. The valve now closes rapidly and the supply column suffers a consequent retardation which gives rise to a rapid increase of

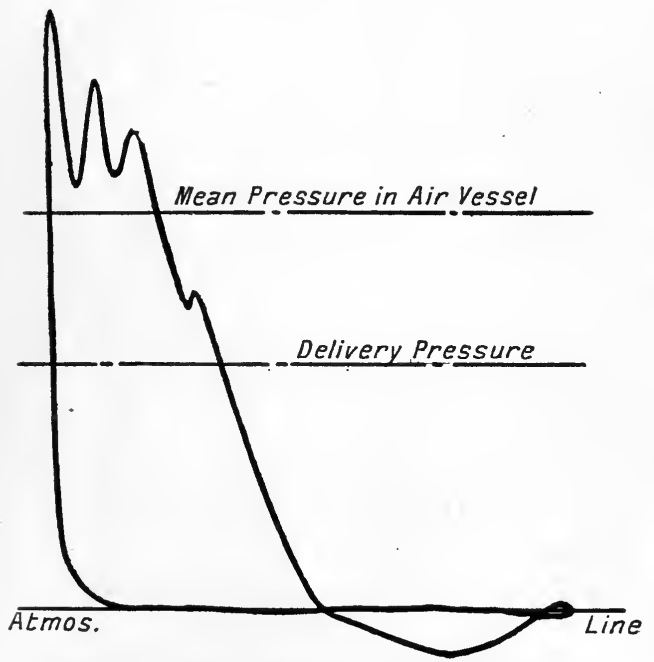

Fig. 266.-Pressure Diagram from Valve Box of Hydraulic Ram. pressure in the valve box until this pressure becomes sufficiently great to open the delivery valve.

Water then escapes through this valve into the air vessel, compresses the air, and flows away in a more or less steady stream along the rising main.

As the air pressure falls the delivery valve closes, the water below the valve partaking of the backward motion thus instituted. This motion, once set up, can only be checked by a reduction of pressure in the valve box below that corresponding to the statical head-in other words, the inertia of the column produces a partial vacuum in its rear-and the pressure in the valve box is reduced rapidly until at some instant the waste valve reopens, and the whole cycle of operations is repeated. Fig. 266 shows a typical pressure diagram from the valve box of such a ram throughout one complete cycle of operations.

The idea that the waste valve reopens because the pressure due to the statical head of the supply column is insufficient to keep it to its seat is quite erroneous. In general, the valve will 
open however light it may be, since the pressure in the valve box is reduced below that of the atmosphere by the reflux action of the water on its rebound. In practice the waste valve is usually made to weigh from $30 \mathrm{lbs}$. to $\cdot 45 \mathrm{lbs}$. per square inch of area, so that a statical pressure corresponding to 1 foot head would be sufficient to prevent any opening.

Advantage is taken of this reduction of pressure to keep the air vessel charged, by the introduction of a snifting valve at $S_{1}$ (Fig. 265 a), air being drawn through this valve into the valve box $B$ when the pressure falls below that of the atmosphere.

The whole cycle, which may only take a fraction of a second to complete, may be divided into four periods, during which the waste valve is respectively opening, wide open, closing, and closed.

Since the force tending to open the valve is equal to its weight together with a much larger force due to pressure differences on its two faces, and since the acceleration of the valve will therefore diminish as its weight increases, the time of opening will tend to increase slightly as the weight increases, and will also increase with the travel of the valve. Also, as the travel of the delivery valve increases with a diminution in the working head, this increases the reflux action and tends to diminish the time of opening.

The period during which the valve is full open depends entirely on the time necessary to produce the required velocity of flow along the supply pipe, and will therefore increase with the weight of the valve, and with the ratio of length of supply pipe to supply head.

Thus, let:-

$$
\left\{\begin{array} { l } 
{ H = \text { supply head in feet. } } \\
{ h _ { d } = \text { delivery. } } \\
{ l = \text { length of supply pipe. } }
\end{array} \quad \left\{\begin{array}{rl}
a=\text { area of supply pipe in square feet. } \\
a_{v}=\text { effective discharge area of waste valve. } \\
=\text { actual discharge area multiplied by the } \\
\text { co-efficient of discharge. }
\end{array}\right.\right.
$$

Then, if $v=$ velocity and $a=$ acceleration in the supply pipe when the valve has been open for a time $t$ seconds, on equating the head in the supply tank to that at exit together with that 
necessary to accelerate the supply column and to overcome frictional resistances, we have:-

$$
H=\frac{l a}{g}+\frac{f l v^{2}}{2 g m}+\frac{v^{2}}{2 g} \cdot \frac{a^{2}}{a_{v}{ }^{2}} \text { feet }
$$

(neglecting losses at changes of section).

Assuming the acceleration to be uniform, as will be substantially the case while the valve is opening and is wide open, so that $v=a t$, this becomes:-

$$
H=\frac{l}{g}\left\{\frac{v}{t}+\frac{f v^{2}}{2 m}\right\}+\frac{v^{2}}{2 g} \cdot \frac{a^{2}}{a_{v}^{2}} .
$$

Solving this quadratic, we get:-

$$
v=\frac{m l}{t\left\{f l+\frac{a^{2}}{a_{v}^{2}} m\right\}}\left\{\sqrt{\left.1+\frac{2 g H\left(f l+\frac{a^{2}}{a_{v}^{2}} m\right) t^{2}}{m l^{2}}-1\right\}}\right.
$$

from which $v$ may be obtained in terms of $t$, when the law of valve closing iś known.

Expressed otherwise, this becomes :-

$$
t=\frac{v l}{g H-\frac{v^{2}}{2}\left\{\frac{a^{2}}{a_{v}^{2}}+\frac{f l}{m}\right\}}
$$

and gives the time necessary to produce a given velocity $v$ of flow in the supply pipe. If $v$ be the velocity necessary to close the valve, $t$ gives the time from the instant of opening, until it begins to close. In practice its weight is usually arranged so as to give $v$ a value between 5 and 8 feet per second, increasing with increasing delivery heads.

The time which the valve takes to close cannot be calculated with any pretension to accuracy. It will evidently depend largely on the form of the valve body and of the valve box, and will increase as the lift of the valve, and its weight, increase.

The total time during which it remains off its seat will thus 'increase with the delivery head and with the length of supply pipe, and will diminish as the ratio $H \div l$ increases, also increasing with its weight and lift.

The time during which it is on its seat increases with the distance from valve seat to delivery air chamber, and increases as the delivery head increases, since the first of these factors 
regulates the time taken by the reflex pressure wave to reach the waste valve, and the second regulates the time at which the delivery valve closes, and hence the time of initiation of this reflex wave.

Eytelwein gives the following values as being approximately' correct for an average case :-

Time during which valve is full open $=\cdot 6$ the period of a complete cycle.

Time during which valve is closed $=\cdot 25$ the period of a complete cycle.

Leaving approximately 10 times the cyclical period for the closing and $\cdot 05$ for the opening of the valve.

\section{Efficiency of Ram.}

If $Q=$ volume leaving supply reservoir, in cubic feet per second, under a head $H$ feet.

If $q=$ volume delivered against a head $h_{d}$ feet, the useful work performed per second $=W q h_{d}$ foot lbs. while the energy in the supply water $=W Q H$ foot lbs.

$$
\therefore \quad \text { Efficiency } \eta=\begin{aligned}
& W q h_{d} \\
& W Q H
\end{aligned}=\frac{q h_{d}}{Q H}
$$

The volume passing the waste valve per second $=Q-q$ cubic feet.

Sources of Loss. - These are due to :-

(1) Leakage at waste valve.

(2) Resistance of valves and of supply pipe.

(3) Eddy production due to sudden changes of section.

(4) Loss of resilient energy.

Leakage from the waste valve increases with the time during which it is off its seat; with the lift and area of the valve; and with the velocity of efflux; all of which, with the exception of the valve area, must be increased with an increase in the delivery head.

The area of the valve is usually made from two to four times that of the supply pipe, an increased area with a correspondingly reduced lift tending to efficiency in working.

This is the most serious of all the sources of loss and usually accounts for between 15 and 25 per cent. of the total energy received. 
To enable it to be reduced as far as possible, the weight and travel of the waste valve should be adjustable to suit any given conditions of working. It should be noted that this loss is not necessarily least when the time during which the valve is open is reduced to a minimum, for with a given supply and delivery head there is a certain valve travel below which the pressure will not exceed the delivery head. Increasing the travel increases

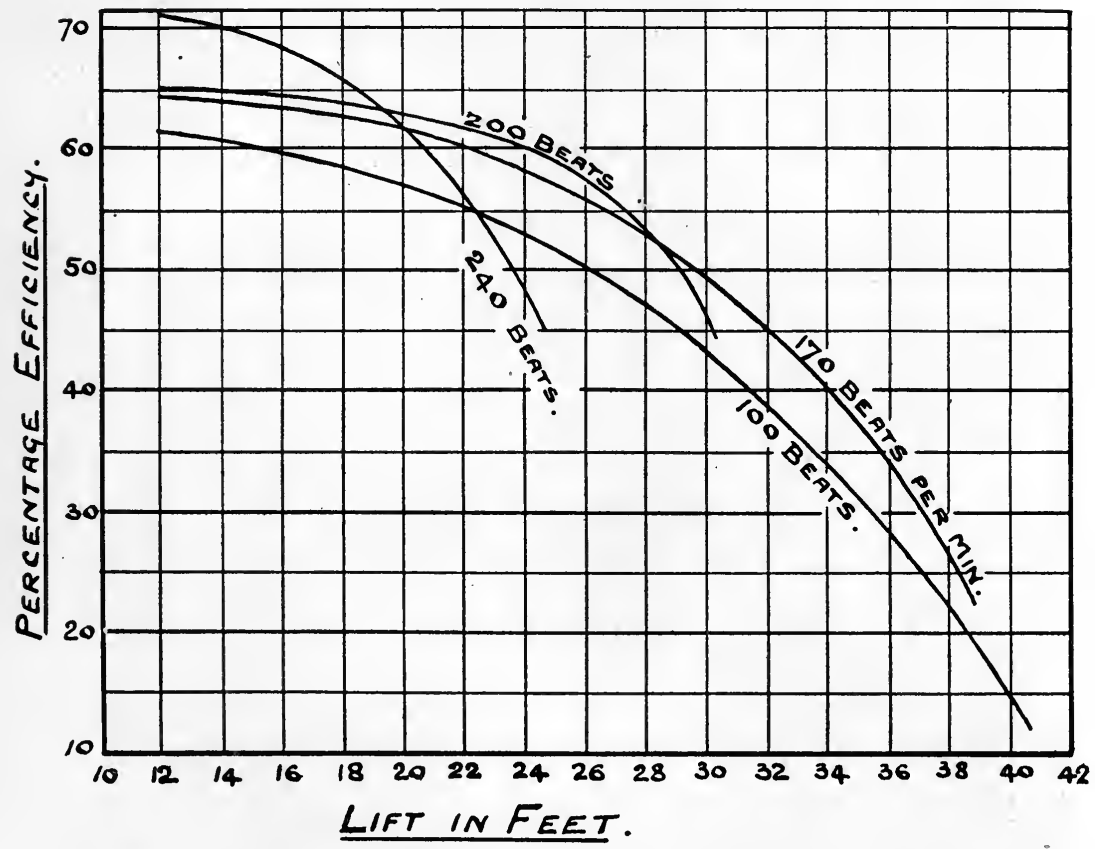

FIcx. 267.-Efficiency Curves for Hydraulic Ram working under a constant head of 4.5 feet.

the velocity of efflux and the time of opening, and therefore the leakage, but at the same time increases the pressure and therefore the proportion of energy entering the air chamber. This goes on up to a certain point, which can only be determined experimentally, where the increased leakage losses counterbalance the proportional gain of energy, and which gives the most efficient working lift.

This point is brought out in Fig. 267 which shows a series of 
efficiency curves obtained by the author from a small hydraulic ram of the type shown in Fig. 265a, having a supply pipe 4 feet 6 inches long and $1 \frac{1}{4}$ inches diameter, and working under a uniform head of 4 feet 6 inches, the waste valve being $1 \frac{1}{2}$ inches diameter. From these curves it is evident that although with low delivery heads the efficiency increased with an increase in the number of beats of the waste valve per minute, as the delivery head was increased the speed for maximum efficiency rapidly diminished.

Valve resistance will be approximately independent of head, while loss by shock and frictional losses in supply and delivery pipes increase as the velocity, and therefore as the delivery head, increases.

The loss of energy due to resilience, being proportional to the square of the pressure of the water at the instant of closing the delivery valve, will vary as the square of the delivery head, and also with the mass of water affected, and will therefore increase with the length of the supply pipe and with the ratio $l \div H$.

Evidently, then, the most economical working is to be expected with a ram in which the delivery head is low and in which the ratio $l \div H$ is small. While the latter factor is not essential for fairly efficient working, it is advisable where possible that this ratio should not exceed 25 . However, where necessary this may be largely exceeded, and a supply pipe length of 1,000 feet with a ratio $l \div H=25$ is well within the limits of everyday practice.

The delivery head may be anything up to about 250 feet and the supply head anything above 18 inches, but the ram becomes very inefficient as the ratio of delivery to supply head becomes great.

The ram will work with this ratio as great as 30 to 1 , but under such circumstances has an efficiency not exceeding about 20 per cent. With lower delivery heads, up to about four times that of the supply, the ram will transform up to 75 per cent. of the energy in the supply reservoir into useful work.

Rankine gives the efficiency in terms of the ratio $\frac{h_{d}}{H}$, as being equal to

$$
1 \cdot 12-\cdot 2 \sqrt{\frac{h_{d}}{H}}
$$


and while this cannot be looked upon as being generally true, it indicates how rapidly the efficiency falls off as $\frac{h_{d}}{H}$ increases.

The curves of Fig. 267 also bring out this fact very clearly.

The simple type of ram already described gives excellent results where the diameter of supply pipe does not exceed about 4 inches.

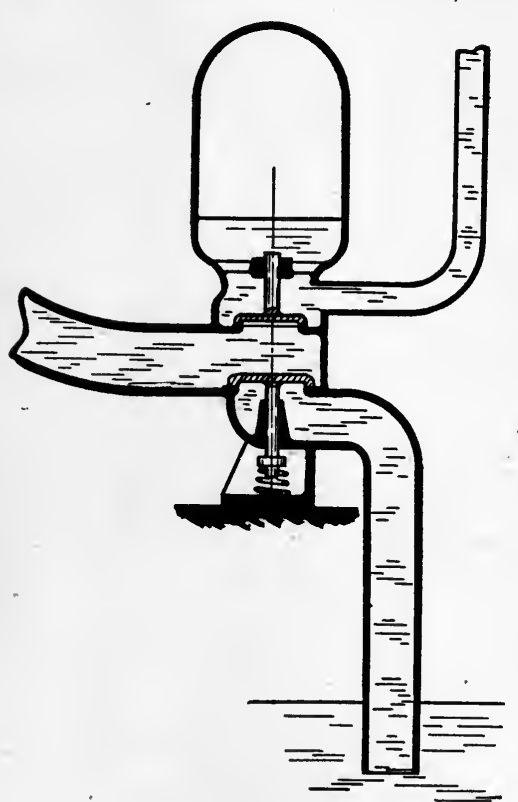

Fig. 268. With larger sizes the shock caused by the sudden closing of the waste valve becomes excessive, and though various devices have been adopted to prevent this, none of them have proved satisfactory as applied to the ordinary ram. One such device is illustrated in Fig. 265 b. Here an air cushion, regulated by the aircock $K$, is provided for the waste valve, but although this effectively prevents shock, it also prevents any high degree of efficiency being obtained. This is clear if it is remembered that the velocity of efflux of the waste water is increasing the whole of the time that the valve is closing and has its maximum value immediately before the valve comes to its seat, so that leakage during this portion of the cycle is more important than at any other time. Slowness of closing is thus particularly detrimental as the valve approaches its seat, and in fact the more quickly the valve reaches its seat after once beginning to close, the less will be the consequent loss of energy in the waste water expressed as a proportion of the whole kinetic energy of the column. A further drawback to the device lies in the fact that because of this leakage it becomes impossible to pump against a head greater than about six times the supply head. 
In the above example the upward pressure of the water on the valve while closing is increased by the provision of a deflector attached to the valve spindle, this deflector also serving as the piston of the air buffer. An auxiliary waste valve $V_{3}$, mounted on the main valve spindle as shown, opens with the main valve, and allows any gritty material or pebbles to escape.

If necessary, the hydraulic ram may be situated well above the tail water level, the waste valve then discharging into a closed chamber which communicates with the tail water by means of a suction tube. The suction head produced in this discharge pipe then helps to increase the velocity of flow on opening the waste valve. This valve must now, however, be aided by means of a

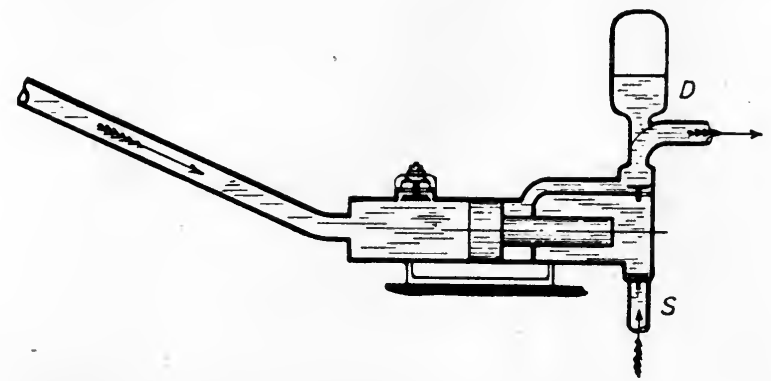

FIG. 269.-Hydraulic Ram for pumping clean water by means of a larger supply of dirty water under a lower head.'

spring so as to be approximately balanced under this suction head. Fig. 268 shows diagrammatically an arrangement of this type devised by Decours, which has given very good results.

Fig. 269 shows the hydraulic ram as arranged for utilizing a large supply of impure water for pumping a smaller supply of clean water. Here $S$ and $D$ indicate the clean water suction and delivery pipes.

In place of the differential plunger pump shown, a flexible diaphragm is sometimes used, dividing the waste valve box from the clean water supply and delivery valve box, the vibration of this diaphragm under the action of the ram acting in the same manner as that of the plunger in the previous sketch.

As thus constructed, the clean water may be lifted against practically any head though the efficiency is only low.

H.A. 
A simpler device, but one not so certain in its action, consists in the provision of a pipe leading the clean water under a head about $\frac{1}{3}$ that of the supply head into that part of the valve box remote from the supply pipe. A check valve prevents flow out of the valve box along this pipe, and a charge of clean water is drawn into the valve box as the pressure falls at each beat. The action may be regulated with considerable nicety by the provision of a regulating valve on this secondary supply pipe.

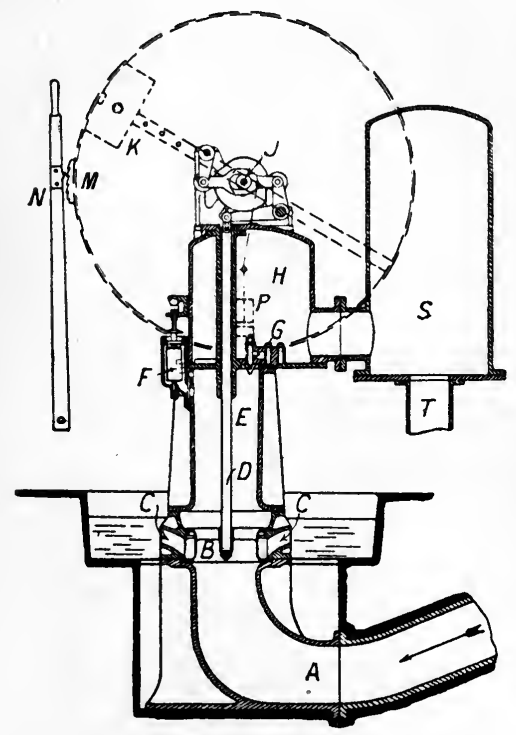

Fig. 270.-Pearsall's Hydraulic Engine or Ram.

It was not until the invention of the hydraulic engine of Mr. Pearsall that the ram attained its highest development and became a really efficient machine for successfully handling large volumes of water. In general terms, this only differs from the ordinary ram in that its valve is opened and closed by mechanical means, this enabling a cylindrical balanced valve to be used, and the periods of the various portions of a cycle to be regulated to suit any given conditions of working.

Fig. 270 shows the general arrangement of the machine. Here $A$ is the supply pipe and $B$ the cylindrical waste valve, which is operated by the valve rod $D$, and which allows water to escape by the ports $C$. On closing this valve water enters the chamber $E$ without shock and drives out before it the contained air, through a valve regulated by the wooden float $F$. When the water reaches a certain height the valve at $F$ closes, and the pressure in $E$ rises until sufficient to lift the delivery valves at $G$. The small remaining volume of compressed air and the water then enter the air vessel $H$, from which the water is led away along the delivery pipe $T$. A second air chamber $S$ is sometimes fitted, but 
is not essential. The valve $B$ is now opened, the rush of water out of $E$ and down the supply pipe is followed by its closure, and the cycle of operations is repeated as before.

The method of working this valve is ingenious. The shaft $J$ carries a pendulum $K$, which swings through an arc of about $240^{\circ}$, and also a can so proportioned as to divide the time of a swing of the pendulum into two parts suitable for the flow and delivery parts of the cycle. 'This cam regulates the motion of the valve rod.

In order to maintain the swing of the pendulum against friction, a crank on $J$ is coupled to a piston in the small single-acting

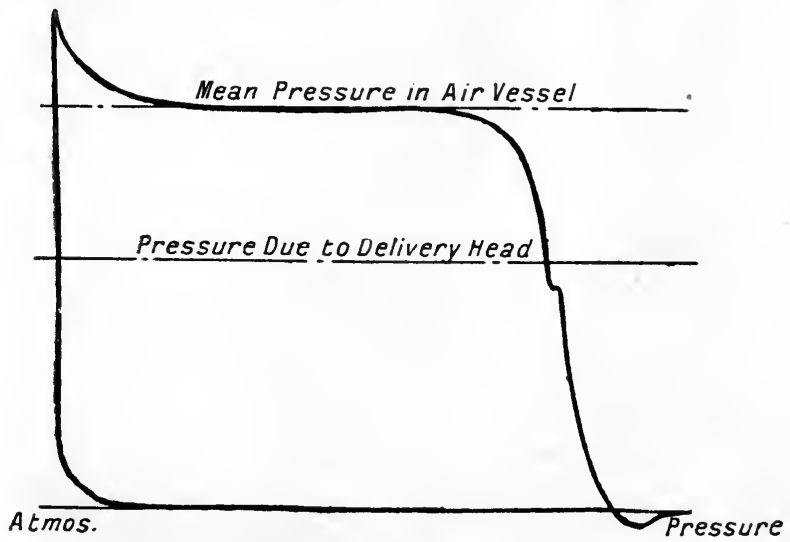

Frg. 271.--Pressure Diagram from Pearsall's Hydraulic lam.

cylinder $P$, into which air is admitted from the air vessel at each double stroke, so as to give a slight impetus to the pendulum at the middle of its swing.

The engine is stopped and started by the bar $N$, which, when held against the pendulum, engages with the rachet $M$ when this begins to descend. This stopping takes place immediately before the end of a working stroke, when the main valve is closed and when the flow of water in the main pump has for the moment ceased. On releasing the rachet, the ram at once engages on a normal stroke.

As thus constructed, the machine is capable of dealing with practically any quantity of water, and works as noiselessly as a pumping engine. The smoothness of its working as compared 
with that of the ordinary type of ram may be inferred from Fig. 271, which shows a diagram taken from the valve box of a ram of the Pearsall type.

The simplicity of the mechanism of the hydraulic ram, its. high efficiency, and the fact that it is capable of working for very long periods without attention, render it specially well fitted for use in a private pumping plant, and there is every indication that at the present time its many advantages are being to an increasing extent realized.

Hydraulic Ram for Air Compression.-With slight modifica-

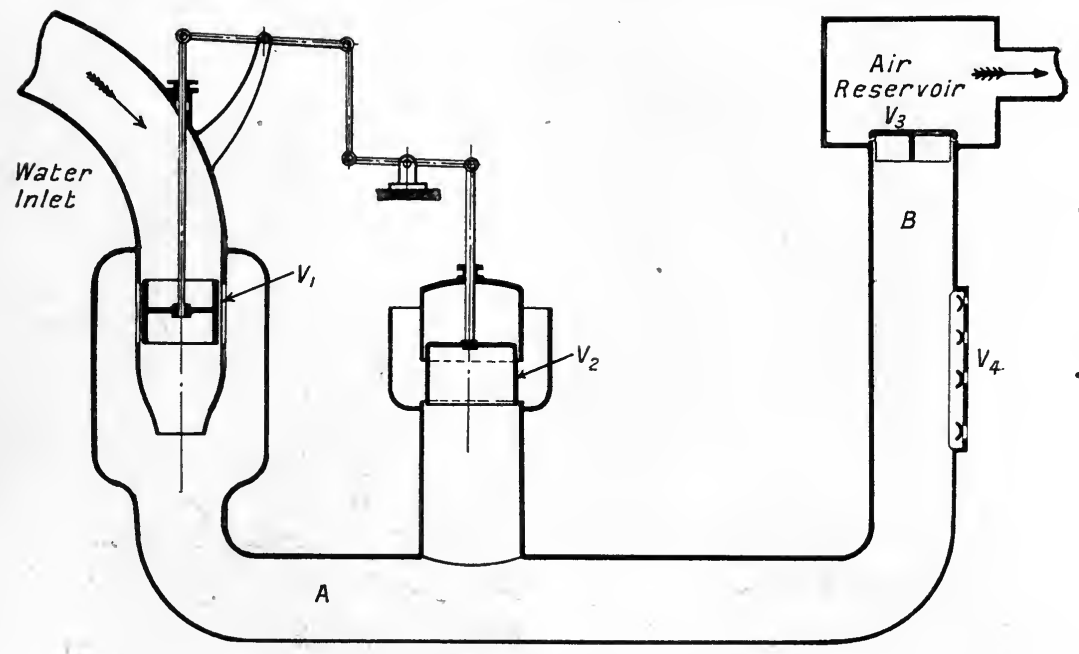

FI(x. 272.-Hydraulic Air Compressor.

tions the hydraulic ram may be adapted for use as an air compressor. Thus in the Pearsall ram the chamber $E$ is enlarged so as to hold the whole volume of air compressed in a single stroke, while the valve at $F$ is modified so as to confine this air, and the delivery valves at $G$ are slightly modified. The cylinder $P$ is also now worked by pressure water from the air vessel $E$ instead of by compressed air. As thus constructed, efficiencies of upwards of 80 per cent. may be obtained.

Fig. 272 shows a type of air compressor on somewhat similar lines, designed by M. Sommellier and used in the work on the Mont Cenis Tunnel. Here the inlet valve $V_{1}$ and the waste: 
valve $V_{2}$ are mechanically driven, and are coupled together. When the valve $V_{2}$ is opened the water in the compressor finds its own level and air at atmospheric pressure is drawn in through the air valve $V_{4} . \quad V_{2}$ is then closed and $V_{1}$ opened, allowing pressure water to flow along the pipe $A$, and in virtue of its pressure and momentum to compress the air in the chamber $B$, whence it passes through the delivery valve $V_{3}$ into the air reservoir. $\quad r_{1}$ is then closed, $r_{2}$ opened, and the cycle of operations repeated as before. Working under a head of 85 feet, this compressor delivers air at a pressure of 75 lbs. per square inch. The machine is, however, somewhat cumbrous for the amount of work which it is capable of performing.

Art. 176.-The Jet Punp.

The fact that the pressure energy of a water supply may be converted into kinetic energy, with a consequent reduction

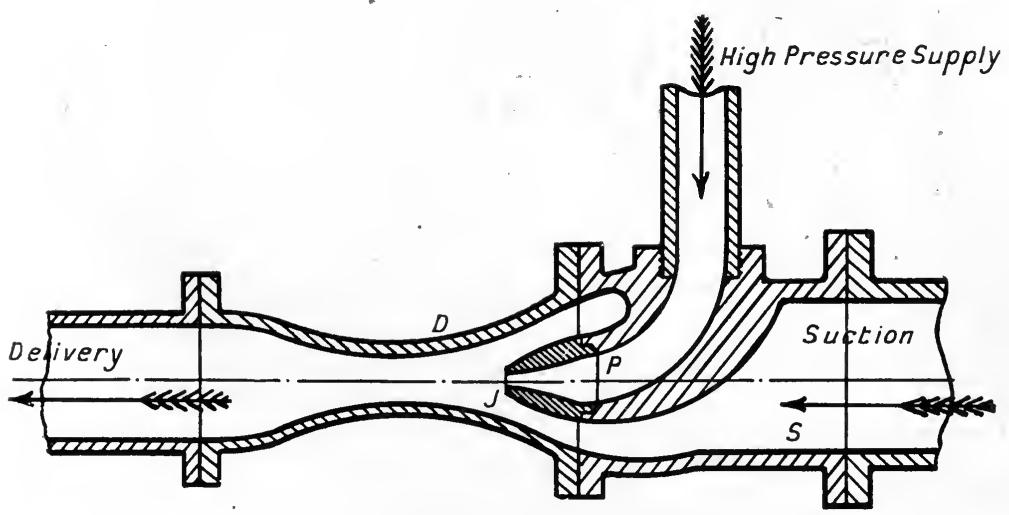

Fĩส. 273. -Jet Pump.

of pressure, is taken advantage of in the type of jet pump devised by Professor James Thomson (about 1852). The pump, as usually constructed, is illustrated in Fig. 273. Here water from the source of supply is led through the converging passage $P$, its pressure diminishing as its velocity increases, and is finally discharged into the delivery pipe through the diverging passage $D$. It follows that at the section $J$, where its velocity is greatest, its pressure may'become considerably less than that of 
the atmosphere. A chamber surrounding $J$ is connected to the supply of water to be pumped, by the suction pipe $S$. The reduction of pressure in this chamber is then accompanied by a flow of water along the suction pipe, which, meeting the high pressure jet, is carried forward as a combined stream into the diverging discharge pipe $D$. Here its kinetic energy is partially reconverted into pressure energy, which is utilized in overcoming the head to be pumped against.

Let $h_{1}=$ height of supply head above jet in feet.

, $h_{s}=$ height of jet above suction supply ,

, $H_{d}=$ height of delivery head above jet ,

, $n_{d}=\left\{\frac{p_{d}}{W}+\frac{r_{d}^{2}}{2 g}\right\}=H_{l}+$ losses between point $D$ and delivery.

, $a_{1}=$ area of nozzle in square feet.

, $a_{s}=$ area of annular suction pipe in plane of nozzle, square feet.

„, $a_{d}=$ area of mixing chamber at throat, square feet.

,$Q=$ volume passing per second, $v=$ velocity, and $p$ the pressure in lbs. per square foot at the point denoted by a suffix.

On the assumption that the sides of the mixing chamber are sensibly parallel from the nozzle until the jets have attained a common velocity and pressure at 1 ), so that, neglecting friction, the sides of the chamber exert no force on the water, we may apply the equation of momentum, which now becomes:-

$$
\begin{aligned}
& \frac{Q_{d} r_{d}}{g}-\left\{\frac{Q_{s} r_{s}}{g}+\frac{\left(p_{1} r_{1}\right.}{g}\right)=\frac{p_{1} a_{1}+p_{s} a_{s}-p_{d} a_{d}}{W} \\
& \therefore \quad \frac{a_{d} v_{d}{ }^{2}-a_{s} r_{s}^{2}-a_{1} r_{1}^{2}}{g}=\frac{p_{1} a_{1}+p_{s} a_{s}-p_{d} a_{d}}{W^{\prime}} \\
& \therefore \quad a_{1}\left(\frac{p_{1}}{W^{T}}+\frac{r_{1}^{2}}{2 g}\right)+a_{s}\left(\frac{p_{s}}{W^{2}}+\frac{r_{s}^{2}}{2 g}\right)-a_{d}\left(\frac{p_{d}}{W}+\frac{v_{d}{ }^{2}}{2 g}\right) \\
&=\frac{1}{2 g}\left\{a_{d} r_{d}{ }^{2}-a_{s} r_{s}{ }^{2}-a_{1} r_{1}^{2}\right\}
\end{aligned}
$$

or $\quad a_{1} h_{1}-{ }^{1} a_{s} h_{s}-a_{d} h_{d}=\frac{1}{2 !}\left\{a_{d} r_{d}^{2}-a_{s} r_{s}^{2}-a_{1} r_{1}^{2}\right\}$

1 Negative sign because $\left(\frac{p_{s}}{W^{-}}+\frac{r_{g}^{2}}{2 !}\right)$ is negative if $h_{8}$ is positive. 
Again, for continuity of flow, we have :-

$$
\text { or } \left.\begin{array}{l}
a_{1} v_{1}+a_{s} r_{s}=a_{d} v_{d} \\
Q_{1}+Q_{s}=Q_{d}
\end{array}\right\}
$$

From which equations, when the dimensions of the pump and the heads are given, any two unknown velocities, and hence quantities, may be determined. For example, if $Q$ is given, $Q_{s}$ and $Q_{d}$ may be determined. If, in addition, we assume that the pressure across the mixing chamber immediately in front of the nozzle is uniform, and equal to $p_{j}$ (an assumption which is only true so long as both streams are parallel), we have

$$
\begin{aligned}
& \left\{\begin{aligned}
h_{1} & =\frac{r_{1}^{2}}{2 g}+\frac{p_{j}}{W^{r}} \\
-h_{s} & =\frac{r_{s}^{2}}{2 g}+\frac{p_{j}}{W^{r}}
\end{aligned}\right. \\
& \therefore \quad \frac{r_{1}{ }^{2}}{2 g}-h_{1}=\frac{r_{s}{ }^{2}}{2 g}+h_{s} \\
& \text { or } r_{s}^{2}=r_{1}^{2}-2 g\left(h_{1}+h_{s}\right) \text {. }
\end{aligned}
$$

Introducing this value of $v_{s}$ in (1), we get:-

$$
\begin{aligned}
& a_{1} h_{1}-a_{s} h_{s}-a_{d} h_{d}=\frac{1}{2 g}\left\{a_{d} v_{d}^{2}-\left(a_{1}^{\prime}+a_{s}\right) v_{1}^{2}\right\}+a_{s}\left(h_{1}+h_{s}\right) \\
& \text { or }\left(a_{1}-a_{s}\right) h_{1}-2 a_{s} h_{s}-a_{d} h_{d}=\frac{1}{2 g}\left\{a_{d} v_{d}^{2}-\left(a_{1}+a_{s}\right) v_{1}^{2}\right\} . \text { (4) }
\end{aligned}
$$

While, by substitution in (2), we have:-

$$
a_{1} v_{1}+a_{s} \sqrt{{v_{1}^{2}}^{2}-2 g\left(h_{1}+h_{s}\right)}=a_{d} v_{d} .
$$

From equations (3), (4) and (5), if the areas of the passages and the various heads are given, the velocities $v_{1}, v_{s}$ and $v_{d}$, and thus the quantities $Q_{1}, Q_{s}+Q_{d}$, may be determined.

\section{Exayple.}

\section{Thomson's Jet Pump.}

$$
\begin{array}{ll}
h_{1}=40 \text { feet. } & a_{1}=\cdot 2 \text { square feet. } \\
h_{s}=15 \text { feet. } & a_{s}=\cdot 4 \text { square feet. } \\
h_{d}=10 \text { feet. } & a_{d}=\cdot 6 \text { square feet. }
\end{array}
$$

Determine $Q_{1}, Q_{s}$ and $Q_{d}$, and also the pressures $\frac{p_{D}}{W}$ at the throat of the mixing cone and $\frac{p_{j}}{W}$ in the plane of the orifices. 
From equation (4) we have :-

$$
\begin{gathered}
-\{\cdot 2 \times 40+\cdot 8 \times 15+\cdot 6 \times 10\} \frac{64 \cdot 4}{6}=v_{d}^{2}-r_{1}^{2} \\
\therefore r_{1}^{2}-r_{d}^{2}=2,790 .
\end{gathered}
$$

Again, from (5),

$$
\begin{aligned}
& \left(\cdot 6 v_{d}-2 r_{1}\right)^{2}=\cdot 16\left\{r_{1}^{2}-64 \cdot 4 \times 55\right\} \\
& \therefore r_{1}^{2}+2 v_{1} v_{d}-3 r_{d}^{2}=4,718 . \quad \text { (ii) }
\end{aligned}
$$

Substituting for $v_{d}^{2}$ in (ii) from (i), we get:-

$$
\begin{gathered}
r_{1}^{2}+2 r_{1} \sqrt{r_{1}^{2}-2,790}-3\left(r_{1}^{2}-2,790\right) \\
\therefore \quad r_{1}^{2}-1,826=v_{1} \sqrt{r_{1}^{2}-2,790} .
\end{gathered}
$$

Squaring both sides, we get, on reduction :-

$$
\begin{aligned}
862 r_{1}^{2} & =3,333,000 \\
\text { or } r_{1}^{2} & =3,866 \\
r_{1} & =62 \cdot 2 \text { feet per second. }
\end{aligned}
$$

Substituting this value in (i), we have :-

$$
r_{d}=\sqrt{3,866-2,790}=32 \cdot 8 \text { feet per second; }
$$

while from equation (3) we have :-

$$
\begin{aligned}
& r_{s}= \sqrt{3,866-3,542}=18 \cdot 0 \text { feet per second. } \\
& \therefore \quad Q_{1}=\cdot 2 r_{1}=12 \cdot 4 \text { cubic feet per second. } \\
& Q_{d}=\cdot 6 r_{d}=19 \cdot 7 \text { cubic feet per second. } \\
& Q_{s}=\cdot 4 r_{s}=7 \cdot 2 \text { cubic feet per second. }
\end{aligned}
$$

Again, since $\frac{p_{j}}{W}+\frac{v_{1}^{2}}{2 g}=h_{1}$, we have :-

$$
\frac{p_{j}}{W^{r}}=40-60 \cdot 1=-20 \cdot 1 \text { feet of water } ;
$$

while since $\quad h_{d}=\frac{p_{d}}{W}+\frac{v_{d}^{2}}{2 g}$, we have :-

$$
\frac{p_{d}}{W}=10-16.7=-6.7 \text { feet of water. }
$$

The actual height through which the water may be forced by the pump is less than the value $h_{d}$ given by $\left\{\frac{p_{d}}{W}+\frac{v_{d}^{2}}{2 g}\right\}$ feet, because of the loss of energy by eddy formation in the diverging discharge pipe, and may amount to between $\cdot 6$ and $\cdot 7 h_{d}$.

This loss of energy is proportional to $r_{d}^{2}$, and will therefore increase-since the necessary value for $v_{d}$ increases-as $h_{d}$ increases. Consequently, since the total work done in pumping 
is proportional to $h_{d}+h_{s}$, while this loss depends only on $h_{d}$, the efficiency will increase as $h_{s}$ is increased at the expense of $h_{d}$.

It follows that with a given total lift, the suction head should be increased as far as possible-up to about 22 feet-at the expense of the delivery head. This conclusion is borne out in practice.

The efficiency of the pump is given by -

$$
\eta=\frac{Q_{s}\left(H_{d}+h_{s}\right)}{Q_{1}\left(h_{1}-H_{d}\right)}
$$

This efficiency is of necessity low, since the action depends on the mixing of two streams moving with different velocities, and hence involves considerable loss by shock. E.g., in the numerical example considered on p. 664, the efficiency (assuming $\left.H_{d}=\cdot 7 h_{d}\right)$ is given by-

$$
\eta=\frac{7 \cdot 2(7+15)}{12 \cdot 4 \times 33}=\cdot 387 .
$$

Actually, frictional losses reduce the efficiency still further, and the maximum efficiency attained in practice, even with a pump placed at delivery level, is about 25 per cent. This is increased to about 30 per cent. where, as when used for delivering a high velocity jet of water for fire purposes, the necessity for converting the kinetic energy of the jet into pressure energy is absent.

For continuous pumping and drainage operations where a fair pressure supply is obtainable, and where the volume to be lifted and the working head are small, the method offers the advantages of simplicity and low first cost, while practically no attention is required. Unless the supply head is large compared with the lift, the ratio $\frac{Q_{s}}{Q}$ is, however, very small-often so low as $\frac{1}{5}$.

The principle of the steam injector, as fitted for boiler feed purposes, is identical with that of the jet pump. The preceding equations; however, need to be modified, since the streams of fluid on impinging are not of equal density, although they become so on condensation of the high velocity steam jet.

The principle of the jet pump has been applied in an intensifier for raising the pressure of a large quantity of low 
pressure water by means of a small supply at high pressure, the delivery pipe $D$ leading directly into the cylinder of the intensifier. While not economical from an energy standpoint, the simplicity and low first cost of the apparatus render it very suitable for such work where its use is only occasional.

\section{Art. 177.-'The Injector Hydrant.}

Since the chief loss of energy in the ordinary jet pump is due to shock at the collision of the two jets, and since this loss

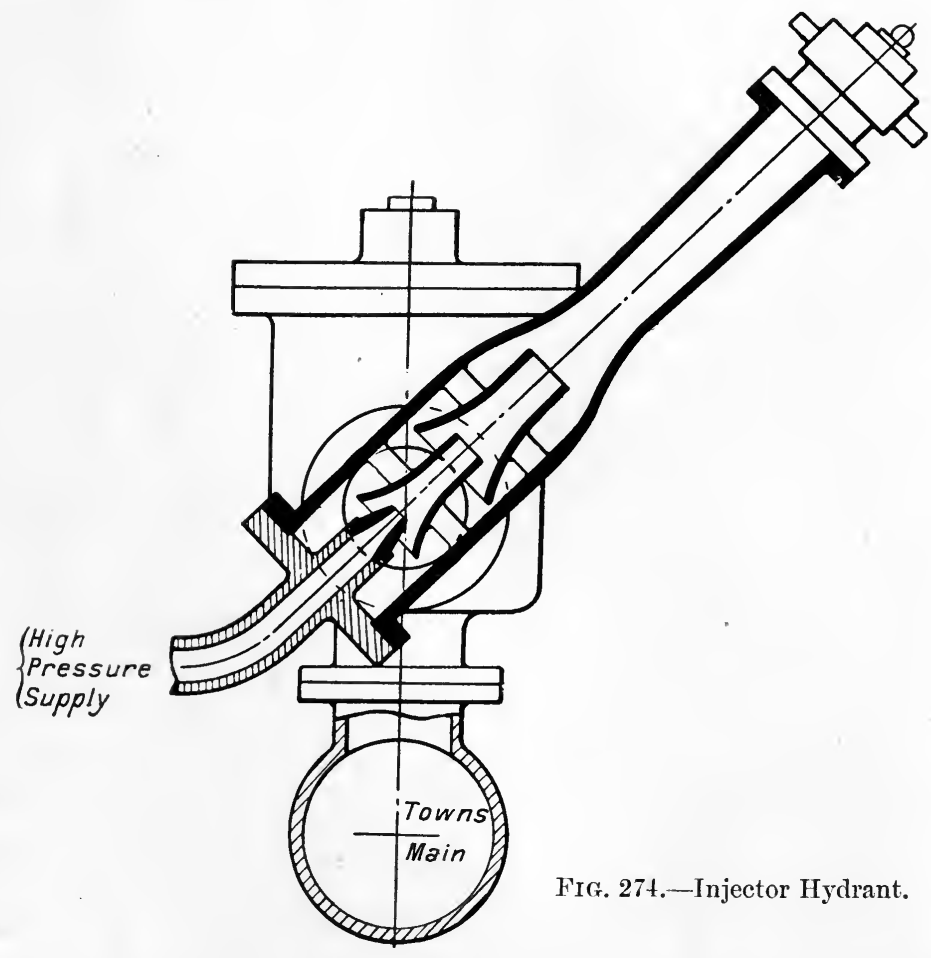

increases with their velocity difference, it would appear that the total loss might be diminished by diminishing the velocity of the high pressure, or increasing that of the low pressure jet in stages, instead of at a single impact.

This method has been applied with success by Mr. Greathead 
in the construction of his injector hydrant (Fig. 274), which is adapted for fire extinguishing purposes where a continuous supply of high pressure water is available, as is the case near the pipe line from an hydraulic power station. By itself, the water in the power main is of insufficient volume to have any appreciable effect on a fire, but when used with an injector hydrant in connection with a low pressure main the advantages of the system are very great As exemplifying the effect of a small jet of high pressure water in increasing the height of the main jet, it is stated that while an ordirary $1 \frac{1}{8}$-inch hydrant supplied from a main at $40 \mathrm{lbs}$. pressure will give a stream about 50 feet high, the same when reinforced by a $\frac{3}{8}$-inch jet of water at $750 \mathrm{lbs}$. per square inch will lift a slightly greater (about 15 per cent.) volume of water from the main and will deliver this as a jet 85 feet high.

The efficiency of the hydrant from an energy point of view ranges from 28 per cent. to 33 per cent.

The following values are given by $\mathrm{Mr}$. Greathead ${ }^{1}$ as being the quantity of high pressure water, at $700 \mathrm{lbs}$. per square inch, required to deliver a jet of $\mathbf{1 5 0}$ gallons per minute through a 1-inch nozzle, through a height variously estimated to be from 75 to 84 feet, and requiring a head at the nozzle of 100 feet. The pressure of the low pressure supply was varied as shown. Here allowance is made for 200 feet of $2 \frac{1}{2}$-inch hose, the resistance of this length of hose being equivalent to 50 feet head.

\begin{tabular}{c|c|c}
\hline \multicolumn{2}{c|}{ I.ow Pressure Supply. } & High Pressure supply: \\
\hline Ihbs per square incli. & Feet. & Gallons per minute. \\
\hline 60 & 139 & $3 \cdot 7$ \\
50 & 115 & $10 \cdot 9$ \\
40 & 92 & $18 \cdot 1$ \\
30 & 69 & $25 \cdot 2$ \\
20 & 46 & $32 \cdot 4$ \\
10 & 23 & $39 \cdot 6$ \\
\hline
\end{tabular}

1 "Proceerlings Institute Mechanical Engineers," 1879, 1. 364. 


\section{Art. 178.-'The Air Lift Puip.}

Among other devices for pumping liquids against a large head that known as the air lift pump is worthy of notice. Invented probably by Carl Löscher about 1797, the system fell into comparative desuetude for many years, and has only recently been revived and improved. In view of its increasing use, and of its adaptability to many difficult cases of pumping, it is worth while considering the system somewhat in detail. Briefly, the method consists in sinking an open vertical pipe with its lower end submerged in the liquid to be raised, and having its upper end arranged to discharge into a reservoir at. the required height. Air from a compressor is then forced through a smaller air pipe into the submerged opening of the lift pipe or rising main. The air bubbles, rising through the water in the lift tube, so reduce the specific gravity of the mixture, and therefore the weight of the column, that the excess pressure at the base of the column, due to the external water pressure, becomes sufficiently great to force the mixture above the supply level and out of the top of the pipe. This excess pressure increases with the depth of submersion of the pipe, and the latter must therefore be regulated to suit the height to which water is to be lifted.

In general, the depth of submersion $h_{s}$ (Fig. 275) is made from (1.5 to 2$)$ times the lift $h_{d}$, so that the total length of lift pipe $=(2.5$ to 3$) h_{d}$. As the depth of immersion is reduced, the relative air consumption increases and the efficiency diminishes. As will be shown later, a further increase in $l_{s}$ tends to more efficient working, and is advisable where this may be obtained without great expense in deepening the bore hole.

To keep down frictional losses, the velocity in the lift tube, calculated on the volume of water discharged, should not exceed 5 feet per second. The efficiency of the system, as calculated from the ratio of the work done in lifting water through a height $h_{d}$, to the indicated work in the air compressor engine cylinder in the same time, is generally between 25 and 30 per cent.- this allowing for a compressor efficiency of 75 per cent.but under favourable conditions may rise to 45 per cent. 


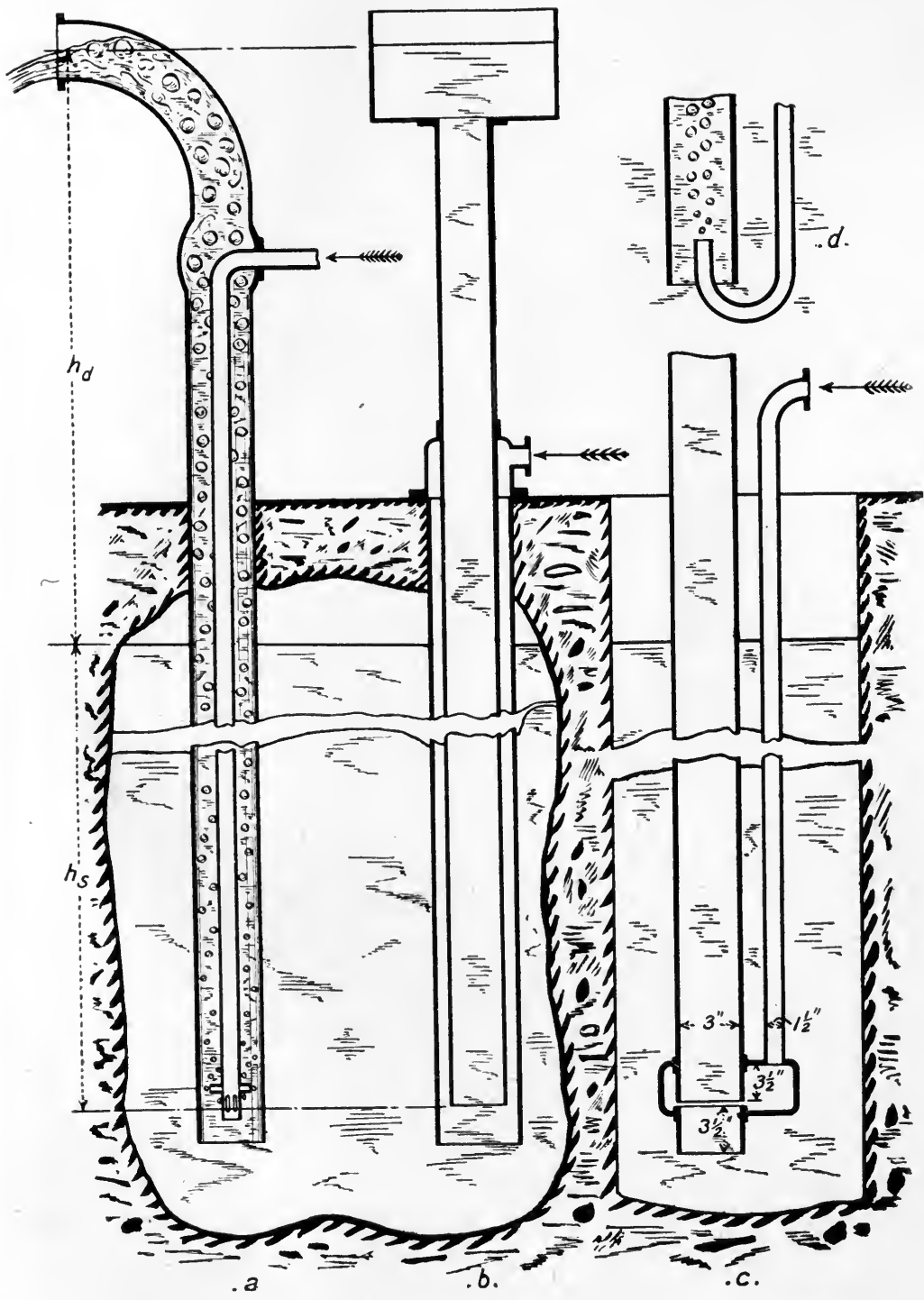

FIg. 275.-Air Lift Pump: 
There are three methods of arranging the pipe lines in a well or bore hole.

(1) The central air tube system (Fig. $275 a$ ), in which the air pipe is suspended in the centre of the lift tube.

(2) The annular air tube system, in which the space between the lift tube and tive bore hole is used as the air line (Fig. $275 b)$.

(3) The side-by-side system, in which the air and lift tubes are carried down the well side by side (Fig. $275 c$ ).

The first of these systems has the disadvantage that the hydraulic mean depth of the water passage in the lift tube is reduced by the air tube, being given by-

$$
\frac{\pi\left(R^{2}-r^{2}\right)}{2 \pi(R+r)}=\frac{R-r}{2}=\frac{D-l}{4},
$$

where $i)$ and $d$ are the internal diameter of the lift tube and the external diameter of the air tube respectively. ${ }^{1}$

This leads to increased frictional losses and so to diminished efficiency. The system is, however, very suitable for application to a small bore hole of suitable dimensions, since the only additional expense is the provision of the comparatively small air pipe, while any alteration in the length of this, to suit different conditions of working, is a simple matter. In general this will be found more advantageous than the second system, the chief advantage of the latter lying in its possibilities of more effective air distribution.

Where the well or bore hole is of large diameter, the side-byside system has many advantages in virtue of its accessibility, simplicity, and flexibility.

In every case provision must be made for distributing the air evenly and in small bubbles among the water in the lift tube, since experiment shows that the system is then much more effective than where the air bubbles are so large as to fill the tube. The diameter of the bubbles at their initiation should be about $\frac{1}{4}$ inch. Some such arrangement of foot box, as shown in Fig. $275 c$, is essential for efficient working. Comparative

1 With a plain tube of the same sectional area the hydraulic mean depth is given by $\frac{D_{1}}{4}$, where $D_{1}=\sqrt{D^{2}-a^{2}}$. 
tests show that this type, in which air enters all around the circumference of the lift tube, gives efficiencies 20 per cent. greater than that shown in Fig. $275 d$, in which the air is supplied in a single central jet. The air pipe should be designed to give a velocity of flow not exceeding 25 feet per second.

\section{Theory of the Air Lift Pump.}

Let $\quad V_{v}=$ volume of water raised in cubic feet per second.

$V_{m}=$ mean volume of air used, in cubic feet per second, during its passage through the rising main.

, $\quad V_{a}=$ volume used in cubic feet per second, at atmospheric pressure, $p_{a}$.

, $\quad p_{1}=$ pressure at base of rising main.

,$\quad h_{d}+h_{s}=l$ feet.

Then, assuming isothermal expansion of the air in the lift tube, we have :-

$$
V_{m}=\frac{p_{a}}{p_{1}-p_{a}}\left(\log _{e} \frac{p_{1}}{p_{a}}\right) V_{a}
$$

The mean specific gravity of the mixture in the tube $=\frac{V_{w}}{V_{v}+V_{n}}$.

$\therefore$ Head producing How $=h_{s}-\left(h_{d}+h_{s}\right) \frac{V_{w}}{V_{w}+V_{m}}$ feet of water,

$$
\begin{aligned}
& =h_{s}\left(\frac{V_{w}+V_{m}}{V_{w}}\right)-\left(h_{d}+h_{s}\right) \text { feet of } \\
& \text { mixture in the lift tube, } \\
& =\frac{V_{m}}{V_{w}} h_{s}-h_{d} \text { feet of mixture. }
\end{aligned}
$$

Equating this to the sum of the friction head $h_{f}$, and the kinetic head $h_{v}$, we have, on reduction:-

$$
\begin{aligned}
& V_{m}=V_{w} \frac{h_{d}+h_{f}+h_{v}}{h_{s}}, \\
\therefore \quad V_{a}= & V_{w} \frac{p_{1}-p_{a}}{p_{a} \log _{c} \frac{p_{1}}{p_{a}^{-}}} \cdot \frac{h_{d}+h_{f}+h_{v}}{h_{s}}, \\
= & \frac{V_{w}\left(h_{d}+h_{f}+h_{v}\right)}{34 \log _{e} \frac{p_{1}}{p_{\vec{a}}}} \cdot\left(\operatorname{since} \frac{p_{1}-p_{a}}{l_{a}}=\frac{34+h_{s}-34}{34}=\frac{h_{s}}{34}\right)
\end{aligned}
$$


This relation enables the volume of free air per cubic foot of water to be determined in terms of $h_{f}$ and $h_{v}$. Writing $h_{r}=$ $\frac{1}{2 g}\left\{\frac{V_{a}+V_{w}}{A}\right\}^{2}$, and putting $h_{f}=\frac{f\left(h_{d}+h_{s}\right) v^{\varepsilon}}{2 g m}$ (where $r^{2}$ is the mean square of the velocity in the tube) this latter term may be taken as approximately equal to $\frac{f\left(h_{s}+h_{a}\right)}{2 g m}\left\{\frac{V_{a}+2 V_{w}}{2 A}\right\}^{2}$, in which case the ratio $V_{a} \div V_{w}$ may be directly obtained in terms of $h_{d}, h_{s}, A$ and $f$.

'The author has determined the values of $f$ for a mixture of air and water (the friction head being expressed in feet of a column of the mixture in the tube) from the published date of a large number of trials on such pumps.

Making the above assumption as to the value of $r$, the value of $f$ varies from 033 in the case of a 3 -inch pipe with $v=12 \cdot 16$ f.s. to 023 in a 12 -inch pipe with $v=6.5 \mathrm{f} . \mathrm{s}$. In every case $f$ has a value very approximately six times that given by the curves of p. 203 for the flow of water alone at the same velocity.

In practice the following approximate values of $V_{a} \div V_{w}$ are found to give the best results :-

\begin{tabular}{l|c|c|c|c|c}
\hline$h_{d}$ (feet) & 10 & 20 & 30 & 50 & 100 \\
\hline$V_{a} \div V_{v \cdot}$ & $1 \cdot 0$ & $1 \cdot 5$ & $2 \cdot 0$ & 2.5 & $3 \cdot 0$ \\
\hline
\end{tabular}

Efficiency of the Air Lift Pump.--Assuming isothermal compression, the work done on the air during compression from $p_{a}$ to $p_{1}=p_{a} V_{a} \log _{e} \frac{p_{1}}{p_{a}}$ foot lbs.

The useful work done by the air in raising $V_{w}$ eubic feet of water through a height $h_{d}$ feet $=62 \cdot 4 V_{w} h_{d}$ foot lbs.

$\therefore$ Efficiency-

$$
\begin{aligned}
\eta=\frac{62.4 V_{w} h_{d}}{p_{a} V_{a} \log _{e} \frac{p_{1}}{p_{a}}} & =\frac{62 \cdot 4 V_{w} h_{d} \times 34 \log _{e} \frac{p_{1}}{p_{a}}}{p_{a} \log _{e} \frac{p_{1}}{p_{a}} \times V_{v}\left(h_{d}+h_{f}+h_{v}\right)} \\
& =\frac{h_{d}}{h_{d}+h_{f}+h_{v}}
\end{aligned}
$$

On the assumption of adiabatic compression from $p_{a}$ to $p_{1}$, the 
work done on the air $=3.463 p_{a} V_{a}\left\{\left(\frac{p_{1}}{p_{a}}\right)^{.29}-1\right\}$ foot lbs., so that the efficiency

$$
=\frac{h_{d} \log _{e} \frac{p_{1}}{p_{a}}}{3.463\left\{\left(\frac{p_{1}}{p_{a}}\right)^{.29}-1\right\}\left\{h_{d}+h_{f}+h_{v}\right\}} .
$$

From expression (2) it appears that as $h_{d}$ is increased from zero (keeping $h_{s}$ constant) the efficiency will increase until some point is reached at which the (velocity of flow) ${ }^{2}$ increases more rapidly than does $h_{d}$. This gives the valve of $h_{d}$ for maximum efficiency. In practice it is found that the ratio $h_{s} \div h_{d}$ then lies between 1.5 and 2.5 , the larger values of the ratio being used with low valves of $h_{d}$.

The following table gives the results of tests on a plant of the type illustrated in Fig. $275 c$, carried out by Professor Jossè at Charlottenburg:-

\begin{tabular}{|c|c|c|c|c|c|c|c|c|c|c|}
\hline Diameter of lift tube, inches & $\cdot \cdot$ & $3 \frac{1}{16}$ & $3 \frac{\pi}{16}$ & $3 \frac{1}{1 \bar{\theta}}$ & $3 \frac{1}{13}$ & $3 \frac{\pi}{16}$ & $7 \frac{1}{2}$ & 23 & 23 & $2 \frac{3}{4}$ \\
\hline Diameter of air pipe, inches & . & $1 \frac{9}{16}$ & $1 \frac{9}{16}$ & $1_{\frac{1}{16}}^{0}$ & $1_{\frac{10}{10}}^{9}$ & $1_{18}^{9}$ & 5 & 一 & 一 & 一 \\
\hline Diameter of bore hole, inches & . & $66_{s}^{1}$ & $6 \frac{1}{8}$ & $6 \frac{1}{8}$ & 61 & $66_{8}^{2}$ & - & 一 & - & - \\
\hline Depth of immersion, $h_{8}$ feet & $\cdot$ & $49 \cdot 2$ & $\overline{49 \cdot 2}$ & $49 \cdot 2$ & $49 \cdot 2$ & $49 \cdot 2$ & 63 & $69 \cdot 2$ & $72 \cdot 9$ & $62 \cdot 0$ \\
\hline Height of lift, $h_{d}$ feet. & $\cdot$ & $24 \cdot 6$ & $24 \cdot 6$ & $24^{\circ} 6$ & $24 \cdot 6$ & $24 \cdot 6$ & $43 \cdot 5$ & $50 \cdot 5$ & 46.7 & $57 \cdot 8$ \\
\hline \multirow{2}{*}{$V_{w}\left\{\begin{array}{l}\text { Gallons per minute } \\
\text { Cubic feet per second }\end{array}\right.$} & \multirow{2}{*}{. $\}$} & $24 \cdot 2$ & $68 \cdot 3$ & $94^{\circ} 0$ & $96 \cdot 8$ & $94 \cdot 8$ & 800 & $17 \cdot 5$ & $51 \cdot 1$ & $39 \cdot 2$ \\
\hline & & .065 & $\cdot 177$ & 251 & $\cdot 259$ & $\cdot 253$ & $2 \cdot 35$ & $\cdot 127$ & $\cdot 137$ & $\cdot 105$ \\
\hline \multicolumn{2}{|c|}{$V a$ cubic feet at atmospheric pressure } & $\cdot 127$ & $\cdot 309$ & 739 & $\cdot 842$ & $1 \cdot 766$ & $6 \cdot 46$ & 316 & $\cdot 334$ & $\cdot 346$ \\
\hline \multicolumn{2}{|c|}{$\frac{V a}{V w}=$ volume of air per cub. ft. of water } & 1.96 & $1 \cdot 75$ & $2 \cdot 94$ & $3 \cdot 68$ & $7 \cdot 50$ & $2 \cdot 75$ & $2 \cdot 49$ & $2 \cdot 45$ & $3 \cdot 30$ \\
\hline \multicolumn{2}{|l|}{ Velocity of flow at entrance, f.s. } & $2 \cdot 54$ & $3 \cdot 46$ & $4 \cdot 90$ & $5 \cdot 07$ & $4 \cdot 61$ & $7 \cdot 65$ & - & - & $一$ \\
\hline \multicolumn{2}{|l|}{ Mean velocity of mixture in tube } & $5 \cdot 16$ & $6 \cdot 64$ & $12 \cdot 46$ & $14 \cdot 87$ & $22 \cdot 80$ & $20 \cdot 8$ & - & 一 & - \\
\hline \multicolumn{2}{|c|}{ Efficiency $\left\{\begin{array}{c}\text { 1.11.P. in compressor } \\
\text { cylinder }\end{array}\right\}$} & $8 \%$ & $44 \cdot 9 \%$ & $26 \cdot 7 \%$ & 21 & $10.6 \%$ & $38 \cdot 4 \%$ & $44 \cdot 5 \%$ & $32 \cdot 9 \%$ & $42 \cdot 3 \%$ \\
\hline
\end{tabular}

For deep well pumping, the air lift pump is very suitable, since it can be used in any bore hole of sufficient diameter to admit the necessary pipe lines, and can take advantage of the whole discharging capacity of the hole.

In addition, the absence of moving parts below the surface, the possibility of installing the compressing plant at practically any

H.A.

$\mathrm{X} \times$ 
distance from the bore hole, and of pumping corrosive liquid or water carrying solid matter in suspension, together with the certainty of operation, give the system very obvious advantages, and in many instances greatly outweigh the disadvantage of moderate efficiency.

With hot liquids, too, the efficiency is augmented, since the volume of air in the rising main is increased by the rise in temperature, while in many instances the aerating effect of the air is an advantage. ${ }^{1}$

\section{Art. 179.-Hydraulic Air Courpressor.}

By reversing the action of the air lift pump, and allowing water under a head $h_{d}$, to flow down a vertical pipe of length $\left(h_{d}+h_{s}\right)$, which has a length $h_{s}$ submerged in the tail race (Fig. 276) a type of air compressor is obtained which is fairly efficient, and has obtained some success from its simplicity of construction.

Water entering at the upper end of the down pipe induces a series of small air jets through suitably placed openings, and, if the velocity is sufficiently great, carries these to the bottom of the pipe, where their pressure $=2 \cdot 3 h_{s}$ lbs. per square inch approximately. The water is then allowed to escape, while the air is collected in an air chamber surrounding the falling main.

The pressure to which the air may be compressed is thus independent of the supply head and depends solely on $h_{s}$. Since, however, the head required to maintain the required velocity of flow increases with $h_{s}$, this limits the pressure attainable.

The velocity with which fine bubbles rise through still water is approximately 9 inches per second, and it is essential that the velocity of flow be greater than this. Experiments show that velocities of from $12-\mathbf{1 6}$ feet per second give the best results.

The volume of air compressed per cubic foot of water used, may be determined as in the case of the air lift pump.

1 Further information in this subject may be obtained from the following papers :-

"Proceedings Institute Civil Engineers," vol. 140, p. 323.

"Proceedings Institute Civil Engineers," vol. 163, 1905-6, part I., p. 353.

"British Association of Waterworks Engineers," 1903.

Engineer, Jannary 10, 1908, p. 26. 
Recently published tests of such a plant as here described are said to have given an efficiency of 82 per cent. ${ }^{1}$ Here $h_{d}=$ 71 feet and $h_{s}=261$ feet. Three vertical shafts are used, each

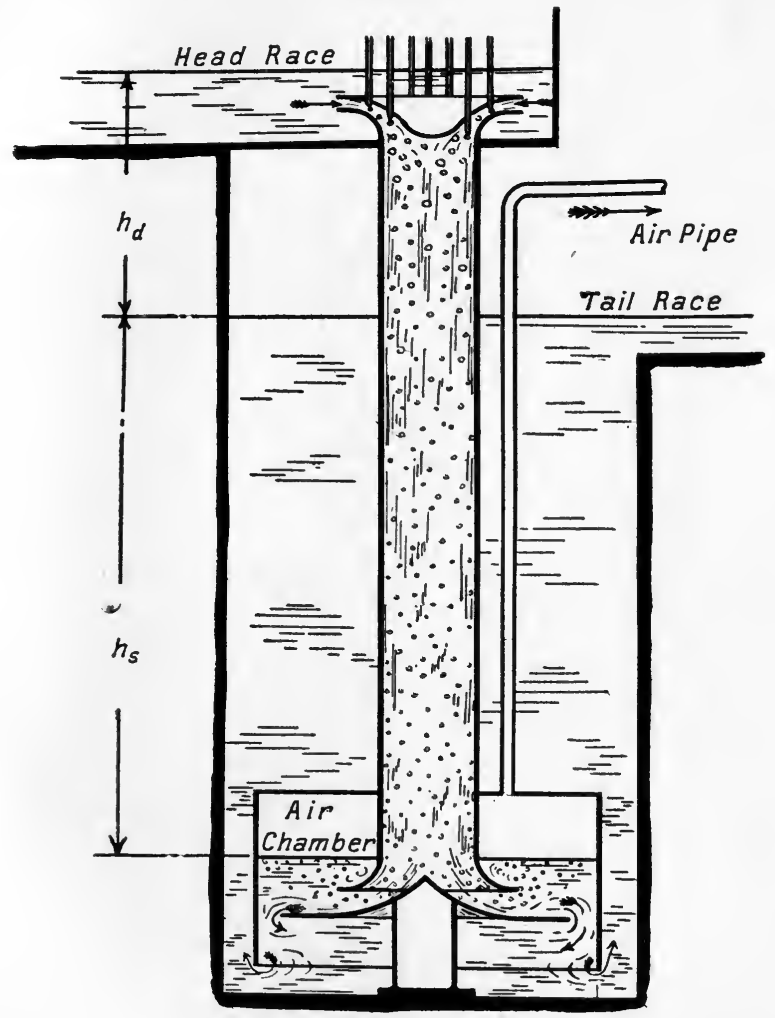

FIG. 276.-Hydraulic Air Compressor.

5 feet in diameter, and the plant is capable of developing 4,000 H.P., compressing the air to a pressure of $117 \mathrm{lbs}$. per square inch alove the atmosphere.

\section{Examples.}

(1) A hydraulic ram uses 50 gallons of water per minute under a supply head of 4 feet, and pumps 5 gallons of this against

1 Engineering and Mining Journal, New York, January 19, 1907, p. 125. See also an abstract in "Proceedings Institute Civil Engineers," vol. 169, p. 500. This value is probably high. 
an effective head of 30 feet. Determine the efficiency of the ram.

Answer. 75 per cent.

(2) A ram uses 900 gallons of water per minute under 10 feet head, and pumps 50 gallons of this through 500 feet of $2 \frac{1}{2}$-inch piping into a reservoir at a height of 80 feet above the ram. Determine the efficiency, taking $f=\cdot 012$.

Answer. 60 per cent.

(3) The waste valve of a hydraulic ram is 4 inches diameter and is required to begin to close when the velocity of flow past the valve itself is 6 feet per second. Assuming the dynamic pressure on the valve per unit area to be given by $1.35 \frac{W v^{2}}{2 g}$ lbs., where $W=$ weight per cubic foot of water, determine the necessary weight of the valve.

Answer. $328 \mathrm{lbs}$. per square inch.

Total weight $=4 \cdot 12 \mathrm{lbs}$.

(4) A jet pump placed 8 feet above the suction reservoir, and 60 feet below the supply reservoir, lifts its water through a total height of 8 feet 9 inches. Determine its efficiency when delivering 100 gallons per minute and when using 36.5 gallons per minute from the supply reservoir.

\section{Answer. $\quad 257$.}

(5) An injector hydrant takes $25 \cdot 2$ gallons per minute of high pressure water at $700 \mathrm{lbs}$. per square inch and delivers 150 gallons per minute at a pressure of $65 \mathrm{lbs}$. per square inch, the low pressure supply being at $30 \mathrm{lbs}$. per square inch. Determine the efficiency.

Answer. $\quad 273$. 


\section{CHAPTER XVIII.}

The Hydraulic Transmission of Energy-Accumulators-Intensifiers-Friction of Leather Collars for Rams-Water Meters.

Art. 180.-The Hydraulic Transmission of Energy.

Since water is virtually incompressible, if one end of an enclosed column be exposed to the pressure of a moving ram the energy of this will be directly transferred to the other end, the only loss of energy being due to pipe friction. With water of a given pressure intensity, the energy transmitted varies directly as the volume of water flowing per second, and hence as the velocity of flow, while with a given velocity the energy varies. directly as the pressure.

It follows, that as the loss due to friction increases as the square of the velocity, the proportional effect of this will diminish as the working pressure increases, and for high effciency of transmission the working pressure is of necessity high.

In many instances the use of water under considerable pressure as a medium for the transmission of energy from a central power station to a private consumer offers decided advantages over other methods of power transmission, and this is particularly the case where the power is required to operate machinery in which the action is either-

(a) Comparatively slow, but in which a considerable force is required, and particularly where the motion is to be regulated with great precision;

(b) Largely continuous in one direction and in which frequent reversals of motion are not necessary; or

(c) . Very intermittent, a large force being required at intervals and for a comparatively short time.

It is thus well adapted for the operation of presses, flanging and rivetting machinery, lifts, hoists, cranes and testing machines. 
The state of high efficiency to which the transmission and utilization of energy in this form have attained is largely due to Lord Armstrong, who was probably the first to develop the use of high pressure energy, and who evolved the many details necessary to make the system a practical success.

In many large towns, of which London, Manchester and Glasgow are notable examples, private customers are supplied by a system of hydraulic mains, taking water under pressure from the supply station to the service pipes leading into the house or works. The pressure adopted varies from 700 to 1,600 lbs. per square inch, being 750 lbs. per square inch in the City of London, and 1,120 lbs. per square inch at Manchester and Glasgow. The arrangement of the Whitworth Street West Station of the Hydraulic Power Supply Department of the Manchester Corporation Waterworks may be taken as typical of such installations.

Here the motive power is supplied by six inverted cylinder, triple expansion, surface condensing engines, having cylinders 15 inches, 22 inches, and 36 inches diameter by 24 inches stroke, and each developing about 200 I.H.P. when working at the normal speed of sixty revolutions per minute, and with steam at 120 lbs. gauge pressure.

The steam raising plant consists of five Lancashire boilers, 30 feet by 7 feet 6 inches, fitted with Vicar's' mechanical stokers, and each capable of evaporating 4,500 lbs. of water per hour.

The engine cranks are set at $120^{\circ}$, each crosshead being direct coupled to a single-acting plunger pump, $4 \frac{1}{2}$ inches diameter by 24 inches stroke. The pumps thus have a mean plunger velocity of 240 feet per minute, which may, if necessary, be increased up to 260 feet per minute, the normal delivery of each set being 230 gallons, and the maximum 250 gallons per minute.

The water supply is taken from the town's mains at a pressure of about $40 \mathrm{lbs}$. per square inch, and before passing into the storage tanks, from which the pumps derive their supply, is utilized to work a direct-acting intensifying pump, by means of which part is pumped directly into the pressure mains. This pump exhausts into the storage tank, and for each 28 gallons which it exhausts, pumps 1 gallon into the pressure mains. 
The water from the storage tanks is circulated through the surface condensers of the engine by means of a special circulating pump, with the result that danger of freezing in winter is largely eliminated.

Air vessels are fitted on all suction pipes and are charged by portable hand pumps. The volume of each air vessel is approximately 3 cubic feet.

The main pumps have no delivery air vessels and deliver into a common pipe, this feeding two accumulators, each 18 inches diameter, and having a lift of 23 feet, the dead weight, consisting of iron slag, being carried in circular iron cisterns 11 feet 3 inches diameter. The total weight of casing, slag, and ram of each accumulator is approximately 129 tons. One of these is slightly heavier than the other, the heavier one being provided with a tappet gear, by means

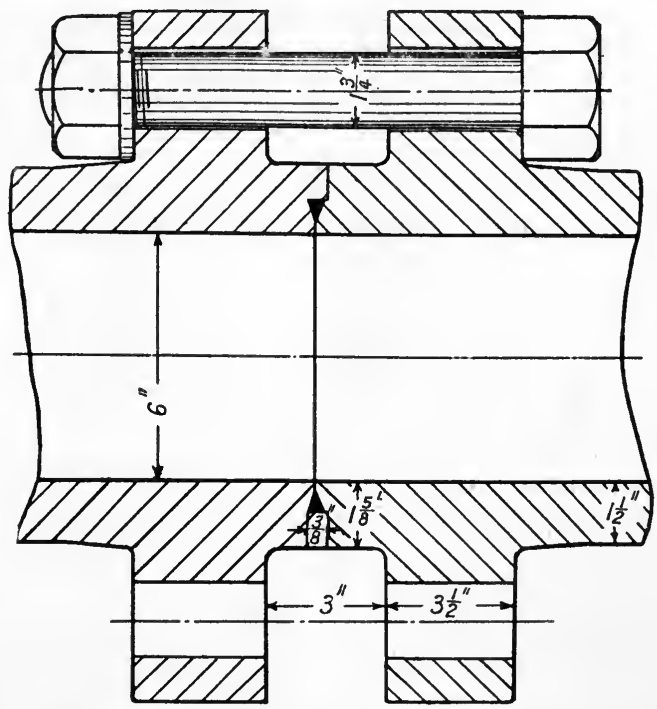
of which the throttle FIG.277.-Joint for 6-in. Pipe, 1,100 lbs. per square in. valves on the main steam pipe are automatically closed as the ram reaches the extreme limit of its upward stroke. The engines under steam are thus brought to a standstill, and remain so until the tappet is released by the descent of the ram. Each accumulator is fitted with an electric bell, which gives warning when the ram is about 10 feet from the bottom of its stroke.

The pressure wate: is led into the streets by four 6 -inch castiron pipes, after which the branch pipes vary in diameter by even inches from 6 inches down to 2 inches.

The joint flanges are oval, the joint consisting of a bevelled 
spigot and faucet union securing a $\frac{3}{8}$-inch gutta-percha ring by two bolts. Fig. 277 illustrates the type of pipe joint as adopted for a 6-inch pipe, and as invented by Mr. E. B. Ellington, the maximum and minimum diameter of the flange being 19 inches and $10 \frac{1}{2}$ inches respectively. These pipes are laid with the longer axis of the flange horizontal for facility in getting at the bolts.

The main stop valves are balanced both ways by the insertion of a small valve $1 \frac{1}{2}$ inch in diameter inside the main valve, the effort required to open the latter only being that necessary to overcome its dead weight. A spring-loaded momentum valve, having a $\operatorname{ram} 1 \frac{1}{2}$ inch diameter, is fitted on either side of every stop valve to minimise any shocks that may occur in the main.

To indicate the condition of the mains and valves, a daily record of the minimum flow during the time the demand is at its lowest (between 11 p.m. and 4 a.m.) is kept by means of an automatic electrical recorder. Should this show an abnormal increase in the output for several consecutive nights the mains are tested. For this purpose certain of the stop valves on the trunk mains are kept closed so that the several circuits are connected only at the power station. Here they can be separated into four sections, and the section in which the abnormal flow is occurring is indicated by the behaviour of the pressure gauge connected with that circuit. The stop valves on this main are then shut down in succession until the defect is discovered, either by the aid of an iron rod which conveys the sound of the escaping water to the ear, or by applying a pressure gauge. Usually the former method is adopted.

All the pressure water is metered by Kent's high pressure rotary meters before reaching the consumer, some 97 per cent. of the water delivered by the pumps being registered on these meter's.

The following abbreviated scale of charges, which comes into force at the end of 1907, may be of interest, as indicating the probable cost of such power. ${ }^{1}$

\begin{tabular}{c|c|c|c|c|c|c|c|c}
\hline $\begin{array}{c}\text { Quantity of water used in } \\
\text { gallons per quarter }\end{array}$ & $\begin{array}{c}2,000 \\
\text { or under }\end{array}$ & 5,000 & 10,000 & 20,000 & 50,000 & 100,000 & 200,000 & 300,000 \\
\hline $\begin{array}{c}\text { Charge per 1,000 gallons } \\
\text { in shillings }\end{array}$ & $12 \cdot 5$ & $s \cdot 6$ & $6 \cdot 4$ & $5 \cdot 2$ & $4 \cdot 5$ & $3 \cdot 7$ & $3 \cdot 10$ & $2 \cdot 83$ \\
\hline
\end{tabular}

1 For these particulars the author is indebted to Mr. L. Holme Lewis, the chief engineer to the Corporation Power Supply Department. 
Power water taken in excess of 300,000 gallons per quarter is charged $2 s$. per 1,000 gallons for the excess quantity so taken, and where the consumer agrees to take a minimum quantity of 500,000 gallons, the price attains a minimum of $1 s$. $3 d$. per 1,000 gallons for a minimum of $3,000,000$ gallons per quarter.

Assuming an efficiency of 75 per cent. for the consumers' machinery, this gives a cost per B.H.P. hour varying from 1.562 shillings, in the case of the smallest consumers, to $\cdot 156$ shillings or 1.875 pence in the case of the largest. Power water for motors running on an average 6 hours per day is charged at $1 s$. $6 d$. per 1,000 gallons.

The following list of costs of buildings and plant of this station may be of interest :-

\begin{tabular}{|c|c|c|}
\hline & $\mathfrak{E}$ & s. $d$. \\
\hline Buildings, tanks, girders, columns, etc. & 16,835 & \\
\hline Boilers, stokers, economisers, elevators, ctc. & 4,023 & \\
\hline Engines, pipes, valves, etc. . & . 20,614 & \\
\hline Total & $. £+1,+72$ & 106 \\
\hline
\end{tabular}

In the London installation the water is taken from the river or from wells; and as it is essential that all deposit should be removed before use, it is allowed to stand for some time in storage tanks. The greater part of the solid matter is deposited here, and the water is then passed through the surface condensers of the engines to a series of filters, in which it is passed first through a layer of broken sponge 18 inches thick, and afterwards through a bed of charcoal. After leaving the filters it is pumped into the clean water tank, from which the main pumps derive their supply.

Among other advantages of hydraulic transmission it may be noted that power is always immediately available; that gearing in the machine is in general unnecessary, the force being transmitted directly from the hydraulic piston; that perfect regulation is easy ; that when applied to the direct working of lifts and of hoists a brake is unnecessary, and that so long as the velocity of flow is kept low the transmission losses are small-with well designed pipe lines this loss should not exceed $10 \mathrm{lbs}$. per square inch per mile.

As compared with electric transmission, it has the advantage 
that fire risks are eliminated, while for slowly moving machinery, intermediate gearing is largely eliminated. Compared with transmission by compressed air, it has the advantage that any leakage is easily detected, while under suitable conditions the hydraulic transmission losses are much the lesser. Each of the three systems of course has its own particular sphere of application. For long-distance work the necessary cost of, and losses in the pipe line, would effectively militate against the application of hydraulic transmission, and a radius of 15 miles from the central station would appear to mark the limit of its effective use.

The various losses occur-

(1) At the power station-roughly about 15 per cent.;

(2) In transmission-about 5 per cent. ;

(3) In use-about 8 per cent.;

leaving a percentage to be utilized of about 72 per cent.

The two last items, however, vary considerably with the type of machinery, and the energy utilized may vary from 30 to 95 per cent., thc latter percentage being obtained with such machines as direct acting coal shoots, where the load, during its descent, may be made to pump pressure water back into the mains.

Losses.-The losses at the power station are those incidental to the use of reciprocating pumps and accumulators, and are considered in detail in that connection.

\section{T'ransmission Losses.}

Let $p=$ pressure at pipe inlet in lbs. per square inch.

,$\quad d=$ pipe diameter (supposed uniform) in feet.

,$\quad a=$, area in square feet.

,$\quad l=$ length of pipe in feet.

,,$v=$ velocity of flow in feet per second.

Then the energy at entrance, per $\mathrm{lb} .=\frac{144 p}{62 \cdot 4}=2 \cdot 31 p \mathrm{ft} . \mathrm{lbs}$.

$\therefore \quad$ Energy entering pipe per second $=H=2.31 p \times 62.4 a v$.

$=144 p a v$ foot $\mathrm{lbs}$.

$=\frac{144}{550}$ p a $r$ H.P.

$=\cdot 262$ p a $v$ H.P. ${ }^{1}$

1 If $\mu=750 \mathrm{lbs}$. per square inch we thus get the approximate rule that two. gallons of water per minute is equivalent to one horse power. 
Again the loss of energy in friction per lb. $=\frac{f l v^{2}}{2 g m}$ foot lbs.

$\therefore \quad$ loss of energy per second $=H_{f}=\frac{f l r^{2}}{2 g m} \times \frac{62 \cdot 4 a v}{550}$ H.P.

Substituting for $v$ in terms of $H$ we get-

$$
H_{f}=\frac{62.4 a f l}{2 g m \times 550}\left\{\frac{H}{262 p a}\right\}^{3} \text { H.P. }
$$

and putting $\quad m=\frac{d}{4} ; g=32 \cdot 2$ this becomes-

$$
H_{f}=\cdot 635 f l \frac{H^{3}}{p^{3} d^{5}} \text { H.P. }
$$

The energy delivered per second- $U$ -

$$
=H-H_{f}=H\left\{1-\cdot 635 f l \frac{H^{2}}{p^{3} d^{5}}\right\}
$$

$\therefore$ Efficiency of transmission-

$$
=\frac{U}{H}=1-\cdot 635 f l \frac{H^{2}}{p^{3} c^{5}}
$$

Differentiating $U$ with respect to $H$, and equating the result to zero, we get the condition that the maximum horse power may be transmitted.

Expressed algebraically this gives-

or

$$
\begin{aligned}
H^{2} & =\frac{1}{3} \frac{d^{5} p^{3}}{635 f^{\prime} l}=\frac{d^{5} p^{3}}{1.90 f^{\prime} l^{\prime}} \\
H & =\cdot 725 \sqrt{\frac{d^{5} p^{3}}{f l}},
\end{aligned}
$$

from which, by substitution in equation (2), we have the energy delivered through the pipe $U=\cdot 483 \sqrt{\frac{d^{5} p^{3}}{f^{3} l}}$ H.P.

$$
=\cdot 138 \sqrt{\frac{d^{5} h^{3}}{f^{3} l}} \text { H.P. }
$$

if $h=$ head in feet at entrance to pipe.

Substituting the value of $H$ from (4) in equation (3), we see that under circumstances of maximum transmission, the efficiency is $\frac{2}{3}$, and that $\frac{1}{3}$ of the energy entering the pipe is absorbed in overcoming friction. On the other hand it is evident that maximum efficiency is obtained when $H$ is as small, and $p$ and $d$ as large as practicable.

The point at which it ceases to pay to still further increase 
the diameter of the pipe line for a given horse power, depends on the relative cost per yard of the pipe line, including excavation, jointing and laying, and of the power production per horse power. ${ }^{1}$ In general, however, a size of pipe which allows of a pressure drop of about 10 lbs. per square inch per mile will be found to give most economical results in practice. In modern practice the largest pipes are about 6 inches diameter, the pipe lines being duplicated for large powers.

\section{Example.}

Let $\quad H=100$ H.P.

, $\quad p=750 \mathrm{lbs}$. per square inch.

Assume $f=\cdot 006$ (this varies with the diameter, velocity of flow, and condition of pipe).

Then allowing for a drop of $10 \mathrm{lbs}$. per mile we have

Efficiency of transmission $=1-\frac{10}{750} \cdot \frac{l}{5,280}$

$$
\begin{aligned}
& =1-\frac{63 E f l H^{2}}{t^{3} d^{5}}, \\
\therefore \quad d^{5} & =\frac{.635 \times .006 \times 5,280 \times 10,000 \times 750}{750 \times 750 \times 750 \times 10} \text { feet } \\
& =\cdot 0358 \text { feet, } \\
\therefore \quad l & =.514 \text { feet }=6.17 \text { inches. }
\end{aligned}
$$

The loss per mile $H_{f}=\frac{10}{750} \times 100=1 \cdot 33$ H.P.

The efficiency of transmission is then given by :-

\begin{tabular}{ll|c|c|c|c}
\hline Length of pipe in miles . & 1 & 2 & 5 & 10 \\
\hline Efficiency of transmission & $\cdot 987$ & $\cdot 973$ & $\cdot 933$ & $\cdot 867$ \\
\hline
\end{tabular}

Also

$$
\begin{aligned}
r & =\frac{H . P . \times 550}{144 p \times a} \text { f.s. } \\
& =\frac{55,000 \times 4}{750 \times \pi \times 144 \times \cdot 2642} \text { f.s. } \\
& =2.45 \text { feet per second. }
\end{aligned}
$$

1 See "Proceedings Institute Mechanical Engineers," 1895, p. 353; also Engineering, May 22nd and June 5th, 1891. 
If $p=1,120$, then for the same fall in pressure per mile we have, for 100 H.P. :-

$$
d=\cdot 4377 \text { feet }=5 \cdot 25 \text { inches }
$$

while $r=2 \cdot 27$ feet per second.

The following table then gives the efficiency for various lengths of pipe line:-

\begin{tabular}{|c|c|c|c|c|c|c|c|c|c|}
\hline Length in & & . & . & $\bullet$ & 1 & 2 & 5 & 10 & 20 \\
\hline Efficiency & . & . & . & • & $\cdot 991$ & (982 & $\cdot 955$ & $\cdot 911$ & $\cdot 821$ \\
\hline
\end{tabular}

The velocity of flow through the pipe should not exceed 4 feet per second, velocities ranging from 2.5 to $4 \cdot 0$ feet per second being usual.

While an increase in the working pressure increases the efficiency of transmission, it also necessitates an increase in the thickness of the pipe walls, which counterbalances the advantages of the reduced internal diameter. Also the difficulty of preventing leakage at all joints increases with the pressure, so that in practice it has not been found advisable to adopt pressures much in excess of 1,100 lbs. per square inch.

Losses in Use.-These are due partly to friction, but, in the majority of hydraulic machines, more particularly to shock at sudden changes of section in valve boxes and supply ports and pipes, and to the necessity in many machines for filling the inlet passages with pressure water before the commencement of each working stroke. The latter loss may be prevented by having separate inlet and outlet passages to the working cylinder, while to prevent the former losses becoming excessive all working velocities must be kept low.

A further loss of energy occurs in some such machines as presses and rivetters, where the maximum force which the plunger can exert is only needed for a short portion of its stroke, but where the expenditure of energy is the same as if this were needed for the whole stroke. Also, in the case of a hydraulic crane, if only one lifting cylinder is provided without any special regulating device, the expenditure of energy is the same whatever load, up to the maximum capacity of the crane, be lifted. 
Certain devices which have been invented to overcome this difficulty will be considered later.

\section{Art. 181.-Accumulators.}

Since the delivery from a reciprocating pump is not uniform and since it is necessary to have some reserve of energy to meet a sudden or abnormal demand, some means of storing pressure energy is a necessary adjunct to the hydraulic power station.

With the high pressures in common use the elevated storage tank is out of the question and the accumulator, devised by Sir W. G. Armstrong, takes its place.

Pressure water from the pumps, then, is not led directly into the supply mains, but first into an accumulator from which it is taken to feed the pipe line.

Stripped of unessentials, the accumulator consists of a vertical cylinder fitted with a weighted ram, the weight and area of this being adjusted so as to give the required pressure in the mains.

$$
\begin{aligned}
\text { Thus, if } A & =\text { area of ram in square inches, } \\
W & =\text { weight of ram in lbs., } \\
\text { we have } p A & =W
\end{aligned}
$$

The energy storage capacity of the accumulator is evidently simply equal to the potential energy of the lifted ram and weight, and if $L$ is the length of its travel in feet, is given by $L W$ foot lbs.

From another point of view, the storage capacity is given by

$$
2.3 p \times \frac{62.4 \times A L}{144}=p A L,
$$

i.e., by the pressure energy in the volume of water stored in the cylinder, and since $p A=W$, this leads to the same result as before.

\section{Example.}

Let $p=1,120$, lbs. per square inch, $L=23$ feet, $A=$ $\pi \times 9^{2}=81 \pi$.

Storage capacity $=1,120 \times 81 \pi \times 23$ foot lbs

$=6,554,000$ foot lbs.

$$
=\frac{6,554,000}{33,000 \times 60}=3.31 \text { horse power hours. }
$$


The accumulator is therefore capable of giving out energy at the rate of $3 \cdot 31$ H.P. for one hour, or $39 \cdot 7$ H.P. for five minutes.

From this example it is evident that the storage capacity is not large and that the main function of an accumulator is not so much to store energy, in the sense that an electric accumulator stores it, as to permit of momentary fluctuations in the rates of supply and demand, or in other words, to act as a flywheel does to a steam or gas engine. It also serves to regulate the delivery pressure, and is usually made to automatically control the motive power. Its efficiency is high, up to $98 \mathrm{per}$ cent. of the energy expended in charging being returned in the delivery.

In its most common form, the accumulator consists of a vertical cylinder, fitted with a ram carrying a platform which is weighted with some heavy material, usually pig iron or iron slag. ${ }^{1}$ Fig. 278 shows this type, the weight here being carried in a wrought iron

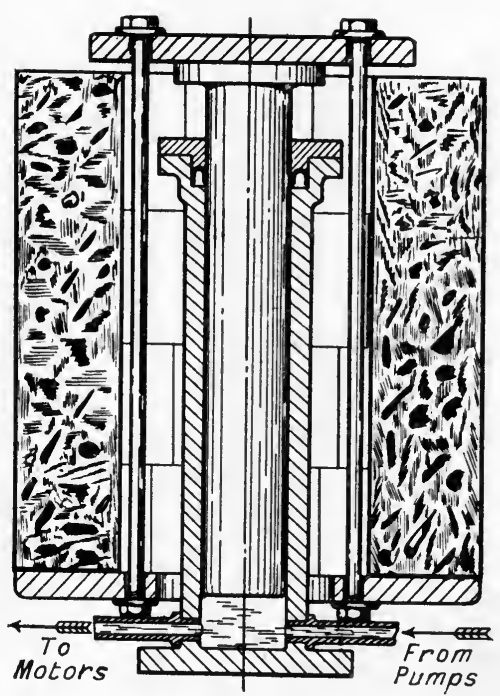

Fig. 278.-Accumulator. cistern suspended from the ram platform.

Inlet and outlet passages are provided in the base of the cylinder, and an air valve is fitted in the top of the cylinder for convenience in first filling. The ram is guided in its travel by a framework not shown in the figure. If the pumps are delivering more water than the motors require, the ram rises, and on

1 The following table shows the approximate volume occupied per ton of weighting material :-

\begin{tabular}{|c|c|c|c|c|c|c|c|}
\hline \multicolumn{7}{|c|}{ Substance. } & Cubic Feet per 'Ton. \\
\hline Pig iron or $w$ & us & ht & iro & $\mathrm{n}$ s & $\mathrm{cr}$ & & $6 \cdot 25$ \\
\hline Broken stone & . & & & & . & & $17 \cdot 2$ \\
\hline Clay or earth & & & . & & & & $18 \cdot 5$ \\
\hline Bricks. . & • & . & $\cdot$ & 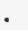 & • & . & $22 \cdot 2$ \\
\hline
\end{tabular}


reaching the upper limit of its travel moves a stop which, by suitable link connection, causes steam to be shut off from the pumping engines. When the ram falls steam is again admitted to the engines, and so on.

Various modifications of this type have been adopted, the ram

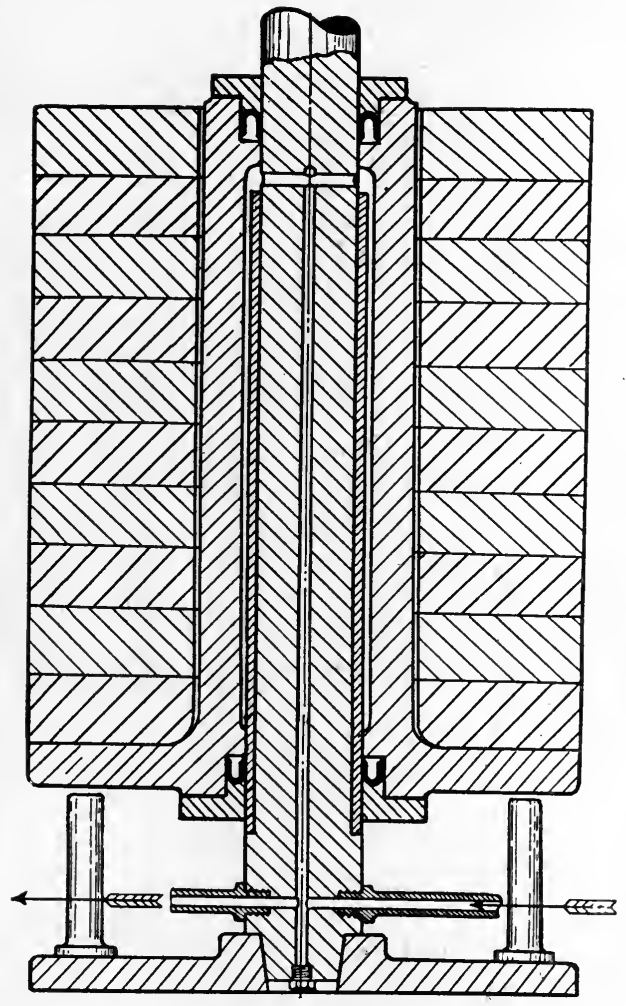

FIG. 279.-Tweddell's Differential Accumulator.

in some cases being inverted, fixed, and fitted with suitable inlet and outlet orifices, and the loaded cylinder moving vertically.

The differential accumulator of $\mathrm{Mr}$. Tweddell may also be noted. As indicated in the sketch (Fig. 279), this consists of a fixed ram of area $A$, surrounded over the lower portion of its length by a closely fitting bush of area $a$.

This bush terminates below the inlet and outlet holes. The ram passes through both ends of the storage cylinder, through glands of area $(A+a)$ and $A$, and the effective cylinder area exposed to upward pressure is $a$.

Thus p a $=W$, and by making the bush of small thickness, a very large pressure may be maintained by a comparatively small weight.

\section{Example.}

If the ram diameter $=6$ inches and the bush is $\frac{1}{4}$ inch thick, we have $a=4.91$ square inches.

$\therefore$ If $p=1,120 \mathrm{lbs}$. per square inch, we have

$$
W=p a=1,120 \times 4 \cdot 91=5,500 \mathrm{lbs} \text {. }
$$


Since the storage of energy is only small, this type of accumulator is more suitable for use with single machines of the rivetter type.

On board ship, and especially for naval purposes, the use of hydraulic machinery for training the heavy guns, rotating

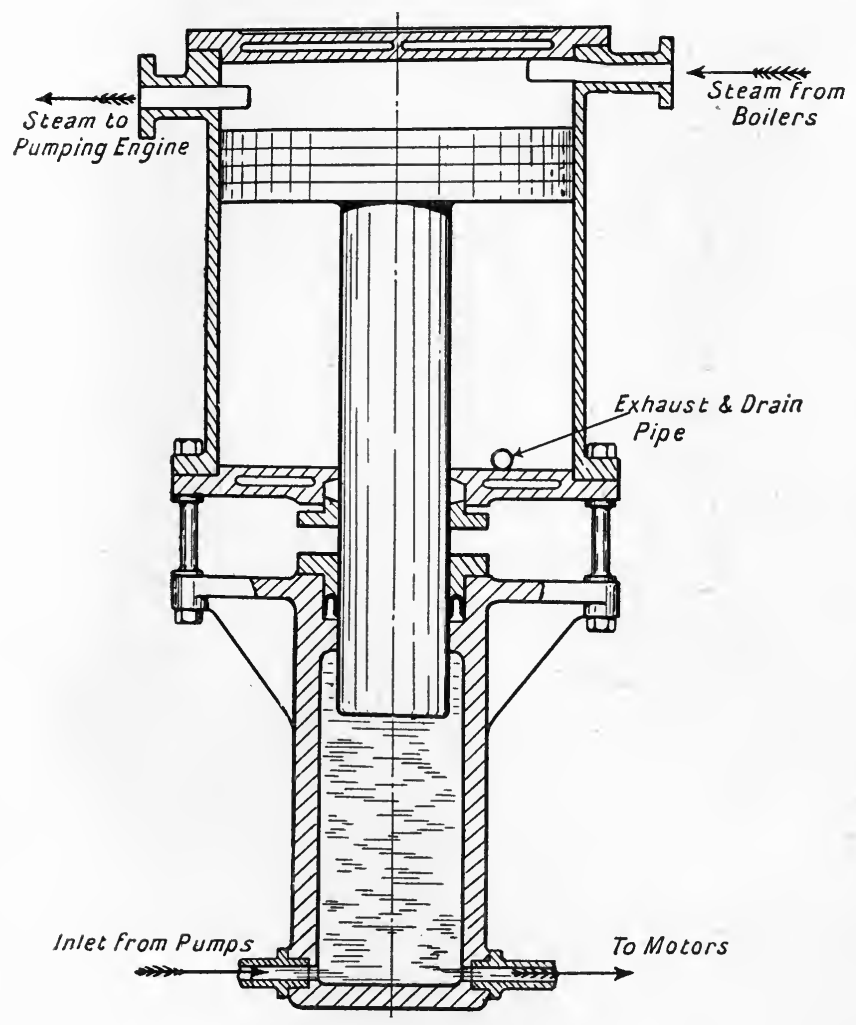

Fig 280.- Steam Accumulator.

turrets, etc., is very general, but here, for obvious reasons, the accumulator loaded with a mass of pig iron weighing many tons is quite inadmissible.

In its place the steam accumulator (Fig. 280) is used. This consists of a steam cylinder fitted with a piston and piston rod or ram, which takes the place of the weight-loaded ram of the ordinary type. Steam from the boiler is admitted to the upper side of the 
piston, first passing through a reducing valve which ensures a constant pressure, and with a given steam pressure $P$, a suitable adjustment of the areas $A_{s}$ and $A$ of the steam piston and ram, will enable any required hydraulic pressure, $p=P \frac{A_{s}}{A}$ lbs. per square inch, to be maintained. The steam supply to the pumping engines is taken through the steam cylinder, and the accumulator piston automatically cuts off this supply on reaching a given height, and thus stops pumping until the ram descends. The steam port is usually designed so that steam may be cut off gradually and the speed of the pumps gradually reduced as the piston approaches the upper limit of its travel.

A drain pipe is arranged to carry away any water or steam leaking past the piston. The storage capacity is $P A_{s} L$ foot lbs., and this type has the advantage that the ram may be placed either horizontally or vertically.

Effect of Accumulator on Working Pressure in Motor Cylinder. -If the motor, piston-area a square inches, derives its supply from the accumulator cylinder alone, then if $A$ square inches $=$ area of ram; $a=$ acceleration of motor piston ; $a^{\prime}=$ acceleration of ram, we have $a^{\prime}=a \frac{a}{A}$.

The force necessary to produce this acceleration is given by

$$
F=\frac{I}{g} a^{\prime}=\frac{I}{g} \cdot \frac{a}{A} \cdot a,
$$

$\therefore \quad$ Equivalent pressure in lbs. per sq. in. on ram $=\frac{W}{g} \cdot \frac{a}{A^{2}} \cdot a$.

$\therefore \quad$ Pressure at entrance to delivery pipe $=\frac{W}{A}\left\{1-\frac{a}{g A} a\right\}$ lbs. per square inch.

If $a_{s}=$ area of this pipe, then $a_{s}=a \frac{a}{a_{s}}$, and if $l$ is its length we have, putting $v=$ velocity of piston:-

Pressure on piston

$=\frac{W}{A}\left\{1-\frac{a}{g A} a\right\}-\frac{62 \cdot 4 l a}{144 g a_{s}} a-\frac{62 \cdot 4}{144} \cdot \frac{a^{2}}{a_{s}{ }^{2}} \frac{f l v^{2}}{2 g m}$

$=\frac{W}{A}-\frac{a a}{g}\left\{\frac{W}{A^{2}}+\frac{62 \cdot 4 l}{144 a_{s}}\right\}-\frac{62 \cdot 4}{144} \cdot \frac{a^{2}}{a_{s}^{2}} \cdot \frac{f l r^{2}}{2 g m}$ lbs. per sq. in. 
With a steam accumulator this becomes:-

Pressure on piston

$=\frac{P A_{s}}{A}-\frac{a a}{g}\left\{\frac{W}{A^{2}}+\frac{62 \cdot 4 l}{144 a_{s}}\right\}-\frac{62 \cdot 4}{144} \cdot \frac{a^{2}}{a_{s}^{2}} \cdot \frac{f l r^{2}}{2 g m}$ lbs. per sq. in. and since $W$ is now comparatively small, the term $\frac{a a}{g} \cdot \frac{W^{r}}{A^{2}}$, which represents the effect of the inertia of the ram, becomes negligible. For this reason the steam accumulator is not subject to the shocks and jars to which the weighted accumulator is subjected in virtue of the great inertia of its moving parts.

To prevent inertia shocks becoming dangerous, a relief valve is sometimes placed on the outlet pipe, this being set to blow off at 10 per cent. above normal pressure. The loss due to the leakage which this necessitates may be avoided by the provision, in the place of the relief valve, of a spring-

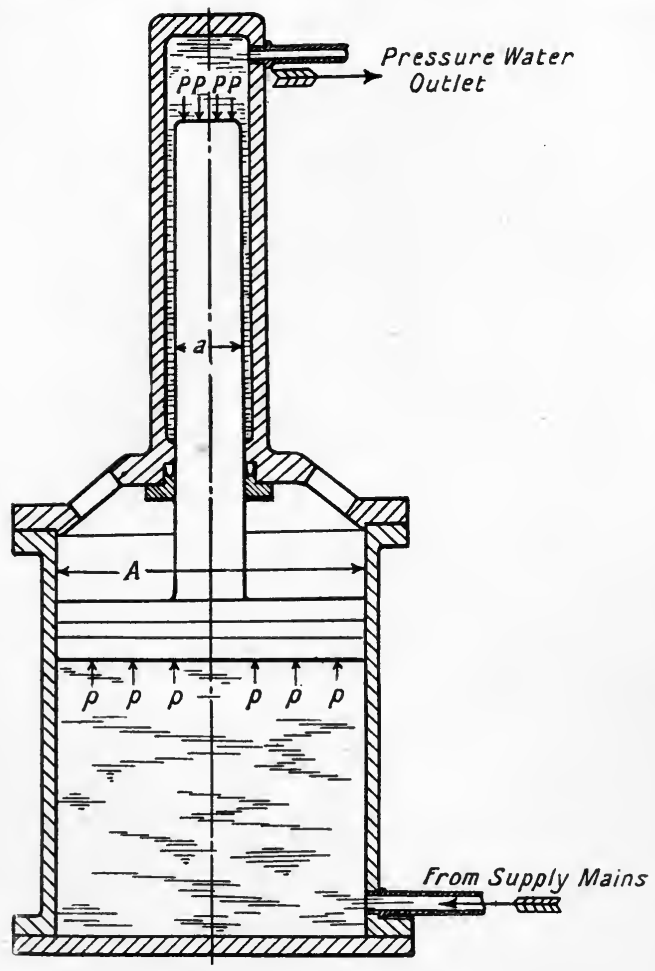

Fig. 281.-Hydraulic Pressure Intensifier. loaded plunger, the kinetic energy of the moving ram then being expended in compressing the spring.

This inertia is, however, an advantage in some cases, as, for example, where fitted to a hydraulic rivetter or similar machine. Here the inertia is utilized to increase the pressure at the end of the stroke, the final and sudden impact thus produced causing the rivet to effectively fill up its hole. 


\section{Art. 182.-The Hydraulic Intensifier.}

Where the main pressure supply is of less intensity than is required to work the hydraulic machinery an intensifier is used. In its simplest form, this consists of a ram of area $a$, carrying a piston of larger area $A$ (Fig. 281). Water from the pressure mains, at pressure $p$, is admitted behind the piston and compresses the water in the ram cylinder to an increased pressure $P$ where $P=p \frac{A}{a}$.

Example.

$p=40$ lbs. per square inch,

$\left\{\begin{array}{l}\text { Piston diameter }=48 \text { inches, } \\ \text { Ram diameter }=8 \text { inches, }\end{array}\right.$

$\therefore \quad P=40 \times 36=1,440$ lbs. per square inch.

This neglects the friction of the packings, and also the weight of the ram and piston. Including these we liave

$$
\begin{gathered}
p A-\left(u+F^{\prime}\right)=P a \\
\therefore \quad P=p \frac{A}{a}-\frac{w+F}{a} \text { lbs. per square inch. }
\end{gathered}
$$

Where $w=$ weight of ram and piston in lbs.

$$
F=\text { frictional resistance in lbs. }
$$

If on the down stroke communication be made between the under side of the piston and the upper side of the ram, the pressure $p^{\prime}$ below the piston becomes

$$
p^{\prime}=\frac{w-F}{A-a} \text { lbs. per square inch. }
$$

Various modifications of this type of intensifier are in use, one of these being illustrated in Fig. 282. Here low pressure water is admitted above the hollow ram $A$, while the intensified water is led away through the smaller stationary ram of area $a$.

If $w$ is the weight of the outer ram, we now have

$$
P=p \frac{A}{a}+\frac{w-F}{a} \text { lbs. per square inch. }
$$

With a single intensifier the supply of high pressure water cannot be made continuous, and delivery only takes place on the in stroke. At the end of this stroke water is admitted to the ram cylinder from the supply mains, while the water below the 
piston is allowed to escape. This type of intensifier is often fitted to testing machines deriving their pressure supply from towns: mains at a comparatively low pressure.

Where a continuous supply of high pressure water is required, this may be obtained by using two intensifiers placed side by side, and each automatically working the valves of the other.

When applied to the working of a hydraulic press, the water escaping from beneath the piston during the down stroke may be utilized to bring the press platform up to its work, and to perform the first part of the compression. ${ }^{1}$

Art. 183. - Friction of Leather Collars for Rams.

One or other of the types of packing illustrated in Fig. 283 is commonly used for hydraulic rams or plungers. 'The material used is leather, and, since the pressure of the water itself forces the packing against the ram, this pressure, and

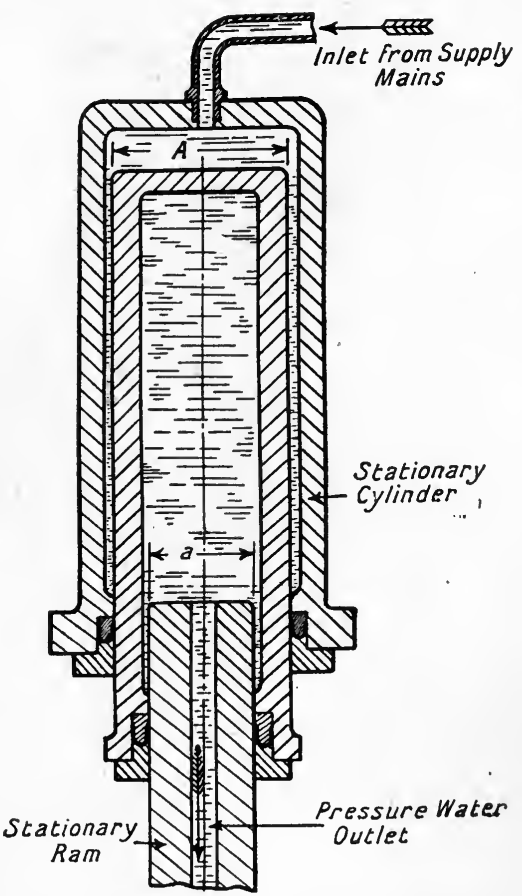

FIG. 282.-Hydraulic Pressure Iñtensifier. the friction produced, become proportional to the pressure intensity of the water. A very complete series of experiments carried out by Mr. John Hicks on rams $\frac{1}{2}$ inch, 4 inches, and 8 inches diameter, and with pressures up to $6,400 \mathrm{lbs}$. per square inch, indicate that-

(1) For pressures above $400 \mathrm{lbs}$. per square inch, friction is directly proportional to pressure intensity.

(2) For rams of different diameters exposed to the same

1 For further details of valve arrangements, etc., the rearler may refer to Blaine's "Hydraulic Machinery," p. 345. 

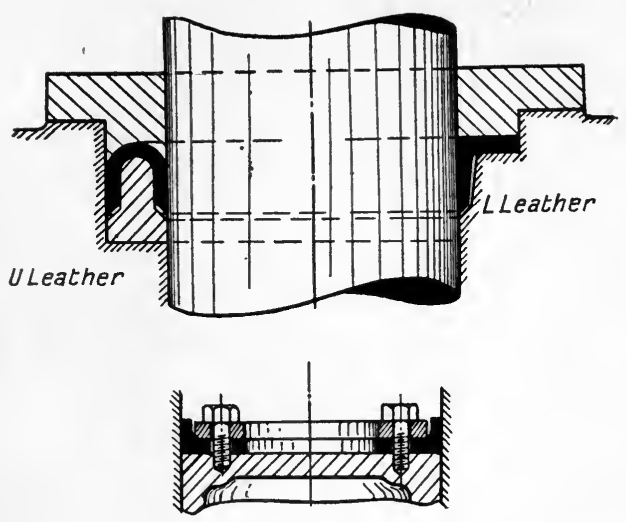

FIG. 283.-Leather Collars for Hydraulic Rams and Pistons. pressure intensity, the friction in lbs. is directly proportional to the diameter, and therefore to the square root of the gross load.

(3) The depth of the collar does not affect the friction. In several of the experiments the depths were reduced from $\frac{7}{8}$ inch to $\frac{3}{8}$ inch without any appreciable effect on the friction.

The following approximate formulae were deduced from the results of these experiments :-

Friction in lbs. $=C \times$ diameter in inches $\times$ pressure in lbs. per square inch. where $\left\{\begin{array}{l}C=\cdot 0471 \text { for new or badly lubricated collars, } \\ C=\cdot 0314 \text { for collars in good condition and well lubricated. }\end{array}\right.$

The annexed table gives the frictional resistance expressed as a percentage of the total pressure on the piston, for rams from 2 inches to 20 inches diameter and for pressures exceeding 400 lbs. per square inch :-

\begin{tabular}{|c|c|c|c|c|c|c|c|c|c|c|c|c|c|c|}
\hline Diameter in i & inches. & 2 & 3 & 4 & 5 & 6 & 7 & 8 & 10 & 12 & 14 & 16 & is & 20 \\
\hline \multirow{2}{*}{$\begin{array}{l}\text { Friction, } \% \\
\text { of total } \\
\text { pressure } \\
\text { on ram. }\end{array}$} & Well lubricated & $2 \cdot 00$ & $1 \cdot 33$ & 1.00 & $\cdot 80$ & $\cdot 66$ & 57 & 50 & 40 & $\cdot 33$ & $\cdot 28$ & $\cdot 25$ & $\cdot 2 \cdot 2$ & $\cdot 20$ \\
\hline & $\begin{array}{c}\text { New or badly } \\
\text { lubricated }\end{array}$ & $3 \cdot 00$ & $2 \cdot 00$ & $1 \cdot 50$ & $1 \cdot 2 \hat{0}$ & $\bullet 99$ & $\cdot 85$ & $\cdot 75$ & $\cdot 60$ & $\cdot 50$ & $\cdot 42$ & $3 i$ & $\cdot 33$ & $\cdot 30$ \\
\hline
\end{tabular}

For lower pressures, the formulae

$$
F \text { (lbs.) }=p d\{\cdot 0467-\cdot 0000139 p\}
$$

gives more accurate results, the coefficients here applying to leathers in good condition and well lubricated.

Where the loading is eccentric, as may be the case in hydraulic jacks, etc., these values may however be increased by as much as 100 per cent. 
Recent experiments by Prof. Martens, of Berlin, ${ }^{1}$ on the packings fitted to the rams of 5 testing machines showed the following friction losses:-

\begin{tabular}{c|c}
\hline Pressure (Atmospheres). & Per cent. Loss per Packing. \\
\hline 50 & $2-5$ \\
100 & $1 \cdot 8-3 \cdot 0$ \\
200 & $1 \cdot 0-1 \cdot 6$ \\
\hline
\end{tabular}

For satisfactory working the U leather should have a ring of metal or other material inserted between the flaps, and should as far as possible have a metal backing over its curved portion.

Hemp packing is also used to a limited extent for hydraulic glands. Here the percentage loss in friction decreases with an increasing load, but since the packing must be tightened so as to prevent leakage at the highest pressures to which it may be subjected, the loss at low pressures is probably three to four times that of a leather collar.

\section{Art. 184.-W Water Meters.}

It is usually important that the volume of water supplied for domestic or power purposes should be accurately measured, and various meters have been devised for this purpose.

These may be divided into the following classes :-

(1.) Low pressure meters.

(2) Inferential meters.

(3) Venturi meters.

(4) Positive meters.

(5) Meters for waste detection.

(1) This type suffers from the disadvantage that all the pressure head of the supply is lost, and that when used for domestic supply it must therefore be placed at the top of the building. It is, however, well fitted for the measurement of small flows. The "Parkinson" meter, which is of this type, is illustrated in Fig. 284, and has given excellent results in measuring the power water delivered by the City of London Hydraulic Supply Company. In this case the meter is applied to measuring the exhaust water from the various machines. Here water from the inlet pipe is 
maintained at an uniform level in the inlet chamber by means of the float and valve $r$. This valve is constructed as shown so as to be balanced whatever the pressure in the supply pipe. On leaving this chamber by the pipe $I^{\prime}$, the water flows into the annular space $S$ formed in the drum $D$. This drum is hollow and contains four compartments formed by oblique radiating plates $R$, which overlap each other to the extent of about $90^{\circ}$. Each compartment opens at its inner periphery into the space $S$, and in turn receives a supply of water. The centre of gravity of
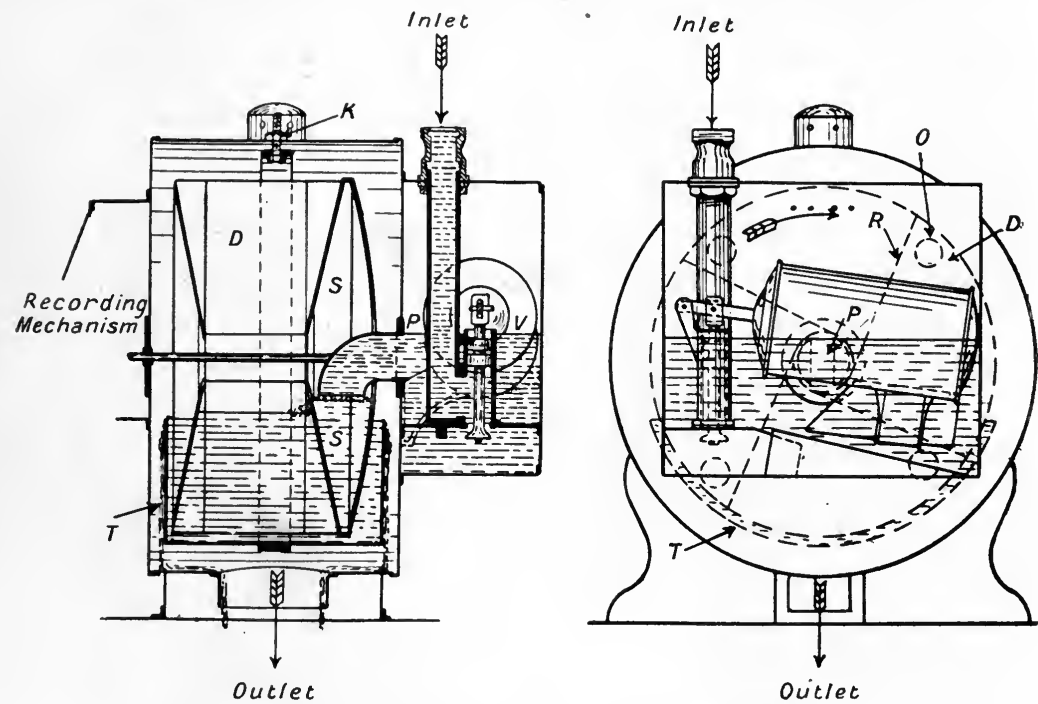

FIg. 284. - "Parkinson" Low Pressure'Water Meter.

the water in the compartment being, on account of its position, to one side of the axis of the drum, this produces a rotation about the axis and brings the next compartment into communication with the space $S$. At the same time the outlet $O$, from the first compartment, which is on the opposite side of the drum to $S$, is brought by the rotation below the water level in the compartment, and discharges the water into the trough $T$ in which the drum rotates, and from which it flows away to the discharge pipe. The height of the trough may be adjusted by the screw $K$, and as the volume discharged per revolution depends on the depth of the immersion of the drum, this enables the discharge 
per revolution to be adjusted. The number of revolutions of the drum, and hence the volume passing the meter, may then be recorded on a suitably engraved dial.

When used for domestic supply purposes, discharge takes place into a cistern from which the water is led over the building. An inlet valve on the supply pipe-not shown in the sketch-is then opened by a ball valve when the water level in this cistern is lowered.

(2) The inferential meter consists simply of a small turbine driving the recording apparatus and through which the whole supply is passed. The water is not actually measured, but its volume is inferred from the number of revolutions of the turbine runner or fan, which is the only moving part. The meter must be calibrated by allowing it to pass a known volume of water per minute, and has the advantage of being small, light, and

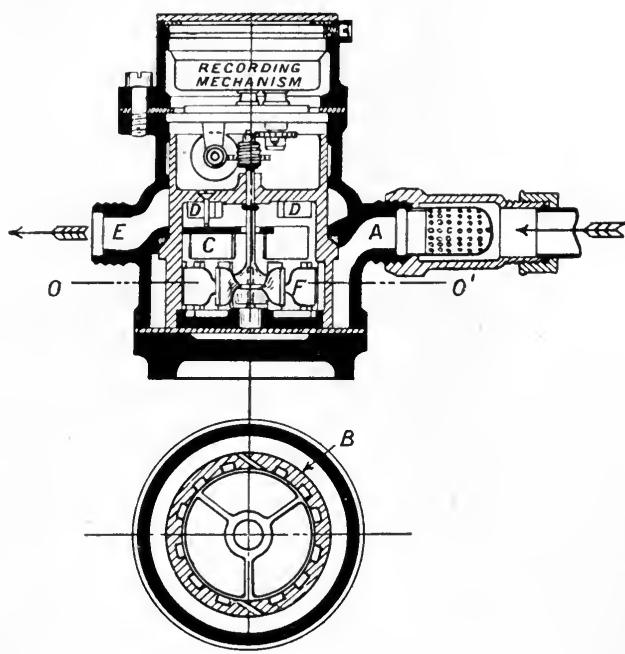

Section on Line $00^{\prime}$ cheap, and fairly accurate for good speeds of flow. Since, however, there is a limiting velocity of flow, below which the reaction on the runner vanes is insufficient to overcome the friction of the bearings and recording mechanism, it is unsuitable for recording small flows. A further point to be noticed is the tendency of the runner to lieep on rotating for some short time after the flow of water has ceased, and thus to over-record the flow. Where taps are opened and closed frequently, this action, unless guarded against, may lead to the flow registered being largely in excess of that actually taking place. 
Tylor's Inferential Meter (Fig. 285) is of this type. Here water enters at $A$, and surrounds the inner casing shown in section at $B$. It then finds its way through two inclined ports in this casing, and, impinging on the vanes of the fan $F$, drives this around with a velocity which depends on the flow of water, and which is recorded on the dial worked by its spindle and worm gear, afterwards escaping through the ports $D$ and pipe $E$. To prevent overrunning, eddy formation is fostered by a series of recesses formed around the inside of the inner casing, and by baffles above the fan at $C$. 'To reduce friction at the lower footstep bearing an oil reservoir is provided in the hollow spindle of the fan.

(3) 'This-the Venturi meter-has been considered in detail in Art. 30.

(4) The Positive Meter consists of a small hydraulic engine, either of the rotary or reciprocating piston type, all the water to be measured passing through the cylinder or cylinders of this engine, and its volume being taken as that of the piston displacement.

The number of revolutions or strokes of the piston being registered on a recording apparatus, this is easily arranged to record the volume passed.

The rotary type is common in the United States of America, and, as usually made, consists of a casing of gun-metal or vulcanite, in which works a rotary vulcanite piston. This has no means of compensating for wear, and, as thus constructed, is very unreliable for small flows, even when new. After being in use for'some time the increased leakage past the piston renders it still less reliable.

The Kent "Uniform" meter (Fig. 286) is one of the best of this type and here compensation is made for the effect of wear of the rotary piston $P$ by an adjustable metal tongue $S$. In this meter water is admitted at $A$ and fills the casing around the working chamber $B$. The vulcanite piston $P$, elliptical in section, rotates and slides freely, but without play, on the fixed hub ?, which is itself eccentric with respect to the working chamber. The piston carries a central pin which describes a circular path as rotation takes place, and which actuates the recording mechanism. 

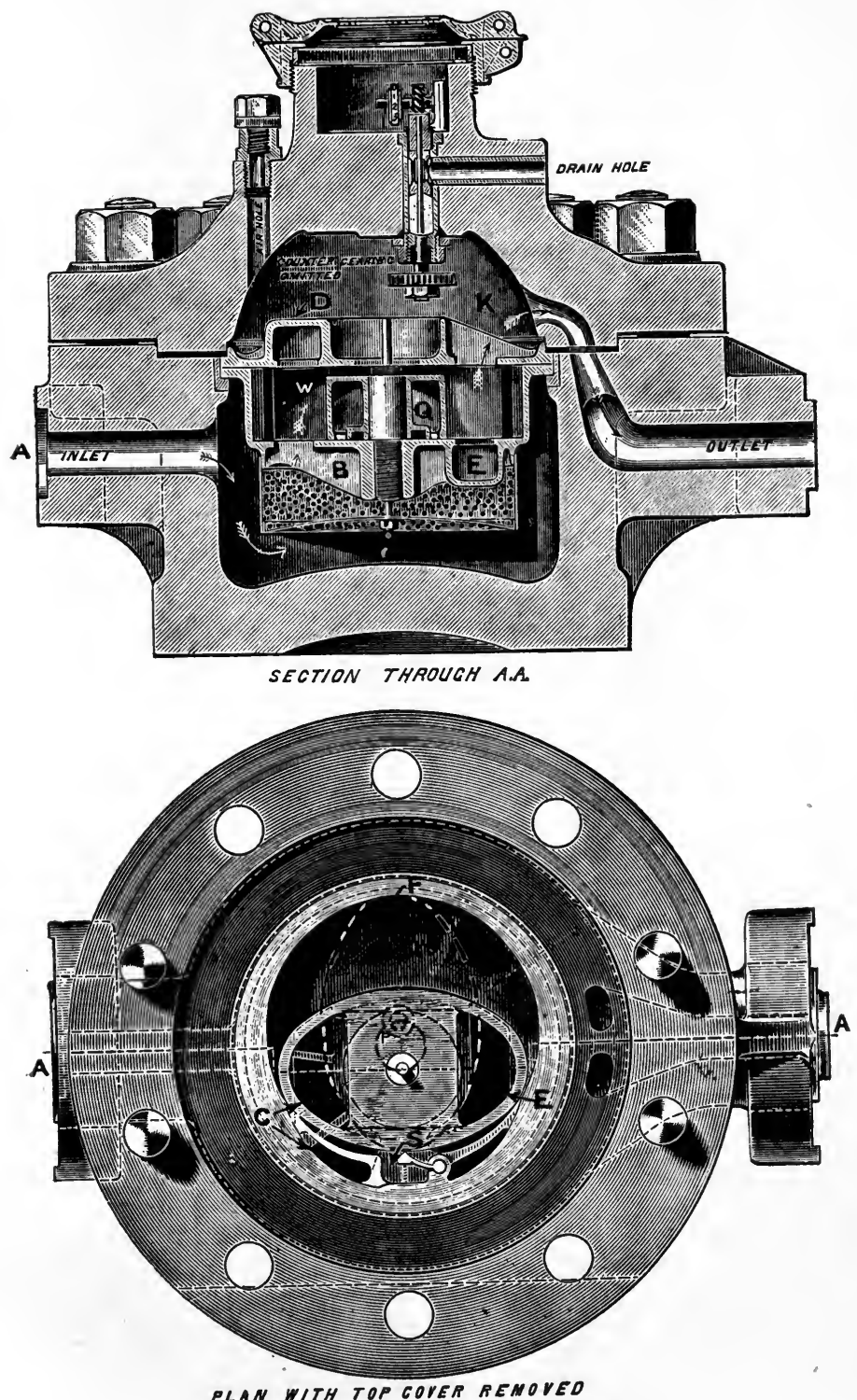

Fig. 286.-- Kent" Positive Water Meter. 
The bottom ports at $C$ are in free communication with the chamber $B$, and the action of the meter is as follows:-

Assuming the piston to be in the position shown in dotted lines, water is admitted through these ports and the upper port $D$, to the space between the chamber, the piston, and the tongue $S$, and to the interior of the hollow piston, thus exerting a pressure between the hub and the inside of the inner end of the piston, as well as on the outside of the piston from $C$ to $S$, and driving the latter round in a clockwise direction. At the end of half a revolution the other end of the piston becomes the driver and so on.

In the meantime water has been filling the space to the left of the piston until the position shown in full lines is reached, when the supply is cut off from the space $F$, this space is put into communication with the discharge port $K$ by way of the lower port $E$ and the hollow piston, and for the remaining half revolution the water in this space and in the outer end of the piston is discharged through $K$, the whole cycle of operations then being repeated. The action becomes much more evident if a tracing showing the piston be rotated, keeping its correct relative position, through a whole cycle. This meter is used exclusively in connection with the measurement of pressure water from the power station of the Manchester Corporation, and has given excellent results. For such a purpose this type has the advantage that in case of a breakdown the flow of water is not stopped, the wastage of water thus entailed being of small consequence when compared with the inconvenience caused by a stoppage of the power supply.

Many types of the reciprocating piston meter have been made, but space forbids the mention of more than one example. The "Imperial" meter of Mr. Schönheyder (Fig. 287) consists of three single-acting cylinders fitted with a single hemispherical gun-metal distributing valve $r$ bearing on a vulcanite seat $S$. The upper side of each piston is exposed to inlet pressure, and according to the position of the valve the lower side of each in succession is put into communication with the outlet passage. The pistons are thus successively forced down and their contents discharged into the outlet. At the same time one or both of the 
other cylinders is having its piston raised, water being admitted below the piston.

Discharge is then practically continuous and the machine has no dead centre, while the smallest flow is registered. The positive type has the big advantage over the inferential meter that whereas the correct registration of the former is unaffected by any increase in the friction of the moving parts, such an increase in friction may seriously affect the speed of the latter for a given flow. Its chief drawbacks consist in its liability to water hammer unless worked at a very low speed, and its consequent large dimensions and high first cost.

(5) One of the best types of meter for the determination of the varying flow in a pipe, and hence of leakage, is that of $\mathrm{Mr}$. Deacon (Fig. 288). Here the water flows through a
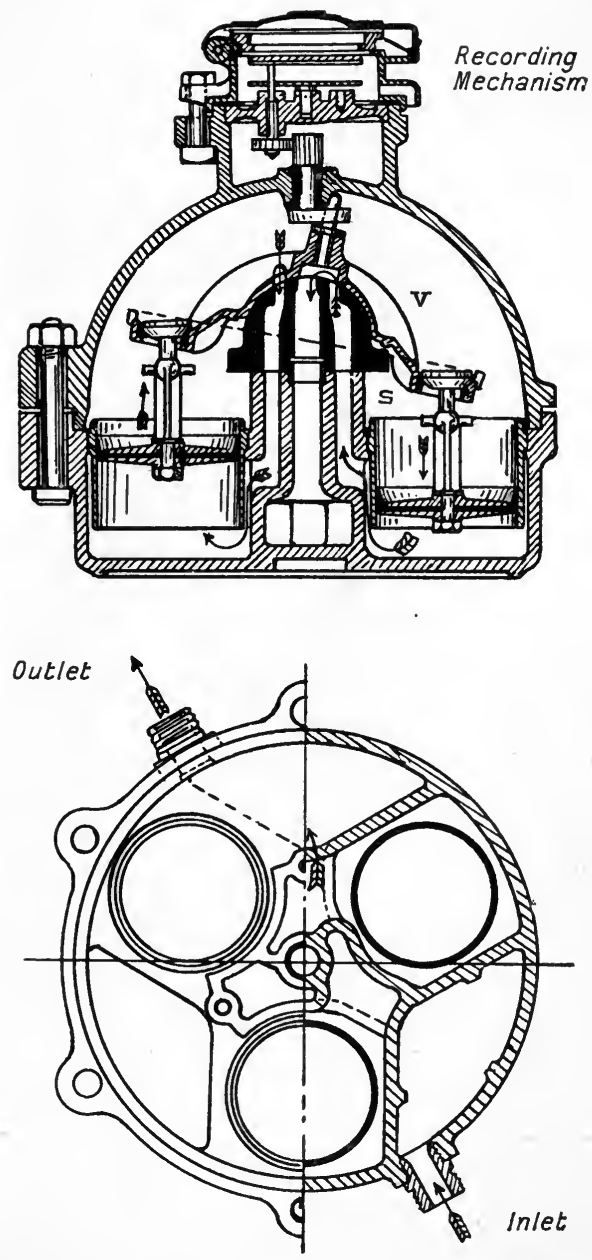

FIG. 287,- Schönheyder Positive Water Meter. conical tube containing an axial rod which carries a circular disc $D$. Any axial movement of this disc is resisted by a spring, and is recorded on the registering apparatus. The varying pressure on the disc, produced by any variation in the rate of How, may then 
be registered, and by suitably calibrating the recording mechanism the position of the rod may be arranged to indicate the flow in gallons per

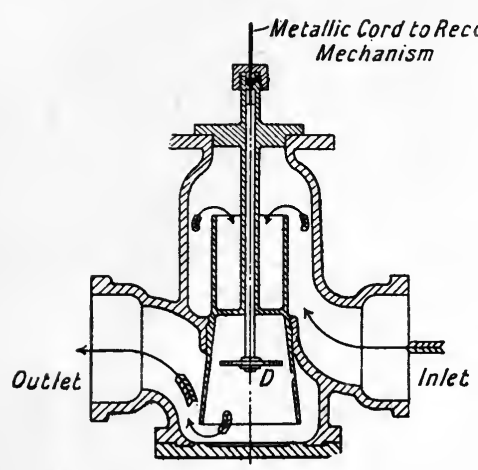

Fig. 288.-. "Deacon" Meter. minute.

For a detailed account of the development of the water meter the reader may consult a paper by Mr. William Schönhey der ("Proceedings Inst. Mechanical Engineers,", 1900, p. 37).

\section{Examples.}

(1) Determine the H.P. transmitted through a 6-inch pipe if the velocity of flow is 3 feet per second, and the delivery pressure 1,000 lbs. per square inch.

\section{Answer. 154 H.P.}

(2) If in the preceding question the pressure at the station is 1,120 lbs. per square inch, determine the efficiency of transmission, and also, taking $f=\cdot 01$, the length of the supply pipe line.

Answer. Efficiency $=89 \cdot 2$ per cent.

$$
\text { Length }=4.7 \text { miles. }
$$

(3) 100 B.H.P. is required from a hydraulic motor having an efficiency of 75 per cent. The motor is two miles from the generating station and only a single 6 -inch pipe line is available. The pressure at the station being $1,120 \mathrm{lbs}$. per square inch, determine the pressure at the motor, the efficiency of transmission, and the velocity of flow.

Answer. Pressure $=1,088$ lbs. per square inch.

$$
\begin{aligned}
& \text { Efficiency }=97 \cdot 0 \text { per cent. } \\
& \text { Velocity }=2 \cdot 392 \text { f.s. }
\end{aligned}
$$

(4) 'Two branch pipes, respectively 4 inches and 3 inches diameter, are supplied from a 6 -inch pipe. The pressure at the delivery end of the 4 -inch pipe is $700 \mathrm{lbs}$. per square inch; that at the end of the 3 -inch pipe is $710 \mathrm{lbs}$. per square ineh. These 
pipes are respectively 500 and 700 feet long. The 6 -inch pipe is 880 feet long. Take $f$ throughout as being $=01$ and determine the pressure at the inlet to the latter pipe.

(5) Determine the maximum H.P. which can be transmitted through a 4 -inch pipe two miles long $-f=\cdot 012$-if the inlet pressure $=750 \mathrm{lbs}$. per square inch. Also determine the pressure at the outlet, and the velocity of flow when this power is being transmitted.

Answer. 564 H.P.

Pressure $=500 \mathrm{lbs}$. per square inch

Velocity $=49 \cdot 4$ feet per second.

(6) An accumulator has an 18-inch ram and 23 feet lift, and is loaded with 129 tons total weight. Taking friction to account for 25 per cent. of the total pressure on the ram, determine the H.P. given into the mains if the accumulator falls steadily in three minutes, the pumps delivering 500 gallons per minute in the meantime.

$$
\text { Answer. Total H.P. }=67+396=463 \text {. }
$$

(7) A steam accumulator is placed horizontally and has a 48 -inch steam piston coupled to a 6-inch ram. The steam piston packings, etc., exert a frictional force of $500 \mathrm{lbs}$., while the hydraulic packings account for 1 per cent. of the total pressure exerted on the ram. Determine this pressure if the steam pressure is $150 \mathrm{lbs}$. per square inch.

Answer. 9,486 lbs. per square inch.

(8) The ram of a differential accumulator is 4 inches diameter, and the surrounding bush is $\frac{1}{2}$ inch thick. Determine the accumulator pressure if the supported weight is 10 tons, neglecting the effect of friction.

Answer. 3,168 lbs. per square inch.

(9) The accumulator of the preceding question feeds a rivetter, whose ram, at the end of its working stroke suffers a retardation of 5 feet per second per second. The rivetter ram is 5 inches diameter. Determine the pressure on the rivet head at the end of the stroke.

Answer. $32 \cdot 1$ tons. 


\section{CHAPTER XIX.}

Hydraulic Appliances-Lifts and Hoists-The Hydraulic Jigger-Cranes-The

Hydraulic Jack-Press-Forging Press-Rivetters-The Hydraulic Brake-

The Hydraulic Dynamometer.

HYDRAULIC APPLIANCES.

Art. 185.-Hydraulit Lifts and Hoists.

Probably in the aggregate more power is used by lifts and hoists than by any other class of hydraulic machinery, and for such work as this, hydraulic transmission is particularly suitable.

Several types of lift are in use, these consisting of modifications of the simple direct-acting or of the suspended type. The former consists of a hydraulic cylinder sunk vertically in the ground; of length slightly greater than the maximum travel of the lift; and fitted with a ram which carries the lift cage as shown in Fig. 289. ${ }^{1}$

Pressure water is admitted below the ram and thus raises the cage. It follows that the ram, considered as a loaded column, must be of sufficiently large sectional area to support the weight without buckling, and this prevents the use of very high pressures in the ram cylinder.

\section{Example.}

If the gross weight to be lifted $=2$ tons.

$״$ pressure in cylinder: $=750 \mathrm{lbs}$. per square inch.

" lift travel $=80$ feet.

$\begin{aligned} & \text { We have the area necessary to } \\ & \text { raise the weight }\end{aligned}=\frac{4,480}{750}=6$ square inches.

$\therefore$ Ram diameter $=23$ inches

1 By kind permission of the makers. 
a diameter which is obviously too small for a column 80 feet long, to support 2 tons without buckling.

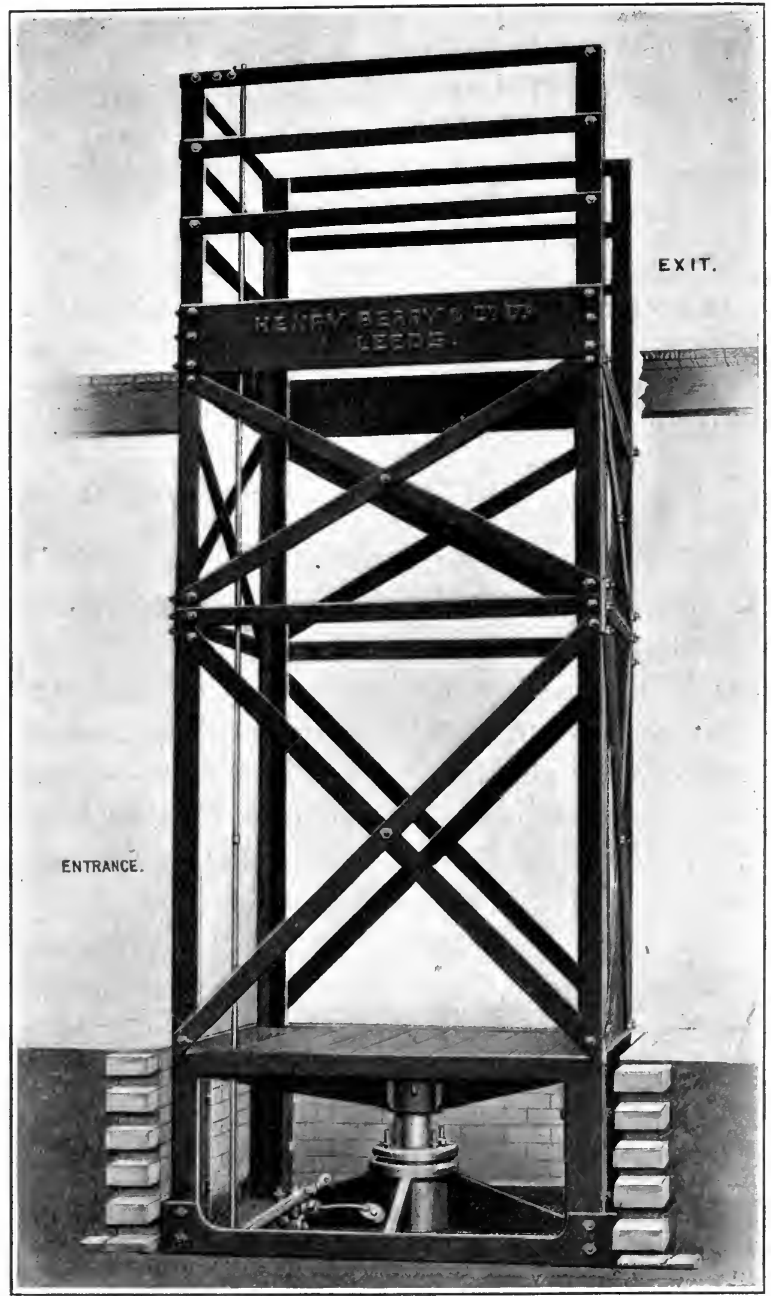

FIG. 289.-Direct-Acting Hydraulic Hoist.

Actually, however, the area of the ram would need to be greater than this in order to overcome friction and to give the necessary acceleration at starting. 
Thus, if $a=$ acceleration of ram and cage in feet per second per second we have

$\left.\begin{array}{l}\text { Force necessary to produce } \\ \text { this acceleration }\end{array}\right\}=\frac{2 \times 2,240}{g} \times a \mathrm{lbs}$.

And if $F=$ force in lbs. necessary to overcome friction of ram, we have

$$
\text { area of ram }=\frac{4,480\left\{1+\frac{a}{g}\right\}+F}{750} \text {. }
$$

Thus, if $a=2$ feet per second per second, while $F=10$ per cent. of the total force on ram, we have

$$
\begin{aligned}
\text { area of ram } & =\frac{4,480}{750}\left\{1 \frac{1}{16}\right\} 1 \cdot 1 \\
& =1 \cdot 17 \times \frac{4,480}{750}=7 \text { square inches. }
\end{aligned}
$$

In practice it is usual to make allowance for friction and acceleration by making calculations for a load about 25 per cent. in excess of the nominal.

If the area of the ram be increased, the working pressure must be reduced to suit, and for this purpose some form of pressure reducer-in effect a reversed intensifier-is used.

Also, since the weight of ram and cage forms a large proportion of the whole load to be lifted, this must be balanced for efficient working.

The energy of the pressure water is then simply utilized in elevating the useful load, in overcoming frictional and hydraulic resistances, and in accelerating the load and balance weights at the beginning of the travel. A further point to be noted is that since the volume of water displaced by the ram diminishes as the lift rises, the effective weight of the ram, which is its own weight less that of the water displaced, increases. In lifts for large powers the effect of this variation is in general unimportant, and becomes of less importance as the working pressure increases.

\section{EXAMPLE.}

$$
\begin{aligned}
& \text { Pressure }=250 \text { lbs. per square inch. } \\
& \text { Area of ram }=\frac{5 \times 2,240}{250}=5=5 \text { tons. } \\
&
\end{aligned}
$$$$
\text { Lift }=60 \mathrm{ft} .
$$ 
$\therefore$ Difference in apparent weight of $\}=\{$ Weight of a column of water 60 feet long

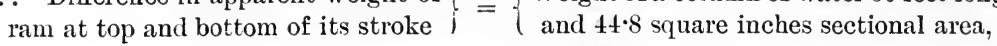

$$
=\frac{60 \times 44 \cdot 8}{144} \times 62.4=1,164 \mathrm{lbs} .
$$

If working pressure $=500 \mathrm{lbs}$. per square inch, the area of ram $=22.4$ square inches and its difference of weight $=\tilde{5} 83 \mathrm{lbs}$, a value which is small in comparison with the weight of five tons.

Various devices have been adopted to overcome these difficulties.

The weight of the ram and cage may be balanced by a counterbalance weight attached to the cage by chains passing over a series of pulleys at the top of the lift shaft, and since, as the lift rises, the length of chain on the balance-weight side of the pulleys increases, this may be made to counterbalance the increasing effective weight of the ram by making the chain of such dimensions that its weight per foot run is half that per foot run of the column of water displaced by the ram. This method suffers from the disadvantage that the upper part of the ram is in tension, and a fracture would cause the cage to be dashed against the top of the shaft. It thus detracts from the otherwise essentially safety features of this type of lift, and also increases the mass to be accelerated at the beginning of the travel.

A second device which is more common in high-class work is that of the balance cylinder, one type of which is illustrated in Fig. 290. Here pressure water is admitted to the interior of the hollow ram $B$. The cylinder. $D$ is in free communication with the pressure supply, or preferably with an auxiliary low pressure supply, through the pipe $C$, and a downward pressure on the annulus at $E$ is thus produced, which, together with the weight of this ram, produces a pressure in the cylinder $F$ sufficiently great to balance any required proportion of the weight of the lift ram and cage. The total pressure transmitted to the water in the cylinder $F$ is then the sum of the weight of the $\operatorname{ram} B$ and of the pressures on $E$ and on $\operatorname{ram} B$, the former performing the balancing, and the latter lifting the load.

A suitable area of lift ram being assigned, the external diameter of $B$ is designed so as to give the required intensity of 
pressure in the cylinder $F$. The lift cylinder is supplied from $F$ through the pipe $G$. On the down stroke of the lift, the ram $B$ rises, the balance water is returned to its own supply tank, and the only water rejected is that originally filling the high pressure ram $B$. In a lift of this type mentioned by $\mathrm{Mr}$. Ellington, the lift ram was $4 \frac{1}{4}$ inches diameter, the lift carrying

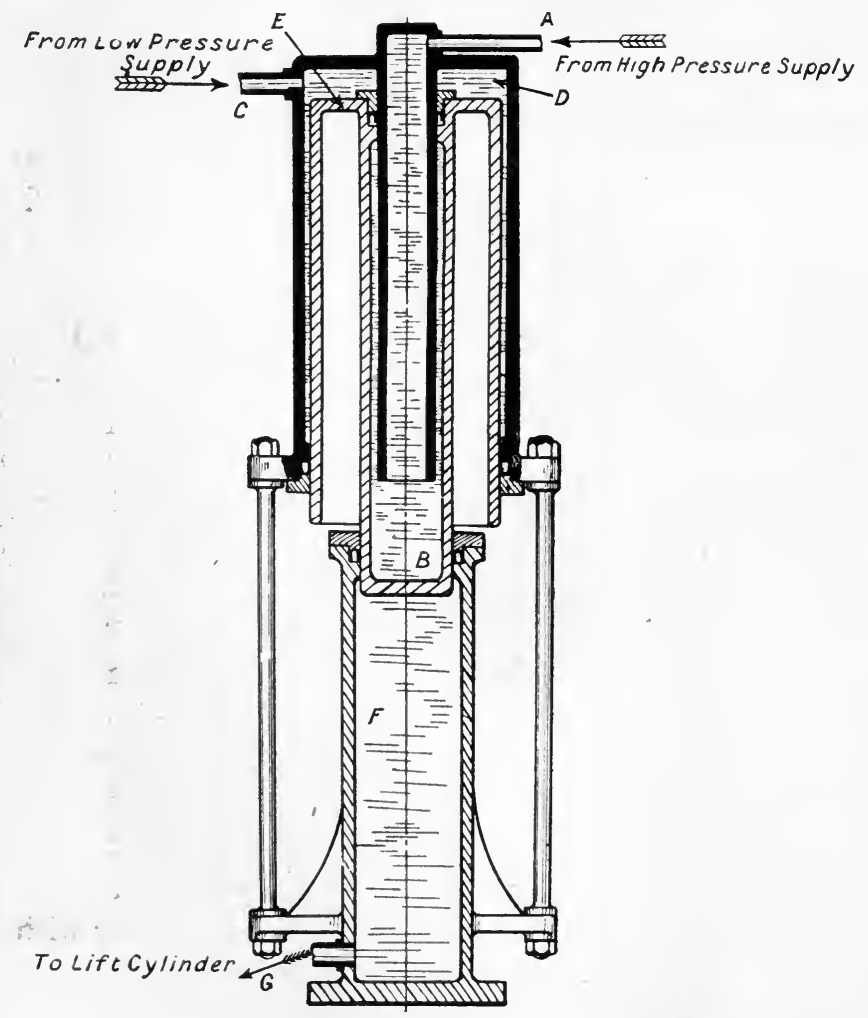

FIG. 290.-Balance Cylinder for Hydraulic Lift.

7 cwts. with a 70 feet rise. The volume of pressure water at 700 lbs. per square inch was $10 \frac{1}{2}$ gallons per trip, as against 43 gallons when working direct acting and without the balance cylinder.

It will be observed that as the balance ram falls, the pressure on the annulus $E$ increases, due to the increasing head to which 
it is subjected, and this to a certain extent counterbalances the difference in effective weight of the lift ram.

A second type of balance cylinder, which has the advantage that all packings are external, is illustrated in Fig. 291. Here pressure water is admitted through the hollow ram $A$ to the movable cylinder $B$, and forces water at a reduced pressure out of the fixed cylinder $C$ into the lift cylinder. The cylinder $B$ is weighted, this weight being proportioned so as to produce a pressure in $C$ sufficient to approximately balance the weight of the lift ram and cage. No attempt is made to allow for the varying effective weight of the lift ram. The weight is always underbalanced, sufficient weight being unbalanced to cause the lift to descend with sufficient rapidity on the down stroke even with an empty cage. A lift of this type, to lift $20 \mathrm{cwt}$. through a distance of 90 feet, carries a 6 -inch ram and uses $24 \frac{1}{2}$ gallons of water at $700 \mathrm{lbs}$. pressure per trip, as against 109 gallons without balance cylinder. With the direct acting or ram-supported lift, hoisting speeds up to 180 feet

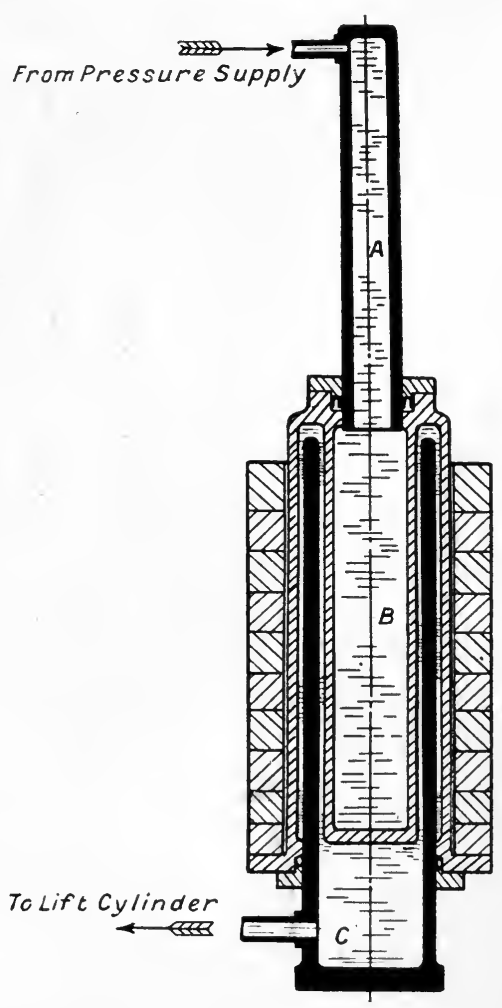

FI(. 291.-Balance Cylinder for Hydraulic Lift. per minute are common, 240 feet per minute being about the maximum.

The second type of lift-the suspension type-is manipulated from a hydraulic ram having a comparatively short stroke. The requisite travel in the wire rope or ropes by which the cage is suspended, is obtained by multiplying this by means of a jigger. The weight of the cage may be balanced by hanging weights, 
the varying immersion of the ram in this case being unimportant. $^{1} \quad$ A hoist on this principle, but with unbalanced cage,

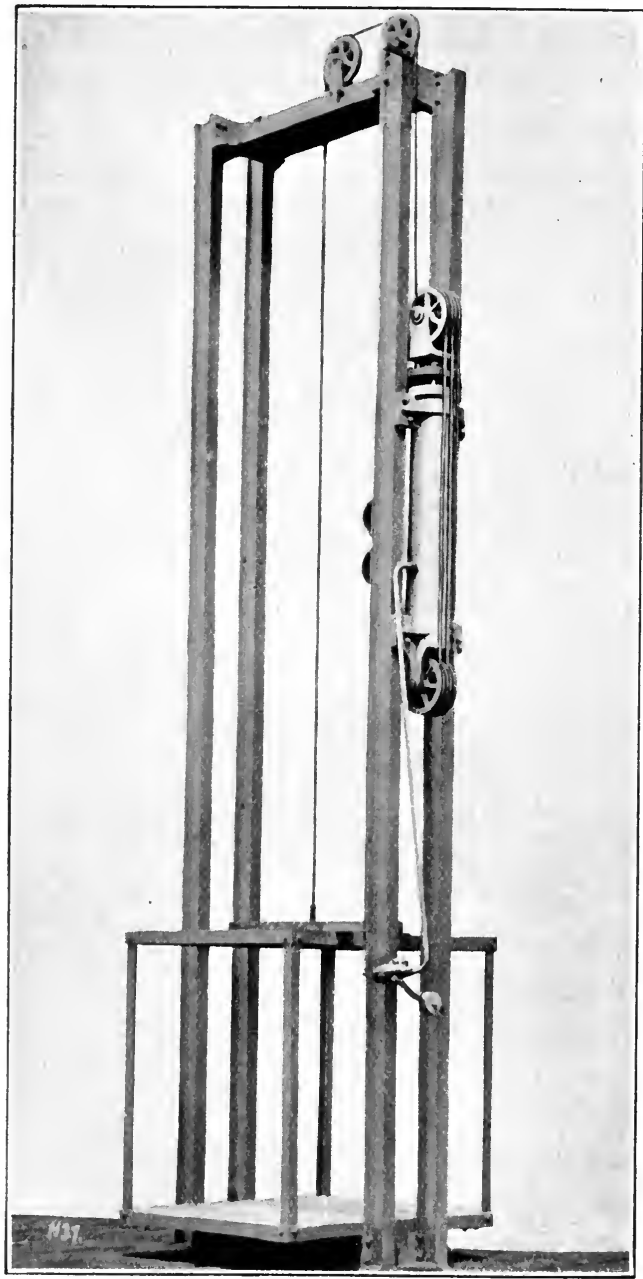

FIG. 292.--Suspended Hoist.

1 In modern suspended lifts, compensation is usually made for the varying weight of the suspending cables as the lift moves up and down. This may be accomplished by coupling up the idle side of the elevator piston or plunger to a low pressure water column whose height varies with the position of the cage. This, and a second method of compensation by the use of hanging chains, are well illustrated in Cassier's Magazine, Sept., 1907, p. 394. 
is shown in Fig. 292, while a balanced lift is shown in Fig. 293. Here two wire ropes are employed for lifting and two for carrying weights which partly counterbalance the cage. The system has certain advantages in virtue of the cheapness of construction of the shorter ram and cylinder, and does not necessitate the provision of a deep well below the lift shaft to contain the cylinder, which may be fixed horizontally if required. Its drawbacks are due to the inefficiency of the multiplying jigger, and to the serious effects which may follow the rupture of a wire rope. If carefully designed, and frequently examined, the latter contingency should, however, be very remote, while the provision of adequate safety catches and brakes renders this almost as safe as the direct-acting system. The speed of hoisting may be made as great as is convenient. For passenger hoists this is usually about 2 feet per second, and for warehouse hoists up to about 6 feet per second.

For very heavy lifting, such as is necessary in canal lifts, etc., where loads up to 1,000 tons may be carried on a single ram, the direct-acting is the only suitable type.

Art. 186.-Efficiency of the Hydraulic Jigger.

The chain, or wire rope, and pulley multiplying gear known as the

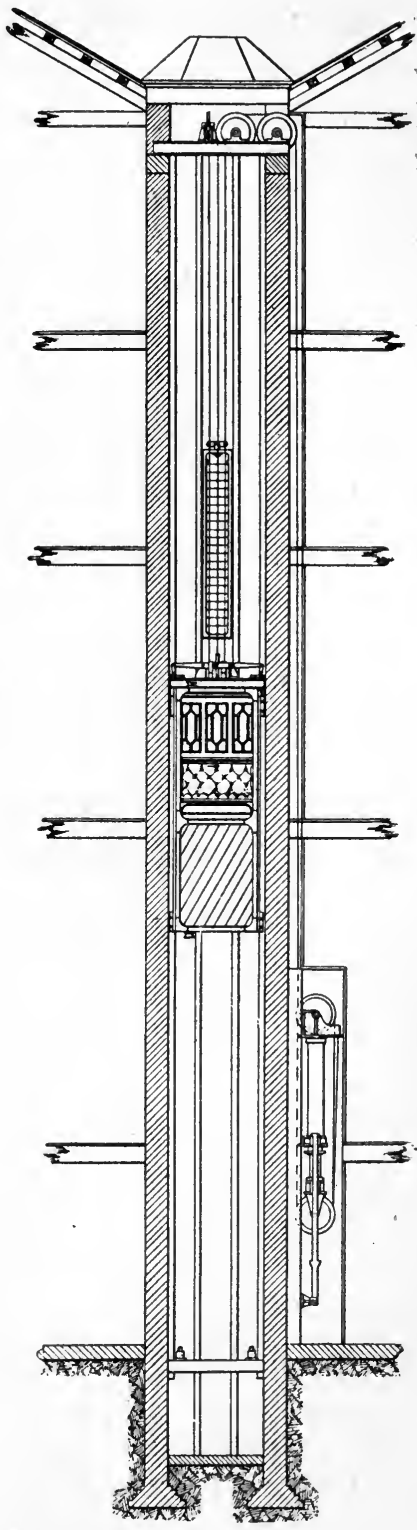

FIG. 293.

Multiple Wire Suspended Lift with Counterbalance Weights. 
hydraulic jigger which is used to multiply the motion of a short stroke hydraulic ram for crane or hoist as illustrated in Figs. 292 and 294, diminishes the mechanical efficiency of the system to a fairly large extent.

The loss thus introduced increases with the number of multiplications, and its magnitude varies so largely with the size and condition of sheaves, bearings, and rope or chain, that no definite law can be expected to even approximately cover each case.

With ordinary well designed gearing, having large pulleys, small bearings, and wire ropes in good condition and well lubricated, the efficiency of the jigger may be taken as being approximately given by the following formula (H. Adams)-

$$
\eta=\cdot 906-\cdot 021 \mathrm{~m}
$$

where $m$ is the number of multiplications of the stroke.

If, then, the friction of packing leathers $=5$ per cent. of the total force on the ram, we have the efficiency of ram and jigger

$$
\begin{aligned}
& =.95\{.906-.021 \mathrm{~m}\} \\
& =.86-.02 \mathrm{~m} .
\end{aligned}
$$

This gives the following values of the efficiency :-

\begin{tabular}{c|c|c|c|c|c|c|c}
$m$ & 2 & 4 & 6 & 8 & 10 & 15 & 20 \\
\hline Eff. & $\cdot 82$ & $\cdot 78$ & $\cdot 74$ & $\cdot 70$ & $\cdot 66$ & $\cdot 56$ & $\cdot 46$ \\
\hline
\end{tabular}

\section{Art. 187.-Hydraulic Cranes.}

Where high pressure water is available it provides a most convenient means of operating power cranes, and in its safety, adaptability to suit varying conditions, and steadiness of operation, offers many advantages over its rivals-compressed air and electricity.

Such cranes are usually operated by hydraulic jiggers, the various operations of lifting, racking, and slewing being often performed by separate rams and cylinders, each regulated by its own separate valve.

Where the load to be lifted may vary within wide limits, some device is usually adopted to economise water at light loads. For 
small cranes, up to about two tons, a differential or telescopic ram is used, the smaller working inside the larger, which itself works in the pressure cylinder. For light loads the larger is held stationary by locking gear, the smaller ram then doing the lifting. For heavy loads the two rams work together as one.

For loads much above two tons, two distinct cylinders, each having its own valves, pipes, chains, and jibhead sleeves, offer many advantages. Thus for a 10-ton crane, one cylinder to lift $6 \frac{1}{2}$ tons and a second to lift $3 \frac{1}{2}$ tons would be suitable, the two working in conjunction for loads above $6 \frac{1}{2}$ tons.

In Fig. 294 a simple type of hydraulic wall crane, suitable for a warehouse, is illustrated. Here hydraulic power is used for lifting only. The jigger may be placed against the wall as shown, or horizontally if preferable, and the controlling valve worked from a hand rope on any floor of the building.

In Fig. 295, a type of hydraulic crane $\mathrm{much}$

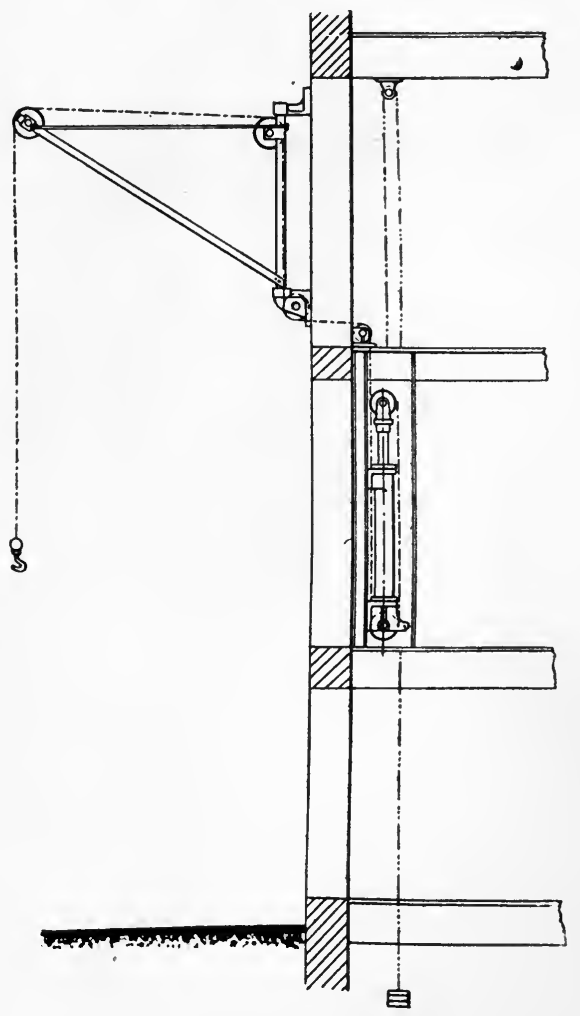

FIG. 294.- Hydraulic Wall Crane. used in steel works for removing ingots from the soaking pit to the rolling mill is illustrated. ${ }^{1}$ The crane is self-contained, hoisting, lowering, racking in and out, and slewing being performed by hydraulic power. The latter operation is performed 
by means of chains, gearing with a chain wheel fixed on the foundation plate.

There are two racking cylinders $R$, with a six-fold multiplying motion, one hoisting cylinder $H$, with an eight-fold multiplication, and two slewing cylinders $S$, with a two-fold multiplication,

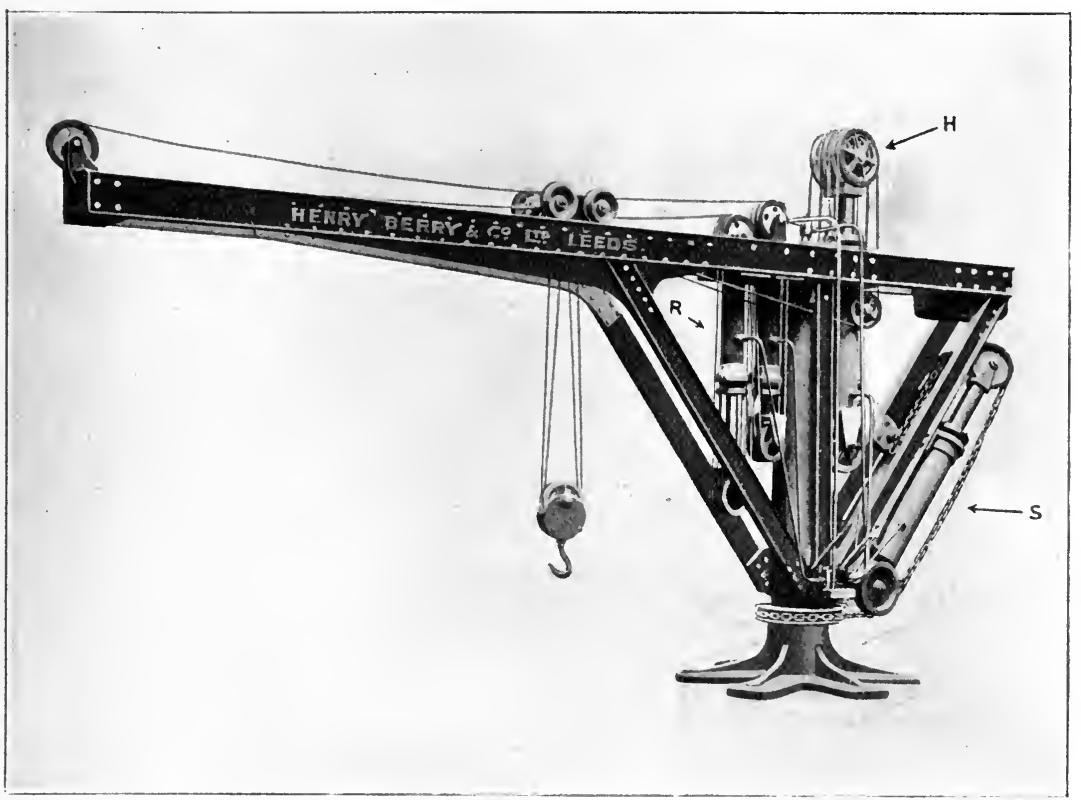

FIG. 295.-Welf-eontained Hydraulie Crane with separate Raeking, Hoisting and Slewing Cylinders.

the valves being so placed that one man has full control over the whole crane.

The illustration shows details fairly well, and is selfexplanatory.

For comparatively small powers and lifts these cranes may be direct acting, such an one, suitable for loads up to two tons, and for lifts up to about six feet, being illustrated in Fig. 296. ${ }^{1}$ Here the lifting hook is attached directly to a carriage running along the jib, which is itself raised and lowered by a direct acting hydraulic cylinder sliding between the cheeks of the mast and

1 By Messrs. The Hydraulic Engineering Co., Itd., Chester. 
working upon a hollow steel ram forming the bottom centre of the crane. For smaller loads and lifts the lifting cylinder may itself be suspended from the travelling carriage, the hook being

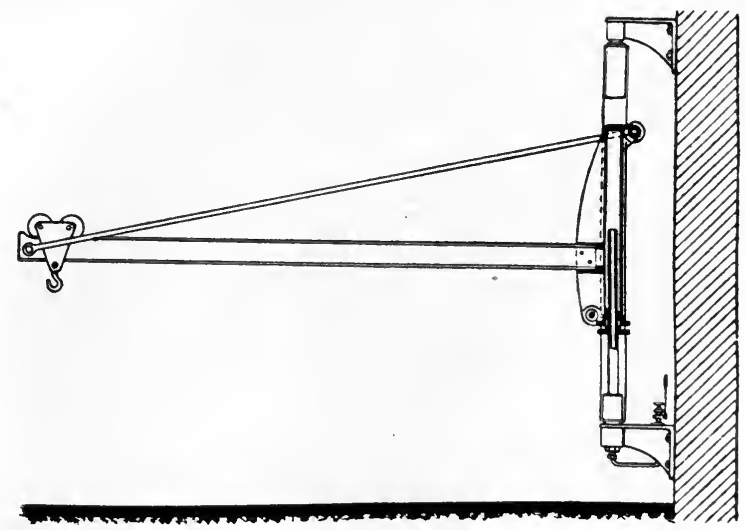

FIG. 296.-Direct-acting Wall Crane.

attached to the ram, and pressure water being conveyed to the cylinder by a walking pipe.

With the hydraulic crane, the speed of the lifting hook may be adjusted up to about six feet per second.

\section{Art. 188.-The Hydraulic Jack.}

For the manipulation of heavy weights by hand the hydraulic jack is of the greatest value. In principle it consists of a Bramah's press on a small scale, and one type of its construction is illustrated in Fig. 297. Here the reciprocation of the hand lever, pumps water from the cistern $A$, through the hollow plunger $B$, past the suction and delivery valves $V_{a}$ and $V_{D}$, into the space $C$ below the lifting ram, and raises the latter. The weight to be lifted is carried either on the ram table $T$, or on a side shoe projecting from the ram casing. Screws are provided for supplying the cistern $A$ with water and for allowing of the inlet of air, while a lowering screw permits of the escape of pressure water from the space below the lifting ram into the supply cistern when it is required to lower the load. The lifting ram is usually packed by means of a cup leather, and the pump plunger by means of a single leather ring. 
If $m=$ ratio of travels of lever handle and of pump plunger, and if $a$ and $A$ are the areas of plunger and ram, the theoretical mechanical advantage of the jack $=m \frac{A}{a}$, so that, neglecting friction, the force $P$ to be applied at the lever handle to support a weight $W^{\prime}$ on the ram table is given by $I^{\prime}=W \frac{a}{m \cdot A}$ lbs.

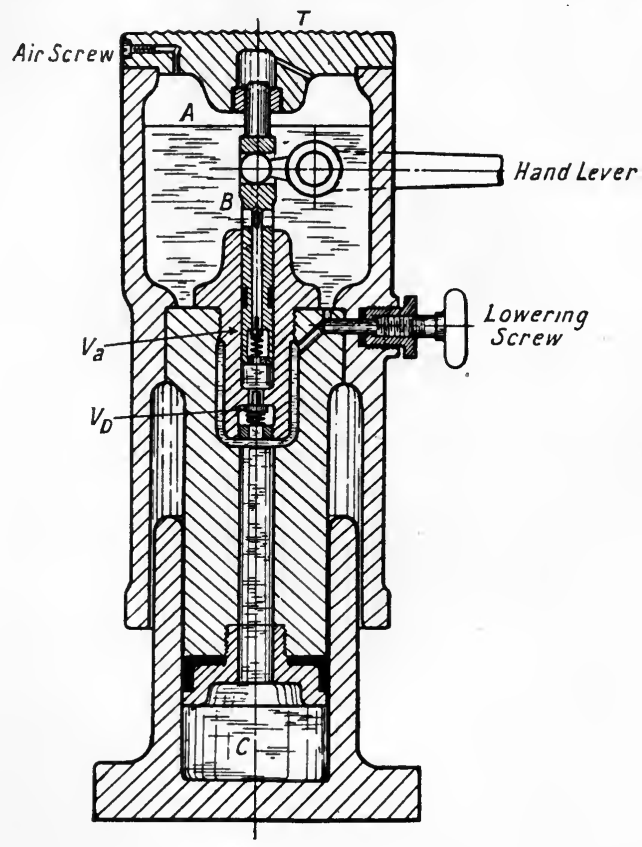

FIG. 297.-Hydraulic Jack.

Thus if

$$
m=\frac{24}{1 \cdot 25}=19 \cdot 2 \text {. }
$$

$\left.\begin{array}{l}\Rightarrow \quad \text { diameter of ram }=5 \frac{1}{2} \text { inches } \\ " \quad \text { diameter of plunger }=1 \text { inch }\end{array}\right\} \frac{A}{a}=30 \cdot 25$,

$$
\text { if } P=50 \mathrm{lbs} ., W=\frac{50^{\circ} \times 19.2 \times 30^{\circ} 25}{2,240}=13 \text { tons. }
$$

Actually the weight lifted is less than this because of friction losses. 'The efficiency of the jack, or the ratio $\frac{\text { actual }}{\text { theoretical }}$ weight lifted by the ram, can only be determined experimentally, and 
varies largely with the condition and size of the apparatus, as well as with the magnitude and position of the load.

Friction losses are proportionately less as the size of the machine increases, and, owing to the fact that the friction is partly mechanical and independent of the load, the losses diminish proportionately as the load increases.

Eccentric loading largely increases the mechanical friction, and may double the friction losses.

The efficiency of a jack in fairly good order may be taken as varying from about 66 per cent. in the case of a small 3-ton jack, with eccentric loading to about 93 per cent. with a 100-ton jack and central loading, and is approximately constant for loads greater than one-fifth of the nominal capacity of the jack.

\section{Art. 189.-T'ine Hydraulic Press.}

Reference has already been made in Art. 8 to the Bramah's press. Its modifications, as applied to such work as cotton baling, boiler-plate flanging, cartridge-case drawing, and heavy forging, are too numerous for detailed mention, and only one or two of such applications will be considered in detail in the present treatise. ${ }^{1}$ Fig. $298^{2}$ illustrates a form of press used for flanging operations, \&c., and capable of exerting a pressure of 420 tons. In this machine two small rams are installed for lifting the head on the up stroke, while the pressure is applied by one central and two side rams, pressure water being supplied to these in succession as additional force is required. During the idle part of the down stroke, water from the exhaust is allowed to fill the space vacated by the rams. The arrangement of valves by which this is rendered possible is simple and worthy of notice, and is shown in detail in Fig. 299. Here the main pressure and exhaust valves $V_{P}$ and $V_{E}$ each carry a closely fitting piston of greater area than the valve itself, so that if the pressure above the piston and below the valve is equalised the effect of the pressure between the piston and valve is to lift the latter from its seat. A small orifice is provided in each piston,

${ }^{1}$ For further applications of the 'press the reader is referred to Blaine's "Hydraulics."

2 By courtesv of the makers, Messrs. Henry Berry \& Co., Leeds, 
so that under normal conditions the pressure above and below the piston is equal, and pressure keeps the valve on its seat. The auxiliary valves $I_{1}$ and $I_{2}$ are worked directly from the

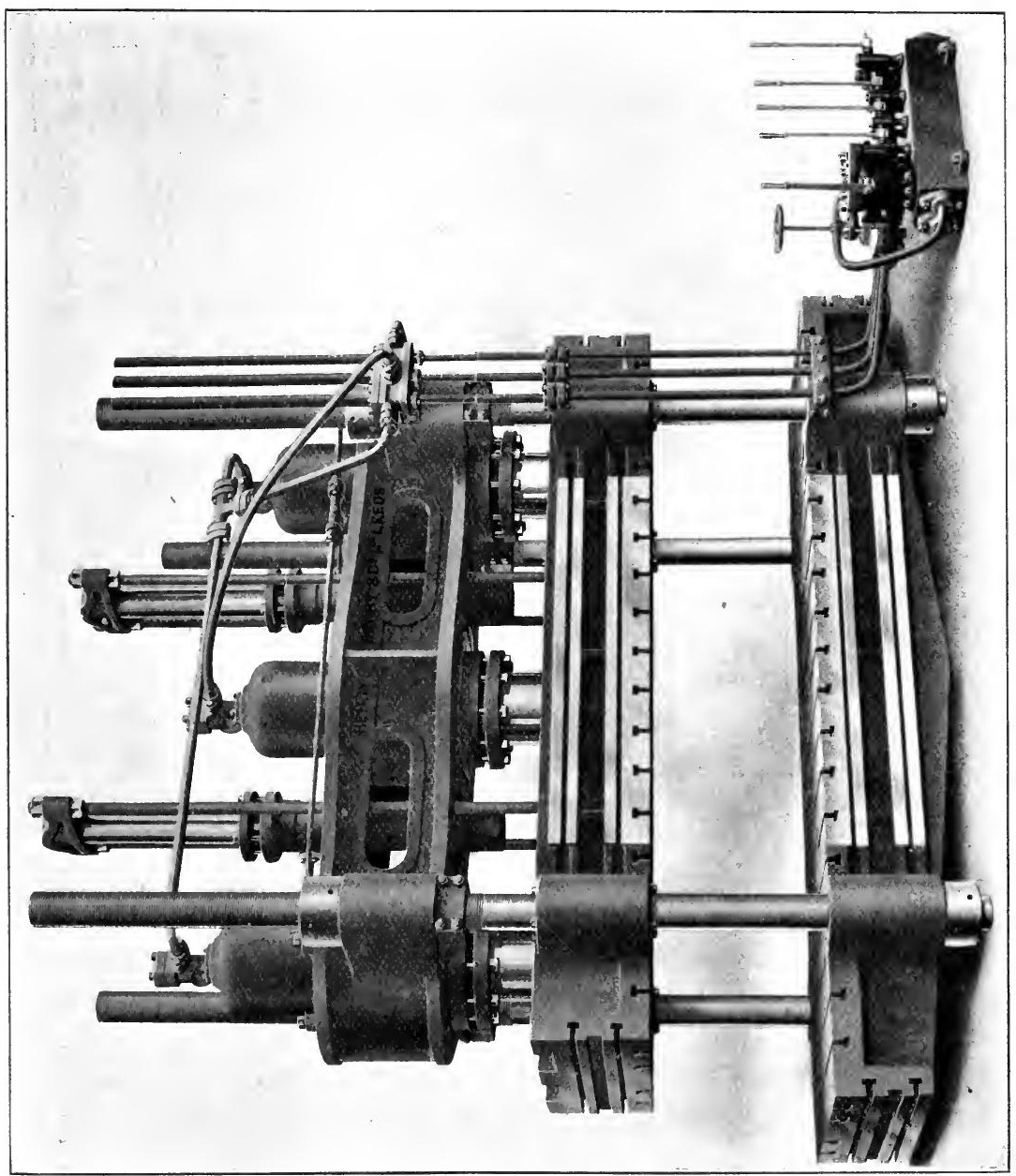

operating lever, and when open give free communication between the under side of the corresponding main valve and the upper side of its piston.

The opening of the auxiliary valve thus leads indirectly to the 
opening of the corresponding main valve. 'During the idle part of the down stroke both auxiliary valves are closed, a partial vacuum is produced above the exhaust valve which opens and water is drawn from the exhaust into the cylinder. When pressure is required, a quarter-turn of the operating lever raises the valve $V_{1}$ and so the pressure valve, and admits pressure water through the passage $P_{1}$ to the cylinder. On the working

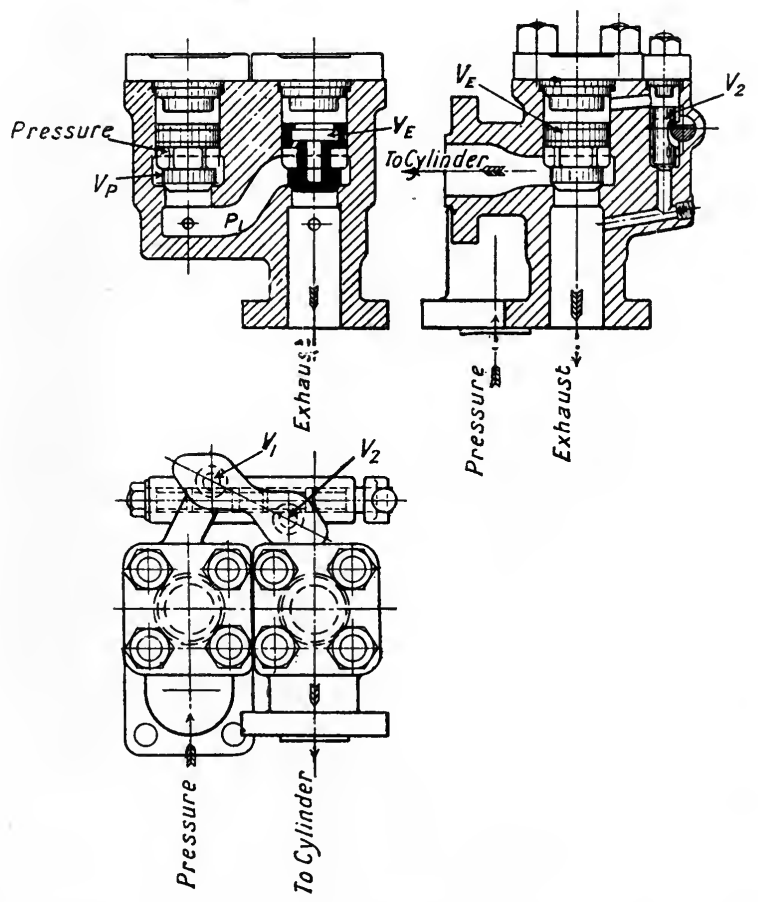

FIG. 299.-Details of $2 \frac{1}{2}$-inch Operating Valve for 420 tons Press.

stroke being completed, a half-turn of the lever closes $r_{1}$ and opens $V_{2}$, thus opening the exhaust valve $V_{E}$ and putting the cylinder into communication with the exhaust, while a second lever and valve admit pressure water to the return cylinders. A smaller press for similar work is illustrated in Fig. 300. 
720 HYDRACLICS AND ITS APPLICATIONS.

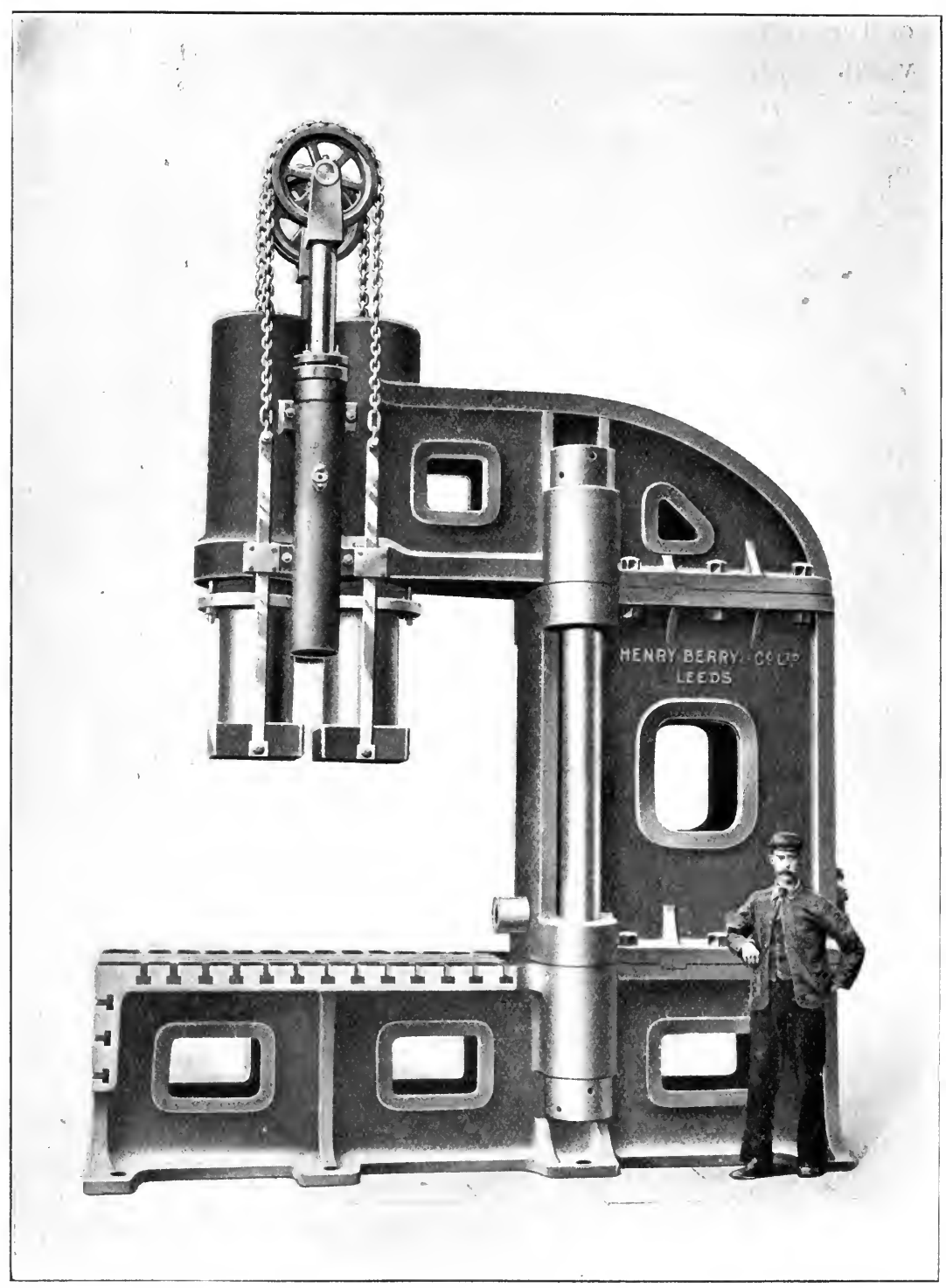

Fig. 300.-Hydraulic Flanging Press 
Art. 190.-The Hydraulic Forging Press.

In the production of heavy forgings for large ingots of mild steel, it is essential that every part of the ingot should be equally worked if the resultant forging is to be homogeneous in structure. Where a steam hammer is used, the energy of the blow

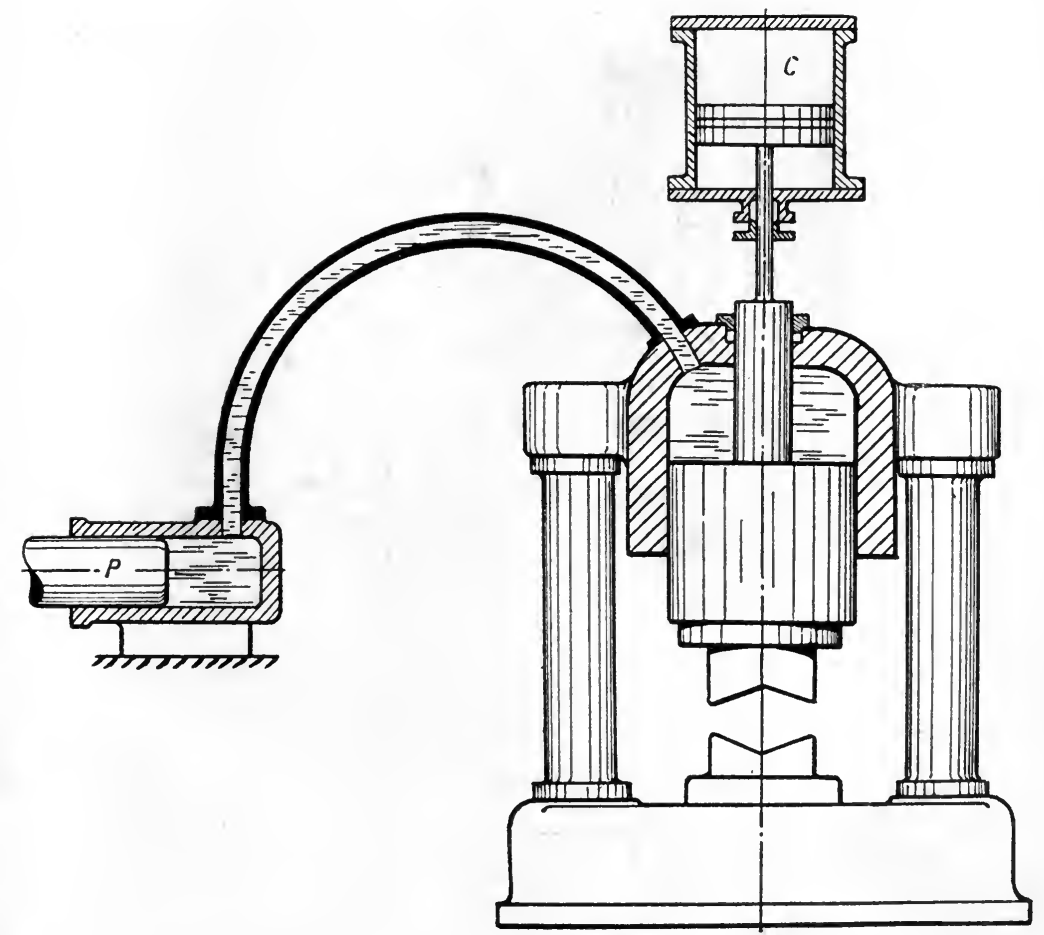

FIG. 301.-Allen's Hydraulic Forging Press.

is absorbed in producing distortion of the outer layers, while the interior is practically unaffected. This disadvantage is overcome by the use of the hydraulic forging press, with its slow and powerful compression, and this is gradually supplanting the steam hammer for the production of very heavy forgings. The principle of the press is the same as that of the ordinary flanging press, and for a very complete account of its 
development and for details of its design, the reader is referred to a paper by R. H. Tweddell. ${ }^{1}$

The Allen press works on a very ingenious principle and is illustrated diagrammatically in Fig. 301. Here a pressure accumulator is unnecessary, as are valves in the high pressure water column. For its operation a low pressure water supply, at about

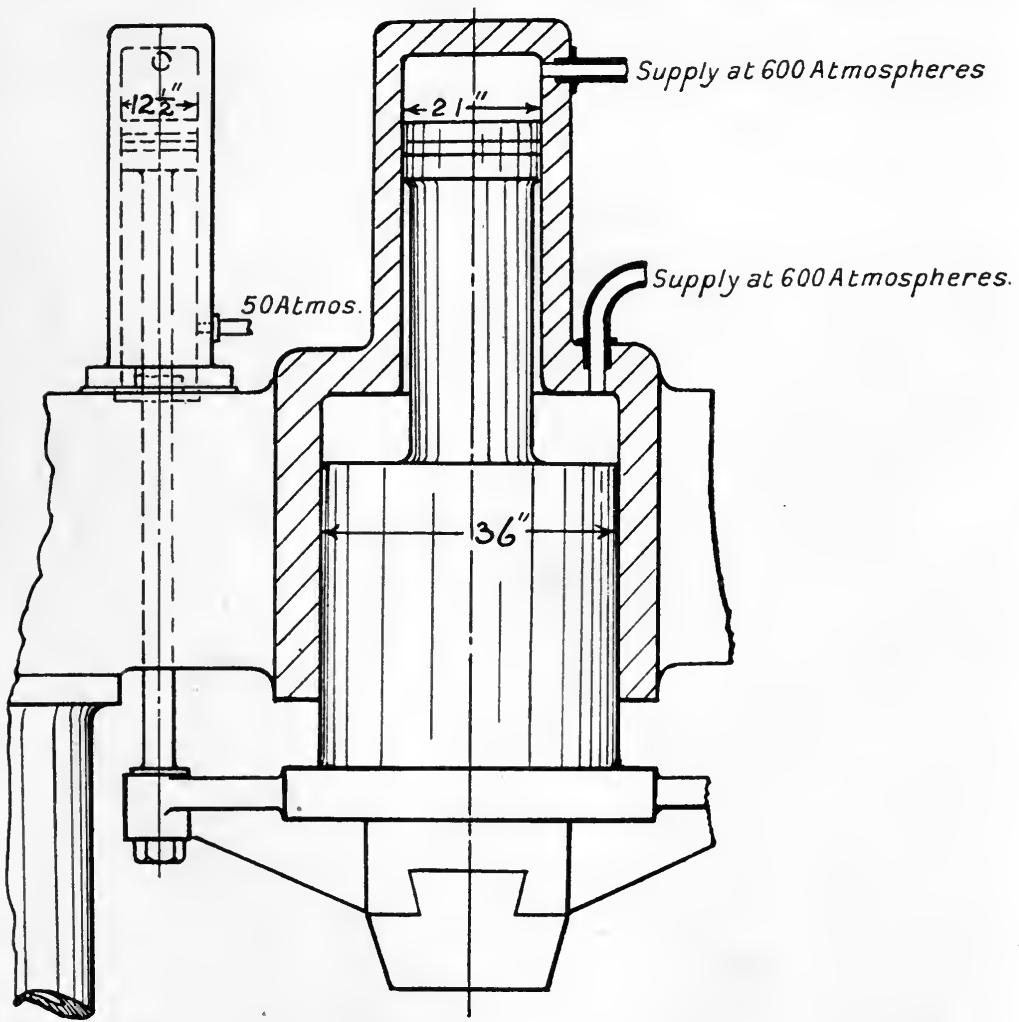

FIG. 302.-Cylinder's of 3-power Hydraulic korging Press for a maximum effort of 4,000 tons.

$200-300$ lbs. per square inch, is necessary, and during the idle part of the stroke this follows up the ram, the high pressure connecting pipe being kept full in the meantime. When pressure is required this supply is cut off and communication is made with the high pressure pump $P$. This has no valves, so that the ram has a

1 "Proceedings Institutê of Civil Engineers," vol. 117, 1893-4, p. 1. 
continuous up and down motion, the water column simply following the motion of the pump plunger. The inertia of this column thus has a useful effect in increasing the pressure on the ram at the end of the working stroke. A steam cylinder $C$ is usually provided for lifting the ram.

A type of forging press to be worked in connection with an accumulator and intensifier is illustrated in Fig. 302. This press is fitted with a differential ram having diameters of 21 inches and 36 inches and supplied with pressure water at 600 atmospheres. By using each cylinder separately, or the two in combination, three powers having the ratio $1: 2: 3$ may be obtained, the effective force varying from 1,300 tons with the small ram to 4,040 tons with the two in combination. 'I'wo lifting cylinders, using water at 50 atmospheres pressure, exert a constant upward force of 70 tons which has been taken into account in obtaining the above values.

As, during the working portion of the stroke of a hydraulic press or similar machine, the ram velocity is required to be only very slow, the diameters of the supply pipes may be very small without appreciable loss of head. A ratio of ram diameter to pipe diameter of about 12 to 1 is usually adopted.

\section{Art. 191.-Hydraulic Rivetters.}

The hydraulic rivetter provides another good illustration of the adaptability of the hydraulic machine to workshop processes. Here the problem is to get a fairly large pressure on the rivet during the first portion of the ram stroke, so as to form the rivet head and to clinch the plates, and a final larger pressure of the nature of an impact to cause the rivet to expand and fully fill its hole. The extent to which this is attained in the rivetter will

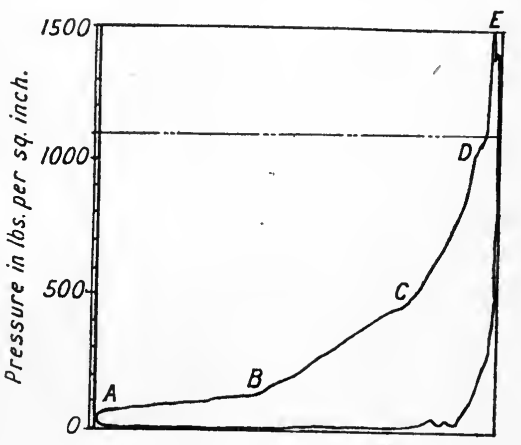

Fili. 303.

Pressure Diagram from Cylinder of Hydraulic Rivitter. Accumulator pressure 1,10 l lbs. per square inch.

3 A 2 
be evident from Fig. 303, which represents a typical pressure diagram taken from the cylinder of such a machine,

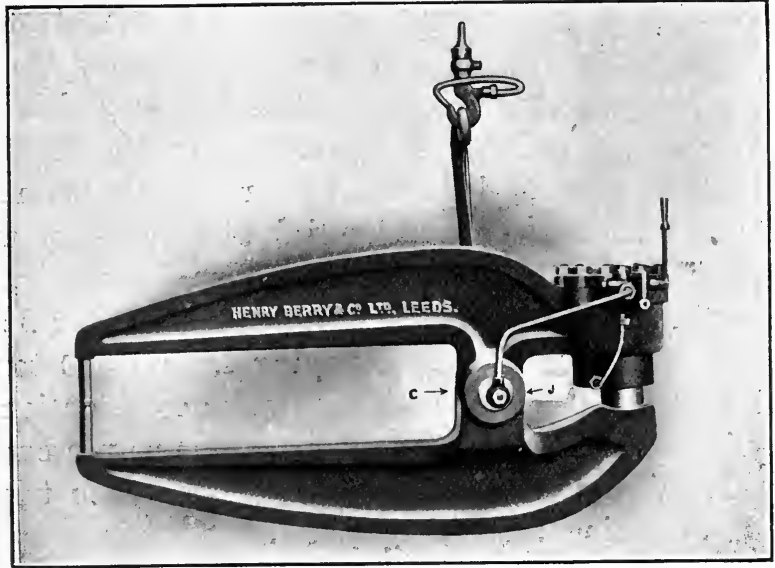

FIG. 304.-Portable Rivetter. Hinged Type.

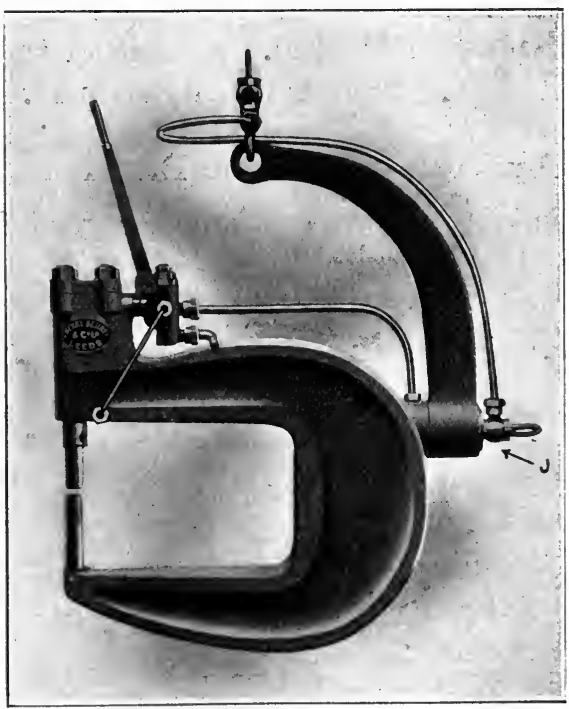

Fig. 305.-P'ortable Bear Rivetter.

supplied from an accumulator under a pressure of 1,100 lbs. per square inch. Here $A B$ represents the idle part of the stroke 
during which the ram is being brought up to its work, $B C$ the setting up of the rivet and the formation of the head, $C^{r} D$ the clinching of the rivet and the closing of the plates, while the sudden stoppage of the heavy accumulator ram is responsible for a further rise in pressure $D E$ above the accumulator pressure, which is depended upon to fill up the rivet hole.

One type of portable rivetter is shown in Fig. 304.' Here the

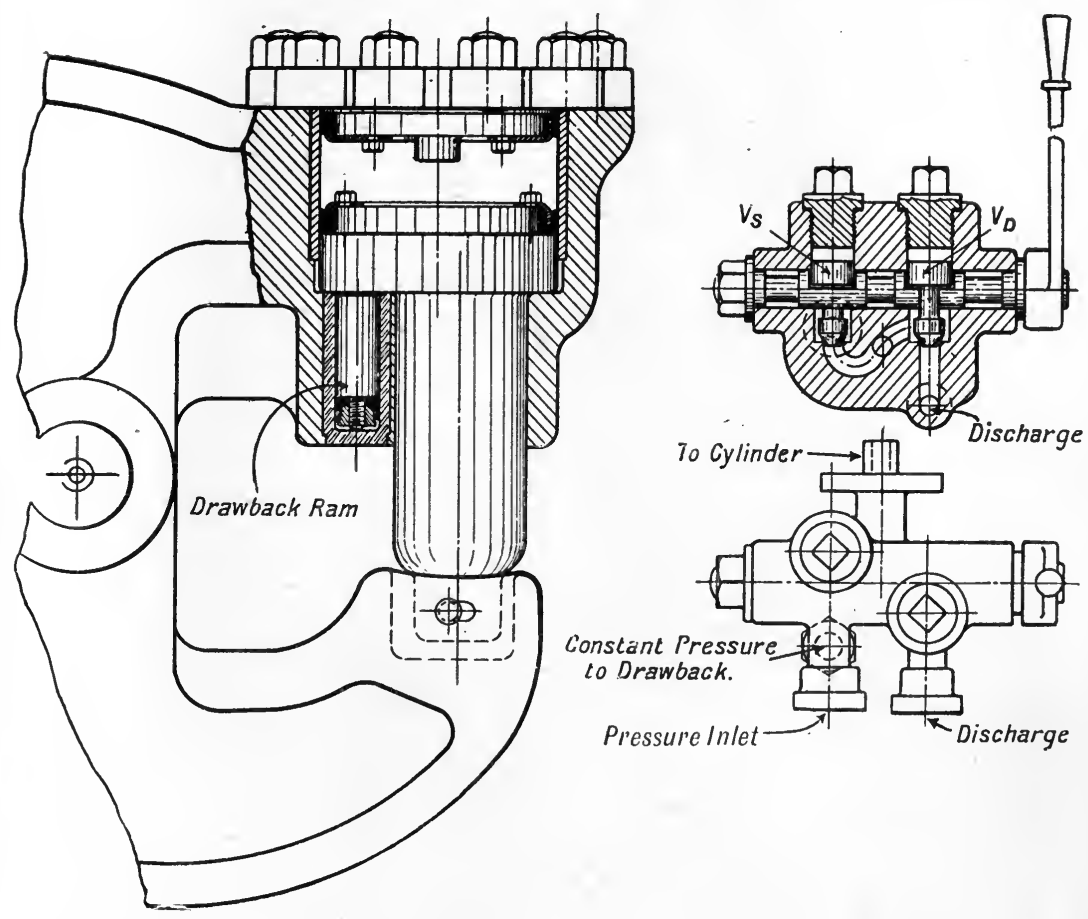

Frg. 306.- Section of Cylinder and Valves of Hinged Type Rivetter.

ram is situated at one end and the rivetting is performed at the other end of a lever hinged at $C$. As thus arranged the machine offers some advantages over the more ordinary type of bear rivetter shown in Fig. 305, ${ }^{1}$ for work in restricted spaces. In the latter type the operation of rivetting is directly performed by the hydraulic ram, the rivetter jaws being formed as a single steel 
casting. In Fig. $306^{1}$ a section of the cylinder and valves of a rivetter of the hinged type is shown. Here water is admitted

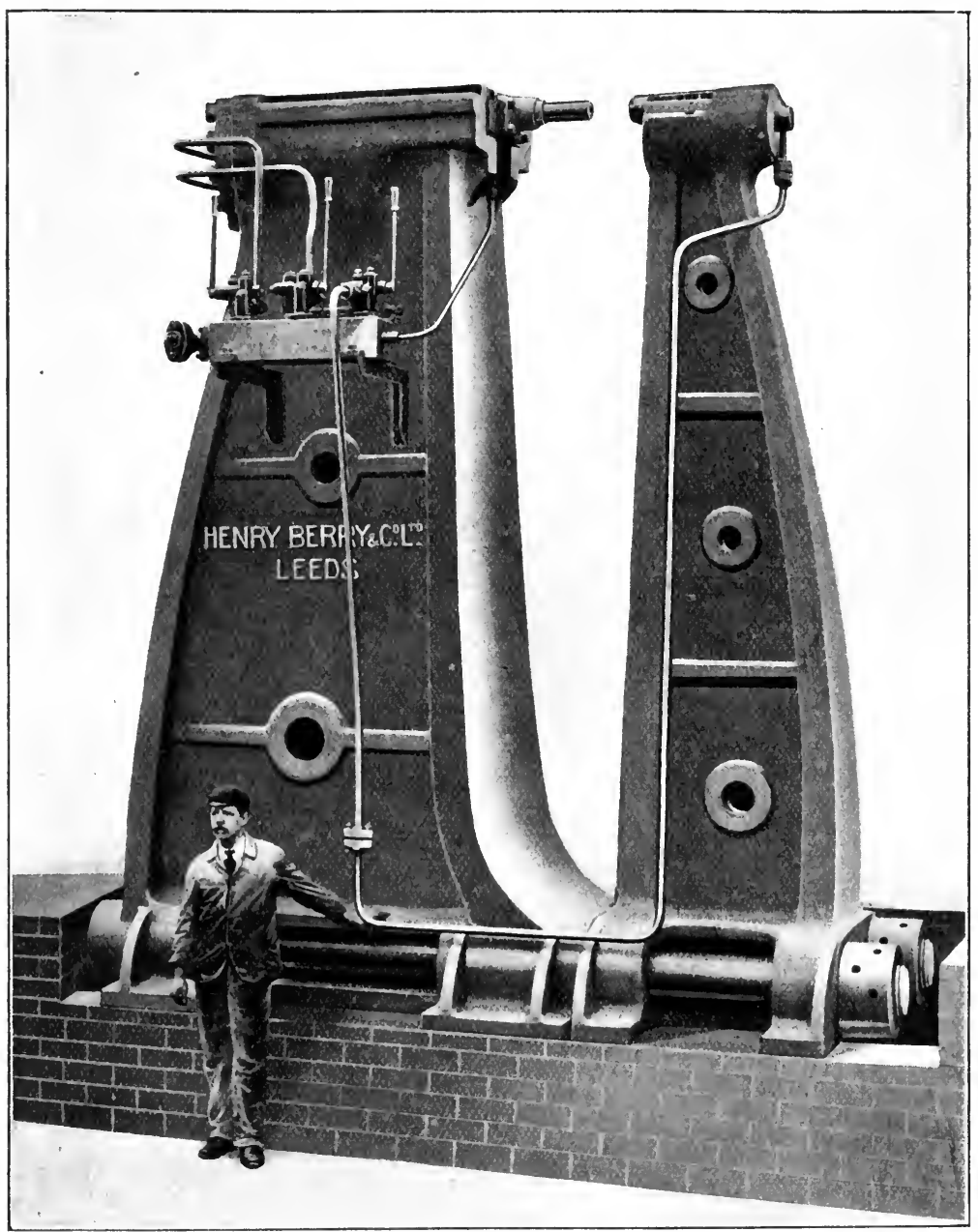

FIG. 307.-Fixed Hydraulic Rivetter.

to or discharged from the ram cylinder by the arrangement of valves shown. Thus a quarter-turn of the regulating lever raises

1 By the courtesy of the makers, Messrs. Henry Berry \& Co., Ltd., Leeds. 
the valve $V_{S}$ and puts the cylinder into communication with the pressure supply. On the completion of the working stroke a half-turn in the opposite direction closes the valve $V_{S}$ and opens valve $V_{D}^{r}$, putting the cylinder into communication with the discharge passages. The main ram is drawn back on its idle stroke by means of a special drawback ram which is always exposed to supply pressure. The method of packing the rams and the general construction is sufficiently well indicated in the figure.

In these portable machines pressure water is supplied to the suspending hook by means of a walking pipe, and provision is made for working at a rivet at any inclination, by means of a watertight swivel joint at $J$. (Fig. 304.)

Fig. $307^{1}$ illustrates a form of fixed rivetter suitable for the circumferential joints of large boiler shells. Here both jaws of the bear are fitted with rams, that to the right of the illustration being adjusted to suit the length of rivet to be handled. The working cylinder, to the left, is in this machine fitted with a differential ram so as to allow a larger range of work to be handled with economy. In this case the pressure supply to each ram is regulated by a separate valve.

\section{Art. 192.-The Hydraulic Brake.}

The necessity for some braking apparatus by which the kinetic energy of a heavy body - such as a moving train or of a gun during recoil-might be quickly and safely absorbed without the recoil effect obtained by the use of spring buffers, led to the invention of the hydraulic brake.

In its simplest form this consists of a cylinder fitted with piston and rod and filled with some liquid, usually oil, water, or glycerine. The two ends of the cylinder are connected, either by one or more small passages formed by holes in the body of the piston itself, or by a bye-pass pipe fitted with a springloaded valve or with a throttling valve by which the area may be adjusted. In its simplest form the brake is extensively used as a dashpot for damping the vibrations of governing mechanism and the like. 
When used as a buffer stop, the body whose kinetic energy is to be absorbed forces in the piston rod and produces a flow of liquid at high velocity through the connecting orifices. The energy of the body is thus partly transformed into kinetic energy of the liquid, which is dissipated in eddy formation, and partly expended in overcoming the frictional resistances of the connecting passages, together with the mechanical friction of the brake. The greater part of the energy is thus ultimately transformed into heat. Since the energy absorbed by the brake is constant for a given mass moving with a given velocity, and is equal to the mean resistance of the brake multiplied by the length of its stroke, it is evident that the pressure in the brake cylinder will have its least maximum value when this pressure, and

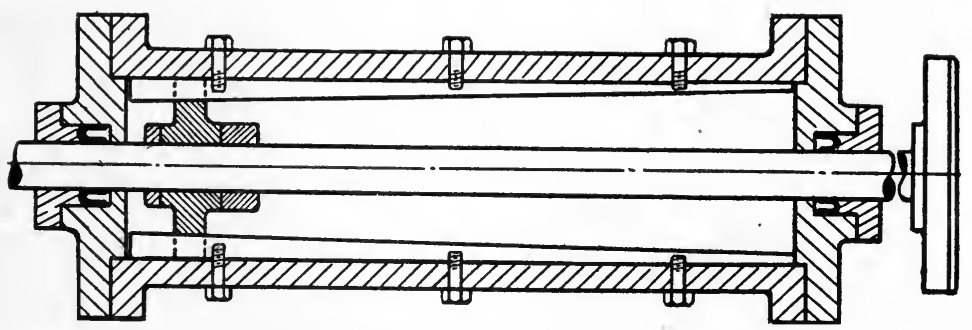

FıG. 308.- Hydraulic Buffer Cylinder.

therefore the resistance, is uniform throughout the stroke, and when in consequence the pressure-displacement diagram forms a rectangle. The brake is therefore preferably designed so as to give as nearly as possible uniform resistance, and since the resistance varies as the square of the velocity of the liquid through the connecting oritices (very nearly), while the velocity of the moving body, and therefore of the piston, varies from a maximum at the instant of impact to zero at the end of the strolie, it is necessary either to make the connecting passages of diminishing area towards the end of the strolie so that the velocity of efflux may remain constant, or to discharge from one side of the piston to the other through a spring-loaded valve set to open at the required pressure. 'The area of the connecting passage may be varied by forming it as a circular' orifice through the piston, and allowing this to work over 
a taper circular spindle fixed longitudinally in the cylinder, the available passage area varying with the diameter of the spindle. A somewhat similar device is applied to a type of buffer stop adopted by Mr. Langley. ${ }^{1}$ This is illustrated in Fig. 308, and consists of a cylinder $4 \mathrm{ft}$. $7 \frac{1}{2} \mathrm{in}$. long, having a $3 \frac{3}{4} \mathrm{in}$. piston rod and a piston $12 \mathrm{in}$. diameter and $4 \mathrm{ft}$. stroke. The annular clearance between piston and cylinder equals $\because 38$ square inches.

Two longitudinal slots 3 inches wide and $1 \frac{11}{16}$ inch deep are cut in the piston body and work over two longitudinal strips 3 inches wide which are fixed inside the cylinder and which vary in depth from $\frac{9}{16}$ inch at the front to $1 \frac{11}{16}$ inch at the back end. The area of the connecting passages thus formed varies from 3.375 square inches at the front to zero at the back end. Adjusting plates are fitted so that this area may be increased if necessary. After being driven home the piston is returned by means of counterweights. With this brake a maximum pressure of $800 \mathrm{lbs}$. per square inch was obtained when stopping a train weighing about 200 tons, and moving at 8 miles per hour. ${ }^{1}$

In a somewhat similar brake at Strasbourg ${ }^{2}$ the piston diameter is 16 inches, and its travel 82 feet. A train of 200 tons moving at 8 miles per hour is brought to rest with a pressure of $580 \mathrm{lbs}$. per square inch, giving an uniform resistance of 52 tons. An automatic valve is fitted to a pipe connecting the two ends of the cylinder so that this may open if the pressure becomes excessive. As an additional precaution against the production of a dangerous cylinder pressure the links supporting the whole stop are designed so as to break under an excessive load.

In the case of a 6-inch quick-firing gun having a weight of recoiling parts equal to $14,800 \mathrm{lbs}$., when firing a cordite charge of $13 \frac{1}{4} \mathrm{lbs}$. and giving a muzzle velocity of 2,150 feet per second to a projectile weighing $100 \mathrm{lbs}$., the velocity of recoil is found to be approximately equal to 17.5 feet per second. The energy of recoil-about 70,500 foot $1 \mathrm{bs}$. - is here absorbed by a buffer of 12 -inch stroke and $7 \frac{1}{2}$ inches internal diameter.

1 "Proceedings Institute Mechanical Engineers," 1886, p. 105.

2 "Proceedings Institute Civil Engineers," vol. 119, p. 4 49. 
Where no attempt is made to vary the passage area it is evident that the resistance falls off very rapidly as the velocity diminishes and, e.g., has only one-quarter of its initial value when the velocity is reduced to one-half. In fact, if the resistance were solely measured by the production of kinetic energy in the contained fluid, the body would never be brought absolutely to rest. Actually, however, the additional resistance in the shape of solid friction at the cup leathers, \&c., together with that of returning springs or balance weights, prevent this state of affairs being realised in practice.

Theory of the Hydraulic Bralie.-Suppose the piston rod to be in compression,

Let $A=$ effective area of piston in square feet, i.e., area of piston minus that of rod and of connecting orifices, if these are through the piston.

$a=$ effective area of connecting orifices.

$L=$ length of piston travel in feet.

$l=$ length of connecting passages.

$R=$ resistance of brake in lbs.

$p=$ excess pressure behind piston in lbs. per square foot.

$W=$ weight of moving body in lbs.

$\mathrm{r}=$ velocity of moving body, and of piston if brake is in operation.

$v:=$ velocity of fluid through orifices.

If the fluid is water we now have

$$
\frac{p}{62 \cdot 4}=\frac{r^{2}}{2 g}\left(1+\frac{f l}{m}\right)=\frac{V^{2}}{2 !} \cdot \frac{A^{2}}{a^{2}}\left\{1+\frac{f l}{m}\right\}
$$

while if $F^{\prime}=$ resistance in lbs. due to mechanical friction and to effect of counterweights, \&c., we have

$$
\left.R=p A+H^{\prime}=\frac{62 \cdot 4 I^{2}}{2 g} \fallingdotseq 1+\frac{f^{\prime} l}{m}\right\} \frac{A^{3}}{a^{2}}+H^{\prime} .
$$

Budles the uniform resistance.-With uniform resistance the retardation of the moving body will be uniform, and will be given by $\frac{b_{1}^{2}}{2 L}$ feet per second per second, where $V_{1}$ is the velocity 
at impact, so that the velocity at any point distant $x$ feet from the beginning of the stroke is given by

$$
\begin{aligned}
V^{2} & =V_{1}^{2}-\frac{V_{1}^{2}}{L} x, \\
& =V_{1}^{2}\left(1-\frac{x}{L}\right) .
\end{aligned}
$$

By equating the work done on the brake, to the loss of K.E. by the moving body we get

$$
\begin{aligned}
R L & =\frac{W V_{1}^{2}}{2 g} \\
\therefore \quad V_{1}^{2} & =\frac{2 R L !}{W}, \\
V^{2} & =\frac{2 R L g\left(1-\frac{x}{L}\right)}{W} .
\end{aligned}
$$

and

Inserting this value in (2) above, we get $p A+F=\frac{62 \cdot 4 L}{W}\left(p A+F^{\prime}\right)\left(1-\frac{x}{L}\right)\left(1+\frac{f l}{m}\right) \frac{A^{3}}{a^{2}}+F^{\prime}$, which finally reduces to

$$
a^{2}=\frac{62 \cdot 4 L A^{2}\left(1+\frac{f l}{m}\right)\left(A+\frac{F^{\prime}}{p}\right)}{W}\left(1-\frac{x}{L}\right)
$$

giving the passage area for any value of $x$, when the form of the orifice is known.

If it be assumed that ram friction is equal to 5 per cent. of the force on the piston, and if the term $\left(1+\frac{f l}{m}\right)$ be taken as unity (i.e., if fluid friction be neglected), this simplifies to

$$
a^{2}=\frac{65 \cdot 7 L A^{3}}{W}\left(1-\frac{x}{L}\right)
$$

\section{ExanpLe.}

Buffer stop-piston, $12 \frac{1}{4}$ inches diameter, provided with two slots of total area $4 \cdot 75$ square inches $-3 \frac{1}{4}$-inch rod-length of stroke 4 feet. Weight of train $=100$ tons.

$$
A=\frac{105}{144}=\cdot 728 \text { square feet; } H^{r}=224,000 \mathrm{lbs} .
$$


Equation (5) now becomes

$$
\begin{aligned}
a^{2} & =\frac{65 \cdot 7 \times 4 \times 386}{224,000}\left(1-\frac{x}{4}\right) \\
& =000453\left(1-\frac{x}{4}\right) \\
\therefore \quad a & =02127 \sqrt{\left(1-\frac{x}{4}\right)} \text { square feet. } \\
& =3.06 \sqrt{\left(1-\frac{x}{4}\right)} \text { square inches. }
\end{aligned}
$$

'I'hus when $x=0$, i.e., at the beginning of the stroke, $a=3.06$ square inches. This gives the effective area of the orifice, and, when the entrant edges are well rounded so as to prevent the formation of a rena contractı, this will be the true area. With sharp-edged orifices, however, the orifice area will need to be greater than this in the ratio $\frac{1}{\text { cuefticient of contraction }}$, and this will depend upon the form and situation of the orifice.

To avoid errors in preliminary calculation it is advisable to make the orifices with well rounded entrant edges.

Assuming, in the example above, that $C_{c}=\cdot 65$, we get the following values for $a_{1}$, where $C_{c} a_{1}=a$ :-

\begin{tabular}{l|c|c|c|c|c}
\hline$x$ feet & 0 & $1 \cdot 0$ & $2 \cdot 0$ & $3 \cdot 0$ & $4 \cdot 0$ \\
\hline$a_{1}$ square inches & $4 \cdot 71$ & $4 \cdot 09$ & $3 \cdot 34$ & $2 \cdot 35$ & 0 \\
\hline
\end{tabular}

An interesting feature of the brake is that the piston travel is approximately independent of the initial velocity of the moving body, and depends only on its weight. The reason for this is that loth the hydraulic resistance and the kinetic energy of the moving body vary as the velocity squared, and their relative value is therefore independent of this. ${ }^{1}$

1 In a buffer stop described by Mr. P. W. Shaw (Proc. Inst. C.L., 1905-6, Part iii., vol. 165, p. 290), the piston came to clead slow at about 9 inches from the end of its stroke, when resisting a mass of 11 tons, for all speeds of collision from 4 to 12 miles per hour. The details of the brake are as follow :-Cylinder, 10 inches diameter, 3 feet 6 inches stroke; piston, 964 inches diameter, with two rectangular slots 3 inches wide $\times 1 \frac{1}{2}$ inches deep, working over strips 3 inches wide $\times 1 \frac{1}{2}$ inches tapering to $\frac{1}{2}$ inch. 
Brake with passages of constant area.-In this case, we have, neglecting mechanical friction,

$$
\begin{aligned}
p \times A=R & =\frac{62 \cdot 4 V^{2}}{2 g}\left\{1+\frac{f l}{m}\right\} \frac{A^{3}}{a^{2}} \\
& =k V^{2} \text { where } k=\frac{62 \cdot 4}{2 g}\left\{1+\frac{f l}{m}\right\} \frac{A^{3}}{a^{2}}
\end{aligned}
$$

Since this measures the mass $\times$ acceleration of the moving body we have

$$
k V^{2}=-\frac{W}{g} \frac{d V}{d t}=-\frac{W}{g} V \frac{d V}{d x}
$$

the negative sign indicating that the motion is being retarded.

or

$$
\begin{aligned}
& \therefore \quad k V=-\frac{W}{g} \frac{d V}{d x} \\
& \int_{V}^{V_{1}} \frac{d V}{V}=-\int_{x}^{x_{1}} \frac{k g}{W} d x
\end{aligned}
$$

The solution of this equation gives us :-

$$
\log _{e} \frac{V_{1}}{V}=-\frac{k g}{W}\left(x_{1}-x\right)=\frac{k g}{W^{\prime}}\left(x-x_{1}\right) .
$$

Writing $x_{1}=0$, so that $V_{1}$ is the velocity at the instant of impact, we have

or

$$
\begin{gathered}
\log _{e} \frac{V}{V_{1}}=-\frac{k g}{W} x, \\
V=V_{1} e^{-\frac{k \cdot g}{W} x,}
\end{gathered}
$$

giving the velocity corresponding to any position $x_{2}$ of the piston.

$$
-\frac{k g}{w} x
$$

Since $e$ never becomes equal to zero, no matter how large the value of $x$, the velocity cannot become zero until a change takes place in the law of resistance, either on account of the effect of solid friction, or because of the motion of the fluid becoming so slow that the resistance becomes approximately proportional to the first power of the velocity.

On taking mechanical friction into account we have, assuming this to be constant,

$$
\begin{gathered}
R=k V^{2}+F=-\frac{W}{g} V \frac{d V}{d x}=-\frac{W}{2 g} \frac{d\left(V^{2}\right)}{d x}, \\
\therefore \quad \frac{d\left(V^{2}\right)}{d x}+\frac{2 g k}{W} V^{2}+\frac{2 g F}{W}=0,
\end{gathered}
$$


or $\frac{d V^{2}}{d x}+b V^{2}=c$,

$$
\text { where }\left\{\begin{array}{l}
l=\frac{62 \cdot 4}{W} \frac{A^{3}}{a^{2}}\left(1+\frac{f^{l} l}{m}\right) \\
c=-\frac{2 g F}{W}
\end{array} .\right.
$$

The solution of this equation is

$$
V^{2}=\frac{c}{b}+D e^{-b x} \text { where } I \text { is a constant. }
$$

Putting $V=V_{1}$ when $x=0$ we have $\left.V_{1}^{2}=\frac{c}{b}+I\right)$,

$$
\therefore V^{2}=\frac{c}{b}+\left(V_{1}^{2}-\frac{c}{b}\right) e^{-b x}
$$

giving the velocity after a piston displacement $x$ feet.

This may be written

or $x=\frac{1}{b} \log _{e}\left\{\frac{V_{1}^{2}-\frac{c}{b}}{V^{2}-\frac{c}{b}}\right\}=\frac{2 \cdot 302}{b} \log _{10}\left\{\frac{V_{1}^{2}-\frac{c}{b}}{V^{2}-\frac{c}{b}}\right\}$ feet,

$$
e^{l, x}=\frac{r_{1}^{2}-\frac{c}{b}}{r^{2}-\frac{c}{b}}
$$

giving the position of the piston when the velocity has been reduced from $V_{1}$ to $V$ feet per second.

It will be noted in Fig. 308 that the piston rod passes through both ends of the cylinder. This construction is necessary where the rod is in compression during the working stroke, since, without the tail rod the displacement of rod plus piston increases throughout the stroke, and if the cylinder were originally full of liquid no motion of the piston would be possible. The difficulty can only be avoided by the provision of an air space inside the cylinder, of volume equal to that of the portion of the rod entering the cylinder during the compression stroke. Owing to this air space, however, the piston during the first portion of its stroke is engaged in compressing air, with the result that the pressure rises slowly and the necessary maximum pressure for a given weight and velocity is increased by about 15 per cent. Where the piston rod is in tension during the working stroke the above difficulty is entirely obviated. The cylinder is filled with liquid 
with the piston run in, and the resistance is now increased by the formation of a partial vacuum behind the piston on its outward stroke.

Fig. 309 indicates the variation of pressure intensity inside the cylinder with piston displacement in the cases $(a)$ where the resistance is uniform ; $(b)$ where a compression buffer without tail rod, liaving passages of uniform area and with air compression is used; and (c) with a tension buffer with passages of uniform area.

With constant pressure and resistance, the maximum pressure attained is

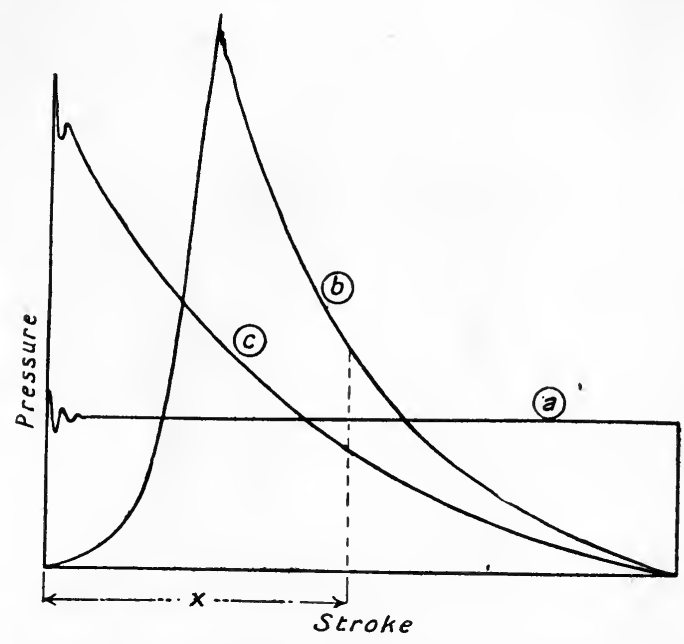

Fig. 309.-Pressure Diagrams from Cylinders of Hydraulic Brakes. about one-third that in the case of the tension buffer with passages of uniform area.

\section{Art. 193.-The Hydraulic Dynamometer}

is certainly the most perfect of all mechanical devices for measuring and absorbing the energy developed by a prime mover at a rotating shaft.

In its modern form it owes its conception-in all but one essential - to the late Mr. William Froude; but the addition of the one detail, which made the brake a practical success was due to Professor Osborne Reynolds.

The Reynolds-or Mather-Reynolds-Dynamometer, shown in Fig. 310, consists of a double disc $D$, fixed to the power shaft by set screws or keys and carrying on its outer faces a series of narrow pockets. 
'These latter are semicircular in section, their plane is inclined at $45^{\circ}$ to the axis of the shaft, and they face forwards in the
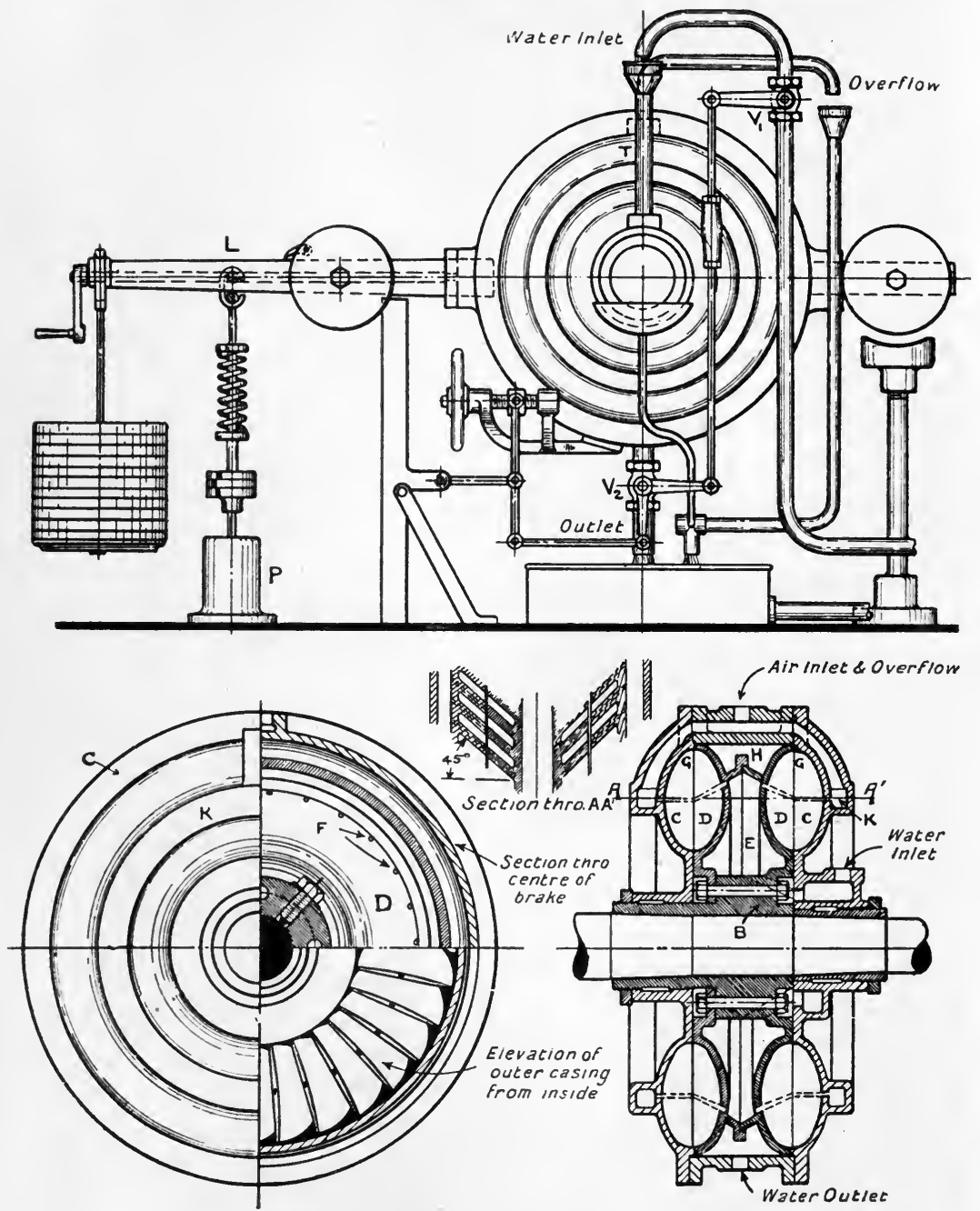

FIG. 310.--Reynolds's Hydraulic Dynamorneter.

direction of motion. A casing $C$, carrying a double series of pockets similar to those on the disc, in the same planes, but facing in the opposite direction, surrounds the disc and carries a 
graduated lever $L$ from which are suspended the weights comprising the brake load.

The shaft passes through bushed openings in the casing which it fits closely so as to prevent undue leakage.

In the case of an 18-inch brake wheel the wheel itself carries 24 pockets on each face, while the casing carries 25 similar pockets. These are $4 \frac{1}{2}$ inches in radial depth and have an axial width of $1 \frac{1}{2}$ inch, the dividing vanes or partitions being $\frac{1}{4}$ inch thick.

Provision is made for supplying the pockets with water, and for allowing this to eseape after having work done on it in the dynamometer, while a small air vent is provided in the thickness of each vane to admit air from an annular chamber $K$ in the casing to the centre of the circle formed when two of the pockets on dise and casing directly face each other. It is to this detail that the brake owes its successful action. As originally designed by Mr. Froude these air vents were absent.

The action of the dynamometer is as follows:-Water admitted to the casing through the flexible rubber tube at $T$ finds its way through a series of four holes in the boss $B$ into the chamber $E$ between the dises, and thence through a series of small holes $F F$, formed in the thickness of the wheel vanes, into the pockets.

To prevent the water being projected into the air holes the radius of the inlet hole circle is made rather greater than that of the air vent circle.

Considering one of the pockets, we have its contained water thrown outwards by centrifugal action, and, guided by the circular boundary of the pocket, projected forwards into a stationary pocket in the casing. Guided back by this pocket it enters a second moving pocket with increased velocity, is thrown outwards and projected forwards with still greater velocity into a second stationary pocket, and so on. Theoretically, but for the effect of friction, eddy losses, and the introduction of fresh water, this velocity would increase indefinitely. To produce the change in the moment of momentum about the shaft which occurs at each reversal of each stream, an equal moment must be applied to the dise and to the easing, and since the friction moment of

H.A. 
the side glands also reacts equally on the casing and on the shaft, there is, so long as the shaft is rotating uniformly, an exact balance between the driving moment on the shaft and the resisting moment on the casing. The latter is provided and measured by the resisting moment of the brake load.

A slight side clearance between the outer circumference of the wheel and of the casing at $G G$ permits of the escape of water into the concentric chamber $H$, which is always full when the brake is working. It escapes from this chamber through the automatically regulated valve $V_{2}$.

Since the change of momentum varies directly as the quantity of water in motion, and directly as its velocity, the brake resistance may be varied by varying either the inflow or outflow, a system of levers being arranged as indicated so as to do this if the brake lever rises or falls, and thus to prevent over or under loading. An oil dashpot at $P$ serves to prevent hunting.

The object of the air vents may now be noted. 'The water in motion in each pair of pockets forms a vortex, and in consequence is at a greater pressure at the outside than at the centre of the pocket. The pressure at the centre may, in fact, in a closed pocket, become less than atmospheric, while in any case there is a tendency for air to accumulate at the points of least pressure, so that in the original type the dynamometer gradually emptied itself of water and became air charged. Further, since with a given speed of rotation the pressure from the inside to the outside of the vortices increases at a fixed rate, any change in the internal pressure is accompanied by a corresponding change in the pressure on the pockets of the wheel and casing, and hence affects the resistance.

By the provision of air vents, however, a constant pressure, equal to that of the atmosphere, is maintained at the centre of the vortices under all normal conditions of working; the brake need not, as in its original form, be full of water for satisfactory working; and for a given speed and water supply the resistance remains uniform.

This type of dynamometer has many advantages in view of its safety, its accuracy, ease of adjustment, and moderate dimensions. 
The resisting moment varies as $A v^{2} r$, where $A$ is the combined cross-sectional area of the streams suffering change of direction, $v$ is their mean velocity which is proportional to the angular velocity of the brake and to its radius, and $r$ is the radius of the centre line of the brake pockets.

It follows that in two similar dynamometers rotating with the same angular velocity, and having corresponding linear dimensions in the ratio $s: 1$, if both brakes are running full we shall have :-

$$
\begin{gathered}
A_{2}=s^{2} A_{1} ; v_{2}=s v_{1} ; r_{2}=s r_{1} ; \\
\therefore A_{2} v_{2}^{2} r_{2}=s^{5}\left(A_{1} v_{1}^{2} r_{1}\right),
\end{gathered}
$$

so that the resisting moments, and, therefore the horse-power absorbed, varies as the fifth power of the linear dimensions.

Experiments show that such a brake as illustrated will absorb a maximum of 29.5 H.P. at 100 revolutions, the dise diameter being 18 inches.

It follows that a brake having a dise 24 inches in diameter will absorb 1,100 H.P. at 300 revolutions, while by mounting two or more dises on one shaft the power which may be absorbed is practically unlimited.

The resistance with a given quantity of water varies approximately as the square of the brake speed so that the brake is specially well adapted for high speed work. Also, since the resisting moment at the instant of starting is zero and gradually increases with the speed, the brake is well adapted for the testing of.internal combustion engines or steam turbines of the pressure type, while once having been adjusted it requires no further attention no matter how the speed or power of the prime mover may vary within wide limits. The only drawback is the somewhat large first cost of the apparatus.

For such high speeds as are common in steam turbines of the impulse type, and for small powers, a simpler type of hydraulic dynamometer: gives good results. This consists simply of a series of parallel dises keyed to the power shift, and rotating with small side clearance between a similar series of stationary dises fixed to the outer easing. As in the previous type of brake, with an uniform speed of rotation the resistance varies with the quantily of water in use, and may therefore be 
regulated by the opening or closing of the inlet or outlet valve. With a constant quantily of water in the brake the resistance depends on the wetted area, and varies probably approximately as the square of the angular velocity. Experimental evidence as to the exact law of resistance at these high speeds is, however, lacking. Assuming the square law, we have the resistance of each face of each disc given by $2 \pi f \omega^{2} \int_{R_{2}}^{R_{1}}{ }^{r} d r$, where $f$ is a coefficient of resistance, probably having a value of about $\cdot 0045$ with roughened metal surfaces; $\omega=$ angular velocity in radians per second; and $R_{1}$ and $R_{2}$ are the outer and inner radii of the submerged portion of the rotating disc in feet.

On integrating this gives $\frac{2 \pi f \omega^{2}}{5}\left(R_{1}^{5}-R_{2}^{5}\right)$ foot lbs.

With $n$ discs the work done against the resistance per second (neglecting the effect of the edges of the discs) is then given by

$$
\begin{aligned}
2 n & \times \frac{2 \pi f \omega^{3}}{5}\left(R_{1}^{5}-R_{2}^{5}\right) \text { foot lbs. } \\
& =\frac{4 n \pi f \omega^{3}}{5 \times 550}\left(R_{1}^{5}-R_{2}^{5}\right) \text { H.P. }
\end{aligned}
$$

\section{Example.}

Brake provided with three rotating dises enclosed on both faces. Outer diameter 9 inches, submerged to an inner diameter of 6 inches. Assuming $f={ }^{\circ} 004$, determine the B.H.P. absorbed at 10,000 revolutions per minute.

Here

$$
\begin{aligned}
\omega & =\frac{2 \pi \times 10,000}{60} \\
R_{1}^{5} & =(\cdot 375)^{5}=\cdot 007413 \\
R_{2}{ }^{5} & =(\cdot 25)^{5}=\cdot 000976 \\
\therefore \quad \text { B.H.P. } & =\frac{12 \times \pi \times \cdot 004 \times 8 \pi^{3} \times 10^{9} \times \cdot 006437}{5 \times 216 \times 550} \\
& =405 .
\end{aligned}
$$

\section{Examples.}

(1) A direct acting hydraulic lift has a travel of 40 feet. The cage and ram weigh four tons, and are counterbalanced to the 
extent of three tons, by hanging weights. The lift is to take a load of three tons, with a maximum acceleration of 2 feet per second per second. The diameter of ram is decided on as being 5 inches. Determine the working pressure in the cylinder, assuming ram friction to account for 5 per cent. of this. If the pressure supply is $750 \mathrm{lbs}$. per square inch, this being reduced by means of a reversed intensifier of the ordinary type and having a stroke of 4 feet, determine the necessary size of this cylinder and of its ram, assuming friction to cause a loss of 5 per cent. Also determine the relative gain in efficiency of this system as compared with supplying the lift cylinder directly with pressure water throttled down to the required pressure.

N.B.-The total weight to be accelerated in this case $=4+3+3=10$ tons.

Answer. $\left\{\begin{array}{l}\text { Working pressure } 554 \mathrm{lbs} \text {. per square inch. } \\ \text { Diameter of reducer cylinder }=15 \cdot 8 \text { inches. } \\ \text { Diameter of reducer ram }=13.95 \text { inches. } \\ \text { Gain in efficiency }=35.4 \text { per cent. }\end{array}\right.$

(2) A direct acting hydraulic lift has a travel of 40 feet. The cage and ram weigh four tons, and the lift takes a weight of three tons, with an acceleration of 2 f.s.s. Assuming a ram diameter of 5 inches, and a hydraulic balance cylinder of the type shown in Fig. 290 to be fitted, this being designed so as to balance three-quarters of the weight of the cage and ram when the latter is at the upper limit of its travel, determine the leading dimensions of this balince cylinder, given that the stroke of its ram is 6 feet; that the ram $B$ weighs one ton; and that the annular space $D$ is supplied from a tank whose level is such as to produce a pressure of $30 \mathrm{lbs}$. per square inch on the annulus $E^{\prime}$ when this ram is at the bottom of its stroke. The supply pressure at $A$ is $1,100 \mathrm{lbs}$. per square inch.
Outer diameter of $\operatorname{ram} B$
$=12.95$ inches.
Answer. $\begin{cases}\text { Outer diameter of stationary inlet ram } & =9 \cdot 0 \text { inches. } \\ \text { Outer diameter of anmulus } E & =44 \text { inches. }\end{cases}$

(3) If in the lift of the preceding example the type of balance cylinder of Fig. 291 is used, determine the necessary diameters 
of $A, B$, and $C$, and the necessary weight of ram $B$. Take into account the acceleration of $B$.

Answer. $\left\{\begin{array}{l}\text { Outer diagram of ram } C=12 \cdot 95 \text { inches. } \\ \text { Outer diagram of ram } A=9 \cdot 0 \text { inches. } \\ \text { Weight of } B\end{array}\right.$

(4) If in example (1) the cage and ram form part of a suspended lift, counterbalanced to the same extent as in that question, and operated through a jigger giving a multiplying ratio of $6: 1$, determine the necessary diameter of the cylinder of the jigger if the working pressure $=750 \mathrm{lhs}$. per square inch; the efficiency of the multiplying mechanism $=75$ per cent.; and frictional losses account for 10 per cent. of the pressure on the ram.

Answer. 12.8 inches diameter.

(5) A direct acting crane of the type shown in Fig. 296 is to lift two tons, with a maximum acceleration of 4 f.s.s. The supply pressure is 700 lbs. per square inch. Assuming an efficiency of 75 per cent., determine the necessary size of ram.

Answer. $3 \cdot 5$ inches diameter.

(6) A hydraulic jack has an efficiency of 80 per cent. The diameter of the ram is 8 inches, of the plunger $\frac{3}{4}$ inch. The leverage of the operating lever is $20: 1$. Determine the pressure on the handle when lifting forty tons.

Answer. $49 \cdot 2 \mathrm{lbs}$.

(7) A buffer stop is to be designed to stop a train weighing 150 tons when going at ten miles per hour, in 8 feet. The cylinder diameter is $16 \frac{1}{2}$ inches, the piston rod being $5 \frac{9}{16}$ inches diameter. 'The thickness of the piston is 4 inches, but the entrant edges of the orifices are rounded so as to make their effective length equal to 2 inches. 'There are two rectangular' orifices, each 3 inches wide by $2 \cdot 10$ inches deep. Determine their effective depth at points where the piston: travel is respectively $0,2,4,6$ and 8 feet, in order that the resistance may be uniform throughout the stroke. Also determine the magnitude of this resistance and the pressure in the cylinder, 
assuming that counterbalance weights and friction exert a constant resistance of one ton.

Answer. Resistance $140,300 \mathrm{lbs} .=62 \cdot 65$ tons.

Pressure in cylinder $=781 \mathrm{lbs}$. per square inch.

\begin{tabular}{l|c|c|c|c|c}
\hline Stroke & 0 & $2 \mathrm{ft}$. & $4 \mathrm{ft}$. & $6 \mathrm{ft.}$ & $8 \mathrm{ft}$. \\
\hline Effective depth & $1 \cdot 30 \mathrm{ins}$. & $1 \cdot 13 \mathrm{ins}$. & $\cdot 92 \mathrm{ins}$. & $\cdot 65 \mathrm{ins}$. & 0 \\
\hline
\end{tabular}

(8) If the buffer stop of the preceding example has the same effective piston area with two rectangular connecting orifices each 3 inches wide, and together having a combined area of 6 square inches, this remaining uniform throughout the stroke, determine the maximum pressure attained in the cylinder, and the length of stroke which would be necessary for the piston to come to rest without touching the end of the cylinder.

Answer. Maximum pressure $=1,320 \mathrm{lbs}$. per square inch. Stroke $\quad=22.45$ feet.

(9) If the area of the passages in Example 8 were 3 square inches, determine the maximum pressure attained, and the length of stroke before coming to rest.

Answer. Pressure $=5,275 \mathrm{lbs}$. per square inch. Stroke $=7 \cdot 53$ feet. 



\section{APPENDIX.}

\section{USEFUL DATA FOR HYDRAULIC CALCULATIONS.}

Multiplier for converting logarithms :-

(Common to hyperbolic (to base e) $2 \cdot 30258$.

( Hyperbolic to common $\quad \cdot 43429$.

One radian $=57 \cdot 296^{\circ}$.

!) $\quad=32 \cdot 1908$ at Greenwich.

One knot $=6,080$ feet per hour.

One metre $=39 \cdot 37$ inches $=3 \cdot 28$ feet.

Standard atmosphere $=29.95$ inches $=750$ millimetres of mercury

$=33.9$ feet of water.

$=14.7$ lbs. per square inch.

Inches of mercury $\times \cdot 4907=$ lbs. per square inch.

Feet of water $\times \cdot 4331=$ lbs. per square inch.

A pressure of $1 \mathrm{lb}$. per square inch is equivalent to a column of water $2: 309$ feet high $=27 \cdot 7$ inches high.

One imperial gallon $=\cdot 1605$ cubic feet.

$=277 \cdot 27$ cubic inches.

$=10 \mathrm{lbs}$.

One U.S. gallon $=231$ eubic inches.

$=\cdot 83254$ imperial gallons.

() ne cubic foot of fresh water $=62.4 \mathrm{lbs}$.

One cubic metre of fresh water $=35 \cdot 32$ cubic feet $=2,200 \mathrm{lbs}$.

(One litre of fresh water

One ton of fresh water

$=2 \cdot 2 \mathrm{lbs}$.

One cubic foot of sea water

$=35 \cdot 9$ cubic feet.

$=64 \cdot 0 \mathrm{lbs}$. 
TABLe A.

Pressure of Water.

The pressure of water in pounds per square inch for every foot in height to 270 feet.

\begin{tabular}{|c|c|c|c|c|c|c|c|c|c|c|c|}
\hline 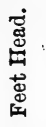 & 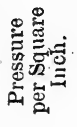 & 导 & 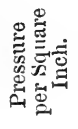 & 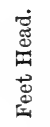 & 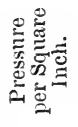 & 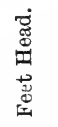 & 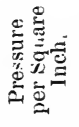 & 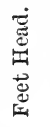 & 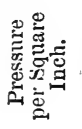 & 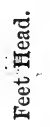 & 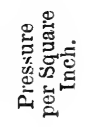 \\
\hline 1 & 0.433 & 46 & $19 \cdot 92$ & 91 & $39 \cdot 42$ & 136 & $58 \cdot 91$ & 181 & $78 \cdot 40$ & 226 & $98 \cdot 10$ \\
\hline 2 & 0.866 & 47 & $20 \cdot 3 i$ & 92 & 3985 & 137 & $59 \cdot 34$ & 182 & $78 \cdot 84$ & 227 & $98 \cdot 33$ \\
\hline 3 & $1 \cdot 299$ & 48 & 2079 & 93 & $40 \cdot 28$ & 138 & $59 \cdot 77$ & 183 & $79 \cdot 27$ & 228 & $95 \cdot 76$ \\
\hline 4 & $1 \cdot 733$ & 49 & $21 \cdot 22$ & 94 & $40 \cdot 72$ & 139 & $60 \cdot 21$ & 184 & $79 \cdot 70$ & 229 & \& $99 \cdot 20$ \\
\hline 5 & $2 \cdot 165$ & 50 & $21 \cdot 65$ & 95 & $41 \cdot 15$ & 140 & $60 \cdot 64$ & 185 & $80 \cdot 14$ & 230 & $99^{\circ} 63$ \\
\hline 6 & $2 \cdot 599$ & 51 & 2209 & $90^{\circ}$ & $41 \cdot 58$ & 141 & 61.07 & 186 & 80.57 & 231 & $100 \cdot 06$ \\
\hline 7 & $3 \cdot 032$ & 52 & $22 \cdot 52$ & 97 & $42 \cdot 0 \mathrm{~L}$ & 142 & $61 \cdot 5 \mathrm{l}$ & 187 & $81 \cdot 00$ & 232 & 10049 \\
\hline 8 & $3 \cdot 465$ & 53 & 2295 & 98 & $42 \cdot 45$ & 143 & 6194 & 188 & 8143 & 233 & 10093 \\
\hline 9 & $3 \cdot 898$ & 54 & $23 \cdot 39$ & 99 & $42 \cdot 88$ & 144 & $62 \cdot 37$ & 189 & 81.87 & 234 & $101 \cdot 36$ \\
\hline 10 & $4 \cdot 331$ & 55 & $23 \cdot 82$ & 100 & $43 \cdot 31$ & 145 & $62 \cdot 81$ & 190 & $82 \div 30$ & 235 & $101 \cdot 79$ \\
\hline 11 & $4 \cdot 764$ & 56 & $24 \cdot 26$ & 101 & $43 \cdot 75$ & 146 & $63 \cdot 24$ & 141 & $82 \cdot 73$ & 236 & $102 \cdot 23$ \\
\hline 12 & $5 * 20$ & 57 & $24 \cdot 69$ & 102 & $44 \cdot 18$ & 147 & $63 \cdot 67$ & 192 & $83: 17$ & 237 & $102 \cdot 66$ \\
\hline 13 & $5 \cdot 63$ & 58 & $25 \cdot 12$ & 103 & $44 \cdot 61$ & 148 & $64 \cdot 10$ & 193 & $83 \cdot 60$ & 238 & 103.09 \\
\hline $14^{\prime}$ & 6.06 & 59 & $25 \cdot 55$ & 104 & $45 \cdot 05$ & 149 & $64 \cdot 54$ & 194 & 84.03 & 239 & $103 \cdot 53$ \\
\hline 15 & 6.49 & 60 & $25 \cdot 99$ & 105 & $45 \cdot 4 \mathrm{~S}$ & 150 & $64 \cdot 97$ & 195 & 84.47 & 240 & $103 \cdot 96$ \\
\hline 16 & $6 \cdot 93$ & 61 & $20^{\circ} 42$ & 106 & $45 \cdot 91$ & 151 & $65 \cdot 49$ & 196 & 84.90 & $2+1$ & $104 \cdot 39$ \\
\hline 17 & $7 \cdot 36$ & 62 & $26 \div 5$ & 107 & $46 \cdot 34$ & 152 & 65.84 & 197 & 8533 & 242 & $104 \cdot 83$ \\
\hline 18 & $7 \cdot 79$ & 63 & 2729 & 108 & $46 \cdot 78$ & 153 & 6627 & 198 & $85^{\circ} 7 b^{\circ}$ & 243 & 105.26 \\
\hline 19 & $8 \cdot 22$ & 64 & $27 \cdot 7 \cdot 2$ & 109 & 4721 & 154 & $66 \cdot 70$ & 199 & $86 \cdot 20$ & 244 & 105.69 \\
\hline 20 & $8 \cdot 66$ & 65 & $28 \cdot 15$ & 110 & $47 \cdot 64$ & 155 & $67 \cdot 14$ & 200 & 86.63 & 245 & $106 \cdot 13$ \\
\hline 21 & 9.09 & 66 & $28 \cdot 58$ & 111 & 48.08 & 156 & $67 \cdot 57$ & 201 & 87.07 & 246 & 106.56 \\
\hline 22 & $9 \cdot 53$ & 67. & $29 \cdot 02$ & 112 & $4 s \cdot 51$ & 157 & $68 \cdot 00$ & 202 & $87 \cdot 50$ & 247 & 106.99 \\
\hline 23 & 996 & 68 & $29 \cdot 45$ & 113 & $48 \cdot 94$ & $15 \mathrm{~S}$ & $68 \cdot 43$ & 203 & S7 93 & 248 & $107 \cdot 43$ \\
\hline 24 & $10: 39$ & 69 & 29.88 & 114 & $49 \cdot 38$ & 159 & 68.87 & 204 & $88 \cdot 36$ & 249 & $107 \cdot 86$ \\
\hline 25 & 1082 & 70 & 2032 & 115 & $49 \cdot 81$ & 160 & $69 \cdot 31$ & 205 & $88 \cdot 80$ & 250 & $108 \cdot 29$ \\
\hline 26 & $11 \cdot 26$ & 71 & 3075 & 116 & $50 \because 4$ & 161 & $69 \cdot\ulcorner 4$ & 206 & 89.23 & 251 & $108 \cdot 73$ \\
\hline 27 & $11 \cdot 69$ & 72 & $31 \cdot 18$ & 117 & 5068 & 162 & $70 \cdot 17$ & 207 & 8966 & 252 & $109 \cdot 16$ \\
\hline 28 & $12 \cdot 12$ & 73 & 3162 & 118 & $51 \cdot 31$ & 163 & $70 \cdot 61$ & 203 & $90 \cdot 10$ & 253 & 10959 \\
\hline 29 & 12.55 & 74 & 3205 & 119 & $51 * 54$ & 164 & $71 \cdot 04$ & 209 & $90 \cdot 53$ & 254 & 110.08 \\
\hline 30 & $12 \cdot 99$ & 75 & $32 \cdot 48$ & 120 & $51 \cdot 98$ & 165 & $71 \cdot 47$ & 210 & $90 \cdot 96$ & 255 & $110^{\circ} 46$ \\
\hline 31 & $13 \cdot 42$ & 76 & $32 \cdot 92$ & 121 & $52 \cdot 41$ & 166 & 7191 & 211 & $91 \cdot 39$ & 256 & $110 \cdot 89$ \\
\hline 32 & 13.86 & 77 & $33 \cdot 35$ & 122 & $52 \cdot 84$ & 167 & $7 \cdot 2 \cdot 34$ & 212 & 91.83 & 257 & $111 \cdot 32$ \\
\hline 33 & $14^{\circ} 29$ & 78 & $33 \cdot 78$ & 123 & $53 \cdot 28$ & 168 & $72 \cdot 77$ & 213 & $92 \cdot 26$ & $25 \mathrm{~S}$ & $111 \cdot 76$ \\
\hline 34 & $14 \cdot 72$ & 79 & $31 \cdot 21$ & 124 & $5: 371$ & 169 & $73 \cdot 20$ & 214 & $92 \cdot 69$ & 259 & $112 \cdot 19$ \\
\hline 35 & $15^{\circ} 16$ & 80 & $34 \cdot 65$ & 125 & $54 \cdot 15$ & $1 ¡ 0$ & $73 \cdot 64$ & 215 & $93 \cdot 13$ & 260 & 112.62 \\
\hline 36 & $15 \cdot 59$ & 81 & 35.08 & 126 & $54 \cdot 58$ & 171 & $74 \cdot 07$ & 216 & 9356 & 261 & $113 \cdot 06$ \\
\hline 37 & 1602 & 82 & $35 \cdot 52$ & 127 & $55 \cdot 01$ & 172 & $74: 50$ & 217 & $93 \cdot 99$ & 262 & 113.49 \\
\hline 38 & $16^{\circ} 45$ & 83 & $35 \cdot 95$ & 128 & 5544 & 173 & 7494 & 218 & 9443 & 263 & 11392 \\
\hline 39 & 16.89 & 84 & $36 \cdot 39$ & 129 & $55 \times 8$ & 174 & $75 \cdot 37$ & 219 & $94 \cdot 86$ & 264 & $114 \cdot 36$ \\
\hline 40 & $17 \cdot 32$ & 85 & $36 \cdot 82$ & 130 & $56 \cdot 31$ & 175 & $75 \cdot 80$ & 220 & 9530 & 265 & $114 \cdot 79$ \\
\hline 41 & $17 \cdot 75$ & 86 & $37 \cdot 25$ & 131 & 5674 & 176 & $76 \cdot 23$ & 221 & 95.73 & $266^{\circ}$ & $115 \cdot 22$ \\
\hline 42 & $18 \cdot 79$ & 87 & $37 \cdot 68$ & 132 & $57 \cdot 18$ & 177 & $76 \cdot 67$ & $2 \cdot 22$ & $96 \cdot 16$ & 267 & $115 \cdot 66$ \\
\hline 43 & 18.62 & Ss' & $38 \cdot 12$ & 133 & $57 \cdot 61$ & 178 & $77 \cdot 10$ & 223 & $96 \cdot 60$ & 268 & $116 \cdot 09$ \\
\hline 44 & $19^{\circ} 05$ & 89 & $38 \cdot 55$ & $1: 34$ & $58 \cdot 04$ & 179 & $77 \cdot 53$ & $2 ? 4$ & $97 \cdot 03$ & 269 & 116.52 \\
\hline 45 & $19 \cdot 49$ & 90 & $38 \cdot 98$ & 135 & $58 \cdot 48$ & 180 & 77.97 & 225 & $97 \cdot 46$ & 270 & $116 \cdot 96$ \\
\hline
\end{tabular}




\section{TabLe $B$.}

Areas of Circles.

\begin{tabular}{|c|c|c|c|c|c|}
\hline $\begin{array}{l}\text { Diameter. } \\
\text { Iuches. }\end{array}$ & $\begin{array}{c}\text { Area. } \\
\text { Squat e inches. }\end{array}$ & $\begin{array}{l}\text { Diameter. } \\
\text { Inches. }\end{array}$ & $\begin{array}{l}\text { Area. } \\
\text { Square inches. }\end{array}$ & $\begin{array}{l}\text { Diameter. } \\
\text { Inclies. }\end{array}$ & $\begin{array}{c}\text { Area. } \\
\text { Square inches. }\end{array}$ \\
\hline$\frac{1}{8}$ & ! 0122 & $16 \frac{1}{2}$ & $213 \cdot 82$ & 38 & $1134 \cdot 1$ \\
\hline $\begin{array}{l}8 \\
\frac{1}{4}\end{array}$ & $\begin{array}{r}0490 \\
\end{array}$ & $17^{2}$ & 226.98 & $38 \frac{1}{2}$ & $1164 \cdot 2$ \\
\hline $\begin{array}{l}4 \\
\frac{3}{8}\end{array}$ & $\cdot 1104$ & $17 \frac{1}{2}$ & $240^{\circ}$ ๖3 & $39^{2}$ & $1194 \cdot 6$ \\
\hline$\frac{1}{2}$ & $\cdot 1963$ & $18^{2}$ & $254 \cdot 47$ & $39 \frac{1}{2}$ & $1225 \cdot 4$ \\
\hline$\frac{2}{4}$ & $\cdot 4417$ & $18 \frac{1}{2}$ & $268 \cdot 50$ & $40^{2}$ & $1256 \cdot 6$ \\
\hline $1^{4}$ & $\cdot 7854$ & 19 & $283 \cdot 53$ & $40 \frac{1}{2}$ & $1288 \cdot 2$ \\
\hline $1 \frac{1}{8}$ & $\cdot 9940$ & $19 \frac{1}{2}$ & $298 \cdot 65$ & $41^{2}$ & $1320 \cdot 3$ \\
\hline $1 \frac{\pi}{4}$ & $1 \cdot 227$ & $20^{-}$ & $314 \cdot 16$ & $41 \frac{1}{2}$ & $13 \tilde{2} 2 \cdot 7$ \\
\hline $1 \frac{1}{2}$ & 1.767 & $20 \frac{1}{2}$ & $330 \cdot 06$ & 42 & $1385 \cdot 4$ \\
\hline $1 \frac{3}{4}$ & $2 \cdot \dashv 0 \tilde{J}$ & $21^{-}$ & $346 \cdot 36$ & $42 \frac{1}{2}$ & $1418 \cdot 6$ \\
\hline $2^{t}$ & $3 \cdot 141$ & $21 \frac{1}{2}$ & $363 \cdot 0 \Sigma$ & $43^{2}$ & $1452 \cdot 2$ \\
\hline $2 \frac{1}{4}$ & $3 \cdot 976$ & $22^{2}$ & $350 \cdot 13$ & $43 \frac{1}{2}$ & $1486 \cdot 2$ \\
\hline $2 \frac{1}{2}$ & $4 \cdot 90 \mathrm{~s}$ & $22 \frac{1}{2}$ & $347 \cdot 61$ & 44 & $1520 \cdot 5$ \\
\hline $2 \frac{3}{4}$ & $\cdot \quad 5 \cdot(939$ & $23^{2}$ & $41 \tilde{5} \cdot 48$ & $44 \frac{1}{2}$ & $15 \overline{5} 50 \cdot 3$ \\
\hline $3^{4}$ & $7 \cdot 068$ & $23 \frac{1}{2}$ & $43: 3 \cdot 74$ & $45^{\circ}$ & $1590 \cdot 4$ \\
\hline $3 \frac{1}{4}$ & $8 \cdot 295$ & $24^{2}$ & $452 \cdot 39$ & $4 \tilde{5} \frac{1}{2}$ & $1626^{\circ} 0$ \\
\hline $3 \frac{1}{2}$ & $9 \cdot 621$ & $2+\frac{1}{2}$ & $471 \cdot 44$ & 46 & $1661 \cdot 9$ \\
\hline $3 \frac{3}{4}$ & $11 \cdot 04$ & $25^{2}$ & $490 \cdot 87$ & $46 \frac{1}{2}$ & $1698 \cdot 2$ \\
\hline $4^{t}$ & $12 \cdot 56$ & $25 \frac{1}{2}$ & $510 \cdot 71$ & $47^{2}$ & $1734 \cdot 9$ \\
\hline $4 \frac{1}{2}$ & $15 \cdot 90$ & $26^{2}$ & $530 \cdot 93$ & $47 \frac{1}{2}$ & $1772 \cdot 1$ \\
\hline 5 & $19 \cdot 63$ & $26 \frac{1}{2}$ & $5.31 \cdot 5 \check{~}$ & $48^{2}$ & $1 \mathrm{~S}(0 \mathrm{~S} \cdot 6$ \\
\hline $5 \frac{1}{2}$ & $23 \cdot 75$ & 27 & 572.5 & $48 \frac{1}{2}$ & $18+7 \cdot 5$ \\
\hline $6^{2}$ & $2 s \div 7$ & $27 \frac{1}{2}$ & $543 \cdot 9$ & $49^{2}$ & $1885 \cdot 7$ \\
\hline $6 \frac{1}{2}$ & $33 \cdot 18$ & $28^{2}$ & $615 \cdot 7$ & $49 \frac{1}{2}$ & $1924 \cdot 4$ \\
\hline $7^{2}$ & $3 N \cdot 48$ & $28 \frac{1}{2}$ & $637 \cdot 9$ & 502 & $196: 3 \cdot 5$ \\
\hline $7 \frac{1}{2}$ & $44 \cdot 17$ & $29^{2}$ & $660 \cdot 5$ & $50 \frac{1}{2}$ & $2003 \cdot 0$ \\
\hline 8 & $50 \cdot 26$ & $29 \frac{1}{2}$ & $683 \cdot 4$ & $51^{2}$ & $2042 \cdot 8$ \\
\hline $8_{\frac{1}{2}}^{1}$ & $56 \cdot 74$ & $30^{2}$ & $706 \cdot 8$ & $51 \frac{1}{2}$ & $2083 \cdot 1$ \\
\hline 9 & $63 \cdot 61$ & $30 \frac{1}{2}$ & $730 \cdot 6$ & 52 & $2123 \cdot 7$ \\
\hline $9 \frac{1}{2}$ & $70 \cdot 85$ & $31^{2}$ & $754 \cdot 7$ & $52 \frac{1}{2}$ & $2164 \cdot 8$ \\
\hline $10^{2}$ & $78 \cdot 34$ & $31 \frac{1}{2}$ & $779 \cdot 3$ & 53 & $2206 \cdot 2$ \\
\hline $10 \frac{1}{2}$ & $86 \cdot 59$ & $32^{2}$ & $804 \cdot 2$ & $53 \frac{1}{2}$ & $224 \times \cdot 0$ \\
\hline $11^{2}$ & $9 j \cdot 03$ & $32 \frac{1}{2}$ & $829 \cdot \tilde{5}$ & $54^{2}$ & $2 \div 90 \cdot 2$ \\
\hline $11 \frac{1}{2}$ & $103 \cdot 57$ & $33^{2}$ & $855 \cdot 3$ & $54 \frac{1}{2}$ & 23328 \\
\hline $12^{2}$ & $113 \cdot 10$ & $33 \frac{1}{2}$ & $+81 \cdot 4$ & $55^{2}$ & $2: 375 \cdot 8$ \\
\hline $12 \frac{1}{2}$ & $122 \cdot 72$ & $34^{2}$ & $90 \div \cdot 9$ & $55 \frac{1}{2}$ & $2419 \cdot 2$ \\
\hline $13^{2}$ & $132 \cdot 73$ & $34 \frac{1}{2}$ & $934 \cdot 8$ & $56^{2}$ & 24630 \\
\hline $13 \frac{1}{2}$ & $143 \cdot 14$ & $35^{2}$ & $962 \cdot 1$ & j6 $\frac{1}{2}$ & $2507 \cdot 2$ \\
\hline $14^{2}$ & $153 \cdot 94$ & $35 \frac{1}{2}$ & $984 \cdot 8$ & $57^{2}$ & $2551 \cdot 8$ \\
\hline $14 \frac{1}{2}$ & $165 \cdot 13$ & $36^{2}$ & $1017 \cdot 9$ & $57 \frac{1}{2}$ & $2596 \cdot 7$ \\
\hline $15^{2}$ & $176 \cdot 71$ & $36 \frac{1}{2}$ & $1046 \cdot 3$ & $58^{2}$ & $2642 \cdot 1$ \\
\hline $15 \frac{1}{2}$ & $188 \cdot 69$ & $37^{2}$ & $1075 \cdot 2$ & j9 & $2734 \cdot 0$ \\
\hline 16 & $201 \cdot 06$ & $37 \frac{1}{2}$ & $1104 \cdot 5$ & 60 & $2827 \cdot 4$ \\
\hline
\end{tabular}


'T'able C.

Theoretical Velocities of Water, $v=\sqrt{2 g h}$, in F'eet per Second, due to Heads from One-tenth to One Hundred Feet.

\begin{tabular}{|c|c|c|c|c|c|c|c|c|c|}
\hline $\begin{array}{l}\text { Head } \\
\text { in feet. }\end{array}$ & $\begin{array}{c}\text { Velocity } \\
\text { in feet } \\
\text { per sec. }\end{array}$ & $\begin{array}{l}\text { Head } \\
\text { in feet. }\end{array}$ & $\begin{array}{l}\text { Velocity } \\
\text { in feet } \\
\text { per sec. }\end{array}$ & $\begin{array}{l}\text { Head } \\
\text { in feet. }\end{array}$ & $\begin{array}{l}\text { Velocity } \\
\text { in feet } \\
\text { per sec. }\end{array}$ & $\begin{array}{l}\text { Head } \\
\text { in feet. }\end{array}$ & $\begin{array}{l}\text { Velocity } \\
\text { in feet } \\
\text { per sec. }\end{array}$ & $\begin{array}{l}\text { Head } \\
\text { in feet. }\end{array}$ & $\begin{array}{l}\text { Velocity } \\
\text { in feet } \\
\text { per sec. }\end{array}$ \\
\hline $0 \cdot 0$ & 0.000 & $\cdot 8$ & $17 \cdot 571$ & 28 . & $42 \cdot 43$ & 52. & $57 \cdot 83$ & $76^{\circ}$ & $69 \cdot 81$ \\
\hline$\cdot 1$ & $2 \cdot 536$ & $\cdot 9$ & $17 \cdot 753$ & $28 \cdot \tilde{5}$ & $42 \cdot 81$ & $52 \cdot 5$ & $58 \cdot 06$ & $76 \cdot 5$ & $70 \cdot 14$ \\
\hline .2 & $3 \cdot 587$ & $\pi$ & $17 \cdot 93$ & $29^{\circ}$ & $43 \cdot 19$ & $53^{\circ}$ & $58 \cdot 38$ & $77^{\circ}$ & $70 \cdot 35$ \\
\hline$\cdot 3$ & $4 \cdot 393$ & $5 \cdot 5$ & $18 \cdot 80$ & $29 \cdot \tilde{z}$ & $43 \cdot 56$ & $53 \cdot \tilde{5}$ & $58 \cdot 65$ & $77 \cdot 5$ & $70 \cdot 60$ \\
\hline$\cdot 4$ & $5 \cdot 072$ & $6^{\circ}$ & $19 \cdot 64$ & $30^{\circ}$ & $+3 \cdot 92$ & $54^{\circ}$ & $58 \cdot 93$ & $78^{\circ}$ & $70 \cdot 83$ \\
\hline 5 & $5 \cdot 671$ & 6.5 & $20 \cdot 44$ & 30.5 & $44 \cdot 29$ & $54 \cdot 5$ & $59 \cdot 20$ & $78 \cdot 5$ & $71 \cdot 05$ \\
\hline .6 & $6 \cdot 212$ & 7 & $21 \cdot 21$ & $31^{\circ}$ & $44 \cdot 65$ & $55^{\circ}$ & $59 \cdot 48$ & 79 . & $71 \cdot 28$ \\
\hline$\cdot 7$ & $6 \cdot 710$ & $7 \cdot 5$ & $21 \cdot 96$ & $31 \%$ & $45 \cdot 01$ & $55 \cdot 5$ & $59 \cdot 74$ & $79 \cdot 5$ & 71.51 \\
\hline .8 & $7 \cdot 137$ & 8 . & $2 \cdot 2 \cdot 68$ & 32 & $45 \cdot 36$ & $56^{\circ}$ & 60.01 & $80^{\circ}$ & $71 \cdot 73$ \\
\hline .9 & $7 \cdot 609$ & $8 \cdot 5$ & $23 \cdot 38$ & $32 \cdot 5$ & $45 \cdot 72$ & $56 \cdot 5$ & $60 \cdot 28$ & $80 \cdot \tilde{5}$ & $71 \cdot 96$ \\
\hline $1 \cdot 0$ & $8 \cdot 020$ & $9^{\cdot}$ & $24 \cdot 06$ & $33^{\circ}$ & 46.07 & $57^{\circ}$ & 60.55 & $81^{\circ}$ & $72 \cdot 18$ \\
\hline$\cdot 1$ & $8 \cdot 412$ & $9 \cdot 5$ & $24 \cdot 92$ & $33 \cdot 5$ & $46+42$ & $57 \cdot 5$ & $60 \cdot 79$ & $81 \cdot 5$ & $72 \cdot 40$ \\
\hline$\cdot 2$ & $8 \cdot 786$ & $10^{\circ}$ & $25 \cdot 36$ & $34^{\circ}$ & $46 \cdot 76$ & $58^{\circ}$ & $61 \cdot 08$ & 82 & $72 \cdot 60$ \\
\hline$\cdot 3$ & $9 \cdot 144$ & 10.5 & 25.98 & $34 \cdot 5$ & $+7 \cdot 10$ & $58 \cdot 5$ & $61 \cdot 34$ & $82 \cdot \tilde{z}$ & $72 \cdot 84$ \\
\hline-4 & $9 \cdot 490$ & $11^{\circ}$ & $26 \cdot 60$ & $3 . \%$ & $47 \cdot 44$ & $59^{\circ}$ & $61 \cdot 60$ & 83 & $73 \cdot 06$ \\
\hline 5 & $9 \cdot 823$ & $11 \cdot 5$ & $27 \cdot 19$ & $35 \%$ & 47.78 & $59 \cdot 5$ & $61 \cdot 81$ & 83.5 & $73 \cdot 28$ \\
\hline$\cdot 6$ & $10 \cdot 145$ & 12 & $27 \cdot 78$ & $36^{\circ}$ & $48 \cdot 12$ & $60^{\circ}$ & $62 \cdot 12$ & 84. & $73 \cdot 51$ \\
\hline .7 & 10.457 & 12.5 & $28 \cdot 3 i$ & $36 \cdot 5$ & $48 \cdot 45$ & 60.5 & $62: 38$ & $84 \cdot 5$ & 73.72 \\
\hline$\cdot 8$ & $10 \cdot 760$ & $13 \cdot$ & $28 \cdot 91$ & $37^{\circ}$ & $48 \cdot 78$ & $61^{\circ}$ & $62 \cdot 64$ & $85^{\circ}$ & $73 \cdot 94$ \\
\hline .9 & $11 \cdot 055$ & 135 & $29 \cdot 46$ & $37 \cdot \pi$ & $49 \cdot 11$ & 615 & $62 \cdot 84$ & $85 \cdot 5$ & $74 \cdot 16$ \\
\hline $2 \cdot 0$ & $11 \cdot 342$ & $14^{\circ}$ & $30 \cdot 00$ & 38. & $49 \cdot 44$ & 62 & $63 \cdot 1 \pi$ & $866^{\circ}$ & $74 \cdot 37$ \\
\hline$\cdot 1$ & $11 \cdot 622$ & $14 \%$ & $30 \cdot 54$ & $38 \cdot 5$ & $49 \cdot 76$ & $62 \cdot \tilde{5}$ & $63 \cdot 40$ & 86.5 & $74 \cdot 5$ \\
\hline$\because 2$ & $11 \cdot 896$ & 15 & $31 \cdot 06$ & $39)^{\circ}$ & $50 \cdot 0 \times$ & 63. & $633 \cdot 65$ & 87. & $74 \cdot 80$ \\
\hline$\cdot 3$ & $12 \cdot 163$ & 15.5 & 31.57 & 39.5 & $50 \cdot 40$ & $63 \%$ & $63 \cdot 90$ & $87 \cdot 5$ & 75.02 \\
\hline-4 & 12.425 & $16^{\circ}$ & $32 \cdot 08$ & $40^{\circ}$ & $50 \cdot 72$ & $64^{\circ}$ & $6+\cdot 16$ & $88^{\circ}$ & $75 \cdot 23$ \\
\hline 5 & $12 \cdot 681$ & 16.5 & 32.57 & 40.5 & $51 \cdot 04$ & 64.5 & $6+\cdot 41$ & 88.5 & 75.45 \\
\hline .6 & $12 \cdot 932$ & 17. & $33 \cdot 06$ & $41^{\circ}$ & $51 \cdot 35$ & $65^{\circ}$ & $64 \cdot 66$ & $89^{\circ}$ & $75 \cdot 66$ \\
\hline .7 & $13 \cdot 179$ & $17 \cdot 5$ & 33.5 & +1.5 & $51 \cdot 66$ & 65.5 & 64.90 & $89 \cdot 5$ & 75.87 \\
\hline$\cdot 8$ & $13 \cdot 420$ & 18. & $34 \cdot 02$ & 42. & $51 \cdot 97$ & $66^{\circ}$ & $65 \cdot 15$ & $90^{\circ}$ & 76.08 \\
\hline .9 & $13 \cdot 658$ & $18 \cdot 5$ & $34 \cdot 49$ & +25 & $52 \cdot 28$ & 66.5 & $65 \cdot 39$ & 90.5 & $76 \cdot 29$ \\
\hline $3 \cdot 0$ & $13 \cdot 891$ & $19^{\circ}$ & $34 \cdot 95$ & 43. & $52 \div 59$ & $67^{\circ}$ & $65 \cdot 64$ & $91^{\circ}$ & 76.52 \\
\hline$\cdot 1$ & $14 \cdot 121$ & $19 \cdot \pi$ & 35.41 & 43.5 & $52 \cdot 89$ & $67 \cdot 5$ & $65 \cdot 89$ & $91 \%$ & $76 \cdot 71$ \\
\hline$\cdot 2$ & $14: 347$ & $20^{\circ}$ & $35 \cdot 86$ & $44^{\circ}$ & $53 \cdot 20$ & $68^{\circ}$ & $66 \cdot 1 \tilde{5}$ & 92 & $76 \cdot 92$ \\
\hline$\cdot \overline{3}$ & $14 \div 69$ & $20 \%$ & $36: 31$ & $44 \cdot \tilde{5}$ & 53.55 & $68 \cdot 5$ & $66 \cdot 38$ & $92 \%$ & $77 \cdot 16$ \\
\hline$\cdot 4$ & $14 \cdot 789$ & $21^{\circ}$ & $36 \cdot 75$ & 45 & $53 \cdot 80$ & $69^{\circ}$ & $66 \cdot 62$ & $93^{\circ}$ & $77 \cdot 34$ \\
\hline 5 & $15 \cdot 004$ & 21.5 & $37 \cdot 18$ & $45 \%$ & $54 \cdot 09$ & 69.5 & $66 \cdot 86$ & 935 & 77.55 \\
\hline .6 & $15 \cdot 217$ & 22 & $37 \cdot 61$ & $46^{\circ}$ & $54: 39$ & $70^{\circ}$ & $67 \cdot 10$ & $94^{\circ}$ & $77 \cdot 85$ \\
\hline$\cdot 7$ & 15.427 & $22 \cdot 5$ & $38 \cdot 04$ & 465 & $54 \cdot 69$ & 70.5 & $67 \cdot 34$ & 94.5 & $77 \cdot 96$ \\
\hline .8 & $15 \cdot 634$ & 23 . & $38 \cdot 46$ & 47 & 54.98 & 71 . & $67 \cdot 58$ & 95 & $78 \cdot 17$ \\
\hline .9 & $15 \cdot 839$ & 23.5 & $38 \cdot 87$ & 475 & $55 \cdots 27$ & $71: 5$ & $67 \cdot 81$ & $95 \cdot 5$ & $78 \cdot 37$ \\
\hline $4 \cdot 0$ & 16.040 & 24 . & $39 \cdot 29$ & 48 . & 5556 & 72 . & $68 \cdot 05$ & $96^{\circ}$ & $78: 58$ \\
\hline$\cdot 1$ & $16 \cdot 240$ & $24 \cdot 5$ & $39 \cdot 69$ & 48.5 & 5.585 & $72 \cdot 5$ & $68 \cdot 28$ & 96.5 & $78 \cdot 78$ \\
\hline .2 & 16.437 & 25 & $40 \cdot 10$ & $49^{\circ}$ & $56 \cdot 14$ & 73. & 68.52 & $97^{\circ}$ & 78.99 \\
\hline$\cdot 3$ & $16 \cdot 631$ & $25 \cdot 5$ & $40 \cdot 50$ & $49 \cdot \tilde{5}$ & $56 \cdot 42$ & 73.5 & $68 \cdot 75$ & $97 \cdot 5$ & $79 \cdot 19$ \\
\hline .4 & 16.823 & $26^{\circ}$ & $40 \cdot 89$ & $50^{\circ}$ & $56 \cdot 71$ & $74^{\circ}$ & $68 \cdot 99$ & 98. & $79 \cdot 29$ \\
\hline .5 & 17.013 & 26.5 & $41 \cdot 28$ & $50 \cdot 5$ & $56 \cdot 99$ & $74 \cdot 5$ & $69 \cdot 22$ & 98.5 & 79.59 \\
\hline .6 & $17 \cdot 201$ & $27^{\circ}$ & $41 \cdot 61$ & 51 . & $57 \cdot 27$ & 75 & $69 \cdot 45$ & $99^{\circ}$ & $79 \cdot 80$ \\
\hline .7 & $17 \cdot 387$ & $27 \cdot 5$ & $42 \cdot 05$ & $51 \cdot \tilde{5}$ & $57 \cdot 55$ & $75 \cdot 5$ & $69 \cdot 68$ & 99.5 & $79 \cdot 90$ \\
\hline
\end{tabular}




\section{TABLE D.}

\section{Fire Streams.}

Pressure required at nozzle and at pump, with quantity and pressure necessary to throw good effective streams various distances through different size nczzles, using 100 feet of ordinary $2 \frac{1}{2}$-inch rubber-lined hose and smooth nozzles.

J. R. FREEMAN.

SIZE OF NOZZLE $\frac{3}{4}$ INCH.

Pressure at Nozzle, in lbs. per sq. in.

Pressure at Pump, ,, ,, ,

Imperial Gallons per Minute .

Distance thrown Horizontal, in feet

Distance thrown Vertical,

\begin{tabular}{r|r|r|r|r|r|r|r}
40 & 50 & 60 & 70 & 80 & 90 & 100 \\
46 & 57 & 68 & 80 & 91 & 102 & 114 \\
86 & 96 & 105 & 114 & 122 & 129 & 136 \\
44 & 50 & 54 & 58 & 62 & 6.5 & 68 \\
. & 60 & 67 & 72 & 76 & 79 & 81 & 83 \\
\hline
\end{tabular}

SizE OF NOZZLE, $\frac{7}{8}$ INCH.

Pressure at Nozzle, in lbs. per sq. in.

Pressure at Pump,

Imperial Gallons per Minute.

Distance thrown Horizontal, in feet

Distance thrown Vertical.

\begin{tabular}{r|r|r|r|r|r|r|r}
. & 40 & 50 & 60 & 70 & 80 & 90 & 100 \\
50 & 63 & 75 & 88 & 101 & 113 & 126 \\
. & 118 & 132 & 144 & 156 & 167 & 177 & 186 \\
. & 49 & 55 & 61 & 66 & 70 & 74 & 76 \\
. & 62 & 71 & 7 & 81 & 85 & 85 & 90 \\
\hline
\end{tabular}

SIzE OF NozzLE, 1 INCH.

Pressure at Nozzle, in lbs. per sq. in.

Pressure at Pump

Imperial Gallon, " "

Distance thrown Horizontal, in feet

Distance thrown Vertical,

\begin{tabular}{r|r|r|r|r|r|r|r}
40 & 50 & 60 & 70 & 80 & 90 & 100 \\
58 & 72 & 87 & 101 & 115 & 130 & 144 \\
.154 & 173 & 189 & 204 & 218 & 232 & 245 \\
.55 & 61 & 67 & 72 & 76 & 80 & 83 \\
. & 64 & 73 & 79 & 85 & 89 & 92 & 96
\end{tabular}

SIZE OF NOZZLE, $1 \frac{1}{\grave{ }}$ INCHES.

\begin{tabular}{|c|c|c|c|c|c|c|c|}
\hline Pressure at Nozzle, in lbs. per sq. in. & 40 & (0 & 60 & 70 & 80 & 90 & 100 \\
\hline Pressure at Pump, & 69 & 86 & 103 & 120 & 138 & 155 & $17 \cdot 2$ \\
\hline Imperial Gallons per Minute & 197 & 221 & 241 & 260 & 279 & 295 & 312 \\
\hline Distance thrown Horizontal, in feet & ธ9 & (6) & 72 & 77 & 1 & 85 & 89 \\
\hline Distance thrown Vertical, & 65 & 75 & 83 & 88 & 92 & 96 & 99 \\
\hline
\end{tabular}

SIZE OF NOZZLE, $1 \frac{1}{4}$ INCHES.

Pressure at Nozzle, in lbs. per sq. in.

Pressure at Pump,

Imperial Gallons per Minute . .

Distance thrown Horizontal, in feet

Distance thrown Vertical,

\begin{tabular}{r|r|r|r|r|r|r|r}
. & 40 & 50 & 60 & 70 & 80 & 90 & 100 \\
. & 84 & 106 & 127 & 148 & 169 & 190 & 211 \\
.246 & 275 & 301 & 325 & 348 & 368 & 388 \\
. & 63 & 70 & 76 & 81 & 85 & 90 & 93 \\
. & 67 & 77 & 85 & 91 & 95 & 99 & 101 \\
\hline
\end{tabular}

SIZF: ÓF NOZZLE, $1 \frac{3}{8}$ INCHES.

Pressure at Nozzle, in lbs. per sq. in.

Pressure at Pump,

Imperial Gallons per "Minute "

Distance thrown Horizontal, in feet

Distance thrown Vertical,

\begin{tabular}{r|r|r|r|r|r|r|r}
40 & 50 & 60 & 70 & 80 & 90 & 100 \\
. & 107 & 134 & 160 & 187 & 214 & 240 & 268 \\
301 & 337 & 369 & 398 & 426 & 452 & 476 \\
. & 66 & 73 & 79 & 84 & 88 & 92 & 96 \\
. & 69 & 79 & 87 & 92 & 97 & 100 & 103 \\
\hline
\end{tabular}

The above pressures are based on the supposition that the hose is coupled direct to the delivery of the pump and while the stream is flowing; if, however, the hose is coupled to a hydrant which is supplied direct from the pump, then the corresponding fire pump pressure must be greater than the hydrant pressure by an amount equal to friction loss, and difference of head between hydrant and pump.

The distances given are for effective fire streams adapted for fire purposes, and are not for mere isolated drops. 



\section{INDEX.}

The Numeruls refer to Pages.

A.

ACCUMULATORS, 686

$\begin{array}{ll}" & \text { differential, } 688 \\ " & \text { effect on pressure in } \\ & \text { motor cylinder, } 690 \\ & \text { steam, } 699\end{array}$

Achard, 5

Adhesion, 3

Air vessels, 21\%

.,,$\quad$ delivery, 591

.. $\quad, \quad$ for centrifugal pump, 630

.. $\quad, \quad$ on pumps, $\mathbf{5 8 6}$

.$\quad$, suction, 586,592

,, charging clevices, 596

Air, flow of, through pipes, 196, 208

, compressor, 674

.. lift pumps, 668

$" \quad " \quad$ " efficiency of, 672

Alexander, 237

Appendix, 74.)

Archimedes, 2

Armstrong, Sir'W. G., 678

Atmospheric pressure, 21

B.

BACKWATER function. 301

Balance cylinder's for lifts, 707

Balancing of end thrust in turbines, 439 .

465

Ball nozzle, 349

pumps, 640

Baı ker's mill, 426

Barnes, H. T., 13, 192

Bazin, 2, 140, 15̃., 186, 269, 270

Bell mouth oritice, 119

Bends in pipe, losses due to, 235

" river, flow round, 312

Bernonlli's theorem, 69

Borda's mouthpiece, 119
Borda's turbine, 431

Bore, 299

Bough, vibration of, 365

Boyilen's diffuser, 435

Bramah, 20

Brake, hydraulic, 727

Branch mains, 245

Breast wheel, 385

Brightmore, 23\%

Brown, Hanbury, 315

Buckets, design of, for Pelton wheel, 401

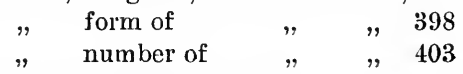

Buffer stop, hydraulic, 727

Bye-pass, flow along, 247

\section{C.}

Calibration of current meters, 327

Capillarity, 6

Castel, 119

Cavitatiın, 572

Centre of buoyancy, 32

$" \quad$ pressure, 21

$" \quad$ on vane, 354

Centrifugal pumps, 600

.. air vessel for, 630

.. for dredging, 601

.. $\quad$ " circulating, 619

.. balancing of end thrust, 640

Buffalo, 638

.. $\quad$ change of pressure in, 612 compound, 625

.. $\quad$ examples of design, 630

.. $\quad$ losses in, 602

.. Mather - Reynolds, 638 
Centrifugal pumps, peripheral speed, 624

\begin{tabular}{|c|c|c|}
\hline$"$ & $"$ & Rateau, 638 \\
\hline " & " & similar, 627 \\
\hline " & ", & $\begin{array}{l}\text { size for given dis- } \\
\text { charge, } 627\end{array}$ \\
\hline " & $"$ & $\begin{array}{l}\text { speed for lifting to } \\
\text { commence, } 626\end{array}$ \\
\hline " & " & $\begin{array}{l}\text { suction and delivery } \\
\text { pipes, } 628\end{array}$ \\
\hline ", & ", & Sulzer, 637 \\
\hline " & " & theory of, 610 \\
\hline & 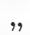 & Worthington, 638 \\
\hline
\end{tabular}

Change of level produced by boat, 310

Channel, circular, 280 ,p piers, 308

" flow through open, 267

" general equations of flow, 287

" most suitable form of, 277

$"$ of constant mean velocity, 281

, rectangular, 277

, trapezoidal, 278

" with horizontal bed, 307

Chezy, 188, 269

Cippoletti, 153

Circular pipe, viscons flow through, 56 ,$\quad$ orifice, 132

Coefficient of contraction, 105, 110

Cohesion, : " $\quad$ " discharge, 106, 110

Coker, E. G., 13, 192

Compounding of jets, 355

Compound pumps, 625 , turbines, 468

Compressibility of water, 4

Contraction of area, 88

Converging channels, 92

Cranes, hydraulic, 712

Critical velocity, 52, 190

Cunningham, Captain, 328

Current meters, 325

Cylinders, strength of thick, 39

\section{D.}

D'ALEMBER'T, 2

Dams, effect on water level, 297 " stability of, 28

Darcy, 2, 187, 206, 269

D'Aubuisson, 188
Delivery pipes for centrifugal pump, $1 ; 29$

Design of centrifugal pumps, 630

,. head and tail races, 485

Diffuser for turbine, 435

, ring for centrifugal pump, 60 , 623

Dise friction, 510

Discharge from pipes, measurement of. 209

" $\quad$, $\quad$ variationsin with diameter, 212

, $\quad$ ", open channels, 322

Displacement curves for reciprocating pumps, 567

Distribution of velocity in pipes, 205 $, \quad, \quad$ open chan-
nels, 313, 316

Draught tubes, 442,482

Dubuat, 182, 269

Dufour, :)

Dynamometer, hydraulic, 7:35 .

E.

ELASTICITY of water, 4

., $\quad$ suction column of pump, 583

, effect of, on water hammer pressure, 213,220

Enlargement of section, 81

Equations of motion for a viscous fluid. 61

, $\quad$,, continuity, 63

Equilibrium of floating bodies, 32

Equivalent diameter of uniform main, 243

Erosive power of water, 320

Eulerian equations of motion, $66 ;$

Eytelwein, 2, 188, 269

FANNING, 251

F.

Fidler, C., 275

Fliegner, 505

Floating bodies, 32

Floats, 325

$, \quad \operatorname{rod}, 330$

" surface, 328

:) sub-surface, 329

:, twin, 330 
Flotation, curve of, 38

Flow in long pipes, 241

, in open channels, 267

$$
\begin{aligned}
& \text {, }, " \text { gauging of, } 322 \\
& \text {, } \quad \text {, non-uniform, } \\
& 291
\end{aligned}
$$

, round river bends, 312

" through nozzles, 251

Fluid, definition of perfect, 16

$$
\text { friction, } 167
$$

Fly wheel effect in turbine regulation, 532

Forging press, 721

Fourneyron turbine, 432

Francis, 2, 140, 155, 330, 504

Francis turbine, 446

Freeman, 201, 251

Friction of U leathers, 693

Froude, 2, 168

Fteley, 140, 143, 155, 156

G.

Galileo, 2

Ganguillet, 189, 270

Gates for turbines, 448,483

Gauging of flow in open channels, 322

field observations, 328

Girard turbine, 421

" pipes, 209

Goodman, Prof. J., 577, 580

Governing of turbines, 455,473

Graphical construction for pressure on turbine vanes, 351

Guide vanes for high lift centrifugal pump, 608, 623

Guide vanes for turbine, 457,462

Guttermuth valves, 566

\section{H.}

HAENEL turbine, 425 ',

Hagen, 2, 188, 213

Head, pressure due to, 18

Head races, 485

Height of jet, 105

Hele Shaw, 47, 173

Hemp packings for rams, 695

Herschell, C., 77, 79, 200

Hicks, 693

High lift centrifugal pumps, 625
Hoists, 704

Hook gauge, 161, 323

Horse power of pumping engine, 252

Hoskins, 201

through pipe line, 257

Hydraulic appliances, 704

air compressor, 674

brake or buffer stop, 727

cranes, 712

dynamometer, $73 \tilde{z}$

engine, 537

" Armstrong, 541

, Brotherhood, 537

, Hastie, 540

" port areas. 543

" Rigg, 540

" theory of, $5+1$

forging press, 721

gradient, 229

jack, 715

jigger, 711

lifts and hoists, 704

mean depth, 184

mining, 362

presses, 20, 717

ram, $6+9$

, diagrams from, 650, 659

"Decœur's, 657

,$\quad$ efficiency of, 653

" for air compression, 660

„. Pearsall's, 658

, sources of loss, 653

relays, 475

transmission of energy, 677

of energy at

Manchester,

678

of energy in

London, 681

losses in use, $68 \tilde{5}$

losses, 682

Hydraulicing, 362

Hydrometric pendulum, 332

I.

ICE formation, 486

Impact of jets, 335

Initiation of motion, 89

Injector hydrant, 666

Intensifiers, 692

Inverted syphon, 264 
J.

JACK, hydraulic, 715

Jets, 335

, actual force of impact, $34 \mathrm{C}$

" compounding of, $3 \tilde{5} 5$

, distribution of pressure across, 344

, form of, 124

$"$ impact of, 335

$" \quad, \quad$ on stationary vanes, 336

$" \quad$ " on curved vanes, 338

$" \quad, \quad$ on surface of revolution, 339

$" \quad$ " on single moving vanes, 349

$" \quad$ normal, on stationary vanes, 336

$" \quad$ " oblique, on stationary vanes, 337

" propulsion, 357

" pump, 661

Jigger, hydraulic, 711

Jonval turbine, 436

Jossè, 673

Jowkowsky, 216

K.

KUTTER, 189, 270

I.

LAW of comparison for orifices, 136

" $" \quad$ pumps, 627

Lawton, 199

Leakage in pumps, 560

Leather collars for rams, 693

Lifts, 704

List of symbols, $\mathrm{xv}$.

Logarithmic homologues, 191

Long pipes, 241

Losses at bends and elbows, 235

, at exit, 238

,, at sudden enlargements, 81

$\therefore$ at tee branches, 237

,$\quad$ at valves, 231

, in pipe lines, 228, 231

\section{M.}

Manning, 271

Marx, 201, 209

Masonry dams, 28

Mean velocity in open channels, 315

,$\quad$, in pipes, 205
Measurement of pipe discharge, 209

Meissner, 442

Metacentre, 33

Metacentric height, 33

Meters, current, 325

" water, 695

Meunier, 425

Mining, hydraulic, 362

Motion of a viscous fluid, equations of, 61

" initiation and stoppage of, 89, 213

Multiple supply, 246

Murphy, E. C., 315

N.

Niagara turbines, 434,453

Normal motion of submerged plane, 363

, $\quad$ impact of jet, 336

Notch, flow over. 137

" rectangular, 138

, triangular, 150

" trapezoidal, 152

Nozzles, ball, 349

" flow through, 250 .

, ring, 251

o.

OBLIQUE impact on plate, 337

" motion of submerged plane, 365

Open channels, flow in, 267

,$\quad$, silting of, 320

Orifice, bell mouthed, 119

" in flat plate, 115

, large rectangular, 129

" $\quad$, circular, 132

" partially submerged, 135

" small, 103

" submerged, 107, 134

$"$ time of discharge from, 121, 162

Oscillations of ships, 37

Overshot wheel, 377

$$
\text { P. }
$$

PAdDle wheels, 180

Parallel plates, flow between, 54

Pearsall's hydraulic ram, 658

Pelton wheel, 258, 396 
Pelton wheel, form of buckets, 401

" " number of buckets, 403

$" \quad$, speed regulation of, 404

$" \quad, \quad$ theory of, 400

Piezometer, 209

Pipe flow, 185, 228, 241

Pipes, strength of, 39

Pipe line losses, 228

Pipes in parallel, 248

" multiple supply, 246

", maximum power through, 255, 683

Pitot tube, 210, 332

Planes, normal motion of, 363

" oblique motion of, 365

Poiseuille, 2, 186, 197

Poncelet wheel, 392

Prentice, 208

Press, hydraulic, 20, 717

Pressure at a point, 17

, energy, 69

.. change of, across stream lines, 94

, $\quad$ exerted on plane by jet, 351

Principle of angular momentum, 60

, linear momentum, 60

" conservation of energy, 61

Prony, 188, 269

Propulsion of ships, jet, 357

$$
\begin{array}{lll}
" & \quad & \text { screw, } 180 \\
" & & \text { power for, } 182
\end{array}
$$

Pumping machinery, 547

Pumps, air vessels on, 586, 592

,$\quad$ air lift, 668

" centrifugal, $600^{\mathrm{L}}$

,$\quad$ jet, 661

., reciprocating, 549

,. $\quad$ screw, 548

, separation in, 572

" theory of, 570

Pump valves, 557

R.

RADIAL flow, 92

Ram, hydraulic, 649

" water, 217

Rankine, 3, 173

Rateau, 601

Rayleigh, 366

Reciprocating pumps, $\check{49}$

,$\quad$ coefticient of discharge of, 567
Reciprocating pumps, displacement

\begin{tabular}{|c|c|c|}
\hline ", & ", & efficiency of, 597 \\
\hline & , & $\begin{array}{l}\text { leakage and slip } \\
\text { in, } 560\end{array}$ \\
\hline , & " & $\begin{array}{l}\operatorname{maximum} \\
\text { of, } \tilde{5} \mathbf{7 t}\end{array}$ \\
\hline , & $"$ & $\begin{array}{c}\text { separation } \\
572,579\end{array}$ \\
\hline & $"$ & theory of, 570 \\
\hline & $n$ & types of, 549 \\
\hline & $"$ & valves, 557 \\
\hline & , & $\begin{array}{l}\text { variation of pre } \\
\text { sure in, } 570\end{array}$ \\
\hline
\end{tabular}
curves, 567

Rectangular notch, 138 , orifice, 129

Relays for governors, 475

Relief valves, 217,480

Resistance of ships, 173

Resultant pressure, 21

Reynolds, Osborne, 5, 46, 51, 87, 190, 195,600

Riedler, 562

Rise in water level produced by dam, 159

$" \quad, \quad, \quad$ bridge piers, 308

$. \quad \quad, \quad, \quad$ vessel, 310

livetters, hydraulic, 723

Rudder action, 368

S.

Scoop wheel, $5+7$

Scott Russell, 175

Scouring and silting of open channels, 321

Screw propulsion, 180

, pump, 548

Sewers, $28 \tilde{5}$

Ships, resistance of, 173

. propulsion of, 179

if - stability curve, 34

;. metacentric beight, 33

,$\quad$ oscillations of, 37

Side wheel, 386

Similar orifices, 136

$\because, \quad$ pumps and turbines, 627

Slip in pumps, 560, 63\%

Small orifices, 103

Smeaton, 390

Smith, Hamilton, 2, 131, 133, 140, 144 
Snoqualmie Falls, 462

Speed regulation of Pelton wheel, 404

" . $\quad$ " turbines, 473

Stand pipes, 217, 478, 529

Standing waves, 293

Stanton, 76, 609

Stearns, 140, 143

Stevinus, 2

Stoppage by gradual closing of valve, 217

" of motion in pipe of uniform bore, 213

Stream lines, 46

$, \quad, \quad$ change in pressure across, 94

, line motion, 46,66

Submerged bodies, resistance to motion of, 369

weirs, 154

" orifices, 107,123

Suction tube, 442,482

Suction pipes for pumps, 628

Sudden stoppage of pipe flow, 213

$"$ enlargement of cross section, 81

, contraction of area, 88

Surface tension, 6

Suspension bearing, 440, 454

Symbols used in book, $\mathrm{xv}$.

Syphon, 259

$"$ inverted, 264

T.

TAIL race, 485

Tee branch-losses at, 237

Thomson, James, 151, 456, 606

Threlfall, 208

Thrupp, 199, 276, 331

Time of discharge from small orifices, 121

, $\quad$,

, notches, 163

" $"$ through pipe line,

Torricelli, 2, 105

Transmissibility of pressure, 19

Transmission of energy (hydraulic), 677

Turbines, American type, 469

" $\quad$ balancing of end thrust, 439

, $\quad$ Barker's mill, 426

, Borda, 431
Turbines, classification of, 419

,. comparison of, 528

, compound, 468

, cone, 441

, $\quad$ fly-wheel effect, 532

" Fourneyron, 432, 518

, Francis, 446,492

" Gates, 448,483

, $\quad$ Girard, 418, 421, 522

, governing of, 473

" Haenel, 425

" impulse, 419

, Jonval, 436, 519

" losses in, 508

" Niagara, 434, 453

" pressure, 418,426

" Shawinigan, 449

,$\quad$ Snoqualmie Falls, 462

" theory of, 489

" Thomson, 456

.. Victor, 472

.. Vortex, 456

\section{U.}

Undershot wheel, 388

Unwin, 31, 198, 213, 511

Utilization of water powers, 373

\section{V.}

VALLOT, 213

Valves, losses due to, \&c., 231

Valves, pump, 557

Van Iterson, 211

Vanes, turbine, effect of thickness, 513

,$\quad$, curvature of, 515

Velocity of approach, 120, 142

" of flow in open channels, 313

, critical, 52, 190

" mean in pipe, 205

. $\quad, \quad$ in open channel, 320

, mid depth, in open channel, 320

Vena contracta, 104

Venturi, 2

, meter, 77

Vessel, change of level around, 310

Victor turbines, 472

"Viper," 360

Viscosity, 11

Viscous flow, 53 


\section{INDEX.}

Volute chamber, 60.5

Vortex chamber, 606, 621

.. compound, 99

.. forced, 96

., free cylindrical, 97

.. $\quad$ free spiral, 98

.. motion, 96;

Vortices, 48

U.

WATER, adhesion of, :

.. bulk, modulus of, $t$

.. cohesion of, 5

.. compressibility of, 4

.. $\quad$ density of, 3

" hammer, $21 \tau$

.. hoisting from mines, 648

.. meters, 695

.. $\quad$.. Deacon, 701

$\begin{array}{lll}. . & . & \text { inferential, } 697\end{array}$

.. $\quad$. low presure, 695

.. $\quad$, positive reciprocating. 700

rotary. 698
Water meters, Venturi, 77

.. physical properties of, 3

.. powers, utilization of, 373

.. ram, 217

.. viscosity of, 11

.. weight of, 3

.. wheels, $3 \pi \tau$

.. .. breast, 385

.. $\quad$.. overshot, 377

$\begin{array}{lll}. . & . . & \text { Pelton, } 396\end{array}$

.. $\quad$.. $\quad$ Poncelet, 392

.. $\quad$, Sagebien, 386

..$\quad$.. undershot, 388

"Waterwitch," 360

Wave-making resistance, 174

Waves, standing, 293

Weirs, submerged, 15t

.. broal-crested, 155

.. use of as measuring appliance, 160

Weisbach, 2, 120, 189, 236

Whirlpool chamber, 606, 621

White, Sir W., 176

Williams, (i. S., 205, 207, 237

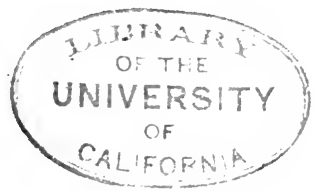

BRADBITY, AGNEW, \& CO. L.D., PRINTGKS, L.ONDON AND TONBRIDGE.

H.A. 
$R$ 



THIS BOOK IS DUE ON THE IAST DATE STAMPED BELOW

AN INITIAL FINE OF 25 CENTS WILL BE ASSESSED FOR FAILURE TO RETURN THIS BOOK ON THE DATE DUE. THE PENALTY WILL INCREASE TO 50 CENTS ON THE FOURTH DAY AND TO $\$ 1.00$ ON THE SEVENTH DAY OVERDUE.

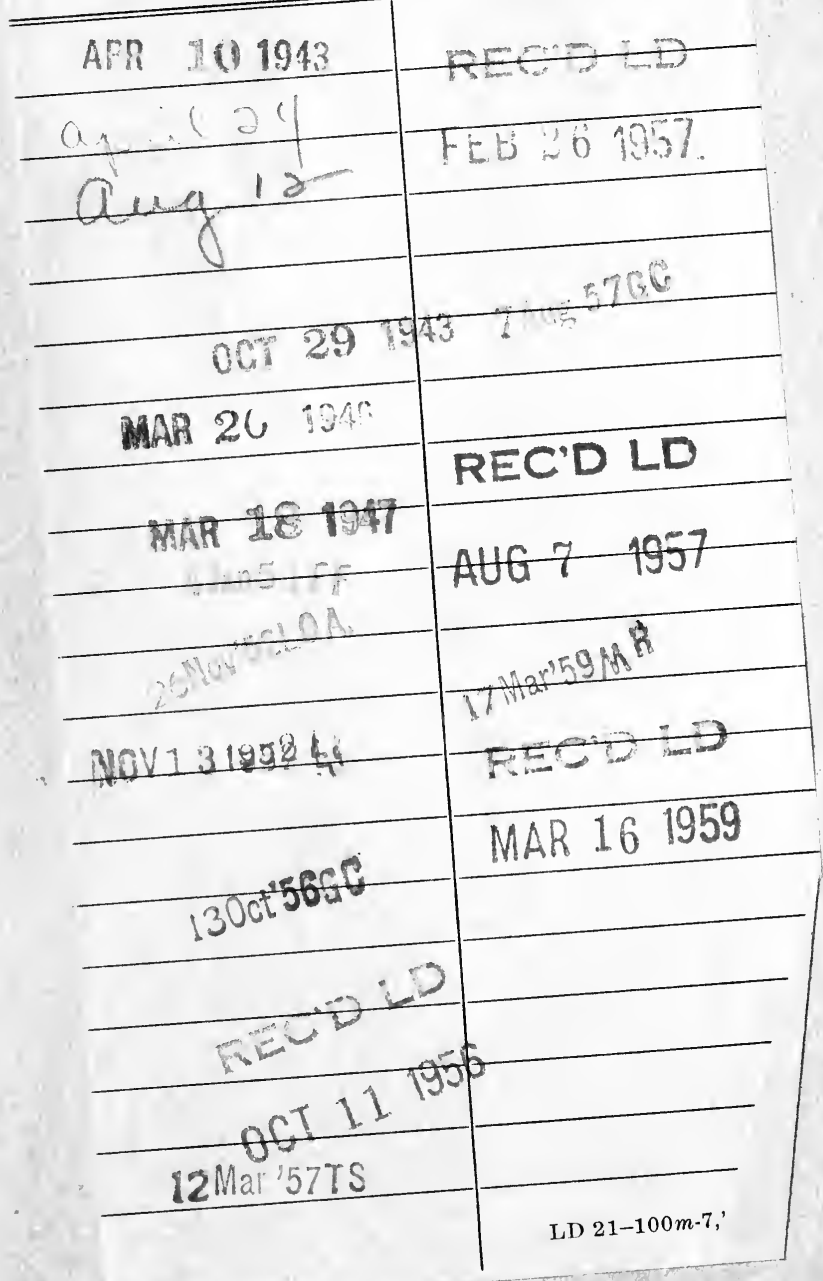




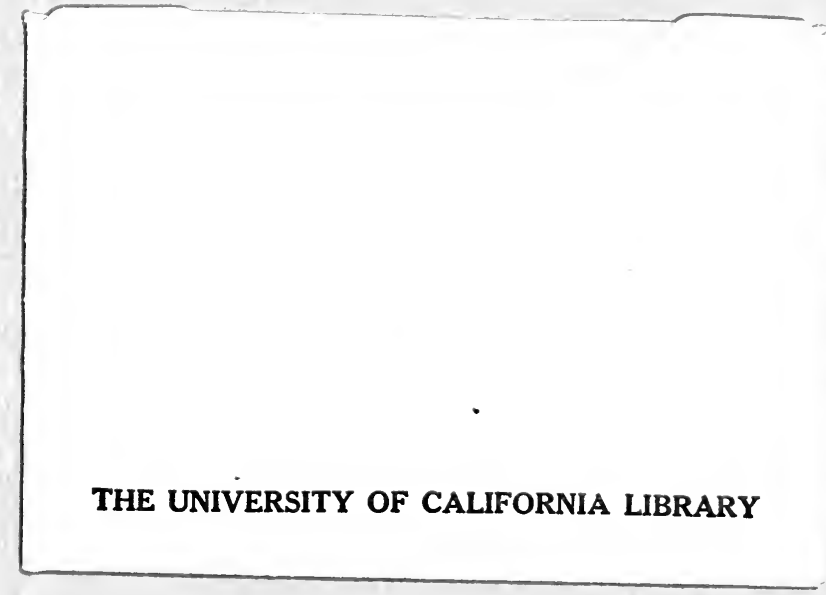




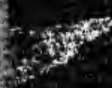

है

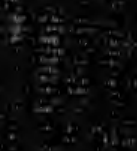

$+\frac{1}{3}$

$2^{-10}$

3

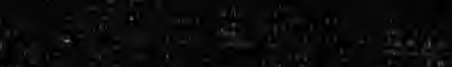

$x_{300}$

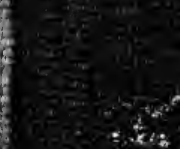

4.

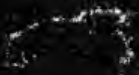

$$
\therefore+t^{4}
$$

tog

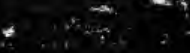

$-2+3$

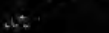

